
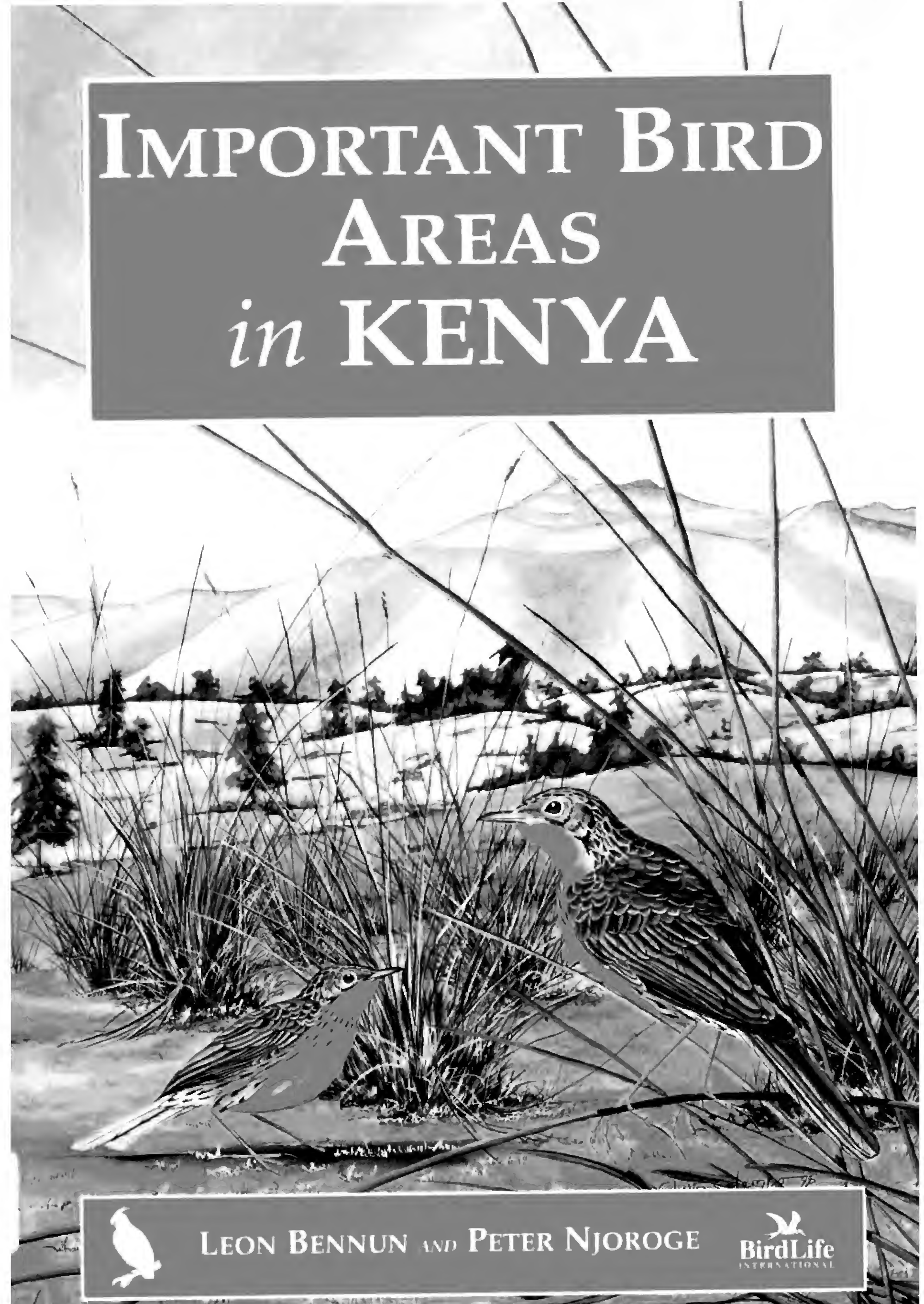


\section{NatureKenya}

Nature Kenya is the Kenyan section of Africa's oldest scientific society, the East Africa Natural History Society.

Since 1909 the EANHS has promoted the study and conservation of nature in eastern Africa. Nature Kenva's diverse membership brings together people who share an interest in the natural wotd, and are concened about its conservation. The society has a long tradition of paticipation and many members play an active robe in its programmes.

These include:

- Conservation of key sites, such as lmportant Bird Areas, through targeted conservation adveracy and action

- Education and raising public awareness, through public proprammes, fiudd outings and participatory activilies

- Publication of handbooks, guides, technical joumala and popular magarines

- Monitoring, research and training, throwgh joitat programmes with the National Museums of Kenya and other institukions.

Nature Kenya co-ordinates the Important Bird Areas programme in Kenva, which involves national and local conservation advocacy and action for Kenya's 60 IBAs.

As well as making a contribution to conservation, Nature Kenya members benefit from regular programmes and outings, a monthly newsletter and a thrice-yearly Bulfetin, free use of an outstanding natural history library and free entry to the National Muscums of Kenya, and reduced rates for books and periodicals.

\section{For more information, contact:}

Nature Kenva

The East Africa Natural History Society

P' O Box 4486, Nairobi, Kenya

Tel. $+25+2749957,746090$

$\mathrm{Fax}+25+27+1049$

E-mail: eanhsoatricaonline.co.ke

httpi//wwwmuseums.orke/eanhs 


\section{Important Bird Areas in Kenya}

\section{Leon Bennun and Peter Njoroge}




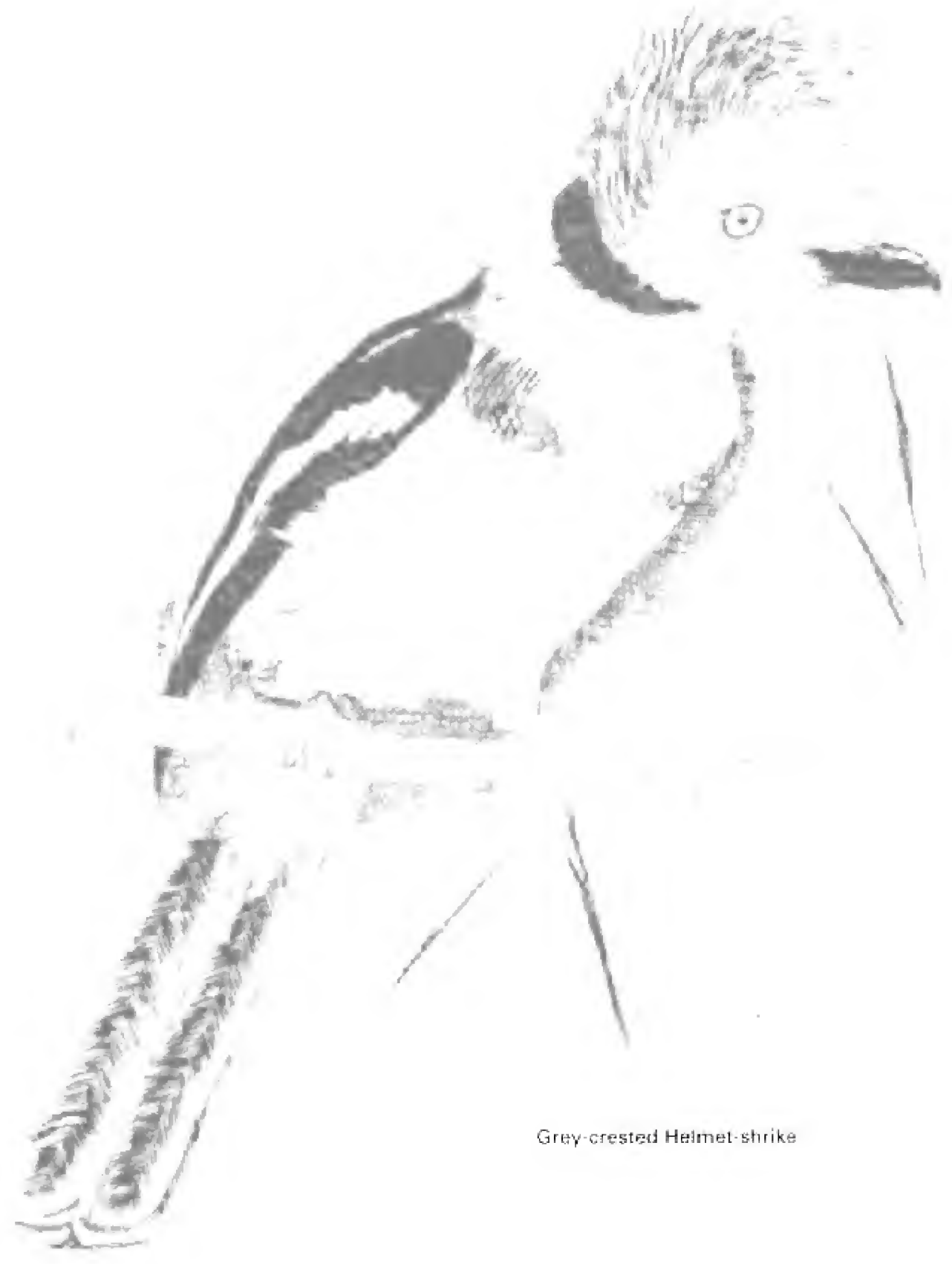




\title{
Important Bird Areas in Kenya
}

\section{Leon Bennun and Peter Njoroge}

\author{
Ornithology Department \\ National Museums of Kenya
}

A joint project of

Nature Kenyaz

(The East Africa Natural History Society)

The Kenyan Partner of BirdLife International

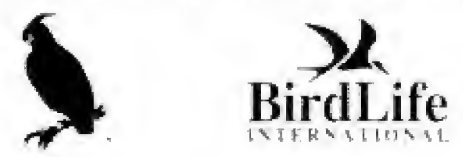

and the Ornithology Department, National Museums of Kenya
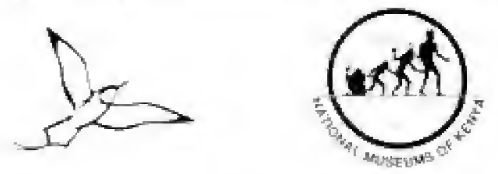

sponsored by the Royal Society for the Protection of Birds, the BirdLife International Partner in the United Kingdom

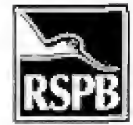

with additional support from the Global Environment Facility through the United Nations Development Programme project,

"African NGO-Government Partnerships for Ststainable Biodiversity Conservation'
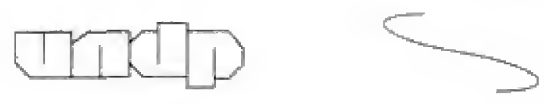
Published by

Nature Kenya

'The Fast Africa Natural History Society

PO Box 44486, Nairobi

Tel. $+25+2749957,746090$

Fix $+25+2741049$

E-mail eanhsoafricaonline.co.ke

(1) East Africa Natural History Society, 1999

ISBN $9966-9921-1-1$

All rights reserved. No patt of this publication may be reprofuced, stored in a retrieval system or transmitted in any form or by any means, electronic, mechanical, photocopying, recording or otherwise without the prior permission of the Fast Africa Natural History Society:

Design and layout by Robert \& Rhoda Burns/Drawing Attention

Printed by Polarprint

Line drawings by Edwin Selempo

and (on Pp. 88, 102, 113, 172, 177, 200, 233, 247) Brian Small

Cover illustration: Sharpe's Longclaw Mncmomar shaper

in the Kinangop Grasslands IBA, by Edwin Selempo. 


\section{Dedicated to}

the late Minnie Gatahi

who worked with unwavering commitment for conservation. 
Important Bird Areas in Kenya 


\section{CONTENTS}

1 Summary $\quad \begin{gathered}\text { Page } \\ 1\end{gathered}$

2 Acknowledgements

3 Introduction _ _ 11

3.1 Overview 10

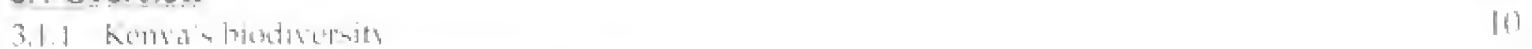

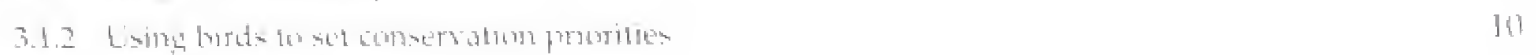

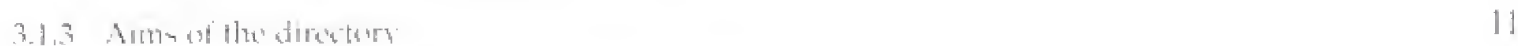

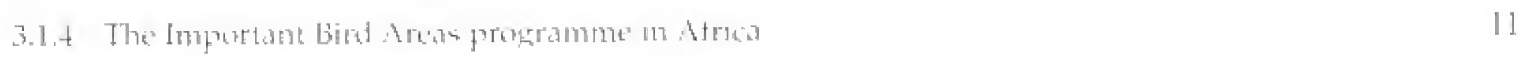

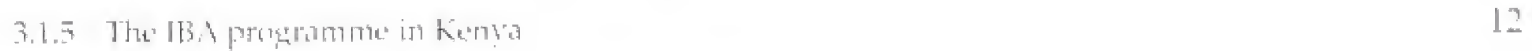

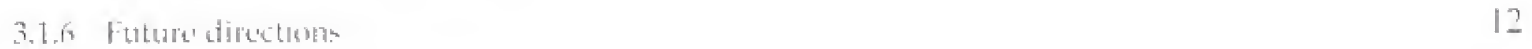

3.2 Kenya: General information 14

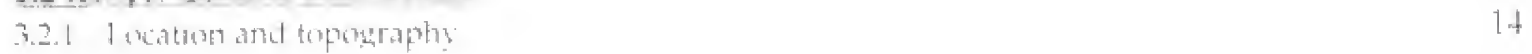

$\begin{array}{ll}3.2 .2 \text { Climate } & 167\end{array}$

$3.2,3$ Water fentireas

3.2.4 lugetation 17

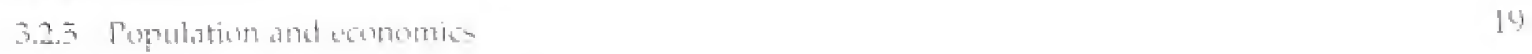

$\begin{array}{ll}3.3 \text { Important Bird Areas in Kenya } & 21\end{array}$

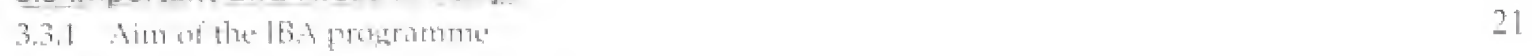

3.3 .2 that ate llih? 21

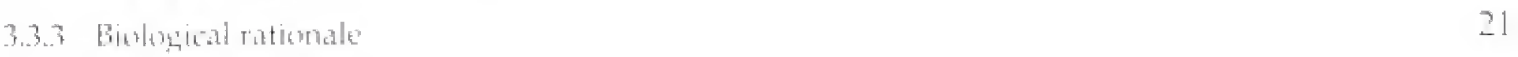

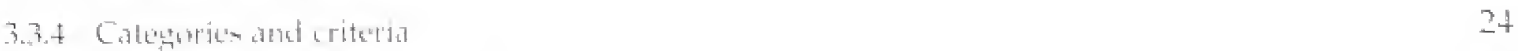

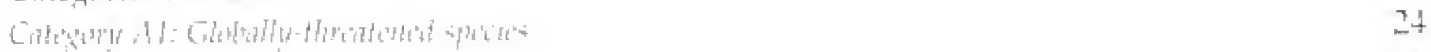

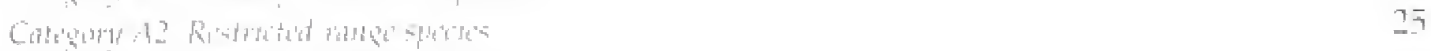

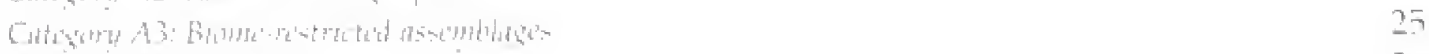

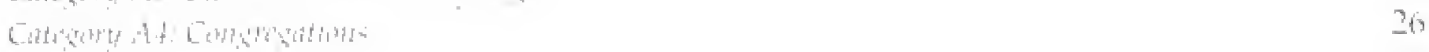

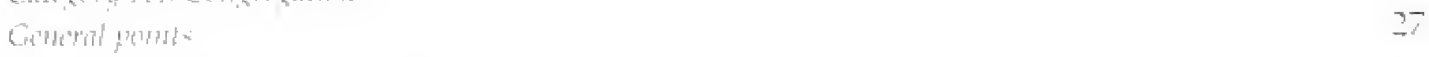

3.35 Kens a

3.3 .65 selectiobs at siten $\quad 32$

3.37 The [Hothetwith

sitclists

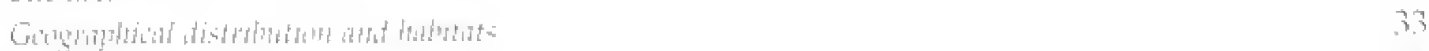

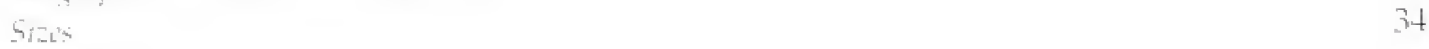

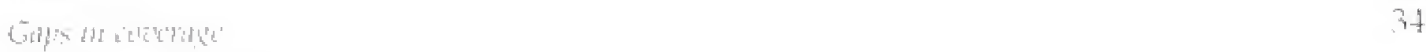

3.4 Conservation issues $\quad 37$

3.5 The institutional, legislative and policy framework for biodiversity conservation in Kenya 38

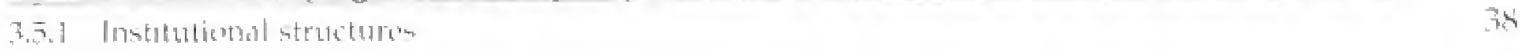

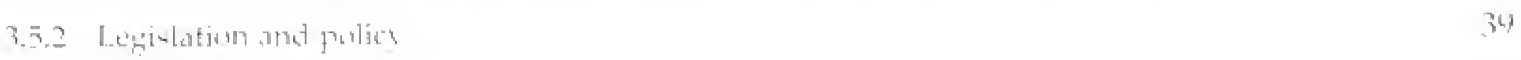

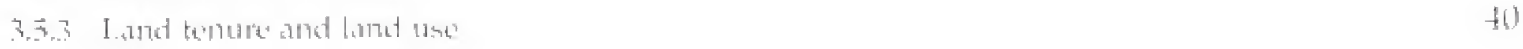




\section{Important Bird Areas in Kenya}

3.5 .4 Widalifumangernent 4

Binckitround

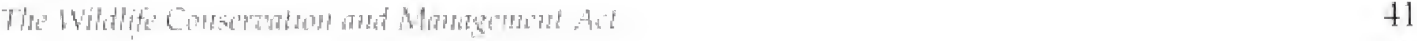

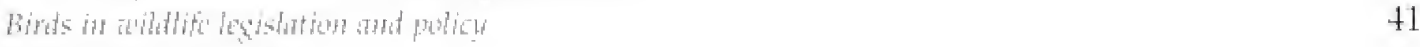

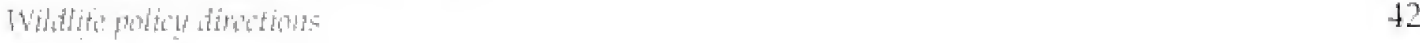

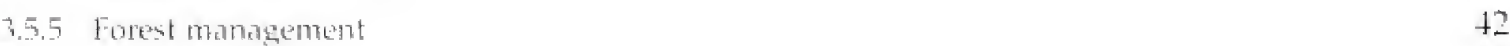

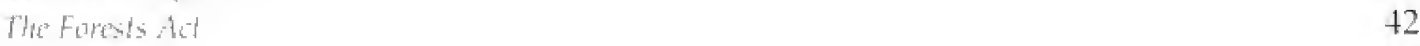

Forist Pellicy

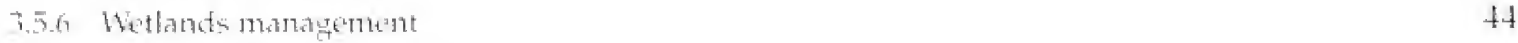

3.5.7 7ha: Protected Areas sistem

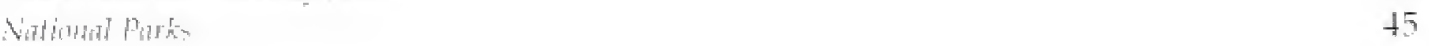

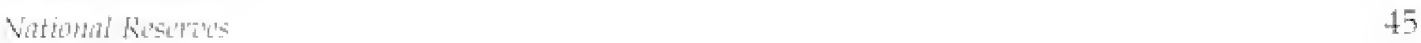

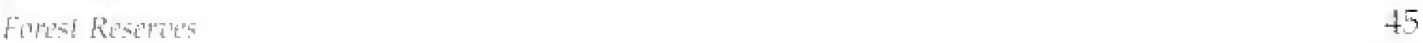

Virtiond Mominterts

3.5.8 International contentions and agreentents 46

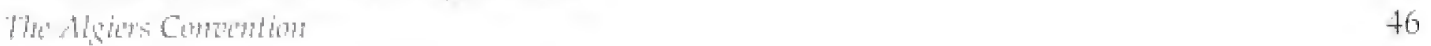

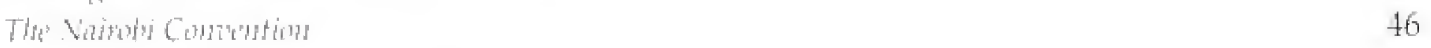

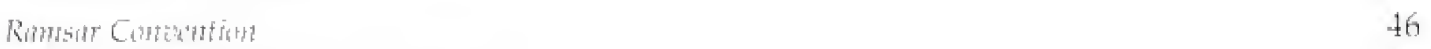

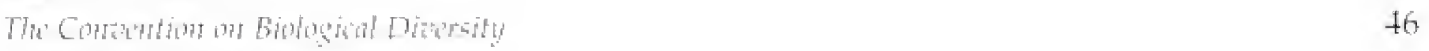

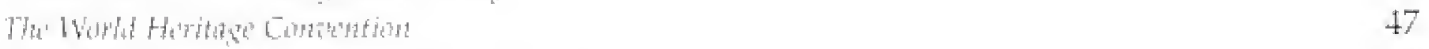

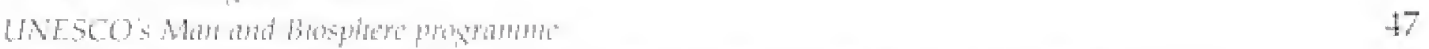

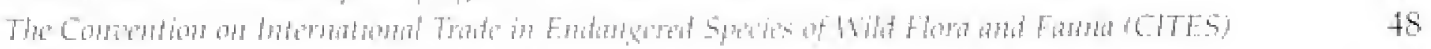

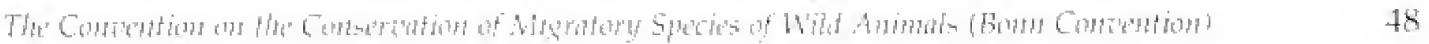

3.6 Priorities for action $\quad 49$

3.6.1 The appreact7 49

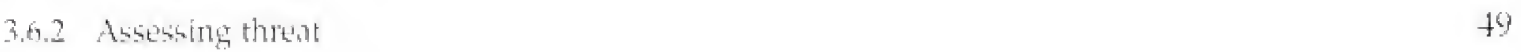

3.6.3 Biological importance $\quad 49$

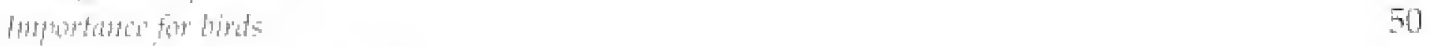

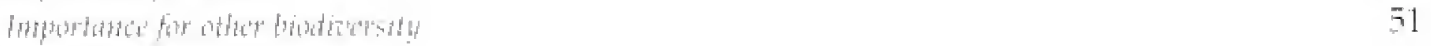

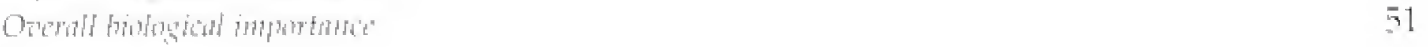

3.6.4 Pribrity sites

Cotequatiation $\quad 56$

Site promitisid as 'critcol' 56

Sites proritistet is' 'urgent' 5

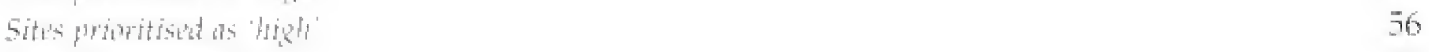

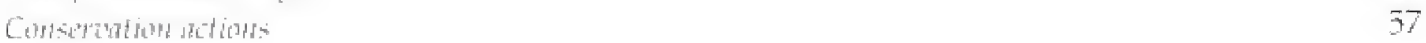

4 Site Accounts $\quad 59-252$

4. 1 Presentation of data $\quad 59$

4.2 Site accounts (in order of site code) 61

4.3 Potential IBAs in Kenya $\quad 249$

5 References 2 253-263

\section{ii Contents}




\section{Important Bird Areas in Kenya}

Appendix I

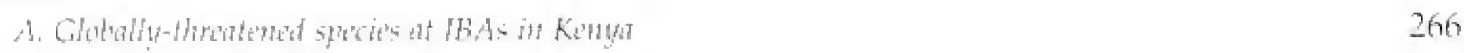

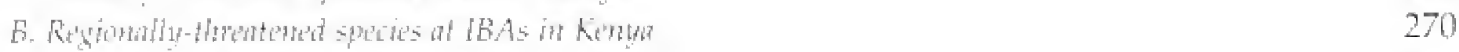

Appendix 2 Restricted-range species at sites in Kenzar 278

Appendix 3 Biome specias at sites in Kerwa

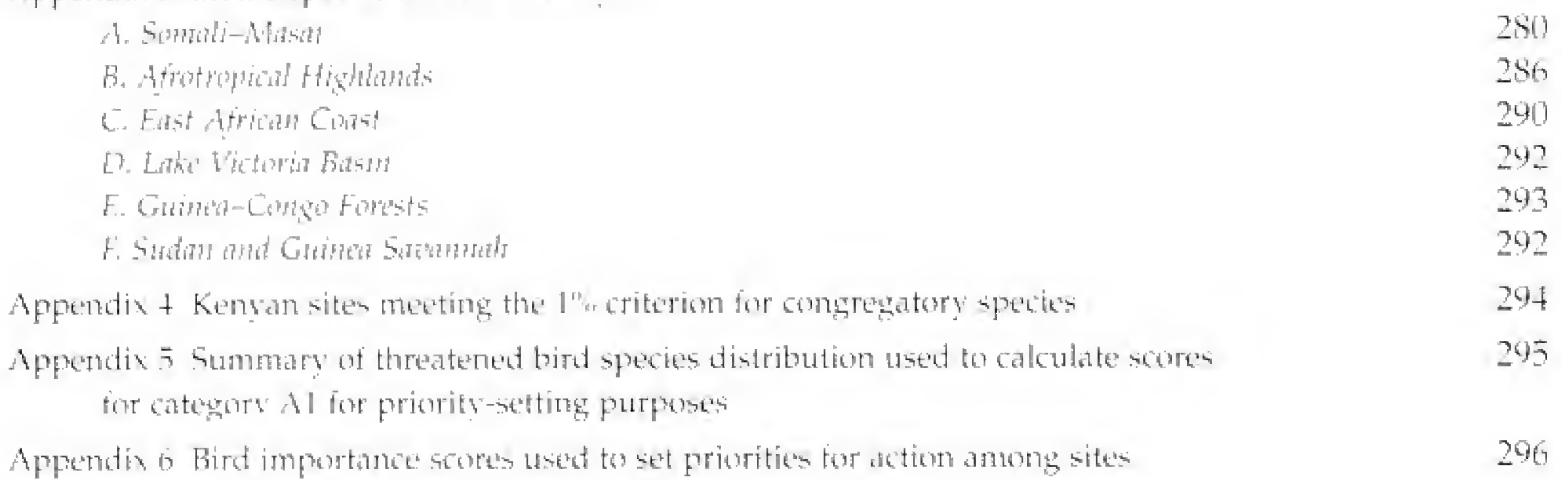

7 Indices 301

Index 1 Bird species in the site accounto and appendices, indexed by scientific name 301

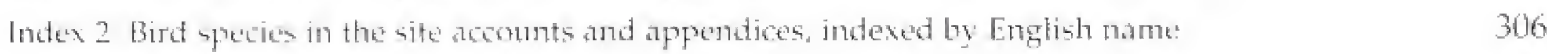

Index 3 Gther animats and plants Bl1

lndex + Site alcerouth

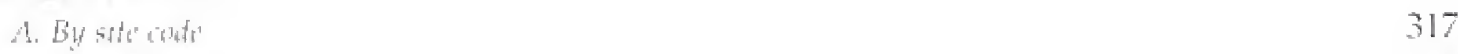

$\begin{array}{ll}\text { Pr. Bustomit } & 318\end{array}$

Tables, figures and maps

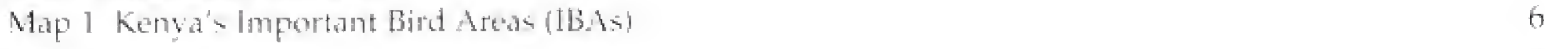

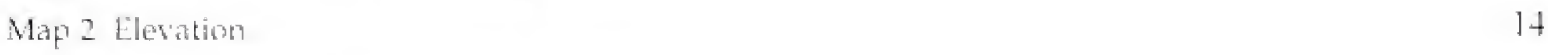

Map 3 antial ramfall 15

Map + Drainage basmis 18

$\begin{array}{ll}\text { Map } 5 \text { Main towns and roads } & 20\end{array}$

Figure 1 treas at Kenvas nolBAs 34

table 1 IBAs in kerya: kocation, categorita, area, profested status and nain habitats 2

Table 2 IBAs: Stmumary of ghobal atewories and criteria 22

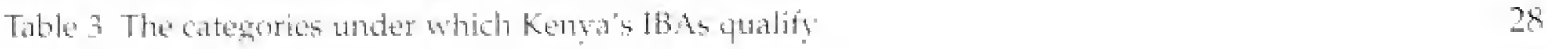

Tables + The number of IBats in tach category

Tables 5 The number of IBAs per protince 35

Table 6 Kenyats IBAs and their level of protection and theat, by dominant habitat 35

Table 7 Classes and sterity of ansoration theats to kingas IBAs 52

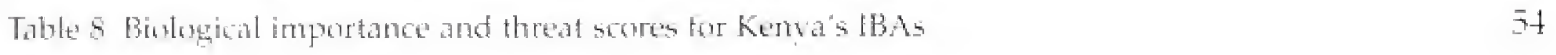


Important Bird Areas in Kenya 


\section{SUMMARY}

This directory is a contribution to identifying Kenya's biodiversity conservation priorities. It presents the results of a three-year study to select and compile information on Important

Bird Areas (IBAs) in Kenya. The Important Bird Areas process uses birds to locate key sites for conservation across the globe. Birds have many advantages as a group to use for biodiversity priority setting. They are widespread, diverse and easily surveyed. Above all. we know much more about them than about other organisms.

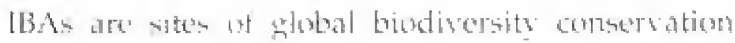
importance that are thosen using intermatienally

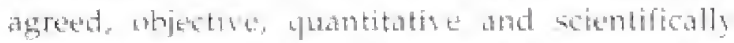

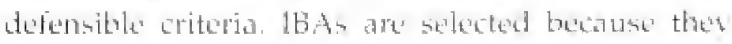
hold bird species that are threaterued with extenction, have highly resiricted distributions, or are characterister of particular biones. Sites holktime exeptionalls

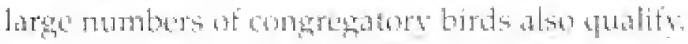

Sixty sites have been identified an IBAs in Kemya (lable 1, Natp I and section 4.2), Sufticient information is lacking for a further tive sibe, which are

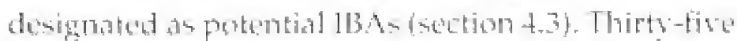
of the 60 IBAs are alreats withe the national protected arase satem; the remainder are unprotected. The 1 BA network coners all Kenya is major

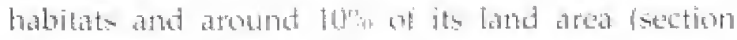

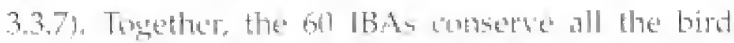

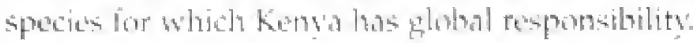

All IBAs are, by defintion, priorities tor coneratafion, but some next more urgent attention than others, A detailed amalyes of threats and biological jmportance was undertaken sithim the set of 60 .

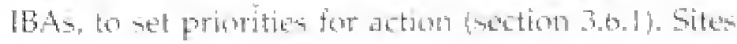
are classified into tritical, urgemt and high priority

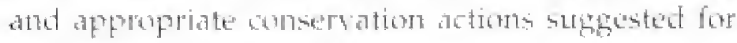

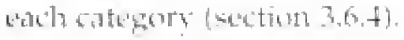

Detailed information en earth site focation, area, alkitude, status, descriftion, key bitd, other wildlife

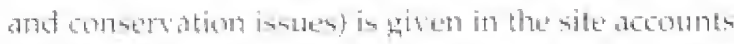
bhat Eorm the bulk of this lrowk (section $t$ ). Some implortant findinge inelude:

(1) The curremt protected area system has at leosis trio major gaps

- The urique highland grasslands on cither side at the contral Ritt balley, on the platean of

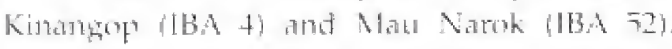
These are homen to the emdemic sharpers

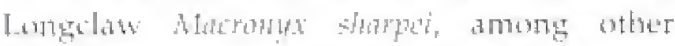
sperialised grassland species. The'se grasislands are hering theded and converted remarkably tast. and the grassiand baun and fora are under zery newere threat.

- The papyrus swamps atround Lake b'ichoria Dunga (IBA 37), Kogruta (IBA 38), Kusa (IBA 36),

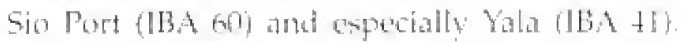
These contain a unique set of parpyrus endemic birds, including the ghobally threatemed Paps rus

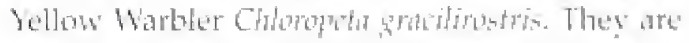
also at crucial ecological importance as water fil-

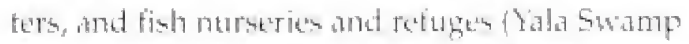

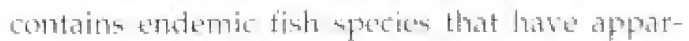
antly become: atinct in the main lake). With increasing fumen pressture an these swamps. they are being burand, harbested tmatstamably, and scared for cultivation. Despite pogr performance of the exinting "reclamation"

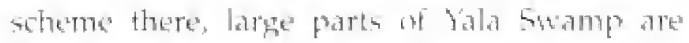
earmarked for draitnage ank consersion.

All these siles are heraly whed by local communitics fand in the case of the hightand grasstand, arte almost entitely prisate landl. Imaginative comberation strategies are reguined to protect these baluable habitats and their biodiversity

(2) One [BA, Dakatcha Woodland (IBA s), lies of presently unadiudionted land in Matineti Districk. Becanse of it biadisersity talue, it should be garested as a Forest Resorve ar other protected

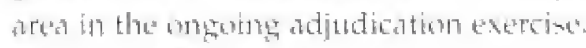

(3) Althrugh 9]"th wh the 22 forest [BAs ale protected areas, $77^{n}$ of of these sites are sewerely or critically threatened. There are severe general problems it forest management that must be tackled if bendisersity is not the loe lost.

Forest Ibds that ale classed as Critical for conservation action include:

- Montane forests on Mt Kenya (IBA 5), Mit Flgon (1BA 59) and the Aberdare Mountains (IB, I). These magnificent forests are impontant for theratened and biome-restrictest birds, als weth as 


\section{Table 1 \\ IBAs in Kenya: location, categories, area, protected status and main habitats}

\begin{tabular}{|c|c|c|c|c|c|}
\hline $\begin{array}{l}\text { Site } \\
\text { code }\end{array}$ & Site names & Province & District & Class & $\begin{array}{l}\text { IBA } \\
\text { Categories }\end{array}$ \\
\hline 1 & Aberdare Mountains & Central & Muranga, Nyandarua, Nyeri & $H F$ & TRB \\
\hline 2 & Kianyauga Valleys & Central & Kirinyaga & 0 & $\mathrm{TR}$ \\
\hline 3 & Kikuyu Escarpment Forest & Central & Kiambu & HF & TRB \\
\hline 4 & Kinamgop Grassland5 & Central/Rift Valley & Nyandarua/Nakura & $H G$ & TR \\
\hline 5 & Mtt Kenga & Central/Eastern & $\begin{array}{l}\text { Embu, Kirinyaga, } \\
\text { Nyeri, Laikipra/Meru, } \\
\text { Tharaka-Nithi, Nyambene }\end{array}$ & $\mathrm{HF}$ & TRB \\
\hline $\bar{b}$ & Mukurmeini Valleys & Central & Nyer1 & 0 & TR \\
\hline 7 & Arabuko-Sokoke Forest & Coast & Kilifi, Malindi & $\mathrm{CF}$ & TRB \\
\hline 8 & Dukutcha Wondland & Chbolst & Kilifi & $\mathrm{CF}$ & $\mathrm{TR}^{2}$ \\
\hline 9 & Dizni Forest & Coast & Kuale & $\mathrm{CF}$ & $T$ \\
\hline 10 & Dentmbo Hill Ferest & Conasl & Kuale & CF & $\mathrm{THR}$ \\
\hline 11 & $\begin{array}{l}\text { Gede Ruins } \\
\text { National Montument }\end{array}$ & Coast & Malinati & $\mathrm{CF}$ & $\mathrm{T}$ \\
\hline 12 & Kaya Gandinj & Coant & Kwale & $\mathrm{CF}$ & $\operatorname{TR}$ \\
\hline 13 & Kaya Waa & Coast & Kwale & $\mathrm{CF}$ & $\mathrm{T}$ \\
\hline 14 & Kisite lstand & Coast & Kivate & $w$ & $c$ \\
\hline 15 & $\begin{array}{l}\text { Kiungt Marine } \\
\text { Siational Regerte }\end{array}$ & Coast & Lamu & W & c \\
\hline 16 & $\begin{array}{l}\text { Mida Creck, Whale lstand } \\
\text { \& Malindi/Watarut Coast }\end{array}$ & Coast & Malindi & m & c \\
\hline 17 & Marenji Forest & Coast & Kwale & $\mathrm{CF}$ & TRB \\
\hline 18 & Mrma Hill Forest & Conast & Kwale: & CF & $\mathrm{TB}$ \\
\hline 19 & Sabaki River Murth & Coast & Matindi & W & $c$ \\
\hline 20 & Shimba Hills: & Corast & Kurate & $\mathrm{CF}$ & TRB \\
\hline 21 & Taita Hills Forests & Coast & Taita-Taveta & EAF & TRB \\
\hline 22 & Tana Ruer Delta & Conat & Tataia River & W & TBC \\
\hline 23 & Tanba River forests & Coast & Tana River & $\mathrm{CF}$ & TRB \\
\hline 24 & Tsand Fast Natiengal Park & Coast/Eastern & Tana River, Taita-Tárets/Kituj & 5 & TB \\
\hline 25 & Tsavt) West National Park & Coast & Taita-Taveta & 5 & $T^{2} B$ \\
\hline 26 & Chyculu Hills Forests & Fustern/Rith Valley & Makuteni/kajiato & $\mathrm{HF}$ & $\Gamma R$ \\
\hline 27 & Dida Gualgalu Desert & Eastern & Marsabit & $s$ & TRB \\
\hline 28 & Lake Turkana & Eastern/Rift Valley & Marnabil Turkana & n & $\mathrm{BC}$ \\
\hline 29) & Machakos Valleys & Eastern & Machakos & S & TR \\
\hline 30 & Masinga Reservoir & Easternt & Embu & W & C \\
\hline 31 & Meru National Park & Eastern & Meru, Tharaka-Nithi & 5 & $\mathrm{~B}$ \\
\hline
\end{tabular}


Codes

Class:

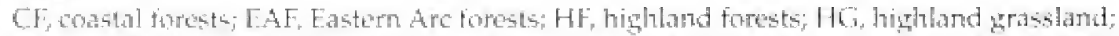

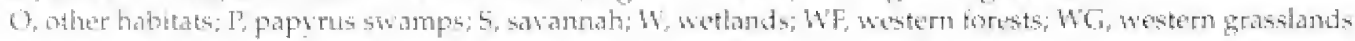

Category:

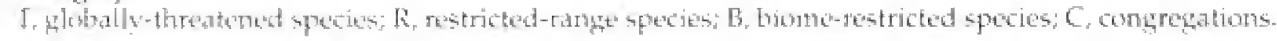

PA protecled-area itatus:

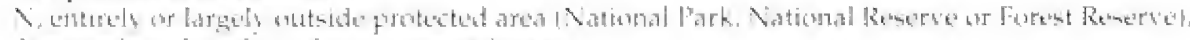

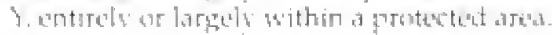

"area appromante

\begin{tabular}{|c|c|c|c|c|}
\hline $\begin{array}{l}\text { Area } \\
\text { that }\end{array}$ & $\mathrm{PA}$ & Main habitats & $\begin{array}{l}\text { Site } \\
\text { code }\end{array}$ & Site names \\
\hline 174,900 & Y & Montane forest, bambon, montane grassland and moorland & 1 & Aberdare Mountains \\
\hline$\cdot 12,0001$ & $x$ & Stcep thier watleys, mon manly culturated & 2 & Kianyaga Valleys \\
\hline 37,600 & Y & Siontant forest & 3 & Kikuyu Escarpment Forest \\
\hline 7.000 & $x$ & Montante frasstand, farmland & 4 & Kingmgong (Grask arots \\
\hline 271,000 & Y & Montane furest, bambou, montane grastand and morland & 5 & Mit Kenya \\
\hline 20,006 & $\therefore$ & 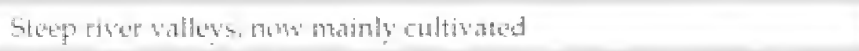 & in & Mukurwein Valleys \\
\hline 41,600 & $y$ & Cirastal evergrem forest and womdland & 7 & Arabuko-Stkoke Forent \\
\hline 32.000 & 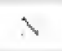 & Cimastat werod land & $s$ & Dakatcha bouthand \\
\hline 80 & $\Sigma$ & Deciduens coral-rag forest & 9 & Diani Frnest \\
\hline 20 & 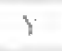 & Linditherentratudet chastal mived torent & 10 & Drimbet Hill Furest \\
\hline+4 & Y & Constal semi-ndecidurus forest & 11 & $\begin{array}{l}\text { Getde Ruins } \\
\text { Vational Montument }\end{array}$ \\
\hline 150 & Y & 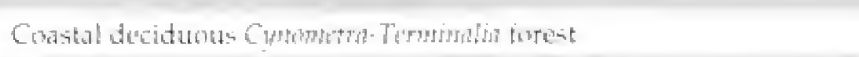 & 12 & Kaya Gurdini \\
\hline 20 & Y & Low coantal Cynommetra-Drupetes forest on conal tag & 13 & Kaya Waa \\
\hline I & i & Coral ruck lais serub & 1.4 & Kiste lifland \\
\hline 25,0000 & Y & Mangrodes, coral islets and platforms, sandy beaches & 15 & $\begin{array}{l}\text { Kiunga Marine } \\
\text { National Reserve }\end{array}$ \\
\hline 26,100 & Y & 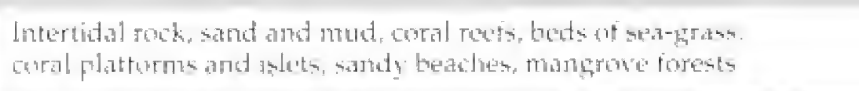 & Lh & 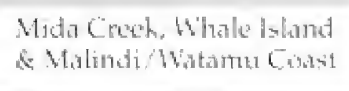 \\
\hline 1.520 & Y & Lindifferentiated cosstal mixed forest & 17 & Marenii Forest \\
\hline 250 & $y$ & Unditierentiated constal noived fortent & is & Mrsma Hill Forest \\
\hline+200 & $x$ & Estuarine samdtanks, mucthanks, dunes, freshwater pools & 19 & Subaki Riter Mouth \\
\hline 21,740 & i & 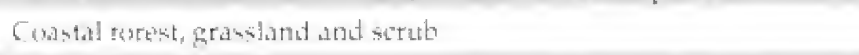 & 31) & Shimbattills \\
\hline 400 & Y & Moist merntane forest & 21 & Taita Hills Forests \\
\hline 130,000 & $>$ & 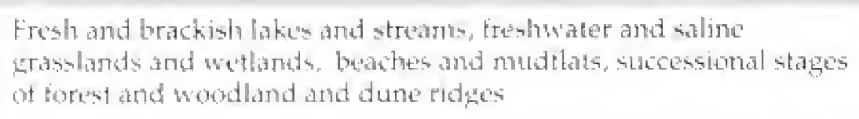 & 22 & Tanat Ribut Dellta \\
\hline$-3,700$ & Y & 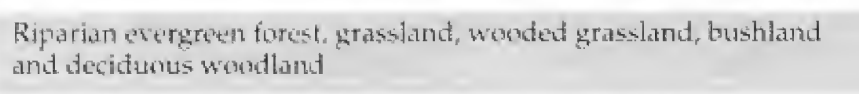 & 23 & Tana River Forests \\
\hline $1,175,000$ & Y & 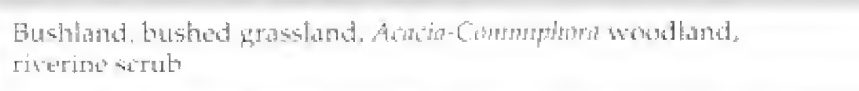 & 24 & Tsarvo East Naticonal Fark \\
\hline 906,700 & Y & Acacin-Commpphra bushland, open grassy plains, riverine strub & 25 & Tşво West National Park \\
\hline 18,07070 & y & Shmtane forest, rough grassland and thicket & 26 & Chyzulu Hills Futest: \\
\hline undefined & $\mathrm{N}$ & Open black-lav'a stesert & 27 & Dida Galgalu Desert \\
\hline 756,000 & $x$ & 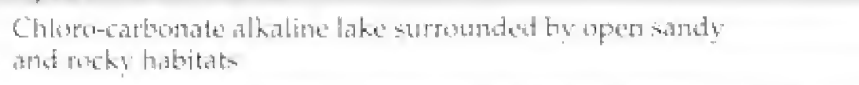 & 28 & Lake Turkana \\
\hline$=5,000$ & $\mathrm{~N}$ & Riwerine bush and thicket, Acacia nantrophtoper woodland & 24 & Machakos Valleys \\
\hline$* 16,000$ & $X$ & 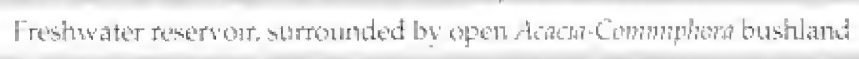 & 30 & Masinga Reservolir \\
\hline 87,000 & $Y$ & Wooded grassland, Awora-Commizhrora busthland & 31 & Meru National Park \\
\hline
\end{tabular}


Important Bird Areas in Kenya

\begin{tabular}{|c|c|c|c|c|c|}
\hline $\begin{array}{c}\text { Site } \\
\text { code }\end{array}$ & Site names & Province & District & Class & $\begin{array}{c}\text { IBA } \\
\text { Categories }\end{array}$ \\
\hline 32 & Mwen National Reserve & Eastern & Embu & 5 & TR \\
\hline 31 & $\begin{array}{l}\text { Sambura/Buffatas Sytrings } \\
\text { National Restres }\end{array}$ & Fasteren i Ritt Valles & Inolv/gimburu & 5 & B \\
\hline 34 & Shaba Natitstal Reserve & Eastern & Isioks & $s$ & TRB \\
\hline 35 & Dandegra Ponds & Vairobi & Viriraliti & $w$ & c \\
\hline 36 & Vairiolui National P'ark & Natrobi & Nairobi & HG & TRC \\
\hline 37 & Dunga Swamp & Nyanzon & kisuthu & [י & $T B$ \\
\hline 38 & Kogguta Swanp? & Nyanza & Kisumu & r & $T B$ \\
\hline 39 & Kusaswamp & Nyanzs & Kisแกาเs & $\mathrm{p}$ & TE \\
\hline 40 & Røm. Natiorsal Park & Nyanza & Suba & WG & T \\
\hline 41 & Yala Swamp & Mianza/Westert & Gaya thesiz & $l^{\prime}$ & $\mathrm{TB}$ \\
\hline 42 & Amboseti Natimal Park & Rift Valley & Kajiato & s & Б5 \\
\hline 45 & Cherangani Hills & Rift Vallew & Elgeyon Hatakwet, West Pokor & HF & F \\
\hline$H$ & Lake Baringo & Rist Valley & Baringu & 5 & $\mathrm{~b}$ \\
\hline .5 & $\begin{array}{l}\text { Lake bargaria Sational } \\
\text { Reserese }\end{array}$ & Rifi Vailley & Kuithate-h, Baringen & w & TC \\
\hline to & Lake Elmenteita & Rift Valley & Nakutru & w & TRC \\
\hline 47 & L.ake Magati & Ritt balley & Kinjasto & w & c \\
\hline 48 & Lahe Nain astat & Ritt Vallay & Nakuru & $\mathrm{H}$ & TRC \\
\hline 49 & $\begin{array}{l}\text { Lake Nakuru National } \\
\text { Park }\end{array}$ & Ritt valky & Nakuru & it & TRC \\
\hline 50 & Masai Mara & $\begin{array}{l}\text { Rift Valley } \\
\text { Rift Valley }\end{array}$ & Narok, Trans-Mara & $H G_{i}$ & TK \\
\hline 51 & What Eureat Comples & & Nakuru, Kerielw, Narok, Bomel & $\mathrm{HF}$ & F \\
\hline 52 & $\begin{array}{l}\text { Mau Narok/Nolo } \\
\text { Grasslands }\end{array}$ & Rift Valley & Nakuru, Narok & $\mathrm{HG}$ & $\mathrm{TR}$ \\
\hline 53 & Nertla Naradi Furest & Rit: Walley & Nanuli & $W E$ & TRES \\
\hline 54 & Ol Donyo Satache & & Simbruru & 5 & $\mathrm{I}$ \\
\hline 55 & South Nandi Fortst & Rift Valley & Nandi & WF & $T 13$ \\
\hline 56 & South Ngurtman & $\begin{array}{l}\text { Rift Valley } \\
\text { Rift Valley }\end{array}$ & Narok & $s$ & TR \\
\hline 57 & Busia Gratshandis & Whatern & Fusisit & $1 \mathrm{Ve}$ & {$[B$} \\
\hline 58 & Katkamega Forest & Western & Kakanuega & WF & TRßB \\
\hline 39 & Mtt Elgon & & Wle Elgon/Irans-Nawa & $\mathrm{HF}$ & TRB \\
\hline 60 & Sio lort Swanip & Western & Busia & r & $\mathrm{TB}$ \\
\hline
\end{tabular}


Class:

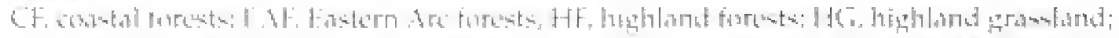

(1) 4.

Eategary:

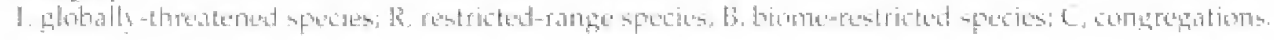

P'A protected area status:

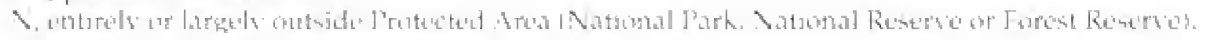

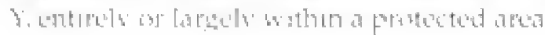

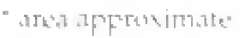

\begin{tabular}{|c|c|c|c|c|}
\hline $\begin{array}{l}\text { Areat } \\
\text { that }\end{array}$ & I'A & Main hathitats & $\begin{array}{l}\text { Site } \\
\text { code }\end{array}$ & Site names \\
\hline,+ 200 & $Y$ & Bushland and woudland with open phates & 32 & 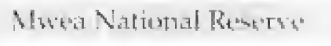 \\
\hline 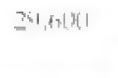 & i & 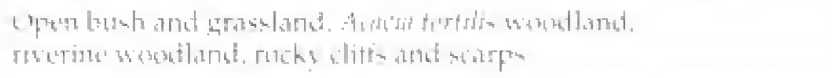 & 33 & 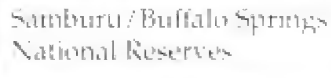 \\
\hline 23,400 & i & 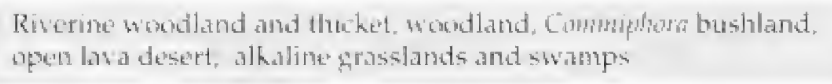 & 34 & Statha Nitianid Reserve \\
\hline 3ilu & $\checkmark$ & 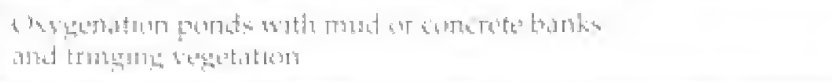 & $\sqrt{3}$ & Quardora lionds \\
\hline $11,7 \mathrm{tal}$ & $Y$ & 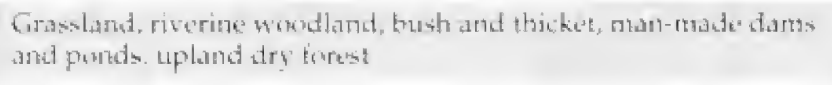 & 36 & Nairnbi Nattinnal Purk \\
\hline "페에 & $\lambda$ & 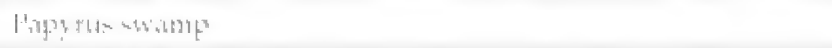 & $3 \bar{t}$ & 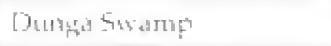 \\
\hline 1,5003 & $\gamma$ & Papyrus swanep & 38 & Kunguta Swamp \\
\hline$* 1 .(2) x+1$ & $\lambda$ & 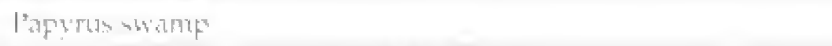 & 34 & kuns sh แтир \\
\hline $12,(140)$ & 1 & Giramshard, open woodland and thickets & $4(1)$ & Ruma National Park. \\
\hline Stlitilit & $\checkmark$ & 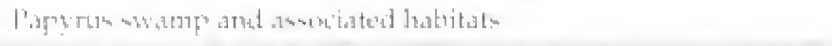 & 41 & ท人 \\
\hline $34,2[y]$ & Y & 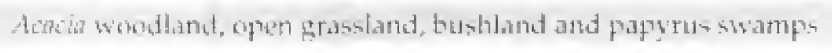 & 42 & Ambuacli National Park \\
\hline $4 \overline{7}, \dot{1} 1)$ & $\mathrm{Y}$ & 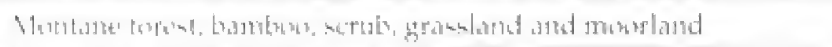 & 4.7 & Chu'Tumbani Hills \\
\hline 28,4130 & 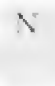 & 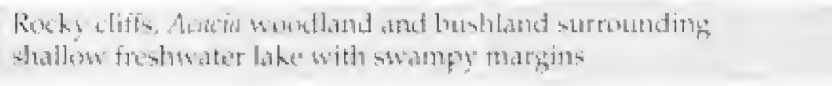 & $4+$ & Lathe baringo \\
\hline $10,-200$ & ) & 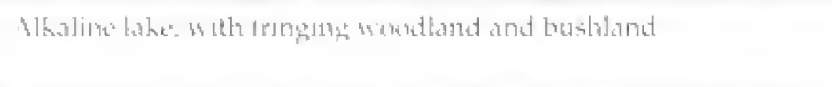 & 45 & 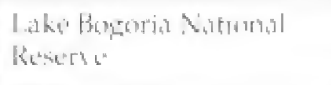 \\
\hline 6,300 & 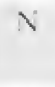 & 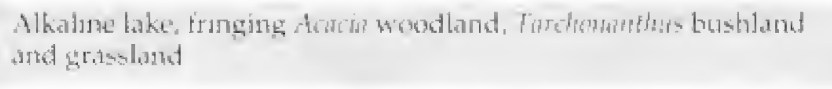 & 46 & l,ake [lmontettit \\
\hline 161,5131 & $\backslash$ & 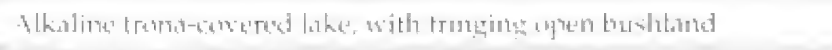 & 47 & Lathe bagati \\
\hline $23,(79,10)$ & $\gamma$ & 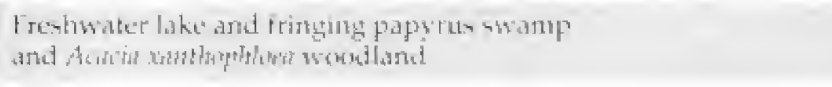 & 48 & Laku Vuivathe] \\
\hline 158010 & $i$ & 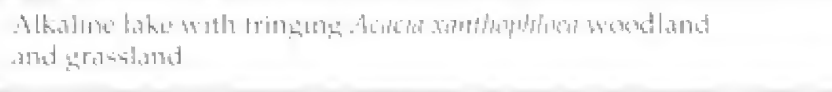 & 44 & 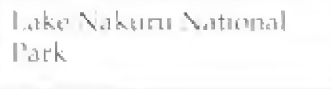 \\
\hline $76(6-7,0)(1)$ & r & 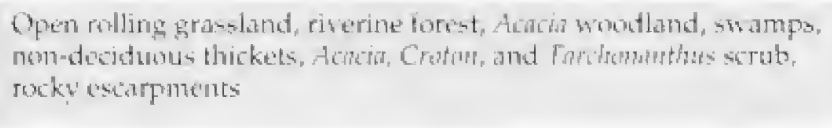 & $\overline{7}(1)$ & Matadi Mark \\
\hline $273,3(1)$ & $Y$ & 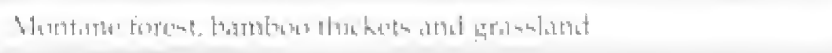 & 31 & Shat fing complex \\
\hline 40,0 , & 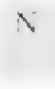 & Montane gramblated, farml.ind & 52 & $\begin{array}{l}\text { Mau Varok/Malo } \\
\text { Gicksalands }\end{array}$ \\
\hline $111,7[4]$ & i & 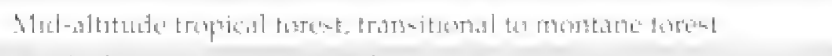 & .79 & Narth Sand lingent \\
\hline 1,600 & $\wedge$ & 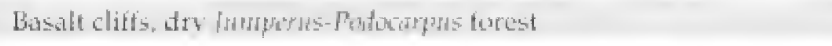 & 5.1 & 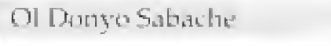 \\
\hline 15,1401 & $y$ & 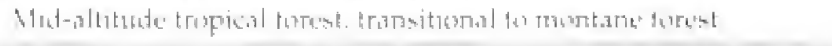 & $5 \overline{5}$ & South Nindi Fisust \\
\hline$=5(1,0) 311$ & $\checkmark$ & 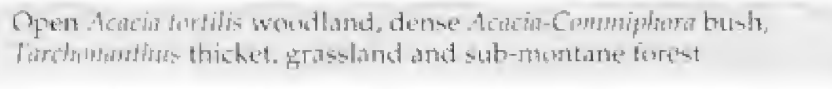 & 56 & Gruth Vixturathan \\
\hline $2 \geq 11$ & 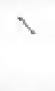 & 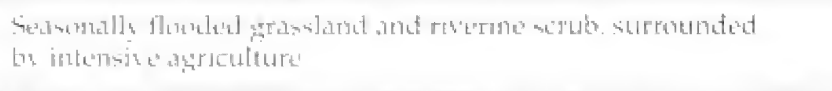 & 3) & 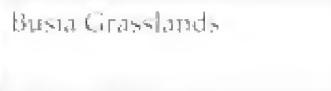 \\
\hline 16,300 & Y & Mat-altitude tropical risingrest. grassy and breshed gilades & 54 & Kakameg, Fortset \\
\hline 447,540 & $Y$ & 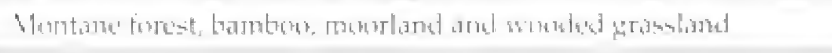 & के & 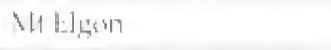 \\
\hline 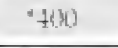 & $X$ & l'apyras swamp & (ख) & Sin Port Swarmp \\
\hline
\end{tabular}


Important Bird Areas in Kenya

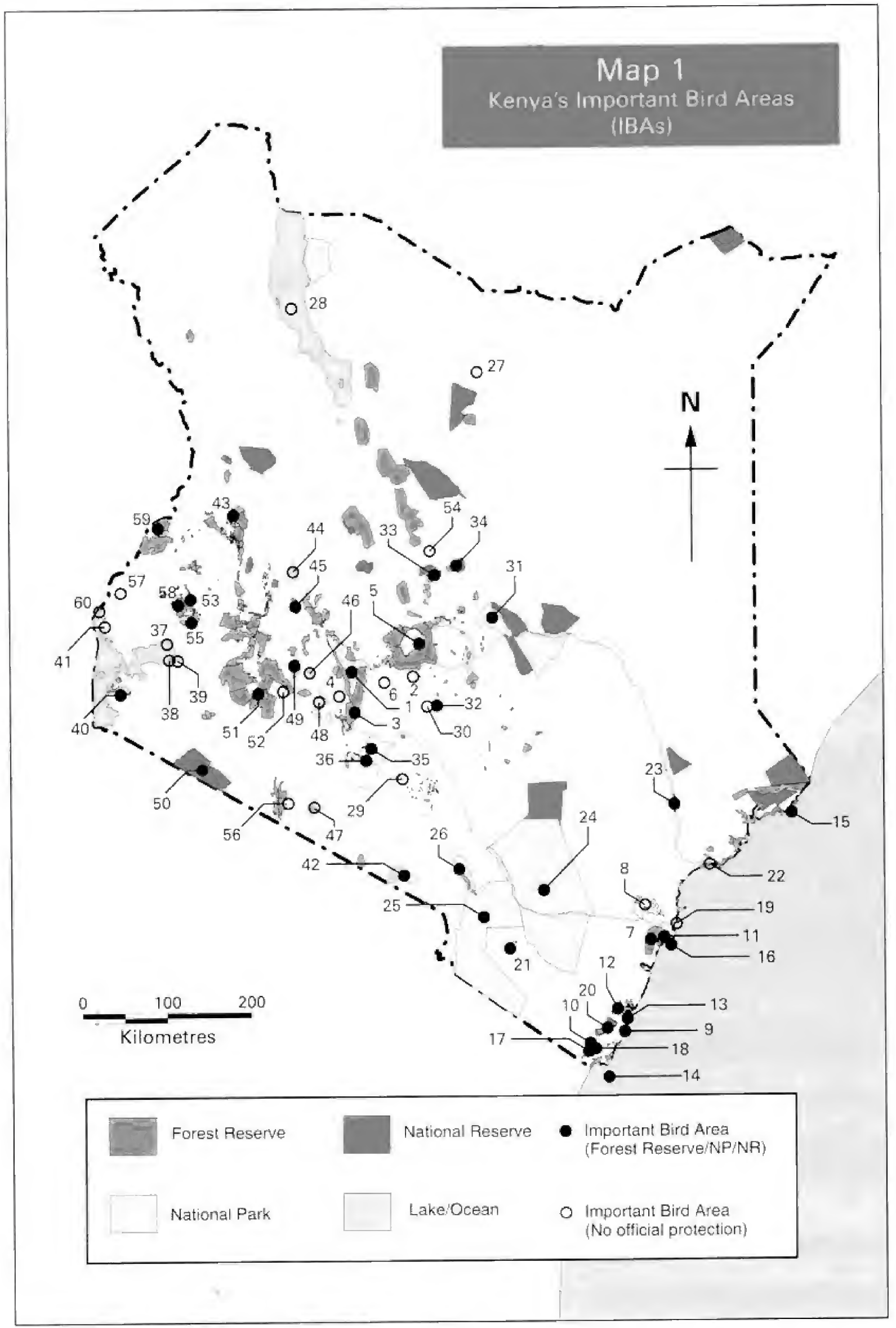

\section{Summary \\ Kenya's IBAs}




\section{Summary}

Key to IBA sites indicated on the map on the opposite page

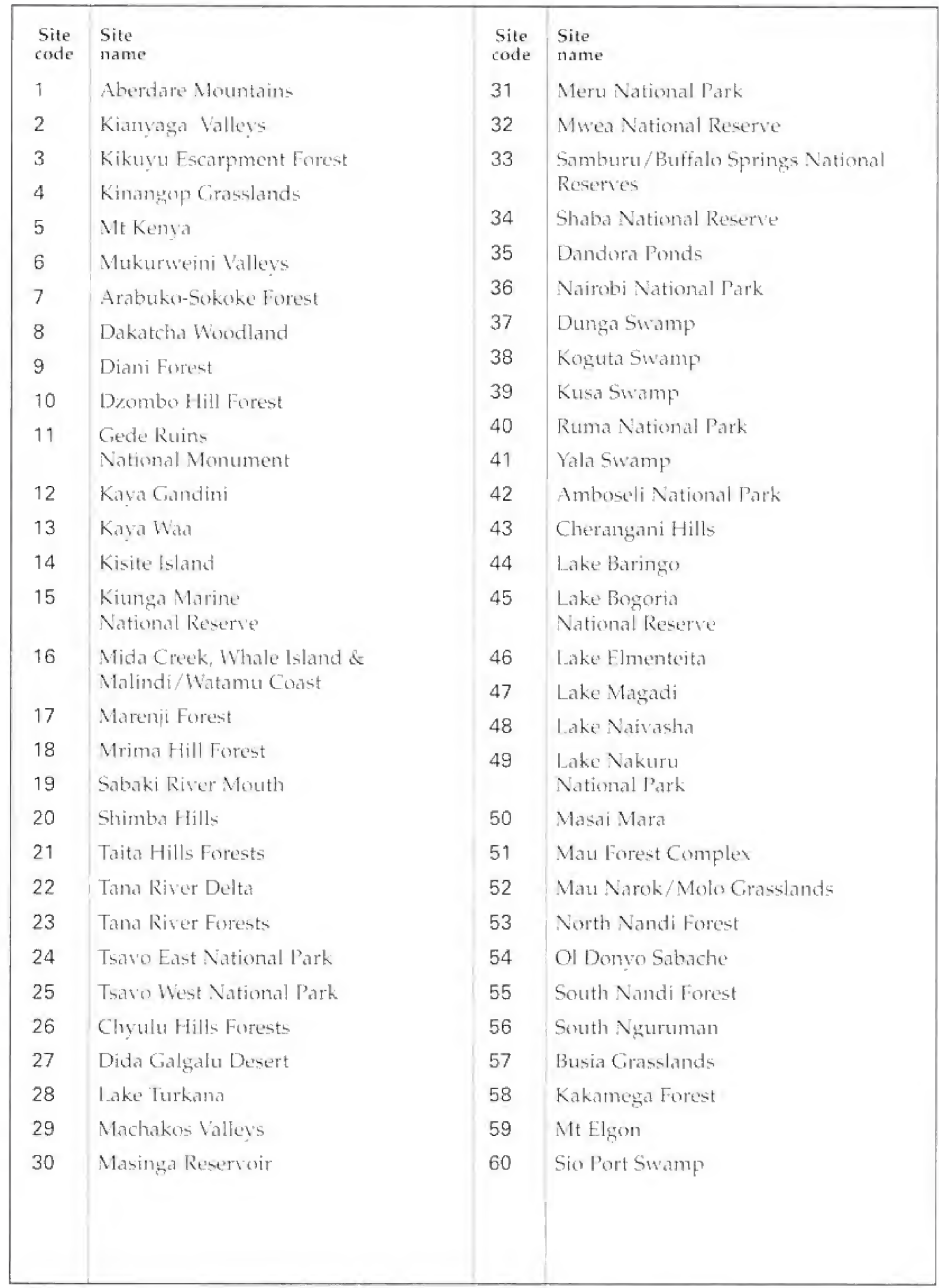




\section{Important Bird Areas in Kenva}

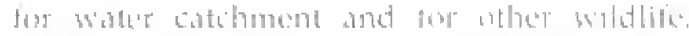

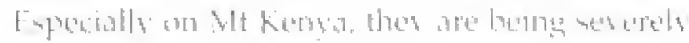

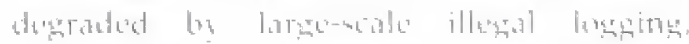

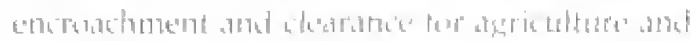

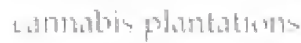

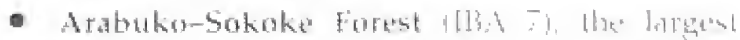

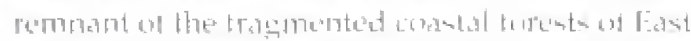

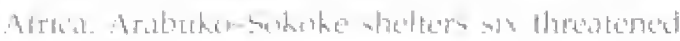

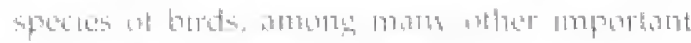

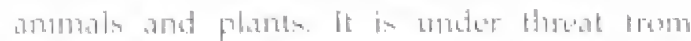

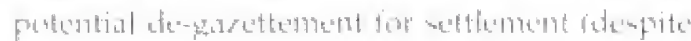

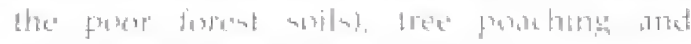

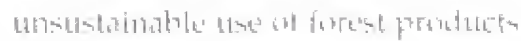

- Tlu bing and dragnented forests of the Taiba

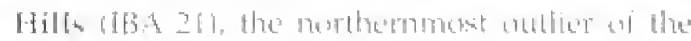

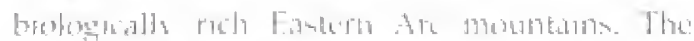

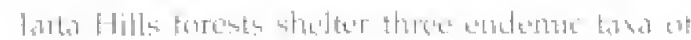

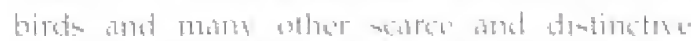

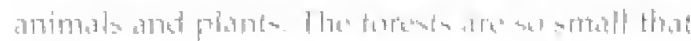

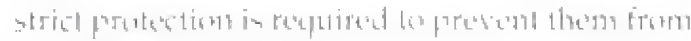

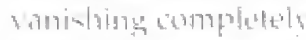

- The wnall riparian forests along the lower Tana

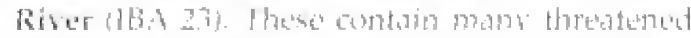

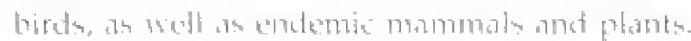

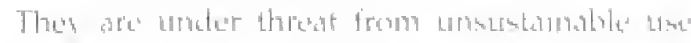

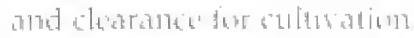

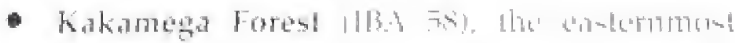

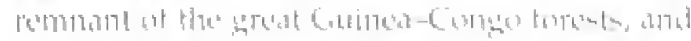

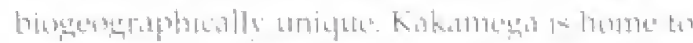

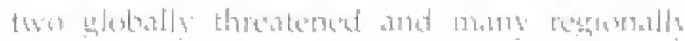

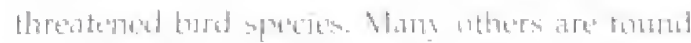

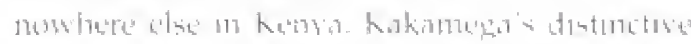

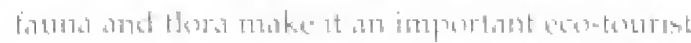

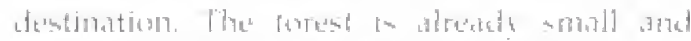

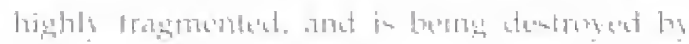

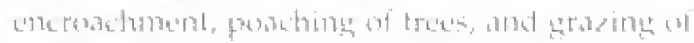

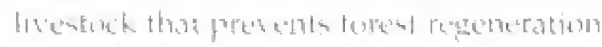

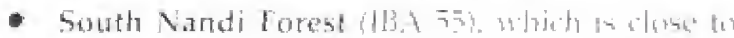

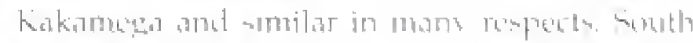

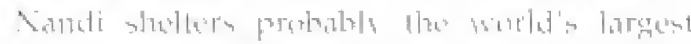

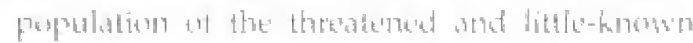

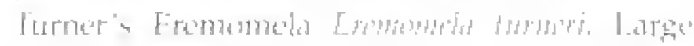

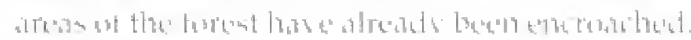

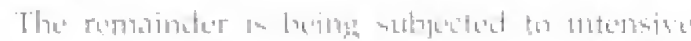

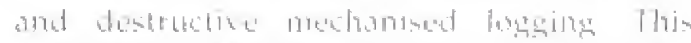

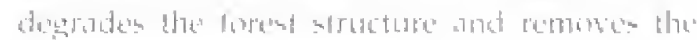

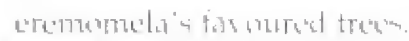

- Foresi remmants on the south Kenya coast. Diani

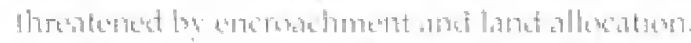

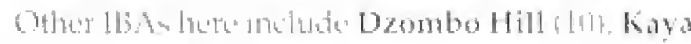

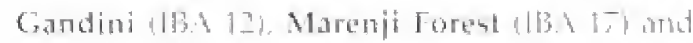

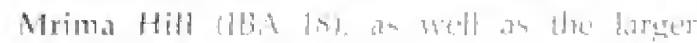

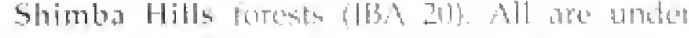

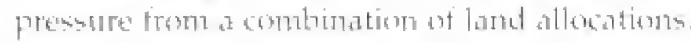

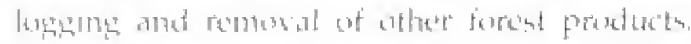

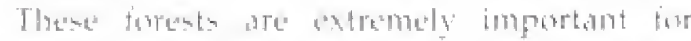

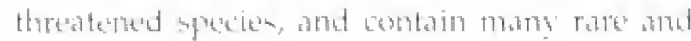

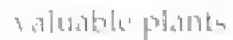

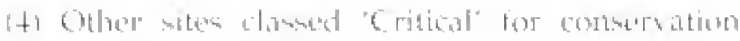
actimu meluste:

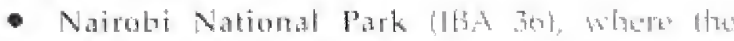

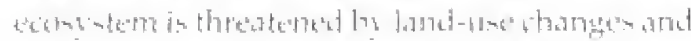

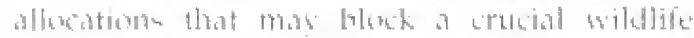

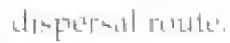

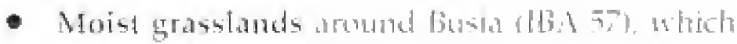

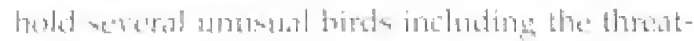

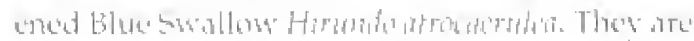

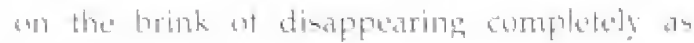

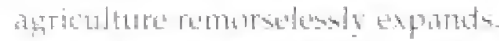

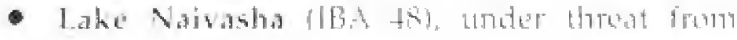

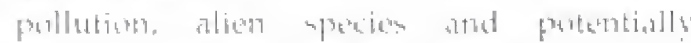

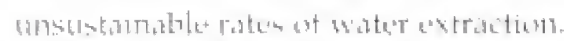

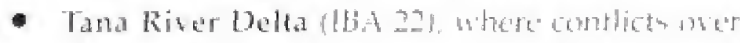

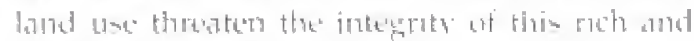

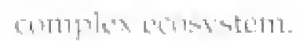

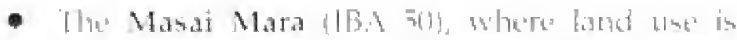

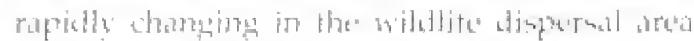

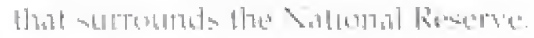

- River valleya arannd Kiambaya (IB) 2) and

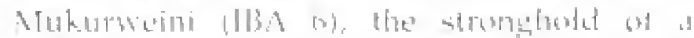

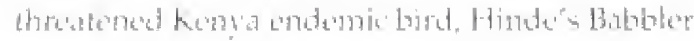

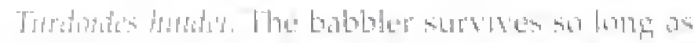

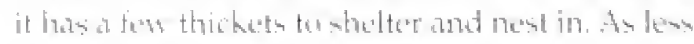

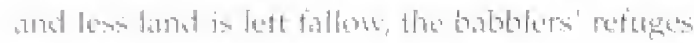
are

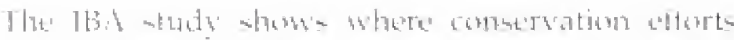

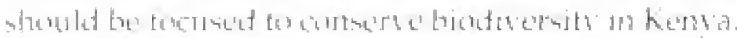

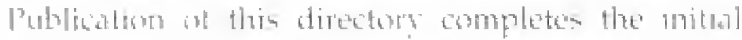

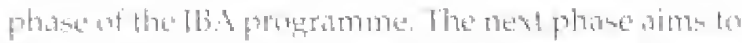

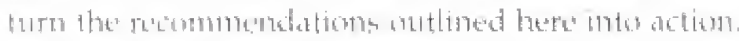

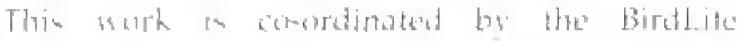

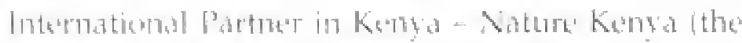

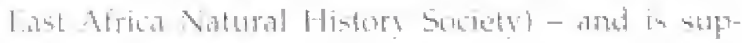

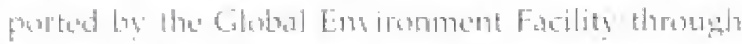

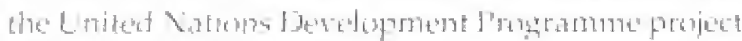

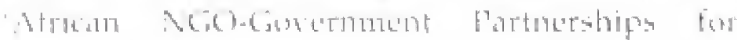

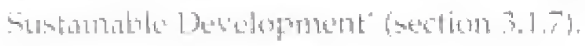

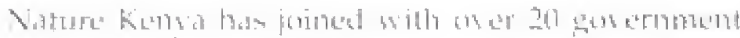

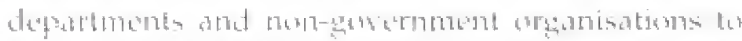

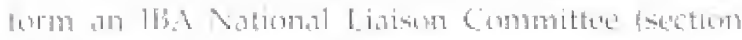

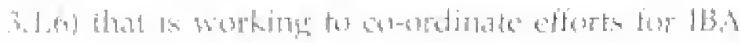

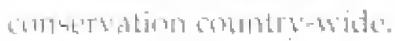




\title{
2 ACKNOWLEDGEMENTS
}

\author{
Compiling and producing this directory has been a long process, to which many individuals \\ and oramisations have made important contributions, If we have onitted aryone in the \\ acknowledgements below, we apologise; it is not for wan of appreciation of their efforts.
}

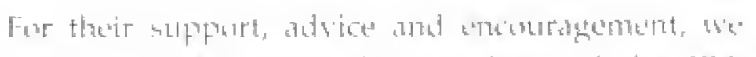

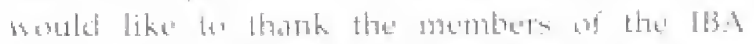

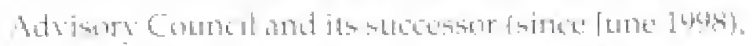

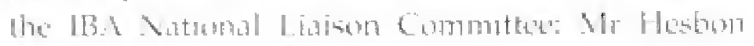

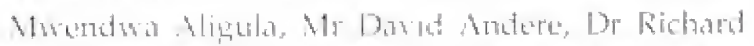

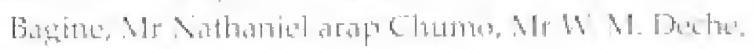

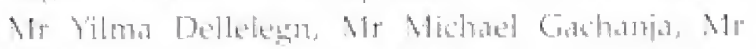

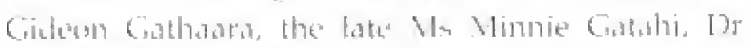

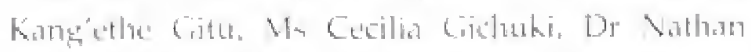

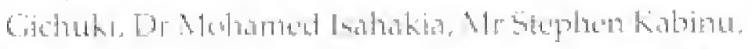

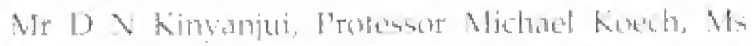

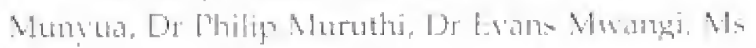

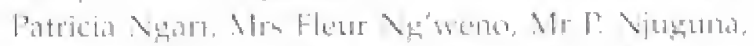

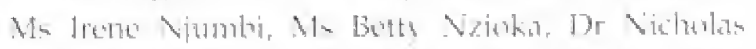

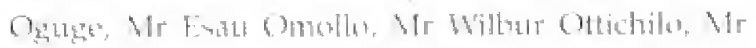

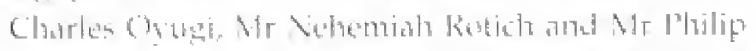

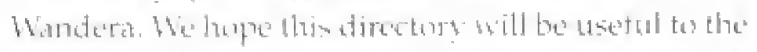
HBA-XLC in turblateity the anme of the IBA promentame.

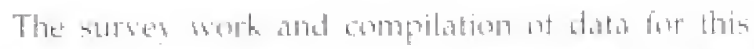

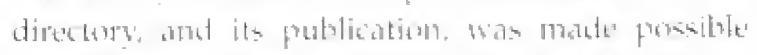

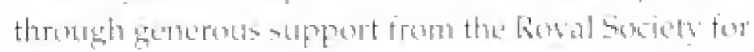

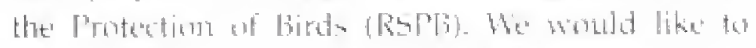
thank all theme in the International Do fartment whe

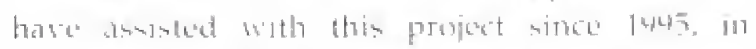
particular l'sul Buthley, Alastair Ciammetl, Dioter

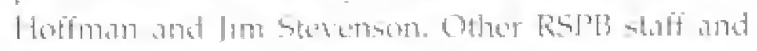

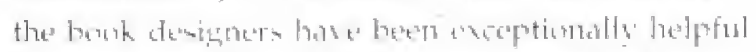

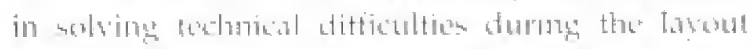

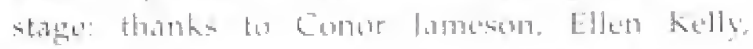

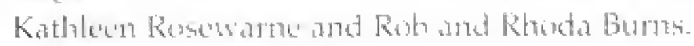

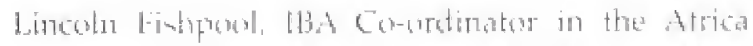
Division of bitallite Intermational. provideat

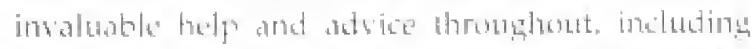

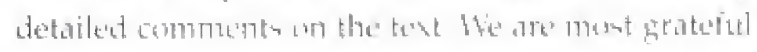

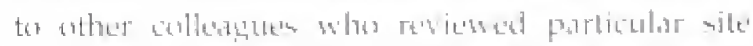

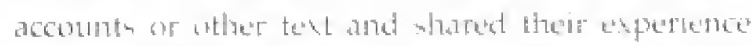

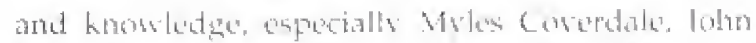

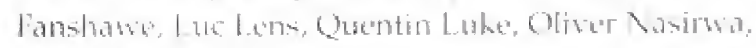

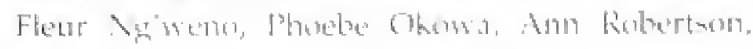

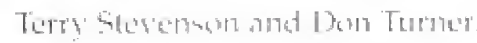

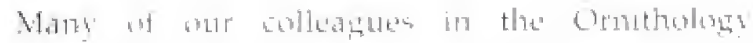
Department iemeributed th steries work to

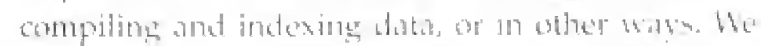

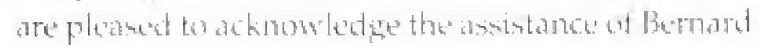

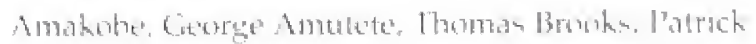

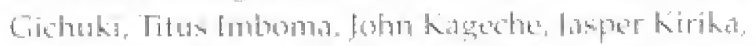

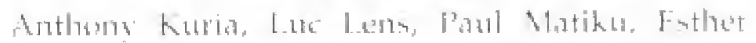

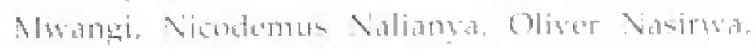

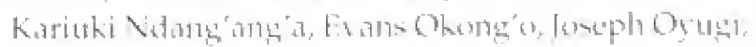

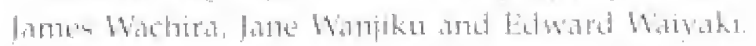

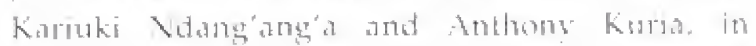

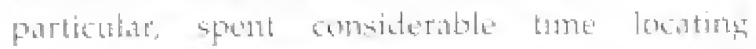
intormathon and reterencess, and checking lists and indices.

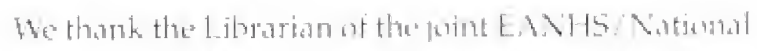

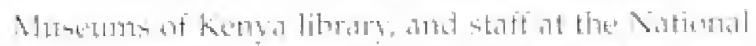

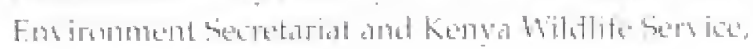

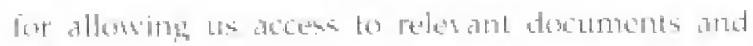

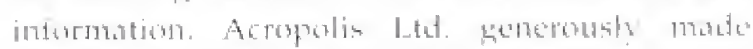

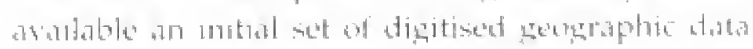
frome which the majes in this dirextery were g't-

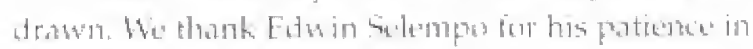

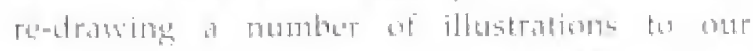

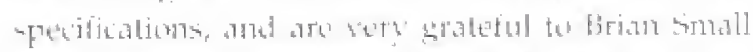

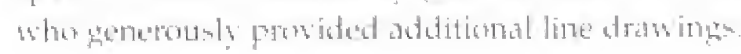

The priofieses tor action reported here, and the"

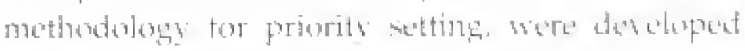

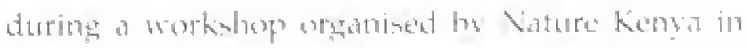

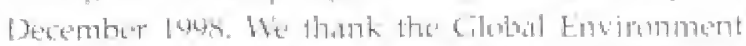

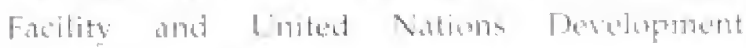

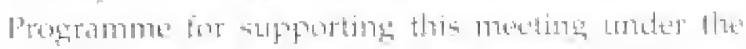

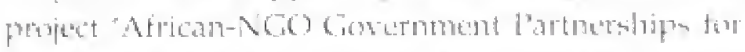

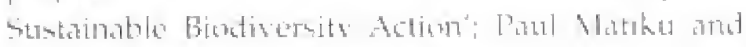

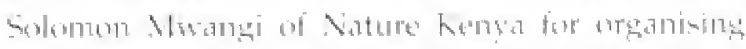

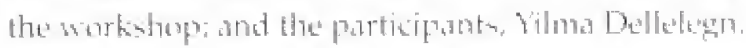

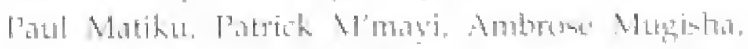

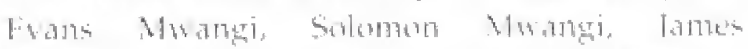

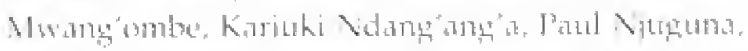
Annita Tapilald, Munir birane and Mengetes

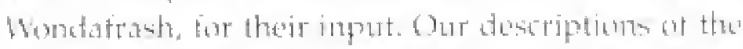

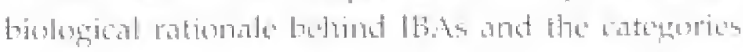

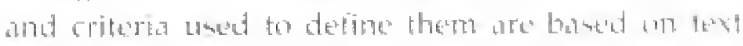

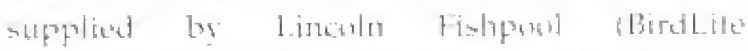

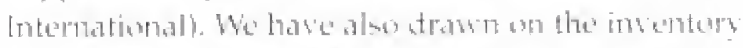

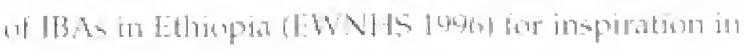
atrerial places.

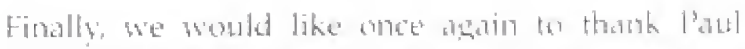

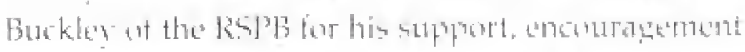

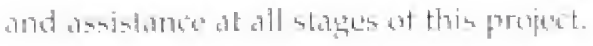




\section{INTRODUCTION}

\subsection{Overview}

\subsubsection{Kenya's biodiversity}

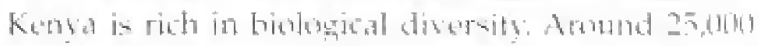

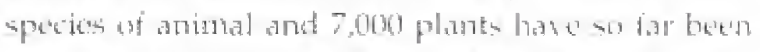

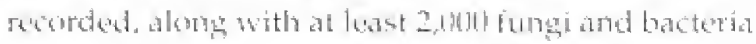
(NBC 1992). An enormous range ut species inflabits

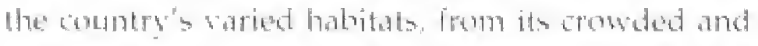

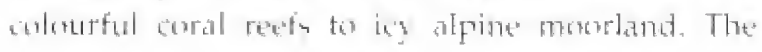

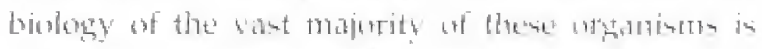
little known. Undoubtedly morry specits have not even been mamed vet by selence. Their salue to

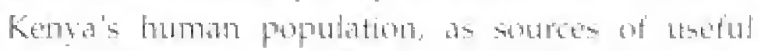
genes, as foot or medicine, or an sital parts of

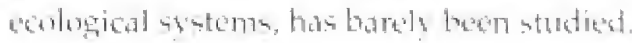

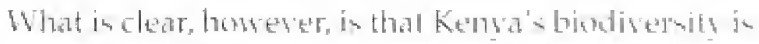
under serioss threat. An exparating poptulation io

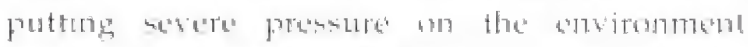

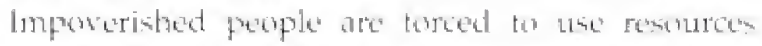
mosustainably. Natural habitats comfinue to be cleared and converted, fand is degrated and water

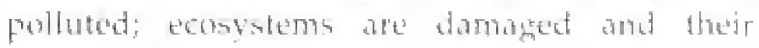
functiuns impaired.

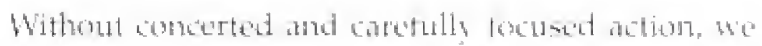

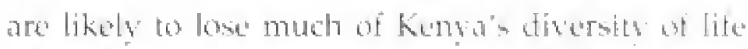

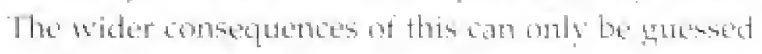
at. The resources be suppert action are sares.

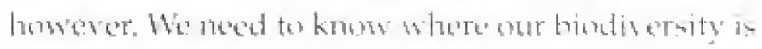
and what state it is ins. Which siles and habitats ate the mest important, whith are the mest thatatemed, ant which therefore require the mome urgent atemition?

Kemfe already has an extensine protected area ny-

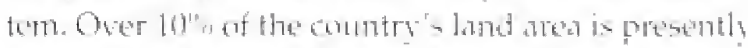

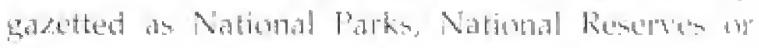

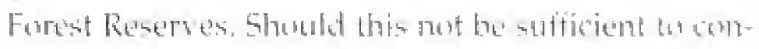

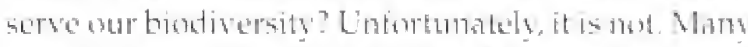

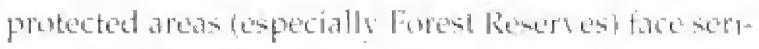

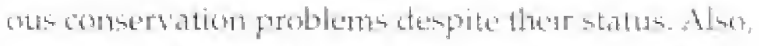
sery few of kenvals proteded areas there choments conserve bindinersiby as such. bur parko and

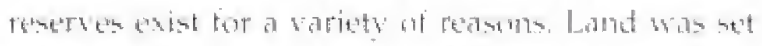

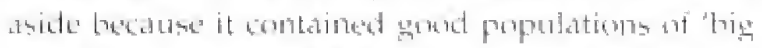
game (i.e. lage mammalat that might attract wiat-

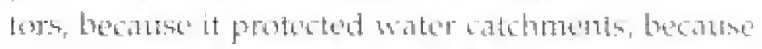
it supported waluable tumber for expleritation. ar simply because at the time very tew feople wante's

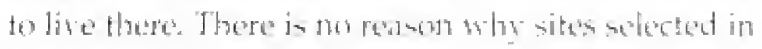
this way should contain excoptionally tich at dictineture fatuna and flora. To select the most impuntank sifes hot biodiversity consertation, we

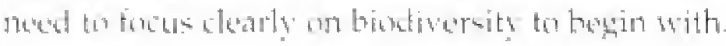

Lntortunately, arstesing biodisursity is dificult,

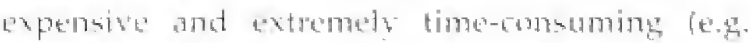

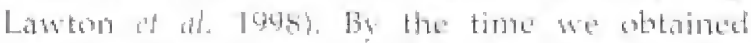
adequate information, it sioud be tero late to make

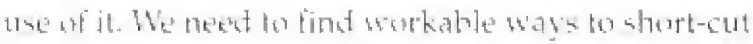

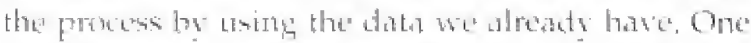
wat to sto this is to ferces an birde.

\subsubsection{Using birds to assess conservation priorities}

This directors on a contribution th identifyng Kengas biodiversty Eonservation priorities, The

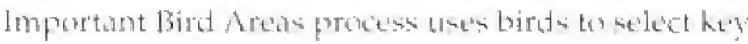
sites for combervation. Important bird Areas (IBAs)

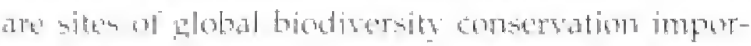

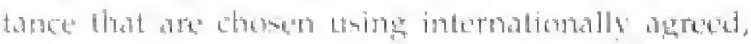

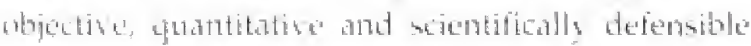

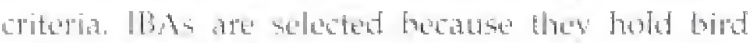
species that are thruaberat with extinction, hate haghly restreted distributions, ort are characteristic of particulat bommen. Sitem holding exceptonally large numbers of compregaters hirds abo gualits.

Birds are an impartant focts for cometrvation

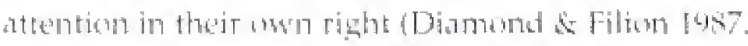

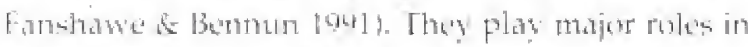

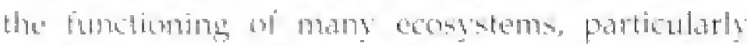

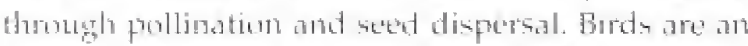
important source of revente through birdsuatsing

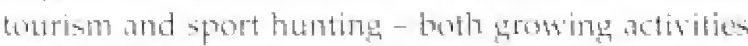
in kenva. As elseshere in the world, birds have sted sisnificame in many Kengan cultuters. Bird also parthe an excollent meaths to create awareness

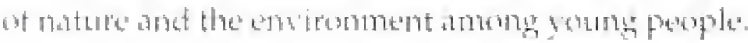

As well as thes antrinate importance, birds as a

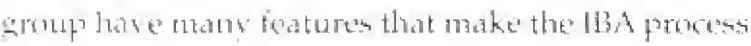
a uneful shortat to sedtitge biodiversity

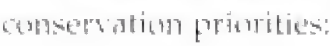

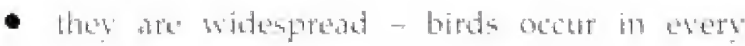
habitue in Kunya

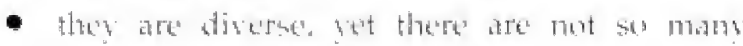
speries than identification becomes a major dificults

\section{Introduction Overview}




\section{Overview}

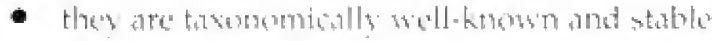

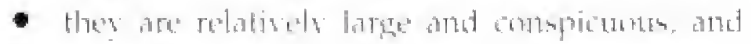

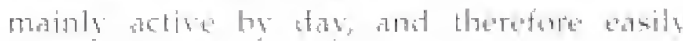
sursered

- thes have wide popular appeal, so many people have wollected urefol information on ther atimtribetion and thats.

As a result, we hathe much more information about

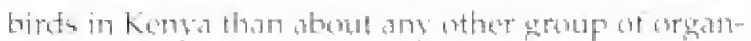

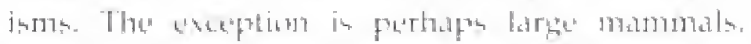

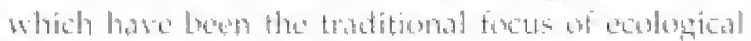

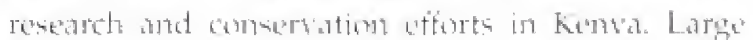

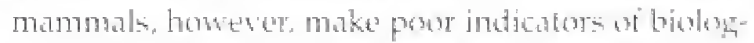
joal diversity at loast in eastertm Africa. First, there are

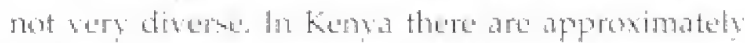

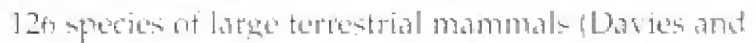

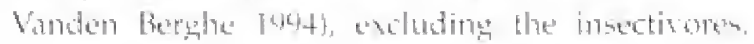

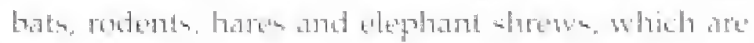

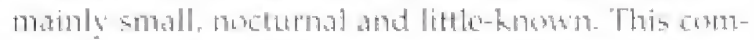

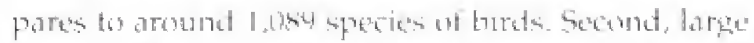

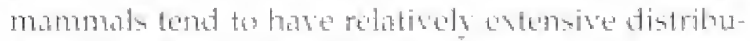

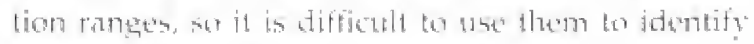

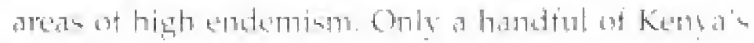

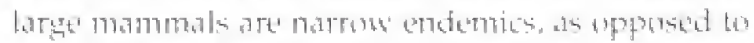

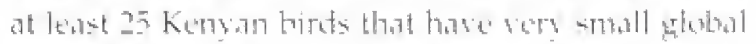

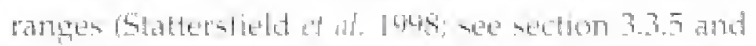
Appencix 2). Thuret, larese mammals ane often in direct contict with perple. Thus particular species

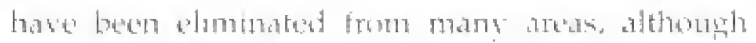

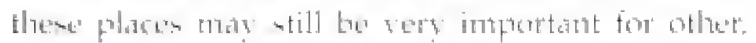

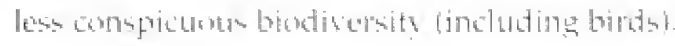

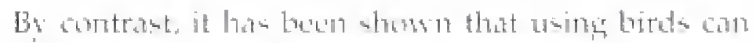

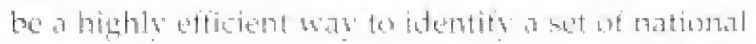

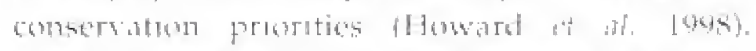

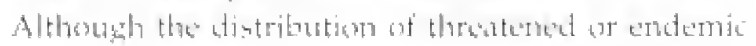

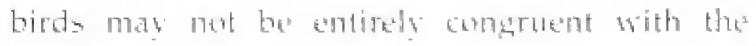
distribution ort कas, Gomilasty important phante

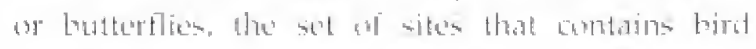

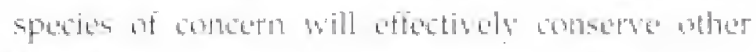
taxta an welll.

This is the appotich taken by the Impertank Bird Areas programme, and prenested in this directory

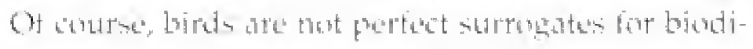

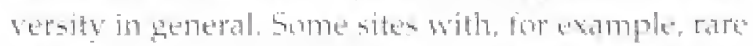

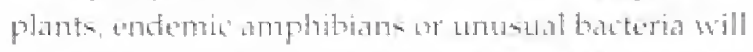

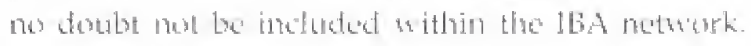
conversely a low sites ate important only tor their rare birkt and nest for other wildite. The need to collect mformation and a moger at

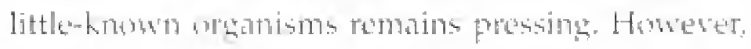

Laking stepe to prostect or sustainatyly manates

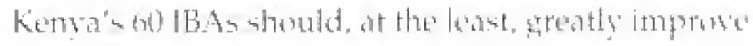

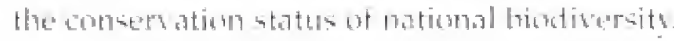

\subsubsection{Aims of the directory}

Thes difectery identitien and dencribes a set w

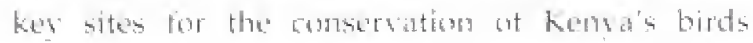

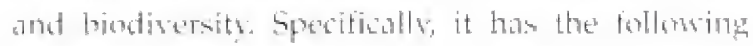
wheretizes:

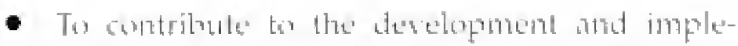

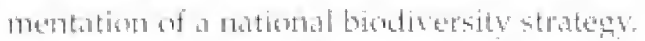

- To assist kenza to meret its ubligations under the

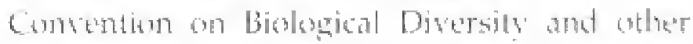

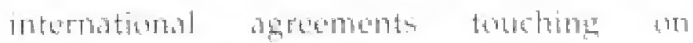
forofinesuth comberstion

- To inform decisinn-makere and techndal

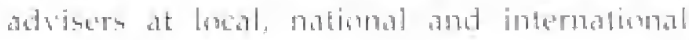

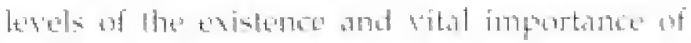
thene sitem, so that apprepriate steps and be haken las their consersiations.

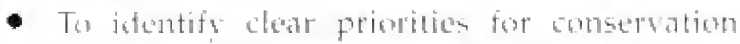

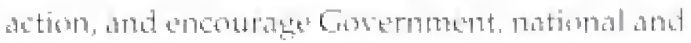

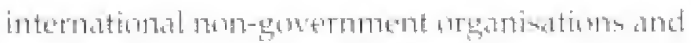

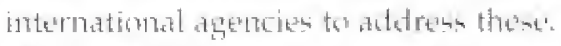

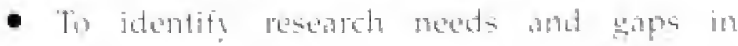
knowidedge that shumlat be actdressed by the seicontific commomits.

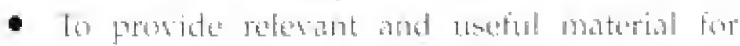
training and extuation.

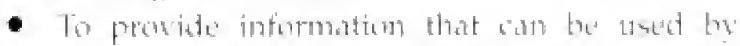

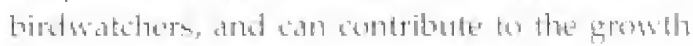

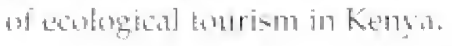

\subsubsection{The Important Bird Areas programme in Africa}

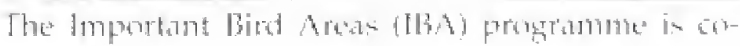

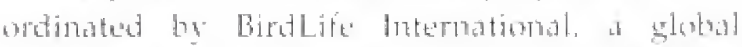

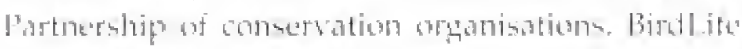

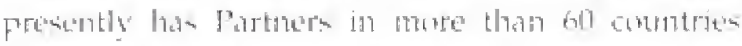
actoris tha sordd, atad is represented in many wthers. The mission of Birdlifo laternational is th abneserve all bird spectess on earth, and the ir habitats. Thraugh this, the organisation work bor the world's biodiver-

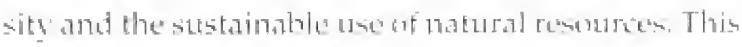

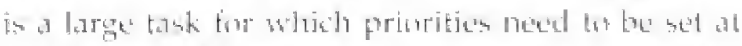
the leveds of speciers, sites and habatats. Thu

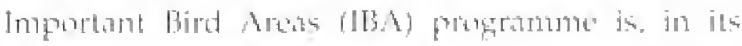

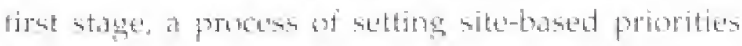
tor bircts bated an exintirg information aboul species

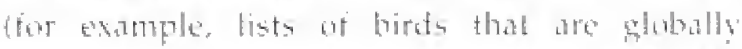

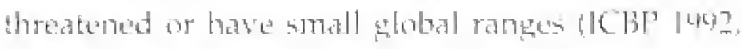

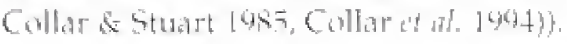




\section{Important Bird Areas in Kenva}

The IBA programme was first begum is betrope in

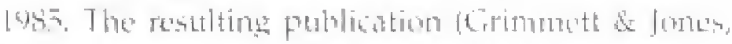

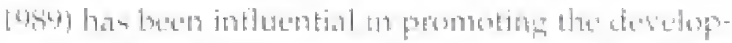

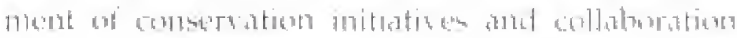

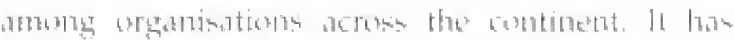

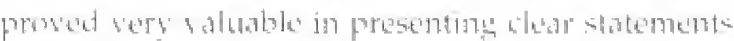

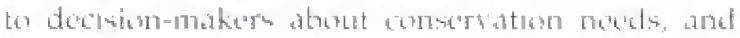

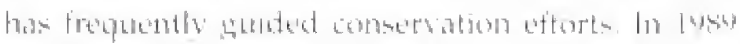

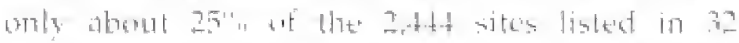

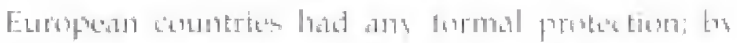

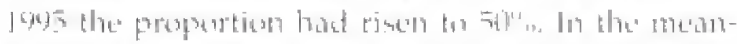

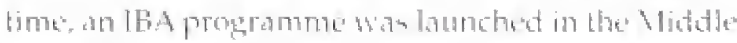

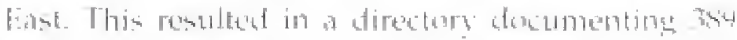

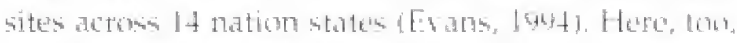

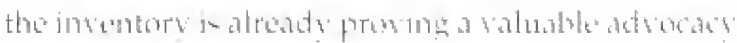
tasel and at lanst tive sites now benefit from formal

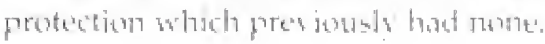

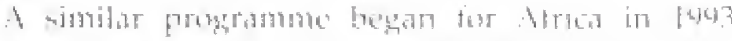

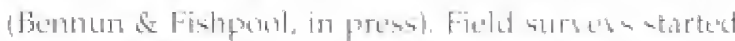

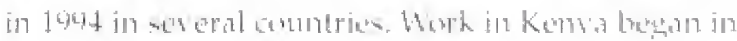

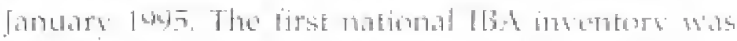
published by the Fothioptan Wildlute and Saturat

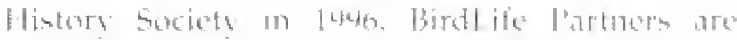

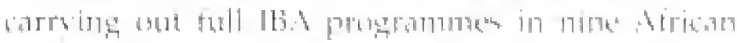

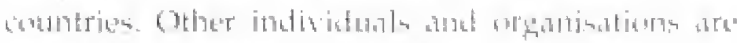

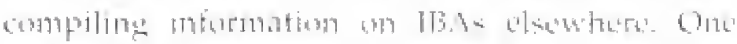

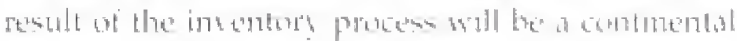

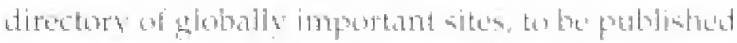

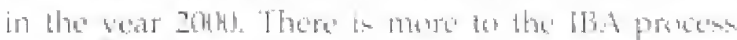

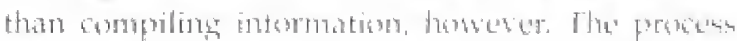

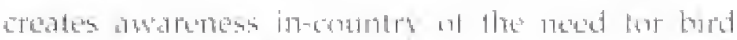

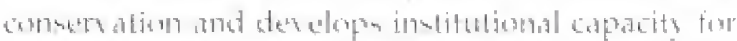

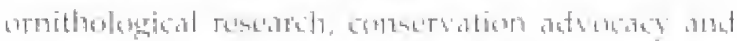

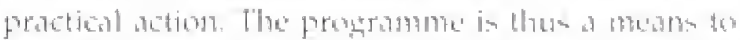

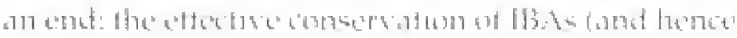
biodiversits') at the nationsal lenedi.

\subsubsection{The IBA programme in Kenya}

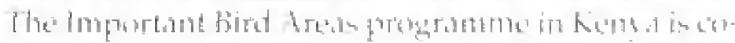

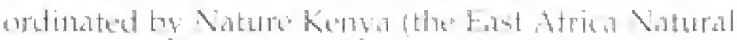

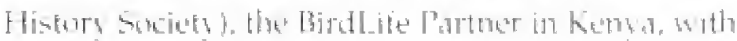

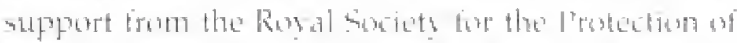

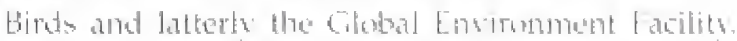

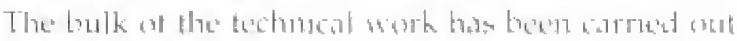

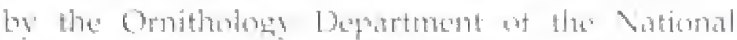

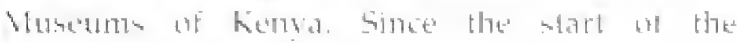

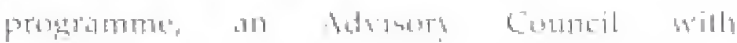
representation fromt 4aribus mimistribe and

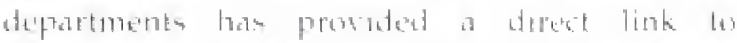

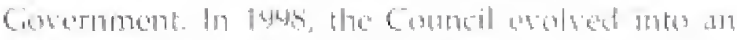

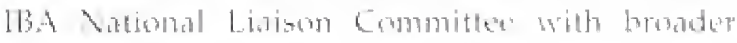

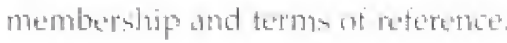

Field surses wath by the Defortment of Onitholog

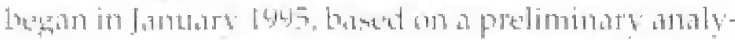

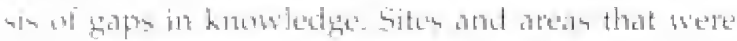

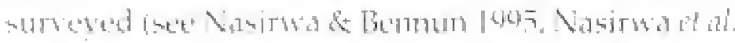

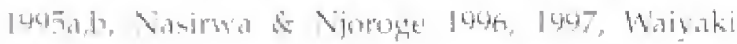

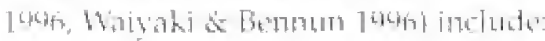

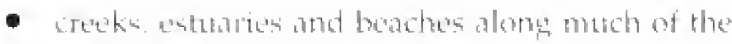
Ritingia colat

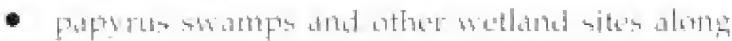

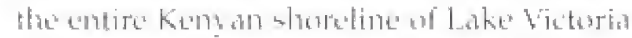

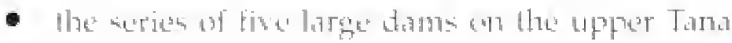
rizer

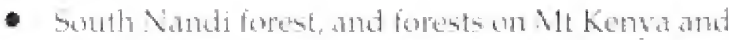
in the cherangani fills, the Taita Hill and south

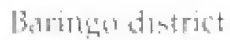

- muint grabelande in Busia and Sulma Districto

Goher published and unpublished inhormation or putential sites was compoiled in paralfed and information entered inte a cumbon-designed

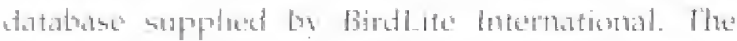

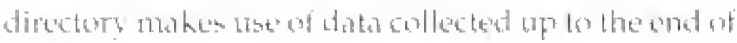

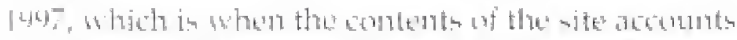

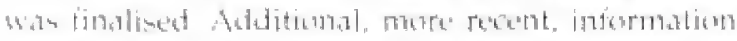

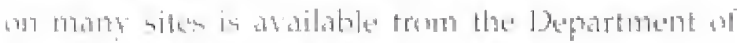

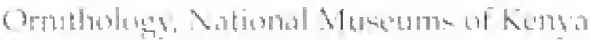

\subsubsection{Future directions}

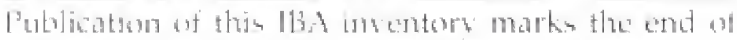

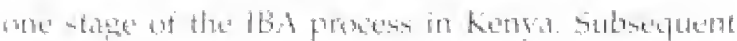

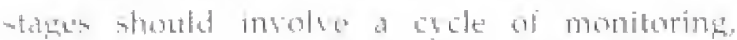

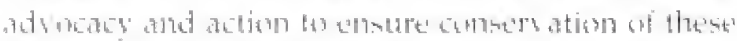
biles in perpentrits Safinal and lexal capacity for

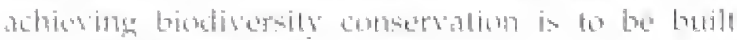
thionuth this promest

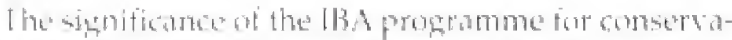

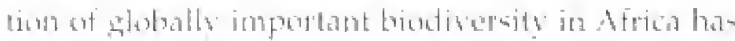

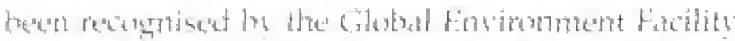

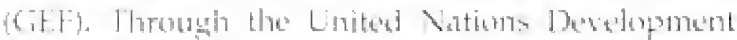

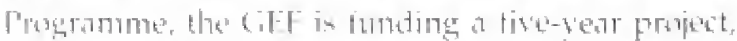

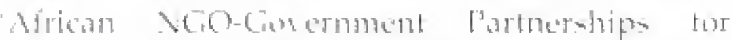

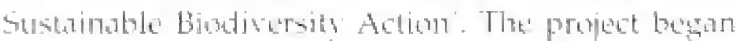

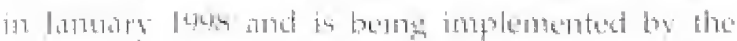

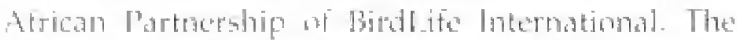

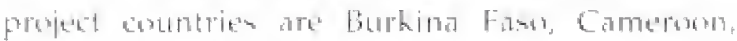

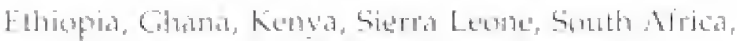
Tanzania, Tunixia and Lgande.

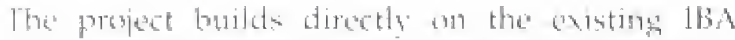

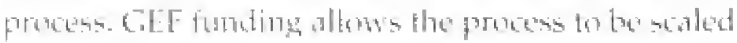

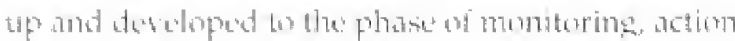

\section{Introduction Overview}




\section{Overview}

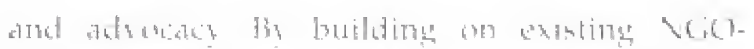

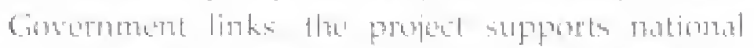

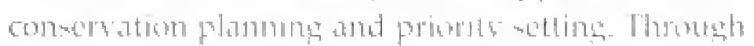

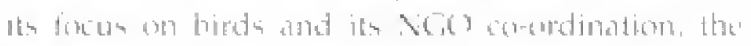

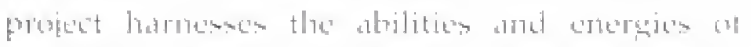

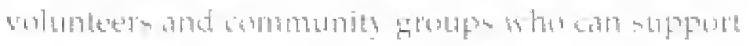

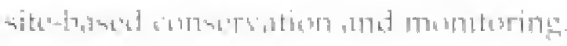

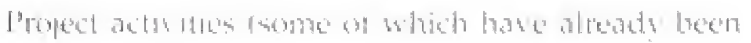
statent bo complated melude:

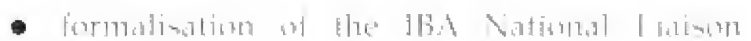
Cinm?

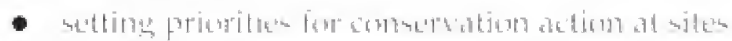

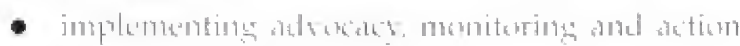

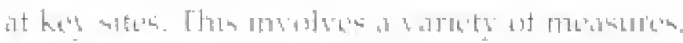
antiludation

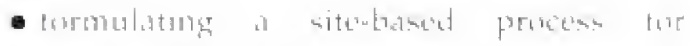
momiturang and comkersatust

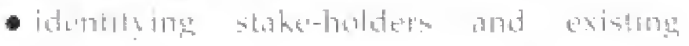

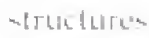

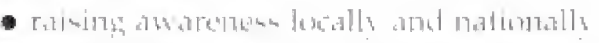

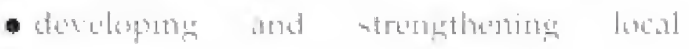

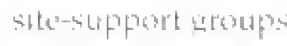

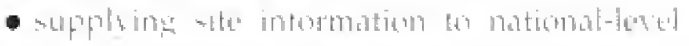

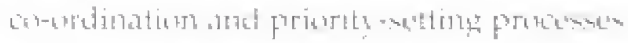

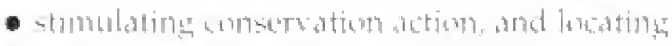

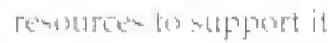

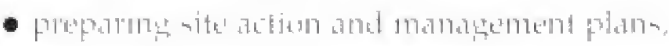

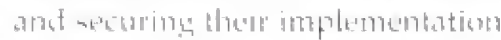

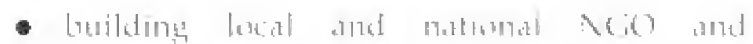

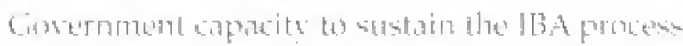

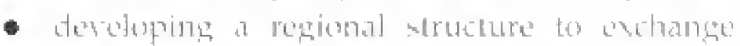

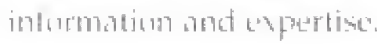

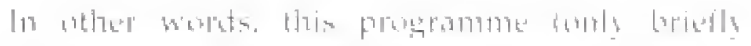

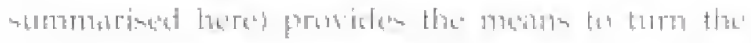

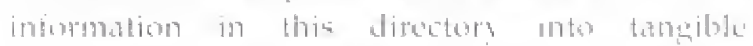

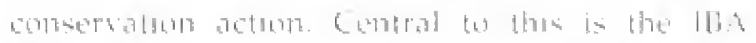

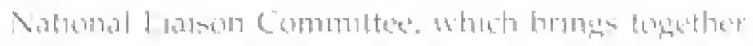

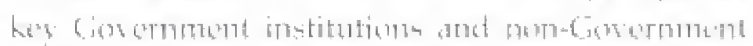

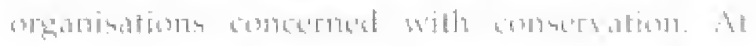

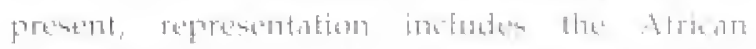

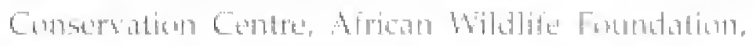

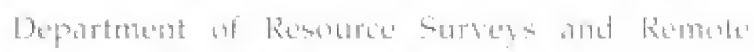

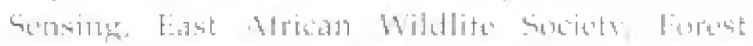

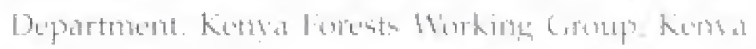

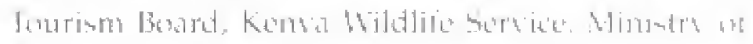

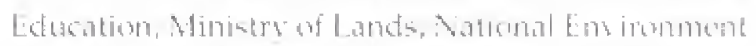

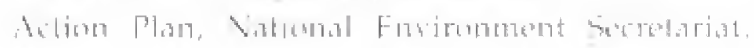

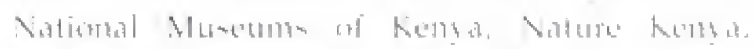

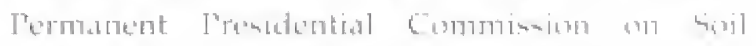

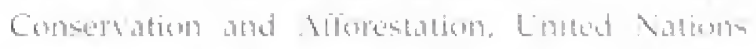

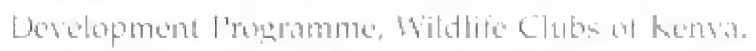

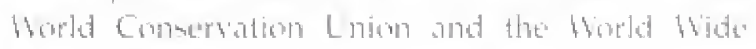
Futrol for Malure. 


\subsection{Kenya: General information}

\subsubsection{Location and topography}

Kenva lies astride the equator on the easter'n cenat of

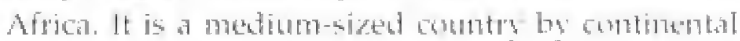
standareds, covering wh areat of about $5806000 \mathrm{~km}^{3}$. Intanet water bodies coner some $10,76 \mathrm{~h} \mathrm{~km}^{2}$, the bulk of this in Lakes Victoria and Turkana. Kenya is bordered by Somalia and the Indian Ocents fo the edat, Exhiopia to the north, Suldath to the north-west, Uganda to the west and Tanzania to the south. The

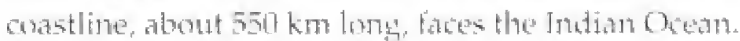

Kenva loas tremendous topouraphical diversity", including ghateded mountains with snow-capped

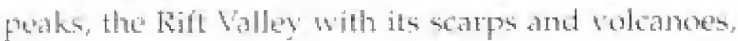
ancient wranitic hilts, flat desert landsapes and coral reets and islets. Howerer, the basic configuration is simple (Map 2). Coastal plains give way to an inland platerat that risen gradually to the central highlands, which art the result of relatively recent solcarnic ackity associnted with the formation of the rift vallev: To the mest the land drops again to the Nvanza




General information

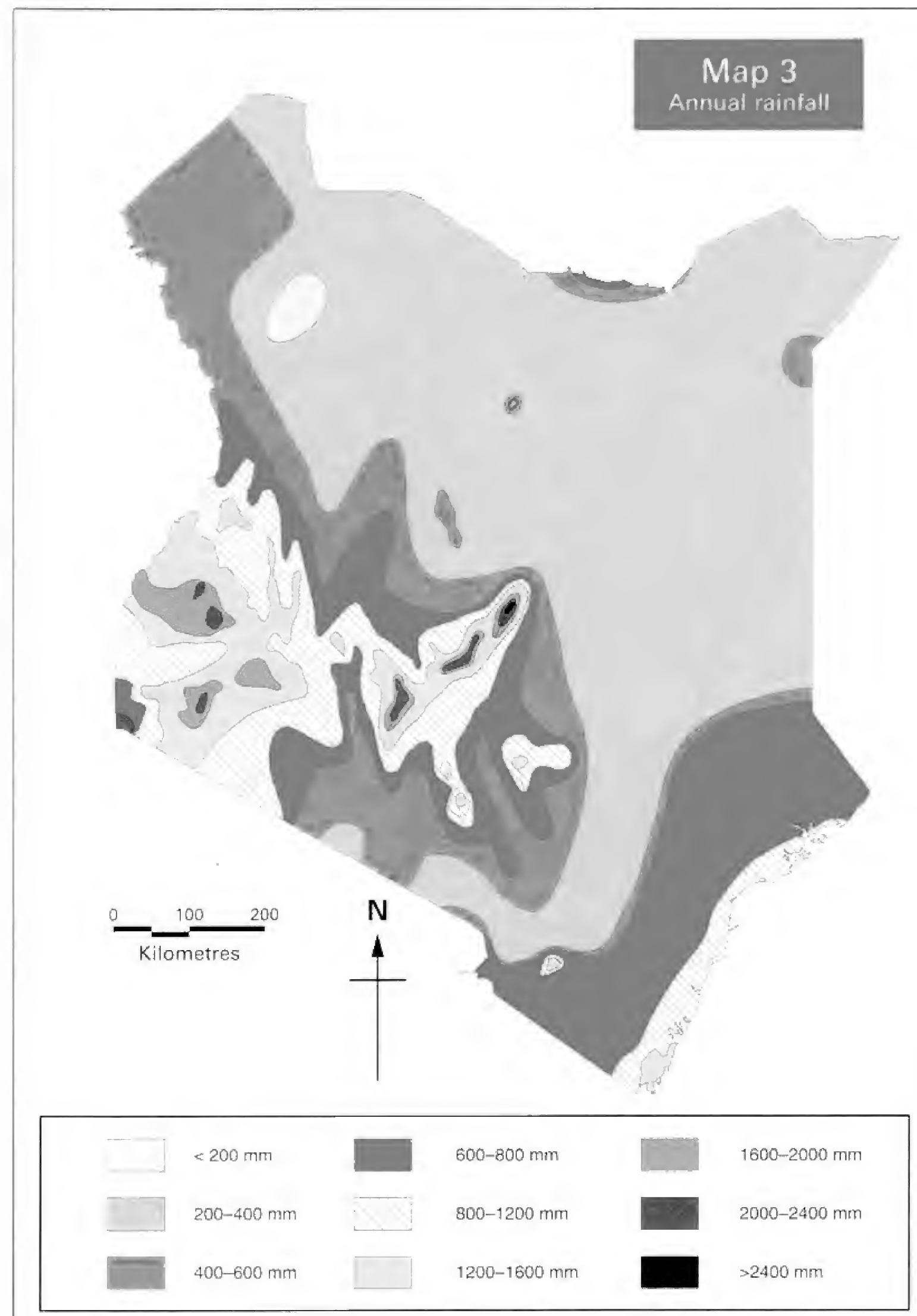




\section{Important Bird Areas in Kenya}

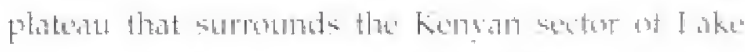

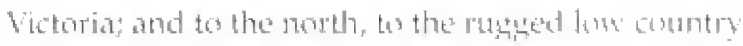
around Lake Turkand.

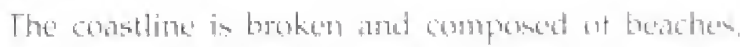

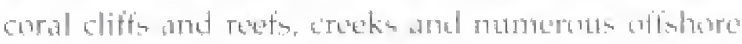

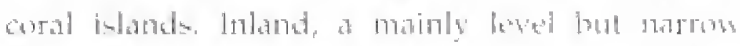

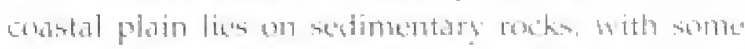

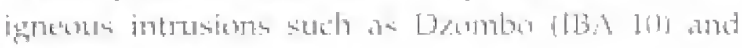

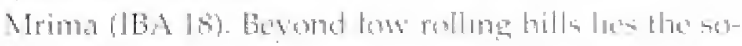

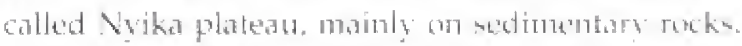

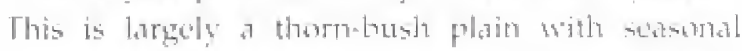
drainase lines and a dew isolated rakeng hille. This

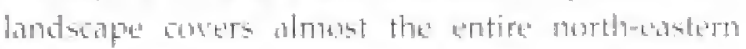

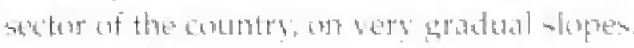

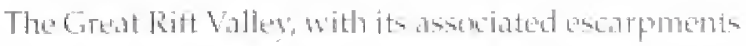
and monulaides, is a major feature. It rum the length of the coumery from Lake Turkand in the nexth to

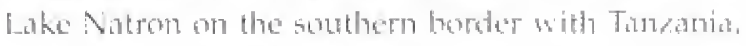
The contral partion of the rift in rabect. with the

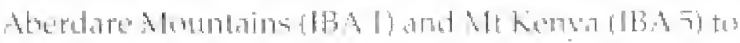

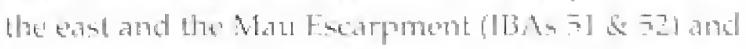

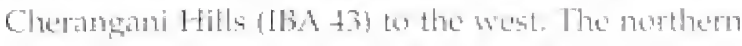
and sosthernmost sectors of the rift ante bro-lyon.

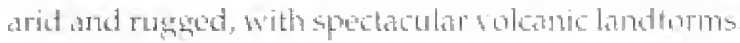

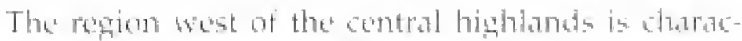

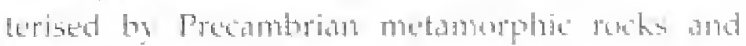

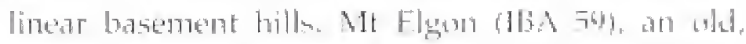

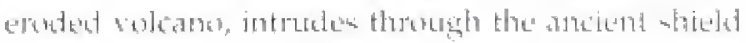

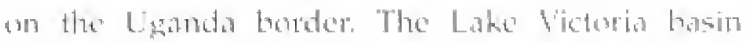

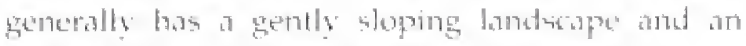

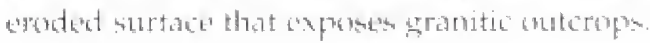

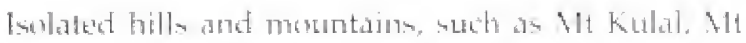

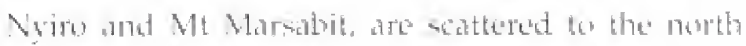
and kat on thes sentral highlande. The Taita Hills

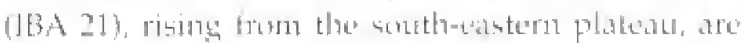

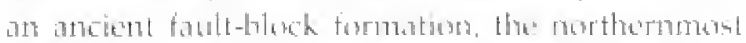
of a chain of isolated parke the eastern aret that

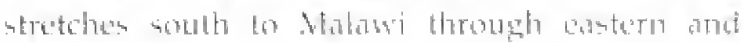

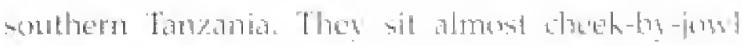

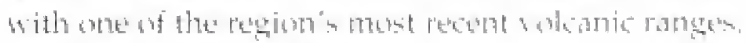
the Chyulu Hi]ls (IB, 26).

\subsubsection{Climate}

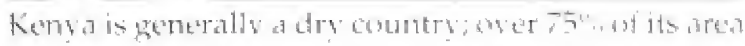

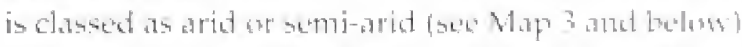

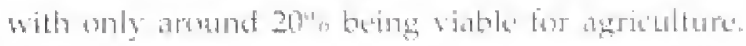

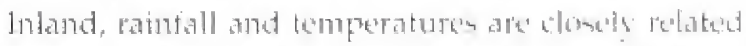

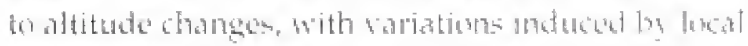

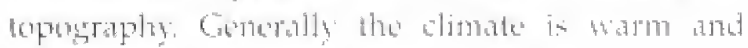

lumid at the coset, exol and humid in the central highlands, and hot and dry 19 the north ard wat.

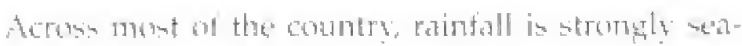

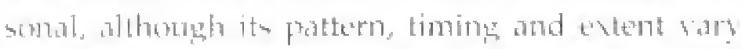

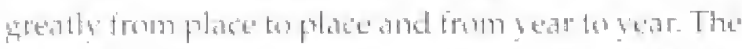

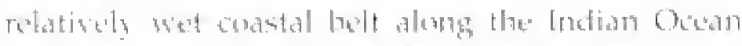

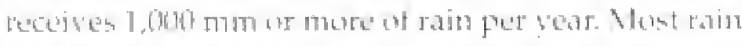

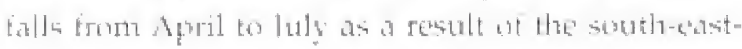

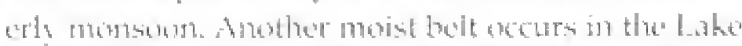
bistoria basin and its surrounding gatpos and

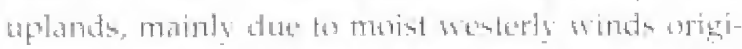

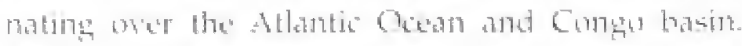

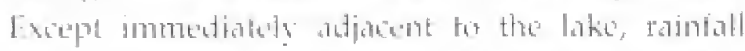
watere rediably from Wareh to Nowember. The

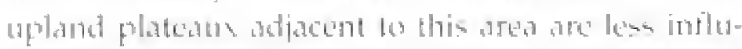
cnced by the lake, and tain talls mainly in Mardi-

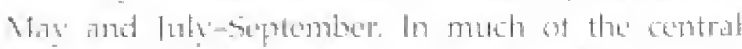

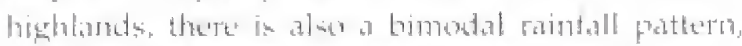

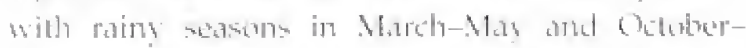

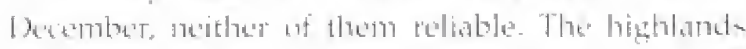

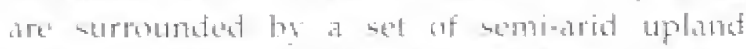

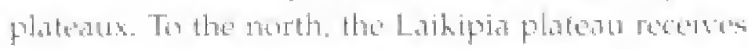

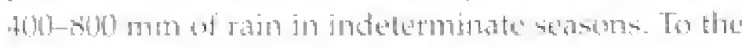

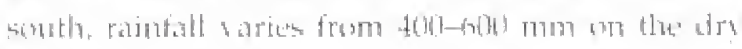

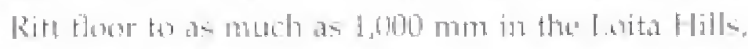

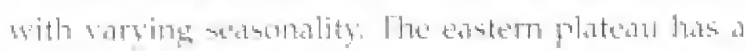

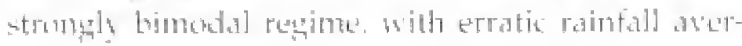

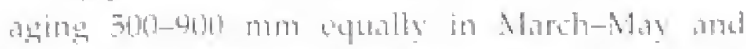

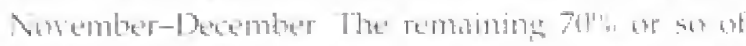

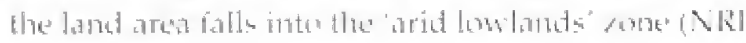

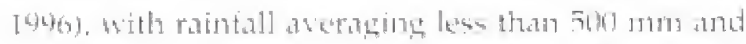

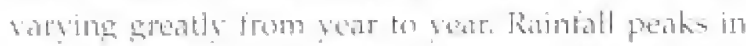

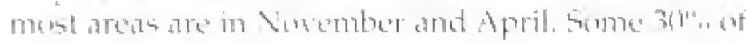

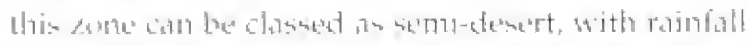

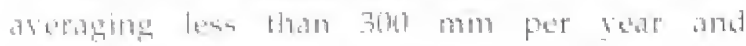

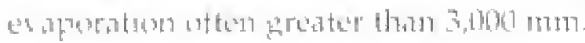

Exicepl tur the coast and lake Victorja region. altitude is the matis determinand uf presipitation. The

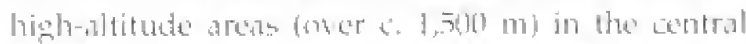

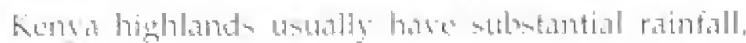

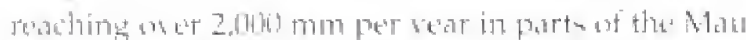

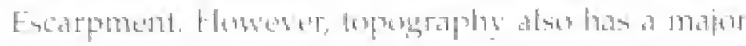

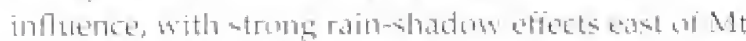

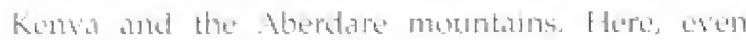

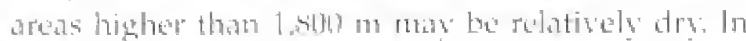

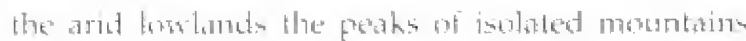

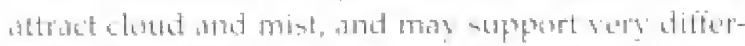

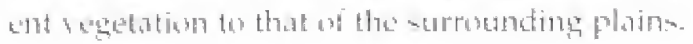

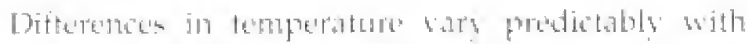

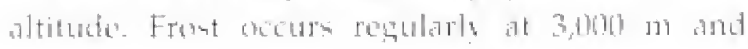

\section{6 Introduction General information}




\section{General information}

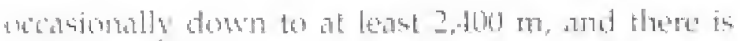

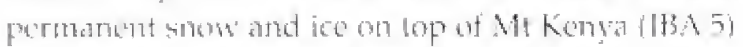

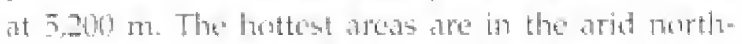

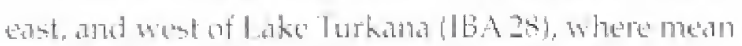
maximum formonatures aberage onet 34 .

\subsubsection{Water resources}

All Kerras major riters drain from the central

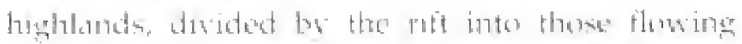

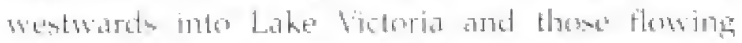

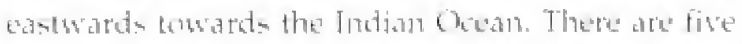

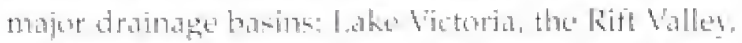

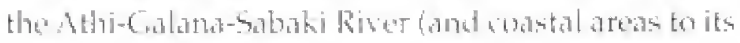

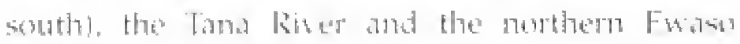

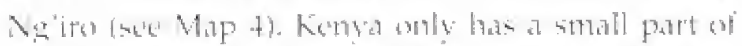
Lake fictoria's water murtace, lout the kenyan

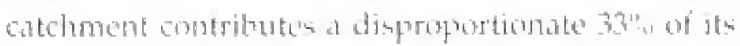

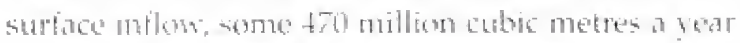

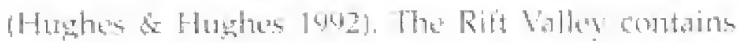

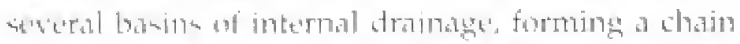

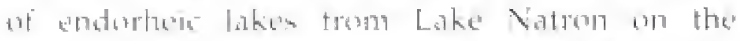

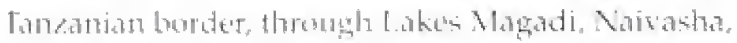
Elmenteda, Vikuru, Bombria, Baringor and Turkana (IBAs $47,48.40,49,45,44$ and 28 respetivedy. These lakes vary in alkatinity from freshuater lake Nairash (IBA th) to the intermedy alkalme Latke

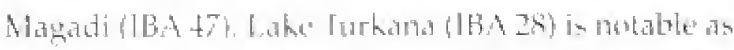

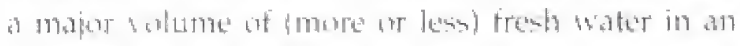

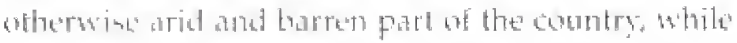
a number of rinters, incluting the Turkwed, Kerio,

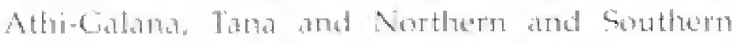

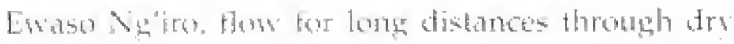

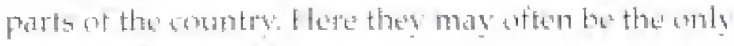

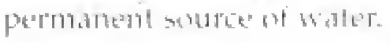

\subsubsection{Vegetation}

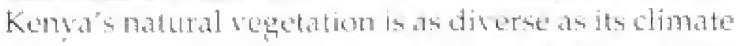
and topography would stapest. Dean \& Irtimp (1963) nupped 19 diatinct biotic communiticas, some of which cant he lumped turder genteral headinges.

Afro-alpine moorland $\left[1.2^{*}\right.$ of of colal land areat

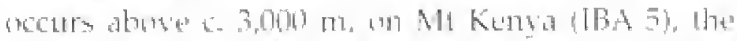

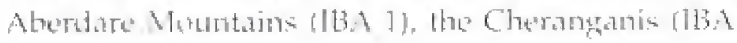

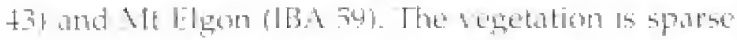

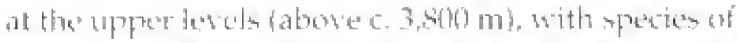

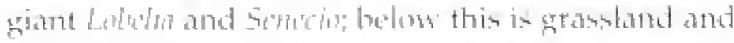

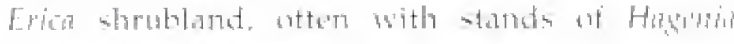

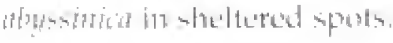

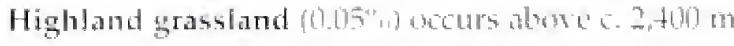

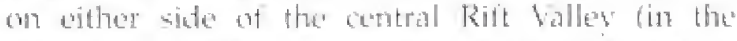
Kinangep and Mau Narok/Molo Grasiands, LBAst

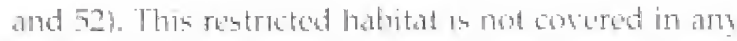

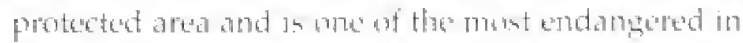

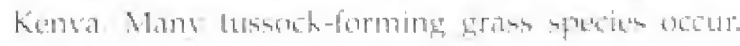

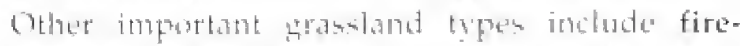

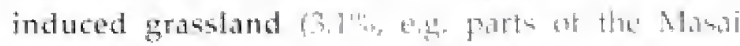
Martis, IBA 5ij) and seasonal floodplain and delta

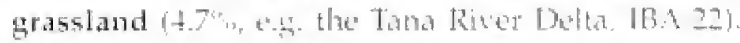

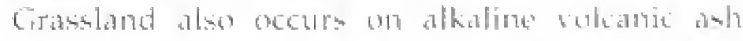

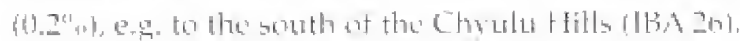

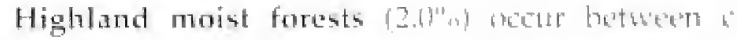

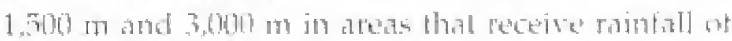
more than 1.200 nom per yeal a momaic of borest and

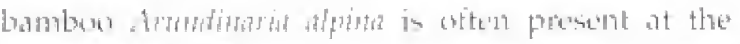

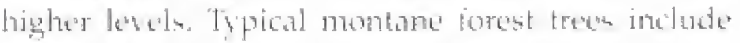

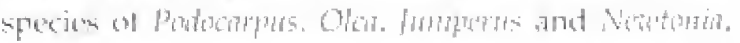
but the forest type vartes greatly acometing to altitende and rainioll,

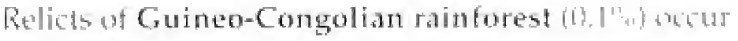

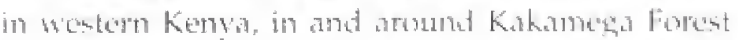
(IBA 58). Dewpite its relatively high altitute (1.6011) m), in terms of biogetograplay Kakame ara is the

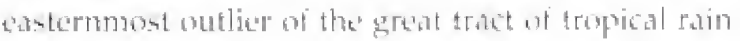

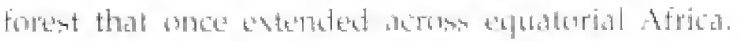

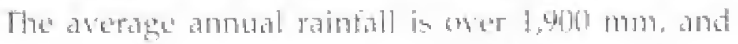

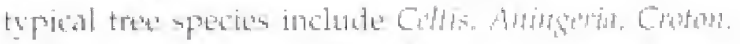

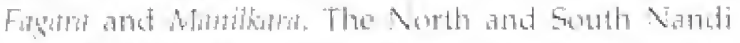
Forests (IBAs 53 and 55 ) are transitional between the Guines-Compoling and mustane forent byes.

Geveral typen of constal forests and whedind

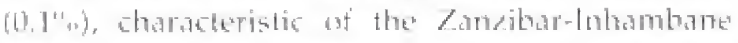

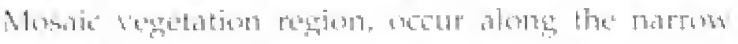

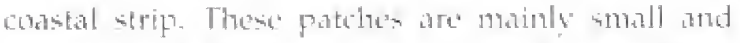

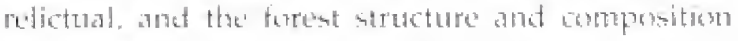
vary yreatly aconding to snil lype and rantioll.

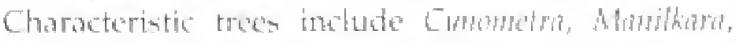

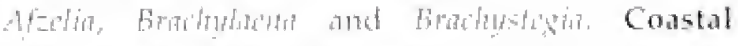

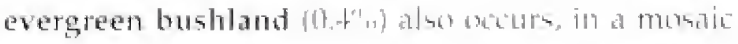

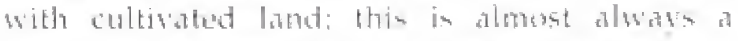
cecomdaty beycetations type. Coastal palmstands.

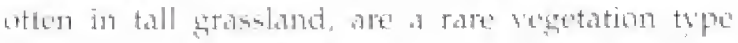

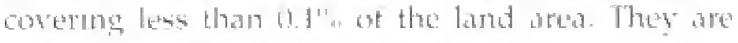
comcentrated near the kambi Riser on the sumth, and

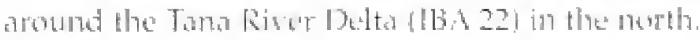

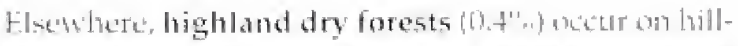

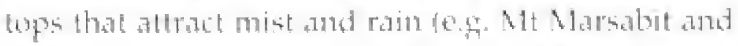

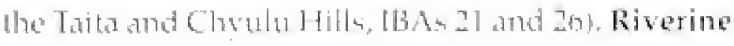

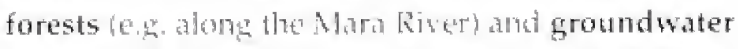

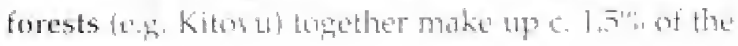
I.and arter.

\section{Introduction General information}




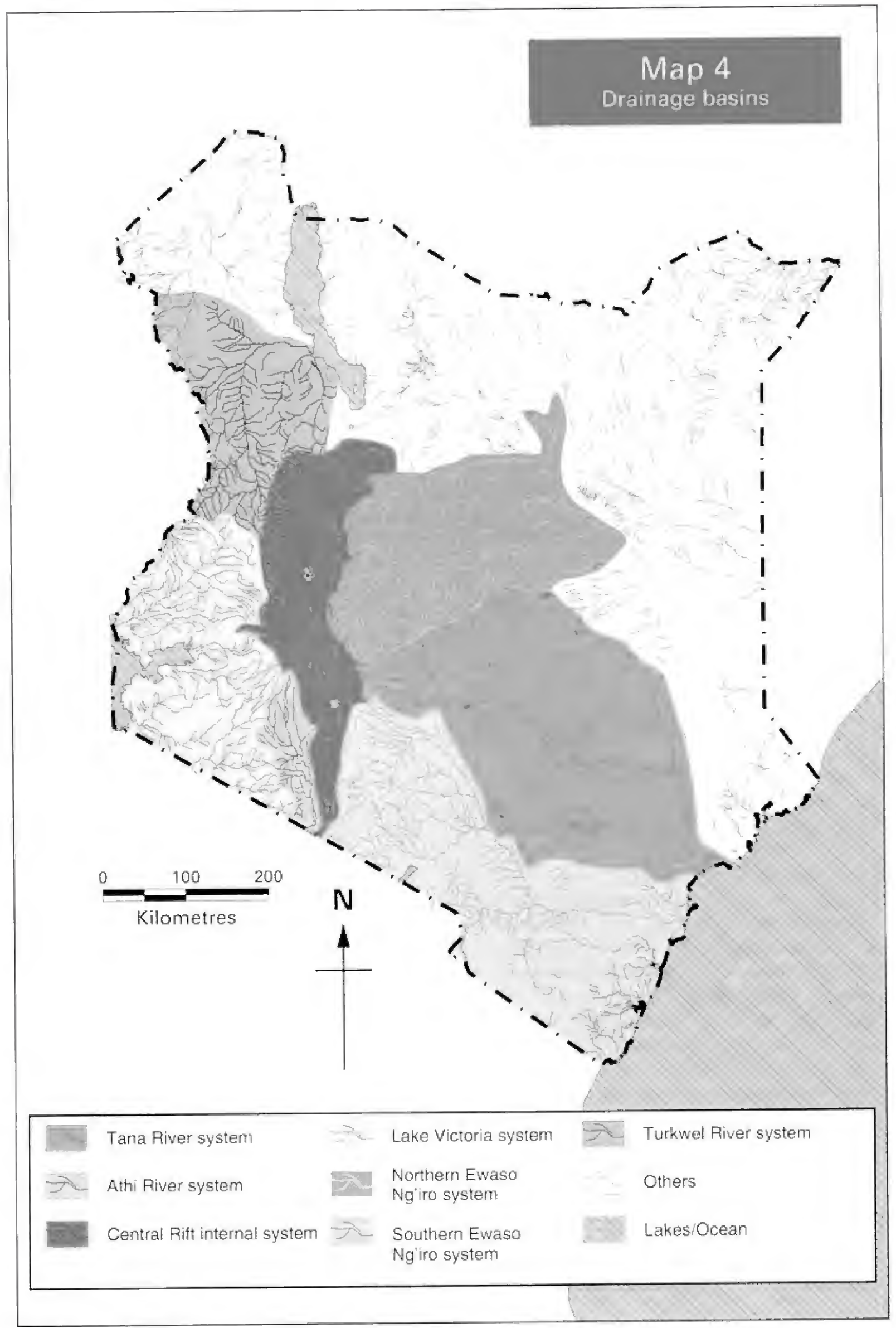




\section{General information}

Thorn bushland and woodland are the most

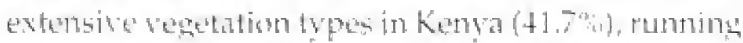
frem amboreli (IBA 42 ) in the sruth throught the

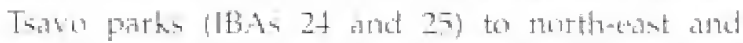

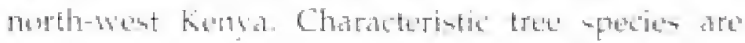

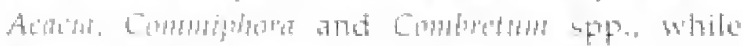

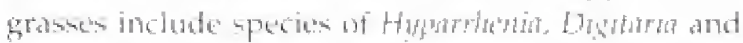

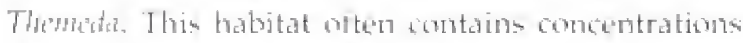
of darge mammalis and many large proberted areas

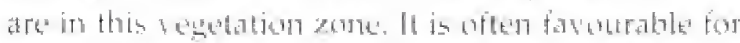

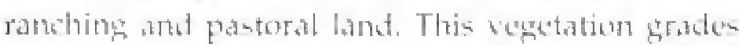
inte semi-arid wooded and bushed grassland $(0,2$, 4,$\}$

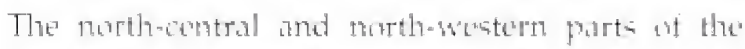
county are conered bo semi-deserl $\left(10,8{ }^{12}\right)$ with characteristic shatoby thumbush sfebies, mainly Acalin. In plates, whe as the Dida Galgalu (IBA 27)

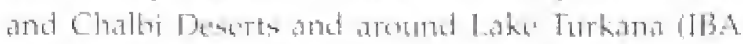

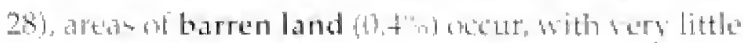
segetathry. Marme beaches and dunes make up another $11.0 f^{\prime \prime}$, ot the land ares.

Wetlands are an important habitat in kernas?

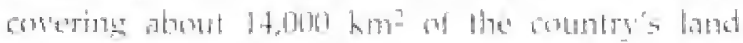

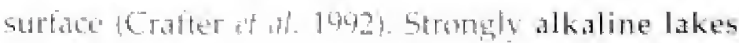

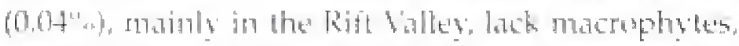

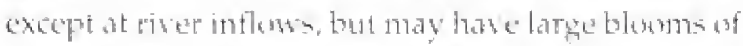

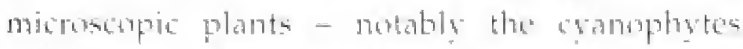

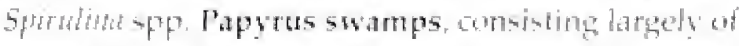

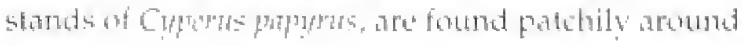
the shorets of l.ake Victoria, mainly allong riner

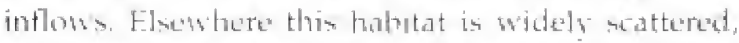

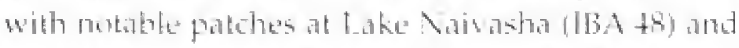

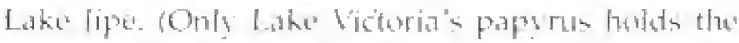

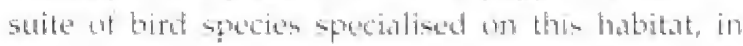

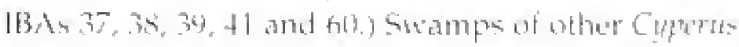

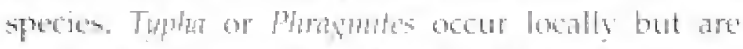
rarely of ang great size. Permanent swamps make? up 0.11 at the land area, while badies bit treshwater

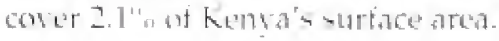

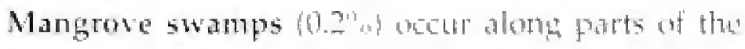

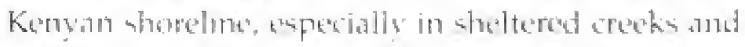

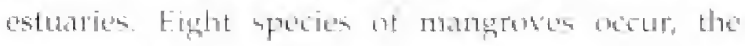

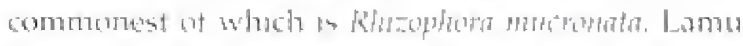

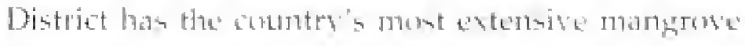
swamps On sandy sherelines are often beds an

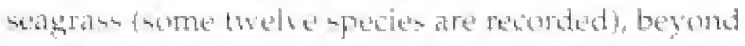
the littoral ame on in seepers bhatureds within it. Coral reefs and islands make up sume 59 , (060) has w $0.1^{\text {th }}$ of the land area.

Human-modified habitats, created at the expense of the natural vegetation, oceur thromghout the country but especally in the hightands. These include

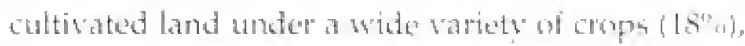
plantations of exotic trets, antondary thisket and

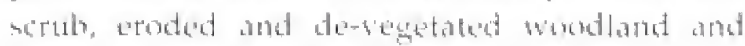
buahland, and osergrabed pastumeland.

\subsubsection{Population and economics}

An a nation. Kerma is ethuically and culturaty

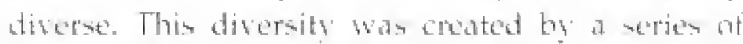
micrations of sarions perples from onther parte of the continasut, mandy Cushites, Nilotes and Bantu. During the 20th century this has bee's arumented Ly the arrival of Asians and Furopeans.

Map 5 shows the major tomves and rond networks in kengat. The combtry population has increased

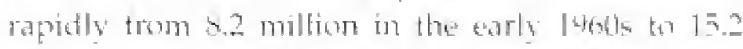
millios porple by 1974 and 22 million in 1487 . Today

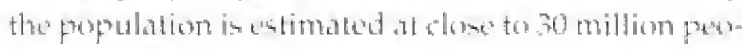

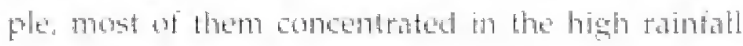
areas. The arerage andul pupulation grmeth rate has tallen from a high of 4 o fone of the hishest rates

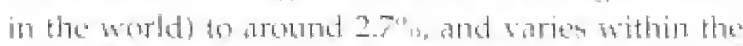
counter Rates of increase" are especially hich in the

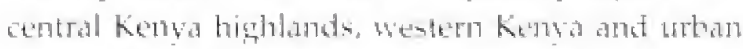

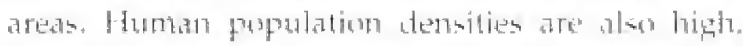

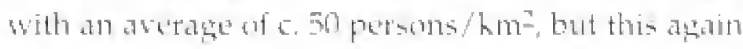

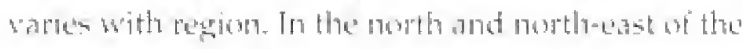
country jast 20 w of the total pupalation creupies so" is arable, with another en" matrinal; the rest is

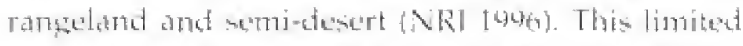

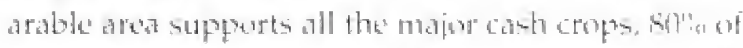

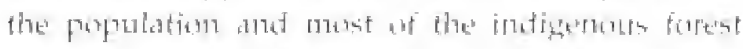
estate (Juman 1989). The rapid groweth in the country" population has subiected this productive land to tremendous pressure. The population increase is now spilling oter to martarinal areas, accelesating

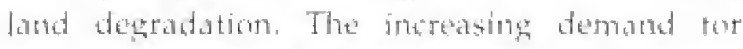
agricultural land and woudtuel has led to high rates

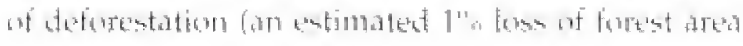
per vear). Savadmath and montane grasslands.

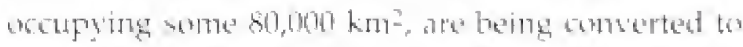
wheat fields and pasture, shile many wothands (especially swamps and narshes) are in danger from drabuges for agriculture. The momentum of population growth is tecreasing but it will tothe a long tinu: before itn impacts itiminish.

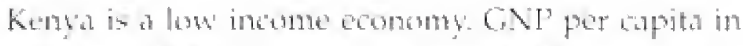
1997 we LSS 330 prer anpita, and has been more or less static since 1942 (Nifrld Bank 1448). Income is very inequitably dintributed. with the highent 20 on of

\section{Introduction General information}




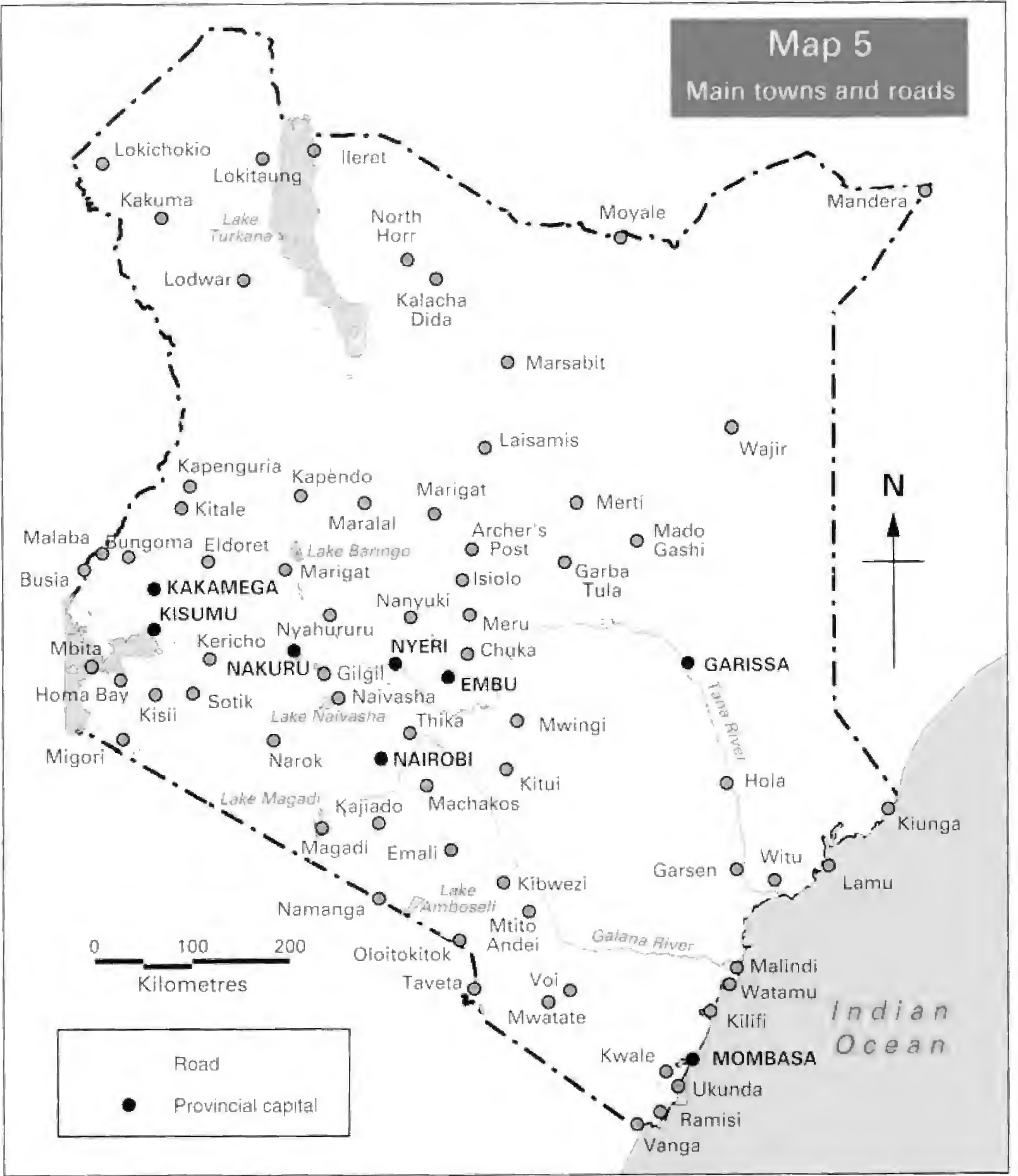

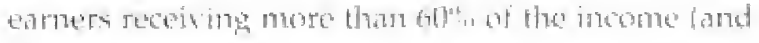
$48^{12}$ of income sering to the top lith the tompared to just 3. $4^{\text {"3. }}$ for the lowest quintile (Wurld Bank Ityos). The economy is mainly asriculture-batsed with oner

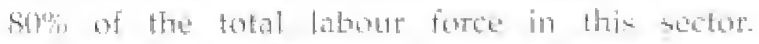
Agriculture presently daras mer 60 'd of Konva's boteign exchange, the traditional export crops of tea and coffee have been joined by but flusess and farious horticuttural crops in recent fears. Tharism is the othet hey sector for toreign exchange earnings, with

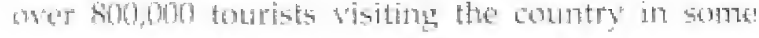
recent yents Major tourist attactions are the coast with its beaches and coral rects, and the varicty we national parks and reserses with their populations of large mammals. Burd tourism appeats to have betn increasing its share of the dotal steadity in recent soars, although ne detailed data are arailatele. and there is substantial usinetised potential in this area. 


\subsection{Important Bird Areas in Kenya}

\subsubsection{Aim of the IBA programme}

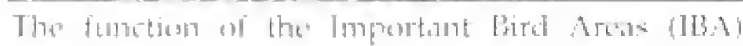

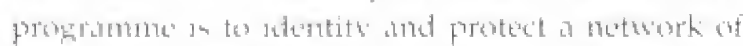

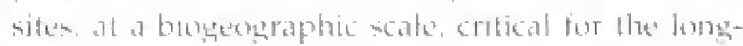

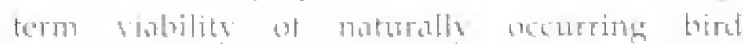

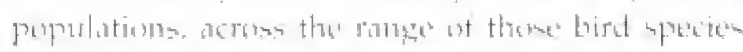

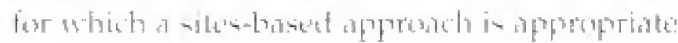

\subsubsection{What are IBAs?}

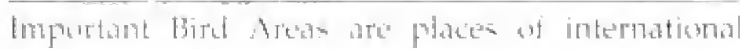

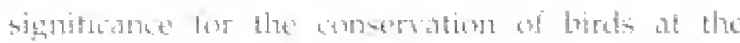

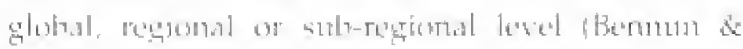

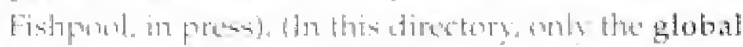

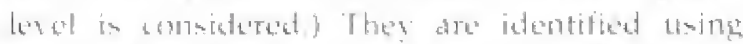

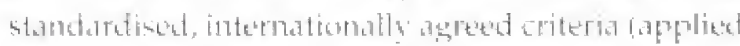

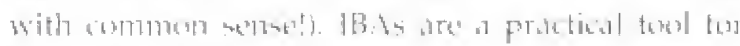

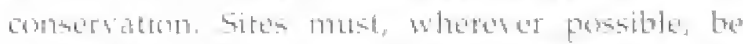

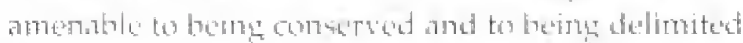

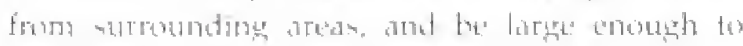

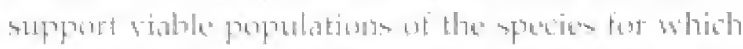

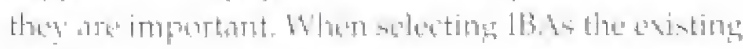

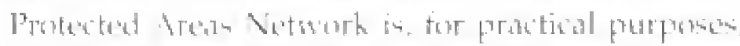

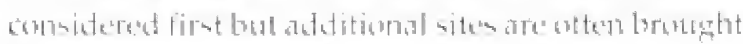

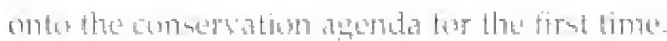

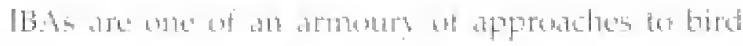

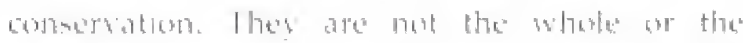

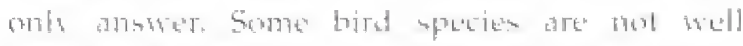

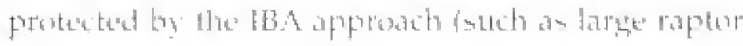

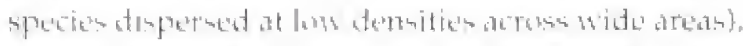

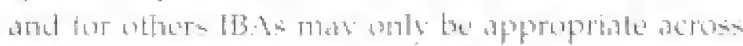
some of their camber ar for parta of thene life arte

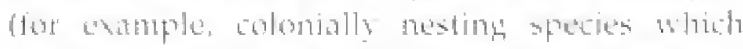

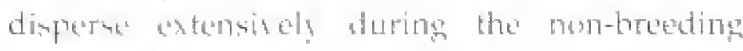

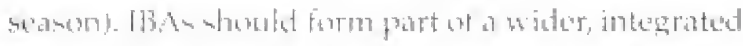

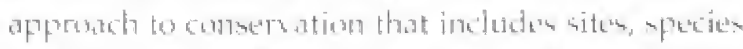

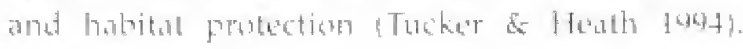

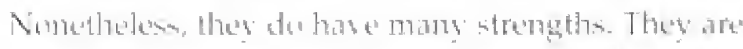

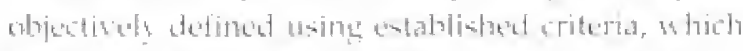

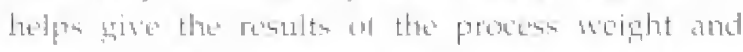

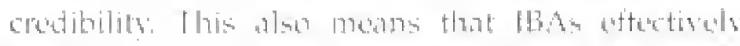

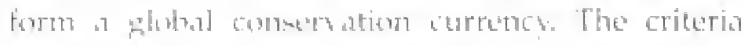

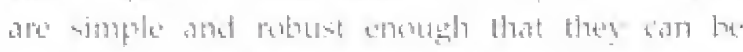

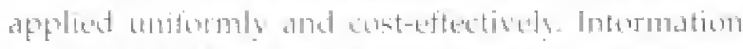

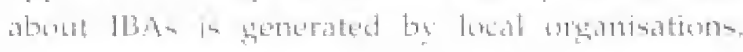
morkinger on the ground. This mesme that the site

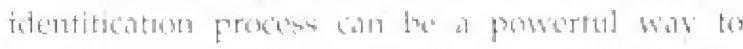

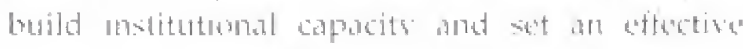

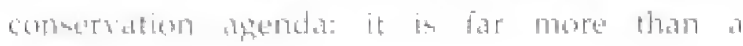

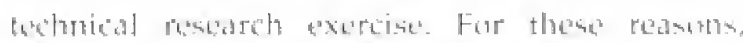
Ilbas an be a practical and affective loser for comseration.

\subsubsection{Biological rationale}

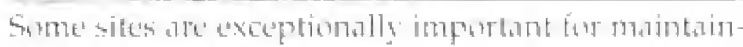

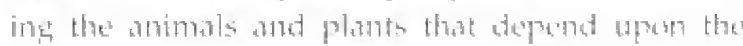

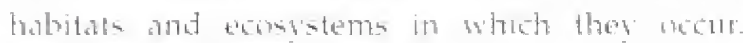

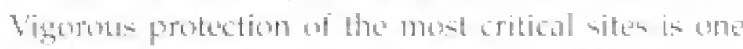
important apprasely to concervatison. Mang Lird species man be effectively consered by this ments l'ateens of bird distribution are such that it is ofter

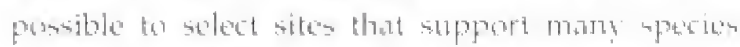

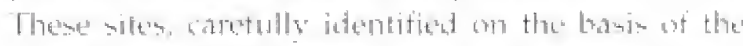

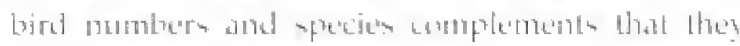

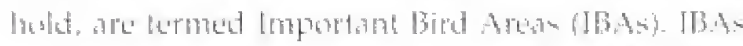

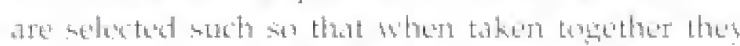

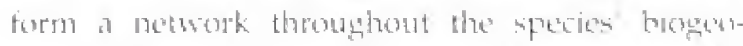

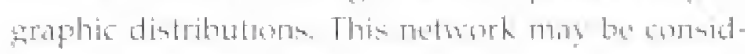

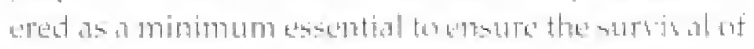

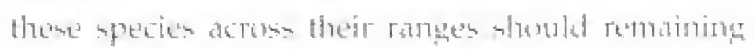

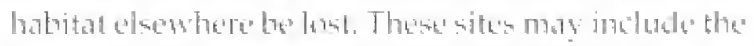

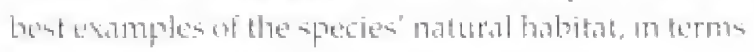

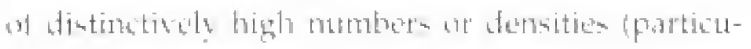

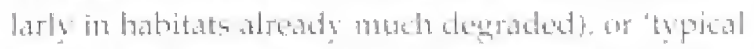

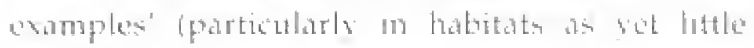

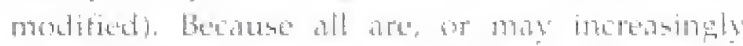
become, reflugess, if ant one of them is lant the

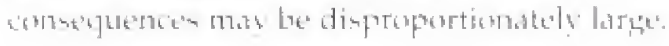

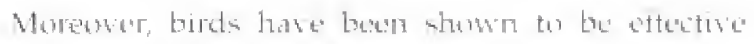
inctiontors of biodsersity in other plant and andmal groups - aspecially when used to define a set of sites

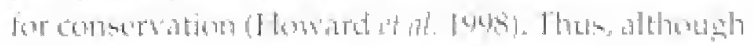

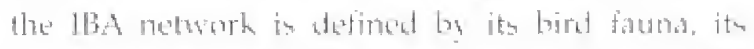

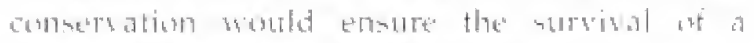

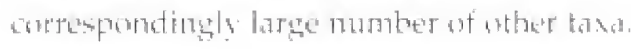

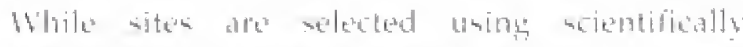

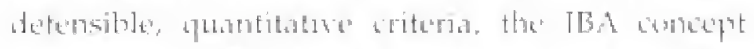

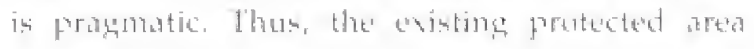

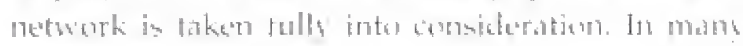

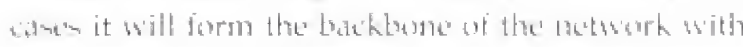

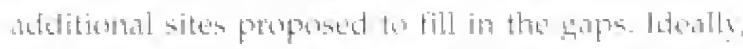

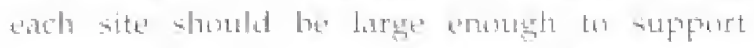

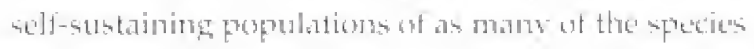

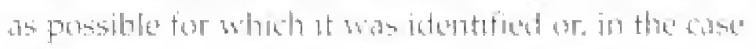
ot migrands, pronde their requirements for the

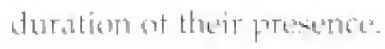

\section{Introduction \\ Important Bird Areas in Kenya}


Important Bird Areas in Kenya

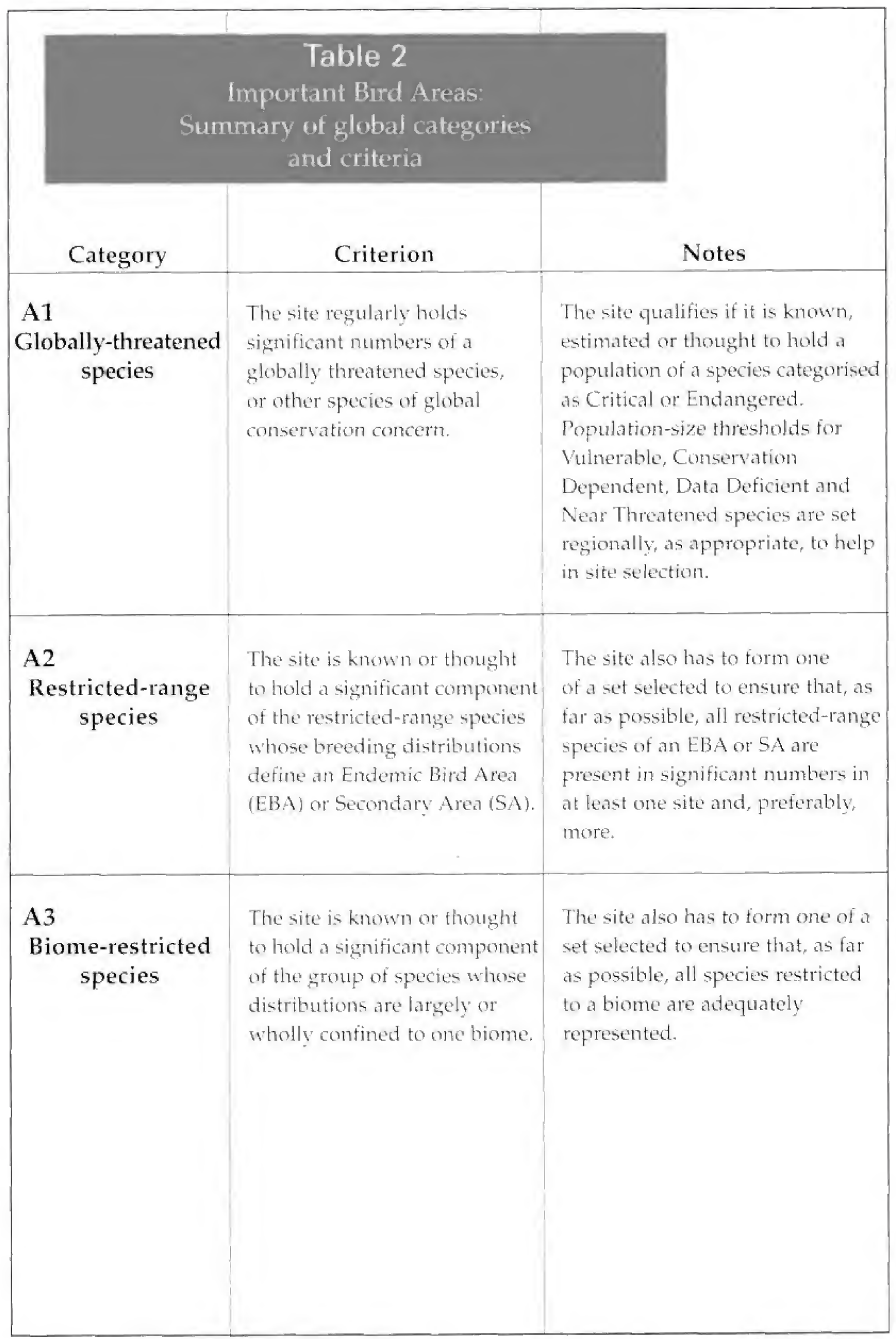


Important Bird Areas in Kenya

\begin{tabular}{|c|c|c|}
\hline Category & Criterion & Notes \\
\hline \multirow[t]{4}{*}{$\begin{array}{l}\text { A } 4 \\
\text { Congregations }\end{array}$} & $\begin{array}{l}\text { (i) The site is known or } \\
\text { thought to hold, on a regular } \\
\text { basis, } \geq 1 \% \text { of a biogeographic } \\
\text { population of a congregatory } \\
\text { waterbird species. }\end{array}$ & 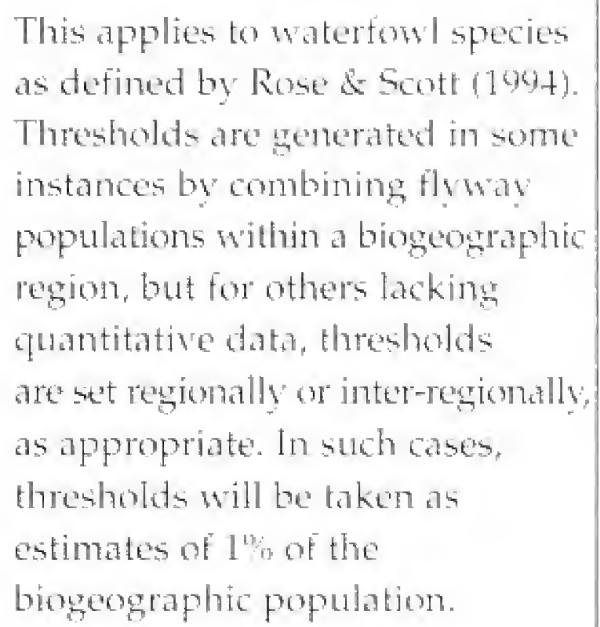 \\
\hline & $\begin{array}{l}\text { (ii) The site is known or } \\
\text { thought to hold, on a regular } \\
\text { basis, } \geq 1^{2 y} \text { of the global } \\
\text { population of a congregatory } \\
\text { seabird or terrestrial species. }\end{array}$ & $\begin{array}{l}\text { This includes those seabird species } \\
\text { not covered by Rose \& Scott } \\
\text { (1994). Where quantitative data } \\
\text { are lacking, numerical thresholds } \\
\text { for each species are set regionally } \\
\text { or inter-regionally, as appropriate. } \\
\text { In such cases, thresholds will be } \\
\text { taken as estimates of } 1^{\circ} \text { 'of of } \\
\text { global population. }\end{array}$ \\
\hline & $\begin{array}{l}\text { (iii) The site is known or } \\
\text { thought to hold, on a regular } \\
\text { basis, } \geq 20,000 \text { waterbirds } \\
\text { or } \geq 10,000 \text { pars of seabirds } \\
\text { of one or more species. }\end{array}$ & $\begin{array}{l}\text { This is the Ramsar criterion for } \\
\text { waterbirds, the use of which is } \\
\text { discouraged wherever data are } \\
\text { gond enough to permit the use } \\
\text { of (i) or (ii). }\end{array}$ \\
\hline & $\begin{array}{l}\text { (iv) The site is known or } \\
\text { thought to exceed thresholds } \\
\text { set for migratory species at } \\
\text { bottleneck sites. }\end{array}$ & $\begin{array}{l}\text { Theresholds are set regionally } \\
\text { or inter-regionally as appropriate: }\end{array}$ \\
\hline
\end{tabular}




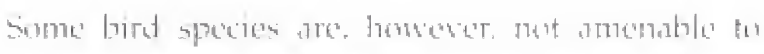
conservation through a siters-bared apponath and

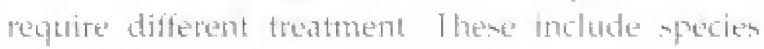

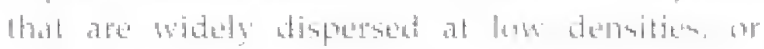

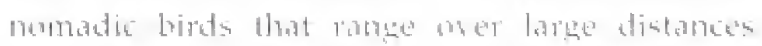

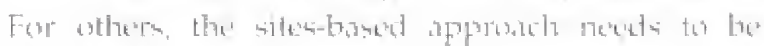

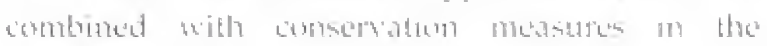

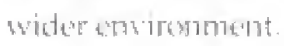

\subsubsection{Categories and criteria}

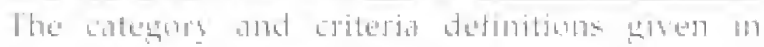

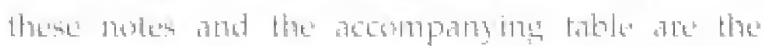

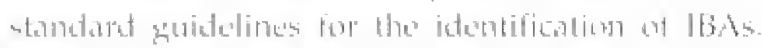

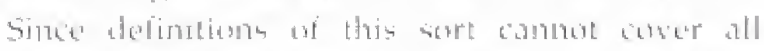

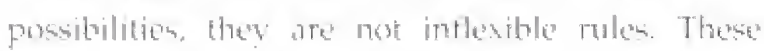

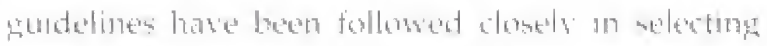

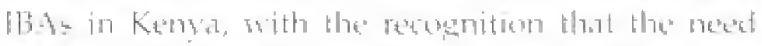

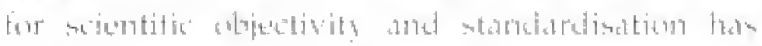

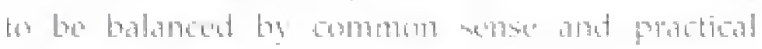

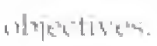

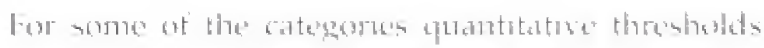

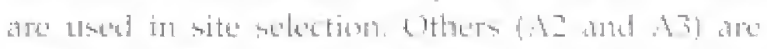

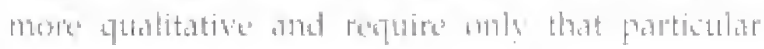

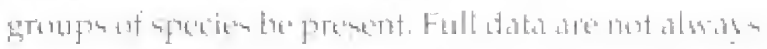

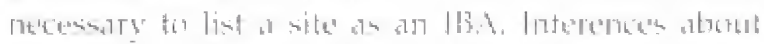

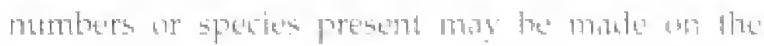

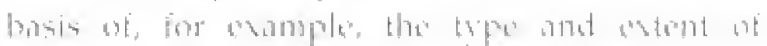

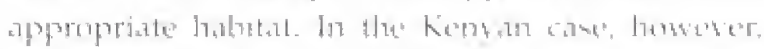

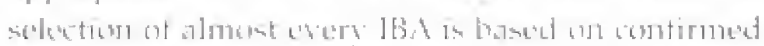

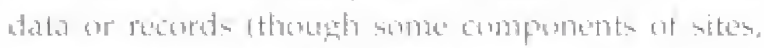

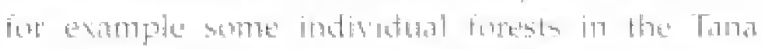

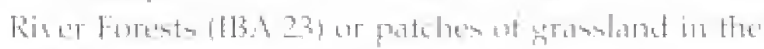

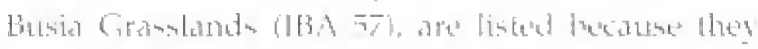

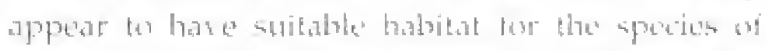

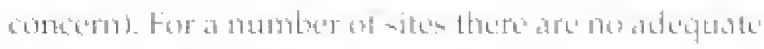

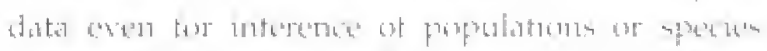

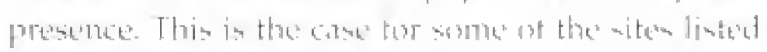

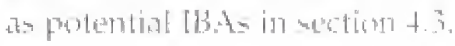

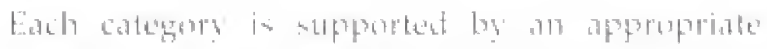

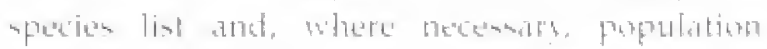

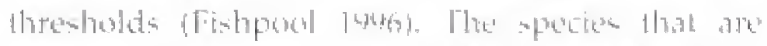

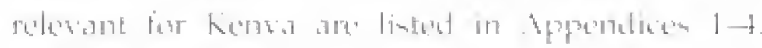
The categries and ariterial are summatiat in Thishen.

\section{Category AI Globally-threatened species}

Gritwh: The site regularly holds significant numbers of a globally-threatened species, ot other species of global conservation concert.
Wathe

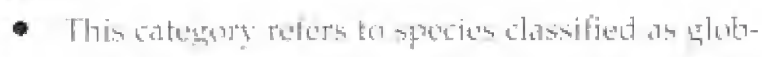

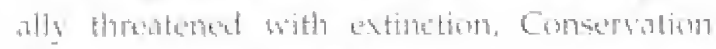
Dependeat ar Data Deficient actording bo the"

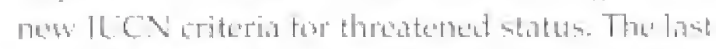

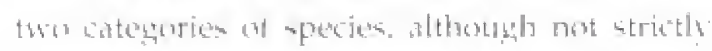

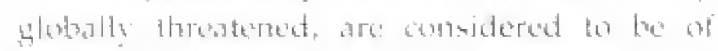

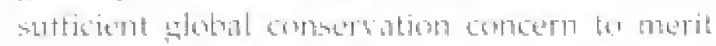

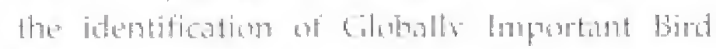

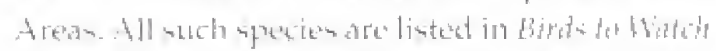

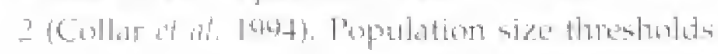
tor site selection and notes on lowe to apply thas lategert are agred regitumally

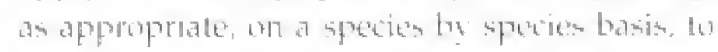

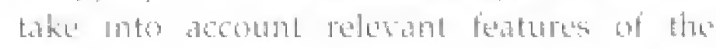

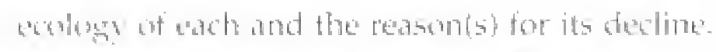

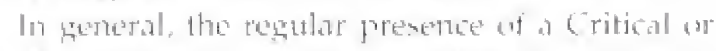

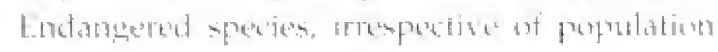

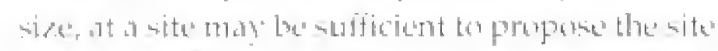

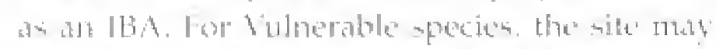

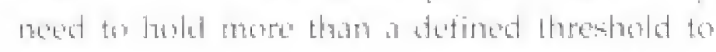

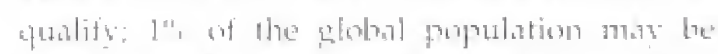
approprate bor somes. For Airical, a propteliation

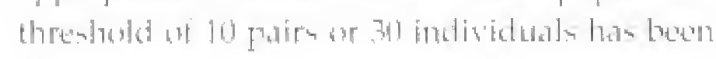

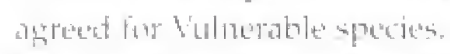

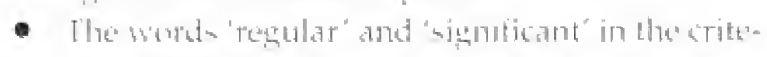

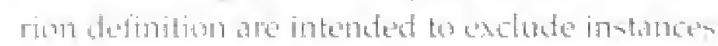

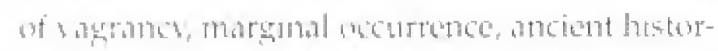

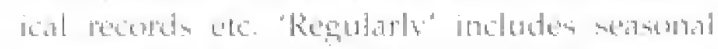

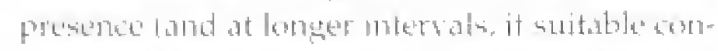

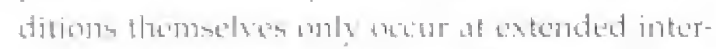

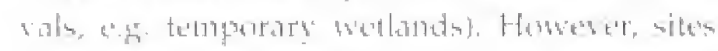

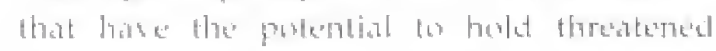

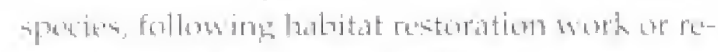

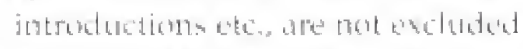

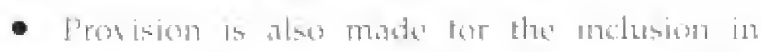

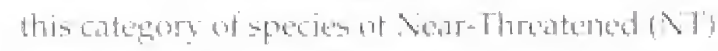

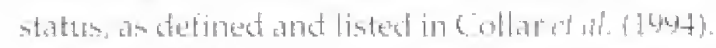

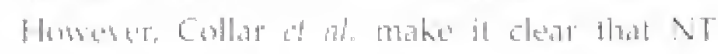

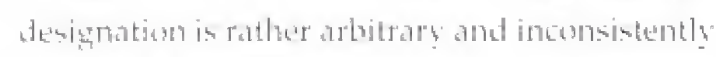
applied anderted to the list of threaterted

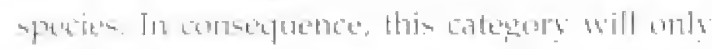

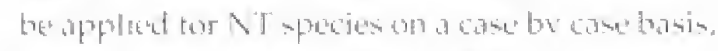

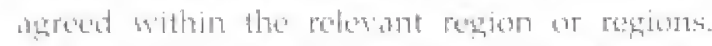

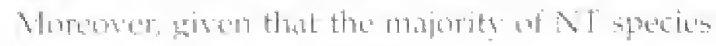

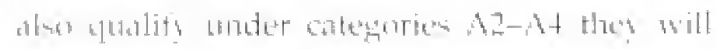

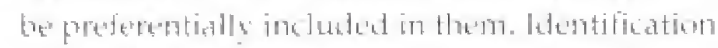

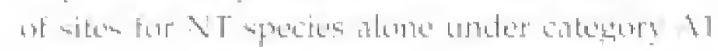
will therefore be tare: none bas been idontifict

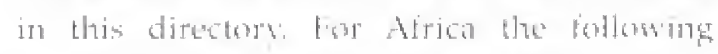
population thresholds hate been acred

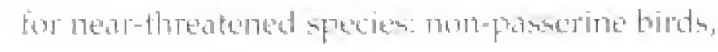




\section{Important Bird Areas in Kenya}

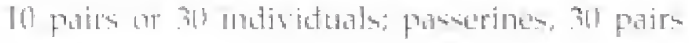

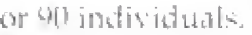

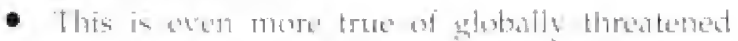

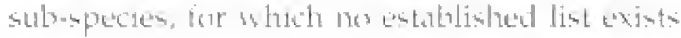

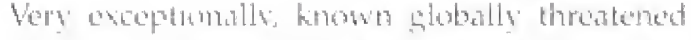

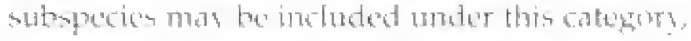

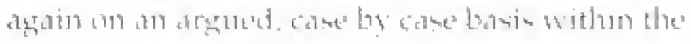

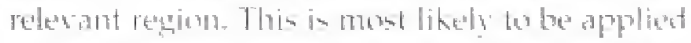

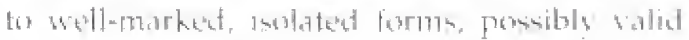

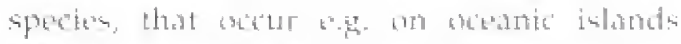

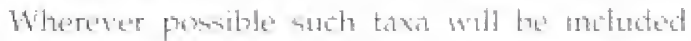

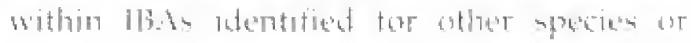
under wher criteria. No JBA hats heen identitiest

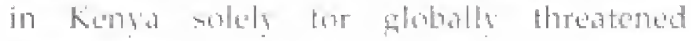
sultisperiens.

\section{Category A2 Restricted-range species}

Cribrum: The site is known or thought to hold a signiticant component of a group of species whose breeding distributions define an Endemis Bird Area (EBA) of Secondary Area (SA).

Notes

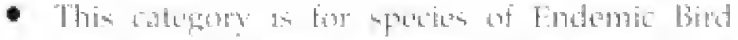

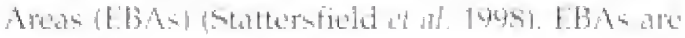

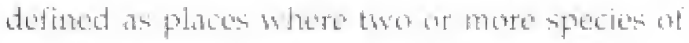
restricted range", i.t. with warted distributions al

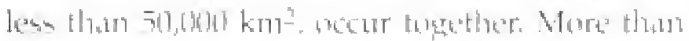

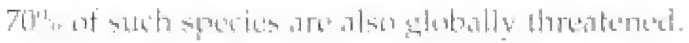

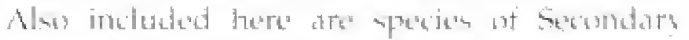

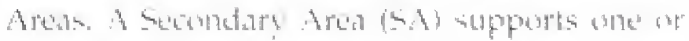

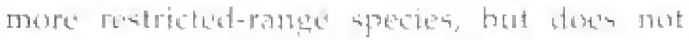

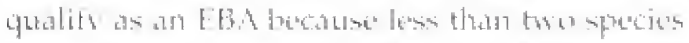

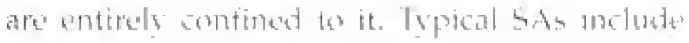

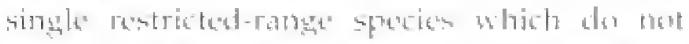
werlaps in stindributions with smy athere - ach

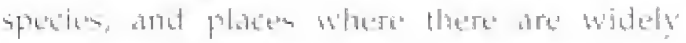

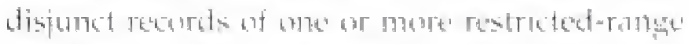

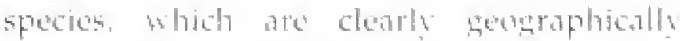

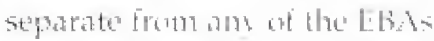

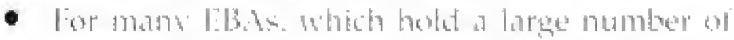
restricked-ranges spectes, it is neceseary that at nethork or sitem be bosen, hy amplementarity

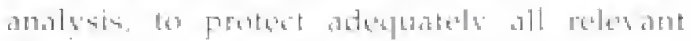

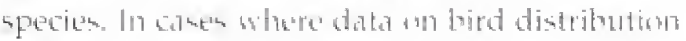

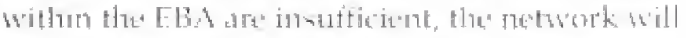

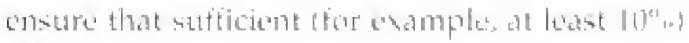
ot the kes hatoitat ty pers ane included.

- The "signitiont componend' term in the criterion

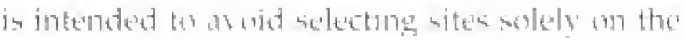

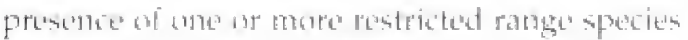

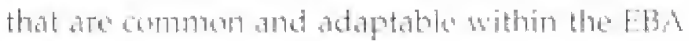

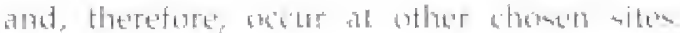

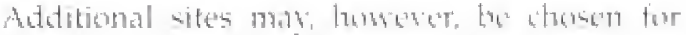

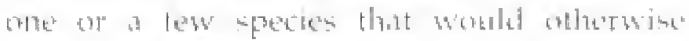
be undur-representert.

\section{Category A3 Biome-restricted assemblages}

Cratrip: The site is known or thought to hold a significant component of the group of species whose distributions are largely of wholly confined to one biome.

Wenters

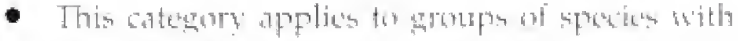

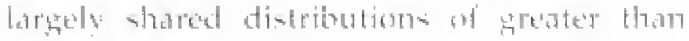

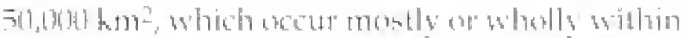
all or part of a particular bisme and are,

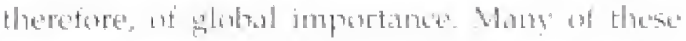

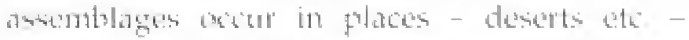

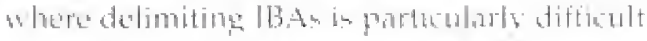

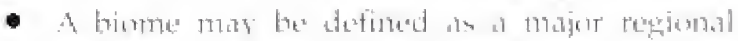

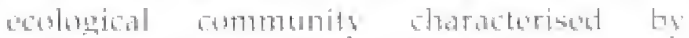

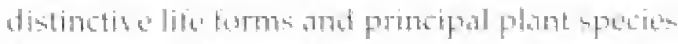

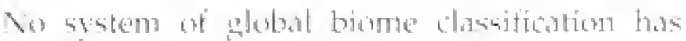

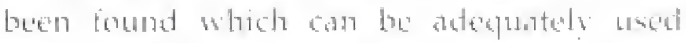

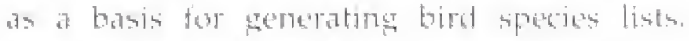

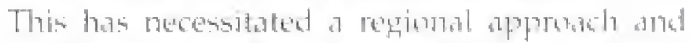
restlted itr intereregional difterences bethestr

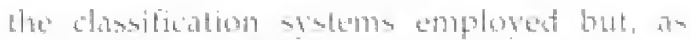

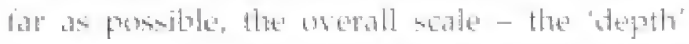

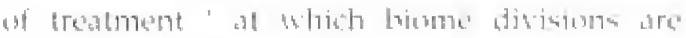
recongriot - is comparable

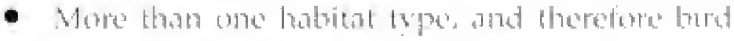

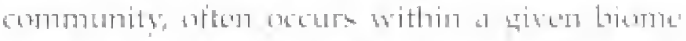

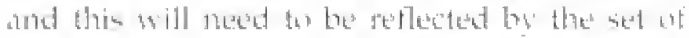

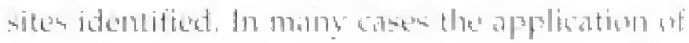

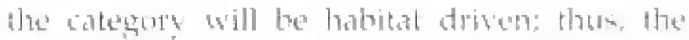

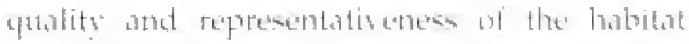

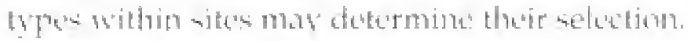

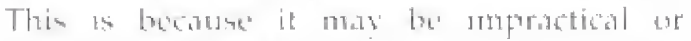

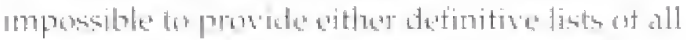

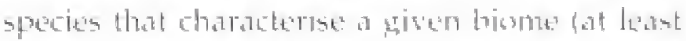

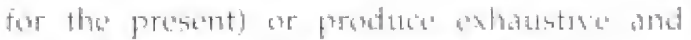

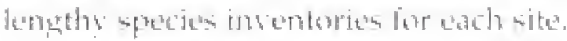

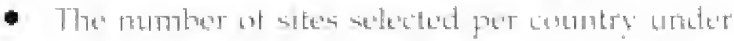
this cabergers should take inter acount both thes size of the country and the redertive ancount of a

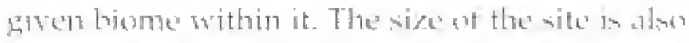

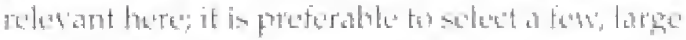

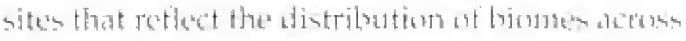

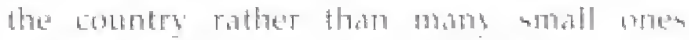
comfined th only a part of it. This will donsate
Introduction

Important Bird Areas in Kenya 


\section{Important Bird Areas in Kenya}

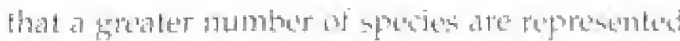

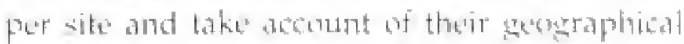

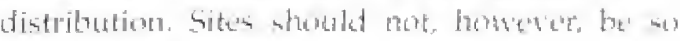

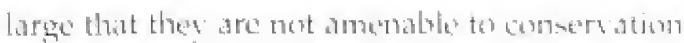
and, in some cases, suball sites sith Jight popolation densitics mas be preferable to lated men with fouver dencition.

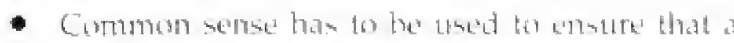

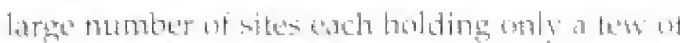
the bome-restricted spectes are that chosen

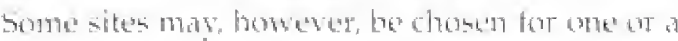

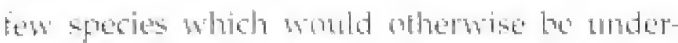
represented such as thime species onnfined to a

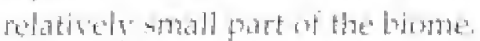

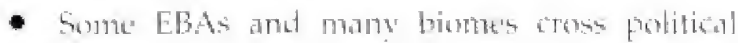
boundatres; where sit, the betwarke of sites

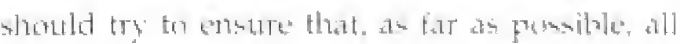

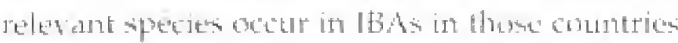
where the EBst of biome is stell represented.

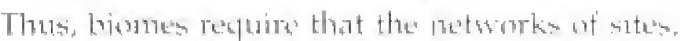
chomes by complementurity analysis, take

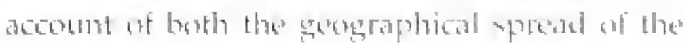

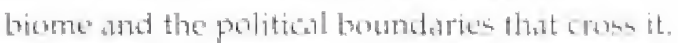

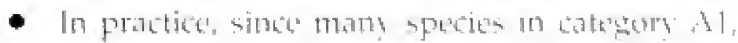

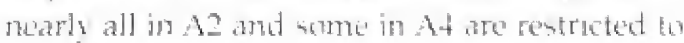

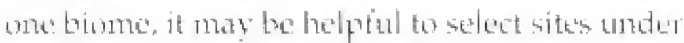
this categort once the waps in conerage of both species and gexpraphical spread that tesult trint application of the othere catestorien hate bert analyact. This will not apply to thame biomes

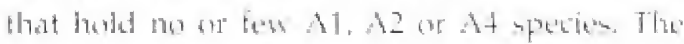
esisting protected area network is a prasmatic

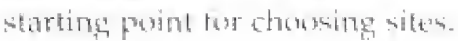

\section{Category A4 Congregations}

\section{Crifriti:}

A site may qualify an any one of the four criteria listed below:

(i) Site known or thought to hold, on a regular basis, $\geq 1^{\text {th }}$ of a biogeographic population of a congregatory waterbird species.

(ii) Site known of thought to hold, on a regular basis, $\geq 1^{t a}$ of the global population of a congregatory seabird or terrestrial species.

(iii) Site known or thought to hold, on a fegular basis, $\geq 20,000$ waterbirds or $\geq 10,000$ pairs of seabirds of ane or more species. (iv) Site known or thought to exced thresholds set for migratory species at bottleneck sites.

Nores

- This category applies to those species that are

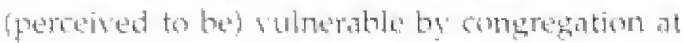

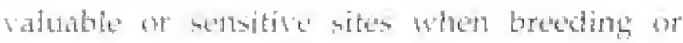
wintering ar while on passage.

- The term "haterbirt" is used here in the sitme sense as the Rambar Comvention whes "waterfunl' and coners the list of families as more pre" cisesy defined by thethands lntormational those o

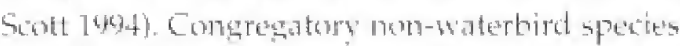
Atii\} inclufer both terrestrial species and these

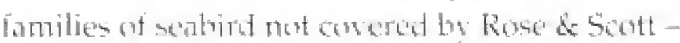

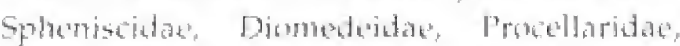

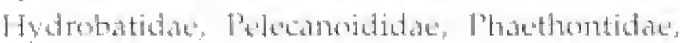

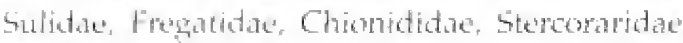
and Alcidiat:

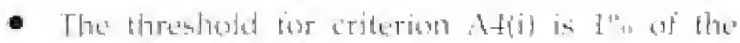
biogeographic pupulation of a congregatery

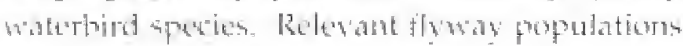

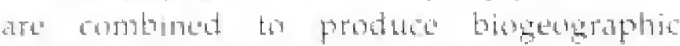
pepulation catimates

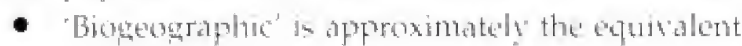
of Birdlites IBA regions bent ditters in some areas due to the genpulitical basis of the IBAcos" erige". This results in some complication in set-

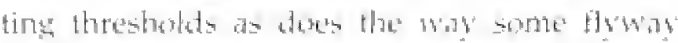
propulationa are shared between regrims in wither breteding or winteriug ranges.

- Threatrold digurus are sel for all congregatom? watkerenct bfocites, including those for which mone" are currently recongised by Rambar. Wethatso International hate collaburated in getme'rating numeric doreshold from range estimates

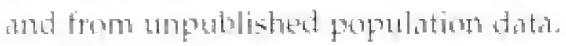

- There is an apparent lugical inconsisteney

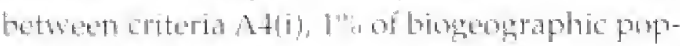
Whation, arsd At(ii), $1^{\text {the of }}$ global population of suabirats. It wan iedt, however, that the alternative

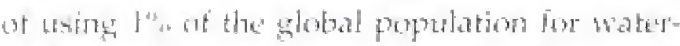

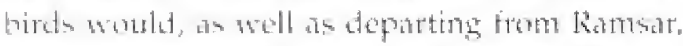
hate insulficient biological justifigativar beatuse

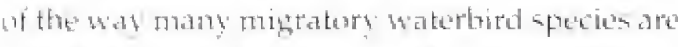
Wistributed and split into well-defined, diserete

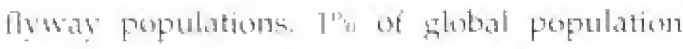
would have the effect of oner-emphasising regrional waterbirat enternics sines were much of

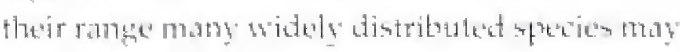
ranely gour at concentrations of $>]^{0}{ }_{1}$. For

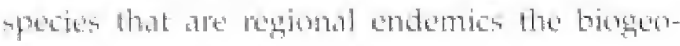
grinphic and global populationo ale the same? 


\section{Important Bird Areas in Kenya}

- Application un criterion At(iii) is to bu discudaged where data quality permits Af(i) and (ii) bis be used.

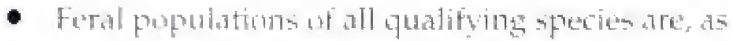

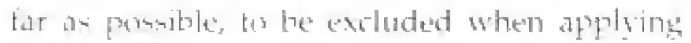
these stiterits.

- This categenry alan embracos sites over which

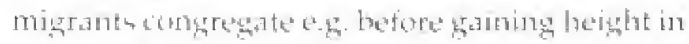
thermak, Althenth it is the agrspace here that is important. consers ation of the land beneath it

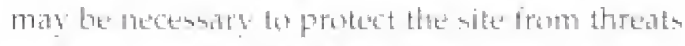

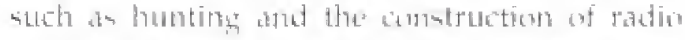

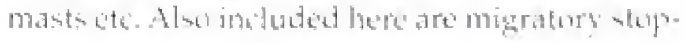

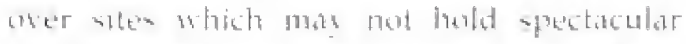

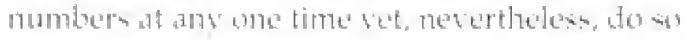

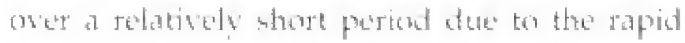
turnover of bists an pasalge.

\section{Gemeral points}

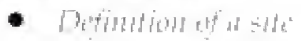

It should an har as formitible

if be different in charater or halvital or arnitionlogical importanese from the strrounding area.

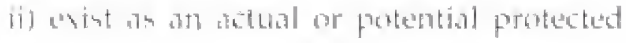
ales, whith or whithent buttel 70 otses, or be an ares which can be mandaged in wime waty for thature comsersation.

iii) ahome or with ather sites be a self sufficiont area which proutios all the

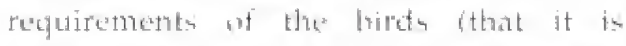
important fort which wase if durine the time? they are present.

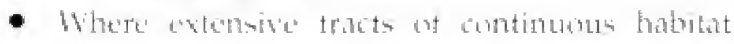
wecur which aro mportant for birdse ondy

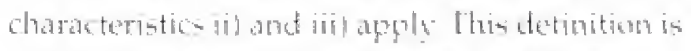

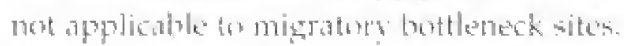

- Here also, it is turt possible to grive exhastige

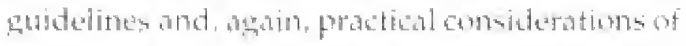

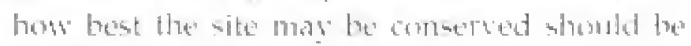
the tomernast bonsideration.

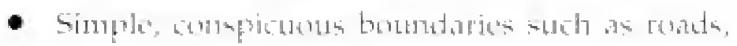
rivers, railma diencete mes be used to delimit site margins whils features steth ats wateraheds

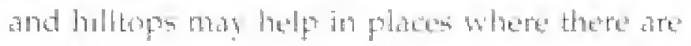

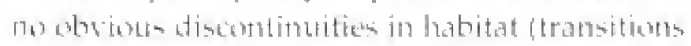
uf regelation or substrates. Bondedates of

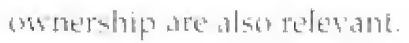

- There are ro fived size maxima or moninina for

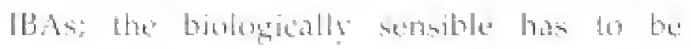
tempered with the practicall. Veither is there a defintixu ansmer bot hom to treat cases where a

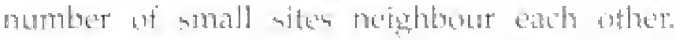

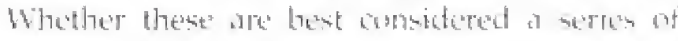

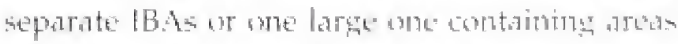
lacking ornitholigrical significance witl depephet upon local comersation realition.

\subsubsection{Kenya's ornithological importance}

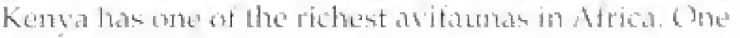
thousand and eichty-nine burd speriem are presently

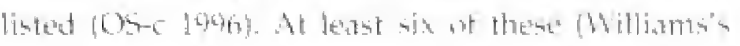
Larh, Sharpe's Iongclans Hindes babbler, Taita

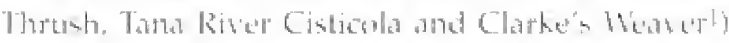

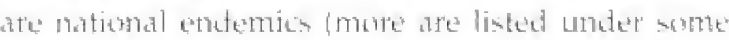

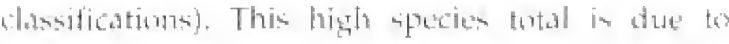

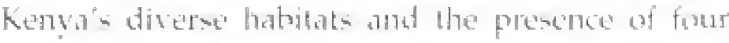
endente bird areas and six avian biomes (Fishpond

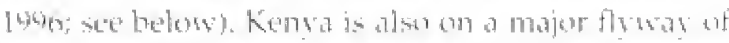
Palatearatic megrante, both land and water-birds,

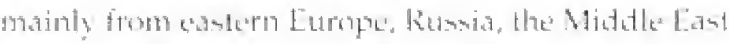

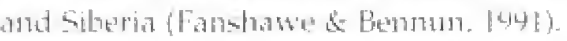

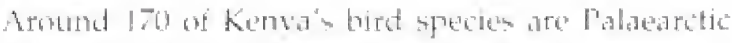

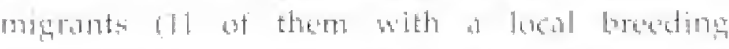

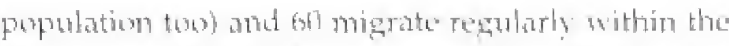

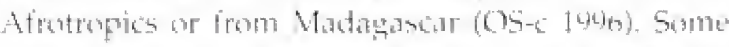

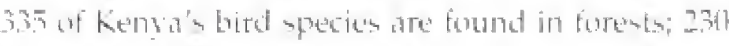
are entire? foreat-dependent, and 110 are "forest specialists, reguiring infact, undjoturled habitat

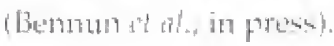

Collar \& Stuart (149-t) list 23 globaly threatened

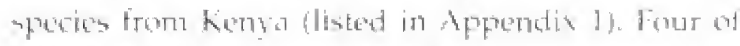
Whese are critically endangered, two endangered ant

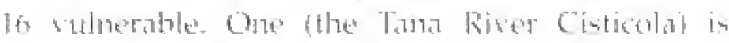
ctarsed as datadedicient. A forther to shercies are clussitind as newrethreaterted.

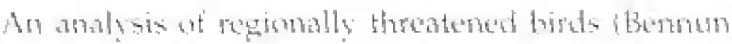

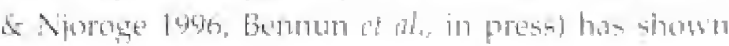

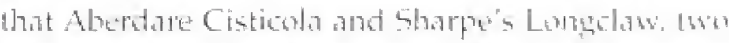

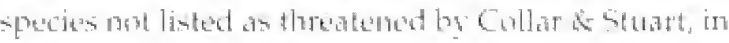

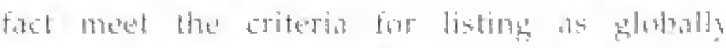

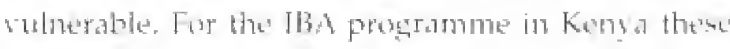

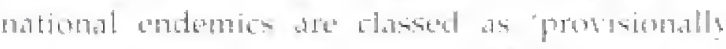
sulnerable and used to delineate bBAs in a simmilar

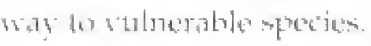

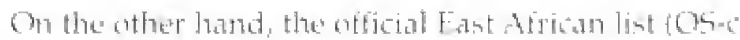

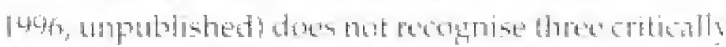

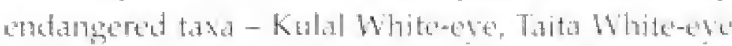
and Taita enpalis - as bull species. The two Taita

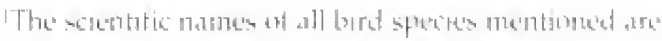
giste con the ondtent's
Introduction

Important Bird Areas in Kenya 


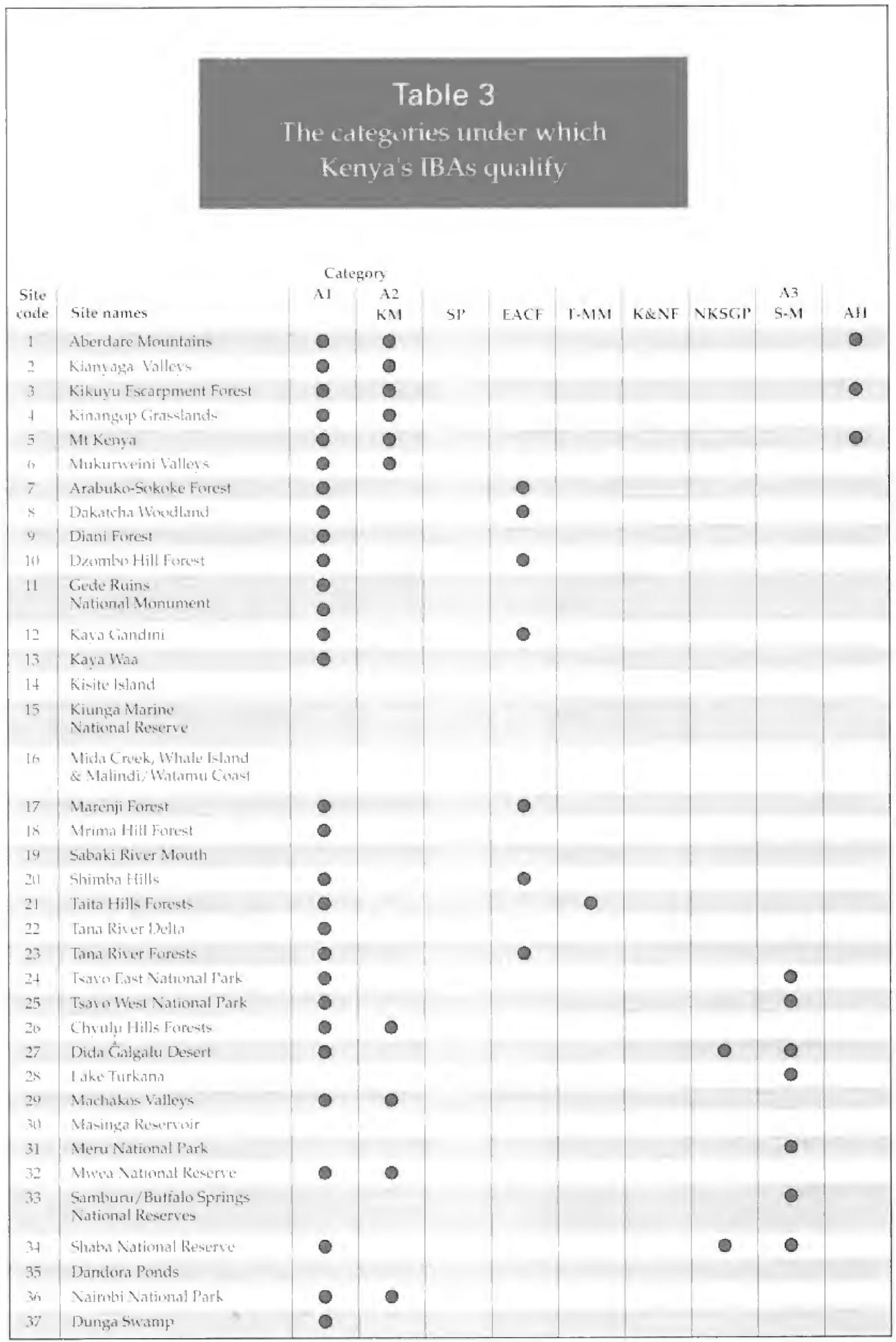




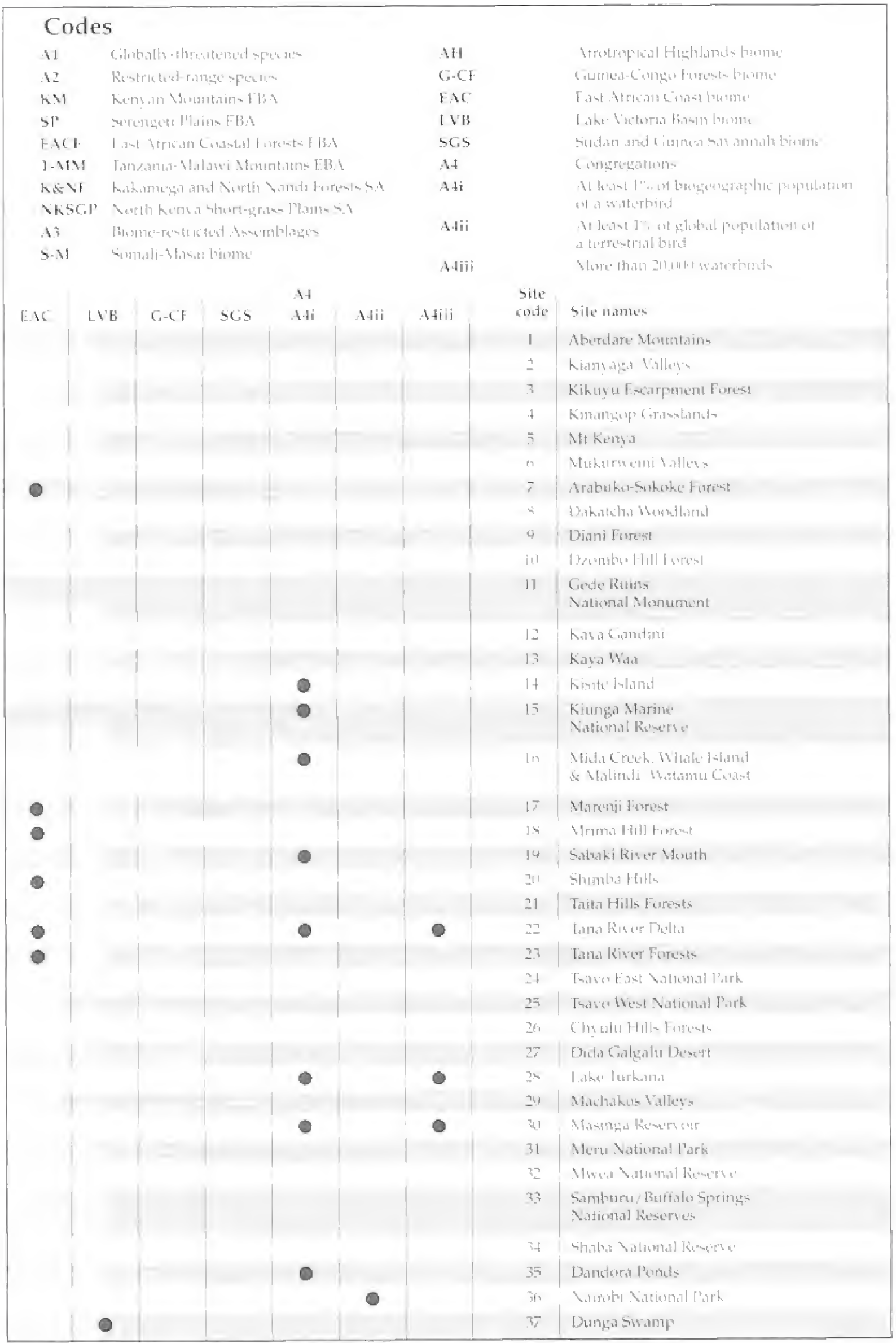

Introduction 
Important Bird Areas in Kenya

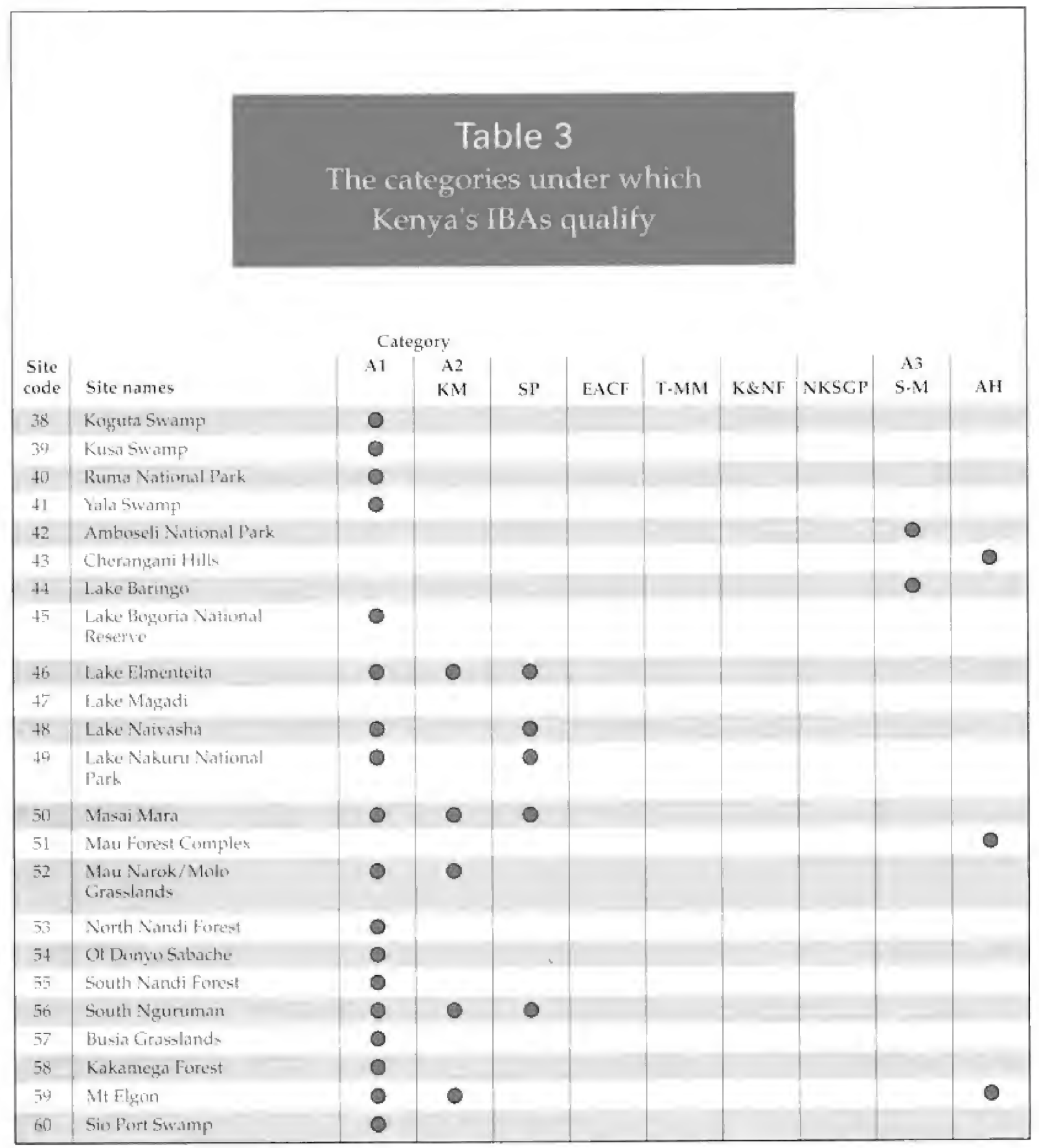




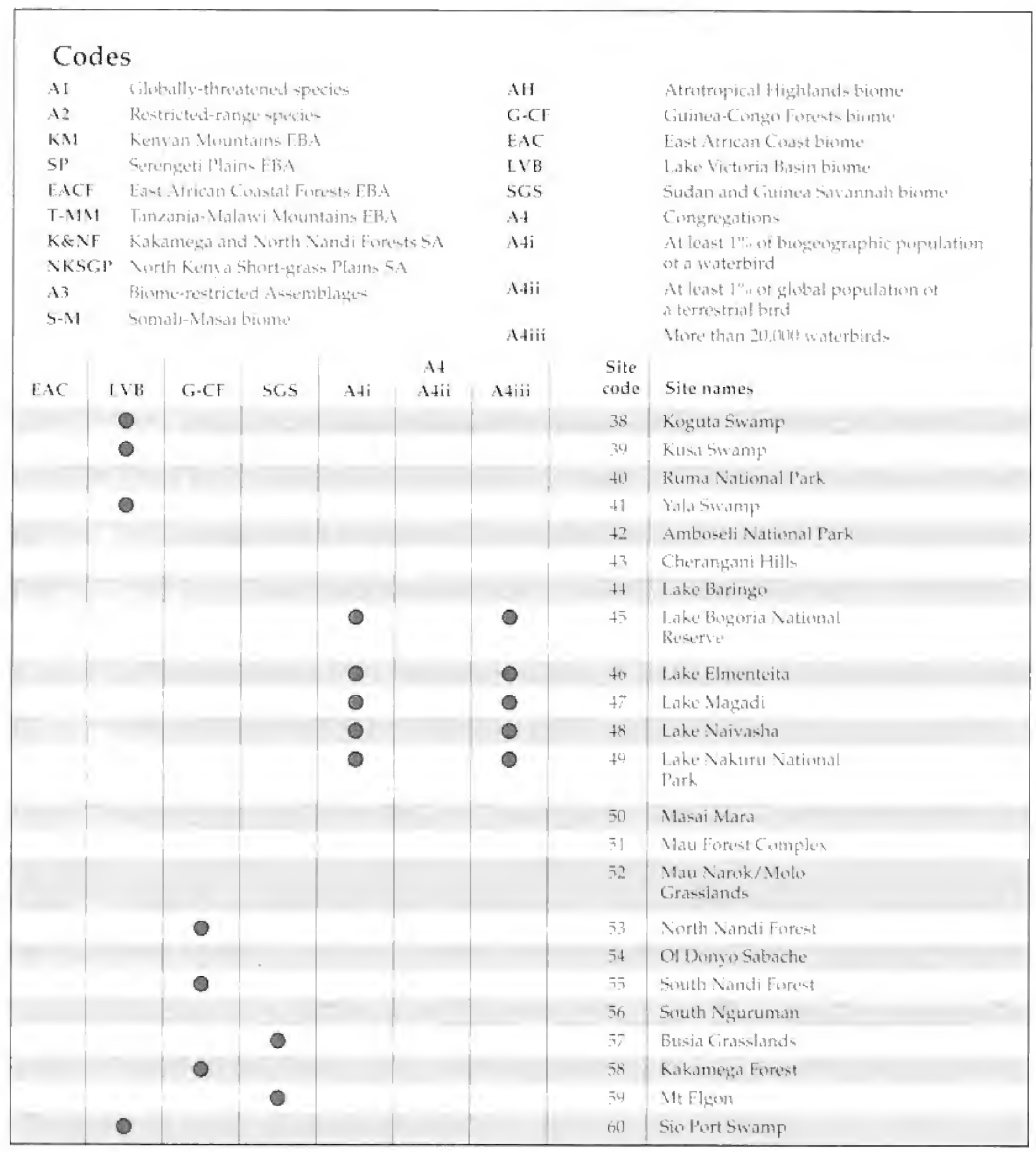




\section{Important Bird Areas in Kenya}

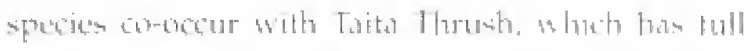

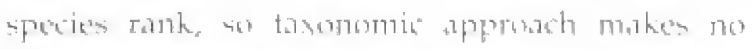

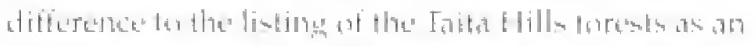

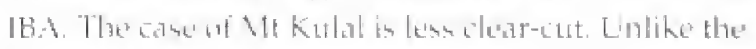

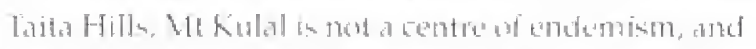

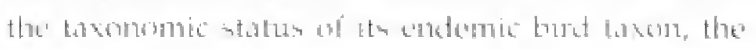
kulal White-eve is undear, It is neat generally

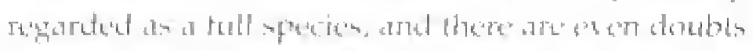

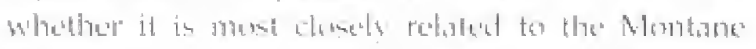

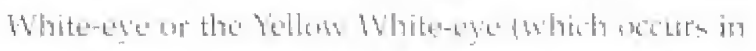

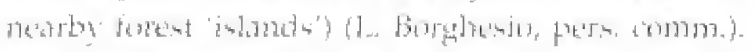

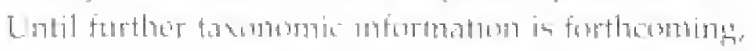

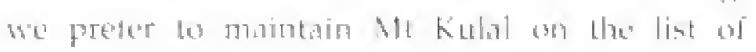
polemetial $13 / \mathrm{s}$.

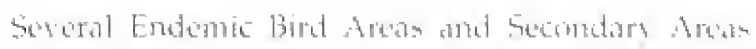

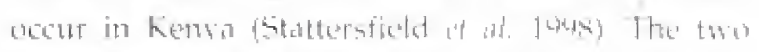

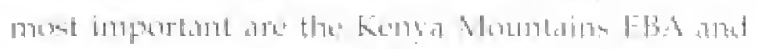

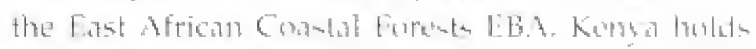

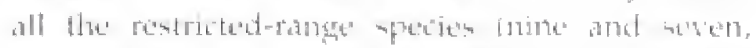

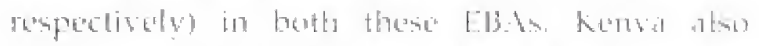

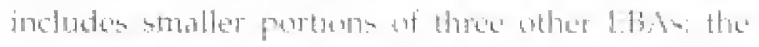

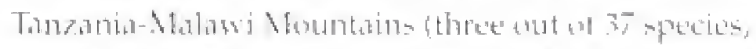

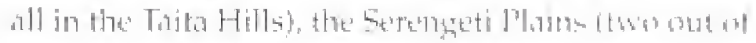

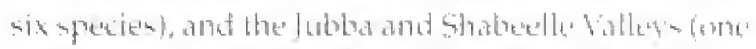

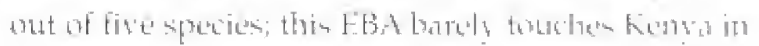

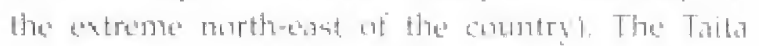

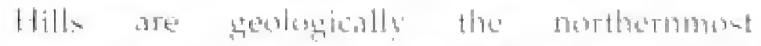

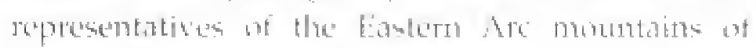

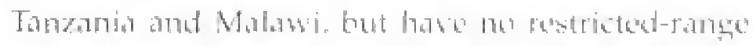

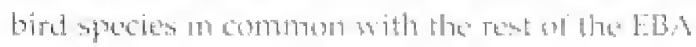

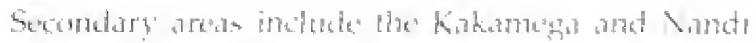

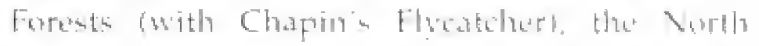

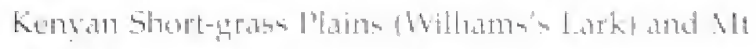

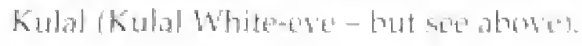

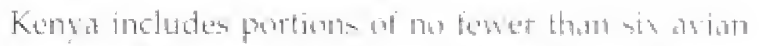

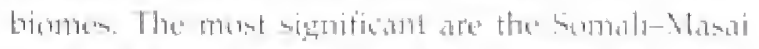

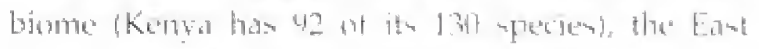
African Const binome (3)

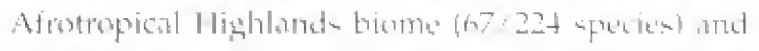

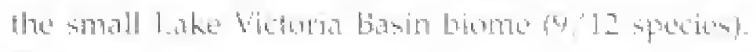

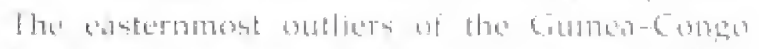

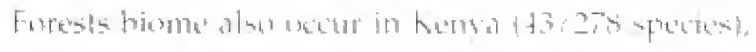

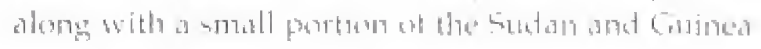

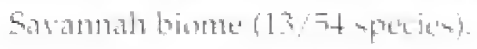

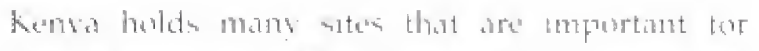

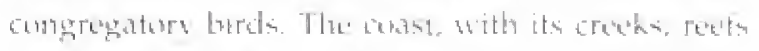

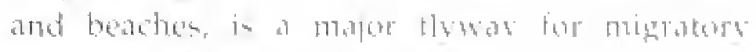

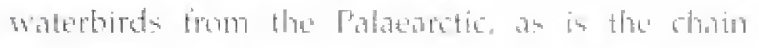
of lakes strefching alones the ritt allew trom

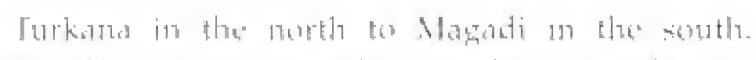

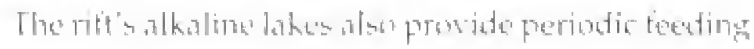

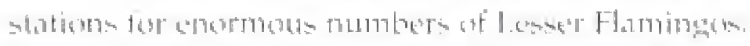

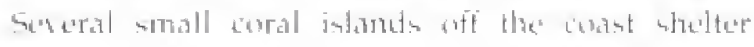

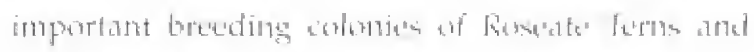
alluer seabitids.

\subsubsection{Selection of sites}

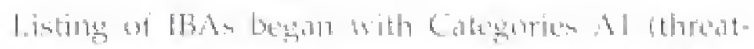

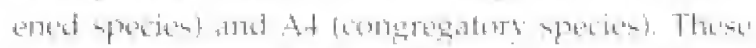

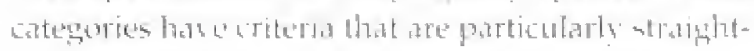

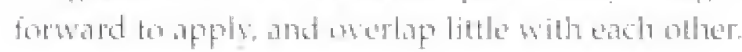

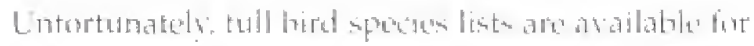

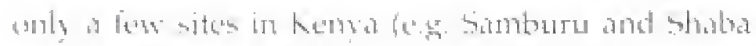

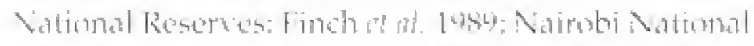

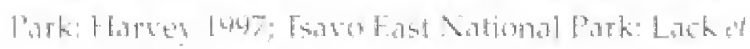

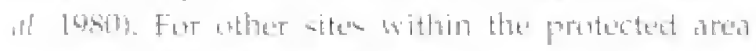

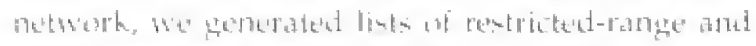

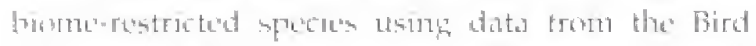

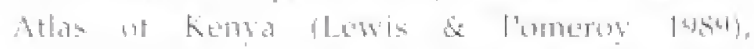
supplemerntert by upteded atlas explate thata and

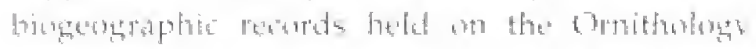

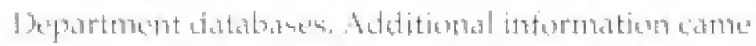

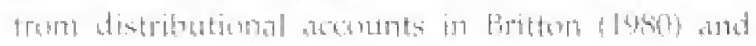

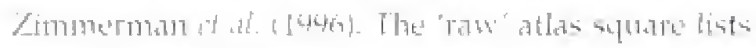

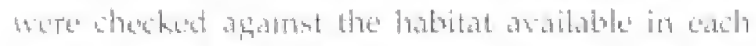

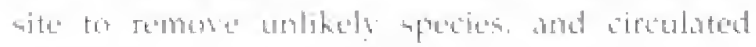

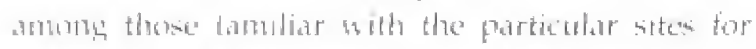

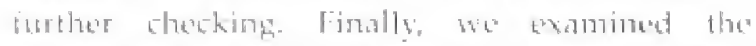

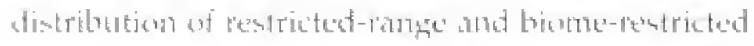

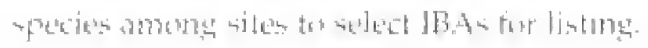

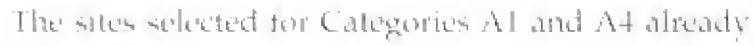

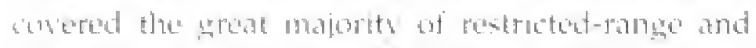

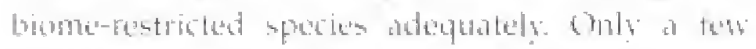

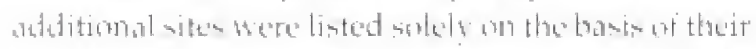

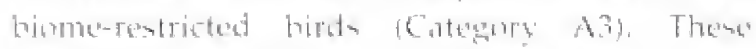
inclated. for tasmpte, Samburu and Buttaluspring

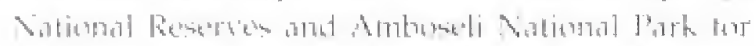

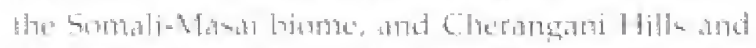

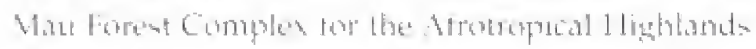

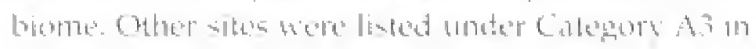

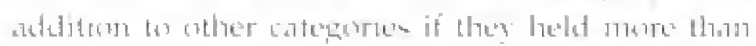

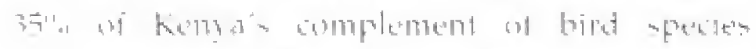

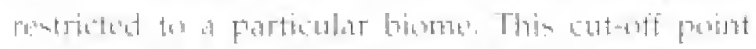

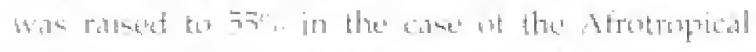

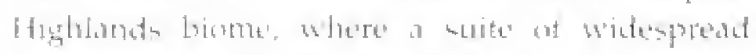

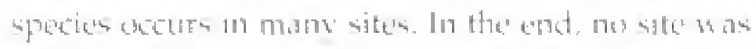

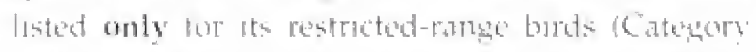

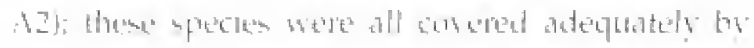

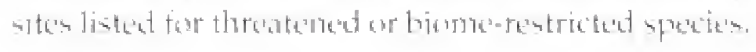

\section{2 \\ Introduction Important Bird Areas in Kenya}




\section{Important Bird Areas in Kenya}

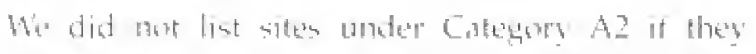

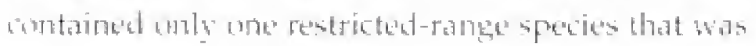

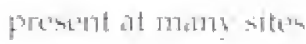

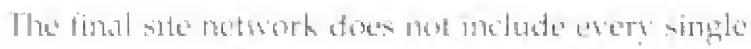

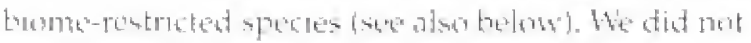

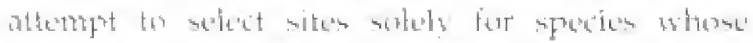
kaspyon range in marginal. comber the rationale that

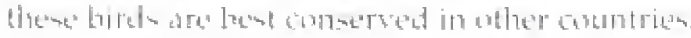

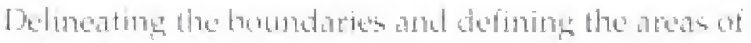

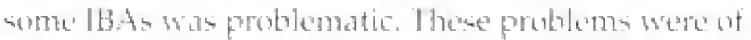
two mian hind

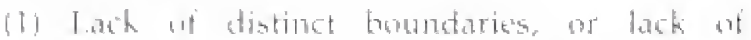
ingormation atomat the extent of a siten. Where

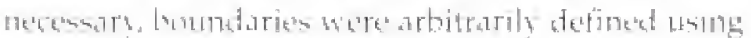

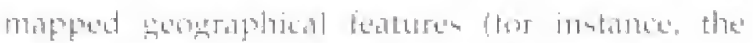

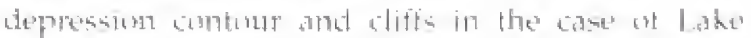

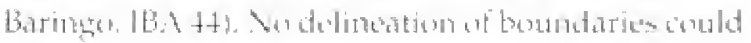

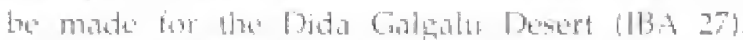

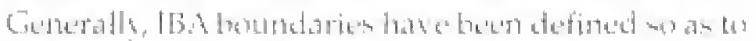
demanofe and area that is potentially amerable to

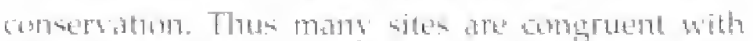
Existing protected areas. Sorne, like Lhe Mosad Mara,

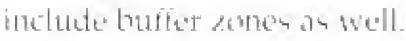

(2) L contions where the hataitat is tragnatented. In

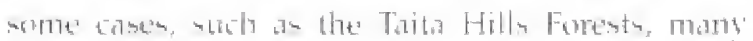

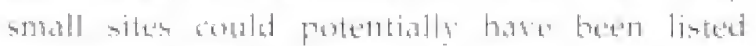

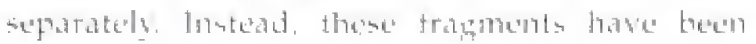

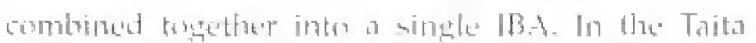

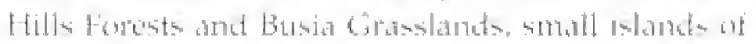
matural hatoitat are surfounded by an agricultural matrix. Here the site benderites follow the

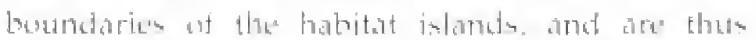

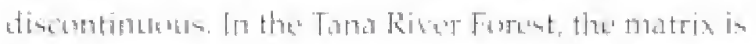

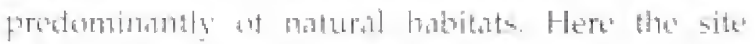

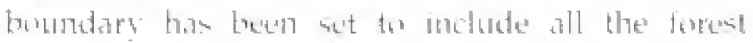

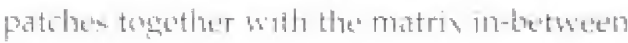

\subsubsection{The IBA network}

\section{Sites list}

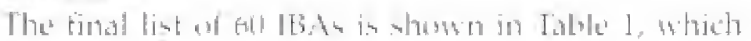
glses summory information about each. Seten aTe

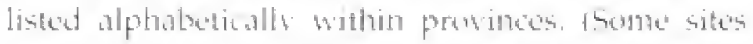

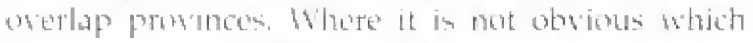

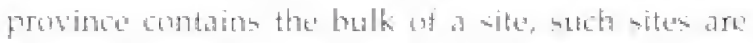

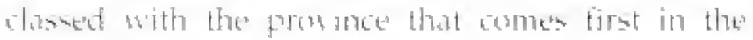

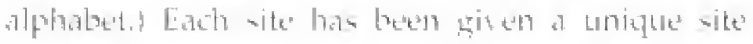

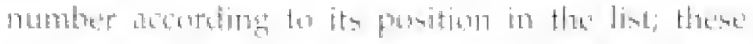

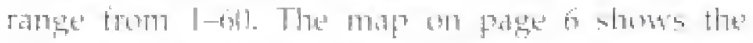

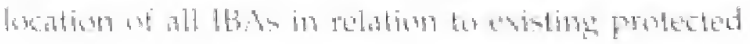

areate and histural festures.

Table 3 indicates in moret detail the caterom or kategories under which particulat sites glatify Substantially mowe sites are listad for ghoballythroatened species (Table f) thane for any ather sing de

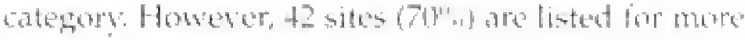
than bate category:

\section{Geographical distribution and habitat}

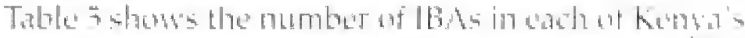

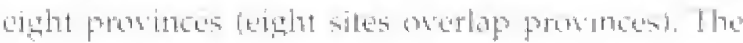

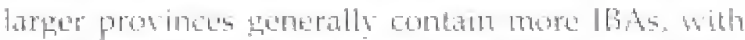

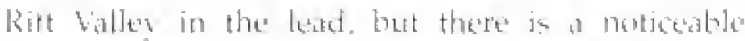
ioncentration of sites in Coast Promince and noves

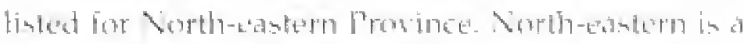

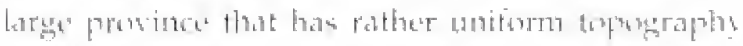

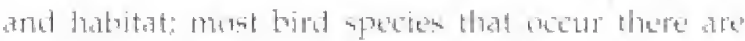
widesprent. and it in difficull to define farticular areas that are more impertant than onthers for biret

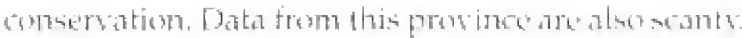

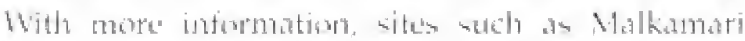
National Pank may qualify as IBAs fit is prlaced on the shadow list at the mument).

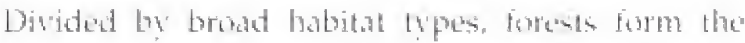
single largent group with wetands clane behind

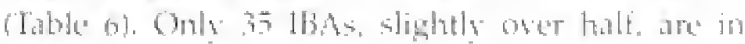

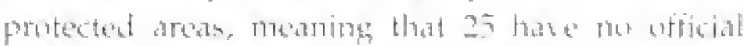
probection. Mast al the" torest, and a majerits of the

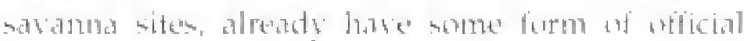

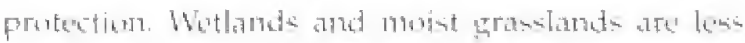

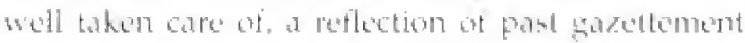
policies that concentrated om impertance for hages

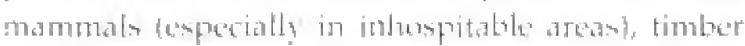

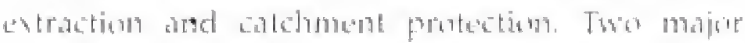

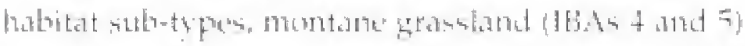

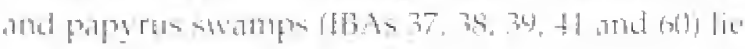

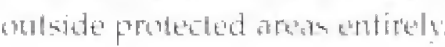

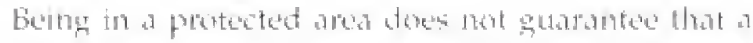

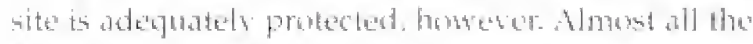

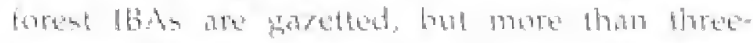

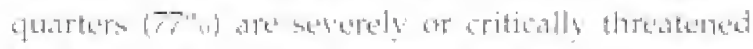

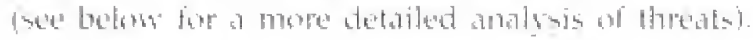
Munt grassland and 'othere' babitat infecilically,

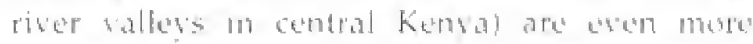

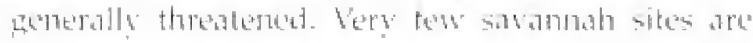

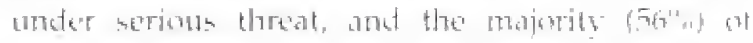

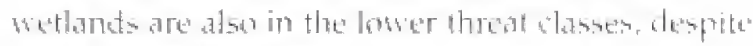
a lack of official protection.

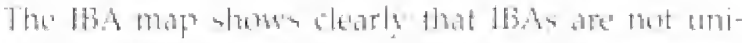

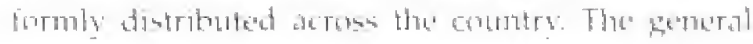

Introduction

Important Bird Areas in Kenya 


\section{Important Bird Areas in Kenya}

\section{Table 4 \\ The number of IBAs in each category \\ (24 sites are listed in two \\ and 18 in three categories)}

\begin{tabular}{|c|c|c|}
\hline Category & $\begin{array}{l}\text { Number } \\
\text { of sites }\end{array}$ & $\begin{array}{c}\text { Number of sites } \\
\text { in this caltegory alone }\end{array}$ \\
\hline A1 Globally-threateneat species & th & 5 \\
\hline A2 Thewtricted-range species & 34 & it \\
\hline A3 Biome-restristed spetins & .32 & h \\
\hline Af Congregationsto & 1.7 & sis \\
\hline
\end{tabular}

picture is of a few large sitem in the semi-ard parts of the exuntry and many, ofter smaller sites in the higher rantall areas - which, unsurprisingly is where all of the forests and moist grinstand, and many of the wetlands, nocur. These parts of Kensa are also dencely populated, generally with intense pressure on matural tesontces. This has obrious implications for the corsersation of many tBAs.

\section{Sizes}

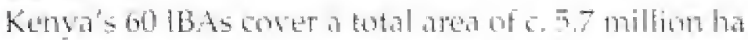
(taking a notional area of 250,000 ha for that Dida Galyalu desert). This is about $10^{13}$ of Kangate land area. Howerem, the size of sites varits oner seren

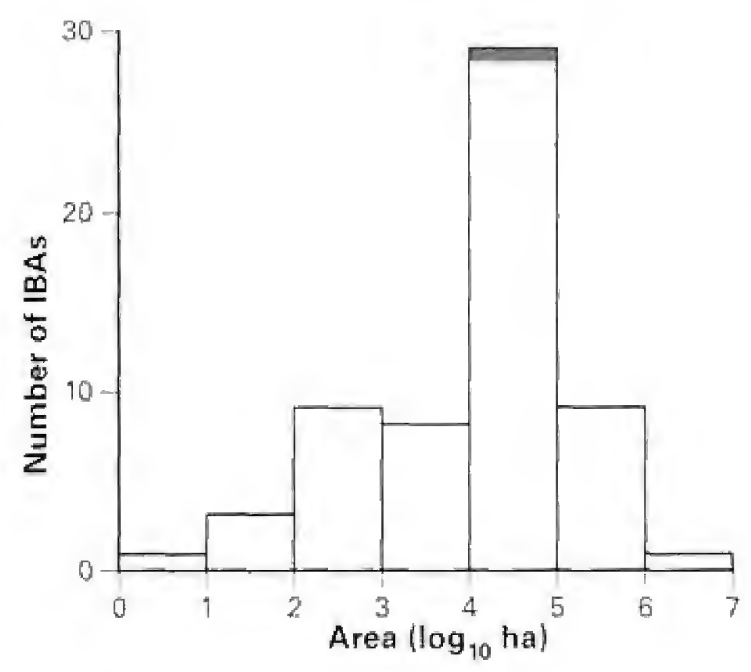

Figure 1

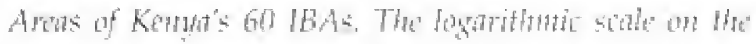

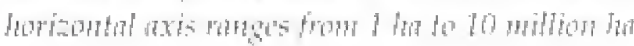

orcters of magnitude, from as small as i ha to more than 1 million th. The nedian size is 18,300 ha, whilt: the nean size is 95,200 hal - shuwing the dispropor= thonate effect of a tew very large sites (Figure 1).

Alemogh the land area corered by the ret of 1 BAs is stibstantial, most of thus $(72.8 \mathrm{~m})$ is alleady within protected areas (PAs). Only around 1.5 milliom ha extra would be reguired to protect the 25 sites that are not yet gaveted as some "ype of reserve. Siter

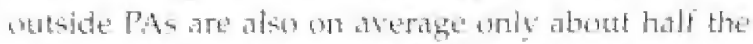

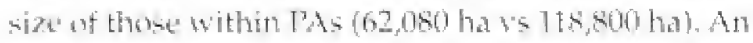
additiona! 20 sites conde be brought into the PA metwork by acquiring, fust 240,000 ha of land.

In practice, the chances of gavetring new $\Gamma^{3} / \mathrm{s}$ in kenya are dsually likely to be poor. For the maknity wif unprofected site's other forms of conservation mest thus be putrited.

\section{Gaps in coverage}

Appentices I - f show our current knomledge of the distribution of threatened, restricted-range, biomerestricted and congregatory species fexceding threshold values anomg Kenya's BAAs. Sites where a spectes has been redorded regularly ane shown with a closed cirter an open circle indicates that the spectes is only a vigrant or irregular visitor. The following species ockuring in Kenya and listed by Fialipos (1906) are not adequately conered by the present IBA network:

\section{Globally-thraterted sifertits}

Shotill: This species has sccurred in three IBAs but is orly a vagrant to Kemva. 


\section{Important Bird Areas in Kenya}

\begin{tabular}{|c|c|}
\hline \multicolumn{2}{|c|}{$\begin{array}{l}\text { Table } 5 \\
\text { The number of IBAs per province } \\
\text { (eight sites overlap provinces) }\end{array}$} \\
\hline Province & $\begin{array}{l}\text { Number } \\
\text { of IBAs }\end{array}$ \\
\hline Rift Vafley & 20 \\
\hline Const & 14 \\
\hline Fanters & 11 \\
\hline Centras & b \\
\hline Nyanza & 5 \\
\hline Wextern & 5 \\
\hline Natrobibi & 2 \\
\hline 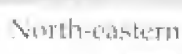 & a \\
\hline
\end{tabular}

\section{Table 6 \\ Kenya's IBAs and their level of protection and threat, by dominant habitat}

\begin{tabular}{|c|c|c|c|}
\hline Doménant liabilat & $\begin{array}{l}\text { Number } \\
\text { of sites }\end{array}$ & $\begin{array}{l}\text { Number in } \\
\text { protecled areas }\end{array}$ & $\begin{array}{l}\text { Number severely } \\
\text { or critically threatened }\end{array}$ \\
\hline Furest & 22 & $20\left(\left.9\right|^{*}{ }_{1}\right)$ & $17\left(7 / 7^{n} n\right)$ \\
\hline Bhtlund & 18 & $5\left(2 \mathrm{~s}^{\circ} \mathrm{st}\right)$ & $8\left(44^{\prime \prime}+1\right.$ \\
\hline Bushland semidarid or arid & 12 & $7(58)$ & $1\left(N w_{0}\right)$ \\
\hline Mosist grasistand & to & $3\left(\overline{3} 0^{63} \cup 1\right.$ & $5(3,3 \cdots \cdots)$ \\
\hline Other & 2 & $0\left(0^{\mathrm{n}}\right)$ & $2\left(100^{n}\right)$ \\
\hline Total & 601 & $3 \overline{3}(\bar{x} 4)$ & $33\left\{55_{0}\right)$ \\
\hline
\end{tabular}

Tana River Cisticola: "The status and distribueion of

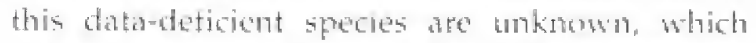
makes conservation action difficult! it is thought

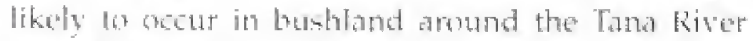
Firesta (13) 23).

Kulal Whitene: This is not recognisted as a tull species in the region, and Mt Kulal (wowere it occurs) is not listed as an 1 BA for the time being. (Gee abotin)

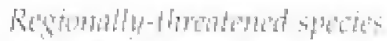

The IBA crikeria do nut make provision for

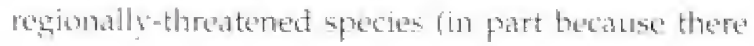

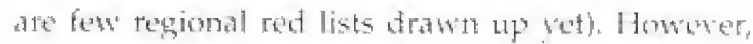
the Kenyan IBA network provides goot conerage of regionally-threatend species (Benmun \& Njoroge: 1496). Those not property condered at the moment include:

Orange River Francolim: This species is at the edge onf its range in Kenge, where it is known form the Huri Hills and Mt Kulat.

Rock Pratincole: Resictent along the Nroja River in western Kenva, but not elsowhere in the comtry. This site deres not quality an an 1BA on premernt incormation. 


\section{Important Bird Areas in Kenya}

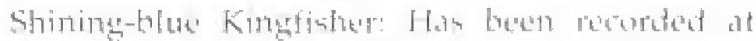

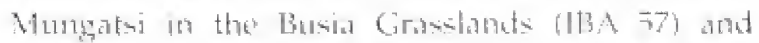
at Kakamega Forest (IBA 5xi). This mpories is at the edge of its ranger in Kency and probaloly nant resistent

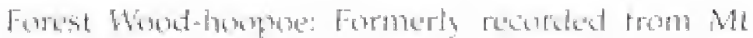

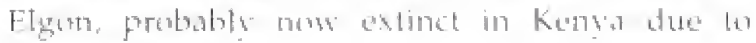
dutereatition

Collared Latk: A Somali-Masti biome specien that is contined tes the dre Home at Africa. This lark is locally antomon in somalia: there are soattered

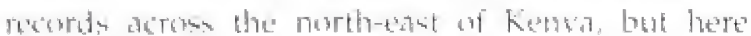

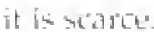

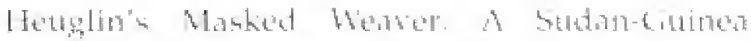

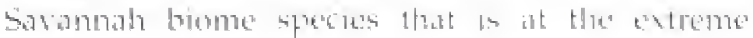

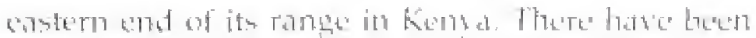

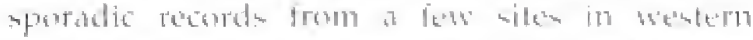

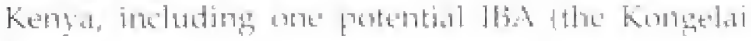
Jisarpment?

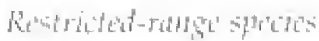

Kulal White-ere: Sec abores.

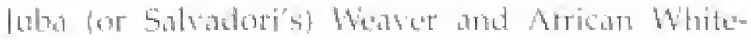

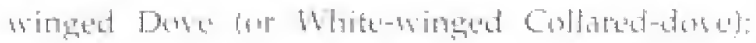

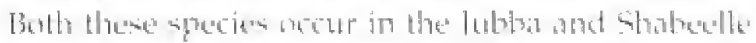

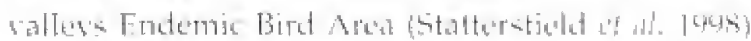

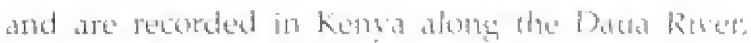
where they are logatle bom a sery matl section of that IBA, along the Ethiogra-kensa burter in the extreme nosth-east on

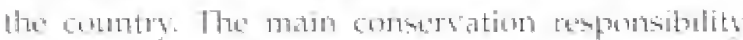
for these and the wher resericted-range species in this EBs rests with Ethinpia and Somalia. Malkamari National Park is thaught likely tor comatin both the these athd the weaver and is listed

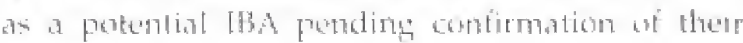
presertite.

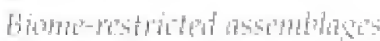

\section{Somali-Masai biome}

For Atrican White-binged Dowe, Gulared Lath and fuba Weaver, ste alone. Two other sperion at murth-watern Kentar are nol lepresented in the

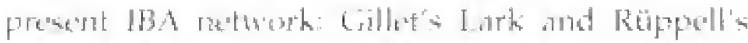

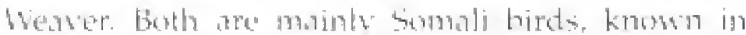

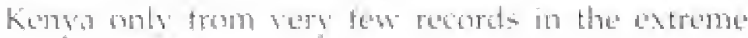

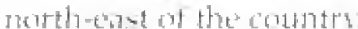

\section{Afrotropical Highlands biome}

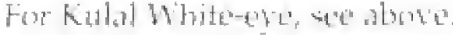

\section{Sudan and Guinea Savannah biome}

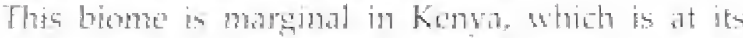
antreme eastern limit. Four spectes, White-crested Turato, Red-pate Cisticola, Bronze-tated Starling

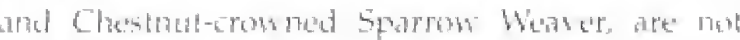

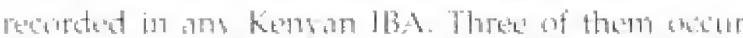

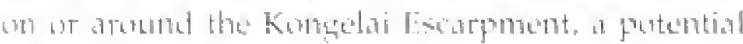

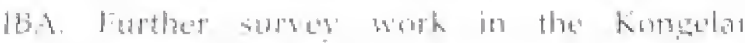

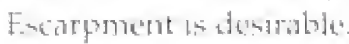

\section{Introduction}

Important Bird Areas in Kenya 


\subsection{Conservation issues}

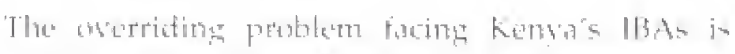

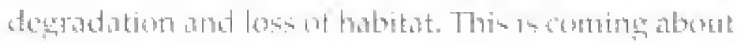

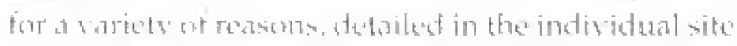

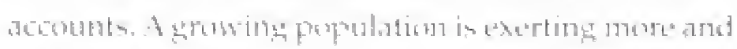

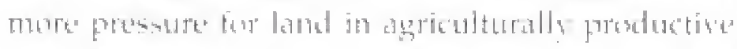

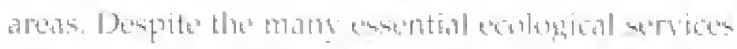

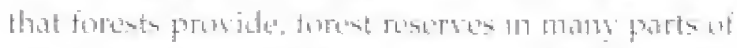

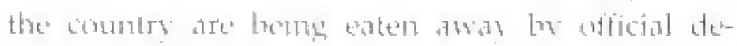

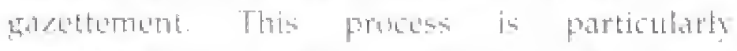

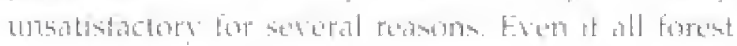

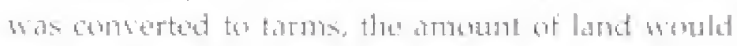

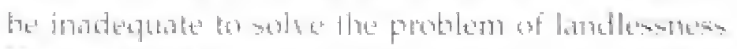

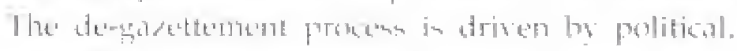

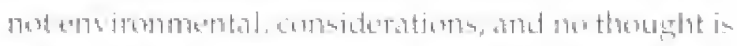

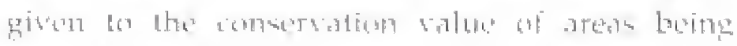

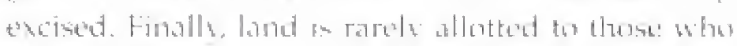

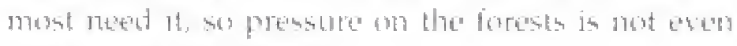

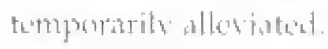

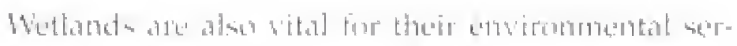

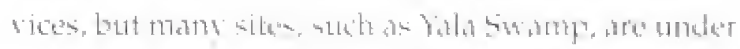

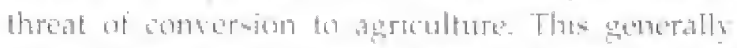

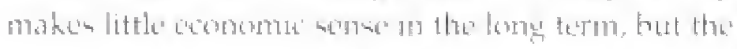

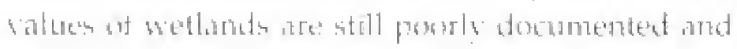

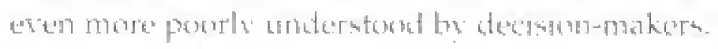

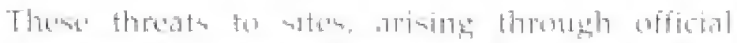

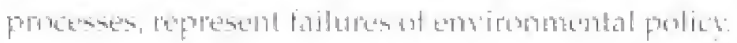

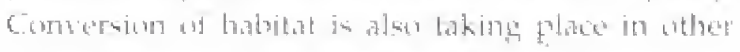

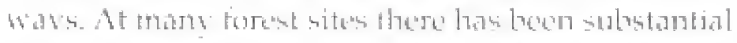

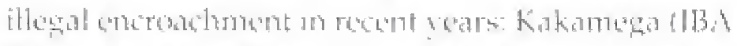

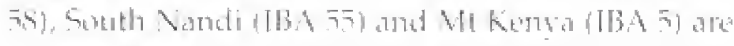

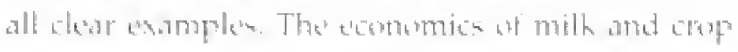

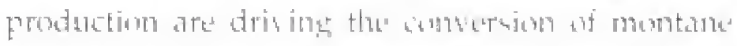

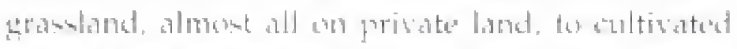

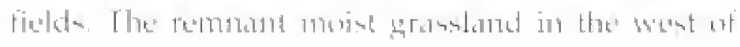

the country being turned into suger cane fiedds for

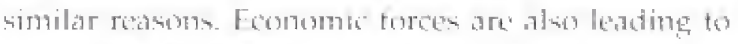
the loss of migration resates and butfer zames for

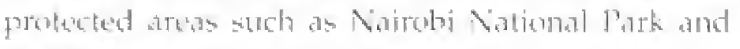
the Masti Mara National Reserse.

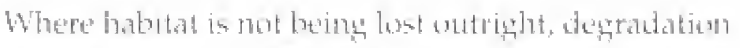
i. when a problem. This takes many forms, ranging

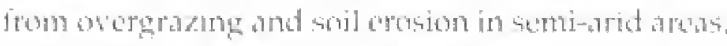
to remoral wh tussock grass species in momitame sprastando ancessise abstration of water from

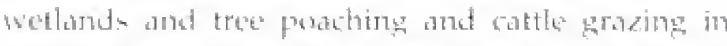

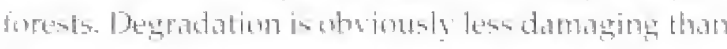

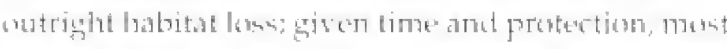

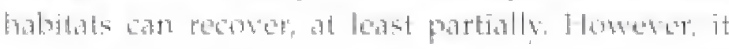
can also leat to the foss at bould species and

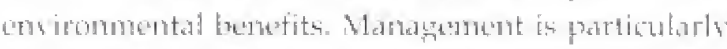
puor in many forest reserves, and degradationd dese to

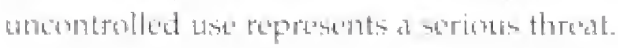

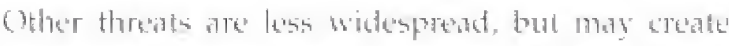

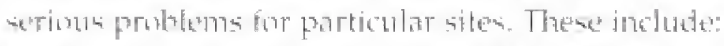

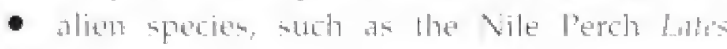

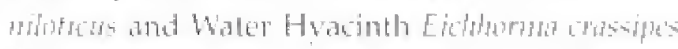

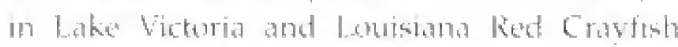

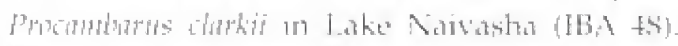

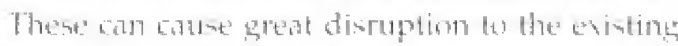

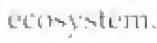

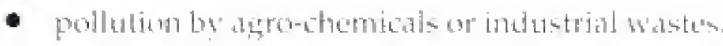
This is a growing problem al many welland siten, and inatequately controbled by existires enforcement mednansms (discusted below t.

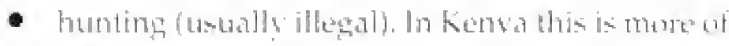

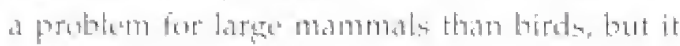

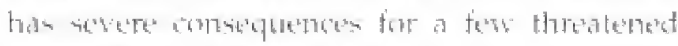

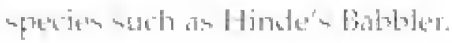




\subsection{The institutional, legislative and policy framework for biodiversity conservation in Kenya}

\subsubsection{Institutional structures}

In Kenca a number of sorerroment departments and organisations have partially usertapping

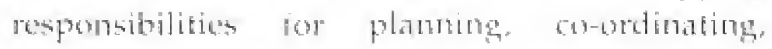
implementimg and monitoring gonernment policiss and pegislation on the edrironment. NBU (1992) lists no fewer than 38 Govermment ministries, departments, and parastatal organisations that alte

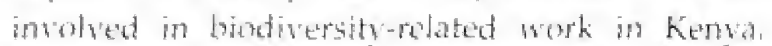
The mandates for differen tacets of ensingmental managenomt change as ministries are steated and others abolished, and the limite of institutional responsibility are nus always cleatr.

An Inter-Ainisterial Committet an the Enviroment

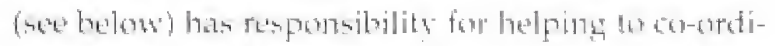
nate institutional roles. In addition, imatitutions and

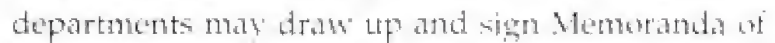
Lnderstanding (Nolsi allowing comperation where their responsibilities onerlap. The most sigrificant example is the NoU between the Forest Depertnemt and the Ketrya Wildlite Services (recototy joined ty the National Musedms of Ketrba and Keorya Fireats Researcly Anstifute, which both have researed

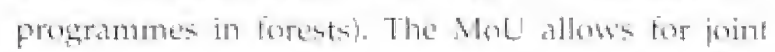
mandgement, and resanch collaboration, at sperilic

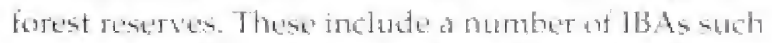

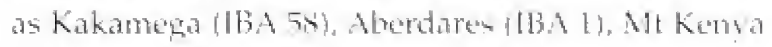
(IBA 5) and Arabuko-Sohoke $\left(1 B_{i}+7\right)$

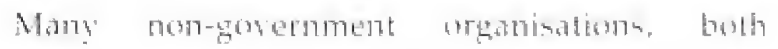
internatiomal and national, plas impartant rolles in bindiversity conmerbation in Kenya. Anomg others, these include the Arrion Combersation Centre UAC a branch of Wildlife Consersation International), African Wildife Foundation (AWF). East Atrican Wildite Societs (FAMLS). Frietrdo of

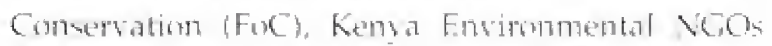
(KENGO), Nature Kenya (the East Artica Natural History Soctets - EAXHsh, thirld Cotmenation

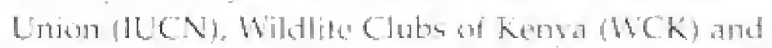

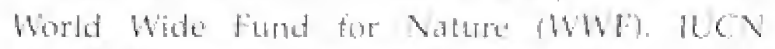

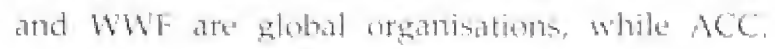

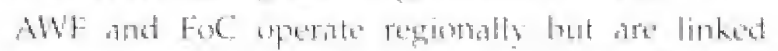
to parent organisations abroat. The EAvis and Nature Kenta are enservation boletien Natute Kenva alad being the national Farther

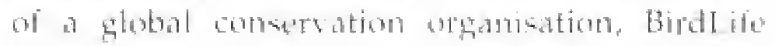
International.
At the time of w riting, the following ministeles land their relevant departments) plated at majur role in biodivesity conseration:

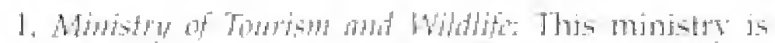
lesponsible for wildlite conterestion (diacussed in section 3.5 .4 and owereight of the tourism industry. Under this Ministry falls the Kerny Wildife Sersice (KWS) responsible for the country's proteded wildlife species, and for the management of National Parks in particular. KWS is a paragtatal (Ir state amporation, meating that it has substantial financial and oprerational antonemen.

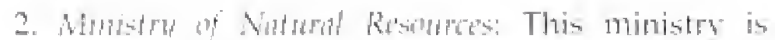

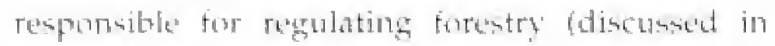
section 355, fisheries and mining. The Forest and Fisheries Deparments fall under this Minister. The Forest Departunent (FD) is responsible for bath indigenous anc plantation forests connervoride.

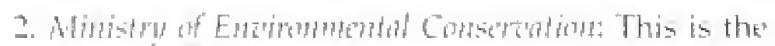

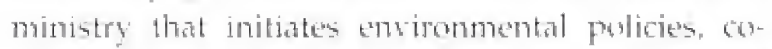
ordinaters the activities of sectoral agencits and

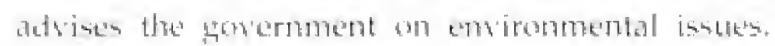
The mingtry atministers the forestry act and mining

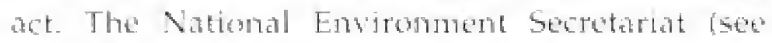
retwon 3,3,2), alse a deportment in this ministry, coordinates enzironmentat pulicies and activities in the combry through an Jnter-Ministerial Comsittes on the Fnyronment. This Ministry hat posted District Envirommental Protection Oficers to most districts.

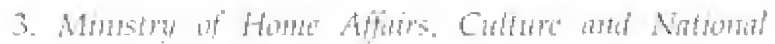
Hertases This is the parent minestry for the National Muneums of kenga, which has an extensive

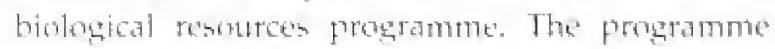

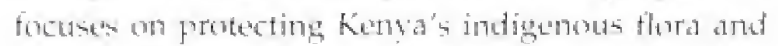
fauna through education, reseatch, curation and

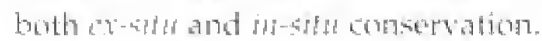

4. Maristry of dond Goternment: Lnder this ministry fall "il sartedy of local authorities. Many of these are respomible for administering national resereses and forest reacerse, and regulating land-use through appropriate byelans within there areas of

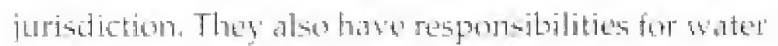
coutiersation and follution combol dulder both the' Public thealth Act and the Local Government Act. 


\section{Institutional, legislative and policy framework}

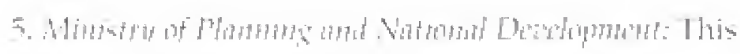

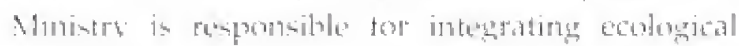
alsedeth intos rational debelopment plannimen. Threugh the Departmetht of Resentare Surveys and

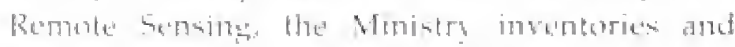

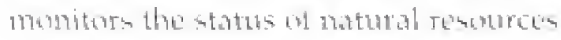

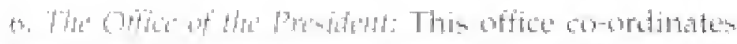

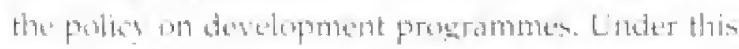

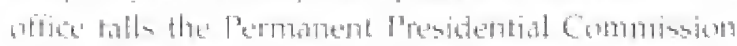

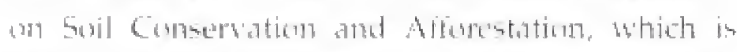

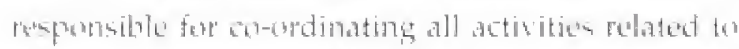

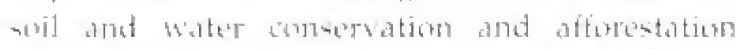

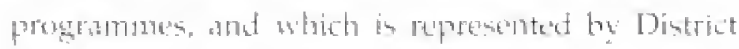

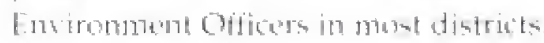

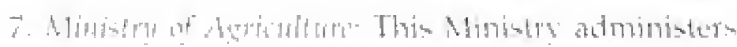
the Agrixulture Aet anet the Pent Gamerol Act

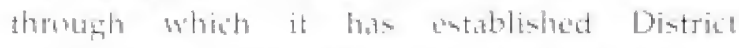
Conseration Commituess responaible for ensuring proper landentse practues.

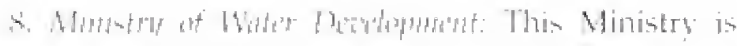

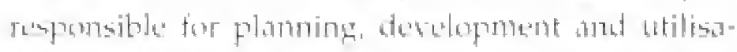

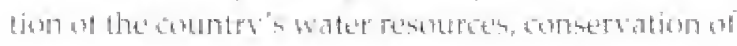

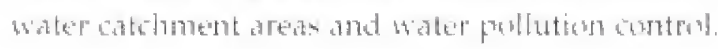

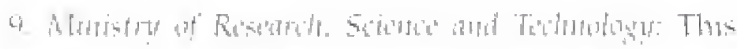

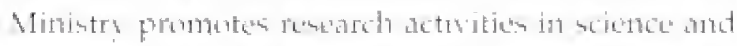

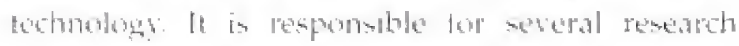

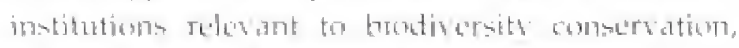

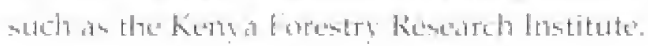

\subsubsection{Conservation Iegislation and policy}

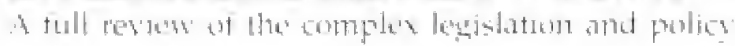

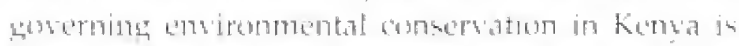

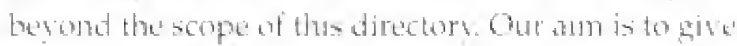
an yuthe of the aserall framemork and its strength

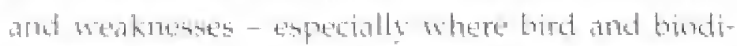

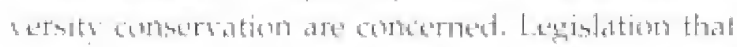

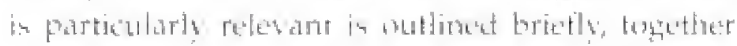
with at decription of the implementing agencice and

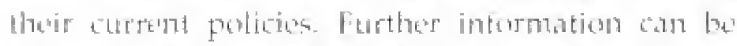

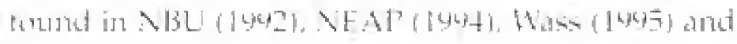
the relevant Acts.

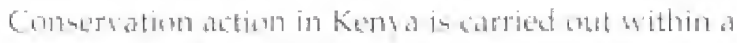

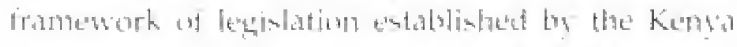

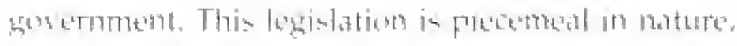

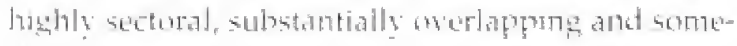

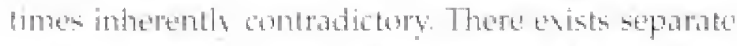

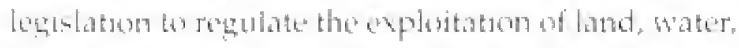

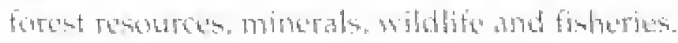

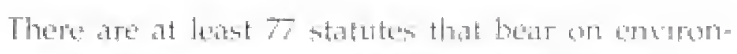

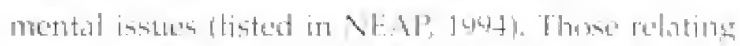

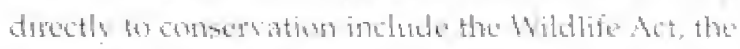
Forentry Ant, the" Antiquitices and Mlonuments Act

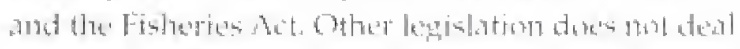

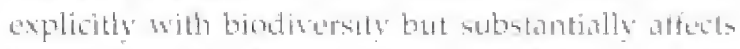

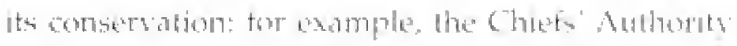

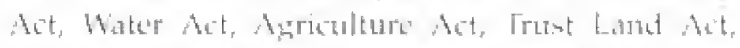

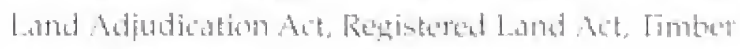
Act and frespits Ant.

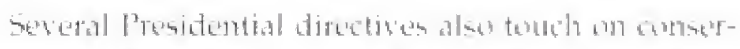

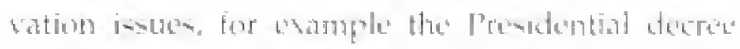

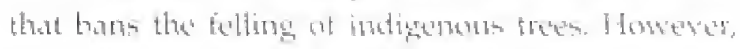

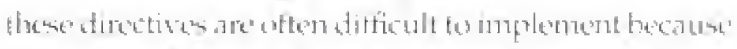

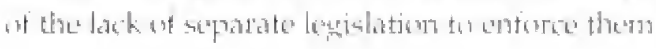

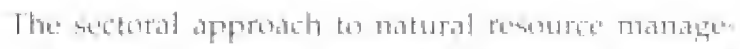

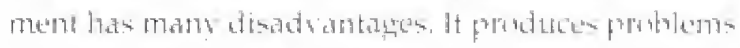

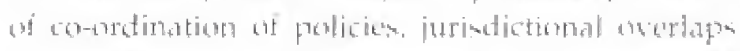

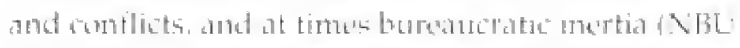

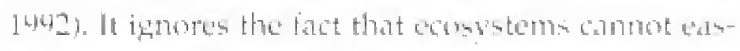
ily the partitioned into indeperedert usits. but must be treated as a tumetional wholde. The implementation.

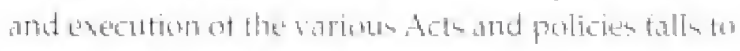

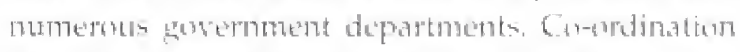

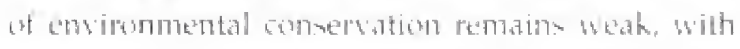
the different institutume working in instation and

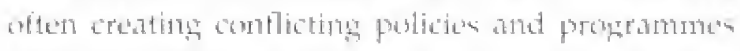

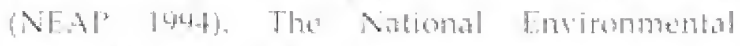

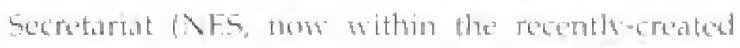

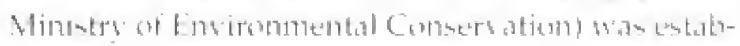

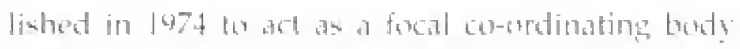

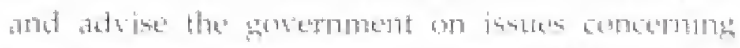

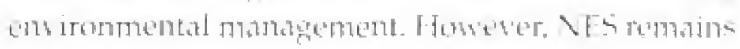
without legriclative bak- kp and independerat authority which considerably diminishes it edfertivenems.

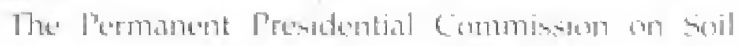

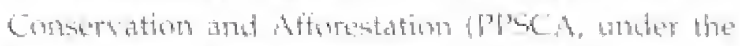

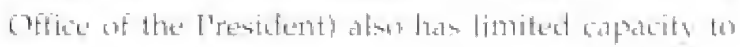

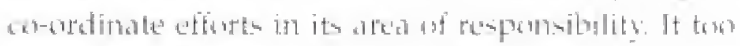

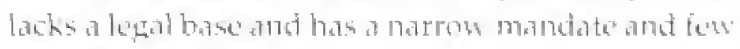

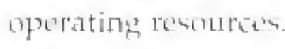

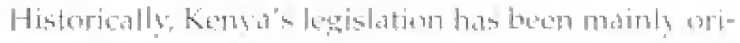

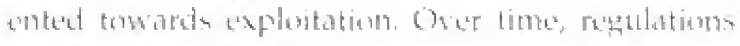

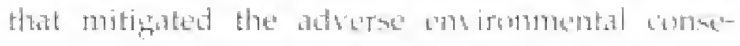

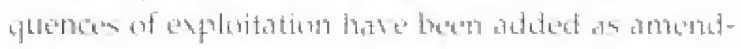

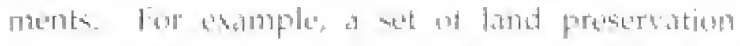
requirements in the Asriculture het were and thet

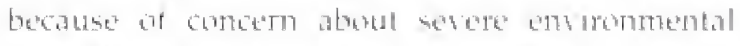

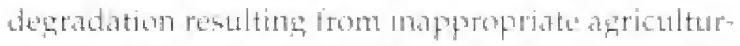

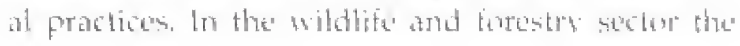




\section{Important Bird Areas in Kenya}

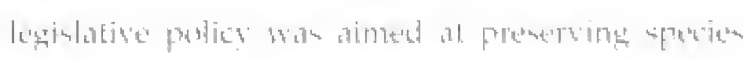

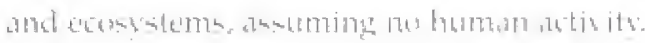

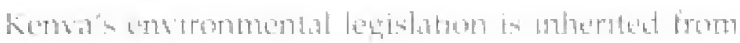

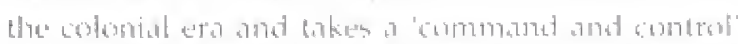

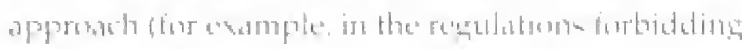

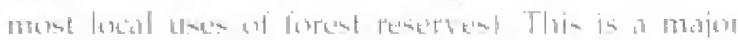

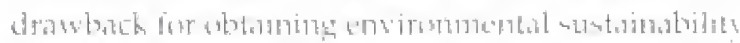

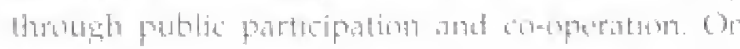

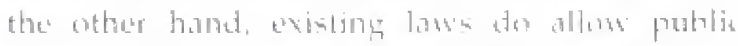

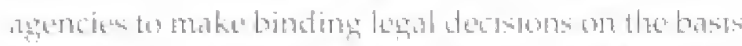

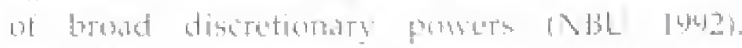

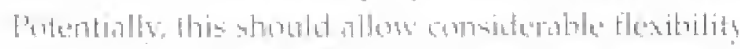

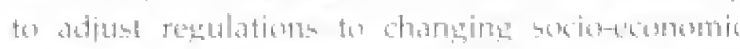

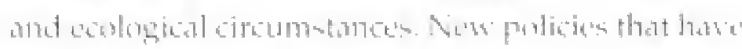

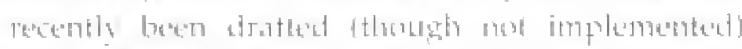

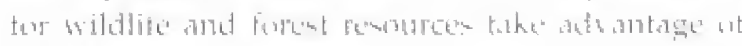

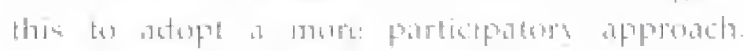

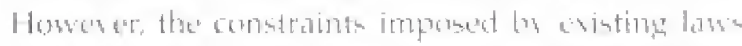

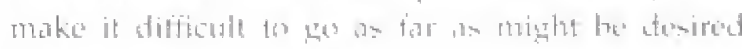
in thim diretions.

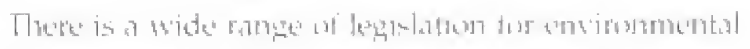

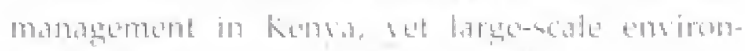

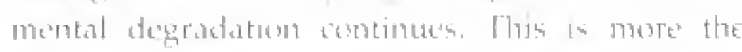

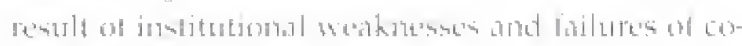

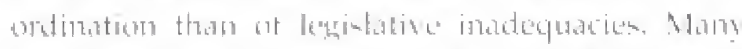

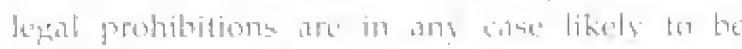

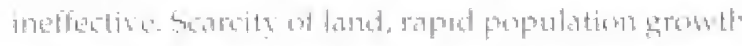

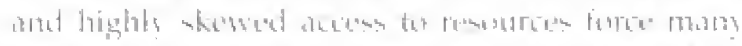

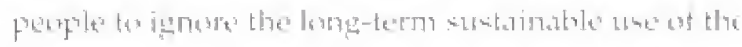

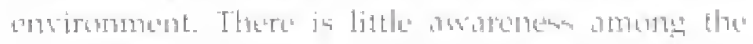

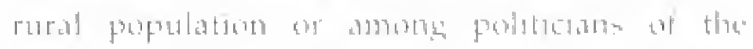

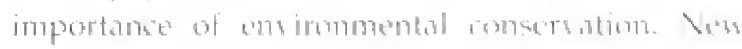

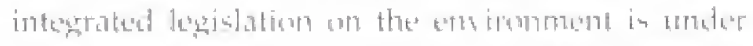

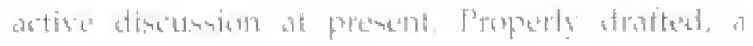

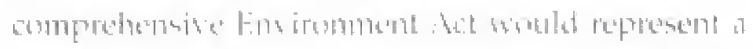

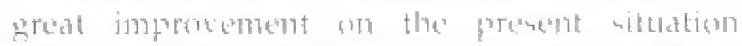

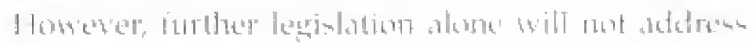
the urderlying probleming.

\subsubsection{Land tenure and land use}

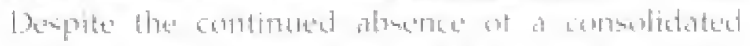

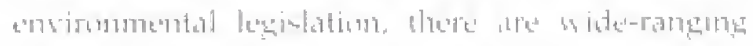

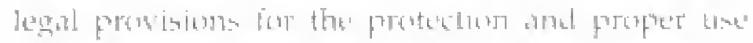

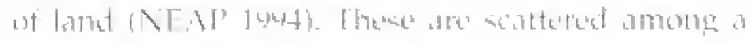

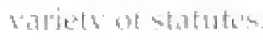

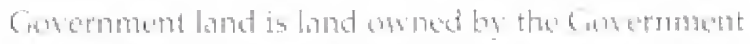

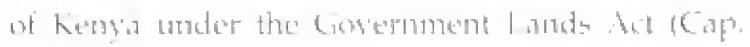

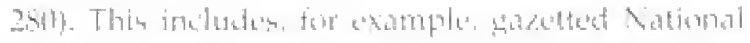

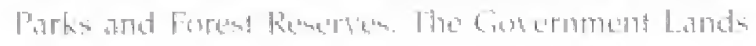

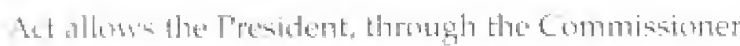

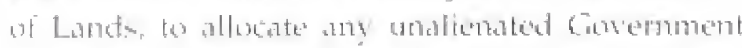
land to any undixidual. lin practice, suth allowations hesce otter been made stithens proper regatel to

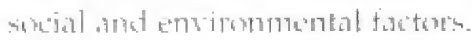

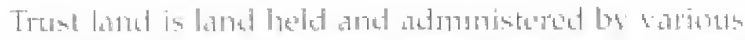
hacal government authurties as trustetes moter the constitutwom at Kensid and the Trust Land Act

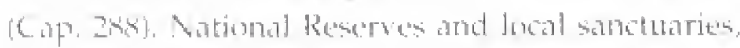

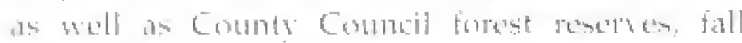

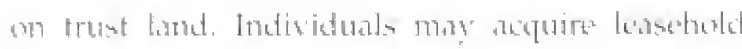

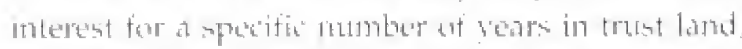

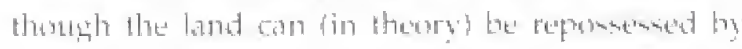

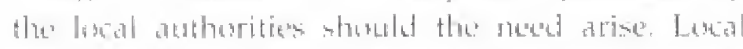

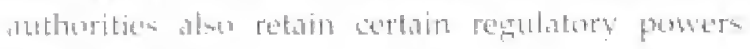
ate tet toul land

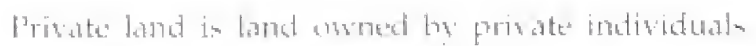

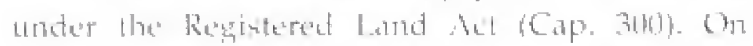
registration an the land moner, an indisiduat

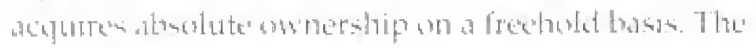
tree of prisate land mas hosener, be limited to provisions mathe in other leysialation, sach as the

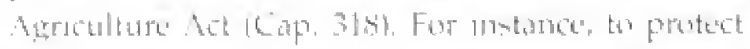

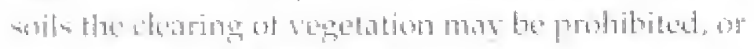

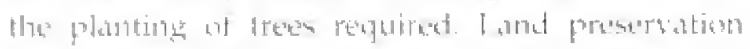

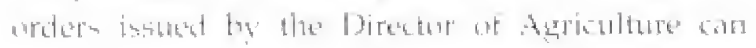

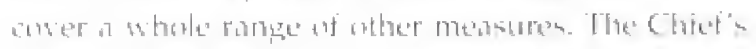

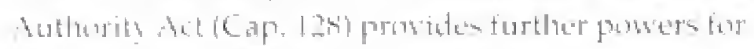

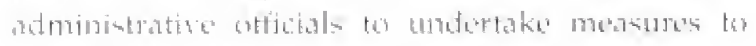

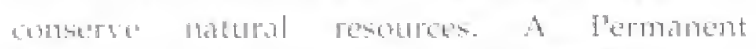

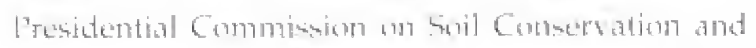

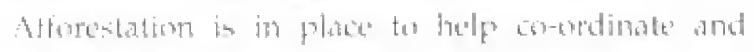

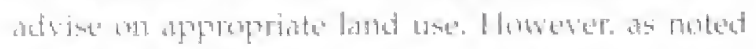

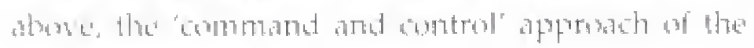

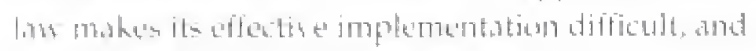
witespresta lanet degradatis continues.

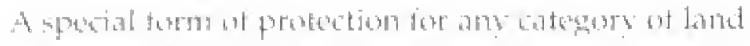

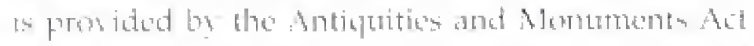

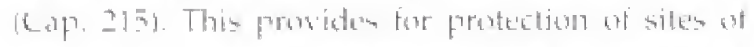

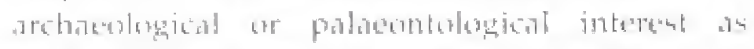

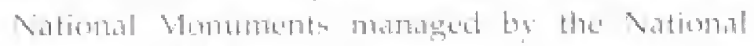

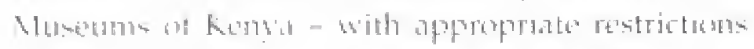

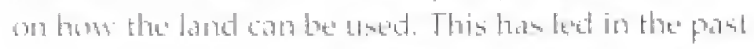
(6) nuciblental conservation af bindisersits at

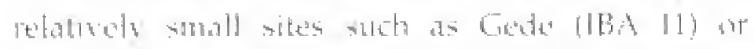

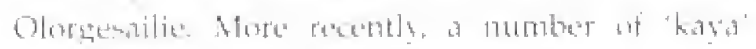

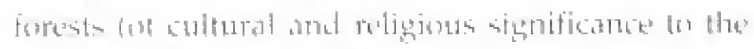

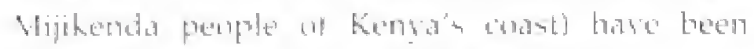

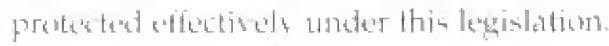




\section{Institutional, legislative and policy framework}

\subsubsection{Wildife management}

\section{Background}

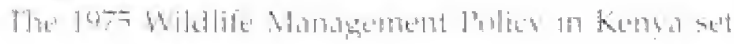

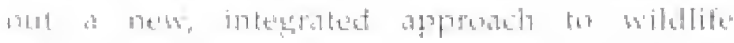

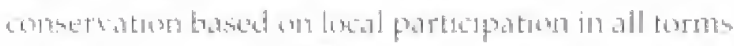

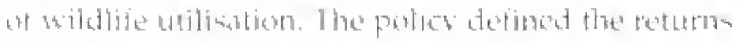

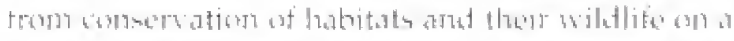

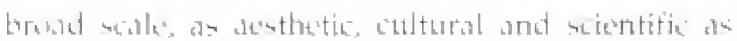

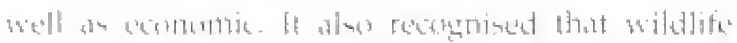

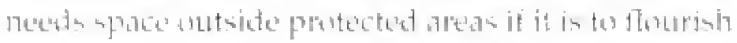

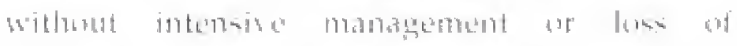

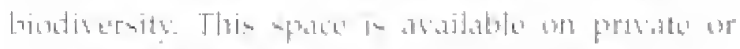

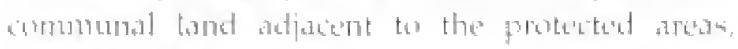

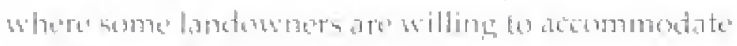

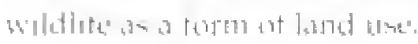

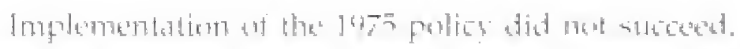

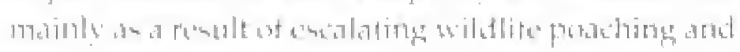

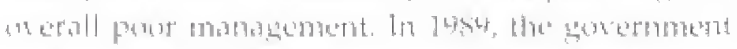

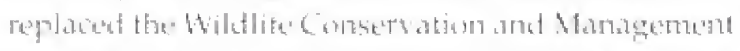

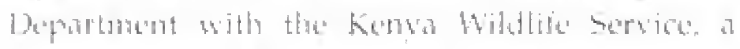

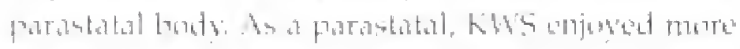

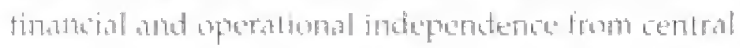

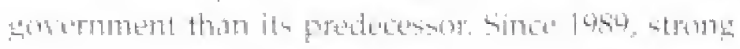

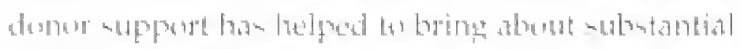

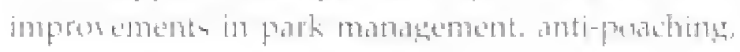

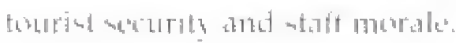

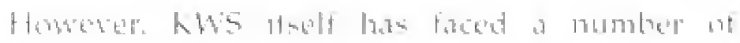

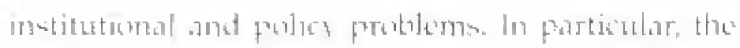

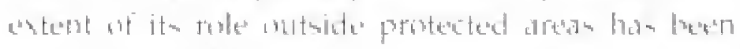
a

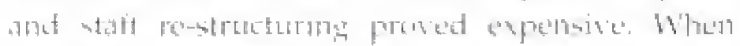

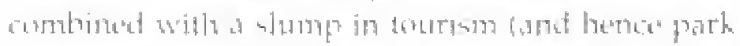

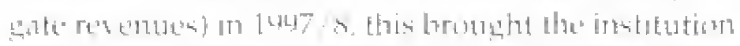

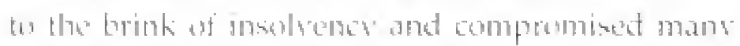

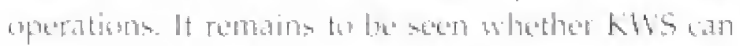

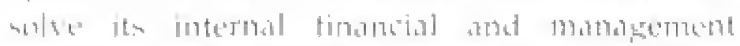

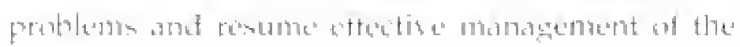
cumbers = wiblitu

The Wildlife Conservation and Management Act

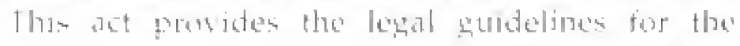

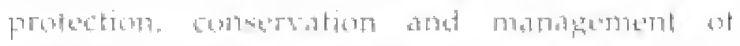

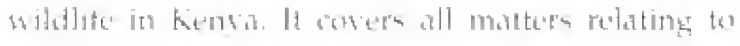

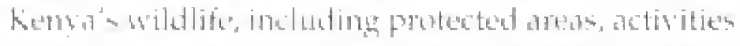

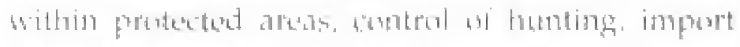

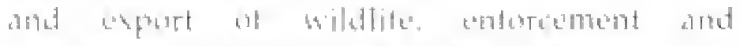

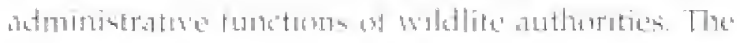

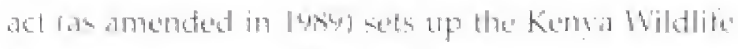

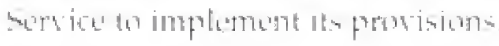

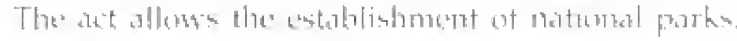

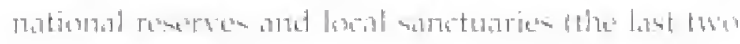

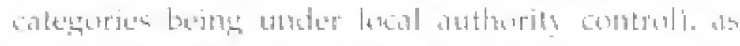

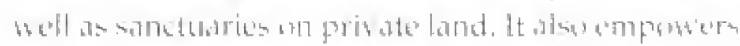

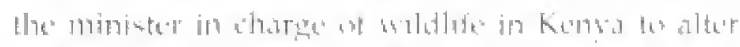

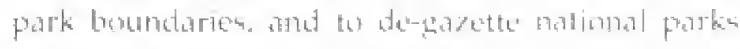

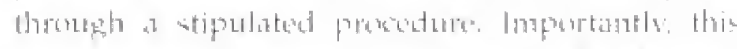

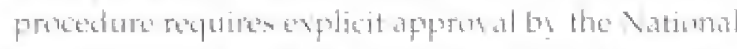

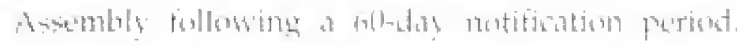

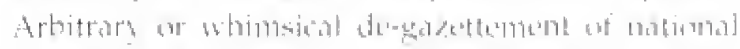

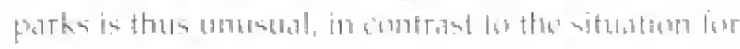

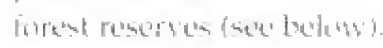

Lnder the at, national parke are mandged La Kho

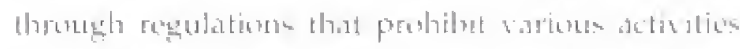

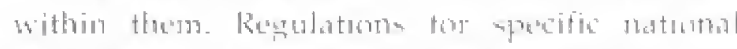

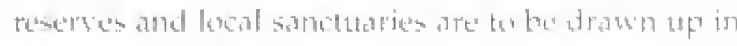

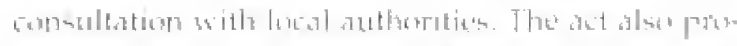

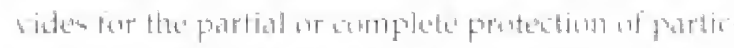

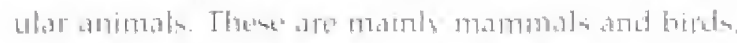

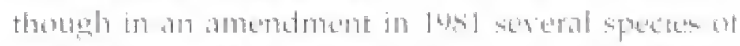

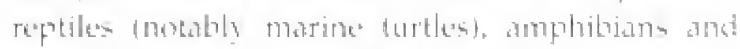

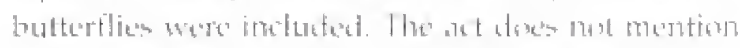

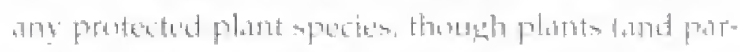

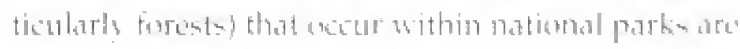

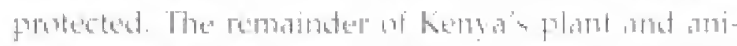

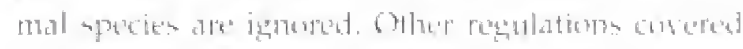

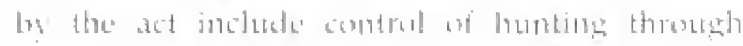

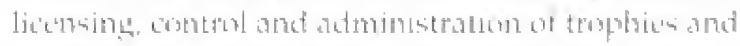

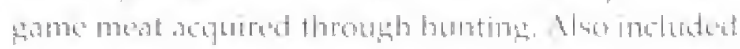

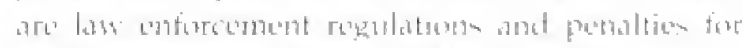

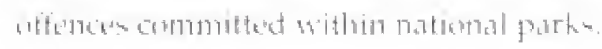

\section{Birds in wildife legislation and policy}

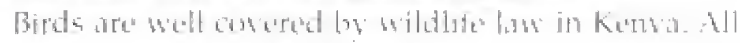

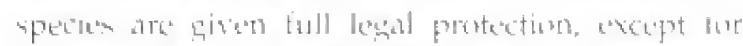

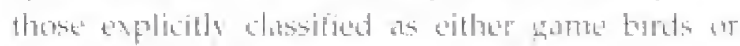

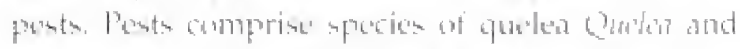

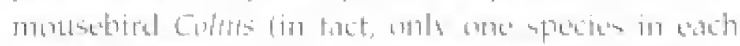

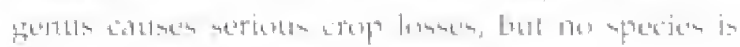

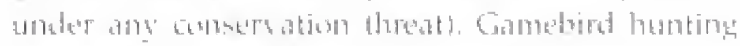

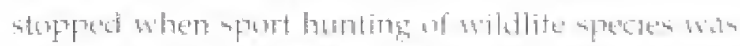

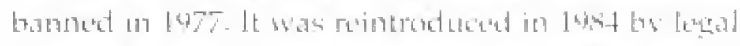

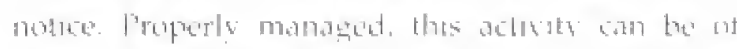

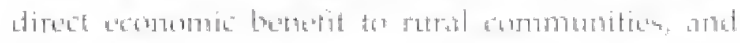

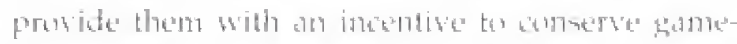

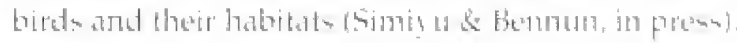

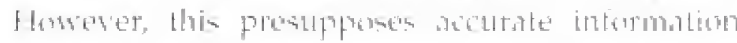
sterul the birds atemograptry and a manowement

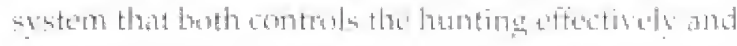

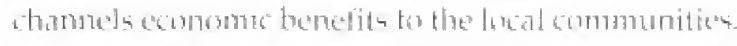

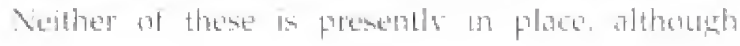




\section{Important Bird Areas in Kenya}

experimental syoteme are benge wested at the local lexel (simiva \& Benthut, in press)

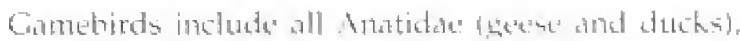
Phasianida (qualil, fromodins and sparfowls

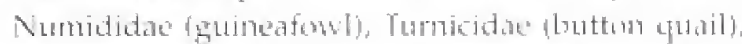

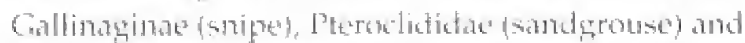

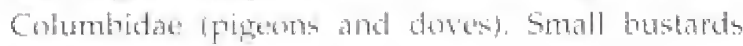

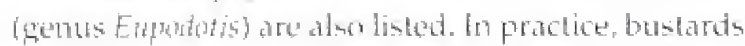
and other regiomally threstered birits fouch as Wacos and white-bucked Duck are not preantly lacinted.

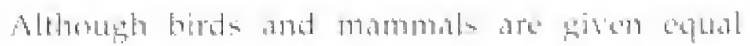
protected status in Komsan mammals have formed

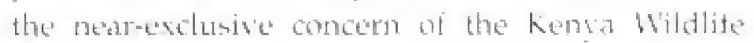
bervice and it preatedestors.

\section{Wildife policy directions}

Khis has drafeded atem wildite forlice to replace that

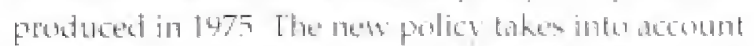

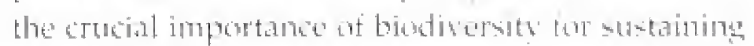
human lifer as well as the inosenger conflicts between wildife and people. It has the tollowing mission statement:

"The froverument of kerapa holds in trust for the prement and juture semerations nathonally and shobally the biological diversity lepertanted by 1ts extrandinary farety of animals, phants and exomstems ranging from comal restes to alpince morlands and from demeres to foremste specisil

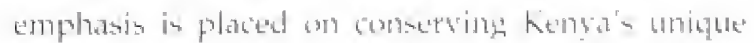

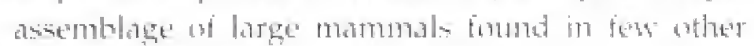
places on sath.

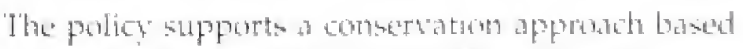

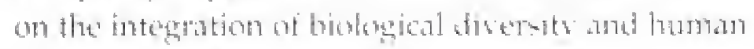
activity: It recognises that the principal guardatas of

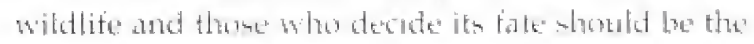
primary beaneficiaries of andersation. The berefita

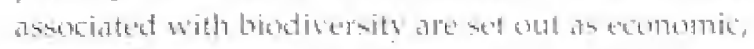
caltural, recreational, atesthetic setentific and

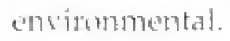

This drafl policy was prepared in 1997 , In 1498, Khs

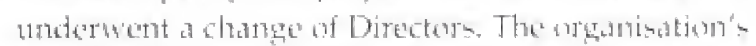
focus remerted to manarement of wilatibe in protected artan, with lemo attention and ither

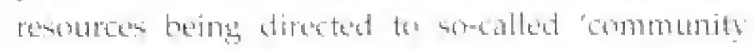
wildife isters. It is as jete undear bow this change

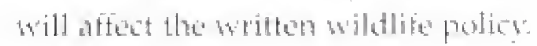

\subsubsection{Forest management}

\section{The Forests Act}

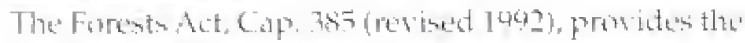

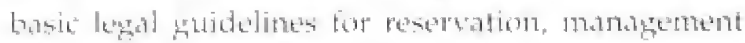

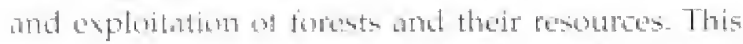
act gistes the goreresment absolute controt of forests

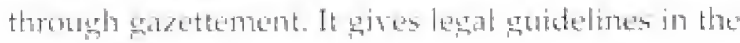
fallowing majur ateas:

1. Gazkdement. alteration of boundaries and de-

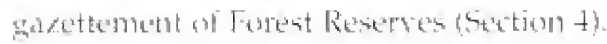

2. Deataration of Nattore Remerves within Forest Resmes, and requiatom of activitis within Nature Remervess (section 5).

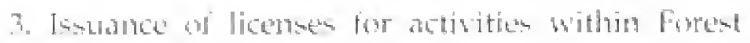

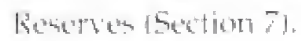

4. P'robibition of activites an Forest Reserves [remasal on forest produce, graring cultivation. hunting etc.) or Government land genceally fremonal at trees, collection of hones, lishting of

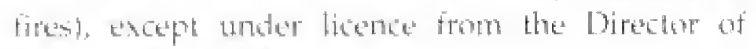
Fonestry (siation 8).

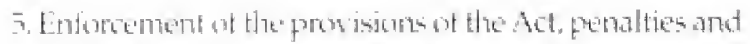

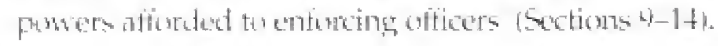

(2. The power of the Minister to make rules with

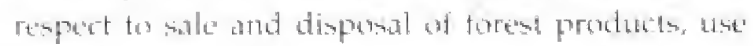
and uretpation of land, licensing and entry into forests (Section 15). This prearogative has been taken with the foren (General) Rules, which set forth rules

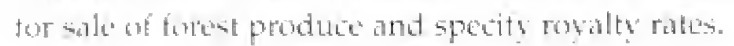

Suberal aspects of the ant are noteworthy

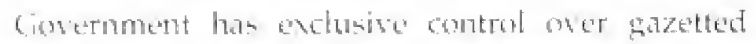
forent ateas. where no dotivitite mas be undertaken

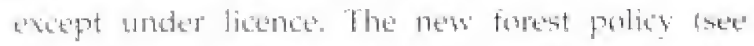
befoss attempto to tackle this insue; until now; howerer, it has meant that ather land uses cannot be integrated anto the gazetted forest estate' By coutrast.

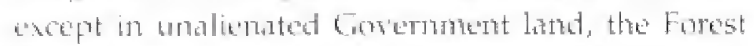
Department (FD) has no managetnent and comberta.

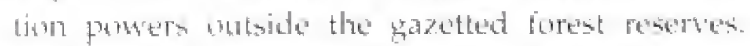
Honserer, the appropriatio management of gareted forteste must inklads the management of surroundIng areas. Alson cansiderable arese of forests and woodands ane an prevate land and on trust land managed be the county councils. The FD must rely.

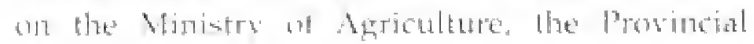




\section{Institutional, legistative and policy framework}

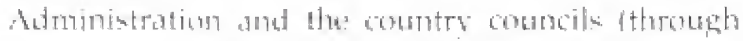

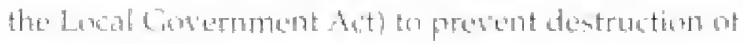

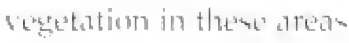

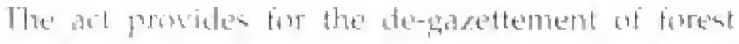

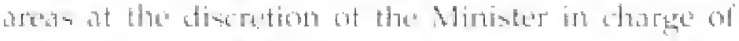

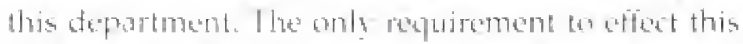

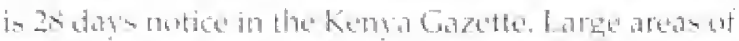

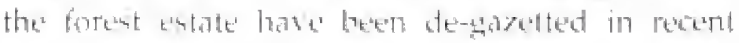

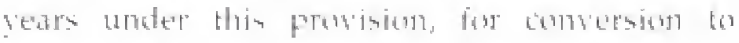

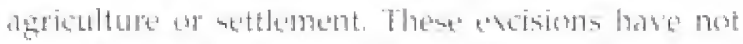

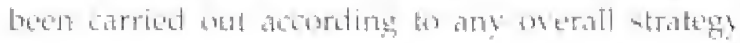

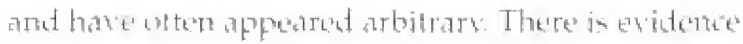
that many areas of porticular mportance for

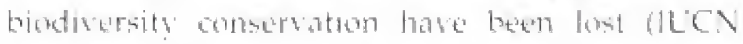

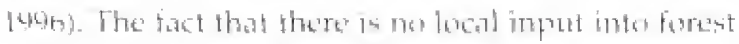

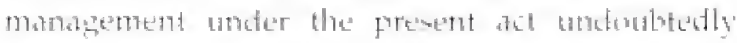

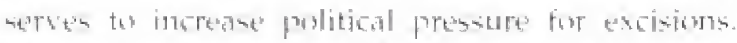

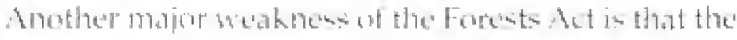
budy set up to implement th, the Forest beportment. is not a state comporation dike kibsp tut smply a

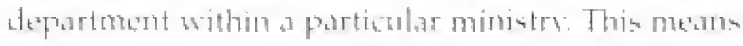

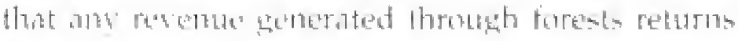

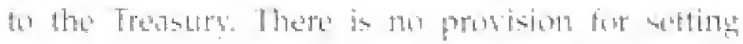

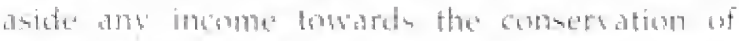

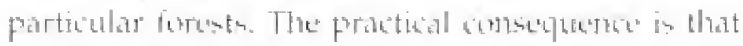

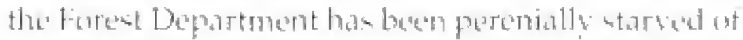

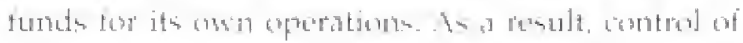

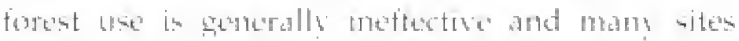

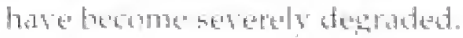

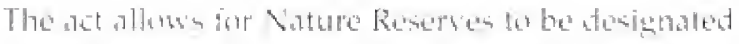

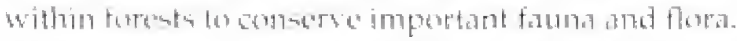
Dinturtsance tos the burent in supponed to be limited withon a roture ruserse a number of such resertes

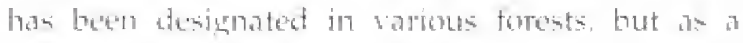

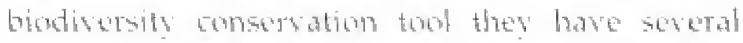

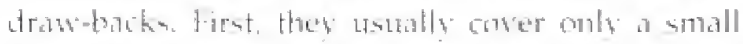

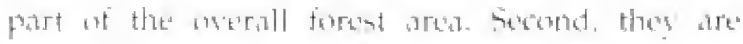

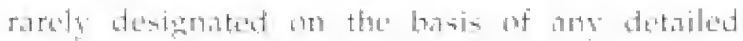
knowledge of the distribution on animals and plants

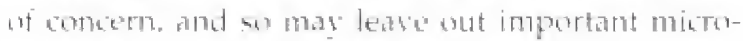
hatritate ser propulateme of particular iperies altogethes. Thide desighation las in pratice

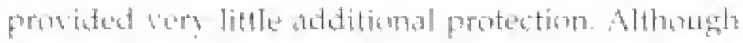

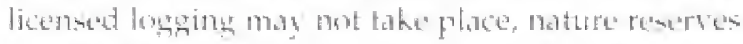

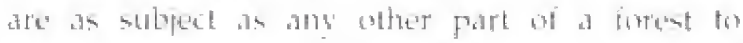

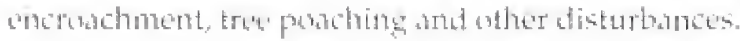

\section{Forest Policy}

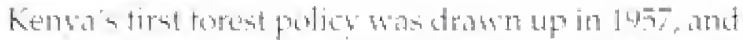

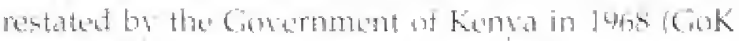

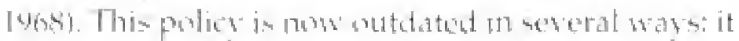

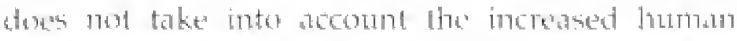

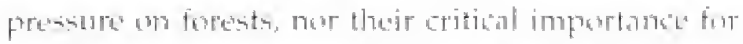

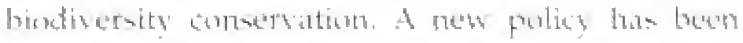

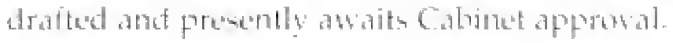

The old forest prolicy sutlined the reacersation af torests for soif and water conservation, and for torat

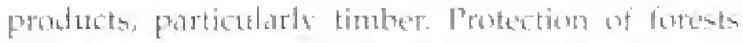

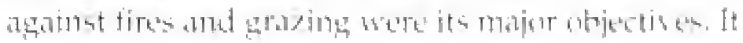
alab exteotraged the deselopinert of the forest industry and mentioned the promotions of

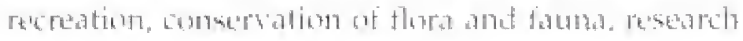

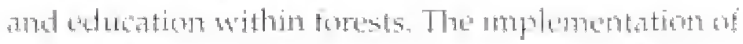

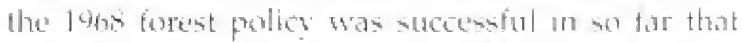
most torests still bxist and fultil their mo in

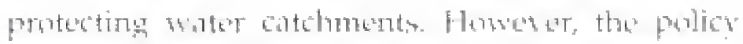

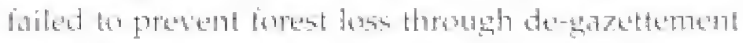

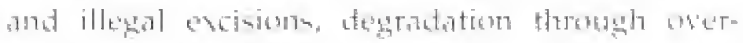

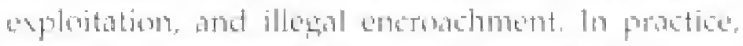

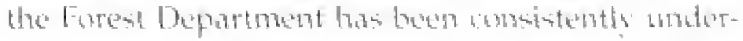

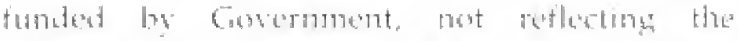

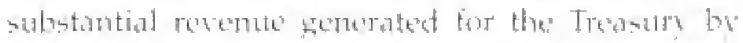

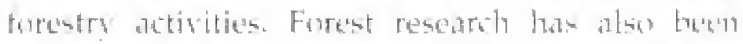

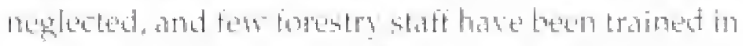

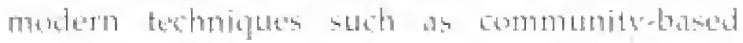

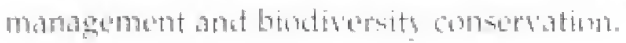

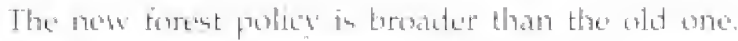
It maintains the impurtant functions of environmental probetion and sustainatole exploitation of

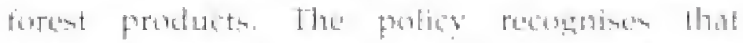

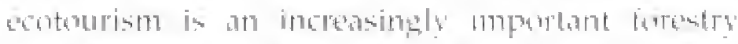

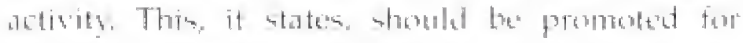

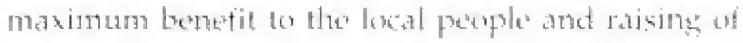

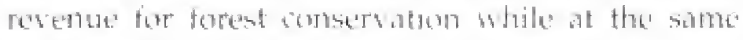

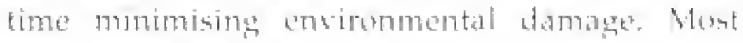
important, the policy states that ofl watertat

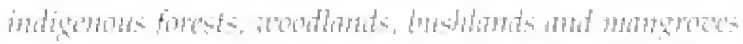

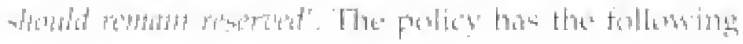
mater oractisen:

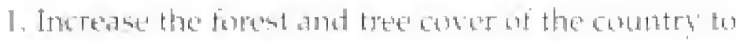

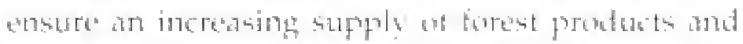
services for meeting the tasic needs of preane and

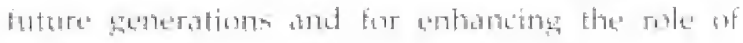

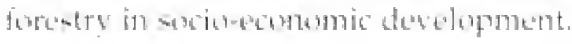

2. Comserve the romaining mitural habitate and the

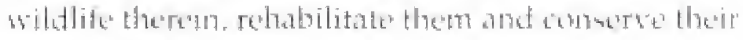
mistiversity:

3. Contribute to sustainable argricubure by con-

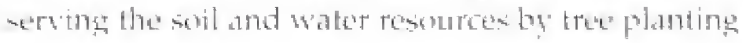




\section{Important Bird Areas in Kenya}

and approprinte forcs mathagement

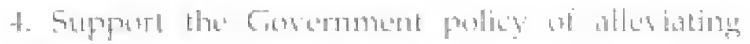

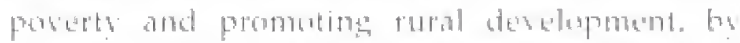

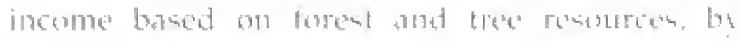

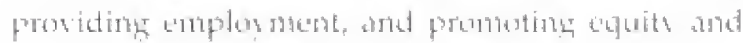
participation by lacal communition.

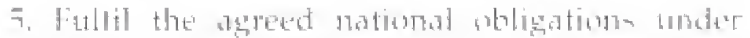

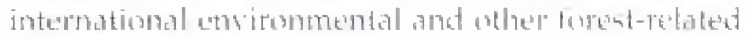
atrugutions.

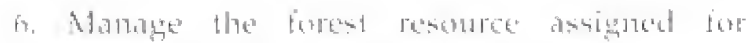

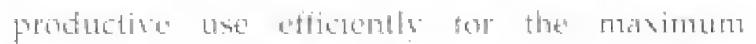

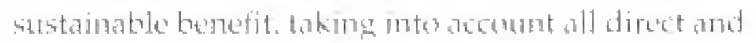

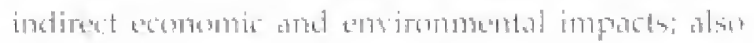

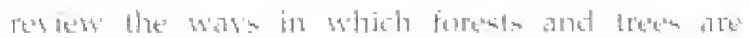

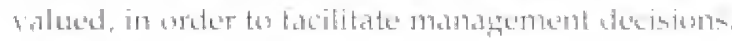

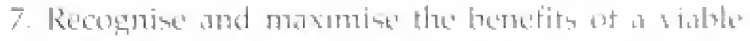

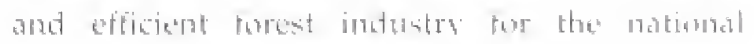
ecomomiz and dexthoment.

\subsubsection{Wetlands management}

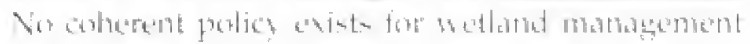

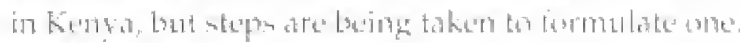

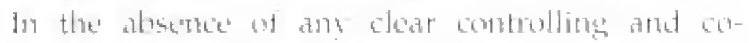

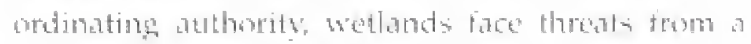

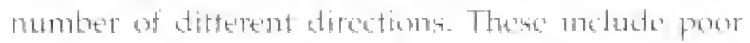

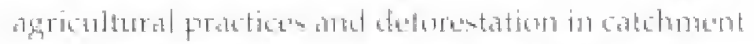

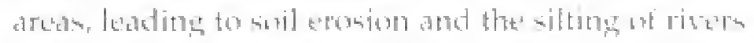

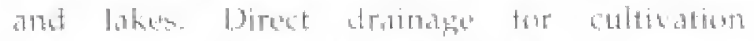

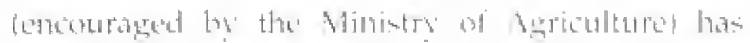

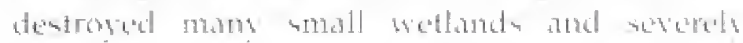

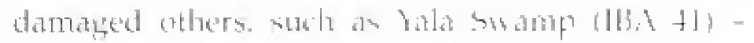

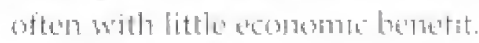

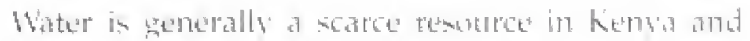

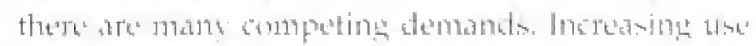

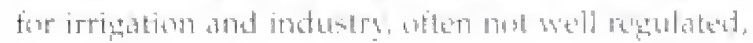

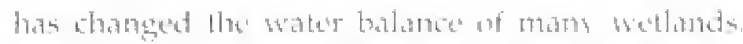

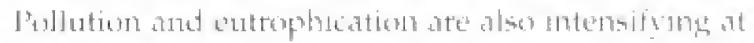

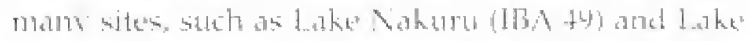

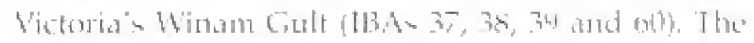

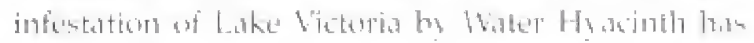

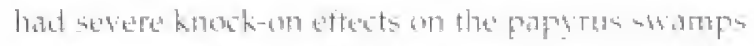

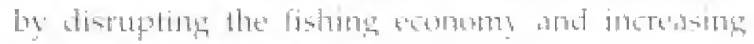

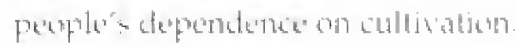

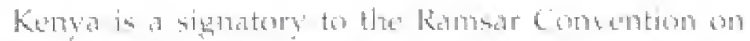

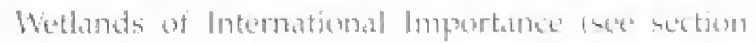

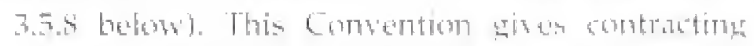

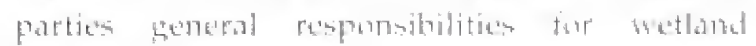

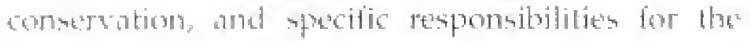

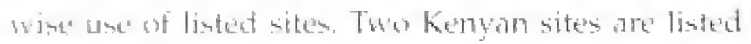

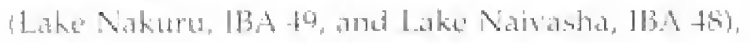
and many more could potentially be addet. The" momerits ot kembats wetland IBAs are poteratal

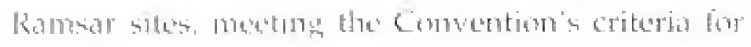
listints

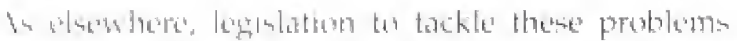

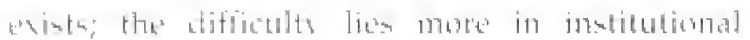

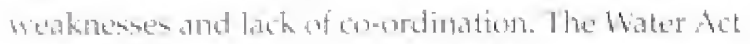
(Cap, 372) lay dum the bank legal framework for the mambenthen of water resources. These dre: sll bested in the state, except where thes lic whalle within a land-anstaer's propurts

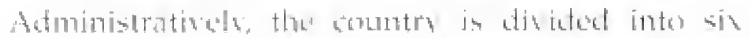

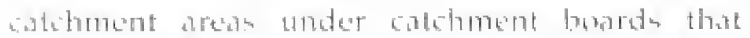

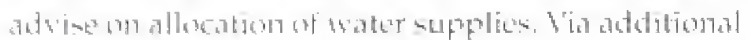

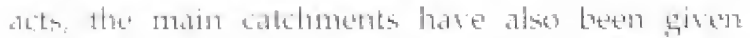

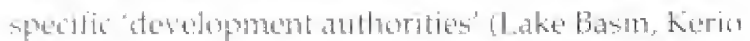

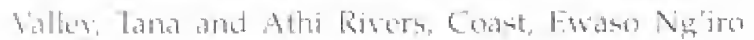

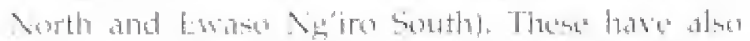

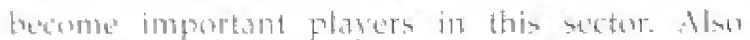

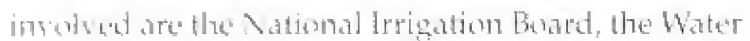
Combersation and Pripuling Corporation, and a number of lexal anthuritica. This proliteration of institutions leats th almost incribable conflic and

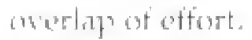

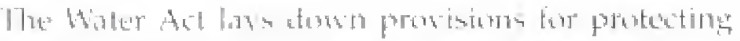

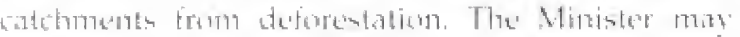

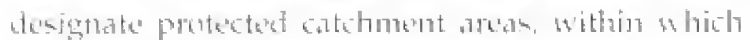

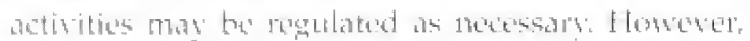

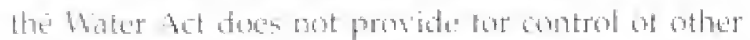

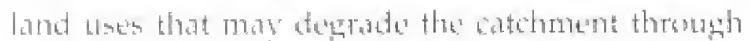

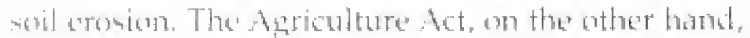

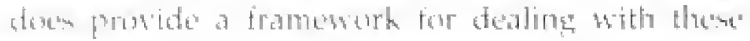

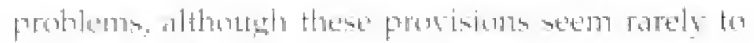
tre implementente

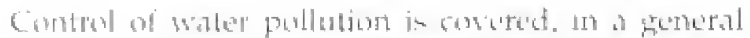

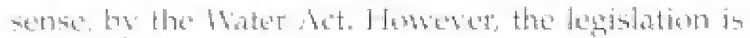

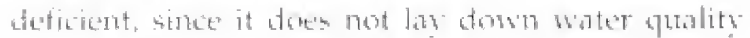

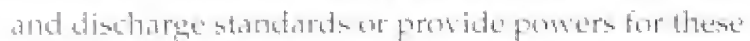

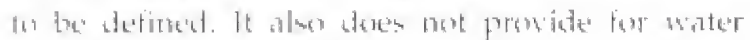

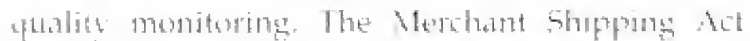

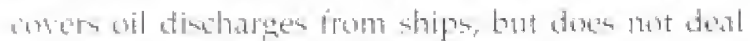
wath ather aspecto of unatine pollution. The T'ublic

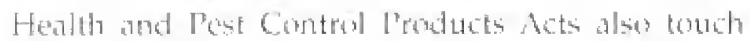

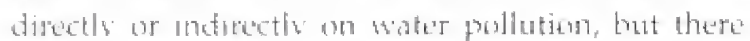
is little institulonal capiaty to implement theit Promismoth. 


\section{Institutional, legistative and policy framework}

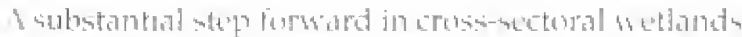

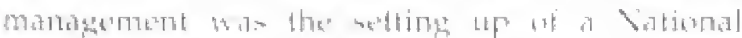

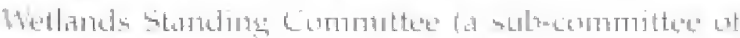

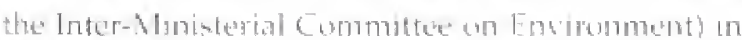
1444. With comatination from the Sational

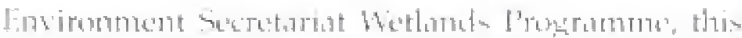

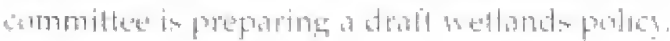

\subsubsection{The Protected Areas system}

['rotected aroas incluato national parkm, nutional

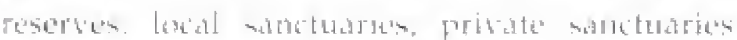
forget reservers, Countr Gouncil forests and natiomial monuments.

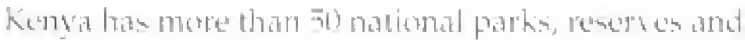

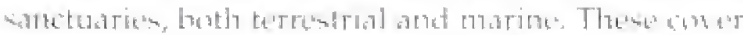

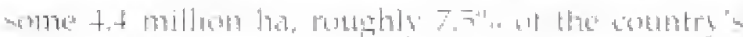

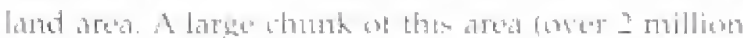

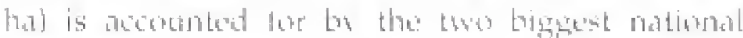

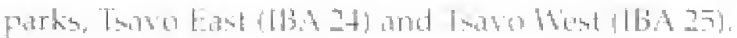

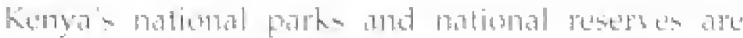

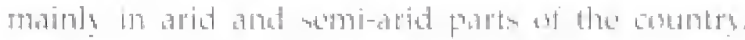

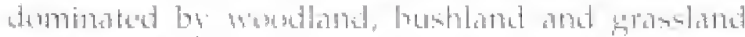

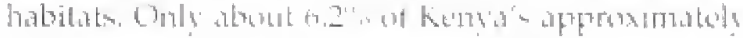

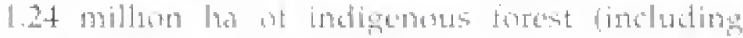

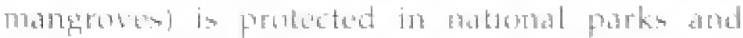

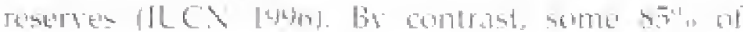

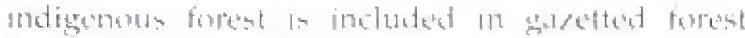

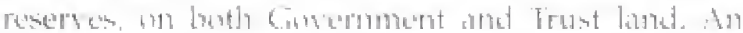

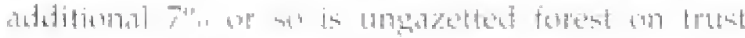

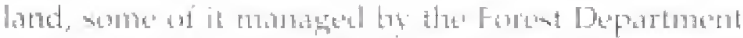

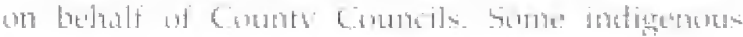

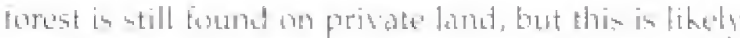

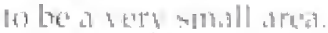

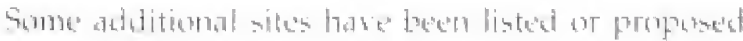

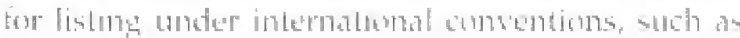

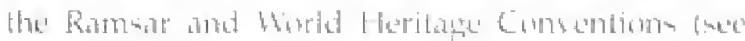

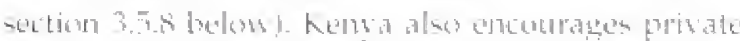

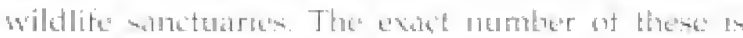
unelear; the ILCN protected areas databolse lists omer

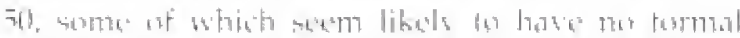

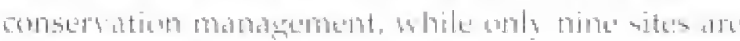

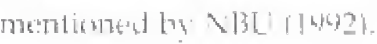

\section{National Parks}

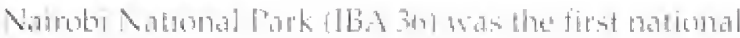

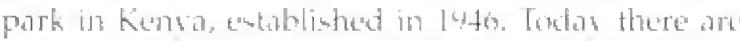

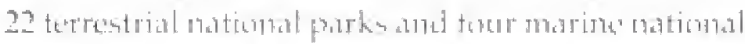

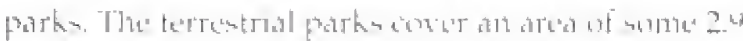

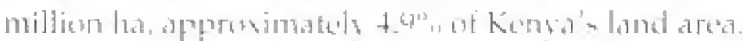

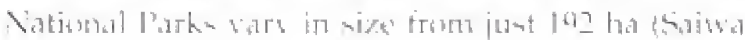

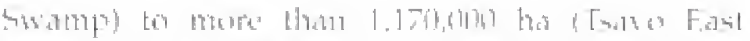

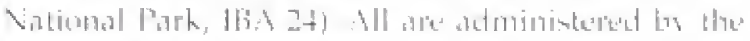

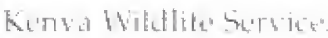

\section{National Reseryes}

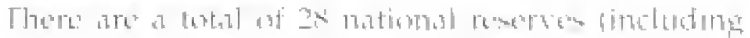

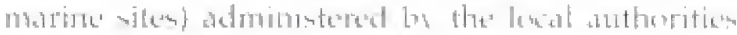

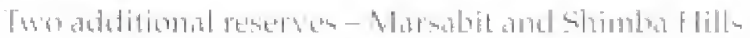

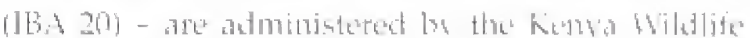

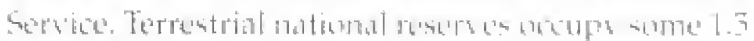

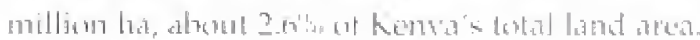

\section{Forest Reserves}

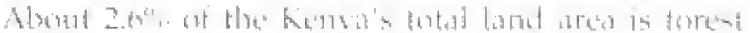

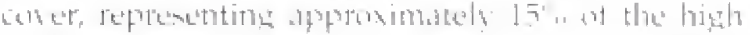

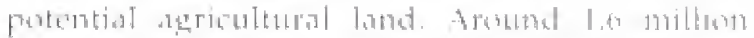

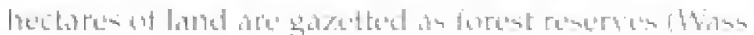

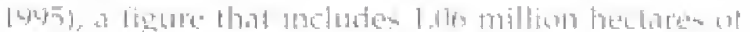

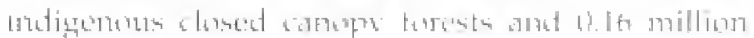

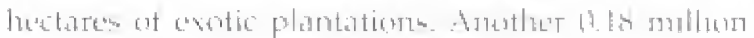

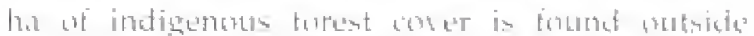

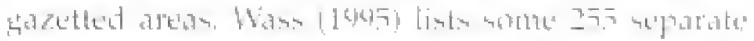

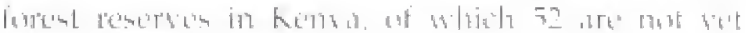

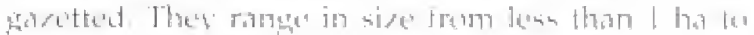

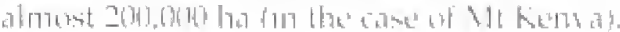

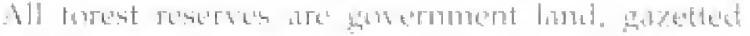

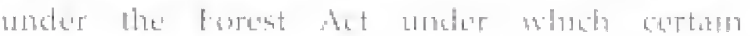

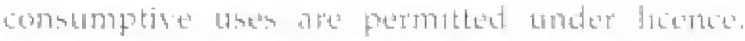

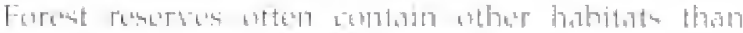

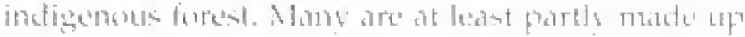

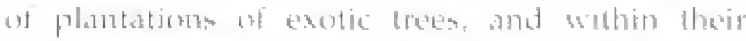

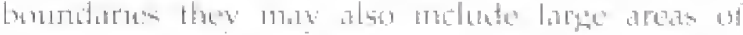

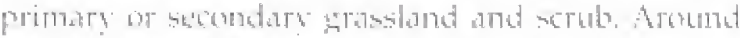

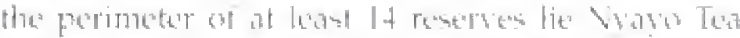

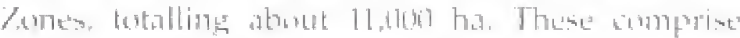

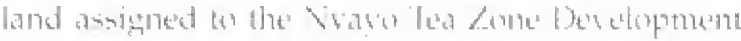

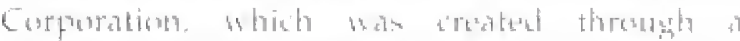

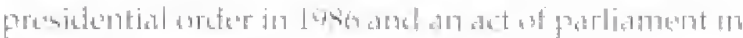

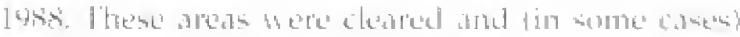

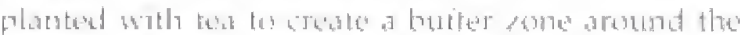

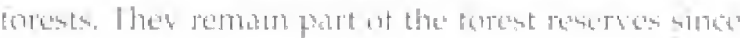

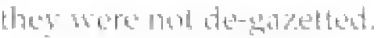

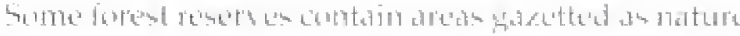

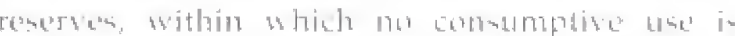

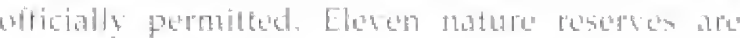

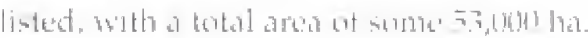

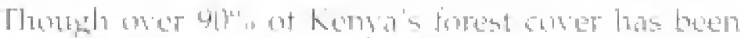

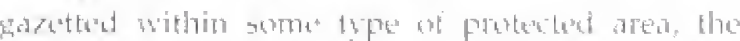

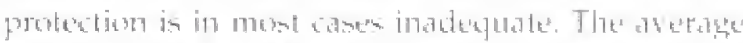
w 


\section{Important Bird Areas in Kenya}

["w, with the highest rates areurrion in forests $117 \mathrm{or}$

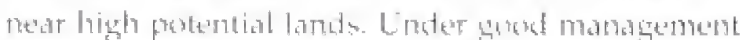

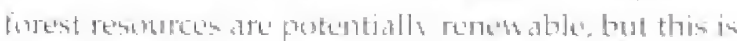
not the eare in keryos at present.

\section{National Monuments}

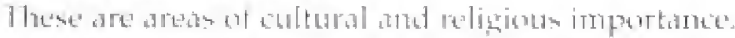

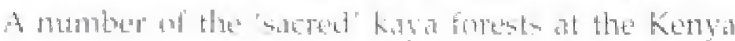

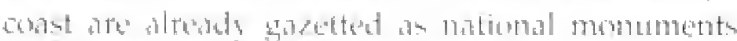

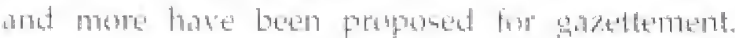
Thungh hatrly small these haresto ald important for

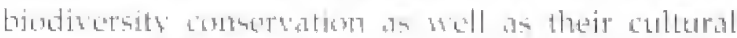
value. National sondoments are administered by the National Alumelums of Kenvit.

\subsubsection{International conventions and agreements}

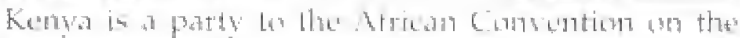

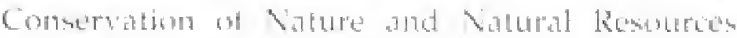

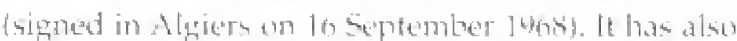
acceded to bres important intermational consentions

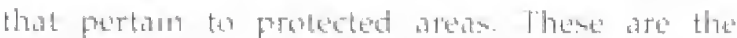

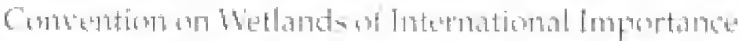

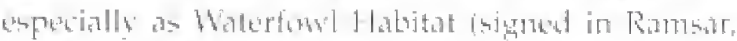
I Protection of the borlat Cultural anat Natural

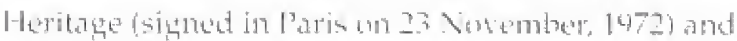
the Consenttem or Biological Dinersity asigned an

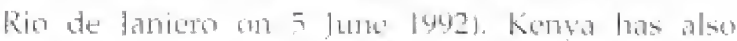

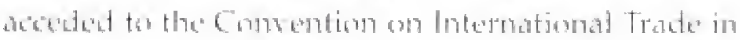

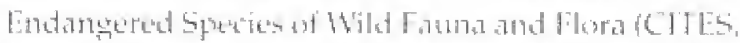

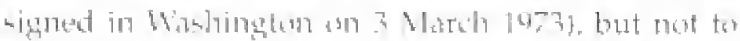

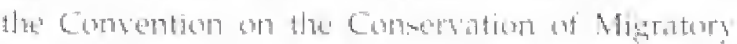

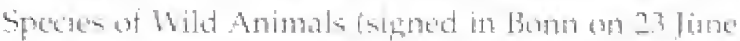

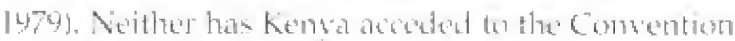

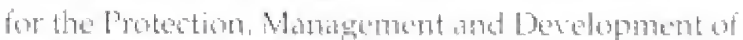
the Varine Foviromment and the Constal Mreat of

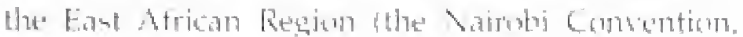

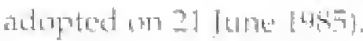

\section{The Algiers Convention}

The African Compention an the conmervation ont

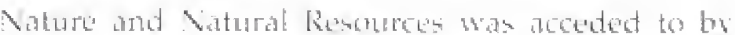

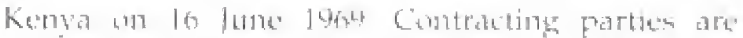

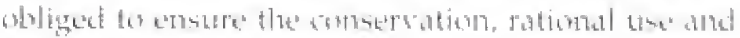
denelopmest of arid, watere floral and batural

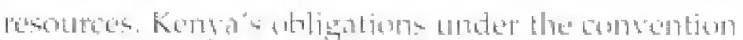

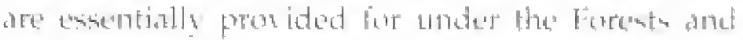

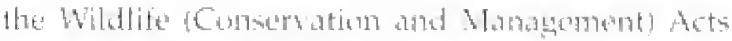

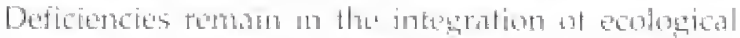

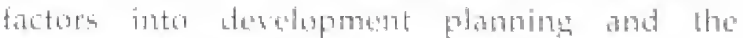

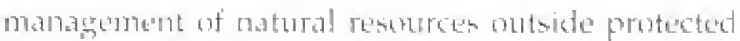
areas ( NBL $\{442\}$

\section{The Nairobi Convention}

this is a regrional framewerk convention atcopters under the United Nations Environmental

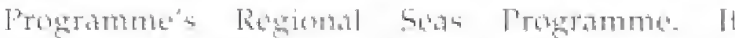
establishes general preteciples for the conkertation of the marinte and constal ensironment ith edstern Africa. Two protocols, at topted at the same time, deal with the cancervation at biondicersity and the establishment af probected areas. The compention

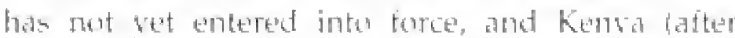
participating in the nemgotiatian wi the cosvention and its two protecola) declined to aigm.

\section{Ramsar Convention}

The Rambar Combention aims to cuture the" "wise" Use. af wetland for the benefit of peoples, in a wat that is compatible with nusintaining the natural properticus af the acoswstem. The compention also

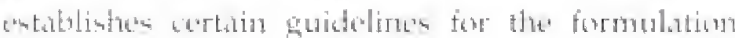
and ianplementeation of wetand policies. Thenes

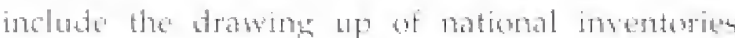

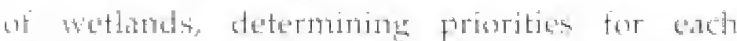

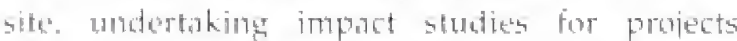
which maty affect wetlands, regulatine the trow of

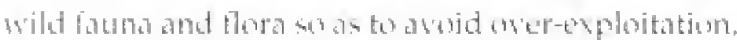

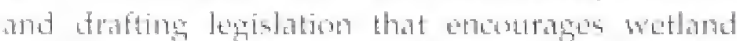
compersation. Contracting parties must designate at leost one site for" inclusion lin the list ut wethends af international importatice. or the basis of the site to international signiticane in terms at erology,

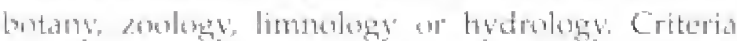

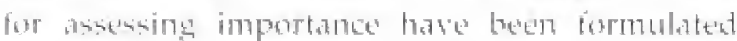
by the Comference of the Partien, and inclute spocific threshohis for waterbird populations.

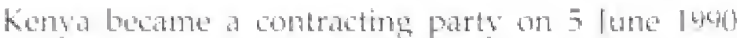
through designation of lake Nakuru National Park

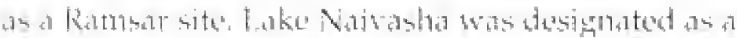

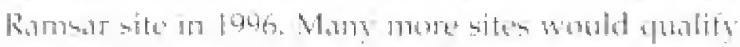
pere designation, atmong them the wethand IBAs isuluted in this directors

\section{The Convention on Biological Diversity}

This sonventiun same inte fores on 29 December 1943. It istentifice the responcibility of states to?

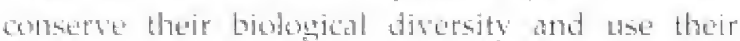
biological resources suxtainably. It reguires that the combactiong parties anticipate, present and attack the

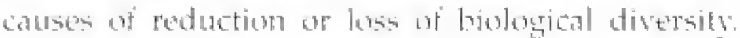
The consention notes that the fundamental fequitement for the consersation of biological diversity is the in sits comervation of ecosvstems and natural habitats and tha maintenance and 


\section{Institutional, legislative and policy tramework}

reconers of riable population of species in the natura] sumpundings." To achiese this, contractung

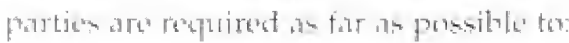

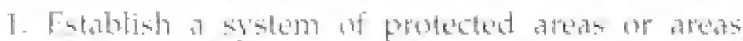

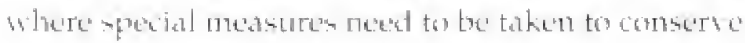
biakgacal dinetsits:

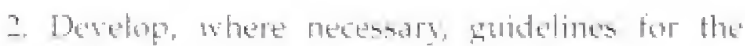
selection, entablishment and managennent of

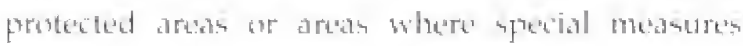

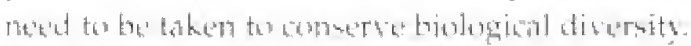

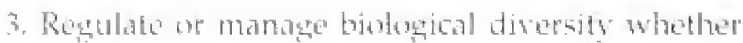
within or oulside prentected areas, with a riew lo

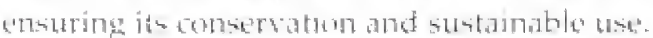

t. Promente the probetion on exosistems, matural

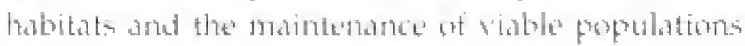
at specieten in nalural surterundines.

7. "romote empiromuncontaly" soumet and sustainable" Ateredopment in areas adjacent to probected areas with a ver to bithering protection of these aldeas.

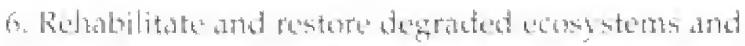

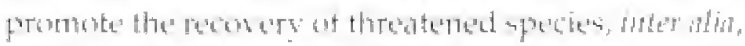
through the development and implementation al plants ar wher manemement strategies.

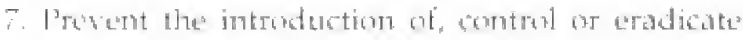
thuse alien speries which thearen ecosysternto bathitaten apesices

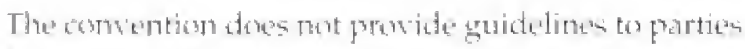
for settme prorities anong sites that are important

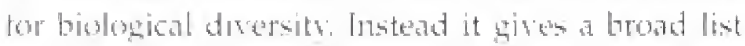
that indeates the componemts of biological diversity that are important for if consersation and

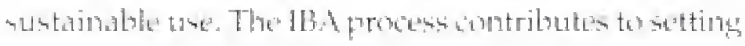
pritritice for consersation action, thus helping tas fuifil mational ublithations under this convention.

\section{The World Heritage Conwention}

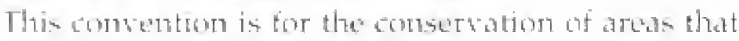
ates of atstatuding global kaltural or natural valbe. The follawing are conpidered as lutural heritage: (a)

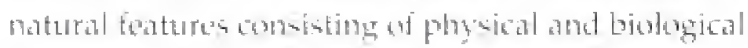
formations or sroups at such formations, which atte

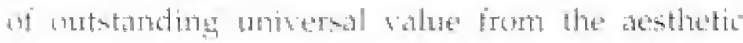
or scientific point of siew"; (b) geolocical and physiographical formations and precisely delineated areas which combitule the habitat of threatened species of andmals and plants of universt salte from the point of vitere of science or conselvation:

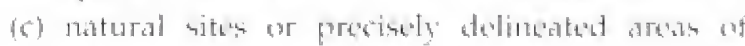

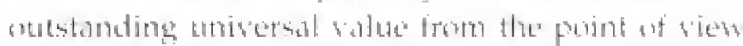

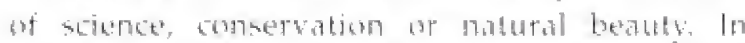
addition the comention requires that eate party recoptrises its duty to ensure the identification. profection, consersatien, presentation and

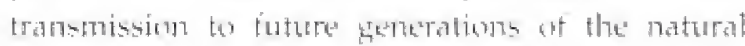
heritane situated within its territurs. The primary

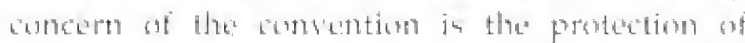

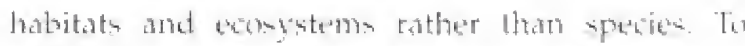

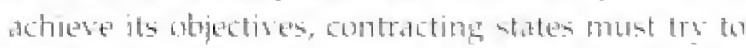
integrate heritage protection inds comprehensise planning programmes, to set up services for heritage

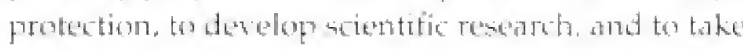
legal, scientific, technical, administrative and

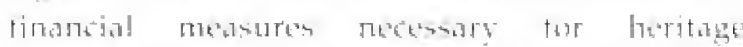
identification, protection, consersation and fresentation. Since the sites combltube a sorld

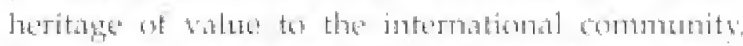

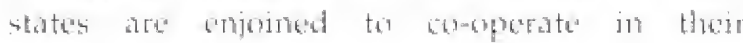
Eonservation and presertation.

By the estat of I942 there were laty patties to the comention, making it the most widely applicabute aredetaced cemeersation traty in the world. It Kenya only sibilot Vational l'ark has bued proponed for incluston in the list of Horld Heritage sites, enem though mang more sitem would quality:

\section{UNESCO's Man and Blosphere programme}

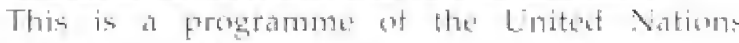

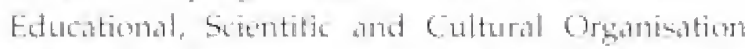
(LNESCO) that was launched in 1670 . Bowphere reserves are areis of terestrial and coastal ecossistems which are internationally recosnined whith the framework of LNESCOs Man and

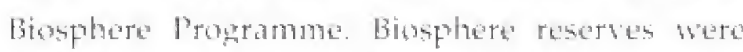
initiated with the anm of develepping tational use and

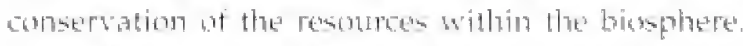
The'y form a world-wede metwork repremenkmg difterent ecossstems and are selected w the bisis of foner categories. These aro (a) representative cxamples of natusal biomes; (b) turique communities wa" areas with wnutual natural feakures of exceptional interest, such as a population of a globally rane species; (b) examples of harmonious landscapes resultimg from traditional patterns of land uste (d) uxamples af modified or degracted consystems capable of being restered to more natural conditions Conservation. scientitic research. monitoring education and araming are tesential pupposes of 


\section{Important Bird Areas in Kenya}

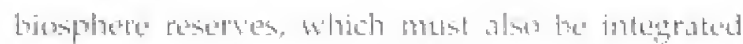

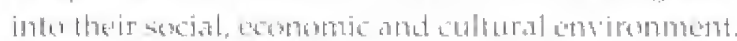

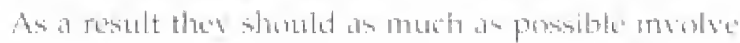

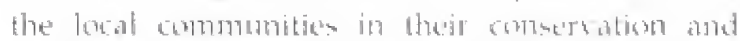

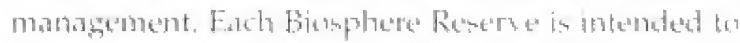

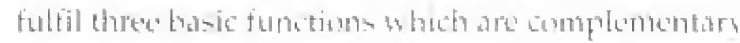

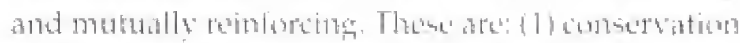

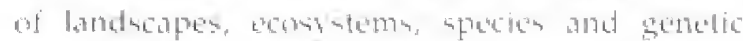

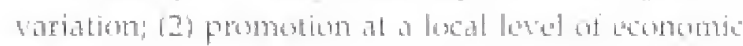
dowelopment which on culturally socially and

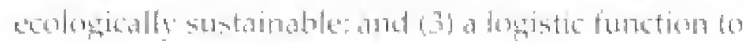

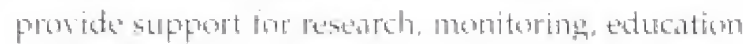
and intormation behames.

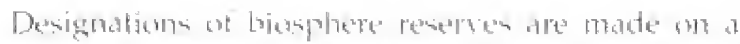

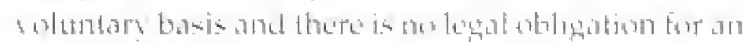

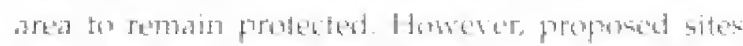

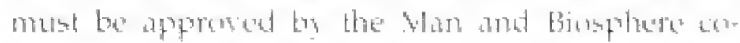

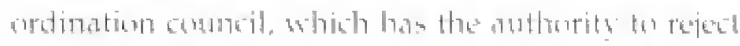

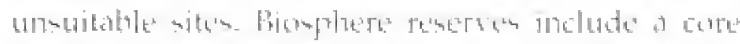

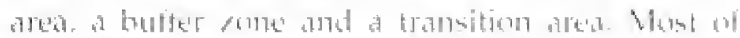

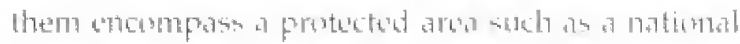

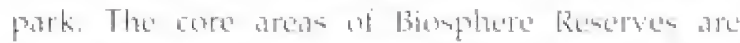

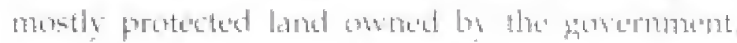

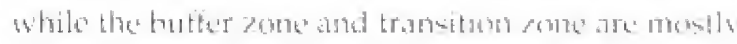

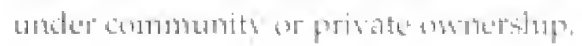

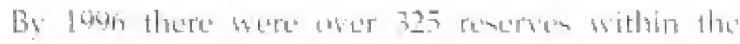

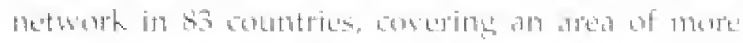

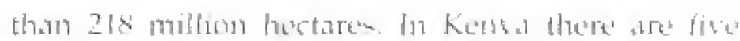

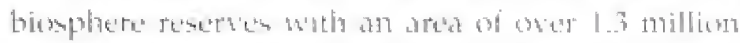

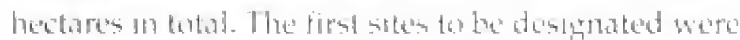

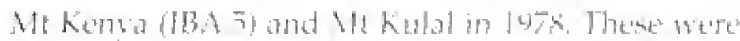

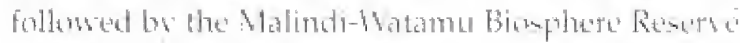

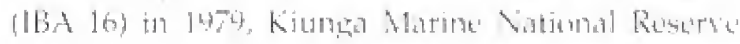

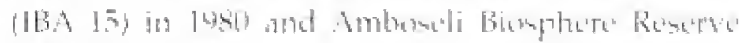
$($ IBA +2$)$ in 7 [is 1

\section{The Convention on International Trade in Endangered Species of Wild Flora and Fauna [CITES'}

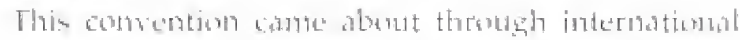

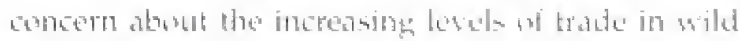

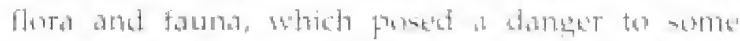

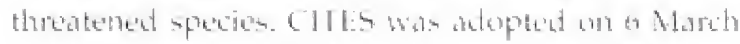

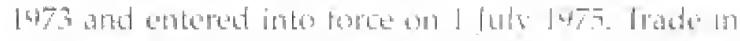
particular apecies, which are linted on the cITES

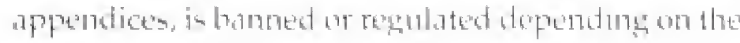

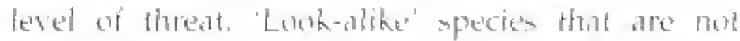

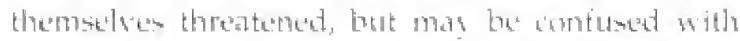

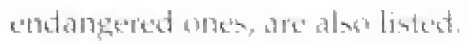

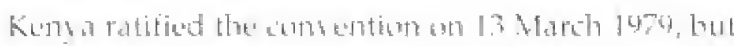

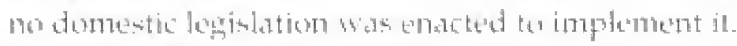

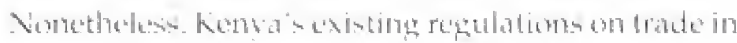
wildtite and its products meen and execed the

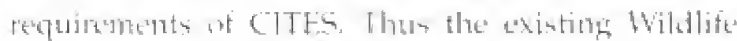

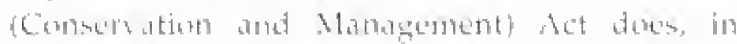

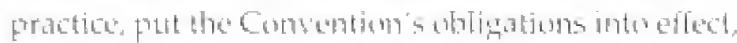

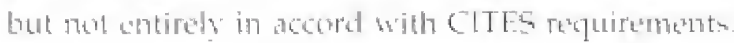
list inatance. the consention reguires that contracting parties designate separate management

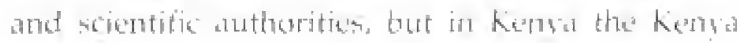

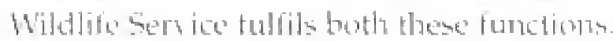

\section{The Convention on the Conservation of Migratory Species of Wild Animals (Bonn Convention)}

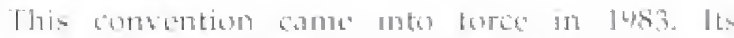

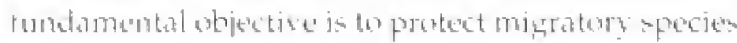

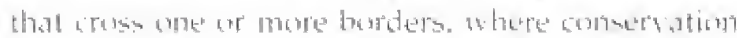

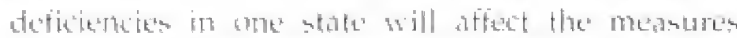

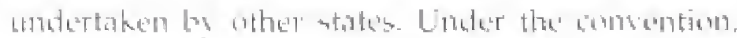

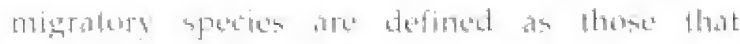

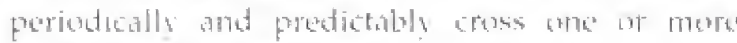
juriadictional boumalares.

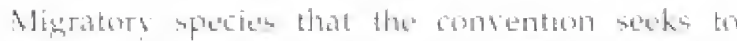

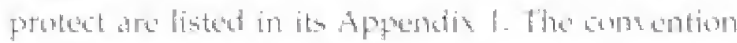

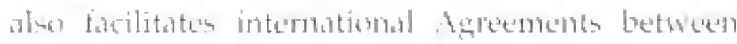

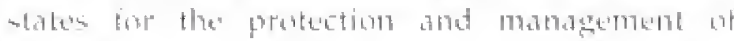

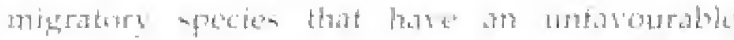

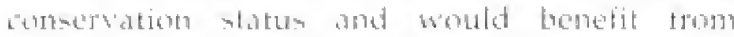

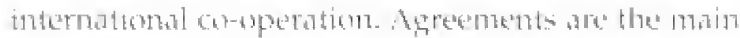

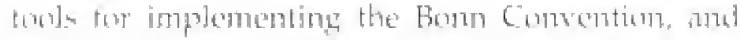

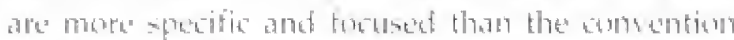

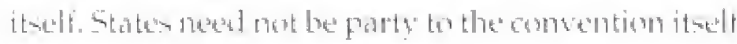

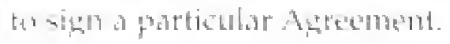

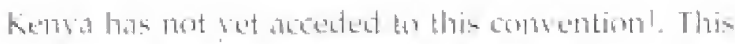
is atespite the bact that the benar Convention's

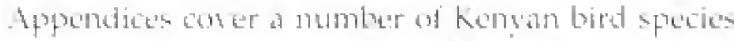

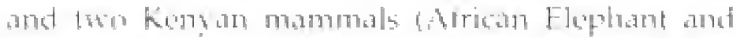

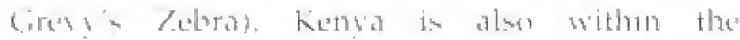

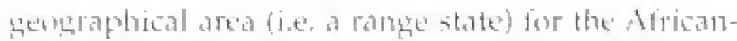

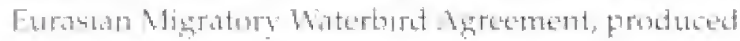
under the combention. This Agretement has bees

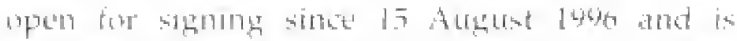
expectede to enter into force in the neas futures. It

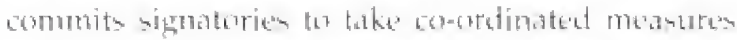

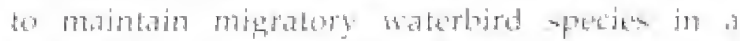

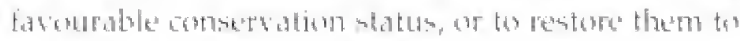
stich a whatus.

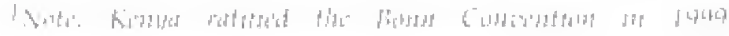

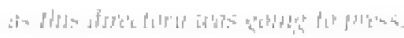




\section{Priorities for action}

\subsection{Priorities for action}

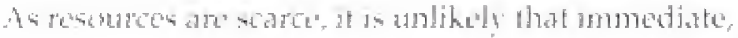
comprethensise comsersation actors can be taken at

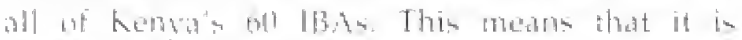

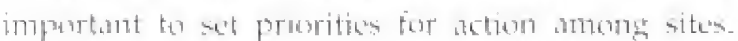

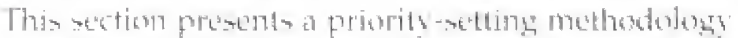

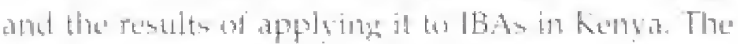

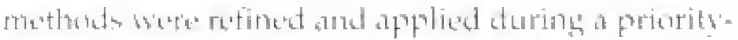

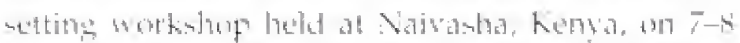

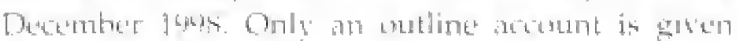

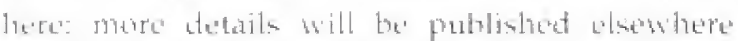

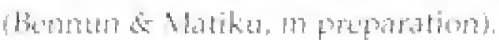

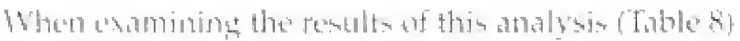

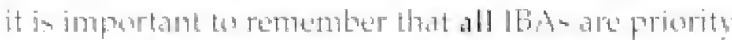
sitem for binthersity consersation. This andysis

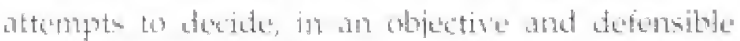

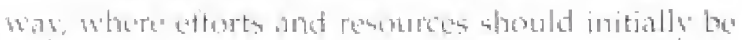
turgeted.

\subsubsection{The approach}

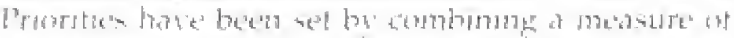
threat with a meanure bot biological importance. As

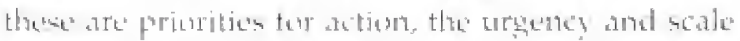

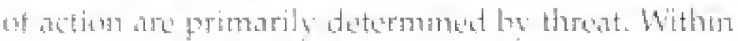

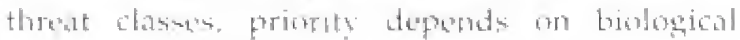
itrpportunces.

\subsubsection{Assessing threat}

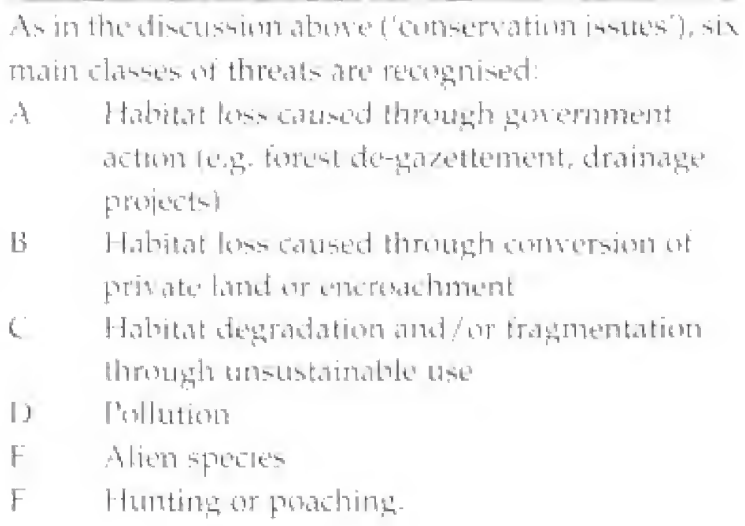

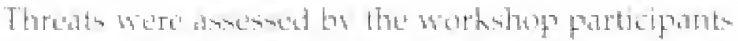

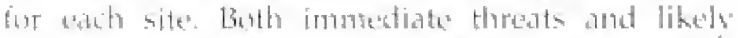
tuture threats (within the nut three years were

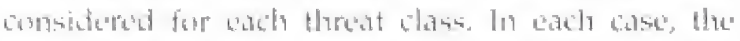

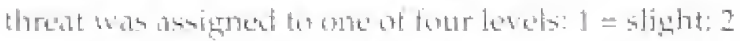

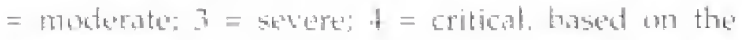
actual impact a particulat Herat would tatye won the situr and its biodisersith. (Thus a threat that was

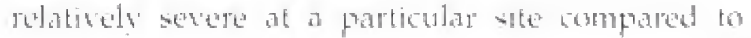
other sites, but triduld have little actual impoct. could be rated slight:

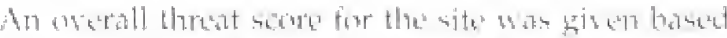

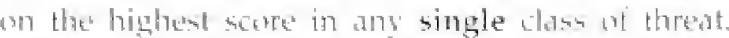

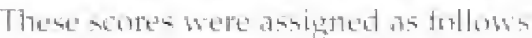

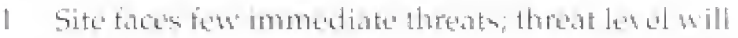
not increase greatly if rou action taken within

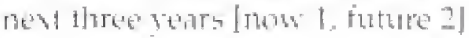

2 Site tates moterate bromediate threst, and/or

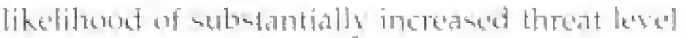

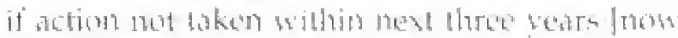
2. iuture 31

3 Site face substantial immeriate threst, and/or

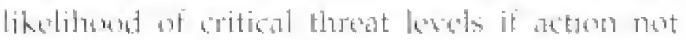

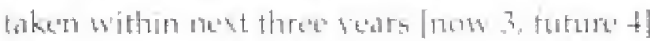

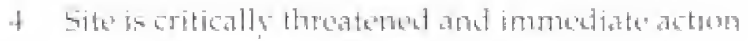
escential] [nom 4 +

The threats assignes for wach sibe, and the onerall

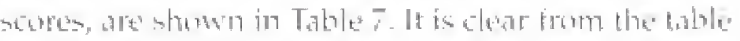

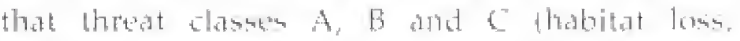
fratementation and destedationt ale by far the mant

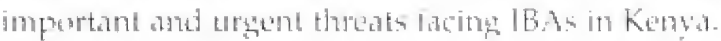

\subsubsection{Biological importance}

Fere, biologisal imfortance hats tur compontunts: importance for birds, and importance for other biodiversity. Importance for binto is the mole

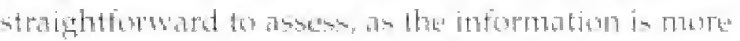

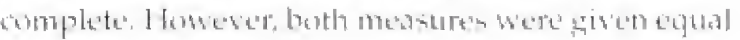
weight in the find ramking

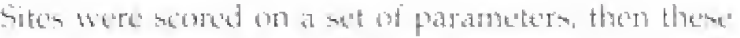

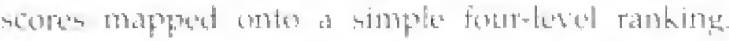

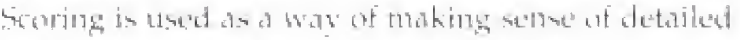

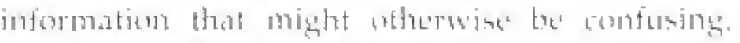

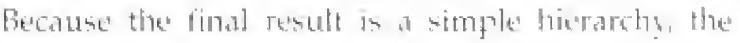

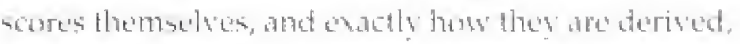

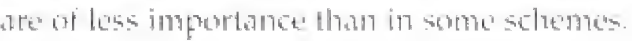

The size of a site" and its global uniqueneses atre not explicity comsidered. Theres aspects and allects

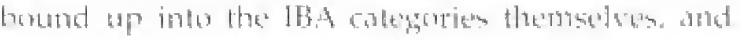




\section{Important Bird Areas in Kenya}

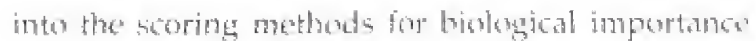

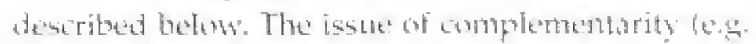
Howard et at 1998) Was alat ignored: thus a particular site's priority was not affectert by the kinds of sites alreakfy listad. Agation, complertentarit. in amplicit at an earlier stage in the process, in the IBA requirements to cover the full bel of restribedrange and biome-gestrictid species in a country

\section{lmportance for birds}

There are four categories in which IBA nav be list-

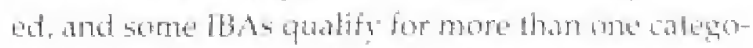
ry All else being aquat, the more catewhifes that an 1BA qualifies for, the mare important it san be con-

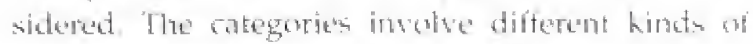

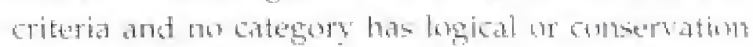
priority ane any other. They are in effect different currencies that need to be combined.

The" apporoch taken here was to socte sites within all

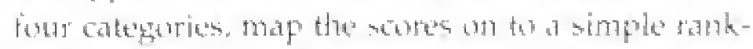

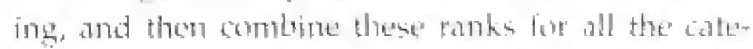
gerien to grive a final lewe of importance. Notte that

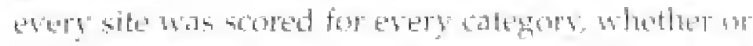

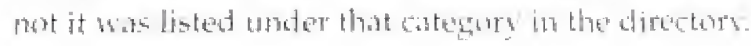

\section{At. Therenteded spectes}

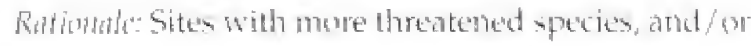

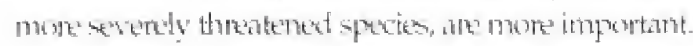

Each throutened species was assigned pointh as follows:

Critically theatered

Endarigered 2

Vultucablate

Near-threatented 0.5

Reghonally threatertud

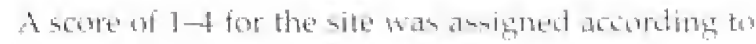
the toutal points. Appendix shows the date used to calculate these scores tor Kemb's I 13 As

$\begin{array}{lc}\text { Total } & \text { Assigned } \\ \text { points } & \text { score } \\ 0-0.9 & 0 \\ 1.1 .-1.4 & 1 \\ 2.0-2.9 & 2 \\ 3.10-3.4 & 3 \\ 3.9 & 4\end{array}$

The notes on applying the [BA triteriat distingulush

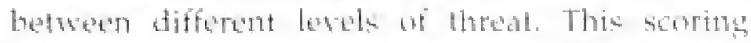
syathon attenupty to weight the different levels. It also introluces a slightly broader fits of importaneo for threaterket binds by including a modest weighting for regionally threatened species.

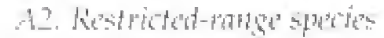

Rathate: Sites with more restricted-range specits art mene impartant.

The number of restricted-range species was fotallet and a soort assigned according to the following sctile:

$\begin{array}{cc}\text { Total } & \begin{array}{c}\text { Assigned } \\ \text { score }\end{array} \\ 6 & 0 \\ 1 & 1 \\ 2 & 2 \\ 3 & 3 \\ >3 & 4\end{array}$

This apporach consicters the absolute number onf restricted-range birds, rather than the proportious ant Whe bora] dordeplement within the EBA. This ferchnises that not ald EBAs are of equal importance as aentres of entismism.

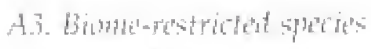

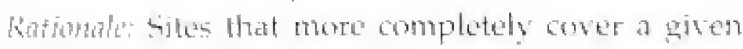
bisme are more important.

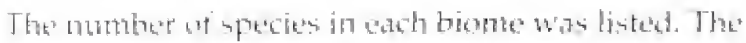
Pereentare that this cortstitutes of the Kenyan total an apretes in that biome was then calculated. The

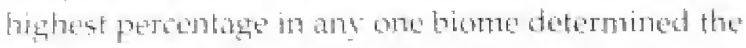
assigned score.

\begin{tabular}{|c|c|}
\hline $\begin{array}{l}\text { Highest } \\
\text { percentage of } \\
\text { biome species }\end{array}$ & $\begin{array}{l}\text { Assigned } \\
\text { score }\end{array}$ \\
\hline$n-1 n$ & 0 \\
\hline $1:-33$ & 1 \\
\hline $3 t-51\}$ & 2 \\
\hline $51-137$ & 3 \\
\hline 67 & 4 \\
\hline
\end{tabular}

Fuin approbsh has the folluming teatures:

- the percentage of woh biome total is considured: L.e. in 'srnall" biome like the Lake Whetoria Basin biome bonsidered a important as a 'large' oner tike the Somali-Masai biome. Biome-testricted assemblages ate among the IBA categnries in order to enotre that no appropriate bire spetith

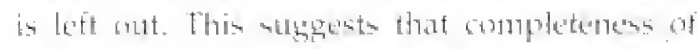
amerage is more signifizant than absolute numbers (i.ce it is not the tact that a site conatains 


\section{Priorities for action}

many species that is important but that thim number constitutes a significant propurtion of the onerall sett)

- For similar reasonas these percentages are not additive acrosh biomes. It would nake littlo sense to have a site that had a fow biome species in several bownes seoring higher than one with alnost all the species in whe biome.

- The percertate calculated is out of the number of bome specits nalionally, not across the bione as a whole. Thin corresponds with the

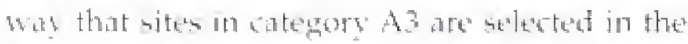

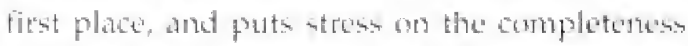
of sorerage actoss the whole geograptrical mpread of a bicme?

\section{At. Gurpretrations}

Ratuphls: Siten that qualin for more species, or that mowe substantially evered the threshold salues for

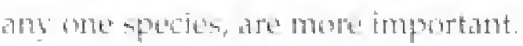

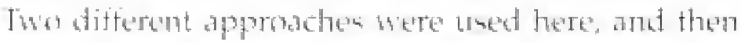
combinged to cteate a simple socrit.

(a) For bach spectes for which the site in listed for for all uperitu combined if linted under category A(iii)\}, the frumbers of that ofecoin reanded was dis ideat by the theshold salue. The highest multiple of the threwhuld was thed to ascign a score, as follows.

$\begin{array}{lc}\text { Multiple } & \begin{array}{c}\text { Assigned } \\ \text { score }\end{array} \\ 01-0.9 & \{\} \\ 1.6-29 & 1 \\ 3.6-6.9 & 2 \\ 7.09-1+9 & 3 \\ 3.9 .9 & 4\end{array}$

(t) The number of individual species for which

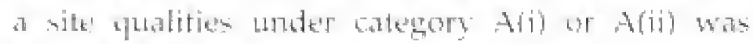

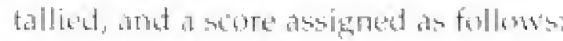

$\begin{array}{cc}\text { Number } & \text { Assigned } \\ \text { score } \\ 0 & 4 \\ 1 & 1 \\ 2 & 2 \\ 7 & 7 \\ >3 & 4\end{array}$

The moan seose of (a) and (b) gave the averall soore for this catemory

\section{Qverall bird importance}

The scores for all four categyories were summed and an onerall ranking giver a follows:

$\begin{array}{lc}\text { Overall summed } & \\ \text { score } & \text { Rank } \\ 1.0-2.9 & 1 \\ 3.0-4.9 & 2 \\ 3.0-6.9 & 3 \\ >0.9 & 4\end{array}$

Appendix 7 shows the dita athat scotes in each ategery for kumbors IBAs.

\section{Importance for other biodiversity}

A rank from I-t was rissigned to ecath site as fullows. based on overall diversity and/or the numbers of (viable populations of) globally threatened or (near)endemic plants, butterflies, fish, reptilew, almphibeans or mammals present. Fonduric or matrendemic specter were bonsidered thost comfined to this site and at most two other knowern siless. ?

\begin{tabular}{|c|}
\hline $\begin{array}{l}\text { Numbers of threatened/endemic } \\
\text { species }\end{array}$ \\
\hline Luw - 11 \\
\hline Minderate - 1-2 \\
\hline H ligh $-3-5$ or $<3$ and nedy diverse \\
\hline lery high - > 5 or $3-5$ and very di \\
\hline
\end{tabular}

Enough information exists on all kempats IBAs to attempe this ranking, though the criteria are necesartily more vague than for the bird importance socese

\section{Overa!l biological importance}

This is a sone trom 2-8 produced by adding the scorem for oneralt bird importance and inportanch for other biudiversily:

\subsubsection{Priority sites}

Table 8 shows the results of the priorits-setting wercioe, with sites ranked within threat dasies

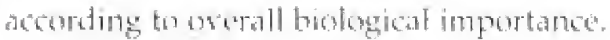

The number of IBAs with particular biological importance scores within each threat class is showen by a filled circle in the tollowing matrix.

\begin{tabular}{|c|c|c|c|c|c|c|c|}
\hline \multirow[b]{2}{*}{ Threat } & \multirow[b]{2}{*}{8} & \multicolumn{5}{|c|}{ Biological importance } & \multirow[b]{2}{*}{2} \\
\hline & & 7 & 6 & 5 & 4 & 3 & \\
\hline 4 & 98 & 6 & es & be & a & ef & \\
\hline 3 & 86 & 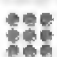 & te & $\Leftrightarrow$ & * & & \\
\hline 2 & $\bullet$ & a & & $8 \%$ & - & 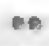 & $\bullet$ \\
\hline 1 & - & $e$ & eet & reet & & ne & an \\
\hline
\end{tabular}

\section{Introduction Priorities for action}




\section{Table 7}

Classes and severity of conservation threats

to Kenya's IBAs

Threat category

\begin{tabular}{|c|c|c|c|c|c|c|c|c|c|c|c|c|c|c|}
\hline \multirow{3}{*}{$\begin{array}{l}\text { Site } \\
\text { cote }\end{array}$} & \multirow[b]{3}{*}{ Sitt" names } & & \multirow{2}{*}{\multicolumn{2}{|c|}{ C }} & \multirow{2}{*}{\multicolumn{2}{|c|}{$\mathrm{D}$}} & \multirow{2}{*}{\multicolumn{2}{|c|}{$E$}} & \multirow{2}{*}{\multicolumn{2}{|c|}{$F$}} & \multirow{3}{*}{$\begin{array}{l}\text { Overall } \\
\text { score }\end{array}$} \\
\hline & & & & & & & & & & & & & & \\
\hline & & r & $\mathrm{F}$ & $\mathrm{l}^{\prime}$ & $\mathrm{F}$ & $P$ & $\mathrm{~F}$ & $P^{\prime}$ & $\mathrm{F}$ & $\mathrm{I}^{\prime}$ & $\mathrm{F}$ & $\mathrm{P}$ & $\Gamma$ & \\
\hline 1 & Aberdare Mountans & 1 & 1 & 2 & 2 & 3 & 3 & 1 & 1 & 1 & 1 & 1 & 2 & 3 \\
\hline 2 & Kianyage valleys & 1 & 1 & 4 & 4 & 4 & 4 & 1 & 1 & $t$ & 1 & 3 & 3 & 4 \\
\hline 3 & Kikuy' Escorphnent Fovest & 3 & 4 & 2 & ; & 3 & 3 & 1 & 1 & 1 & 1 & 1 & 1 & 3 \\
\hline+ & 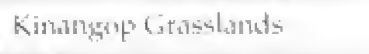 & 1 & 1 & 4 & $t$ & 4 & 4 & 」 & 1 & 2 & 3 & 1 & 1 & 4 \\
\hline 5 & Wt Kenya & 3 & 3 & 3 & + & + & 4 & 1 & 2 & 1 & 1 & 2 & 2 & + \\
\hline n & Mukurwentu balles' & t & 1 & 4 & 4 & t & 4 & 1 & 1 & 1 & 1 & 2 & 2 & 4 \\
\hline 7 & Arabuko-Sokoke Forent & 4 & 4 & 2 & 3 & 3 & 3 & 1 & I & 1 & 1 & 2 & 2 & + \\
\hline 8 & Dakatha Woontland & I & । & 3 & 3 & $?$ & $\xi$ & 1 & 1 & 1 & 1 & 2 & 2 & 3 \\
\hline 9 & Diasni Forest & 1 & 1 & 3 & 4 & 4 & 4 & 2 & 2 & 1 & 1 & 1 & 1 & 4 \\
\hline 16 & Damborlo Hill Forsent & 1 & 1 & 1 & 1 & 2 & 2 & 1 & 1 & 1 & 1 & I & I & 2 \\
\hline 11 & $\begin{array}{l}\text { Gede Kuins } \\
\text { National Mennument }\end{array}$ & 1 & 1 & 1 & 1 & 1 & 1 & 1 & $b$ & 1 & 1 & ] & 1 & 1 \\
\hline 12 & Kaya Gandini & 1 & 1 & 1 & 1 & 1 & 1 & 1 & 1 & 1 & 1 & 1 & 1 & 1 \\
\hline 13 & Kaya Wat & 4 & 4 & 1 & 1 & 3 & 3 & 1 & 1 & 1 & 1 & 1 & 1 & 4 \\
\hline 14 & Kinite |siand & 1 & 1 & 1 & 1 & 1 & 1 & 1 & 1 & 1 & 1 & 1 & 1 & 1 \\
\hline 15 & $\begin{array}{l}\text { Kitunga Mhrine } \\
\text { Mational Renerve }\end{array}$ & 1 & 1 & 1 & 1 & 1 & 1 & 1 & 1 & 1 & 1 & 2 & 2 & 2 \\
\hline 16 & 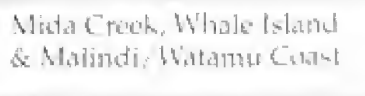 & 1 & 1 & 1 & 1 & \pm & 2 & 1 & 1 & 1 & 1 & $\beth$ & 2 & 2 \\
\hline 17 & Maremji Fonest & I & 1 & 1 & 1 & 1 & 3 & 4 & 1 & 1 & 1 & 1 & 1 & 4 \\
\hline 18 & Мглла Hall Furect. & 1 & 1 & 2 & 3 & 3 & 3 & 1 & 1 & 1 & 1 & I & 1 & 3 \\
\hline 14 & Sabaki River Wouth & 1 & 1 & 1 & 1 & 2 & 2 & 2 & 2 & 1 & 1 & 1 & 1 & 2 \\
\hline 20 & Shimbatsils & 1 & $\mathbb{1}$ & 1 & 1 & 2 & 3 & 1 & $t$ & 1 & 1 & 3 & 2 & 2 \\
\hline 21 & Thital Hills Foressty & 1 & 1 & 2 & 2 & 4 & 4 & 1 & 1 & 3 & 4 & 1 & 1 & 4 \\
\hline 22 & 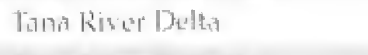 & 3 & 4 & 2 & 2 & $I$ & 2 & 1 & 2 & 1 & 1 & 1 & 1 & 3 \\
\hline 23 & Tana River Fonests & I & I & + & 4 & 4 & 4 & 1 & 1 & 1 & I & 1 & 1 & 4 \\
\hline $2-1$ & 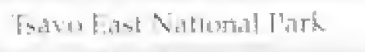 & 1 & 1 & 1 & I & 1 & 1 & 1 & 1 & l & 1 & 1 & 1 & 1 \\
\hline 25 & Traved West Vational Pork & 1 & 1 & I & 1 & 1 & 1 & 1 & 1 & 1 & 1 & 1 & 1 & 1 \\
\hline$I_{i}$ & Chvulu I tills Foresests & I & I & 1 & 2 & 1 & 2 & 3 & 1 & I & 1 & 1 & I & 2 \\
\hline 27 & Dida Galgalu Desert & 1 & 1 & 1 & 1 & 1 & 1 & 1 & 1 & 1 & 1 & 1 & 1 & 1 \\
\hline 26 & Lathe Tushumbl & 1 & 1 & 1 & 1 & 1 & 1 & 1 & 2 & 1 & 1 & 2 & 3 & 2 \\
\hline 29 & Machalasos Valleys & 1 & 1 & 2 & 3 & 2 & 3 & I & 1 & 1 & 1 & 1 & 1 & 2 \\
\hline 30 & 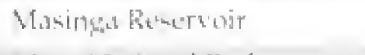 & 1 & 1 & 1 & 1 & 1 & 1 & 1 & 2 & 1 & 1 & 2 & 3 & 2 \\
\hline 31 & Meru National Park & 1 & 1 & 1 & 1 & 1 & 1 & J & 1 & 1 & 1 & 1 & 1 & 1 \\
\hline 32 & 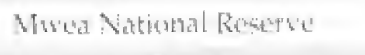 & 1 & 1 & ] & I & 1 & 1 & 2 & 2 & 1 & 1 & 1 & 1 & 2 \\
\hline 33 & $\begin{array}{l}\text { Sunbura/Buffalo Springs } \\
\text { National Rematses }\end{array}$ & 1 & 1 & 1 & 1 & 1 & 1 & 1 & 1 & 1 & $i$ & 1 & 1 & 1 \\
\hline 34 & Shaba Natumal Restorte & 1 & 1 & 1 & 1 & 1 & 1 & 1 & I & 1 & I & 1 & 1 & 1 \\
\hline 35 & Dandiora fonts & 1 & 1 & 1 & 1 & 1 & 1 & 1 & 1 & 1 & 1 & 1 & 1 & 1 \\
\hline 30 & 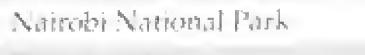 & t & 4 & 1 & 1 & 1 & 1 & 2 & 3 & 1 & 1 & I & 1 & 4 \\
\hline 37 & Dunga Swamp & 1 & 1 & 2 & 3 & 3 & $\frac{1}{4}$ & 2 & 3 & 2 & 2 & 2 & 2 & 3 \\
\hline
\end{tabular}




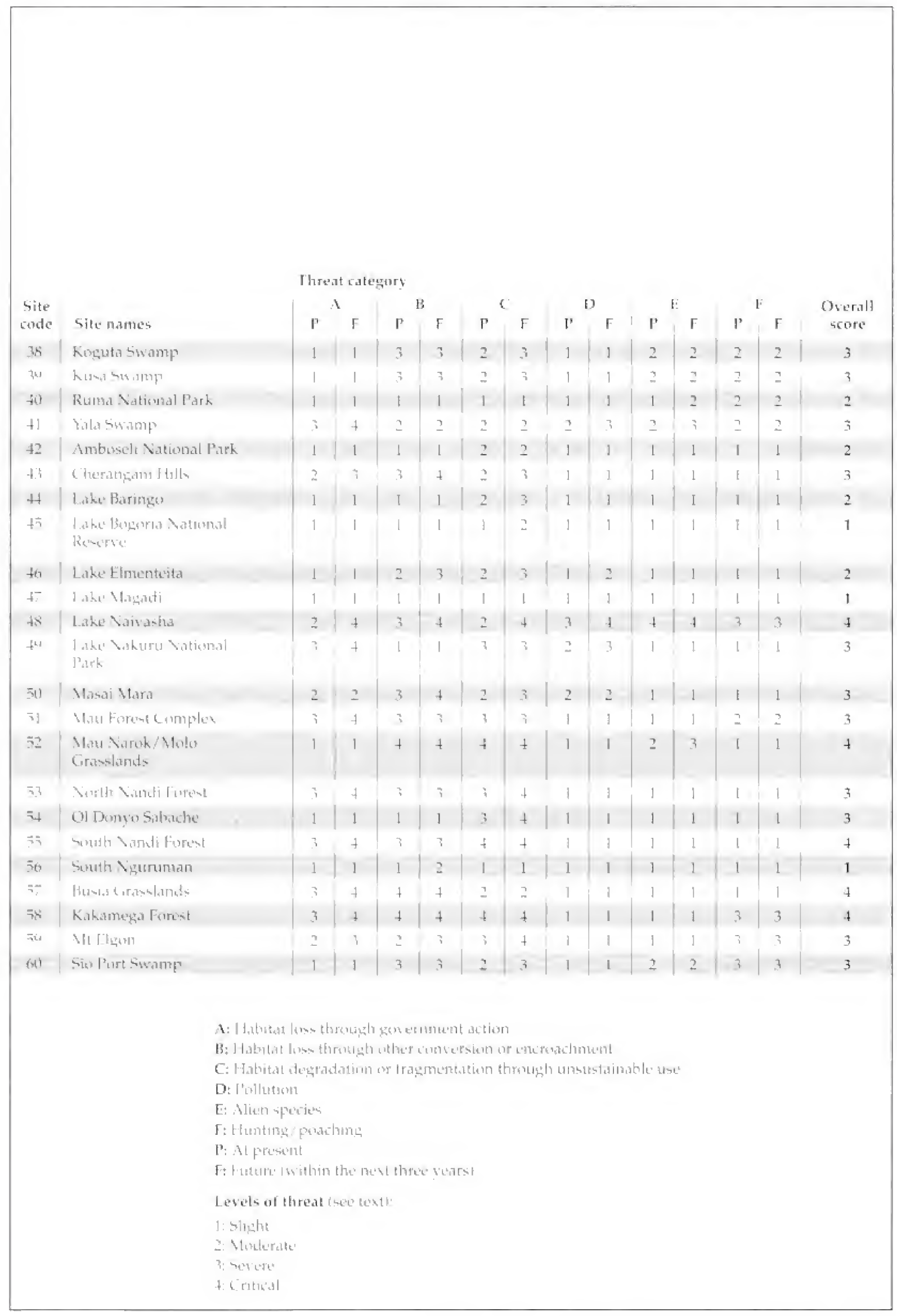




\section{Table 8}

Biological importance and threat scores for Kenya's IBAs

Sites are ranked by biuhgical importance score

(minimum 2, maximum 8) within threat classes

(most threatened 4, least threatened 1)

(tied sites ire listed in order of kite number)

\begin{tabular}{|c|c|c|c|c|c|c|c|}
\hline $\begin{array}{l}\text { Site } \\
\text { code }\end{array}$ & Site names & Birds & Biodiv. & Overall & Threat & P'riority for action & $\begin{array}{l}\text { Site } \\
\text { code }\end{array}$ \\
\hline 5 & Mt Kenya & 4 & 4 & $s$ & 4 & CRITICAL & ; \\
\hline 7 & Aratuluk-Solkoke forest & 4 & 4 & s & 4 & CRITCAL & 7 \\
\hline 21 & Tuitu Hills Fortests & 4 & 4 & 8 & 4 & CRITICAL & 21 \\
\hline 23 & Tana kiser Foreus & 4 & 4 & s & + & CRITICAL & 23 \\
\hline 36 & Nairobi Vational Park & 4 & 4 & 8 & $t$ & CRITICAL. & 36 \\
\hline 58 & Kahamega Fores & 4 & 4 & 8 & 4 & CRITICAL & 58 \\
\hline 32 & Mau Namk/alolo Girasilands & 7 & 3 & 7 & 4 & CRITICAL & 52 \\
\hline 55 & South Nandi Forest & 3 & 4 & $\bar{i}$ & 4 & CRIJICAL & 55 \\
\hline 4 & Kinangep Grasslands & 3 & 3 & 6 & 4 & CRITICAL & 4 \\
\hline 9 & Diam Furest & 3 & 3 & 6 & + & CRITICAL & 4 \\
\hline 13 & Kaya Waa & 2 & 3 & 5 & 4 & CRITICAL & 13 \\
\hline 57 & Bstasia Grasslomuts & 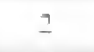 & 3 & 5 & 4 & CRITICAL & 57 \\
\hline 48 & Lake Nairasha & 2 & 2 & 4 & 4 & CRIICAL & 45 \\
\hline 2 & Kiarygaga biallens & 2 & 1 & 3 & 4 & CRITLAL & 2 \\
\hline 6 & Mukurweint Valleys & 2 & 1 & 3 & 4 & CRMC IL & 6 \\
\hline 1 & Aberdares Monntain & 4 & 4 & $b$ & 3 & CRITICAL & 1 \\
\hline 22 & Tanา Kiver Delta & 4 & 4 & 8 & 3 & CRITICAL & 22 \\
\hline 50 & Matali Mard & 4 & 4 & 8 & 3 & CNITICA. & $5 t$ \\
\hline 59 & Mt Elgon & + & 4 & s & 3 & CRIICAL & 59 \\
\hline 3 & Kikusu Ecarpment Fonest & 4 & 3 & 7 & 3 & LIRGENT & 3 \\
\hline s & Dakotutas Werdland & 3 & 4 & 7 & 3 & URGENT & 8 \\
\hline 17 & Marenil Forest & 3 & 4 & 7 & 3 & URGINT & I7 \\
\hline 18 & Mrima Hill Forst & 3 & 4 & 7 & 3 & LHGENT & 18 \\
\hline+1 & Yalla swane?" & 3 & 4 & 7 & 3 & LRGINT & +1 \\
\hline+3 & Cherathani Hills & 3 & 4 & 7 & 3 & LRGENT & 43 \\
\hline+4 & Lahe Nahuru Vatioral Park & 4 & 3 & 7 & 3 & LIRGTST & thit \\
\hline 51 & Mau Forest Comples & 3 & 4 & 7 & 3 & URGENT & 51 \\
\hline 53 & Nurt]r Nuntíli Futust & 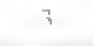 & t & 7 & ; & LRGFNT & 53 \\
\hline 37 & Dunga Swamp & 3 & 3 & 6 & 3 & URGENT & 37 \\
\hline 54 & (9] Domyes sabnetwe & 2 & + & to & 3 & URGENT & $5+$ \\
\hline 36 & Koguta 5 w. & 2 & 3 & 5 & 3 & URGENI & 38 \\
\hline 60 & Siw [rart Shamp? & 2 & 3 & $\overline{3}$ & 3 & LIRGENT & ti) \\
\hline 34 & Kusa Swamp & 1 & 3 & 4 & 3 & LIRGENT & 39 \\
\hline 20 & Slimba Hills Eorent & t & 4 & 8 & 2 & LKGFNT & It) \\
\hline 10 & Dzombo Hill Forest & 3 & 4 & 7 & 2 & LRGENT & 10 \\
\hline 28 & Lake Jurkatna & 3 & 4 & 7 & 2 & LRGEYT & 28 \\
\hline 46 & Lake Elmenteita & 4 & 2 & 6 & I & LRGENT & 46 \\
\hline 15 & Kiunga Marine Sational Reserve" & 1 & 4 & 5 & 2 & $\mathrm{HtCH}$ & 15 \\
\hline 16 & $\begin{array}{l}\text { Mida Creek, Whate bland } \\
\text { and Malindi/Watamu Const }\end{array}$ & 2 & 3 & 5 & 2 & $\mathrm{HICH}$ & 16 \\
\hline $2 \nmid 7$ & Cherulu Hills- Foreats & 1 & + & 5 & 2 & Hons & 26 \\
\hline
\end{tabular}




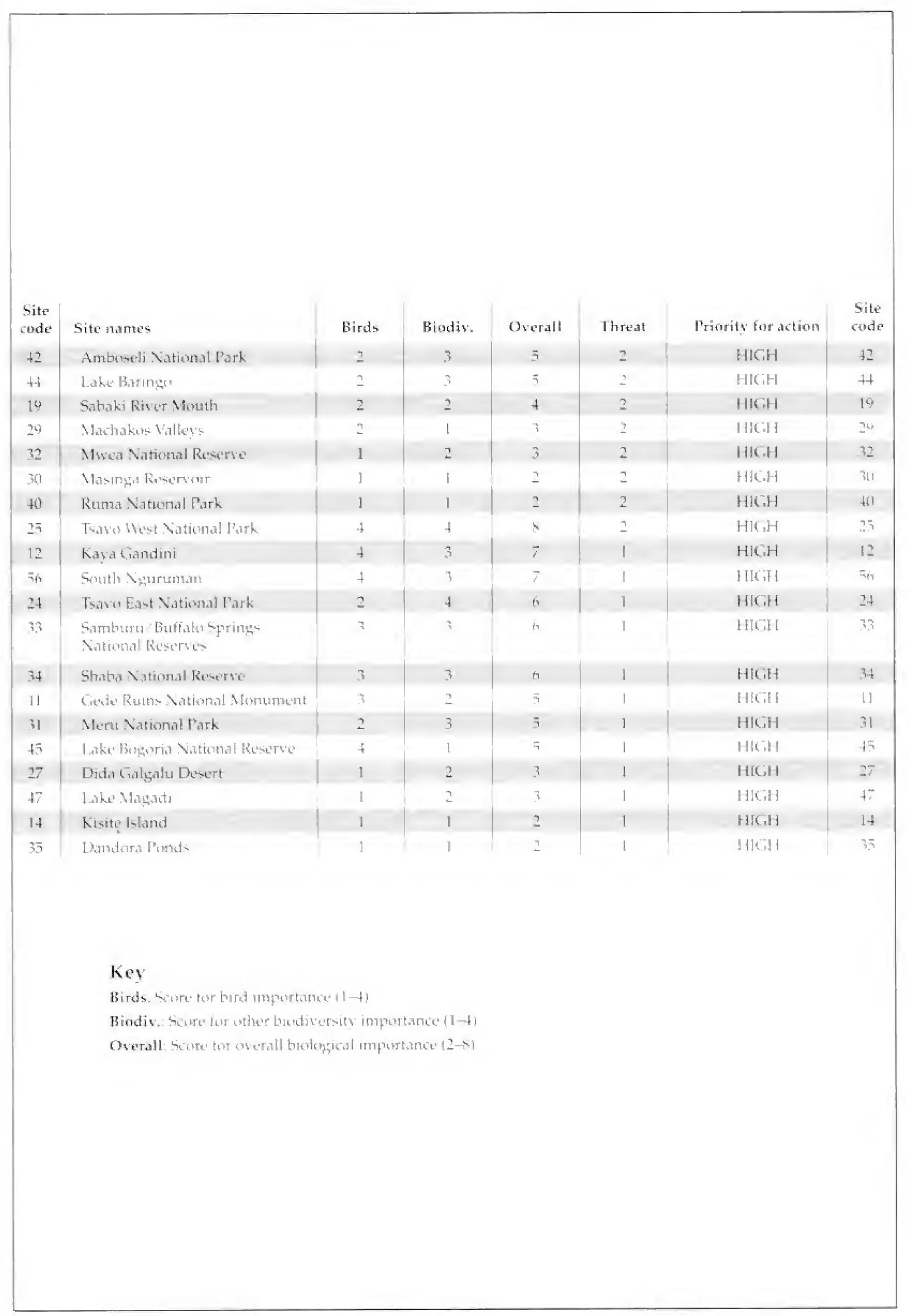




\section{Important Bird Areas in Kenva}

\section{Categorisation}

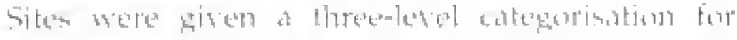
conseration action pribrity an follow

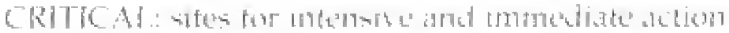

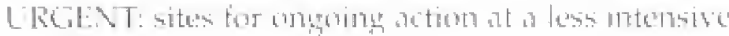
laxid

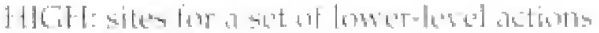

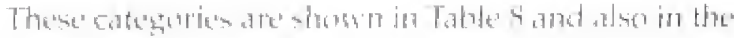

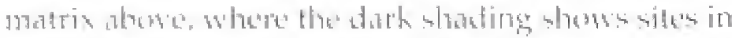

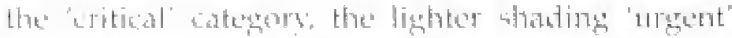

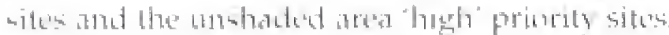

\section{Sites prioritised as "critical'}

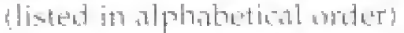

\begin{tabular}{|c|c|}
\hline Site no. & Site name \\
\hline 1 & aberdare vounataino \\
\hline 7 & 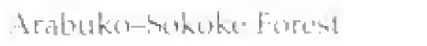 \\
\hline 57 & Buming Cirastand o \\
\hline 4 & Dhani Foresat \\
\hline नis & 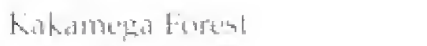 \\
\hline 13 & Kand kad \\
\hline 2 & 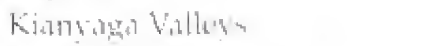 \\
\hline$f$ & Kinatgang Girabaland \\
\hline tis & 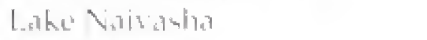 \\
\hline 30 & Mathai Matra \\
\hline 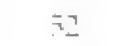 & 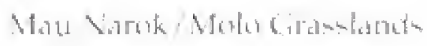 \\
\hline 34 & Wt Elom \\
\hline$\overline{7}$ & Vit Ke'riba \\
\hline 19 & 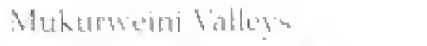 \\
\hline int & Vairngtri Satiatma] l'ark \\
\hline $5 \overline{7}$ & Senuth Nandi Forest \\
\hline 21 & 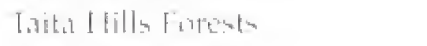 \\
\hline 22 & Tama Rusto Dolko \\
\hline 23 & 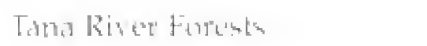 \\
\hline
\end{tabular}

\section{Sites priorifised as 'urgent'}

thintert in alphabelicall irder)

\begin{tabular}{|c|c|}
\hline Site no. & Site name \\
\hline 43 & Cherangatoi flills \\
\hline 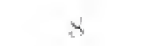 & Dakath ha Wekdlamet \\
\hline 37 & Dunge Sหr.tmp \\
\hline 10 & Danmba Hill Furesel \\
\hline 3 & Kikuyu Facarpment Foresi \\
\hline 38 & Koggula Swamp \\
\hline 39 & Kusa Swamp \\
\hline+6 & Lake Elmentexita \\
\hline 49 & I.ake Nakuru Natiunal laurk \\
\hline 26 & Lake Turkama \\
\hline 17 & Marenji Fure'st \\
\hline 51 & Whu Furest Garmplex \\
\hline 18 & Meima Hill Forest \\
\hline 53 & Vertly Vartali Forest \\
\hline 7.1 & 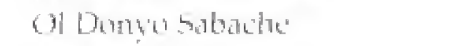 \\
\hline 20 & Shimba Hills Emesi \\
\hline$b(1)$ & Sio lent 5 w \\
\hline 41 & bila siramp \\
\hline $\begin{array}{l}\text { lites priori } \\
\text { listatel in al }\end{array}$ & $\begin{array}{l}\text { i as 'high' } \\
\text { etical oruler' }\end{array}$ \\
\hline Site no. & Site name \\
\hline 42 & Ambureli Nationat Park \\
\hline 2 & Chyculu Hills Forente \\
\hline $3 \overline{7}$ & Dandera Pands \\
\hline 27 & Ditar Collyalu Dutert \\
\hline 11 & Giede Ruins Nationsl Stmument \\
\hline 12 & Kina Gandini \\
\hline 14 & Kisite Island \\
\hline 15 & Kiunga Marine Vational Remerse" \\
\hline$H$ & Lake Barimpo \\
\hline $4 \overline{7}$ & Lake Buscoria National Reserve \\
\hline 47 & Lake' Magadi \\
\hline 24 & vachatas balleys \\
\hline 30 & Naxitga Reservotr \\
\hline 3] & Vere National l'ark \\
\hline
\end{tabular}

Elack-capped Socłal Weaver

\section{Introduction Priorities for action}




\begin{tabular}{|c|c|}
\hline 16 & 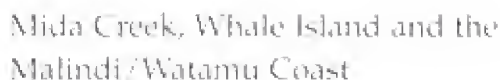 \\
\hline & 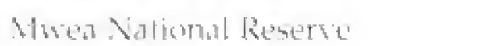 \\
\hline thi & Ruma Natiunal f'ark \\
\hline 14 & 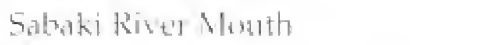 \\
\hline 3 & Santoura Buftale Springs \\
\hline & 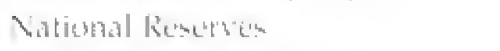 \\
\hline it & 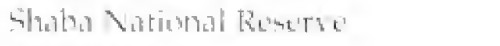 \\
\hline $5 t$ & South Nyctrumutr \\
\hline & Isatho Past Vational Park \\
\hline & Tsane hest Sational Park \\
\hline
\end{tabular}

\section{Conservation actions}

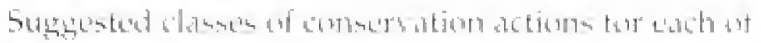

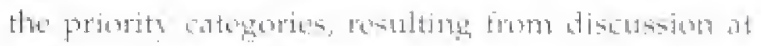

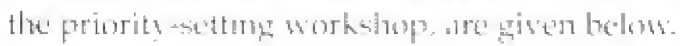

\begin{tabular}{|c|c|c|}
\hline 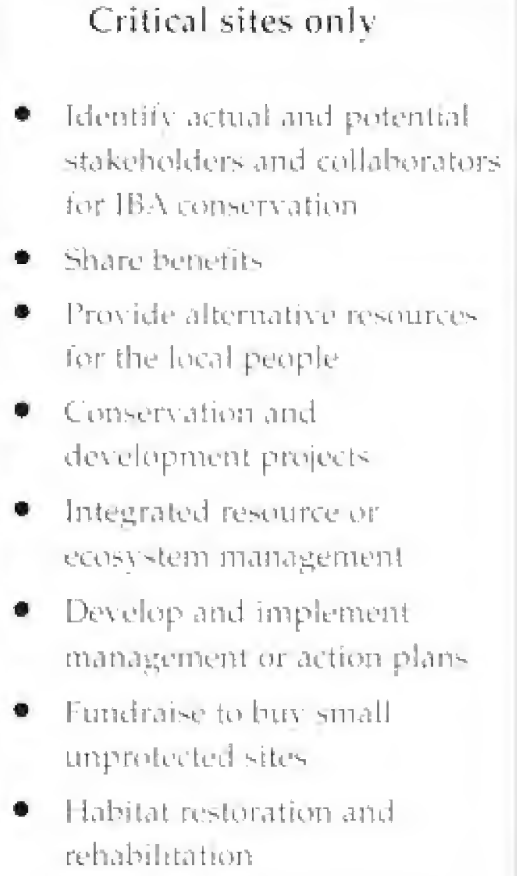 & 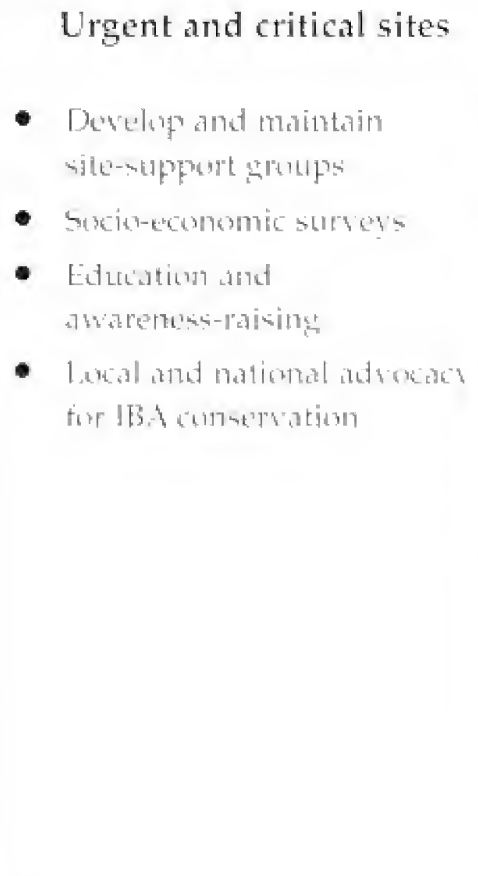 & 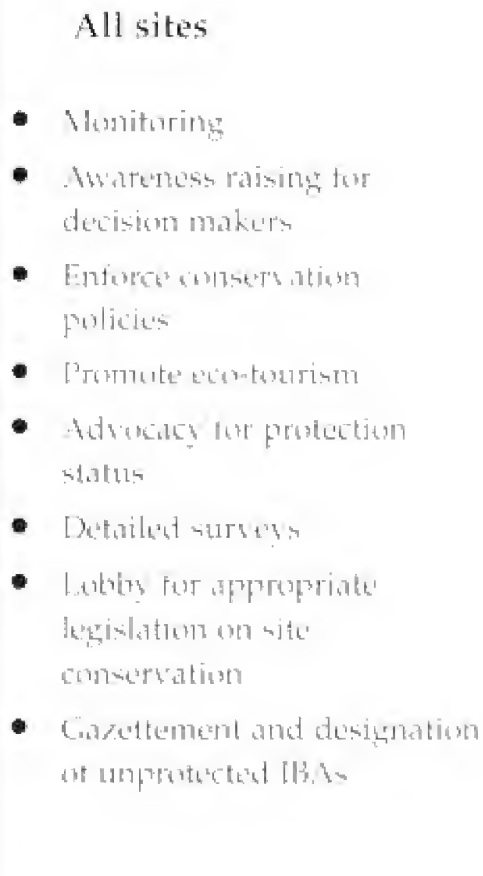 \\
\hline
\end{tabular}


Important Bird Areas in Kenya 


\section{Site Accounts}

\subsection{Presentation of Data}

The main part of this directory consists of detailed descriptions for the 60 sites listed as Important Bird Areas in Kenya. Seven potential IBAs are described at the end of the main site accounts.

Site dacounte we presented atplabetically by administrative province. Each site account Eontains the fesllowing actions

\section{Heading}

Thin Includs the site reterence number, site name.

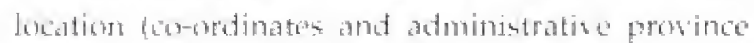

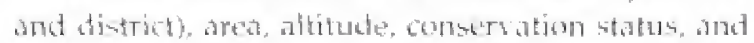
the entegories urdes which the ritu in Tisted.

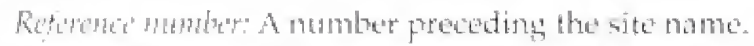
Sites ate numbered in the sequetere in whin the are

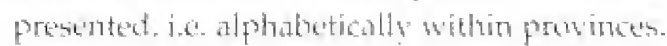

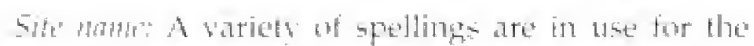
mames of sorne of the siles listed. We lanse chosen the" spellone that atppeared to be most appropriate. Lisually this is exthes the garetted fome ffor a protected areat), ar the name in most common use.

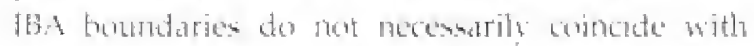

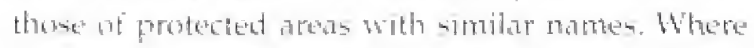

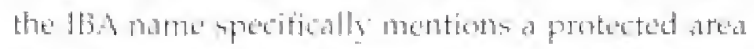
(e.e. 'Shoba National Reserve') then the [Bet and the' protedted area are congremertit.

Lotabs: The latitude and longitude (to cone minute

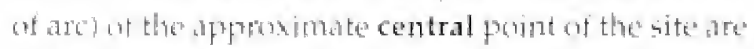

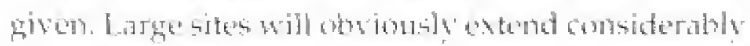

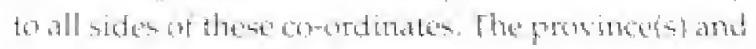
diatrictic) in which the site to located ate atso given.

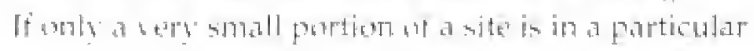
provinete ar district, thes in indicated.

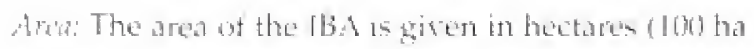

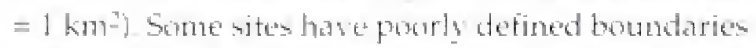

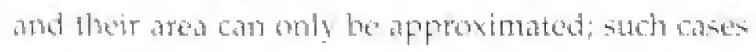
are inutiated. For wher sites, we hase used a sariby uf soures to obtain areas. Theme include the Wortat Constration Moritoring Cenere"s database of

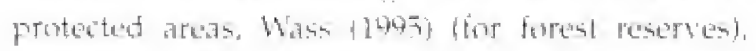

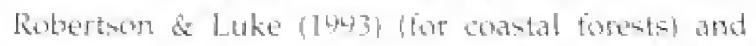
topographacal maps isuree of Kenva 1:250,omo

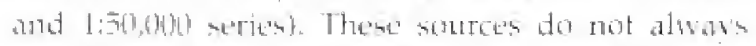

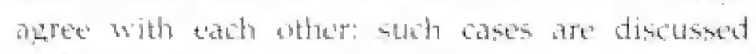
unter the sife deaription section.
Some IBAs combain a tartety of habitats, not all w

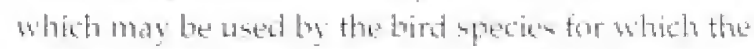

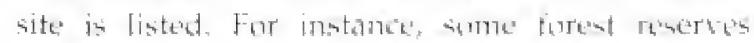

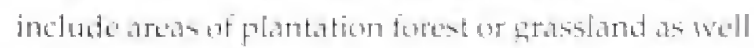
as indigenous torest. Where approprite, the artas of key habitats within the IBde are indicated: Further Atail may be givere undes the site description" atction.

Altwite: The approvimate altitudinal range of the site is grixen in metres ahorie sea lesel. derived from similar suturces to the site area.

Shotus: The conerersation statum ot the site is brietly indicated. Types of site may include Natnonal Park Nationial keserse, forrest Reserts, National

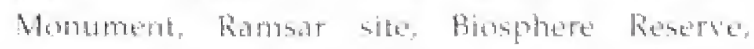

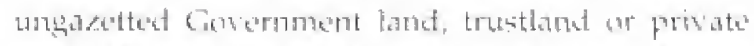
land. We were not able to clarify the status of ame sites an public land ti.e. whether (romerament land

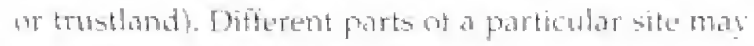

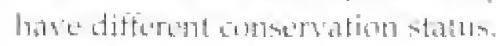

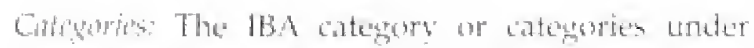
which the site is listed ate indicaterf lgloballythreatered specion, restricted-rames spectes.

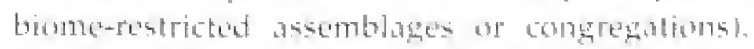
For biotretestricted assemblates, the bione in guestion is gat

\section{Site description}

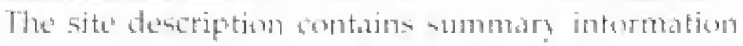
ost hestbiats, focation and borandaries, history (where

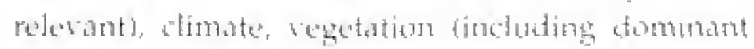

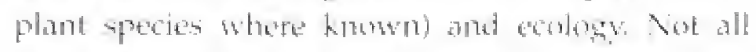

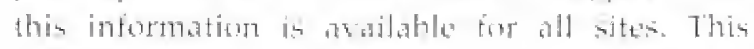
information is compled from on wide wariety of sounces, lathenced in the text shet umber 'Further reating'

\section{Birds}

This sectom gives a brief explanation sof the ornithologicat importance of the site. The text

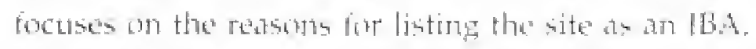

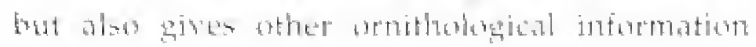




\section{Important Bird Areas in Kenya}

where appropriate. Details of the species for which

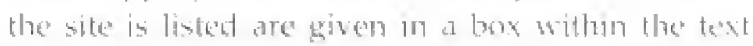

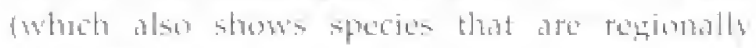

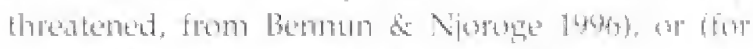

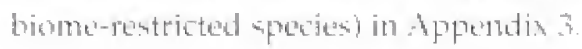

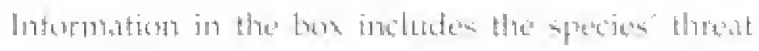
statum (where appropriales and status at the site (where knowry). Numbers crunted fimcluding maximum and mean counts shere avalabley are given for congregatory species. Fill referestes th the

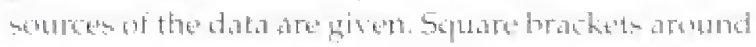

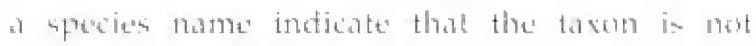

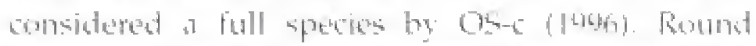

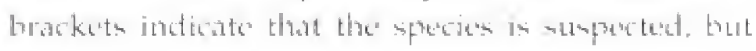
not known, 60 oceur.

At gome sites, particular grobally-threatemest.

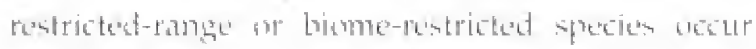

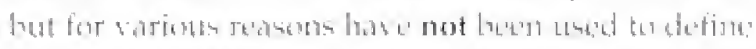

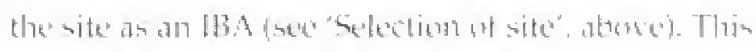

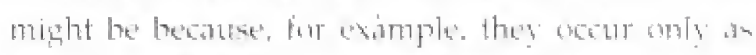

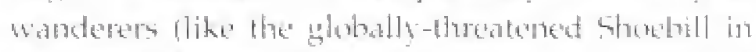

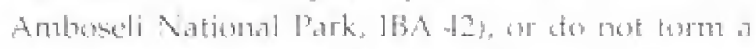

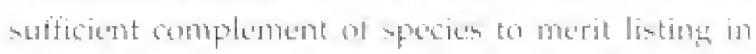

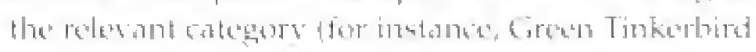

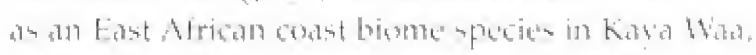

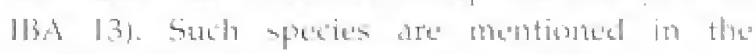
sitenaccount tent, trut not shown in the bess.

\section{Other wildlife}

This section brietly antine the importance as the site for wher tanna and ilora, specieicatly womety and

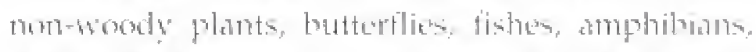

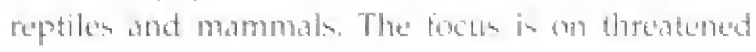

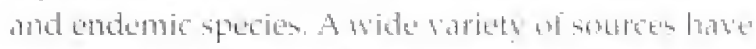

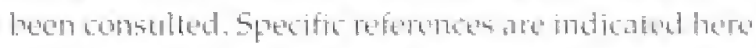

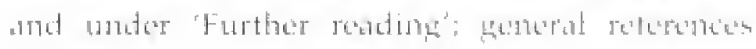

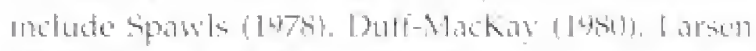

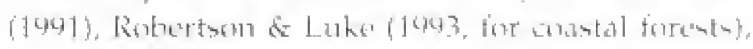

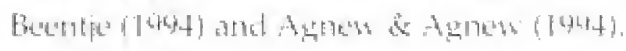

\section{Conservation issues}

This sections outlines the matis threats currently facing the site and problems that soom likely to ariat in future. Recommendations are mate for specific conservationations.

\section{Further reading}

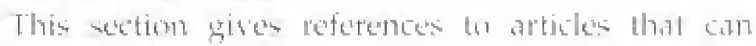

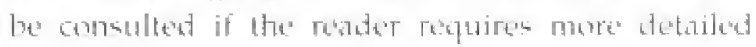

Intormation on the sile. The further reading list is not

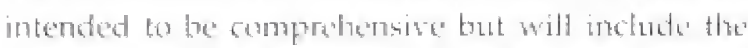
main sorurces of information sesed to compile the

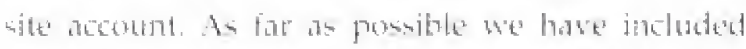

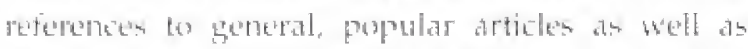
the more kehongal works. A bull reference list for the entire directery appears at the end of the

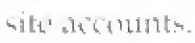

\section{Appendices}

Appendices i-t stmmatrise the spectes relesant th

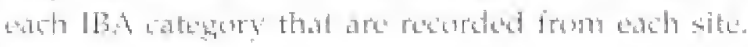

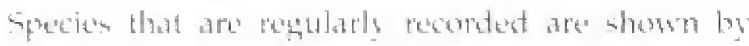
a clumet circle; speties that are reorded from the

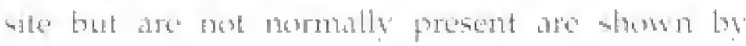
an opertitele.

\section{Maps}

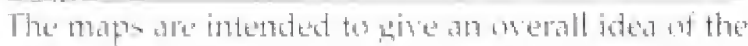

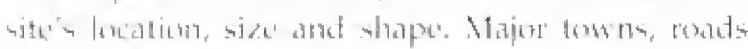

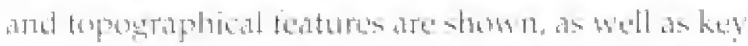

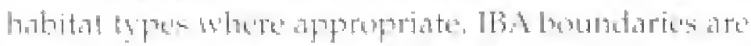

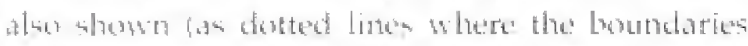

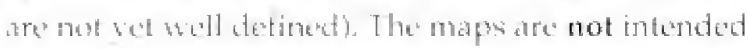
to be defintio for demarating the boundartes

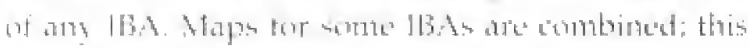

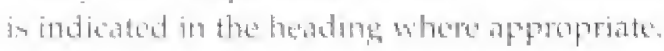

\section{Nomenclature}

Dnly the English name of bird species are griven in the text and Appendices 1-t. Sicutitic names of bricts can bu bound in Indexes 1 and 2. Except where

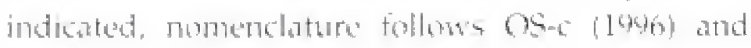

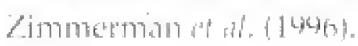

For whther rauma and Bura, shisutific names are giver in the text, fugether with the English name it "appropriate. Nomemelature follows these standard tents:

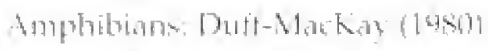

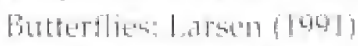

Herbs: As

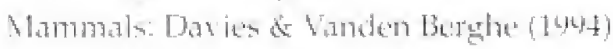

Snakes: Spawin (1478)

Moody planks: Beentien (1494). 
Site accounts

\subsection{Site Accounts}

(ordered by site code) 
Important Bird Areas in Kenya 


\title{
1 A BERDARE MOUNTAINS
}

\author{
$0^{\circ} 08^{\prime} \mathrm{S}, 36^{\circ} 55^{\prime} \mathrm{E}$, Central Province, Murang'a, Nyandarua and Nyeri Districts \\ 179,900 ha $(76,600$ ha National Park, 108,400 ha Forest Reserve) \\ Altitute: $1,800-4,000 \mathrm{~m}$ \\ Status: National Park and Forest Reserves \\ Categories: Globally-threatened species, restricted-range species, \\ Afrotropical Highlands biome species
}

\section{Site description}

The" Aberdare or Vyundarua mountaine are ant isolated folcanic range that form the easternmost wall of the Gregory Rift Valles, to the esat of the hiyh

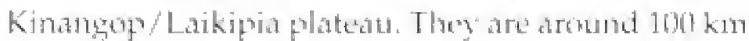
long from north or soxth the northeth end almus reaching the equatorto. There are two main peaks, O] Dongy Lesatinta (3,449 m) to the florth and

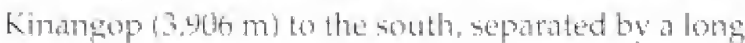

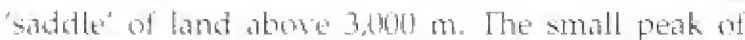
Kipipiri $(3,349 \mathrm{~m})$ lanks the main tiunge to the west, linked to it by a formerty forested valley at arourat 2,700 wh. Dexp rawines ent through the forested wastem and weterts slopes and there are many clart streame and waterfalls. Mist and rain becur througlout nuch as the seur, with precipitation Sarking rom around 1,000 mon on the drier nothlyesterm slopes to as mush as 3,000 mom in the south-ast. The vegetation viries with altitude. A rich alpine and sub-alpine flora, including species

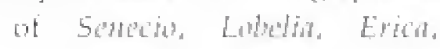
Helchrwam and tussock

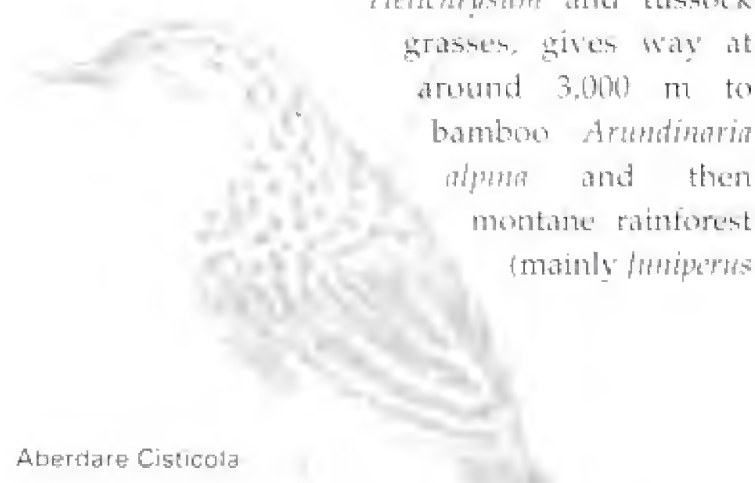

Aberiare Cisticola

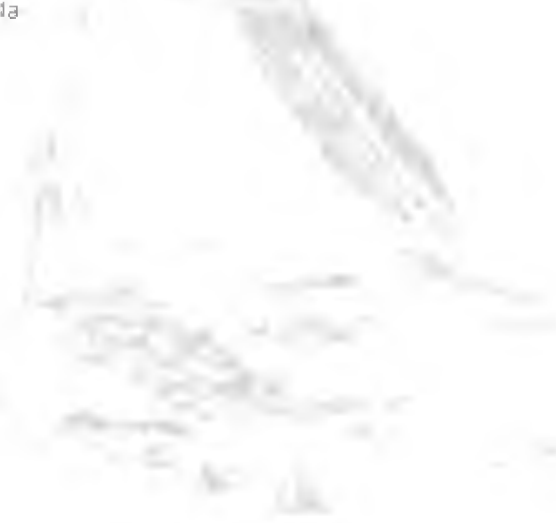

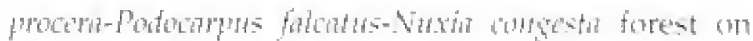

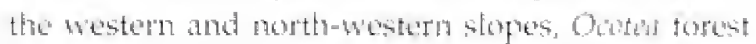

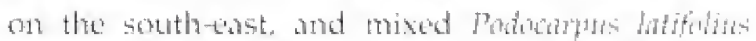

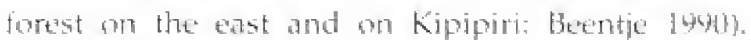

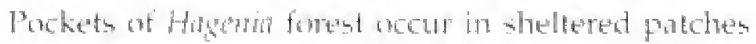
bre the tolling momalat.

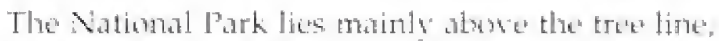
with some forest and serelr at lower altitude in the

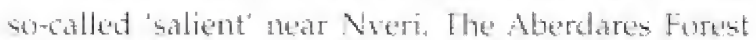

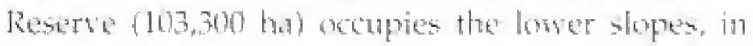
three main blocks that almost surround the Park, with Kipipiri Forese Reverve $(5,100)$ ha $)$ tached on to the west. The southerts boundats of the Aherdates Forest Reserve adjoins the Kiktyu Escarpment Forest (IBA 3). The Alotedares are an extremely mportant water catchment for the Tana River system, for the northerm Finats Nviro Rister and For Lake Naisasha, and provite muth of the water supply tor Natrobi and adjoming districts.

\section{Birds}

See hox and Appendix 3 for hery species. Tha Aberdares hold 52 of Kenxa's 67 Arotropical Highlands species, and six ot the ejght restrictedrange spectes in the Kencian Mountains Fademic Bird Area. Over 20h species are recorded in all. including African Green Ibis, African Cuckon Hawk, Mountain Buzzard, Jackson's and Moorland Frakeline, Hartaub o Turaco and Cape Faglesowl. The Scarlet-tuited Malachite Sunbird is found on the high feaks, foraging largely on labelias, while wher montane stonbirds (including Tacare, Golden-

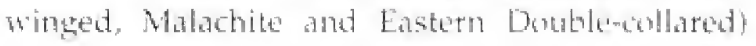
are common at slightly lower altitudes. The restricted-range Aberdare Cisticulat appeare to be locally common in tassock mortand. 


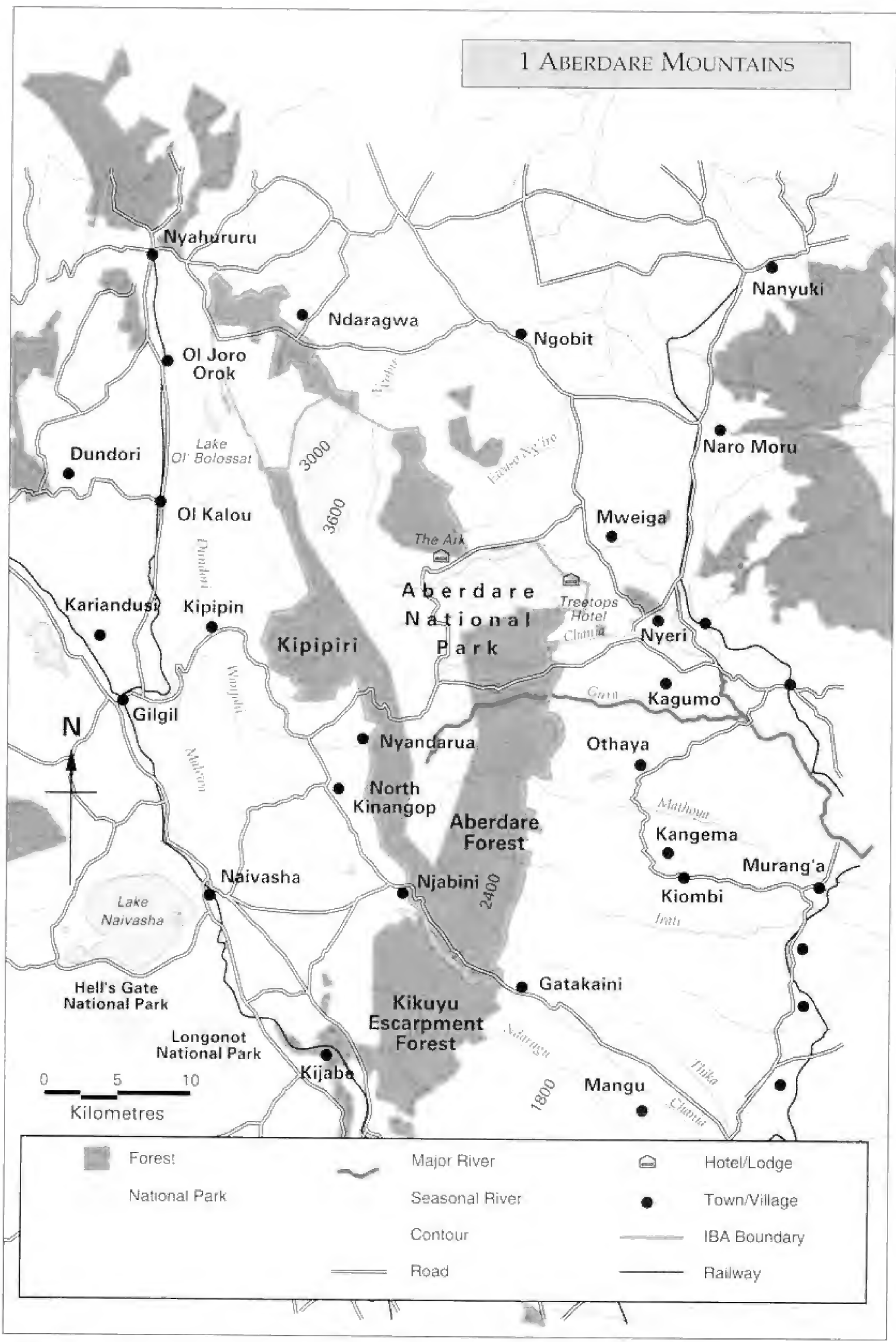




\section{Aberdare Mountains}

\begin{tabular}{|c|c|c|}
\hline \multicolumn{3}{|c|}{ Globally-threatened species } \\
\hline *Sharpe's Longclaw & $\begin{array}{l}\text { Provisionally Vulnerable: } \\
\text { (Bennun \& Njoroge 1996) }\end{array}$ & $\begin{array}{l}\text { Uncommon resident of the southern } \\
\text { slopes, in grassland (Zimmerman et } \\
\text { al. 1996). Current status uncertain }\end{array}$ \\
\hline "Abbott's Starling & Vulnerable & $\begin{array}{l}\text { In forest. Status uncertain; may be } \\
\text { only a rare visitor }\end{array}$ \\
\hline *Aberdare Cisticola & $\begin{array}{l}\text { Provisionally Vulnerable: } \\
\text { (Bennun \& Njoroge 1996) }\end{array}$ & Locally common in tussock grassland \\
\hline •Jackson's Widowbird & Near-threatened & Status uncertain \\
\hline \multicolumn{3}{|c|}{ "also restricted-range species } \\
\hline \multicolumn{3}{|c|}{ Other restricted-range species } \\
\hline Jackson's Francolin & & $\begin{array}{l}\text { Common in high forest and moorland } \\
\text { scrub }\end{array}$ \\
\hline Hunter's Cisticola & & Common at the forest edge \\
\hline \multicolumn{3}{|c|}{ Regionally-threatened species } \\
\hline African Green Ibis & Vulnerable & $\begin{array}{l}\text { Few records; in forest and on wooded } \\
\text { moorland streams }\end{array}$ \\
\hline Ayres's Hawk Eagle & Vulnerable & Scarce in mantane forest \\
\hline African Crowned Eagle & Vulnerable & At low densities in montane forest \\
\hline Striped Flufftail & Vulnerable & In alpine grassland; rarely recorded \\
\hline Baillon's Crake & Vulnerable & Uncommon, in montane swamps \\
\hline African Grass Owl & Vulnerable & In àlpine grasstand. No recent records \\
\hline Cape Eagle Ow! & Vulnerable & Local, on moorland eliffs and crags \\
\hline Long-tailed Widowbird & Vulnerable & Status uncertain \\
\hline
\end{tabular}

\section{Other wildlife}

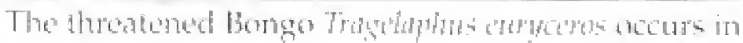

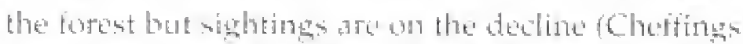

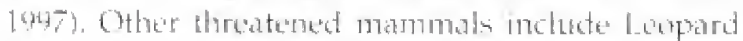

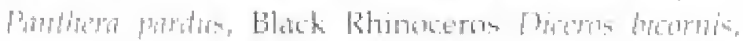

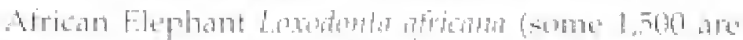

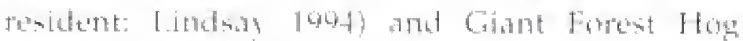

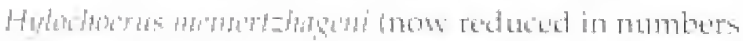

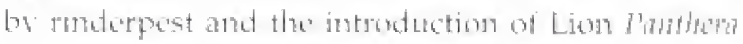

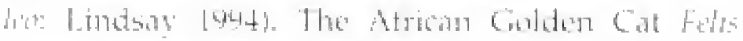

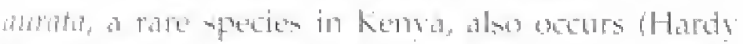

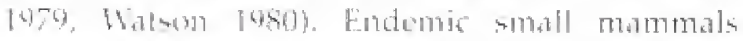

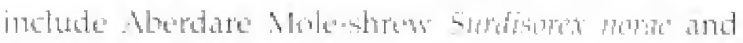

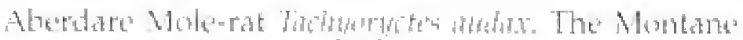

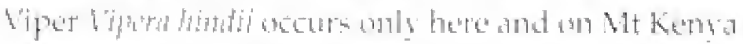

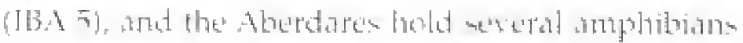

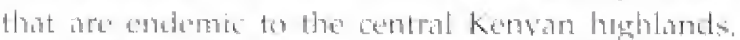

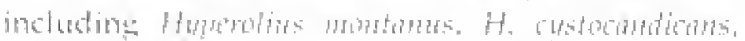

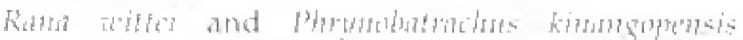

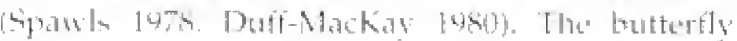

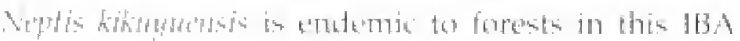

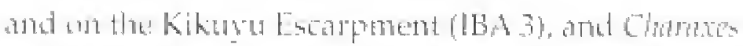

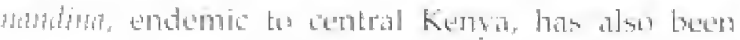
rexirded [Latsen layl).

Vitable plants include the Aburatare endomics

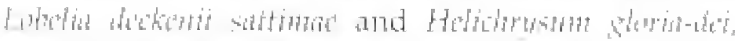

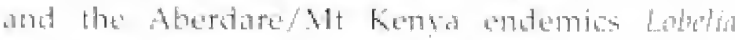

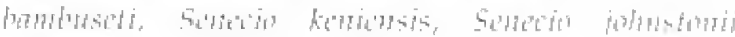

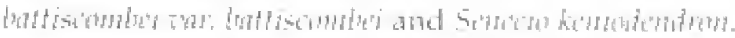

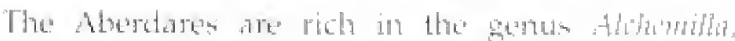

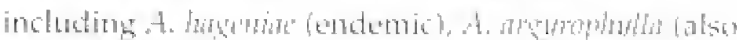

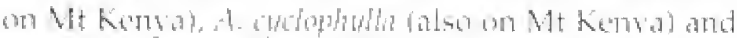

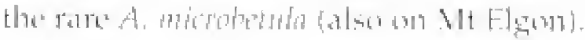

\section{Conservation issues}

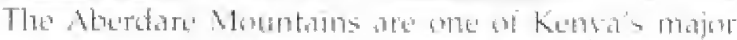

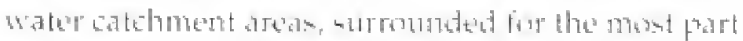
bs intensive, small-scale agrieculare. "The" hichlo momeland in swell protected and appearo retatively

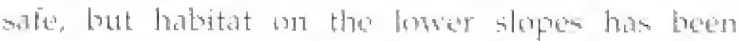
wererely domaged in recents gearo. Forest destructiom 


\section{Important Bird Areas in Kenya}

and degradation ss the major threat to the site, thrometh agricultural encroachment, illegal Combors

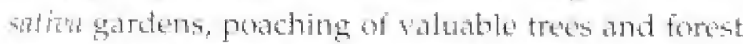
grazing of livestock. Forest fires, eithes actidental or deliberately set (esprecialy by homey collectors), have destared or damaged large tacts of forest during recent dry periods. Human-widifife conflict has long been internse around the borders of the National l'ark 'salient' and the forest reserves. Marauding animals regularly damage erops, and occasionatly kill or mijure peoples. Parts of the saliente are already tenced, and there are plans to continute fencing sections of the forst reserves where problems are most severe. As in many ol Kenya's indigenous forests, management is presently imadequate, and is likely to remain so uniil an integrated management plan is supported by sufficient resources to put it into action.
On the mertand, the states of twe of the throatened species - Sharpes longctans and Aberdate Cisticola - remains litte know mo and nesds investigation.

The unusual regetation, rugered terrain, strants and waterfalls combine to create an area of great scenje beatuty tri the National Park, which has tremendous potential for eor-tomerism. This potential is only partially realised at present thanks to pou acoess roads and a lack of safe walking trails.

\section{Further reading}

Agnew 1985, ILCN/LNEP 1987, Lindsay 1994

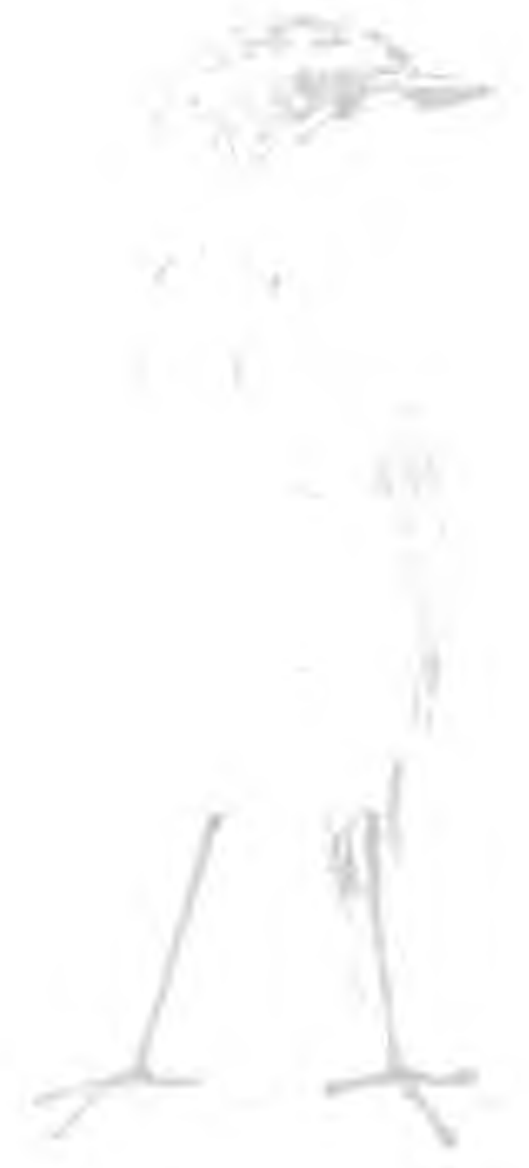

Alpine Chat 


\title{
2 Kianyaga VAlleys
}

\author{
$0^{\circ} 30^{\prime} \mathrm{S}, 37^{\circ} 20^{\prime} \mathrm{E}$, Central Province, Kirinyaga District \\ Area undefined: minimum 12,000 ha \\ Altitude: $1,200-1,600 \mathrm{~m}$ \\ Status: Unprotected private land \\ Categories: Globally-threatened species, restricted-range species
}

\section{Site description}

Kinnaga comprises a landscape of ridges and stewp-sided valleys on the southerasterm stopes of Mt

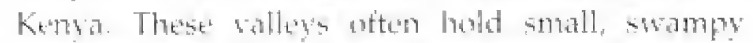
atreams, which dtatin inter tributaries of the Tanel River: The area is entirely settled and cultivated. predomanatly witherfoe and maize; arrostroot and other conps are srown in the salless. The remaining small areas of maturat wetland include plants steh as

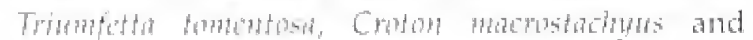

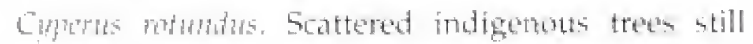

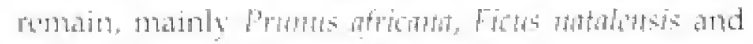

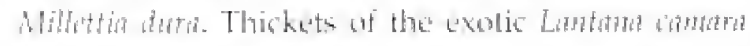
accur at the buges of cullivation, in fallow tarmand and unweedect coffere plantations, and in inacessible parts of the vallews. wetland regetation has been cleared for cultixation, and smalter and smaller areas are left ballow: Batober breeding succoss is already low, appartently as a result of human disturbance (Noproget \& Mutinata 1966), athd the birds are alab hunted for ford (Nomge 1994). With adequate thicket cover. the babblers an perstst in habidals severely modifind by peoplex. However, their conservation in this agricultural setting will require innowative approaches - purthape the seting aside of a number of small sanctuartes centred on schools and obther public institutions, as well as an extensive public aducation campaign.

\section{Globally-threatened species}

"Hinde's Babbler
Endangered
Babbler groups occupy many of the valleys and swamps, with an estimated total population size of $500+$ (mear group size 4.5 birds, men density 3.8 birds $/ \mathrm{km}^{2}$ ?

Niongge \& Bennun, in press)

\section{Birds}

See bou for hes apecies. This is the contre of abuntance for the threatened, restricted-range Hinde's Babloler a species endemic to central keny. The diversity of other birth is lows: a six-month study carried out in 1493 recorded ondy 94

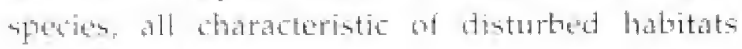
in the central highlands.

\section{Other wildlife}

Very little other wildifien in mote occurs.

\section{Conservation issues}

This site ts critically important for the conservation of Hinde" Babloler. The habblers lise in groups in the river walleys and swamps, and depend om small

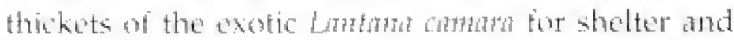
mest sites. The fast-groming human population is intensitying pressure on the lathd. Almost all antural

\section{Further reading}

Plumb 1979. Turner 1692, Noroge 1945, Niotoge \& Bemnum, in press, Noroge ed al, in press 


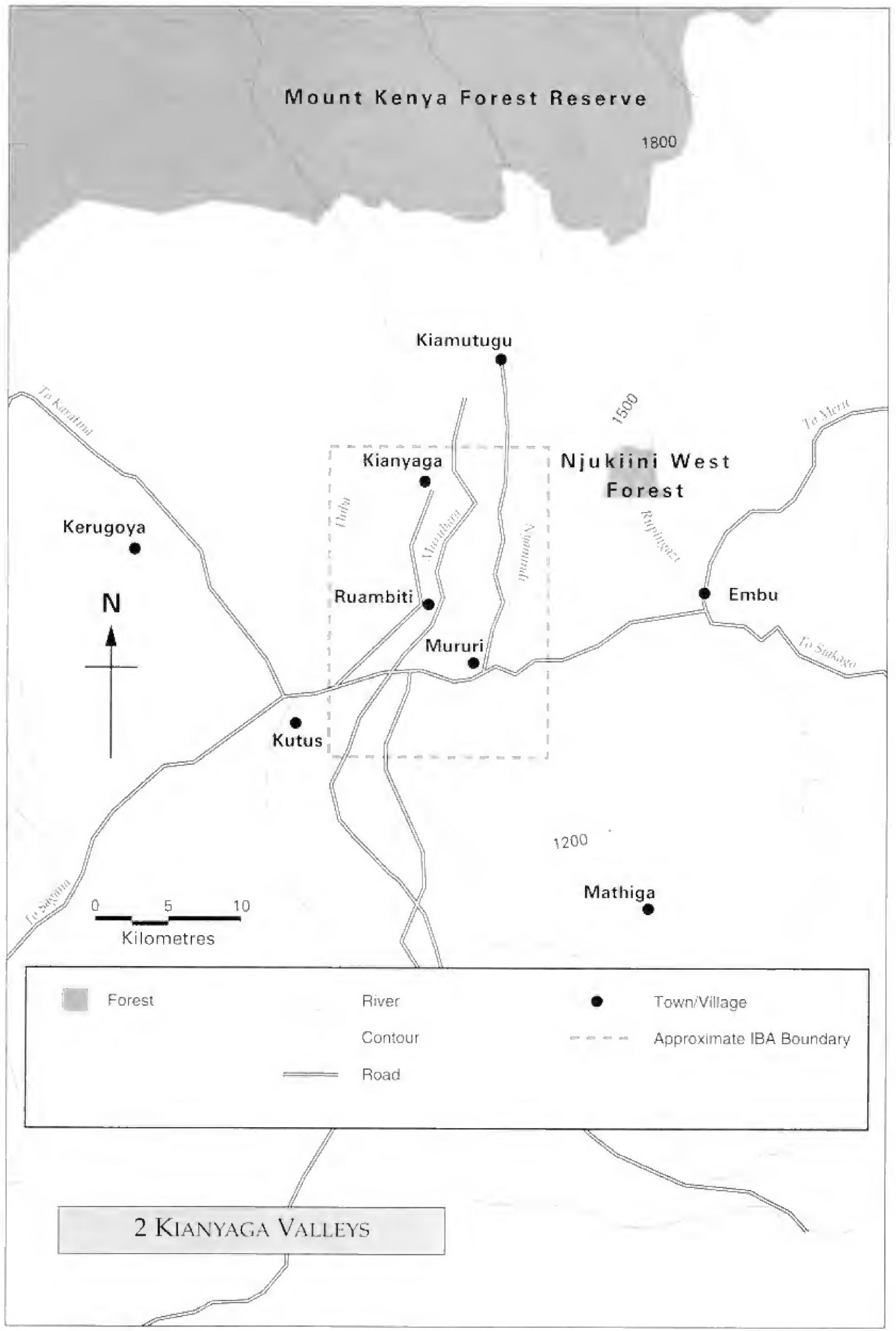




\section{KIKUyU ESCARPMENT Forest}

$0^{\prime} 56^{\prime} \mathrm{S}, 36^{\circ} 40^{\prime} \mathrm{E}$, Central l'rovince, Kiambu District (with very small sections in Nyandarua and Murang'a Districts, and in Rift Valley l'rovince, Nakuru District)

\section{7,600 ha}

Altitude: $1,800-2,700 \mathrm{~m}$

Status: Forest Reserve

Categorios: Globally-threatened species, restrictud-range species.

Afrotropical Highlands bome species

\section{Site description}

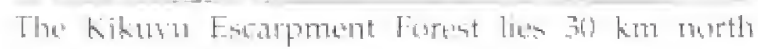

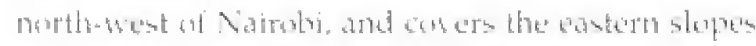

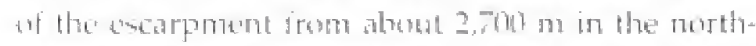

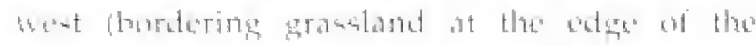

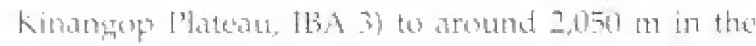
east, where it borders anglicultural land. The man

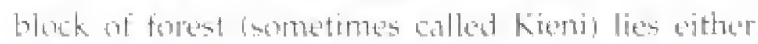

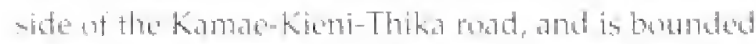

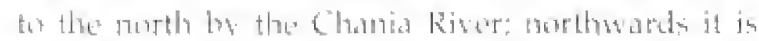

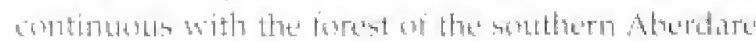

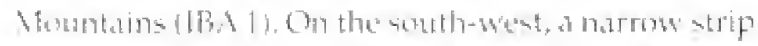
antends along the wall of the Rift valley, bevend

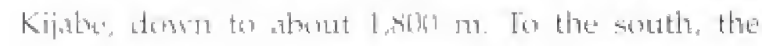

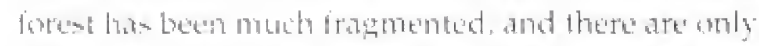

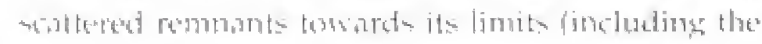

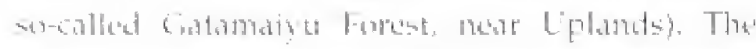

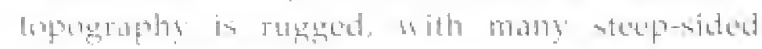

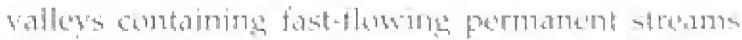

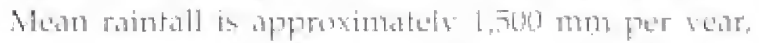

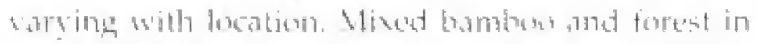

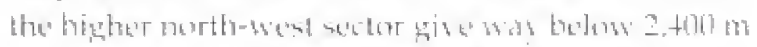

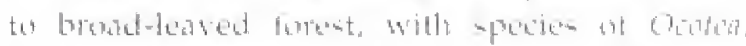

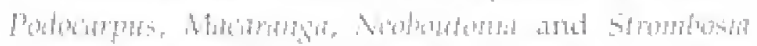

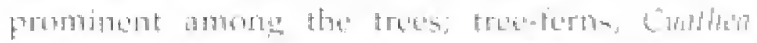

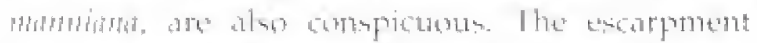

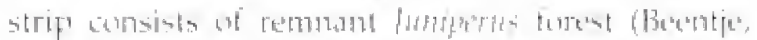
1940). The forest was logest an ter extersindy in the 1971 and 1900 , but many parts in the main blath hater regtiouated wedl.

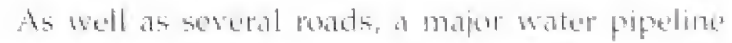

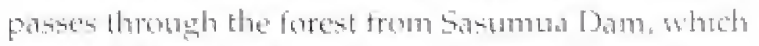

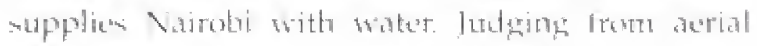

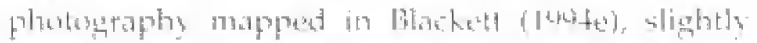

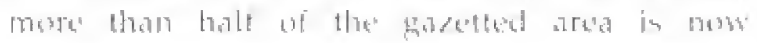

\section{Globally-threatened species}

\section{* Abbott's Starling \\ Vulnerable}

The main block (Kieni) is one of the most consistent sites for this littleknown species, where it has been recorded almost the whole vear around (Taylor \& Taylor 1988); it is atso regutarly recorded in the southern remnant forest patch. Gatamaivu

\section{"also restricted-range species}

\section{Other restricted-range species}

Jackson's Francolin

Hunter's Cisticola

\section{Regionally-threatened species}

Aftican Green lbis

Ayres's Hawk Eagle

African Crowned Eagle

Red-chested Owlet
Vulnerable

vulnerable

Vulnerable

vulnerable
Common in the higher forest

Common at forest edge and in scrub
Regularly recorded; probably resident Scarce resident Resident in small numbers Appears to be resident (Taylor \& Taylor 1988\} 
Important Bird Areas in Kenya

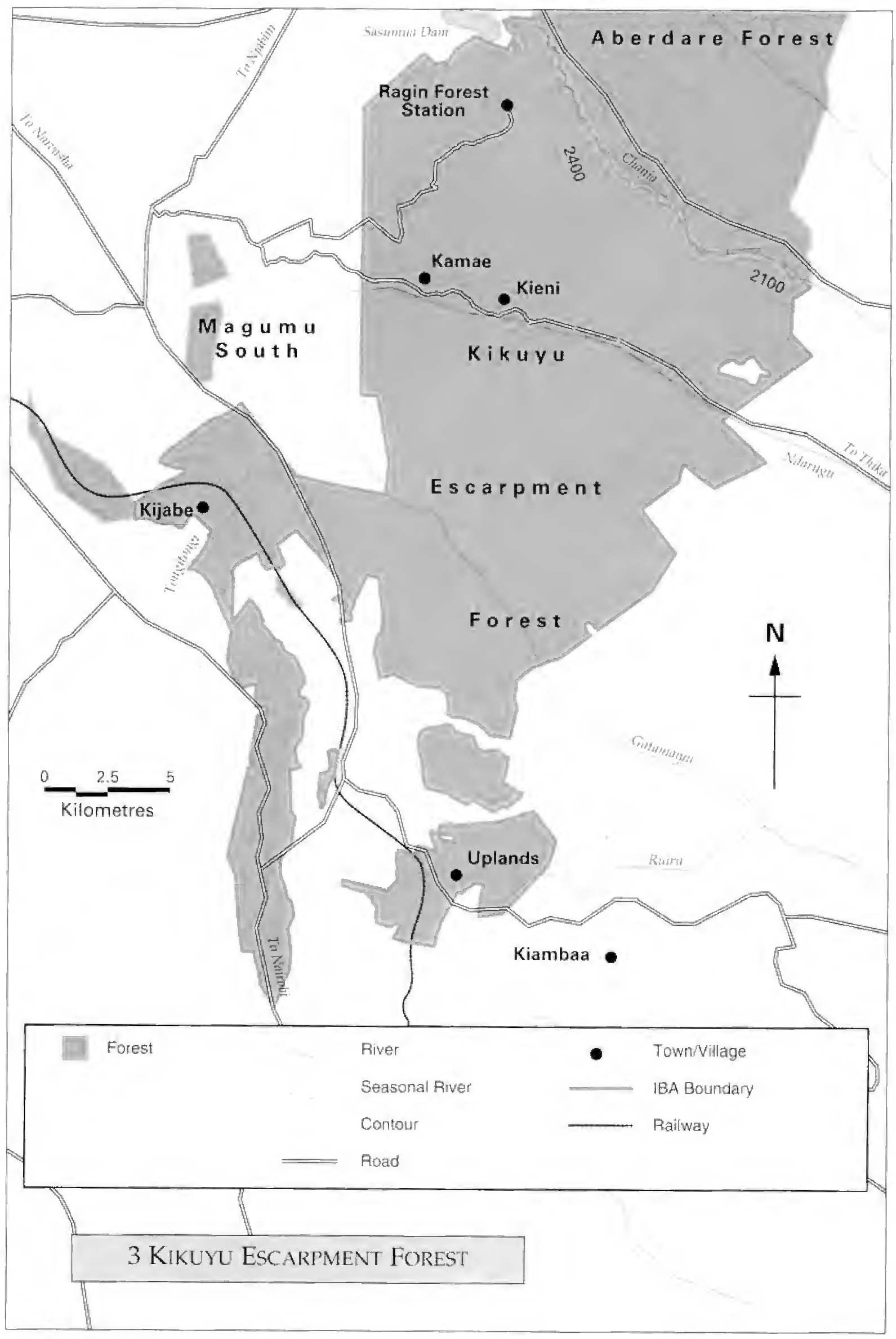




\section{Kikuyu Escarpment Forest}

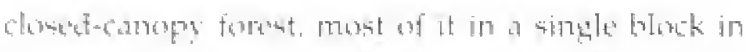
the east-central part of the Reserve. There are "stensine aress of plantatioth and cleated land on the"

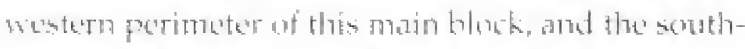
wentern strip and southern sections are a patchy mosate of degrated forest tempanes. scrub. cultiration and pountation

\section{Birds}

Ser hax and Appendix 3 for key specter. This forest

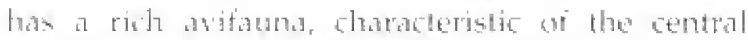
kensan bightands but with a componition difterent

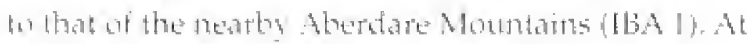

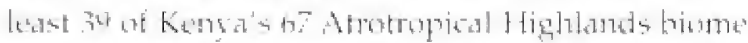
specien acter. The forest forme the weateren limit in kemya for Barted Long-tailed Cockos, Trumpetter Iforabill, Orange Grubard Thrush and Abboti"s

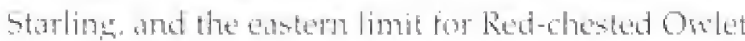

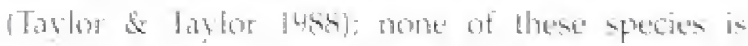
knuwn to be preant an the Aberdare. Mountains.

\section{Other wildlife}

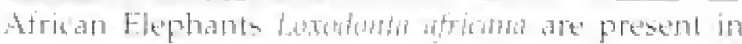
suad numbers at timase thin foputation appears to noxis buck and forth between the Kikutu Escaspment Forest and the Aberdare Moundatis.

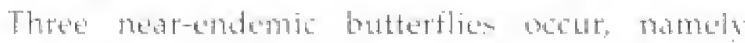

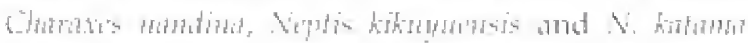
(1.ursen 1991), little is known about the wher bistiversity values of this site.

\section{Conservation issues}

The buminn pressure on this forest has been

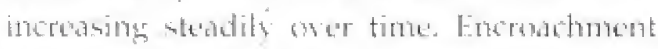

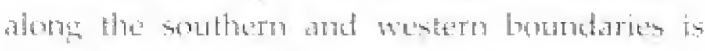

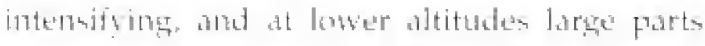

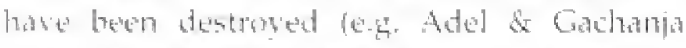

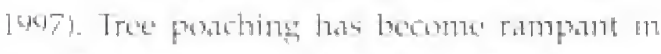
the forest bordering the min Kieni-Thekil roat, and in the southem remoments. It is esident that the porest Deforartment is able to tewert hery little controb. Ilye consersiation ialew of the forest must te more widely Juchentisted, and

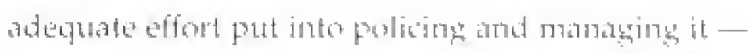
preterably as a joint aptration hetween foremb Department and Konya Hildate bervide under Ehe

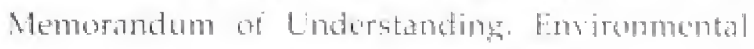

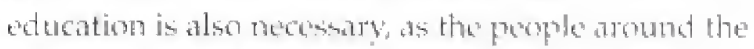

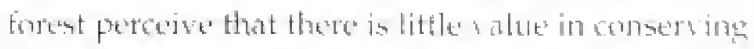
it, and are generally very hestile tor the Fortat Department (Adel \& Gachania 1947). This borest in

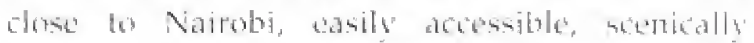

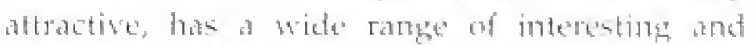

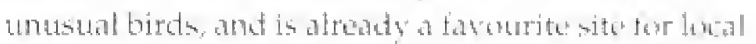
und toregn birdwathers. It has axcelledet potential tor eccotourbm.

\section{Further reading}

Taylor \& Taylor 1948, Backett 196ad, Malcolm-Com 1994, Gardner 1696 


\section{KINANGOP GRASSLANDS}

$0^{\circ} 42^{\prime} 5,36^{\circ} 34 \mathrm{E}$, Central I'rosince, Nyandarua District

(with a small section in Rifl Valley Province, Nakuru District)

77,000 ha

Altitule: $2,400-2,700$ n

Status; Linproteded, manly private latud

Categories: Globally-llneatened species, restricted-range species

\begin{abstract}
Site description

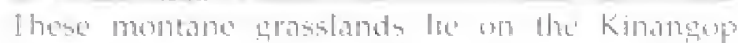

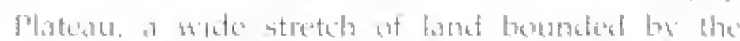

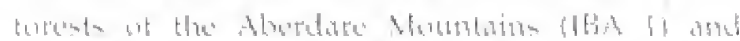

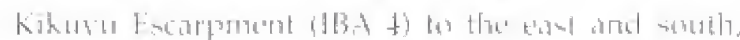

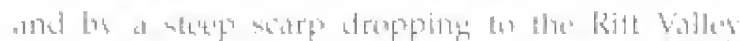

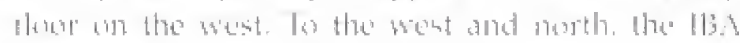

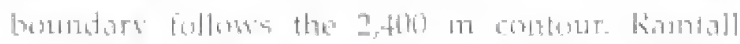

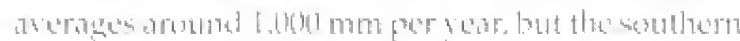

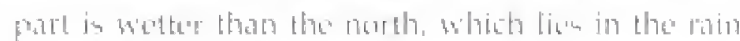

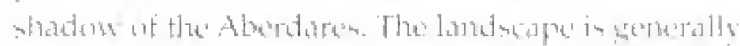

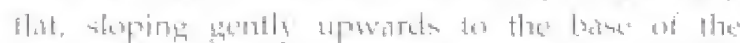

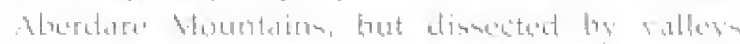

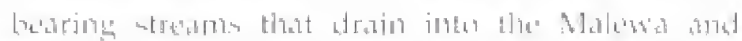

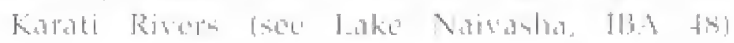

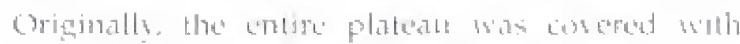

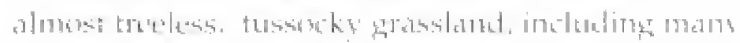

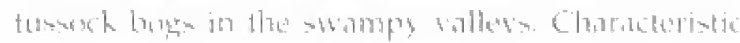

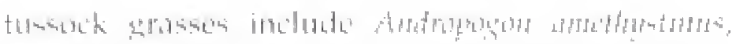

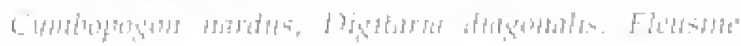

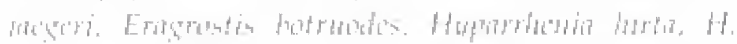

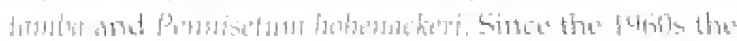

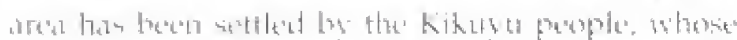

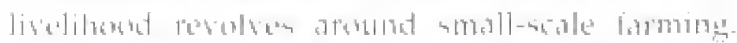

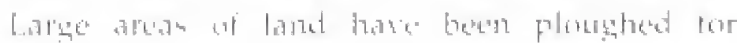

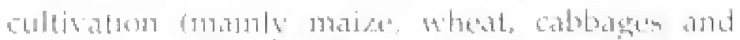

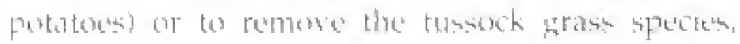

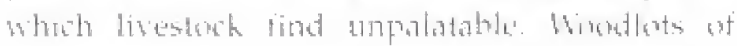

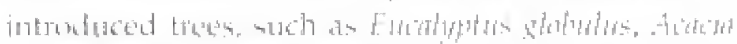

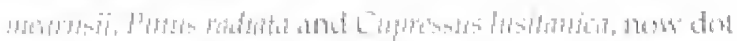

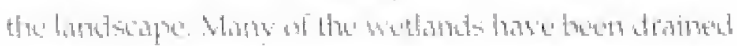

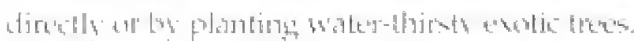

Birds

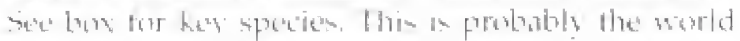

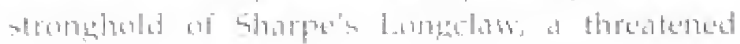

\section{Globally-threatened species}

Pallid Harrier

Sharpe's Longclaw

‘Jackson's Widowbird

Near-threatened

*also restricted-range species

\section{Other restricted-range species}

Hunter's Cisticola

Provisionally Vulnerable (Bennun \& Njoroge 1996)

Provisionally Vulnerable

(Bennun \& Njoroge 1996)

\section{Regionally-threatened species}

Great-crested Grebe Critical

Long-taited Widowbird Vulnerable
Gccurs on passage

Confined to grassland, preferring short grass fields with tussocks, with densities of 0.8 individuals/ha in good habitat (Muchai 1997)

Expected to occur in the higher parts of the plateau

Seasonal visitor; nests in tussock grasslands, and at times in wheat fields \{L. Bennum, unpubl. data\} 
4 Kinangop Grasslands

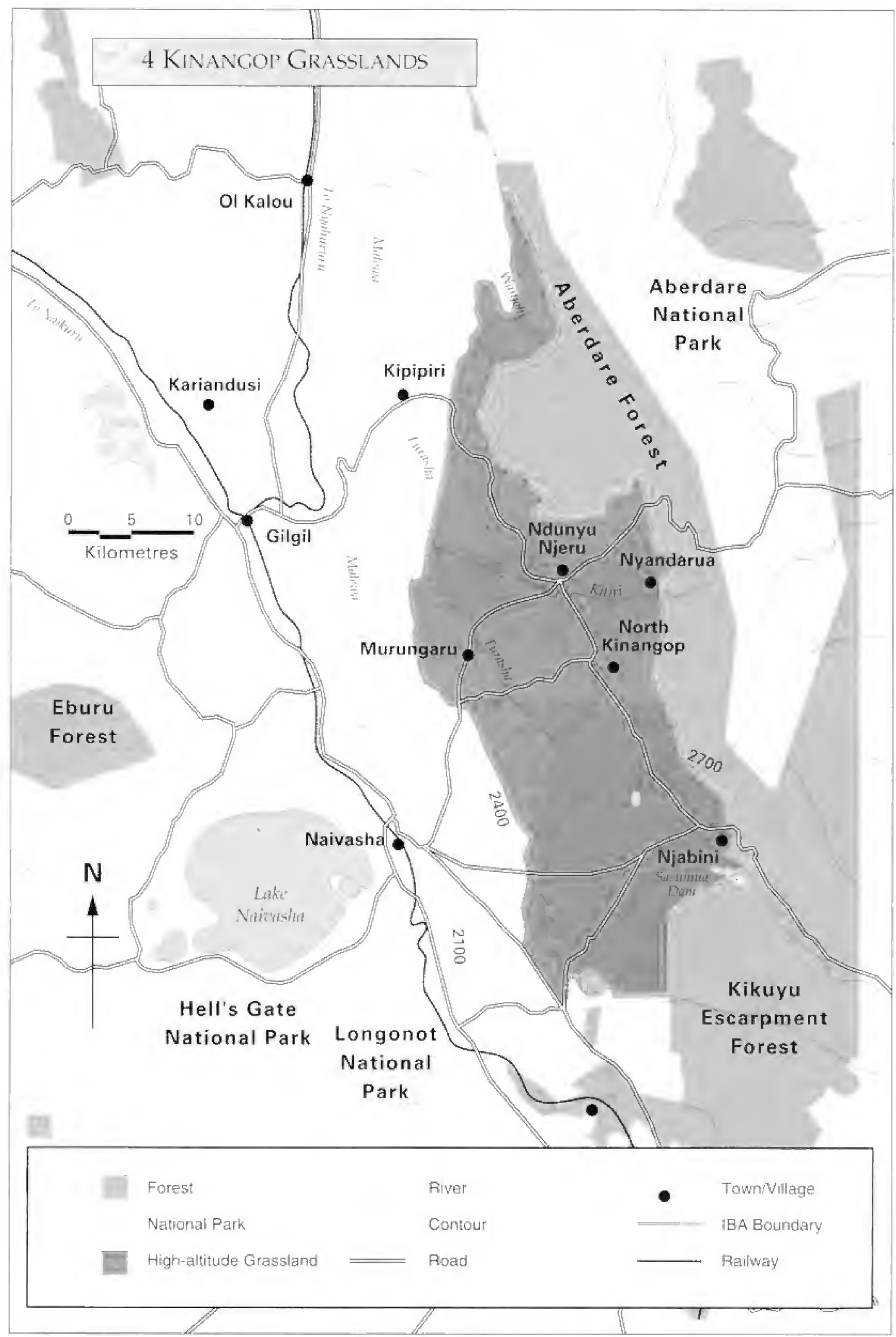




\section{Important Bird Areas in Kenya}

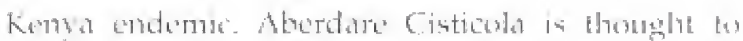
ocetrin in the hisher parts of the IBA. close to the

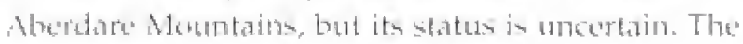

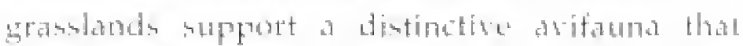

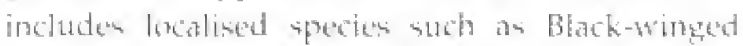
Plorer, Wing-smappong Cistionla and lackant's and Lomg-tailed Widumbirds. Larges numberte of Palaearctic migrants use the anca on passage, notably

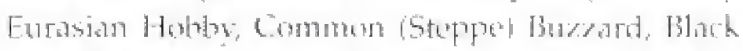

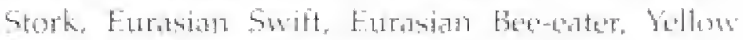
Waztail and Northem Wheatear:

\section{Other wildlife}

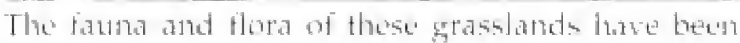

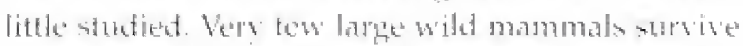
ma the kinamgor, but many smaller spectes that ast

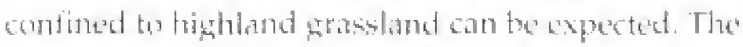

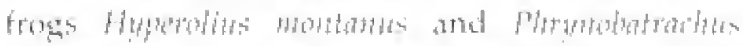

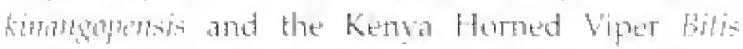

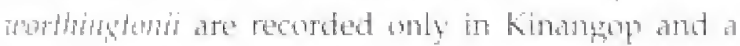

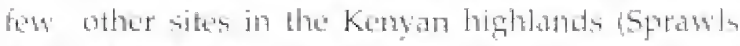

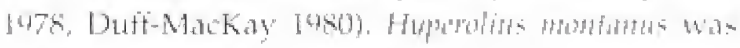
combidered securte in 1980 , Lut it is a montane

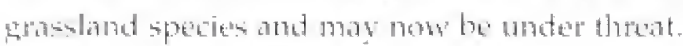

\section{Conservation issues}

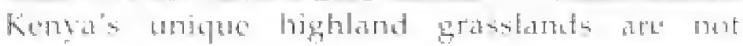

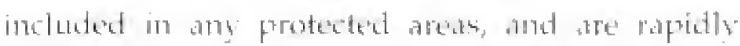
ranshong. The remaining grasiland on the

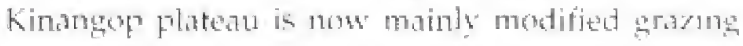
land, sometimes in rotation suith arable croppingr The ecological character of the platean is changing

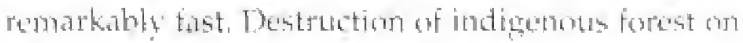
the sherdare slopes. drainage of wetlands and

Sharpe's Longclaw

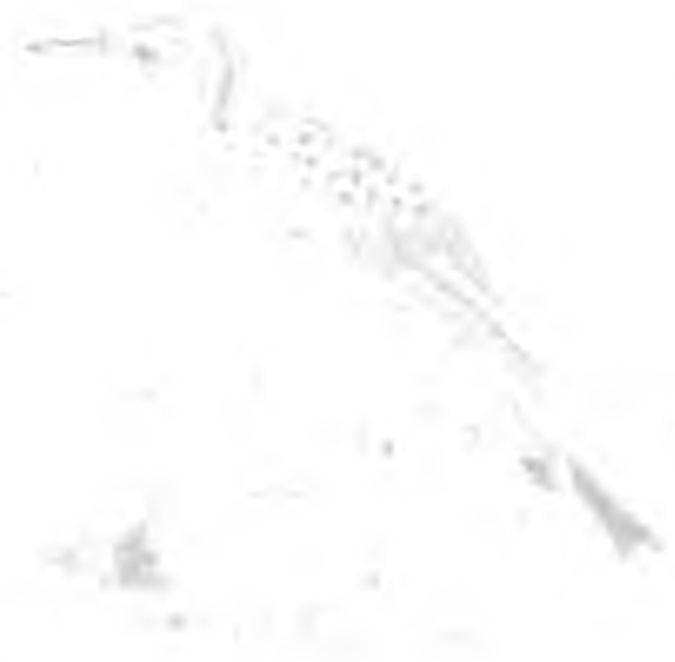

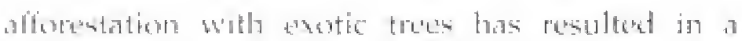
warmer, drier timate, with lesis frequent frosts and

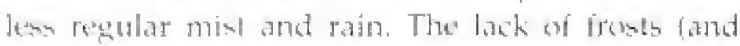

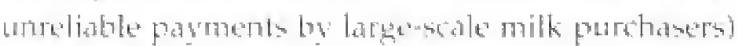

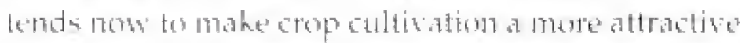
land use than liseoterk rearing. Incteasing human

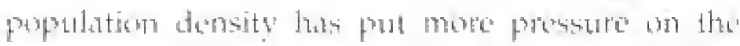

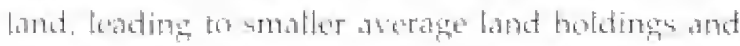
linghen stockines rates. These torbes have pesulted in a raplet ruduction an the area of grassland, especially tussock grassland, and that which remorime is incerasingly fragmented (Afluched |497). Bonth these"

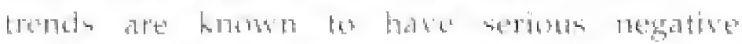

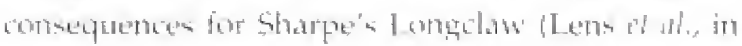
press, at. The longelate seems able to comedist with

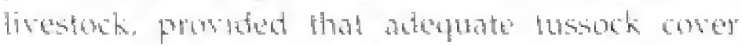
remains, bul banmol surting in farmed fichds. it

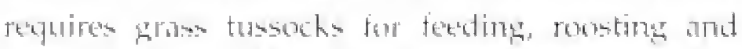

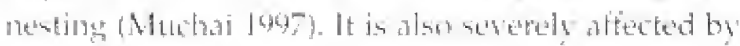
labitat frognentation (Lene d at. in press, b).

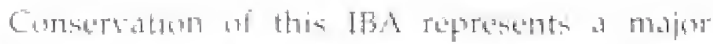
thallenge. Foological and eoonomic studies are

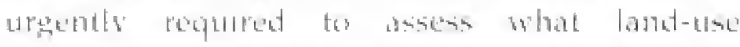

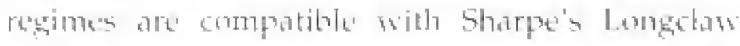

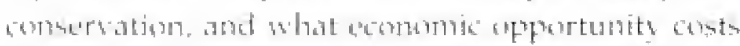

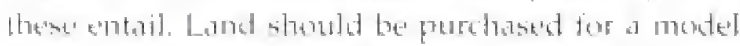

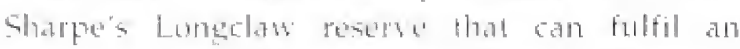
atucational and demonstration function. Potential small nature renereses on common land, or in justilutions sucts ats schosls, need to be identified and dusinated. Forlunately enxironmental awareness

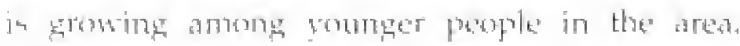
A conastation action stoup. 'Friends of Kinangop

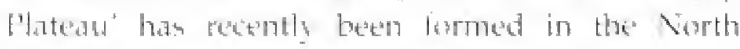
Kinangop area, and deserese at tive support.

lints of this 1B3A have now been well surveyed. but aditional surver work is neded in the morthers

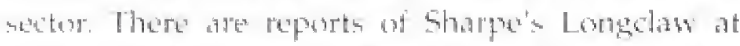

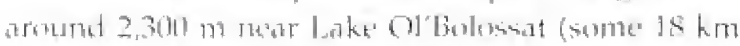

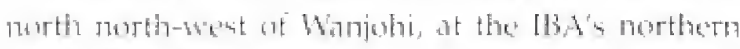
limit). If confirmed, these would make it appropriate? to extednd the morthem boundiary of the IBA. The premence of aberdate Cisticola at the bated of the

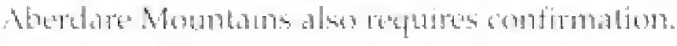

Further reading

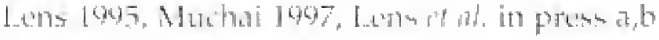




\title{
5 M T KENYA
}

\author{
$0^{\circ} 10^{\prime}$ S, 37 $20^{\prime}$ E, Central Province, Kirinyaga, Nyeri \\ and (marginally) Laikipia Districts, and Eastern P'rovince, \\ Embu, Tharaka-Nithi, Meru and Nyambene Districts
}

271,000 ha

\author{
Altitude: $1,600-5,200 \mathrm{~m}$ \\ Stufus: Forest reserve $(199,500$ ha) and National Park $(71,500$ hat \\ Categorics: Globally-threatened species, restricted-range species. \\ Afrotropical Highlands biome species
}

\begin{abstract}
Site description

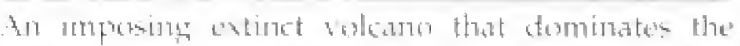

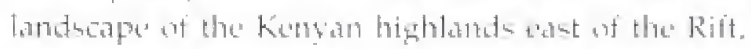

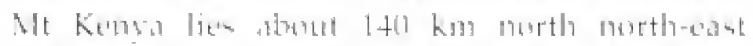

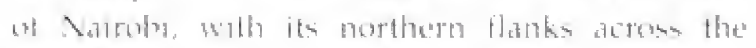
expertor. The musuntan's sprawling slopes are

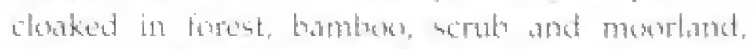

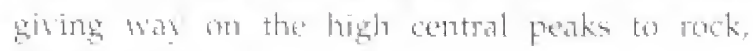

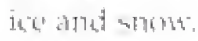

Mt Kermyal is an extremely important water Latehment ares, supplying the lama and Nortbern

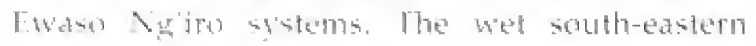

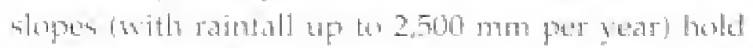

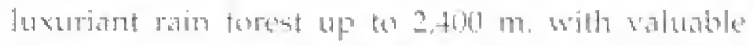

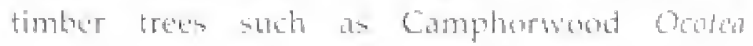

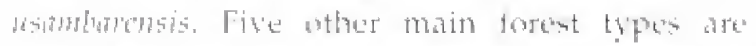

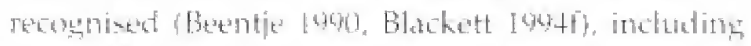

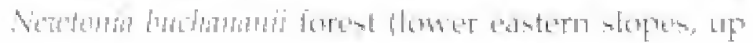

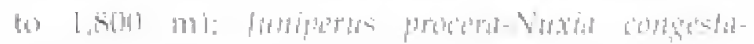

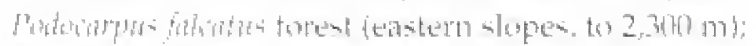

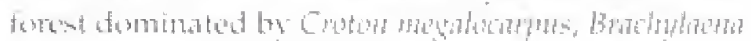

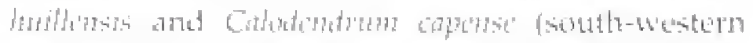

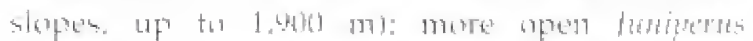

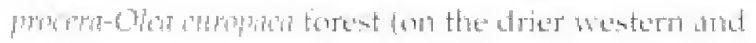

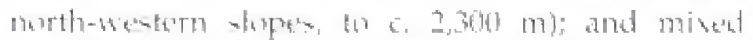

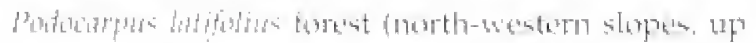
to 2.600 m). From appromimatedy $2,400 \mathrm{~m}$ altitude,

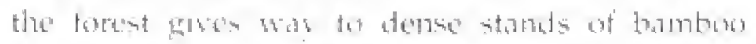

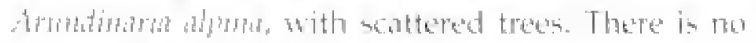

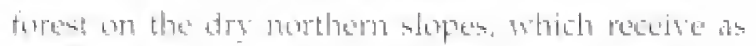

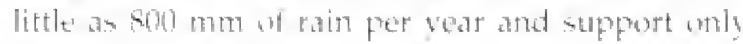
acrubbat vegetation.

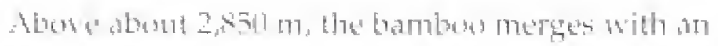

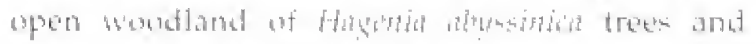

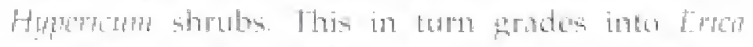

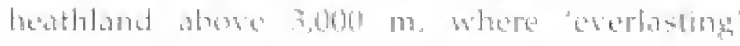

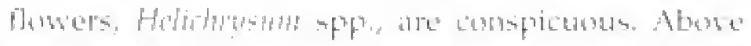

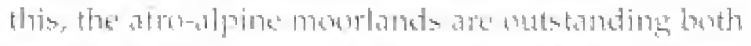

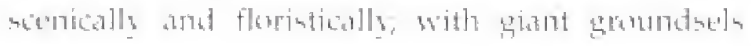

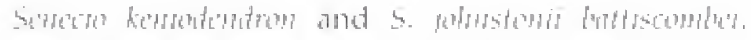

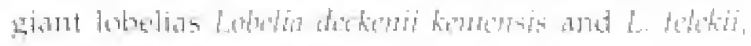

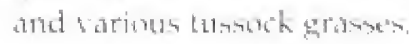

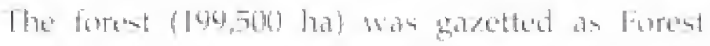

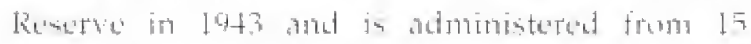
Forest stations in six administrative distrits.

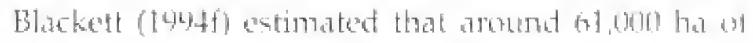
the gazeted ared was closed-candops horest. Almost

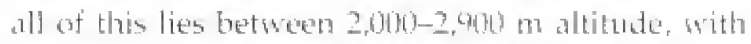
(ว)

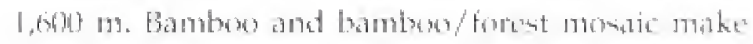

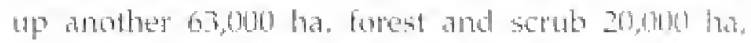
with sonet 20,500 ha af plantations and 35,000 hat of non-forest, includimg serub, grassolated and cultivation. The National Park conere 71 , 5 ma he.

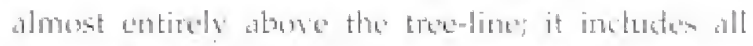

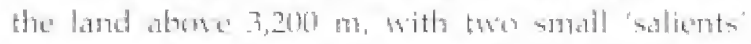

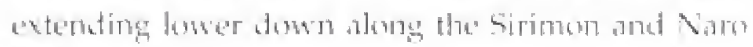
Noru tracks.

\section{Birds}

See box and Appendin 3 for key species Mt Kenst is undoubtedly a stronghold for the threatented and

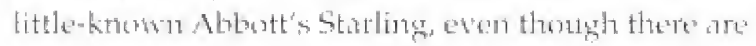

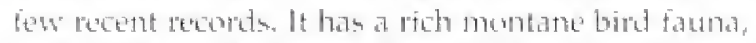

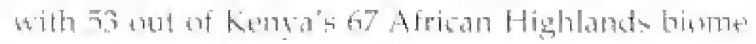

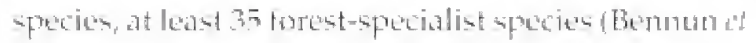
wh, ty presed, and sid of the edght species lhat maken up the Kemsan Mbuntains Endemic Bird Area. Thet Arican Gieen lbis feedo in marshy fortet glades and

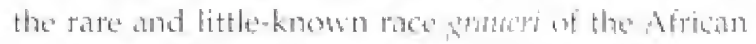

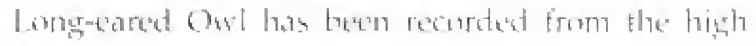

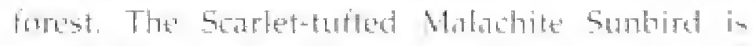
particularly sommon an the high menotand.

\section{Other wildtife}

keat Data Book mammat specien lownd on the furest

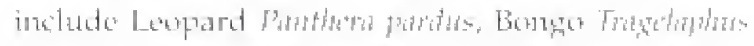

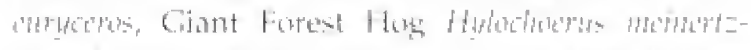

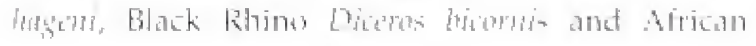




\title{
Important Bird Areas in Kenya
}

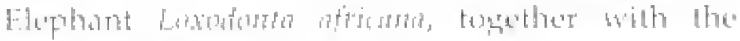
toncomnom bentral kenya race of Blach-frombut

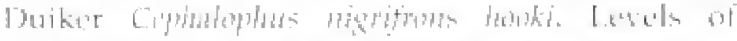

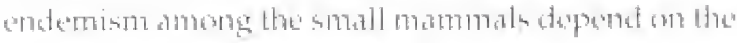

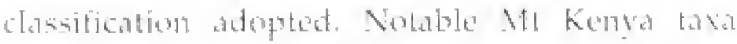

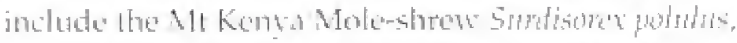

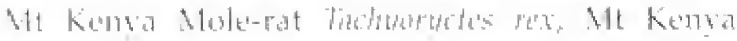

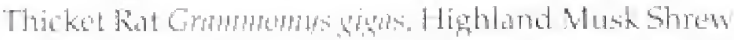

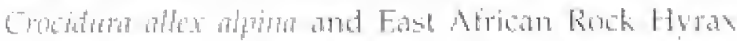

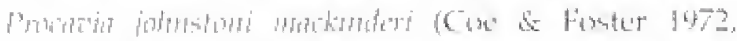

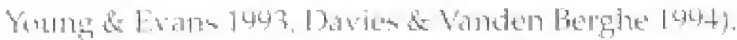

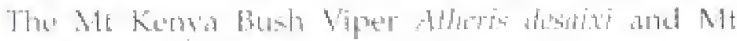

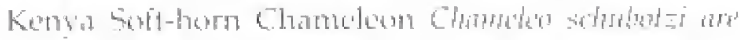
meatole snatemic reptiles, while the Mentane Vipere

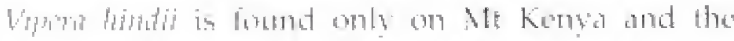

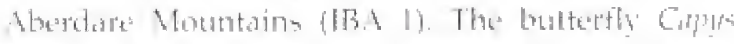

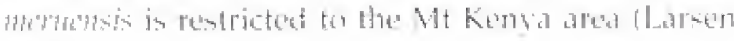
(6)

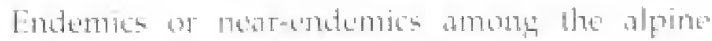

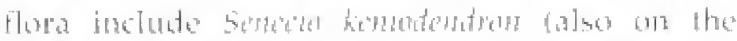

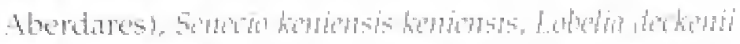

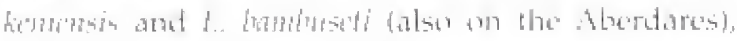

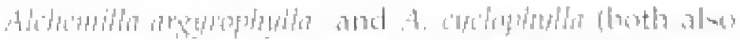

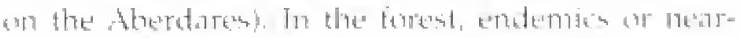

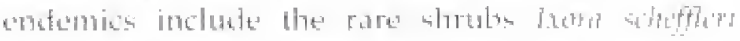

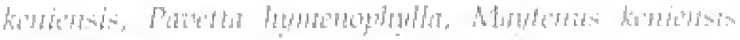

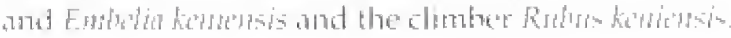

\section{Conservation issues}

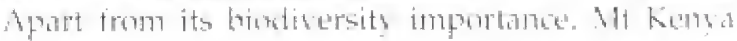

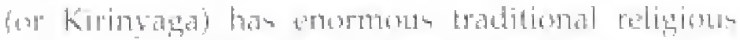

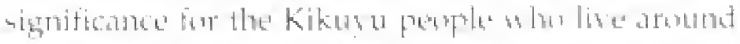

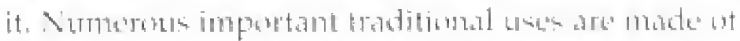

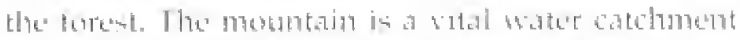

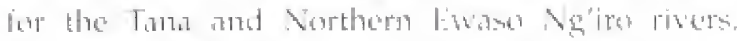

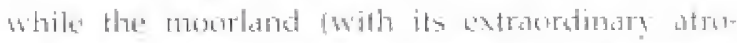

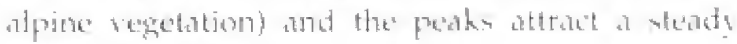
alresan of toraristis.

Lnforeunatery, flace Findert

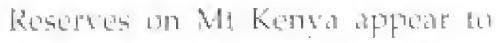
havio suffered an almose costrphlate

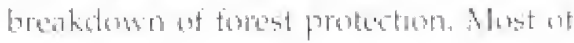
the furtest has alpeats beren lapiged or ter for

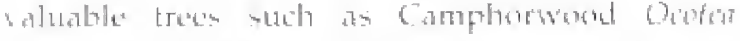

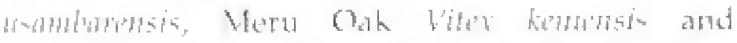

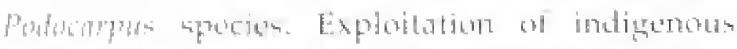

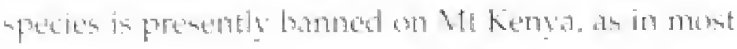

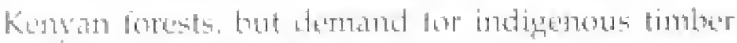

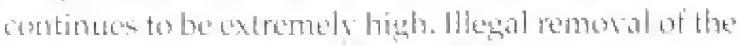

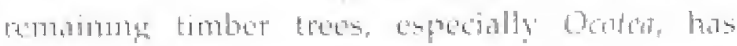

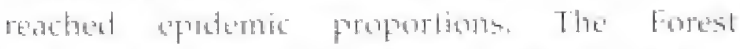

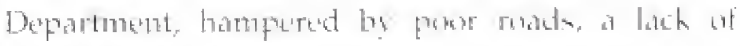
transpert and exupmeme, and underpaid and

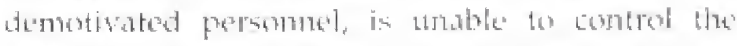
situation. The result is much tominuing damage to the torest.

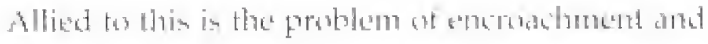

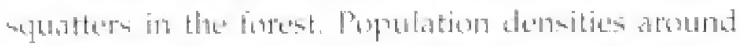

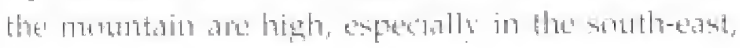

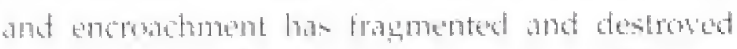

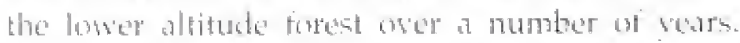
Mane lormer Forest Dopartment stafi anet sawmill

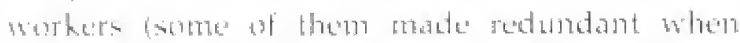

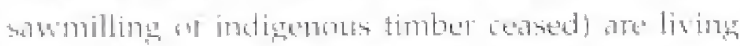

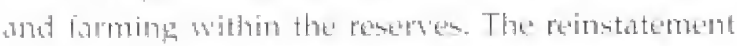
的 |

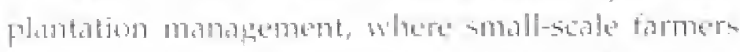
plant crops alompalde soplings, has remelted in farms

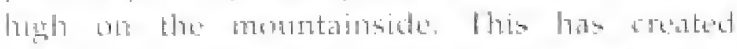

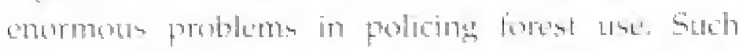

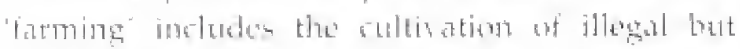

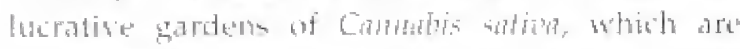

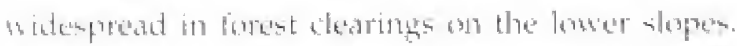

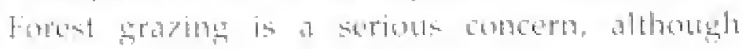

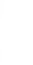

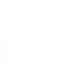

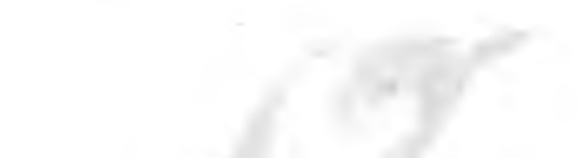

.

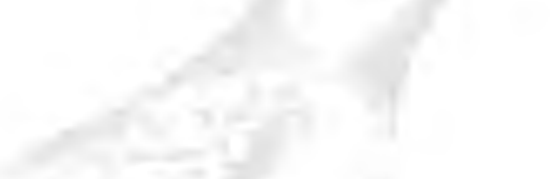




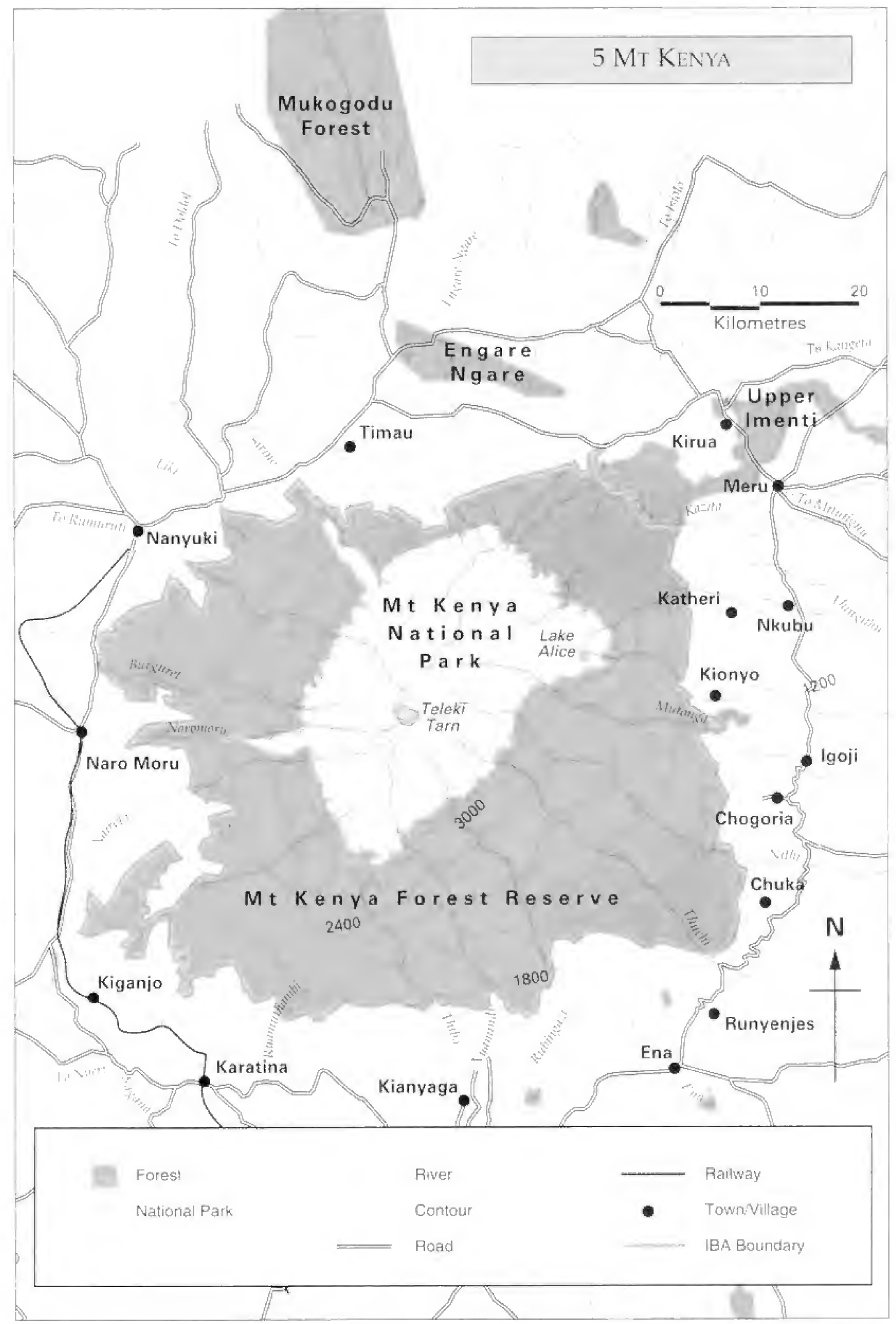




\title{
Important Bird Areas in Kenva
}

\author{
Globally-threatened species \\ Lessin Kestrel \\ "lacksotis Widowbird \\ "Sharpe"s Longclitw \\ *Abbutt's Starling

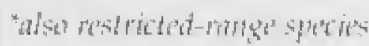 \\ Palatearctic passage migrant, on the \\ mocirland \\ In grassland up to $3,000 \mathrm{~m}$ \\ Known from the north-isest slopes \\ (Zimme"rman at ail. 1996), \\ but present status uncertain \\ Sporadic records; probably nomatic in \\ search of fruting trees (Bennum 199410)
}

\section{Other restricted-range species}

Jackson's Francolin

flunter"s Cisticola

Kenrikk's Starling

\author{
Commun in montane forest and scrub \\ Common in fortestedge habitists and \\ scruls \\ Apart from the nearby Nyanbeni Hills, \\ It Kenva is the only Kengan site for this \\ specties
}

\section{Regionally-threatened species}

\begin{tabular}{|c|c|c|}
\hline Arricall Greest lbis & Vulnerable & $\begin{array}{l}\text { Sarce resident, foraging in forest glades } \\
\text { and along streams }\end{array}$ \\
\hline Iammetrgeiter & Vulnerable & $\begin{array}{l}\text { Formerly nested on momoland alifs; no } \\
\text { fecont tecords }\end{array}$ \\
\hline Arres $=H_{a}$ wa E Eigla: & Vulnerable & Soarce fosident \\
\hline African Crument Eagle & Vulneriable & $\begin{array}{l}\text { An important site for this low-density } \\
\text { specits }\end{array}$ \\
\hline Afrian Grass Ow & Vulnerable & No recent records \\
\hline 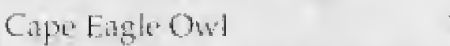 & Venlunerable & Nests an moorland crags \\
\hline l'urple-throated Cuckou=shrike & Vulnerable & Uncommon in montane forest \\
\hline Long-tiiled Widowbird & Vulinerable & $\begin{array}{l}\text { In tall grassland outside forest; stutus } \\
\text { uneedtain }\end{array}$ \\
\hline
\end{tabular}

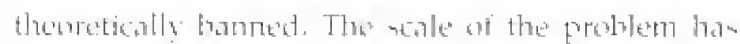

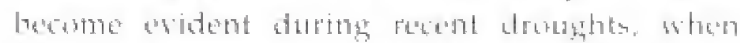

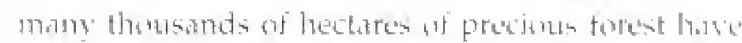

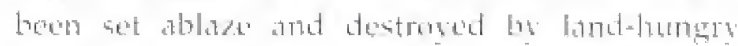

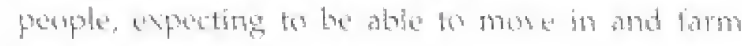
Alt area therester.

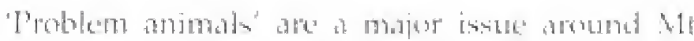

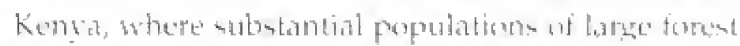

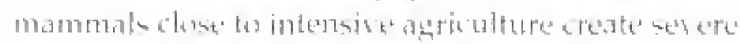

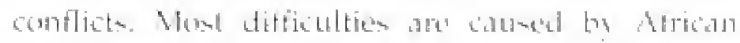

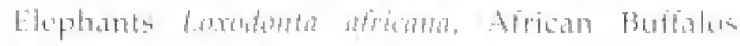

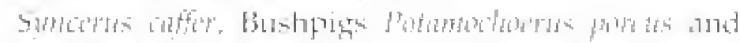

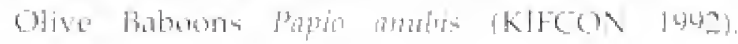

Lilephants account for the buth or bith orop damane

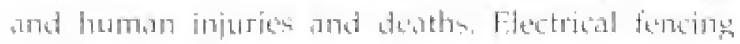

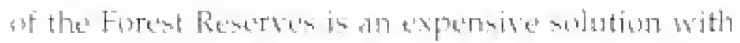

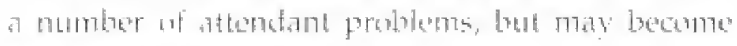
necusary in shme areas.

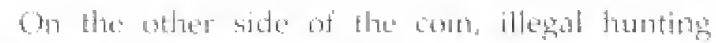

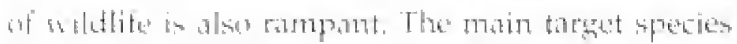

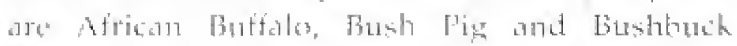

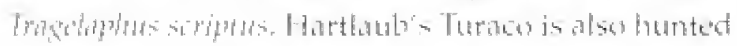

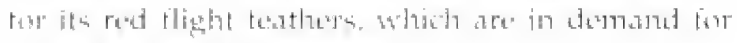
metrabactoring tiathing flies

Elephame presside on the indigenous tomest is 


\section{Mt Kenya}

alas high, whe that these traditional migration rontes butside the? monatain are cut wft, and detrarking and

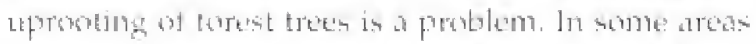

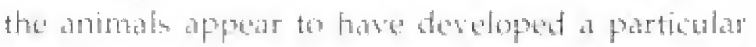

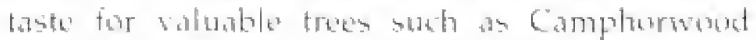
(KJFCOx luge), Butbato similarly damage the

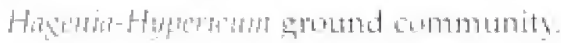

Threats within the Vatiomal [rark are math lebs severe, whough there has been conceros about

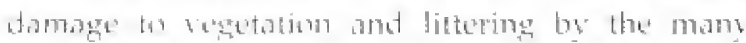

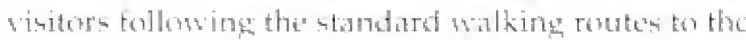

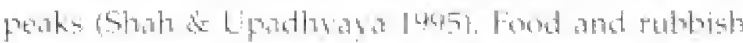

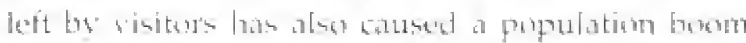

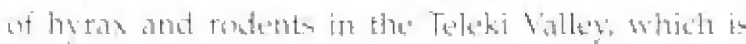

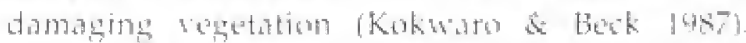

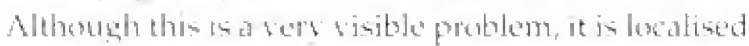

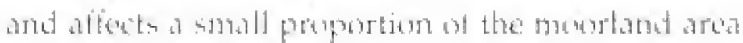
Padbing at wildite in the Park has beter exacerbated to the remolated "shamba" statem.

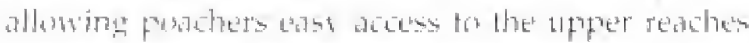

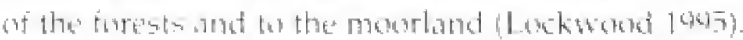

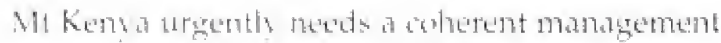

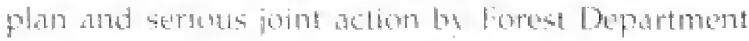

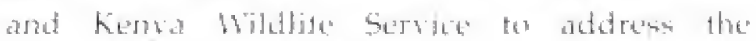

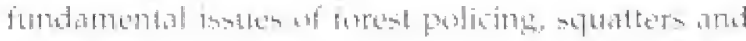

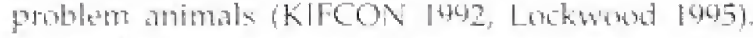
some at this work was to hase been undertaken by the Eurapesn Unian-funted project CONIFOR

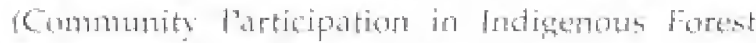

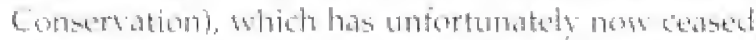

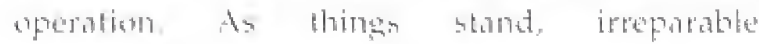

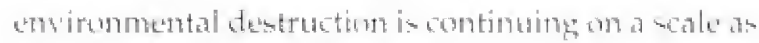
sroud as the forountain itsedf.

The aviatun of Mt Kerra is reasthaty well-known but there is very lietle intormation tor the

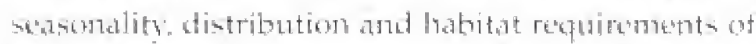

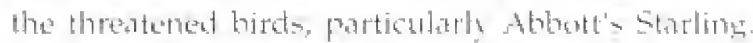

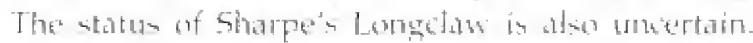

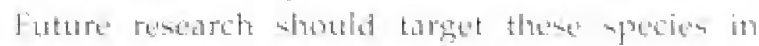
particular

\section{Further reading}

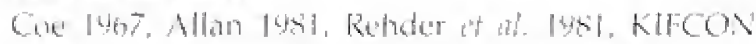

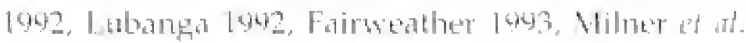

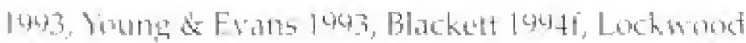
1445

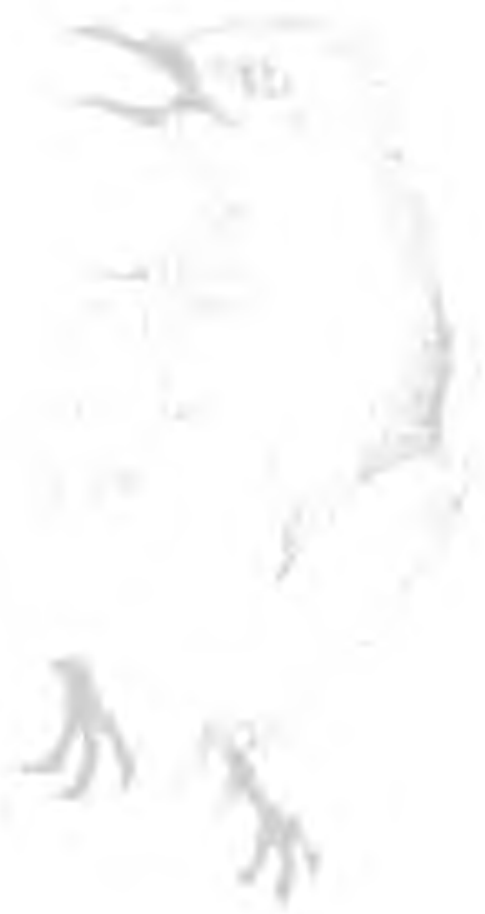




\title{
6 Mukurweini VAlleys
}

\author{
$0^{\circ} 30^{\prime} \mathrm{S}, 37^{\prime \prime} 07^{\prime} \mathrm{E}$, Central Province, Nyeri District
}

Area undefined: at least 20,000 ha

Altitude: $1,500-1,600 \mathrm{~m}$

Status: Unprotected private land; 10 ha private bird sanctuary

Criteria: Globally-threatened species, restricted-range species

\section{Site description}

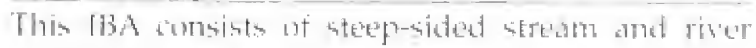
walleys with Lantana thickets, an the lonverr

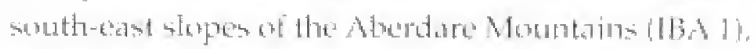
in the upper watement at the "Tana Riset. The" antes ont the. IBA canmot be pacisedy detined withent further

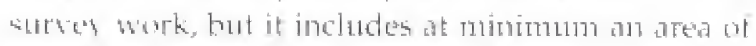

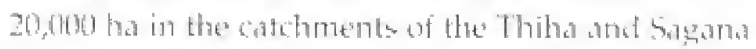

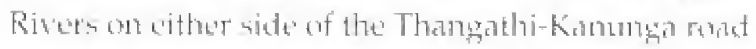
near Mukurweini tematr. This is a zante of high ascricteltural potential and is intensinedy cultivaled, the major coups inclading culfee majas and arrowroot. Little natural fengetation remains in most

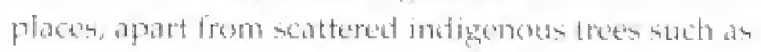

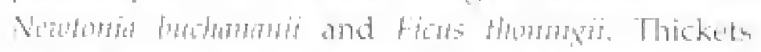

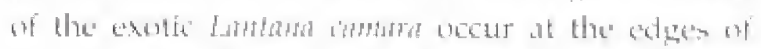

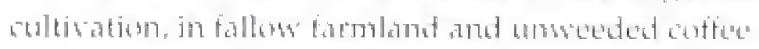

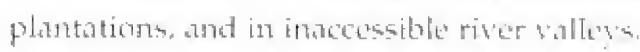

\section{Birds}

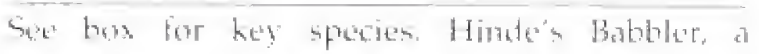

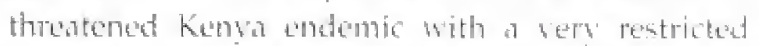
range, accotos at relatively high densition. It is

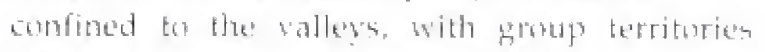

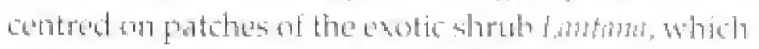
the babblers deperted on for sholter and nest sites. Othere birt spectes are typical of forest edpe and scrub habitats in the central Kenya highlande, but

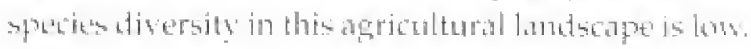

\section{Conservation issues}

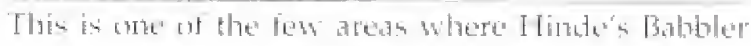

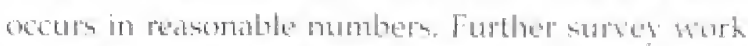

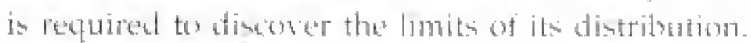
Comservation bstes are similar to theme dencribed

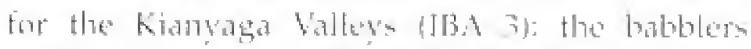

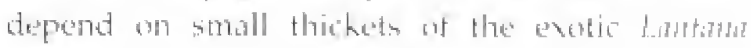

imere for shelter and nest sitem and fouter and tewer

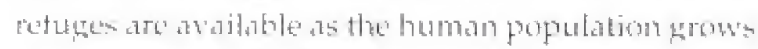

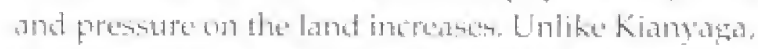

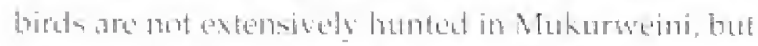

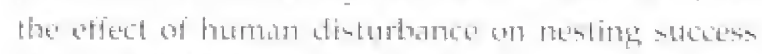

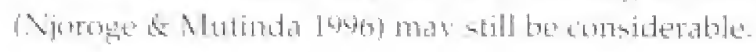

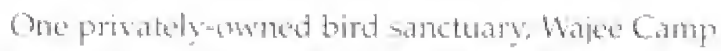

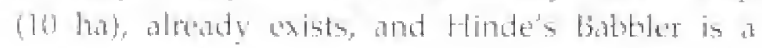
major wisitor attraction here.

\section{Further reading}

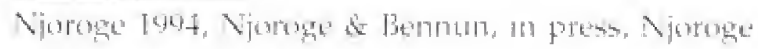
it al., in press

Cinpamon-chested Bet-enter

\section{Globally-threatened species}

"Hinde's Babbler

Endangered
Grotips oceur in many wif the river valleys (mean group sizes 3.8 birds, mean densities 3.1 birds/ $\mathrm{km}^{2}$ :

Noroge \& Bennth, in press)

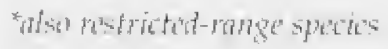


6 Mukurweini Valleys

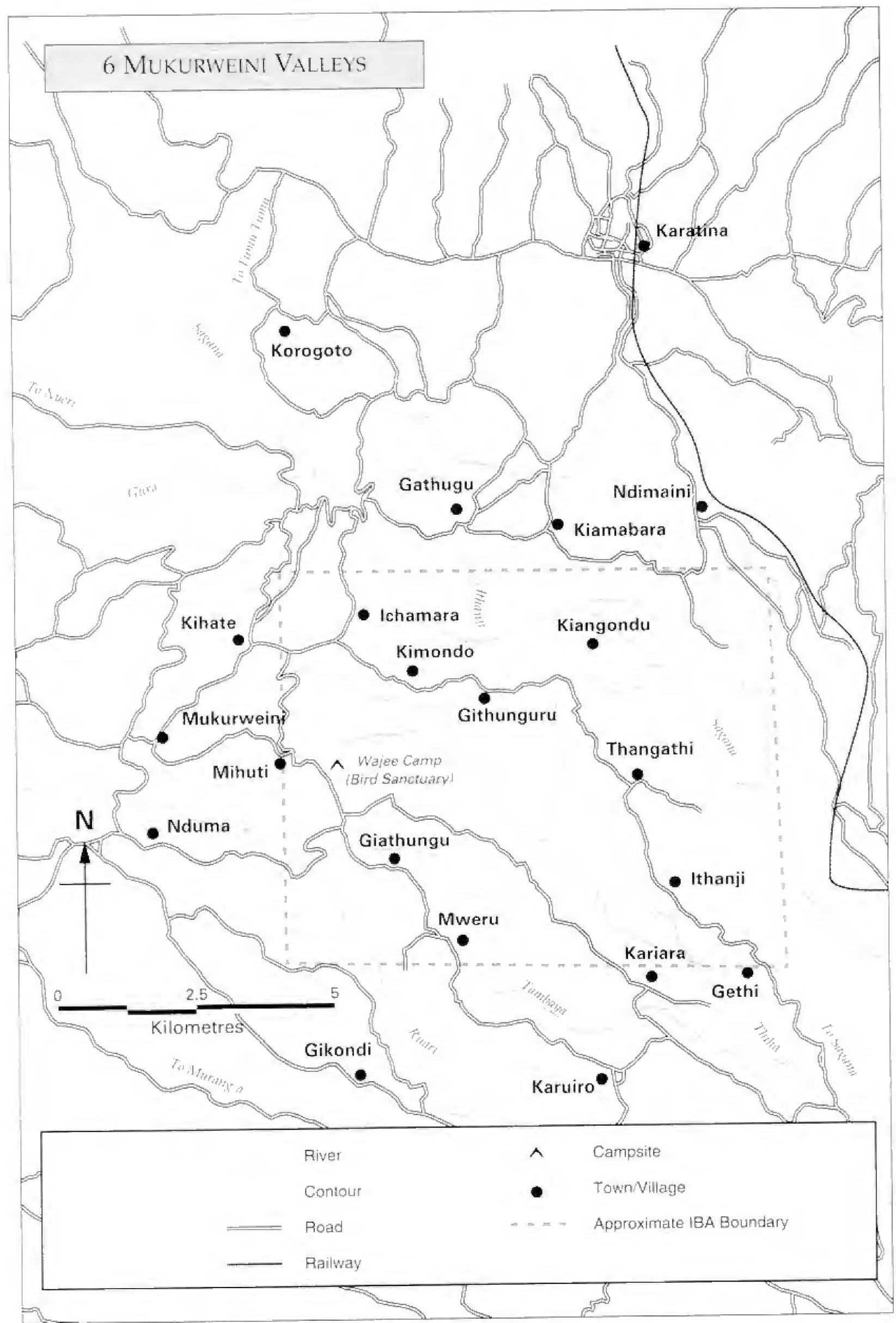




\title{
7 ARABUKO-SOKOKE Forest
}

\author{
$3^{\circ} 20^{\prime} \mathrm{S}, 39^{\circ} 55^{\prime} \mathrm{E}$, Coast Province, Kilifi and Malindi Districts \\ 41,600 ha (c. 38,200 ha indigenous forest or thicket) \\ Altitute: 0-210 $\mathrm{m}$ \\ Status: Forest Reserve \\ Cotegories: Globally-threatened species, restricted-range species, \\ East African Coast biome species
}

\section{Site description}

Arabuko-sakoke lies a few kilometres inland on the Kenyan coast, betsuen the towns of Kilifi and Malindi and somes $110 \mathrm{~km}$ north of Nombers. It is the largest watant fragment of the forents that unce conered muth of the last African coast, and whone [emmants comstitute the East Africin Coastal Porests Endemic Bird Ares. Aratukb-Sokoke wiar

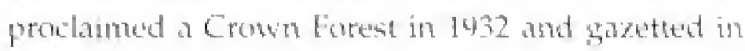
1443. coverizg an area of 39, 100 law. The Karatatha extension (2.700) ha) to the solith-east, which imbutes imporkent tracts of hey habitats, was addegt ir 1948 .

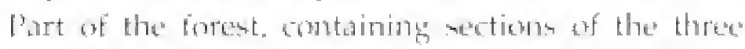
main babitat types, was gazelted as a strict nature

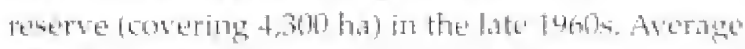

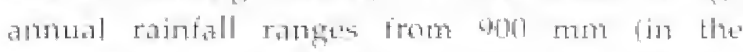

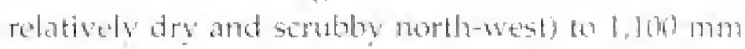

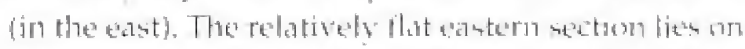

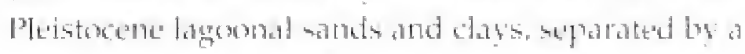

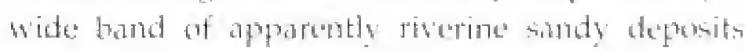
from the ridge of red Wagarini sands that tomo the western port of the Reserve (Robertson \& l.uke 1903) Three very distinctive forest wpes, each with its

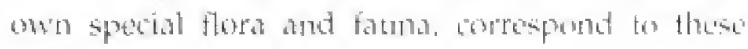
soil types:

1 Nixed torest $(7$, oug ha) in the cast, onf grey sands. This rabitat is rebatively deranc, tall and undifferentiatud, with a diversity ot tree species.

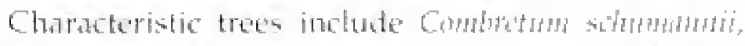

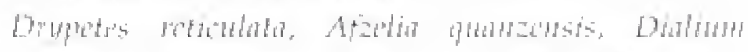

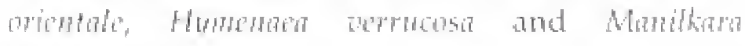
smsibritusis.

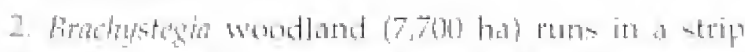
through the approximate antres at the forest, wh white, sery infertide soil. This retatively open hatritat

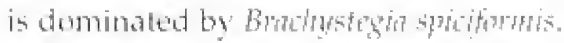

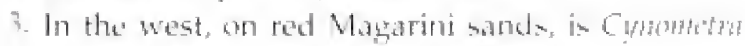

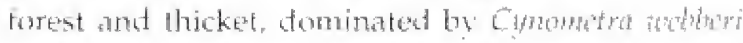

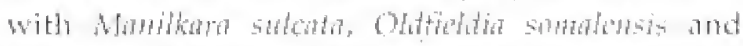

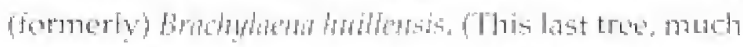
in demand for the barsing tracte, ham been almost logged aut from mach of the fortat.) The tratenition hertwen white and rod soil is sudden, and marked

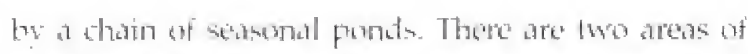

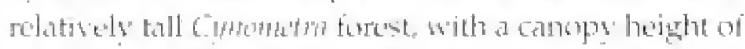

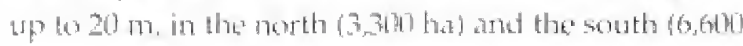

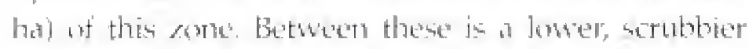

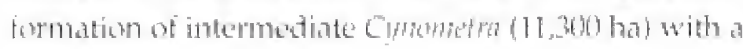

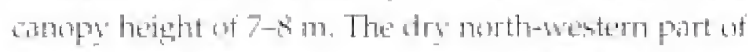
the Reserve in conered by a low: chense, and often

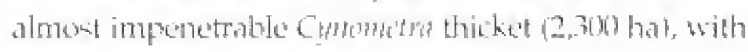
the comply no nore than $5 \mathrm{~m}$ hich.

\section{Birds}

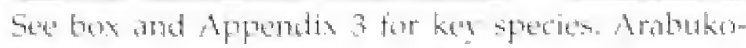

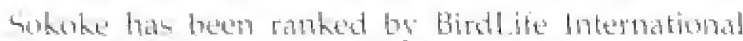
as the second mont important fortent for birst comperpation in mainland Atrica (Cotlat \& stuat 1988 . Siv globally-threateded species, and bive out of the surem specirs in the East Afrom Comatal Forest

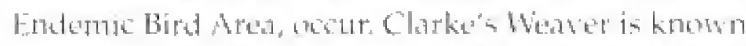
anly from Arabukn-Sonkoke and the litte-tudied

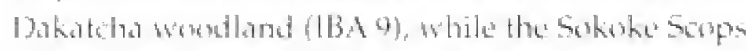
Ond is andy knemen from this forest and one whther site in nerth-east Tanzania. Where than 230 bird species we recorded (Fonshane 1465\}, induding 23 al Kerba's all Afran East Coast biome species (24 rengularty As wedl as the species listed ith the bow, Circat Einret. White-backed Duch and African Finferot,

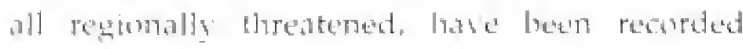

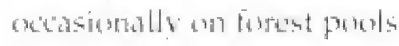

\section{Other wildlife}

Arabuko-bokake is rich in rave ant endemio whithes especially among the famma. Six taxe of butterties endemic on the East ditican chast are

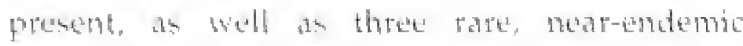

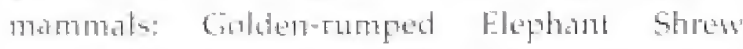

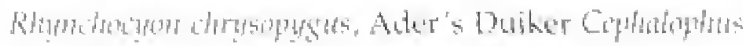

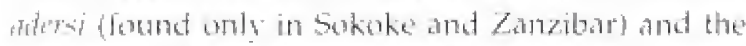

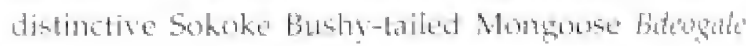

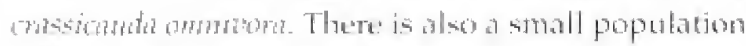

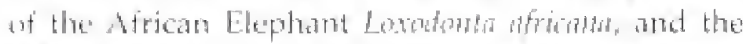

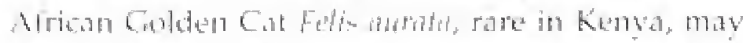
weur (Viranj 1463). Lnusual reptiles include the?

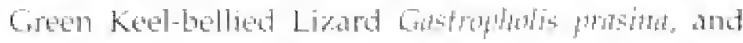
the turest is exceptionsuly rich in amiphibia, incleding 
7 Arabuko-Sokoke Forest

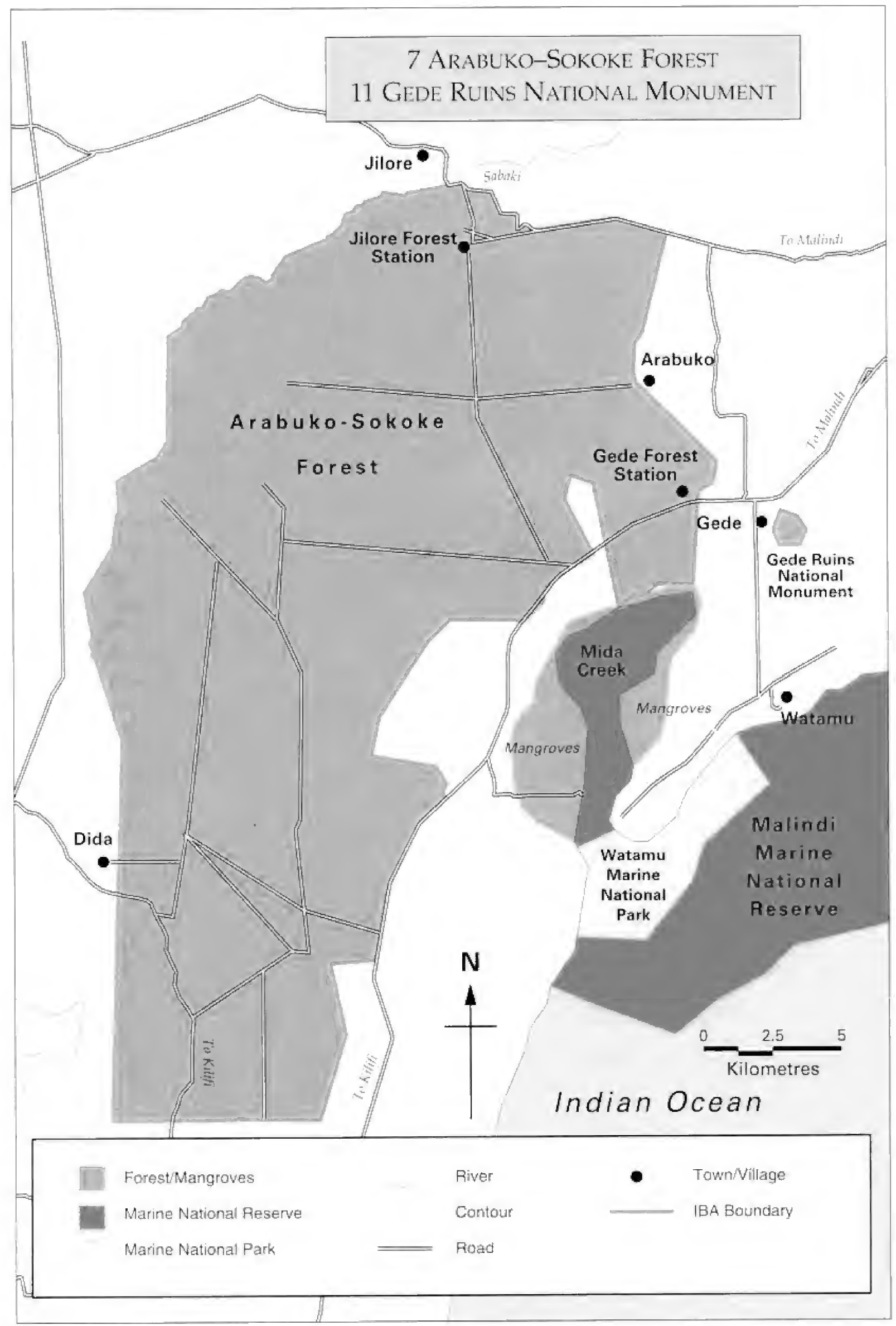


Important Bird Areas in Kenya

\section{Globally-threatened species}

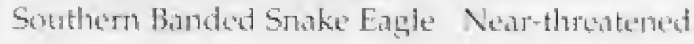

"Fitichers Turates

"Sokoke Scops Ow:

Spotted Ciround Thrush

Endangerod

Sokoke l'ipil

Vulnerable

East Const Akstat

Vulinerable

* Amani sustirird

Vulnerable

Plain-backed Sunbird

*Clarke's Werser

Viduerable
Resident

Commen in the taller forest

Confined to Cumornetrar forest and hat much lower densition intermediant

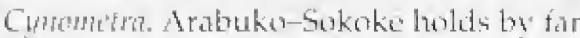
the butk of the world's population, with an estimanted 850-1,200 pairs (britlon \& Zimmerman 1979, Virani, in prexis)

Regular intra-African migrent, March-Ocloben (Mann 1976, Brittom \& Zimmerman 1974y

Uncommon resident of the ground stra-

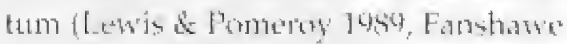
1065)

Arabukw-sukoke moy hold the world's laresest population. As mant as 9,1$) 00$ pairs may be present, mainly it the Cunirmitha forest (13ritton \& Zimnerman 1974, Matiku et at., in press

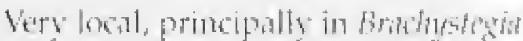
woodland (Turner 1977, Britton \& Zimnerman 1474, Fanshawe 1495, in ISIt.)

Relatively commom, especially in mixed forest

Mainly in Braclustegh woudland. An engignatic species whose numberts fluctuate (Mann 1976, Britton \& Zimmerman 1979. favfor 1984) and whose nest is unhnowin

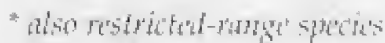

\section{Regionally-threatened species}

Ayres's I liwnk Eagle

Arican Crowned Eagle African Pitta

Scaly Babblet

Little Yellow Flycateluer
Vulnerable

Vulnerable

Vulnerable

Vulnerable

Vulnerable
Scarce resident

Resident

Sarce non-breeding visitor, fen recont records

Local and rarely recorded Mainly in mixed forest, anoids disturbed areas (Fanshow' (- 1965)

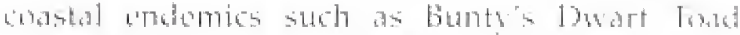

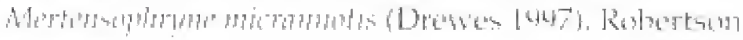

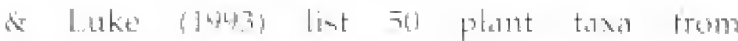

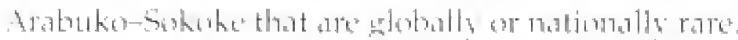




\section{Arabuko-Sokoke Forest}

\section{Conservation issues}

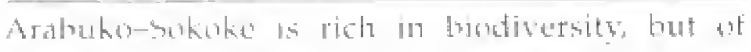

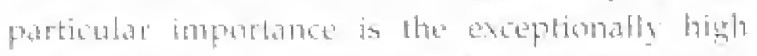

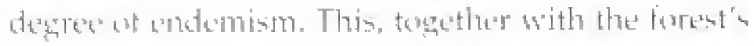

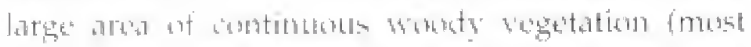

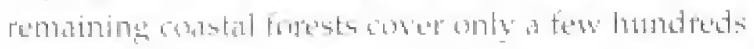

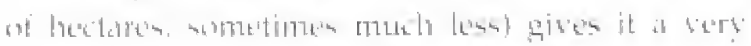

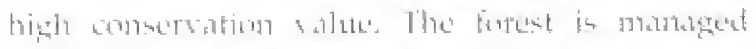

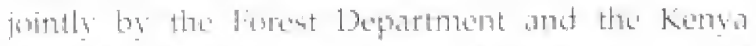

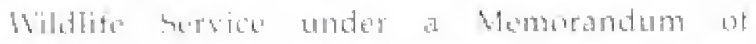

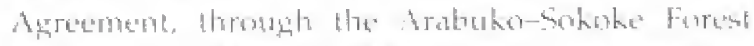

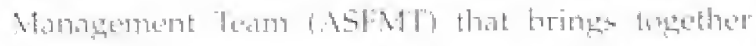

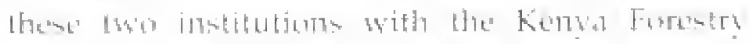

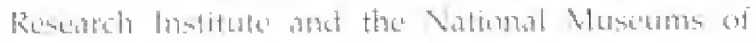

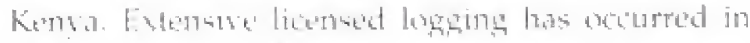

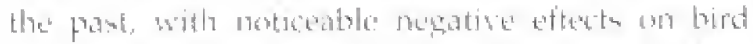

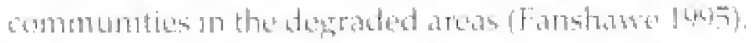

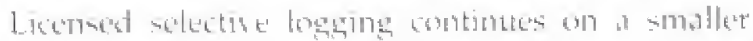

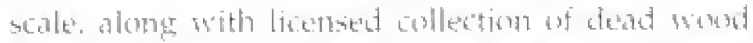

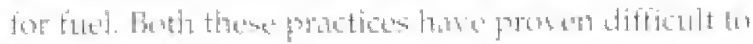

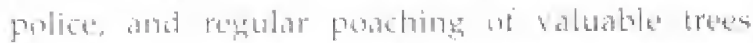

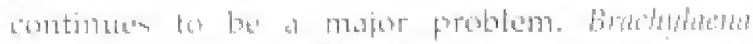

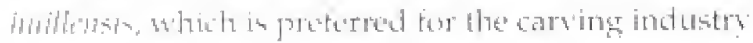

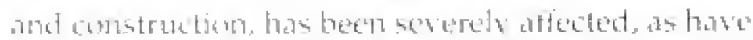

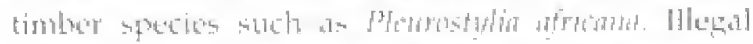

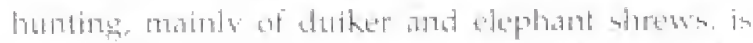

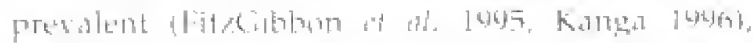

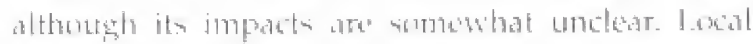

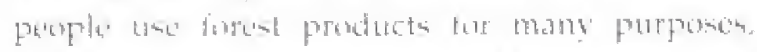

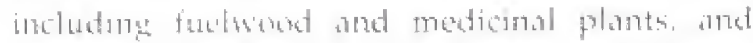

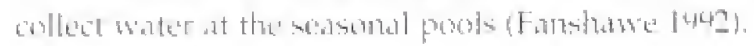

seile there are extremaly infertila and quite unsulable tor agticulure. Mare protitable and

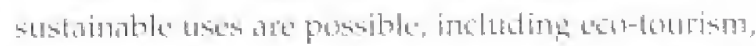
which is alreads growing in soble, and butterty brming. These ativitiss are being promotad by ASFMT through a Etropean Linim-funded Aralsuks-Sokoke Forest Hanagement \&

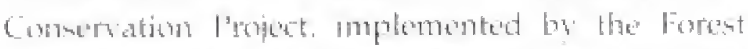

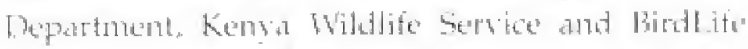
International. and by the werk of the Kiperear

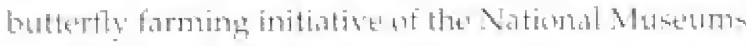
and Lat Aricia Vatural History Sexety. The Latter

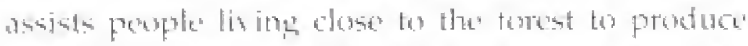

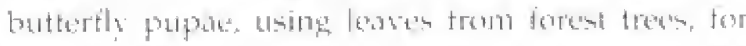
export to Eumope and anderical. Fundine from the ELL

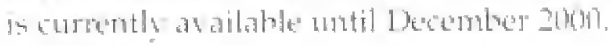

\section{Further reading}

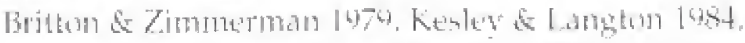

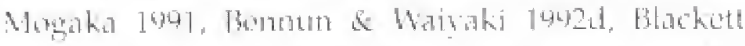

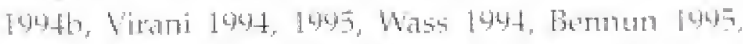

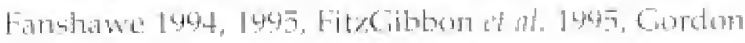

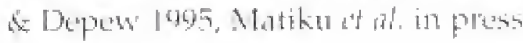

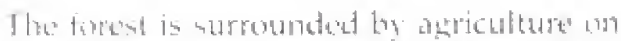

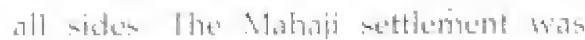

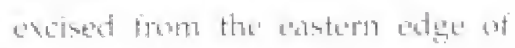

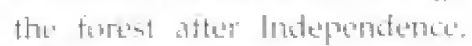
and pressuge remation high from

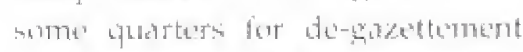
and sottlement of thes southerasterts

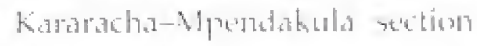
- despigte the fact that the

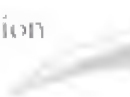




\title{
8 D AKATCHA WOODLAND
}

\author{
$03^{\circ} 01^{\prime} \mathrm{S}, 39^{\circ} 51^{\prime} \mathrm{E}$, Coast Province, Malindi District \\ c. 32,000 ha \\ Altitude: $90-230 \mathrm{~m}$ \\ Status: Unprotected, unadjudicated Government land and private fand \\ Catcgories: Globally-threatened species, restricted-range species
}

\section{Site description}

Dakateha is an extemaive tract of rebativels intalet coastat woodland itr constal Kencia, north of the Sabaki kiver and between 25 and $5(1) \mathrm{km}$ inlend irom the coast. The vegetation is simitar to that on the western boundary of Arabukor-Sokoke Forest

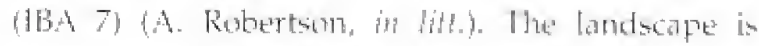
gently undulating, with woodtand dominated

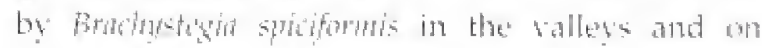

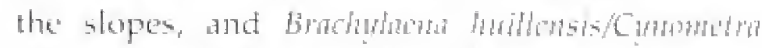
weblyey associations on top of the low luills, the

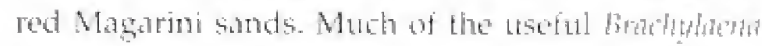
has already becen cuts, since it is in great demand as a long-bumirut smbeless fuelwowd and as

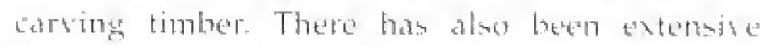
clearing of the hildteps for cultivating pintapples, which dor well in this soil type.

The IBA coners two tracts of woodland that nearly adjom each other, one of around 25,000 hal, nomtly-west of Baricho town, wp to and beyond the Galana Ranch boundary to the base of Dakubuko Hill (A. Roberton, wh litt), and ancther of 7 , thot has. noth and west of Maratia loms. These talong with another trat. Adu, coverimg 17,000 ha north and sobt of Ads village some 22 kn north of Marafal, have been proposed for gazettemeint an Forest Reserves in the Kilifi District Forestry Master Plan (Foment Department 1992). Galanes Ramth is the property of the stgricultural Deselopment Comporation.

\section{Birds}

Sete box forl hey species. Datalcha appears to hold sulmantial population of the shobally threatened sukoke Jipit and Clarke's Wveaver IT. Butynksi, umpubl. datal. Both these specius prosibly bread here the breeding grounds of Clarke s Weaver are undiancoredt), and this is the ondy site autside Arabuks-Sokoke Forest (IBA 7) where Clarken Weaser is known to oncur. That rimainder of thes arifauna is an vet incomplestely hrowen, but is typical

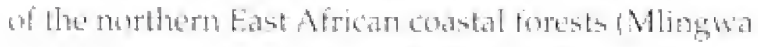

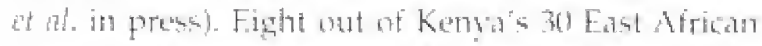
Codst biome species are so far recorded, and more will certaink be found with iurther surveys.

\section{Other wildlife}

There is 170 information an other tauna. The

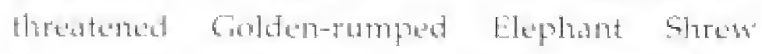

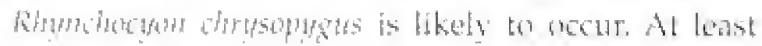
11 plant taxa in the area are slobally or

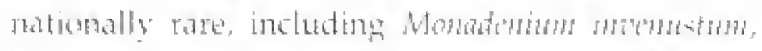

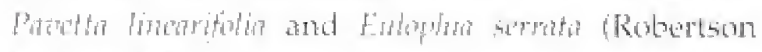
\& Luke lygit).

\section{Conservation issues}

Dempite its irimpufance as one of the last patehes onf

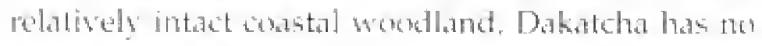
formal probeted status. It has already sulfered some damage from unomtrolled logging and clearing

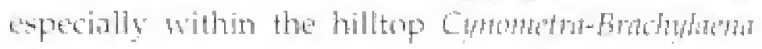
forcat. In the ongoing abjutieation of land in the

\section{Globally-threatened species}

Southern Banded Snake Eagle Near-threatened

Fischer's Turaco

"Sokoke Pipit

"Clarke's Weaver

"ulso pestricted-range species
Probably resident

Probably resident

Status uncertain, probably a sizeable resident population

Status uncertain; may nest here 
Dakatcha Woodland

\section{DAKATCHA WOODLAND}

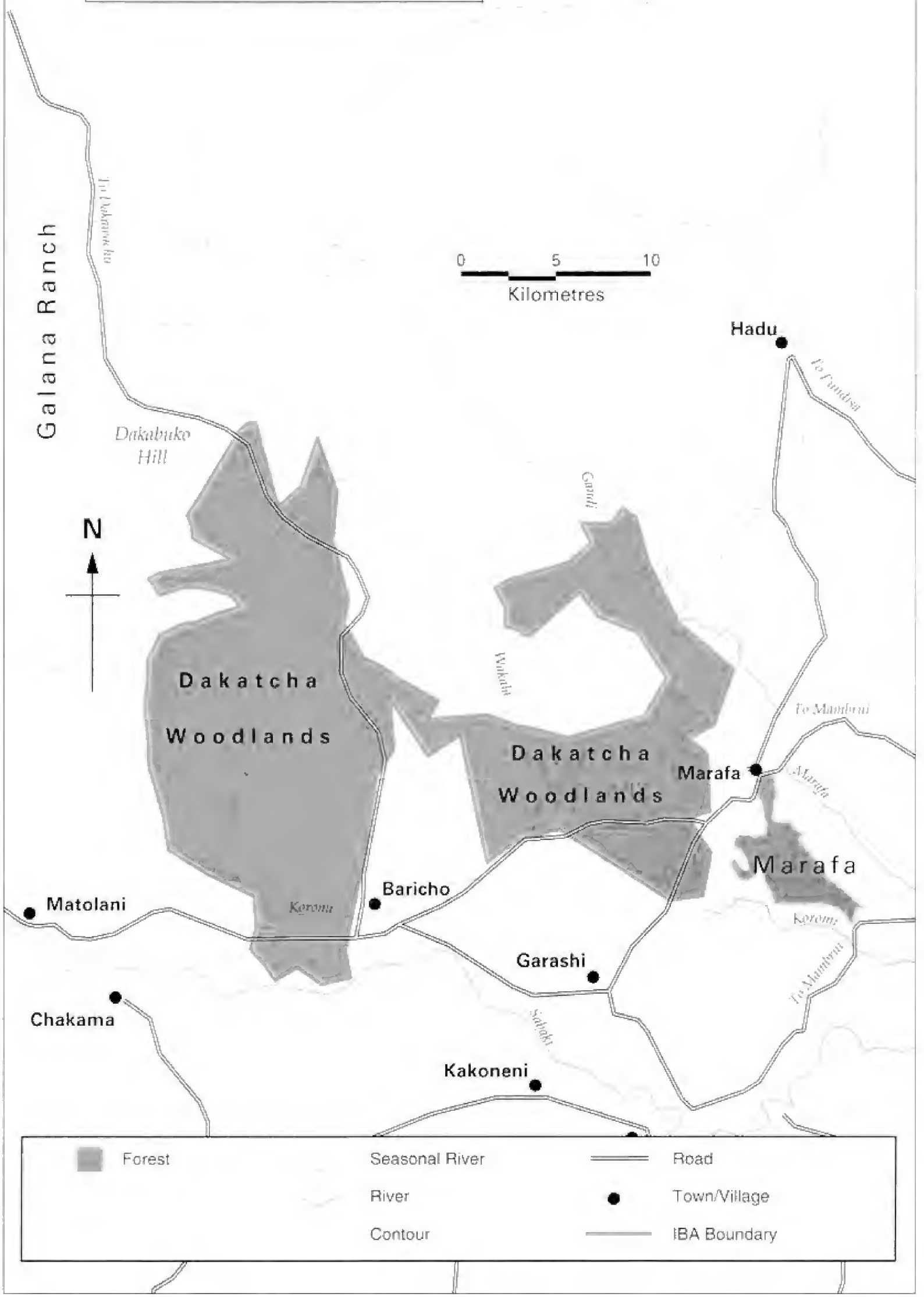




\section{Important Bird Areas in Kenya}

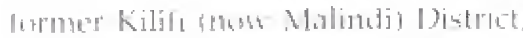

HAys a

Fonest Reserve to protedet ity

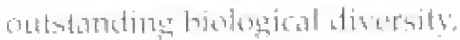

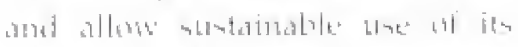

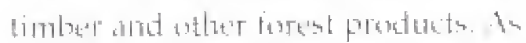

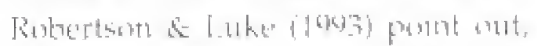

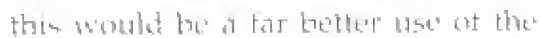

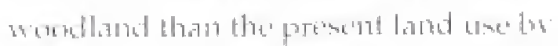

nominal as proposed wrous randers

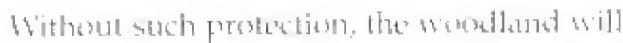

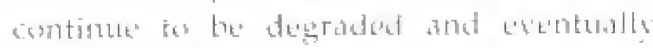

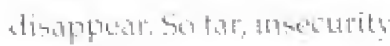

Inis maite the ares

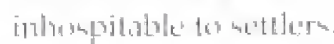

Winen the proposed

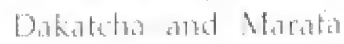

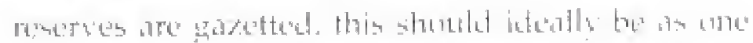

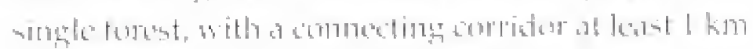

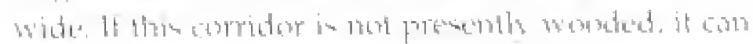

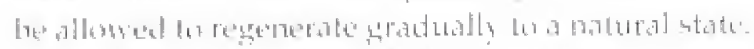

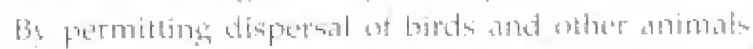

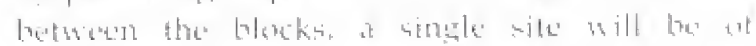

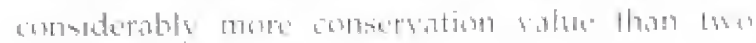

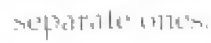

\section{Further reading}

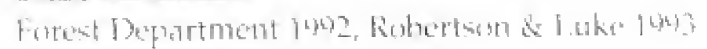




\section{Diani Forest}

$04^{\circ} 19^{\prime} \mathrm{S}, 39^{\circ} 33^{\prime} \mathrm{E}$, Coast Province, Kwale District (sete map in site ncount 20)

c. 80 ha

Altitude: $5-20 \mathrm{~m}$

Stafus: P'rivate land and (part) National Monument

Catcory: Globally-threatened species

\section{Site description}

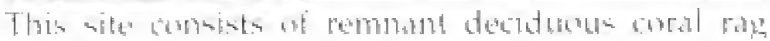

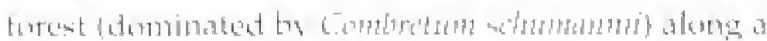

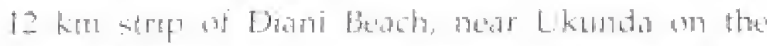

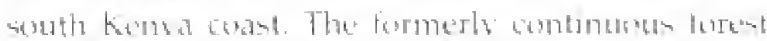

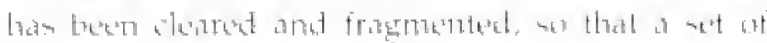

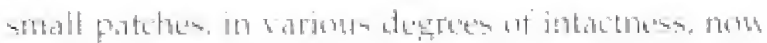

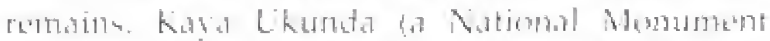

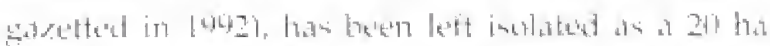

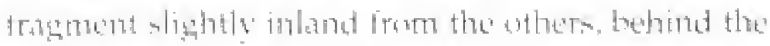

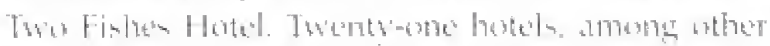

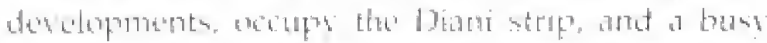

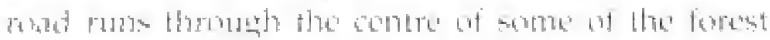

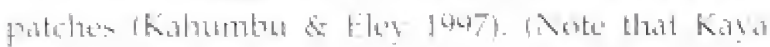

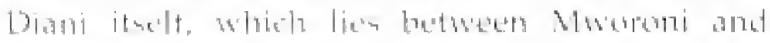

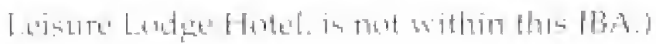

\section{Birds}

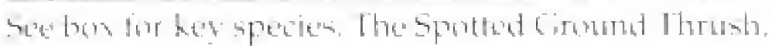

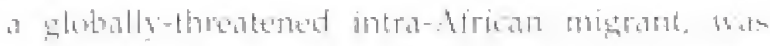

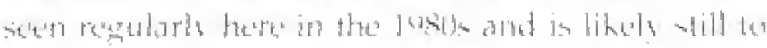

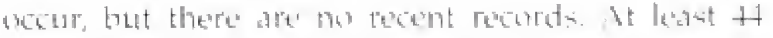

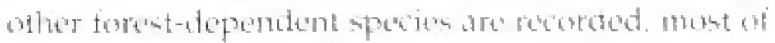

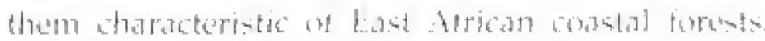

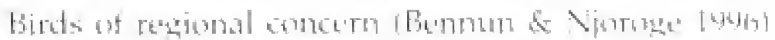

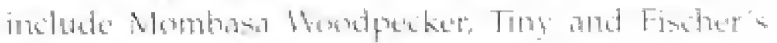

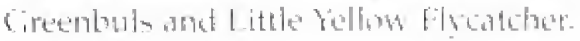

\section{Other wildlife}

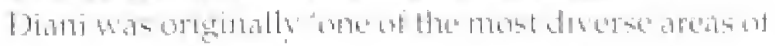

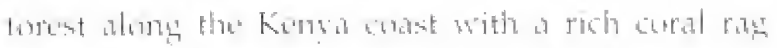

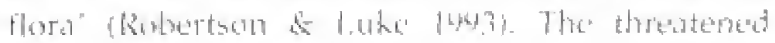

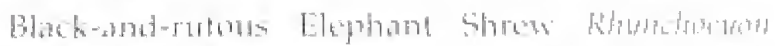

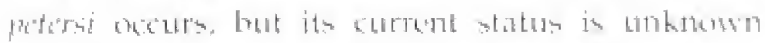

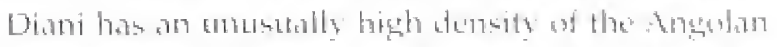

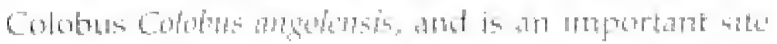

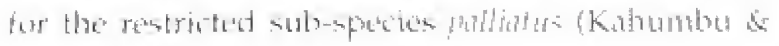
Elan, 1447$)$

\section{Conservation issues}

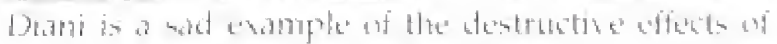

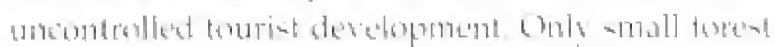

\section{Globally-threatened species}

Southern Banded Snakia Eagle Near-thratered

"Fischer" furacon Near-threstenest

Spotted Ground Thrual $\quad$ Enctangered

l'lain-bucked Sunbird

Near-threatened
Stutus unknomin

Status unknow'n

Formerly" regular (e.s. Irime so Isvine 1977a, 1988. 1991); Diani may still be an important 'steppoing stone' lorest for this migrant spacies

Status tuthknomen

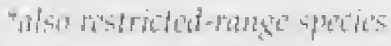

\section{Regionaliy-threatened species}

Artican Cromoned Eigle

Atrican Pitta

L.itlle Yellow Flaratheht

Uluguru Violet-inacked Stimbirt
Vulnerables

vilnerable

Vulnetable

Vulnerable
Status unknown

An intra-African mierand; 190 rectunt records

Statts unknown

Stakus unknown 


\section{Important Bird Areas in Kenya}

patches are now left and rompant culting at

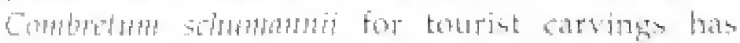
alesraded muth of what remains. Apart from the

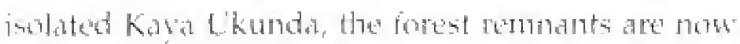
andatived on private land. Though many owners have destruged their forest or permitted it to be

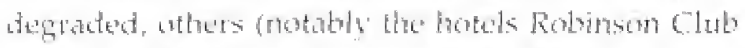
Babbats and Nomads have protected their holdings. The remaining forest, althughd fragmernted, is atill

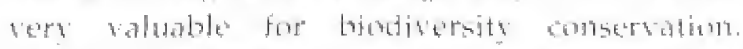
Recently, rout killis of colotous hase hicused local atteatious the the plight of this

speries and the forest as a

whole, resulting in the

gexistratien of a lowe

Lithirommeshtal group;

Wakuluzu, Friends

uf the Coldows Trust.
Wakuluzu's soncern coners Diani in particular azd

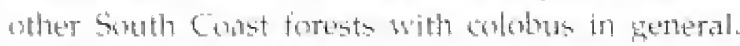
At Diani, work te restore the habitat and the connestions between fores patches is urgently neded. I, noal retouniticn of the value of the forests. for thurishe as well as for biodiversty conservation, mos he?p to britg thes about.

\section{Further reading}

trune \& Irvine 1970, 1477a,b, 1988, 1991, Vy'weno

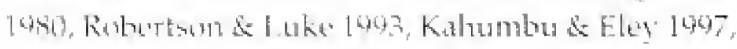

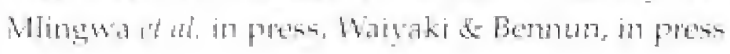

Little Yellow Flycatcher 


\section{Dzombo Hill Forest}

$04^{\circ} 26^{\prime}$ S, 39 $32^{\prime} \mathrm{E}$, Coast Province, Kivale District (see may in sife account 17)

295 ha

Altitude: $100-470 \mathrm{~m}$

Status: Forest Reserve and National Monument

Categories: Globally-threatened species, restricted-range species

\section{Site description}

Dzombo Hill, like Mrima (1BA IS), is an igneentes intusion inte the Triassic sandstones of the surrounding coastal plain. The hill tises abruptly to around $470 \mathrm{~m}$, with a lower summit at $400 \mathrm{~m}$ to the west. The tainfall is too-1, 100 mm per year, with considerable additional mist and dew ton the upper slopes, Dzombo is conterest by undifferentiated constal mixed horest. wettest on the southerastern slopes. To the north and murth-3west, the forest grades into atrien womblarut and sorub.

Pale Batis

\section{Globally-threatened species}

* Sokoke ripit

Vulnerables

Plain-backind Sumbirt

Near-threatened

*Fischer's Turaco

Near-threatened

"also restricted-ramge species

\section{Regionally-threatened species}

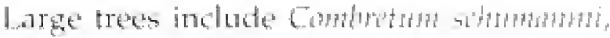

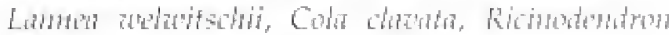

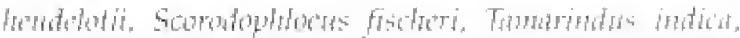

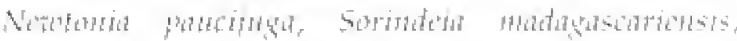

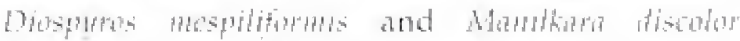
(Robertion \& Luke 1493). Dzombo was gazetted as a Forest Reserve th 1941, and Kave Damber as a

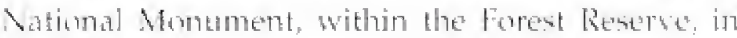
1992. The forest in comsidered macred by the local

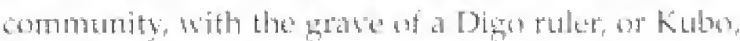
near the smmonit.

\section{Birds}

See bux for key spercies. The globs lly-threatered and resiricted-range sukuke Pipit has recently besth disconered here. Dronnbo has a rich asifauma, with
Recent records; presumably resident (Waivaki 1995)

Probably fairly abundant

Probably tairly" abundart 


\section{Important Bird Areas in Kenya}

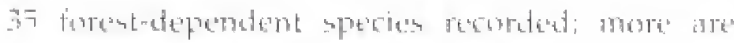

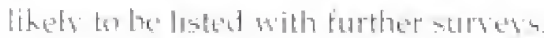

\section{Other wildlife}

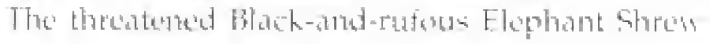

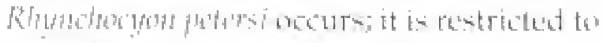

if stmall number af Fast African

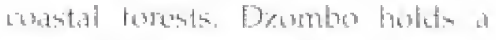

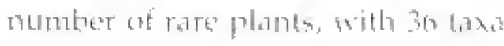

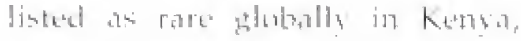

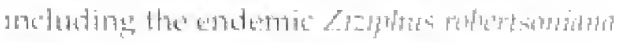

and an unctereribert apasion

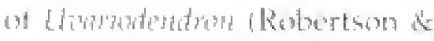

lake 15013, O. I tuke

with.

\section{Conservation issues}

1) ramber in designateid is as

matiomal momement anct

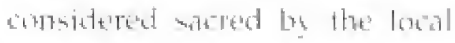

semmunity: The afferete it somet

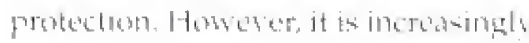

wander threat from arricultural

ancrometument intich in aleats

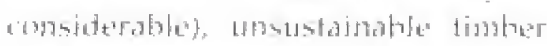

and pute extration, bark wripping a d

freas bir binding materials and firenwend

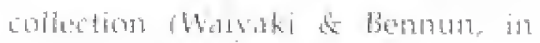

pressit, At present, vehicle areses into the fordest

is dificult and forest befartment control in

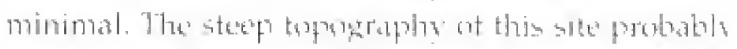

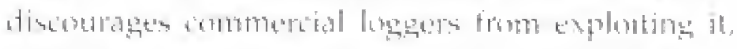

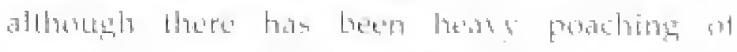

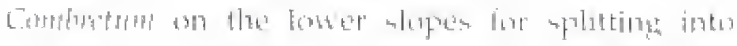

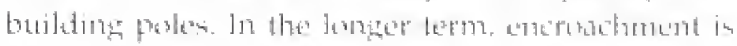

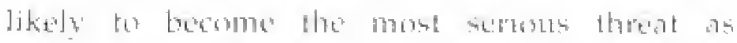

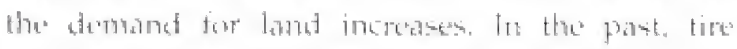

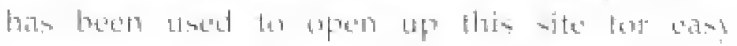

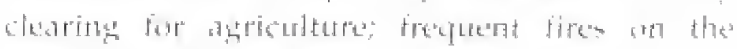

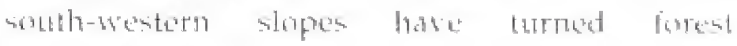
into grasslatid.

\section{Further reading}

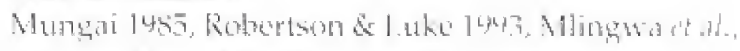

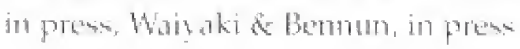




\title{
11 GEDE Ruins National MONUMENT
}

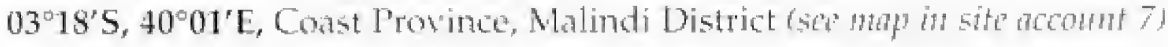 \\ 44 ha (c. 35 hat Erest) \\ Altitude: $15 \mathrm{~m}$ \\ Status: National Monument \\ Category: Cilobally-threatened species
}

\section{Site description}

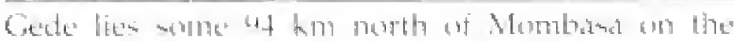
Mombasa-Malincti romat. A gaserted Xational

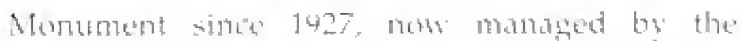

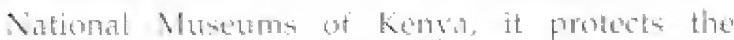

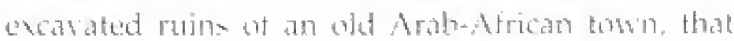
was abandoned in the $17 \mathrm{fth}$ centurs. Ohe the statns,

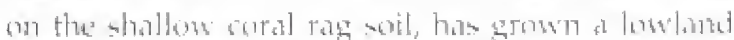

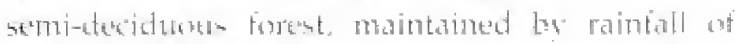

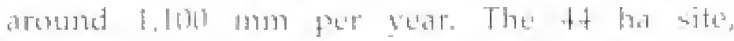
serfoumded by farmland, is entirely tereded, and

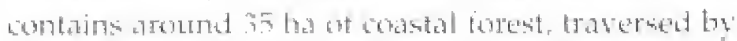
nartow fothe that wind between the exasated

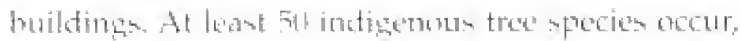

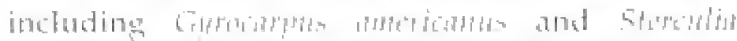

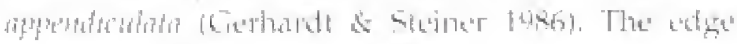

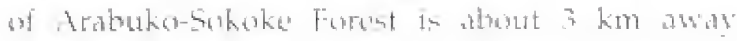
tor the west.

\section{Birds}

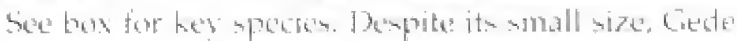
is an important spe for the grobally-thentemed

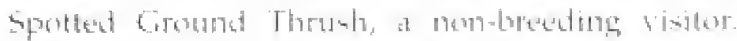
is matry an 110 birde mas be present between

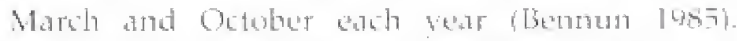

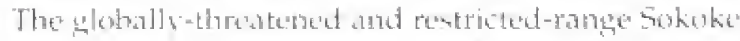

Spotted Ground Thrush Pipit hats also been recterded heres. therugh thex a): 17 ?

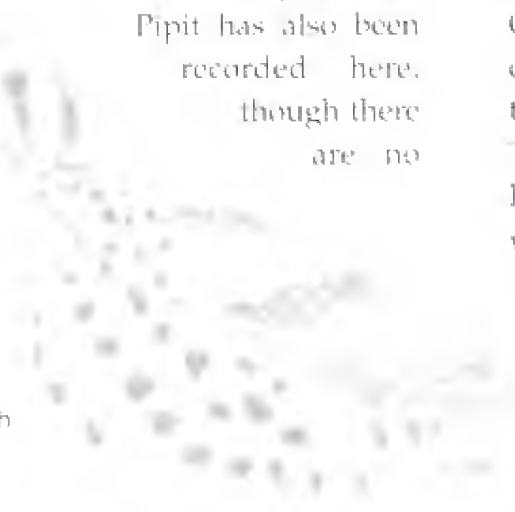

rexent recordes and the poypulation, if atill entank,

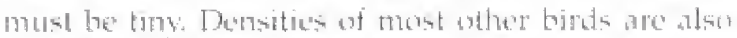
low, although the listed avilown is semprisistgh

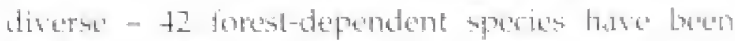

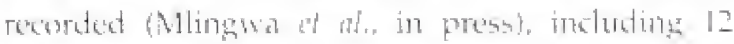

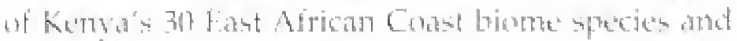
the restricted-rathe Fincher's Turace, which is

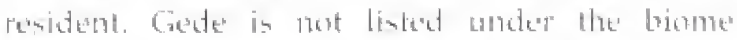

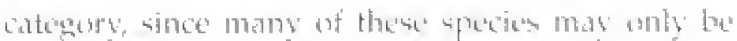

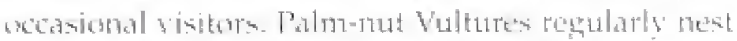

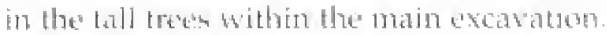

\section{Other wildlife}

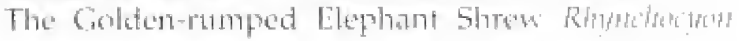

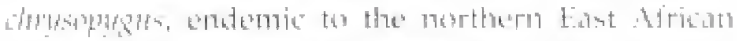

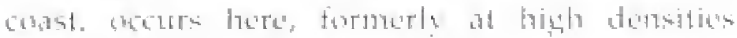

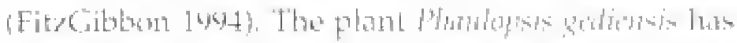

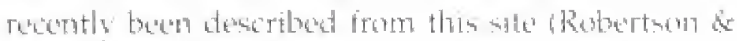
Luke 1945).

\section{Conservation issues}

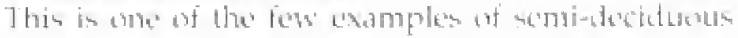

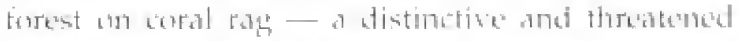
furest type - that in lormally prosterted it Ketry. The ruinget walls and buitalings, overgrown wath

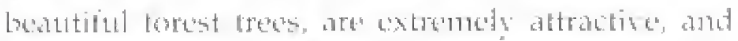

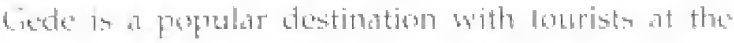

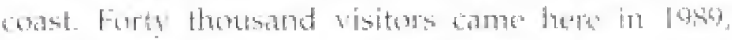

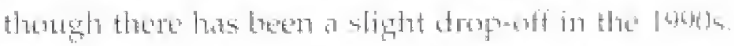
The forest is now completely surdounded by fartmed land, bot up until aroumd the tath tosos there were corridots lo wher tortest pateches in the Salindi-kbatame arda (A. kobertsom, ne lith small trese and

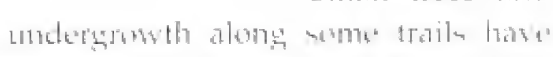

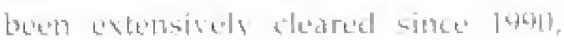

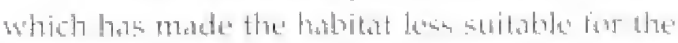

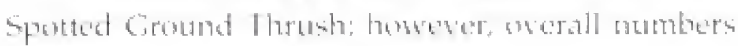

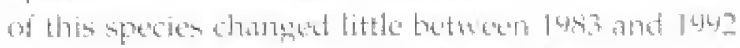

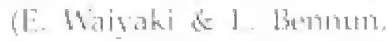
unputat. datsit.

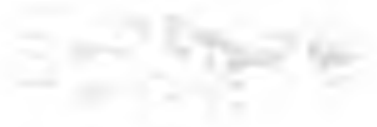




\title{
Important Bird Areas in Kenya
}

\author{
Globally-threatened species \\ Southern Bandad Suake Eagle Near-threatened \\ Status unknom \\ "Fischer's Tutaco \\ Near-threatened \\ Faitly common \\ Spotted Ground Thrush \\ Findangered \\ A reyalar non-breeding visitor; at \\ relatively high densities (bennun 1985 , \\ 1957 \& 19926 ) \\ 4sokoke Pipit \\ Vulnerable \\ No recent records: may have been an \\ occasional sisitor in the past \\ Plain-backed Sunberd \\ Noar-threatened \\ Fairly cotmmon \\ *also restricted-range surtios

\section{Regionally-threatened species} \\ African [ritta \\ Scaly Babbler \\ little Yellow Flvatcher \\ vulnerable \\ Vulnerable \\ vulnerable \\ Non-breeding visitor (Rathbun 1978): no \\ recent records \\ Status unknowr \\ No recent records; may have beere an \\ occasional visitor in the past
}

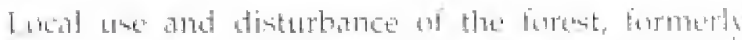

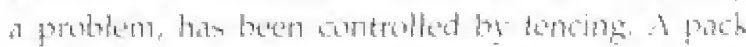

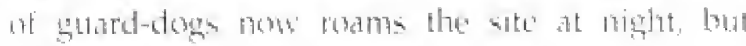

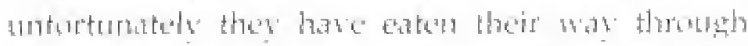
mus, and purthape all, of the ciofdem-rumped

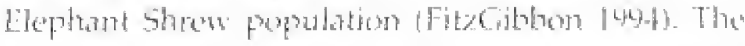

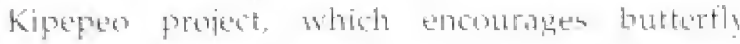

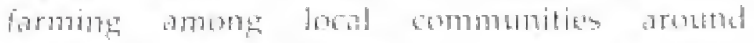

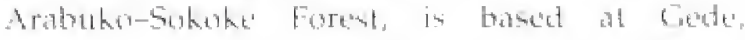

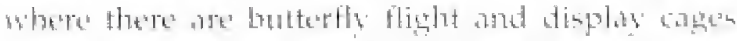

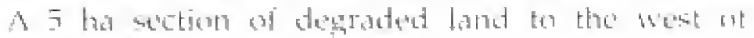

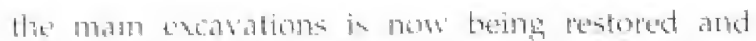
shoming up as forest throum the Code Niational Nomument Forest Restomatum Project foriginally the

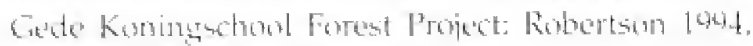

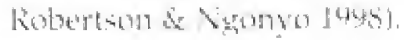

\section{Further reading}

Fanden \& Fadum 1472. Kirkman 1973, Rathbun

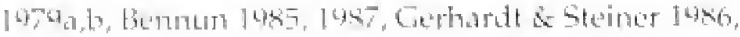

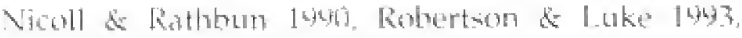

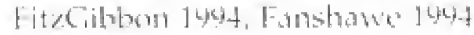




\section{KAYA GANDINI}

04.01'S, 39³0'E, Coast Province, Kwale District (see map in site account 20 )

150 ha

Altitude: $140-200 \mathrm{~m}$

Status: National Monument

Categories: Globally-threatened species, restricted-range species

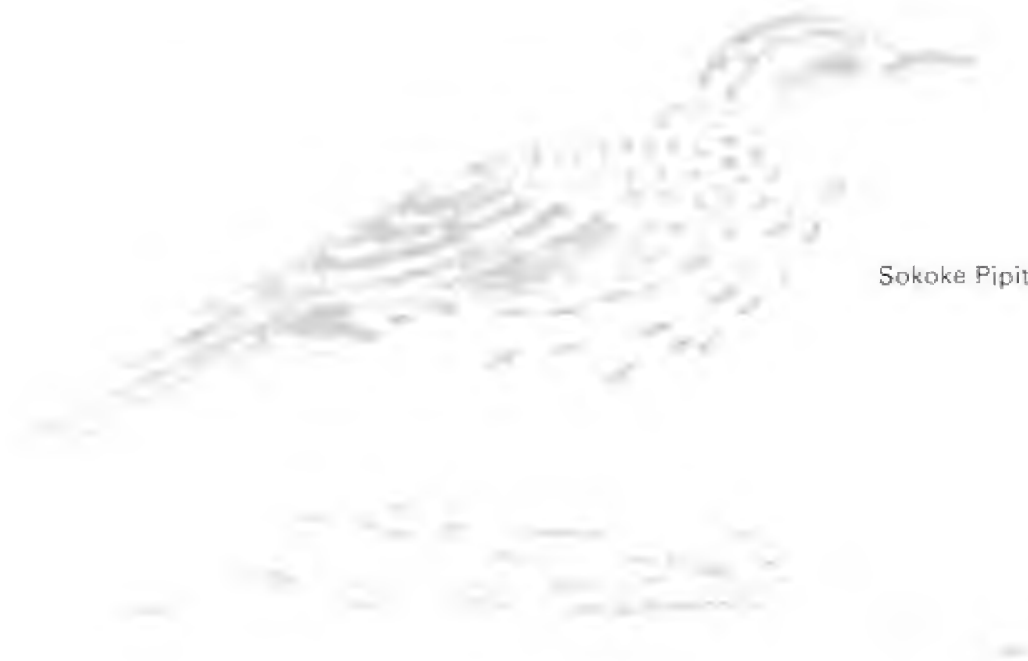

\section{Site description}

The Kayas are small, radict palclues of forest flat

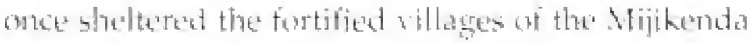
Pehple wa the Kengath corast. They have spiritual

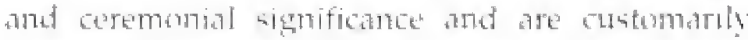
protected by a Commeil of Elders. Kaya Gandini tatos known as Takawa or Durumat and sabed to the

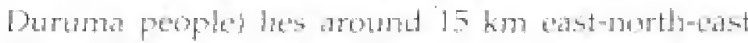
of Mombasa, near the bowne of Gandini, and

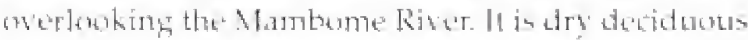

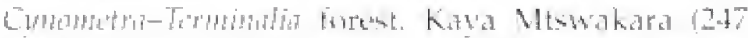
hat, which is simidar flotistically, is situinted ancund 2 kn awas acoss the Vambsme river, anet is in turn

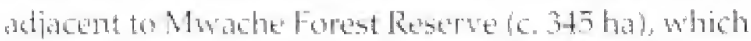
is anmesw hat weter forest acromo the Nwathe River. The Karat tain gaverted as a National Monument

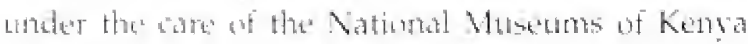
in 1459 ?

\section{Birds}

Soe box tor key species. This is a potentialls important site tom the tluteatered spotted ciround Thrush and the threatemest and restrinted-ange

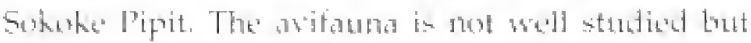

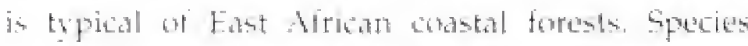

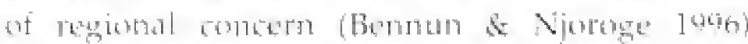
inducte Fiother's Turaco, Thick-bilked Cuckor,
Dastern Green Tinkertird, Mombas Womdpecker. Chestntit-honted Helmest-shrike and Plabin-backed Sunbinat.

\section{Conservation issues}

As in other Kayas, an increasing distegant for traditional values the led to anservation problems in Guatini. The Kaya has recently beer the" subpext of a land dispole? where certain elders marked out and cut parts of the forest for farming. They have thow been requested tor move ont by the Councit of Elders (Q. Luke, its int.). There bas beent some cutting of building poles, wspectally" Termandiat, but on the whole the forest is in fairly gond condition. Gandini is relatisely acessible from a main roat, making it potentially attractive for

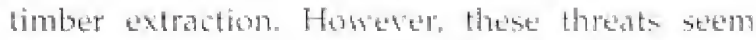
to be under control at prement, and fwith the support of the National Alusems Coant lont Consertation Unit) the eders ate taking a more atctive role in mathaging use of the torest. Trapping of animals (possibly unsustainathe and sollection

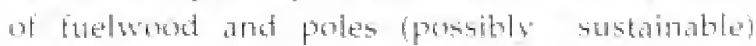
antinue.

Kaya Mtswakara (gazeltent as a National Nonument in 1997) is separated from Gandini by 


\title{
Important Bird Areas in Kenya
}

\author{
Globally-threatened species \\ "Tischer's Turaco Nent-threatened \\ Spotted Gromed Thrush Endangered \\ Non-breeding visitor, March to October \\ (Waiyaki \& Beronun, is press) \\ *Sokoke ripit \\ Vulnerable \\ Recent records, presumably resident in \\ small numbers (Waiyaki \& Betrnum, in \\ press) \\ Plain-backed Surbird \\ Near-threatened

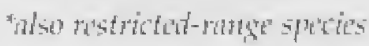

around $2 \mathrm{~km}$ orf settled latid afong the Marnborme River. Restoring a strip of matural vegetation allong the river would form a dispertul corrider for brids and other animals, Survers shented Mtowskara's

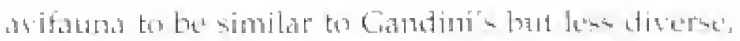
and apparently latekng the two threatened specien

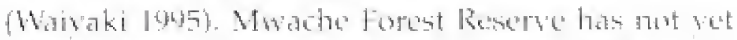
been surveged tor birds. It could form a third fart of

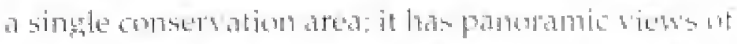

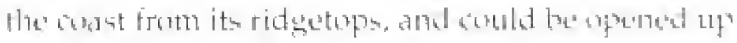

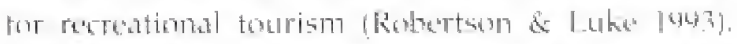
Additional surees ane needed to determine we het the the theretened birds de nocur in Mtsuakerat and Nwache, in shich aase the sites should the

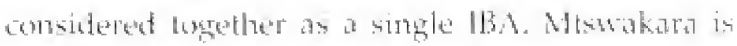
wel] conserved at present and lecal control oner forent une apperars to be effective, fut the ore in

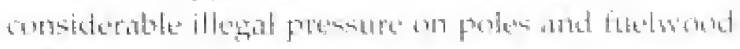
in Muache.

Further reading

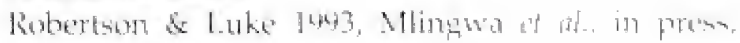

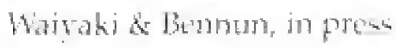




\section{KAYA WAA}

$04^{\circ} 11^{\prime} \mathrm{S}, 39^{\circ} 36^{\prime} \mathrm{E}$, Coast ['rovince, Kwale District (so' may in site acom 20 )

20 ha

Altitude: $15 \mathrm{~m}$

Status: National Monument

Category: Globally-threatened species

\section{Site description}

The Kayse are small, rolict patches of torent that

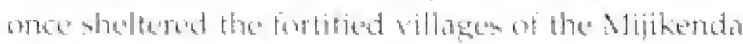

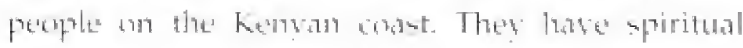

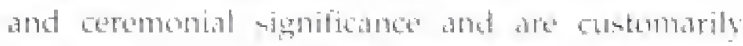

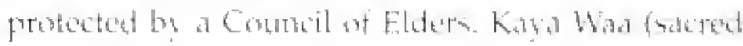

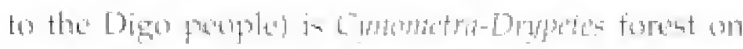

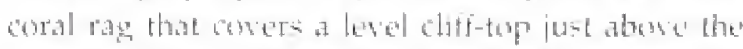

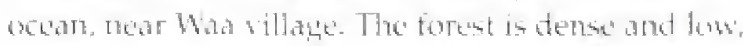
practically a thether in many places, and diticult to watk thrometr. Lnter the corat ctith there is

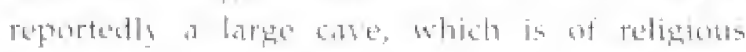

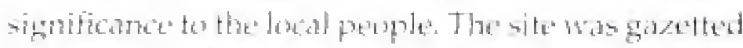

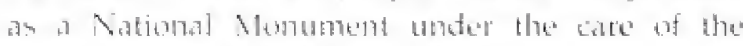

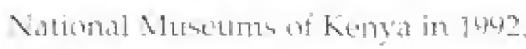

\section{Birds}

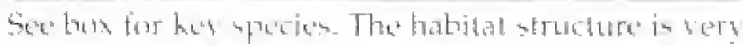

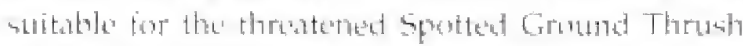

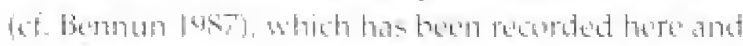

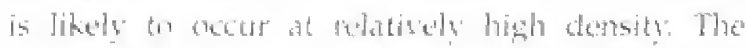

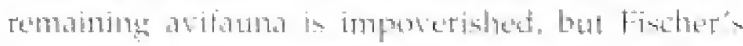

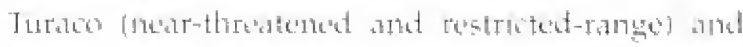

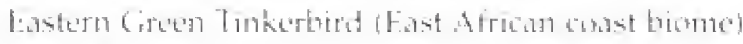
have aboberes recorded.

\section{Other wildlife}

The threatened Bhach-and-rutous Elephant Shrew

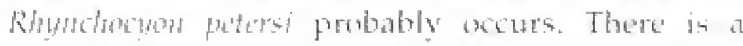
healthy population of the coratsal fortest tree

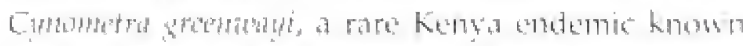
anly from lexe and the Watamu area (Rotwethon \& luke 14t3, O. Luke m hith h.

\section{Conservation issues}

The main threat to thes seafremt kity in of the land being "allocited" to private developers. This kayt was the centre of a land allecation contronersy in 1988 , which led, exentually, to a surecestul Private

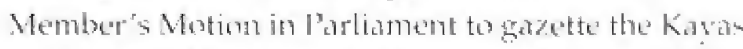

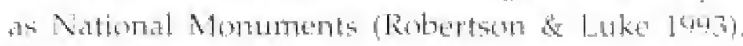

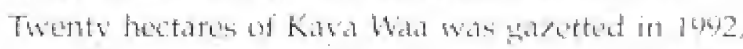
but the forest remains divided into some 77 plots tor

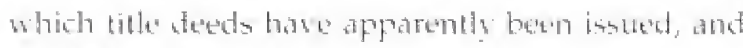
ito fate in uncertion.

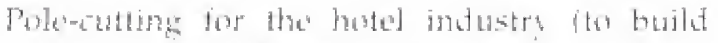
tradilionallatyo Mijikenda huts has caund

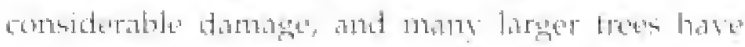

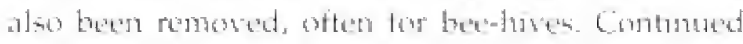

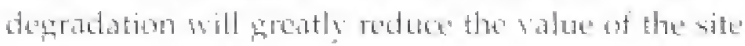




\section{Globally-threatened species}

spotted Ground Thrush

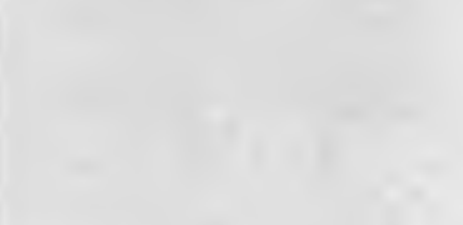

"Fischer's Jurnco

"also restricteri-range species
Endangered

Neat-threatened
Recorded during survey work in 1994

(Waiyaki 1995). The habitat appears to fit this species' requirements very well, with dense shade, thick leaf litter and an open understorey interspersed with tangles of vegetation (Bennum 1987)

Status unknown

for the Spotted Ground Thrush, which avoits areas with an sper canopy. Fires deliberately sot to cleat the survounding agrictluaral land also pose a threat (1) the forest. Hunting pressure appears to be very high, with numersus traps and snares. apparently exacerbated thy the settlement of Tharaka immigrants in the area some 15 years ago (Waisaki \& Bernum, in Fress?

\section{Further reading}

Robertion \& Luke 1943, Wasaki 1994, Ningwe a d in press, Waiyaki \& Bennun in press 


\title{
14 KISITE ISLAND
}

\author{
$4^{\circ} 43^{\prime} \mathrm{S}, 39^{\circ} 22^{\prime} \mathrm{E}$, Coast Province, Kwale District
}

c. 1 ha at low tide

Altitude: $0-5 \mathrm{~m}$

Status: Protected within the Kisite-Mpunguti Marine National Park

Category: Congregations

\section{Site description}

A small, waterless corab island 8 km affohore in the Kisite-Npunguti Marine Vational Park. The Park lies surth of Wasinj Istand of Shimoni, wh the south Kenyan coast near the Tanzanian border, and novers an area of 1,100 ha. Kiste itself 15 that and tredest. covered in low grass and herbs coral plattorms around the rained central portion are posed at low tide. There are three other coral islets in the Park
Mpunguti va luu, Mpunguti ya Chini and Jise la lathasi, bui these lie toser to the larger Wasmi Fsland, are ucrub-ionered, and support no significant numbers of nesting waterbirds.

\section{Birds}

Soe box for key species. Between July and October, Kisite supporth an assemblage of pelagic-feeding birds. In many (but not all) years this includes it

\section{Congregatory species}

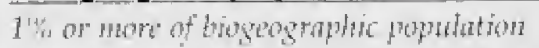

Roseate Tern (400)

Up to 1,000 pairs (Lewis and Pomeroy 1989); 400 adults and c.100 young birds in October 1997 (F. Ng'weno in litt.); numbers fluctuate greatly from year to year

Rosate Terns 
Important Bird Areas in Kenya






\section{$1+$ Kisite Island}

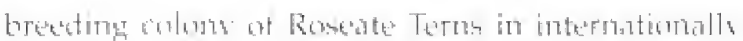

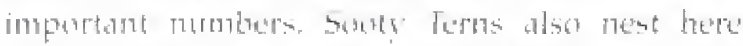

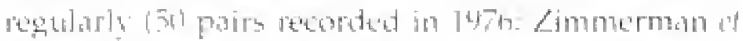

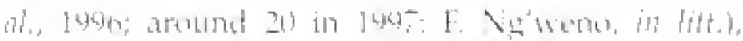

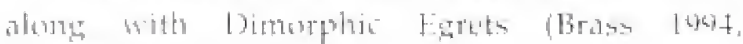

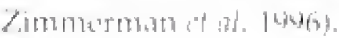

\section{Conservation issues}

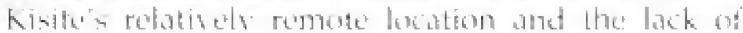

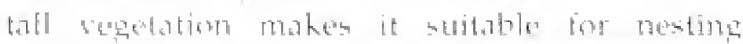

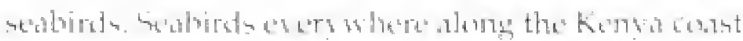

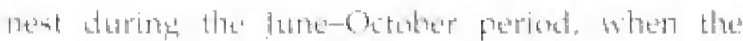

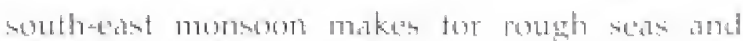

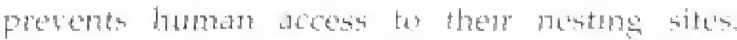

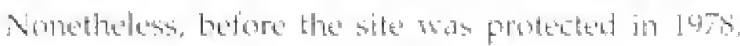

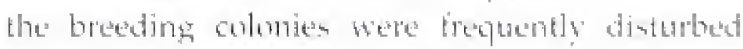

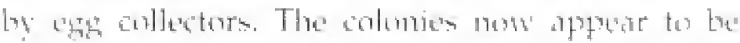

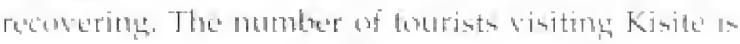
increasing, and careful manngement is metedest tos masure that they do thet disrupt the collonters.

\section{Further reading}

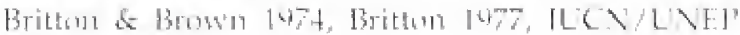

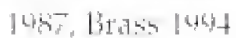




\title{
15 KIUNGA MARINE \\ NATIONAL RESERVE
}

\author{
$1^{\circ} 50^{\prime} \mathrm{S}, 41^{\circ} 26^{\prime} \mathrm{E}$, Coast Province, Lamu District \\ 25,000 ha \\ Altitude: $0-30 \mathrm{~m}$ \\ Status: National Reserve \\ Category: Congregations
}

\section{Site description}

Kiunca Marine National Reserve (4azetted in 147ty incoporates a chain obout 50 alcarengs of

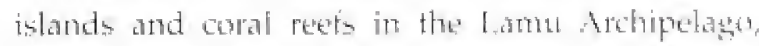
running for some bo $\mathrm{km}$ parallel to the coustline off

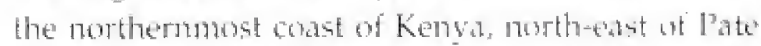
Island Componed of old, eroded coral, the ishand. mainly lie around $2 \mathrm{~km}$ offshore, and insthore of the fringing reet: they sary in size from a tew husdred square metres to log ha or more. Their stalls rise sheer from the surrounding seabed and ate usually d $e^{2} e^{2} p l y$ undercut on the landwat side: The langet islands and the more thellered inner islands are covered witl low, tangled, thom vegetation, including grass, aloes and creepers. The smatl outer istands, conal blocks up to 18 m high, prowide nest sites for seabirds. They consist of bare, sharpeditest spikes and ridges of coral on the seaward side, with only a little stragsling begetation, such as saliopribl and the succulent somsereria. On the landwart wite there is mote vegetation, including stumed, thumy

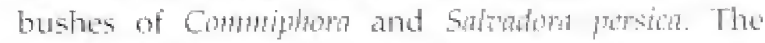
cosst itself has sandy beaches, some with mangtoper swamps, and mangroses ofterm grom in the shelter of the targer islatnds. Rainfatl is around zolo mm pret vear. Part of the mainland contline is protected in the 87,700 ha Doburi National Reserte gareted in 1976 .
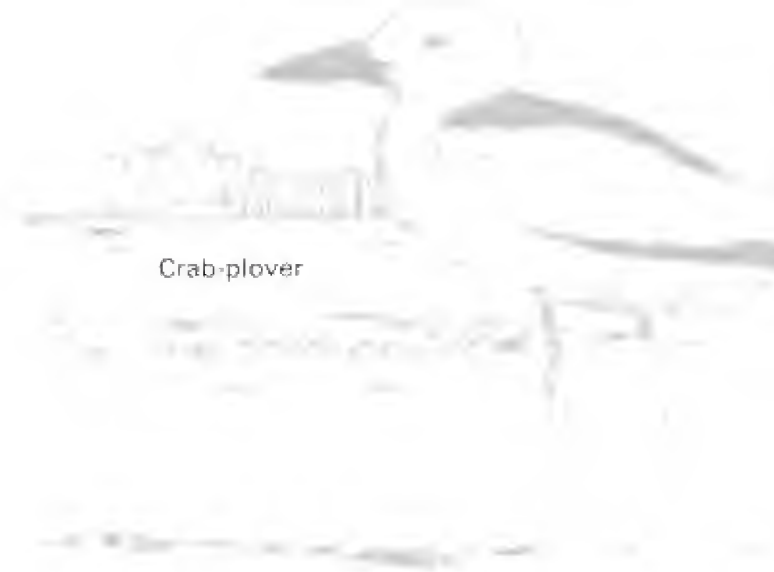

\section{Birds}

Ser box har hey sperem. The small onter islands are rich in seabirds. As well as Rosedte Ferns in internationally important numbers, species nestime herre inclute Soroty Gidl, White-cheeked Tern, Bridled Tern and Brown Nodety (Fogden 1964. Britton A Brown 1971). The seabirds nest from Itune to September, when pough ses and strong winds make humber acces to the istands difficult. Ther do not use the larget or more resetaled foland. Crab-plovers (in internationally inportint numbers and other migrant waders frequend the ntore shelered thats and creeks, mainly from Augest to April.

\section{Other wildlife}

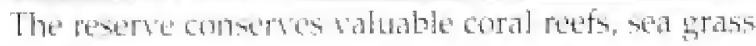
meadows and extensive mangrove forests, with thes attendant biodintersily. Three species of turtes, Cheromin mulas, Eretmothelys imbricata and Lepratoldelys ofitatia, nest on the beaches.

\section{Conservation issues}

The humath population around the reserve is lows and the porm security situation in recent years means that fur peoplex line pormanently in the alea. This, combined witls difficult access, has alon limed the deselopment of eco-tourism. Loat pishermern traditionally collect seabirds eggs when they can.

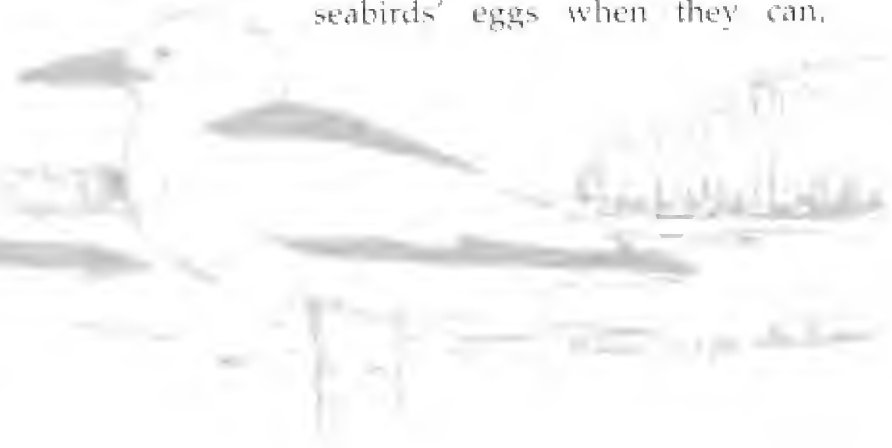




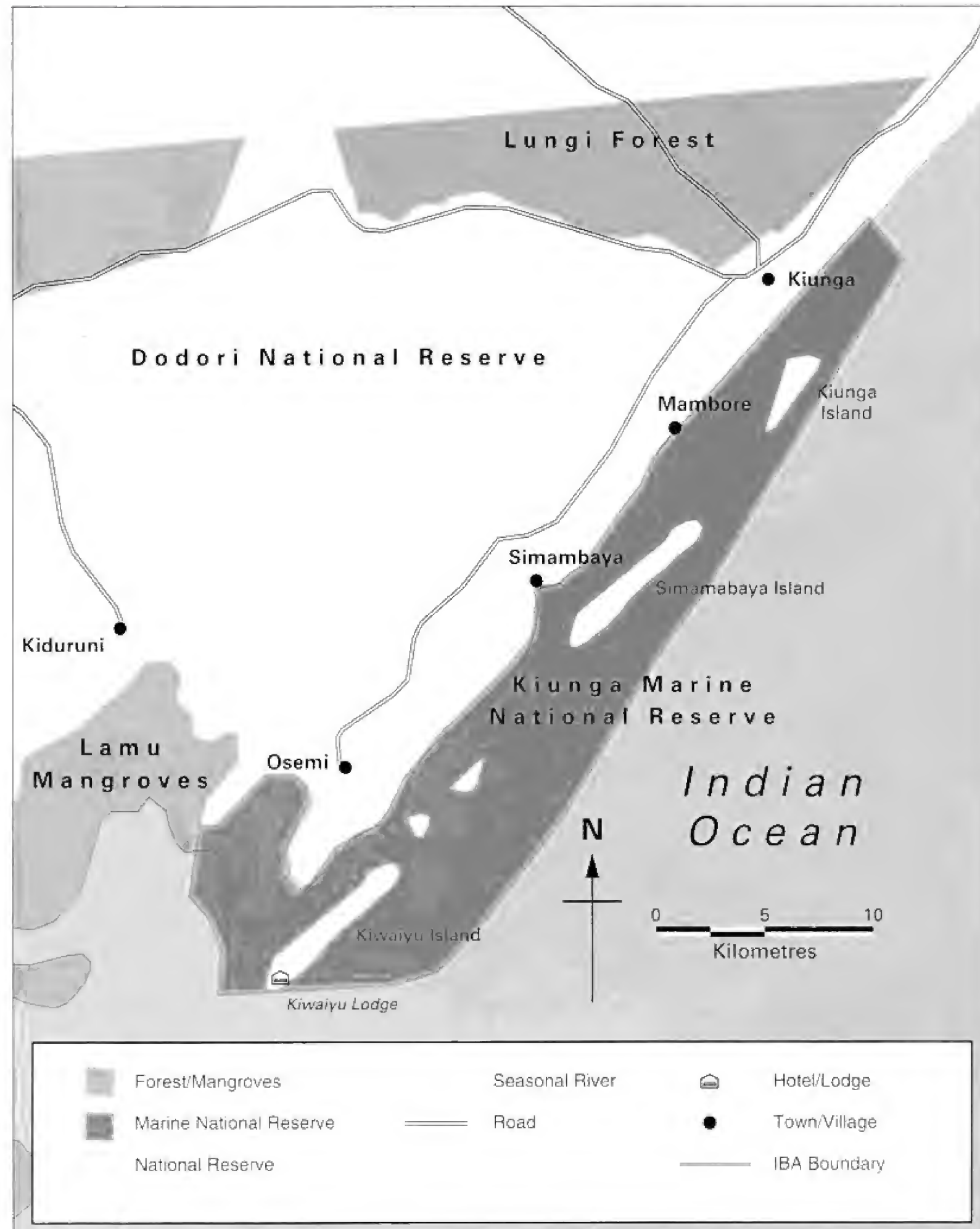

15 Kiunga Marine National Reserve 


\section{Important Bird Areas in Kenya}

\section{Congregatory Species}

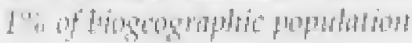

Crab-plover (300)

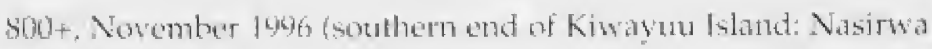
at at, 1048 )

Risente Tern $\{(+1) 0\}$

1,195 pairs an iskets mear Kiunga, 1961 (Fogden, 1964), 5,1000+ pairs, Mlamger wa Hindi, August 1970 (Britton \& Brown. 1971)

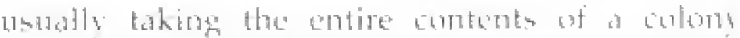

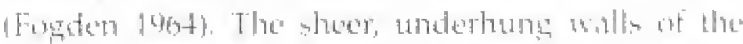

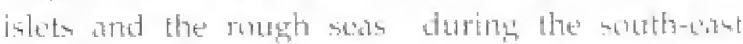

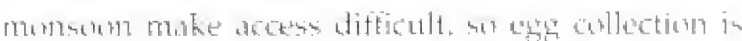

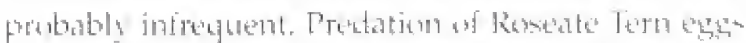

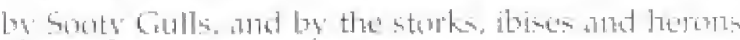

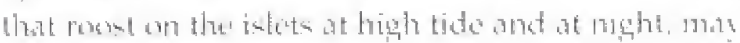

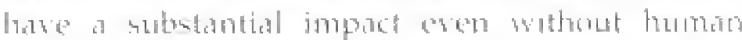

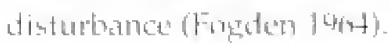

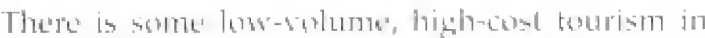

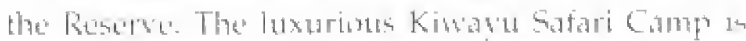
based at Stembend the the minland, and there are

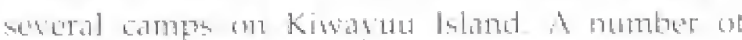
atchaentogical sites on the mainland and matin

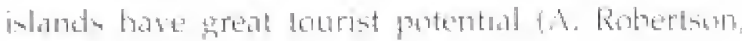
in tith.

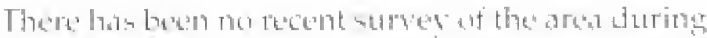

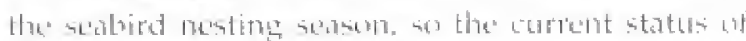

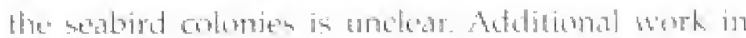
this little-known site is needed.

\section{Further reading}

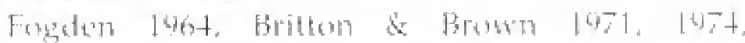

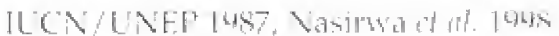




\title{
16 Mida CREEK, WhALE ISLAND And ThE Malindi-WATAMU CoAst
}

\author{
$03^{\circ} 20^{\prime} \mathrm{S}, 40^{\circ} 05^{\prime} \mathrm{E}$ \\ 26,100 ha \\ Altitude: $0-10 \mathrm{~m}$
}

Status: Protected National Park and Reserve; mangrove areas also gazetted as Forest Restrves, and coastal area designated as Biosphere Reserve

Category: Congregations

\section{Site description}

A complex of marine and tidal habitate on Reray a north coast, strotehing from just south of Malindi toms southward to beromet the entrane to Mida Creek. Holvitate include intertidal rock, sand and

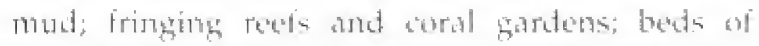

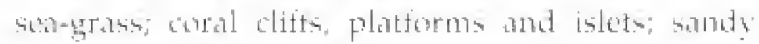
beaches; and mangrone forests blida Creek.

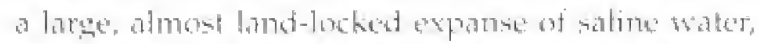

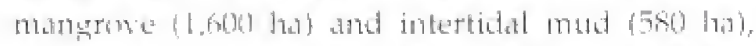
is is the stuthern stetor of the IBA nuar Watamu town and Midas rildige, and protected by the 3,200 ha Whambt Matrine National kemere (gazeted in 1468 ).

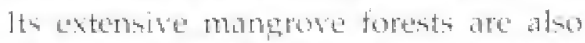
Garetted at forest Reserves, and the extreme westem tip of Mida Crest is part of the Arabuku-5oksthe

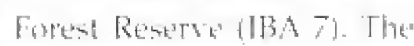
remulinimg part of the"

[BA, aling the

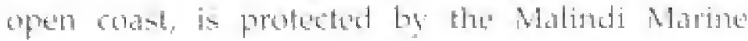

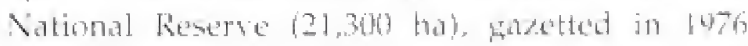
and designated as a Binsphere kemerne in 167\%. Fnclased withon the Reserve are the Watame and

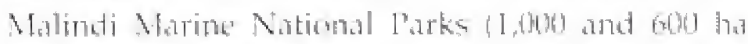

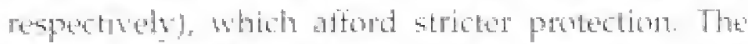

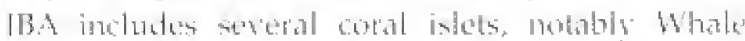

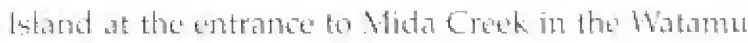
vatine National Park.

\section{Birds}

Sece bux hor key species. Mida Creek is an important pastage and wintering anear for Palaearetic migrant waters, while the coastline and Whale filatat stopport significant beeding and nesting pipulations of terpas. The populations bit

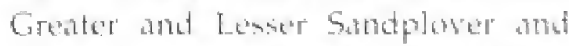
Crab-plasers at Wida Crech are internationally important, and mams

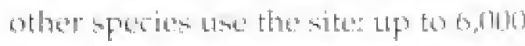
mactere mas be present at any she time (D. I. Pearson, ne list.).

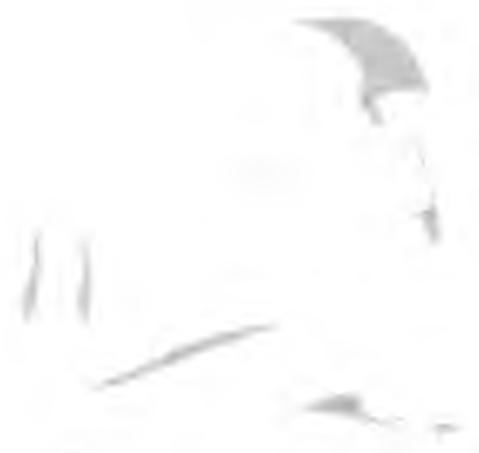

Saunders"s Tern 


\section{Important Bird Areas in Kenya}

The areek is atse of signaficant feading aren for Western Reef Herom. Leser Cresked Fern and Romente Tern. Common misant shoredude here indedes Sanderling, Curlew Sandriper, Whimbel,

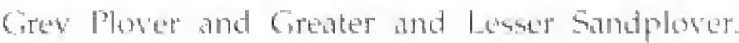
Romeate and Fridled Terns nest on Mhale baland

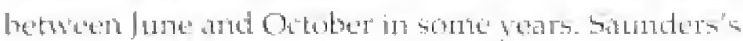
Tern occurs in internationally important numbers along the constine, usually betsing chese to short

\section{Other wilidlife}

Nida Crenk has improtant mangrove foresty, with a

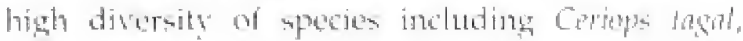

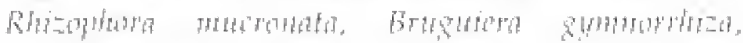

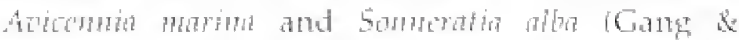
Agatsina 1942). It is a key spawmeng gromed for

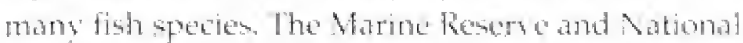
Parks are important for the comedeation of the

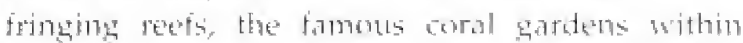

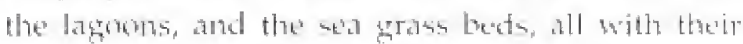
attendank, diverse marine fatuna and blora.

\section{Conservation issues}

The Matindi-Watamul coast is a populan" tourint testination, with numeroum berach hotels, and coral reds ate one of the major attrations. Management of visiturs has been inateruate in the pasta delicate sorals were trampled on by tourists, on smathed by boat anchers. The situation is inprosing through tourist eduction, prosision of moorings, and better policing, Anothere, more insidious, threat to the rexts

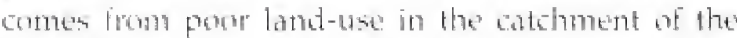
Sobaki Ruete Soil eronitu has greatly inereased ult lowas, which ane deposited in the sea just morth of Nalindt, killing corats or reduciag their groweth.

Finhing, and other tese of marine resources in permitted, under licence, in the Reserves but not

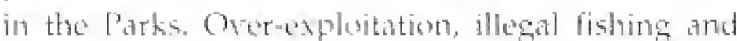
collection of coral and shells femain problems, and are ditficult to controt. Disturbance by tormists visiting the tern colmies on Whale lsland needs to be monitored and resulated, expedalty now that fucal hoted are advertising the nesting terms at an attraction.

Thu' integrity of vid Creek depends on

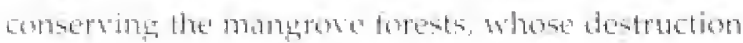
appears to be acrederating $x$ in whe mangrose areas, cuttung for building poles is rife and lingedy uncontrollest. The creek dack a collutemt managentent plan, and is threaterted by alhoabion of

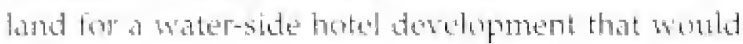
hate a majur ansironmental impat. Nida needs to

\section{Congregatory species}

$1 \%$ ar mow of lrogergaphic population

Crab-ploser (300)

A nom-breeding visibor, present mainly August-April. Mida Creek max.: B00; mean of five counts, Decmber-April, 540 (Seys as al. 1945)

Lesser Sandplover (250)

Mida Creek max: 1,506) (Geys at al. 1995)

Greater Sandplover (650)

Mida Creck max: 1,250 (Soys at al. 1995)

Roseate Tern ( 400$)$

1,500 pairs nesting on Whale Island, 1967 (Britton \& Brown 1971); several hundred pairs frequently nest hert, though not each year (Zimmerman (1) 19. 1996)

Satunaters : Tern (200)

5,700 in Malindi Marine National Resere, February 1495 (Nasirwa of a! 1995a)

\section{Regionally-threatened species}

Great Eyret vulnerable
Occurs in small and variable numbers (max. 15 Mida Creek, five Malindi Marine National Reserve: Sers of al. 1995.

Vasirwa ef al. 1995b) 


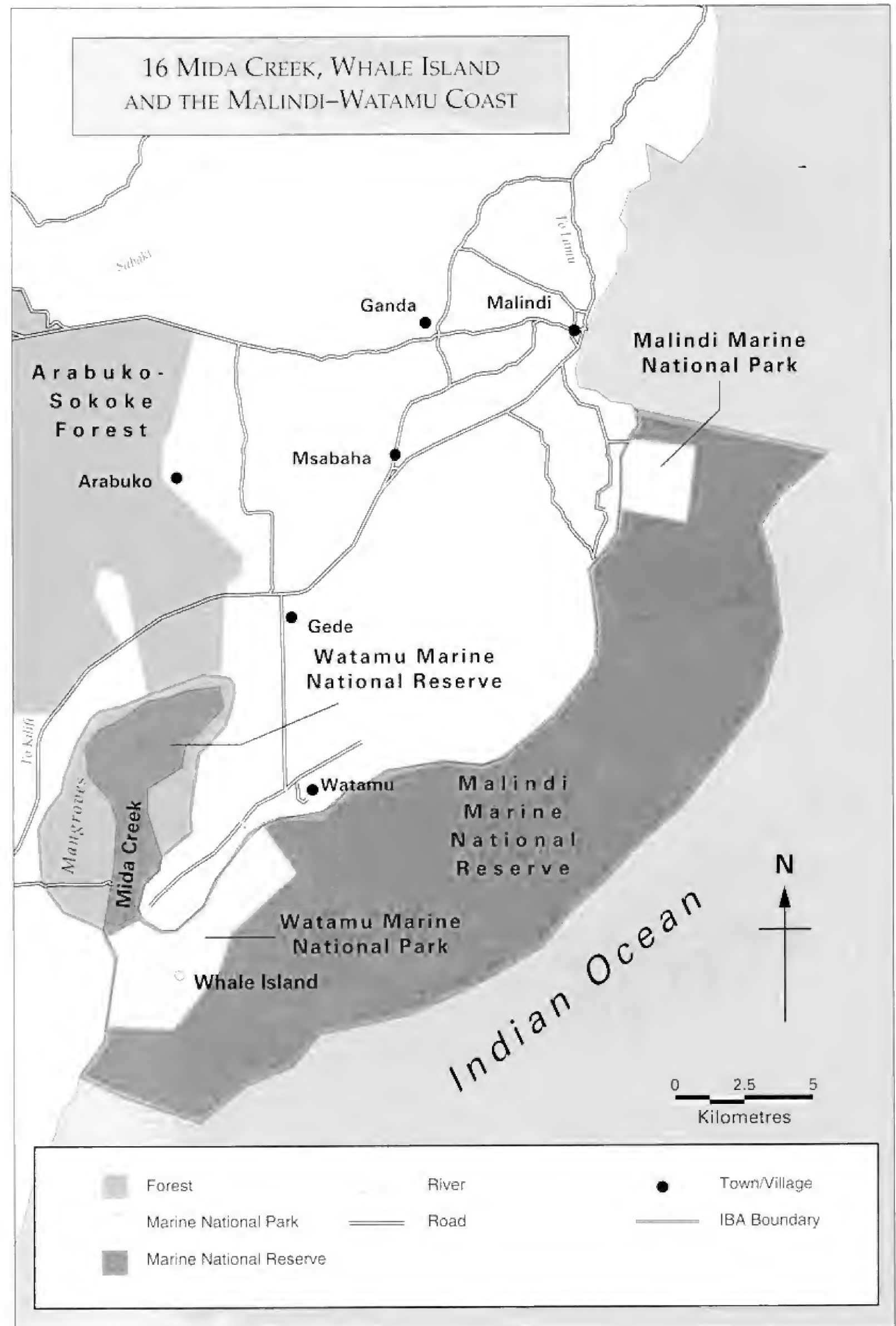




\section{Important Bird Areas in Kenya}

Whimbles

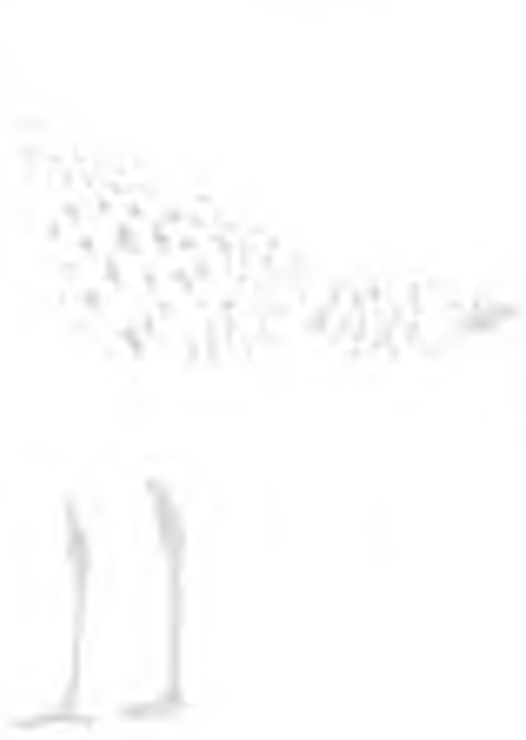

tas incorgorated into conservation planning fur thas entire comples of protected areas a comprehersise and integrated plan for use and connersation sould

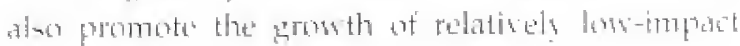

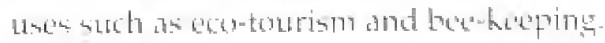

\section{Further reading}

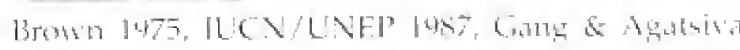

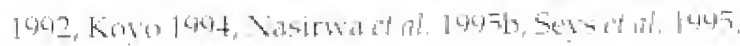

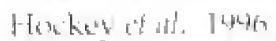




\title{
17 Marenji Forest
}

\author{
$04^{\circ} 29^{\prime} \mathrm{S}, 39^{\circ} 14^{\prime} \mathrm{E}$, Coast l'rovince, Kwale District \\ 1,520 ha $[6,1,480$ ha forest) \\ Altitude: 30)-160 m \\ Status: Forest Reserves \\ Catcgories: Globally-threatened species, restricted-range species, \\ East African Coast biome species
}

\section{Site descriptian}

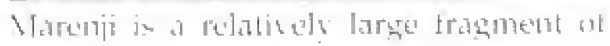

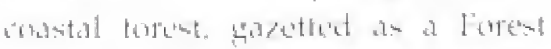

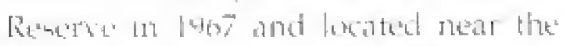

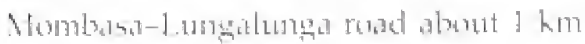

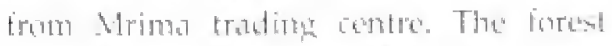

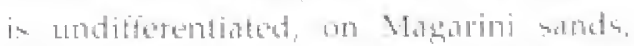

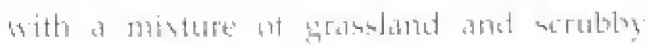

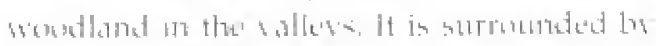

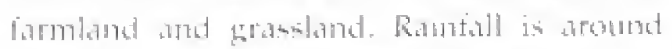

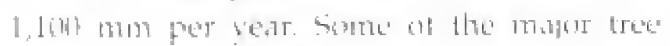

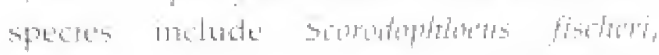

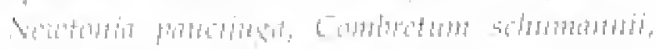

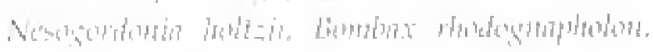

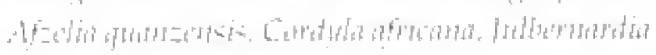

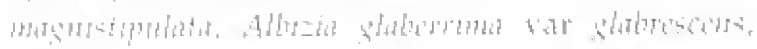

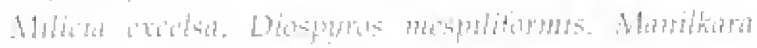

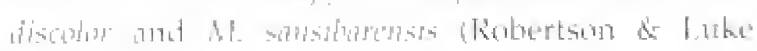
[4.45).

\section{Birds}

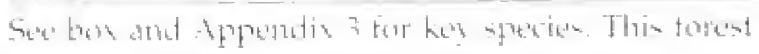

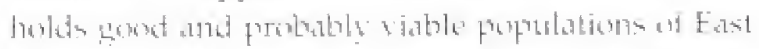

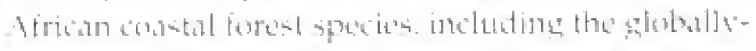

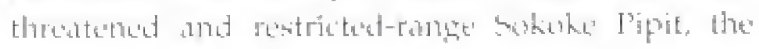

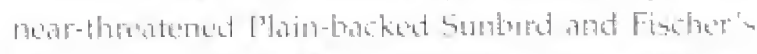

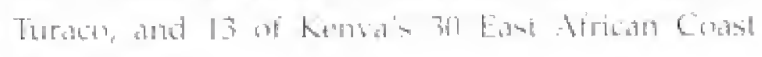

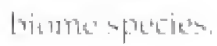

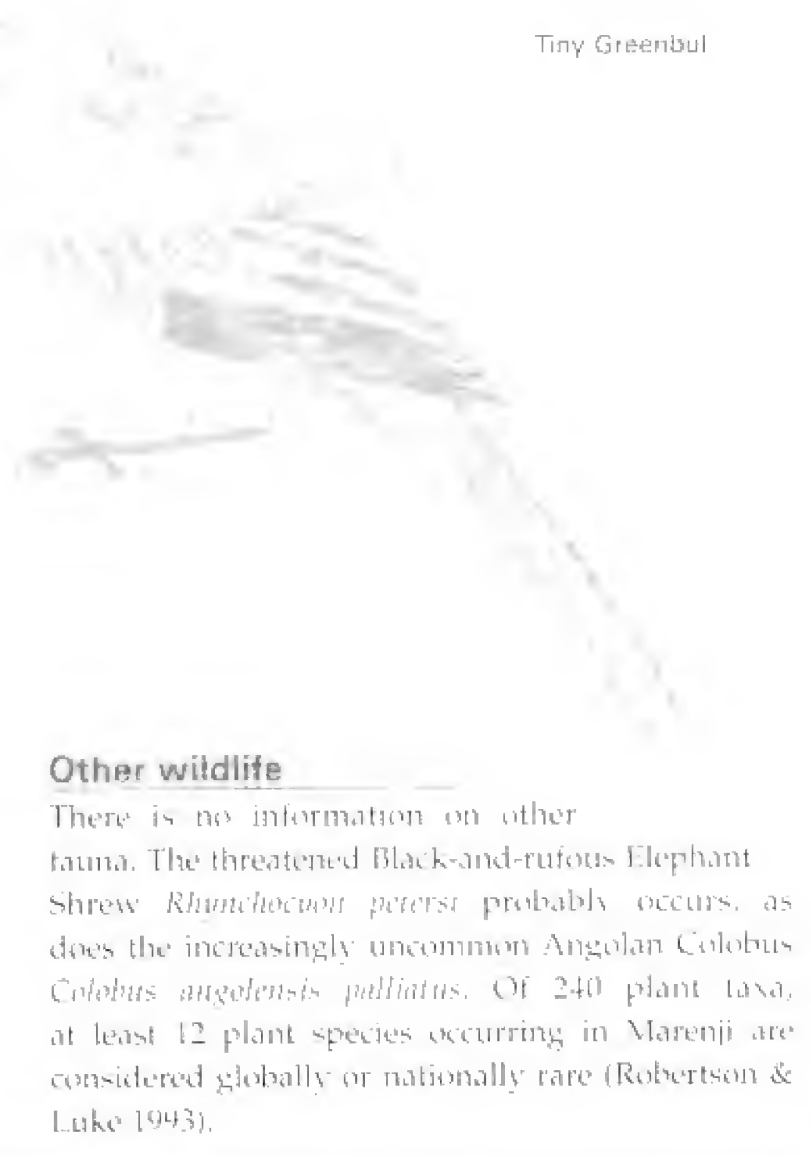

\section{Globally-threatened species}

"Fischer's Turaco
"Sokoke Pifrit

[Pain-backed Sunbird

Near-thatatened
Waiyaki (1995)

Recorded by Whigki (1965). The poputhtion size is not known, that this ts likely to be an important site for this species

Wriyaki (1995)

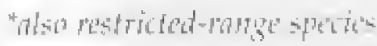

\section{Regionally-threatened species}




\section{Conservation issues}

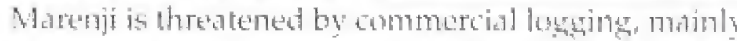

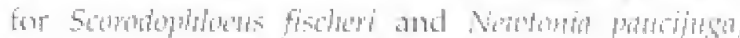
which has been heary and dambing to the seruchume of the torest. Tree poaching (especially of Combrim

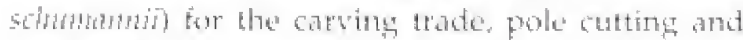
chareoal burnimg are also prowlent, especially atoth the eastern and south-eastern boundaries (Robertson

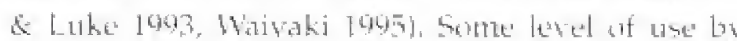

local people is probalbly sustatinble, but this shoutd be controlled and based on a manamement plan that

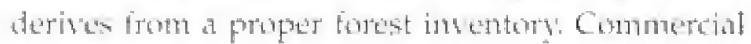

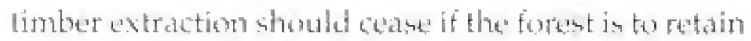
its biodinersity values.

\section{Further reading}

Robertion \& Luke 14y3. Allingwa at at. in press. Waryaki \& Benmun, in prest

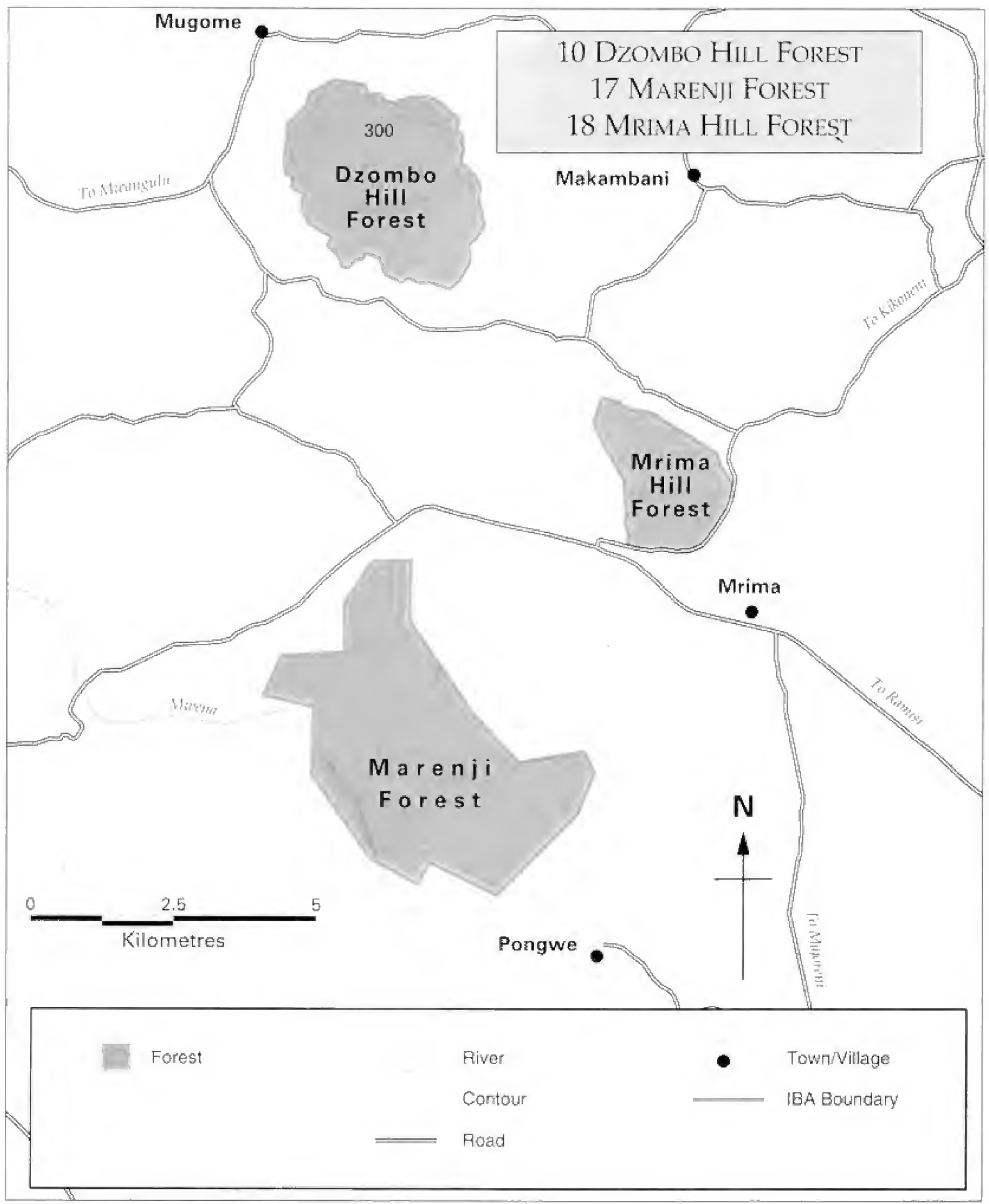




\title{
18 Mrima Hill Forest
}

\author{
$04^{\circ} 28^{\prime} \mathrm{S}, 39^{\circ} 16^{\prime} \mathrm{E}$, Coast Province, Kwale District (sex map in sile accom 17) \\ 250 ha \\ Altitude: $100-300 \mathrm{~m}$ \\ Status: Forest Reserve, Nature Reserve and National Montment \\ Categories: Globally-threatened species, East African Coast biome species
}

\section{Site description}

This IBA combints of wet coastal torest on at mall hill rising trome the cosintal plain some on km

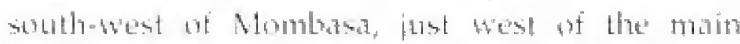

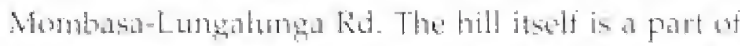

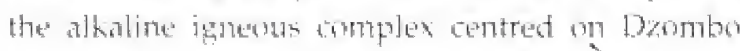
Hill (IBA lon and is knowen to hase signiftcant

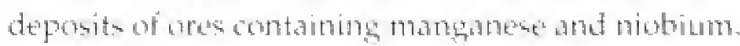
Nrima has been proppected oner several bimes by gevologists and there are many deep lest hollen,

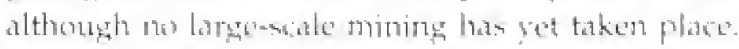

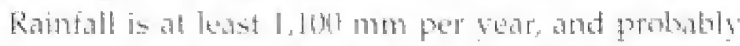

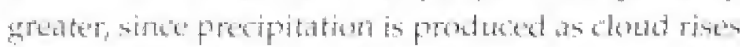
over the slopes during the south-exst monsoom. 'The' forest is andifierentiatad, with exoptional plath spertes diversing harge trees include Cumbretsm sohtomanti.

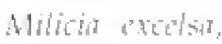
Terminalia

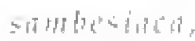

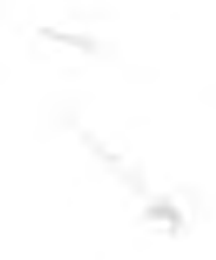

Plain-backed Sunbird

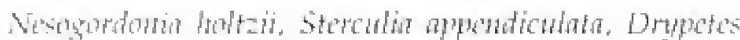

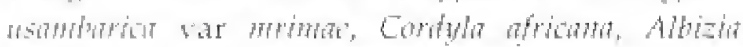

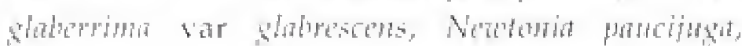

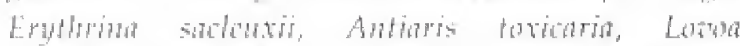

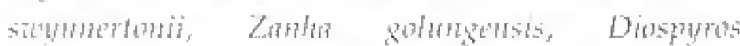

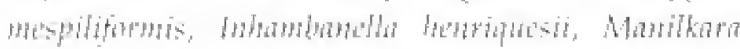

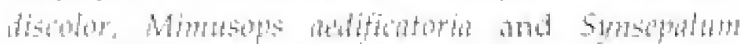
brevige (Robertson \& Luke 1943).

Mrimi Forest Regerse was goneted in 1901, and the site wis made a strict Nature Renerse under the Forests ate in the early 1480s. Nrime Hill is also a Kaya, recogriated by the site's yazettement as Mrima Hiil Sncred Grose National Nonument in 1992. The Kavas are relict patches of forest that ance wheltered the fortified villages of the Nijikenda people dirs

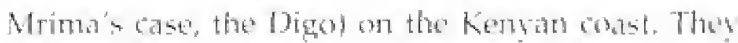
have spiritual and ceremenial significande and are customarily protected by a Council of Fders

\section{Birds}

Sec box and Apperatix 3 for key species, Mrimat holds a non-breeditig population of the glotiollyHoratened Spatted Ground Thrush, and stpports a rich avifatma sharacteristio of the Fast African

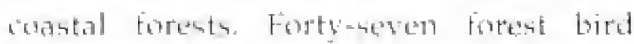
species ane recorded, including 11 us

Kenyas 30 African Fast Coast biome species. White-starred labin and Orange Gronet Thrush are known from Mrima (Britton ctal. 1980) but no other 


\title{
Important Bird Areas in Kenya
}

\author{
Globally-threatened species \\ "Fischer's Turaco \\ Spotted Ground Thrush \\ Enctangered \\ Plain-backed Sunbird \\ "also restrictet-range spectios \\ Near-threatenct Firly common \\ Recorded by Britton at al. (1980) and \\ recently by Waiyaki (1995); may be a \\ significant site for this non-biresding \\ visitor \\ Fairly common \\ Near-threatened
}

\section{Regionally-threatened species}

1.ittle Yellow Flycatcher Vulnerable Status unkınown

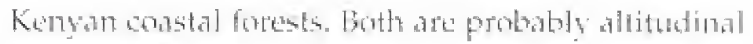
migrants.

\section{Other wildlife}

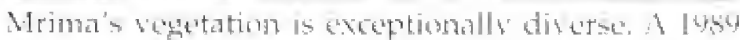
expedition recorded aser 270 taxa, indating 25 that

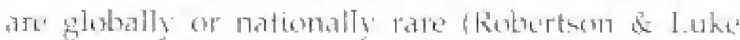

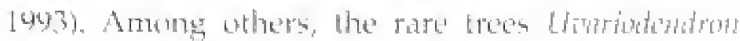

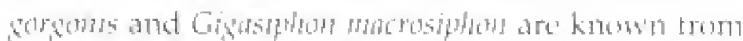
thas site, though the latter was nust re-tocated in a

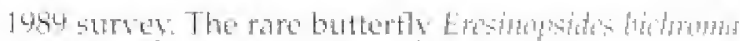
also aceurs (Larben 1941). Mammals inchuste the

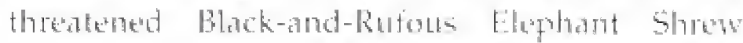

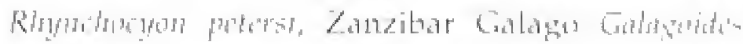

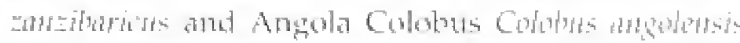

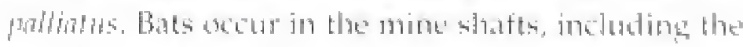
rare and lacalised Gerater Collared Fruil bat Mwometeria roticto

\section{Conservation issues}

Despite its status an a strict Nature Reserve, and now

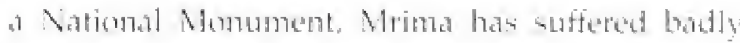
hom selectise logring and pole cutting. Numerom

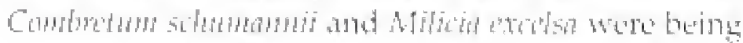

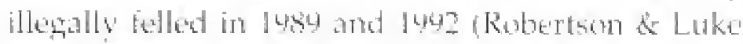

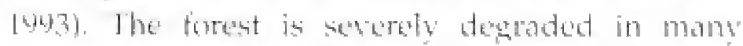

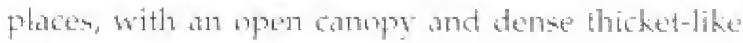

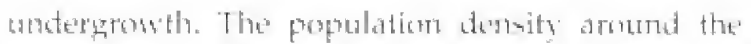
hill is hysho and there has been stome encrobchment

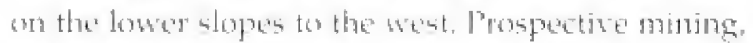

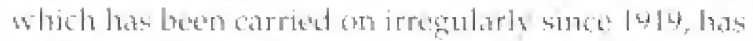
alson affocted the fortst structure. Himes is now

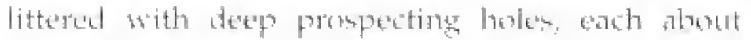
half a metre in diameler. Thatigh some of these

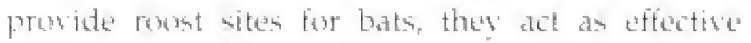
piltall traps tor wother vertebrates finduding biolomists!')

The main threat to the forest pemanes the persibility of intensise mining for manganese a d

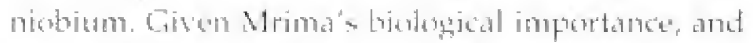
its signitionace ar a Kaya (or 'bacred forest') to the'

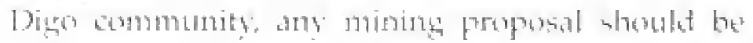

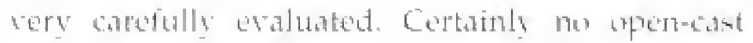
miniong should be andentenand, an this rould be extremety destructive to the forest. In the meantime.

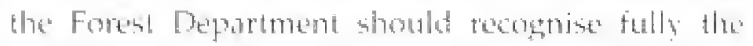
cunseration importance of this site, and work with the Vatiomal Museums Coast Foreat Comertation

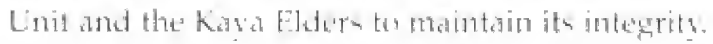

\section{Further reading}

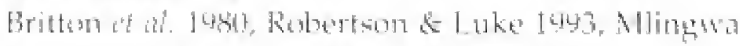

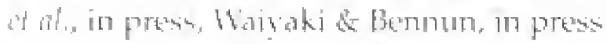




\title{
19 S A B AKi River Mouth
}

\author{
$03^{\circ} 09^{\prime} \mathrm{S}, 40^{\circ} 08^{\prime} \mathrm{E}$, Coast Province, Malindi District \\ Area undefined: c, 200 ha \\ Altitude: $0-5 \mathrm{~m}$ \\ Status: Unprotected \\ Category: Congregations
}

\section{Site description}

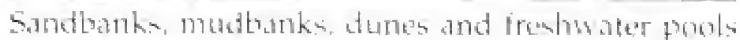

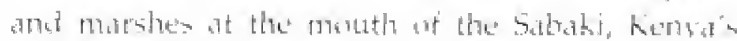

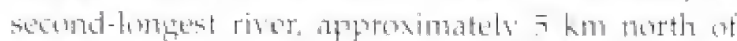

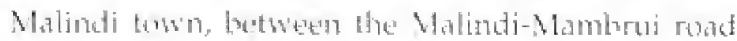
pridge and the sen. The state and sire of the eatuary

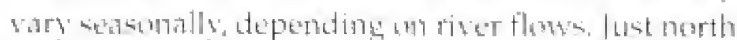

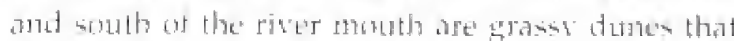

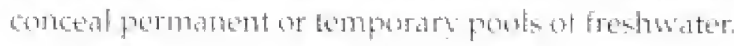

\section{Birds}

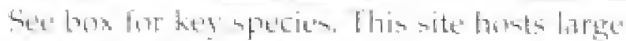

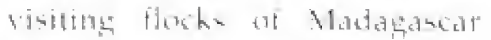

Pratincols, and 1 an ampertant

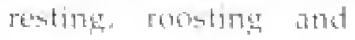

fordifis soumat tor galls

and terms. Gived

numbers on

Patacatetic

waterera alon

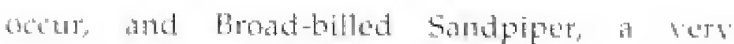
unommon biat in Kenya, winters here in flocks at up to st. The near-thederned, restricted-range Malindi d'ipit is resident in and around the grassy dumes.

\section{Other wildlife}

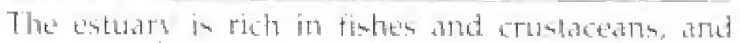
supports an important fishery:

\section{Conservation issues}

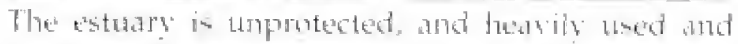
dinturbed by papple, althesugh there is tittle dited

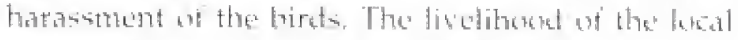

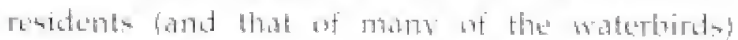

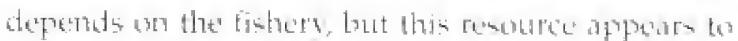

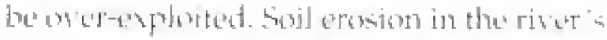
catchonent is incrabine silt lonts in the Sathaki, pat the ethat of thes an the

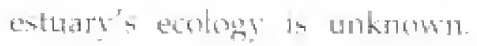


Important Bird Areas in Kenya

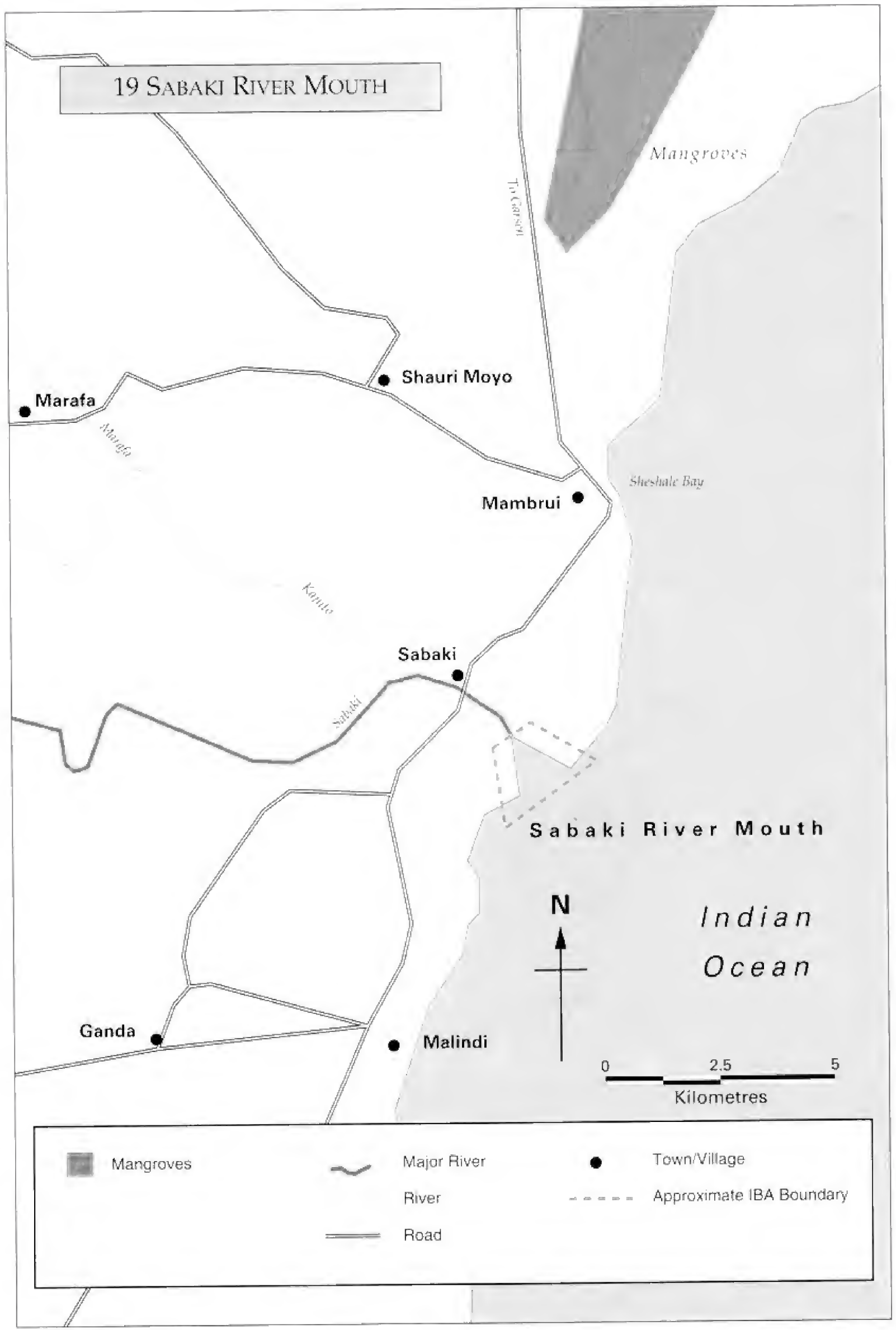




\title{
19 Sabaki River Mouth
}

\author{
Congregatory species

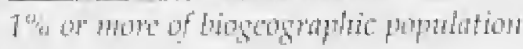 \\ Madagascar Pratincole (50) Regularly up to 2,500; maximum 9-10,000 in 1978 (more than the current \\ population estimate!) (1-ewis \& Poneroy 1989) \\ Sooty Gull $(400)$ \\ Sanders's Tern (200) Max: 900 \\ Lesser Crested fern (250) Max: 270

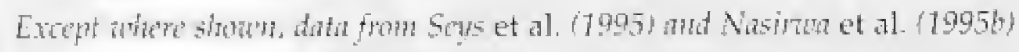

\section{Regionally-threatened species}

Great Egret Vulnerable?

African Skimmer

Vulnerables

Usually present in small numbers

Regular -isitor, mainly August-March (Lewis \& Pomeroy 1989)

The river mouth is at grteat attraction tobordwatehers.

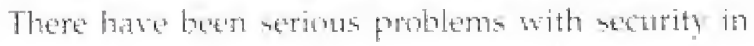

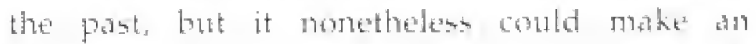

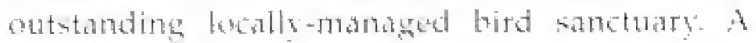
reacarch centre is being ate up by Mui University an the sath batth, between the fout bridge and the river mouth.

\section{Further reading}

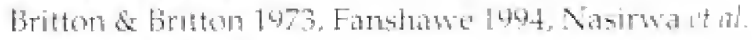

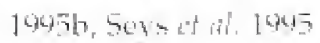




\title{
20 Shimba Hills
}

\author{
$4^{\circ} 15^{\prime}$ S, 39 $25^{\prime} \mathrm{E}$, Coast Province, Kwale District \\ $21,740$ ha (c. 9,500 ha forested $)$ \\ Altitude: $120-450 \mathrm{~m}$ \\ Status: National Reserve and Forest Reserve \\ Categories: Globally-threatened species, restricted-range species, \\ East African Coast biome species
}

\section{Site description}

The Shimba Hills are a dinceted phatean that sseends sleeply trom the echstal plains, some $30 \mathrm{~km}$

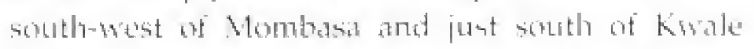
town. The surrounding exarpmext rises from around 120 no clexation to c. 300 m acrose the bulk of the plateau, and as high as $454 \mathrm{~m}$ at Varare alut

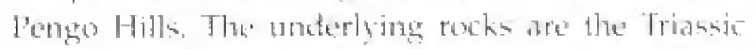

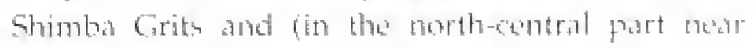

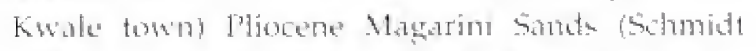

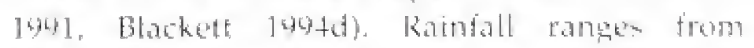
you-1,200 mom per vear, and risere flowing trom

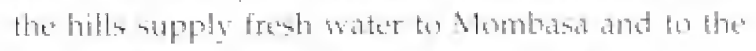

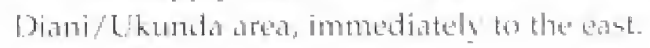

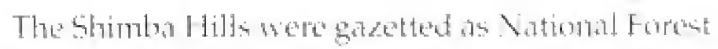

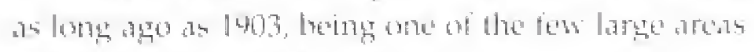
on the south coast that was still well horested. Gitassiand areas were incorforated in Ib2t, and seteral sillomequent

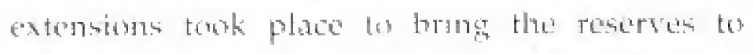

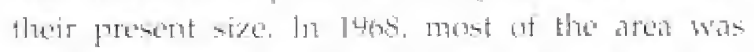
domblengerted as the Shimba Hills National

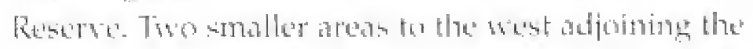
National Rearse and almont entigedy forested,

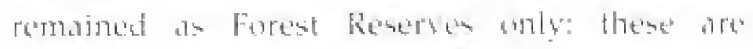
Nangant Nith TR (1.110 hal) and Mkongani Weme FR $\{1,370$ hat

The hith hate a beterogerachers mosaic of

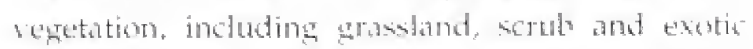
plantations as welt as corest. Blackets (109tad)

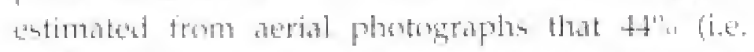

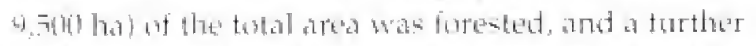

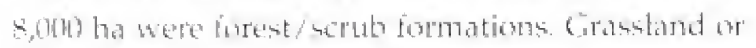
pritsind/scrub covered 3,40) ha. the remainder heing plantations (old ha) and other cover. The hill

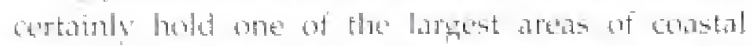

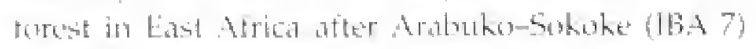

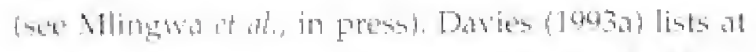

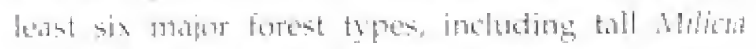

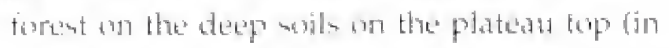

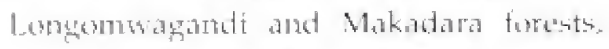
and near kwale town', and on the western escarpment:

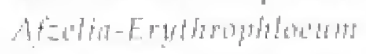
forest, towering muth of the castern and southern

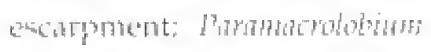
torest on particularly step scapp shopes to both east and wast;

Green-headed Oriole 







\section{Important Bird Areas in Kenya}

and Minnibara-combretwm forest in the lower,

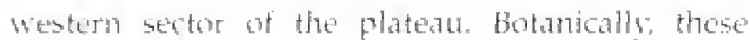
hatuitat assegiations alle rather more compoles, and this dassibation mav be an oversimplification (Q) luke, in ith). The bigrest single pateh of farese in in the south-western sector, including Mkongami North and West. Funther east and north, the forest breaks up intor a complex mosaic intersperaed with sorut and grassland. Verv few forest patches atte

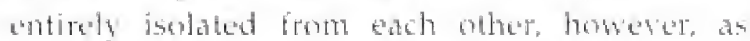
corridors of forest or forest/scrub fortmations remain

At loast two Kayas, Kunate and Longomwagandi, are situated within the National Reserve. (The Kast forest have spiritual and cerenonial simnibicance to the Mijikenda people of the Kempa conat.) is fenced elephant ontribor comnets the shimba Hillo with Mwaltganji Forest Resurve to the north.
Birds

See box and Appendis 3 for key speries shimber Hillo has a rich coastal ferest bird fauma, jneluding

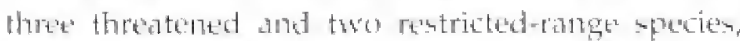
and holds 18 of Kentad 30 East Codst biome species. The relativef lare area of torest means that papulation of raset of these species are likely to be viable. In late March and early April, spectacular concentrations of certain Palaeactic nigranto sucth

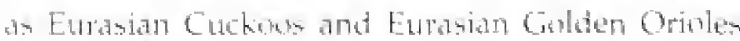

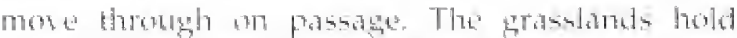

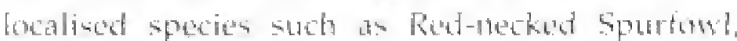
Croaking Cisticala and Zanzibar Red Brshop. Tlu'se

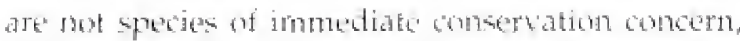

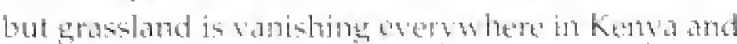
the habitat protected in the Shing a fillo is saluable.

\section{Other wild dife}

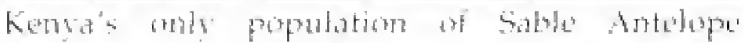

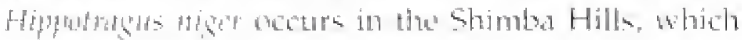

\section{Globally-threatened species}

Southern Banded Snake Eagle Near-threatened

*Fischer's Turaco

Nent-threaterned

Spatted Ground Thrush

Endangered

*Sokoke P'ipit

Vulnerable

East Coast Akalat

Vultertable

Plain-backed Sunbirt

Near-threatered

*also restricted-rimger species

\section{Regionally-threatened species}

Avres's Hawk Eagle

African Crowned Eagle

Little Yellow Flycatcher

African Pitta

Vulnerable

Vulnerable

Vulnerable

Uluguru Violet-backed Sunbird Vulnerable
Fairly commong resident

Conmon in taller forest

Recorded in Nkmmani (Benmun \& Waiyaki 1902e)

Recorded in Mkongani (Bennun \& Waivaki 1992t)

Patchily distributed, in tall closed-canopy forkst. Known from Mkongani (Bennun \& Waibaki 1992e; densities estimated as 0.4 territories/ha by Nemeth 1996); from Longomwagandi, where fairly common in 1992 (Bennum \& Waitaki 1992e) but wot relacated in 1996 (Nemeth 1996); and from Makadara (Nemeth 1996).

Fairly common in closed-canopy forest
Recorded, but status unknown Several pairs probataly resident Fairly common in closed-canopy forest Rarely-recorded non-breeding visitor Probably the Kenvan stronghtuda of this little-known species 


\section{Shimba Hills}

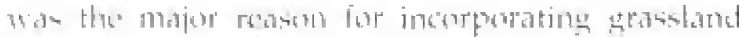

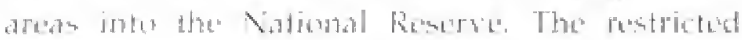

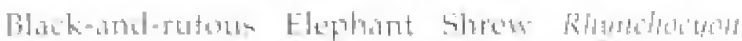

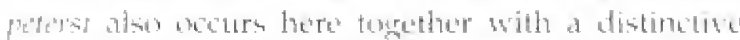

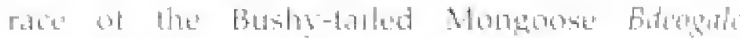

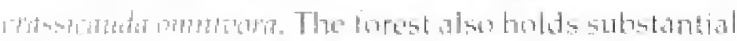

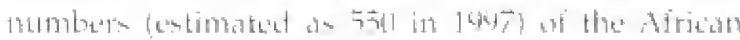

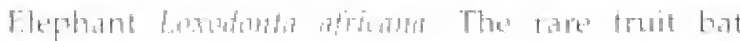

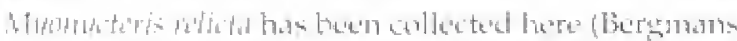

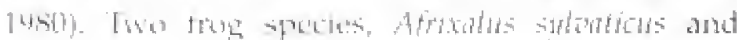

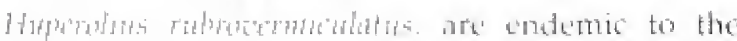
shimbar Hille forests and are helleved to be

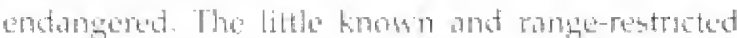

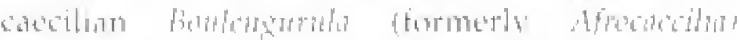

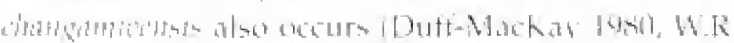

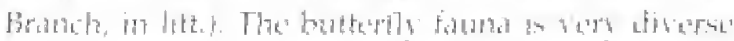

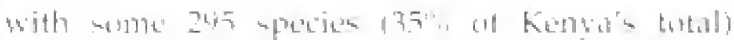

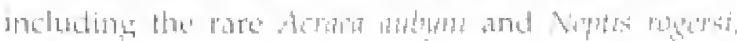

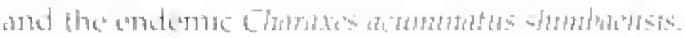

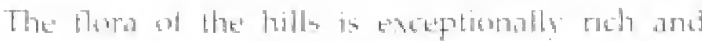

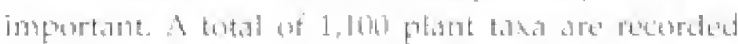

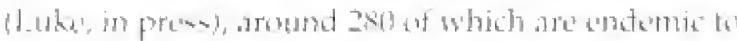

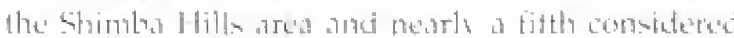

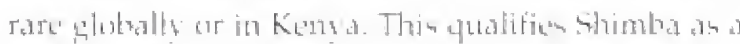

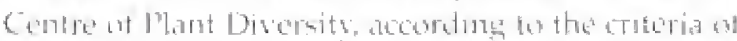

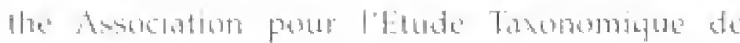

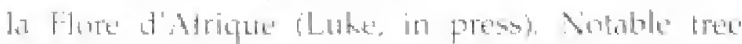

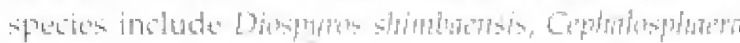

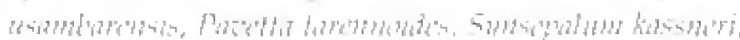

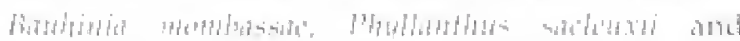

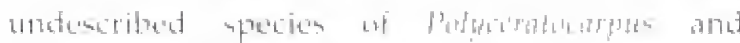

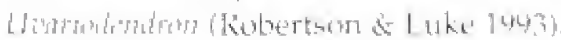

\section{Conservation issues}

Lihe wher andal borads, the shombal Hills

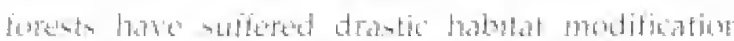

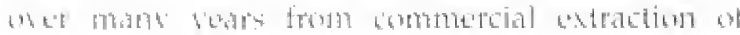

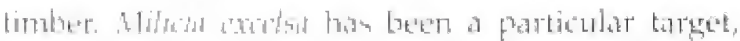

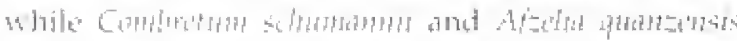
have been heavily expluited in the drier formet

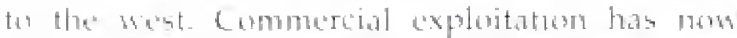

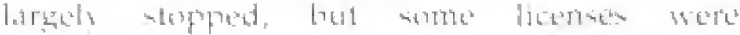

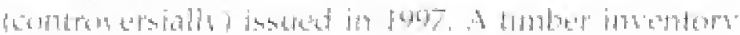

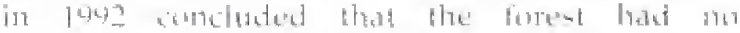

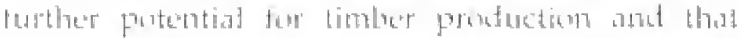

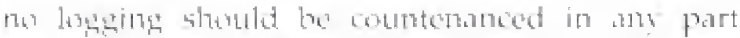

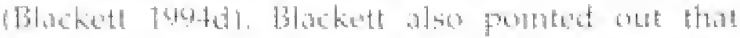

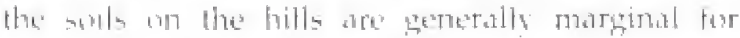

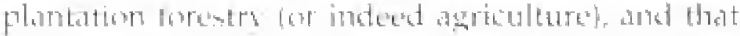

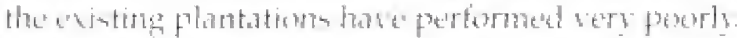

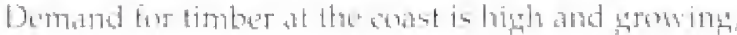

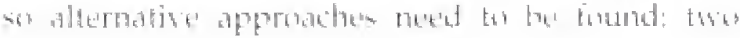

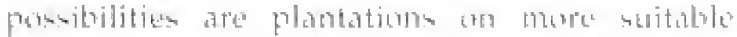
disumed lande and making befter une of e'visting

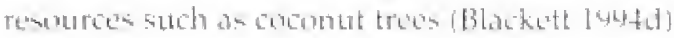

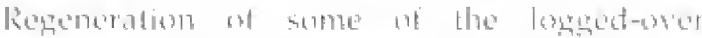

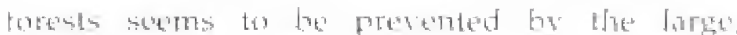

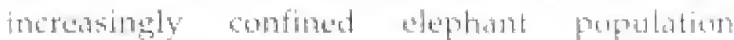

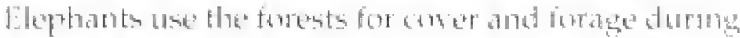

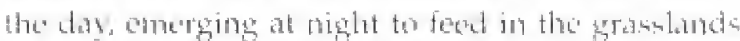
and to raid farms outside the Reserves Sortu forest areas, strch as the Mifror forest in l.tulgommagund and Combetam torest on the southern edige of the

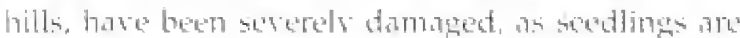

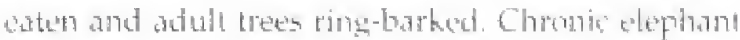

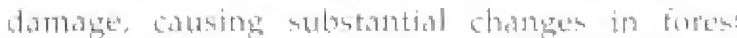

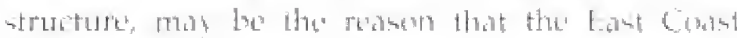

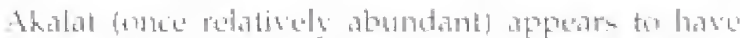

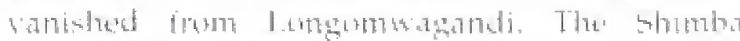

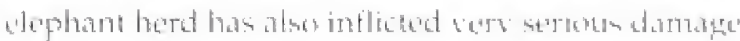
(n) adjacent forests such as kay Lumguma arut

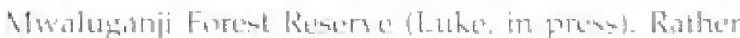

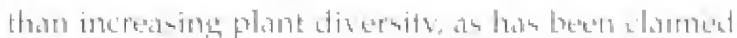

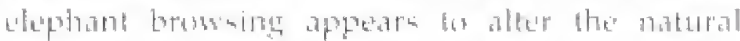

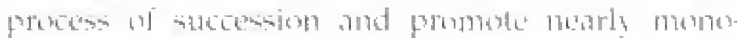

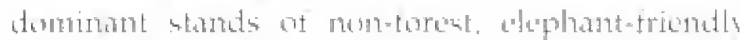

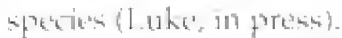

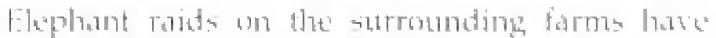

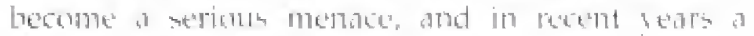

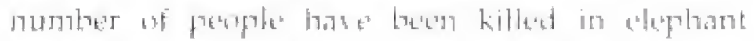

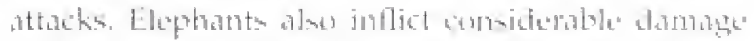

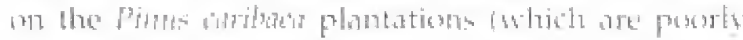

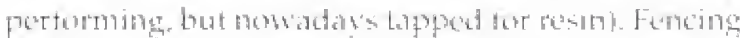

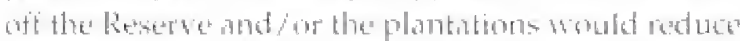

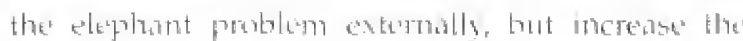
interthal pressute on the fortests trom a contined and

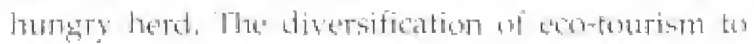
include forest walks, birtwatching shet 560 on. to which shimbs is well-suited, is prescutly impossible

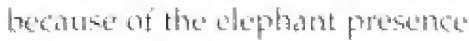

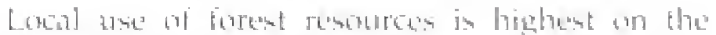

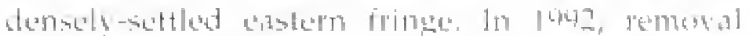

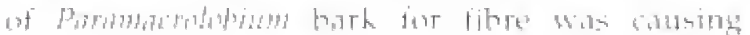

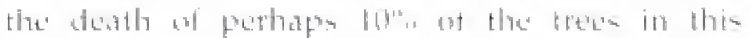

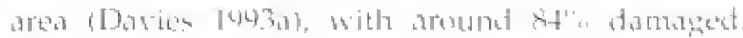

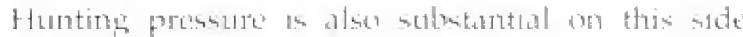
int the" forent.

The combinted arts burning of the plateat

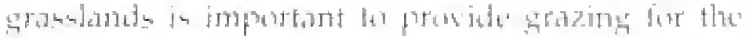

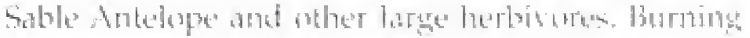

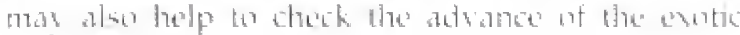




\section{Important Bird Areas in Kenya}

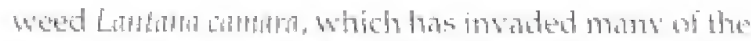

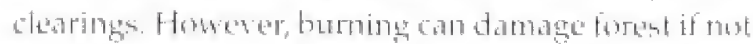
carefulf controlled, and mas inhibit borest regeneration.

The Ehereats facing shimba forint th the need for a

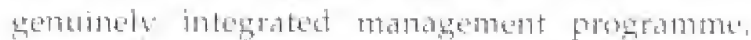
thit deale with forest comberration. eramaland

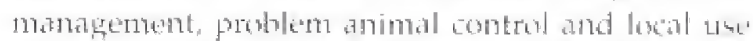
of forest products. The Forest Department and

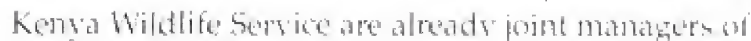

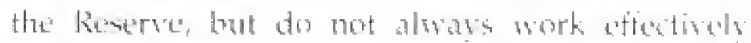
tospether. Some recommendations mates by prexious

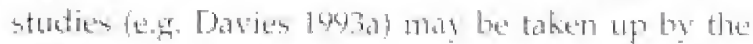
Girz-funded Shemba Hillo Inegrated Natural

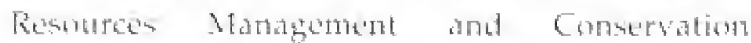

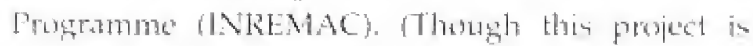

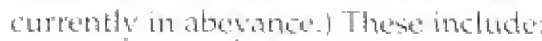

- caretuldy planned elephantefencines of sectemas

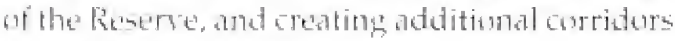

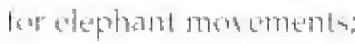

- gascotement af Nature Reserves in 1.angonwasandi and bakadra, perhaps as the aore of a well destined protection rone:

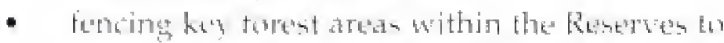

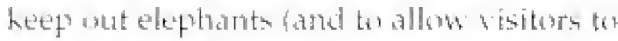
walk and louk at plants, butterflies and birast:

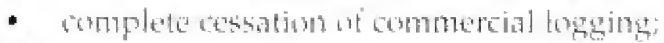

- doar-felling of the current plantations, allowing netural vegetation to regenerate, and a thift to agroforestry and forentro extengion on barms arotind the foresti

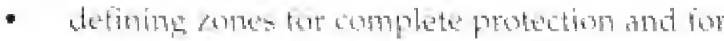
antrofled firest use, and ensuring that bontrols are appropriate and etfectise.

\section{Further reading}

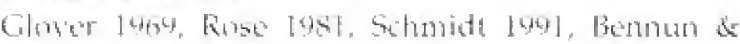
Wainal f \& Luke 14ts, Nemethe guks, Luke, in press, Nlingwa

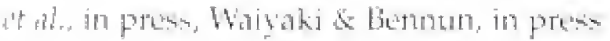

Ulugur U Violet-backed Sunbird 


\title{
21 Taita Hills Forests
}

\author{
$3^{\circ} 25^{\prime} \mathrm{S}, 38^{\circ} 20^{\prime} \mathrm{E}$, Coast Province, Taita-Tavesta District \\ c. 400 ha
}

Altitude: $1,350-2,228 \mathrm{~m}$

Status: Guzetted or proposed Forest Reserves

Categaries: Globally-threatened species, restricted-range species

\section{Site description}

The Taita Hids lies in south-eastern Kenca, south and west af boi tomb, and rise abouptly abose the

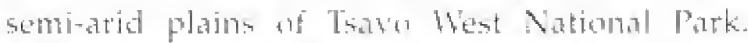

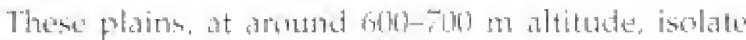

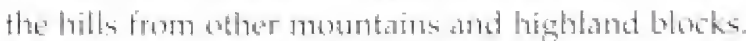

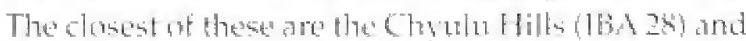

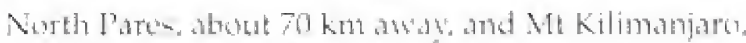

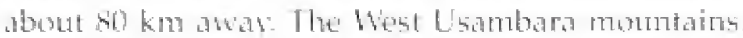
with wheth the Taita Hills have sone botanical affinities, ate atound $120 \mathrm{~km}$ atistent.

The Taita Hills are disided into thee main blocks. Sogalla fill (peak 1, $4=0 \mathrm{~m}$ ), directly south of Voi, in separated from the rest of the hills by the voi liver on the plains. "The main body of the hills. Dabida, lies $25 \mathrm{~km}$ north-1west of vol, including the high peaks

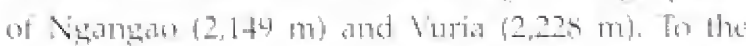

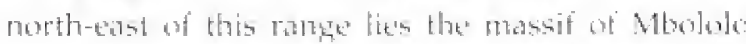

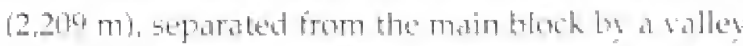

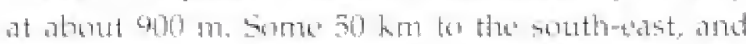
mon incteded within this IBA, lime Mt Kasigat. The



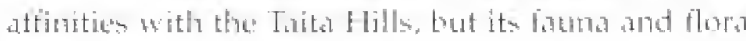
are as vert littlestudied.

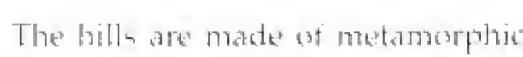

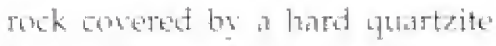
cap. Geologicalty, they are the northermest wutposit of

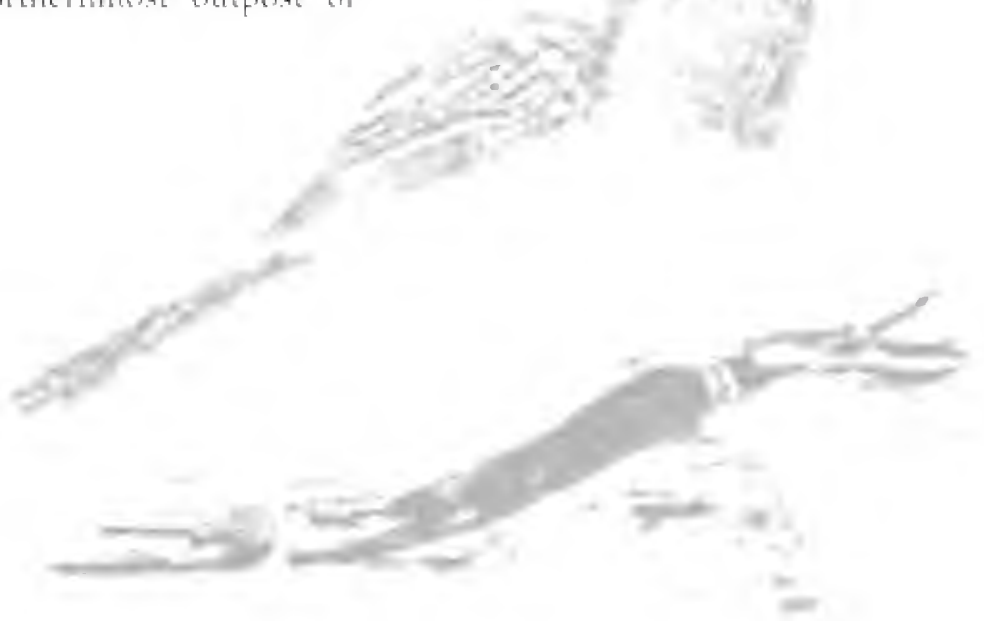

the ancient Eastern Are mountains of Tanzania and Malawi. At the bast of the hitls, rainfall is mly somen

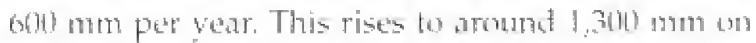

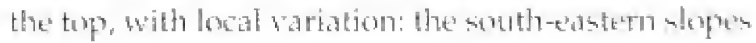

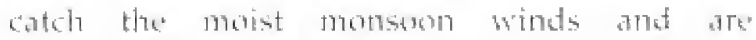

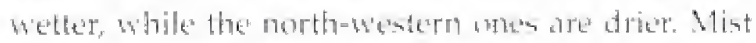
and cloud form throustasut the yent, and there is substantial "ocoult" precipitation, especially within Hinc: firrestion.

Dry bushland rame up the riank of the hills. gising way ather abreapty near the top tis small-hulder caltiwation and remnant patehes of must forest. The area is heavity settled. The torth porsulation is around 250,000 , and densities reacth 1.400 people per $\mathrm{km}^{2}$ in places. Cultisation in intensive, with maine the mest conspicusus crop.

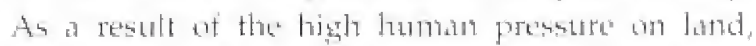

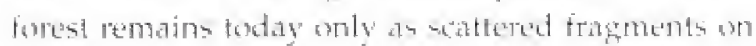

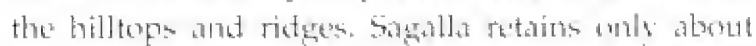

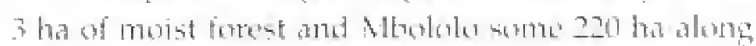

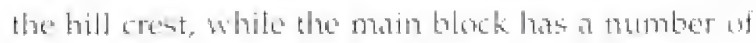
tims remonats, ancluding Fururu (12 has), Nhacheral

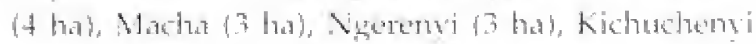

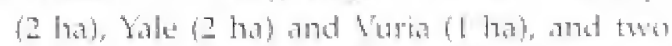

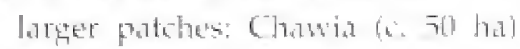
and Vyangaco ic. 92 hil) (L. Lens, in Mat.; areas based on mapping carred went in 1997). The" garettement of all these sites as Forest Rescruces has besa approsed by Wumatany County Council for many ytars. Howerer, while a number ab smaller patches (incluting plantation

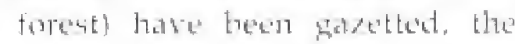
main blocks to bugallat

$$
\text { Taita Thrush }
$$




\section{Important Bird Areas in Kenya}

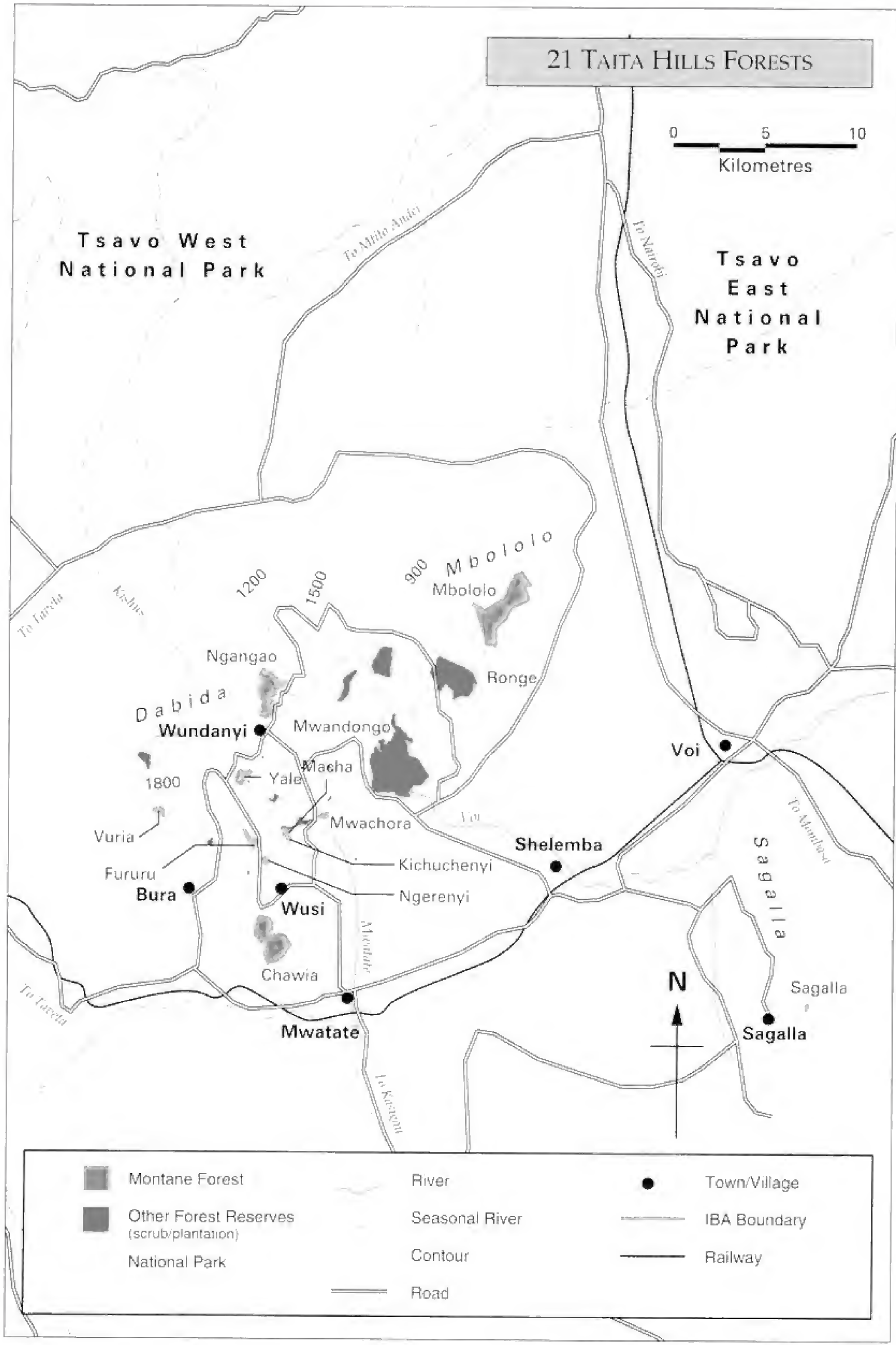




\section{Taita Hills Forests}

Chawia, Nyaggan and Nboloto though managed by the Forest Department) still lo.in met.

All the forsaty hate been longed oner for saluable timber trees, and substontial portions lame been

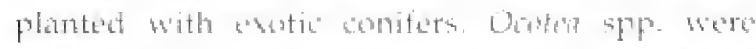
ones common in the loresen, but have bed alingst

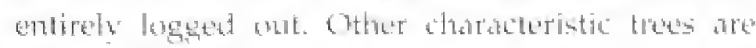

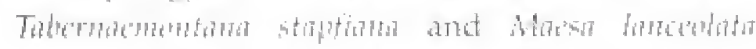

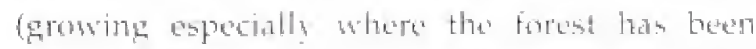

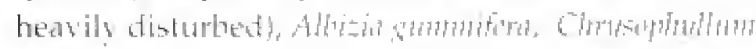

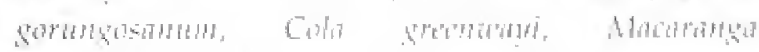

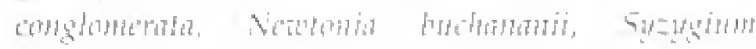

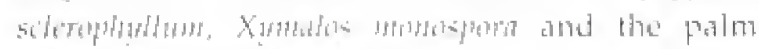

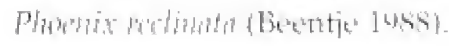

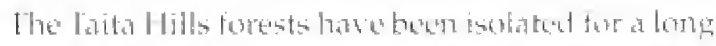

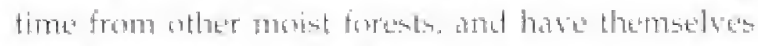

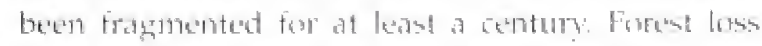

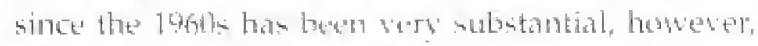

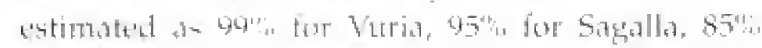

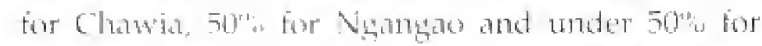
Mhotolu (Brouks e? sid, it press, a). Despite their small size. the furests ase jmportant for water catchment supplying the ver finer and various bocal

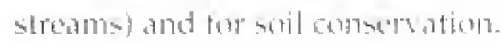

\section{Birds}

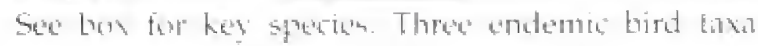

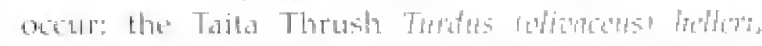

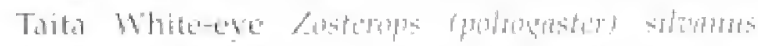

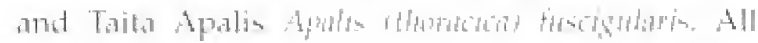

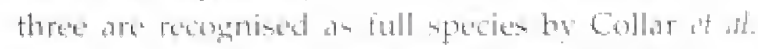

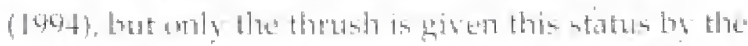

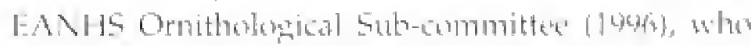

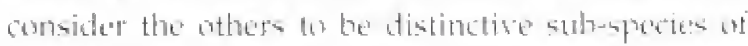
the Montane White-ene and Bar-breanted Apalis.

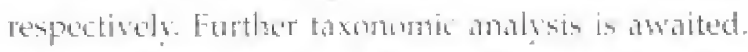

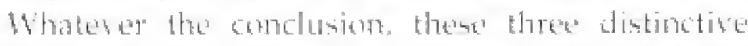

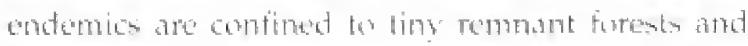

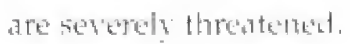

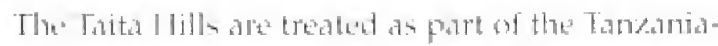

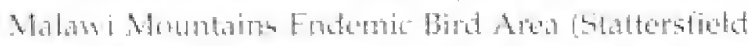

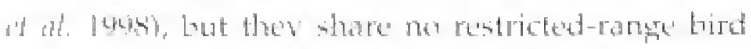
species with the othere siter in this F.BA. Itr fact, the th

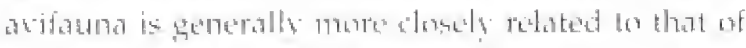

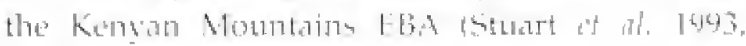

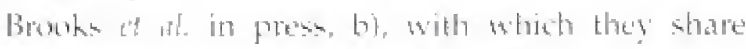

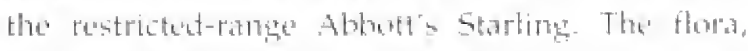

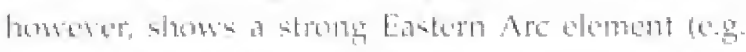

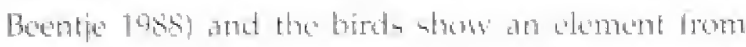

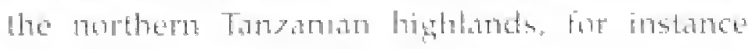

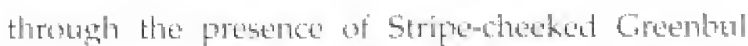

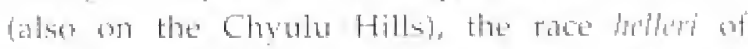

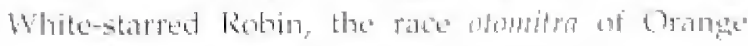

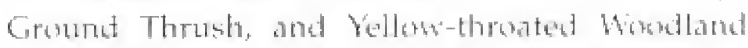

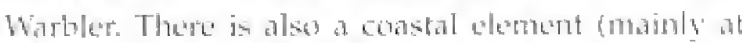

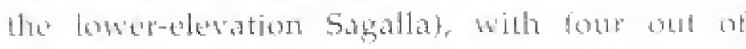

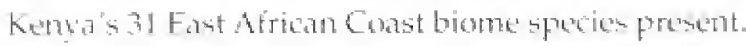

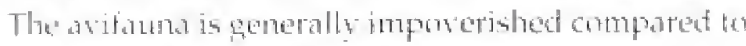

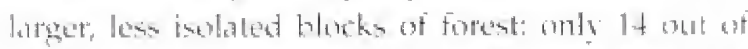
the. 67 African Highland Biome speciens fegularly acase theurgh Taita Thrush. Taita Apalis and Taita

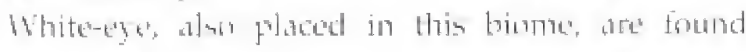
(n)ly here).

\section{Other wildlife}

Lexels of endemism in the Taita blills are genterally

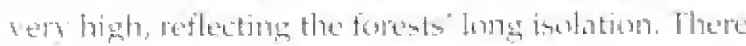

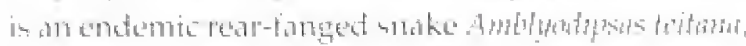

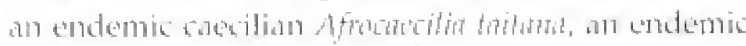

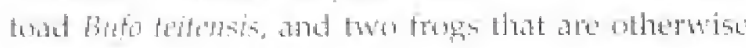

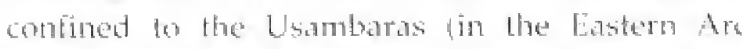
monntains of Tom/anial. Three butterflies, the Taita Glider Cumofher teita, Taita Charanes Chathere

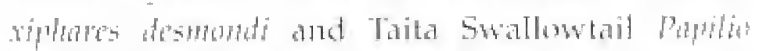

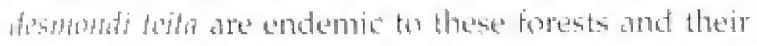

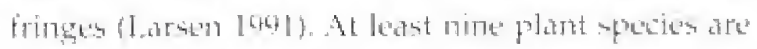

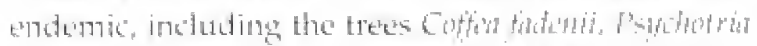

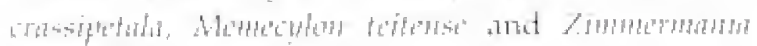

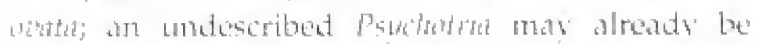

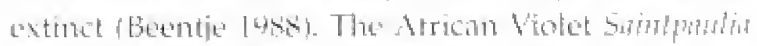

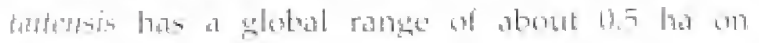

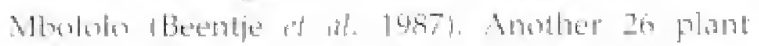

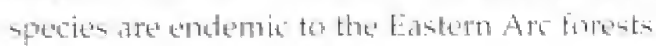

\section{Conservation issues}

The ting sire of the Tata flills forests, ant the dernet human population surrounding them, make them extremely sulnerable. Wost of the framente are aready thatily distubed. The hargent and least

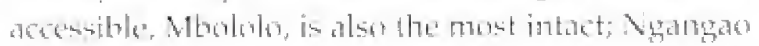
bur suffered substantiol tantage; and in the smetl]

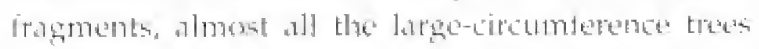

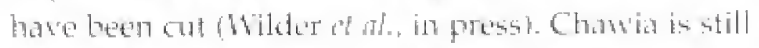
lossing its small and easily-cut treen. The bindisersity importance of the borests in still litte appreciated by

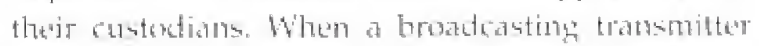
pust thas buill on the hill erent in Chandia in 1497,

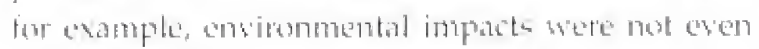

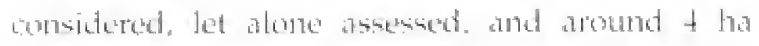

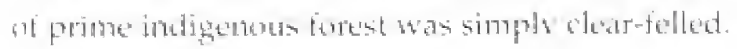

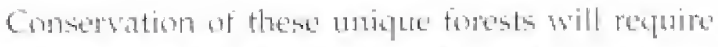
integrated plannisg and action. Serveral inikial stepm 
Important Bird Areas in Kenya

\section{Globally-threatened species}

\begin{tabular}{|c|c|c|}
\hline Taita Falcom & Vulnerabli: & $\begin{array}{l}\text { Early specimens collexted at the base of } \\
\text { the'se hills (Zimmermath of al. 1946); no) } \\
\text { recent records }\end{array}$ \\
\hline "Thita Thrush & Critical & $\begin{array}{l}\text { Taita forests endemic, a forest-specialist } \\
\text { species presently known from Mbololo } \\
\text { (where most commont, Ngangan, } \\
\text { Chawia and Yale (Brooks et al in press, a, } \\
\text { E.M. Wabaki, fil litt.) }\end{array}$ \\
\hline "Taita White-eye & Critisal & $\begin{array}{l}\text { Taita forests endemic, but the most } \\
\text { adaptable of the three, recorded at most } \\
\text { forest patches apart from Silgalla, and in } \\
\text { scrul and remnant trees (Brooks ot al. in } \\
\text { press, a). Its status should perhaps be } \\
\text { downlisted to Endangered. }\end{array}$ \\
\hline *Taita Apatis & Critical & $\begin{array}{l}\text { Taita forests endemic, only recorded on } \\
\text { the main massif (in Ngangao, Chawia, } \\
\text { Fururu and Vuria), not on Sagalla or } \\
\text { Mbotolo (Brooks id al. in press, a) }\end{array}$ \\
\hline *Abbott's Starling & Vulnerable & $\begin{array}{l}\text { Lf to } 20 \text { recuded at Chawa (Brouks it } \\
\text { al. in press, a); maly be an overlooked } \\
\text { seasonal visitor }\end{array}$ \\
\hline Southern Banded Snake Eagle & Near-thrateract & $\begin{array}{l}\text { One record from Chawia (Brooks it al, in } \\
\text { press, a) }\end{array}$ \\
\hline
\end{tabular}

Regionally-threatened species

\author{
Ayres's Hawk Eagle Vulnerable
}

Status unknown; no confirmed records

(Brooks ef at, in press, a)

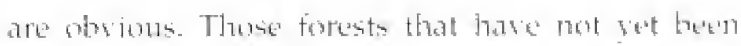

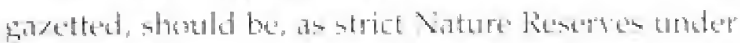
thu Forests act. Even this status may not aftent

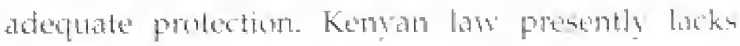
prosiagon for small nature reseraes to be designated

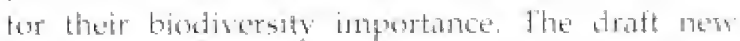

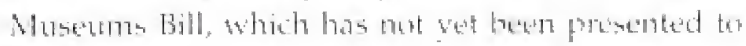

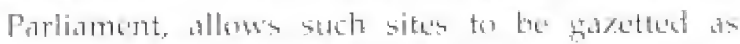

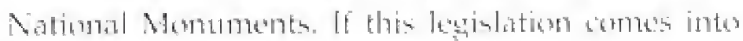

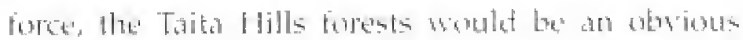
place to apply it.

Plameations of exotic tress, mainls conders, make up a substantial area of all the rmalge frasmembs.

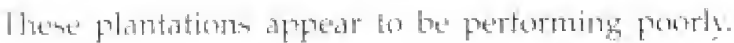

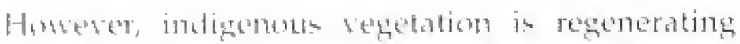

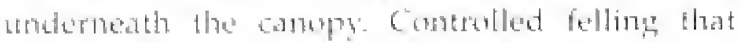

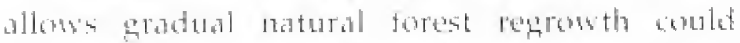
acentualy morease the indigenous forest atea substantially of the same time', it in clear that the

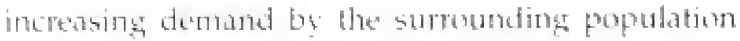
for fuehowd, poles and obther forest protucts simply cannot be met stastainabiy from the natemal forests. Serict controt of torest ure is neeted, combined with

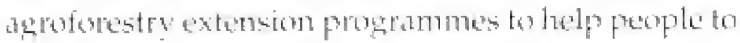
med these needs on-tarm. Extraction of meticinal plante and bork might till be possible under a caretul ticenas system. 
Some Eampenation for the lase of forrest

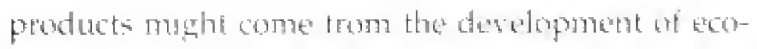
tourism in the Taita Hills. The hills are accensible and corventently hacatert mear the mation

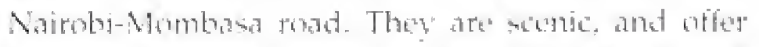

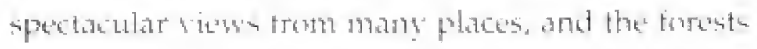
hase much to interest any maturalint fard certainly ans birdwalchert. The foresto are casy and safe to walk in - เnlike man sthe'r sites, the're are mep

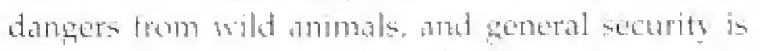
groont in the areas. Sites like Ngangate and Chawias

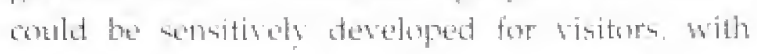
walking trails, information boards, trained local

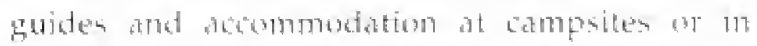
local gout lasustion. Such an approach has been

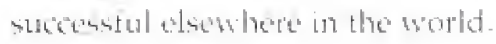

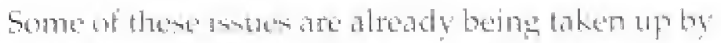
He" Fast Arikan Mildite Society, which is ruming a

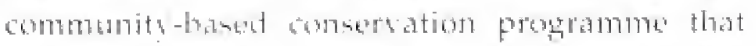
should wentually give rise 60 an integrated

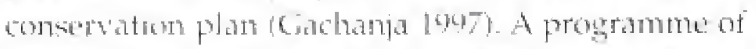
resentely inte the toremb biodsersity and the effects of traspuntation is alon underway, run fondly be the National Museums of Kemba, Kernyatta Lnibersity

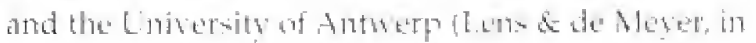

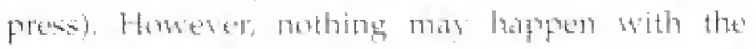

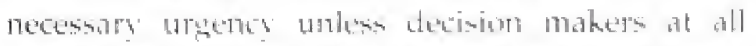
levers are comsunes of the exeptional inportance (b) the he situ's.

\section{Further reading}

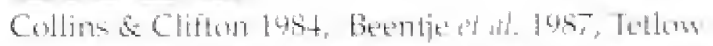

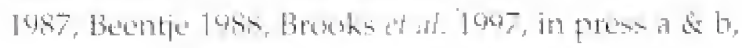

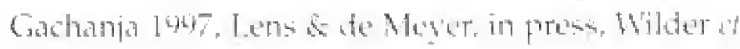
at., ith press 


\title{
22 TANa River Delta
}

\author{
$02^{\circ} 30^{\prime} \mathrm{S}, 40^{\circ} 20^{\prime} \mathrm{E}$, Coast Province, Tana River and Lamu Districts
}

Area: 130,060 ha

Altitule $0-37 \mathrm{~m}$

Unprotected

Catrgories: Globally-threatened species,

East African Coast bione species, congregations

\section{Site description}

The Tana Delta is the mame lousels grinen to the

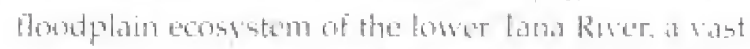

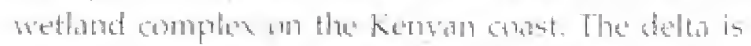

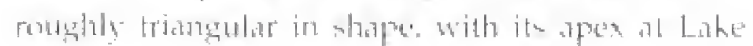

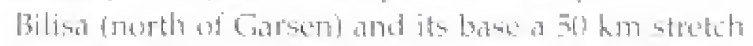

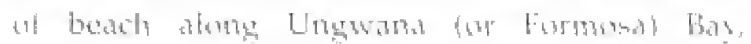

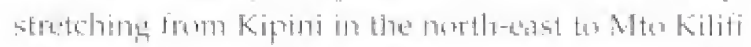

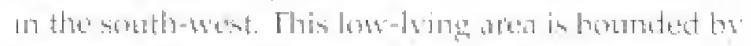
hightere band to the east and west, and to the senth by a dune shatem borderiog the Indian bearen. It ferms

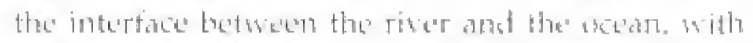

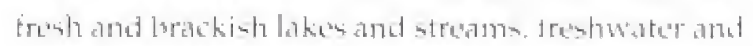

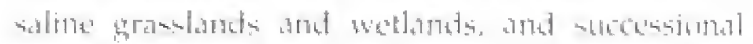

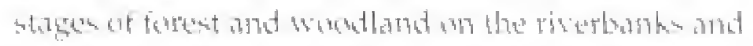

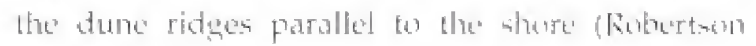

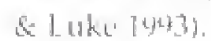

the anouth of the raser has shitted mang times.

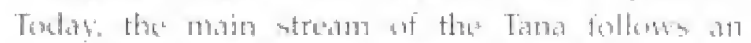

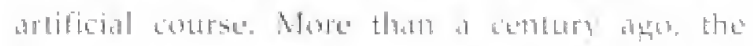

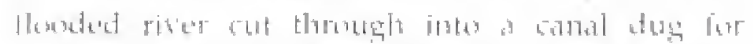
navigation from Bsifurat, on the main

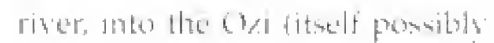

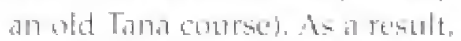
the riter nus flowe difects

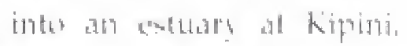

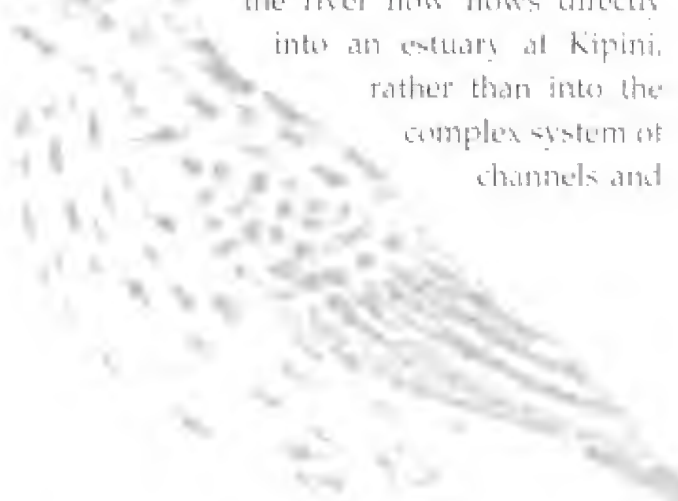

Matind Pipit

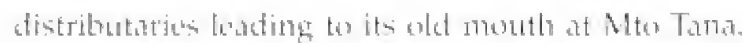
Lntil recentla, some tresh watter at ill flowed intes the

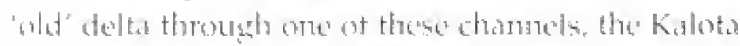

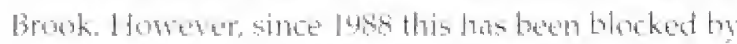

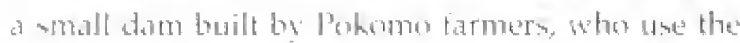

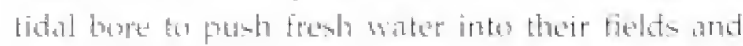
irrigate thate croph.

The entire floodplan in the fower parts is onsed

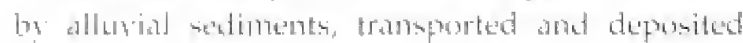

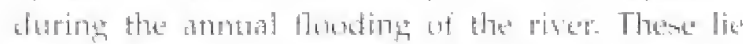

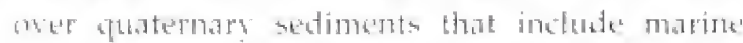

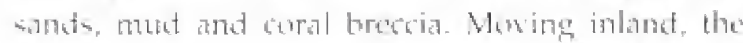

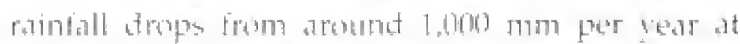

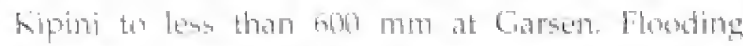

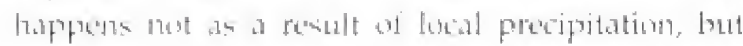

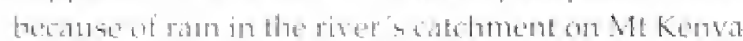

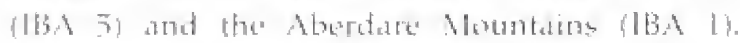
sormalls; the major flunde oceur in Aprol-Nay,

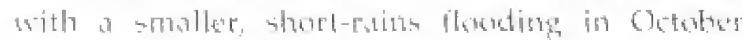

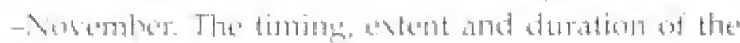

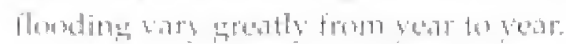

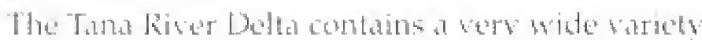
af habitats, inctuding roterine liorest, grassland,

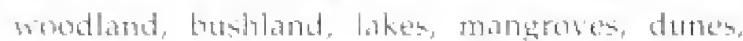

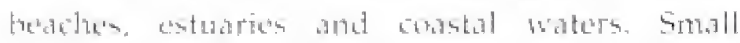

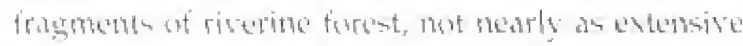

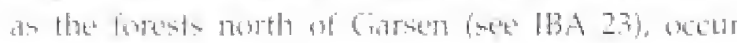

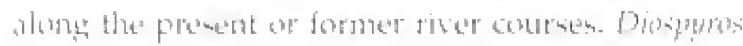

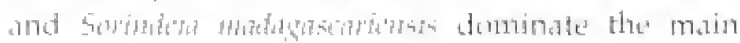

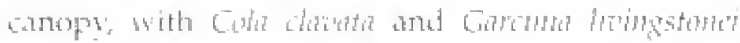

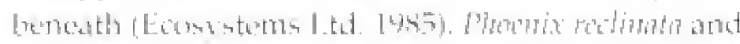

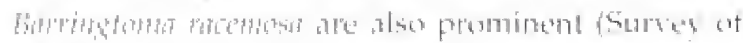

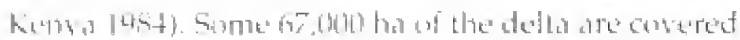

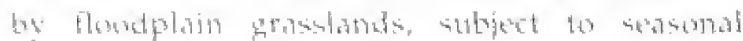

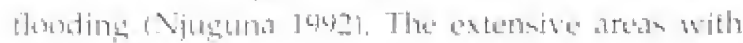

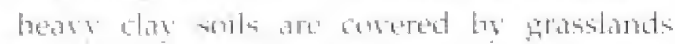

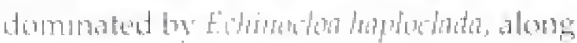

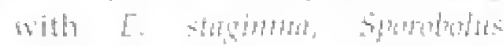

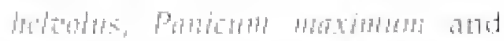

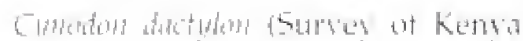

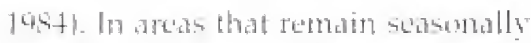

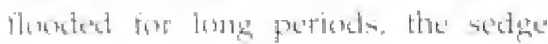




\section{Tana River Delta}

\section{Globally-threatened species}

Southem Banded Srake" Eagle Near-threatened

"Basta Reed Warloler

Near-threatered

Malindi I'ipit

Near-threatened

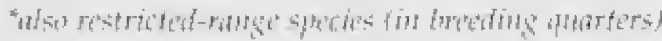

Uncommon in riverine forest

(Zimmerman et al. 1966), but not recorded in recent surbegs (O. Nasirwa, in lift. k; exact status unclear

Common non-brexding visitur in floced platn thicket, espercially salte-bush vegetation, from Nowember to April: this is probaloly the main wintering ground for this species (Parson it al. 1978. Lewis \& Pomeroy 1989. Zimmerman ef at. 1946)

Widespread at low densities in the flood plain (Zimmermaty dis 1906 )

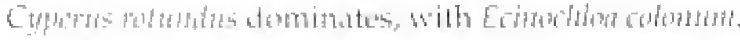

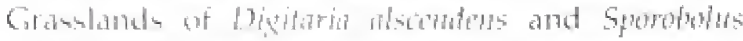

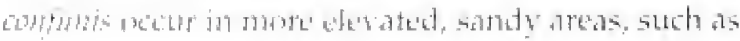

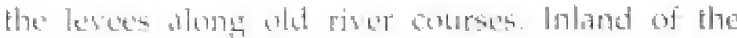

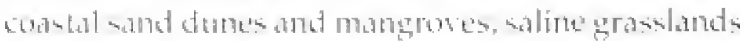

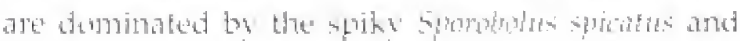

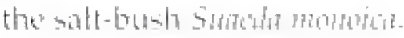

Nest al the flowdplatio to bushland, with a diverses

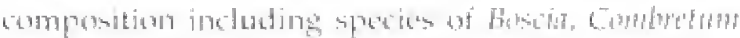

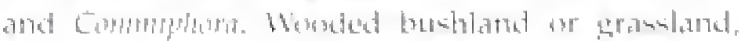

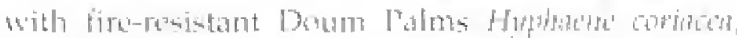

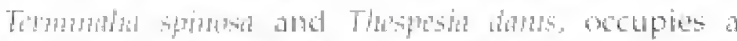

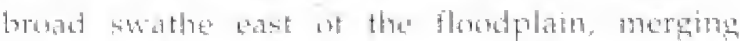
(througth an intermediate wordland that indedes

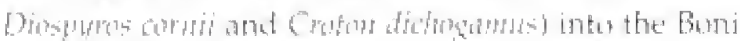
Forent atedation to the north.

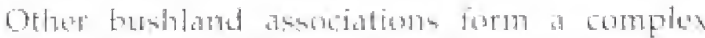

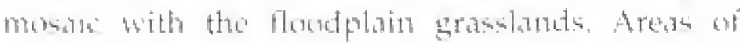

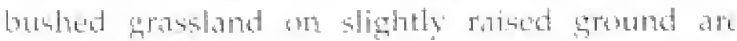

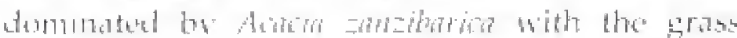

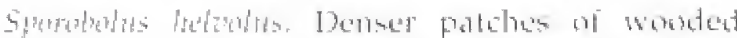

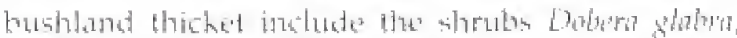

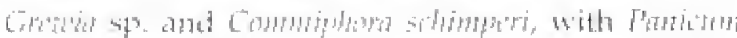

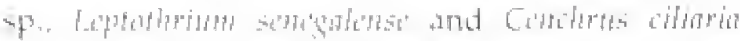

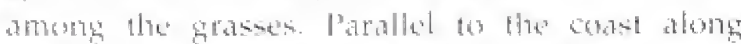

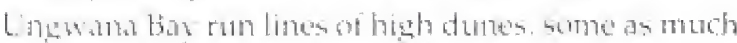
at 37 m atrone the sed. These are conered by

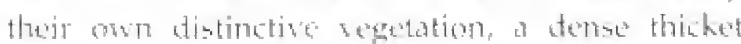

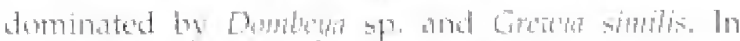

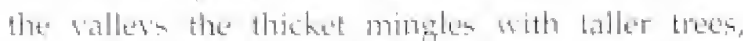

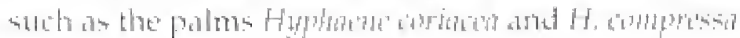

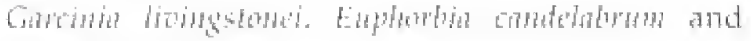
Afoth whangersis.

Palms are prominent in mares places, with

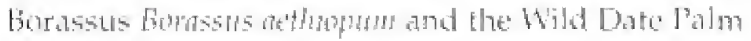

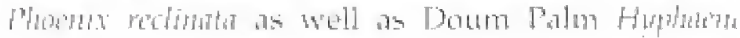

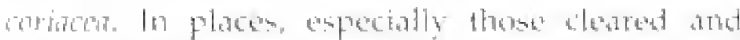
burnext in the: past. thexe form stubstantial tracts on

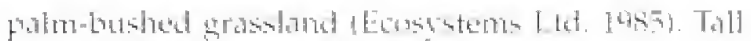

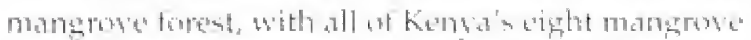

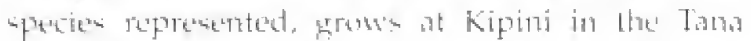

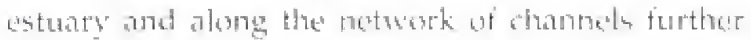
south.

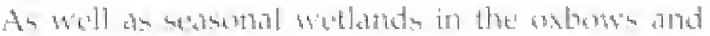

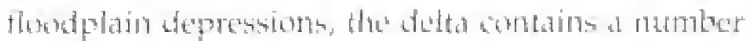

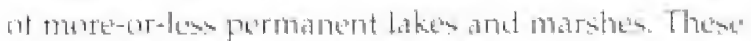
include Lakes Bilim, Dida Warede. Harakina,

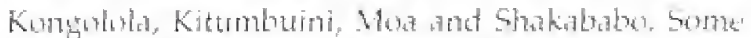

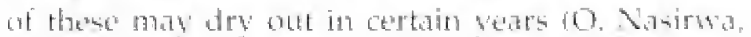
in lid.). but others, like Lake shakatrobo and

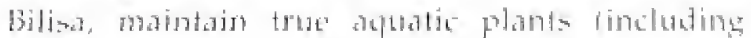

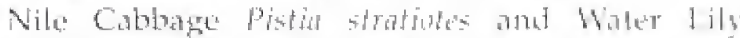

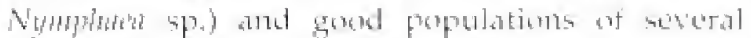

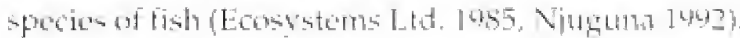
but and Luhra immigration to the area are responsible for an actise and thrivimg bishers. white Orma pastoralists use these wetlands a druseason grazing areas for their livestoch.

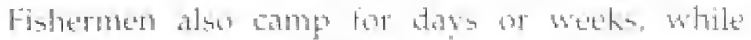
catching, salting and aldithe fish, wh the cotal cruterops of Nwamba Ziwantul, some $10 \mathrm{~km}$

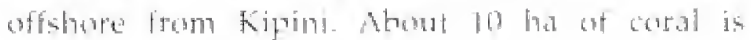


Important Bird Areas in Kenya






\section{Tana River Delta}

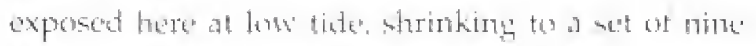

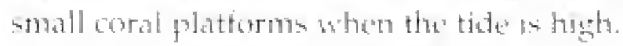

The wher mais ethatic sorthe in the arease the

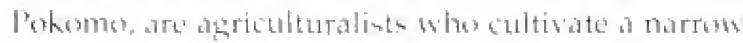

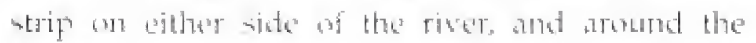

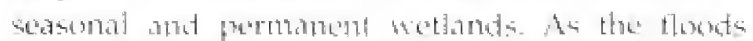

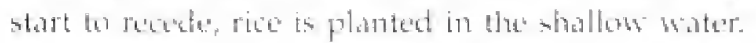

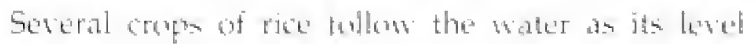

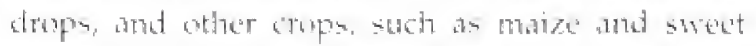

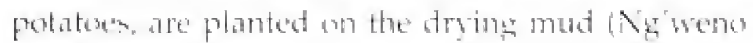
1493) As witl as omall-acale cultixation, there is an

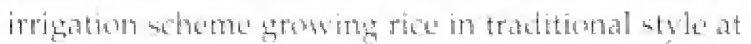

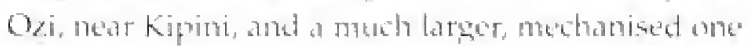

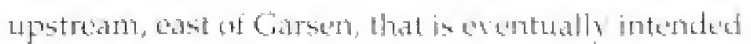
to cencer as much as 16, bith hat

\section{Birds}

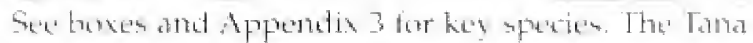

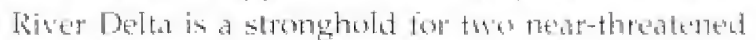
species, Malindj l'iprit and Baska Reed Warblor, and

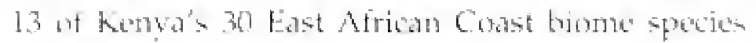
actur. The wetands, meluding the combtine and siffshore inters, at times hosd exaptional concentrations of waterbirds, Internationalls -

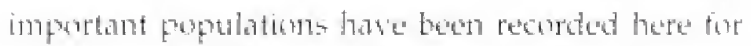
nur ferter than 22 species, during the flomd enand,

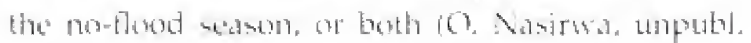

\section{Congregations}

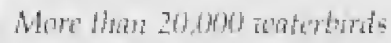

Wesn: 70,000; no-floud seison 75,000, flood season, 65,000.

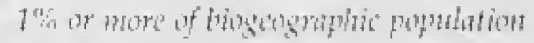

Great White P'tican $(1,800)$

Pink-backed Pelican $\{1,000\}$

Cattle Egret $\{10,000\}$

Yellow-bilket Fgret (1,000)

Creat Egret $\{500\}$

African Open-billed Stork (1.000)

bellow-billed Stork (F)0).

Arrican sponbill (15i)

Greater Flamingo $(1,250)$

Spur-isinged Goose $(3,750)$

White-fronted Plover (1,000)

Iteser Sandplover (250)

Litte Stint $(10,000)$

Curlew Sandpiper $(7,500)$

Marsh Sandpiper (550)

Sorty Gull (400)

Slender-billed Gull (100)

Gull-billed Tern (270)

Caspian Tern (250)

Lesser Crested Tern (250)

Saunders's Tern (200)

Whiskered Term $(1,0070)$

" 470 counted on Nwamba Ziwayuu

**2,440 counted on Mwamba Ziwayuu

$\begin{array}{rrr}\text { Medj } & \text { No-flood } & \text { Flood } \\ 1,215 & 360 & 2,070 \\ 1,385 & 2,500 & 270 \\ 6,230 & 11,270 & 1,190 \\ 1,010 & 2,000 & 20 \\ 1,440 & 320 & 2,560 \\ 2,470 & 3,530 & 1,410 \\ 845 & 970 & 720 \\ 2,165 & 3,680 & 650 \\ 1,170 & 2,240 & 90 \\ 2,805 & 210 & 5,400 \\ 650 & 230 & 1,070 \\ 1,205 & 701 & 2,340 \\ 10,150 & 15,310 & 4,940 \\ 6,875 & 740 & 12,960 \\ 855 & 1,690 & 20 \\ 690 & 830 & 550 \\ 380 & 490 & 270 \\ 1,120 & 1,450 & 790 \\ 860 & 1,340 & 380 \\ 1,275 & 1,670 & 880 * \\ 1,840 & 70 & 3,610 * * \\ 950 & 1,450 & 450\end{array}$

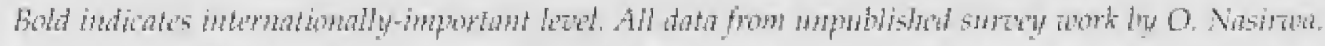

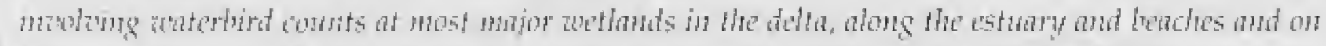

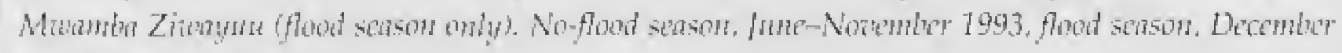
$1993-F$ idmuary 1994. 
data). This makes the Della one of the kuy sites un the country for waterbind conserbation, The Tand Delta

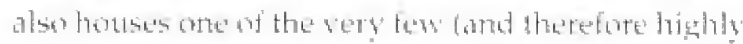

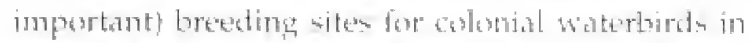

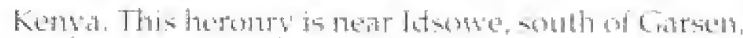

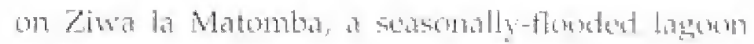

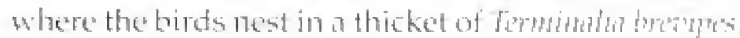
usitally beewerm May and september but alses at

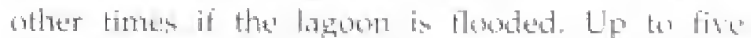
thousind colonial waterbyeds an at kat thirtedn species have been recotded nesting here. including Arican Darter, Grey, Purple, Black, Cammon Squaces atad Blackeremened Night Herons, Gireat Yellow-billed and Litte Furebs, Arrican Open-billed Stork, bacred and Gloss lojess, and African Spoonbill (North 1459, Briaton 1474. Concerdale at abl. 1483, o. Nasirwa in fift.). Nwamba Ziwastu, a

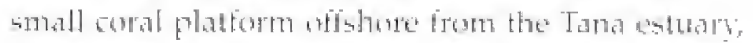
is a renting site for significant numbers of Satunders's Tern and Lesser Crested Term that feed in Lnghama Bay (O. Vasirnta, in lith.).

\section{Other wildlife}

The inportange of the Tana River Delta lite in the exponse, intectness, warkets and proutuctivity of its

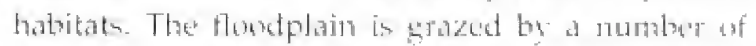

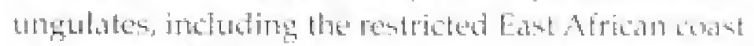

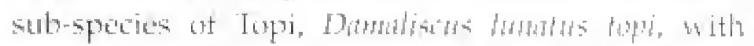

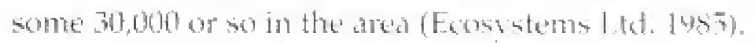
The rixers and thannels support large numbers on

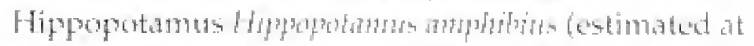

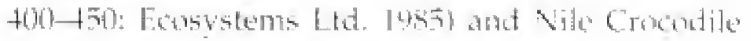

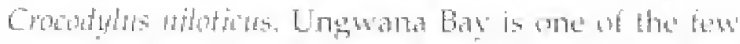
places where Dugung Duroug atsom, eritiontly endangered in the region, has been recorded

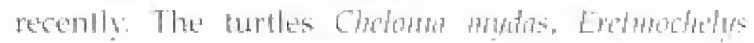

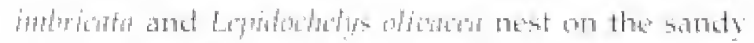

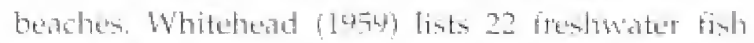

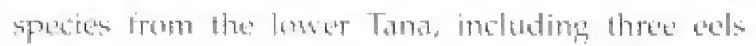
Angwill spp. and a distinel sub-speries of

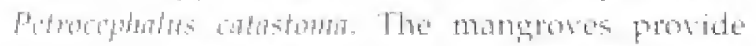
sitally inportant spawning and nusery grounds for many species of tish and crustaceant.

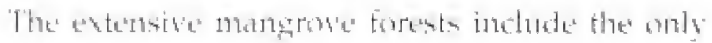

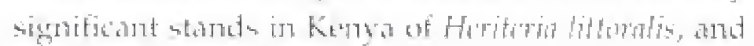
two other species that are considered threatened,

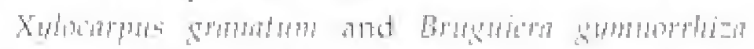
(Was 1695). 280 plant taxa art recorded for the delta,

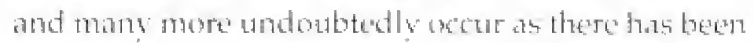
Int thwomgh botonical supreys of these. 18 aft

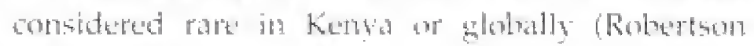
\& 1.uht 1903).

\section{Conservation issues}

Disputes wer the rights to water and land are at the berat of the complex corservation problems facing the Tand Riser Delta. The importance of the area for cothers ation is thety recominged. but there is little areetenten on the appropriate steps formard, and the site remains withent any formal protection.

The numerous patches of riverine torest, wromeleand and bushland in the della are edaphic in

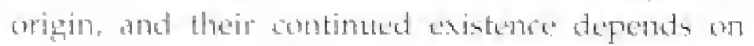

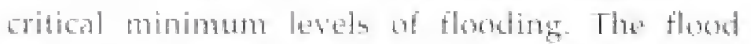
resine has already been distupted by five datge

\section{Regionally-threatened species}

African Darter

Creat Egret

Scaly Babbler
Vulnerable

Vulnerable

Vulnerable

Vulacrable
Still present in moderate numbers (O. Nasirwa, in lift.), and up to loo pairs have bred at the Idsowe heronry (Coverdale ol arl. 1983).

An important site for this species, with more than 2,500 recorded in the flood season 10. Nasirwa, unpubl. data), and steral hundred pairs breating at the Id sowve heronry (North 1959)

Regular visitor in mall numbers, May to September (Zimmerman of al. 1496)

Local and uncommon in thickets (Zimmerman (1) al. 19963 


\section{Tana River Delta}

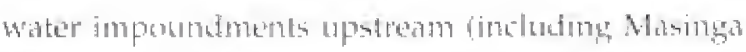

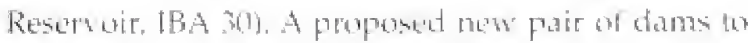
be built by the Hutuncha-Ciand Falls Hydropower Project has the potential for tevern more serious

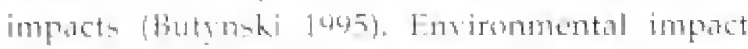

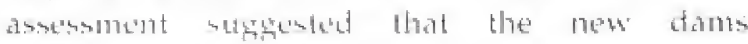

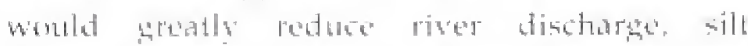
deposition and the level of grostodwater. Honeser, the present design of the low Gitud Falls dam incorporates an artiticial flowat and

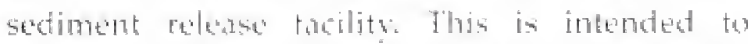
release sediments and artificial flonds twice

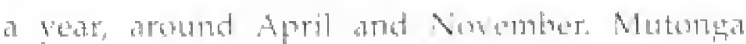

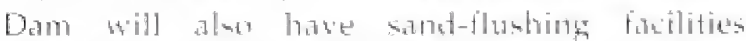

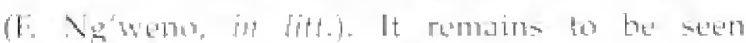

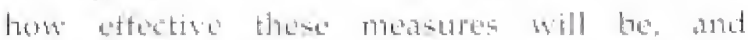

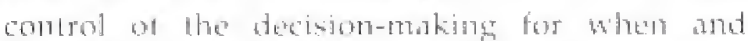

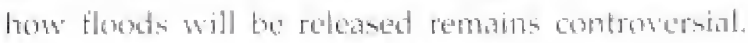

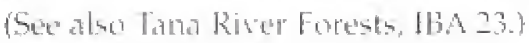

A nuajur rice irrigation scheme has berele initideted it the Tatra Dentat by the Tand and sthi Rivers Dewelopment Authorits, with Iunding from the dapanese Conemment. Four thousand hectares are

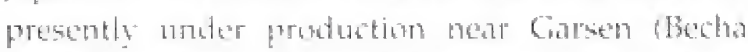
1497), and the wherme is cruatiog considerable tomplomernt. It low also damaged riserine forest

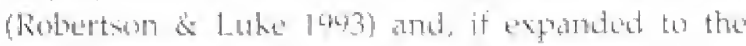

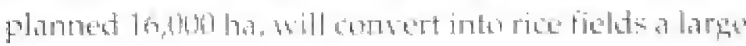
swathe ant tratitiond dry-seanon grazing land relied upon by pistomalists. Other concerns bamilias to

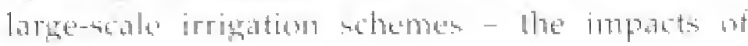

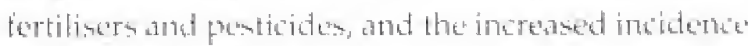

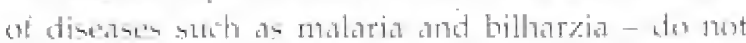

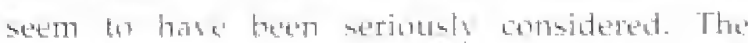

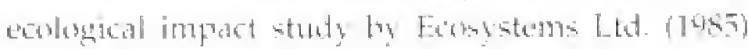

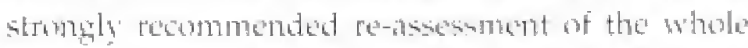
propect andept, painting out that there were more officient wase of producing rice and

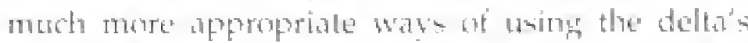

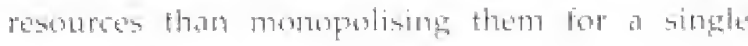
agriculturat ateivits:

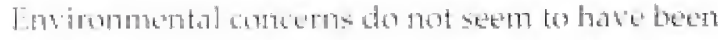

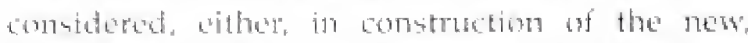
embanked road to Lamb ancose the fomdplain

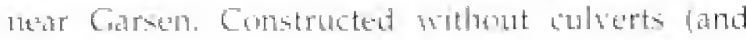

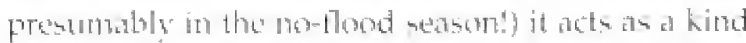
of Aam, and the sabstantially altered flombling

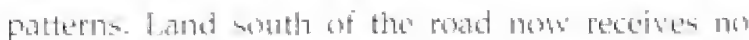

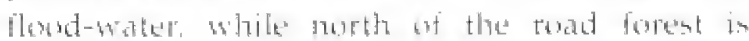
drowned and dring (Rothertson \& Luke 1943) Norwe 1493\%.
The two major distributaries ints the della proper. the Furaha and Kalota Brooks, are nam blocked by bartages constructed by the local penpl he". The Furith was cut off in the late 1970 s and the kalota in 1988, in both sases after a drowght year. The opi rise scheme depends on natural irrigation fom riveg" water pustud back by the tidal bore at Kipini, and the luwerod river volume in time of trought raised fears that the lana might change its course once again, abandoning the Kipani mouth entirely. Evem if this did net happen, it was thenght that the outhow of iremb water to the distributaries might result in salt

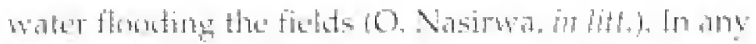
cast: the barrages have comperted the Kalotia and Furabatinto saline creeks, and proboundy altered the walogy of the southern dedta. With thetr fresh wated

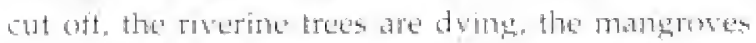

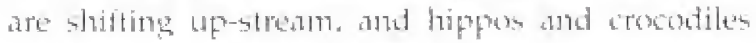
have been forct to mote. [ he freshwater grass]and are bocoming siline and unsuitable for gratimg $A$

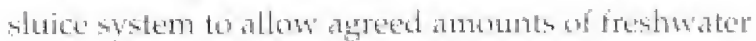
into this part of the delta would probably solve these problems fairly simply.

Who owns the deltat, and whe should control its resources? The With suttlement seheme is

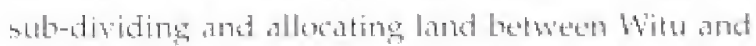
Kipini, in the noth-eastern part of the site. The landles in Lamul and Fana River Districts are yet to be given priarity whet the plats are issuted

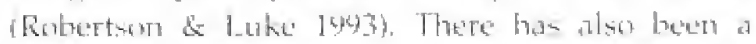
bong-runding and acrimoniats dispute over the section of the dedta woth of the rivere frontions

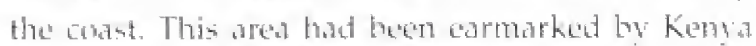

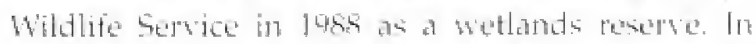
part, thes was in recognition of the entestanding

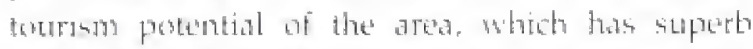
scenic and wildtile interest, a remute lecel and unspoiled landsopes and beaches te.g. Chenting 1987. A purtion of 20,000 ha was allotted in 1990 to a sertap ranch, Kon-Dertu, mate up of around 100 people living neate the delta. Kon-Dertu, saying that it lacked the funds to develep the nrea, promptly sold half the allocationt to Constal sequaculture latt.. who intended to develus their pirat as a commercial prawt fatm. Prawn farmo are notorious for the'it extremely negative ensirommental feffects, and the allacation was hotly disputed by many comeromed for the comberation tof the delda. Almost a year's raging comtersersy culmidated in an anmouncentent Ly Ketrats President Mos in July l69) that the Tana Dela should be protected as a hetland of infertational importance. The" lind allocation was 


\section{Important Bird Areas in Kenya}

nullified, and a gorermmental Tana Delta Ketland

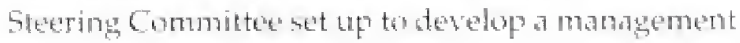
plan.

Five vears later, progress tomards this is monimal. An emviromenental awareness profect has beten carriced out, which may hase helped to defube some of the strongly-held antipathy fell by the local

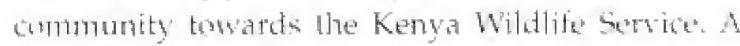
literature surver has bean completed. Howeret, there has been row headway on Ramsar designation, and no management plan has bean begun. This is due to lack of funds and, more seriously the continued absence of a consensuls statement on lanet from the Ministrs of lands and Gettlement (Beche 1997. Neconthile, Coastal Aquaculture thallanged the nutlification of the land allocation in coust and, in Warch 1996, won their case. Orere a vear later. the Govermment finally appealed for an injunction out of time a gainst this ruling (Becha 1697 ).

The contsensus statement is essential, becallse it will clarify the issue of land am nership and temure, and help to settle unresolued questions of access, mashagement responsibitities, enviroumetrted frestection and sustainable use of resourchis. Without it, little progress is possible.

Neanwhile, in the continuing state of contusion,

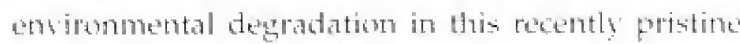
habitat continues apace. Destruction of wortland and mangroues, slash-and-burn agriculture, illegal lumting of wildlife and unregulated off-shore trawling are all diminishing the delta's resources. Wese disturtingly, there are reports of allowations of latge drunks of land to seriar government oficiats and civic leaters trom the district (Becha 1947). The

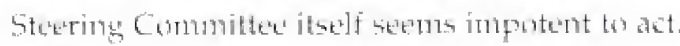

The local communtits amain extremely homile to the idea of their ancestral land being hived off as at widulife reserse, Irosically, frotection tender the Ramsar Comvention means something quite diferent to this. The Comverition enshrines the: itsea of wise use - and central to this is that the theteds of the lowat people must be mest, in a sustainable way The resoures of the delta atte botmeiful thumgh that it can be managed is the interests of those who lise theres. while ensurng the long-term consersation of its birets and other bodisersity. The smoner the planumg process be piths, the leetter.

\section{Further reading}

Andrews dit. 1075, Ecosysteme 1.td. 1985, Cheifings 1987, Cometal ASAL Derehoment Proverel 1941. Niuguna jo42, 1993, Nkako 1492, Ng'weno 1993. Opala 1693, Robertson \& 1.4ke 1963, Becha 1497 


\title{
23 Tana River Forests
}

\author{
$2^{\circ} 30^{\prime} \mathrm{S}, 40^{\circ} 30^{\prime} \mathrm{E}$, Const Province, Tana River District \\ Area untefined: $c, 60,000$ ha, including 3,700 ha of forest
}

Altitule: $40-70 \mathrm{~m}$

Status: Part National Reserve, part unprotected

Categories; Globally-threatened species, restricted-range species, East African Coast biome species

\section{Site description}

These are riparian forests ahos the meandering course of the lowe Tand laver, some $350 \mathrm{~km}$ east of

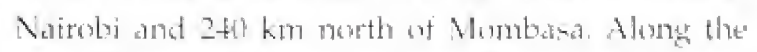

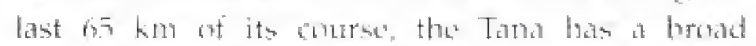

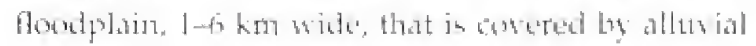
sediment ateposited during flaxds. Flooding takes place in response to hears rang on the Aherdate Mountaina (IBA I) and Mt Kanca (IBA 5) watershedo

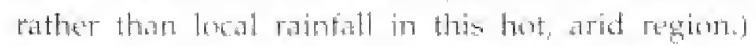

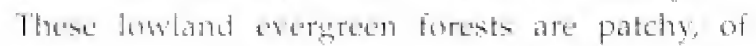
different stacesional states, and are dependent on groundwater supplied by the river. Characteristic

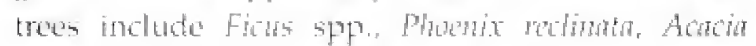

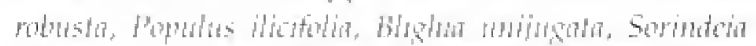

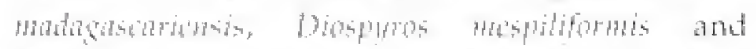

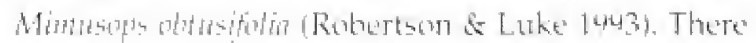

are about 7 distinct forests, ranging in size from $1-1,100$ he and cosering around 3,700 ha in total (Bubraki \& Mwand 1995). They form part of al mosatio of habitats that theludes grassland, norded

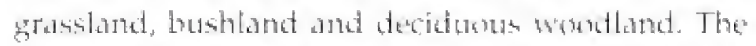
forests lie ou both bank af the Tans. Of tha" 7 I potehes, 16 (covering $I, 000$ hat tall within the 17.100 ha Tama River Primate National Reserser which extends for about 3 h km alomg the river"s

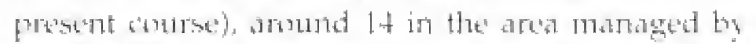

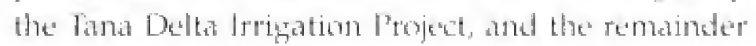
on Trust land (Geal of on, 1997).

\section{Birds}

See box and Appendix 3 for key species. At least two andoblly-threatened bird species occur, atong with at least two and possibly three of the species in the East

\section{Globally-threatened species \\ Sruthern Banded Snake Engle \\ "Fischer"s Turaco \\ Near-threatenest \\ Malindi Pipit \\ East Coast Akatat \\ Vulecrable \\ * White-winged Apalis \\ vulnerable \\ ("Tana River Cisticola \\ Data-deficient \\ Basra Reed Warbler \\ Plain-backed Sunbird \\ Near-threatened \\ Probalbly farly common \\ Fairly commor in taller forest \\ In open yrassland south of the Primate Reserve \\ Status unknown \\ This is the only site for the nominate race chariess, which may now be extinct: the last record was in 1961 (Zinnmerman et at. 1996) \\ This enigmatic species may accur in bushland in the National Reserve) \\ A non-bretding visitor, November to April; may be numerous on the lower Tana, in riverine thickets (Dearson ed al. 1978) \\ Probably fairly common in closed-camopy forest




\section{Important Bird Areas in Kenya}

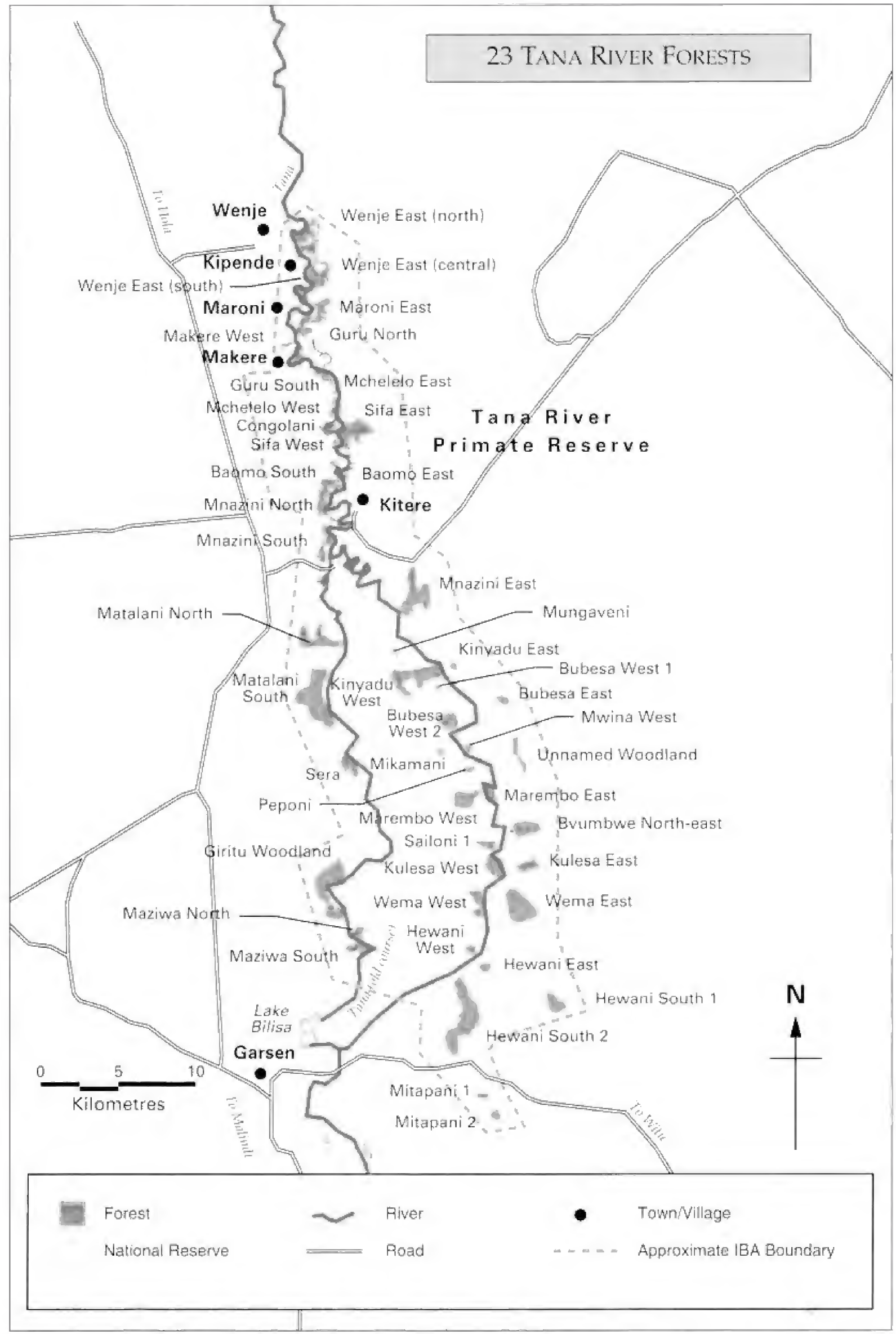




\section{Tana River Forests}

\section{Regionally-threatened species}

\begin{tabular}{|c|c|c|}
\hline African Darter & Vulnerable & On river and lakes \\
\hline Siddle-billed Stork & Vulnerable & On river and likes \\
\hline Ayres's Hawn liagle & Vulnerable & Status unknown \\
\hline African Crowned Eagle & Vulnerable: & 5 tatus unknown \\
\hline Arrican Finfoot & Vulnerable & On watlasuds with well-vegetated bonks \\
\hline Pel's Fishing Onl & Vulnerable & $\begin{array}{l}\text { In fortest along waterourses; a stronghold } \\
\text { for this speciss in Keny }\end{array}$ \\
\hline Violet Wond-twopoe & Vulnerable & In riverine stoodland \\
\hline Scaly Batbbler & Vulneratile & In thicket vegetation \\
\hline Litle Yellobs Flyciatcher & Vulnerable & $\begin{array}{l}\text { Trobathly tairly commun in closed-condopy } \\
\text { forest }\end{array}$ \\
\hline $\begin{array}{l}\text { Uluguru Violet-backed } \\
\text { Sunbird }\end{array}$ & Vulnerable & $\begin{array}{l}\text { Recent records front Kitere Forest } \\
\text { (Butynski 1994) }\end{array}$ \\
\hline
\end{tabular}

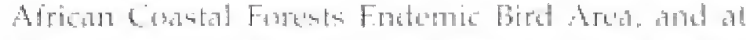

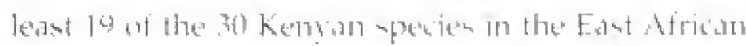

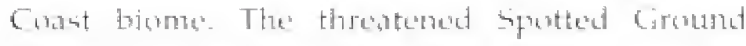

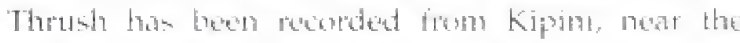

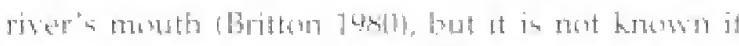

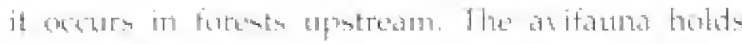

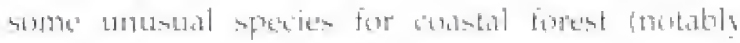

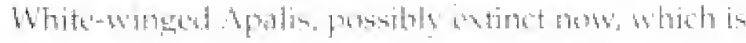

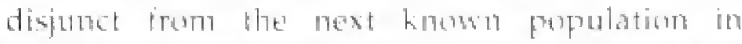

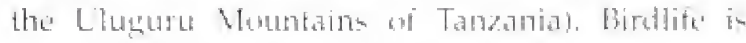

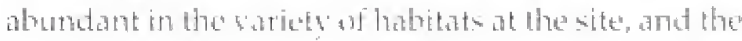
ox-howe lakes and riverheraks harbour a saricty of

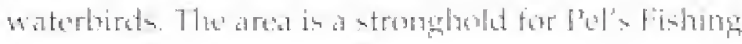

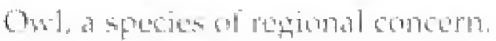

\section{Other wildilife}

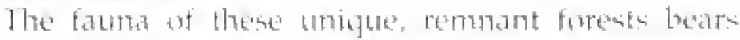

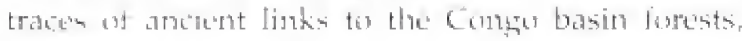

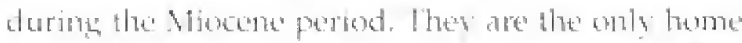
of tho distinctine primates, the Tand Riser Reat

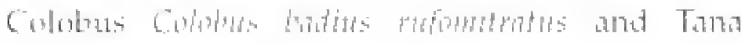

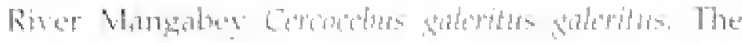

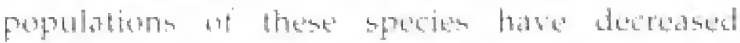

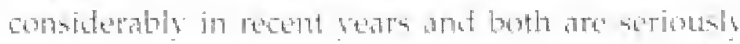

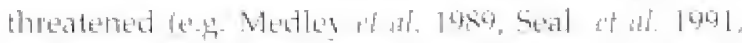

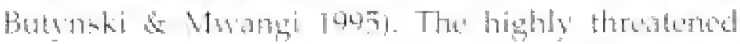
and restricted Hunter's Anteltope the flipota

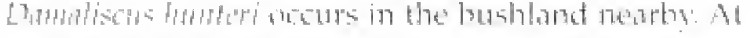

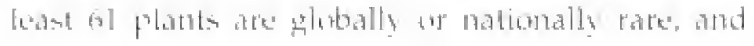

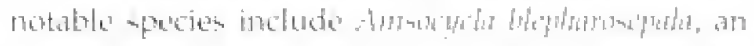

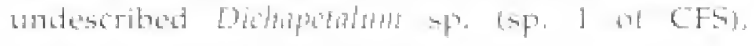

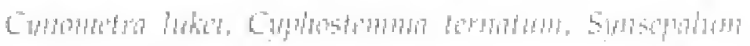

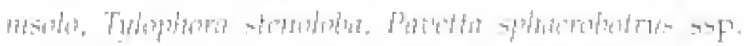

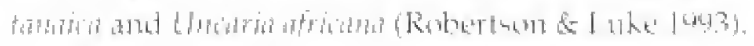
Or sandy bars and bartas in the ris cor gromes a papplar

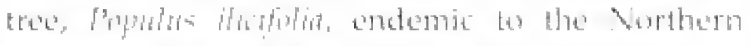

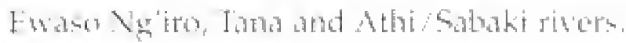

\section{Conservation issues}

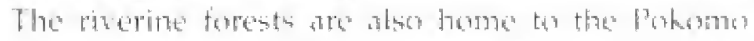

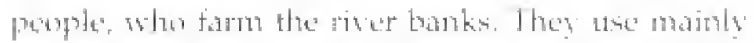

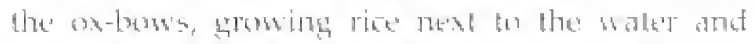
maize furthere track. The forest in expluited Ly them for fuevorut, kimber and tratitional medicines.

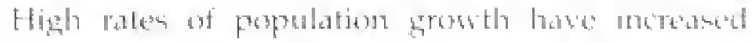

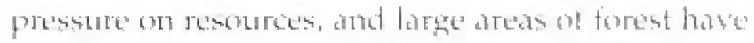

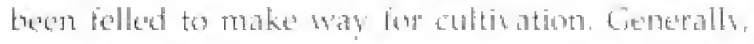

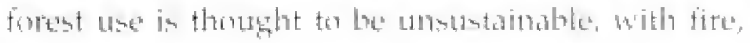

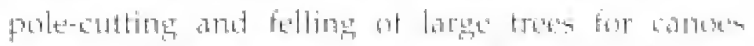

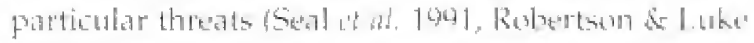
1643\%. Insecurity on the castern bank of the river has helped protect the foreses there, which are gemerally in beter condition. Many important homets, and an

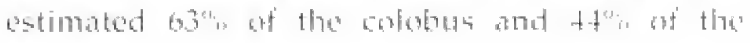




\section{Important Bird Areas in Kenya}

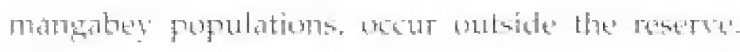

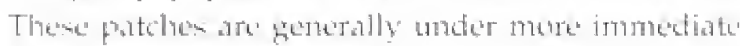
persture than thest sithin it.

A major Gel-funded project, which began in 1497.

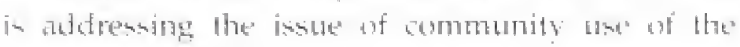

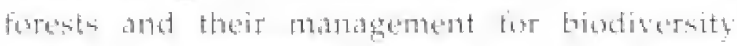

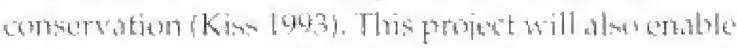

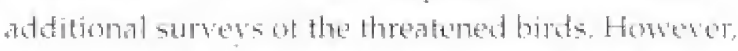
it will nus tack the other major potential these to

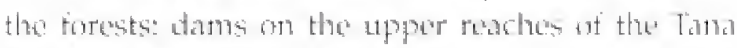

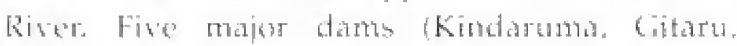
Kiambers, Kamburu arad Aasinga $(1 B$ A 301) alread:

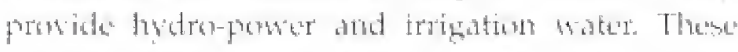

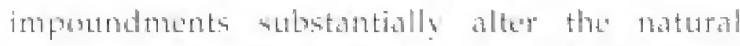
Flomilng resgime of the river, an which continuent survival or the forests atepends.

Recernt experen has foruted on the planded

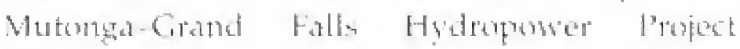

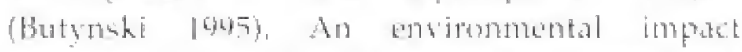

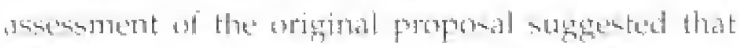
the new dams at Menomiga and Gamal Fallo would gratly reduce river discharge. silt depositim and the lewel of groundwater. The would last directly to the lase ot the ritiserabe fortent and the spereites that it holdk.
Henteres, folluning further debate, the present design of the Low Grand Falls dam incorporatem art ortificial ferod and sediment release facility. This is intended to redeate sediments and artificial forods

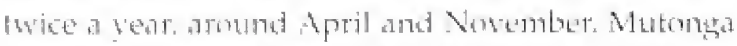
Dan will atar hate sand-flushing facilities. Athough thin increases the conks they are calculated

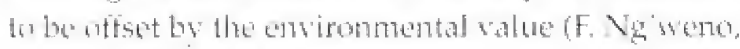
an latt.). (onstruction is to begin in 2003, for commisioning in 2008 . befone ctetriled design and londering begins, an additional envirumental ansessment will measure thont patterns bor at leant

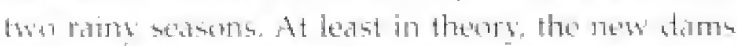

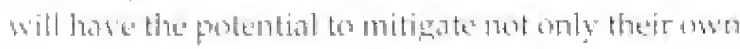
impact but that of the find existing dames as well. Howerer, who decides whether, when and how

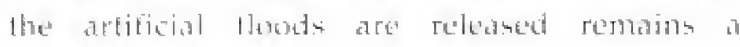
contentions ismute.

\section{Further reading}

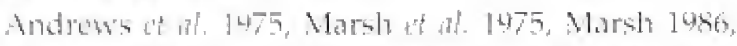

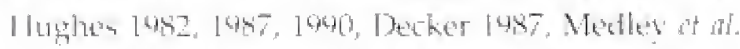

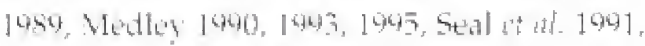
Neuc 1642, Kahumber 1493, Davies \& Kathumbu 1943, Rotuertson \& L.ske: 1403. Butgmakj 1445, Butwnski \& Nwangi 1995.

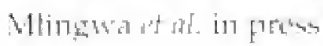




\title{
24 Tsavo East National Park
}

\author{
03'22'S, 38 35'E, Coast Province, Tama River and Taita-Taveta Districts: \\ Eastem Prosince, Kitui District \\ $1,175,000$ ha \\ Altitude: $300-910 \mathrm{~m}$ \\ Status. National Park \\ Categories: Globally-threatened species, \\ Somali-Masai bione species
}

\begin{abstract}
Site description
This vast somona National lark lies in lom,

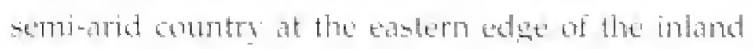

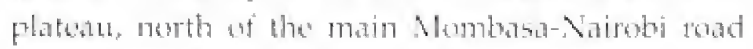

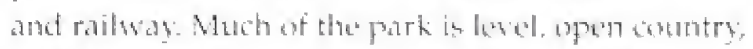
with scatered rocky outerops. The hatta lHateats, a lome, flateropped law ridge, runs alome the western bumatary and beneath it Hurs the Athi River; this joins the Than River to berme the Galana Riner, a Permanent strean that culs righe Bcross the Park. The stastsal Tha and viri Risers are important teatures of the nowthern and soblhern secturs, respectively. slong the rivers is a fortum fringe

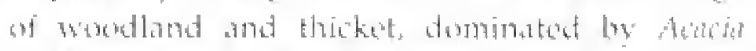

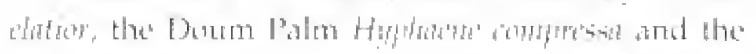

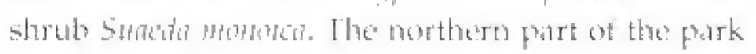

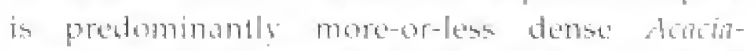

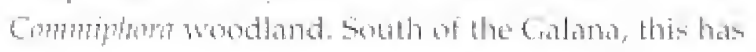

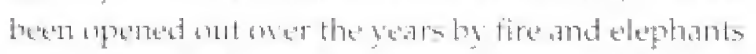

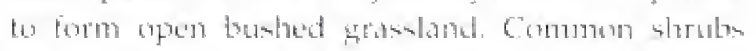

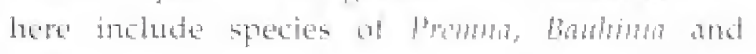

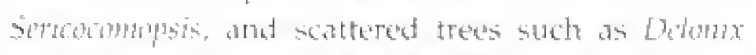

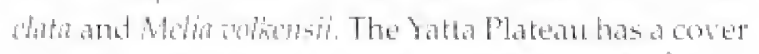
of dense buthland, with shands of Baobab Adamson

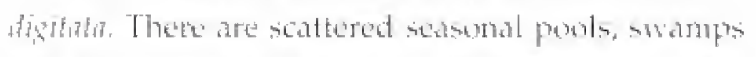

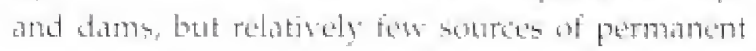

\section{Globally-threatened species}

Lesser Kestrel

vulruerable

Friedmann's Lark

Near-hitizatened

Basra Reed Warbler

Near-threatemed water. The regetation is generally denser in the west, where rainfall is around $450 \mathrm{~mm}$ per year, than in the drier east, which matrecise only around 250 mam.

\section{Birds}

See box and Appendix 3 for key species, Sixly at the 42 Kenyan spectes in the Smmali-Masti biome have berse recorded. The enirmatic neas-threatened Friedmann's Lark probably neste here. The Park's huge area of natural habitat supports impurtant populations of resident spexcies, and it is alson an sery signiticant stop-ovet and wintering ground for Palneatic migrants.

\section{Other wildlife}

The park lrodds substantial populations of a diversity of large mammals. Threatened species

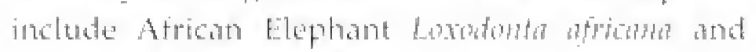

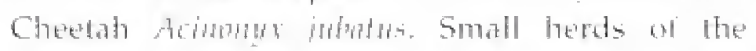

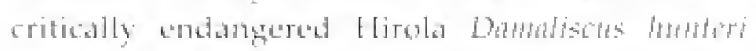
wete translocated in the 1960 s and again in 1996 , from Arawale National Reserver and are managing to sustain their numbets. Twe amphibian towat

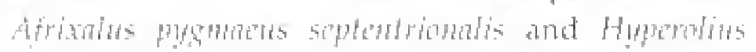
sheditich, ate endemic to the lsate atea (Duff-Makkas 1980).

\footnotetext{
Baranest Hather
} 
Important Bird Areas in Kenya

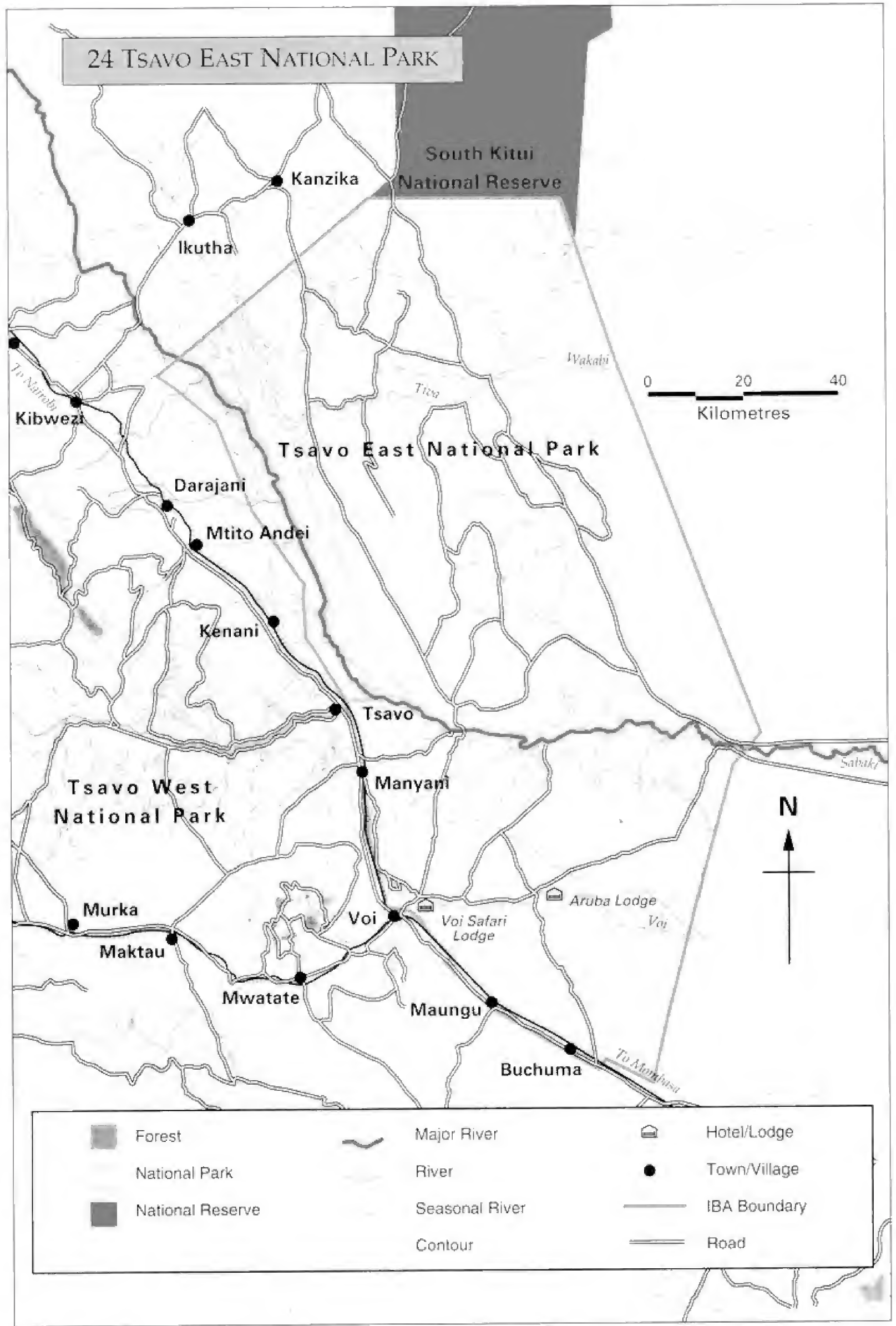




\section{Tsavo East National Park}

\begin{tabular}{|c|c|c|}
\hline \multicolumn{3}{|c|}{ Regionally-threatened species } \\
\hline African Darter & vulnerable & Occasional visitor to dams \\
\hline Great Egret & Vulnerable & Regular visitor, along rivers and on dans \\
\hline Saddle-billed Stork & Wulnerable & Regular at waterboles and on Tiva River \\
\hline White-hended vulture & Vulnerable & Residert in small numbers \\
\hline Martial Eagle & Vulnerable & $\begin{array}{l}\text { Tsavo East is regarded as a stronghold of } \\
\text { this species, although the total numbers } \\
\text { may be small as home ranges cower a } \\
\text { great area (Srmeerak 1974) }\end{array}$ \\
\hline African Finfoot & Vulnerable: & $\begin{array}{l}\text { Recorded along the Tsavo and Galano } \\
\text { Rivers, where the banki are well } \\
\text { vegetated }\end{array}$ \\
\hline Vinted Wond-furepoe & Vulnerable & $\begin{array}{l}\text { Unconmon in riverine werodland on the } \\
\text { Galant and Tiva Rivers }\end{array}$ \\
\hline
\end{tabular}

\section{Conservation issues}

Wildite pondhing was a serous problem during the

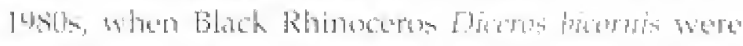

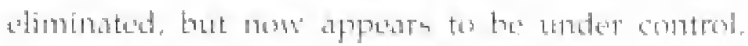

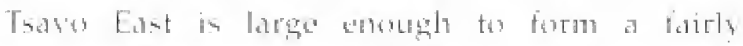

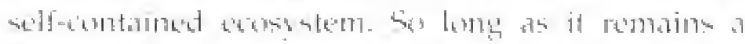

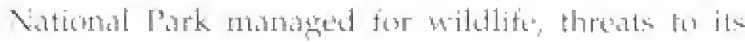
biodiversity are minimal.

\section{Further reading}

Hax 1903 , letuthold \& Leuthold 1976 ,

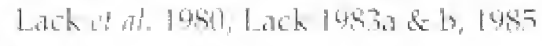

Vulturine Guneatowl 


\title{
25 Tsavo West National Park
}

\author{
$02^{\circ} 50^{\prime} \mathrm{S}, 38^{\circ} 10^{\prime} \mathrm{E}$, Coast IProvince, Tata-Taveta Districts; \\ Eastern Province, Kitui District
}

\author{
906,500 ha \\ Altitude: $600-1,800 \mathrm{~m}$ \\ Status: National Park
}

Categories: Globally-threatened species, Somali-Masai bione species

\section{Site description}

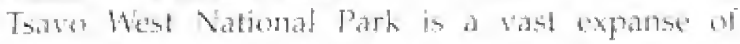
somannat stretching from the Athi Riser, borth of the Mombasa-Nairobi roak, seuth tes the Tanzanian bordere. The northeastern buandary along the sute atjoins Thavo Fast National l'ark (IBA 24), but Thave West has a more varied topagraphy and a nowe diverse array of habients than itm neighlasur. Must of

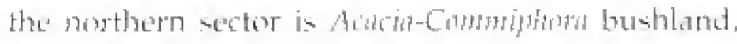

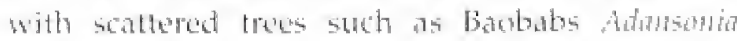

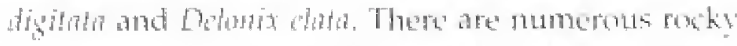
nutcops and rideses, and, tomards the Chyulu Hills, ast cones and lawa Bows - some th them bers fecent. In the Ninulia area. a range of cragers hill

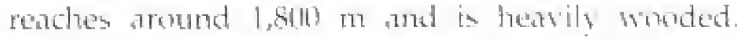
The suthern stetor compists af open grassy plains. The permanent Trase River rums theough the northere part of the l'ark, with a fribge ort riserine

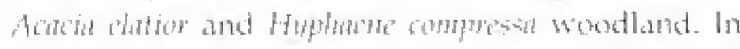
the far" south-western corner on the Kenga-Tanzusia border is laske lipe, part bit whith is in the Park. This

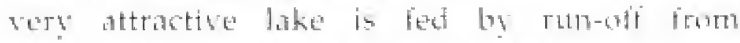
Me Kilimandaro and the North Pare muluntaind it is

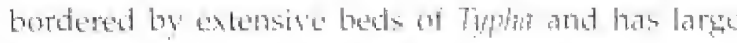
permanent swamps at its edstern and westerments At Mzima Springe, in the nerth of the Parh. Water that has riltered undergenund from the Chytu Hills

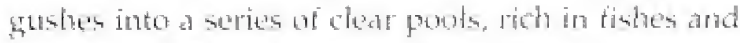

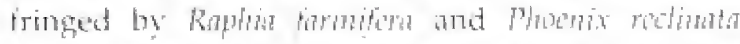

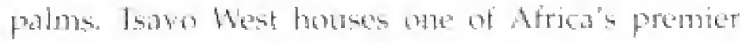

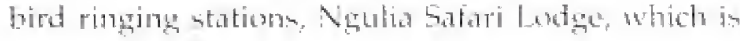

focated on the eatge of a dramatic escampment at the

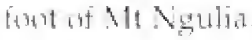

\section{Birds}

See box and Appendix 3 for key species. Tsavo West

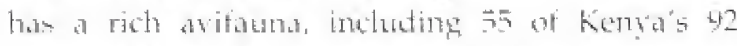
Simali-Masai biome upteice. The enismatio, near-threatened Friedmann's Lark his been recorded

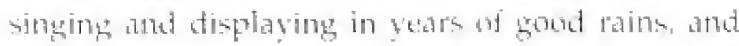
presemably mesth here. Tsino biost forms part of a corpidar of matural habitat in eastern kenya through which wast numbers of Palatartic birds migrate, especially iп Nowember/Detmber. Thest include tha threaterued Cormerake and

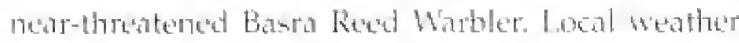
conditions at \gtalia safari Lodge are often such that thupterand we migating birds are altracted to the lodge lights at wight, and arumel 220,000 inctividuals have been renged here since fore. Lathe lipe supports an importunt heromes, where

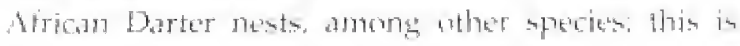

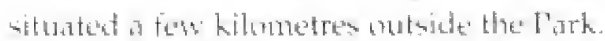

\section{Other wildlife}

Fom thest in well known for its population of large sabanmah mammale particularly African Elephant

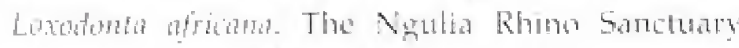

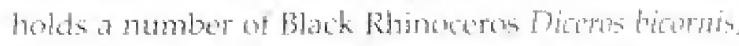
and other theatened wildife include Cheteth

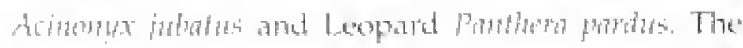

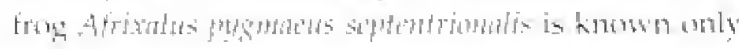

\section{Globally-threatened species}

Comcrake Vulnerable

Bastar Reed Warbler

Near-threatened

Friedmann's Lark
Near-theatened
Palaearctic pastage migrant, regularly saught at Ngulia Lodge (Zimimerritun et a. 1996, Pearson 1996)

Regularly ringed at Ngulia (Backhurst 1966), and a number of birds mav overwinter (Pearson 1997)

Probably nests in years of goot rains (Pentson it al. 1992, LAB umpubl. data) 


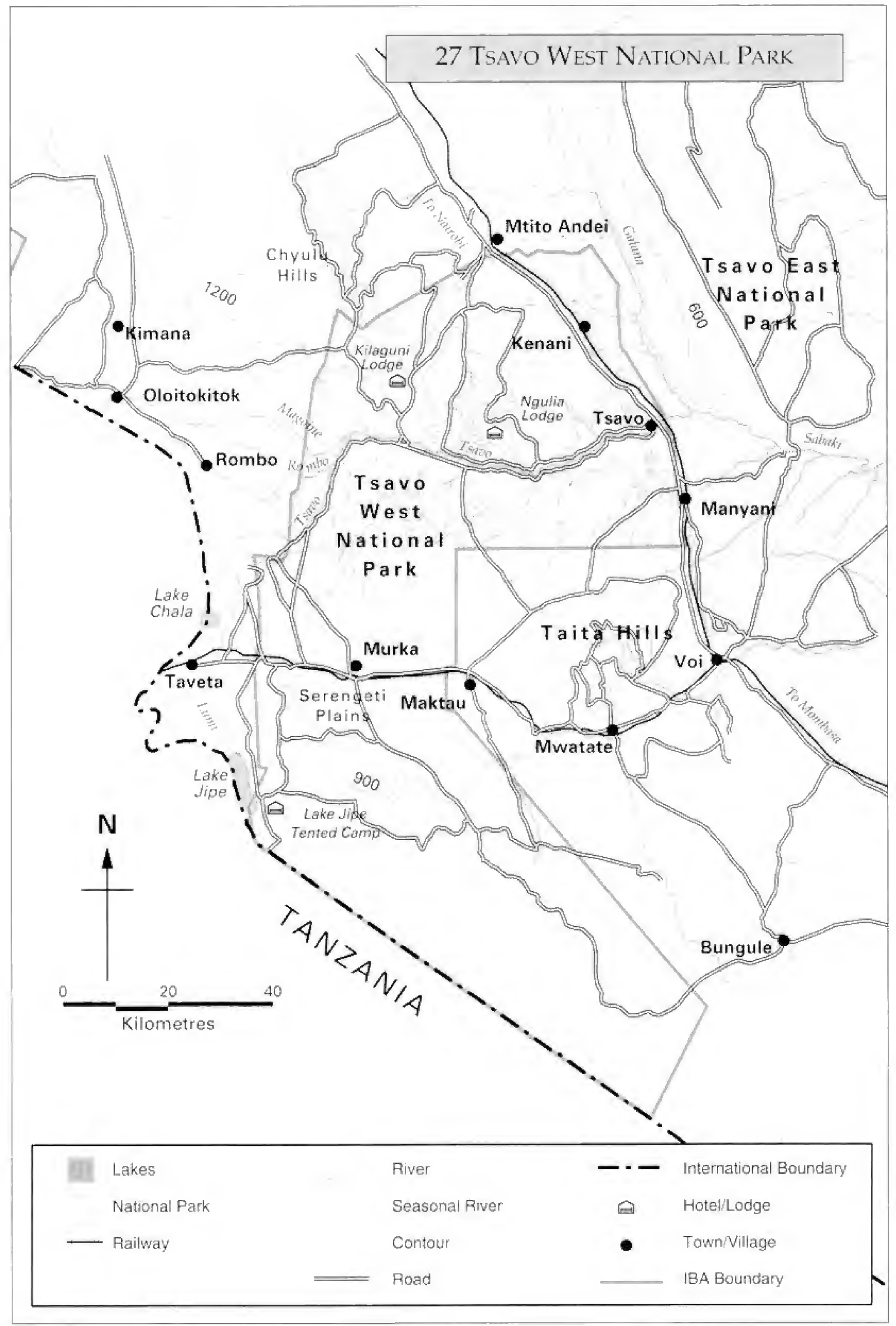




\section{Important Bird Areas in Kenya}

\begin{tabular}{|c|c|c|}
\hline \multicolumn{3}{|c|}{ Regionally-threatened species } \\
\hline Common Ostrich & Vulnerable & Common within the l'ark \\
\hline Great Egret & Vulnerable & $\begin{array}{l}\text { Small numbers necur at Lake lipe and } \\
\text { Maima Springs }\end{array}$ \\
\hline African Darter & Vulnerable: & $\begin{array}{l}\text { Small numbers occur at Lake lipe (where } \\
\text { they have nestet) and Mzima Springs }\end{array}$ \\
\hline White-headed Vulture & Vulneratile & Resident in small numbers \\
\hline Martial Eagle & Vulnerable & Resident in small numbers \\
\hline Blue Qนลว। & Vulnerable & Uncommon intra-African migrant \\
\hline African Finfogt & Vuliwerable & $\begin{array}{l}\text { Uncomnon on Tsavo River, where there } \\
\text { is thick fringing vegetation }\end{array}$ \\
\hline Violet Wood-hoopoe & Vulnerable & $\begin{array}{l}\text { Local and unammon in riverine } \\
\text { woodland }\end{array}$ \\
\hline Yellow-billed Oxpecker & Vulnerable & Fairly comrnon in extreme south \\
\hline
\end{tabular}

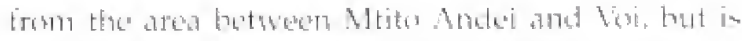

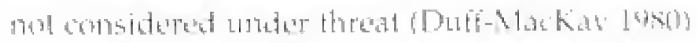

\section{Conservation issues}

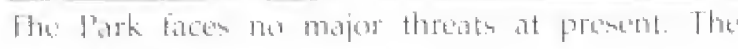

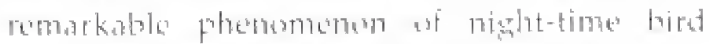

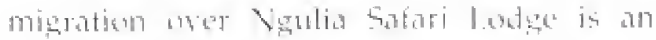

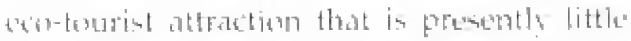

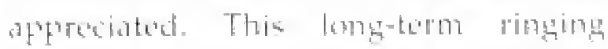

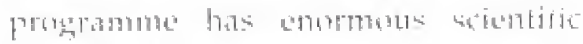

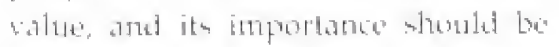
better recentised by the laak andminditration.

\section{Further reading}

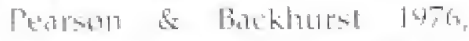

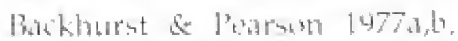
$1474,1480.1481,19832,12,1484$,

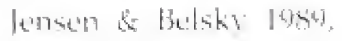

Backlukst 1940,

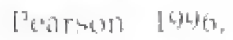

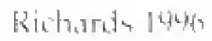




\title{
26 Chyulu Hills Forests
}

\author{
$02^{\circ} 35^{\prime} \mathrm{S}, 37^{\circ} 50^{\prime} \mathrm{E}$, Eastern Province, Makueni District and Rift Valley Province, \\ Kajiado District \\ 18,000 ha (c. 7,900 ha forest) \\ Altitude: c, $1,500-2,160 \mathrm{~m}$ \\ Status: National Park and (ungazetted) Game Conservation Area \\ Catogories: Globally-threatened spectes, restricted-range species
}

\section{Site description}

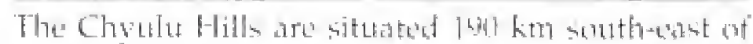

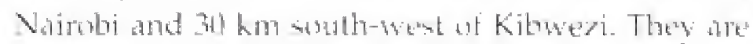
af rebatively recent valcance orighe and the ragege is

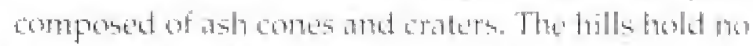
permanemt surtace water, but romatil percolatingt through the porous renck feeds many permantent fresh water sourem in the sterbanding plains,

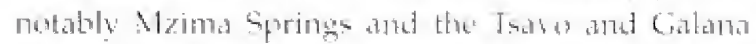
Riters. The hills are relatibly turdisturbet and still shelter indigenous regetation and witdlite. Router srassland and thicket yove wat to pateher of montane borest alomg the spirbe of the hills, mainly above the 1 , sod no contour: the largest ract of forest is around the hishest peaks in the central-southerr portion. Chateteristic trees include fictes sp..

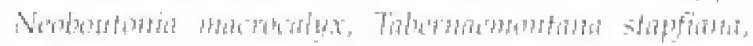

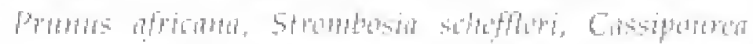

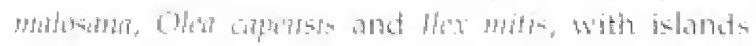

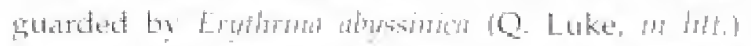

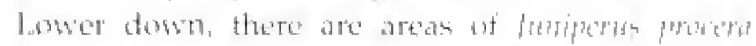
torest and. patticalarly on lava flows. Forest dominated by the bles-stemmed Comminhorat beluersis. The castern flank we the hills inclading about thalf eloe forent ared, is in the $+7,100$ ha Chyolus East National Park, administered by Kensa Wildife

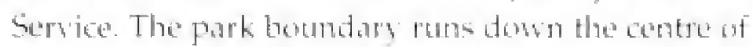
the hills along the lifne of the peaks. The western halt is part of the ungazeted Wost Chytulu Come? Consersation Area, owmed by sereral Maana group

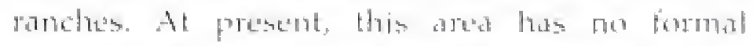
compervation status. The notiomal boundary of the IBA follows the $1, \overline{300}$ the combuter includime an area of bome 18000 ha of which around half is included in the Park and slighty under hate is torested.

\section{Birds}

see box her key' ipecies. 'The chytu Hills may bes an important stopping-point for Abbott's Statings as the mene betwerem forests on Ne Kilimanjare and the central Kenyan highlands. The hills hold

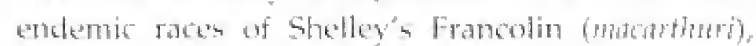

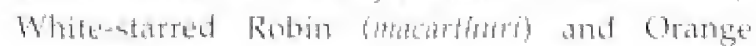

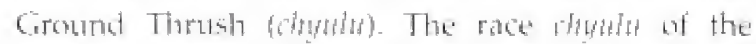
Cinnamen Bracken Warbler ho nuw generally merged with the race refophtitus of northern Tanzania,

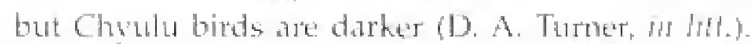
Var S(rmeren (1939) named 22 furthes Chylu Hills stib-spentes, trost of which are no longer considered alid, bes definitise taxonomic work remaitom to be dome.

\section{Other wildlife}

A harken of large mammals occurs on Chyulu 10. Luke inf fith), including African Buftalo Statome cathy Commen Fland Towrotraps orya, Bushbuch

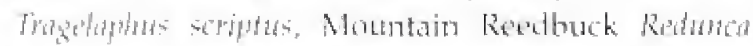

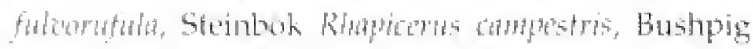

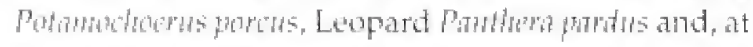

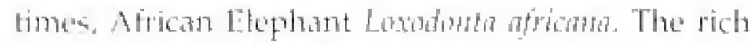

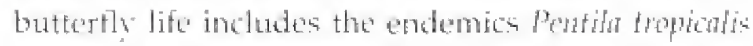

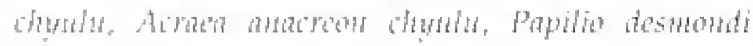

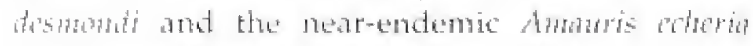

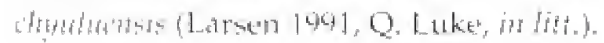

Onguing research on the vengetation (Luke, in prep.) gises some 5 s0 taxa, excluting the rumerous

\section{Globally-threatened species}

\author{
"Abbott's Starling \\ Vulturable
}

In the forest canopy, at times in large flocks; probably a seasonal visitor when forest trees are in fruit (van Someren 1939, Turner 1977, OS-c 1983a)

*also restricted-mine spateds 
Important Bird Areas in Kenya

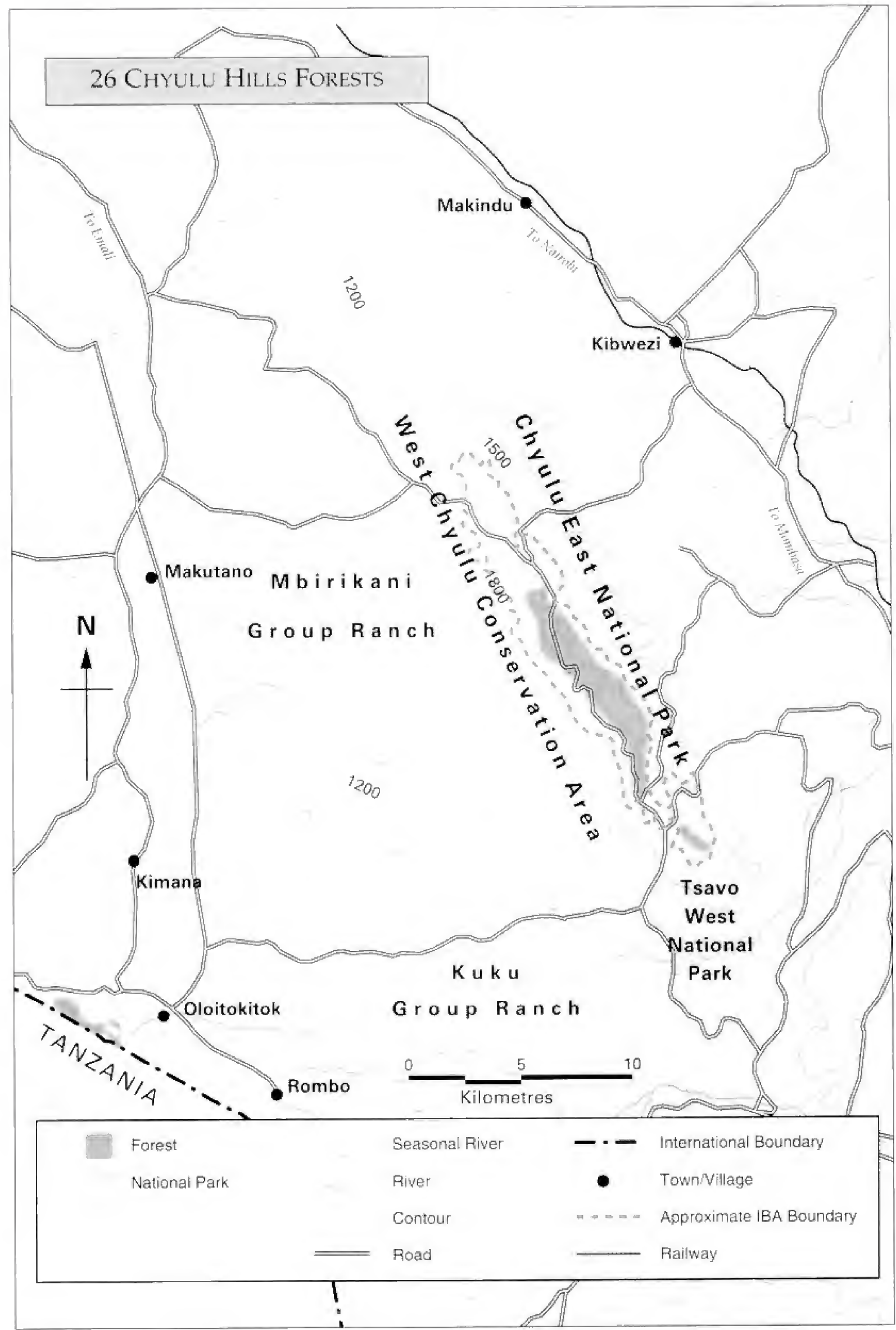




\section{Chyulu Hills Forests}

\section{Regionally-threatened species}

Ayres's Harsk-Fagle

African Crowned Faglo

Martial Eagle vulnerable

Vulretable

Vulnerable
Status unknown

Status unknown

On the lower slopes, outside forest grass"s, fmonget these ane 37 speries of ordids mostly epiphytes supported by the heave mists and

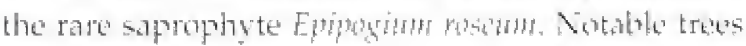

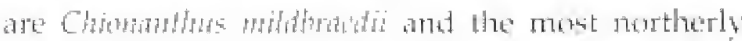

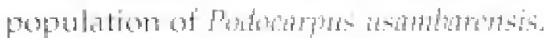

\section{Conservation issues}

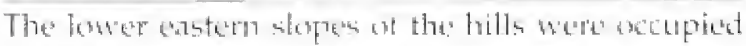
in the past by agricultural setalers, who were displaced in 1988 w make way for the National Park. This has fert tor libgering restentment amony those evicted, and difficultes in palicing use of the park. There hate been particular problems watti forest burning and eliting to eroourage sprouting af

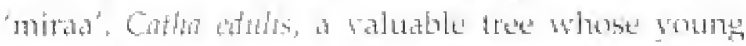
shoots ane used an a stimulant in parts of Kensa and Somalia. "Nirda" harbeting coutla well be' stataivathle it properly mamaged,

but National Park regulationes

do not allow for it to be

whacted lewatly. The hills

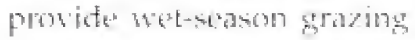

tor Natasi pastaralists from

the neatby group ranches

Recrutar burming of the

srassand is also caused by

meat pouchers eibher drixing:

game into snare lines or using the rusultant new grass as bait (O. T.zoke, in lith). It is debatable how destructive these annual grass fires are to the forests, guite possibly protecting them in the long run by enstung that Hammable bruste and litter do not bujd up to dangerous lesels. The hills bave gatat postertial for eco-tourism, but difficuties of aceess and the lach of surfake water tare kept this on a fimited scales sor. Chy dulu Lodge, on the northwest alopes, caters for sisitors interested in walking or harse-fiting in the hills, or bird-shooting on the plains below:

There has been little ornitholugical work in Chyulu sinct an East Afrita Natural History Suciety expedition in the 1930s (van Sumeden 1939). A bird conservation sursey of the forests is crevertue. It should fores on the status and reapuifemeston of Abtontt's Starling and the endemic sub-specios of robin and thrush.

\section{Further reading}

หan Somemen 1439

White-stared Robin

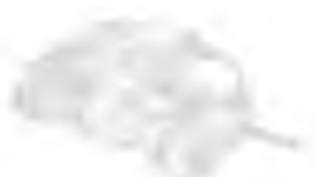




\title{
27 Dida Galgalu Desert
}

\author{
$02^{\circ} 40^{\prime} \mathrm{N}, 38^{\circ} 05^{\prime} \mathrm{E}$, Eastern Province, Marsabit District \\ Area whdefined
}

Altitude: $600-800 \mathrm{~m}$

Status: Unprotected

Categorics: Globally-threatened species, restricted-range species,

Somali-Masai biome species

\section{Site description}

A wat expanse of arid, inhopitable black lava deapert, stretching form arount $20 \mathrm{~km}$ north ar Matsabit town for at least 60 km ore cither side of the main Marabit-Moyale roat. The destributional limits of the special birds, and therefore the limils of the IBA, are unknowm. Rainfaly is erratic anct unpredictable, averaging less than 20 m m per veat Thu area is inhabited by the nomadic Cablora people. but there are no permanese settlements in thic latrols. waterlese terain. The desert's southern trimge is jub within the Marsabit Nafional Reserve, but it is otherwise unprotected.

\section{Birds}

See bux and Appendix 3 for key specien. Thin

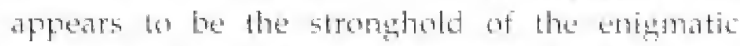
near-threatened, restricked-fange Williams's Isark, and most resent records have been trom this ares Other notable species include Heuglin"s Bustard, Somali Bee-eater, Masked Lark (probably the laryest population in Kenya: D. a. Turnes, in Pits.h, Theklas Lark, Chestnut-headed Sparrow-lark and fon bhe northerm fringes) White-cowned Starling Some +4

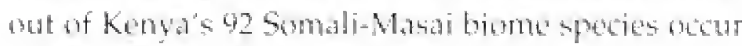

in the gemeral ared of Narsabit fand are listed in Appendix 1), but it is not known how many are plesert in the desert itself.

\section{Other wildlife}

No intortation is a wailable.

\section{Conservation issues}

Litele information is avalable, but this habitat is undikely to be under threat. Yery little is known. about Williams" Lark, and an ecological survey of this species would field useful information. The lath should also be fooked for on the slopes of Ne Narsabit the trpe lecalits if a ninter population is present there, the National Park could be incorpurated intin the $13, \mathrm{~A}$.

Star-sppotied Nightjar
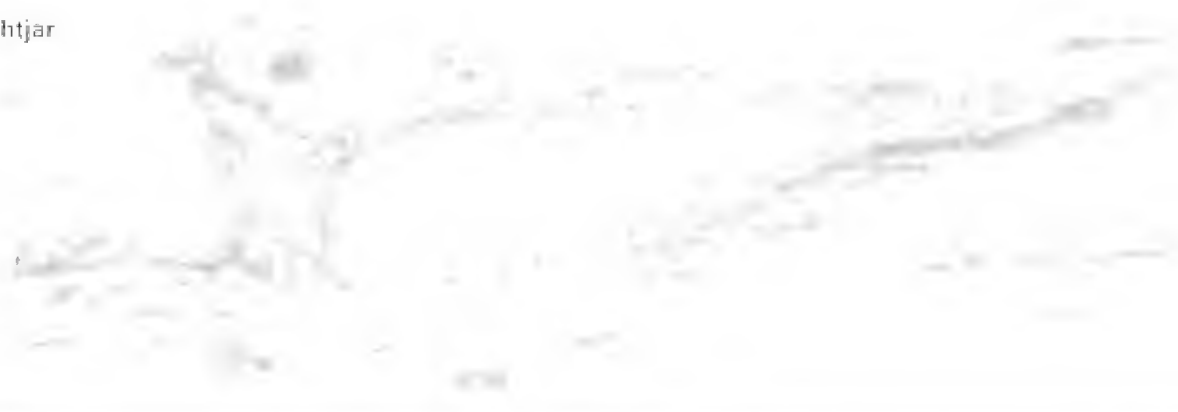

\section{Globally-threatened species}

\section{*Williams's Lark}

Near-threatened
Locally common but inconspicuous in racky lasa desert with sparse grass and low Butleria shrubs (Citmmerman it al. 1996, Turner 1998)

"aloo restricted-rame" species 
27 Dida Galgalu Desert

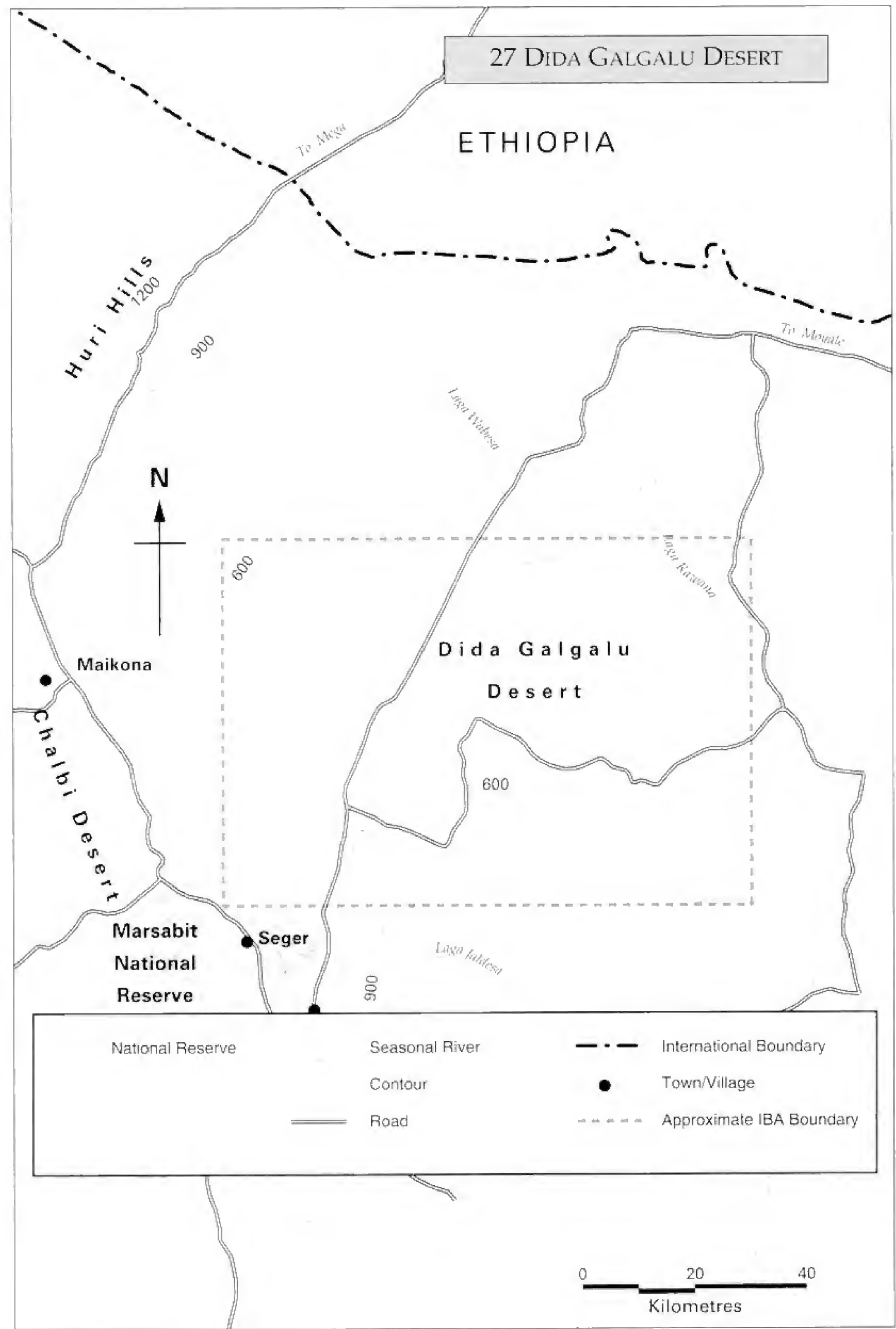




\title{
28 LAKE TURKANA
}

\author{
$2^{\circ} 27^{\prime} \mathrm{N}, 36^{\circ} 04^{\prime} \mathrm{E}$, Eastern \& Rift Valley Provinces, Marsabit and Turkana Districts \\ 756,000 ha \\ Altitude: $355 \mathrm{~m}$
}

Status: Mainly unprotected; two islands and c. $13 \%$ of shoreline are National Parks

Category: Somali-Masai biome species, congregations

\section{Site deseription}

A very large, isolated, chloro-carbonate alkaline lake, the rorthermonost ard by far the laryest of tha shain of Raft Valley lakes in keswa. The Ono kiver delta at the extreme northern sond of the liske lies within Elhiopta. Turkana's water is brackish, with a pH w 9.5-0,7, but drinkable, and the lake holds freshwater fish. The $600 \mathrm{~km}$ or so of lake shore vary greatly in substate, from rock most of the southern sector, the central castern shore, and North. Central and South Islands) to pebble, sand (most of the norbli-mestem shore, and patches whowheres athd mat bat

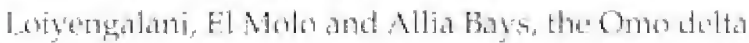
and the inlets of the Turkwel and Kerio Risers?. Beds

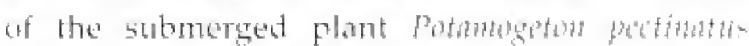
occur in the most shellered mudds bays. The country surmombting the lake is somi-ctesert with sparse vegetation: armat rainfall averages less than 250 mm (sulpstantialty less in some platest, alt it mav not ratu for several vears at a stretch. South and Central 1stands are Natomat Parks, and part of the norla-east shore is problected withon Sibilot Vational Park

\section{Birds}

See box for key species. Turkana is an watremely important waterbird site: 84 waterbird species, including 34 Palaearetio migrants, have berer

\section{Congregations}

More thin 20,000 whterhirts 220,000

$1 \%$ or more of biogentaphical population

P'ink-backed ['elican $(1,600)$

1,060

Greater Flamitgo $(1,250)$

2,580

Sptir-winged Plover (5,000)

6,930

Ringed Plover $(3,000)$

13,600

Caspian Plover (200)

500

Killitz's Ployer (1,000)

8,600

Little Stint $(10,000)$

113,000

Estimates from acrinl conms in Februtry 7992

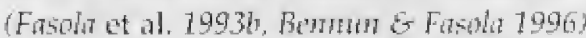

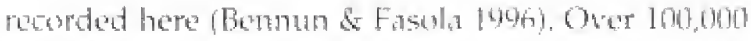
Little Stints may winter, representing more than I0 of the tentie East Arrican/South East Asian wintering fopulation (cl. Rose \& Satl 1997). As well as supporting many wintering Polateartic mistants,

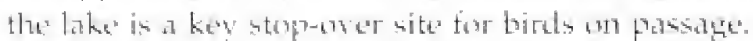
Waterbirds ate distributed all aresunt the lake, but the lighes dersities are on mud and pebble shores; particular concentrations occur in sheltered mudds

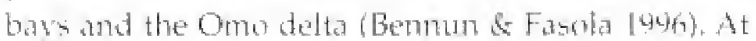

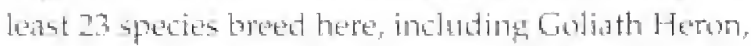
and up to 50 pairs of African Skimmex have bred on Central fatand (Lewis \& Poneros 1989). Thirty-four of Kentar 92 Sumali-Masai biome species occur, indading somali spatrow, wheth is net listed hot ans ather IBA. Fox Kestrel for the Sudan de Guinea

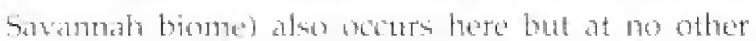
$1 B_{i}$ in Kentat.

\section{Other wildlife}

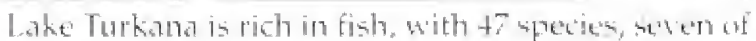

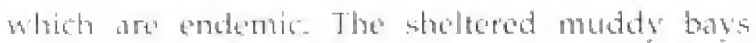

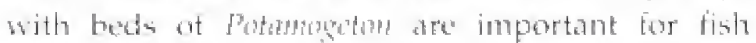

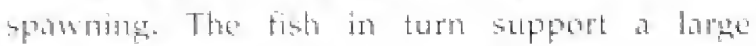
population cestimated at some 14,000 in 1968)

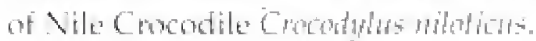

\section{Conservation issues}

Most of the lake has no formal protection, and in this wild and remote area the protection given by National Park status is largely nominal. Fortunately. firect human pressure on the lake is relatively fom. Turkana's water level has been droppitig stedily for

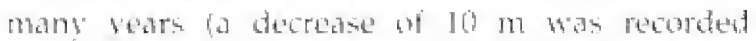
between 1975 and 1942), mainly due to ratuced intlom from the Omo River that supplies $90^{4}$ of of the lake' water. The Omo Dows trom the Ethispian Highlands where its flow has been diminished by irrigation projects and the effects of prolonged drought. Important Kenfan inflows, such as the Turkwel, have also bent stibntantially reduced in rexerty yous by hydropower and irtigation schemes. Some is and that supportet nesting waterbirds are

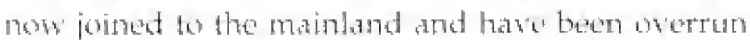
by guats. Disturbance by fisluermen seems to be a gencrat problem for the ishand-nesting birts (Davey 


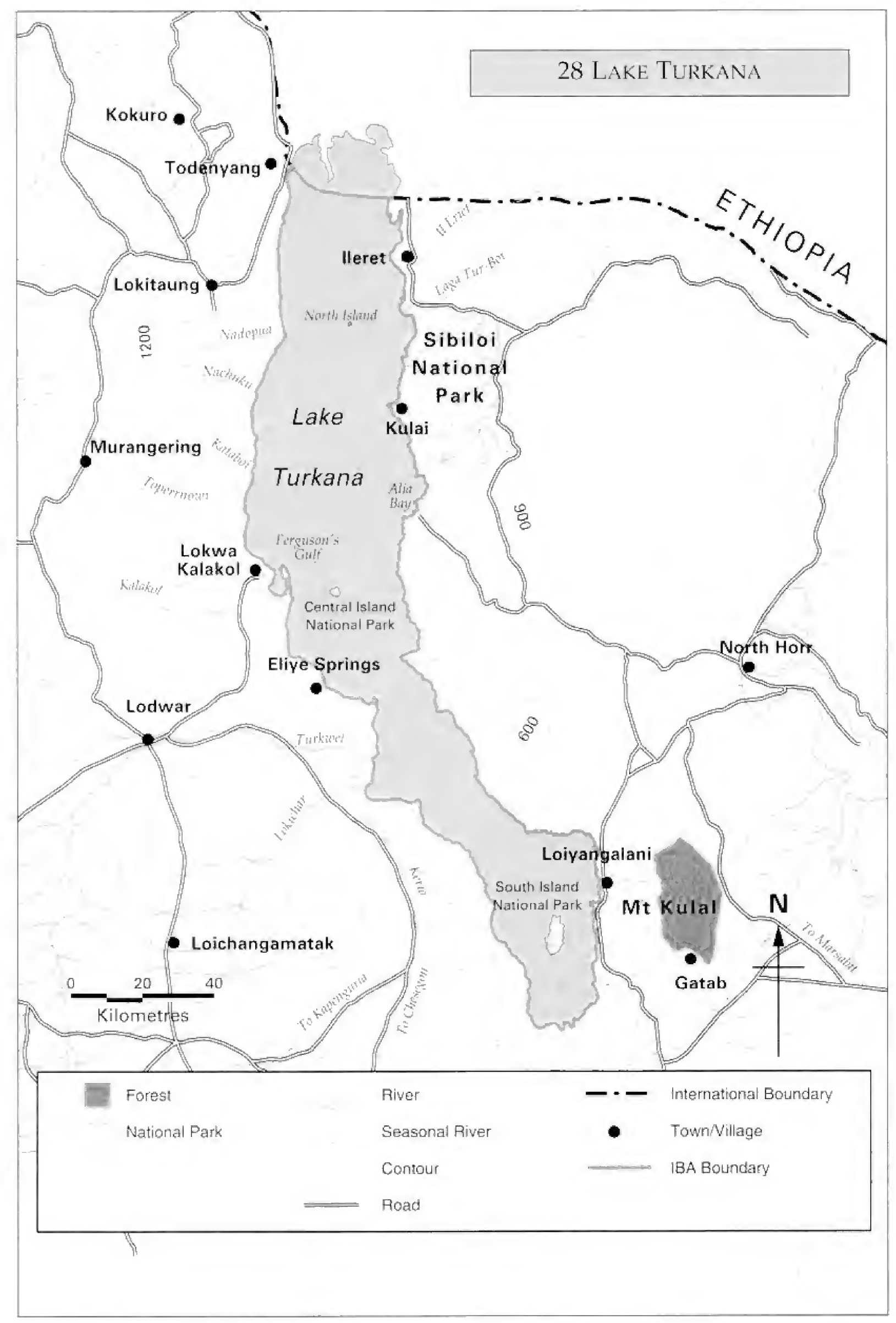




\section{Important Bird Areas in Kenya}

\section{Regionally-threatened species}

Great Egret

Saddle-billed Stork

Banded Snake Eagle

African Skimmer
Vulnerable

Vulnerable

Vulnerable

Vulnerable
Occurs in small numbers; 60 estinated in February 1942 (Fasola ed al. 1993b)

Nine estimated in Febrtary 1992 (Fasola at al. 1993b)

In riverine thickets

(1ewis \& Pomeroy 1989)

Up to 50 pairs once nested on Central Island, but have now shilted to less disturbed localities (Lewis \& Pomeroy 1989)
1982): in particutan", fistuermen's camps ot central lshand hase forced the African Skimmers that hested

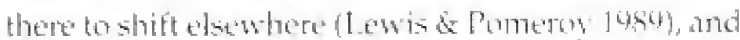
the present status of this breeding colomy is anctear.

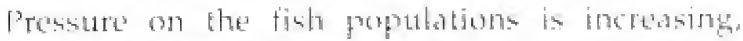
although attempts to institule industrial-seale

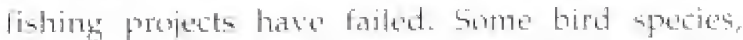

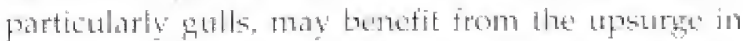
fishing and fishedring. Haman populatimo around

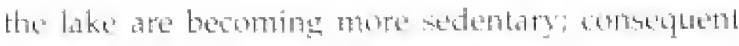

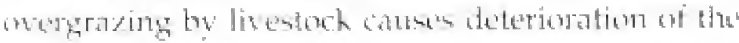

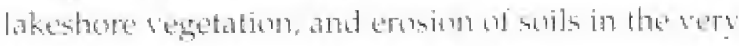
stures sind that characterise this artect.

\section{Further reading}

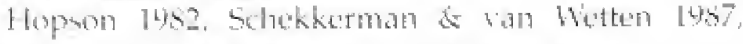

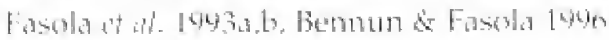




\section{MACHAKOS VALLEYS}

$1^{\circ} 35^{\prime} \mathrm{S}, 37^{\circ} 14^{\prime} \mathrm{E}$, Eastern I'rovince, Machakos District

Area undefined: at least 5,600 ha

Altitude: $1,300-1,600 \mathrm{~m}$

Status: Unprotected, private land

Categories: Globally-threatened species, restricted-range species

\section{Site description}

kiver and strean yalles is the catchment of the

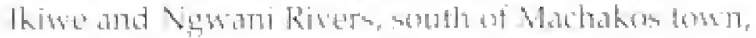

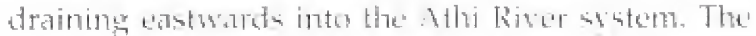

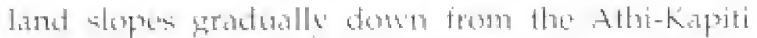

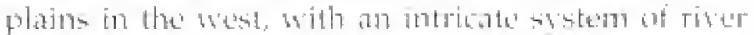

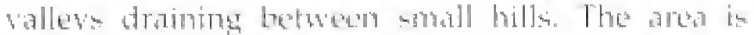

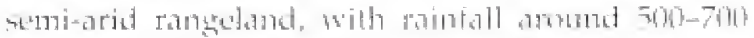

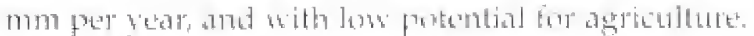

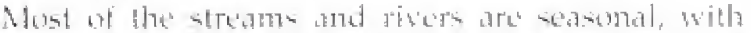

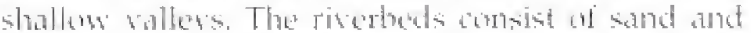
ruck, with a dense baent or bush and thicket forr about

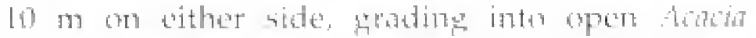

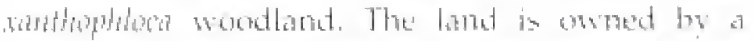

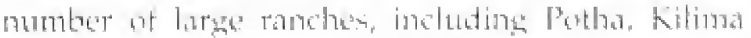

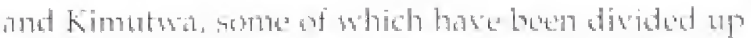

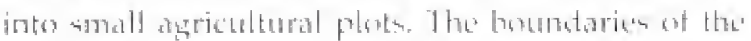

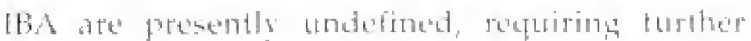

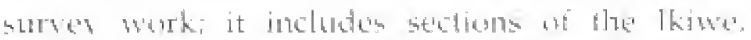

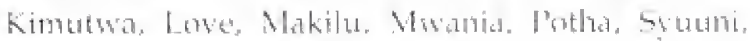
Wamua and bamuli Riters.

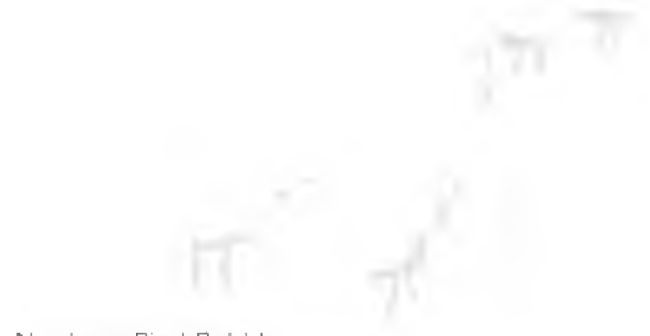

Northern Pied Babbler

\section{Birds}

The riverine thickets and wothdand shellet the

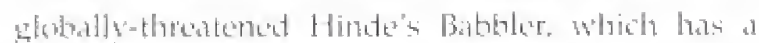

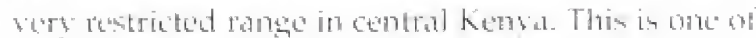
the lew sitter where it is knomen to occul in

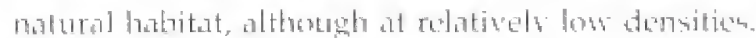

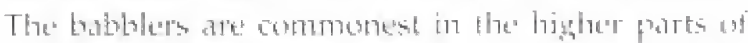

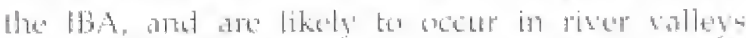
immediatels" to the south as well. The resit of the

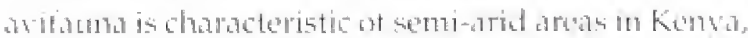
with 18 species from the Somali-Masil homb".

\section{Other wildlife}

So intormation in as allable.

\section{Conservation issues}

the area is mainty ramethand, but bs in ather

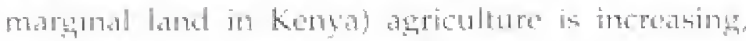
with athendant desteuction ont habitat. Of partictalar concern is the sub-disision of Jaget romethes inter

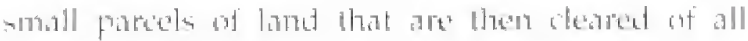
natural segetation. The riserime soudland anet

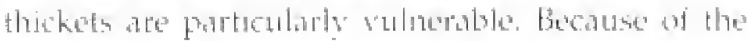

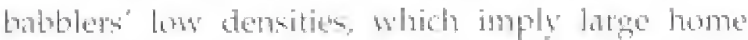

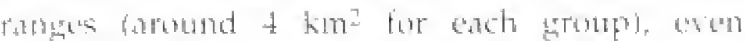
moderate habital lows might loat to serious pepulation detines comtrats the siluation in the

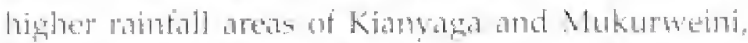
IBAs 2 and 6). Subsiatence hunting is also likely whe a threat to the babblete in this ares. More sursey whon is meded tometablish the extent of distribution af the babblers in this area, to muestigate land

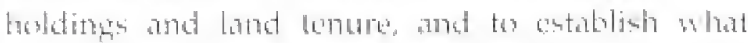
consereation mosures mat be fosible.

Further reading

Viorige \& Bentum, in presin

\section{Globally-threatened species}

"Hinde's Babbler

Endangered
Recent records but at relatively low densities (c. 0.5 birds and 0.24 groups per kn²; Njoroge \& Bennum, in pass)

"irlso restrictot-ramet spertes 


\section{Important Bird Areas in Kenya}

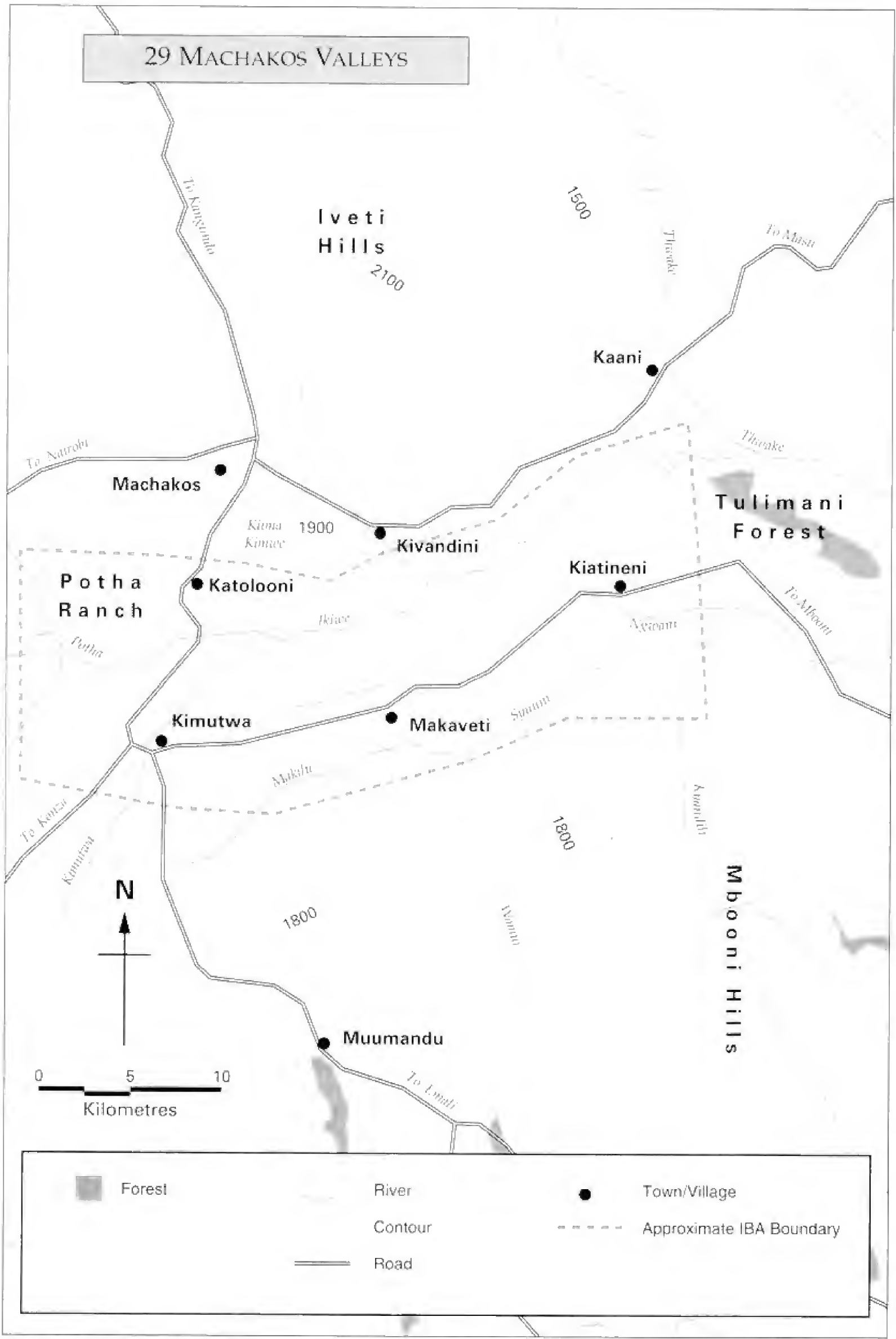




\title{
30 Masinga Reservoir
}

$0^{\circ} 49^{\prime} \mathrm{S}, 37^{\circ} 46^{\prime} \mathrm{E}$, Eastem Province, Embu District (set map in site acount 32)

\author{
c. 16,000 ha \\ Altitude: $1.050 \mathrm{~m}$ \\ Status: State owned \\ Category: Congregations
}

\section{Site description}

This is by ar the targest reservoit of the fire impoundments alome the upper Tana Riser; and

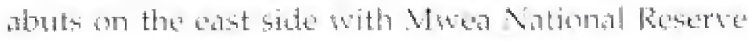
(IBA 32). Maringe dam. which is matraged by the Tand and Athi Risers Detelopment Atalnority

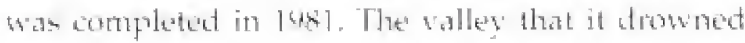
has a highly comoluted shomeline and contains a number of sizuble islands. The maximum depth is

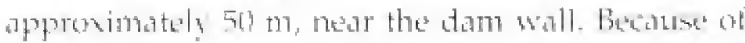

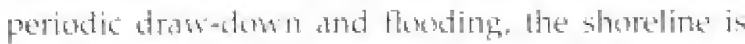

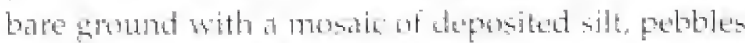
and mud. Further troug the sater, a torrose strip

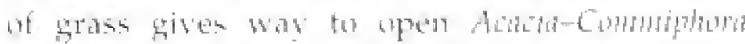
bushlatid. The ares is semu-arid with an annual raindall of between 250 and $5(40)$ mom.

\section{Birds}

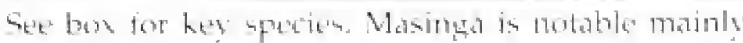

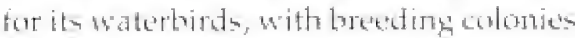
of Gormuratsts and Africin

Dartors. The atertstrial arifaura is not particularly distroe. but the threatened and restricted-range Hinde Bablabler lats beren pecorded in Acition thickets lon-20th m from the" existert shares of the aesersit

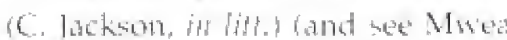
Nationall Reserse, IB, 32 ).

Great Cormoran:

\section{Other wildlife}

The dam supports substantial puputatione of

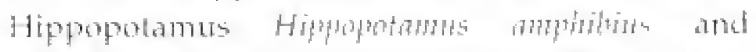

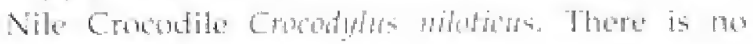
information on enther fauna ar hora.

\section{Conservation issues}

Subsistence fishing is widespread in the danc. And

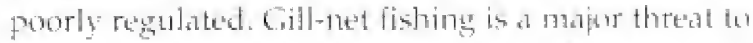
diving biats, particularly the African Darter - the 1995 sures found theer that had beet kntangled in

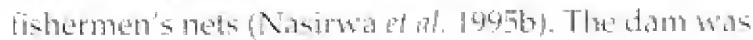
built in part to prowide irrigation water for agricultule, but poor land-uste in cultivated antas nuarty for teading to siltation and eutrophication from fertiliser run-oif. The waterbirt nesting colonises neted regular momitoringe and any constration plan for the Africas Darter slould focus on Masingat a d a key site.

\section{Further reading}

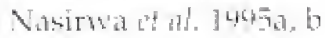

\section{Congregations}

More that 20,00) ataterlorits

27,900

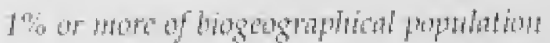

Great Cormorant $(1,000)$

2,150

White-winged Tern $(2,000)$

2,120

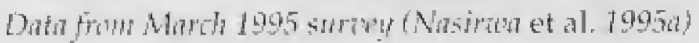

\section{Regionally-threatened species}

African Darter

Greint Egret

Nartial Fagle vulnerable:

Vulnerable

Vulnerable
80 counted in March 1945 (with 360 attogether on the five Upper Tand dams).

Masinga is one of the few known

Kenyan nesting sites

The count of 260 in March 1995

(Nasithat at al. 1945a) is notable

Status tmknown 


\title{
31 MERU NATIONAL PARK
}

\author{
$0^{\circ} 18^{\prime} \mathrm{S}, 38^{\circ} 25^{\prime} \mathrm{E}$ \\ 87,000 ha \\ Altitude: $370-910 \mathrm{~m}$ \\ Status: National lark \\ Category: Somali-Masai biome species.
}

\section{Site description}

Veru is a satantha National Park, $85 \mathrm{~km}$ wat of Nert

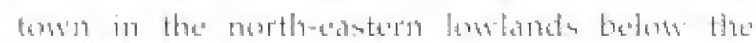

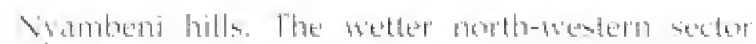

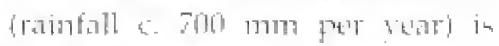
hills; with rich voteonic millis. The land Hatteds tomards the exat, wheres

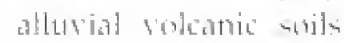
apperar. Thim alrea is orisent by

Eastern Yellow billed Hornbild numerous permanent streans, draining from the

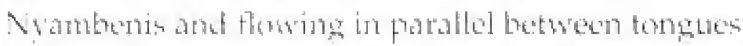

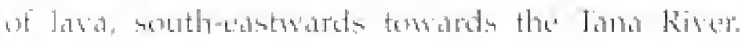

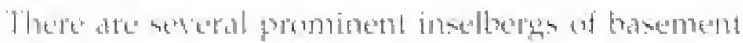

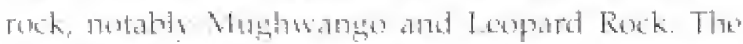
vengetation we the ridgtes is Compretum wooded

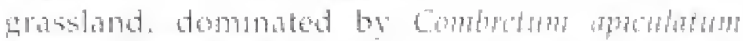

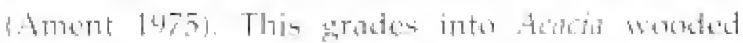

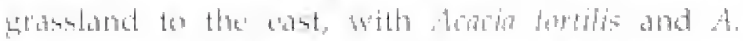

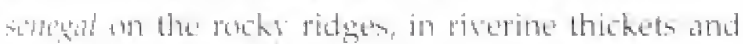

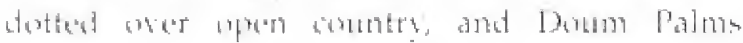

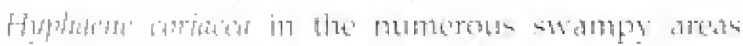

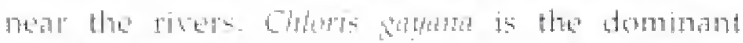

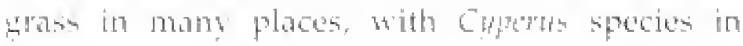
the sumps.

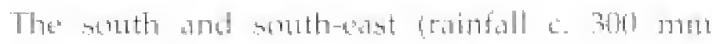

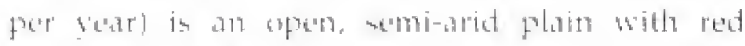
lateritis suil. This ares is anderes with rather

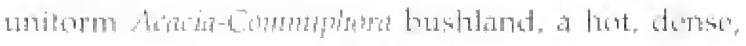

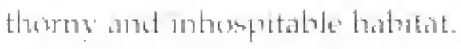

As well the that many streams that crose it, the

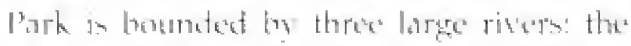

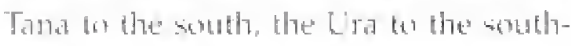

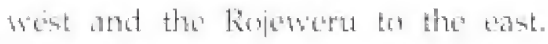
Riveriote trese inelude the palme

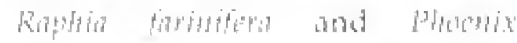

\section{Regionally-threatened species}

\begin{tabular}{|c|c|c|}
\hline Sadtle-billed Stork & Vulnesalote: & $\begin{array}{l}\text { Is knowir to brest (Zimmerman et al. } \\
\text { 1996) }\end{array}$ \\
\hline Martial Eagle & Vulnerable & Status unktrown \\
\hline African Finfout & vulnerable & $\begin{array}{l}\text { Recorded from rivers with thisk fringing } \\
\text { vegetation (Willians } 1967 \text {, } \\
\text { Dothoherty } 1994 \text { ) }\end{array}$ \\
\hline Tel's Fishing Oml & Vulnerable & In dense riverine forest \\
\hline Violet Wood-hoopore & Vulnerable & In riverine forest and woodland \\
\hline
\end{tabular}


31 Meru National Park

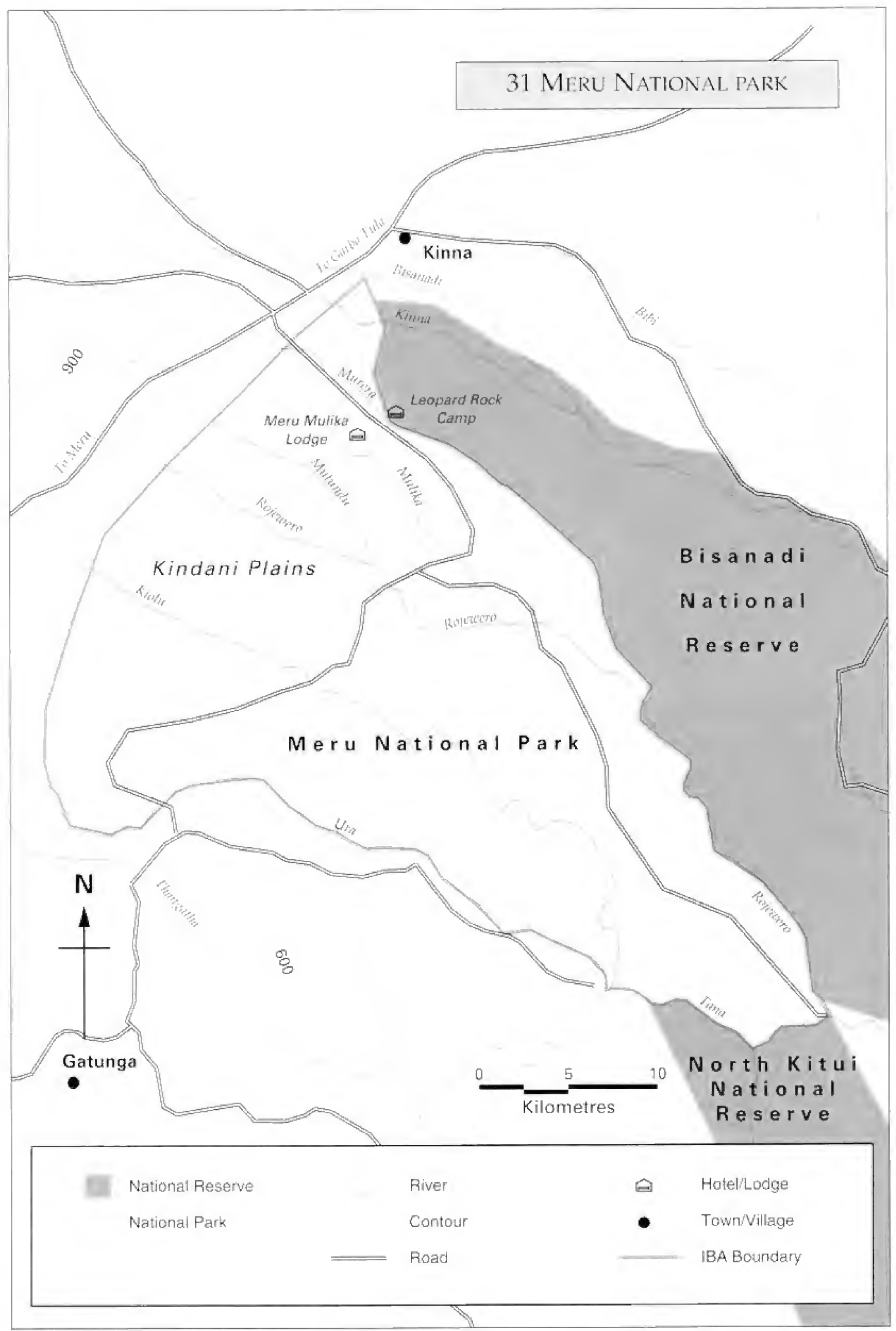




\section{Important Bird Areas in Kenya}

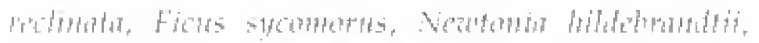

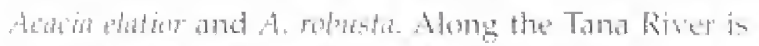

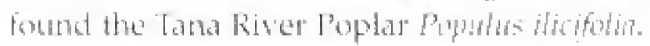

Mera is part of a complas of protected areas along the Tana Rivel that includes the adiacent Bisanadi and North kitui National Reseres th the cant and south, respectizely), Kota Nationsal Jark and Ralude Naticulal Re'se'tset. Thene areas are beter protected on paper than on the ground, hoserer, and their birdlife though generally little homant appears lese rith thath Meru's

\section{Birds}

Fift-sesen Somali-Masdi biome species necur ont of

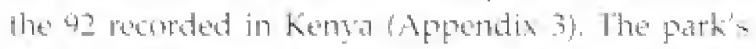
alsifiand is gemerally diserese with around 280

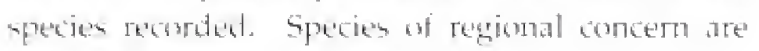
shown in the bos.

\section{Other witdlife}

The park hoddengod poputations of harge manmals. Encluding the theatened Arican Elephant Lowotenta

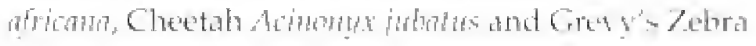

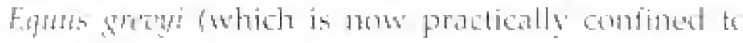

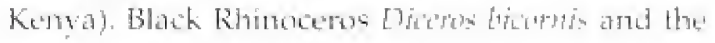
introduced Square-lipped or

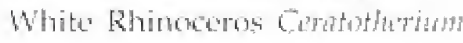

simn have been eliminated

by poraterest.

\section{Conservation issues}

Lithe othere National Parks, Neru is protected by

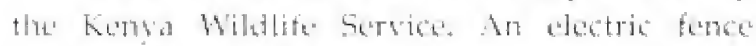

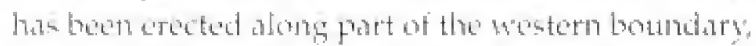
(1) prevent mintals moning inter farmand. One section of the pate has berey designated at a

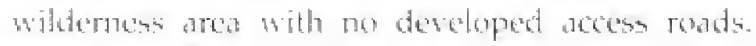
Fere has mudh to attract the wistore and was a popular toutist destimation in the past but insectrity and porching (now both undet comtrol), freme accese roate and dilapidation of facilitien hase catsed sisitor mumbers to decline

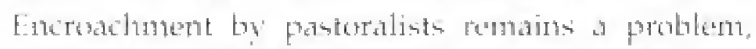
but is reneral this site races fow immediate agnerosation threats.

\section{Further reading}

Ament 1975, Dumbery 1494

Rosy-patched Bush-shrike

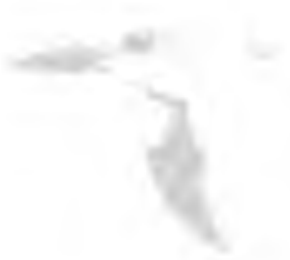




\section{Mwea National Reserve}

$0^{\circ} 50^{\prime} \mathrm{S}, 37^{\circ} 40^{\prime} \mathrm{E}$, Eastern Prowine, Mbeere District

\section{4,200 ha}

Alfitude: $1000-1,100 \mathrm{~m}$

Stofus: National Reserve

Categories: Globally-threatened species, restricted-range species

\section{Site description}

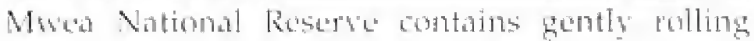

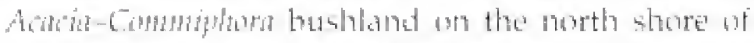

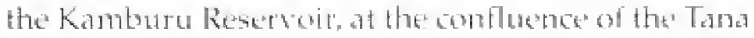
and Thiba Rivers, $100 \mathrm{~km}$ north-west of Nairobre This mall Remerve lies just cast bit Nasinga Reservoir (IBA .32). Within its borders are 700 ha (including two

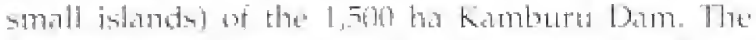

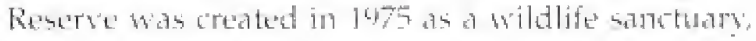
and is ownet by the Hbeere Gounty Councit (permating District condirmation' and managed by the Kenva Wildife Serke. The area is semi-arid with an anumat raintal] of between 250 and $5(30$ mm. Thick bush and scattered treas, including Bantab

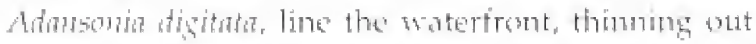

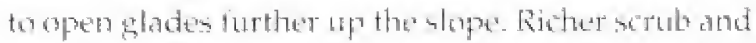

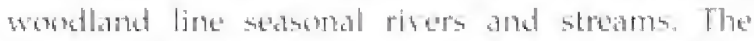
Reserve is essentially undevelopent. There is a campsite and around $45 \mathrm{~km}$ of rough roads, but nor accomombdation.

\section{Birds.}

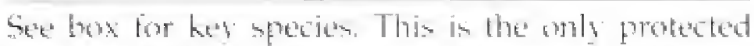
area in which the shoully threatened Hinde"s

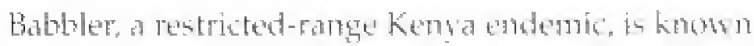
to oceur. Populatione ore the babbler have riot be'te assesed. This is also a rich localiby isy birds gernerally, especially birds of prey, and is close bo

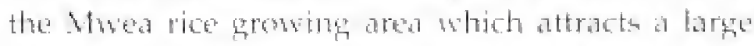

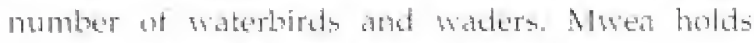

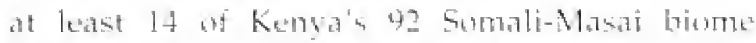
speries. and more are likely to be added in futures.

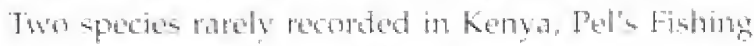
Owl and White-backed Night terom, ocus here in fiverine soodland.

\section{Other wildlife}

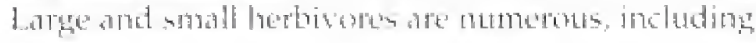

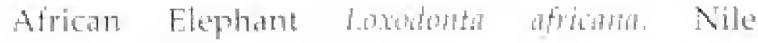

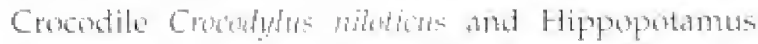

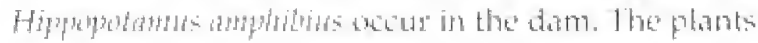

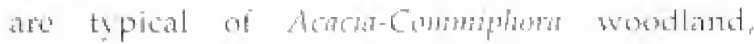
including many Combertism species and trees of

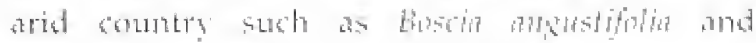

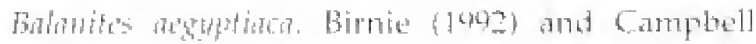
(1042) grive further infomation an the flost.

\section{Conservation issues}

Conservation problems in Musea sentre on

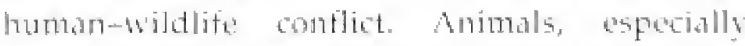

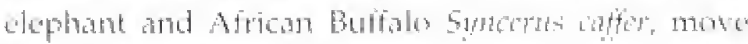
oet the Reserve is deratroy cops in the settled areats nuarby: The kenya widdife Gervice is in the process of preparting a Marbegenent Plan fore the Reserest, fotlowing an bom a plinning workshop held in June 1994, One of the steps then agreed was du construct an eletric fence around the lamet boundary, both to prexent crop damase by anmals and to present wanthorised access, destruction of wees and puaching by people. Whork on the fence and on a new Park Headgunters building and

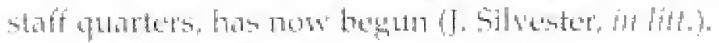

The wephant papulation, which numberes about 45 in 1955, is in any case felt to be tou large for the sise of the Reserse. Translocation of ellophanty brogan in late 1405, when a family stotap of eive and a singlge bull were

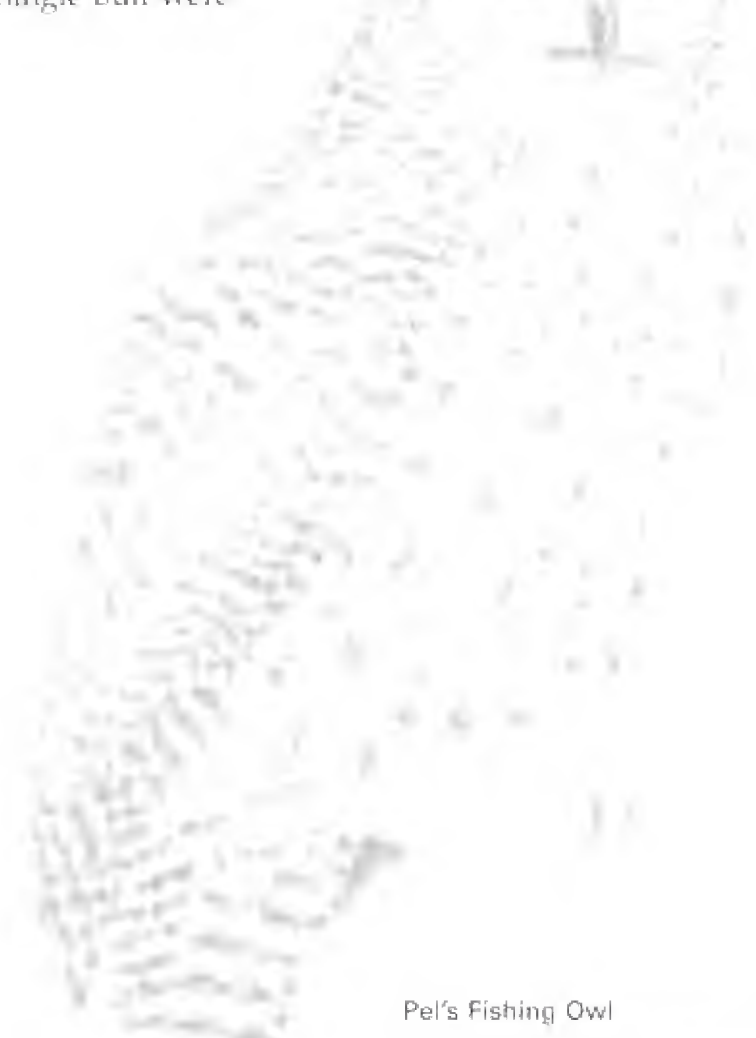


Important Bird Areas in Kenva

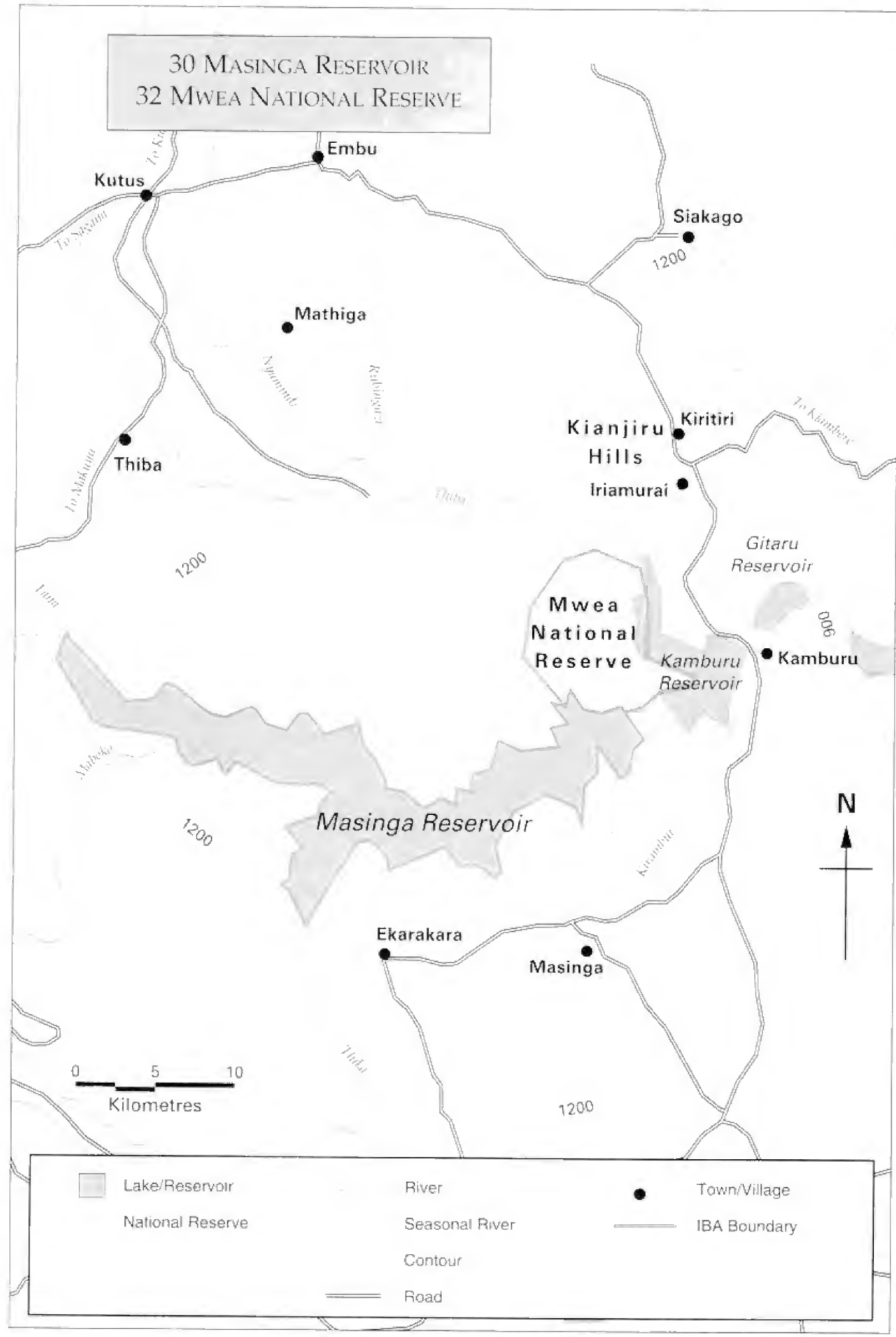




\section{Mwea National Reserve}

Globally-threatened species

"Hinde's Babbler

Near-threatemed
Recently observed (C. Jackson, in lifh. Zimmerman of at. 1996)

An uncommon non-breeding visitor, May to October (Zimmerman et al. 1996)

*atso lestricted-ramere species

\section{Regionally-threatened species}

African Ditter

Martial Eagle

Pel"s Fishing Owl vulimable

Vulnerable

Vulnerable
Nests on Masinga Reservoir to the west

Status unknown

Reconded in dense tiparian woodland

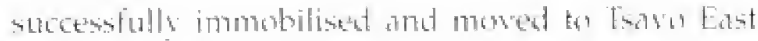

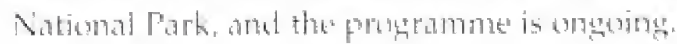

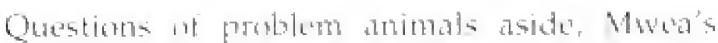

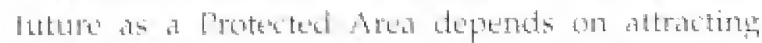

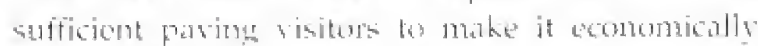

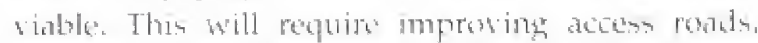

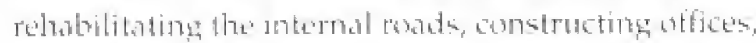

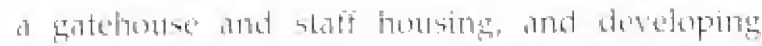

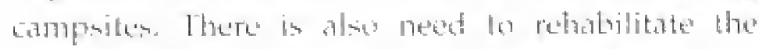

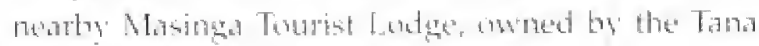

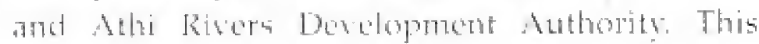

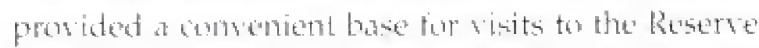
but is premently alosed. Given the impartase of

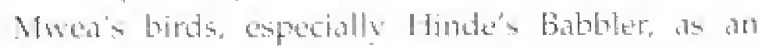

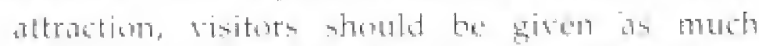

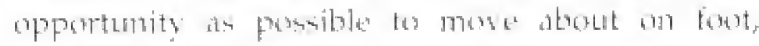

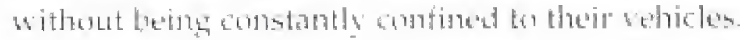

Pollutiog of the lasa Riser by articultural motustrits upstratm is a problem, causing concerm

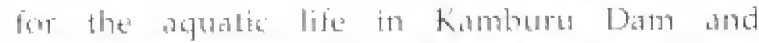

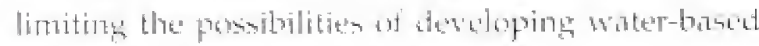

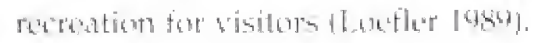

Tha density distribution and habitat selection of flinde's bablser in the Reserve are presently

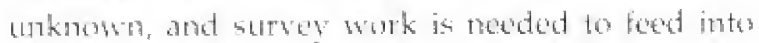
the manotgentent process.

Marea is fortumate in that a comedration charite

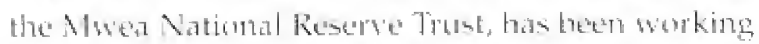

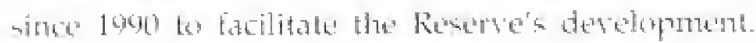

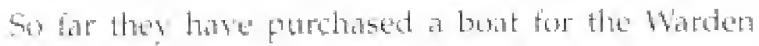
and stafi, to us for patrots and for waterfont

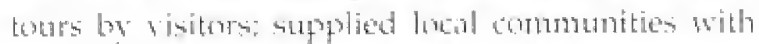

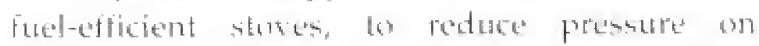

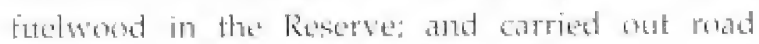

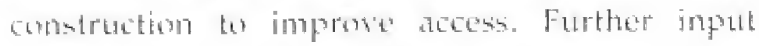
from the linst must await finalisation of the managenent planning process.

\section{Further reading}

Loetler 1489, Bimie 1992, Camplodl 1492, Le Pelley 1492. Näaแ-joา 1496 


\title{
33 Samburu and Buffalo Springs NATIONAL RESERVES
}

\author{
$0^{\circ} 40^{\prime} \mathrm{N}, 37^{\circ} 30^{\prime} \mathrm{E}$, Eastern P'rovince, Isiolo District and Rift Valley Province, \\ Samburu District \\ 29,600 ha (Samburu 16,500 ha, Buffalo Springs 13,100 ha) \\ Altitude: $850-1,250 \mathrm{~m}$ \\ Statws: National Reserves \\ Category: Somali-Masai biome species
}

\section{Site description}

These small, adponing sationa National Reserten lite e'ther side of the Northern Ewase Ne'ino River,

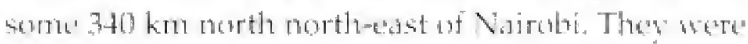
established in 1948 as the Samburu-lsiolo Came Remerte, pat of the ontee extersine Marsabit National Reserve. Now they are managed by their respective County Councils, Samburu and Isinto. Buffaio springs consists of gently rolling plains of holcanic arigin, while 5 ambure is more ruged, with outcreps of ancitat basement rocks forming isulated

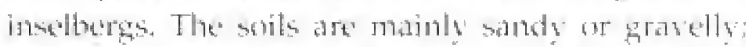
rainfall is around 350 mom per year:

Despite their relatively small size, the reserves contain cemtrasting habitats: rocky clifts and sorps:

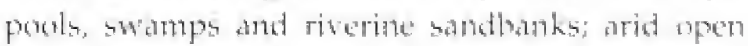

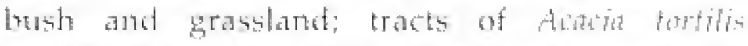
woodland; and, along the banks of the Ewase Ng'iro and its tributaries (the Isiolo and Ngare Mara) of narfow band of riserine forest with

magnificent Achotio rofios, Tana

River Poplar Paptules idofolda

and Doum Palm Huwhere

atrmposin. The Buflalo
Springs themselves, with therie pools and streame of

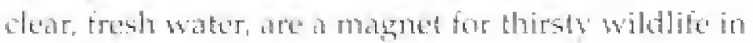
the drat setastor)

\section{Birds}

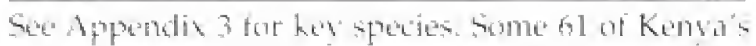
92 Somali-Masal biome spectes ocent here, and biadlife is generally abundant and colourtul; oret

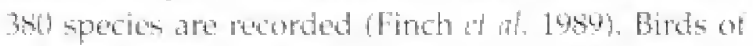
the arid northern buth country are augmented by a number bf riverine forest species. Numerous raptors

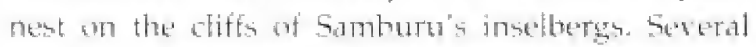
aridecontry birde reath their southern limil here, including Donaldson-Smith's

Sparrow keaver, shinimer

Sunbird and Briste-crowned

Starling, Rexgionally

throatemest species

are shown in the

box. In addition, 


\section{Samburu and Buffalo Springs National Reserves}

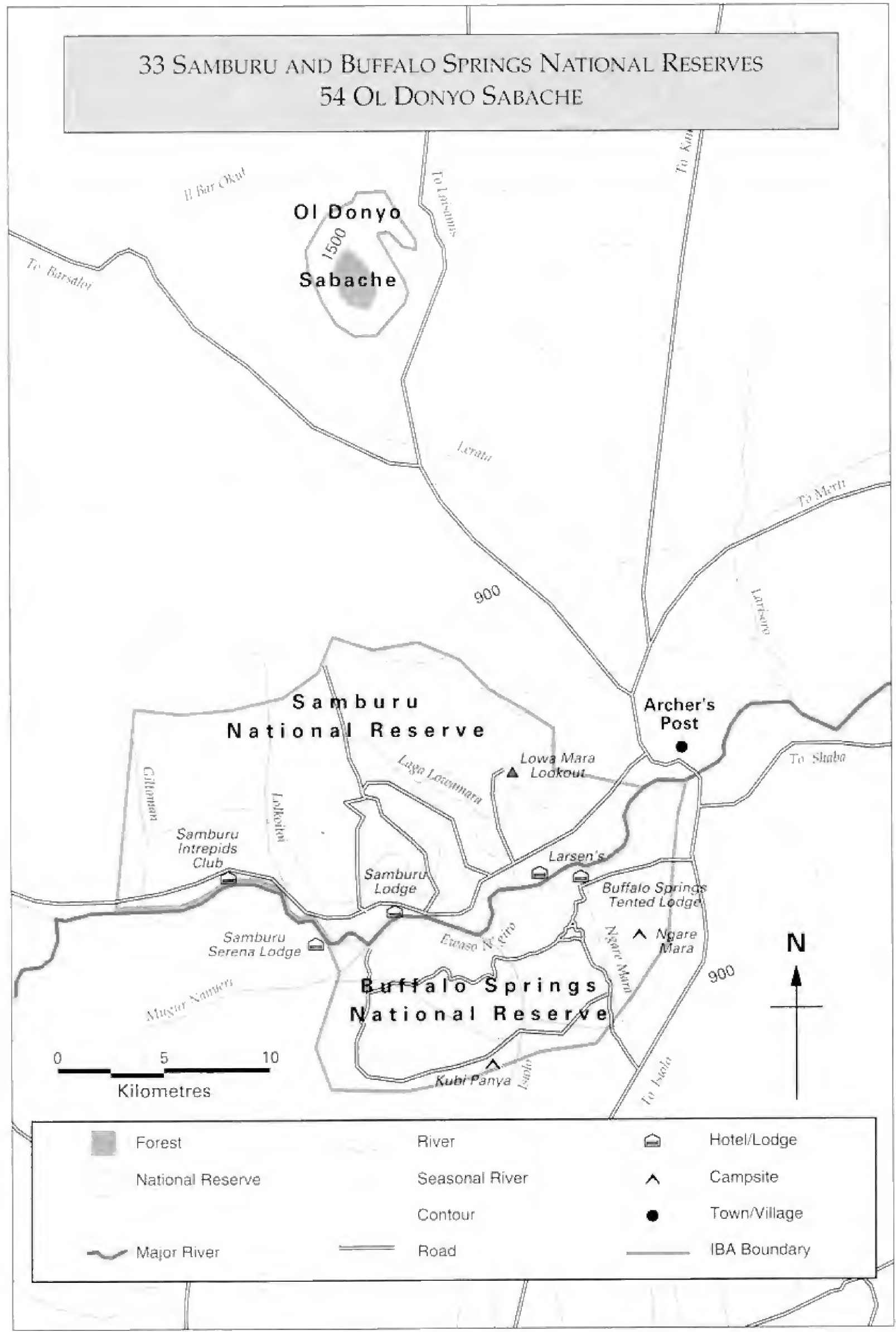




\section{Important Bird Areas in Kenya}

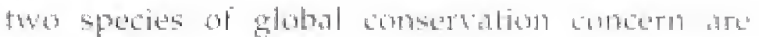

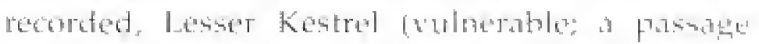

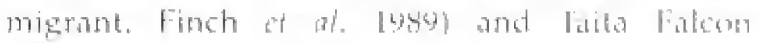

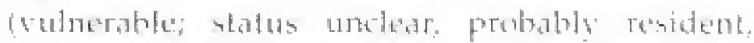

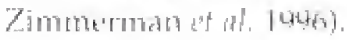

\section{Other wildlife}

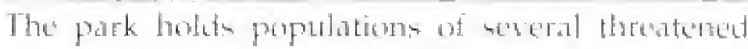
mammal species, including Grev's Zutar Eapus

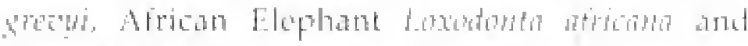

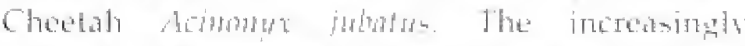

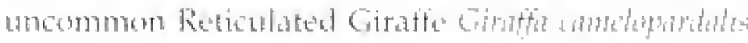

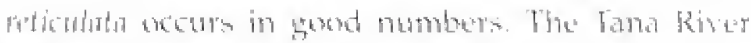

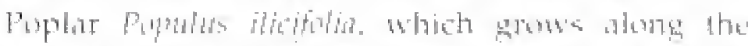

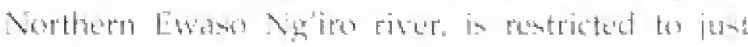
three Kengan riger stotems.

\section{Conservation issues}

The renteres an a popdat tourist dentination, the

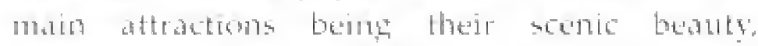
remotences allat compentrations of photengente

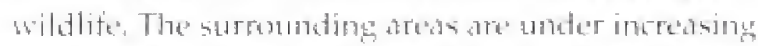

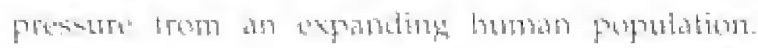

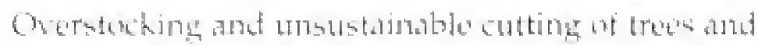

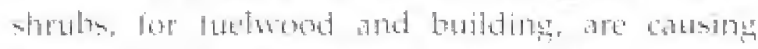

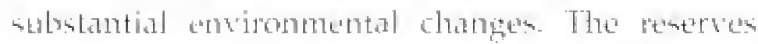
Hencelses remain wall protected, and there are fen

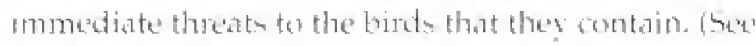

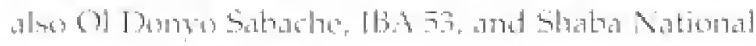

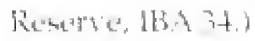

\section{Further reading}

Wilsun lastr, Richards lyos

\section{Regionally-threatened species}

\begin{tabular}{|c|c|c|}
\hline African Darter & Vulnerable & Sporadic visitor \\
\hline Great Eygret & Vulnerable: & Sporadic visitor \\
\hline White-headed vulure & Vulnerable: & Resident in small numbers \\
\hline Martial Eagle & Vulnerabt: & Resident in small numbers \\
\hline Yellow-billed Oxpecker & Vutnerable & Fairly common fesident \\
\hline
\end{tabular}

From Find et al. (1989), Zimmemm et al. (1996) 


\title{
34 Shaba National Reserve
}

\author{
$0^{\circ} 34^{\prime} \mathrm{N}, 37^{\circ} 57^{\prime} \mathrm{E}$, Eastem Provine, Isiolo District \\ 23,900 ha \\ Altitude: $700-1,500 \mathrm{~m}$ \\ Status: National Reserve \\ Catcories: Globally-threatened species, restricted-range species, \\ Somali-Masai biome species
}

\section{Site description}

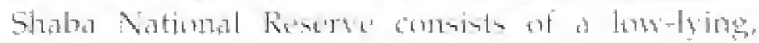

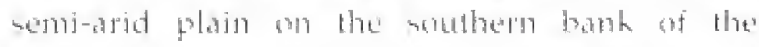

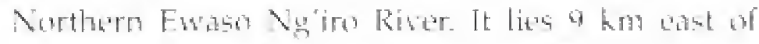

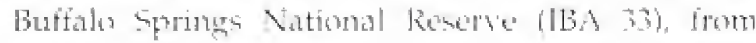
which it is separated by the main road trom fsiolo to Marabit. Shabo was mazetted in $197 t$ and is administered by Ho Findo Count Council. It5 northern section indudes a $3 t$ hm stretel

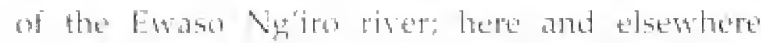
in the Restrie are numetols spribes and swampy areds, although some hase bitter-tasting water. The starkly treatiful landsape is demunated by shabre Hill to the suthth, at the

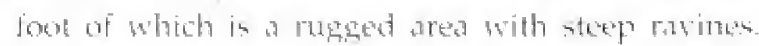

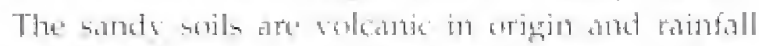
is some $250-5170$ mom pese veats. Habifats in the Reserse include rovertic bendand and thicket

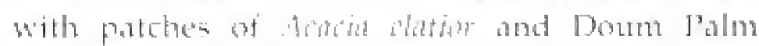

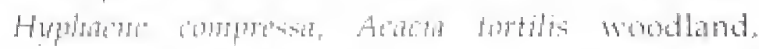

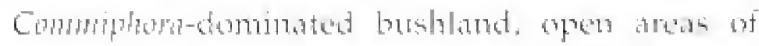
lasa rock sith soatlered grass and shrutes,

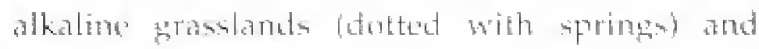
swamps.

\section{Birds}

Sex box and Appendix 3 for key spectes. This is the waly protected area in whith the wongatic near-threatered. restricted-range Williams's Lark is knower to octur. Out of the 92 Somali-Masai

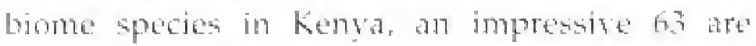

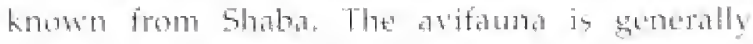
singiar w that of the neaby samburu-Bultala Sproting National Reserves (IBA 33). Sumall numbers of the globally threatened Leser Kestrel (rulnerables) prose through shatm on migration each bear from the Palateartic (Find et al. T486)

\section{Other wildlife}

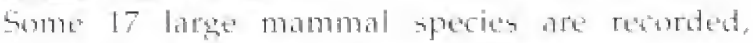

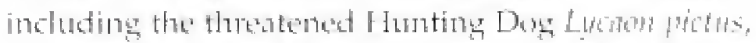

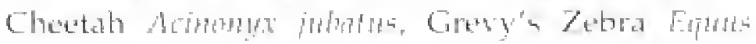

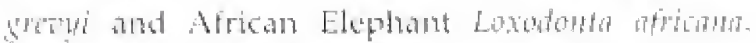

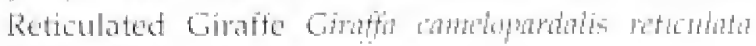
also ocours.

\section{Conservation issues}

Apart from ite dinersity of Somali-Masal biome

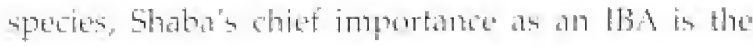

\section{Globally-threatened species}

\begin{tabular}{|c|c|c|}
\hline "Williams's Lark & Near-threatened & $\begin{array}{l}\text { Local in rocky lava semi-desert with low } \\
\text { Bartorin strubs (Tumer 1998) }\end{array}$ \\
\hline
\end{tabular}

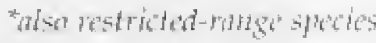

\section{Regionally-threatened species}

African Darter

Great Egret

White-headed vulture?

Martial Eagle

Yellow-billed Oxpecter
Vulnerable

Vulnerable

Vulretable

Vulnerable

Vulnetable
Sporadic visitor

Sporadic visiter

Resident in small numbers

Resident in small numbers)

Fairly common resident

From Find et al (1989) nut Zimmerman at al (1996). 
Important Bird Areas in Kenya

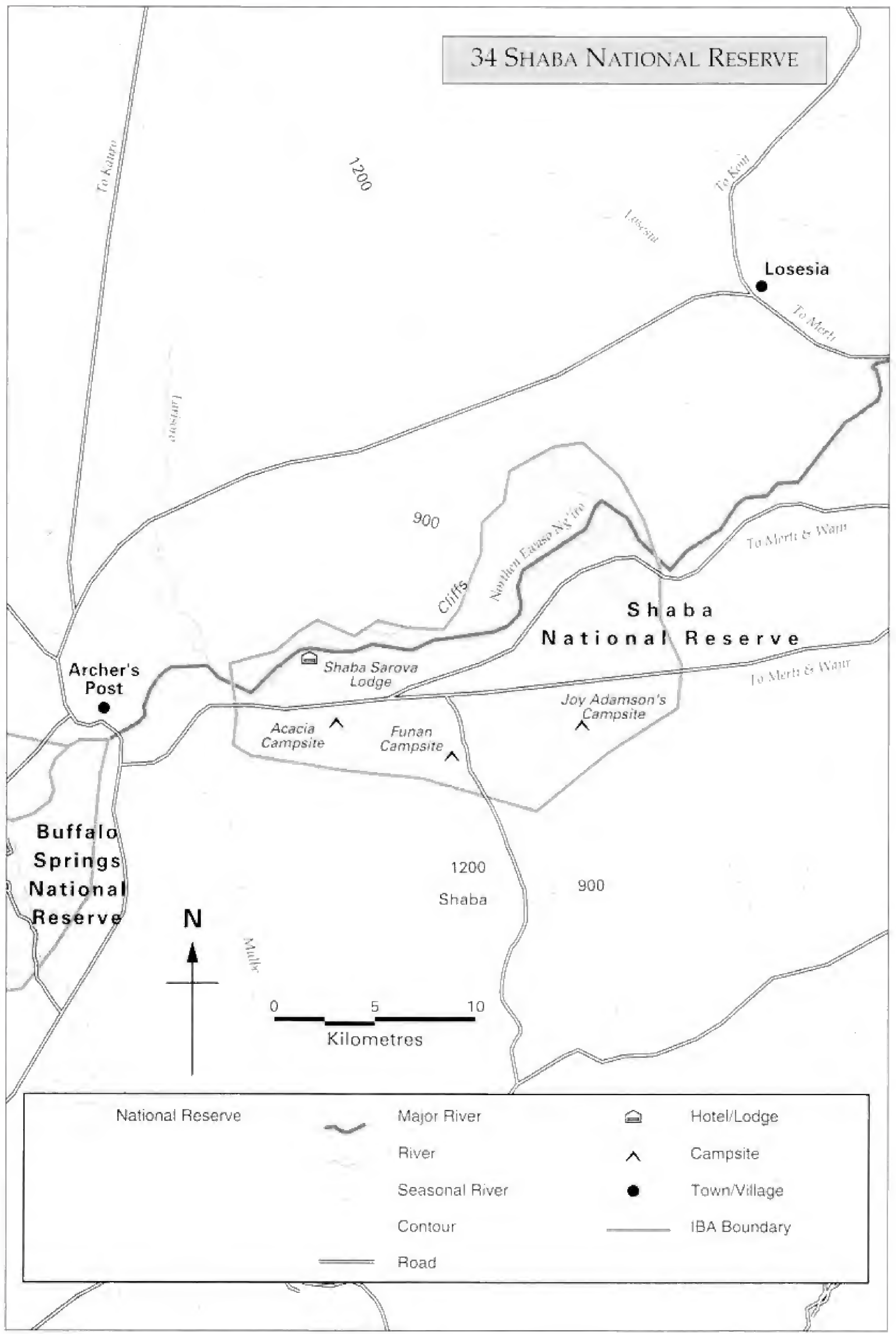




\section{Shaba National Reserve}

presence of twiliams's lark. Vers little is known

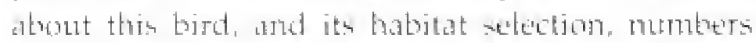

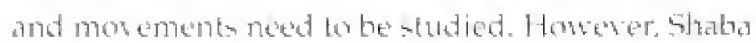

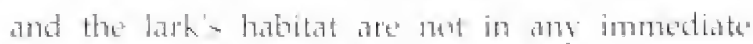

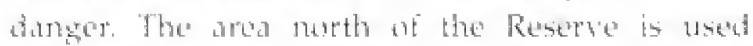

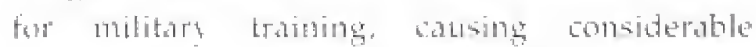

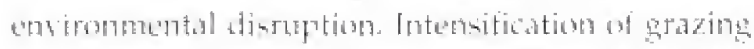

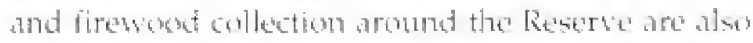

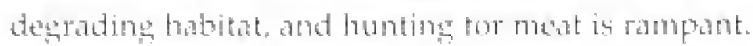
These problems at times splll were inter the Reserese

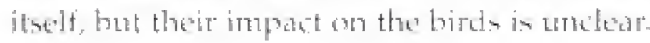

Shaba is a populat touriat destinatuon thecause of its actme beates, and is world famous as the location of fory Adamson's last adventure, the reterase at a

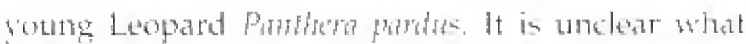
amount of sisitur pressure the Reserte can sustain

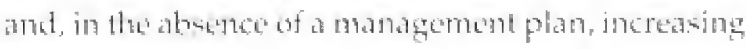
tourist nembers mat hecome" it problems in the future.

\section{Further reading}

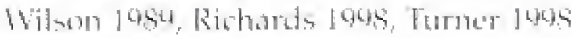

Williams"s Latk 


\section{DANDORA PONDS}

$01^{\circ} 16^{\prime} \mathrm{S}, 37^{\circ} 02^{\prime} \mathrm{E}$, Nairobi Protince, Nairobi District

Area: c. 300 ha

Altitute: $1,500 \mathrm{~m}$

Status: Owned and managed by Nairobi City Council

Category: Congregations

\section{Site description}

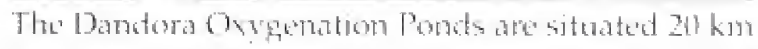

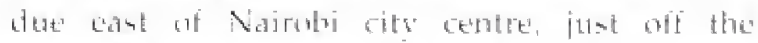
Nairobi-Kangundo roat but within the city linuts.

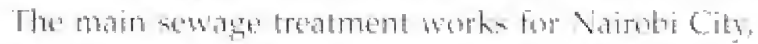

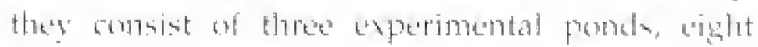
facultation ponds and 27 maturation ponds, all bounded by embarkments. The first phase on

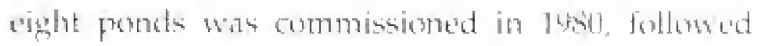
by the mud darerer secombl phase in byy2. The alder set has mud bonks, with asubetated grawh of

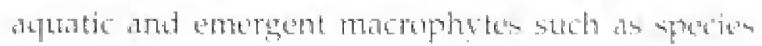

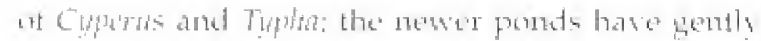
shoping concate broks. Water quatity and the

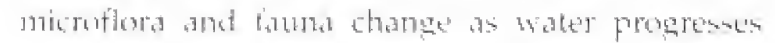

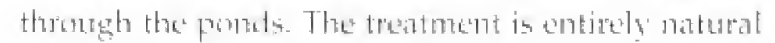

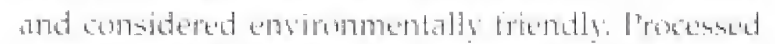

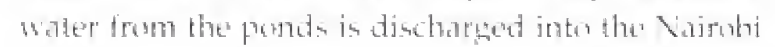
Risut:

\section{Birds}

5o. Lrox tor key spectes Dandora lobles

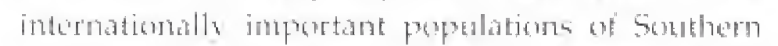
focharet and (in the twotheres winter, from

\section{Congregations}

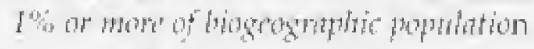

Southem Pochard (500) [ [994-1997 me:an: 1,480 (four counts); max: 3,200 (1994)

Northern Shoveler (1600) 2,300 (1996)

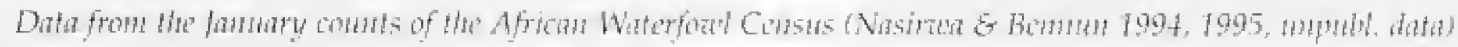

\section{Regionally-threatened species}

Cricit Fytet

Vulneratele
Sporatic visitor, in small numbers 
35 Dandora Ponds

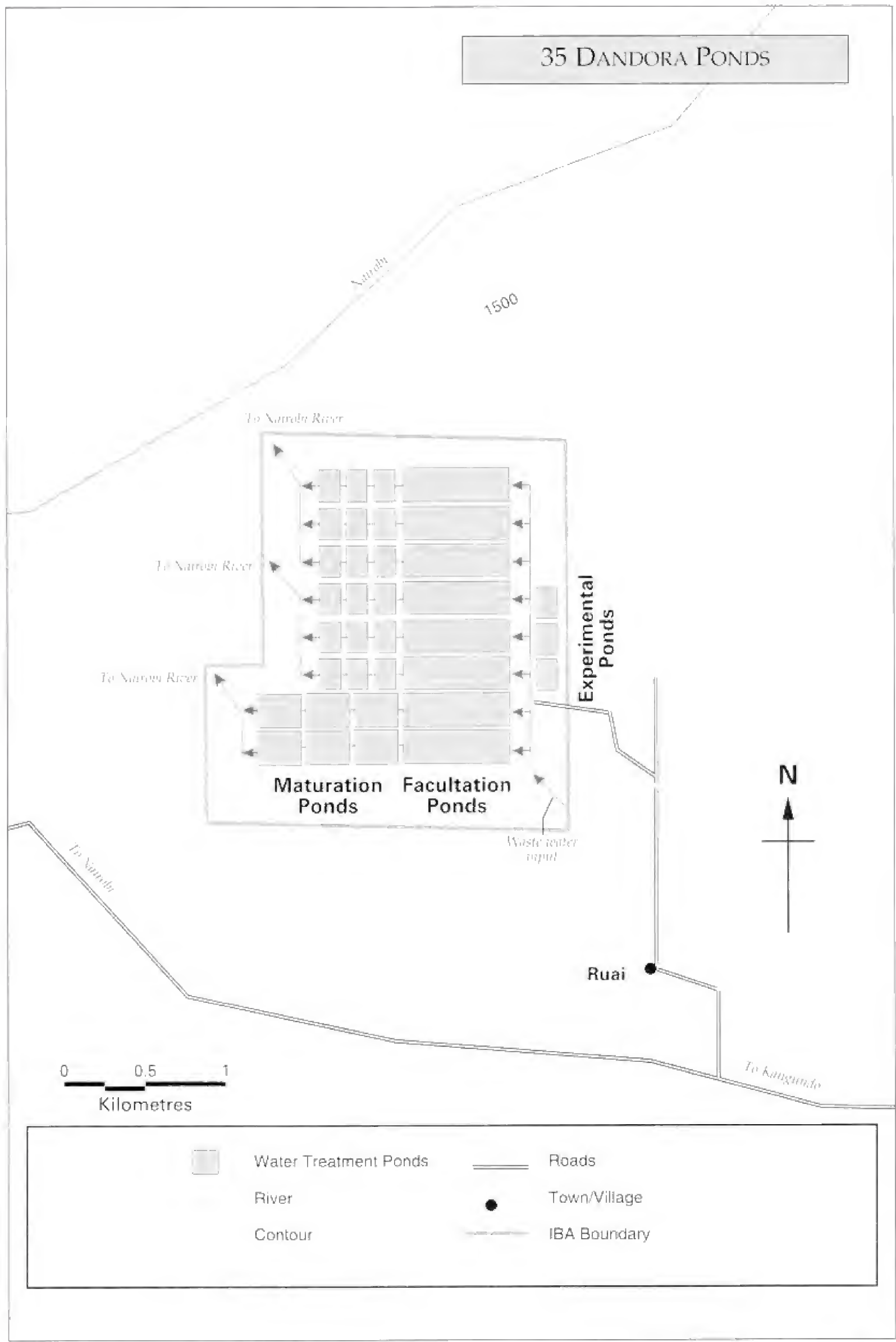




\section{Important Bird Areas in Kenya}

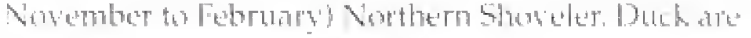

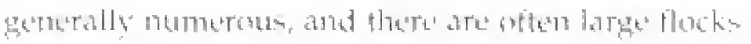

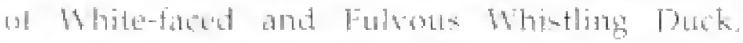
Reret-billed and Yellow-billed louch, Hentented Teal

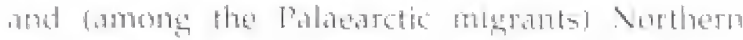

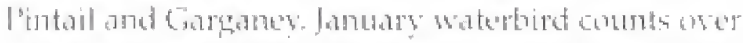

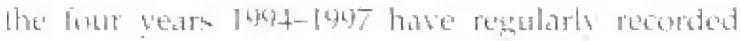

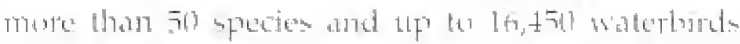

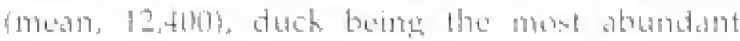

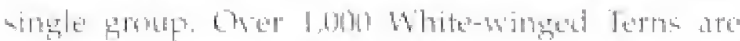

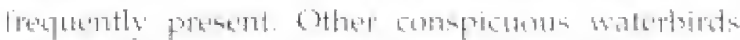

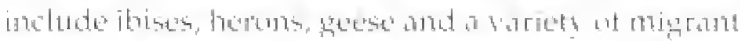

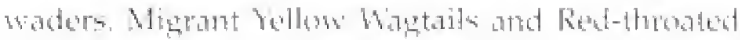

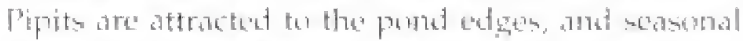

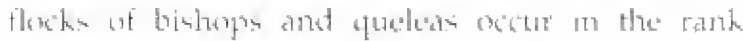

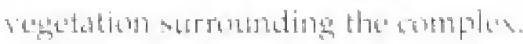

\section{Conservation issues}

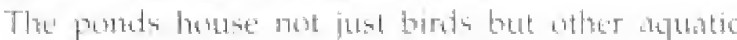

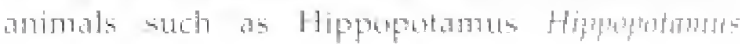

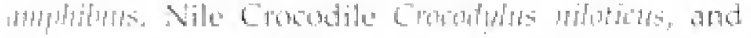

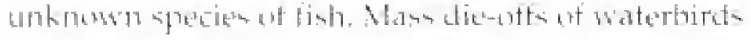

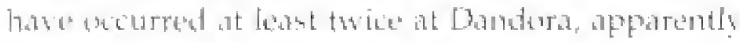

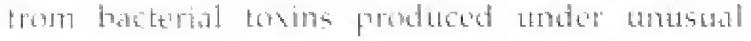

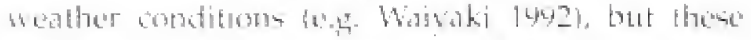

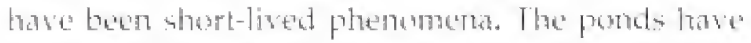

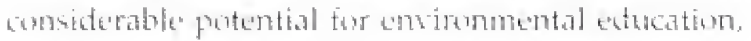

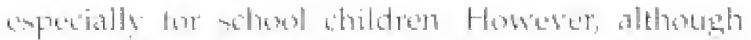

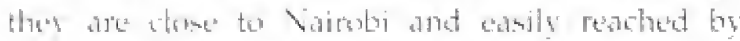

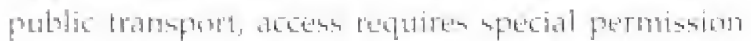

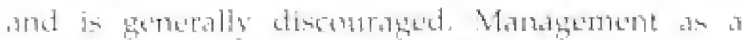

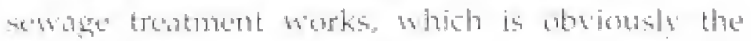

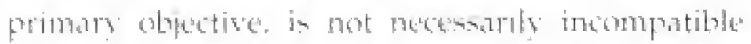

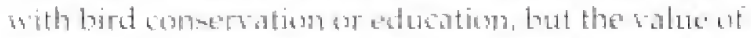

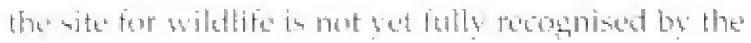
Cits Coruthet.

\section{Further reading}

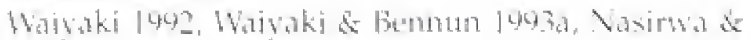

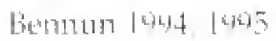




\title{
36 Nairobi National Park
}

\author{
$01^{\circ} 17^{\prime} \mathrm{S}, 36^{\circ} 49^{\prime} \mathrm{E}$, Nairobi Province, Nairobi District
}

11,700 ha

Altitude: $1,540-1,780 \mathrm{~m}$

Status: National Park

Categorics: Globally-threatened species, restricted-range species, congregations

\section{Site descriptian}

An ared of hatural landicoper at the grasstand-femest bountary, only $7 \mathrm{~km}$ from the gentre of Kervats capital cits, Nabubi. The Parks saried habitate indude epen, rolling grase plains, riserine woodtant, walke thicket and bush, man-made dams and pords, rocky goryes and upland dry forest. The lark is lenoed atong thete sides, wheres it is adpacent to urban housing, industry, roads and airports; only the southern border, along the Embakasi and Athi rivers, is opten fur animal dispersul. Eoblogrioally, the Park is utimatedy linkind to the Kitemgela and Athi-Kapiti phama, which adjoin it to the south, forming a single ecolugical unit. Bentres close to the city centre and housing a watedy of large mammals. this Park in a popular dentination and a substantial

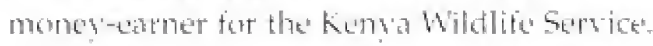

\section{Birds}

Sec boxes for key species Nairobi National Park is on important foosting site for Lesser Kestrel bocks on fasage although numbers hase dectined martiedly in recent forar. The substantial area of undisturber grassland is of great importance for species such as the restricted-range facksoln: Widowbird, which breds here regularly after gont rains. African Cromned Faglen 17est in the forest. The axifauma in diveres", with a remarkatole 516 species recorded Haresy 1947), including 27 of Kenva's

\section{Congregations}

More than in of popratation (at roost)

Lesser Kestred (600) Rousts of up to 5,000 have been recorded (Smalley 1983, Lewis \& Pumeroy 1989, Zimmerman it al. 1996)

92 Somali-Masali bomm specien (23 of sthich are regulary, and 25 of Kenyes 67 Arican Highland biones species. The globelly-threatened Cormenat is a sarae ristot from the Palantetic and the near-threatemed Shobill and Basta Reed Warble: hate both beetr recordes ance (Harrey 1997).

\section{Other wildlife}

Nambi National Park has healthy populations of an array of large mumbals. The Park is a thinc sanctury and numbers of Blach Rhinoceros Deverto

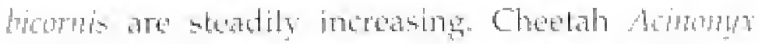

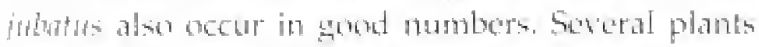
growing on the rocky hillsides are urnilume to the

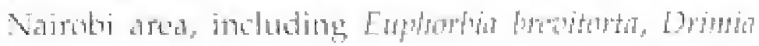

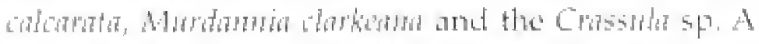

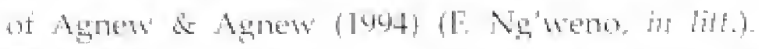
The lark profecto an important area of

\section{Globally-threatened species}

Madagacar Squaco Herom Nar-threatened

Lesser kestrel

Vulnerable

Red-throated Tit

Near-threatened

"Jackson's Widombird

Near-klaraterned
Regulat non-breeding visitor in smatl numbers, May-October (Zimmerman at al. 1996, Harvey 1997)

Regular on migration; see below

Fatrly common in riverine Atatio woodland (Harvey 1997, umpubl. data)

Nests regularly in tall grassland after good rains (Harvey 1997, unpubl, data) 


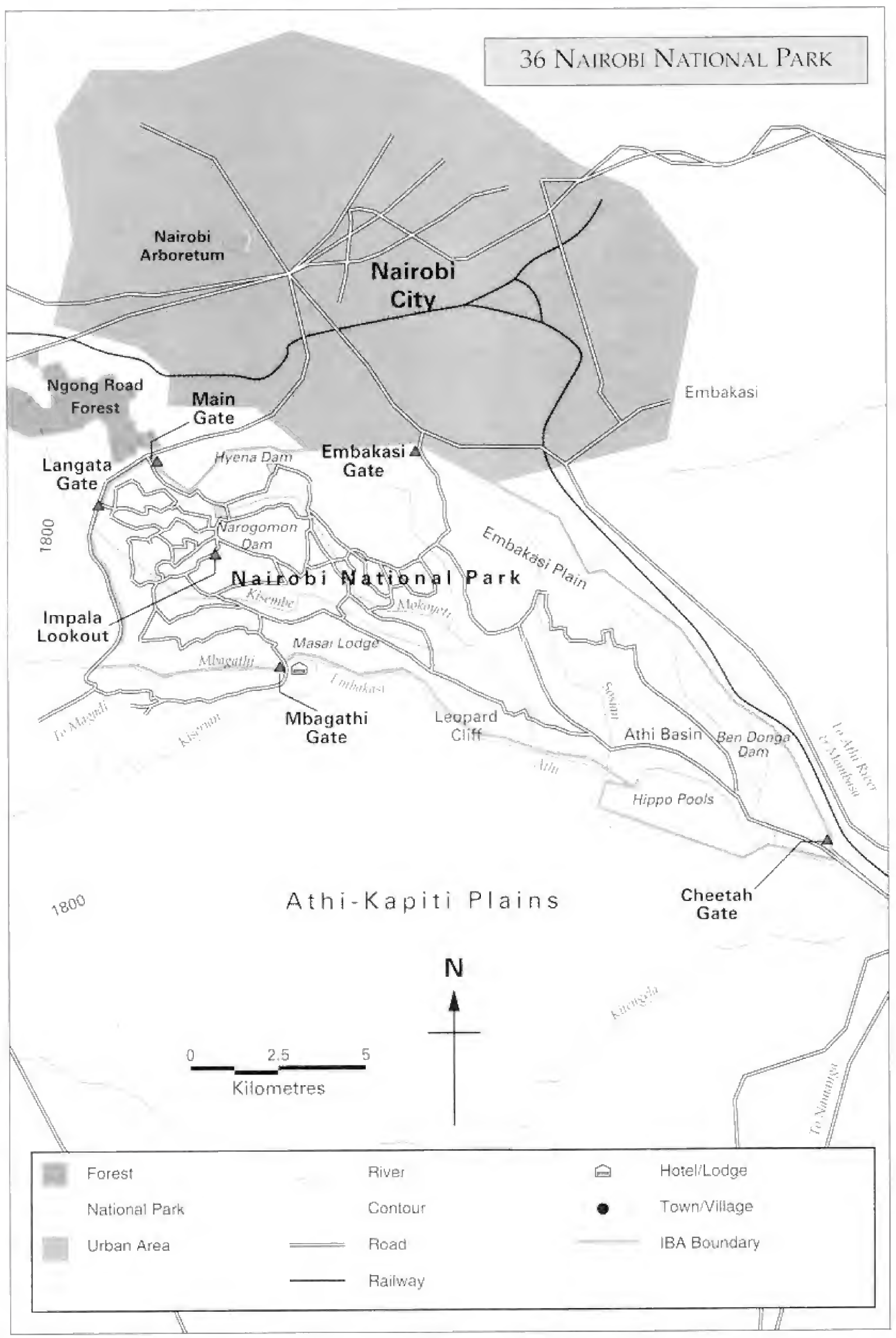




\title{
36 Nairobi National Park
}

\author{
Regionally-threatened species \\ Common Ostrict? Vulnerable \\ Conmon on the open plaim \\ Alrican Darter \\ Vuinemble \\ Scared sisitur \\ Great Egre: \\ Vulnerable \\ Regolar visitor to dams and punde \\ Sadsle-billud Sturk \\ Vininemalse \\ Resident in small numbers \\ Auress Hawk Eagle \\ Vulnerable \\ Scarce resident it the furent \\ African Crowned Eagke \\ Vulnerable \\ At least bone parir neste in the forest \\ Martisl Euslo \\ Vulneralule \\ Several pairs have home ranges that \\ include the Park (C. Ong, umpubl. data) \\ Airican Finfout \\ Vulneratid: \\ Resident in small mumbers on thickly- \\ iringed sections of the rivers \\ Yellew-billed Oypeckel \\ Vultierable \\ Hocterately common

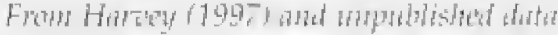

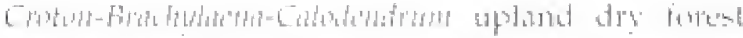

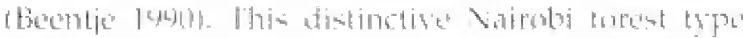

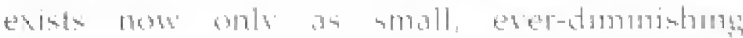
fritgmentitio

\section{Conservation issues}

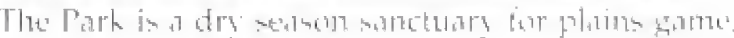

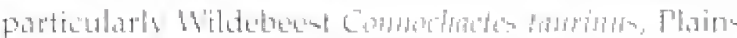

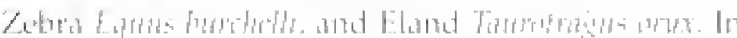

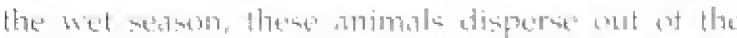

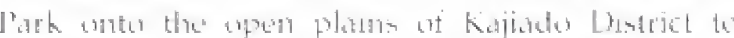

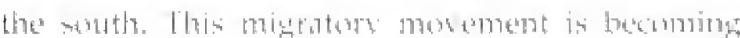

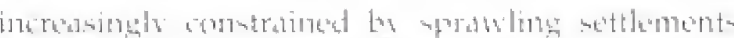

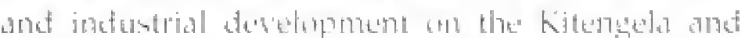

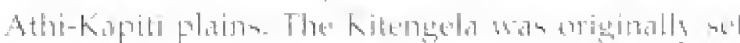

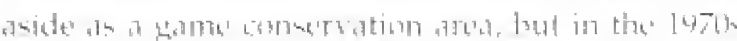

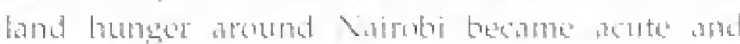

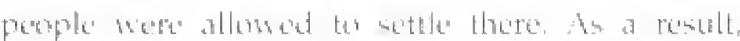

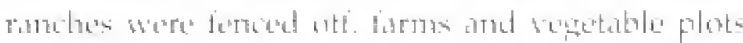

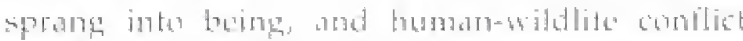

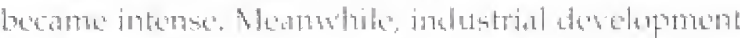

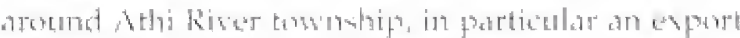

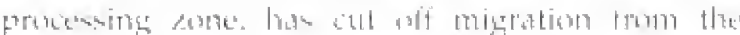

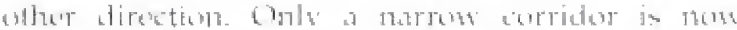

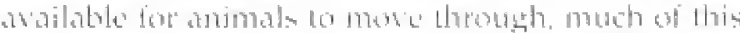

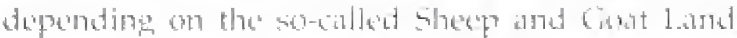

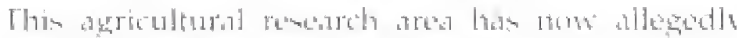

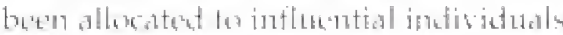

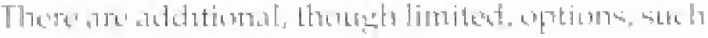

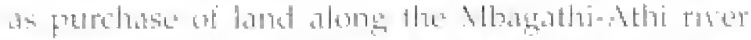
proutage, and encouraging combers aticn-minded

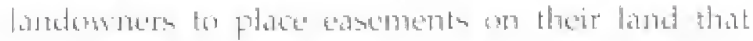

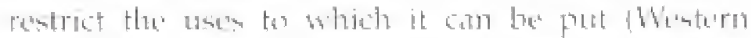

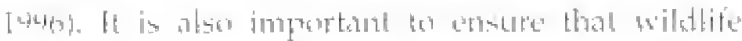

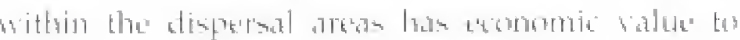

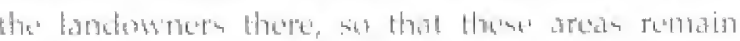

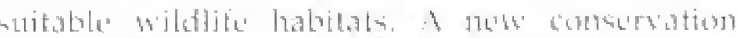

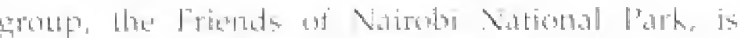

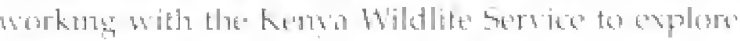
thesentissues.

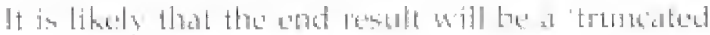

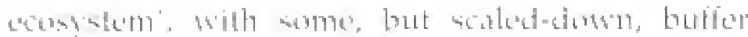

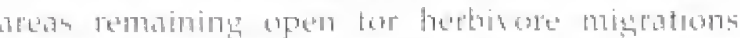

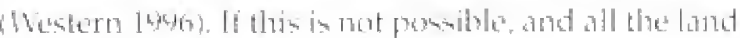

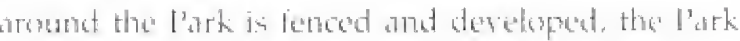

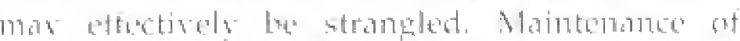

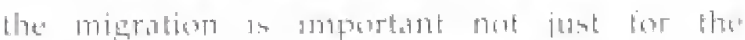

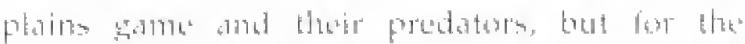

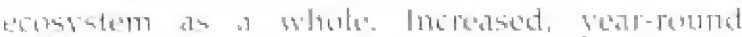

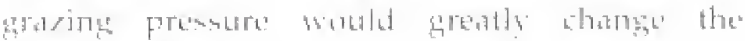

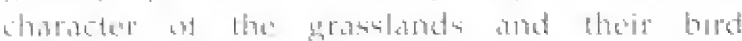

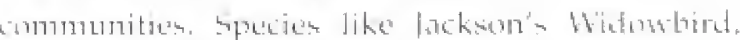

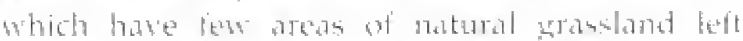

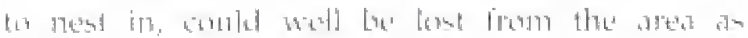
aresiale. 


\section{Important Bird Areas in Kenya}

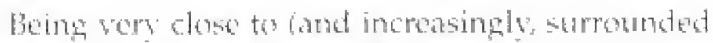

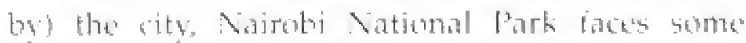
ubutoum atditional problems. Waste from some

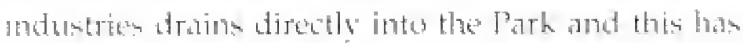
been ditficult to contrenl, Patt on the Park witl be hised off for a major bypass rodd in the neder future,

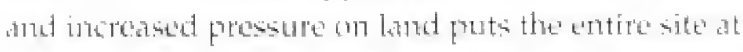
potertial risk. On the olther hand, its proximity the cits makes varbori Nathomal Path an unparalleled facilits for public butation about wild life and consernations.

Further reading

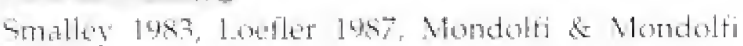

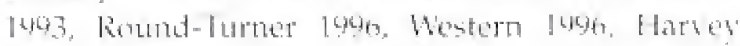
1447 


\section{Dunga SWAMP}

\section{$01^{\circ} 10^{\prime} \mathrm{S}, 34^{\circ} 47^{\prime} \mathrm{E}$, Nyanza P'rovince, Kisumu District}

c. 500 ha

Alitude: $1,130 \mathrm{~m}$

Status: Unprotected

Categories: Globally-threatened species, Lake Victoria Basin biome species

\section{Site description}

Dunga for lake River muthith is a methat sibuted

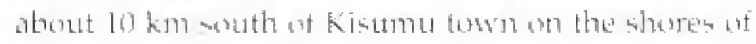
Wimam Gutt, I ake Vigtoria. At the westerm lamit is a

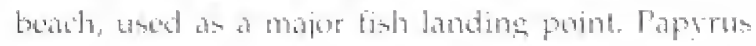

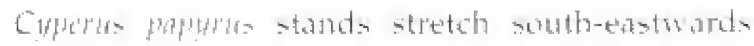
along the there from bere for about $5 \mathrm{hm}$, in a

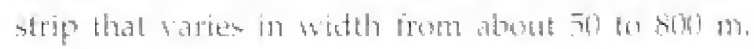

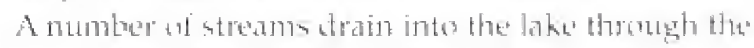
suratop, the math tome being lako Rives.

\section{Birds}

see box and sppendix 3 for key apecies. This site is

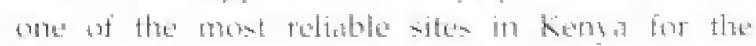

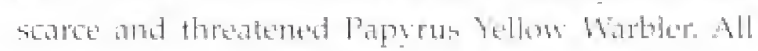

Pawyrus Yellow Warbler

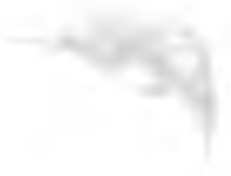

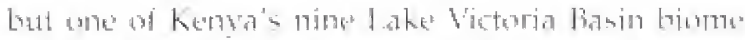

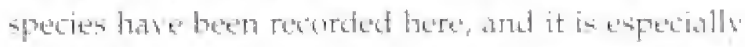

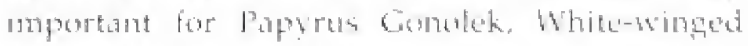

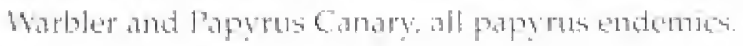

\section{Other wildlife}

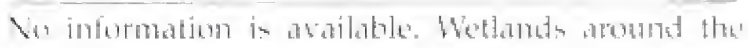
sheres of Lake victoria are knomin to be important reduges tor a number of the lake's endemic laphothomine tisth species.

\section{Conservation issues}

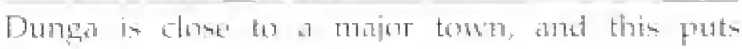

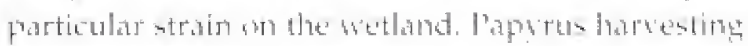

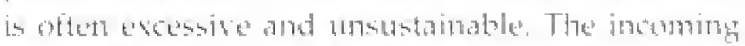

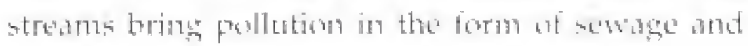

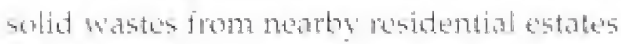

Lake bictorias papurus stamps are under

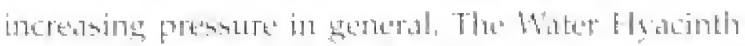

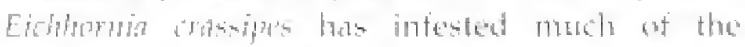

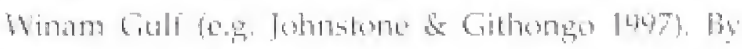
presentiong fishermen from fishing, it fartem them

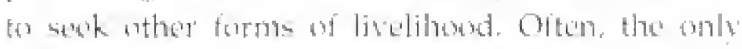

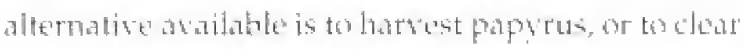
it in ardes to cultivate eroph.

Dumparenty requires better hormal protection,

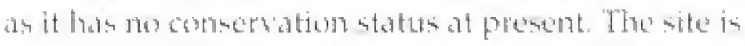
already a populat area for recreation. Its proxirtity to

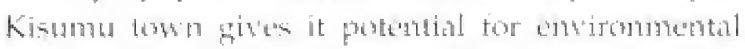
aduation and bird watisen focused on bhe papyerus undemics.

Further reading

Nasirwa $\mathrm{d}$ Noment 1497

\section{Globally-threatened species}

Papyrus Gonolek

Papyrus Yellow Warbler
Near-threatened

Vulnerables
Relatizely common

(Nasirwa \& Njoroge 1997)

Often seen along the lakeward side of the swamp (B. Finch, in litl, ) 
Important Bird Areas in Kenya

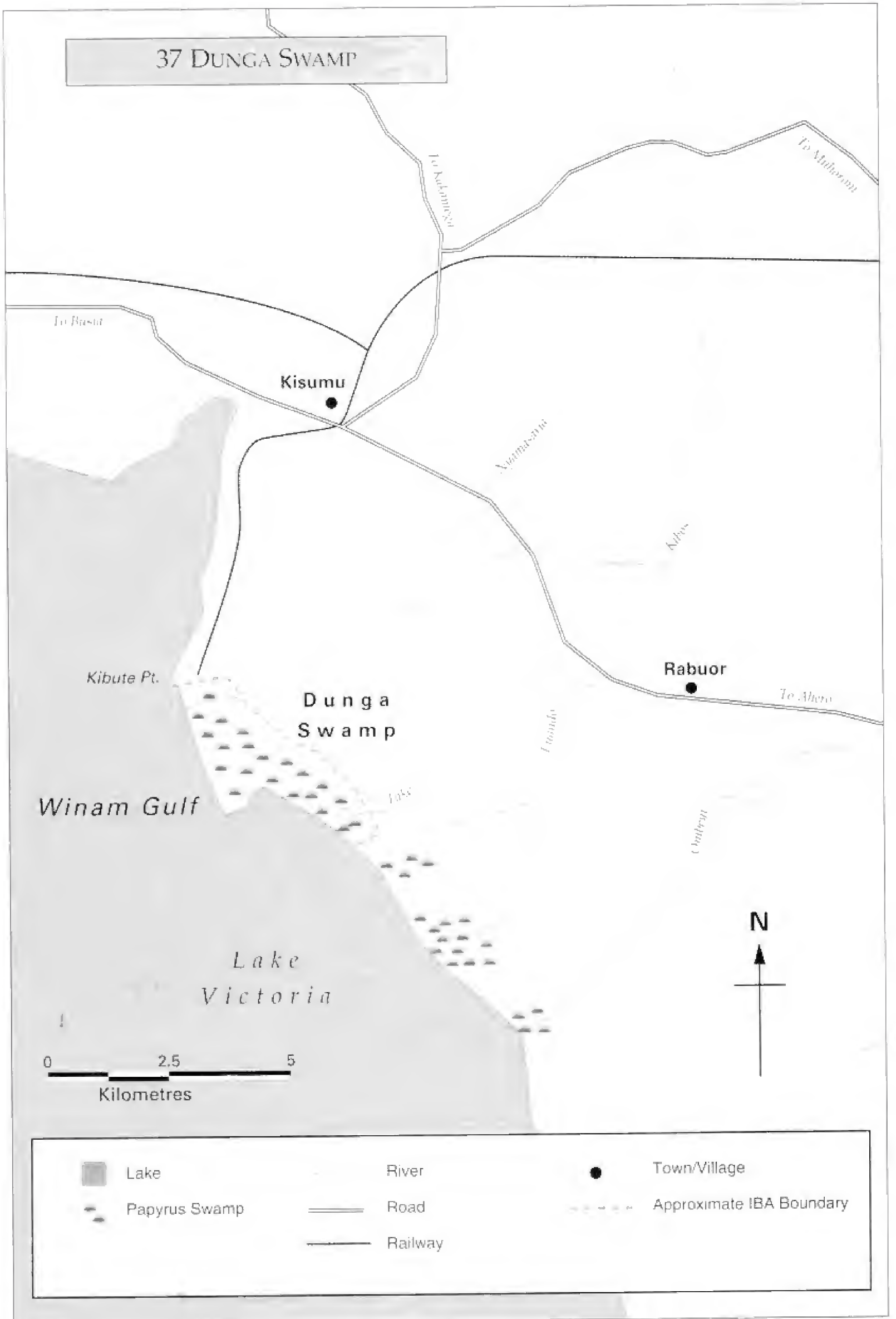




\title{
38 Koguta Swamp
}

\author{
$0^{\circ} 17^{\prime} \mathrm{S}, 34^{\circ} 36^{\prime} \mathrm{E}$, Nyanza Province, Kisumu District \\ c. 1,800 ha \\ Altitude: $1,130 \mathrm{~m}$ \\ Status: Unprotected, public land \\ Categories: Globally-threatened species, Lake Victoria Basin biome species
}

\section{Site description}

Kogruta Suramp is a setland at the mouth af the

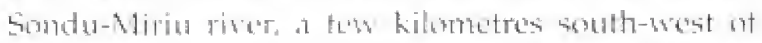
the Viander river moulth an the shores of Lake Victoria. The vegetation gomeints mainly of reds

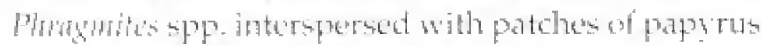

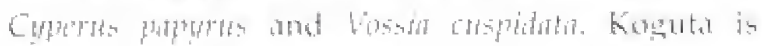
Fooded during the wet wasom, while during the try

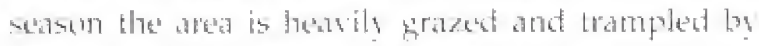

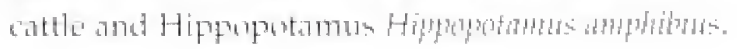

\section{Birds}

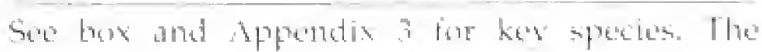

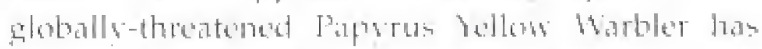

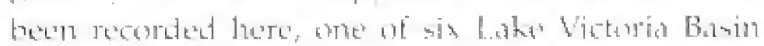

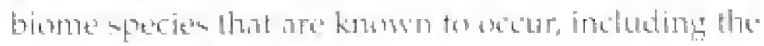

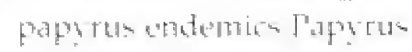
Giancilek, White-ringerd Worbler and Carruthers's Eintionla.

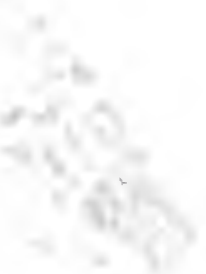

\section{Other wildlife}

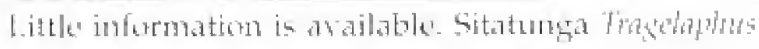

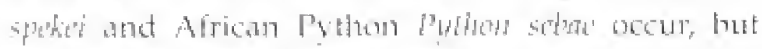
there staters is unkmown. Like other wetland around the shoues of latke Vistoria, Kostuta is probably an

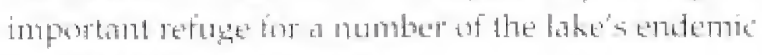
buplochmomine fisto species.

\section{Conservation issues}

The ijte is almose inacessiblo trom the lakeward side whe to dense mats of Water Hacinth Eridhish

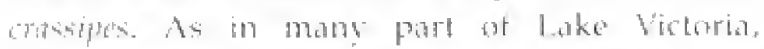
infertation by this watic weed has presented

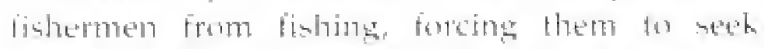

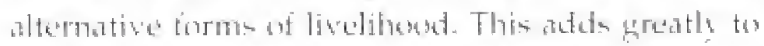

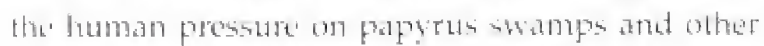

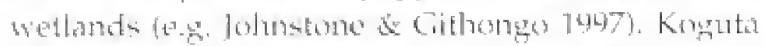

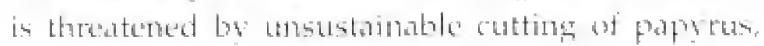

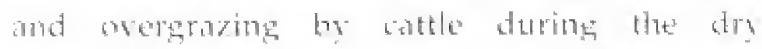
season when the sater lavel recedes. It requires formal protedtom, and a community consersation

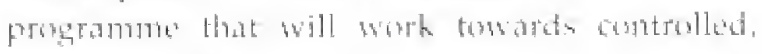

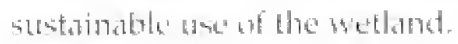

Further reading

Nasirwa \& Noroge $154 \overline{7}$

Whitewinged Warbler

\section{Globally-threatened species}

Papyrus Yollow Warbler

vilnerable

Papy'rus Gonole'k

Neabl-threatened

\section{[resent but uncommon}

(Namirtwa \& Noroge 1997)

Relatively abundant

(Nasima \& Noroge 1947) 
Important Bird Areas in Kenya

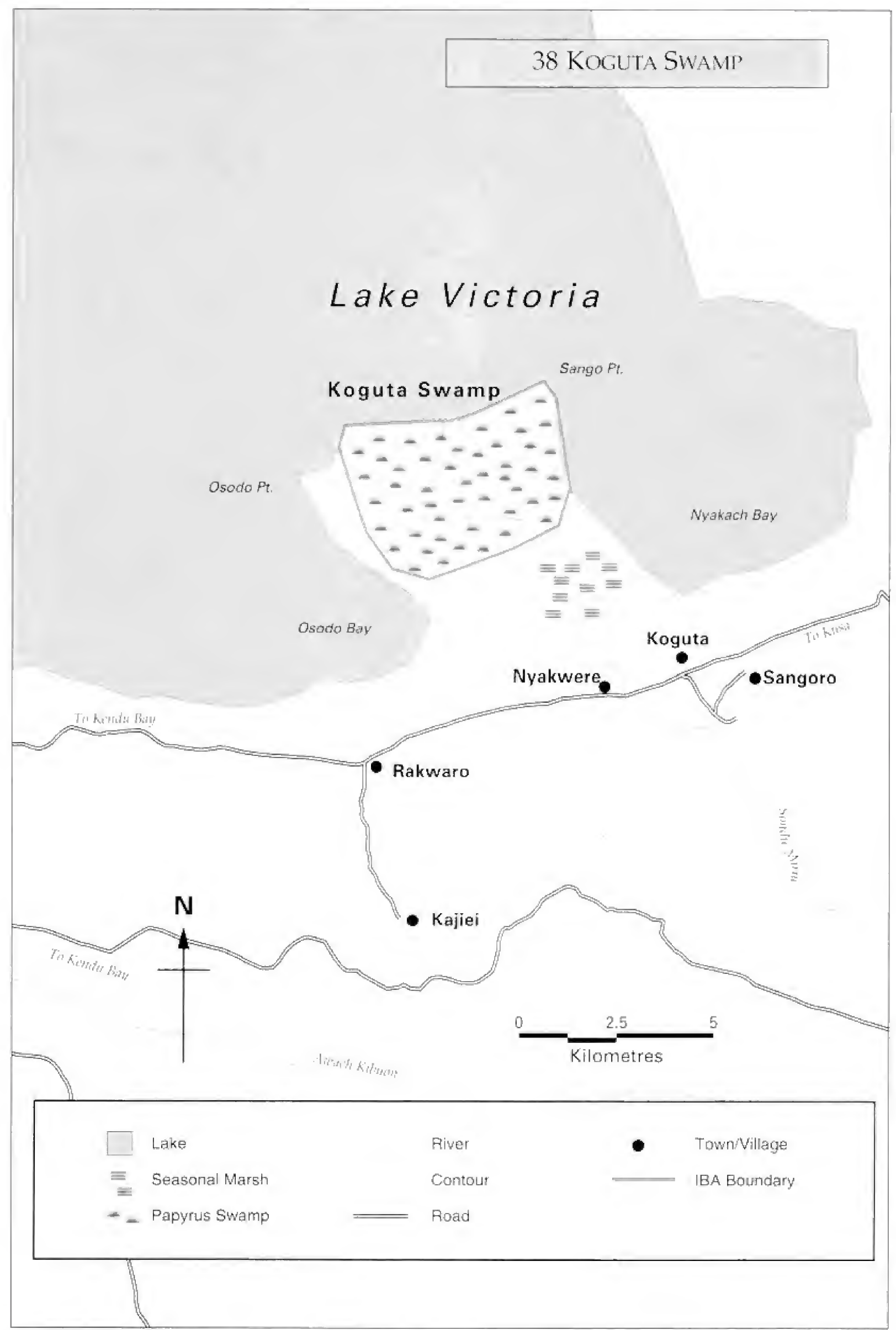




\section{Kusa Swamp}

\section{$01^{\circ} 19^{\prime} \mathrm{S}, 34^{\circ} 51^{\prime} \mathrm{E}$, Nyanza Province, Kisumu District}

\section{c. 1000 ha}

Altitude: $1,130 \mathrm{~m}$

Status: Unprotected, public land

Categories: Globally-threatened species, Lake Victoria Basin biome species

\section{Site description}

Kusa Swamp, the mouth of River Vyando, is a wettame at the casternmost end of Lake Victeria's

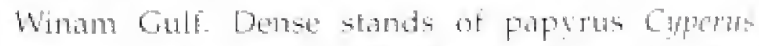
poptwes are tringed on the lakeward side by the water grass Finsial cosphtata and along the shore by reeds Phropmitas sply.

\section{Birds}

Sev trox and Appendix 3 for ke't species. Kust includers a substantial sand of papsters, and the near-threaterted l'apytus Gonolek is thuly abundant here, Other lake Victoria Baxin bome species inclede the papyras endemics thiterwinged Warbler and Carruthers's Cistionla, and if is suspected that Papsrus Camary and the gloteally threaterned l'aprese vellow Warbler are likely lo occu:

\section{Other wildlife}

Litte information is avalables. The sitatunga

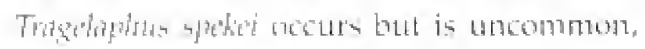
and African Pythom pythos sepros is recorded. Like other wetlants atound the sheses of lathe

Victoria, Kusa is probably

an important refuge for

a number of the lake's

endenic haplobhomine tisto speciem.

\section{Conservation issues}

Kusa remains a sizeable enough papros swamp to be of consett"ation importance.

However uncontrolled cutting of Fapras by the local residents and poollation atud siltation from the Nyando Riker are serisus threats. Thurugh papyras showe remarkable poners of regemerafion. unatstainable cutting for the local mat-making industry has the potential to destroy the wethend. Local residerts are atso opening up the swamp for rice beming. Sitatumga, thumh alfeady rare, are hunted. The lake'ward side is almost thoted by the

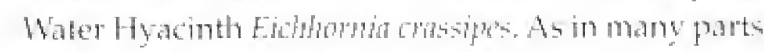
of Lake Victoria, infestation by this exotic wed bas presented fishermen from fishing, forctus them to seck alternatise forms of livelihood and adding grevely to the buman prosure on wetlands lohstone \& Githonge 1997).

\section{Further reading}

Visinta \& Nioroge 1097

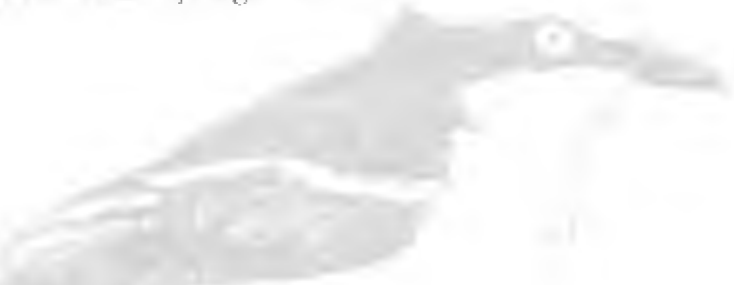

\section{Globally-threatened species}

Papyrus Gonolek
Near-threatened
Relatively comnon

(Nasirwa \& Njoroge 1997) 
Important Bird Areas in Kenya

39 KUSA SWAMP

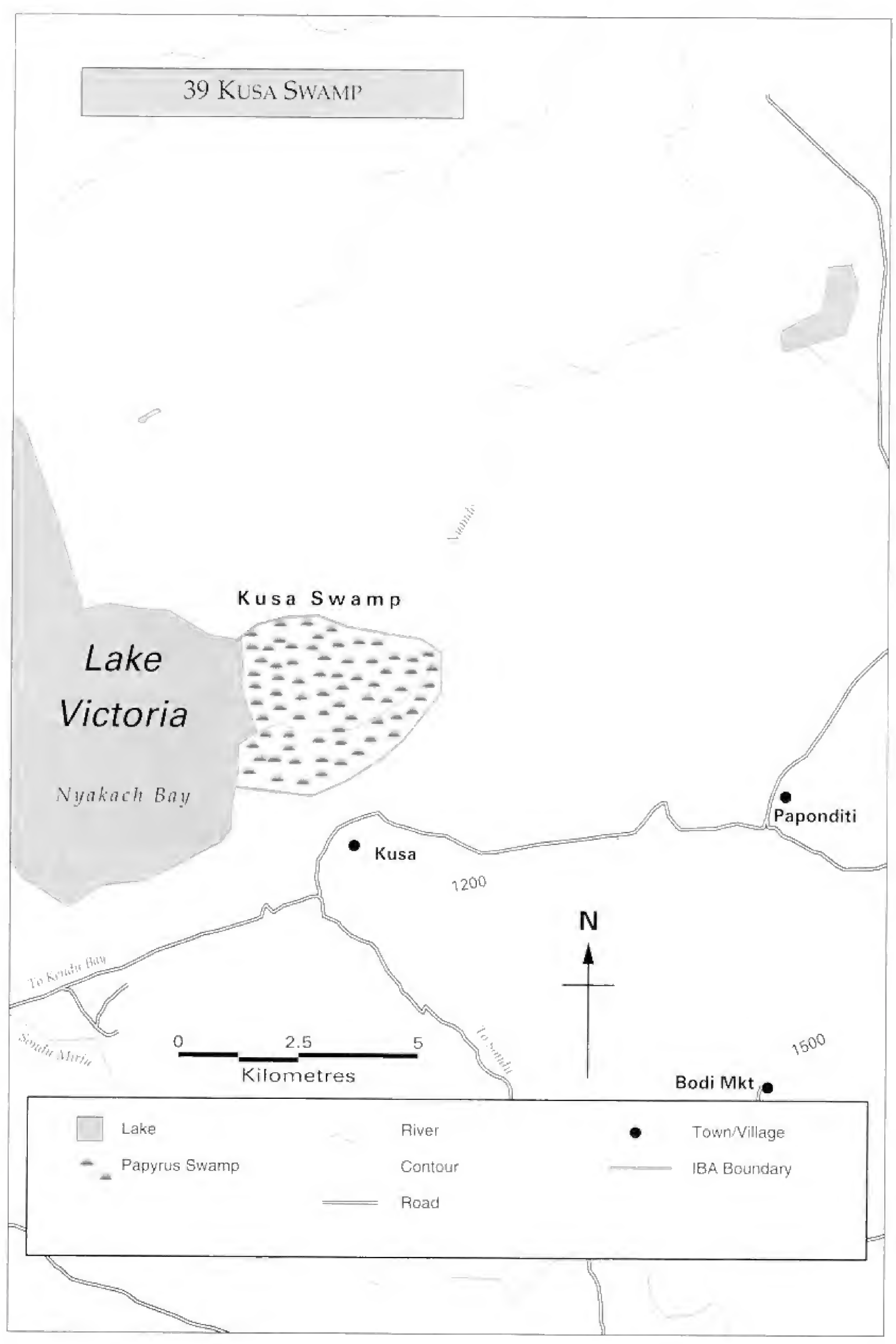




\title{
40 Ruma National Park
}

\author{
$0^{\circ} 35^{\prime} S, 34^{\circ} 12^{\prime} \mathrm{E}$, Nyanza Province, Suba District \\ 12,000 ha
}

Altitude: $1,200-1,600 \mathrm{~m}$

Status: National Park

Category: Globally-threatened species

\section{Site description}

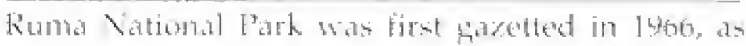

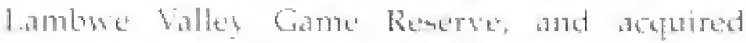

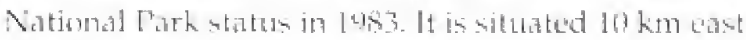

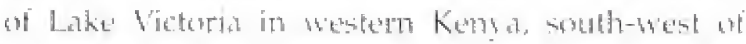

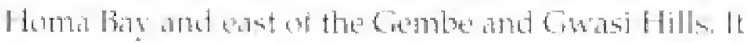
lien on the flat flow of the Lambure kalley bortertad by the Kanfama ksappment fand induding a

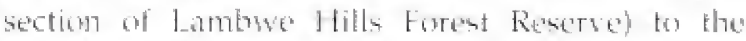

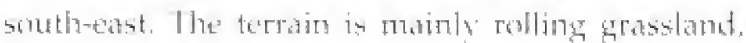
with tracts of orem woodlated and thekets

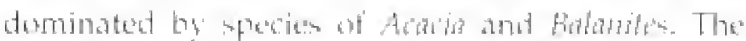

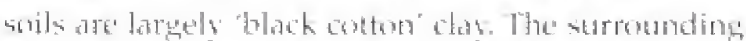

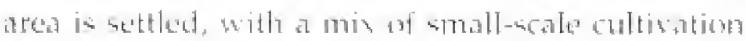
and errassin pasturestant.

\section{Birds}

Ruma is the only protected area ic kensa where the

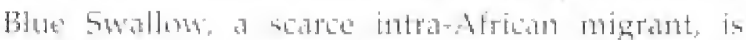

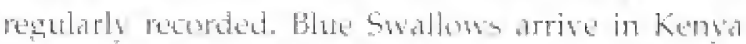
from their brecding gremols in southern Tanzania armuth April and depart in September. They depend

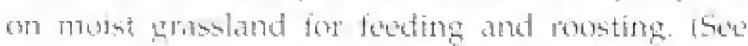
also Ibat 57, Busis Grasslands.) Black-backed Cisticula, a speries thought to be extinet in

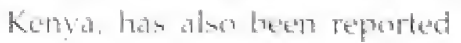
From Ruma, but these necords

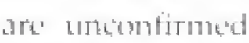

(1).A. lutner, sh lill.).

\section{Other wildlife}

A saritety of manumalon occur in the park but the most notuble is

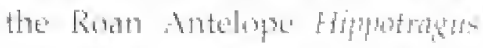

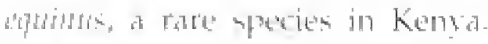

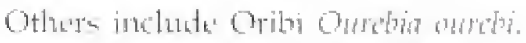
Little is knomen about cother tamo and Fora.

\section{Conservation issues}

Ruma represents a saluable island of natural hatoitat in a sea of foman settement. "The strrounding population demeity is high, lout perple and their

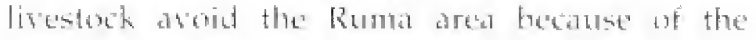
presence of thetse fly. Wildite parthing alumg the boumbarien has been reported in thet past.

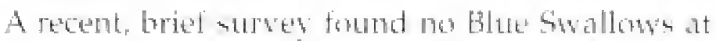

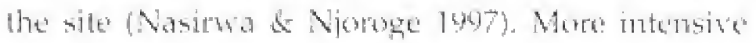
work is needed to atetermine the staters of this

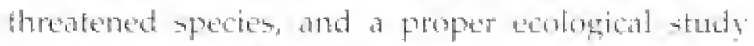

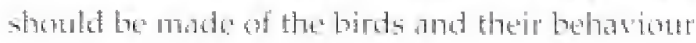

\section{Further reading}

Nasirwa \& Noroge 1997

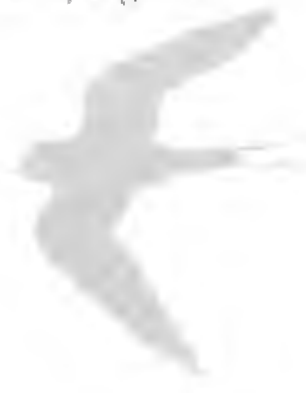

Blue Swallow

\section{Globally-threatened species}

Blue Swallow Vulnerable

Several recent records, April-September (Zimmerman of al'. 1996), but not seen during a 1946 survey (Nasirwa \& Njoroge 1497) 
Important Bird Areas in Kenya

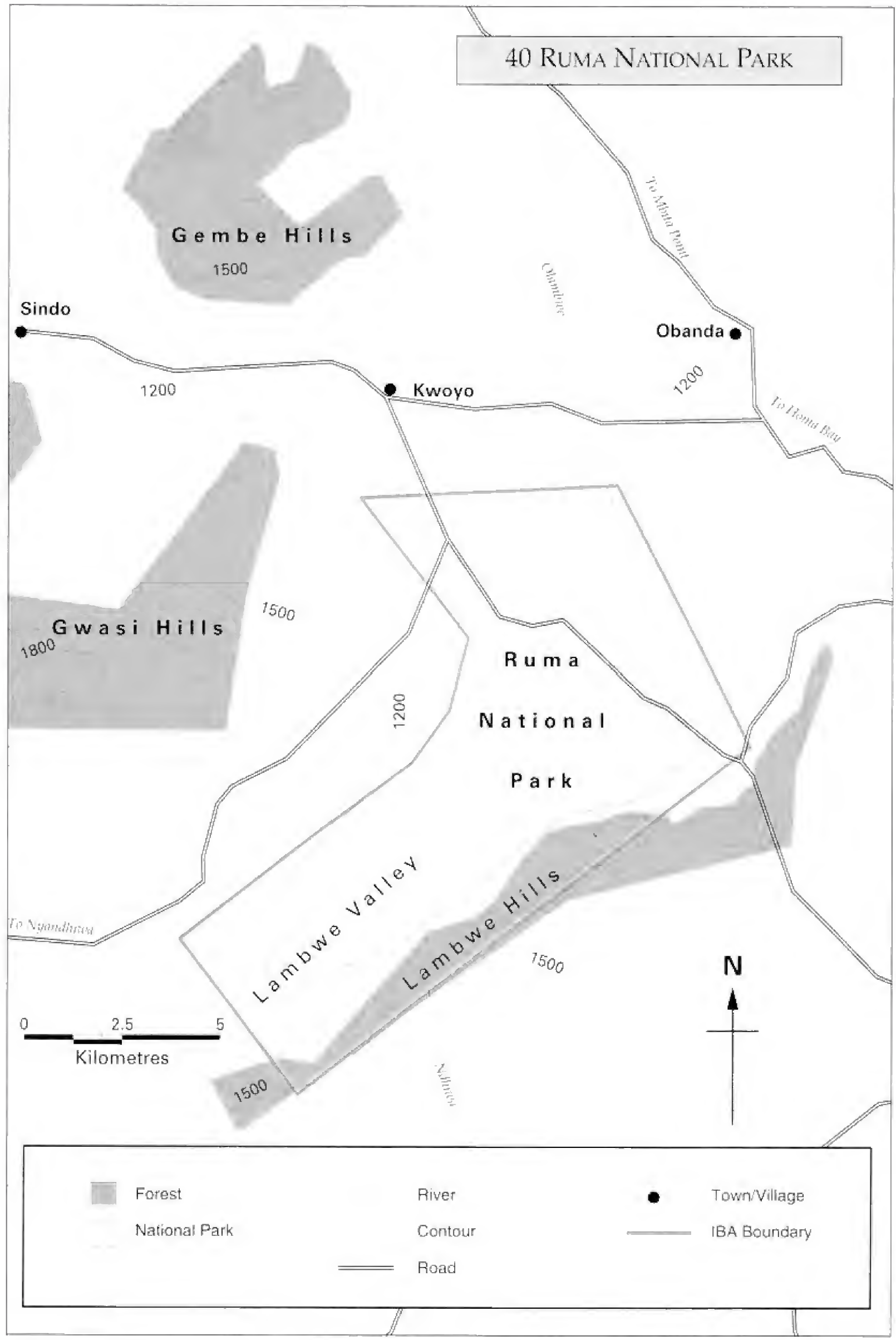




\title{
41 Yala Swamp Complex
}

\author{
$0^{\circ} 05^{\prime} \mathrm{N}, 34^{\circ} 11^{\prime} \mathrm{E}$, Nyanza Province, Siaya District and Western Province, \\ Busia District \\ c. 8,000 ha
}

Altitude: $1,130-1,160 \mathrm{~m}$
Status: Unprotected

Categories: Globally-threatened species, Lake Victoria Basin biome species

\section{Site description}

This in a comples of wetland in the deth of the Yala Riser, sh the north-east shore of lake Viteoria. The site has there main componemes: the Yala Swamp itself correndy c, 6,500 ha after drainage of the eastern fibth): Lake Kanvaboli in the north-eastera comer, a 3 m deep lakt of around 1 , 1000 hat and Lake Sare, the most southerly of sereral butlets of the Yala Risere jontu lake Victorid, around 5 m deep and 500 ha in area. Formerly, the Yala kiver flowed theough the edstern sump (now "reclaimed') into Late Kanyoboli, then into the main swanap, ind findly into lake Victoria sia a small gulf. The rala thom is now diserled directly into the main swamp, and a

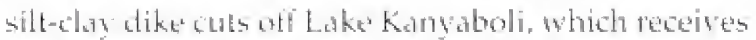
its water" from the survounding catchment and through back-sepage from the stramp. A culdert across the moutto of the Yala, some netres above the lew of Lake Victorta, has cut of the rult on the lake and, through back-flooding, created Lake Sart (Maruti 1942).

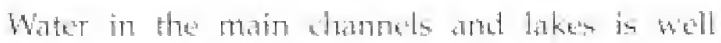

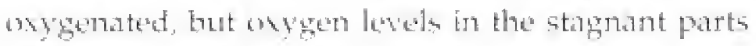

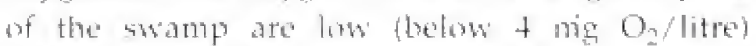

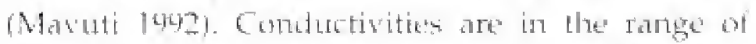
$10(1-130$ HS/om in the chantuls and Lake Sare, bat considerably higher in lake Karvabuli dite to the latk of fresh water inflows from the riser for feeder cacal has fallen into disteparir).

The predominant vegetotion is papytus

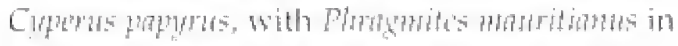
shallower arests and stamp grasies around the periphery. Both Lake Kanyaboli and Lake Sareate surrounded by a thick tringe?

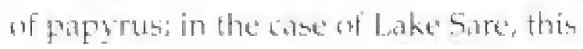
mextses with the main stamp.

The Yala swampe complex is by far the largest papsus swamp in that kenyan sector of lake Vickoria. making up more than g(t), wit the total areat of papyrus
(Nasirwo \& Noroge 1497). The swamp acts as a matural filter for a variety of bricides and ather agriculatul pollutants from the surrounding catchment, and also effectively remoses silt before the water enters Lake Victoria. The site supports an important lowal fishery tor the Luro and Luhya people whe live to its south und north, respectivels:

\section{Birds}

See bux and Appendix 3 for key species rala swamp holds bight onet af Ketrya's nine Lake Victotia biome species, including the globally-threatened Papyrus Yellow Warbler. The nent-threatered Creat Snipe, a Palaentetic migrand, probably atsa accurs. Becalse of its sige and the generally gond condition of the papyrus, the Yala Sxamp complex is an important site for East Africa's papyrus endemics. These include Papstus Yellow Warbler, Carruthers's Cisticola, White-winged Whrbler and Papsrus Canary, Many other wetland bids also oceur (Britton 1475, Nasirna \& Noroge 1997). Reports of accasional sighting of showill by the lexal rewdents have nut beren confirmed.

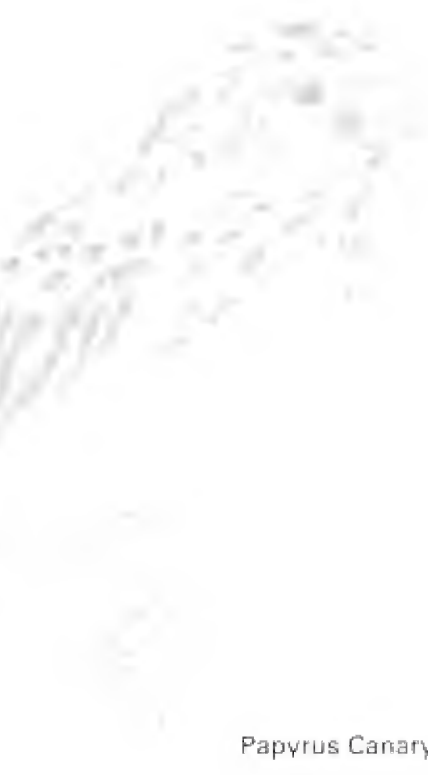


Important Bird Areas in Kenya

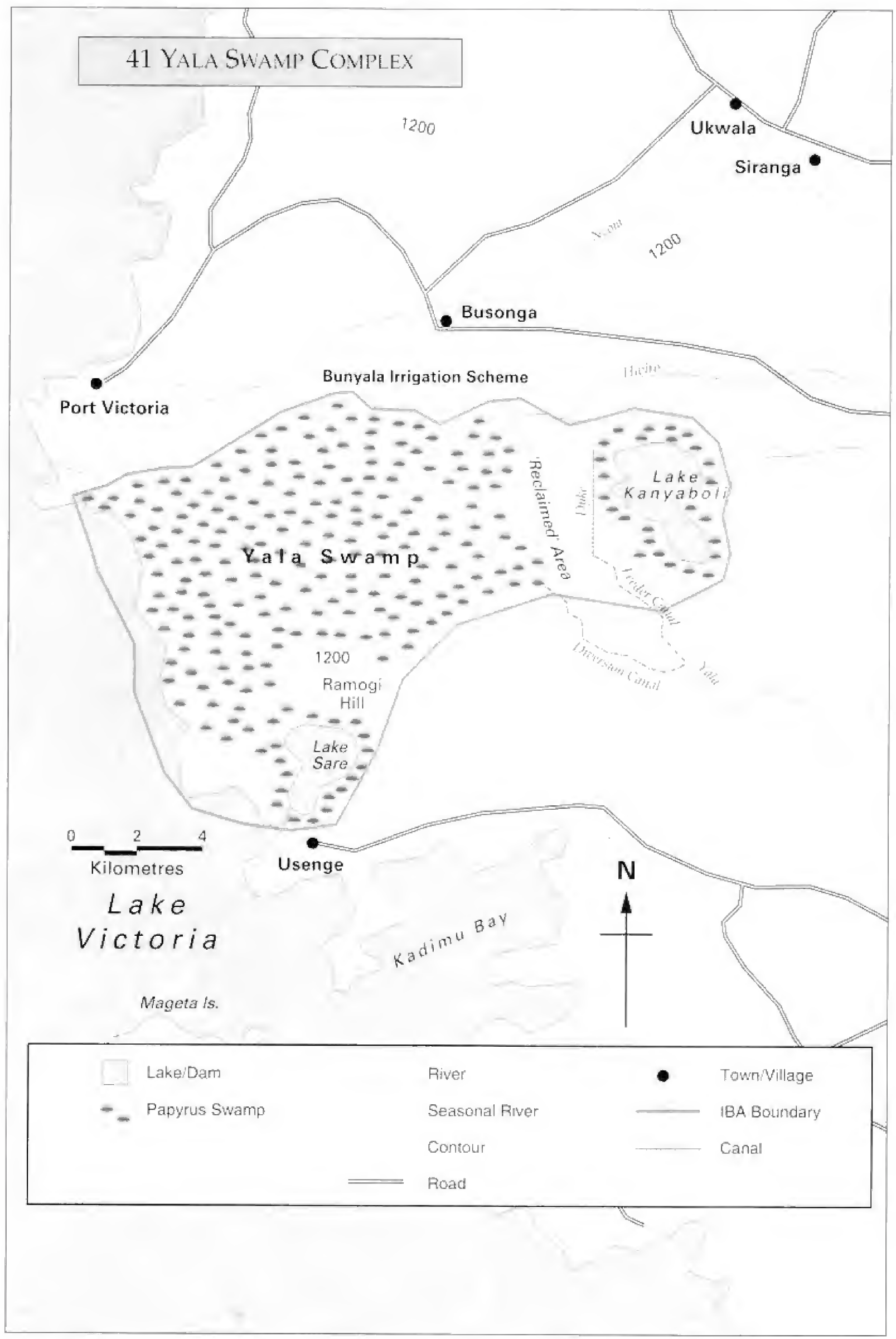




\section{Yala Swamp Complex}

\section{Globally-threatened species}

\begin{tabular}{|c|c|c|}
\hline Papyrus Gonolek & Neat-threatened & $\begin{array}{l}\text { Reasonably coumon (Britton 1978, } \\
\text { Nasirwa \& Nporoge 1907) }\end{array}$ \\
\hline Papyrus Yellow Warbler & Vulteriable & $\begin{array}{l}\text { Resident in small numbers (Brittom } 1978 \text {, } \\
\text { Nasirwa \& Nornge 1397) }\end{array}$ \\
\hline \multicolumn{3}{|c|}{ Regionally-threatened species } \\
\hline Great Egret & Vulnerable & $\begin{array}{l}\text { Present it small numbers } \\
\text { (O. Nasirwa } a_{r} \text { unpubl data) }\end{array}$ \\
\hline Baillun's Crake: & Vulnerable & 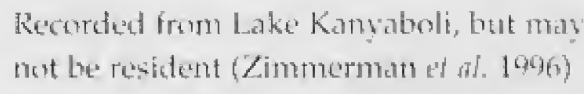 \\
\hline
\end{tabular}

\section{Other wildife}

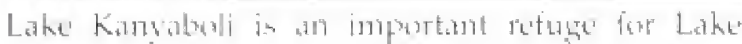

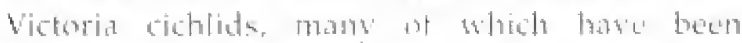

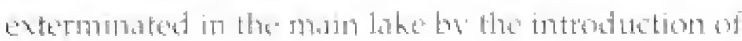

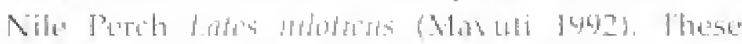
include ecomomically inportant spectes suth as

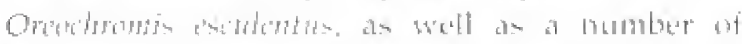

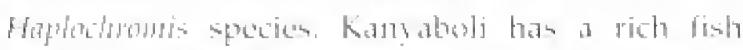

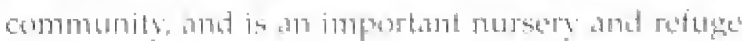

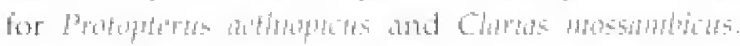
The Nile Ferch in frement in Like sore, which has an imponeristued fislo faume compared to Kanyaboli. Othex common tish species in the lakes and swalmp

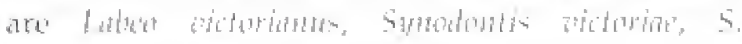

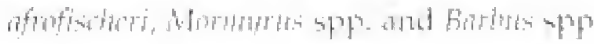

\section{Conservation issues}

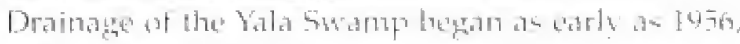

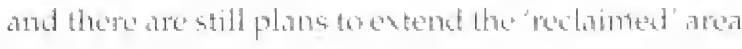
oyer much of the prosent swamp. Thes is ant

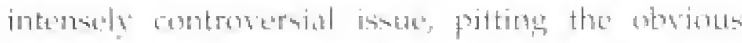

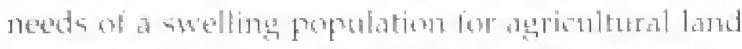

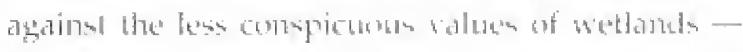
bot instance, water biltration, flenol soutrol, and

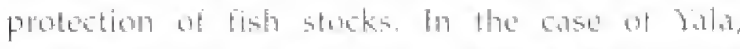

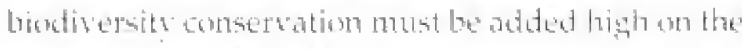
list of allues. This is a nery important site for

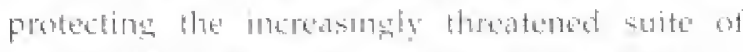
papyos birds, as wodl as ane of the last remuants of

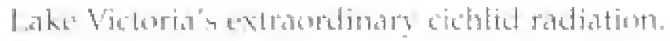

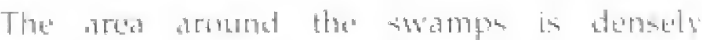

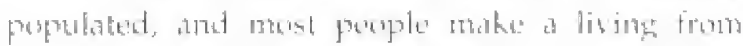

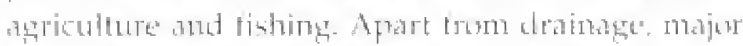

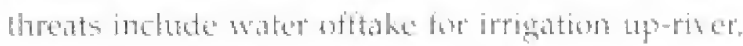
mtersificatenn of fertiliser and biocide inputs, and

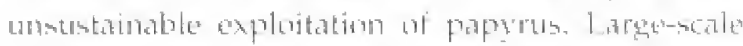

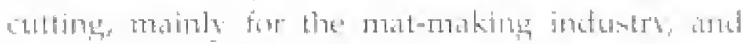
extetisige buang to apen up land for cultixation

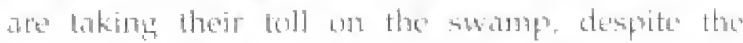

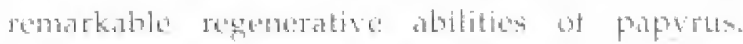

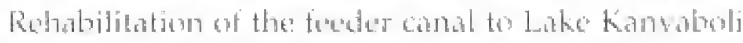

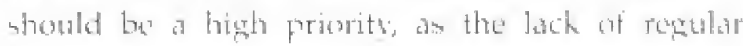
influss from the river are chamging its water chemintry and may interfere with its function

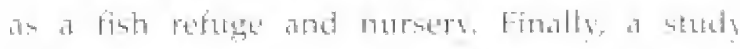

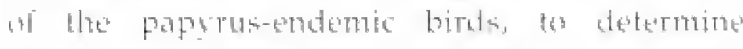

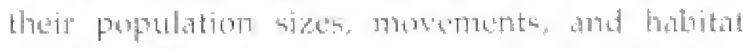

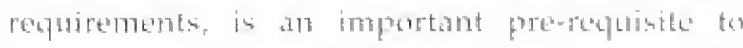

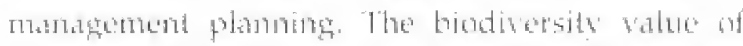
Yata Swamp should be recognised thy attorting the

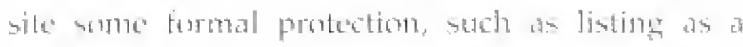

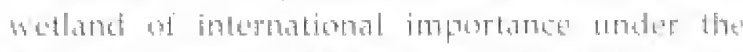

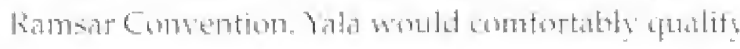

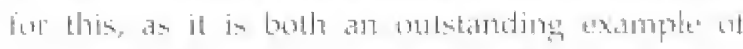
a specific befe of wetland and sipplorts an

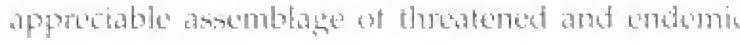
specticts.

\section{Further reading}

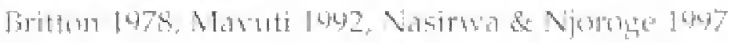




\title{
42 Amboseli National Park
}

\author{
$2^{\circ} 33^{\prime} \mathrm{S}, 37^{\circ} 06^{\prime} \mathrm{E}$, Rift Valley Province, Kajiado District
}

39,200 ha

Altitude: $1.100-1,200 \mathrm{~m}$

Status: National Park,

Category: Somali-Masai biome species

\section{Site description}

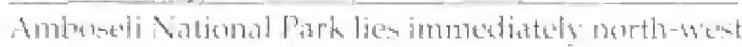

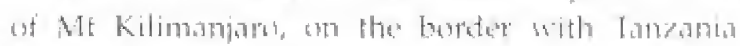

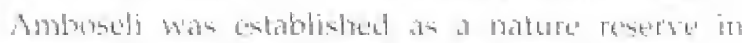

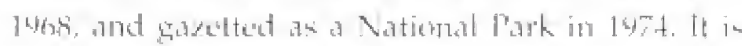
stermunded ly sid communally-ommed cimup

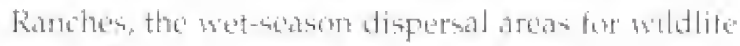
whome mathapenut has diret nothenes on the encolompal stabilits or the park.

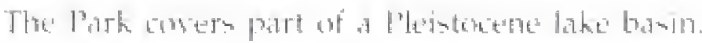

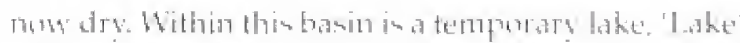

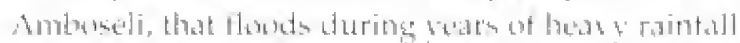

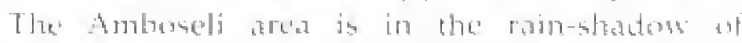

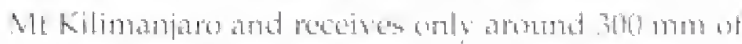

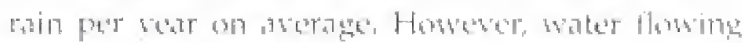

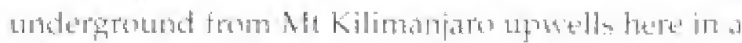

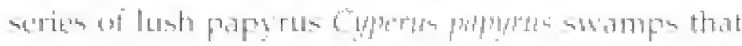

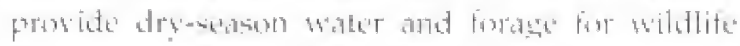

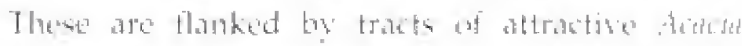

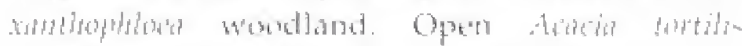

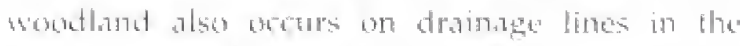
sisuthern part of the Park. The basin as surromated by disto-cimmithm bushland, while the lesel

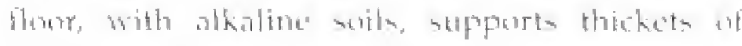

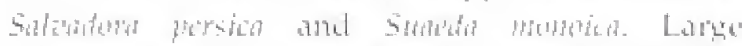

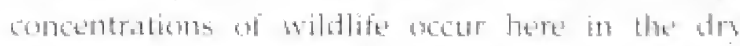
sention. This, with the preturestuc stromending dommated by the imposing bulk of Wt kilimamarob. Gase made Amboselt a morjer

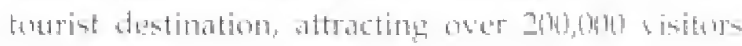
enach teitr.

\section{Birds}

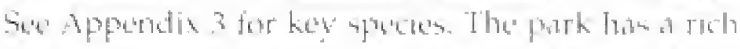

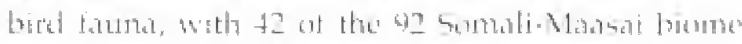

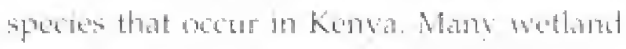
birds une, and at timen nest in, the

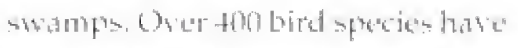

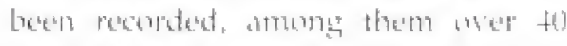

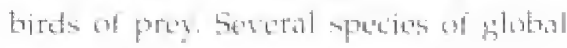

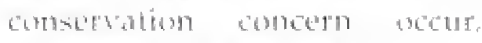
ineluding Leseren Kestrel (vedremable; on passagel. somall numbers or mon-

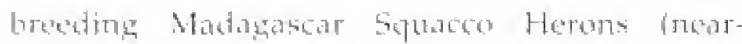

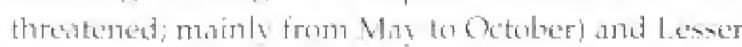

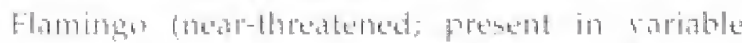

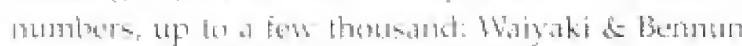

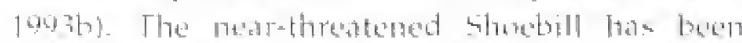

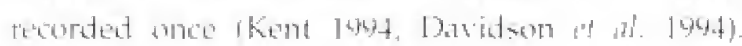

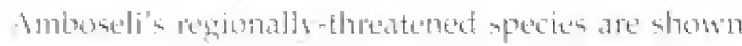
11. the buth.

\section{Other wildlife}

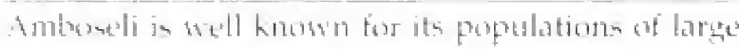

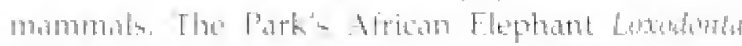

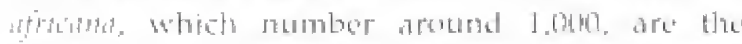

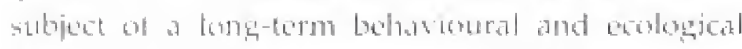
siluts: The Park's population or liack Rhinowerom

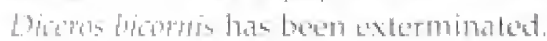

\section{Conservation issues}

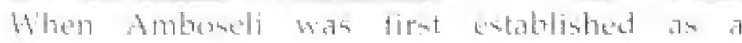

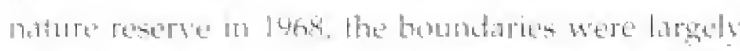
artitrars and thiled to coner the wet-seasen wildtite

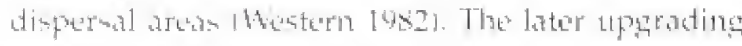
to Vationat l'ask shatus wats in part an atterspt to arrest the onetring condict betreen wildlife and the

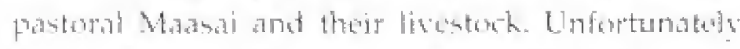

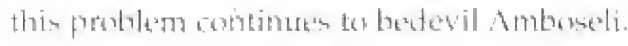

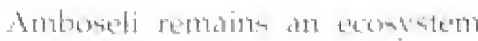
in the. The l'ark depends hearihy (i) sublteranean batker from

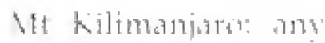

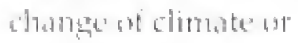
fand $-21=\mathrm{t}$ Black-Fined Sandegrouse 


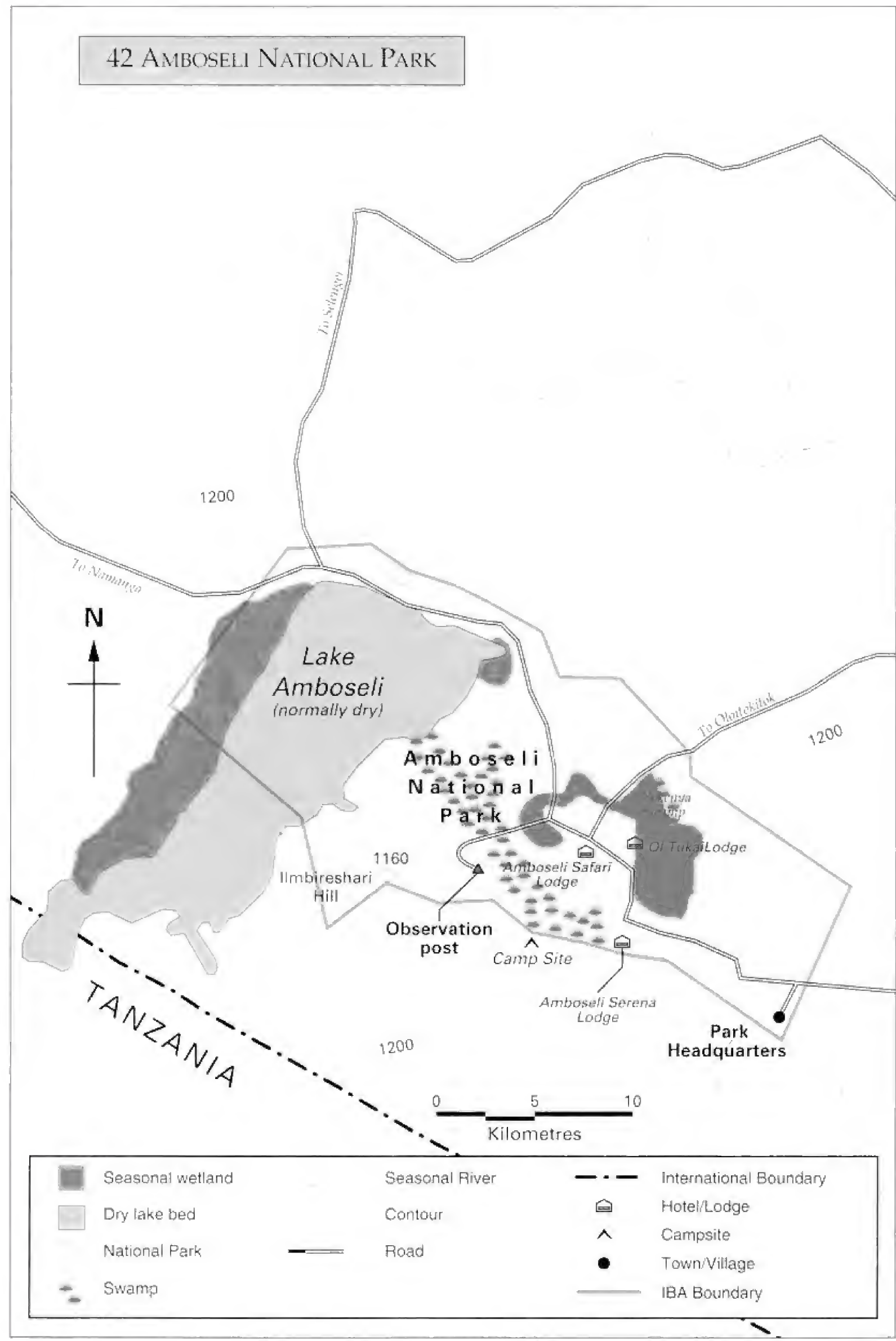




\section{Important Bird Areas in Kenya}

\begin{tabular}{|c|c|c|}
\hline African Dartet & Vulnerable & A scarce non-breeding visitor \\
\hline Great Egrel & Vulinerable? & $\begin{array}{l}\text { Usually present in small numbers in the? } \\
\text { swamps }\end{array}$ \\
\hline White-backed Duck & Vulnetritse & Occasional visitor \\
\hline White-headed Vulture & Vulnerable: & Uncommmon resident \\
\hline Martial Eagle & Vulnerable & Resident in small numbers \\
\hline
\end{tabular}

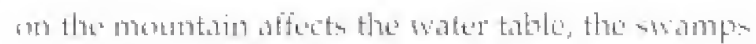

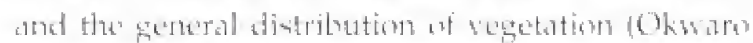

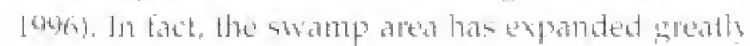

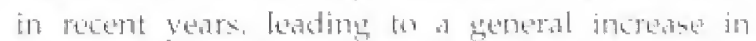
numbers of waterbisds, on the ather hathd, the Alater

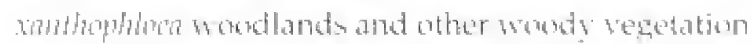

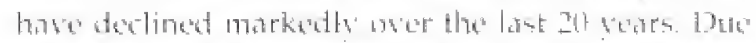

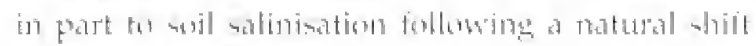

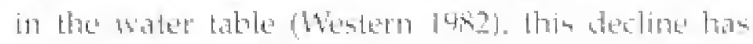
been hastened by heary bromsing preseure foum

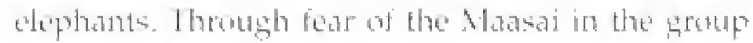
manches outside the porte the Antrmeli vephants hate gonfind themselves to the park bodndertes.

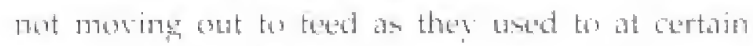

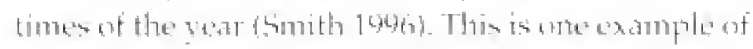
the park's major problem: haman-sildtite contlict.

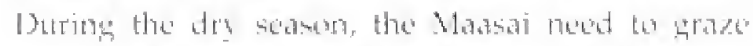
thent cattle around the smamper chring the wet

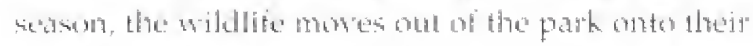
land in bearch of pasture. This problem appears to hove been at terst partially resolved in recent years.

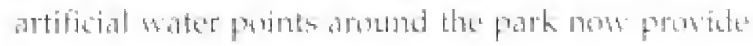

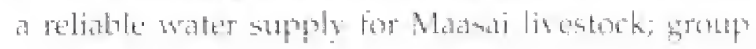

faraches are cumpensaled for the presence ot wilktlife

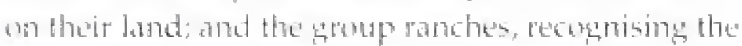

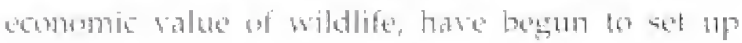

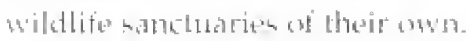

The large number of touriste sisiting anbuseli.

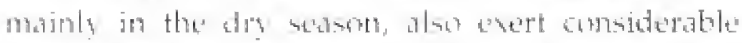

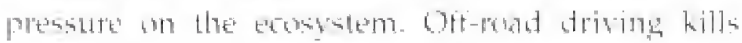

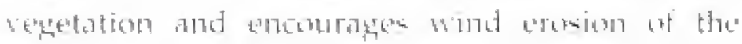

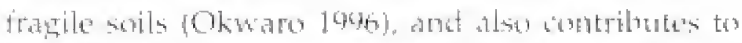
hadasment of semsitive specien suth as Chexthoth

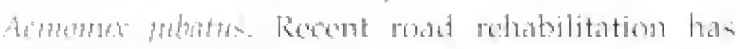

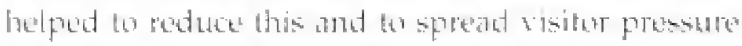
to lesarfoquented ports of the park. Accommedation fort sintemen in comcentrated ith the of Tukai areas

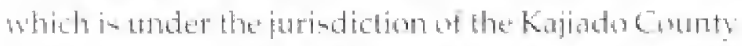

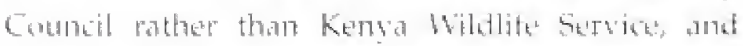

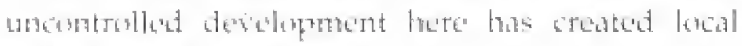
ent tronamental froblems.

\section{Further reading}

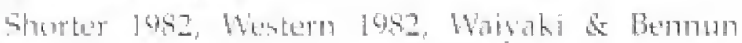
logab, Okwaro kata, Smith 1640 


\section{Cherangani Hills}

$01^{\circ} 16^{\prime} \mathrm{S}, 35^{\circ} 51^{\prime} \mathrm{E}$, Rift Valley Prowince, Elgeyo Marakwet, West Pokot and Trans-Nzola Districts

c. 95,600 ha

Altitude: $2,0001-3,365 \mathrm{~m}$

Status: Forest Reserve

Category: Afrotropical Highlands biome species

\section{Site description}

The Cherangani Hills, ath ald fault-binck formation

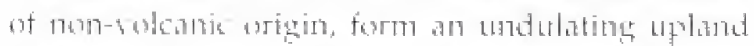

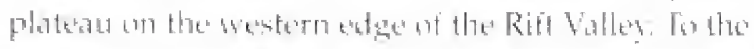

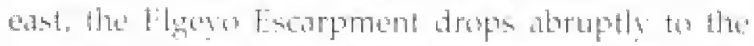

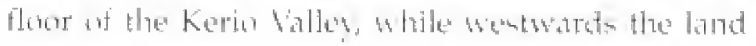

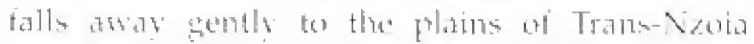

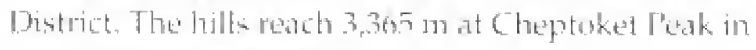
the north-terntmal setion.

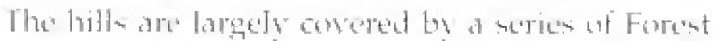

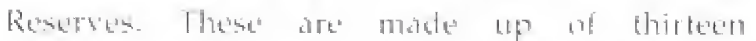

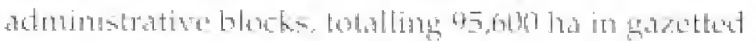

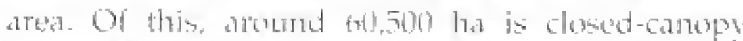
format, the remainder beirg tormutions of trambers.

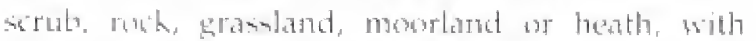
about tomp he ch cultisation and plastations

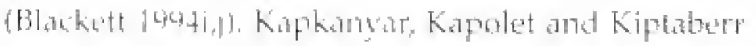

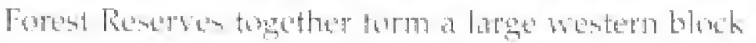

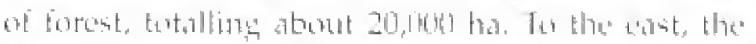

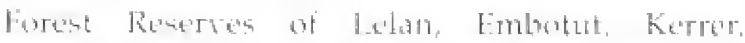
Käinumar, Toropket, Chembrokoi, Kupkunture.

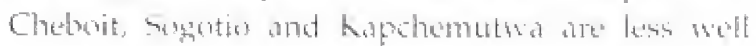

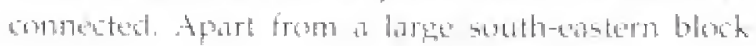

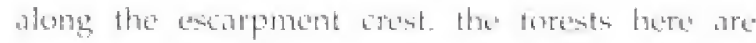
fragmented and separated by exteribine natural

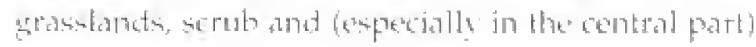
tirmlamet.

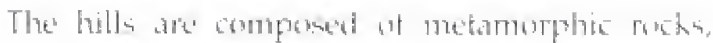

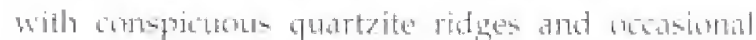

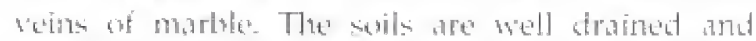
menterated tertile, and anmol ramfall varies from

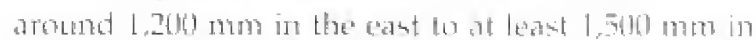

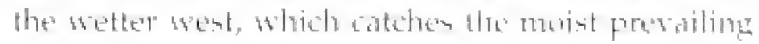

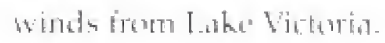

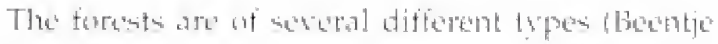

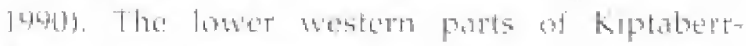

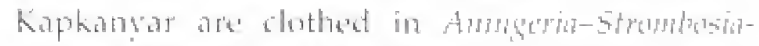

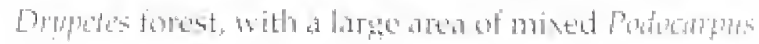

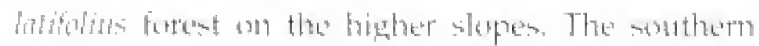

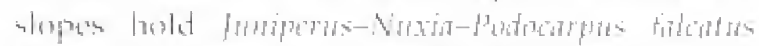

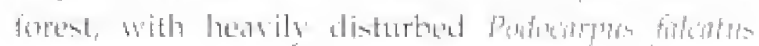

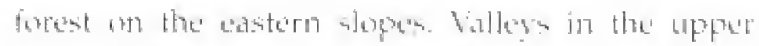

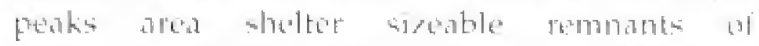

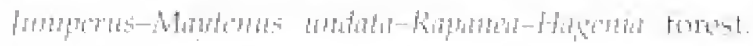

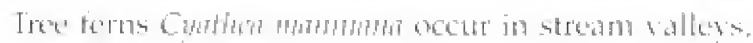

\section{Regionally-threatened species}

\begin{abstract}
Lammergeier Vulnurable?
Arrican Crownet Eagle Vulnerables

Red-hested Ontet Vulnerable
\end{abstract}

Гuгple-throated Cuckoo-shrike" Vulnerable

Thick-billed Honeygutide Vulneratse
The bills shelter one of the last breeding populations in Kenya nesting on the high poaks (5. Thomsett, in litt.)

Widespread in small numbers

Recently recorded in Kapkanyar (Waivaki 1996)

Uncommon and local (Zimmerman at al. 1996); recent records from Kapkanyar (Waigaki 1996)

Uncommon in forest interion (Zimmermanel as: 1996) 
Important Bird Areas in Kenya

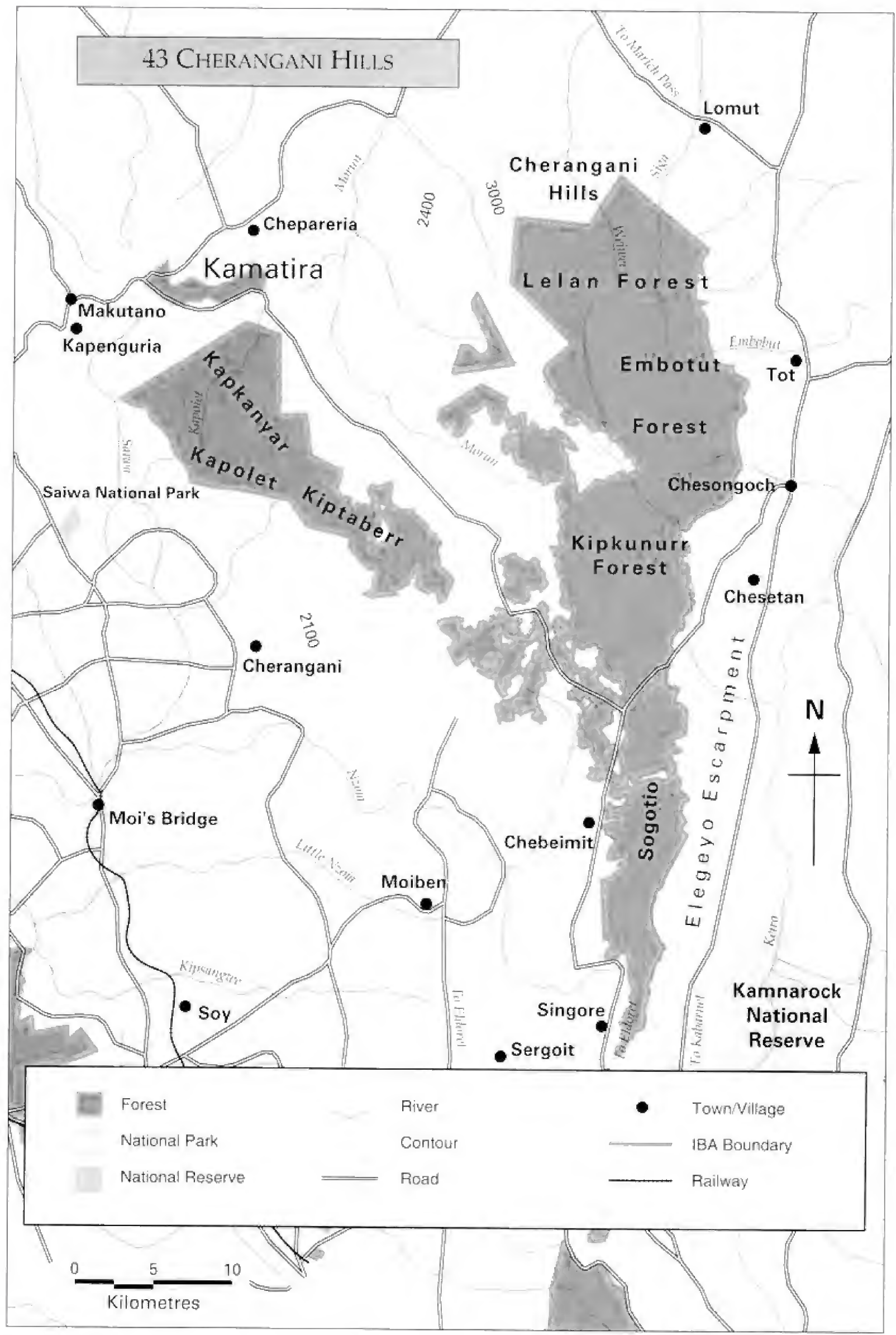




\section{Cherangani Hills}

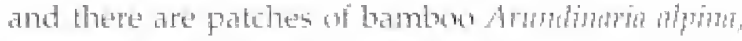
though no bamber zonte as such.

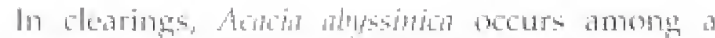
scrubty grablind with a distersity of flowering plants. An huther aleitudes, the torest in interspersed with a mixture of heath vegetation and swamass, the

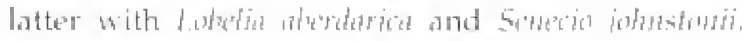

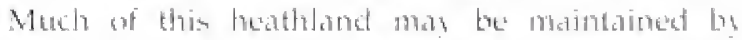

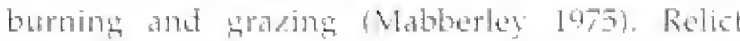

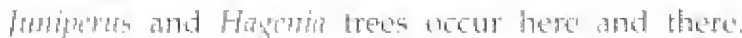
Fafextially an the dast, the

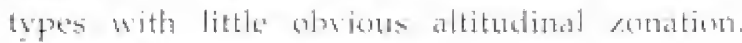
Mabberley (1972) ascribed thin to the hills' wriegt topogriphy and the lotpe histery at cultisation,

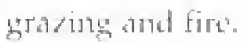

The Cherangani foreste are impertane for wate cateliment, and sit astride the waterathed between the

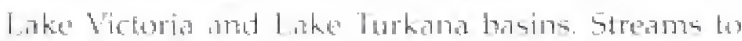
the west of the witerstued fored the Varia Riser sybem, which fous into Lake Victoria; streame tar the edst flew into the kerio River sytem.

\section{Birds}

Forts-nine of Konva's 67 African Hiphland biom species ocer here (see appendix 3). The andatan of the Cheramands is characteristic of the hightand forests of Kemat west of the Ritt Valles; which

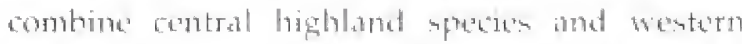

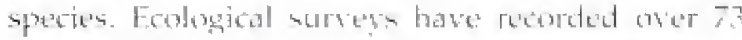

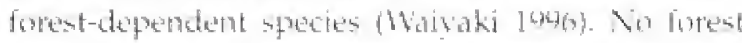

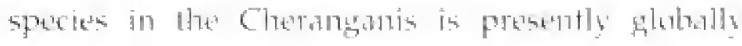

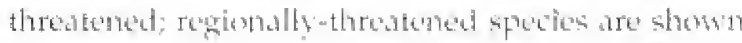
in the bay.

\section{Other wildlife}

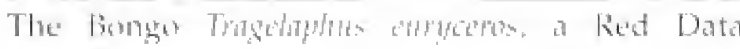
Book-listed speries, has been recorded here in the

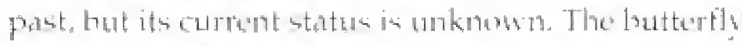

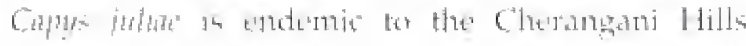
(Latsen 1641). Tho Giant Senceion taxa, sumpto

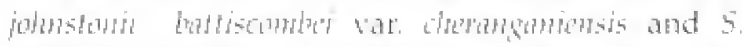

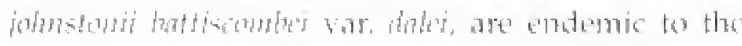

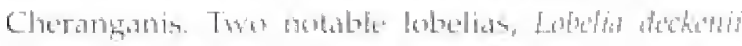

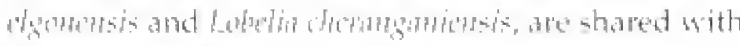

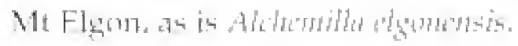

\section{Conservation issues}

The andition of many of the remuining foremt

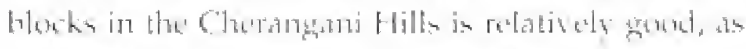
indicated lys the presente of masty torest-dependent

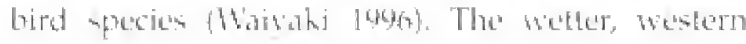

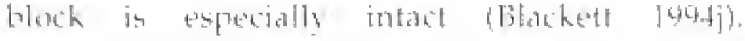
Nonetletess, there are a number onf berinus

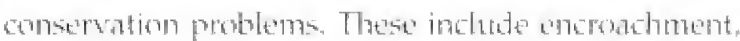
de-gazeltement for sellement, puaching of trees

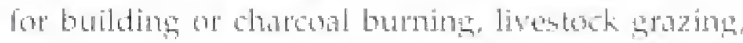
and tree-felting by hothey antherers ffor honey,

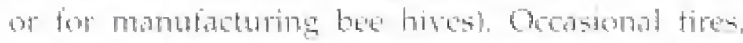

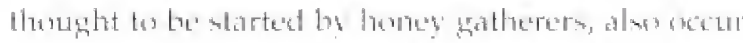

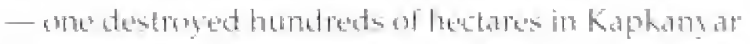

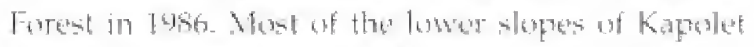

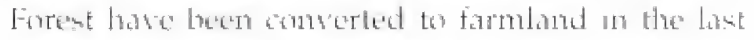
twenty seats, and similas threats liace mont of the forest blochs: subedisision and clearance of

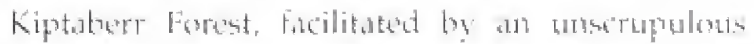
Forest [department emplaree, was recently halted

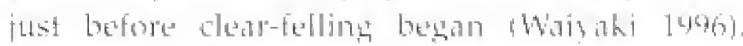

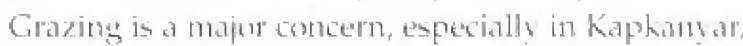
which burders land wecupied by the pastoralial

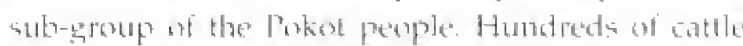

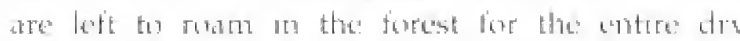
season period, cansing enormuls damage. As

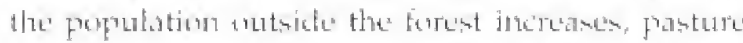
land diminishes and pressure on the forest riats.

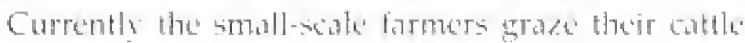
in panture land outside the burest, and the large luerds in the forests apparently bedomg to wealthy individuals whe are influental focally

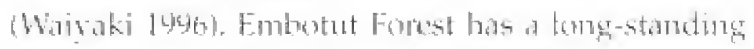
spuatter problem, with around 5, und people lixing

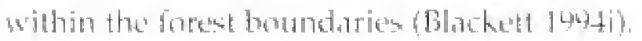

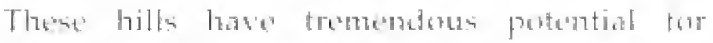

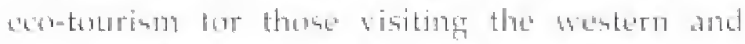
north-westem part of Kegry. They are scenically

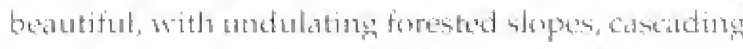
risers and a

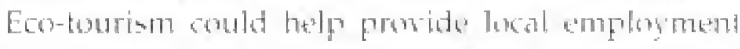

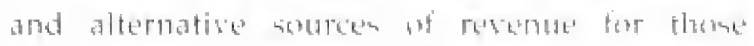
living atsond the forest. The Forest Depatmesn needs to be better whate of the natiomal and slobal significatice of the Cherangani forests and to protect them nome effectively. Education of the

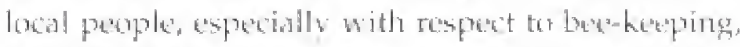
is important tors, and can help to present forent

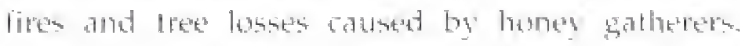
Heney collution, it properly contrenled and mashiged, can be a sustainatile use of the forest and indeced provide substantial incontines for latbitat conservation.

\section{Further reading}

Hancok 1971, Cheke 1472, 1978, Hablowley 1475,

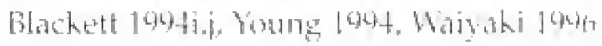




\title{
44 LAKE BARINGO
}

\author{
$0^{\circ} 38^{\prime} \mathrm{N}, 36^{\circ} 05^{\prime} \mathrm{E}$, Rift Valley Proxince, Baringo District \\ c. 28,400 ha, (lake 16,800 tha, surrounding area 11,600 ha)
}

Altitute: $975-1,070 \mathrm{~m}$

Status: Vational Reserse and inprotected

Catcgory: Somali-Masai biome species

\section{Site description}

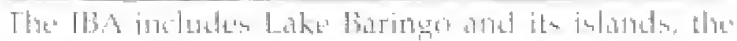

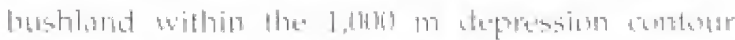

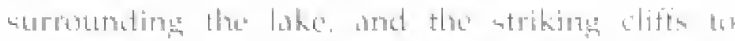

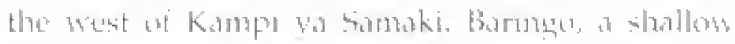

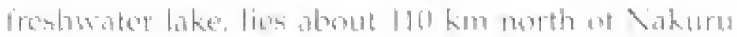

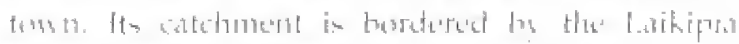

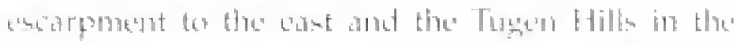

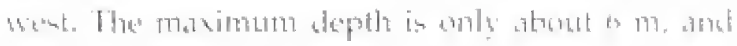

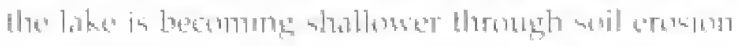

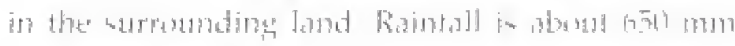

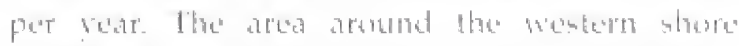

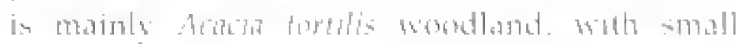

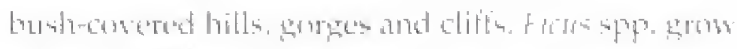

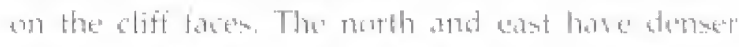

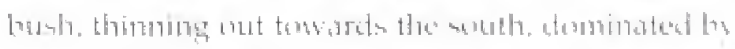

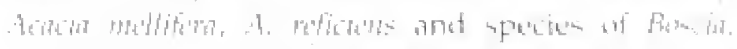

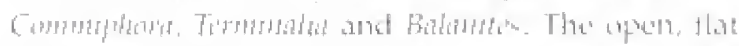

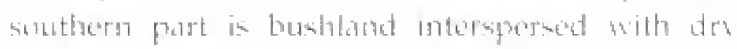

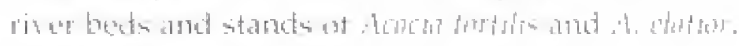

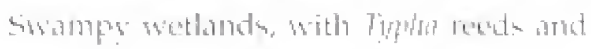

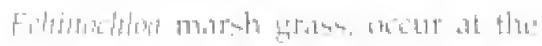

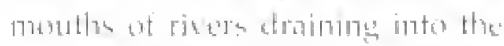

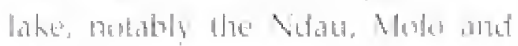

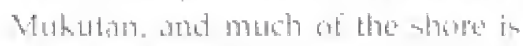

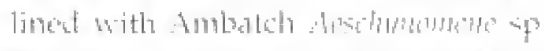

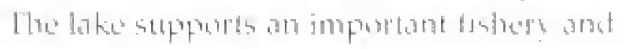
is a major tourist demtimatum.

\section{Birds}

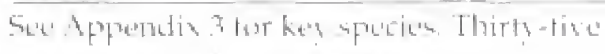

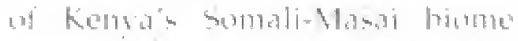
species ucero, including sereral tound al tew other site's. Votable specese ind lute the

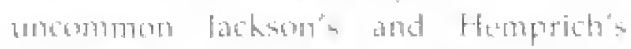

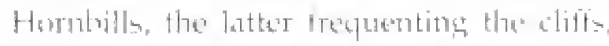

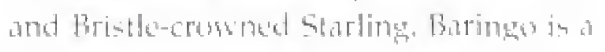

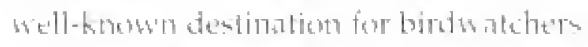

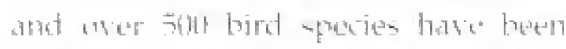

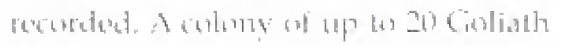

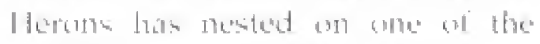

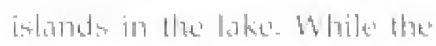
dintersy of waterbirts in concisterable,

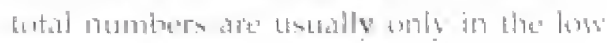

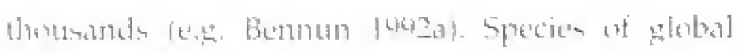

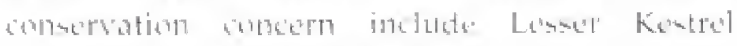

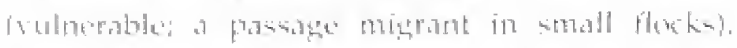

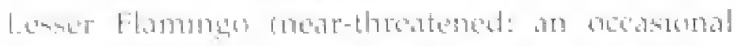

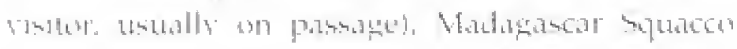

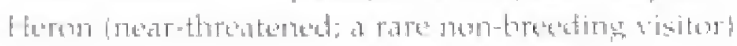

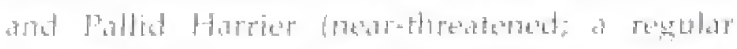

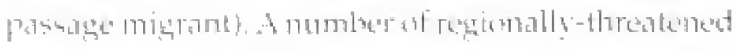

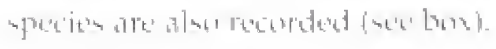

\section{Other wildlife}

Thu lake supperte harge populatioms of vile

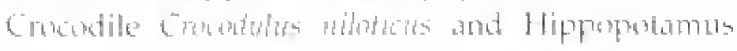

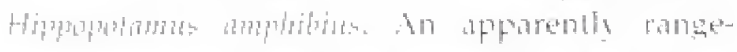

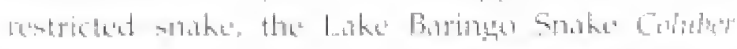

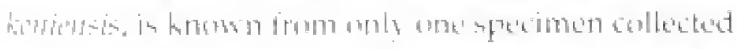

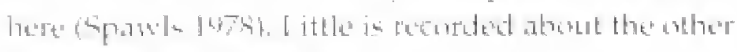

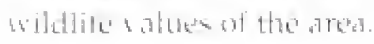

\section{Conservation issues}

The main somateration isate in baringe District is

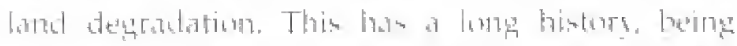

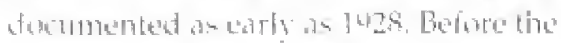

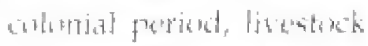
thumbers were kept at

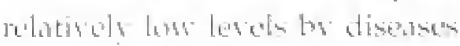

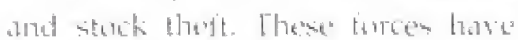

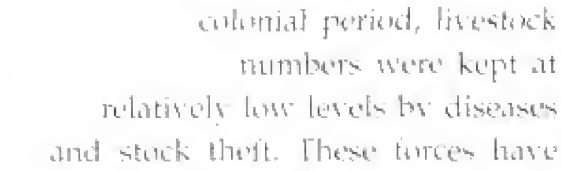

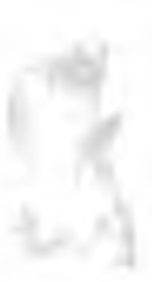

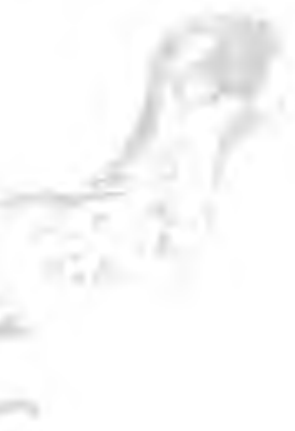

Homprichs Hernbill 


\section{Lake Baringo}

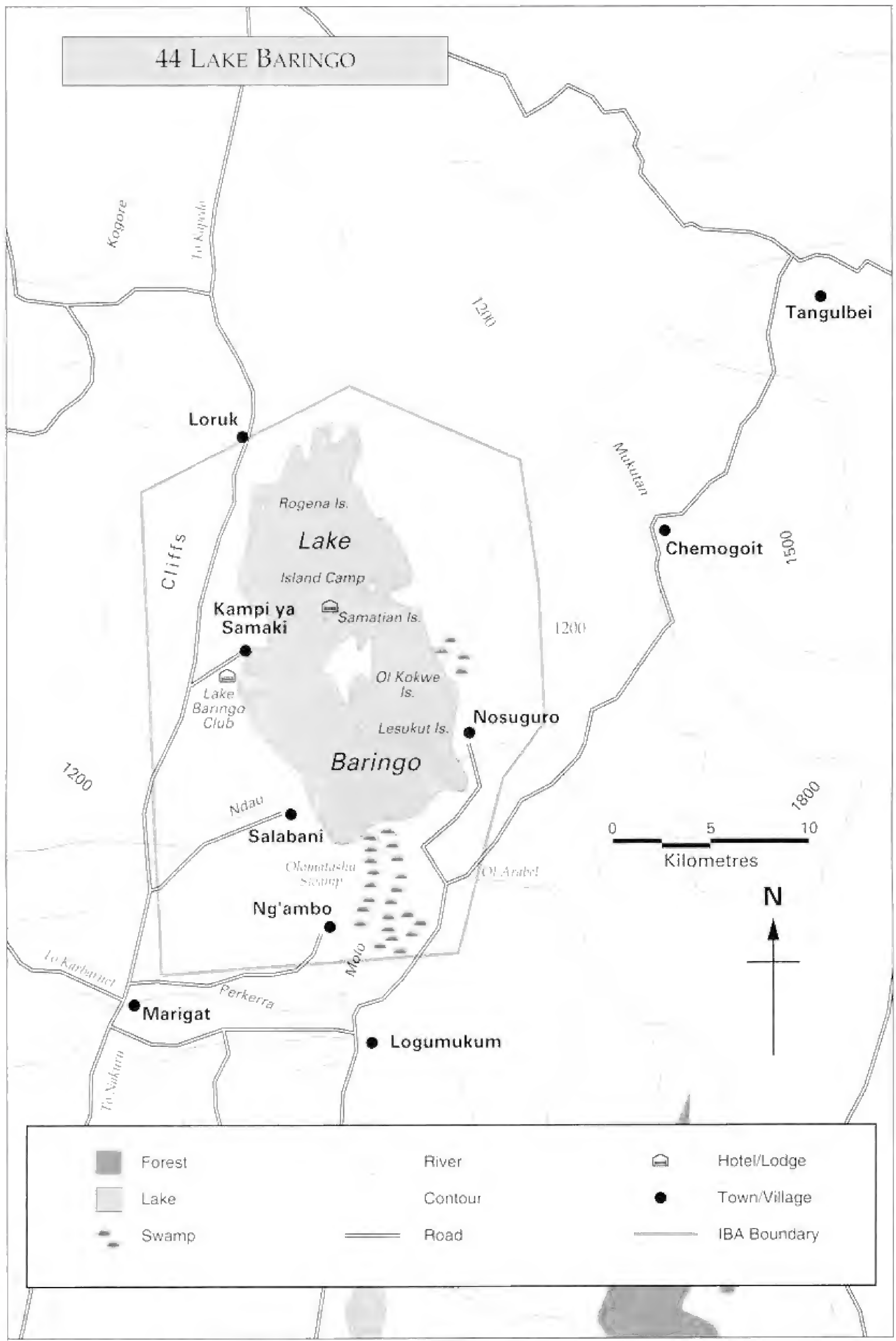




\title{
Important Bird Areas in Kenya
}

\author{
Regionally-threatened species \\ Great Crested Grobe Critical \\ Alrician Dartét \\ Vulnerable \\ Great Egret \\ Vulnerable \\ Saddle-billed Strork \\ Vthinerable \\ White-backed Duck \\ Vulnerable \\ White-headed Vulture \\ Martial Eagto \\ Bailon's Crake \\ African Skimmer \\ Vulnerable \\ Vulnerable \\ Vulntrable \\ Vulnerable \\ Data from Sterenson (1980), Zimmerman et al. (1990) \\ No recent records \\ Small numbers resident, has bred on \\ Natu lstand in the lake \\ Regular, up to 100 recorded \\ (Stevenson 1980) \\ Ocasional risitor \\ Occasional tisitor \\ Occasional k'isitor \\ Occasional visitor \\ Irregulas wisitor \\ Irregulas visitor
}

boen checked since then, and livestuck numbers have steadily inceded. Orerstomking and high srazing presters have reduced ground coter and

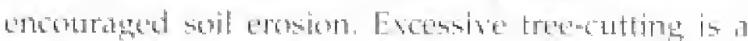

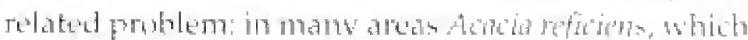

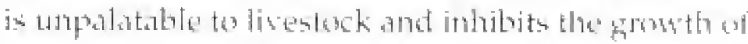
wher platuts, has replacted the oritimal trees and

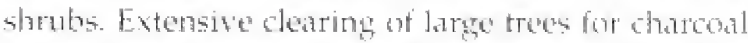
has seriousty deyraded some of the ment moportant and well-knewy birdhatehing areas, and chathed the neiture of the lecal avifauna.

These environotiontal changes have eontributed to periodic flasoding that causes massine epporates of eromion, with most of the soil beidg washere donen into bake baringo. The lake is much mate turbid thine in past years and fisth stock three declined.

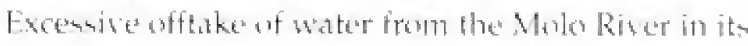
catchment is also a proditem, sime it has greatl

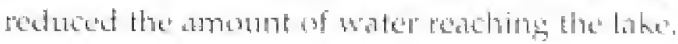

Mamy ensibumental propects have atempent to solve these problens, but memat have beren complicuarasty tenculecentul. One of the mome promising attompts is the Baringu Fut and Fodder fropect, wheth started in 1981, and until recently was funded by the Vethertancts genermment. It aims at rehatulitating parts of the degraded western shores. by 1942 the propect hat mand a ha, by creting plenghed suriaces to trap water and replanting with garassos and troes in fenced-ond plath.

The lake and part if iss hinterland are now a Vational Reserte under the matagement of the

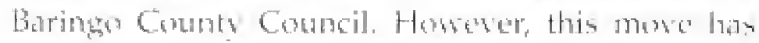

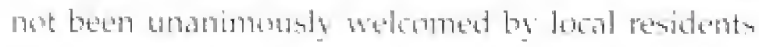
and horteldedegers, some of whern clam that

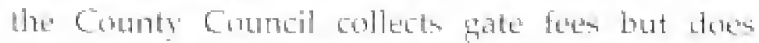
Jittle to deal with pressing conservation ancerns.

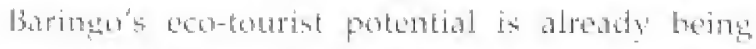
explaited. This needs to be botter tied in to the local teomomy, so that those living around the lake lawe more of a stake in conserting its special birds what there habitals.

\section{Further rearding}

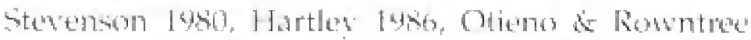
[4his, Gitoger 1453 


\title{
45 Lake Bogoria National Reserve
}

\author{
$0^{\circ} 15^{\prime} \mathrm{N}, 36^{\circ} 06^{\prime} \mathrm{E}$, Rift Valley Prowince, Koibatek and Baringo Districts \\ 10,700 ha, (lake c. 3,000 ha, maximum 4,250 ha)
}

Altitude: $1,000-1,600 \mathrm{~m}$

Status: National Reserve

Categories: Globally-threatened species, congregations

\section{Site description}

Bogaria is a narroms, shallow, athaline lake (maximum depelin 8.5 mi) on the Rite Valley floot: To the east, the Siratho excarpment rises ahruptly" from the lakeabore, while on the relatively flat western shore is a serves of spectucular hot springs and meysers. The reserse wa gayelted in 1481 and incfudes the entire lake ard its immediate surroundings. The water 1 PH \& 10.0 , conductivity

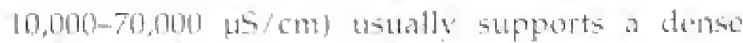
bloom of the cramophste spration sp. The terrestrial beyetation is mainly thom bushand,

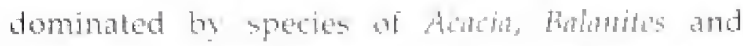

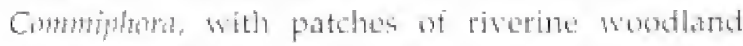

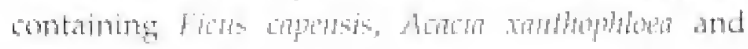
A. tortilis. The spen shore, often littered with lavia butcters, is dominated by alkaline-tolerant grasslands of spomolus spronts, with the sedge

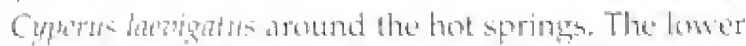
slopes of the siruche esarpment are correred by

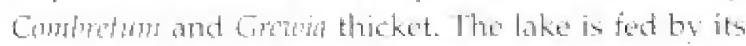
apring and by the Sandai (on Whatese) River, whide rises on the eastern sadp of the Rith Valles. The Sandai mow's pat the lake and them turna through $180^{\circ}$ to conter it from the noth through the Kisilwor Swamp, a sizentale freshwater wetland dominated by Tinitit.

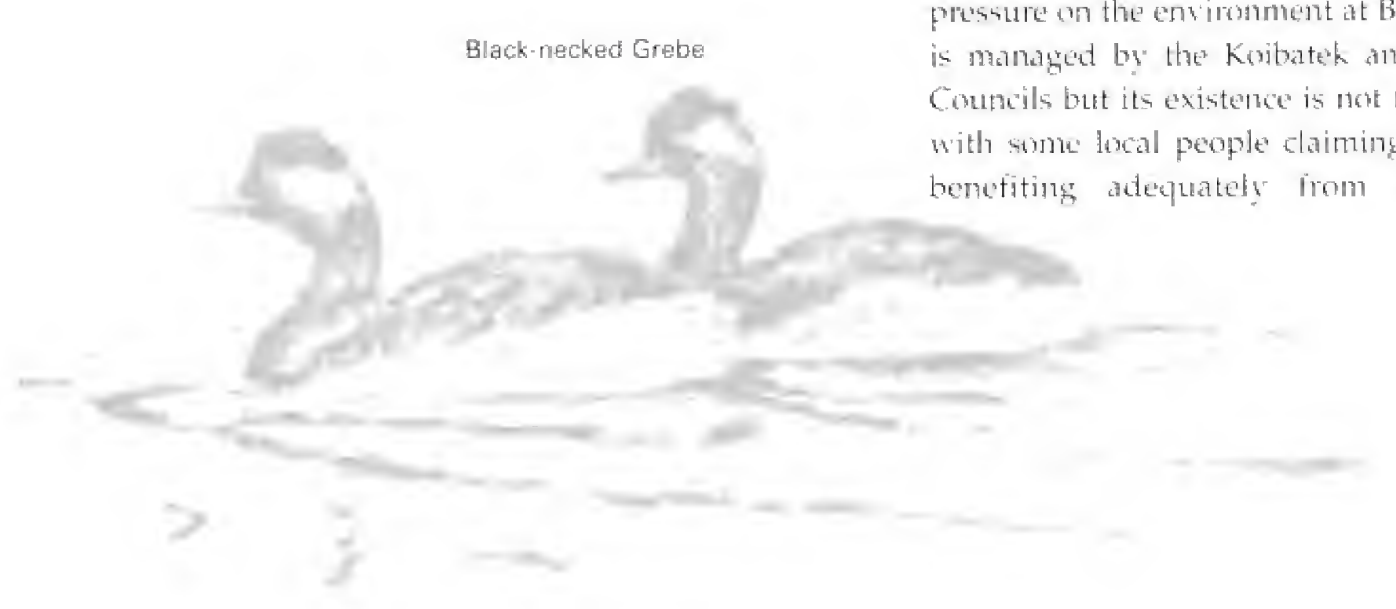

\section{Birds}

Sere box bor key spectes. Bogroria is a key feediag ground for the itherant Rift Valley population of the near-threaterned Lesser Flamingo. Spectacular constegations (estimated at up tos 2 million birds) uceur at times, and several humdred themsand birde are often present. Bhach-nerked Grabe and Cape Teal are ustatly present in gout numbers. Thirty-une of Kenya's 97 Somali-Masai biome species occur in the beshland and wondland around the lake (Appendix 3). Other speries of ghobal

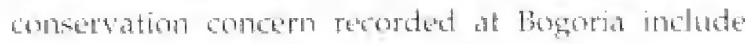
Pallid Harrier (neatr-flareatened; octurs an pasadge) and Lesset Kestrel (rulneralble; also ucours on pasage in small numbersi.

\section{Other wildife}

Apart from the enormous concentrations of flamingos, another teason for desisnating Lake

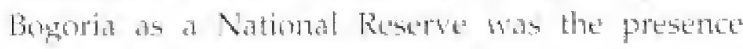

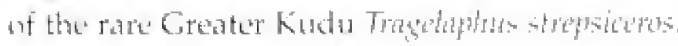

\section{Conservation issues}

L.ake Bogoria is spectaculat. The entratordinary hat springs and geveres, the rugised faulted landscape, the huge numbers of flamingos and the thances of secing Greater Kudu arte great attrations for visitors. At present, kourises exert no obrious pressure on the enviroment at Bongria. The reserse is managed by the Koibatek and Baringe Count Coundils but its existeme is not free of controxersy with some local people claiming that they are not benofiting adequately from the income that 
Important Bird Areas in Kenya

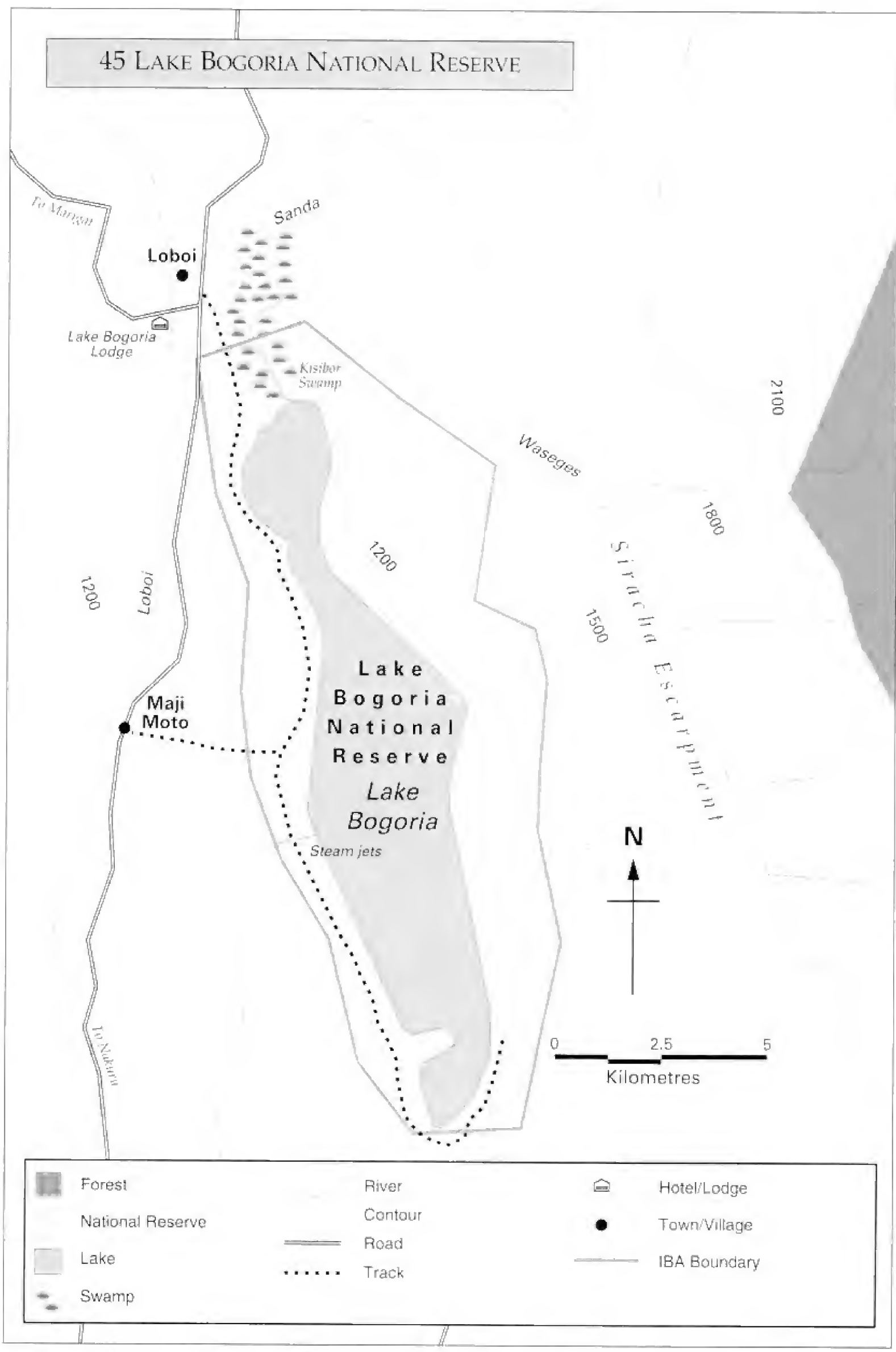

194 


\title{
45 Lake Bogroria National Reserve
}

\section{Globally-threatened species}

Lesser Flamingo Near-1hutened

\begin{abstract}
A key feeditig site for this speries; numbers fluctuate less than on other Rift Valley lakes. Although large numbers of very young birds may be present at times, this species has not bred successfully here (Brown 1973, Bemmun 1993, Nasinwa \& Bentun 1994, 1495, umpubl data)
\end{abstract}

\section{Regionally-threatened species}

\begin{tabular}{|c|c|c|}
\hline Great Crested Gretse & Critical & No regent records \\
\hline African Datter & Vulnerable & las occurred in swamp to north of lake \\
\hline Maccou Duck & Vulnerable & Nor recent records \\
\hline White-backed Duck & Endangered & No recent records \\
\hline White-feacted vulture & Vulnerable & Stantus uncertain \\
\hline Martial Eagle & Vulnerable & Probably resident \\
\hline
\end{tabular}

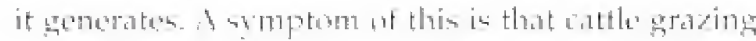

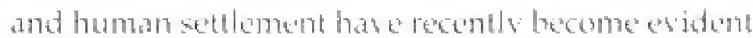

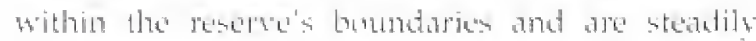

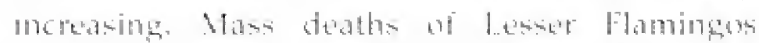

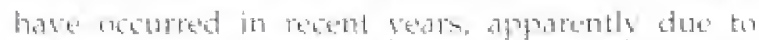

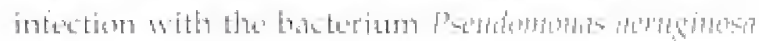

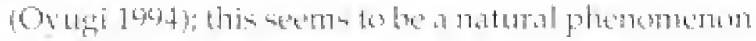
and not cause for great alarm.

Further reading

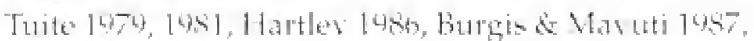

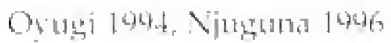

\section{Congregations}

More than 20 oOd woterlinds

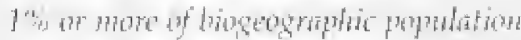

Greater Flamingo $(1,250)$

Lesser Flamingo (20,000)

Black-necked Cirebe (250)
1992-1997 January mean 425,600 ; max: 874,000 (1994): 1.5 million estimated July 1904 (Nasirwa \& Bennun 1995).

1992-1997 lanthary mean: 4,$400 ;$ max: 10,700 (1995)

1992-1497 January mean: 415,400 ; max: 865,000 (1994). 1.7

milliom csimated in a grounct count on 23 July 1994

(Nașinta \& Bennum, 1495)

1992-1997 Janนary mกานา: 1,$600 ;$ max: 3,700 (1997)

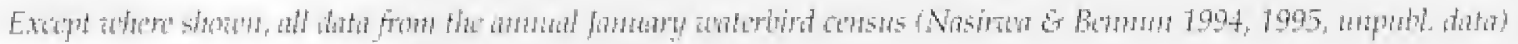




\title{
46 LAKE ELmenteita
}

\author{
$0^{\circ} 27^{\prime} \mathrm{S}, 36^{\circ} 15^{\prime} \mathrm{E}$, Rift Valley Province, Nakuru District \\ 6,300 ha, $(1,800$ ha lake, 4,500 ha Soysambu Wildlife Sanctuary) \\ Alfitude: $1,775-1,950 \mathrm{~m}$ \\ Stafus: Part unprotected, part private wildlife sanctuary \\ Catcgories: Globally-threatened species, restricted-range species, \\ congregations
}

\section{Site description}

Elmenteita is a shallow alkaline lake (maximum deptly $1.4 \mathrm{~m}$ ) lying on the Rifl valles flow some 20 km south-east of Nakuru town. It in fat by the Kokopey hot springs at its southern end. and two small streams. the Mertroni and Kariandusi. flowing from the eastern plateau. The sumounding landscape is chancterised by dramatic rothy faults,

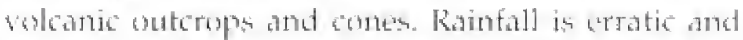
lebs than 600 mon on average per year. To the ceast, the lake is tlanked by small-scale acriculture, while several large rathes surround the remainder. The northerr and southantern hakshores are opem and Fat, a spectacular cliff tises to the mortly-e ant, and the western shores are broken and rocky. The matural

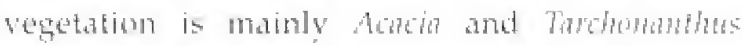

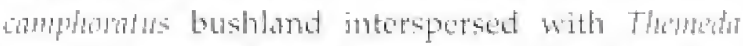

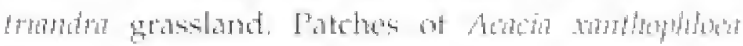
werdiand occur near the shore, and formerty comered a large area south of the lakn. The" [DSA conmist of the laki and it surrounding sherelines. including the hot spretnes and the clifts, and the Saysombu Wildife Sanctuary (part of the Delamere? Estates that alute the lake to the norkh and west.

Greater Flaminge

\section{Birds}

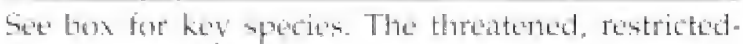
range Gres-osested Hedmet-shrike oceurs in the surrounting wostand, and Tackson's Widowbird nests in the prasshant. The lake cunsistently holds internationally important populations of Greater and Lesher Faning and liegt Avocet. At least 49 waterbird specien are recorded, including 10

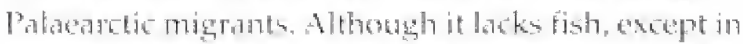
the peripheral bot oprings, Elmenteita at times is also host to large numbers of Great Whife relicans. Lp to

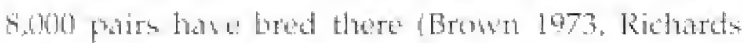
1491) when whor levels are high and noky outcrops

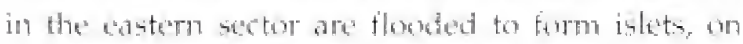
which the birets can safely nest. The pedicarion mete

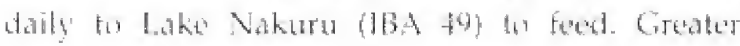

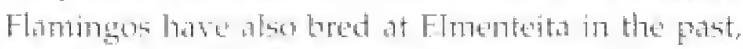
but have been displaced by pelicans in recent

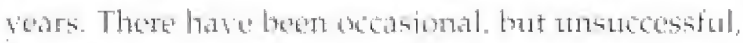
breeding attempts by the near-threatened Iesent Flamingo (Brostly 1473). The woodland and

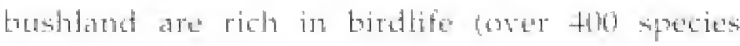
hate been retarded), particularly raptors, As well an the specites bhown in the box, Lesser Kestrel frulnerable accurs on passace, and varant Groater spotted Fagle (volnerable) and Redthrodted Tit (ruas"-threatemed) hate betan recorded (D.A. Turner, in Jill.). 


\section{Lake Elmenteita}

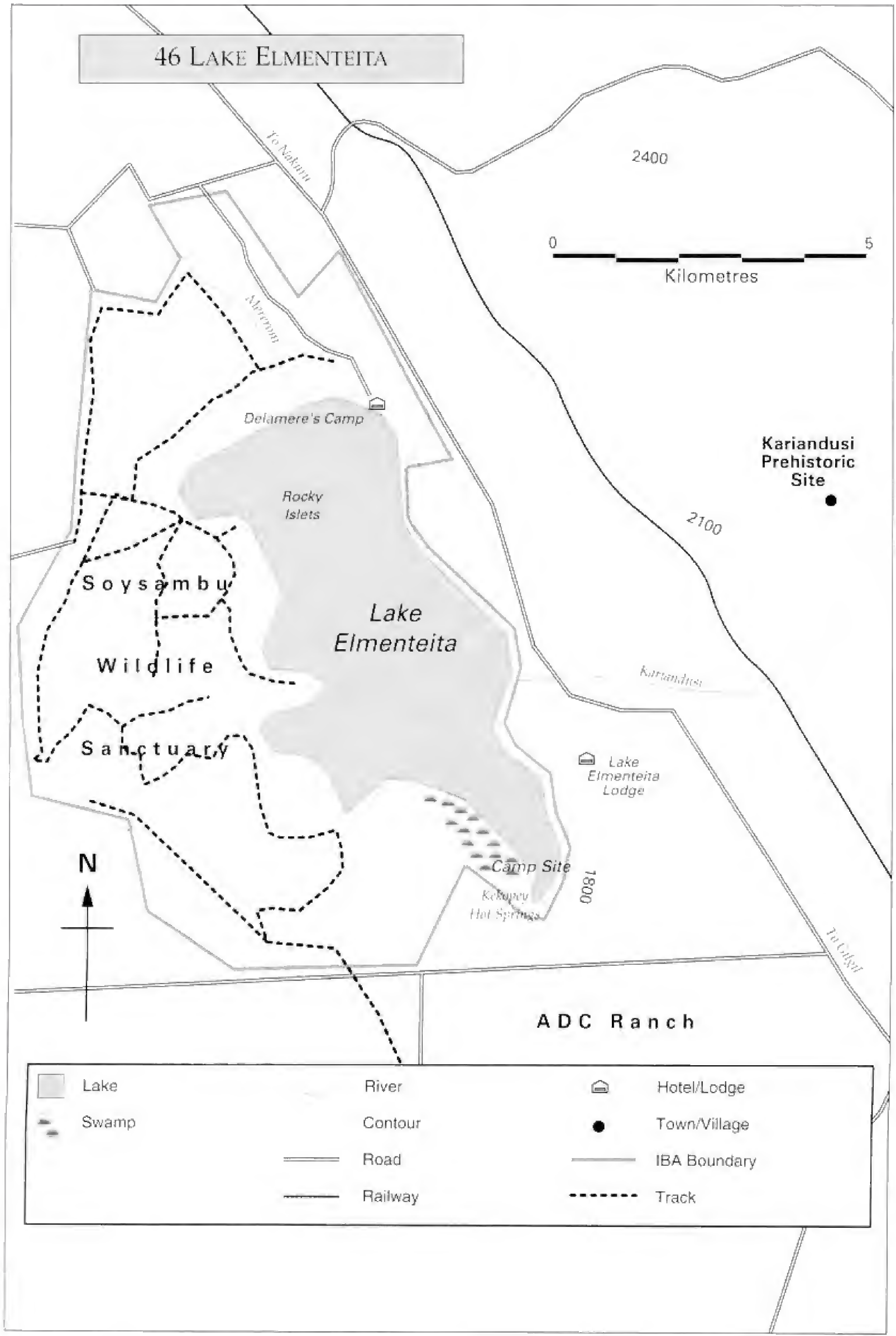




\title{
Important Bird Areas in Kenya
}

\author{
Congregations \\ Wore than 20,000 andering \\ 1992-1997 January mean: 171,000. Max: 486,000 (1944)

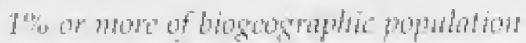 \\ Black-necked Grebe (250) 3,000 in lanuary 1490 (M.A.C. Combrdale, in litt.) \\ Great White Pelican (1,800) $\quad 11,800$ in January $1942,1,400$ in lanuar 1997 \\ African Spountilt (150) $\quad 260$ in January $199-4$ \\ Greater Flamingo $(1,250) \quad$ 1992-1997 January mean: b, 1007 ; max: 23,810$)(1993)$ \\ Lesser Flamingo $\{20,000) \quad$ 1992-1997 January mean: 150,$100 ;$ max; 666,61$)(1494\}$ \\ Pied Avocet (250) 1992-1997 lanumry mean: 2,200) max: 4,200 (1997)

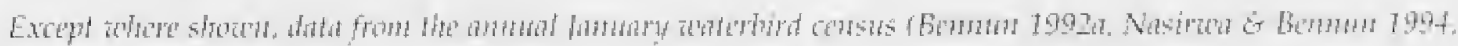 \\ 1995. amulli, data?
}

\section{Other wildlife}

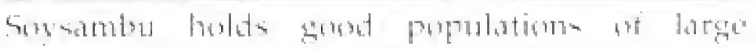

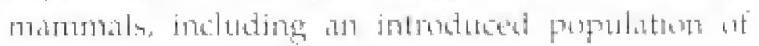

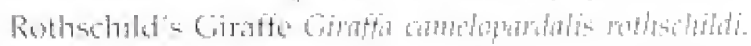

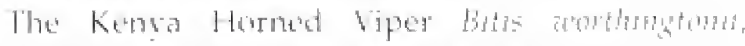

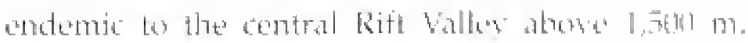

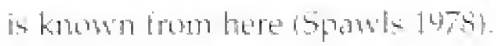

\section{Conservation issues}

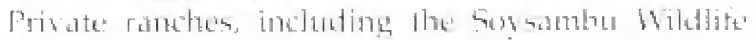

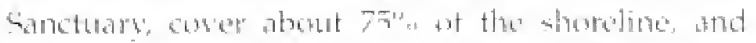

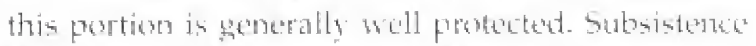

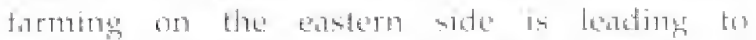
increased siltation of the lakt from soil teromon, while

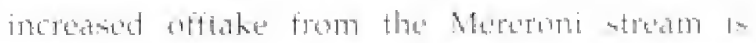

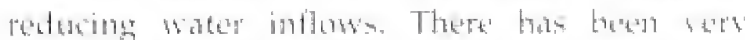

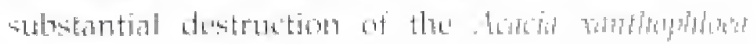

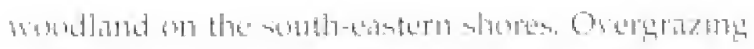

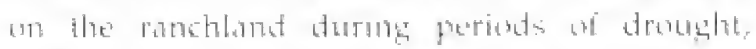

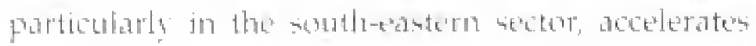

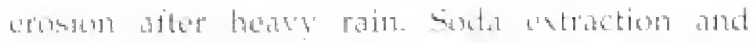

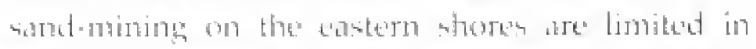

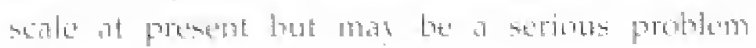
in tuture:

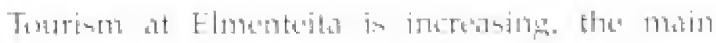

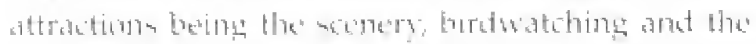

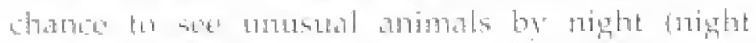

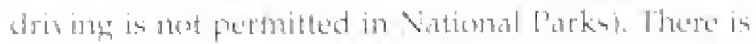

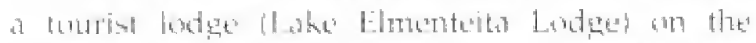

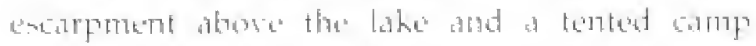

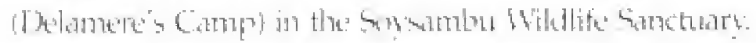
At the momene temorisn exerts little pressure and the

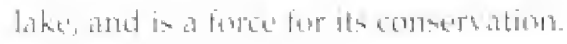

\section{Globally-threatened species}

Lessur Flamingo Near-threatened

*Grey-crested Htanel-shrike Vulnerable

"Tackom's Widowbird Near-threatened

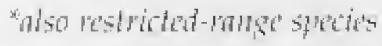

Usually present in tems or hundreds of thousantis (see betow); has attempted nesting (Brown 1973)

Several recent records in whothand; may bes resident (Bennาum 1494c)

A seasomal risitor that inests in tall grassiand 


\title{
46 Lake Elmenteita
}

\author{
Regionally-threatened species \\ Great Crested Greber \\ Critical \\ Cireat Esret \\ Vulnerable \\ (Nasirwa \& Bemmun 1994)

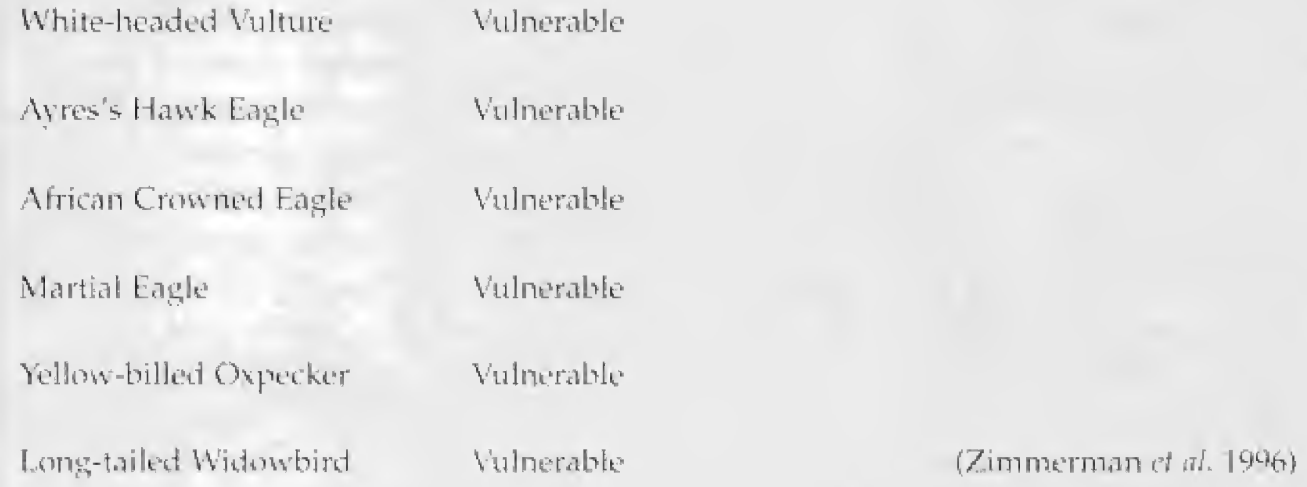

Several pairs have neated retently

(Ormithology Dept, umputil. data)

An ingertant non-breeding site for this species, with regularly more than 50 and as many as 156 counted in January 1994 


\section{LAKe Magadi}

$01^{\circ} 52^{\prime} \mathrm{S}, 36^{\circ} 17^{\prime} \mathrm{E}$, Rift Valley Province, Kajiado District

$10,500 \mathrm{ha}$

Altifude: $580 \mathrm{~m}$

Status: Unprotected; part private land

Cafeyory: Congregations

\section{Site description}

This is a shallem alkating lake", its surface larged

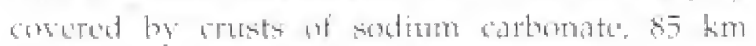

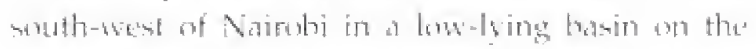

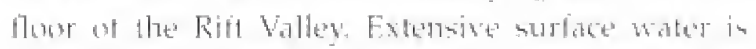

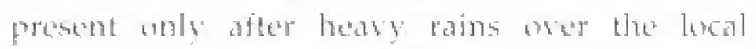

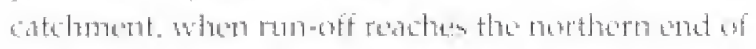

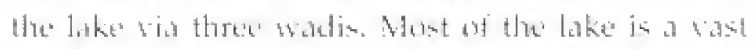
exparse of sulid sudium carbomate (tromat and alliest salts, some $15-30 \mathrm{~m}$ thick. This is mined by the Nagda Sudid compang, whose factory and

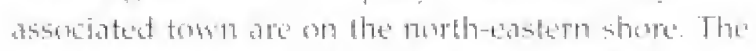

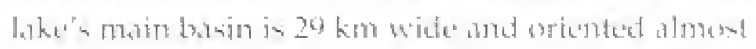

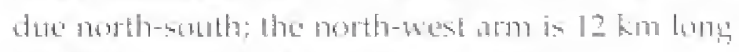
and $2.5 \mathrm{~km}$ widt. The lake is somponded ind fod with siater by a number ont hout

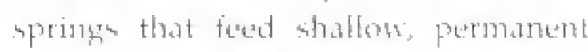
letgesses at the northere, sontheren and

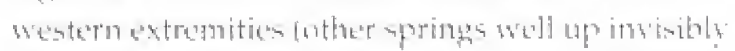

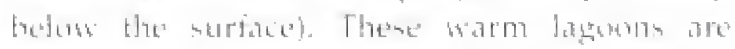

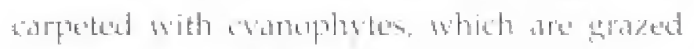

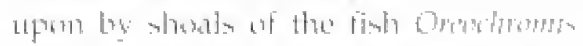

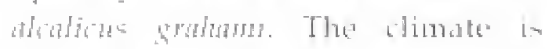

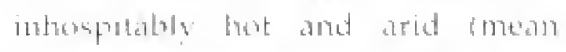
maximum temperatules $2.35 \mathrm{C}$, ramtall aramet the mom pere fearb. and the" Negeatation eneroundimg the lake is sparac, tputed bumblant.

\begin{abstract}
Birds
hee box for kets species, Birdlite is conchentrated at the

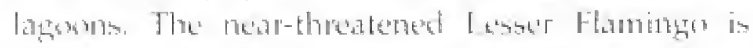
aften present in internationally-important numbers. thorgh Nagadi in a meth less significont feeding site

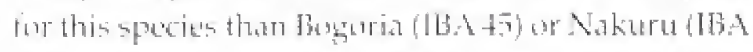
49). Very latge number st thin spocies may hred here on rare bocasions. pethant omece at
\end{abstract}

Chestnut-banted Plover

\section{Congregations}

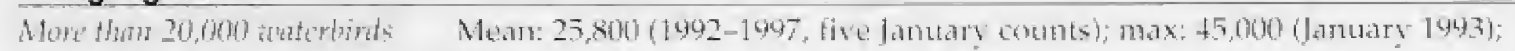
$50,040(1+$ counted from the air July 1904

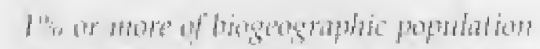

Lesser 1:lamingo $(20,000)$ Men: 25,450 (1992-1997, the July and five January counts):

max: 50,000 (aterial count, July 1994 )

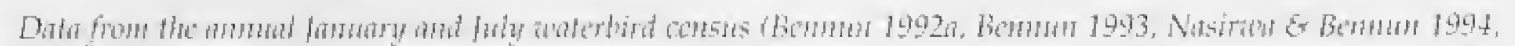
1995, wmpuld. Atatis) 
47 Lake Magadi

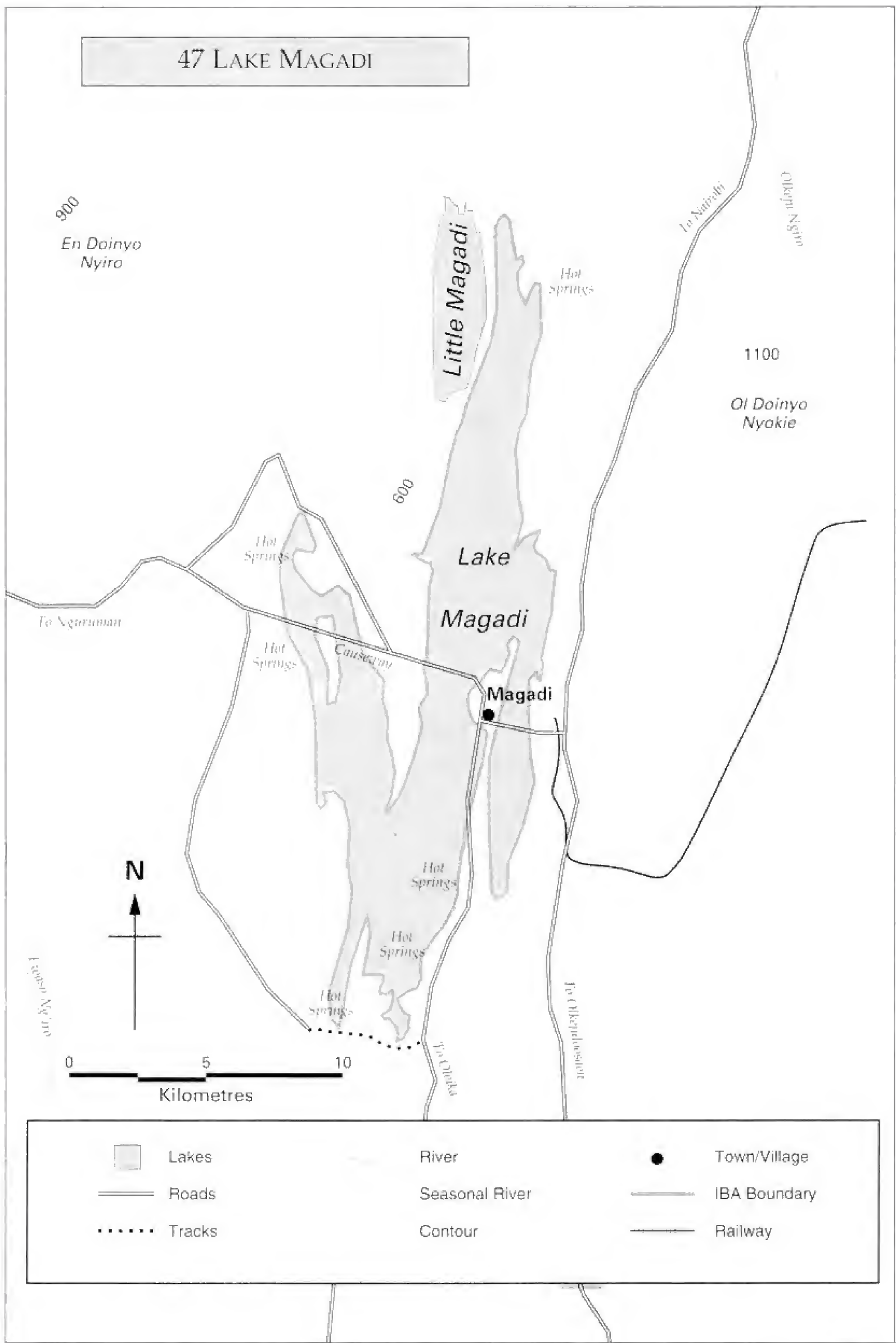


Important Bird Areas in Kenya

\section{Regionally-threatened species}

Great Egret

Occasional visitor

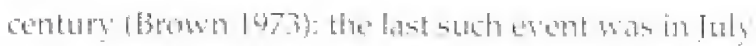

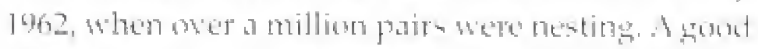
saricty of othes watertirets is present, including at sizeable resident population on Chemblnet-bantited Phower. Other birds nesting at Magati include

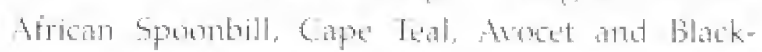
winged stilt. Mant Pallearctic migrant watere winter here tow, notatis I ittle stint. The butehland wround the late supports 28 at kens as 4 s SomaliMasal bismone apecien.

\section{Conservation issues}

The: lake is tomprobeted and is commercially motud

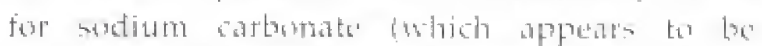

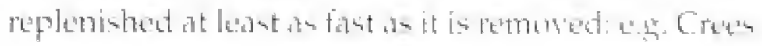

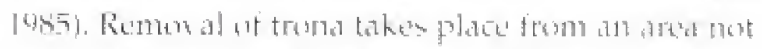

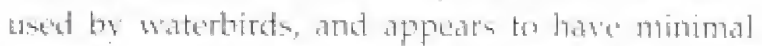

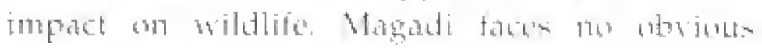
comservation problems at preserent.

Further reading

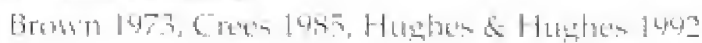




\section{LAKE NAIVASHA}

$0^{\circ} 46^{\prime} \mathrm{S}, 36^{\circ} 21^{\prime} \mathrm{E}$, Rift Valley Province, Nakumu District

23,600 ha (15,600 ha lake, c. 7,000 ha woodland)

Altitude: $1,890 \mathrm{~m}$

Status: Ramsar site (no other formal protection):

surrounding land privately-owned

Categories: Globally-threatened species, restricted-range species, congregations

\section{Site description}

This site lies on the floos of the Rilt Vallew; $80 \mathrm{~km}$

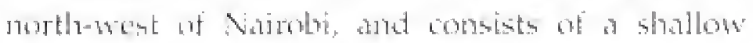

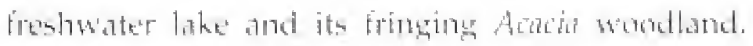
lake Najastha is of recent geological origin, and is rimged by extinct ot dormant rolcances, including Mes Longonot, Ol Karial amal Eburu. Naiknsha's water is supplited by the permanent Malembal and Cilgill rivess which respectinedy drain the Mberdate Moundans (IBA I) and the Rift valley foom to

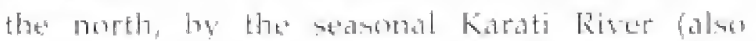
draining from the Aberdares) atud from substantial sround-water seepage. The Malewa comers 1,730 $\mathrm{km}^{2}$ at the $2800 \mathrm{~km}^{2}$ catchone and antributes $90^{\mathrm{a}}$ in of the surface water entering the lake. Nabasho has no surface coutlet. It is thosisht that a combination of undergrond ondflew and sedimentation of salte keps the lake treside, unlike other endorlowe lakes in the tastern Rift Valley:

Fied-knobbed Coot
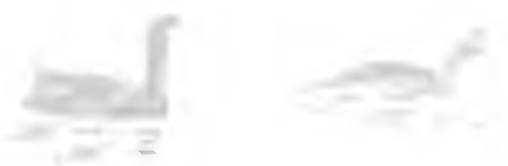

Naisuthancluden three chemically distinet water

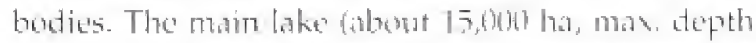

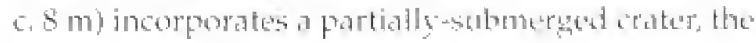

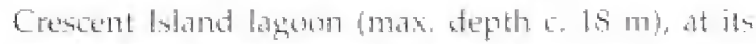

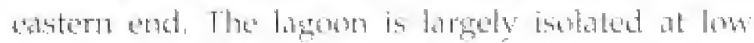

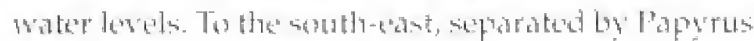

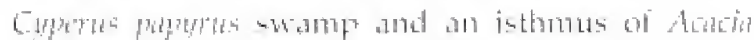

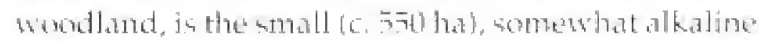

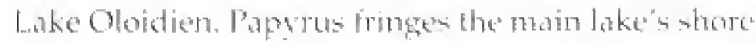

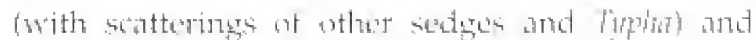

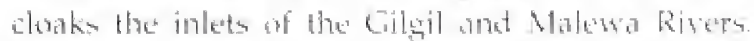

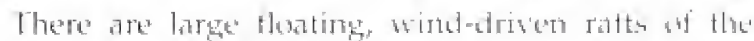

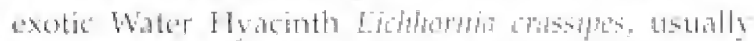
concentrated in the south-weat acctur. Submerged

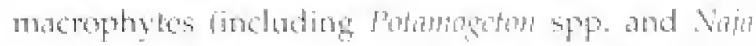
phetimato sometimes oceur in large beds. mainly in the shallow wastem part, but these vary orently in

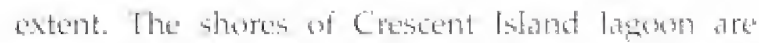

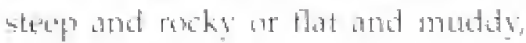

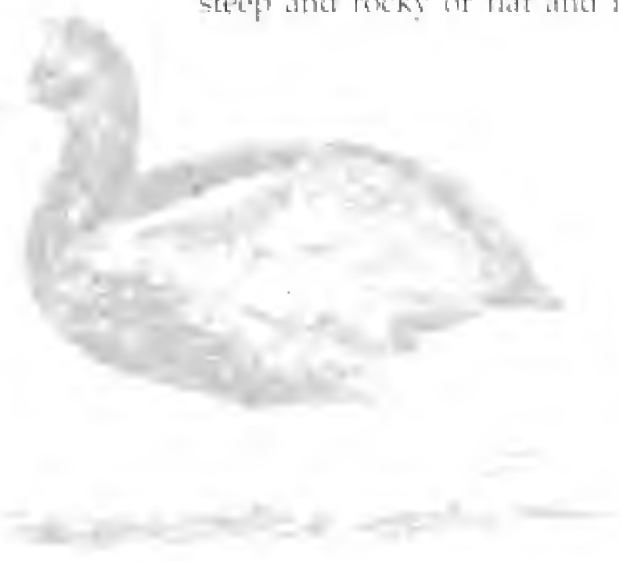

\section{Globally-threatened species}

"Grey-crusted Helmet-shrike Vulnerable?

Basta Resd Watbles

Near-thicatened
A few records from the woodlands around the lake (Lew is 1983), especially to the north. It is probably resident in small numbers (T. Stemenson, it litt, )

Winter visitor and passige migrant; exact status unknown 
Important Bird Areas in Kenya




t8 Lake Naivasha

\section{Congregations}

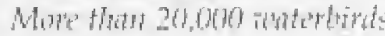

$1991-1997$ mean: 22,000; n7ax: $30,600\langle 1991\rangle$

Tos or more of biogrographic population

Red-knobbert Coot $(5,000)$

$1991-1697$ mean: 6,$500 ;$ max: 19.400 (1991)

African Spoonbill (150)

1991-1997 mean: 180; max: $412(1997)$

Little Grebe (500)

[99]-1997 mestา: 750; max: 1,500 (1997)

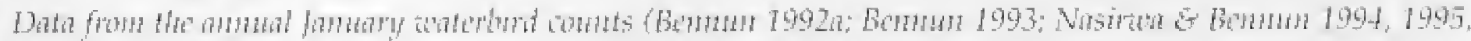
(Howble data)

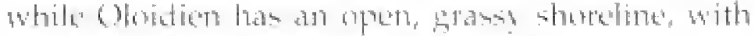

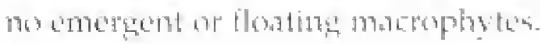

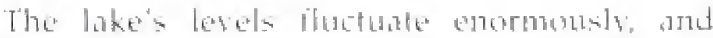

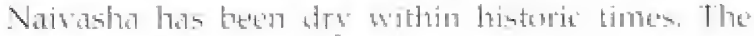

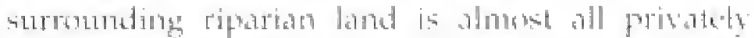
ouned, much of it mone whed fort interminge horticulture and therienlene tasing water tram the

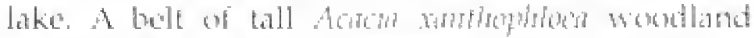
tringtes the lake and extends along the risers to the nowth, theough pentions have been cleared for

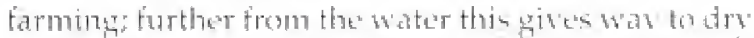

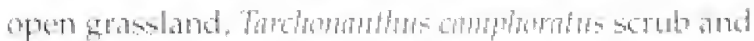

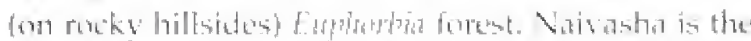

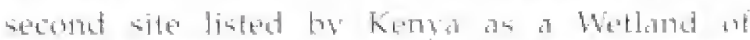

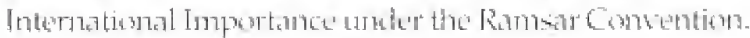

\section{Birds}

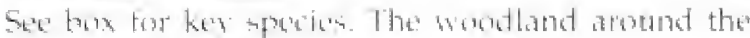

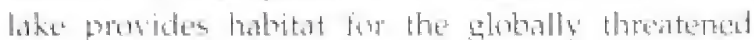

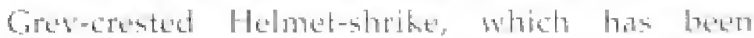

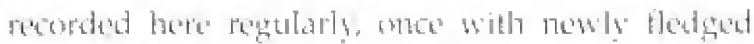

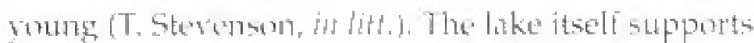

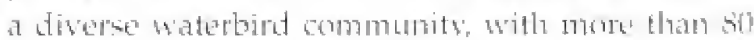

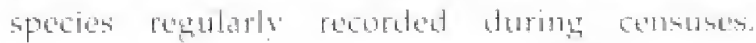

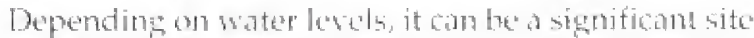

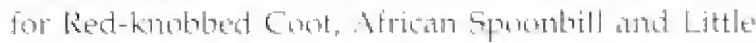

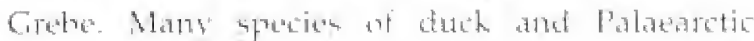

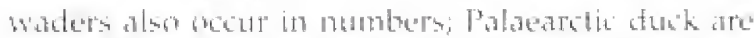

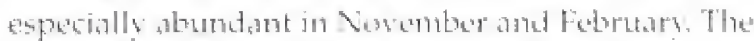

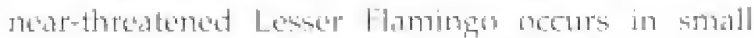

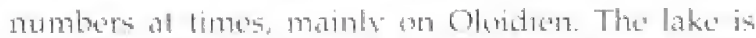
known for ith high density of Atrien Fish Eagles,

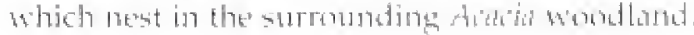

\section{Other wildlife}

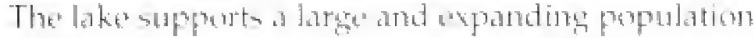

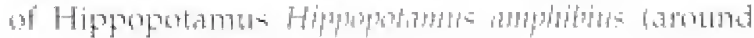

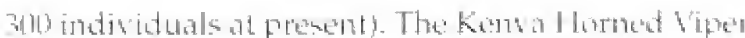

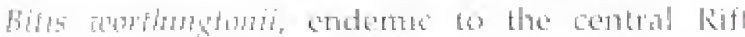

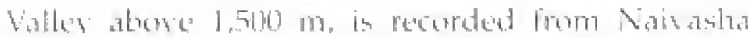

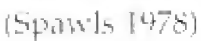

\section{Conservation issues}

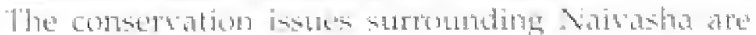
preticularly complex and tangled, with the effects of human pressures superimposed on a maturally fartiblessetem.

The lake has seen dranatio ecolengical thanges through the effects of introblexed upecies. lit the

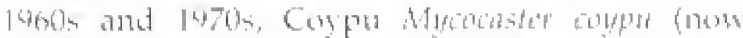
probably sunct) and Ionticiand Red Crayfish

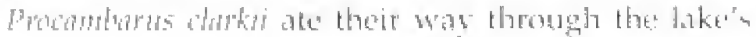
submerped plants and externsine buds of water litice

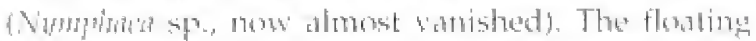

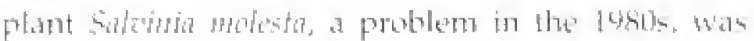
ceplaceat in the 1990s bu the notoriaus Water

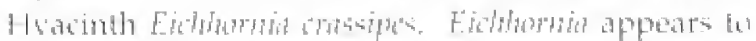
provide acellent shether for soung and adtell craytialy, couttibuting bo a cruskicean population borm that is presenting any recosers of the sulmorged macroptrites (D.M. I tarpert, in fid.s. This

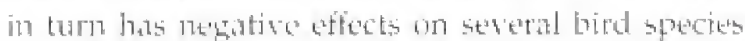

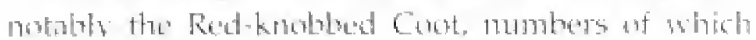

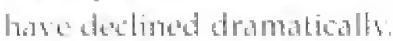

All the lake"s fine tish species are introduct. Then

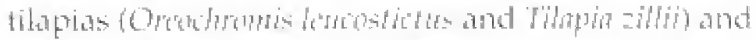

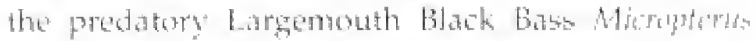

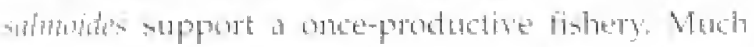

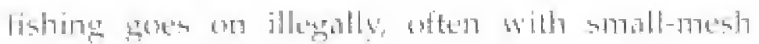
gill-nets that iatch undeg-sized fish, and

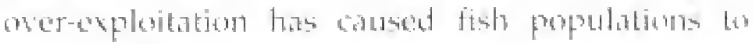

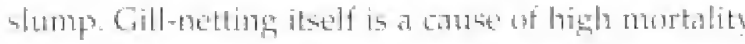
in dixime birds such as Great Crested Girebe and 


\section{Important Bird Areas in Kenya}

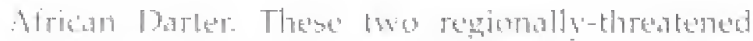
4)

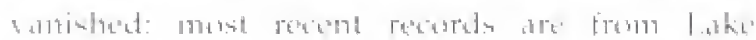

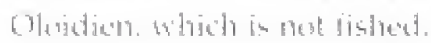

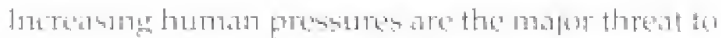

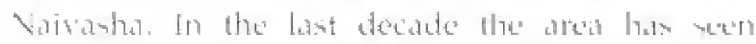

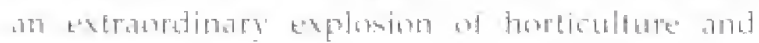

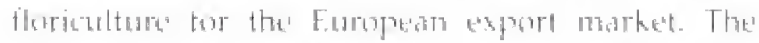

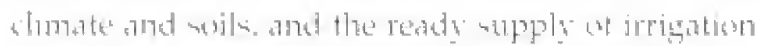

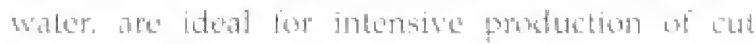

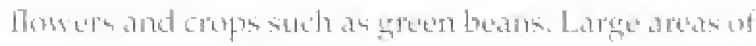

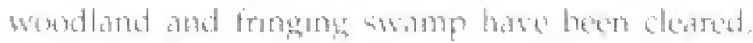
with cultustibn semetumes extending right domb to

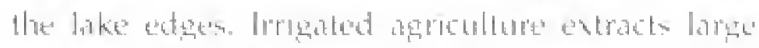

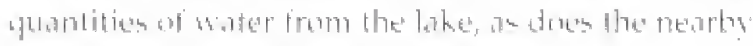

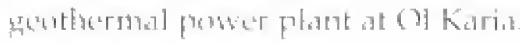

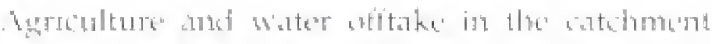

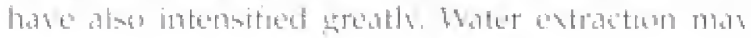

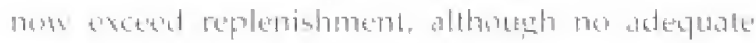

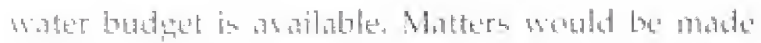

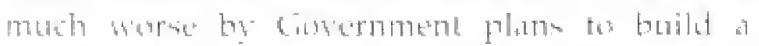

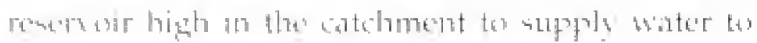

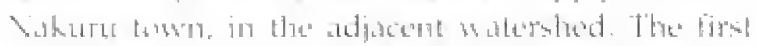

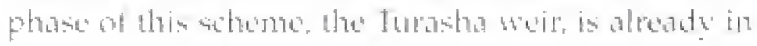
pilact.

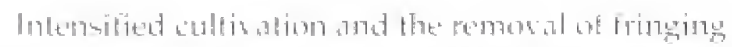

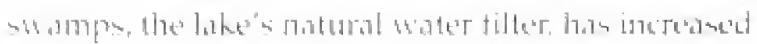

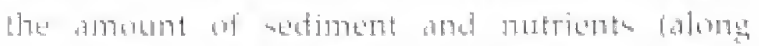

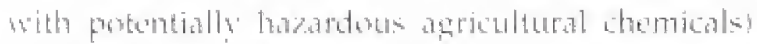

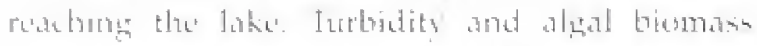

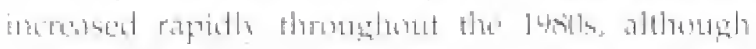

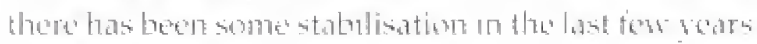

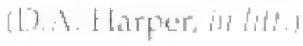

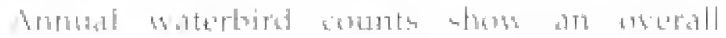

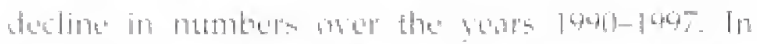

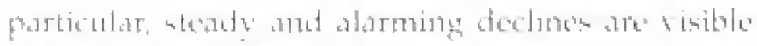

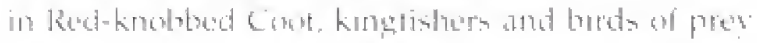

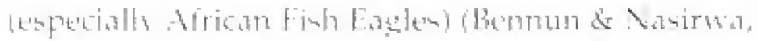

\section{Regionally-threatened species}

\section{Grent Crestent Grebo \\ Critinal}

Marcoa Duck

African Darter

Vulnerable:

Grent Egret

Vulnerable

Saddle-billad Stork Vulnerable

Whita-backed Duck Vulnerable

Baillom's Crake Vulnerahle

Arican Skimmer Vulmerable
Most recent Kenvan recordis bif this scarce species, which has been severely redused by gill-netting, are from Ofoidien, with seven binds seen in lamaty 1406

Regular on Otoidien, with 170 in lanuars 1494 and January 1497

Severely restuced by gill-netting, it survives in bery small numbers: one recorted on Oloidien in Jamuary 1497

Retgular at Naivasha, which is an important fesding site; 73 counted in |аเาแสม 1997

IRtsident in sery snall numbers: two counted in lanuarv 1997

Ocatsignal:12 counted on Olvidien in

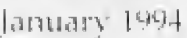

Status uncertain

Irregular visitor

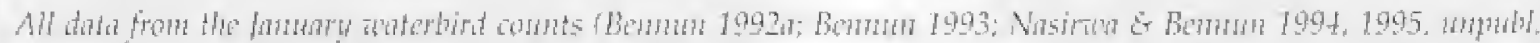
(fiata) 


\section{Lake Naivasha}

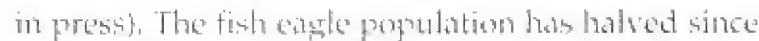

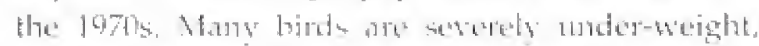

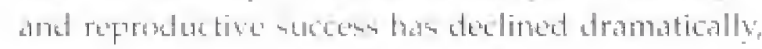
suggesting that the birds have problems finding

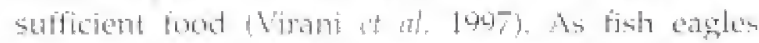

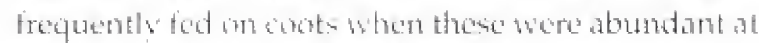

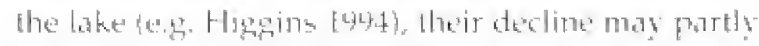

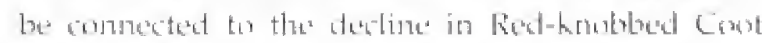

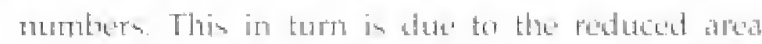

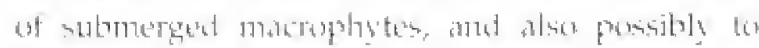

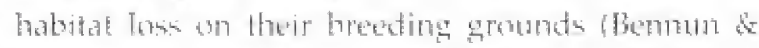

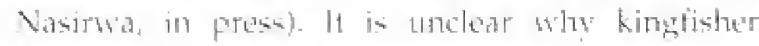
nombers and declining.

With spectacular scemery, a tine climate tramclail

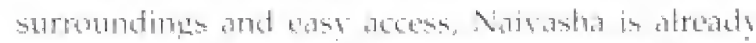
un imprortane site for hakal and international tourism.

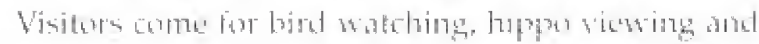
watersforts, and to wisit the mearby National laarke

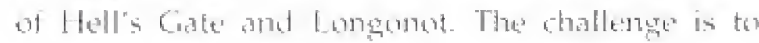

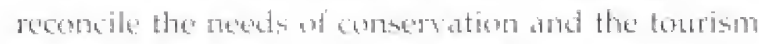
influstry with the" onthet use of the lake - which

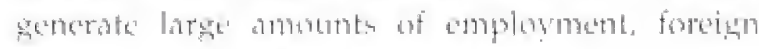

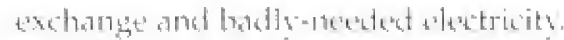

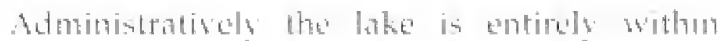
Nain and beswn, but almost all the land around it in

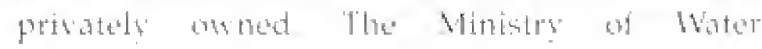

Development and Departmeat of Fisherien atre in

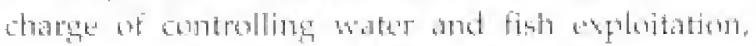
rempetively, but it is the riparian land-omeders wo ban contribute most to management efforts. The Lake Natuatha Riparian Assotiation was

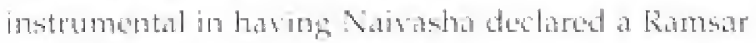

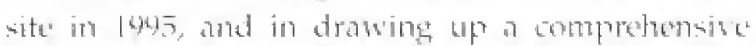

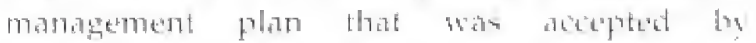

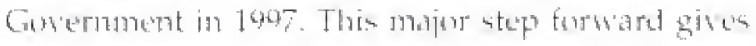
emcouragenent that the lake can be mandged wisely tor multipla usis.

The woodland north of the lake las generally been well conserved by land-owners, who fam under the

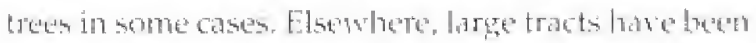

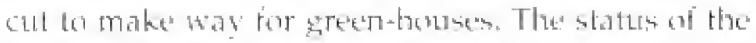

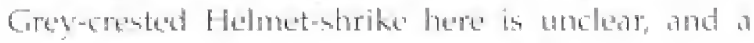

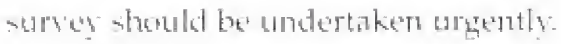

\section{Further reading}

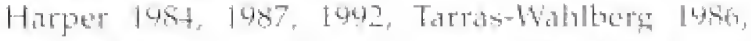

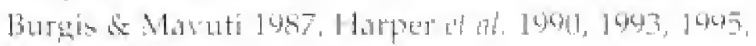

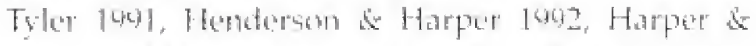

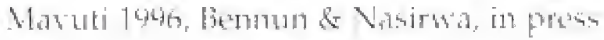




\title{
49 Lake Nakuru National Park
}

\author{
$0^{\circ} 22^{\prime} \mathrm{S}, 36^{\circ} 05^{\prime} \mathrm{E}$, Rift Valley Province, Nakttru District \\ 18,800 ha (lake 3,300 ha) \\ Altilude: $1,750-2,070 \mathrm{~m}$ \\ Status: National Park and Ramsar site \\ Categories: Globally-threatened species, restricted-range species, \\ congregations
}

\begin{abstract}
Site description
A very thatlow, stromgly alkatine lake, with strpounding woudland and grasiland. Sot in a picturesque landscape, the l'ark alouts Nakuru toust an important and expanding agricultural and industrial centre. The lake catchment is hasaded by Benengaci Crater to the anorth, the Balati fills to the north-east, the Lion Hill ranges tor the east, Elotalt Crater ter the woth and the Mate escalpement bo the west. Three thojer rivers, the Xjoro, Makeflia and Fnderit, drain intes the lake, toggether with brated water fom the town's sewaye works and the outtem foom several springs aloug the shore.
\end{abstract}

Vakuru was first gazetted at at bird wnctuary in

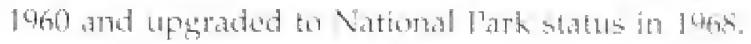
A mothern extemsion to the park was added in 1974 .

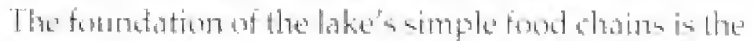

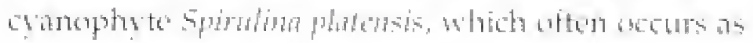
a unialias blomen. At such times it can suppert huge rumbers of Lesen flaminges and the finh

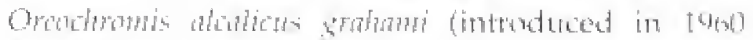

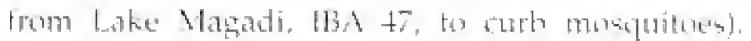
The tistr in tum supportl a number of secondary consunters.
The lake shores are mainls open alkatione mud,

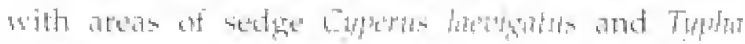

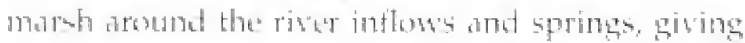

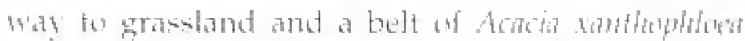

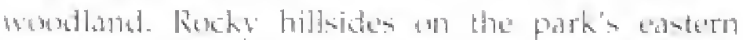

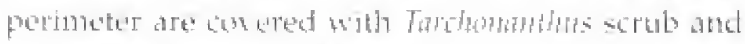

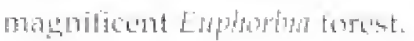

\section{Birds}

Set bex for key afeciem. The lake in internationally

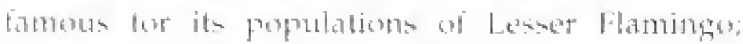
numbers can resth 1.7 mollion at times, though drastic and unpredictally fluctuations oceur.

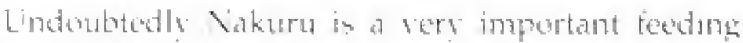
site for them species; attempts by flamingon tor breed here hase not been stecesstud. Other suaterbirts hate increased corloderably th numbers and

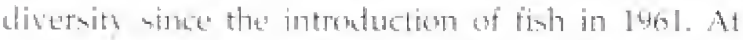
limes Nakuru is a major fueding ground for Great

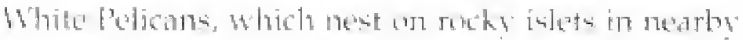

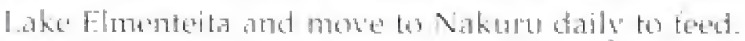
Large numbers of Palacartic waters wignter al Nakuru or use the site on passace, and Nakure fat

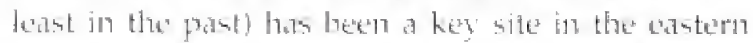
Rift valles tlywa, Nakuru is rich in birds generally"

\section{Globally-threatened species}

Madagacar Squacon Heron Near-threatened
Lesser Flamingo
lesser Kestres
*Gear-threatened
*alnerable

Non-breding visitur, May to October (Zimmerman et m/. 1996)

A key fueding site for this species

Passage migrant, relatizely common in the past (e.t. os 19836 )

Probably resident in the Acacin stood land, where it has nested (Bennun 1494a, OS-C 1981)

"also restricteht-range" spectes 
49 Lake Nakuru National Park

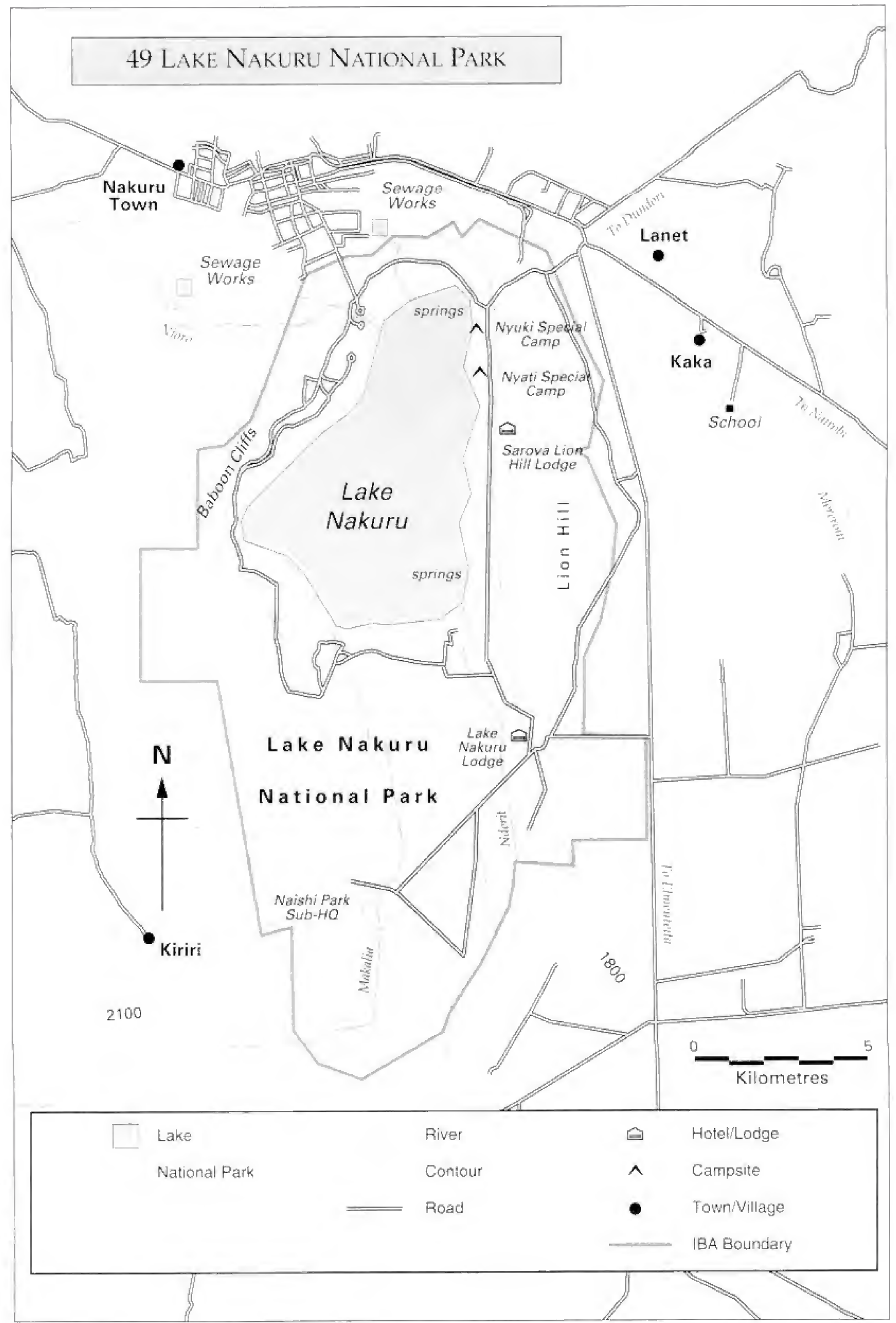




\section{Important Bird Areas in Kenya}

\section{Congregations}

Mere than 20,000 anderhinds

1990-1947 mean: 260,000 (15 counts); max: 1,48,000 (July 1993)

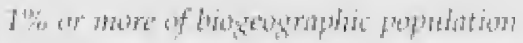

Greater Flattingo $(1,250)$

l.esser Flamingon $(20,000)$

Black-nuched Grebe (250)

Litule Grebe $(500)$

Great thite Pelican $(1,800)$

Yellow-billed Stork $(500)$

African Spronbill (150)

Black-winged Stilt (1,000)

Grey-headed Gull $\{1,0(0))$

Gull-billed Tern (270)
1994-1947 mean: 1,700 (15 counts); max: 9,440 (fan 1941)

1900-1997 mean: 280.200 (1.5 sounts); max: $1,+48,000$ (1993)

(600) (1an 1903)

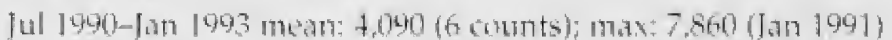

Jul 1990-Jul 1993 mean: 22,460 (7 counts): max: 44,430 (Jan 1992)

ful 1490-Jan 1492 mean: 1,010 (4 coutnts); max: 1,620 (†ul 1991 )

Ful 1990-Ju] 1993 mean: 230 (7 counts); max: 580 (Jan 1992)

3,120) (lan 1991), 2,140 (Jan 1992)

Jul 1940-Ju1] 1993 muan: 3,720 (7 counts); max: 9.040 (Jan 1991)

1,390 (1) 1917 1942)

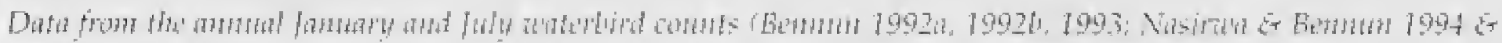
1995. anyinl. datut

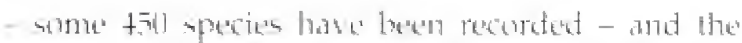

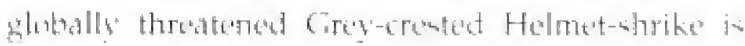

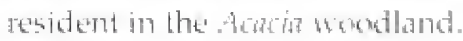

\section{Other willdlife}

The pork is a sanctuary for the: Bhatk Rhinaserom

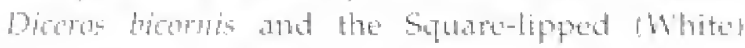

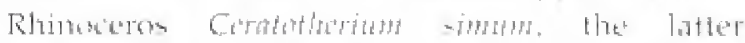

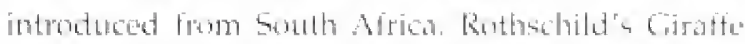

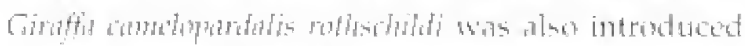

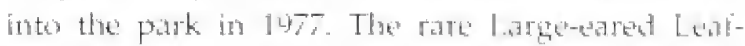

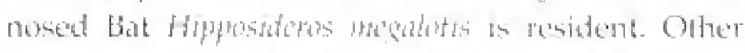
harge matmmalis, some recently re-introduced.

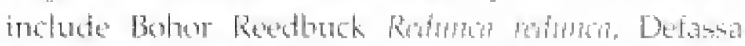
Waterdouck Koders dipsipurbaras, Bushouck

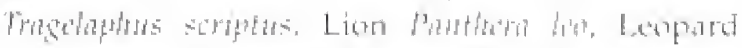

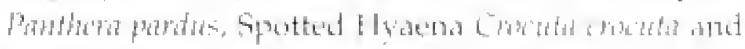

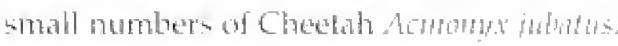

\section{Conservation issues}

Conflicts betreern conbersation and development

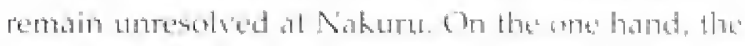
National l'ath is a major tometist attation, with up th

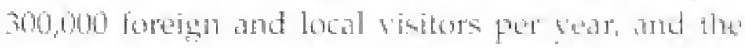

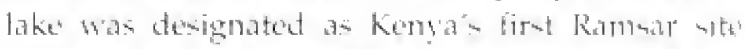
in 19y. On the other hand. Nakuru tassin is an important industrial and agrionltural entre, whese yrowsth diretly affects the lake Lintil recently, treatment of waste water entering the lahe from the town was inadequate. for expanded semedse treatmetal woth is now in commission, but

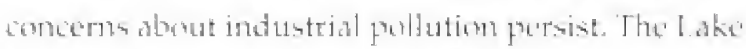

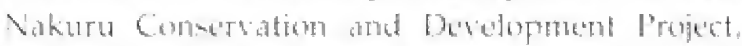
rust by the World Wide Fumd for Nature, Iras

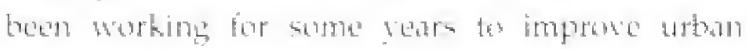
2017

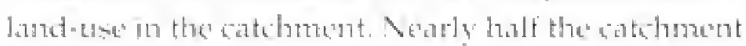

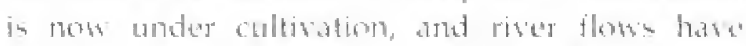

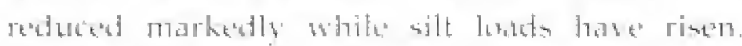

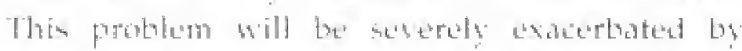
recent deforemation in the Lastern Mat Fotest Restere \{pote of IBS 51). which providus the catchment tog muth of Nakutu's water. Encroachement and settlement in this porest (repportedly by as mang as 280001 penpley rueds to be reversed and natural vedetation allowed to regetnerates wo the late mat have lietle future.

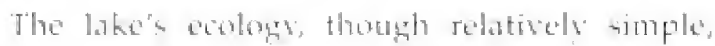

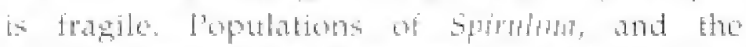

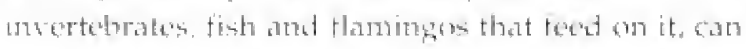

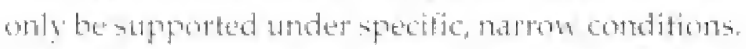

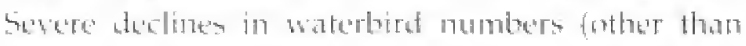

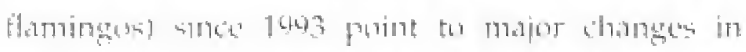
the foos bhain - specithatly, a kack at fish and ianethorates - associated with a periog of low lakt lewes. Lake Nakuru's lewed fluctuate maturally due wo listle-anderstond interations between hydrology,

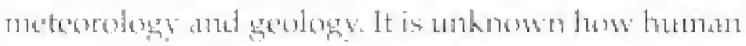

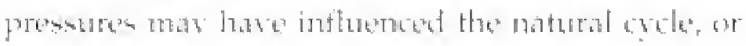




\section{Lake Nakuru National Park}

\begin{tabular}{|c|c|c|}
\hline Great Crested Grebe & Critical & $\begin{array}{l}\text { Used to occur in numbers (29 recorded } \\
\text { in July 1990); no recent records }\end{array}$ \\
\hline Mactoril Duck & Endangered & No recent records \\
\hline Great Egret & Vulnerable: & $\begin{array}{l}\text { Lp to } 84 \text { recorded (January 1992); } \\
\text { numbers have declined in recent years }\end{array}$ \\
\hline Martial Eagle & Vulnerable & Sparse resident \\
\hline African Skimmer & Vulnerable & No recent records \\
\hline Long-tailed Widowbird & Vulnerable & Seasonal visitor, in long grassland \\
\hline
\end{tabular}

if the lake's bird populations will tethenond if and

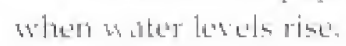

Itwe National Park is mow entindy surrounded lys

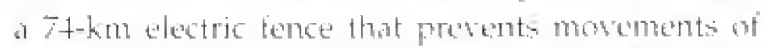

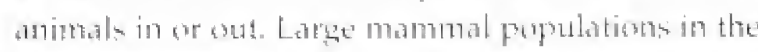

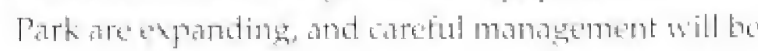

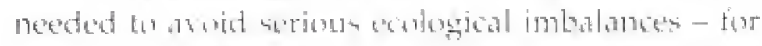

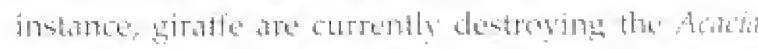

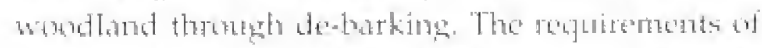

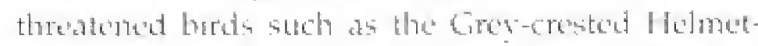
whike sthould athe bo considered in mamagement

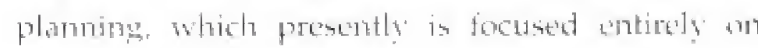
listue matmothula.

\section{Further reading}

Mburtgu 1974. Myers 1974, Varenchi 1478, Vareschi

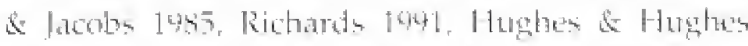

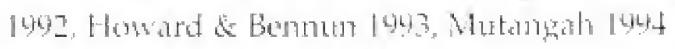

Lesser Flamingo 


\section{Masai Mara}

$01^{\circ} 30^{\prime} \mathrm{S}, 35^{\circ} 00^{\prime} \mathrm{E}$, Rift Valley Province, Narok and Transmara Districts

664,000 ha $(181,200$ ha National Reserve

and 482,800 ha wildlite dispersal area)

Altitute: $1,500-2,170 \mathrm{~m}$

Status: National Reserve and Group Ranches

Categorics: Globally-threatened species, restricted-range species

\section{Site description}

This IBA includes the Masal Mara National Remetrat and the strrounding wildife dispersal areats in south-western Kerya. (Collectively; the Renerse and its surpolnds are often called the Greater Mara; here, Masai Mara reters to the entire IBA., The site adjoins the Serengeti National Park alomg the Kenya-Tanzania border, and is considered part of

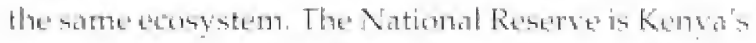
most-risibed proteded ared, world fomous for its

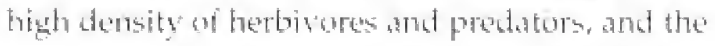
annual migrations of Wildoberest

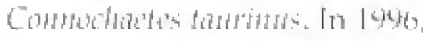

it was mommanted for designation as a thorld Heritarise Corncrake Siter. lis the north. east and usent ant large piacelo wit

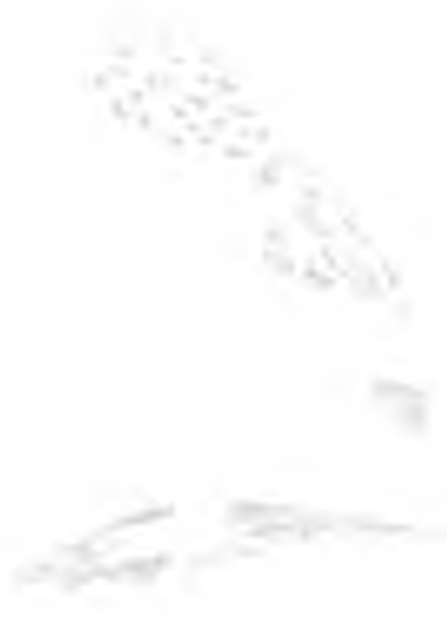

lemd dematosted as group ramelnes, ommed and inhatrited by the semi-nomadic pastoral Maradi people. This commumal land forms an extensivet widlife dispersal area for the Reserve, comprising the group ranches of Siana $(152,000$ ha). Kowaki
(94, (100) hat), Olkingej (80), (000 ha), Lemek (66, (90) ha),

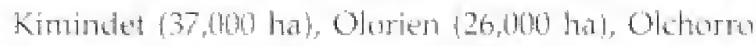
Ourwa $(11,800$ ha), Kerinkani $(8,100$ hat $)$ and Angata Barayoi $(7,9) 0$ hat.

Habitats in the Masai Mara are varied, including open rolling grassland riverine forest, Aoriag woodland, shamps, non-deriduous thickets,

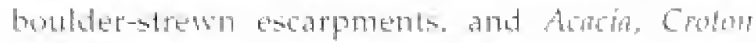

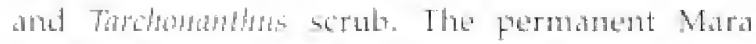
and Talek Risers, and their tributaris. Alow throush the Reserse and approximately trised it. There is a promouncos rantall sradient from the dried east with co sog mm rain per year) to the wetter west (with $5,1,20$ ) num per seart.

\section{Birds}

See box for key speries. The Mara's extemsine grasslands are a stronghold for the threatomed, migratory Corncrake and the near-threatened. restriated-range lackan's Widowbiret. The woodlands around the reserve are probably the centre of alomadarace for the threatemed, restricted-ranger Grey-rested Helmuthrike, More than 500 wher

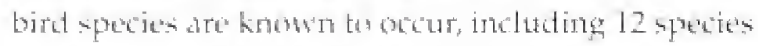
wof Cistiolio and 53 bird of prey Graseland birats a uspecially woll represented. I arge numbers of Palaearctic migrants sinter in the area, moluting Canzan Plovers and White Storks. The Olonlolo or Siria Escapoment is one of the few Kunsan sites for Roch Cisticolat and wther tocal and unusual birds in the Masal Mara include Rutous-bedlied Herom, Dentham' B Buntard, Bjack Cuscal, Reat-tailed Chat, Pale Wren Warbler: Tabora Cisticola, Teterine Wabler fin the northeres wintert, Yollow-bedlied Haliosta, Gren-capped Lremonela and Maspie Shrike. There is a single recond at shoubill, from the Nusiara Smanap (Kahindi loc4).

\section{Other wildlife}

The Masai Mara is lematkable for ils great concentration of large herbisores and their atdendatit predatom. The density of herbivones is estimated as nuarly $240 \mathrm{per}^{2} \mathrm{~km}^{2}$ with a biomass of just 


\section{Masai Mara}

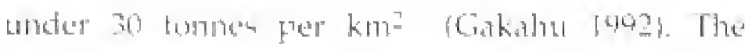

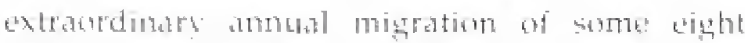

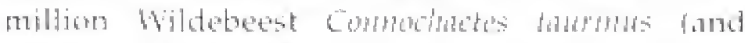

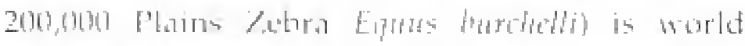

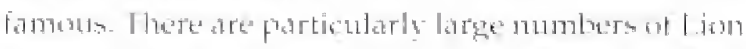

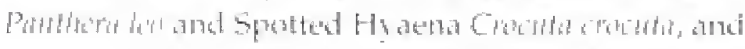
pespulations of the threatened Black Rhinuceros

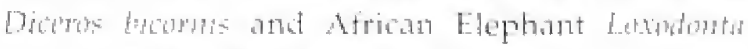

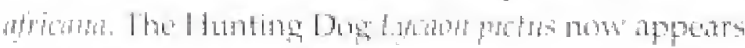

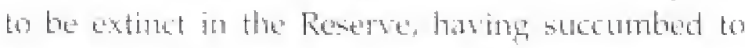
epridemics of rabies and canine distemper stow

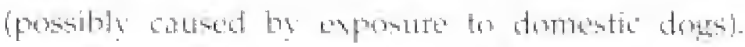

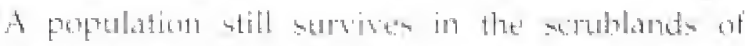

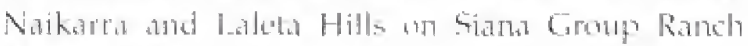
(Martin |atsa).

\section{Conservation issues}

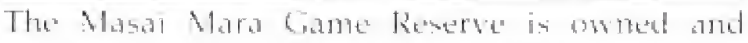

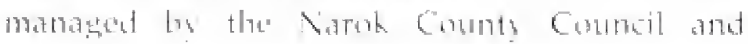

Jransmand County Council, which cullect gate

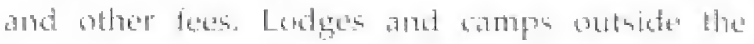
Reserse romit occupation, concusion and youring tees directy to widelite and touriom associations componed of the lonal communition.

Wildlife generates very suthitantial revenue in the Masai Mara An estimated KSh 174 millios wa

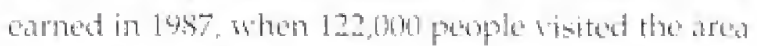

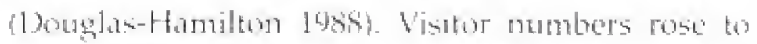
almost 159.1000 by: 1964 (Wasilus 1947/, with revetrue" prenumatioly increasinut in proportion. at 527 dollars

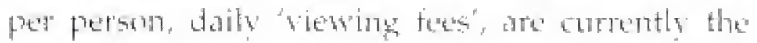
hizhest in Kengo. Howesere only a small amount of

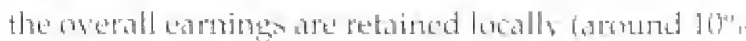

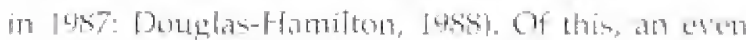
maller atrount appeats to trickle dowa he the laxeal

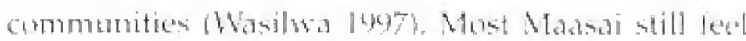

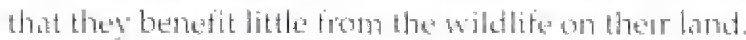

\section{Globally-threatened species}

\begin{tabular}{|c|c|}
\hline Mindagascar Squuaceo Heron & Near-threatend \\
\hline Lessert Kestrel & Vulnerable \\
\hline Cortucrake & Vulnerable \\
\hline
\end{tabular}

Grey-crested Helmet-shrike Vunerable

"Red-throated Tit

Near-throatened

Tackson's Widowbird

Near-threatened
Regular nom-breding visitor (Finch 1994)

Regular passige migrant (Zimmerman at (1). 19961

A regular non-breding sistor to extasland areas. This is an important wintering population (D.A. Turner, in lin., Collar d al. 1994)

Regularly recorded but apparently nomadic, in woodland and bushland ith the eastern dispersal area and western escarpment (OS-c 1981, Benmun 1994c, Finch 1994)

Common resident in Acricia woodtand

Probaty the largest breding population of this species

"also pestrictul-range" species

\section{Other restricted-range species}

[Dsambiro Barbet Trachylomis Ustmbiry]
Common in bushed and wookted grassland with termite mounds. This is treated as a full spereies by Statterstield el al. (1998), but considered a sub-sperits of D'Armald's Barbet T. dammediz by the East Africa Natural History Society Ornithological Sub-committere (OS-c 1046) 


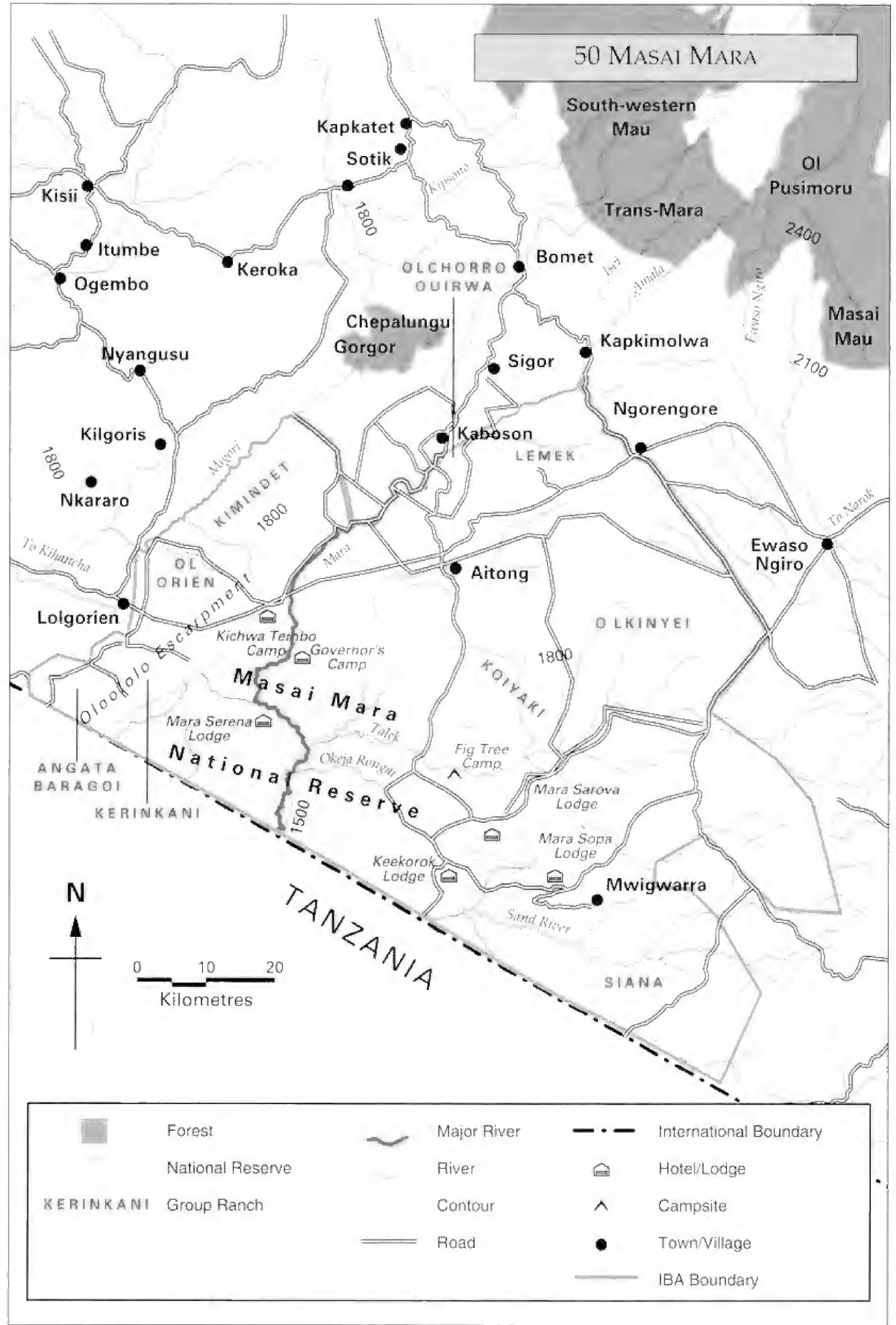




\section{Masai Mara}

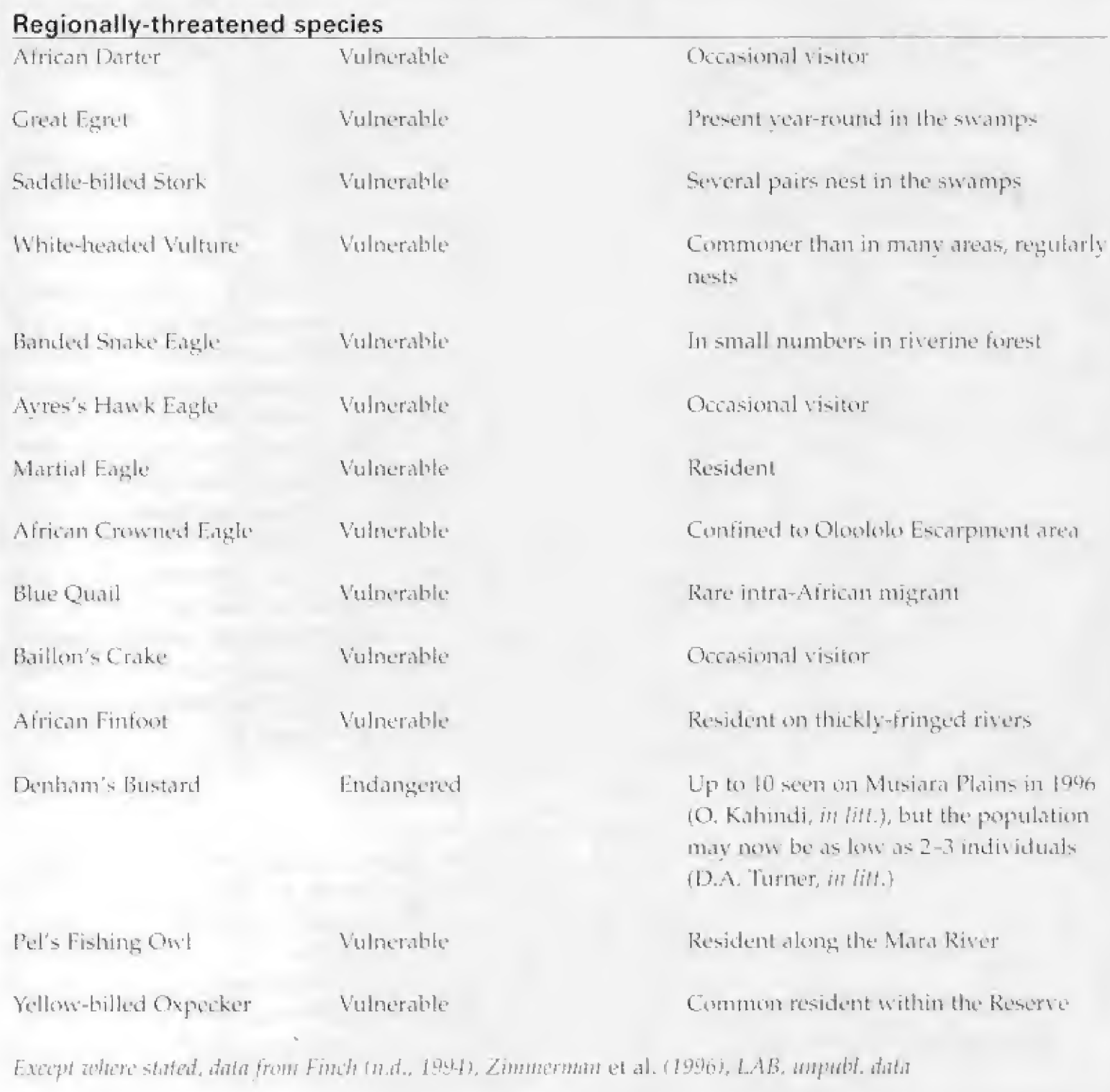

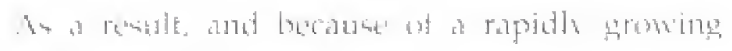

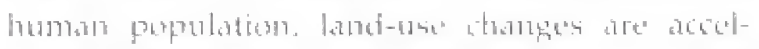

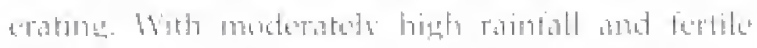

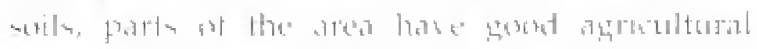

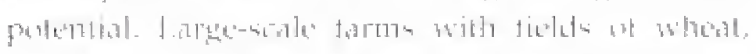

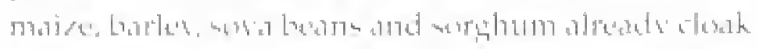

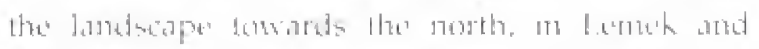

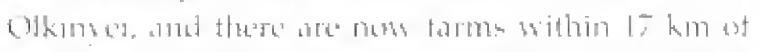

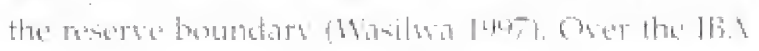

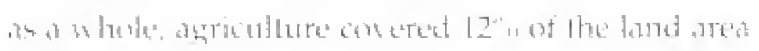

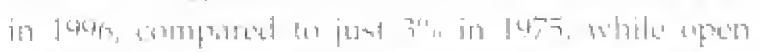

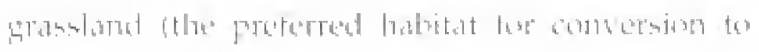

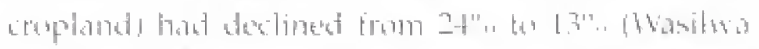

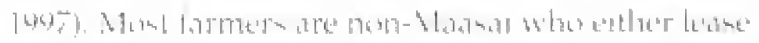

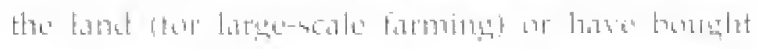

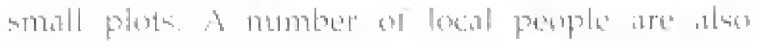

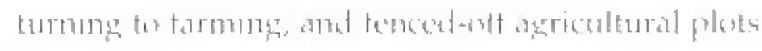

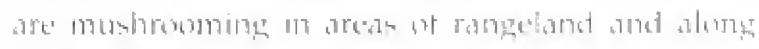

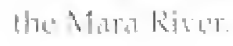

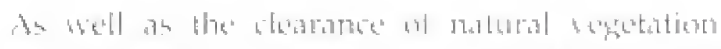

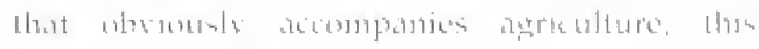

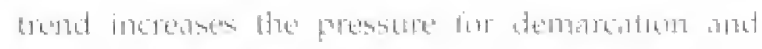

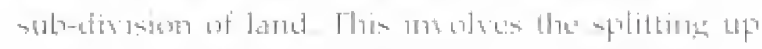

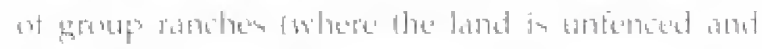

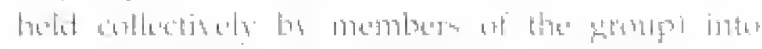

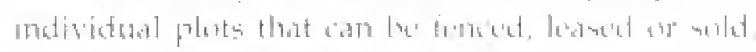

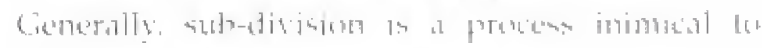

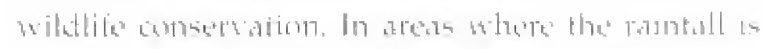

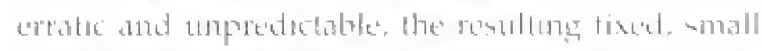

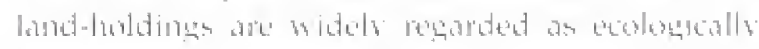

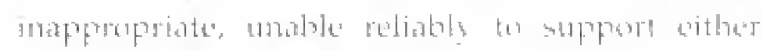




\section{Important Bird Areas in Kenya}

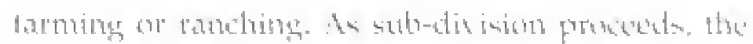

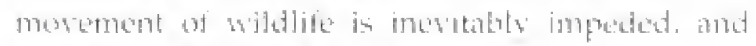

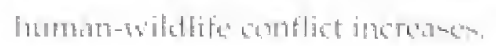

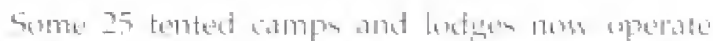

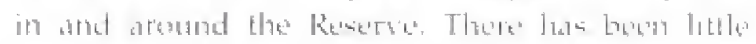

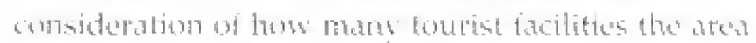

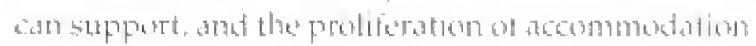

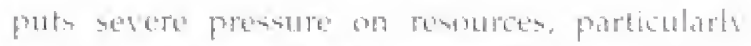

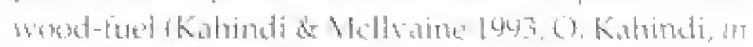

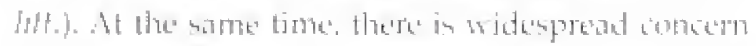

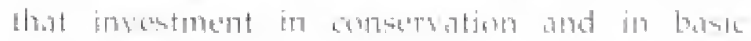

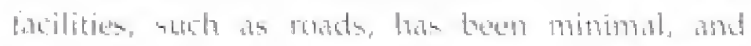

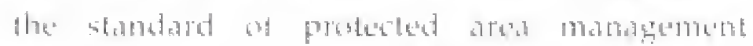

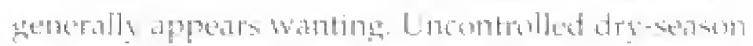

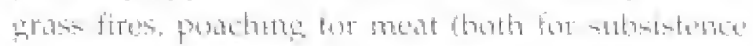

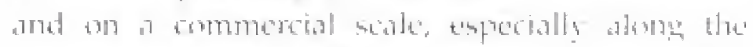

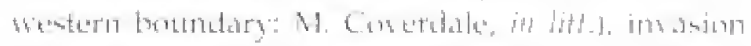

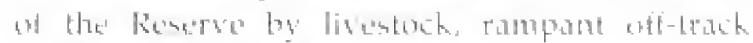

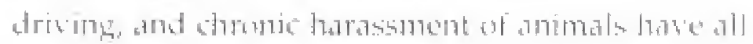

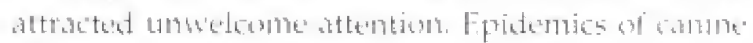

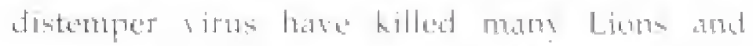

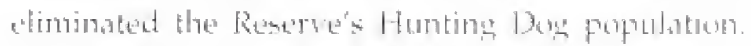

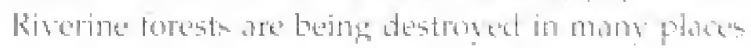

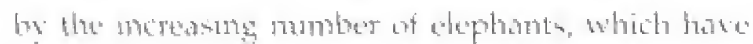

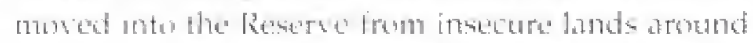
it fintlading the Sermedi valional Park in T.11าวania).

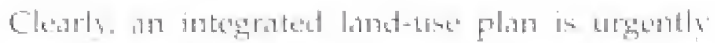

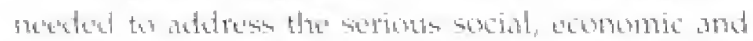

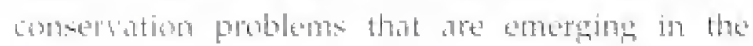

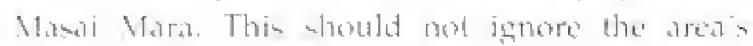
birdlite. The seremeti-Mara tompotem is alrods

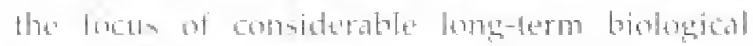

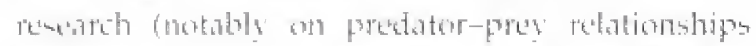

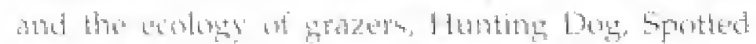
Hห⿻一𠃋十

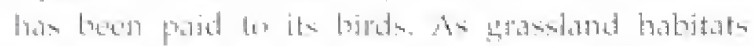

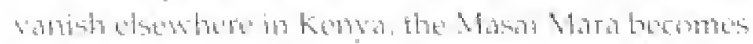

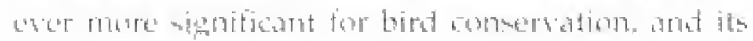

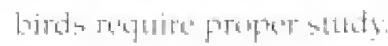

\section{Further reading}

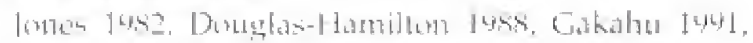

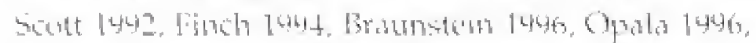

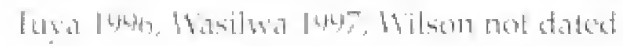




\title{
51 Mau Forest Complex
}

\author{
$0^{\circ} 30^{\prime} \mathrm{S}, 35^{\circ} 20^{\prime} \mathrm{E}$, Rift Valley Province, Nakurt, Kericho, \\ Narok and Bomet Districts
}

270,300 ha $(224,300$ ha gazetted, 46,000 ha ungazetted $)$

Altitude: $1,800-3,000 \mathrm{~m}$

Status: Forest Reserves and ungazetted forest

Category: Afrotropical Highlands biome species

\section{Site description}

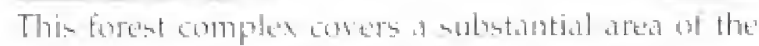

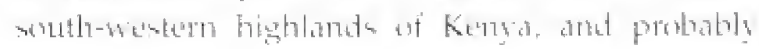

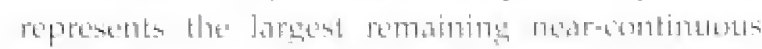

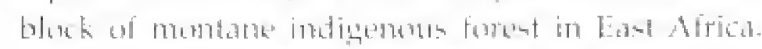

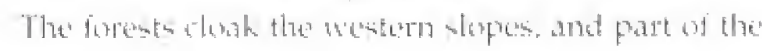

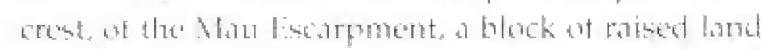
that forms the wented wall at the Giregoty kift

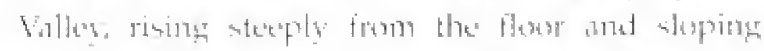
awa mote gratually th the went. There ate

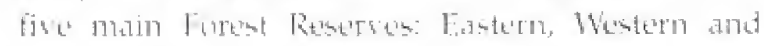

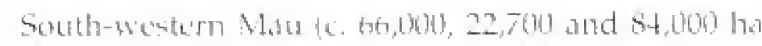

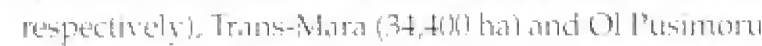

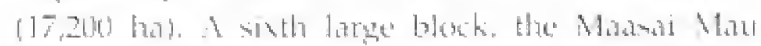

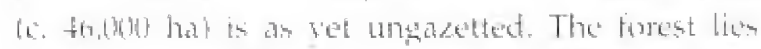

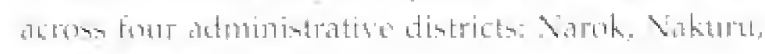
Bomet and kerithet.

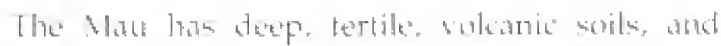

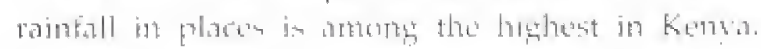

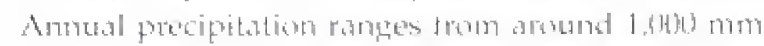

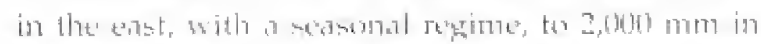

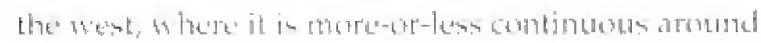

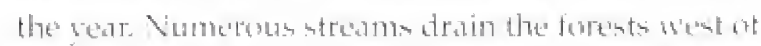

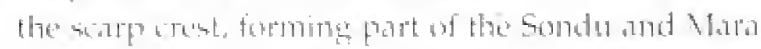

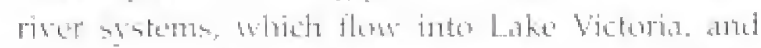

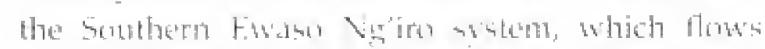
inter I ake Sabore. The Fasteren Mau is the main

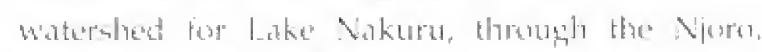
Matkilia and I-nderit kisters. The surroumding artats

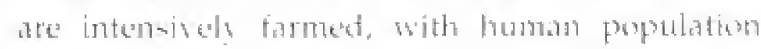

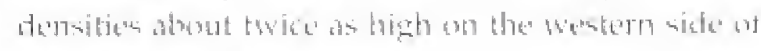
the borest an and llte dast.

Vépetation patterpo are complex, hest

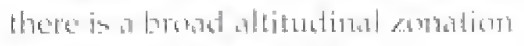

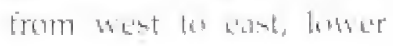

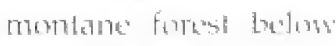

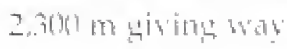

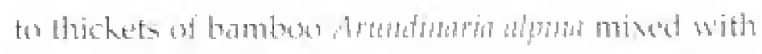

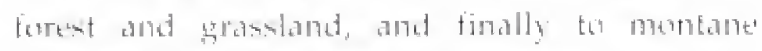

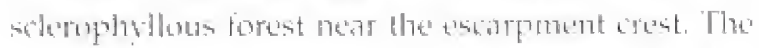

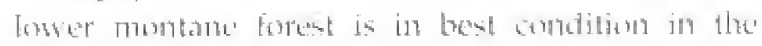

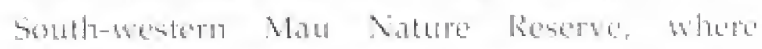

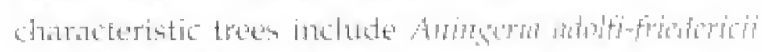

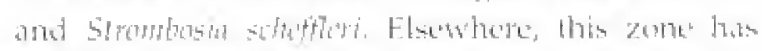

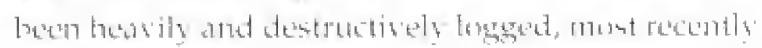

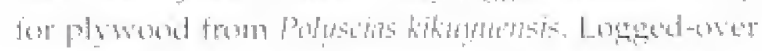

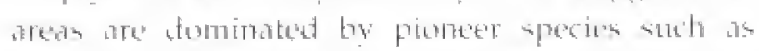

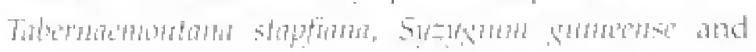

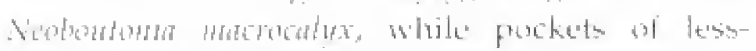

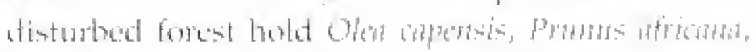

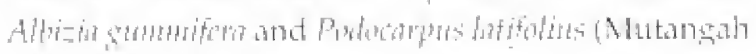
(1) wh. 1942\%. Substantial parts on the high

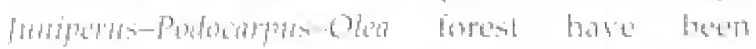

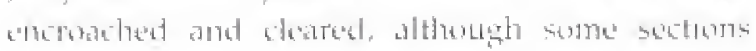

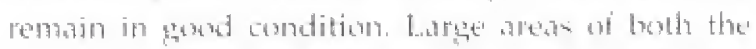

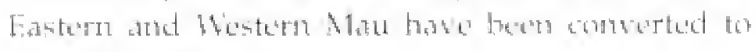
plantakion forest.

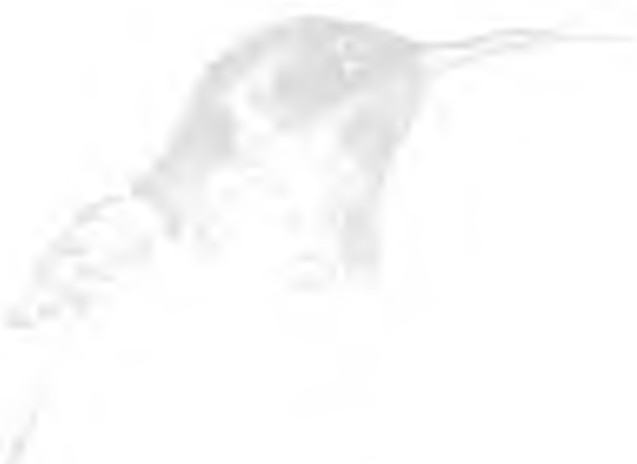




\section{Important Bird Areas in Kenya}

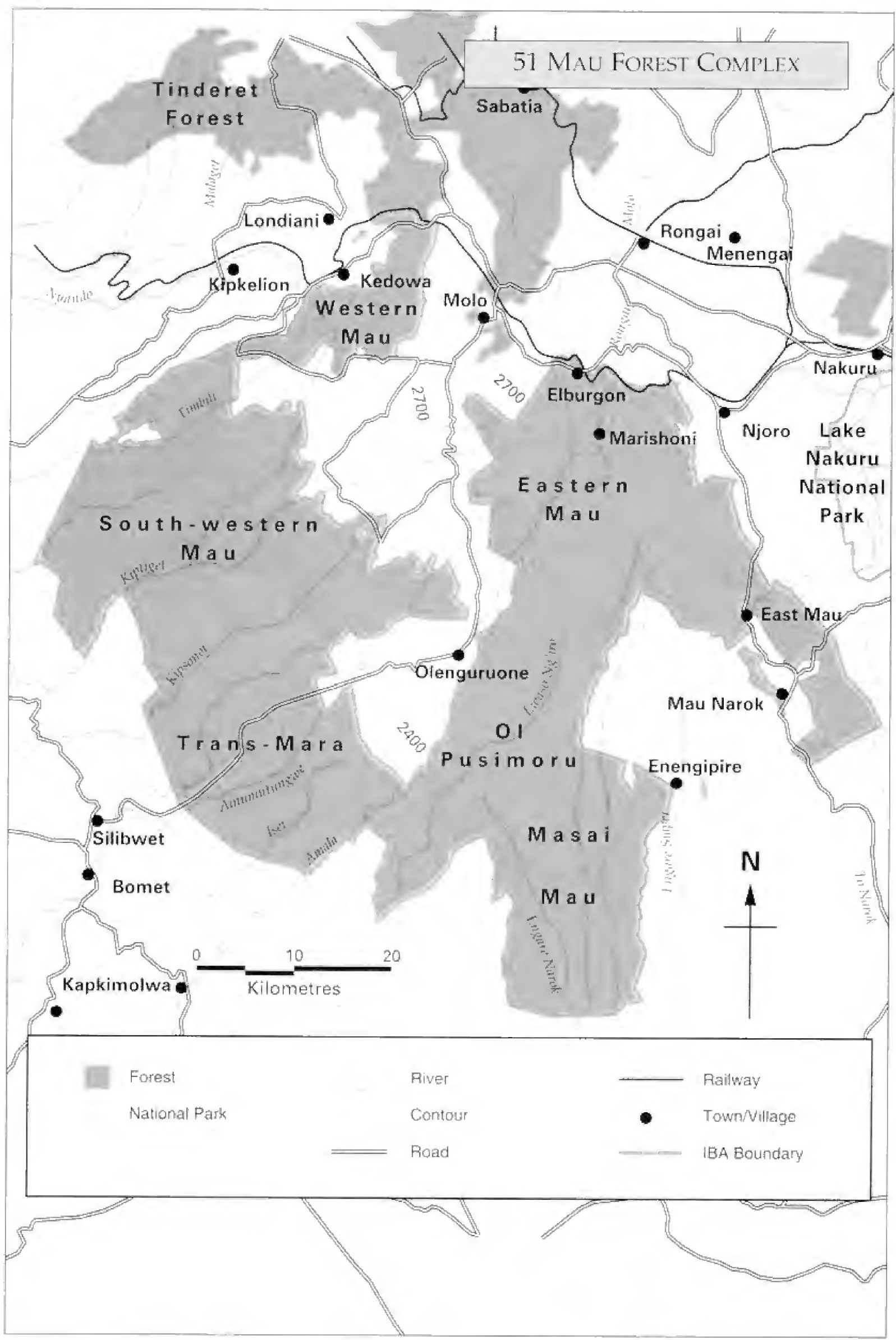




\section{Mau Forest Complex}

\section{Regionally-threatened species}

\begin{tabular}{|c|c|c|}
\hline Ayros's thank Eagle & Vulneroulale: & Scarce and lowal \\
\hline Arican Cromod Eatgle & Vulneralyle: & Rexident in small numbers \\
\hline Africatim Gratsi Ond & Vulnerable & $\begin{array}{l}\text { No recent records; may necur in high } \\
\text { gritsibands of the Sulth-uventern Mau }\end{array}$ \\
\hline Cape Eagle Oni & Vulneralb]t & $\begin{array}{l}\text { Mainly in high, rocky ravines in the } \\
\text { Eastern Mau }\end{array}$ \\
\hline Red-chested Owtel & Vultueratile' & 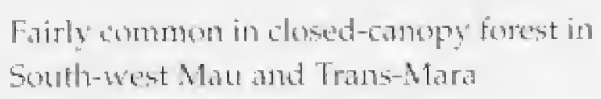 \\
\hline Leant Honeyguide & Vulaerable: & $\begin{array}{l}\text { In closed-canopy fortent in Sunth-westetn } \\
\text { Mau and Trans-Mara }\end{array}$ \\
\hline Gere-winged Robin & Vulnerable & $\begin{array}{l}\text { Uncommon anct local, only on the } \\
\text { western trimge }\end{array}$ \\
\hline P'urple-therodted Cuckoomhrike & Vulnerable & Uncummon resident \\
\hline
\end{tabular}

\section{Birds}

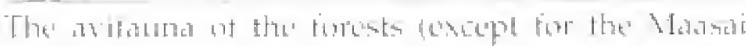

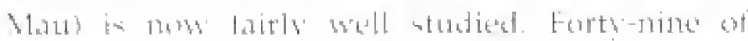

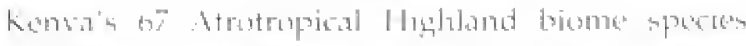

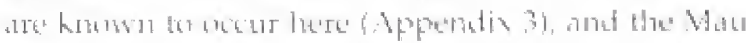

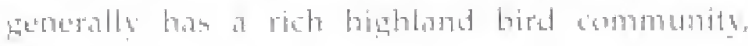

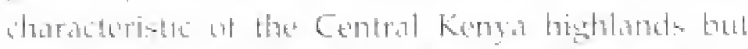

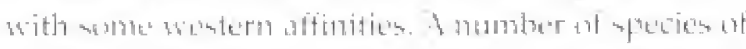

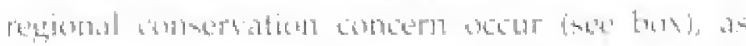

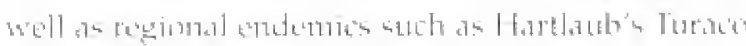

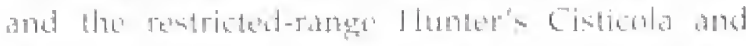

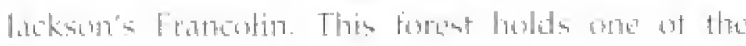

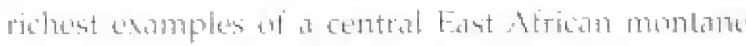

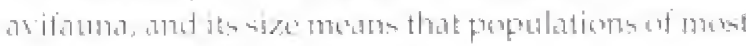

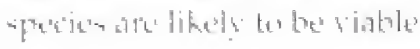

\section{Other wildlife}

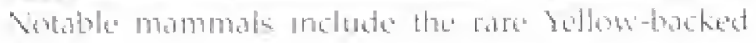

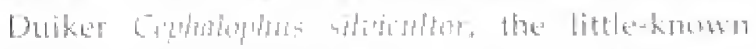

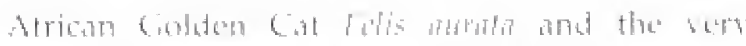

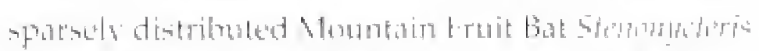

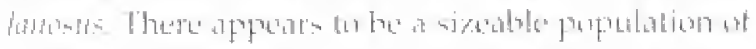

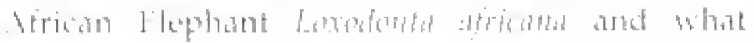

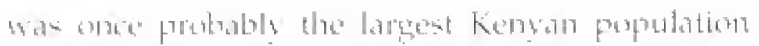

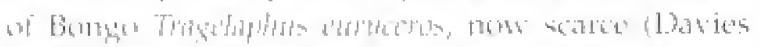

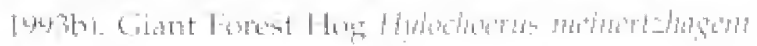

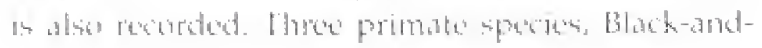

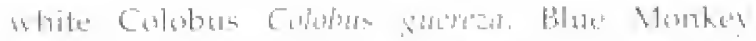

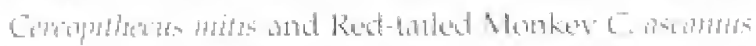

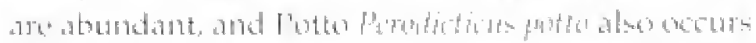

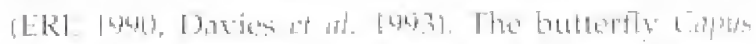

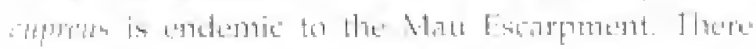

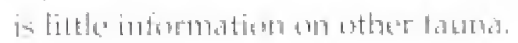

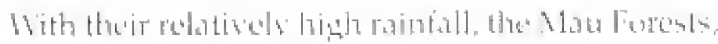

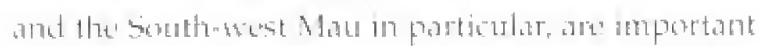

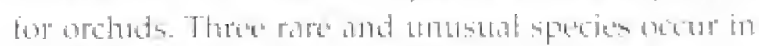

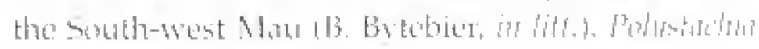

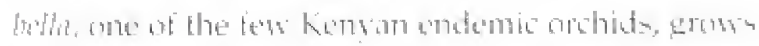

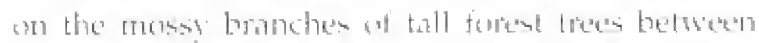

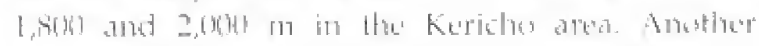

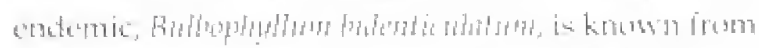

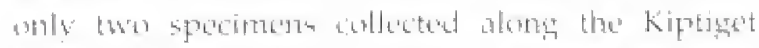

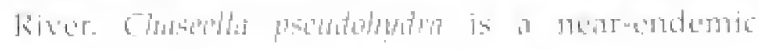

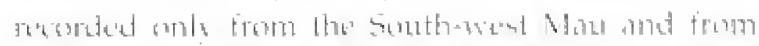

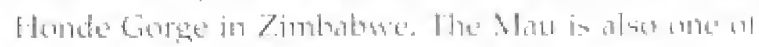

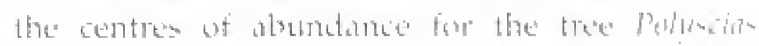

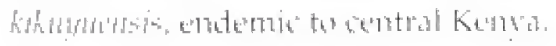

\section{Conservation issues}

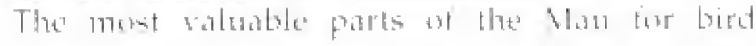

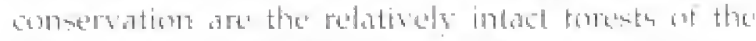

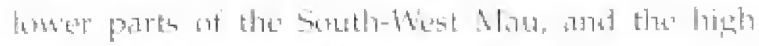

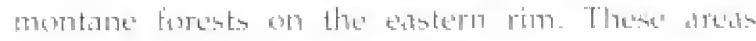




\section{Important Bird Areas in Kenya}

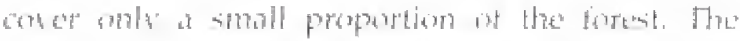

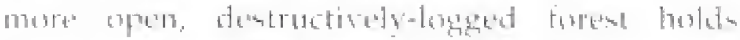

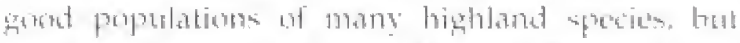

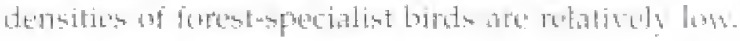

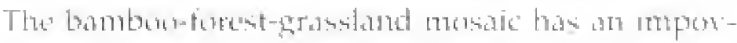

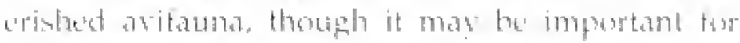

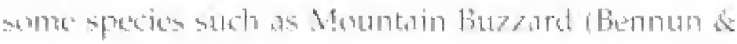

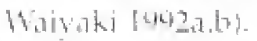

The main consertation problem is the Mau in that

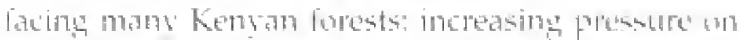
produtice land from an expanding population. A patticular complicatien in this cose bs the presene

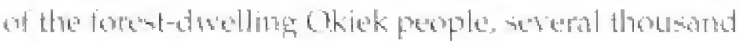

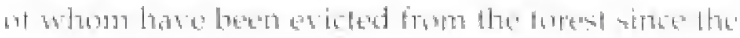

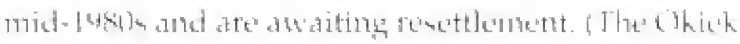

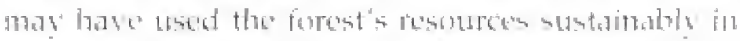

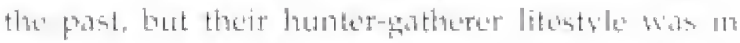

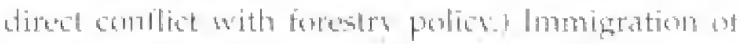

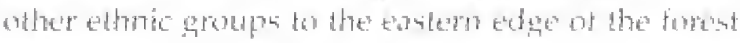
(particularly from the demsety populated bestern houdersi has added to the number at perple

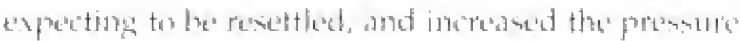

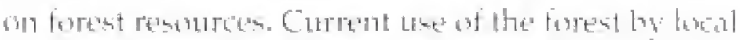

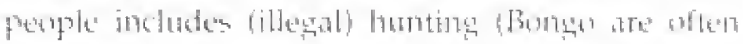

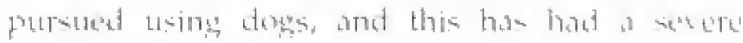

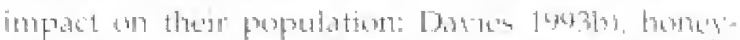
sathering threst tipes are cut and ate-batked to

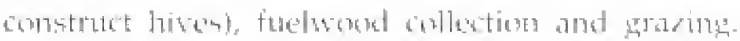
These ontigites, which might he surred aut

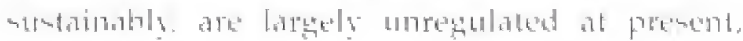

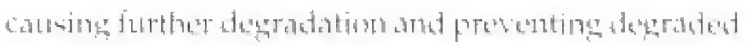

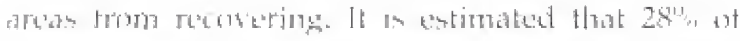

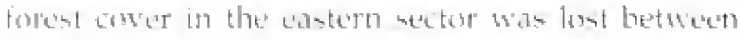

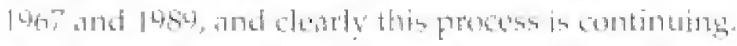

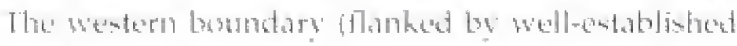

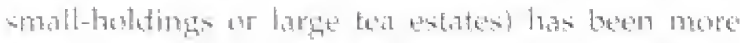

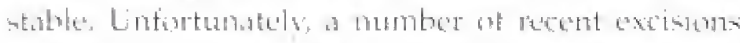

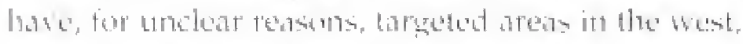

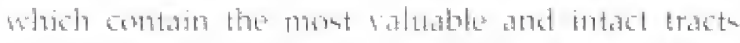

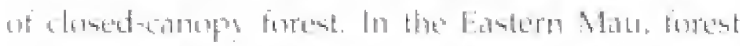

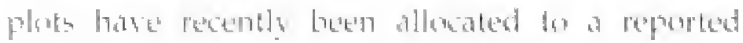

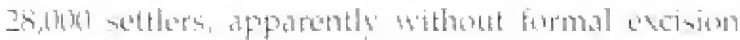

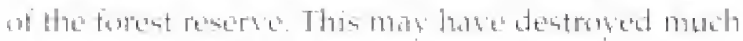

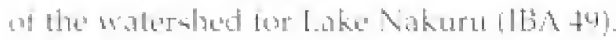

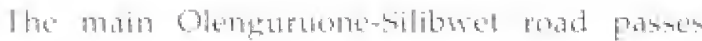
throusth the centre of the Trange-Mara forest. The

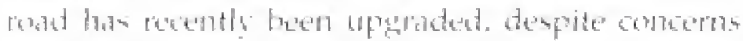

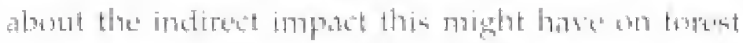

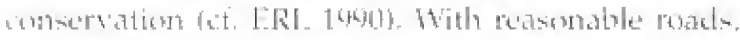

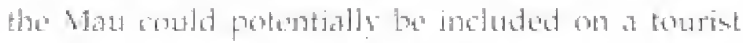
ciacut that induded l.ohe Makuru and the Matad

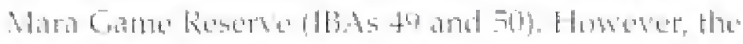

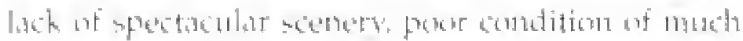

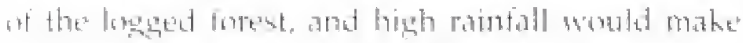
(⿻上丨匕日)

\section{Further reasting}

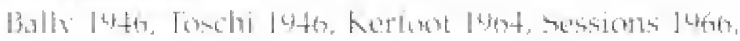

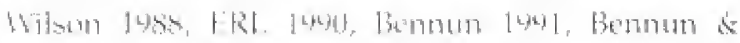

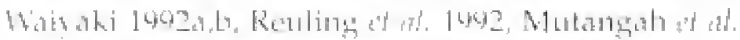

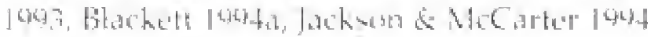




\title{
52 Mau Narok-Molo Grasslands
}

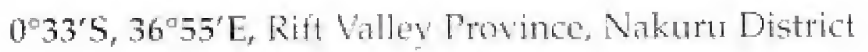 \\ c. 40,000 ha
}

Nitude: $2,700-3,100 \mathrm{~m}$

Status: Unprotected, mainly private land

Categories: Globally-threatemed species, restricted-range species

\section{Site description}

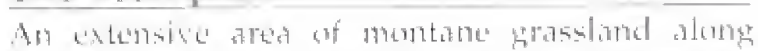

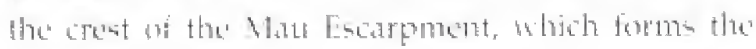
western wall of He central kill Salley un Ketrya. This high, open platwe stum for around sit hut south-east

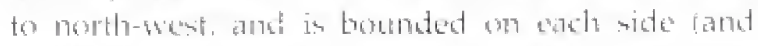

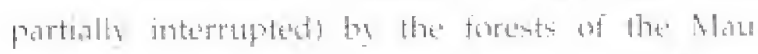

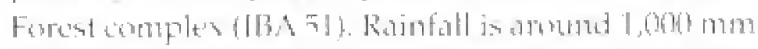

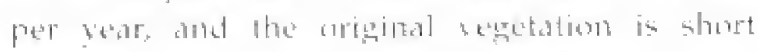

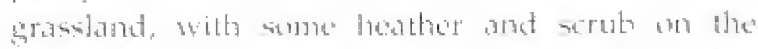
ridges where the soil is deeper. The" areat hat high

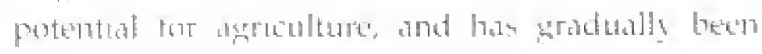

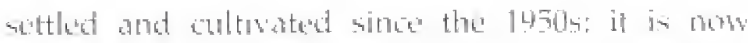
hasidy propulated, with a landscape sereredy

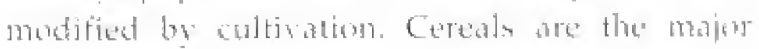

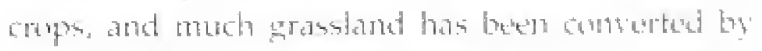

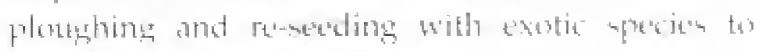

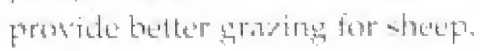

\section{Birds}

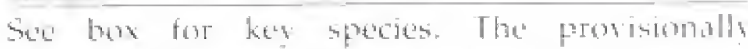

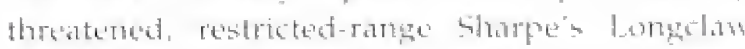

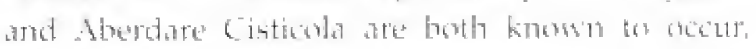
but their statum and dintribution sithin the IBA

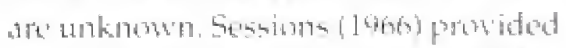
a bird list comprileat in the lasos, but there is lible reant intormation wa the bircts on this area. The prescontedas arifuma is Jikely tu be similar to that al the Kinamera? I'latean (IBA +b) an the copperates side ot the Ritt valles: 
Important Bird Areas in Kenya

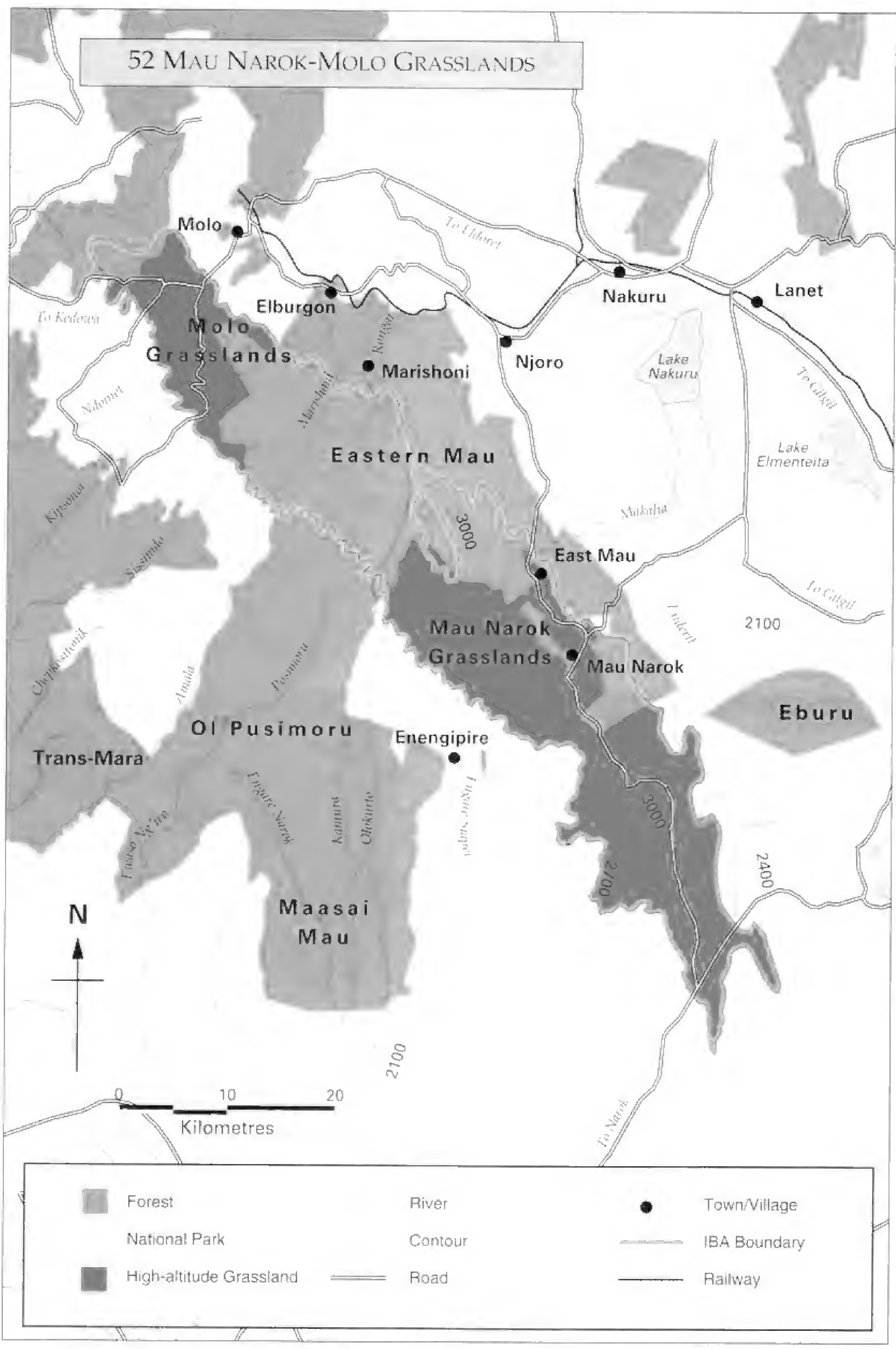




\section{Mau Narok-Molo Grasslands}

\section{Globally-threatened species}

Lesser Kestrel

Great Snipe

*5harpe's Longclaw

* Aberdare Cisticola

"Jackson's Widowhird

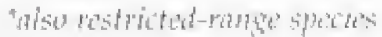

Vulnerable:

Nerar-thraterined

Provisionaly vulnerable

(Bemnun \& Njoroge |996)

Prosisionally wulnerable

(Bemmun \& N Nortge L966)

Neatethroatenest
Palateartic pasange migrant, formerly regular (Sessions 1966)

Uncommon P'alaearctic winter visifor (Sussions 1966. Zimmerman at al, 1946)

Status unknown, formerly common (Sessions 1966)

Status unkmown

Status Lunknown

\section{Regionally-threatened species}

Greall Crusted Grebe

Demhame \& Eststard critical

Endangered
Status unknown: may still brest on upland dams (ci. Sessions 1966)

Probilyly extirpated (Zimmerman of al $1996)$

\section{Other restricted-range species}

Jakknon's Francolith

Hunter" \& Cistichla
Formerly common at forest margins Likely to be common in scrubby areas

\section{Other willlife}

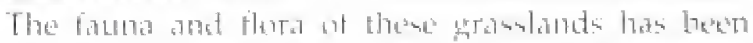

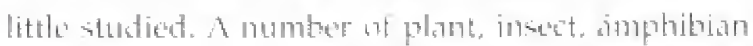
und reptite upeces that are andined to highland

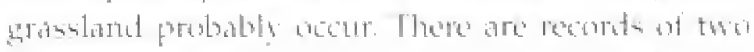

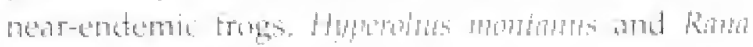

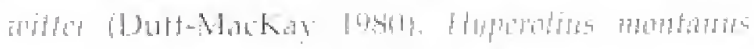

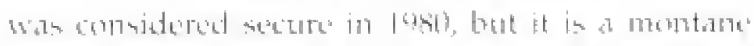

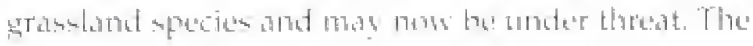

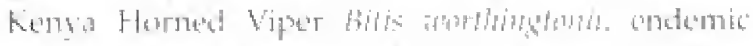

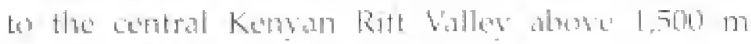

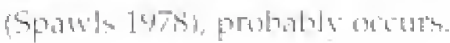

\section{Conservation issues}

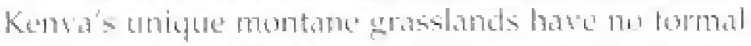

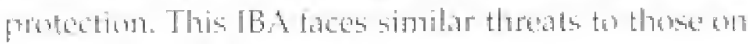

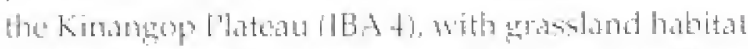

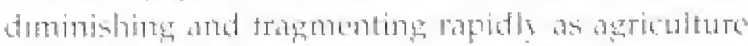

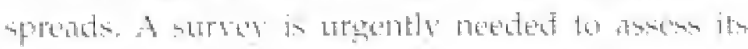
status and that wit the theseatened birds

Fusther reading

bessions 1966 


\title{
53 NORTH NANDi Forest
}

\author{
$00^{\circ} 00^{\prime} \mathrm{S}, 35^{\circ} 00^{\prime} \mathrm{E}$, Rift Valley Province, Nandi District \\ 10,500 ha \\ Altitule: $1,700-2.130 \mathrm{~m}$ \\ Status: Forest Reserve \\ Catgories: Globally-threatened species, restricted-range species, \\ Guinea-Congo Forests biome species
}

\section{Site description}

This is a strip of hing-cancopy forest on the adge of the Vadudi escapment, sbore and immediately east

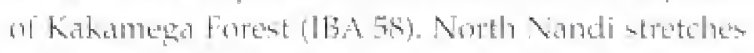
for more than $30 \mathrm{~km}$ from worth to south athe is $3-5 \mathrm{~km}$ wide for mokt of its lemgth. Drainate in mainly eastwarets jnto the kiswat and Kimondi River systems, which How through the South Vandi

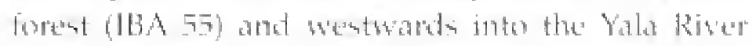
mont Take Vhetoria.

Biogeograptrically, North Nundi is transilional between the lowland forests of West and Coneral

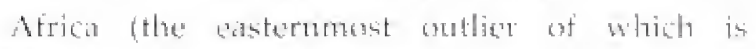
kakamuera) and the montane torests of the entral Kenya hishtands. It is higher in altitude than

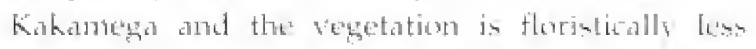

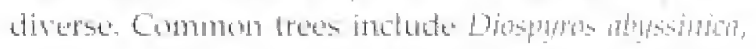

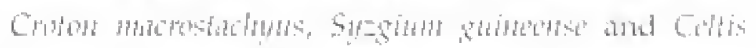

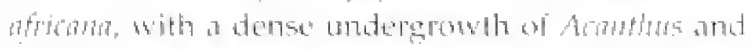

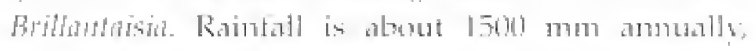
and the wellodraned, triable soils are moderately tertile.

Ninctla Nandi was first

gareited in 1936 as a

Trust Foremit comerings 11,850 lat.

In 1968 the Nonth Nandi Nature

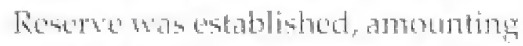

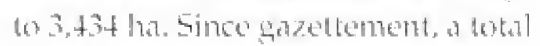

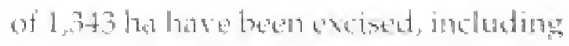

part of the nature reserve. An additional th th ha

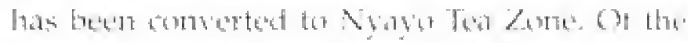

prosent garetted forest alea $(10,5(70)$ hat.

approximately some ha is indigemous.

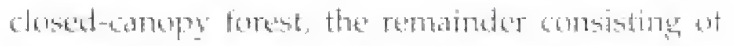
cultization, scrubs grasidand. plantations and ten

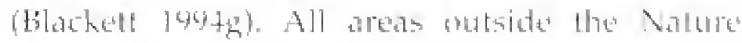

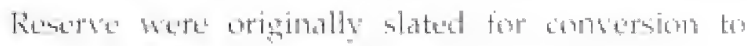

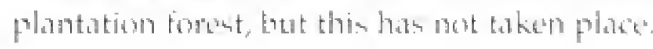

\section{Birds}

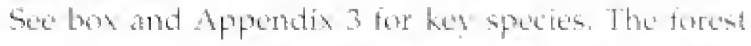
belongs to the Kakamega and vand lorests
Secondaty Area of endemism, terfined by the

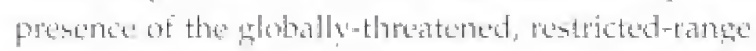

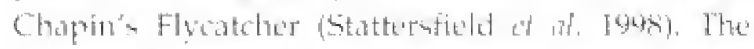

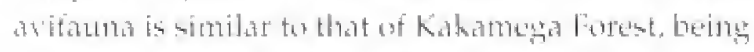
a mixture of species chatacteristic of 1 wo bomes: the

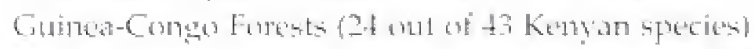

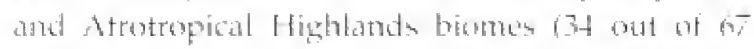
spoctes including (Chapin's Flycatcher) around 160

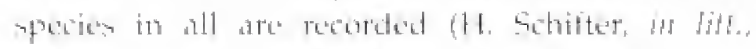
Zimmerman ot al. 1966). North Nond is lecos rich in species than Kathamesa and its bird bommunties

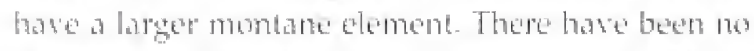

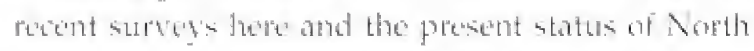

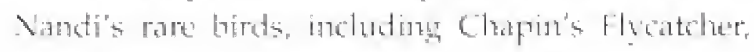
is unknowin.

\section{Other wildlife}

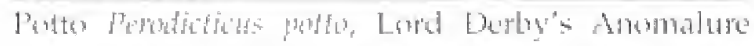

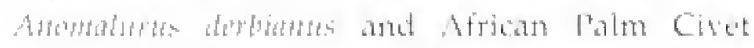

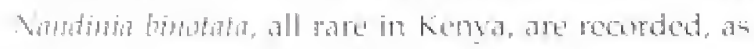

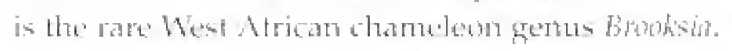

Chapin's Flycatcher 


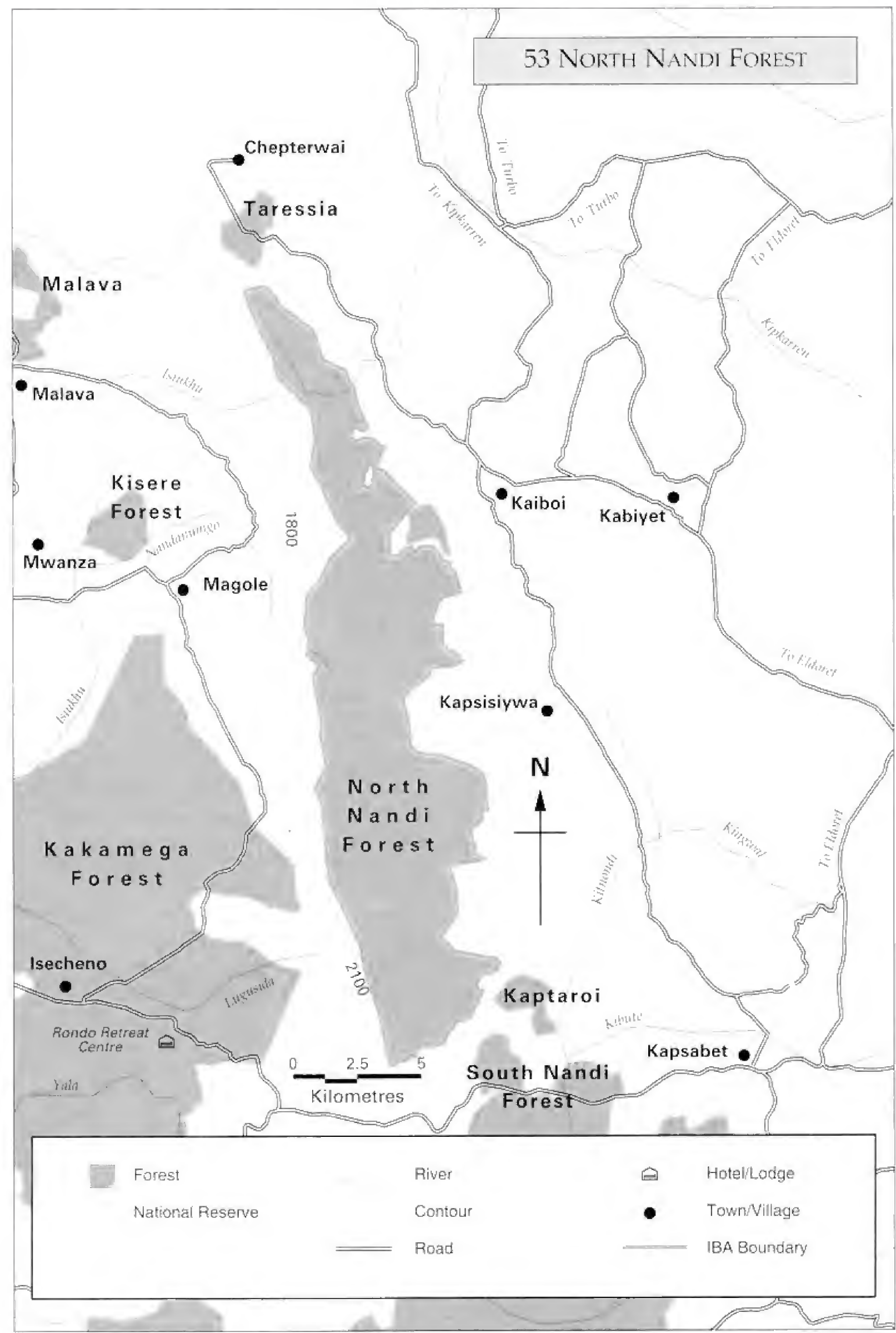




\section{Important Bird Areas in Kenya}

\section{Globally-threatened species}

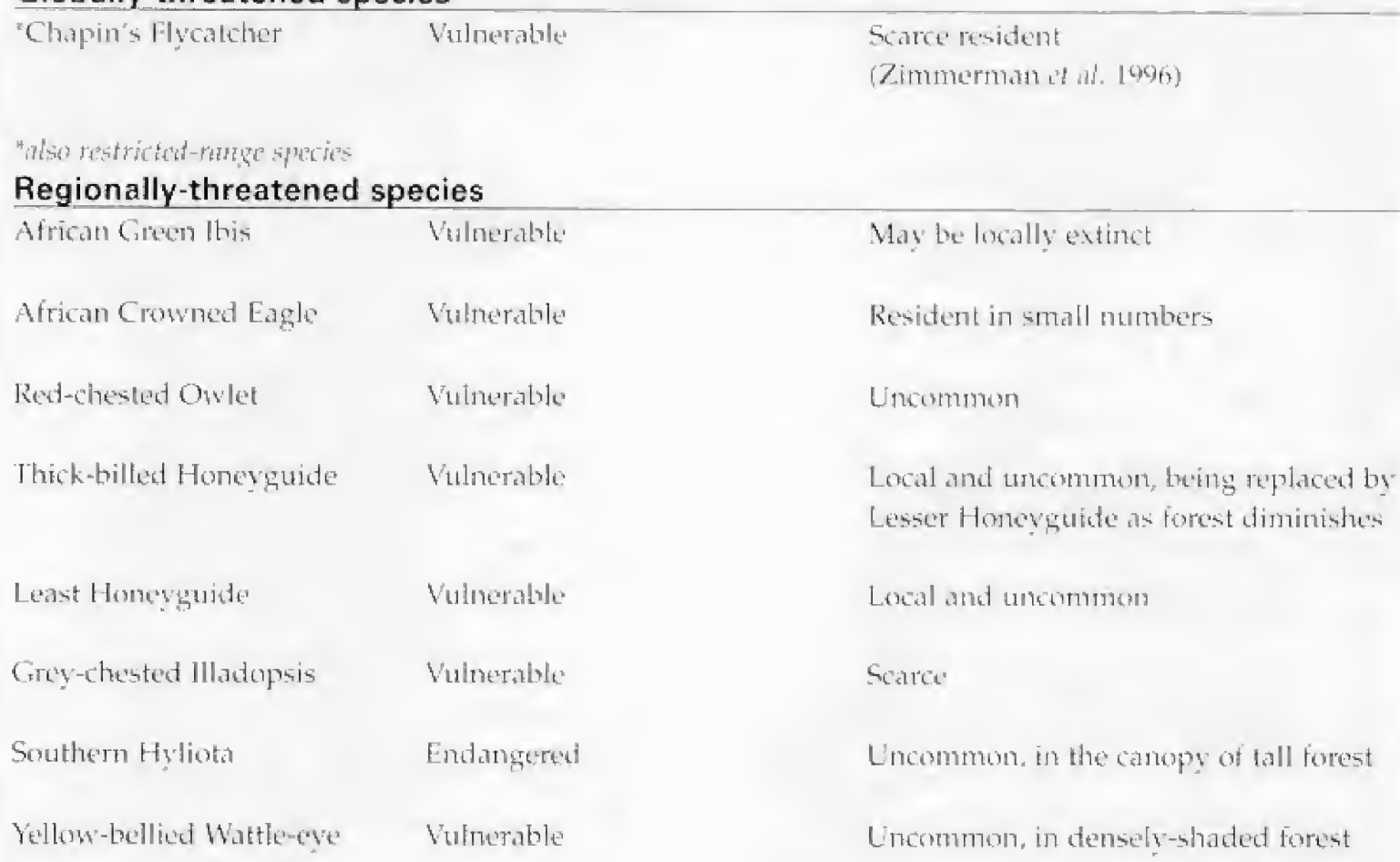

Stats informaticus from Zimmerman et al. (1956)

\section{Conservation ișsues}

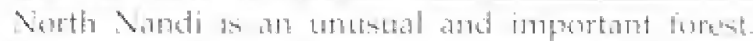

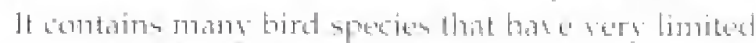

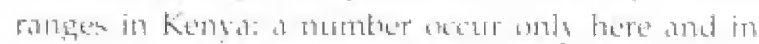
Kakancega. Compersion of the formest to plantations.

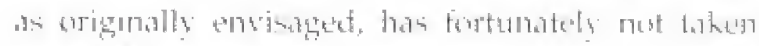

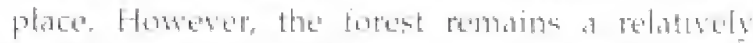

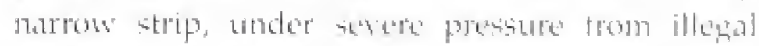

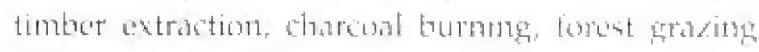

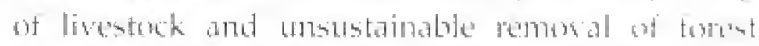

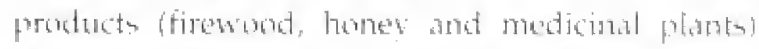

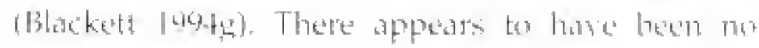

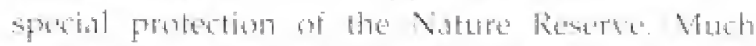

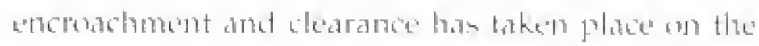

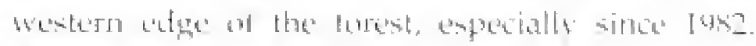

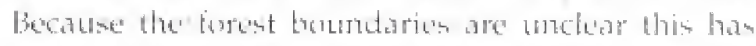

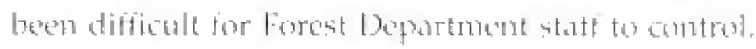

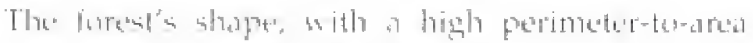

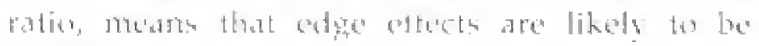
sothetantial.

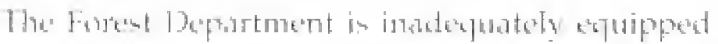

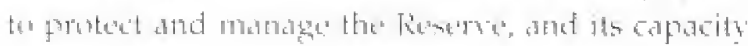

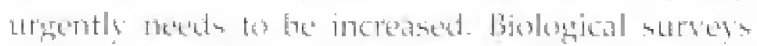

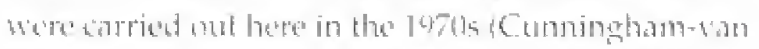

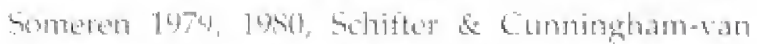
sumeren. in preps but there is little up-tastate

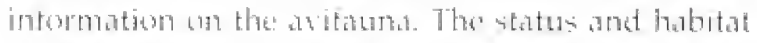

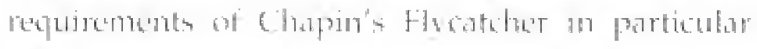

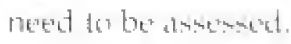

\section{Further reading}

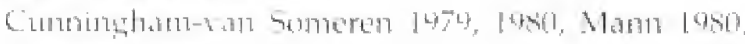
biakete butg 


\title{
54 Ol Donyo Sabache
}

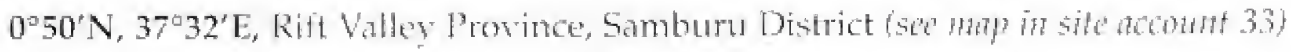

\author{
1,000 ha \\ Altitule: 1.880 tm \\ Status: Unprotected \\ Category: Globally-threatened species
}

\section{Site description}

An isulated hasalt mountam with dramatic dift facus

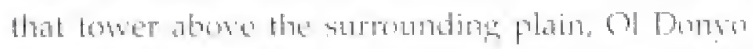

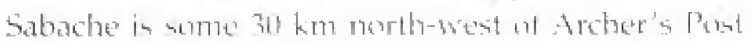

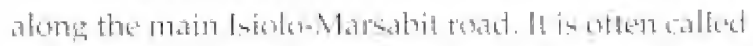

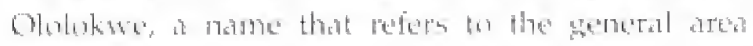

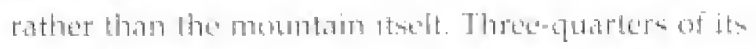

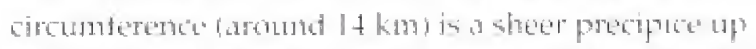

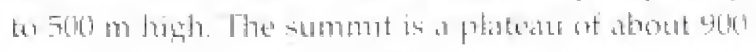

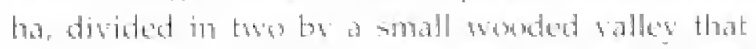

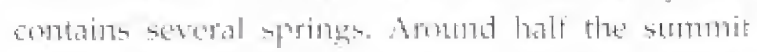

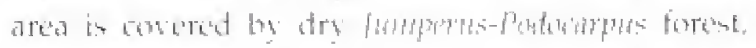

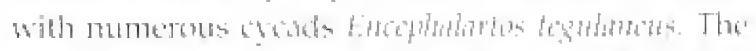
steve cliffs are sparsedy sugheated. The semi-arid

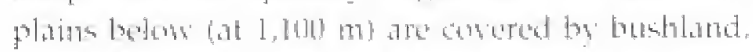

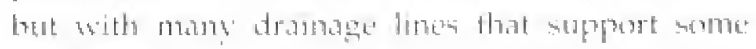
tollder totetes.

\section{Birds}

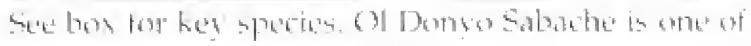

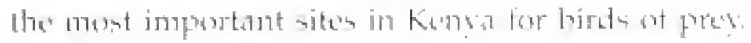

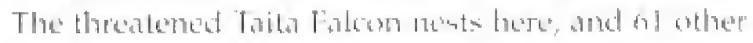

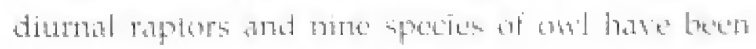

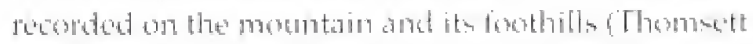

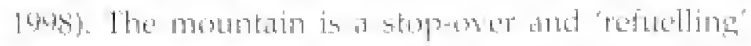
point for mumerous Padaterethe migatats, ineluding

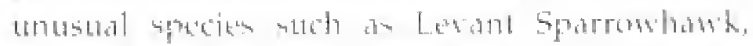
Eurasian Sparrowhowh, Saker Falcoun and Eurasion

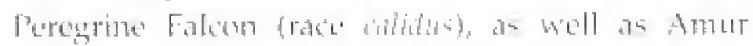

Fatcou, Commen bteppes Buzard and Eurasian

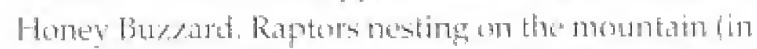

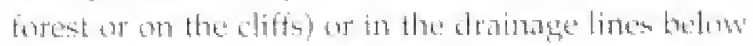

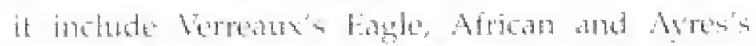
Hawh-Eagles, Martial and Tawny Fatgles, Arrant Harrier Hawh, Bateledi", Pereerine and (probably) Batban Falcons, and sape Late owl. Atrican

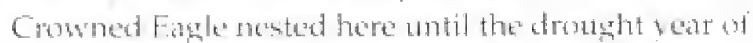

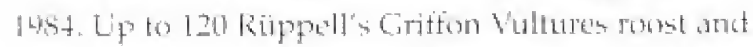

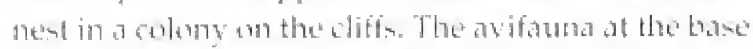

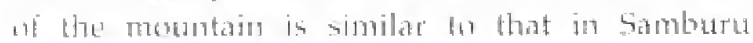
National Rererve (1BA 33), while that an the summit contains an imporerished set of specios charateristic of the central Kenvan highlands. inclusting the restricted-ranger 1 dunter's Cisticala [Zimmeromand at. 1496).

\section{Other wildife}

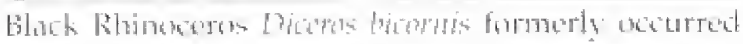

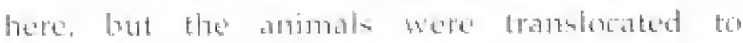

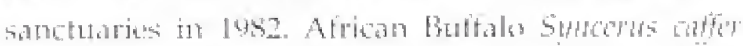
are losally extinct since the drying up of

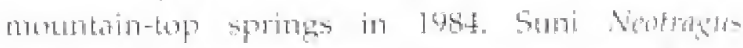

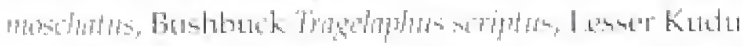

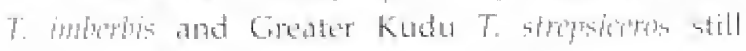

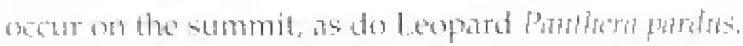

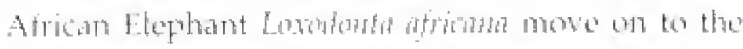
masunting from the platis during the wet season, and

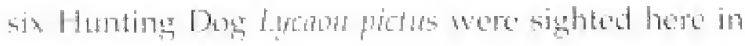

\section{Globally-threatened species}

vuluirable

Regionally-threatened species

Lammergeier

Ayres's Hawk Eagle

Martial Eagle

African Crowned bighle

Data from Thansirt (1998)
At least one nesting pair

\section{Rare visitor"}

Nests in the forest

Nests near the base of the hill

Not recorded sinet 1984 


\section{Important Bird Areas in Kenya}

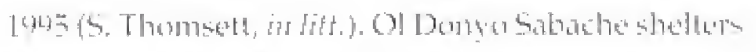

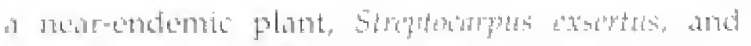

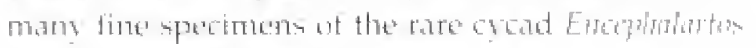

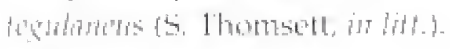

\section{Conservation issues}

The forest on ol bonyos Sabathe has beerm

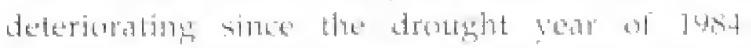

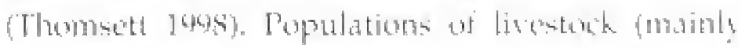
cattle and gototo hat built up an the plains, thanks to the prox ision of new watering points. When dorkght

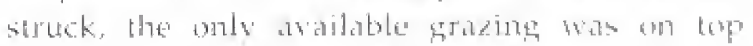
of the mountain, the hill withe atral

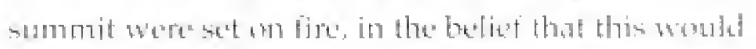

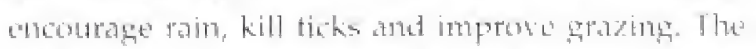

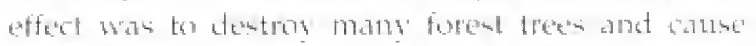
the springs to dre up, in kurn wasting the death of

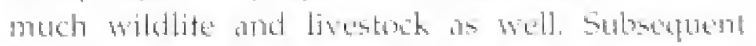

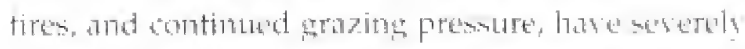

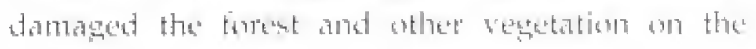
summit. The guats ale rire-resistant, but mamb

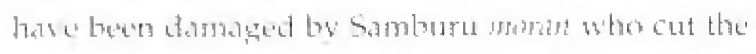

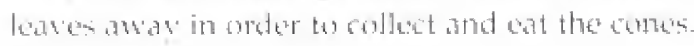

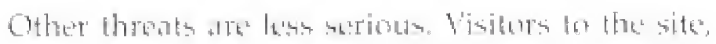

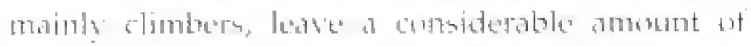

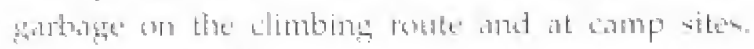

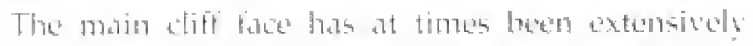
mortaget and thelled for military praction. thes discupting nesting mapters.

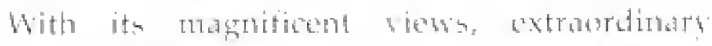

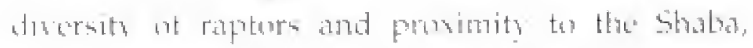

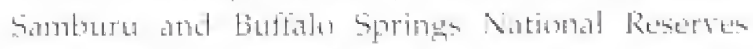

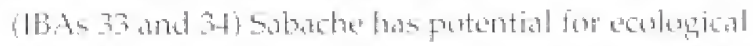

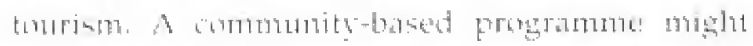

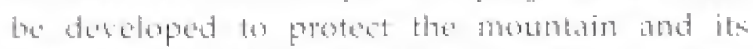

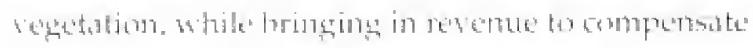

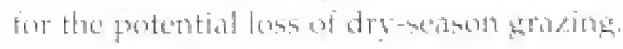

\section{Further reading}

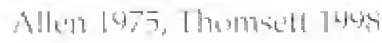




\title{
55 SOUTH Nandi Forest
}

\author{
$00^{\circ} 05^{\prime} \mathrm{S}, 35^{\circ} 00^{\prime} \mathrm{E}$, Rift Valley l'roxince, Nandi District \\ 18,000 ha (c. 13,000 ha forest) \\ Alfitude: $1,700-2,000 \mathrm{~m}$ \\ Status: Forest Reserve \\ Catcgorics: Giobally-threatened species, Gunea-Congo Forest biome species
}

\section{Site description}

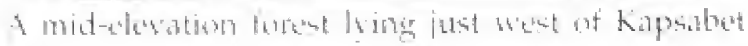

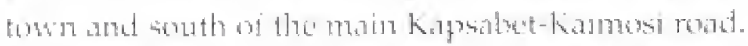

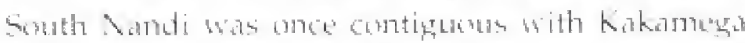

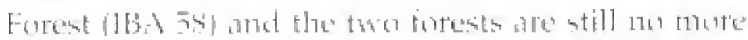

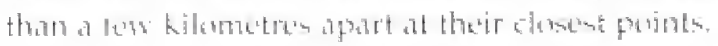

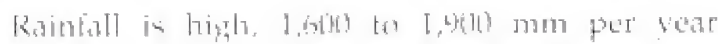

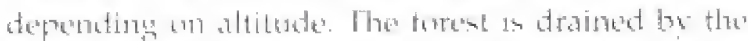

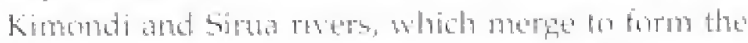

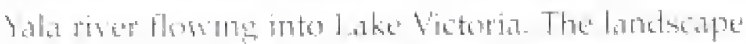

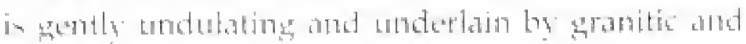

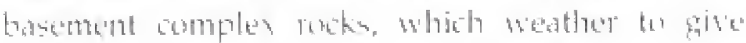

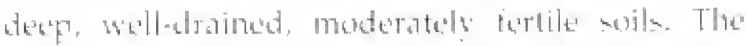

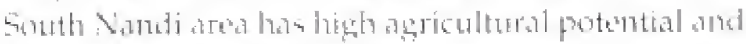

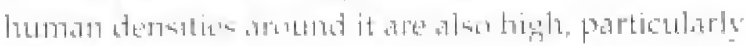
wo the west.
Biongeographialty, South Vandi is aften

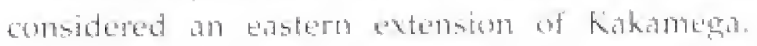

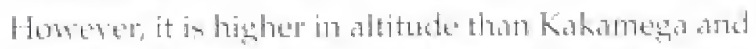

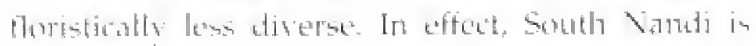

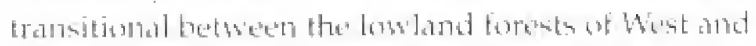
Central Sirica (the tasternmest outline ot which is

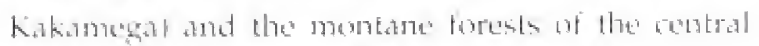

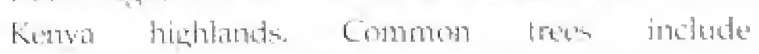

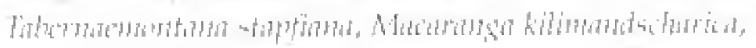

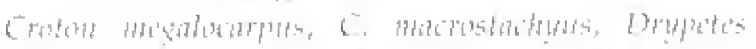

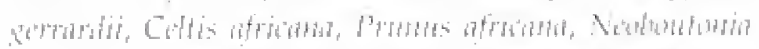

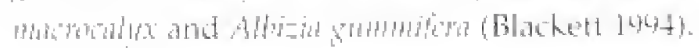

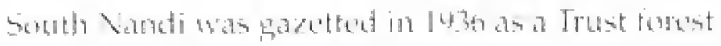

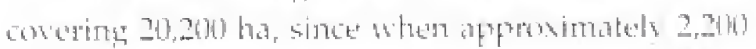

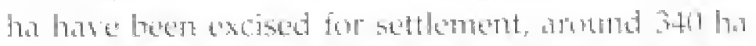
planted with tea, and 1, thet ha planted with exatic

\section{Globally-threatened species}

Turnot's Eremoาม
Vulnerable

\begin{abstract}
South Nandi has exceptionatly high densities of this littlo-knewn species (around 0.27 groups/ha, equating to 1.1 birds/ha), and is probably its world strotighold. The estimated population is 13,000 hirds (Kosgey 1998).
\end{abstract}

\section{Regionally-threatened species}

\begin{tabular}{|c|c|c|}
\hline Sirican Crowned Engle. & Vulnerable: & Uncommon resident \\
\hline Red-chesterd Owlet & Vulnurable & P'resenent but status unknomin \\
\hline Thick-billed Honeyguide & Vulnurathle & Local and tuncommon \\
\hline Least Honeyguitita & Vulnerable: & Lotal and uncommon \\
\hline Groy-chested Illadopsis & Vulinerable & Scarce and very loal \\
\hline Grey-kinged Robin & Yulne'rable & Local and uncommon \\
\hline Yollow-bellied Wattle-esc & Vulnerable & Sarrae in dentidy-shaded forest \\
\hline Sotullaern Hy-tionta & Endangered & Uncommor in forest canopy: \\
\hline
\end{tabular}

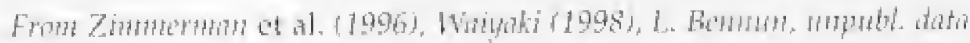




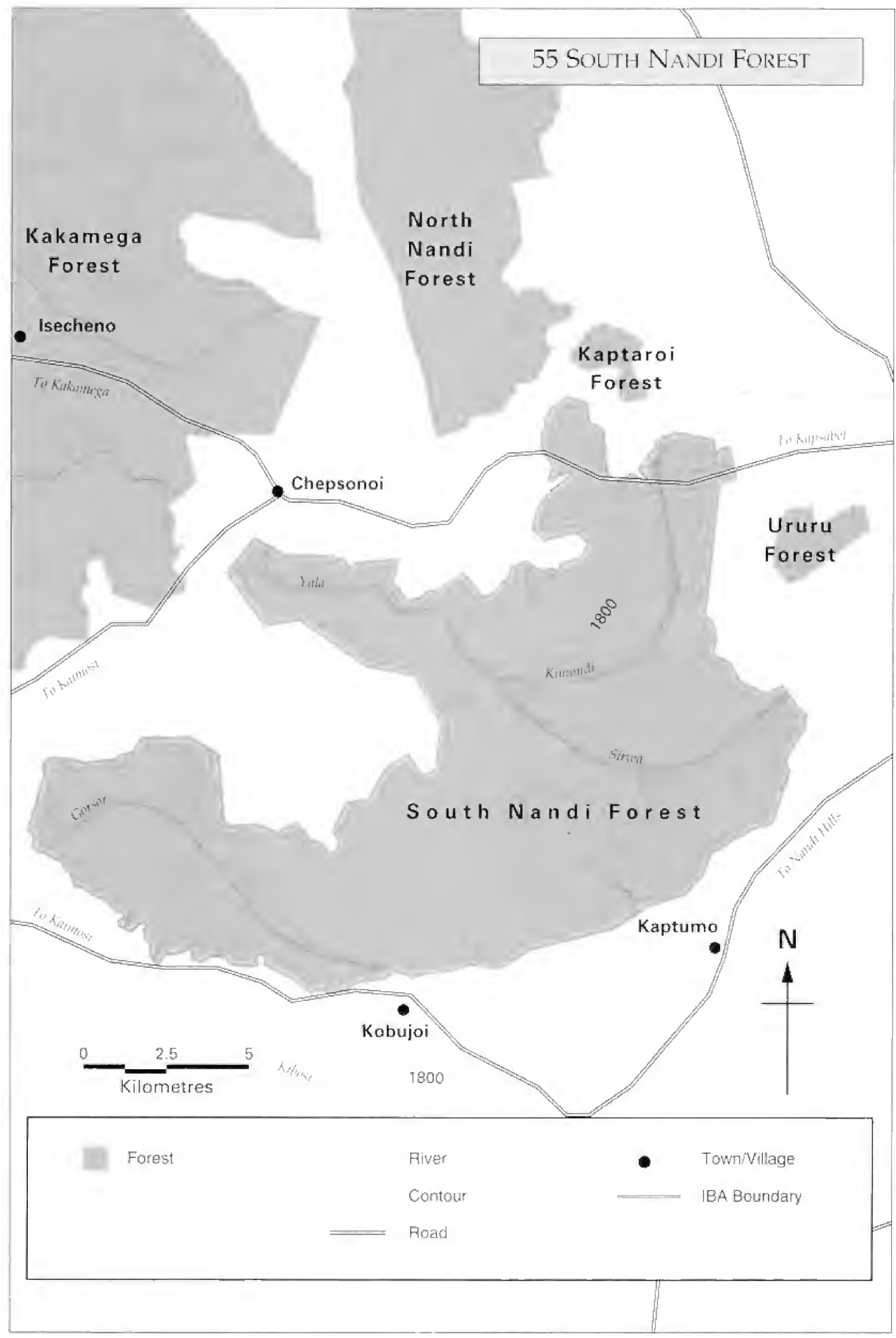




\section{South Nandi Forest}

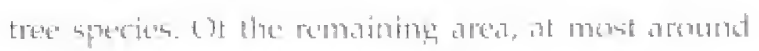

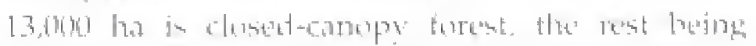

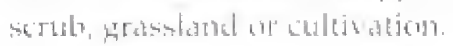

\section{Birds}

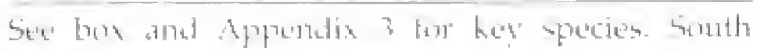
Sandi foreset is alment certaindy the mest important

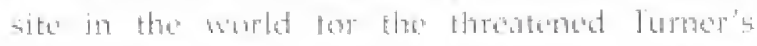

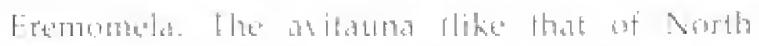

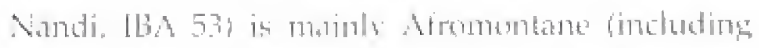

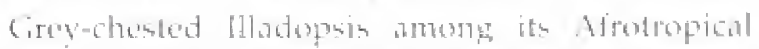

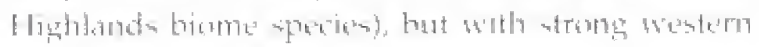

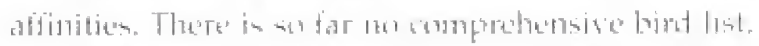

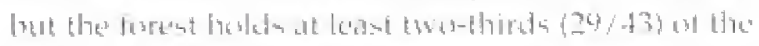

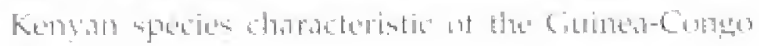

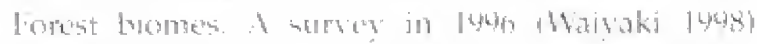

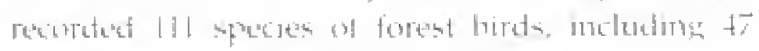

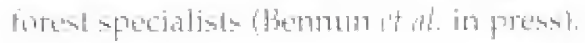

\section{Other wildlife}

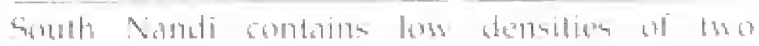

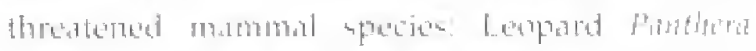

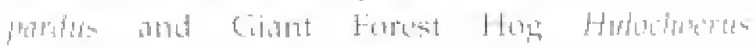

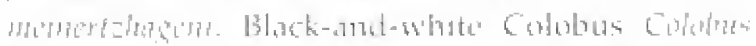

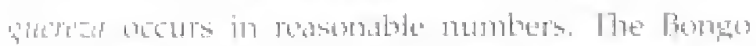

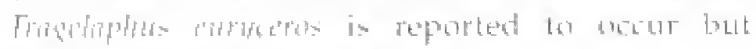

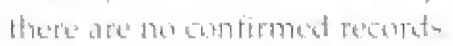

\section{Conservation issues}

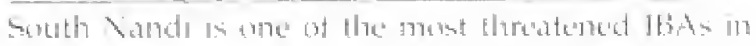

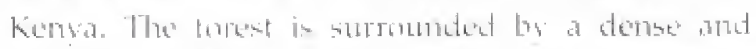

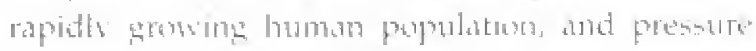

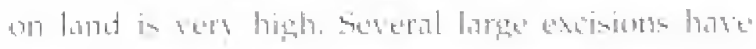

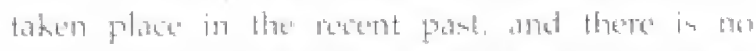

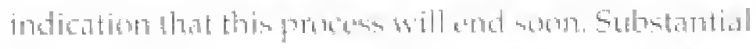

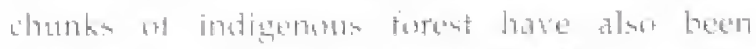

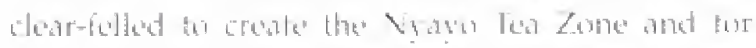

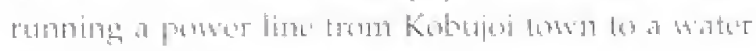

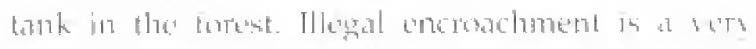

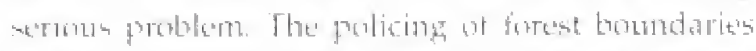

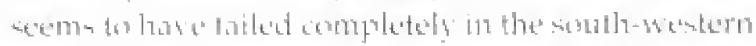

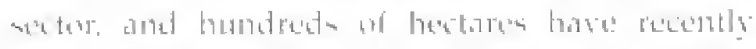

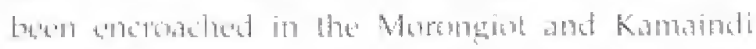

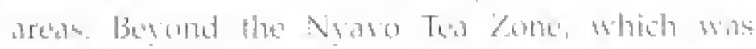

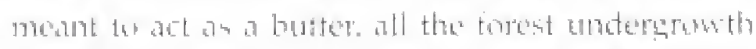

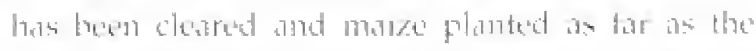

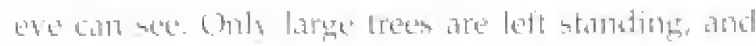

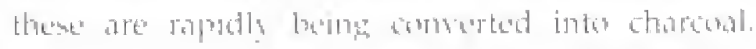

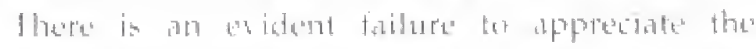

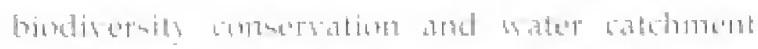

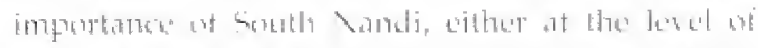

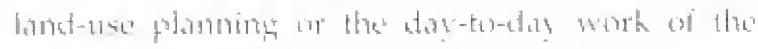
Forest Department.

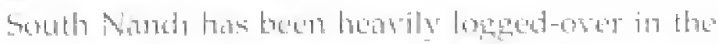

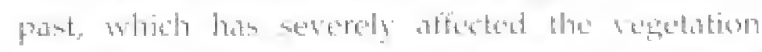

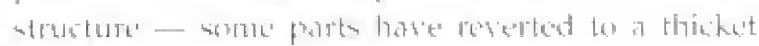

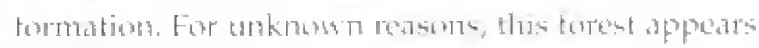

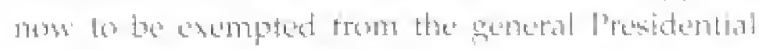

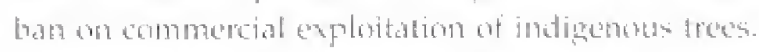

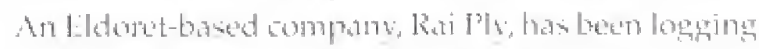

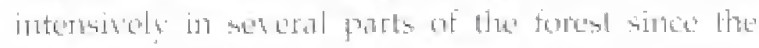

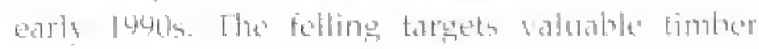

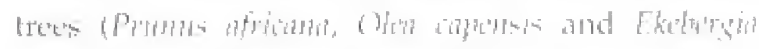

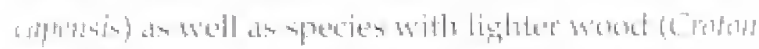

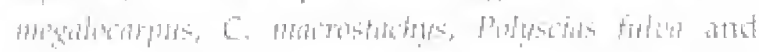

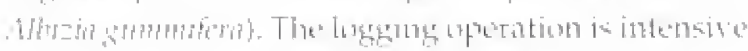

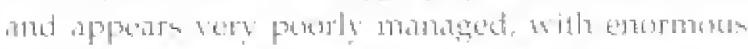

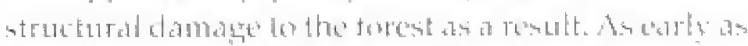

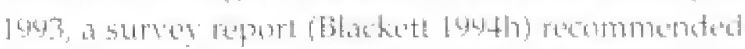

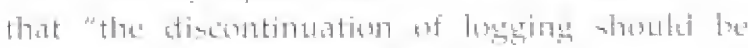

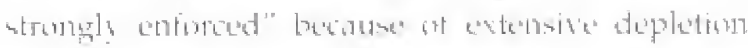

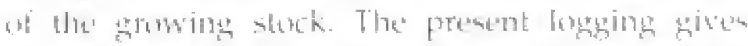

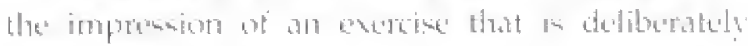

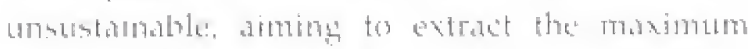
anoune of timber before commercial explontatum in

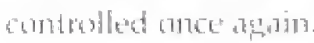

Mang of the other probleme Faced by bouth Vandi

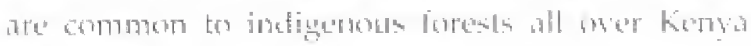

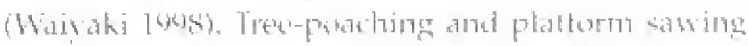
are rampant in the Kaimus ated. and mear wother majur setelemests. Forest anteleype are hunted

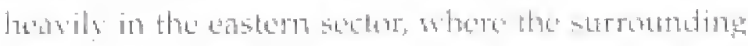
humatr populatign is lowent; the lath of hunding

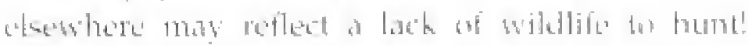

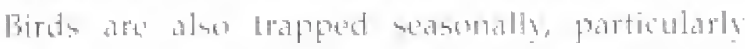

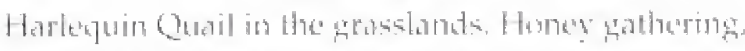

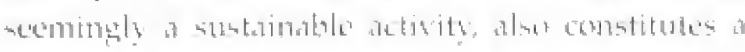

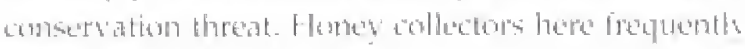

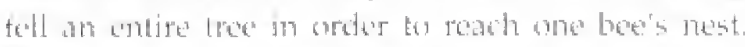

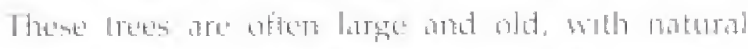

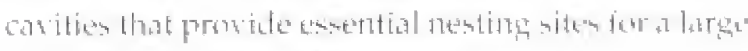

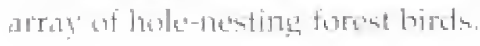

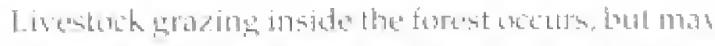

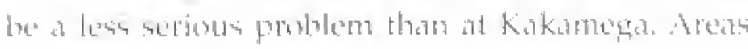

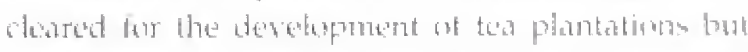

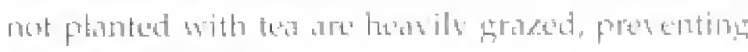
toresel regenteration.

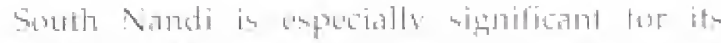

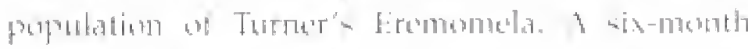

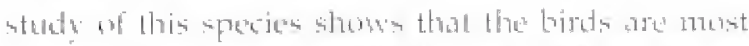
abundiant is the lasw-altitude parte of the terest

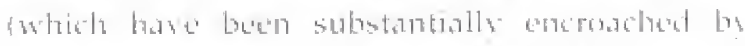

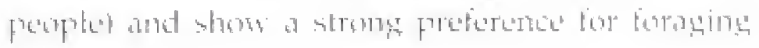




\section{Important Bird Areas in Kenya}

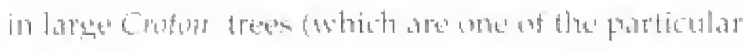

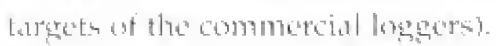

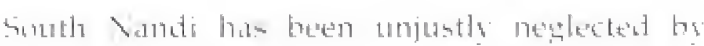

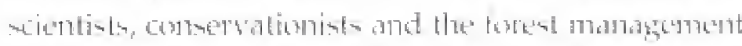

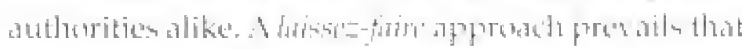

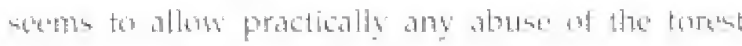

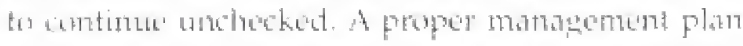
for the forest, produciol with the whollenerated

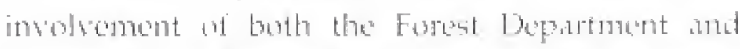

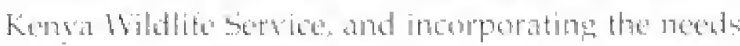
of domal prople as opponed to industrial timber anmpanters, is

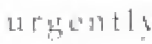
nected.

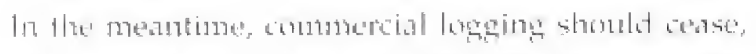

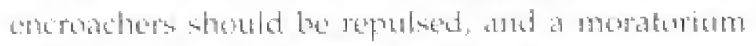

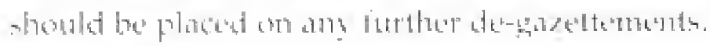

\section{Further reading}

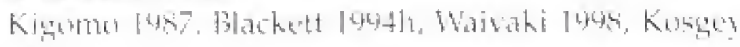
104 


\title{
56 South Nguruman
}

\author{
$01^{\circ} 50^{\prime}$ S, 35 50’E, Rift Valley Province, Narok District \\ Area tuntefinet: c. 125,000 ha \\ Altifude: $900-2,300 \mathrm{~m}$ \\ Status: Unprotected \\ Catcgories: Globally-threatened species, restricted-range species
}

\section{Site description}

The Nisurumat Exarpment forms the bestern

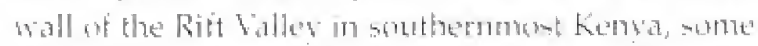

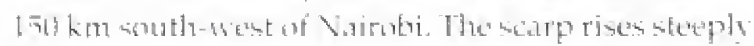

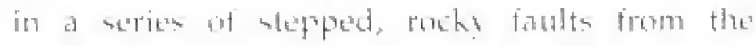

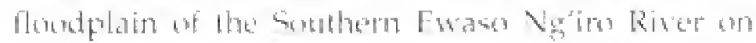

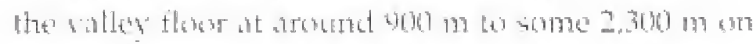
the exatponest crest. From here the land fallo more

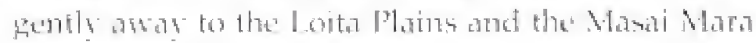

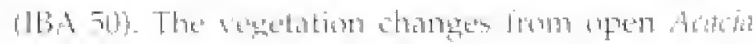

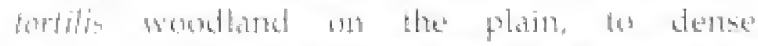

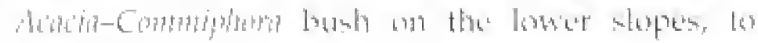

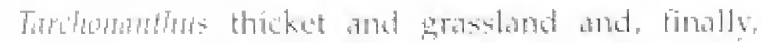

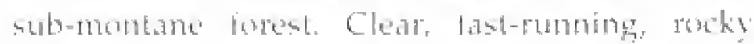
strams flow domb the eseapment. frimed en their lower ferathes with tall riparian Furest of fings

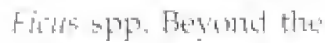
thaspersent crust, tho rolling country is a ImLsale of prasidand, sorub ant torent,

Grey-crested Helmet-shrike

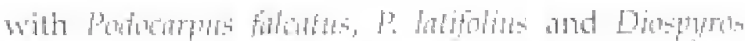

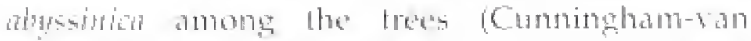
Sorgeren [977). Rainfald at the hase of the easteren

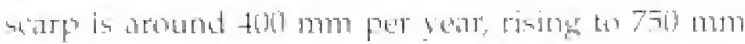
on the forested ridges and perike. Mist and debs an bes hease in the highest areas

Land on the excarponent and hille is comonumally

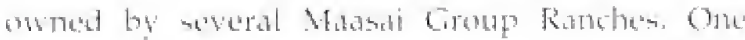

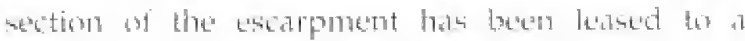
private compang for the deselopnient of lusury tolirism.

\section{Birds}

bet bon for key speciem This it likely to be an important site for the threatened, restricked-ratnge

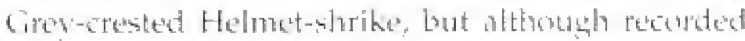
bere its statum is utecertain. It is most likely to be

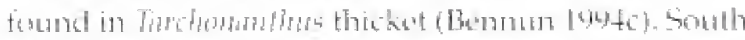

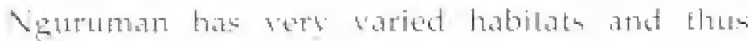

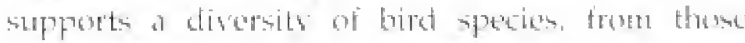

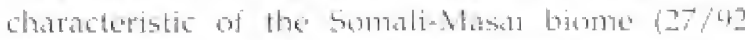

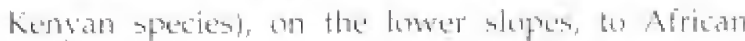

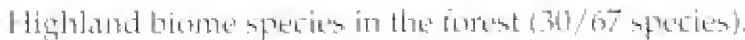
South Nguruman is the only Kenyan sitte for

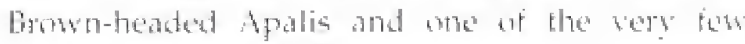

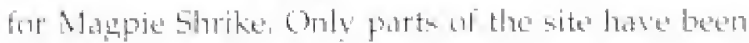
survered, and atditionsl sperien will doubtlest

ber astated to the list.

\section{Other wildlife}

The site hotds subatantial propulation of

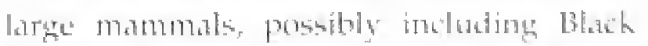

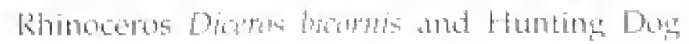

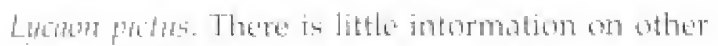
fatura and trora. 
Important Bird Areas in Kenya

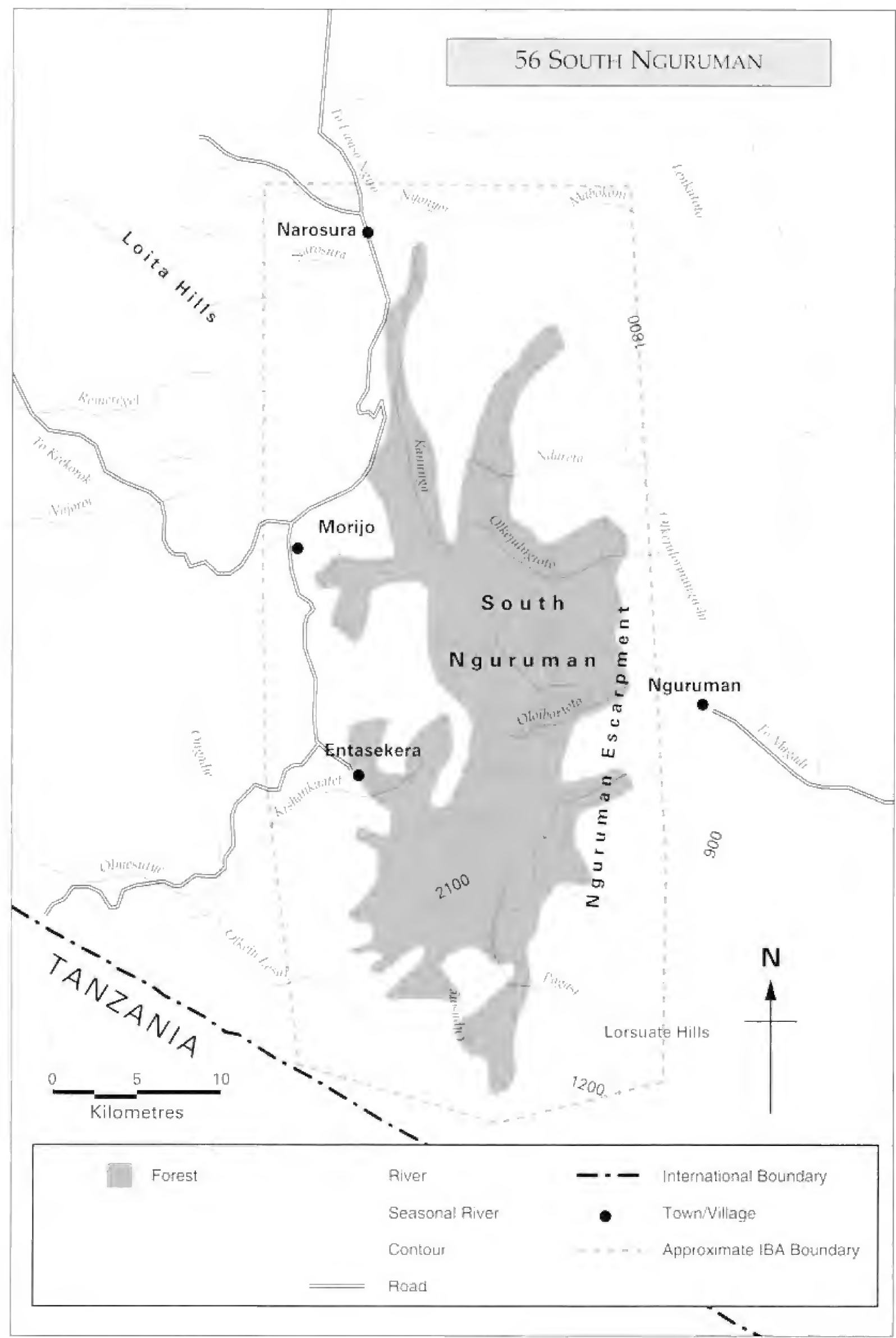




\section{Sotth Nguruman}

\section{Globally-threatened species}

"Cirey-crested I lelmet-shrike Vulnembles

Probably at uncommon resident in

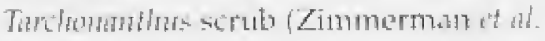
1996)

Red-thrmated 'lat

Near-thecatened

Probably residene, in Atritat woodland

"Jacksm"s Nidombind

Near-theratened

Nest in long grasslund abore the escatpmont crest

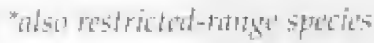

Other restricted-range species

I funter"s Cisticontia

At forest expes and in sctub on the escolspment crest

\section{Regionally-threatened species}

Lammergoiker

Atrican Crontred Eagle

Striped Flutitail

Purple-throbated Cuchoo-shrike Vultionable

Datio from Zimmormm et al, (1996)

vilneratolo

Vulneralve

Vulpexalye
Reterded from thigh grabsiand

Uncommon, in highland forest

Statts uncertain, may be only an occasional visitor

Uncommon resident in forest

\section{Conservation issues}

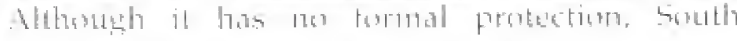

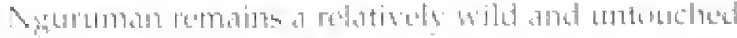

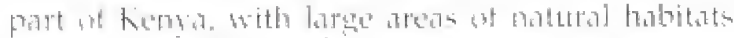

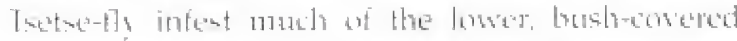

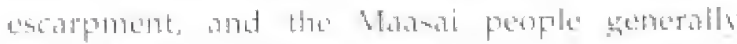

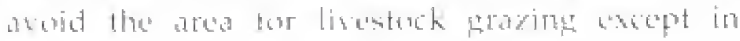

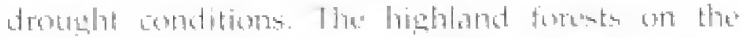

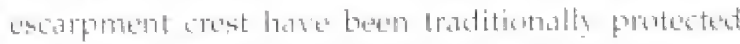

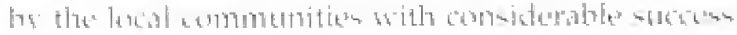

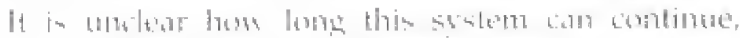

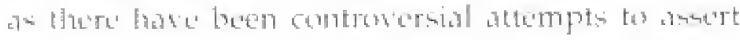

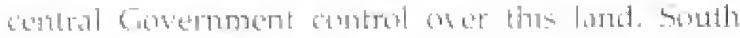

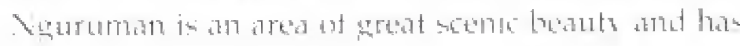

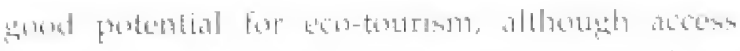

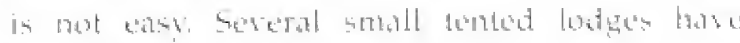

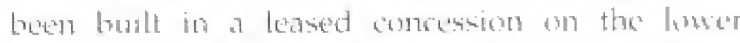

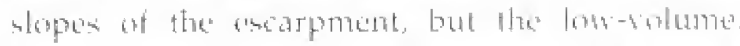

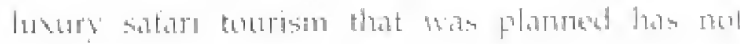
Fot lowetum.

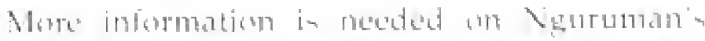

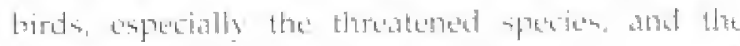

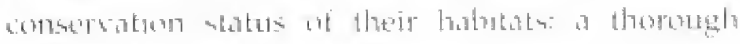
axitamal surver is recommoteded.

\section{Further reading}

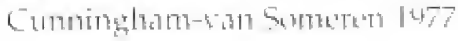




\title{
57 BUSIA GRASSLANDS
}

\author{
$00^{\circ} 25^{\prime} \mathrm{N}, 34^{\circ} 15^{\prime} \mathrm{E}$, Western Province, Busia District
}

250 ha

Altitude: $1,200-1,220 \mathrm{~m}$

Status: Unprotected, private or communal land

Categories: Globally-threatened speries, Sudan-Guinea savamah biome species

\section{Site description}

A. hain of small grassland patathe (some neasonally

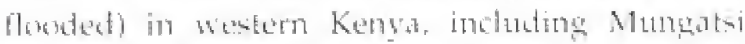

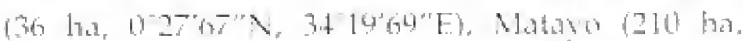

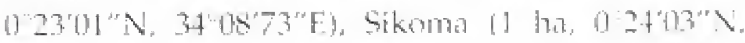

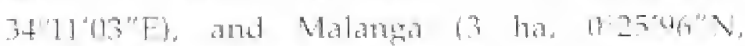

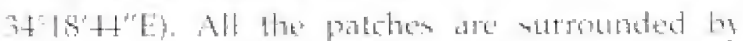
intensive agriculture, mainly" maize and stygarcane, and are grazed by livestok. The most important of the markel alomg the Wungataj-Munami road. Tho privately-owned site lies on either side at a small utream a tributary of the Rivel siob, which in

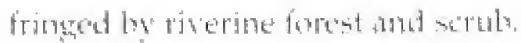

\section{Birds}

Gove has and Appendiv 3 for key species. The blue Swallew, a globally threstemed intra-African

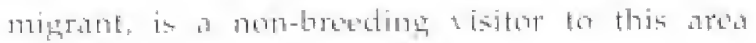
from April to September. Blut Swallens teed

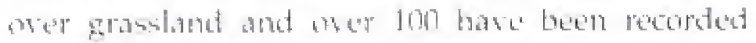
reranting at the blowded grasstand patch neat Vungatsi (D.A. Thener, it lit.). The riverine forest and screb at Mumatsi alas holds several Sutan

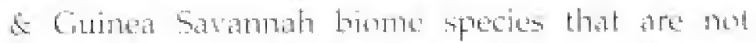
foumd in other IBAs. This is also the soly Kenyen

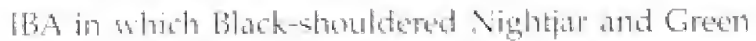

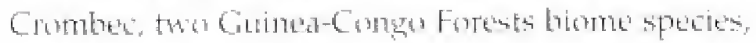
are recorded.

\section{Other wildiife}

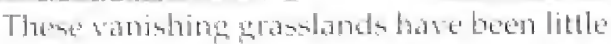

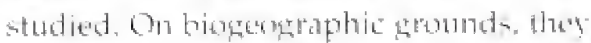
ate likets to hase dose links with Lrandang gratationds and to contain

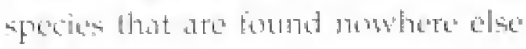
in kiting.

\section{Conservation issues}

The time grisstintrd patehes in Busin Disterat are under sovere and immediate threat. Busia has a high lyeman population destsity that is increasing sury fast, and thete is great prostate on land. A styant factury is now being tatsolished in the area and cane growbing is being promotes - in 1946, 4. were slated for confersion intes sugar-cane plantations

Nasirata de Noroge T447\%.

Harlaub's Marsh Widuwbird 
57 Busia Grasslands

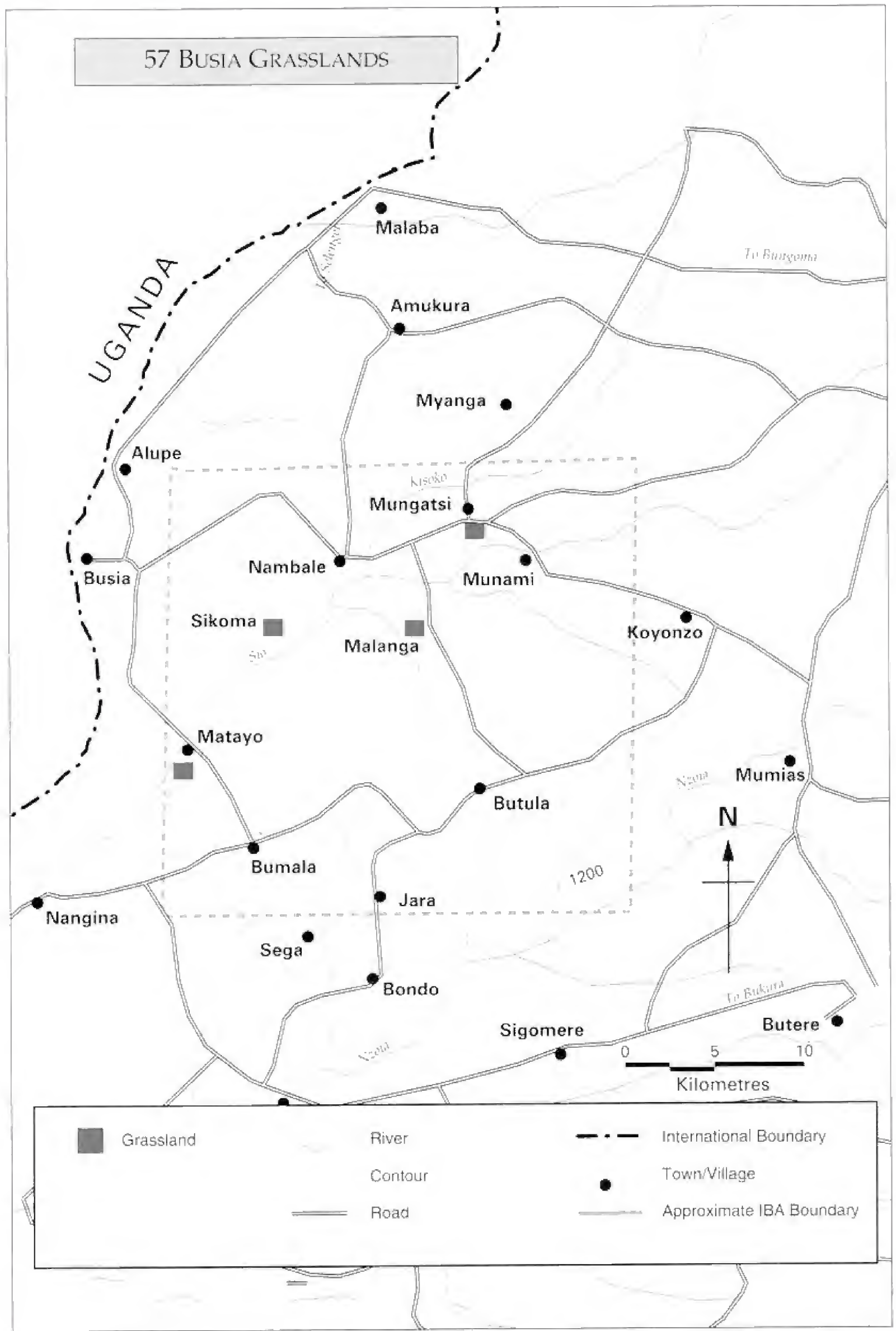




\section{Important Bird Areas in Kenya}

\section{Globally-threatened species}

Blue suallow vilnerable

Resulaty recorded between April and Suptember (Zimmerman of mi. 1996, Finch. 1489y

Great Snjpe Near-threatened

Uncommon Palatedratic migrant, likely to

Regionally-threatened species

Banded Snake Eagle Vulnerable

Shining-blus Kingtishes

Hartaub's Marsh Widowbird Vulnuable accur here regularty but status uncertain

In rizerine wastland

Has been recorded at wungalsi

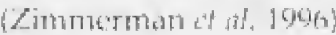

Netsts in flouded grastand

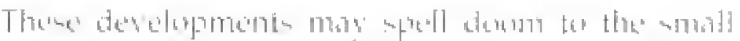

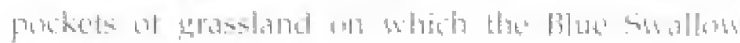
dependis.

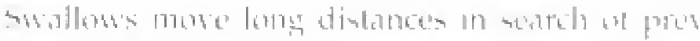

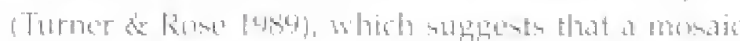

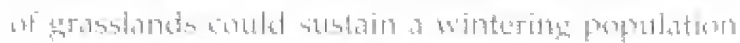

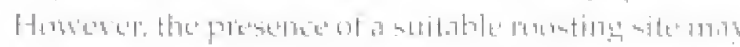

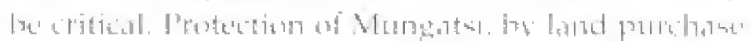

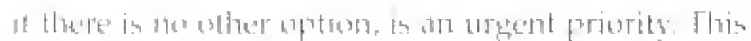

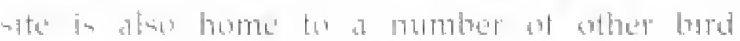

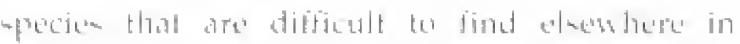

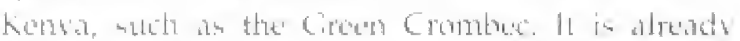

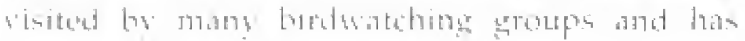

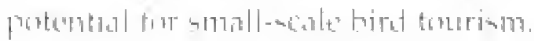

\section{Further reading}

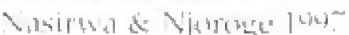




\title{
58 KAKAMEgA FOREST
}

\author{
$00^{\circ} 17^{\prime} \mathrm{N}, 34^{\circ} 53^{\prime} \mathrm{E}$, Western I'rovinee, Kakamega District \\ 18,300 ha (c. 12,000 ha forest) \\ Altitude: $1,550-1,650 \mathrm{~m}$ \\ Status: Forest Reserve \\ and part National Reserve \\ Categories: Globally-threatened species, restricted-range species, \\ Guinea-Congo Forests biome species
}

\begin{abstract}
Site description

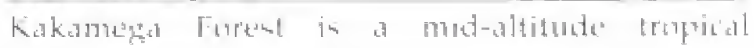

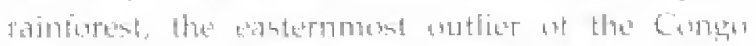

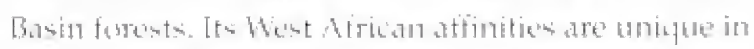

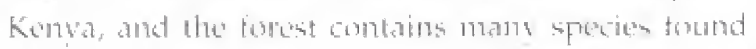

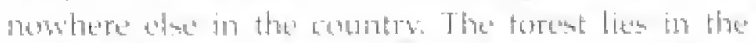

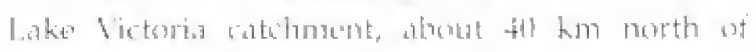

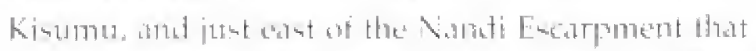

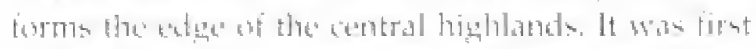

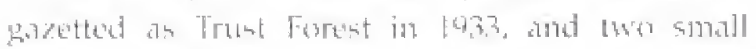

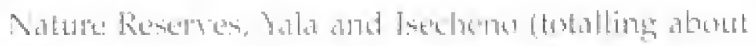

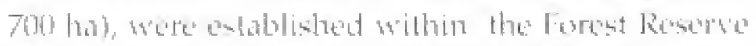

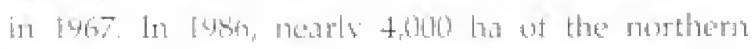

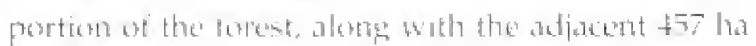

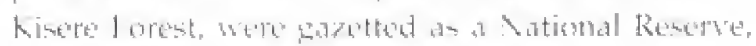

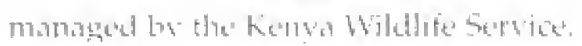

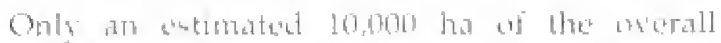

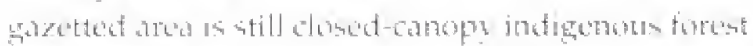

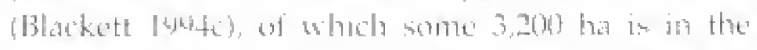

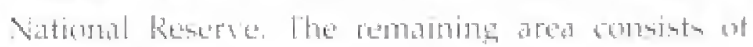

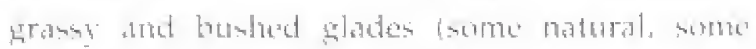

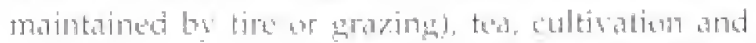

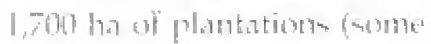
mome than $5(1$ wars ald

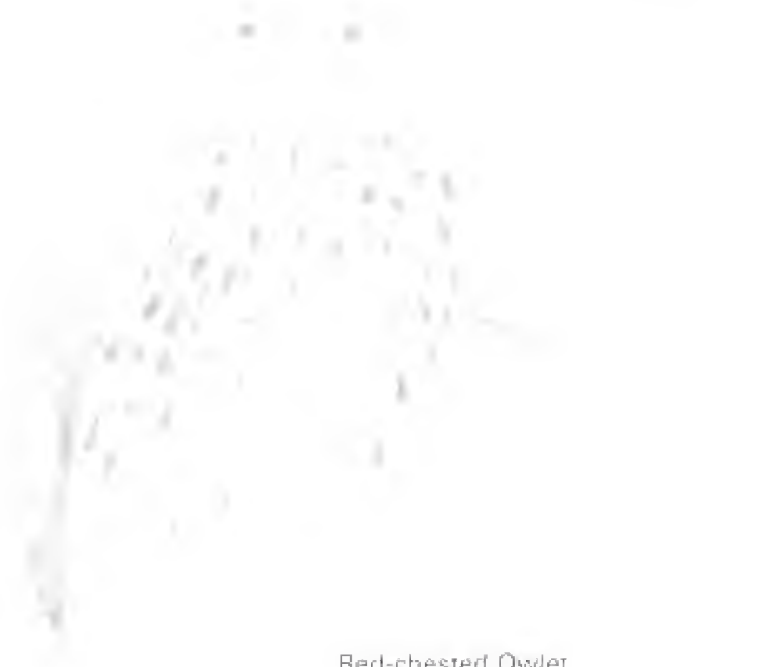

Red-chested Owlet

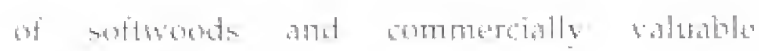
hatduguds.

Kakamega Forest is an anportand water

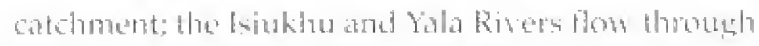

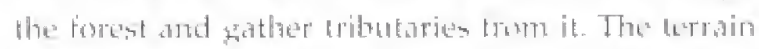

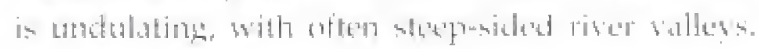

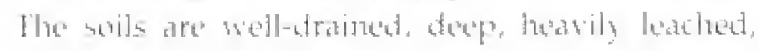

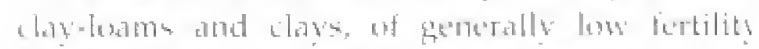

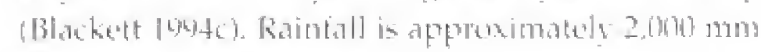

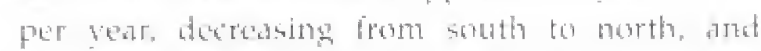
apparenty declining due the detorestation.

\section{Birds}

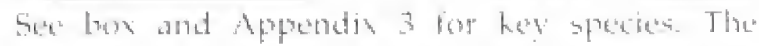

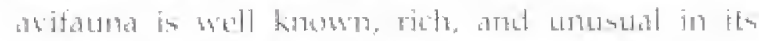

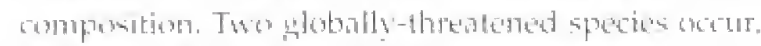

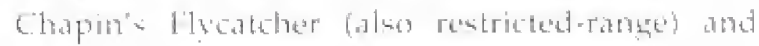

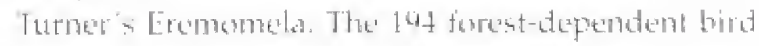

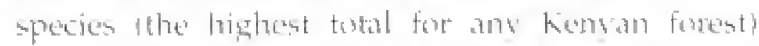

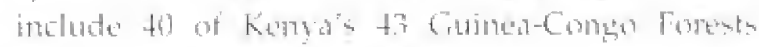

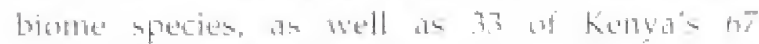

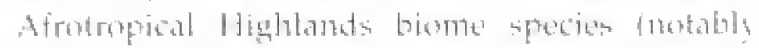

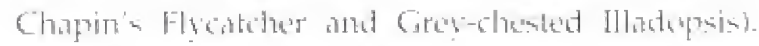

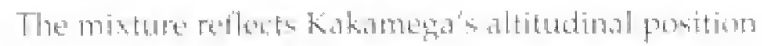
betwerem hos lond and montane borest.

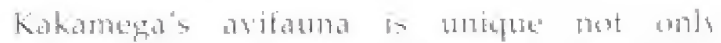
nationalls, but continentally sesteral specien have"

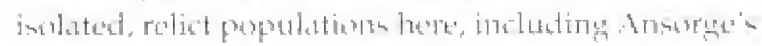

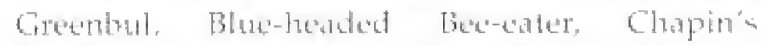
Flyoutcher and Turner's Eremomela. which are absent trom all it neats ald of the superticinlly similar midederation foresto in Lganda. Chapitis Plycatcher in a restricted-ranger species that

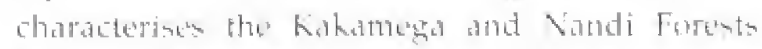

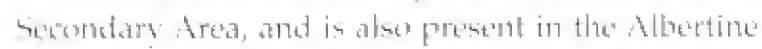

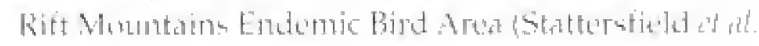

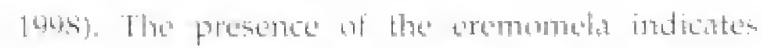
biogerestaphic links the the Eastern Laire Lowlands

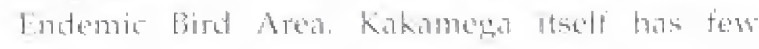
"ndemic taxa; among birds, there is an endertite

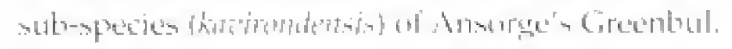


Important Bird Areas in Kenya

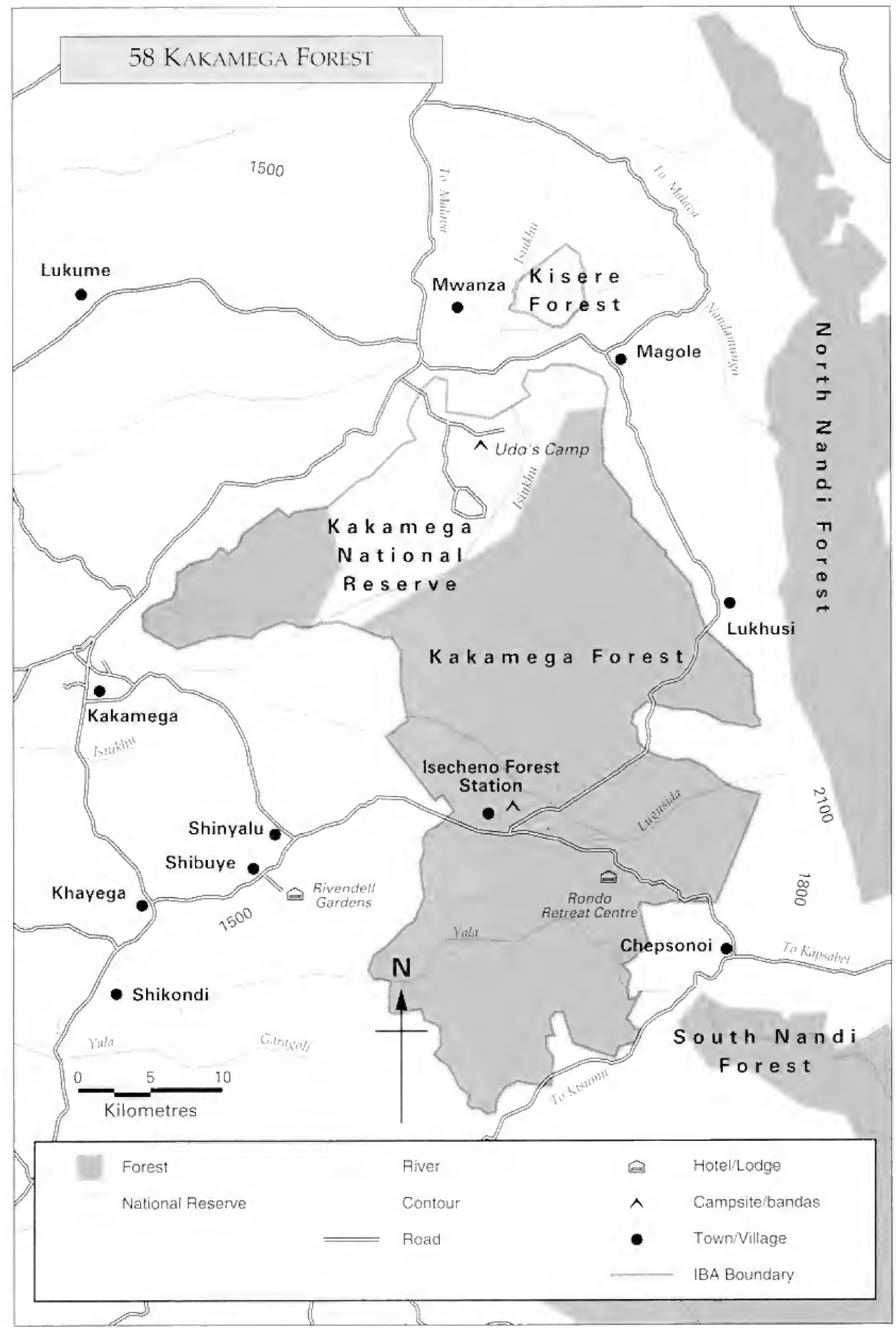




\section{Kamamega Forest}

Globally-threatened species

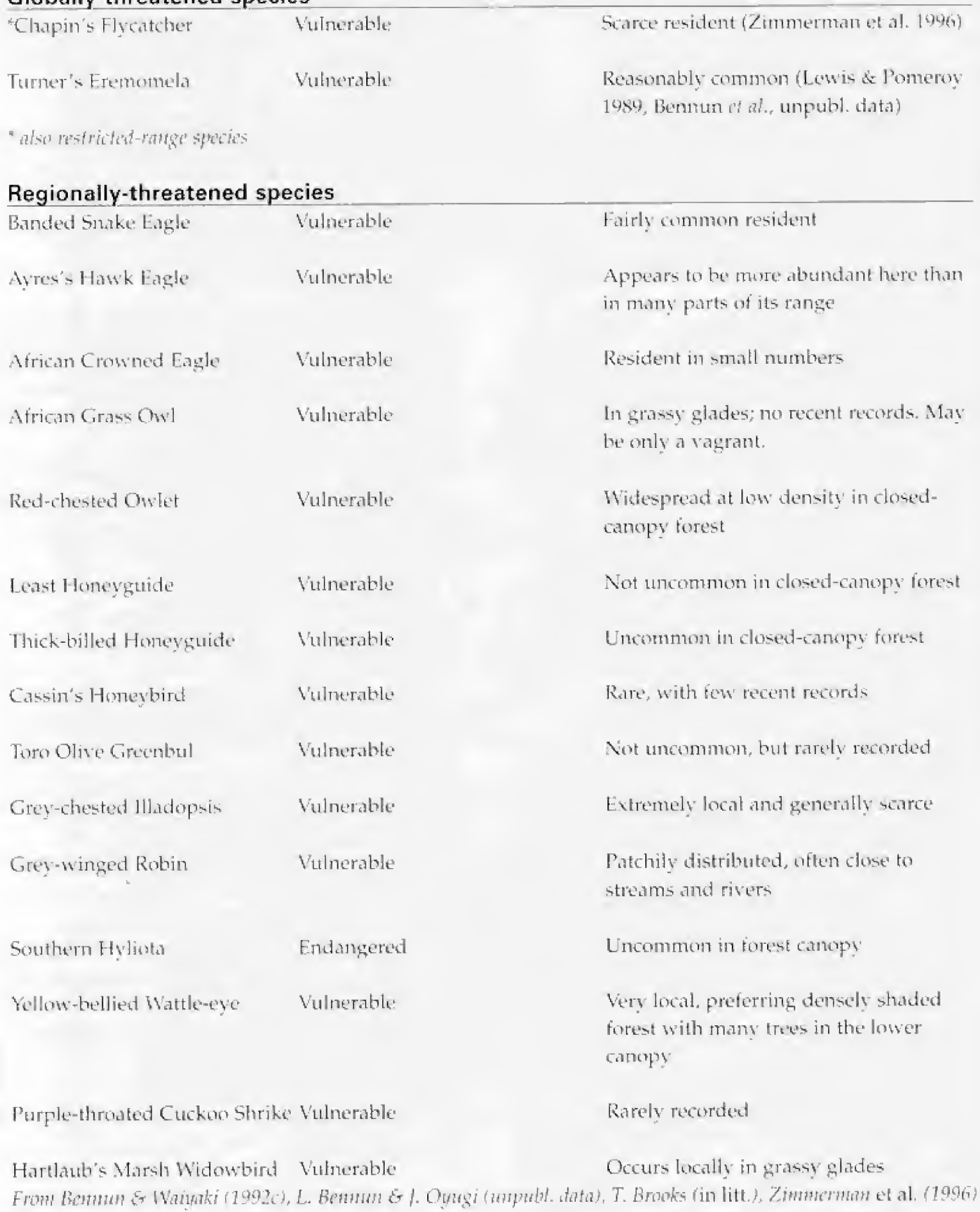

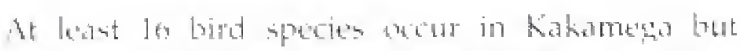

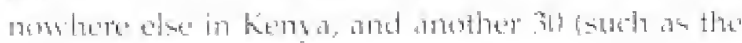

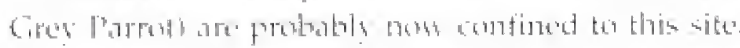

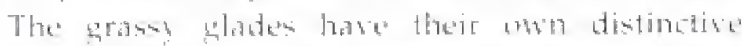

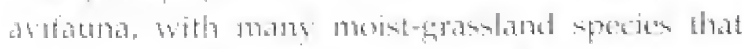

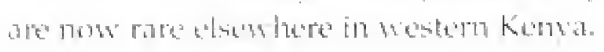

\section{Other wildlife}

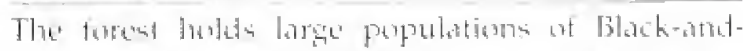

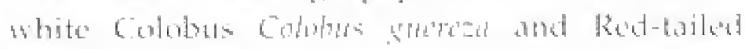

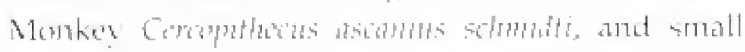

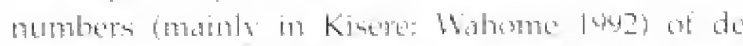

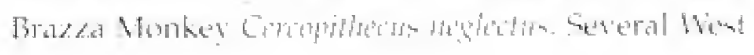




\section{Important Bird Areas in Kenya}

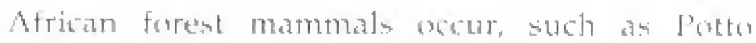

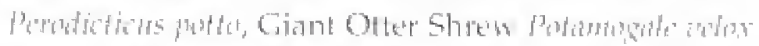

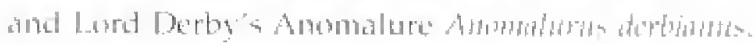
The simall mammal communits is aloo berty rich and shows stromes affinitios to the Zaire basm.

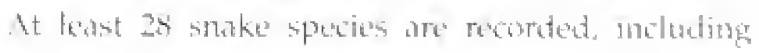

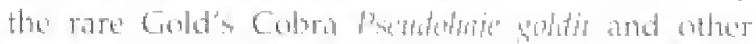

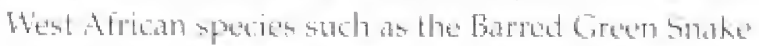

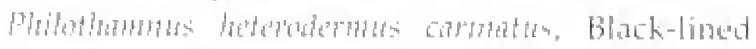

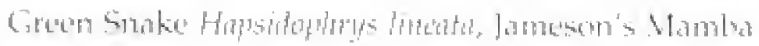

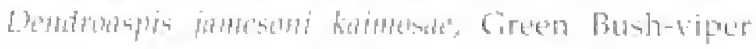

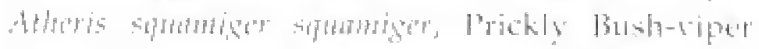

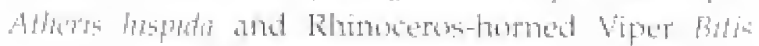
matomis apawls 1478). Tho notable and probubly.

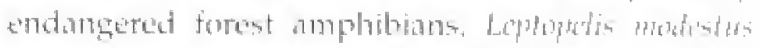

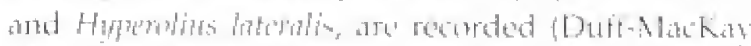

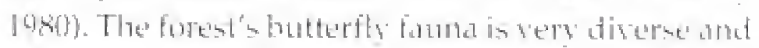
important, both regionally and continentally, around 330 species atre thought th wetur, meluding at hillat

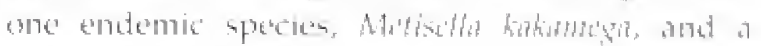

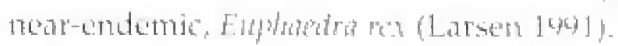

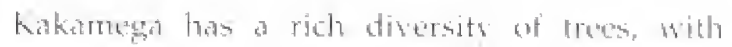

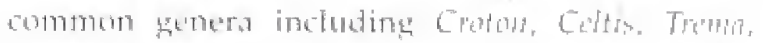

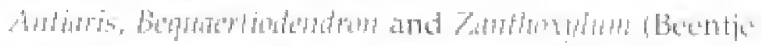

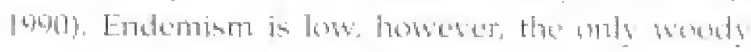

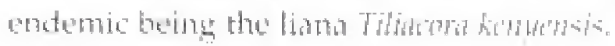

\section{Conservation issues}

Kakamega is a comples and tragmented forest, and

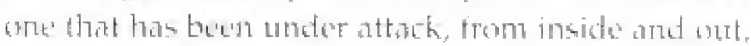

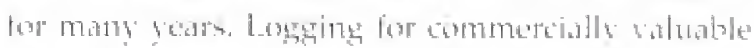

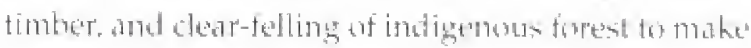
way for plantations, was externsise toneter the

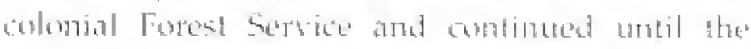
late 1ybuts. This began the process of isolationt

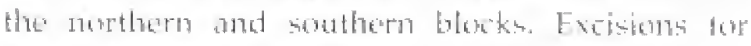
settlement, shorols and tea plantations lihe "Vyaro

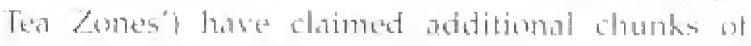

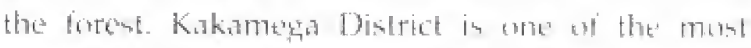

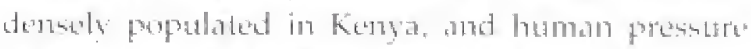

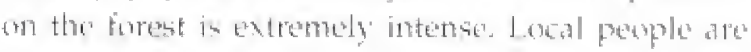
cstimated to derive prodtets worth KSh 10li nillion

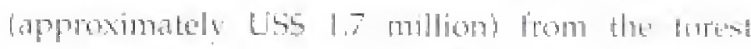
each year (Emerton 1994). Forest protection remains

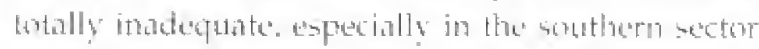

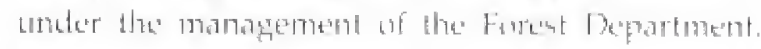

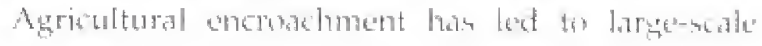

destruction [e.ge within hald Nalure Reserve' in

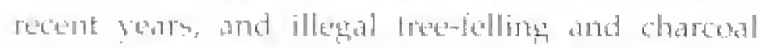
furning are rampent 69 yosi 1990 . L. Bernoum,

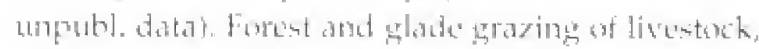

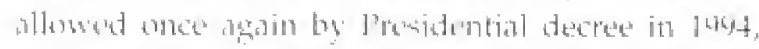

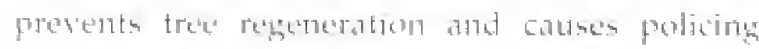
problems. Hunting tor bush-mat, debarking of artais trees for traticional mediane and firewost

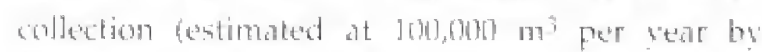

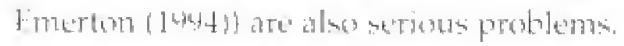

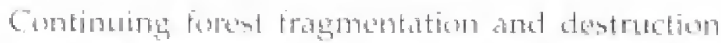

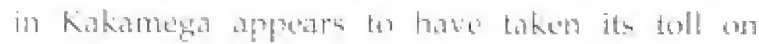

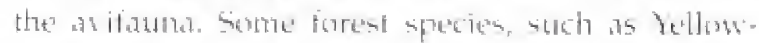

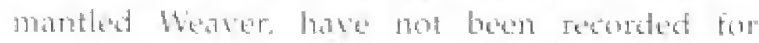

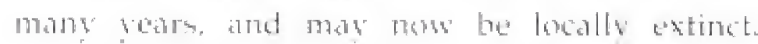
A nambers of montane forest binds that formerly

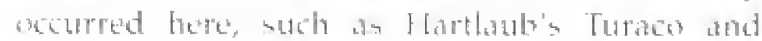

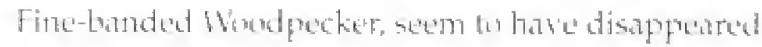
sine the sesering of forest annedions with the nearby, higher altitude North Nandi Forest (JBA 53 )

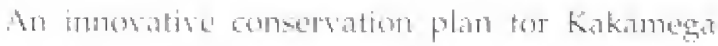

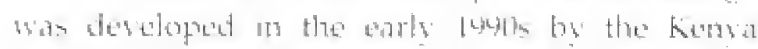

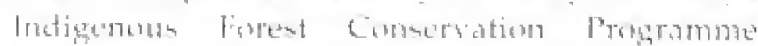

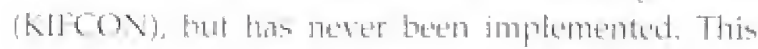

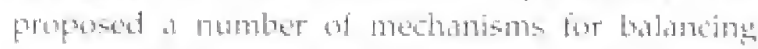
the reed of bichtiversity tomsersation and torest.

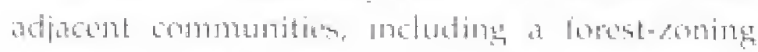
approach (than 1945). Theser ideas should be

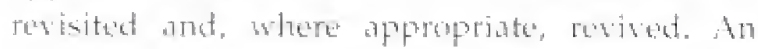
integral part of this plan was eco-loutist

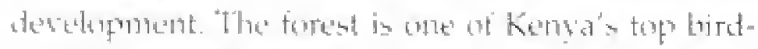

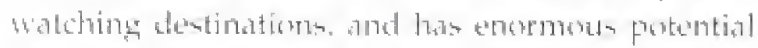

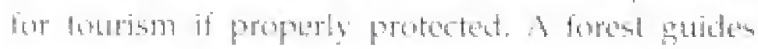

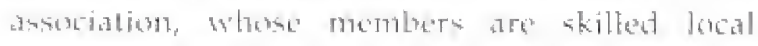

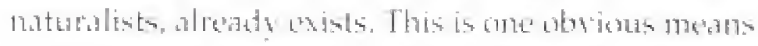

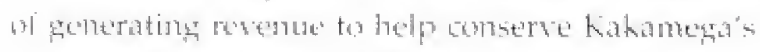

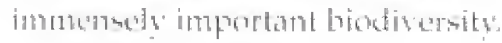

\section{Further reading}

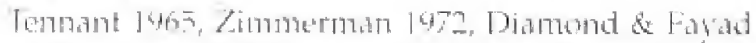

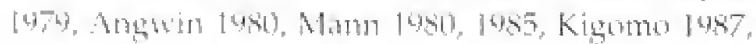

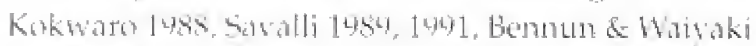

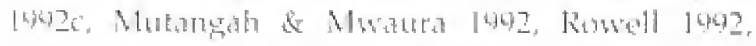

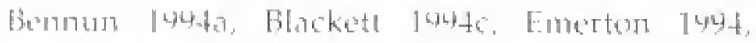
() 


\section{Mt Elgon}

$01^{\circ} 02^{\prime} \mathrm{N}, 34^{\circ} 48^{\prime} \mathrm{E}$, Westem Province, Mt Elgon District

and Rift Valley Province, Trans Nzoia District

c. 129,000 ha

Altitude: $2,100-4,280$ m

5tatus: National Park (16,900 ha). Forest Reserve (73,000 ha)

and umprotected fexact area unknown, c. 17,000 ha of moorland and c, 31,000 ha of

wooded grassland)

Categories: Globally-threatened species, restricted-range species,

Afrotropical Hightands biome species

\section{Site description}

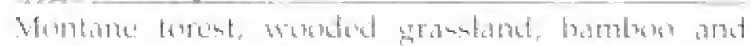

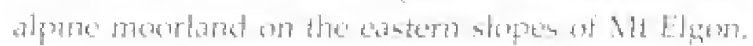

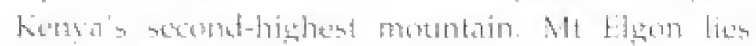

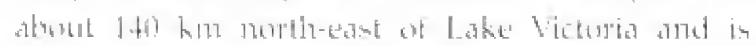

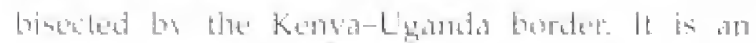

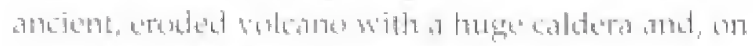

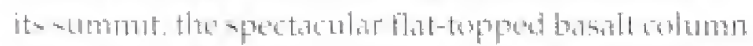

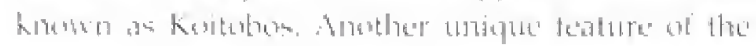

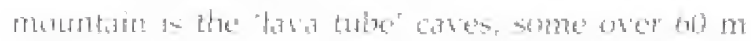

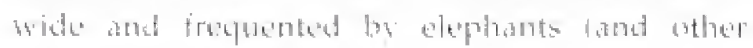

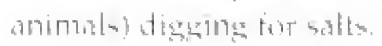

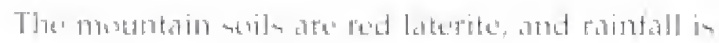

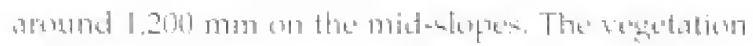

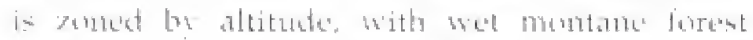

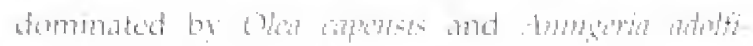

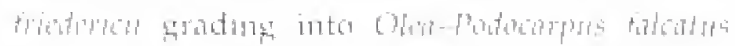

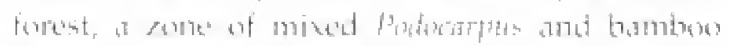

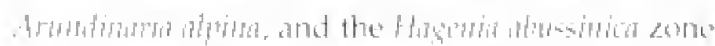

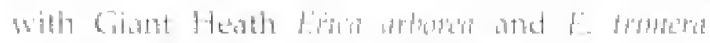

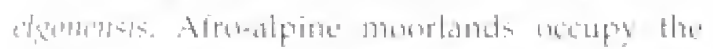

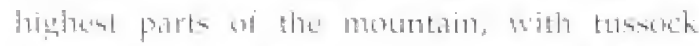

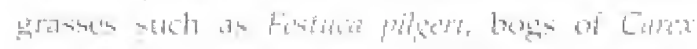

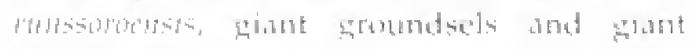

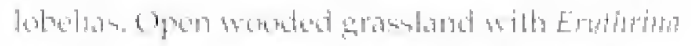

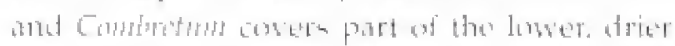

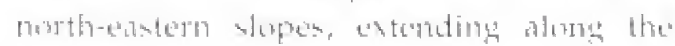

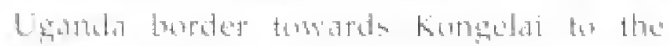
nowtherat.

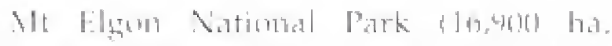

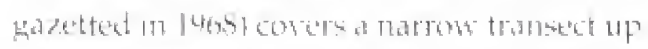

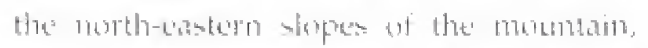

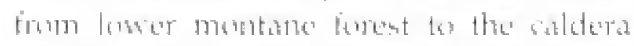

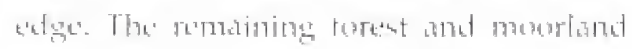

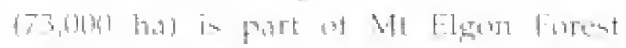

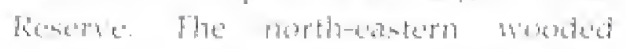

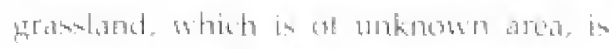
unfrotected. The Limandan side of the

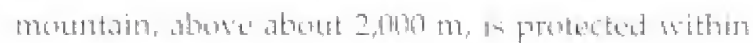

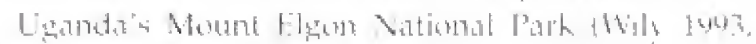

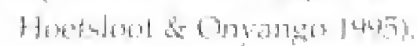

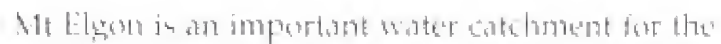

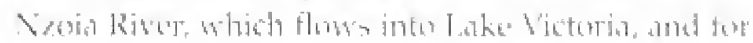

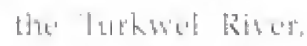
which flanes into I.ake Turkathes $(113+24\}$.

Stender bulled Starling 


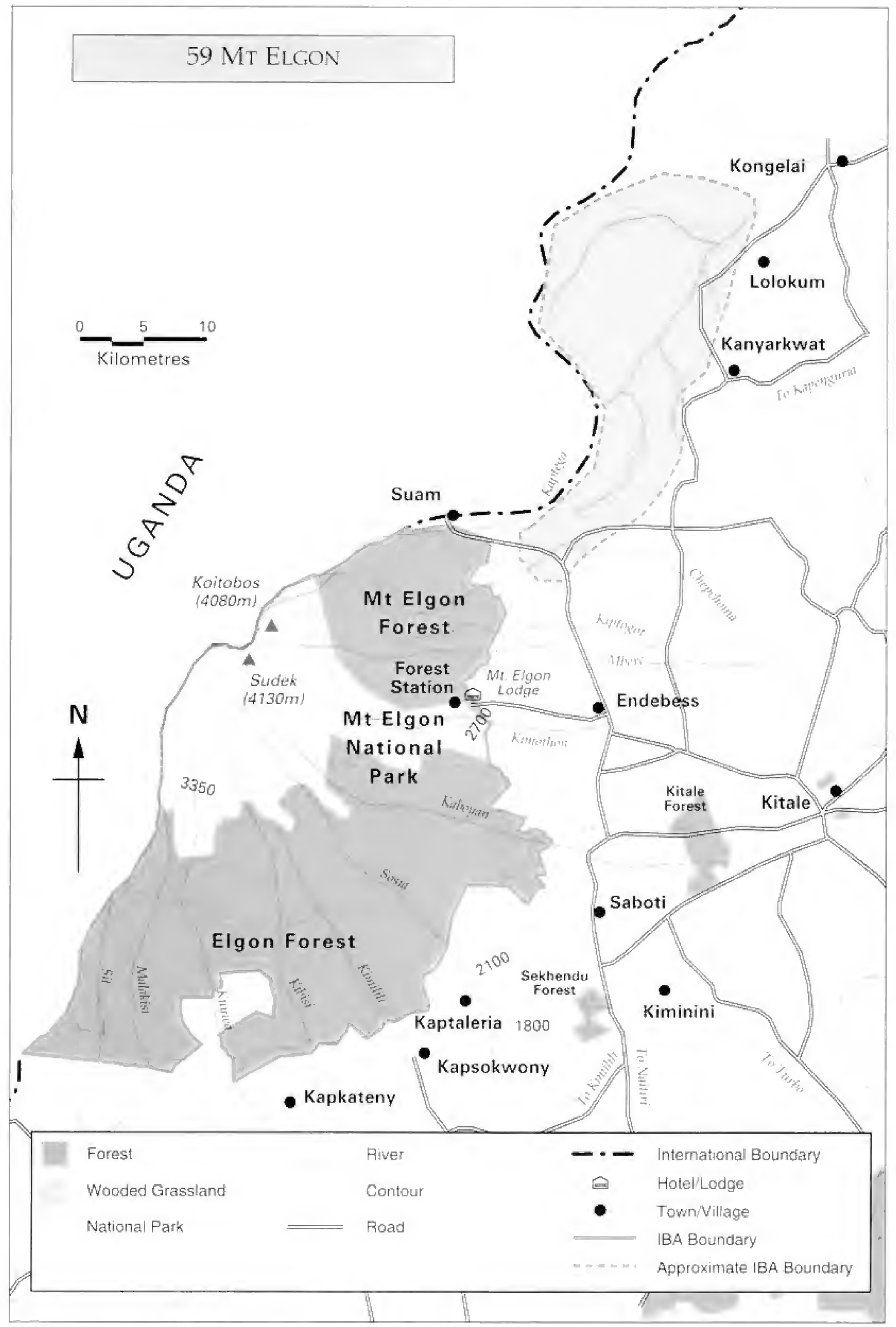




\section{$59 \mathrm{Mt}$ Elgon}

\section{Globally-threatened species}

\begin{tabular}{|c|c|c|}
\hline "Sharpe" = Longedam & $\begin{array}{l}\text { Prowisionally vulnerable } \\
\text { (bentrun \& Njoroge } 1496 \text { ) }\end{array}$ & 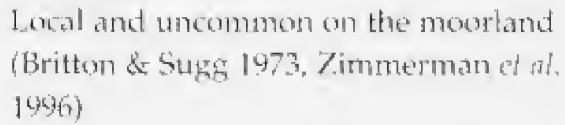 \\
\hline
\end{tabular}

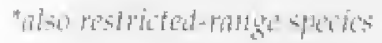

\section{Regionally-threatened species}

\begin{tabular}{|c|c|c|}
\hline Lammeterefer & Endangered & l'robahly nest on the high eraysi \\
\hline African Crowned Fagle & Vulnerabte & Resident in small numbers \\
\hline Ring-necked Francolin & Vulneratole: & 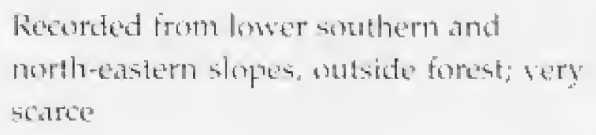 \\
\hline Striped Flufftail & Vulnerabls & Scarce resident of moorland \\
\hline Cape Eaglu Owl & Vulnerable & Nests on hight mororland crages \\
\hline Redtchested Oulet & Vulnerable & Unemmonon in clased-canopy forest \\
\hline There-billed Honeyguide & Vulnerable & Uncommon in closed-canopy forest \\
\hline Toro Oliv't Greenbul & Vulnerable & $\begin{array}{l}\text { Formerly ocurred in the fourer forest: } \\
\text { may be extinct }\end{array}$ \\
\hline Gridrchested llladopsis & Vulnerable & $\begin{array}{l}\text { Formerly accurred in the lower forest: } \\
\text { may be extinct }\end{array}$ \\
\hline Gref-winged Robin & Vulnerable & Uncommon at the fringes of lower forest \\
\hline \multicolumn{2}{|c|}{ Purple-throated Cuckoo-shrike Vulnerablo } & Lncommon in closed-canopy forest \\
\hline
\end{tabular}

\section{Other restricted-range species}

Hunter"s Cisticoh?

Jackson's Framoblin
Common abore $2,500 \mathrm{~m}$, below which it is replaced by Chubb's Cisticola (Britton \& sugis 1973, Britton 1980)

Status uneertain; one sight record (Zimmerman ant al. 1996)

\section{Birds}

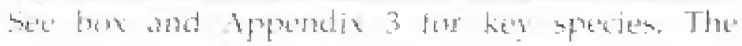

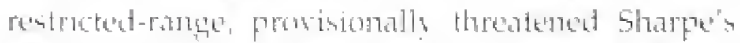

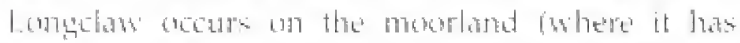

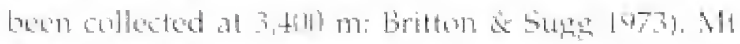

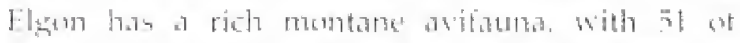

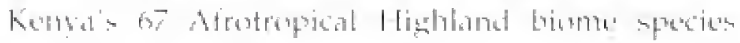

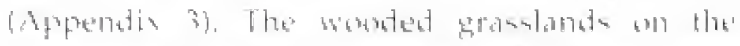

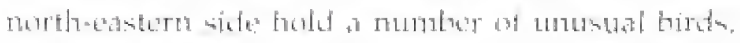

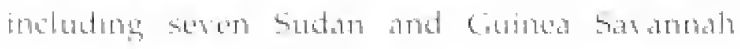

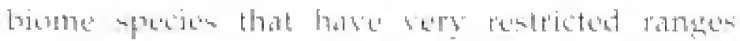

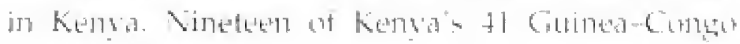

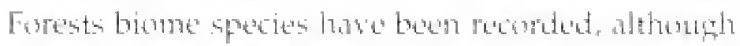

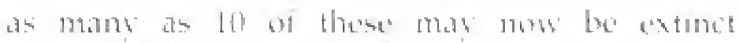

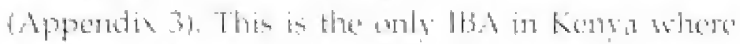

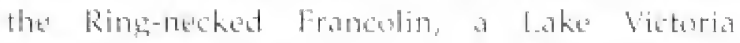

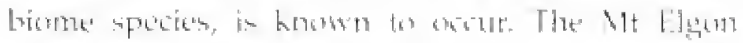

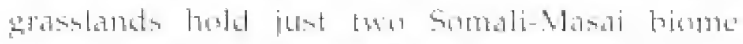

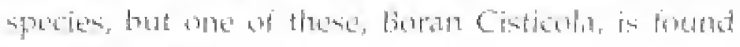
int monthersite 


\section{Important Bird Areas in Kenya}

\section{Other wildlife}

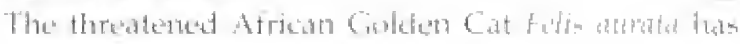

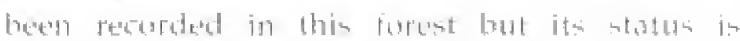

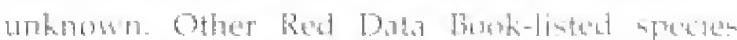

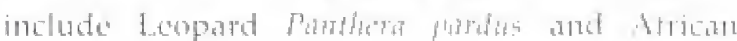

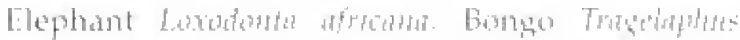

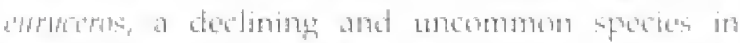

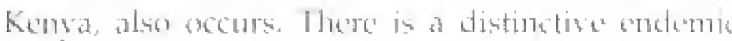

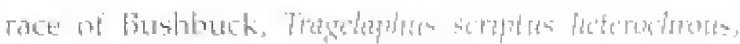
and several endemic smatl manmonts, including the"

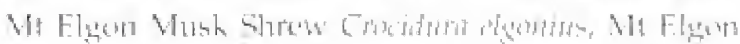

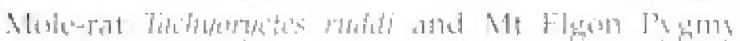

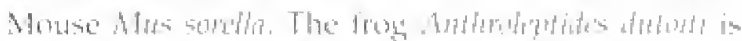

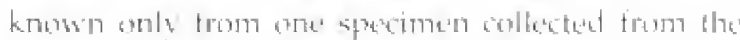

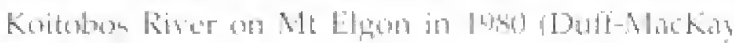
I내니․

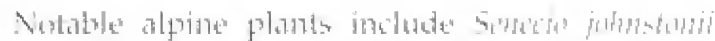

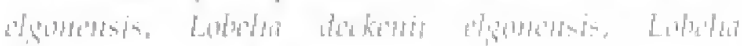

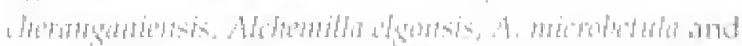

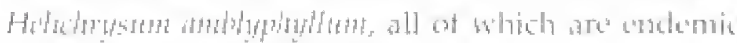
ar near-trultaric to Alt Elyon.

\section{Conservation issues}

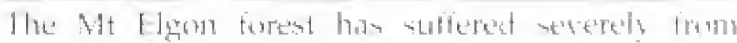

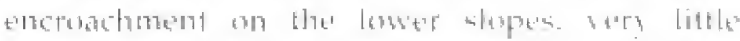

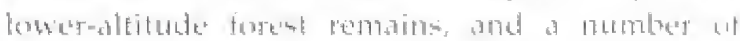

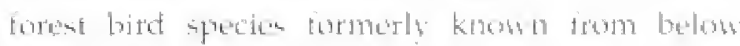

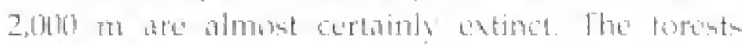

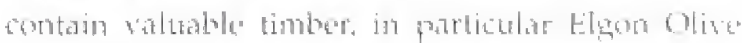

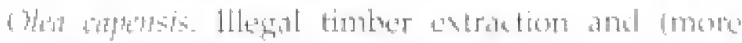

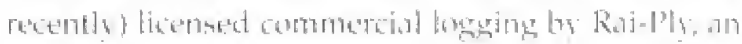

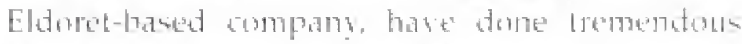

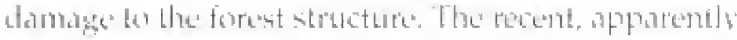

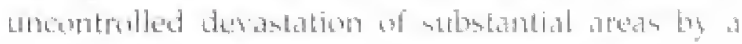

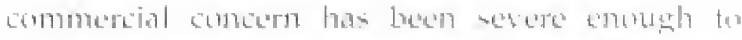
spork peotests and demumatrations by thome lising around the toreat.

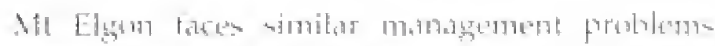

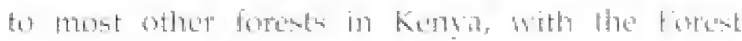

Department finding dilicultics in controlling

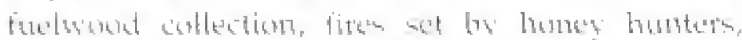

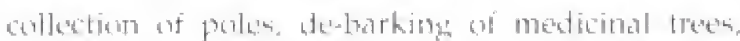

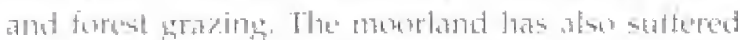

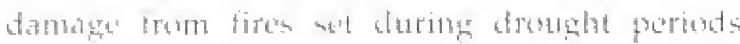

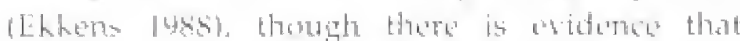
sonke of the veretation communities there sas

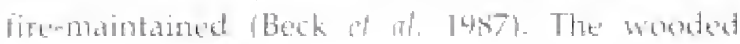

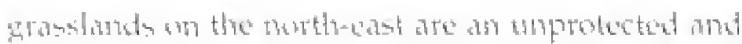

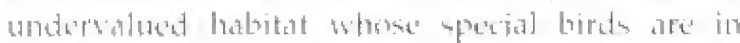

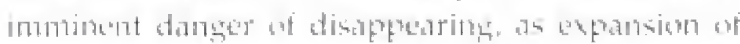

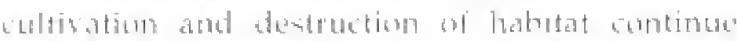

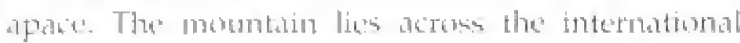

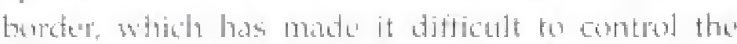

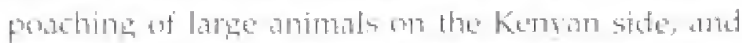

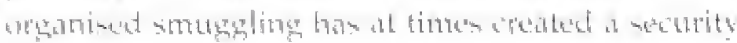

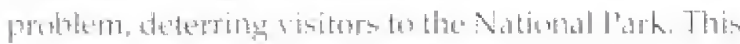

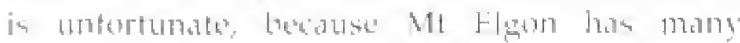

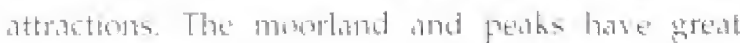

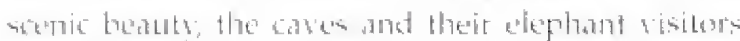

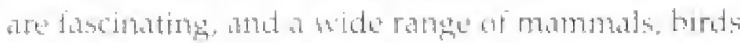

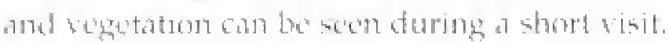

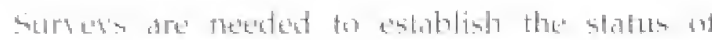

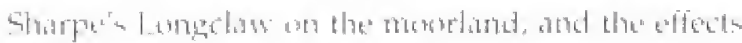

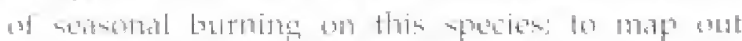

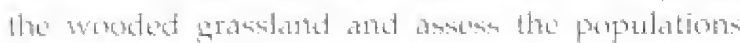

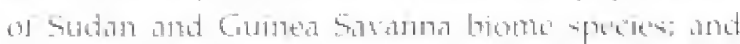

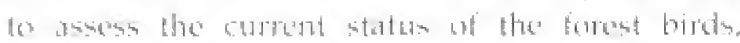

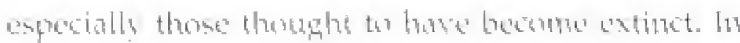
the mesntime, commercisl heseing in the forst varuld ceast. An integrated management plan for

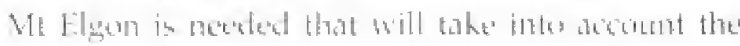

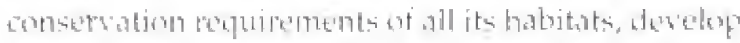

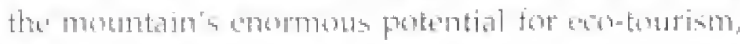

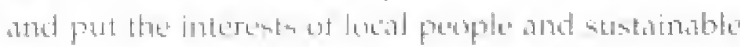

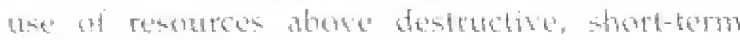
exploitation.

\section{Further reading}

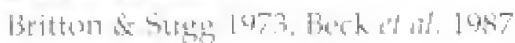




\section{Sio Port Swamp}

$00^{\circ} 4^{\prime} \mathrm{N}, 34^{\circ} 51^{\prime} \mathrm{E}$, Western Proxince, Busia District

c. 400 ha

Altitude: $1,130 \mathrm{~m}$

Statts: Uimprotected

Categories: Globally-threatened species.

Lake Victoria Basin biome species

\section{Site description}

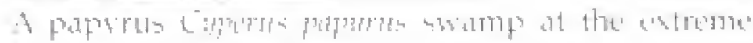

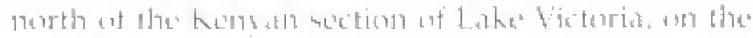

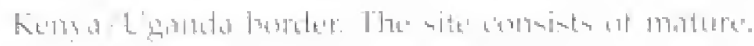

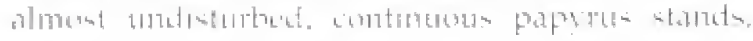

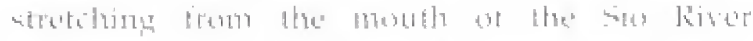

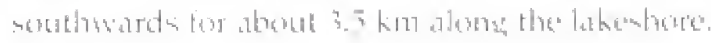

\section{Birds}

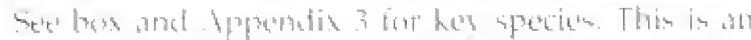

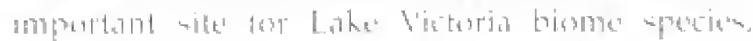

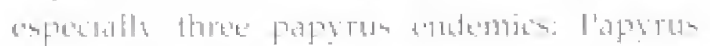

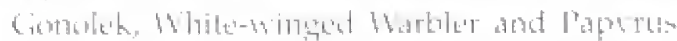

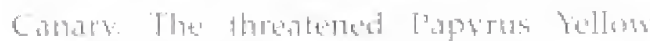

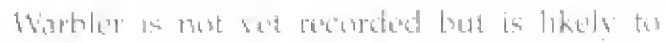
14.

\section{Other wildlife}

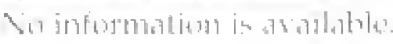

\section{Conservation issues}

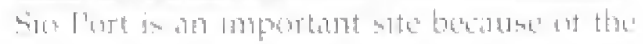

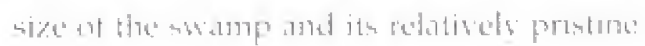

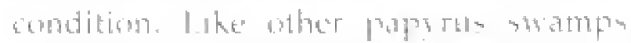

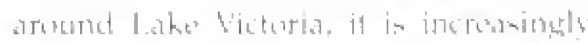

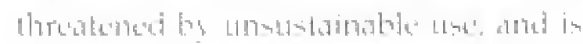

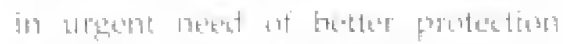

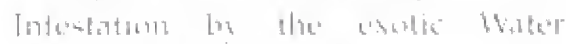

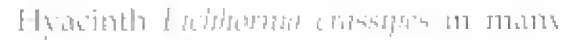

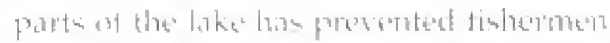

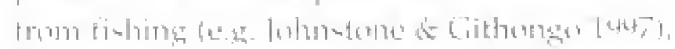

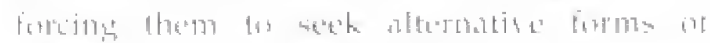

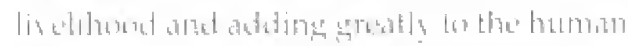

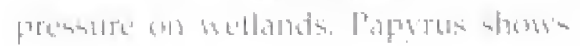

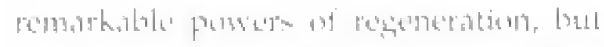

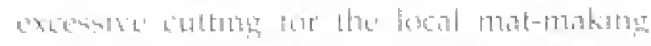

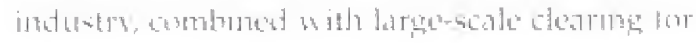

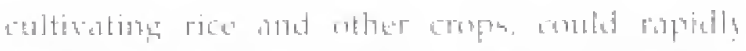

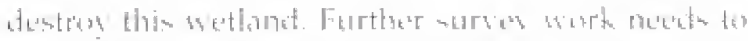

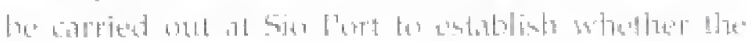

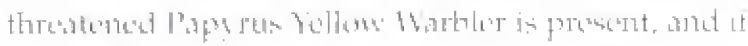

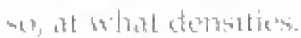

\section{Further reading}

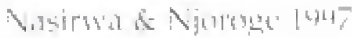

\section{Globally-threatened species}

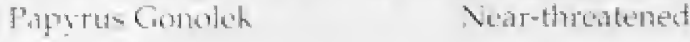

Vuluerable
Relatively commom (Natirat \& Njoroge 1997 
Important Bird Areas in Kenya

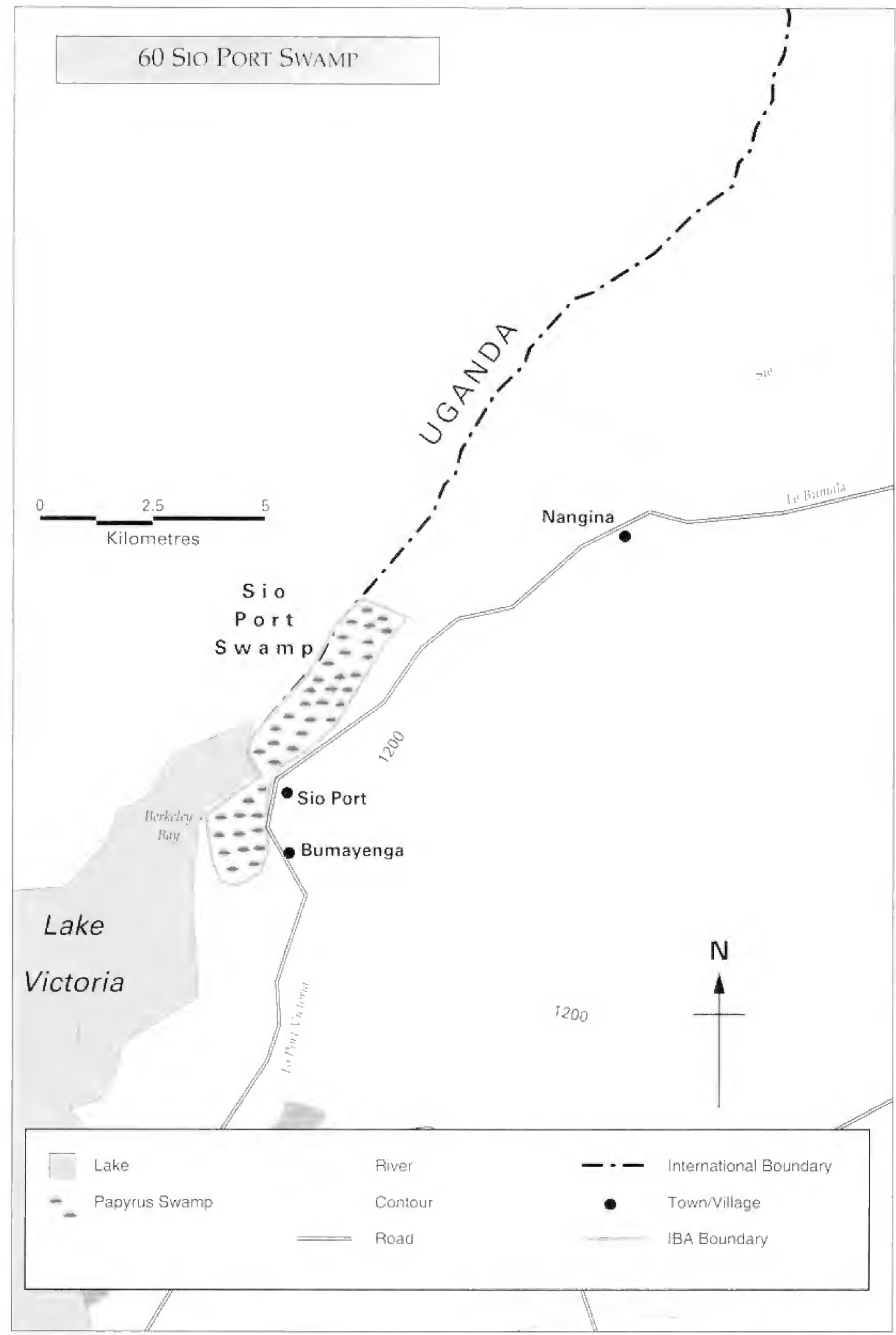




\subsection{Potential IBAs}

At least five sites in Kenya are thought likely to qualify as Important Bird Areas,

but for a variety of reasons have not been included in the main inventory. For the time being they are classed as potential IBAs because of lack of sufficient reliable information on their avitanth, or because the taxonomic status of key species is unresolved.

These (and other) sites may be adted to the list of Kenya's IBAs when more information becomes available.

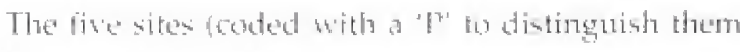
Erom the bol listed (B, As) are:

Pl 13uni and Dondari Fencoste

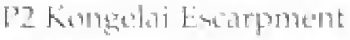

P3 Mathamari Mational Park.

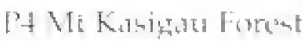

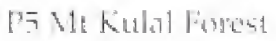

\section{P'1 Boni and Dodori Forests}

$01^{\circ}+3^{x} 5,41^{\circ} 10^{\prime} \mathrm{E}$, Const Prominces, Lamu Diatrict

249,600 ha (extent of forest miknown?

$$
\text { Atitude: (1)-\$0 Tा }
$$

Status: National Reserte $(221,600)$ ha) anct

proposed, angalytted Forest Reserie (28.000 ha)

Potenthat catories: Clobally-thratened species, East African Contat biome spectien

\section{Site description}

Boni and Doderi lie wh the extreme north-east cont

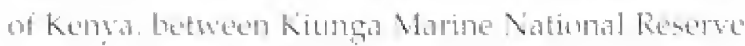

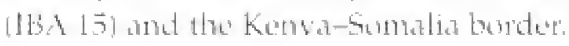

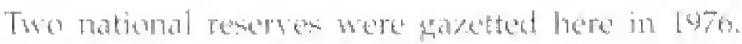
Boni Dational Remerse (133, solu ha) abuts the

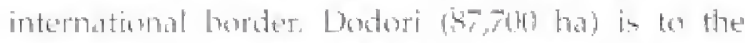

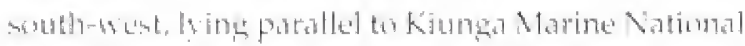

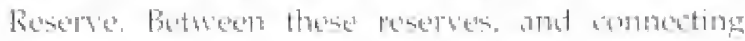

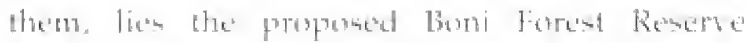

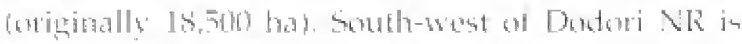

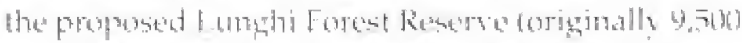

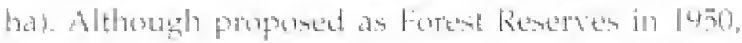

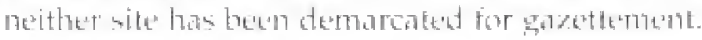

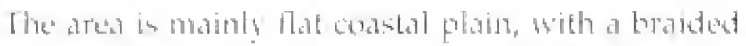

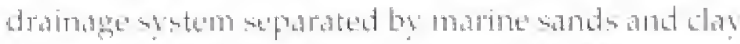

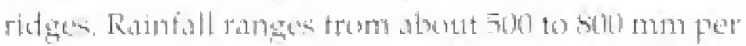

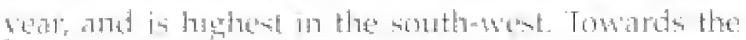

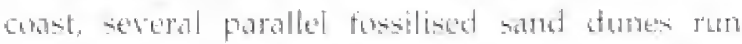

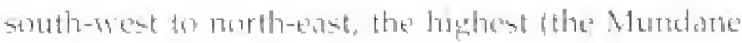

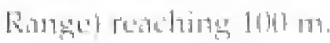

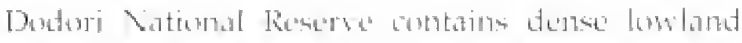

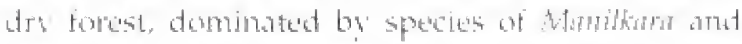

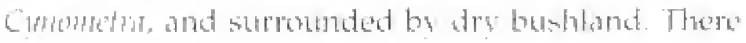

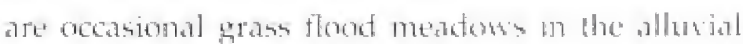

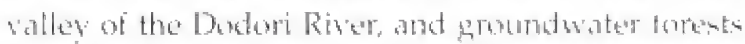

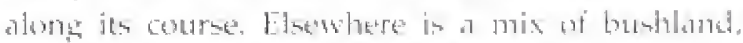

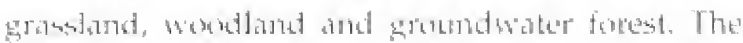

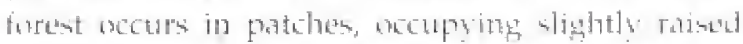

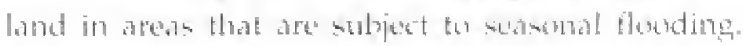

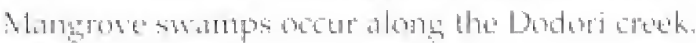

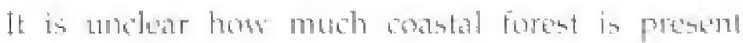

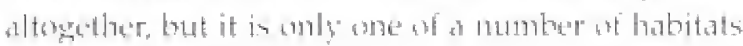
in these restatist

\section{Birds}

Very little is know atrut the birds in these forentor.

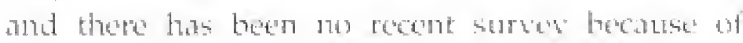
security concerns. As thamples wt Fast African

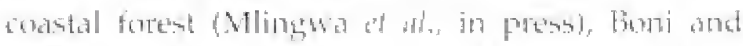

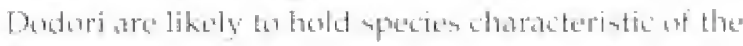
East African Cost biomes poraibly ineluding globally-threnterned species such as Sokuke T'ipit. The Cheyer Expodilion in the darly 1970s (Ancho, 1973) recorded the restricted-rathe Fisther" Turaco,

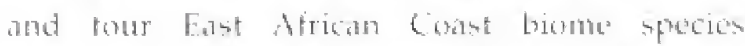

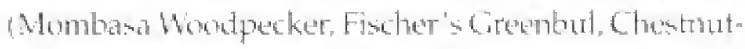

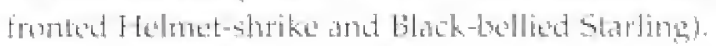

\section{Other wildilife}

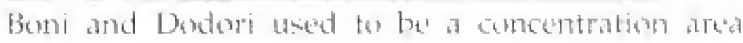

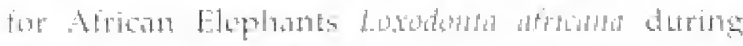
the dis sedsin. Elephants are nom protobly extimpated by porching. Notalyle trean 117 the

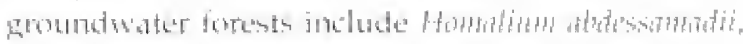

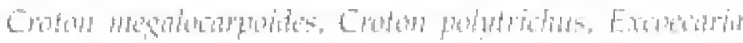

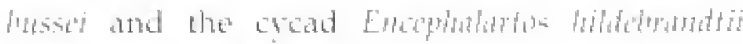

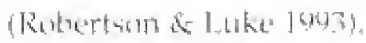

\section{Conservation issues}

The National Reserses are under the puriatiction of Lamu Country Council but it is unclear what

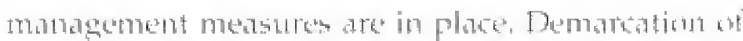

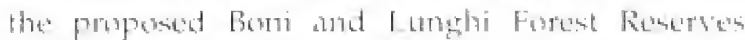

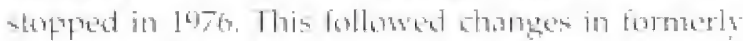

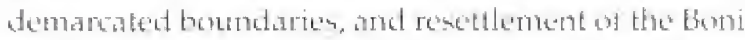

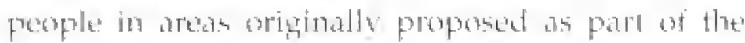

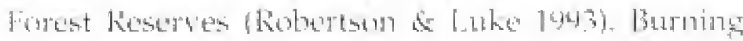




\section{Important Bird Areas in Kenya}

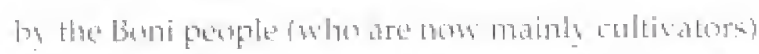

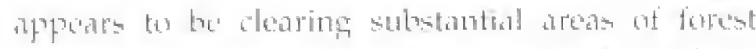

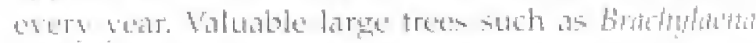

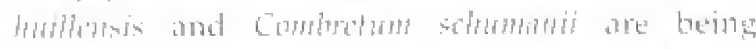
extracted for the carsing industry funthere south on

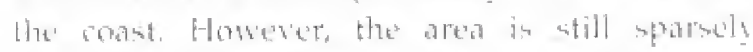

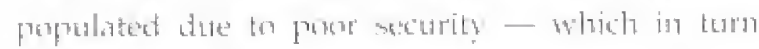

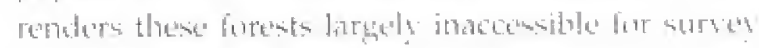

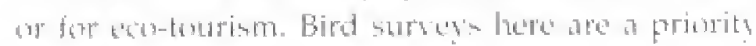
when the situation allows.

\section{Further reading}

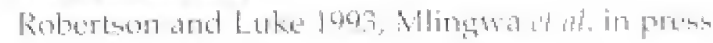

\section{P2 Kongelai Escarpment}

\author{
$01^{\prime \prime} 18^{\prime} \mathrm{N}, 35^{\circ} 04^{\prime} \mathrm{E}$, Rift Walley Prowince. West l'okod \\ District \\ Area undefined \\ Altitude: $1,360-2,100 \mathrm{~m}$ \\ Status: Unprobeted \\ Potential calcerories. Sudan and Guinea Savamiath \\ bieme species
}

\section{Site description}

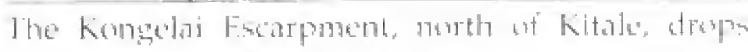

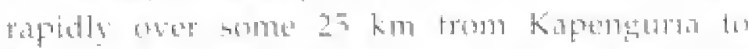

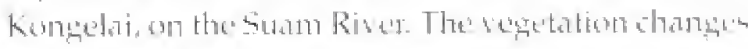

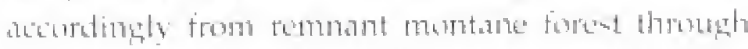

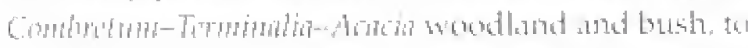

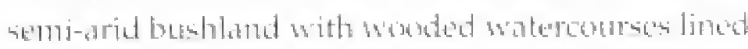
with alethat trats.

\section{Birds}

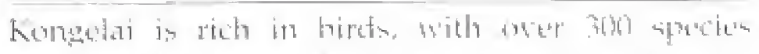

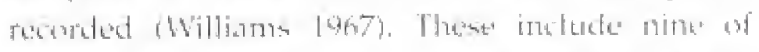

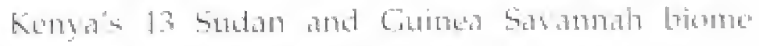

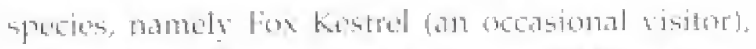

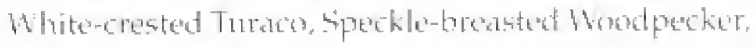

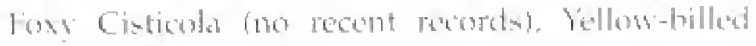

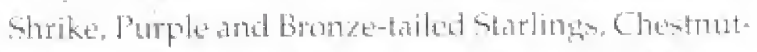

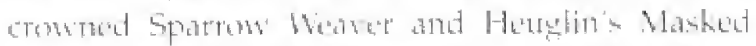

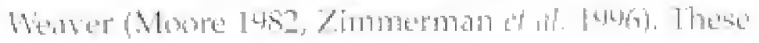

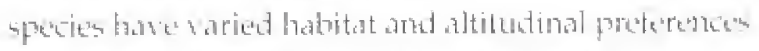
and are rot necessalily to be found wegether.

\section{Conservation issues}

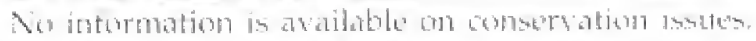

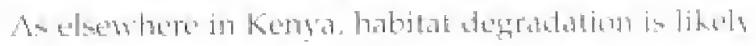

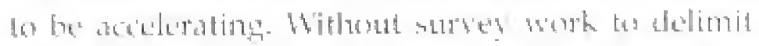
hotritats and bied stintributions, it is impossible tor

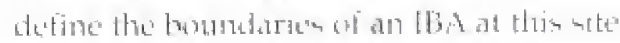

\section{P3 Malkamari National Park}

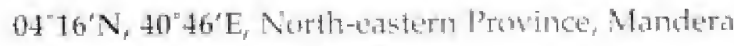 \\ District \\ 87,600 ha \\ Altitute; $360-770$ m \\ Stafus. Natiomal Park \\ Potential categories: Restricted-range spectes. \\ Somali-Masai biome species
}

\section{Site description}

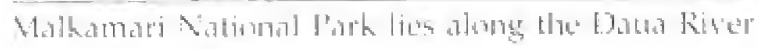

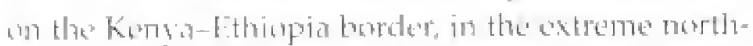

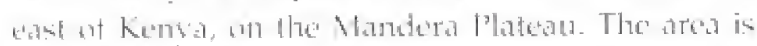

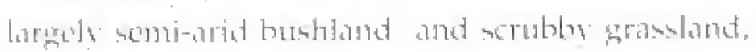

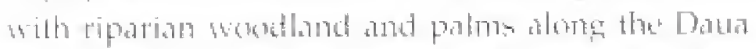

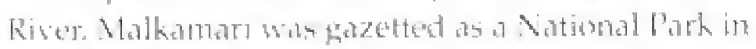

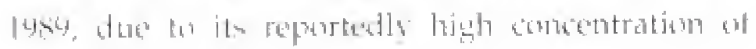
wildite.

\section{Birds}

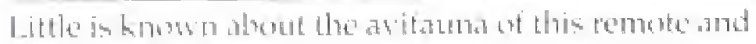

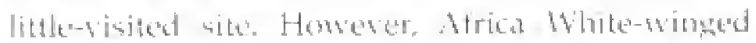
Done and labat Wearer. twa spectes of the fubta and

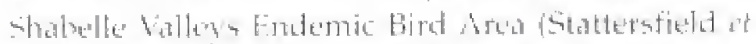

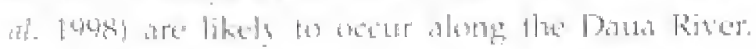

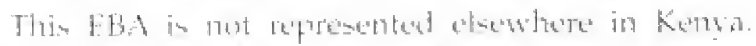

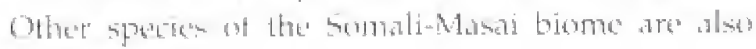
likely wo chetur.

\section{Other wildlife}

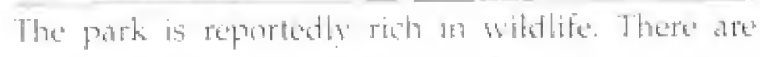
uncubstintiated records of African Elephant

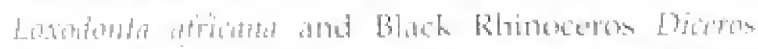

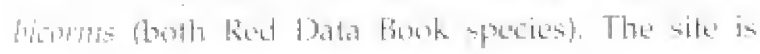

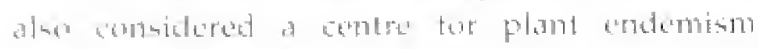
(VBL 1402!)

\section{Conservation issues}

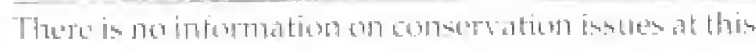
nite".

Chestrut-throated Apalis 


\subsection{Potential IBAs}

\section{P4 Mt Kasigau Forest}

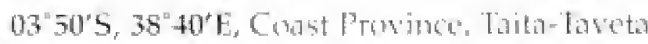

District

c. $230 \mathrm{hl}$

Altitite: $1,250-1,6+1) \mathrm{m}$

Status: Firest Reserve

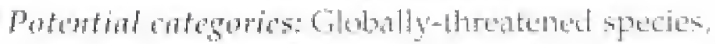
resilricted-range apecies

\section{Site description}

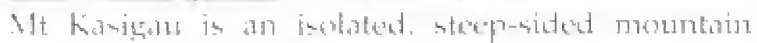

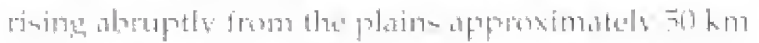

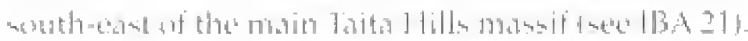

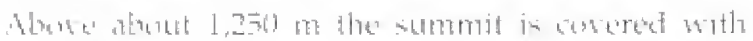

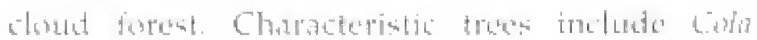

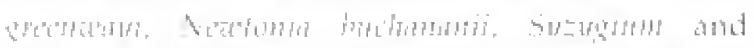

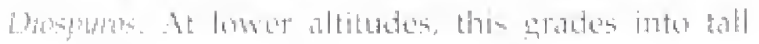

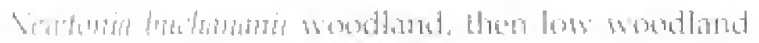

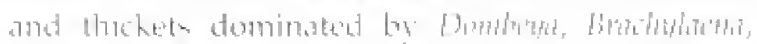

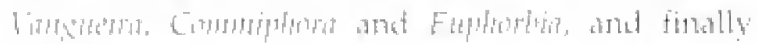

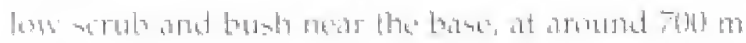

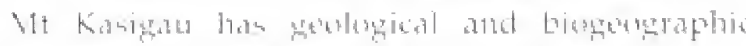

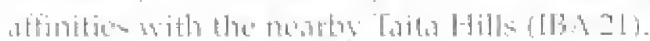

\section{Birds}

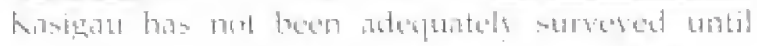

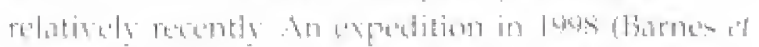

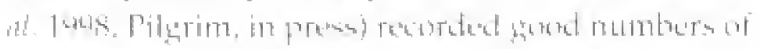

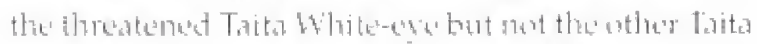

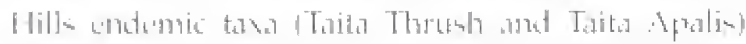

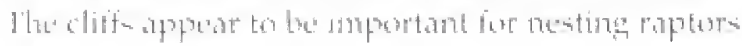

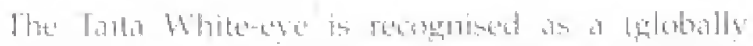

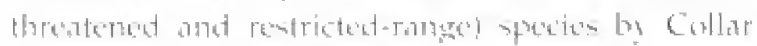

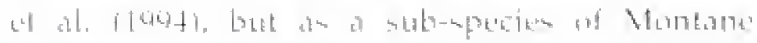

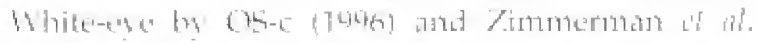

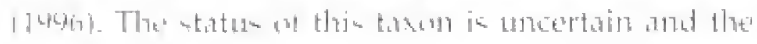

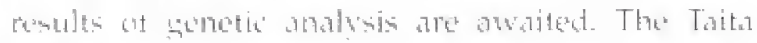

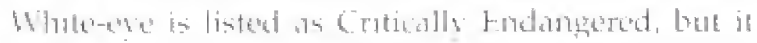

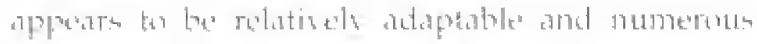

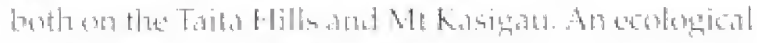

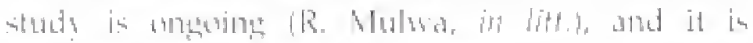

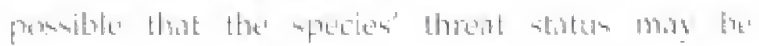

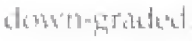

\section{Other wildlife}

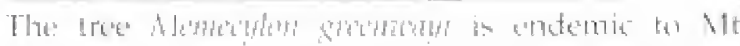

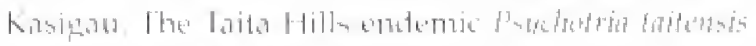

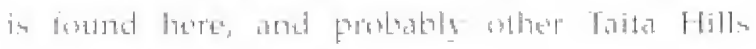
uperialitiens alson chocur.

\section{Conservation issues}

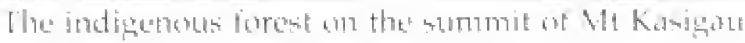
ss relatively undisturbed (Tilygrim, in prosed, thanks

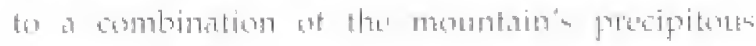

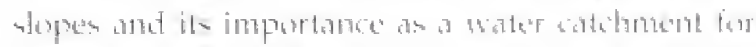

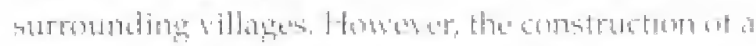

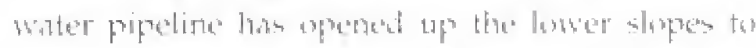

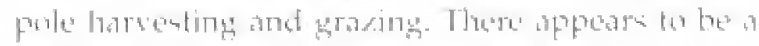

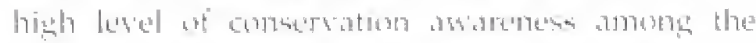

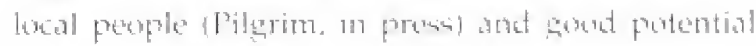

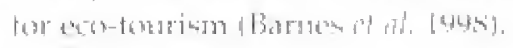

\section{Further reading}

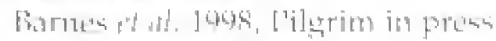

\section{P5 Mt Kulal Forest}

\author{
$02^{\circ} 40^{\prime} \mathrm{N}, 36^{\circ} 50^{\prime} \mathrm{E}$, Eastern l'ourince, Marsatit \\ District
}

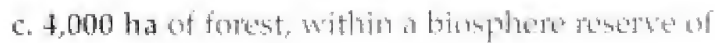
$700,000)$ hat

Altitute: 1, son - C. $2,40(1) \mathrm{m}$

Status: Bitoghlyere Reserve

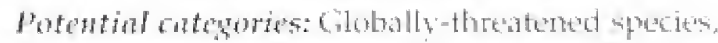
restricted-ratige spectess

\section{Site description}

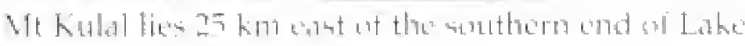

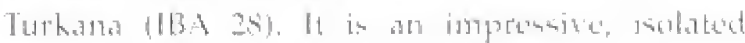
solcanic mountain rising abruptly from the semidesert plain, with a substantal central crater and

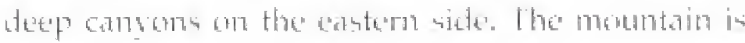

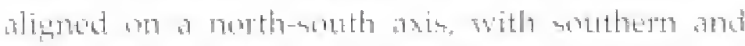

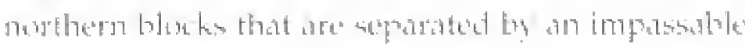

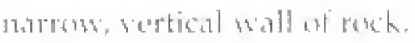

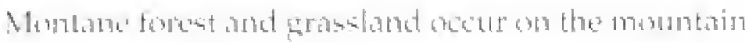

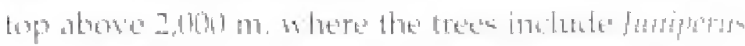

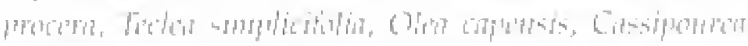

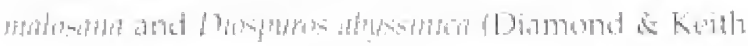

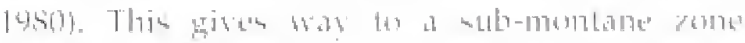

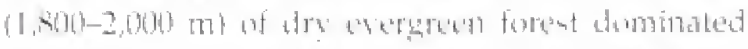

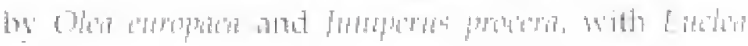

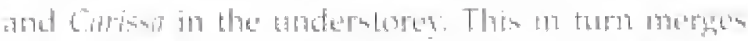

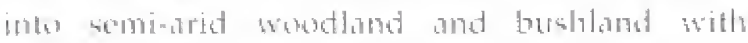

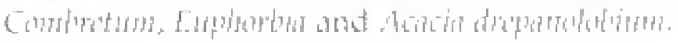

\section{Birds}

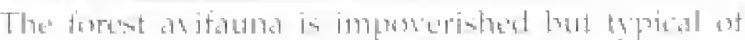

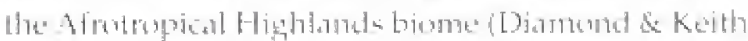

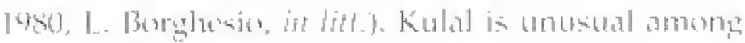

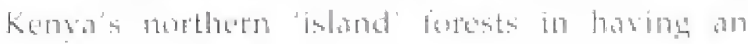




\section{Important Bird Areas in Kenya}

endeme herat tax

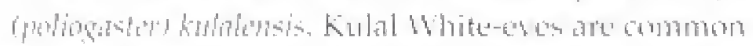

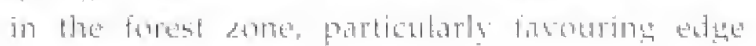
habitats (L. Borghesias, tonputal, datat. This is

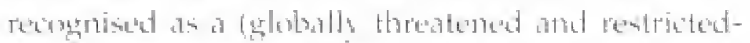

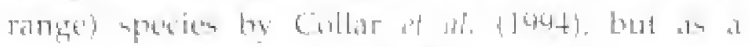

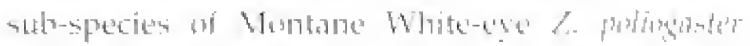

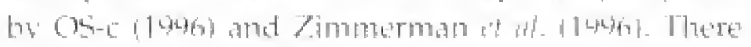

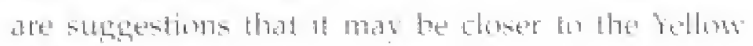

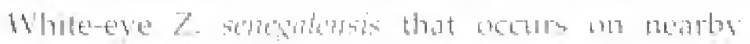

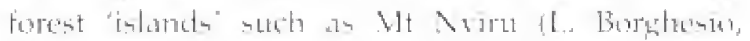

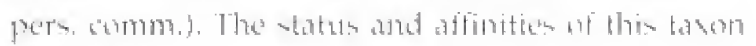

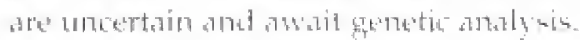

\section{Other wild life}

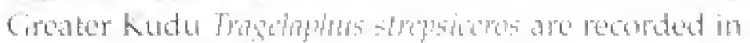

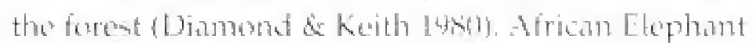

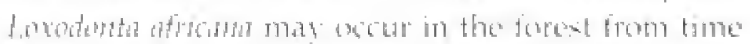

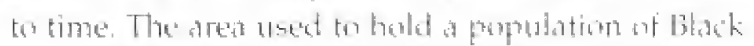

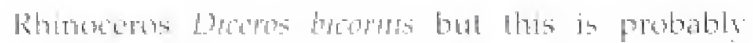

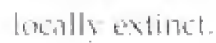

\section{Conservation issues}

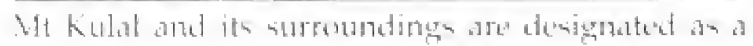

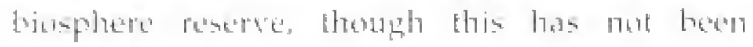

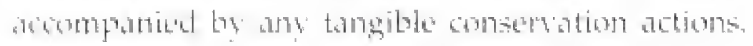
The forrest is under moderate pressure from lizestock

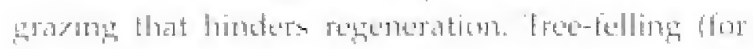

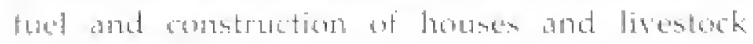

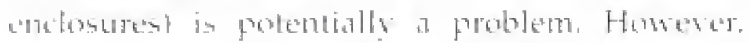

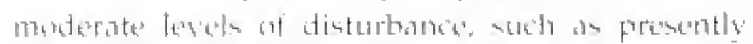

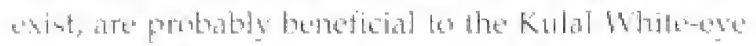

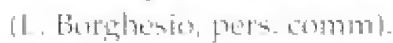

\section{Further reading}

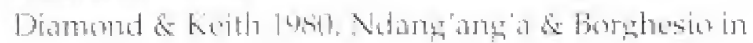
press
Scarlet-tuted Malactrite Sunbird 


\section{REFERENCES}

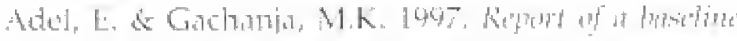

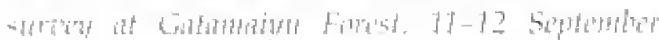

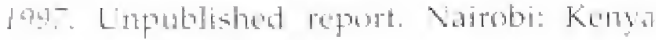

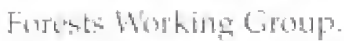

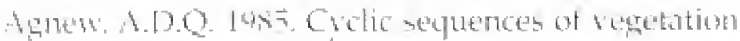

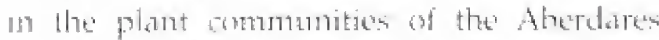

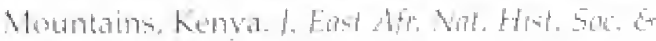
Nit? Mus $75,176,183$

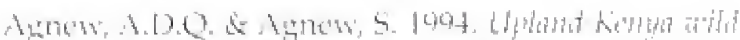

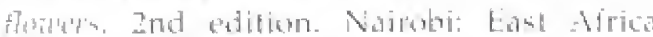
Vitual Hintery Societs:

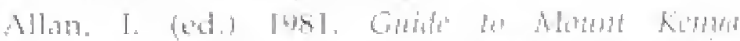

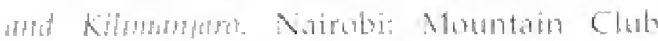
of kempor.

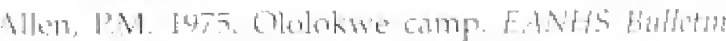
1낙: 4

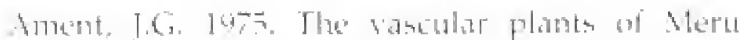

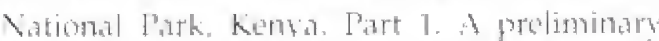

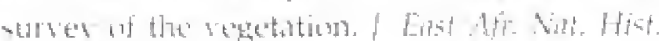
40.: 179: 15: 1-10.

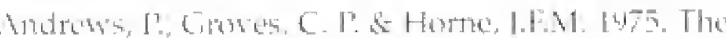

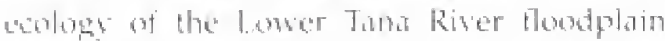

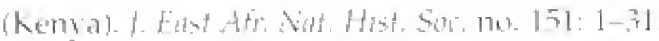

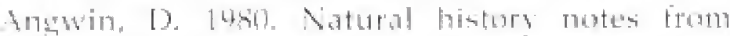

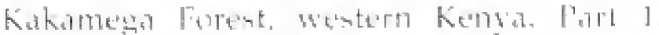

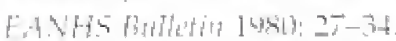

Angmanch Iy Ferent. Lnpublished manes heded in the

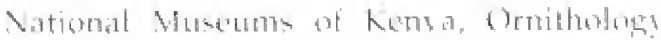
Derpatiment.

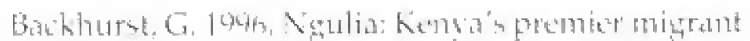

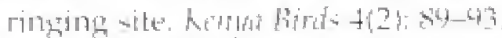

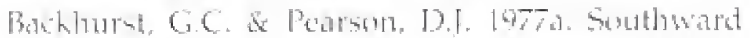

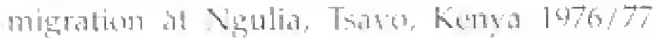
5oin? $1: 12-17$

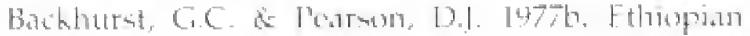
regrion birde attracted to the lights of ventilia

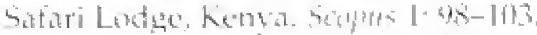

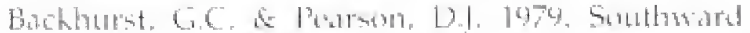

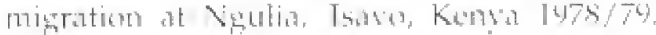

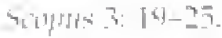

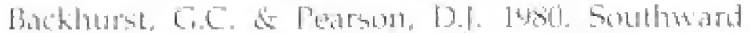

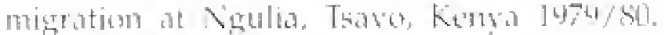

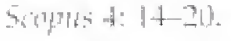

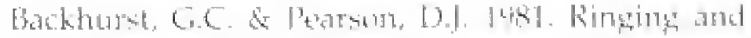

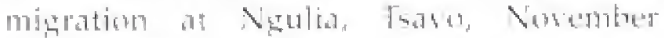

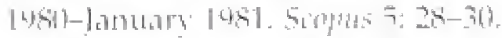

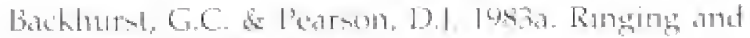

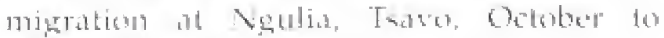

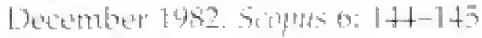

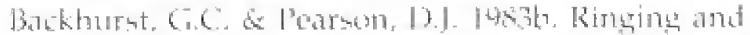
migration at Nesulia, Thavo, atemem 1483. sicentis $7: 136-1.38$

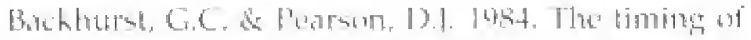

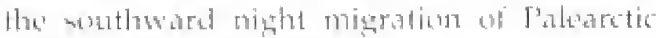

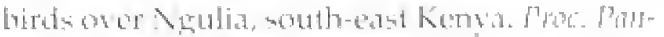

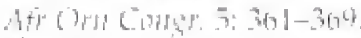

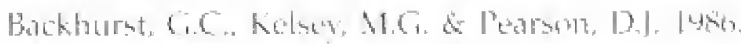

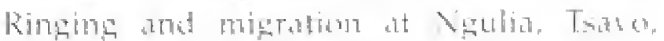

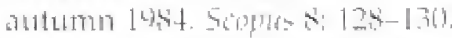

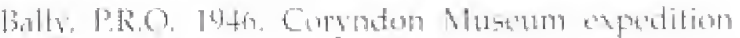
tis the Waul Firest. Part I. Butatiot's diars:

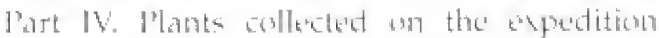

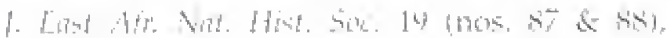
$[\because]-91,45=1011$.

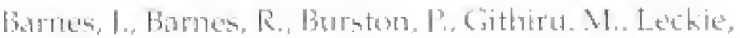

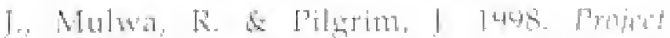

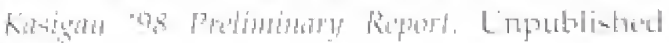
Te'plett.

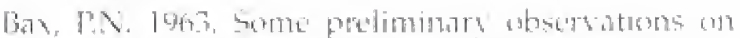

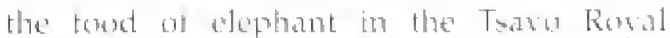

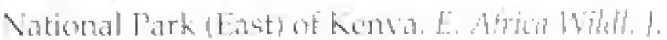
$1: 40-43$.

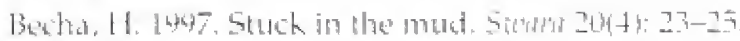

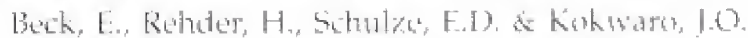

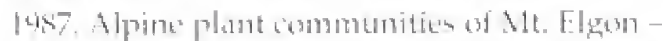

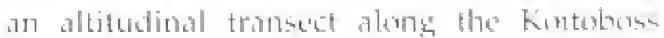

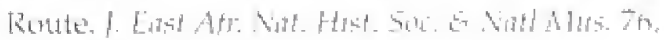
|นต. $|9|: \mid-12$

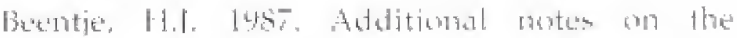

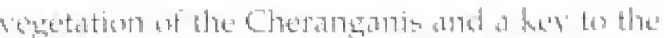

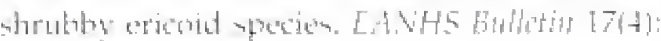
$71-\overline{7} 2$

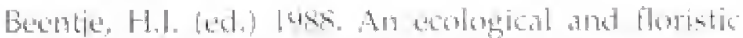

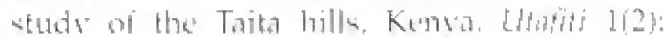
$23-64$

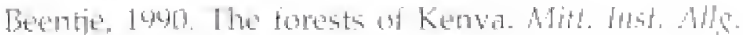

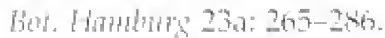

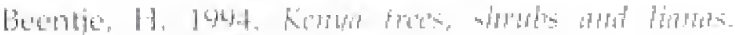

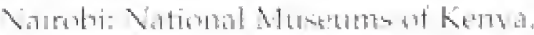

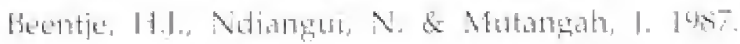

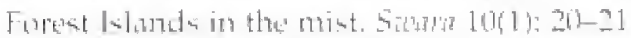

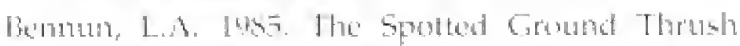

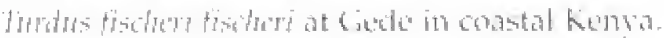
Sompts: 4. 47-107

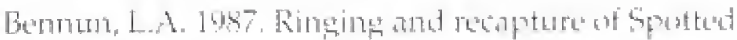

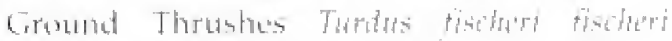

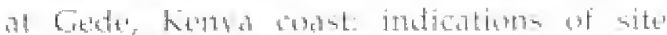

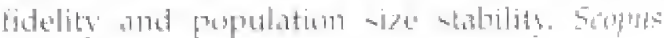
$11(1): 1-5$,

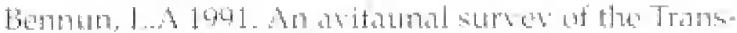

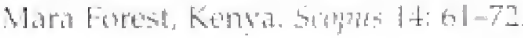

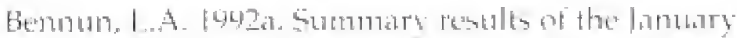

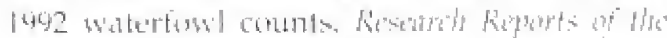

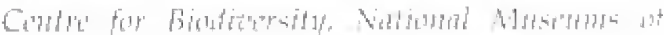
Remlat: Ormithestege 


\section{Important Bird Areas in Kenya}

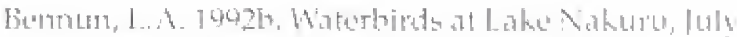

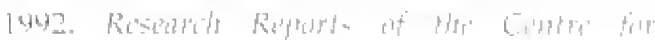

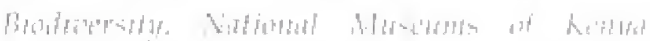
Ormitholution

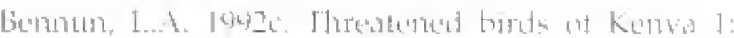

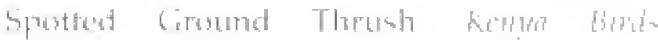
1(1):19-21.

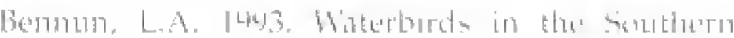

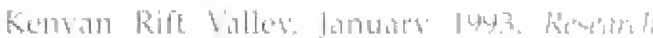

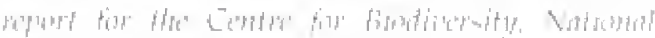

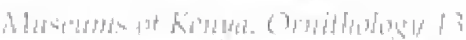

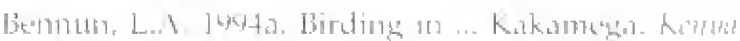
Bint: 20?: ] 녀 -27

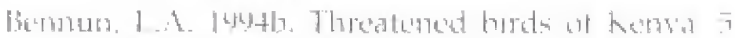

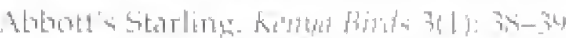

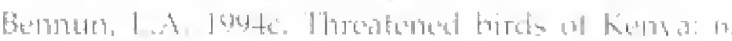

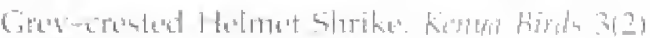
$5+8$

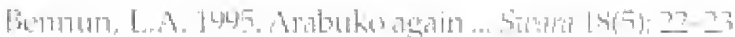

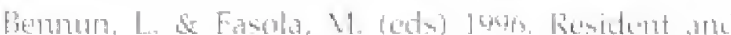

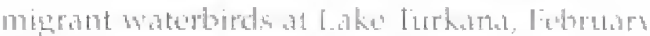

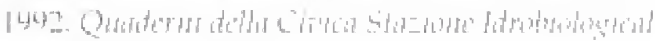
di Miluster 21:7-40.

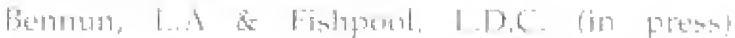

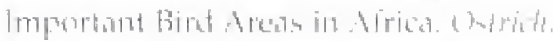

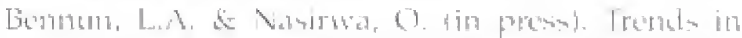

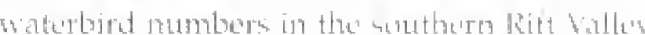

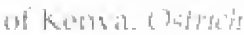

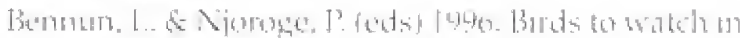
Fint Africas: A preliminare Red Data limb.

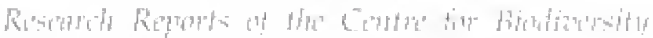

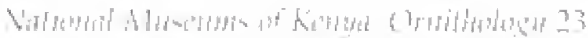

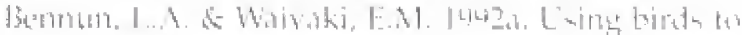

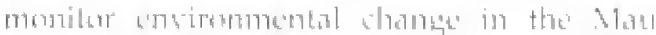

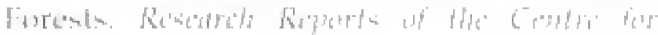

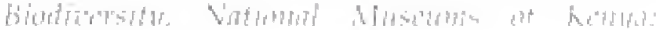

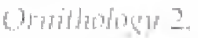

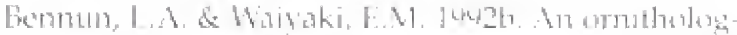

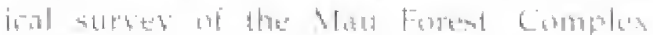

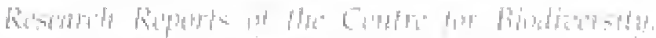

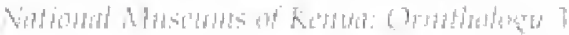

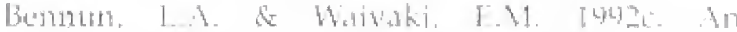

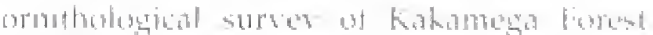

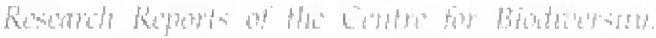

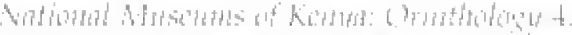

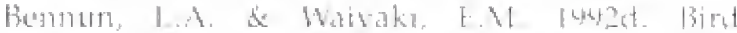

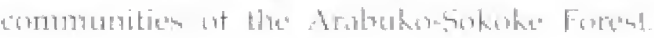

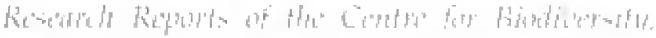

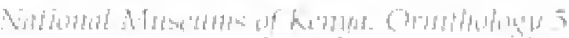

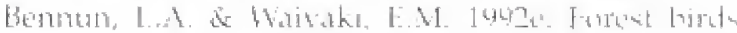

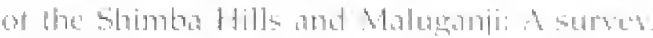

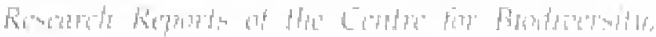

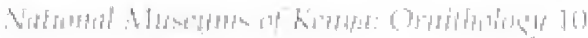

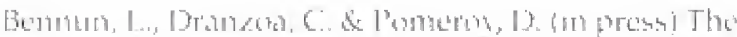

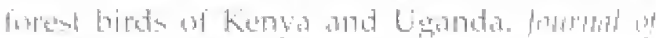

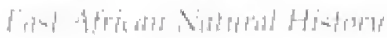

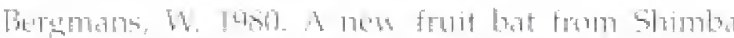

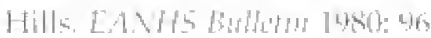

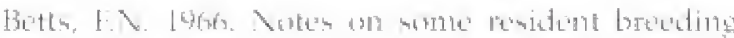

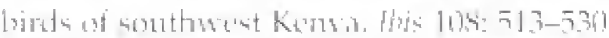

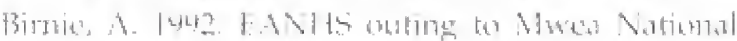

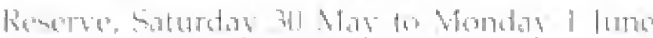

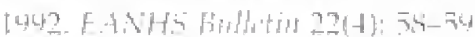

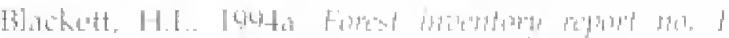

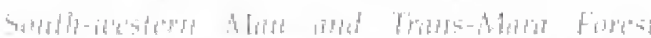

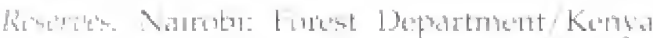

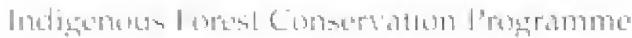

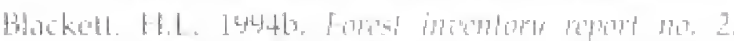

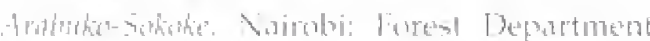

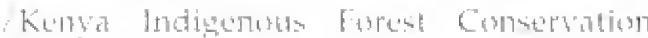
Progitamme.

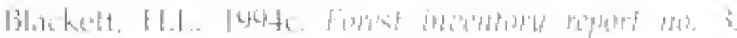

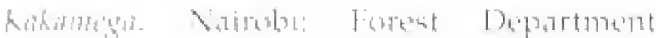

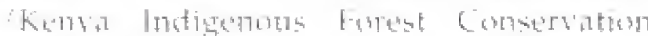

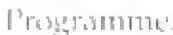

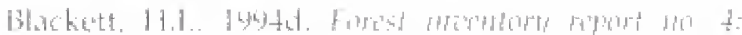

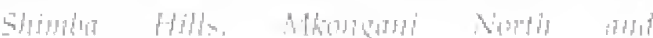

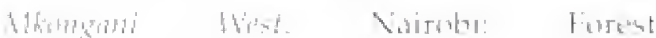
Depirlument Kumba Indigetions forest

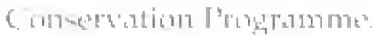

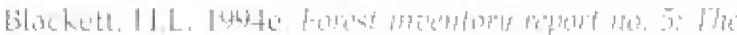

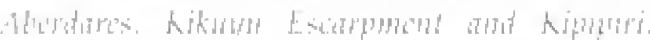

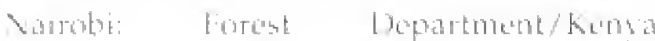
Indigenus Forrent G

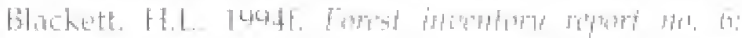

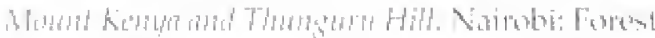

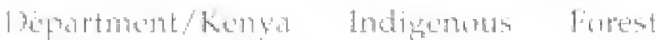

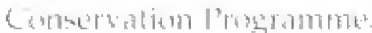

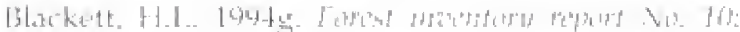

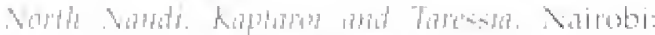

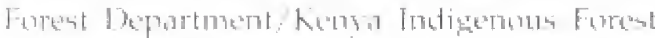

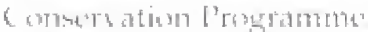

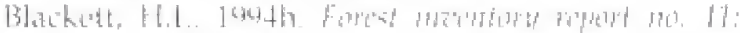

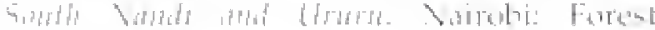

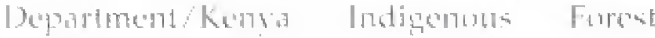
Comereation l'rogrammen.

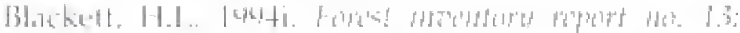

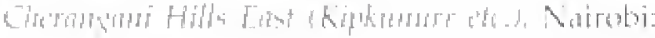

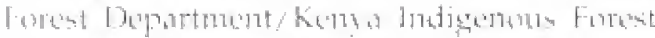

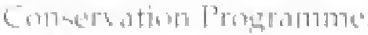

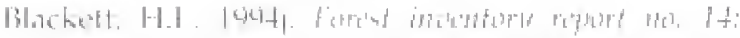

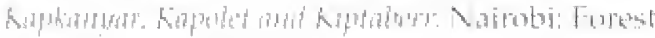

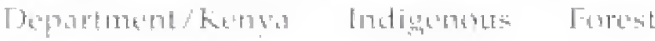

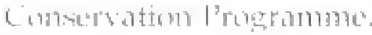

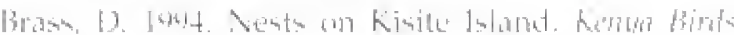
(2) 2 : is-36

\section{References}




\section{References}

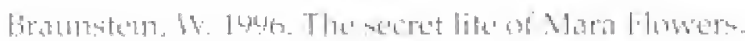

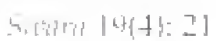

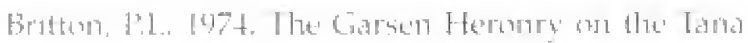

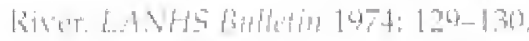

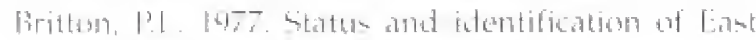

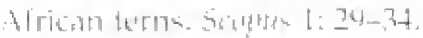

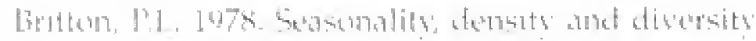

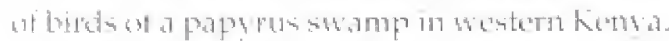

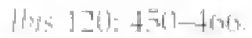

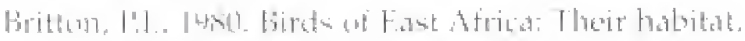

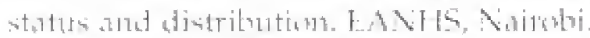

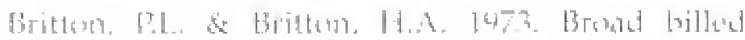

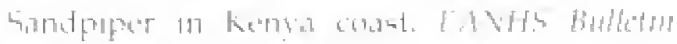

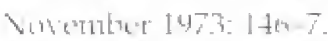

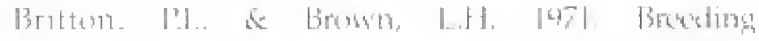

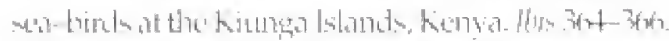

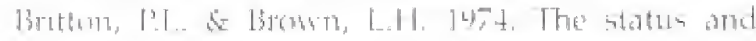

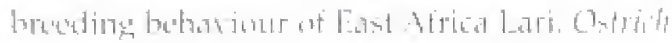
4. $45: 47^{3}-42$.

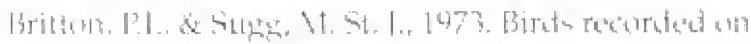

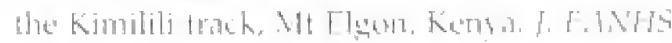

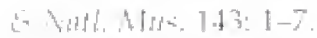

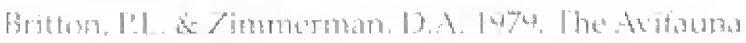

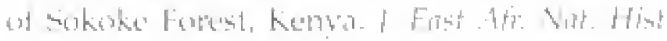

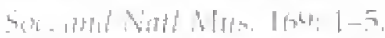

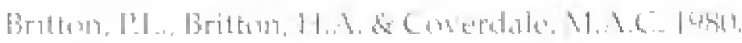

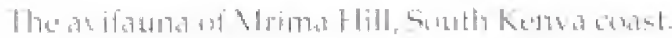
setprest.73-70.

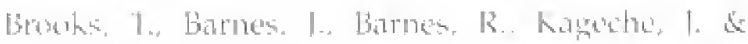

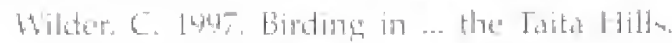

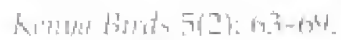

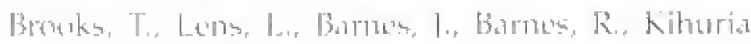

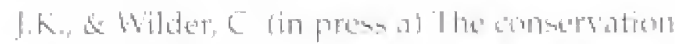

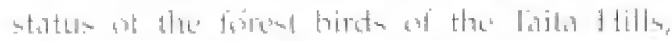

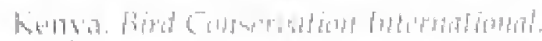

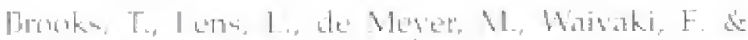

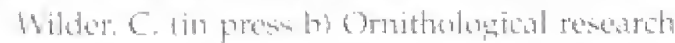

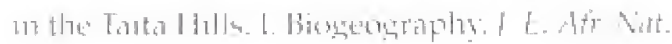
lisis

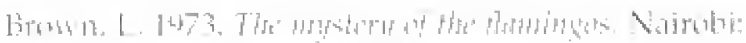

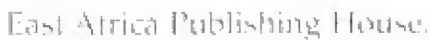

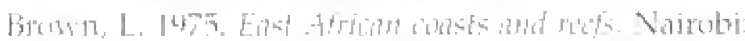

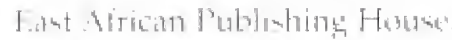

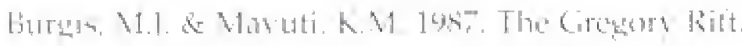

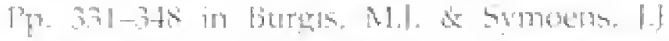

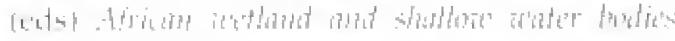

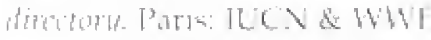

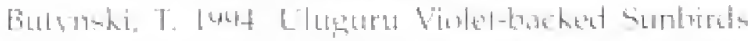

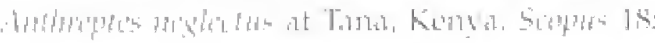
17. -174 .

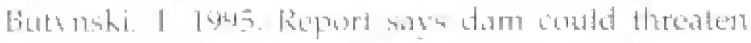

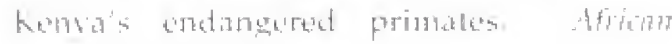

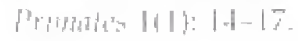

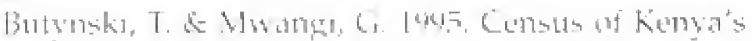

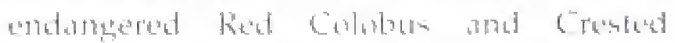

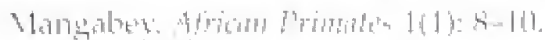

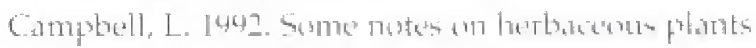

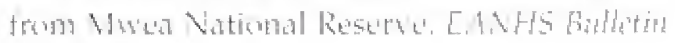
$22(4): 54-617)$

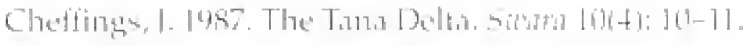

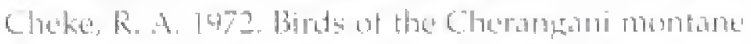

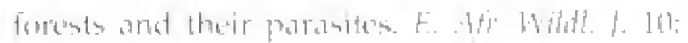
$3+5-7+4 \times$

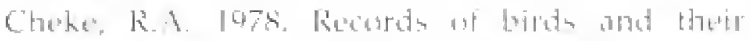

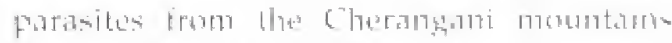

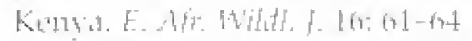

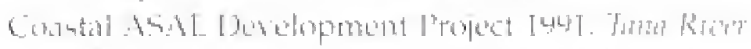

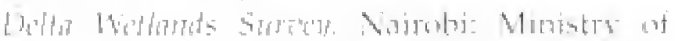

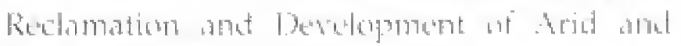

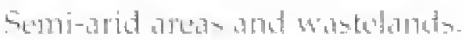

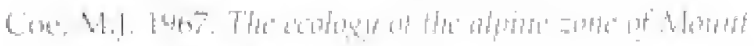

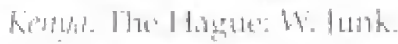

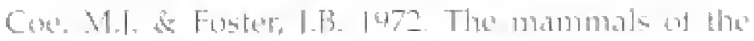

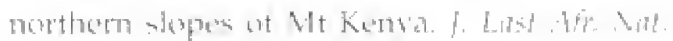

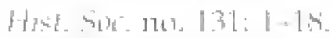

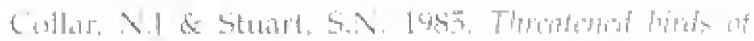

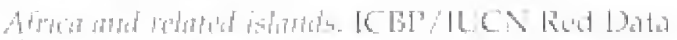

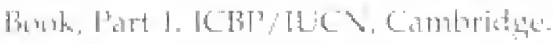

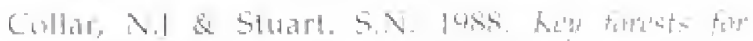

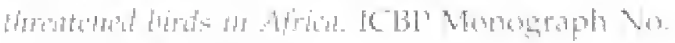
3. Combridure: Ke BII"

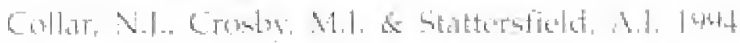

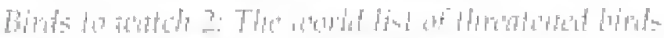

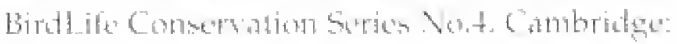
birdlifedeternationat.

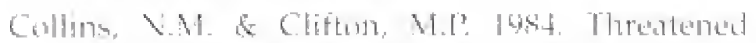

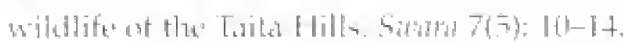

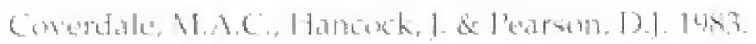

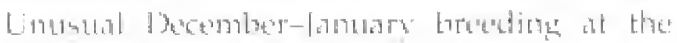

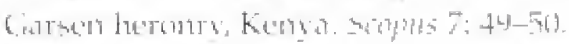

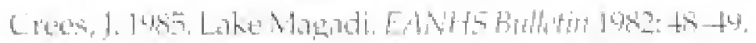

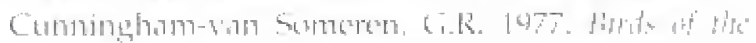

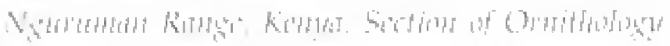

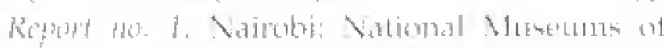
Kungar [exdestrited].

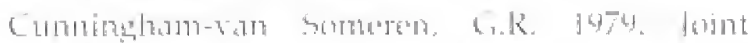

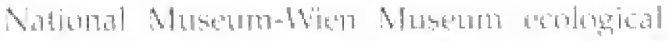

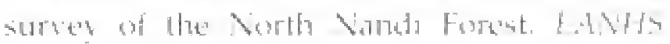

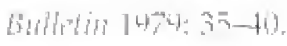

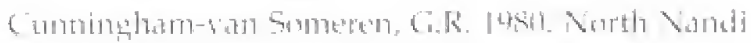

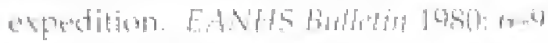

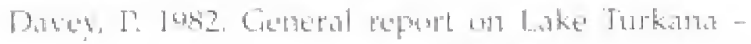

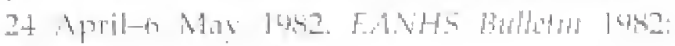
$7 k-74$

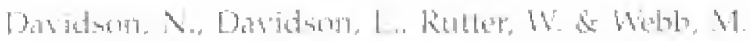

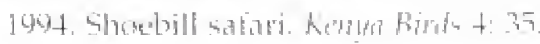




\section{Important Bird Areas in Kenya}

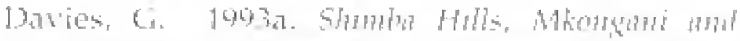

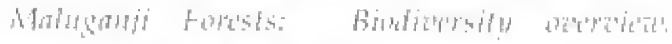
Nairobi: KIFCON/NMK.

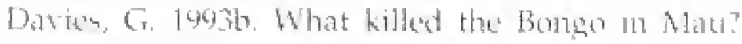
EANHSBultetin 2301) 3-6.

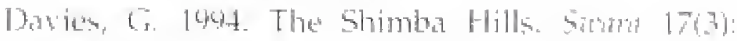
2̄-27

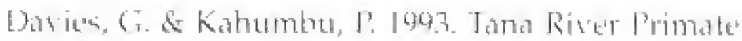

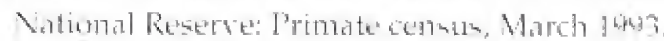
LANHS Buld $23(3): 35-44$.

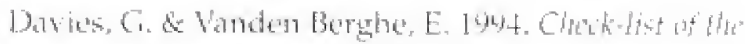

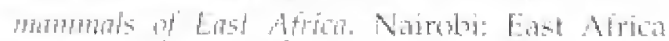
Natual Hintory Society.

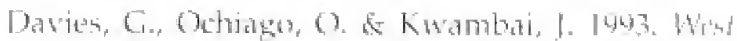

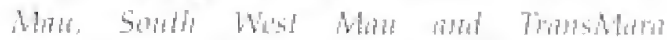

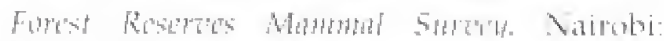
KIICON/ Matimal Muarums of Kenven.

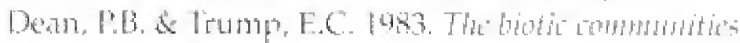

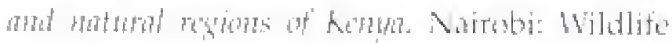
Planning Unit, Wildite Comsertation and Mashagertento Departoment.

Decher, B.S. 1987. The Tana River Natimal Primale

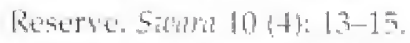

Deamend, A. \& Fatad, VC, 1974, Proliminary

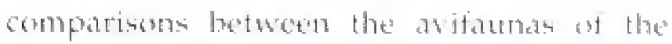

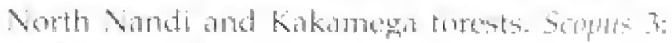
93-14it.

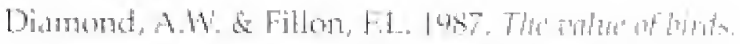

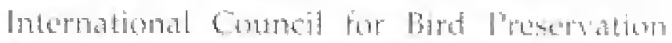
Technical P'ublicatian No, 6. Cambridge": ICIBI'

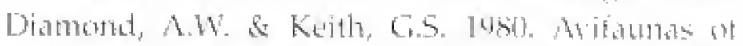

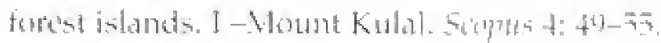

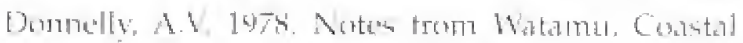
Kenva. LANHS brithen 1978: 122-125.

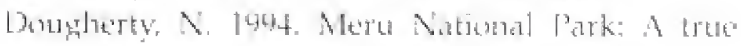

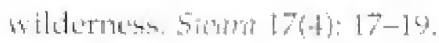

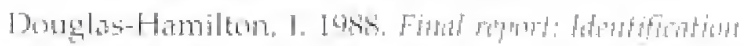

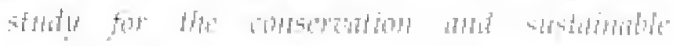

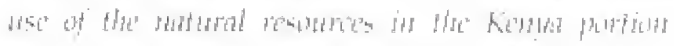

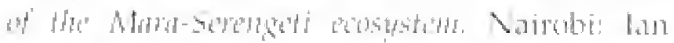

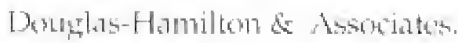

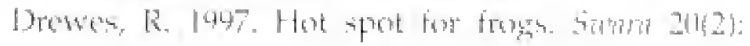
$27-3 h$

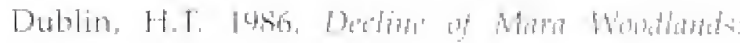

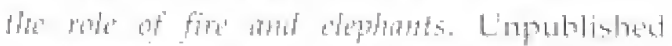

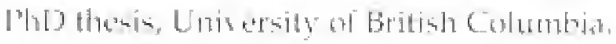

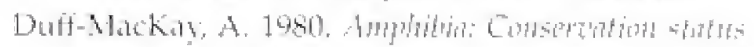
repart H. 1. Niarobi: National Musetme of Konva.

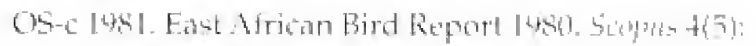
$101-140$

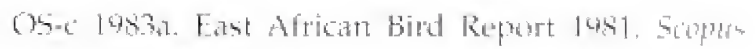
$5(5): 129-153$.

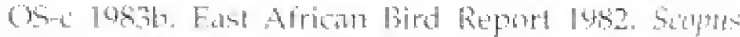
h(

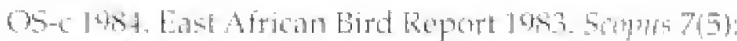
$105-1+8$

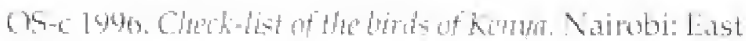

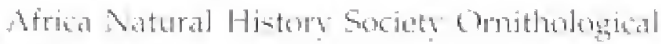
sublommitted.

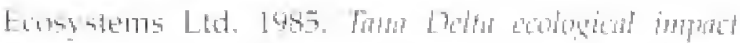

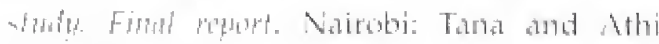
Rovers Denelopment Authority

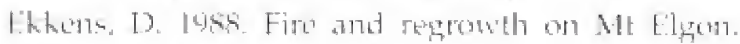

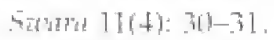

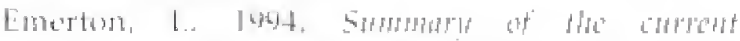

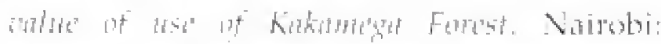
Kenga Indigenous Foreat Conservation

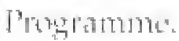

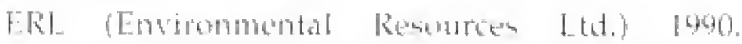

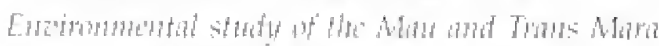

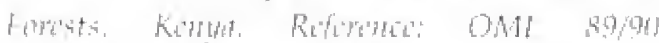

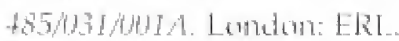

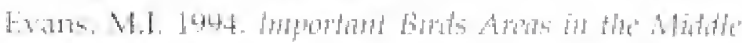
Eirst, Bintlife Comberation Seriles Vo. 2. Catnbrictade: Biralite International.

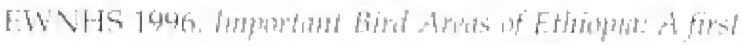

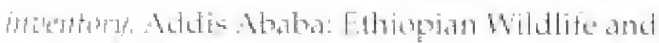
Vatural Jistory simerets.

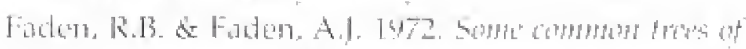

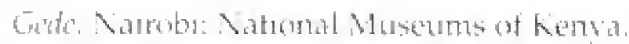

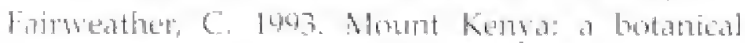

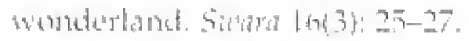

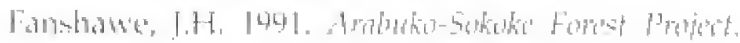

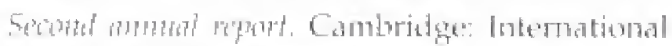
Conncil for Bind Pritateration.

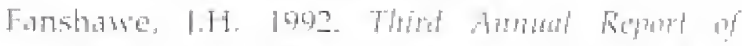

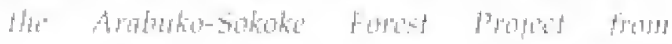

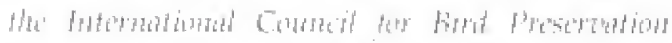

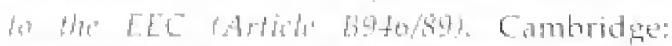

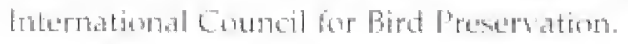

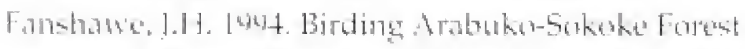

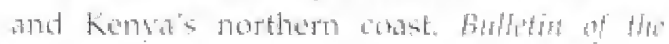

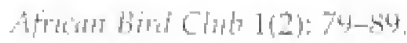

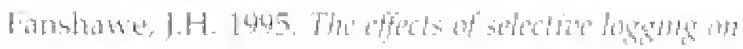

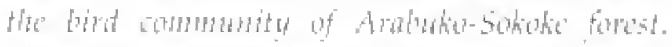
Lippublished Iho thesis, Lniversity of oxford. L'.

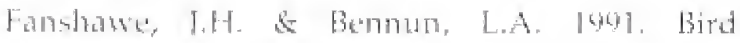

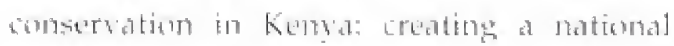

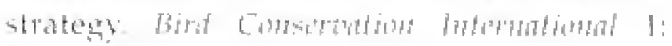
$243-315$

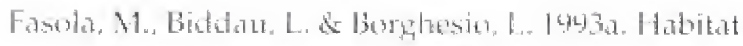
preference of resident and Palearetio waterbirts

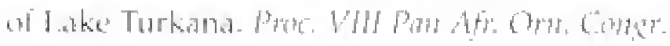
$534-545$.

\section{References}




\section{References}

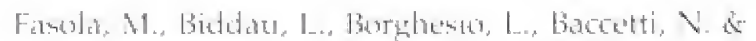

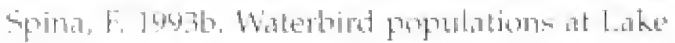

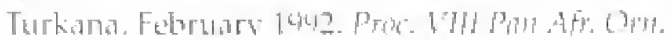
Cimptis, 524-532.

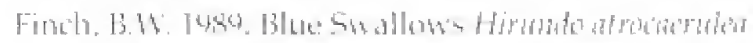

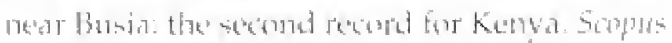
$13: 125-126$,

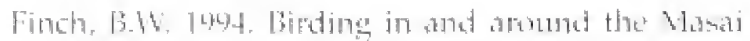

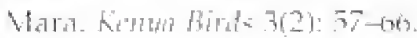

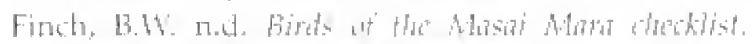
Natrolvi: Friende of Conseryation.

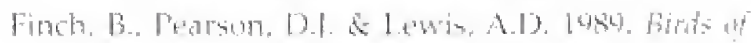

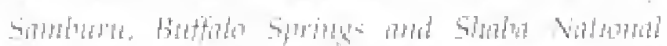

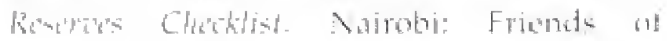
Combersation.

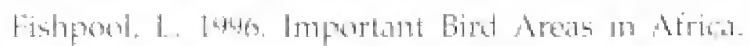

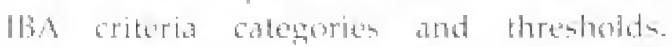

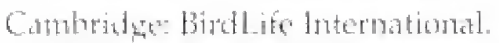

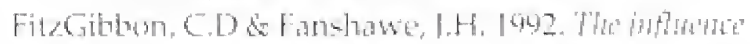

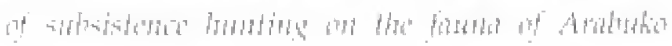

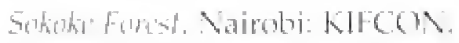

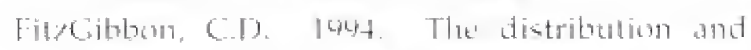

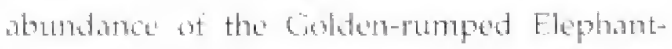

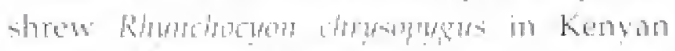

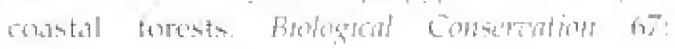
Iวิ>-164.

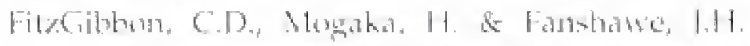

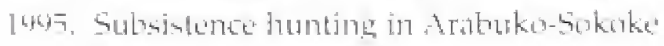
Fonet, Kermad, and its aflects on populations of

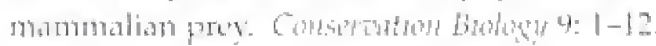

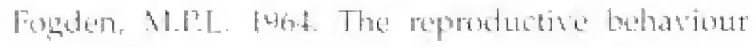
and taxinomy of Hempricts's Gill baters

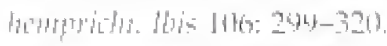

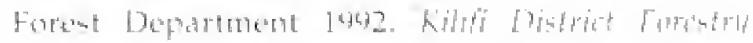

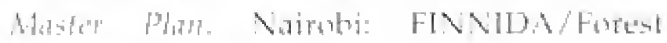
Department.

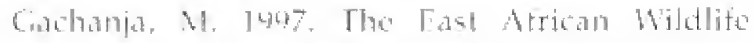

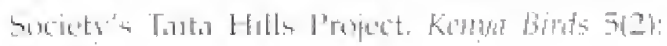
$43-45$

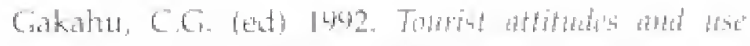

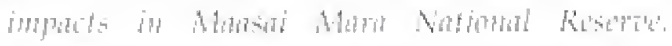

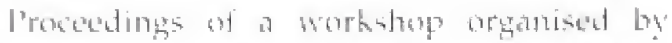

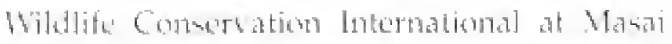

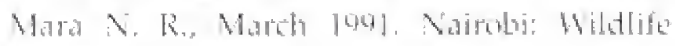
Comsertation foterdaticosid.

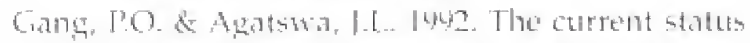
of mangroves alorse the Kensan Coast.

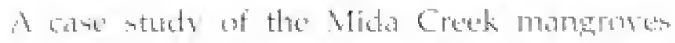

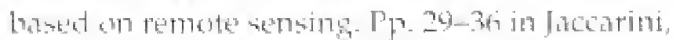

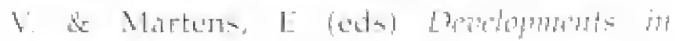

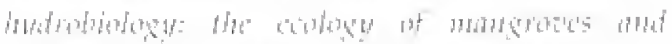

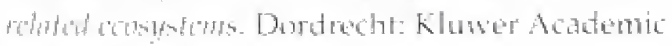
L'ubleshests.

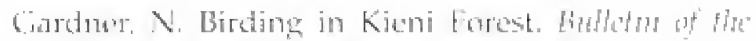

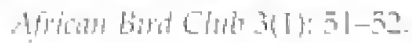

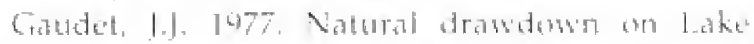
Navastra (kenva) and the furmatiout as

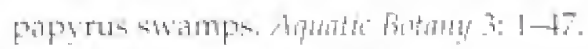

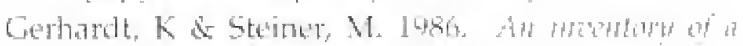

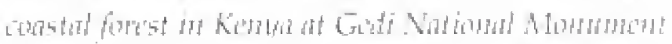

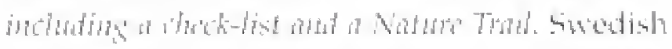
Lniversily of Agricultural scimeses. International Rearal Dexedopment Centre

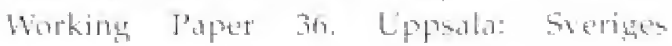
Lambratenniversideit.

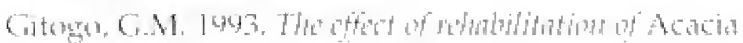

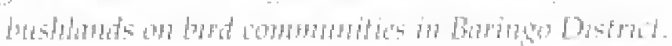

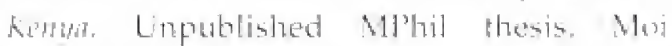
University; Eldnred, Kenva.

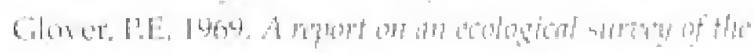

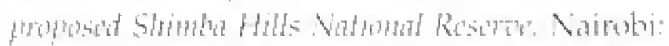
Suil Survey Unit, Department af Agricultume.

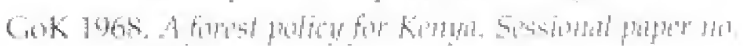
I w Ihos. Darobi: Covernment Printer.

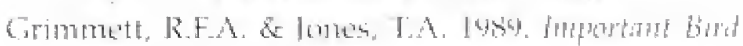

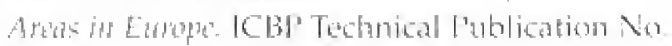
4. Combrider: ICBI?

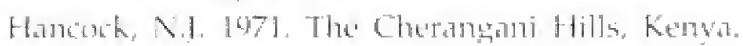

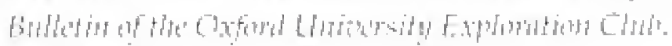
18: $55-86$

Hardy, 1. 1979. Golden Cat in 11ue deerdane National

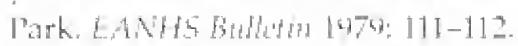

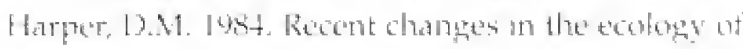

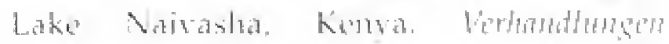

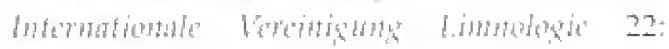
$1193-1197$.

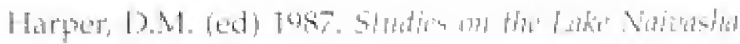

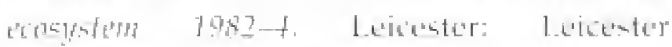
Lniversity Zaxlogy Department.

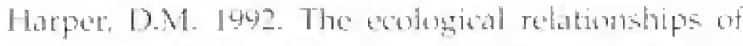
aquatic plants at Lake Narastad Kenra.

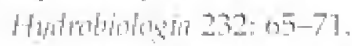

Harper, D.M. \& Masuti. K.M. Iblif. Freslubater

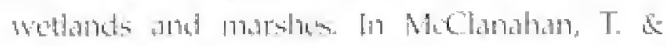

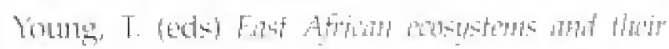

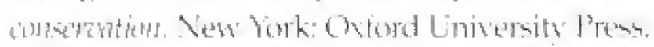

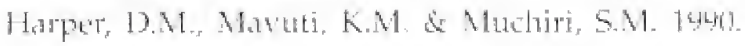
Foblogy and management on lake vairasha. kenga, ir relation to climate champen afier species intendutions and agricaltumal

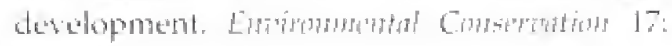
$328-335$

Ihaper, D.M., J'hillips, G., Chilyters, A. Kiteks, N. \&

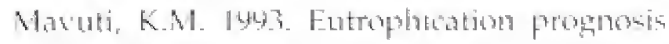

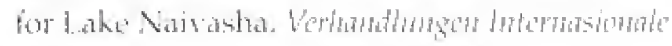
Vorits 


\section{Important Bird Areas in Kenya}

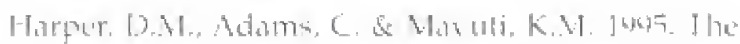
anjuatic plant communites of the laket

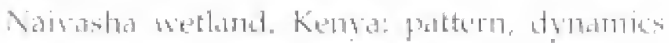

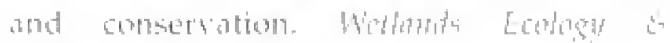

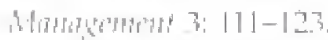

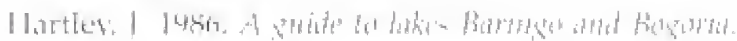

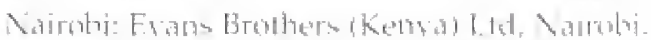

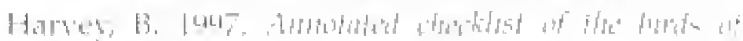

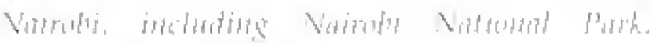
Nainto British Gombil.

Hendersom, I.Ci \& Haper, D.M. phes Bird distribution and hathitat structure wathe

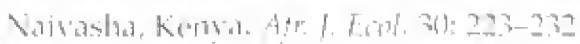

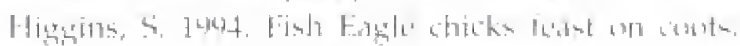

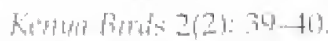

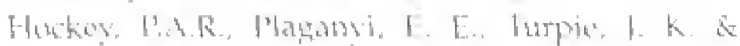

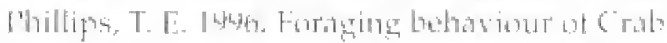

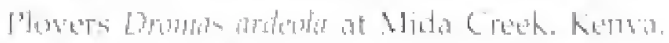

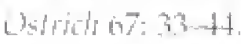

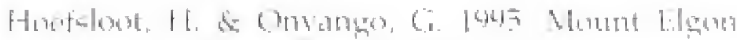

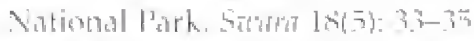

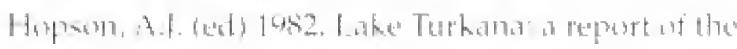

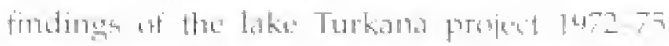

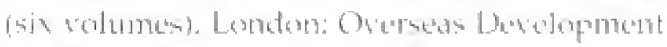
s.tmengistation.

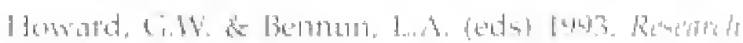

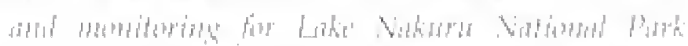

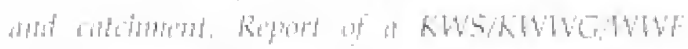

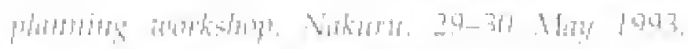

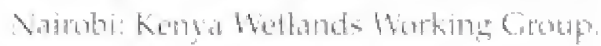

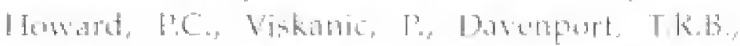

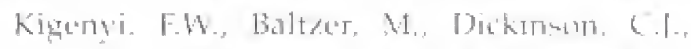

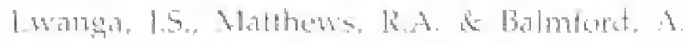
pugs. Complemententify and the" whe of indicastor

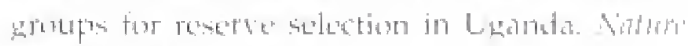
$344: 472-475$

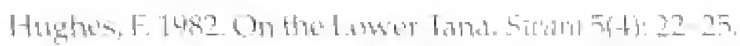

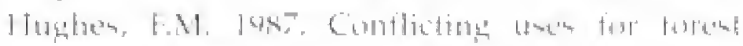

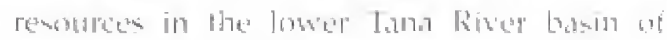

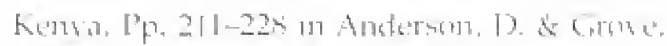

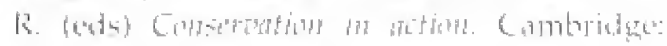
Combridge Lininersits flows

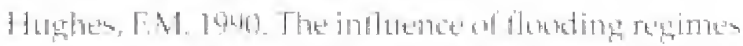

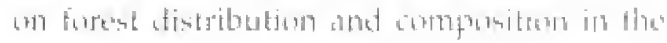

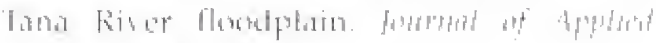

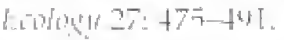

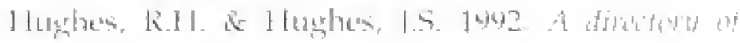

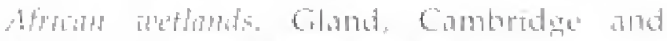
Natrolv: ILCN/LNIT/MCNC

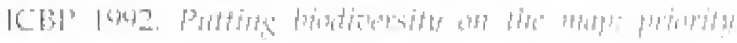

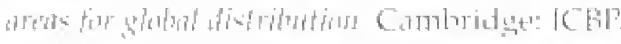

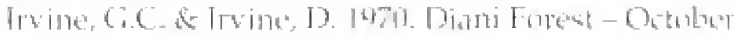

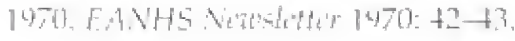

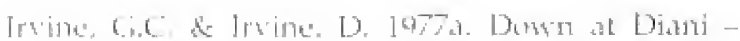

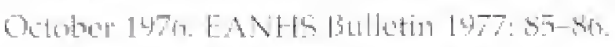

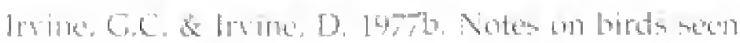

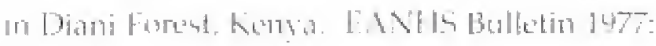
Wh-10ki

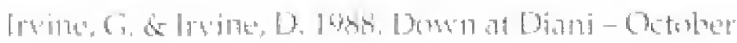

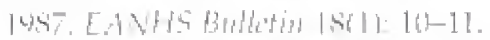

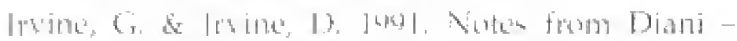

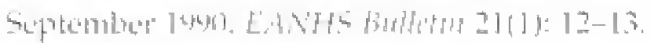

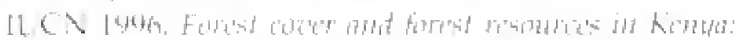

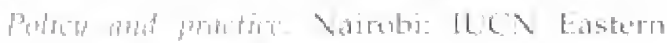

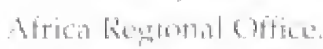

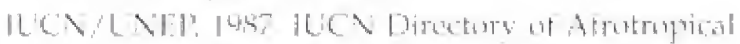

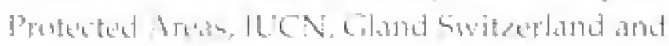

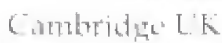

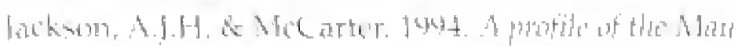

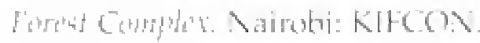

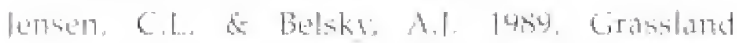

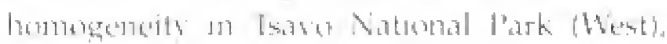

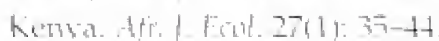

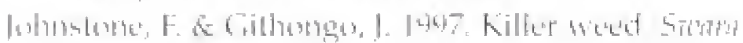
$2044: 24-24$.

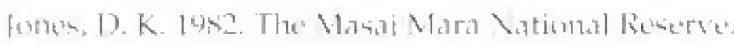

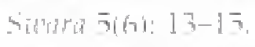

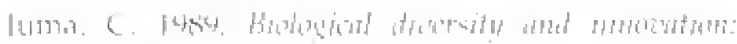

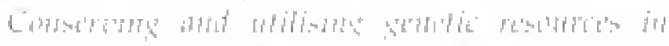

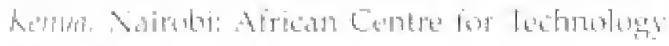
Studitus

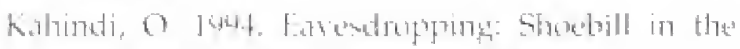

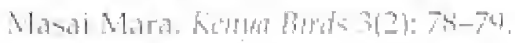

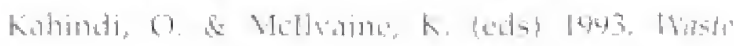

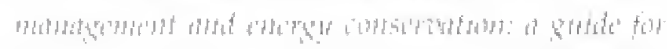

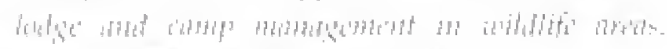

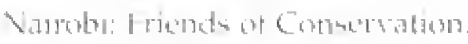

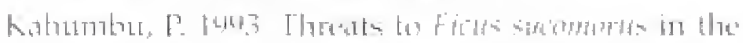

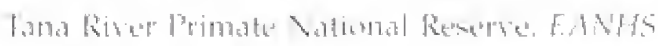

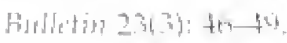

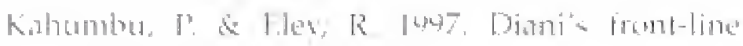

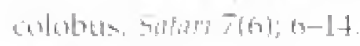

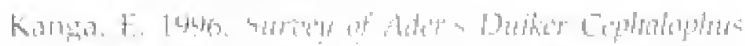

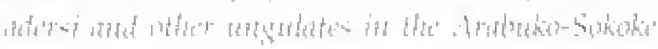

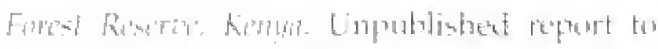

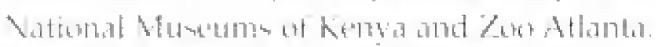

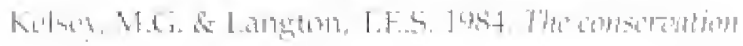

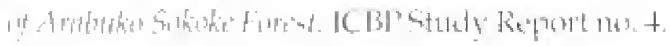

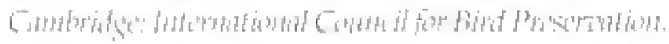

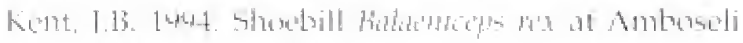

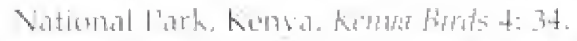

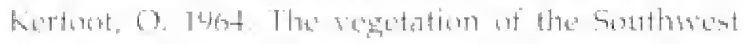

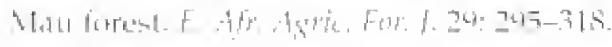

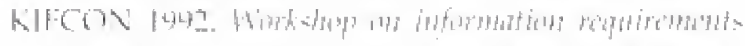

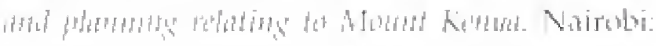

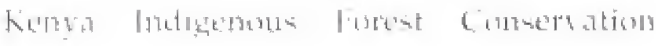

\section{References}




\section{References}

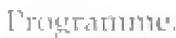

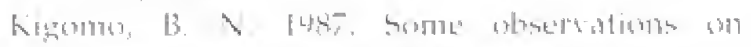

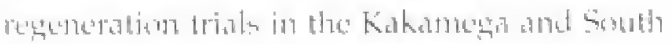

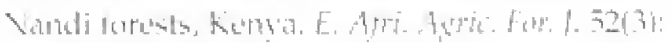
$18+145$

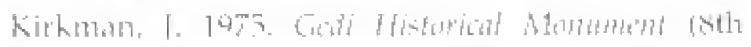

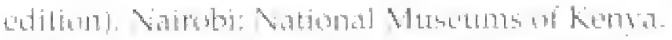

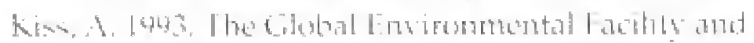

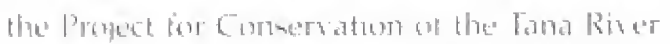

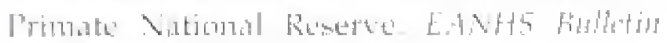

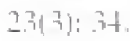

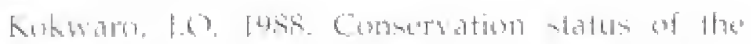

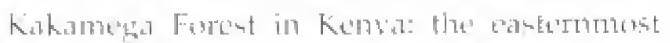

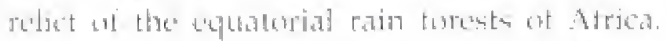

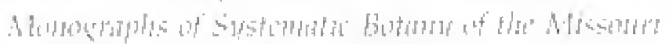

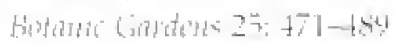

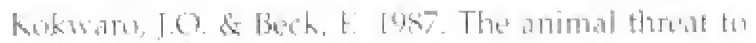

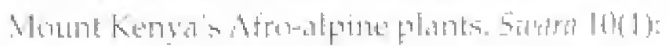
$30-3$.

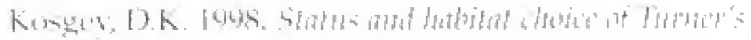

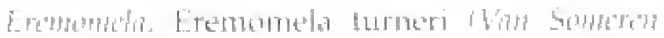

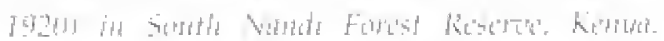

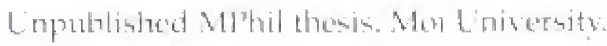

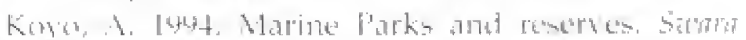
[7, 7 ) 1 1. 15 .

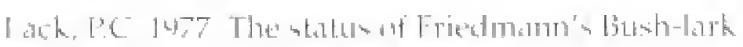

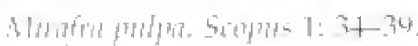

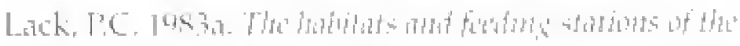

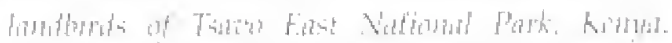

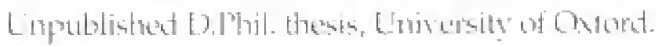

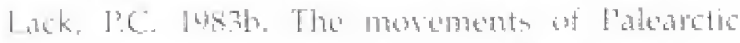

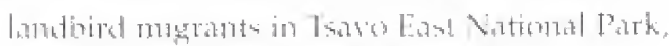

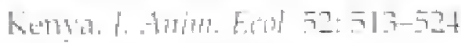

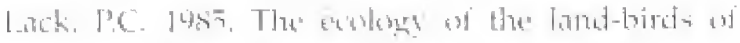

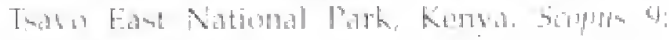
2-23. $77-96$

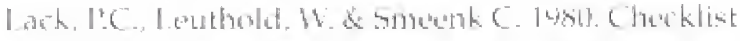

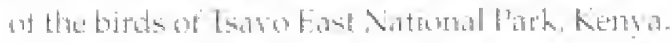

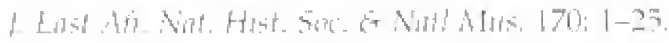

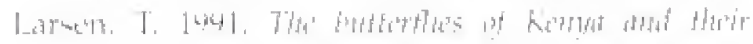

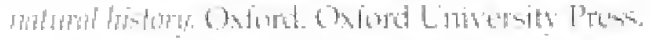

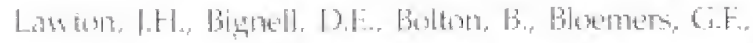

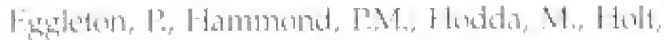

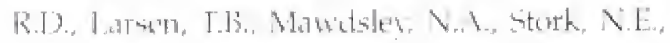

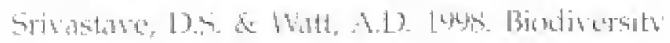

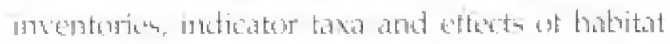
modification in tropical forest. Not?

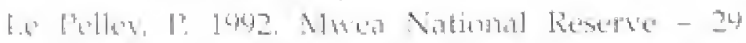

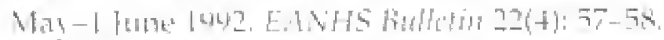

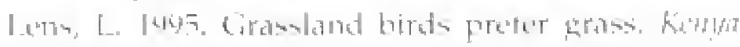
Hirits til.

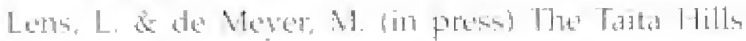

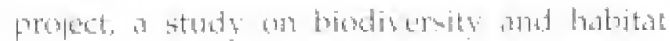

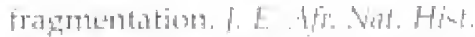

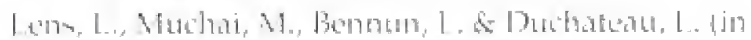

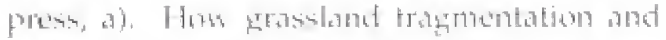

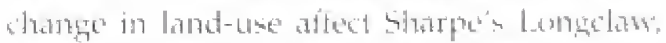

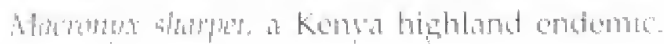
Ostrots

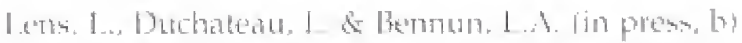

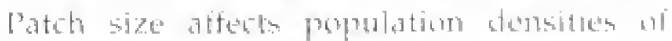

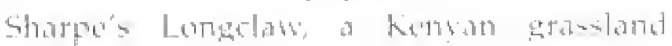

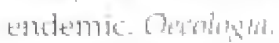

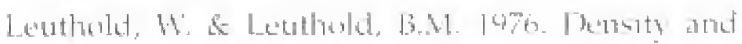

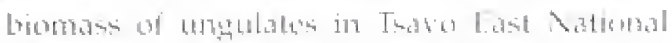

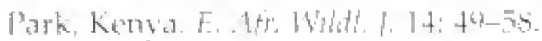

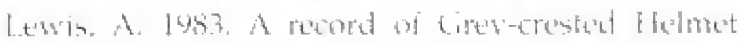

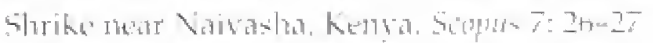

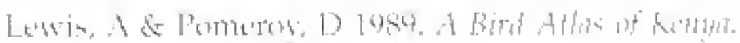

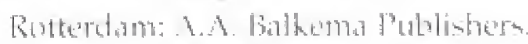

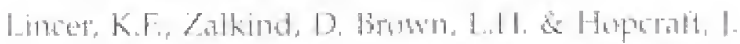

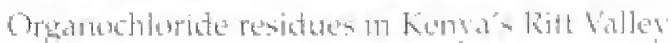

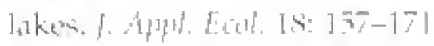

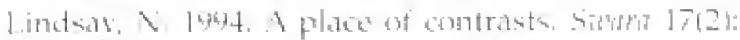
$28-31$.

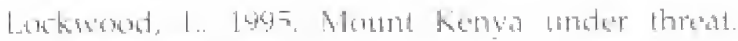
5 -

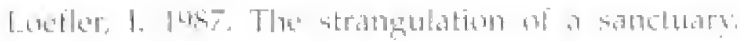

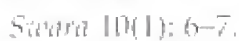

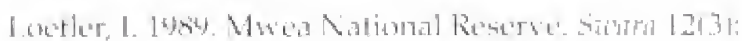
$8-10$.

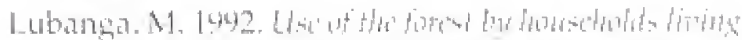

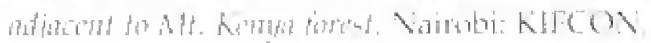

Luke, Q. (in prest) Flephant, the Katas and plant

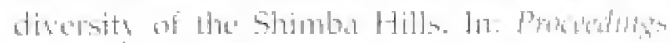

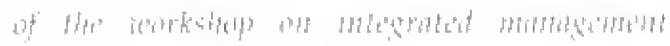

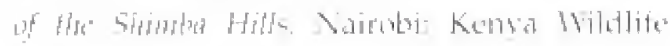

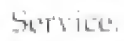

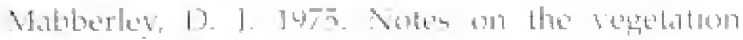

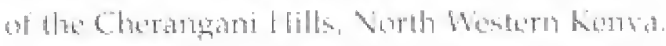

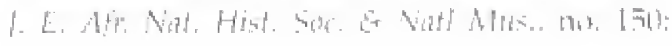
$1-11$

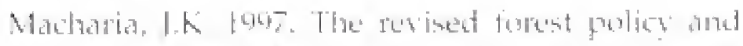

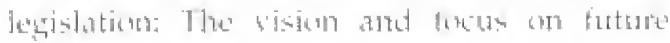

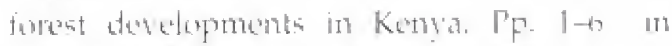

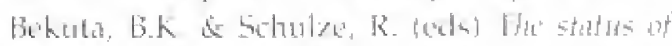

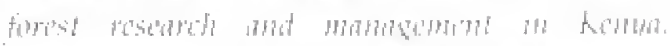
Jrocereditege of a joint REFRI-FD National

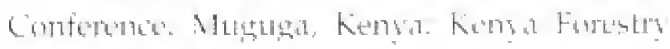

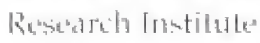

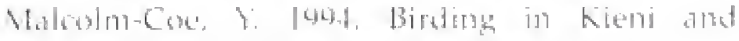

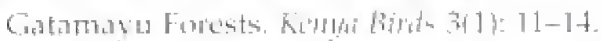

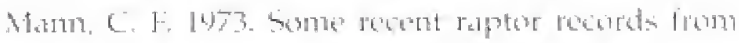

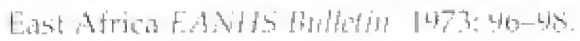

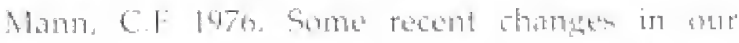

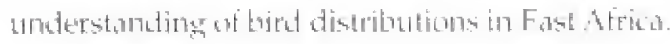




\section{Important Bird Areas in Kernya}

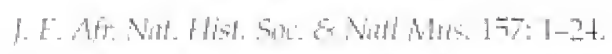

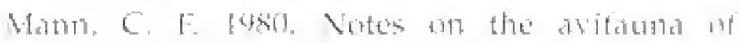

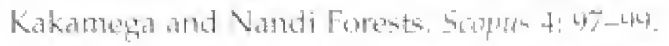

Mann, C.F. 1485. An arifoumal stuty in Kakanogit Forest, Kenga, with particular reterence w species diversity wedght and mult. Oafrito 5 to $236-262$.

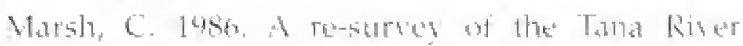

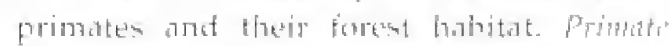

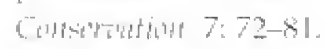

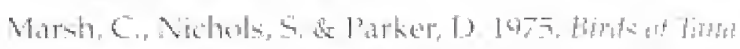

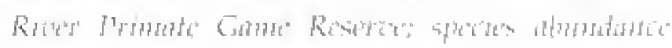

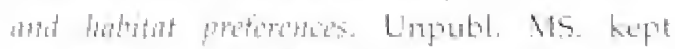
be East Africa Vatural listors siciets. Naraboi.

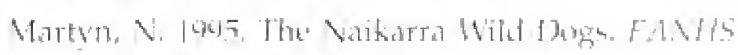

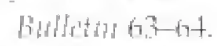

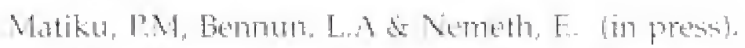
Distribution and pupulation size on the Tast

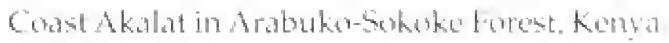
Ustrichts.

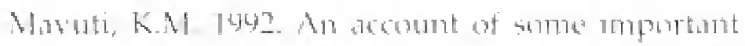

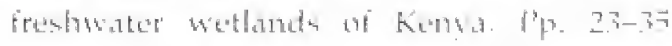
in Crafter, S.t. Nituguma SC. \& Hombat.

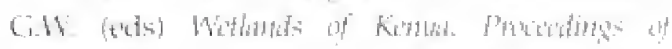

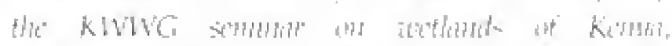

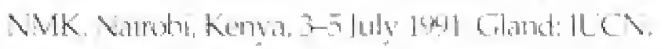

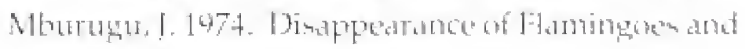

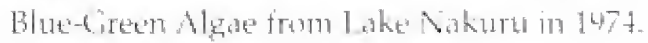

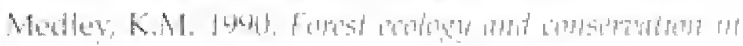

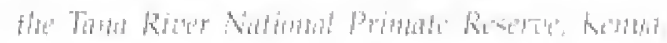

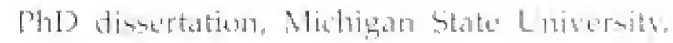
East L 147sing, LSA

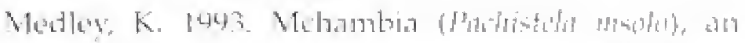
indicater of forest condition in the han Rincer.

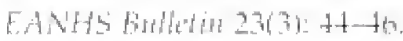

Aladler, K.E. Iys Forest regenterition in the land

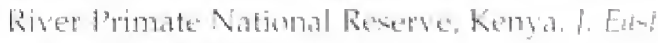
Afr. Nor. Hit. 84:77-4h.

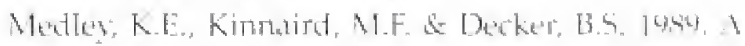

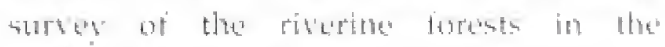
Wema/Hewani veinets, with references to

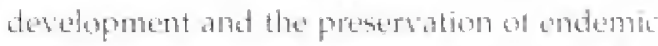

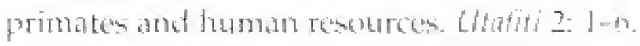

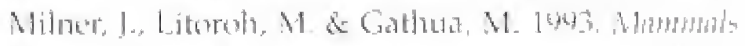

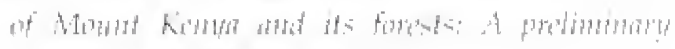
strete KIFCON, Nairebi.

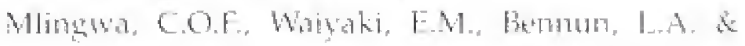
Burgess, N.19. (in press). Binds. In X.1). Lutrentis

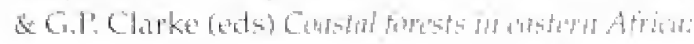

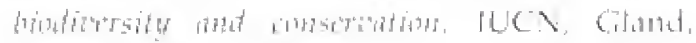
Suitzerland.

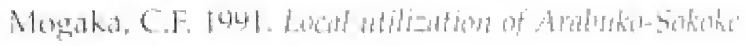

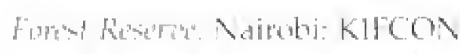

Awdulfi, E, \& Mondolfi, K. 1993. Less commen antelepes found in virubi National Park. Sictirit lin (1): 24-27.

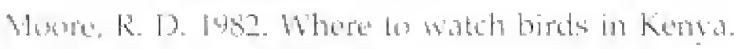

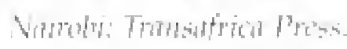

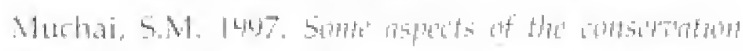

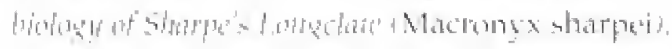

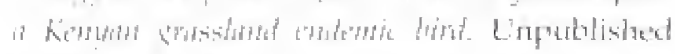

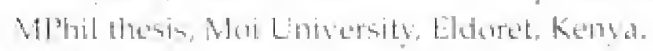

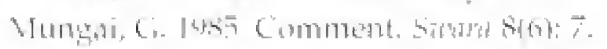

Vutangah, I. G. temt. The segetation of Lake Nokuru National loark, ketrya. A smopsim of

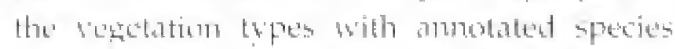

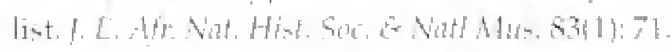

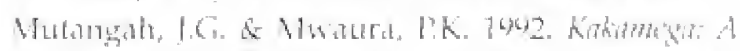

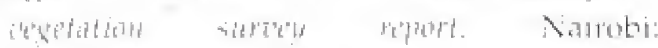

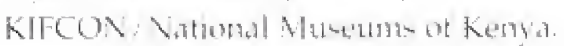

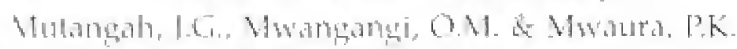

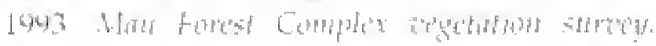

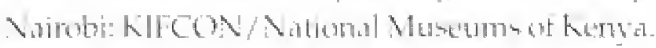

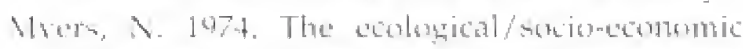

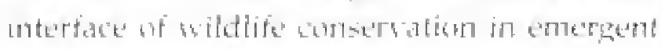
Avica: Lake" Nakuru and lake Nirsatha in

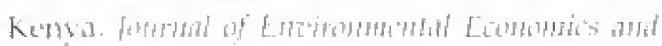

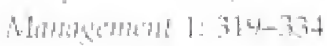

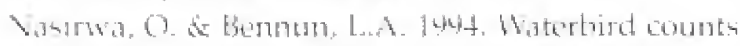

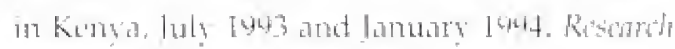

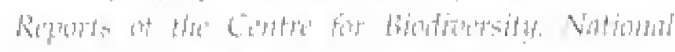

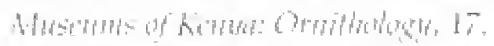

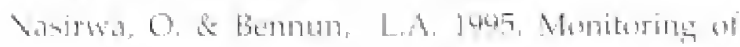

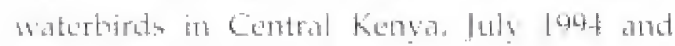

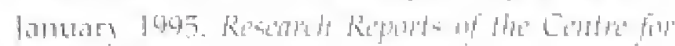

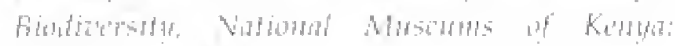

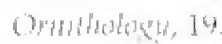

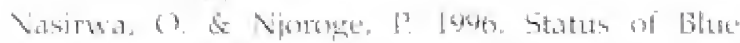

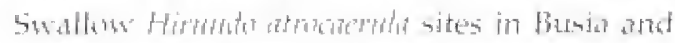

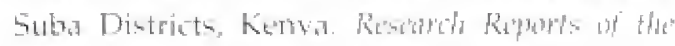

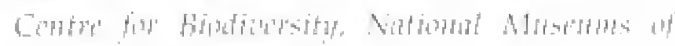

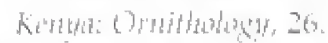

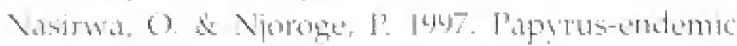
birds in the fromeing atamps of Lake Victoria,

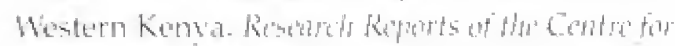

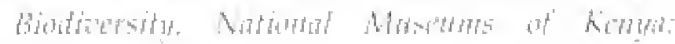
(M)rithethen, 28,

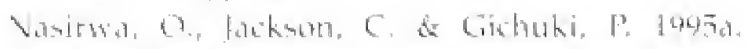
Waterbirds on the Lepere Tama River Dams.

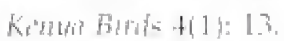

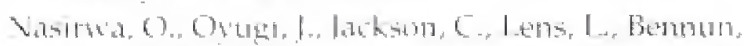

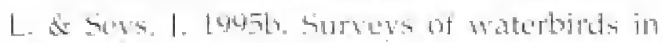

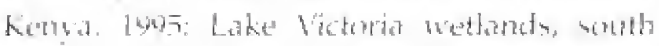

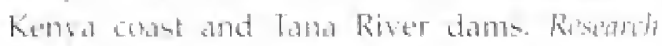

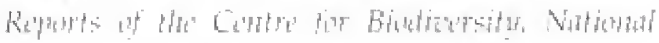




\section{References}

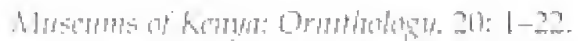

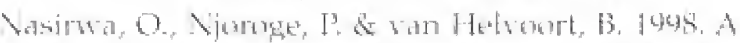

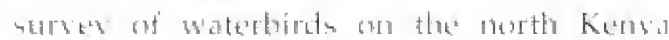

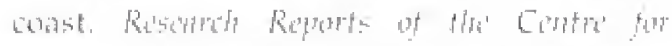

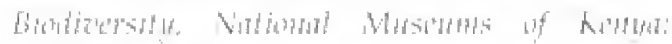
(T)rithology, 31.

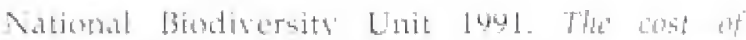

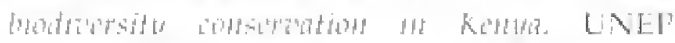
Kema Biolizersity Country Study. Nairobi: LVEP.

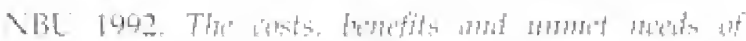

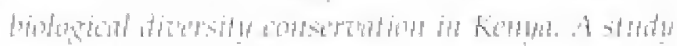

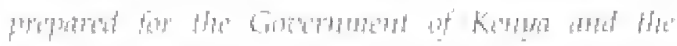

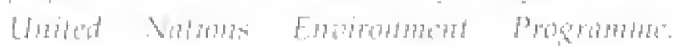
Vuirabe: National Biontincesity Lnit.

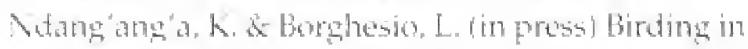

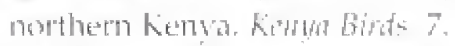

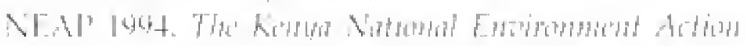

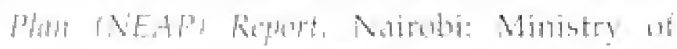

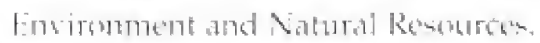

Nemeth, E. 1990. Diatrilsution, habitat selection and behariour of the Last Coust Akalat Sheppondti

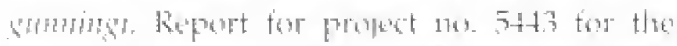

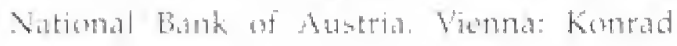

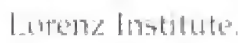

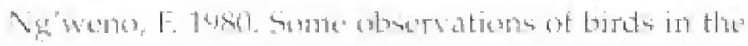

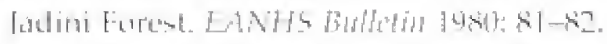

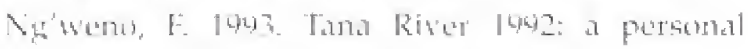

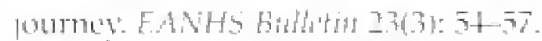

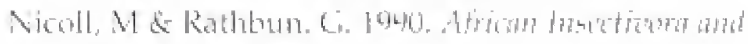

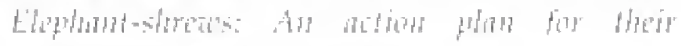

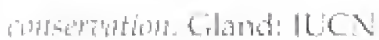

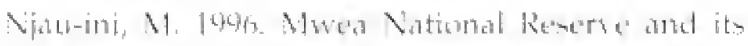

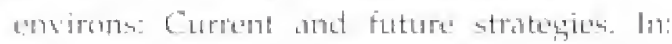

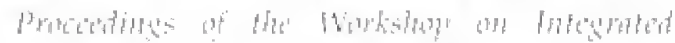

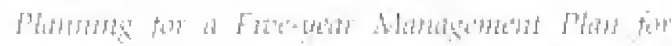

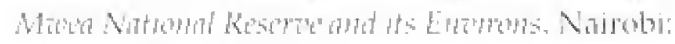
kenyar Mildite seor ice.

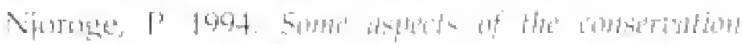

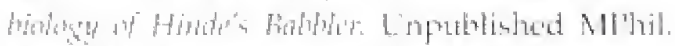

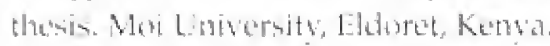

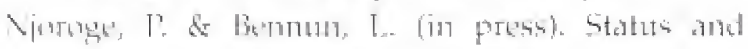
conservation of Hinde' Babbler Thaturater irime's a throatened specios in an agriculturad

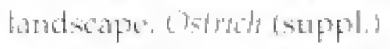

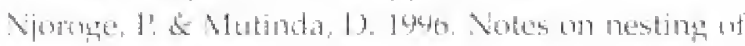

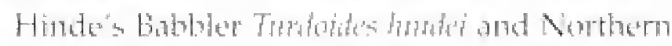

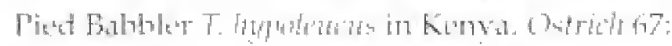
$170-172$.

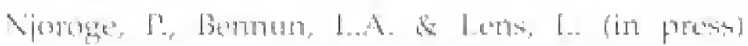
Iabilia ues by the stobally endangered

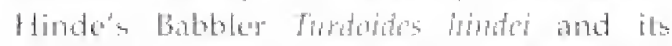
stimpatere retative, the Nottherty l'jed babbles

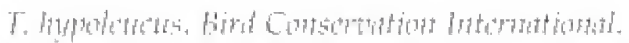

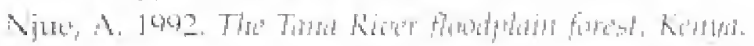

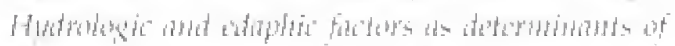

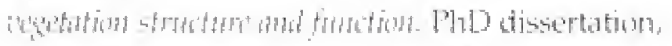
Lniversity of California, Davis, LSA.

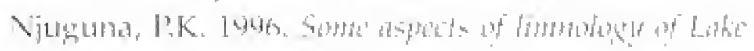

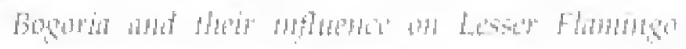

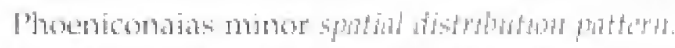
Lnpublished Mrhil thesis, Mui Lnisersits. Eldirel, kimba.

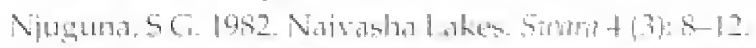
Nitugha, SG. 1492, Tana River Delta wetlanda. I'P. 139-140 in Crafter, 5.A. Niuguna b. C. \&

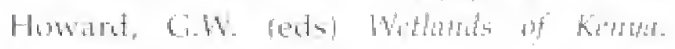

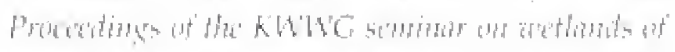
kivin, NMK, Nairobi, Kenga, 3-5 luly payt. Giland: IUCN

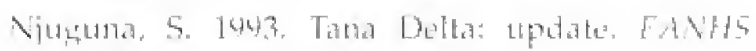
Bullitin 23(3): 46-51.

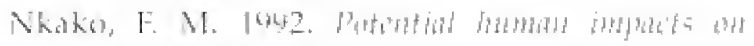

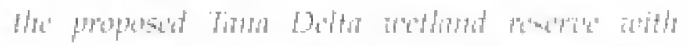

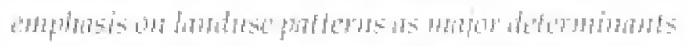

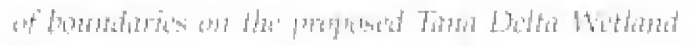

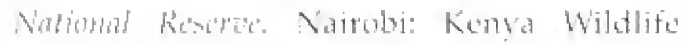
service.

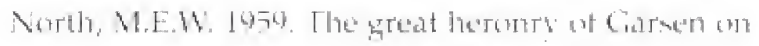

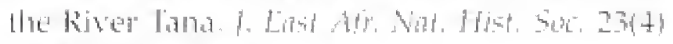
179. 101: 150. 160.

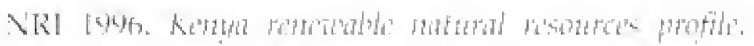
Chatham, Lh: Natural Resurces lastitute.

Okwanc, P. 1696. Wild jewel: anboseli's babitats and

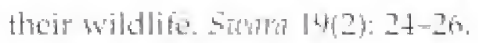

Opata, K. Jugz. National Park or farme for prawne? Simulh(2): 12-13.

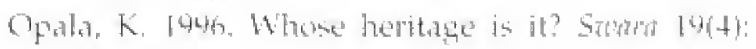
$14-16$.

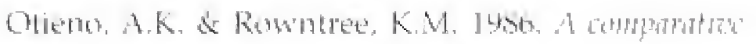

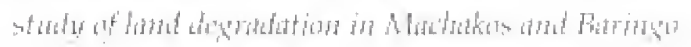

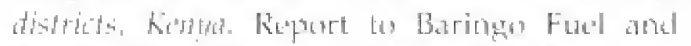
Fodder I'noject. Kanpri sa Samaki: BPFP.

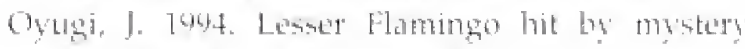

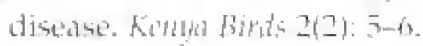

Oyugi, 10. 1690, Kakamega Forest is dyim, EANHS Bulditi $20(3)+4): 47-46$

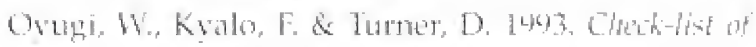

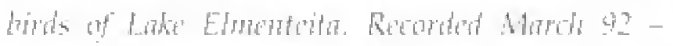

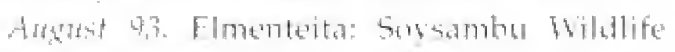
santulary.

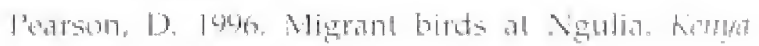
birts $4(2)$ : 93-69.

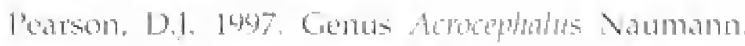
Pp. 98-122 in Lrtan, E.K., Fr, C.H. L Keith, S.

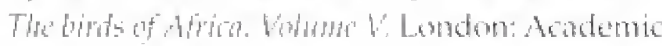




\section{Important Bird Areas in Kenya}

Presis.

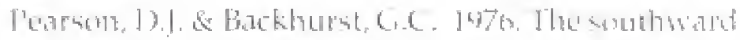

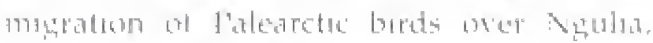

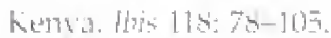

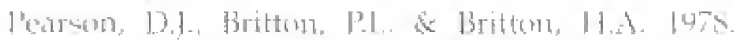
Substantiat wintering population on the Basa

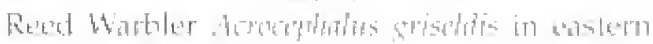

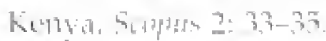

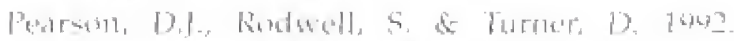

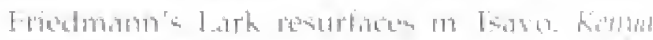
Hirit: 1120: 4,-14

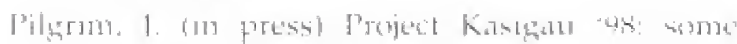

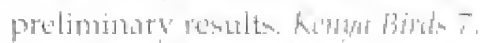

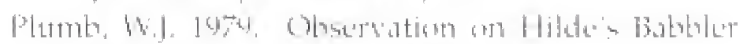

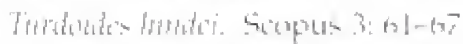

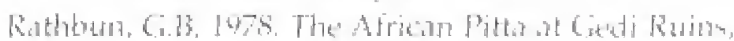

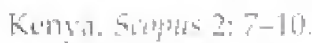

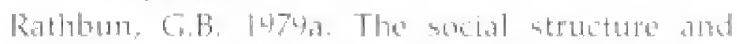

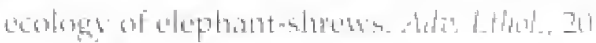

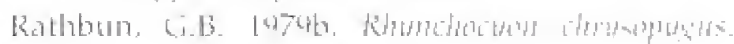

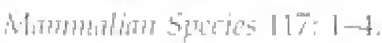

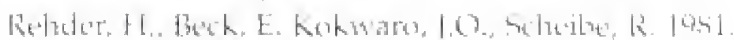

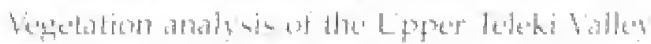

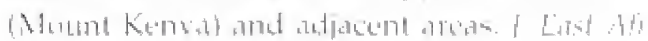

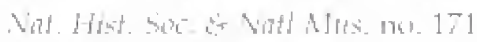

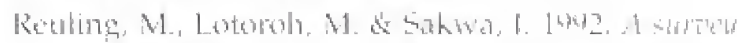

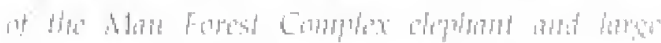

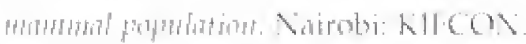

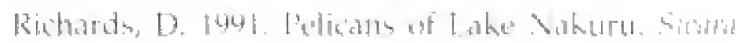
$1+(i): \int 4-21$

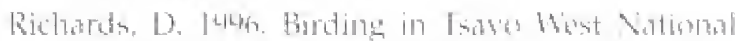

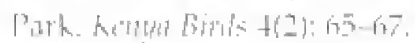

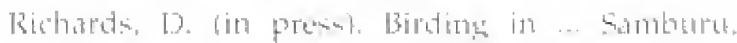

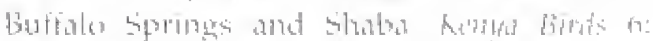
$24+35$.

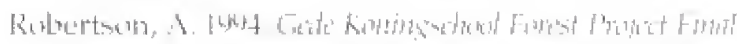

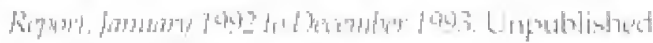

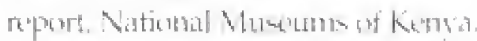

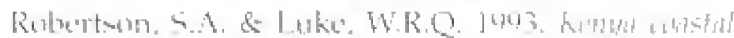

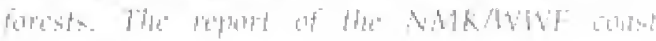

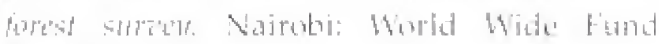
Ior Nature".

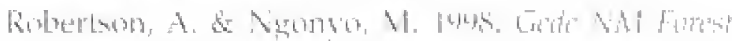

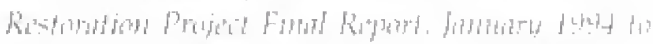

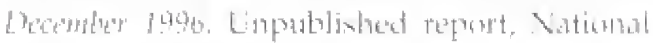

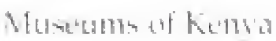

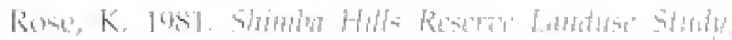

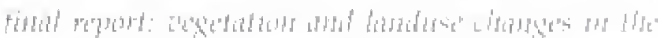

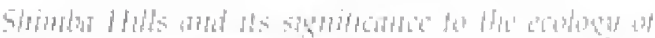

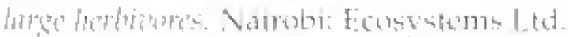

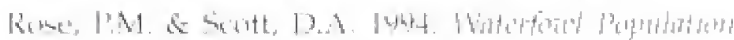

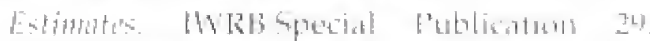

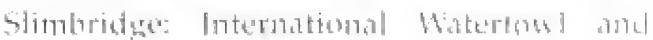

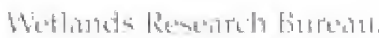

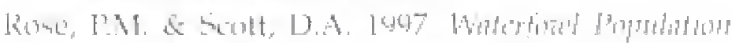

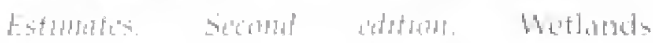

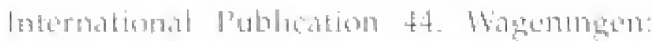

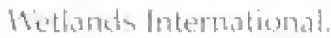

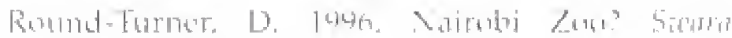
19(6):2(4) 1): 12-1:

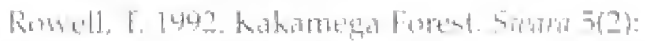

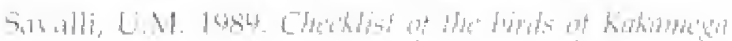

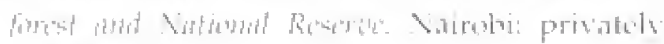
probliationt

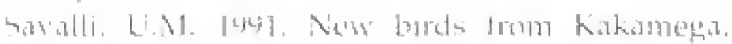

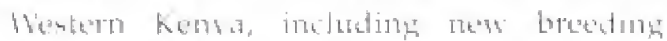

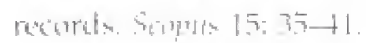

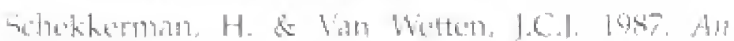

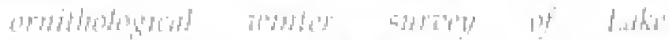

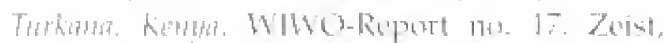
Nethordanda: Butch Working Goup an

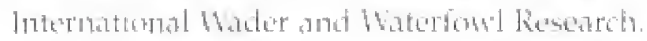

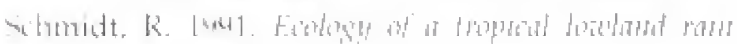

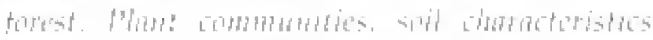

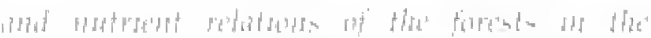

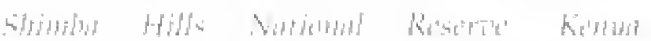

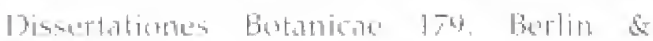

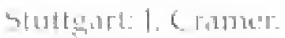

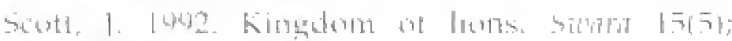

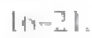

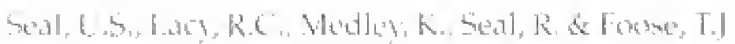

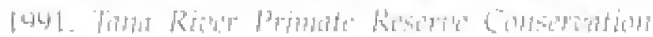

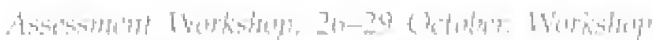

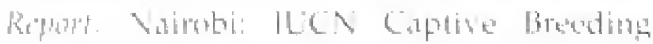
Spretialis Gorotup.

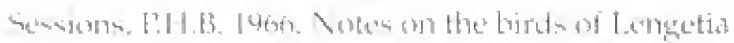

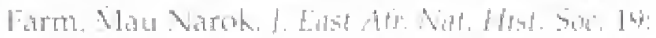
$43-6$.

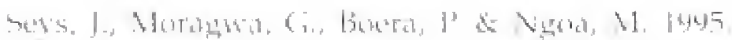
Distributang and abundance af birds in titat

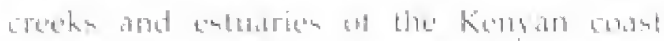

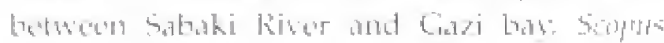

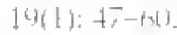

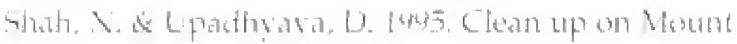
ketrua. me

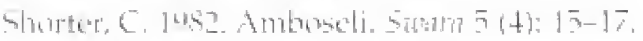

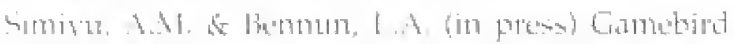

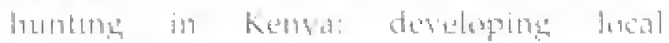

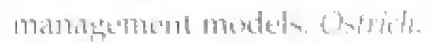

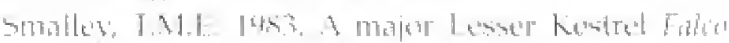

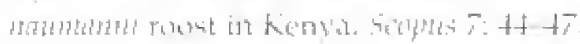

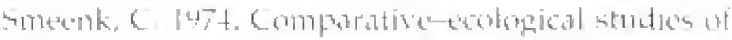

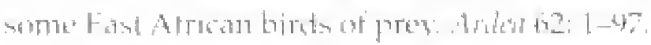

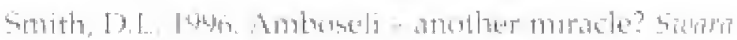
$10(2)=18-21$.

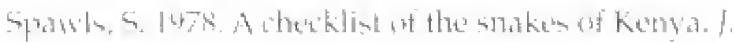

\section{References}




\section{References}

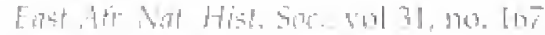

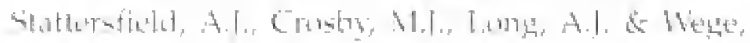

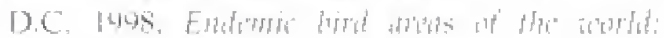

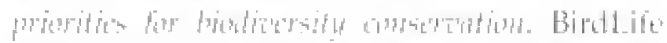

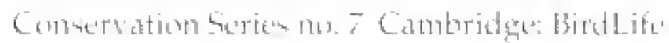
Inturmationtel.

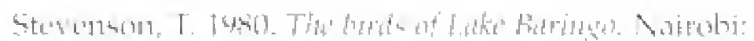

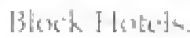

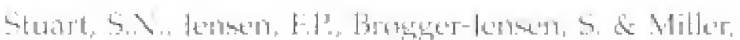

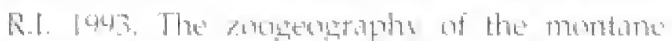

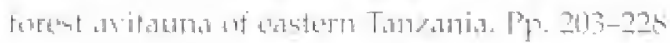

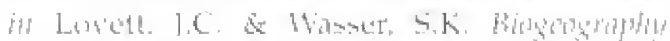

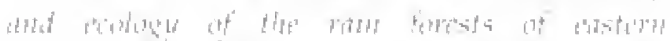

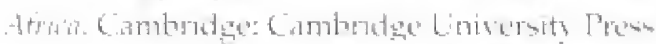

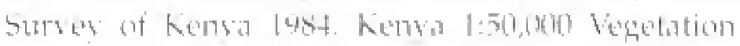

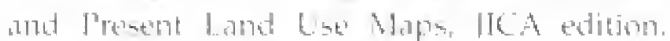
Vairatri burs's al kens.

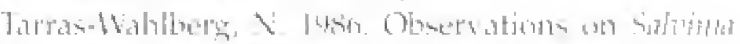

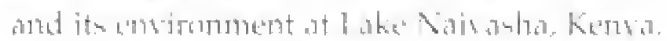

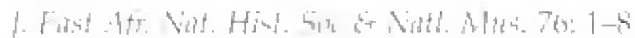

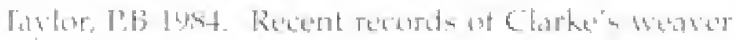

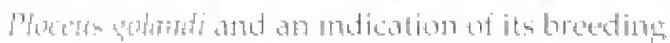

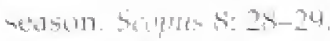

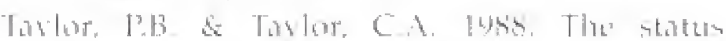

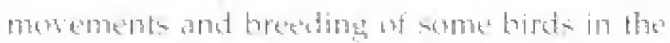

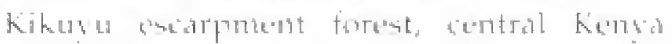

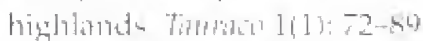

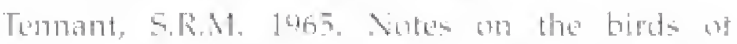

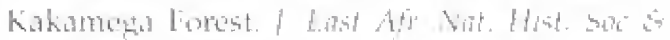

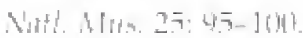

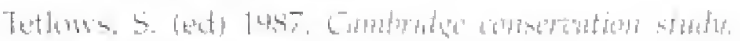

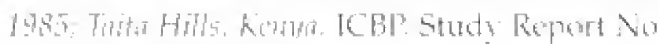
14. Cambridge: IC BP.

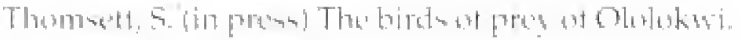

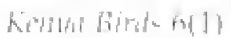

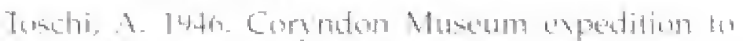

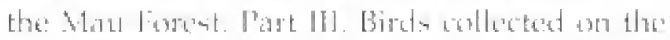

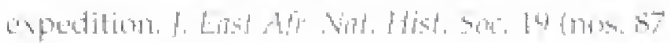
\& $885,43-94$

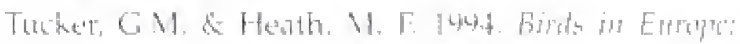

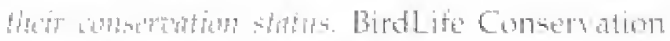

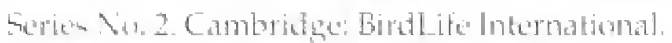

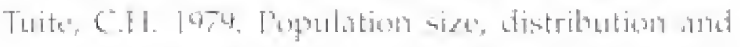

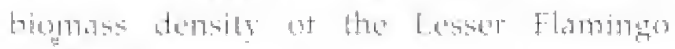

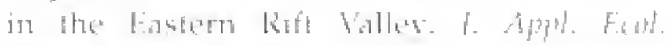
In: $70,7=5$

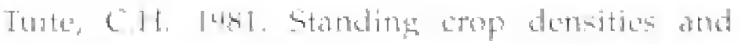

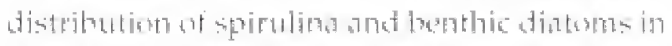

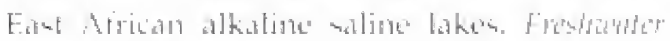
Bithente 11:3-35-3601

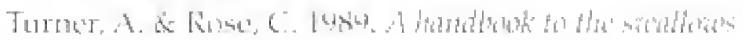

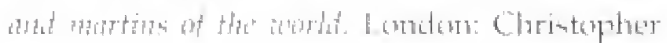
Hellon.

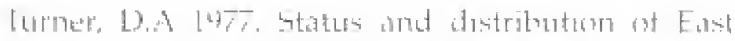

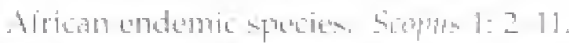

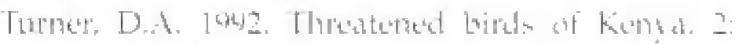

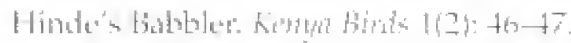

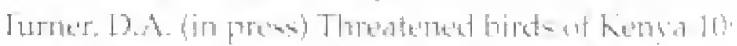

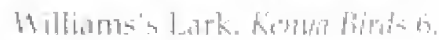

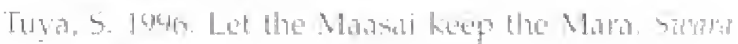
14t: :

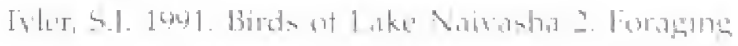

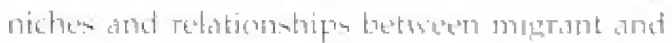

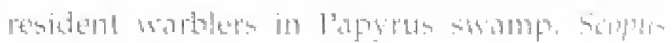
$1+(2): 117-124$.

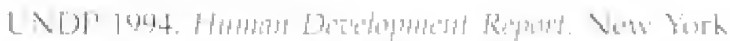

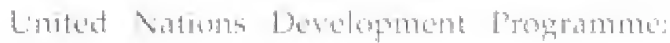

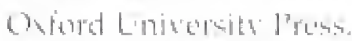

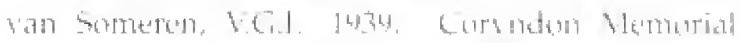

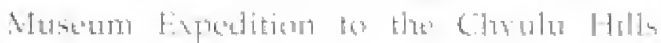

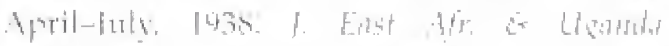

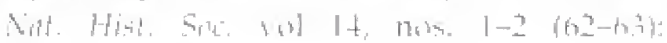
$1-151$

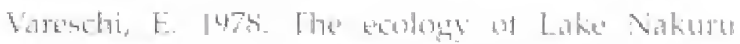

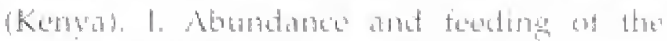

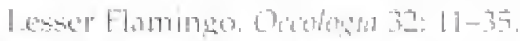

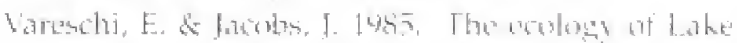

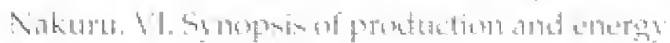

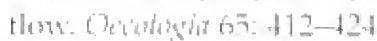

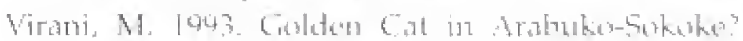

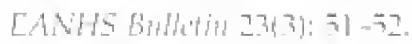

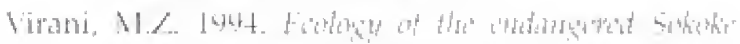

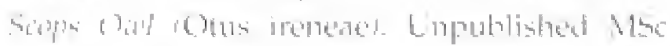

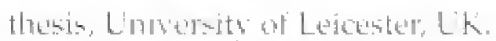

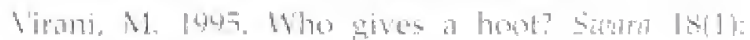
$24-2-$

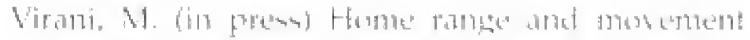

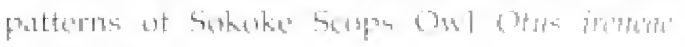

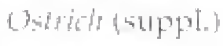

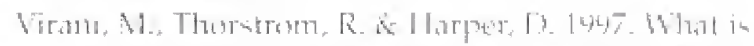

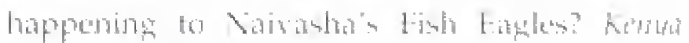
Birts $523: 45-100$.

Whanente, 1.M. 1642, Kistere Forest: a land of rart

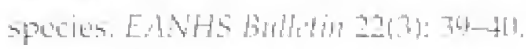

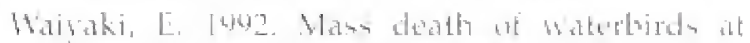

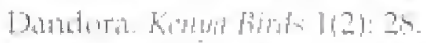

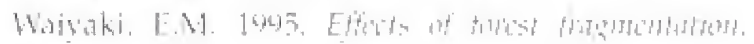

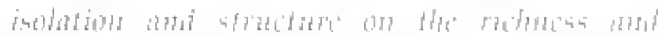

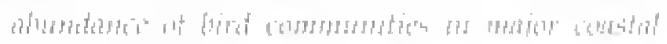

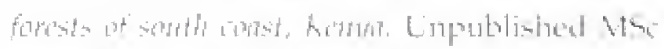

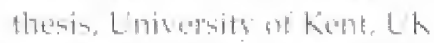

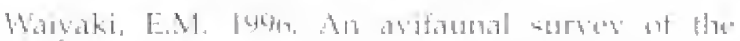

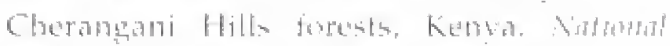

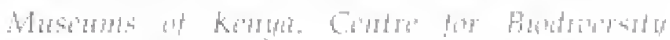

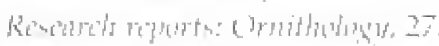

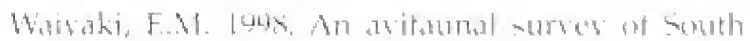




\section{Important Bird Areas in Kenva}

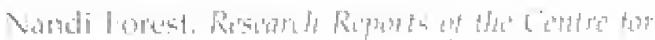

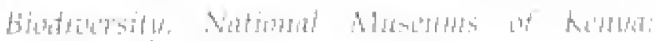

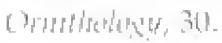

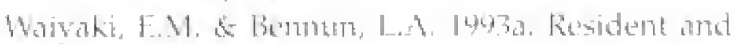

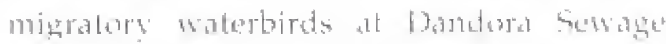

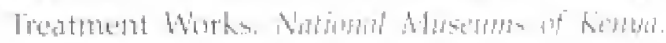

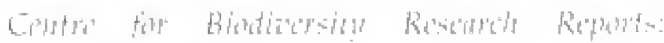
Ornithading, II

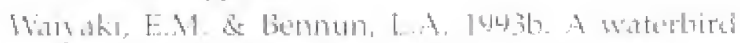

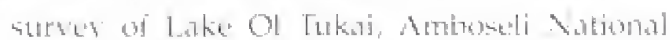

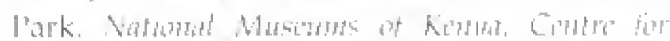

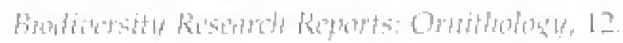

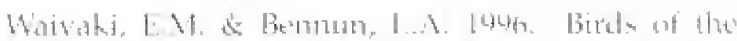

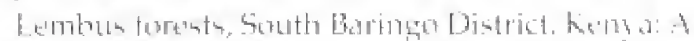

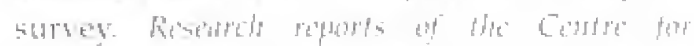

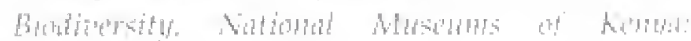
chmillowit. 24

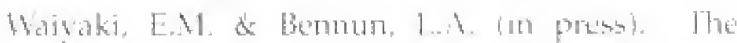
an ifauna of comstal fortats in southerm kimas:

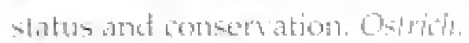

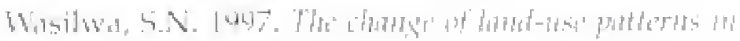

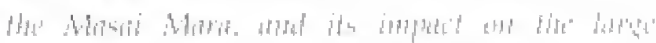

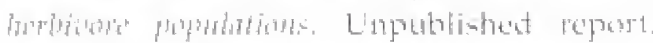
Wirabi: Wustat Wide Fund tor Vature.

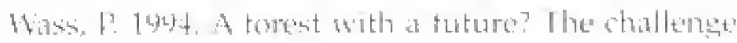

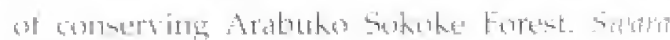
$17(3): 28-11$.

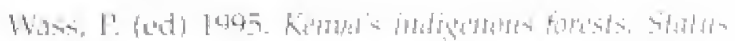

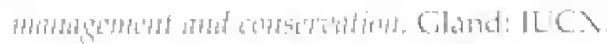

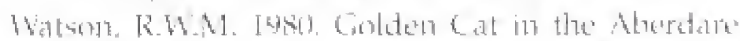

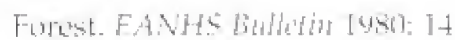

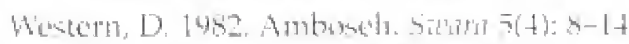

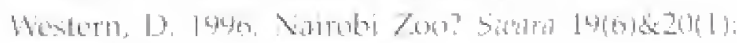
$14-20$.

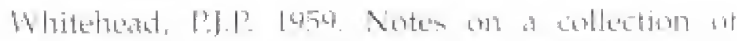

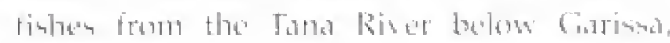

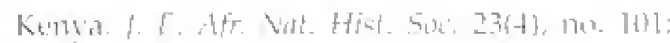
$167-171$.

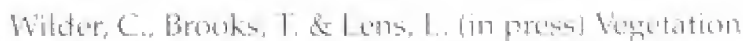

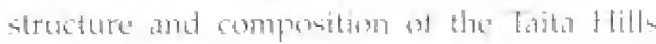

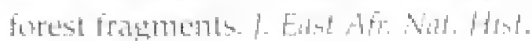

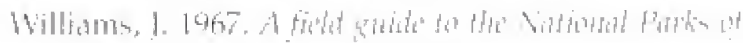

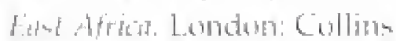

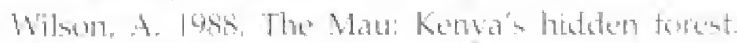

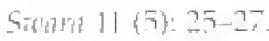

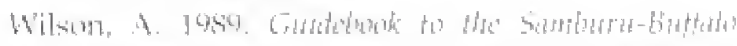

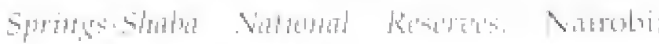

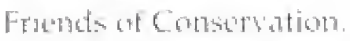

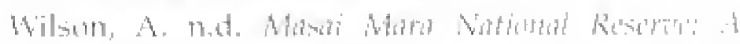

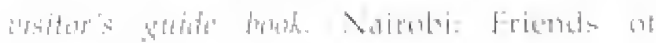
Corthetration,

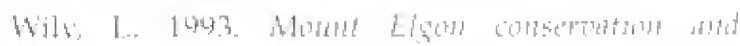

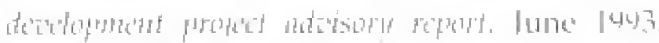
Nanoti: KIICON

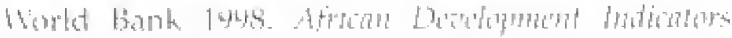

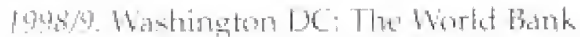

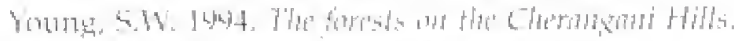

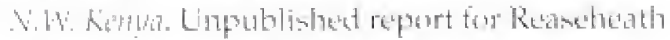
Callews: Nantsich Chethire, LK

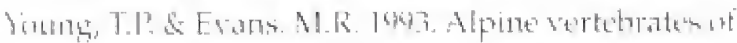

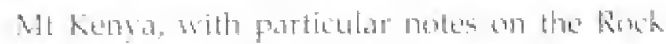

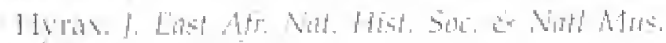
เด1 $82,062.202$.

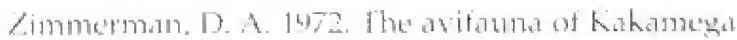

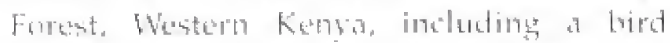

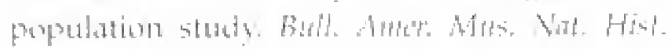
1-44: $254-339$.

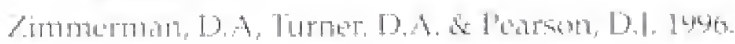

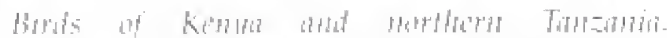

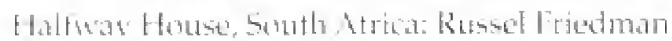
Books.

\section{References}


6 A pPEndices

Appendices 265 









Appendix 1A 267 


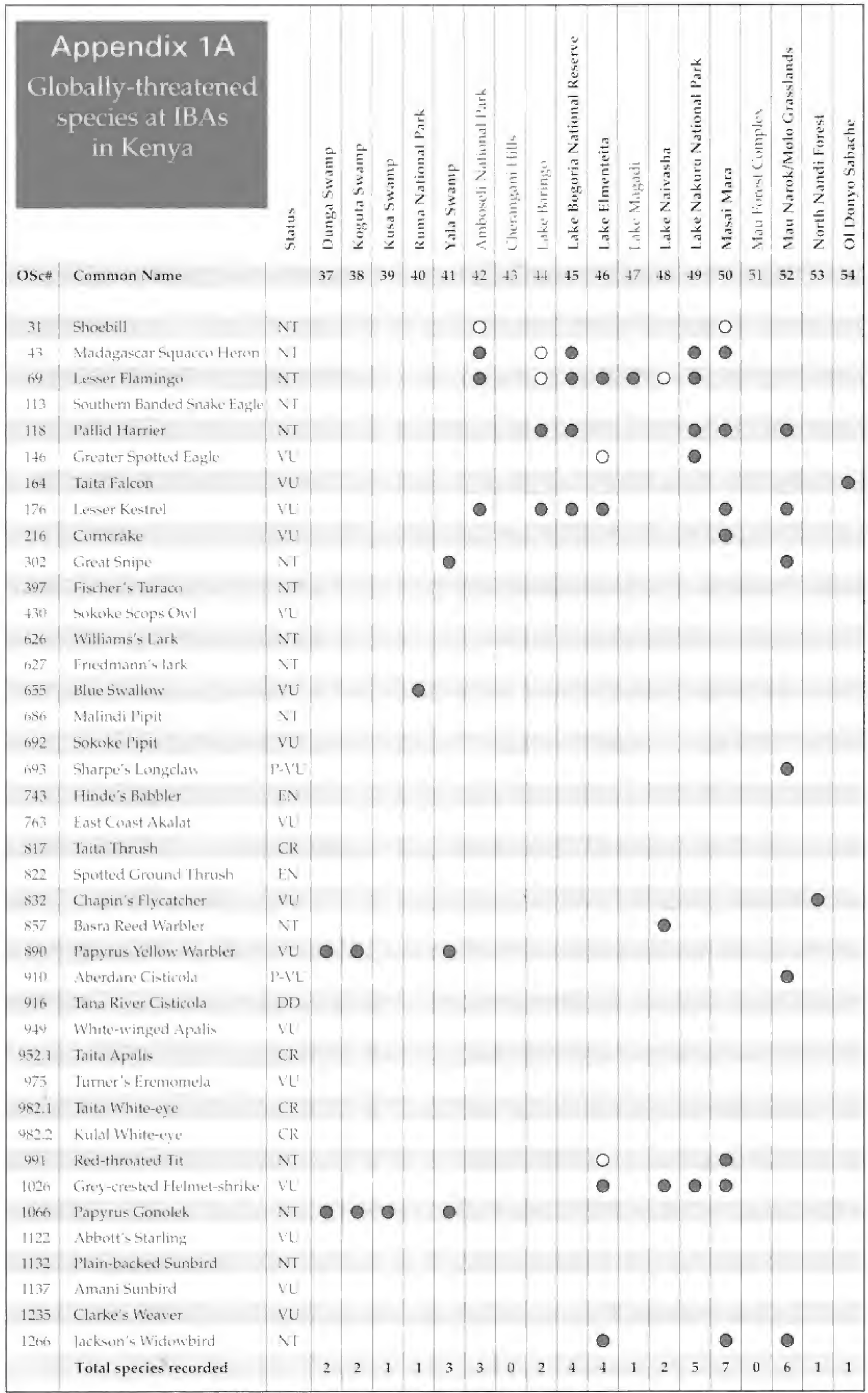




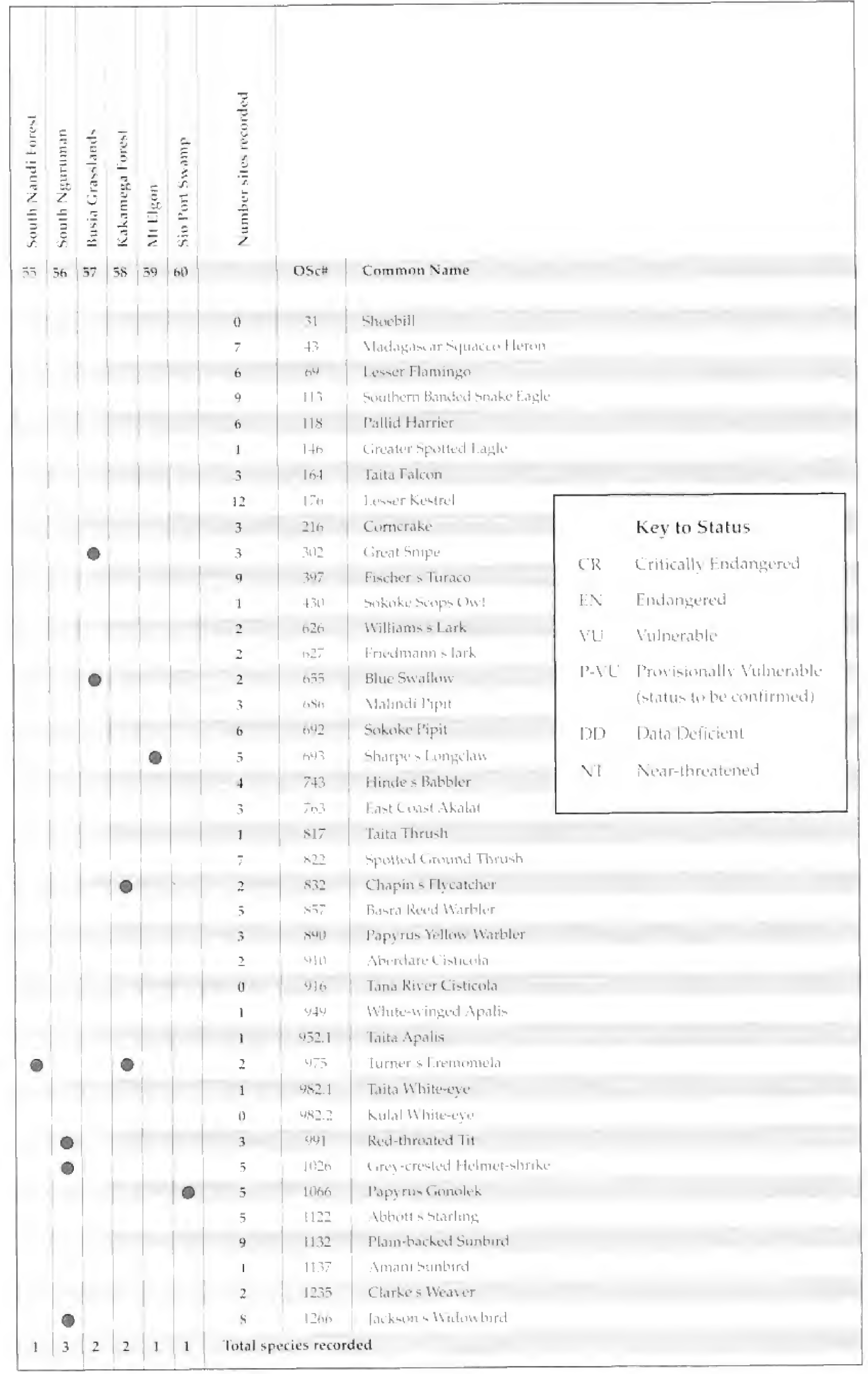

Appendix 1A 269 


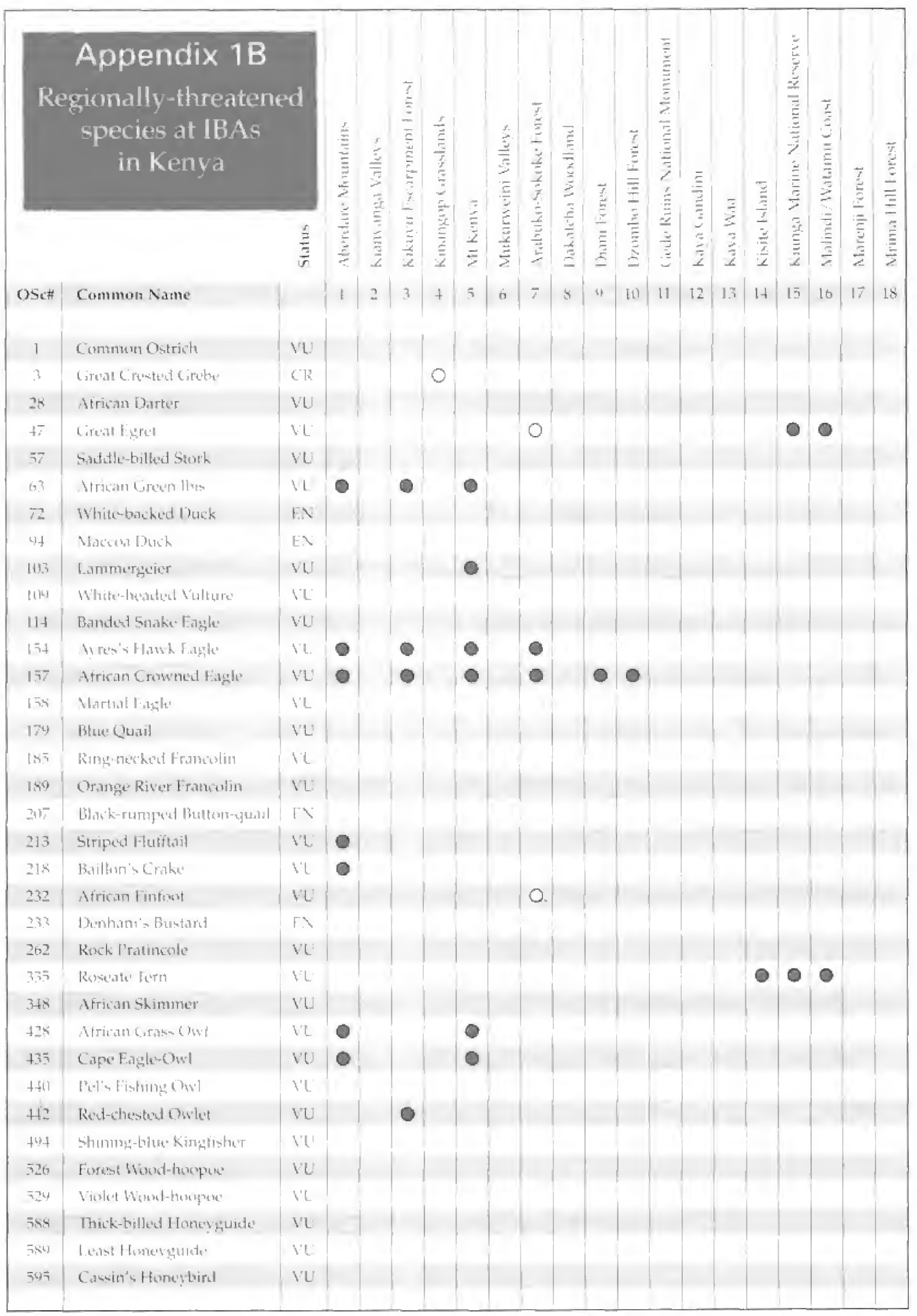




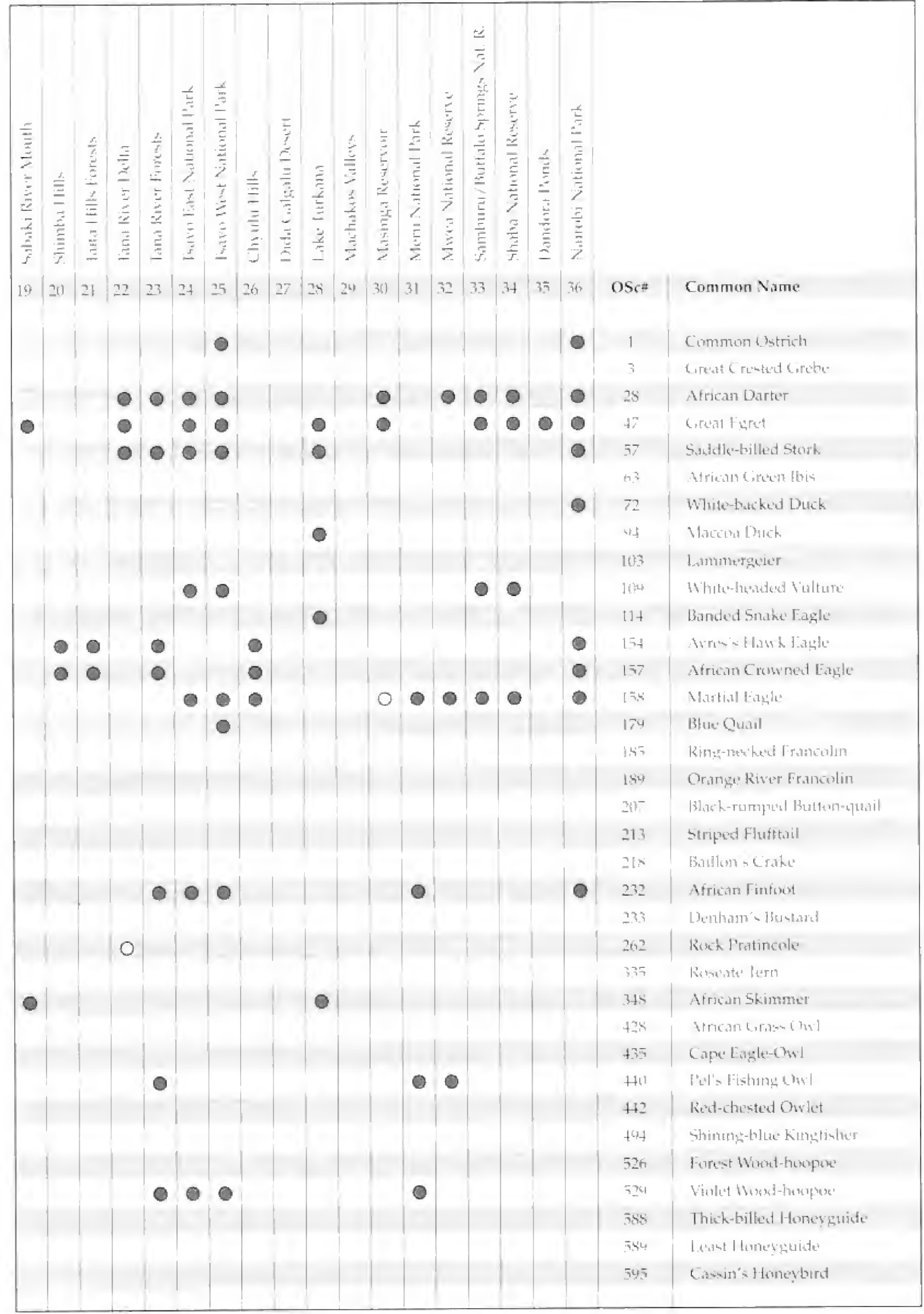




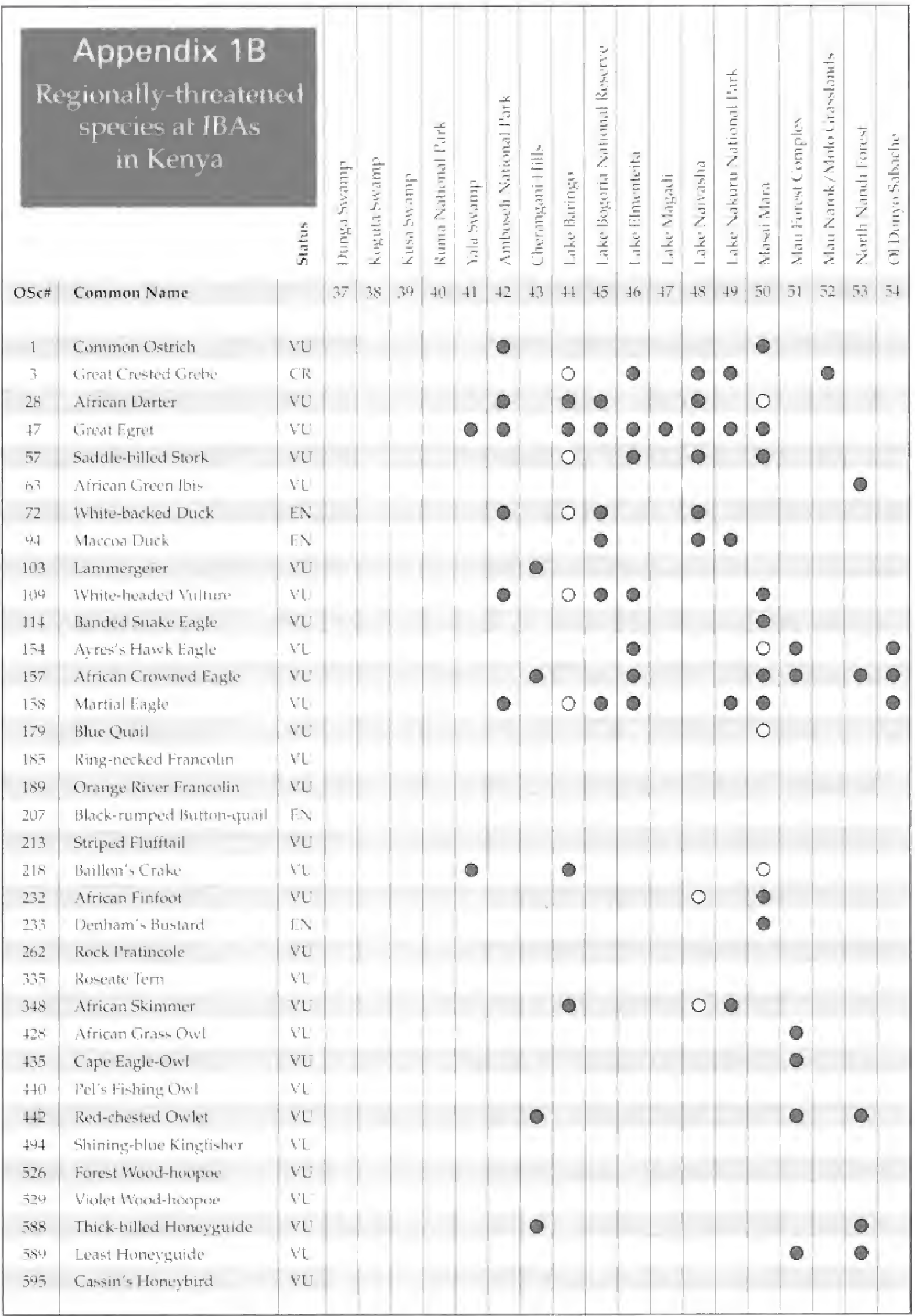




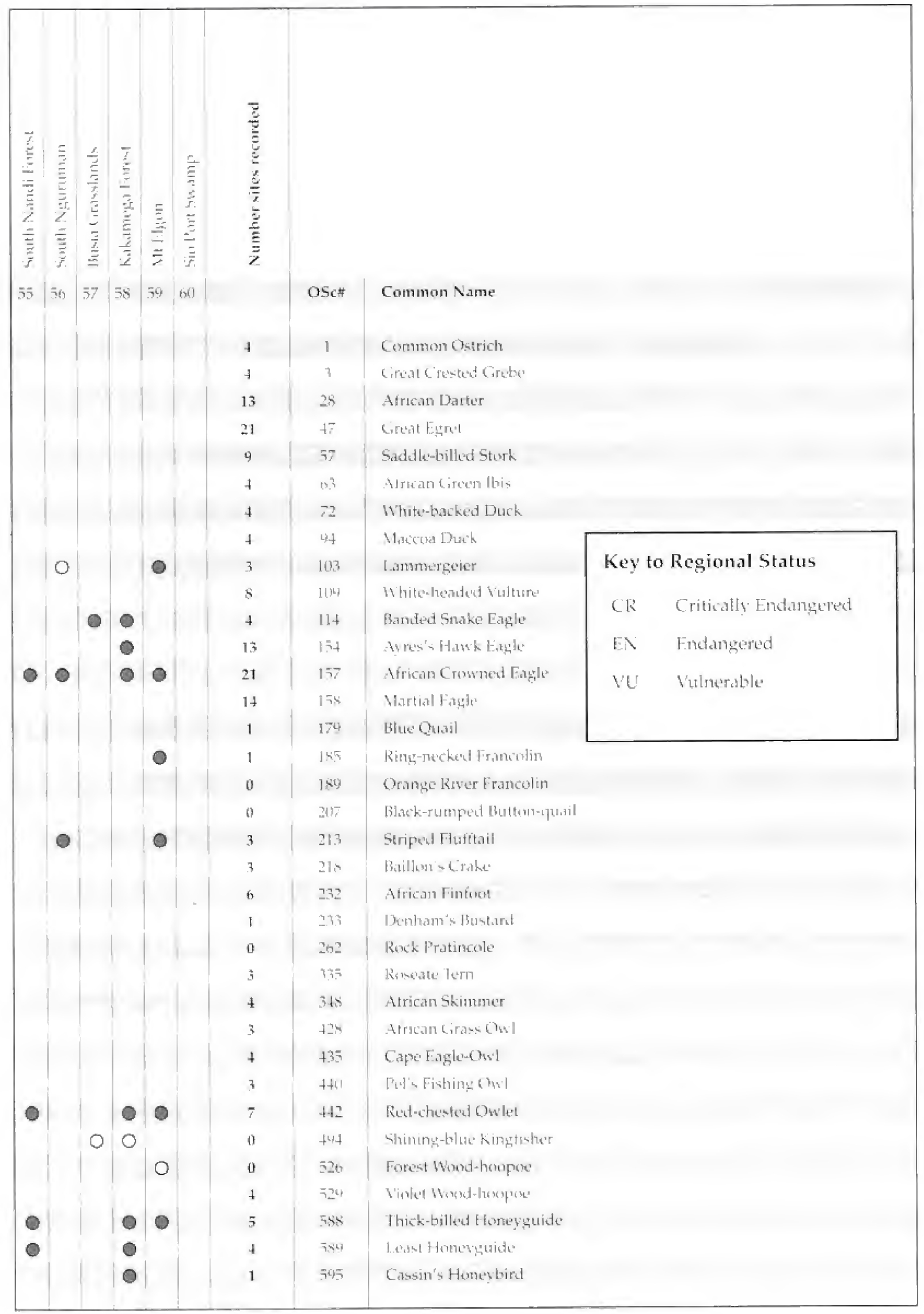




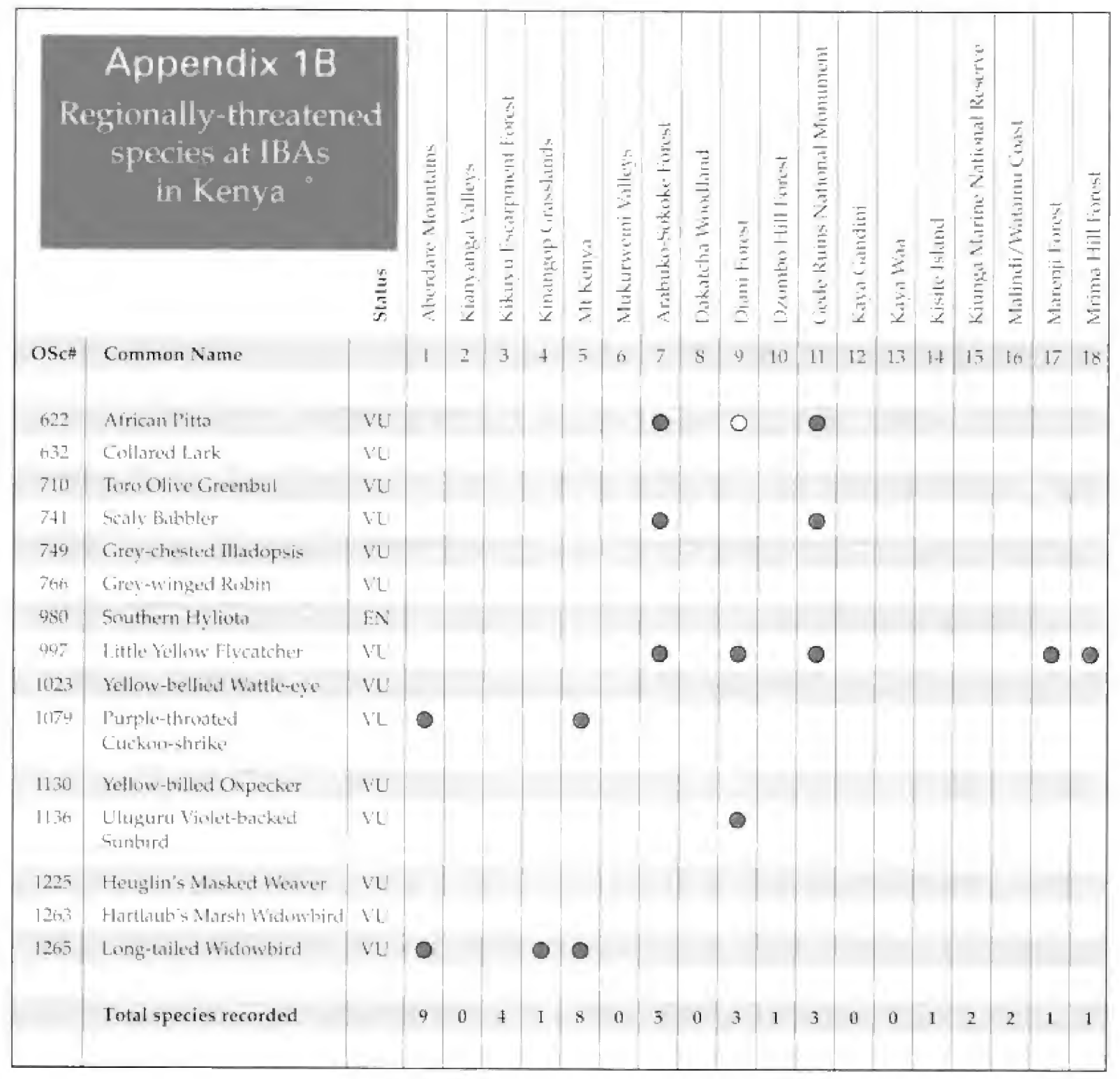




\begin{tabular}{|c|c|c|c|c|c|c|c|c|c|c|c|c|c|c|c|c|c|c|c|}
\hline 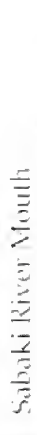 & 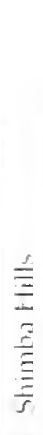 & 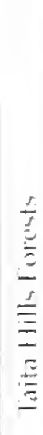 & 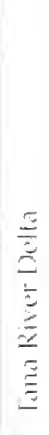 & 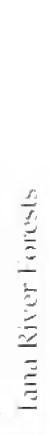 & 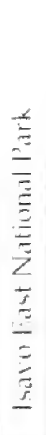 & 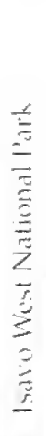 & $\begin{array}{l}\stackrel{5}{\equiv} \\
\equiv \\
\equiv \\
\equiv\end{array}$ & 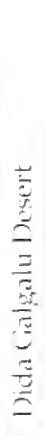 & 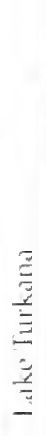 & 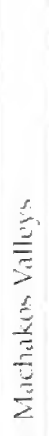 & 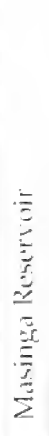 & 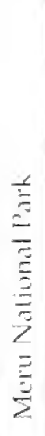 & 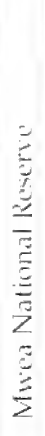 & 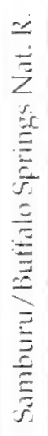 & 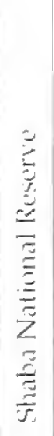 & 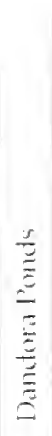 & 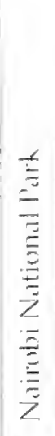 & & \\
\hline 19 & 20 & 21 & 22 & 23 & 24 & 25 & 26 & 27 & 28 & 29 & 30 & 31 & 32 & 33 & $3+4$ & 35 & 36 & $\mathrm{OSc} \#$ & Common Name \\
\hline & 0 & & & & & & & & & & & & & & & & & 622 & African Pitta \\
\hline & & & & & & & & & & & & & & & & & & 6.32 & Coflared Lark \\
\hline & & & & & & & & & & & & & & & & & & 710 & Toro Olive Greenbul \\
\hline & & & 0 & 0 & & & & & & & & & & & & & & $7+1$ & Scaly Babbler \\
\hline & & & & & & & & & & & & & & & & & & 749 & Grey-chested Illadopsis \\
\hline & & & & & & & & & & & & & & & & & & $7(x)$ & Grey-winged Robin \\
\hline & & & & & & & & & & & & & & & & & & 980 & Southern Hyliota \\
\hline & 0 & & & 0 & & & & & & & & & & & & & & 947 & Little Yellow Flycatcher \\
\hline & & & & & & & & & & & & & & & & & & 1023 & Yellow-bellied IVattle-eye \\
\hline & & & & & & & & & & & & & & & & & & $1(1,-4)$ & $\begin{array}{l}\text { Purple-throated } \\
\text { Cuckoo-shrike }\end{array}$ \\
\hline & & & & & 0 & 0 & & & & & & & & 0 & 0 & & 0 & 1130 & Yellow-billed Oxpecker \\
\hline & 0 & & & 0 & & & & & & & & & & & & & & 1136 & $\begin{array}{l}\text { Ulururu Violet-bached } \\
\text { Sunbird }\end{array}$ \\
\hline & & & & & & & & & & & & & & & & & & 1225 & Heuglin's Masked Weaver \\
\hline & & & & & & & & & & & & & & & & & & 1263 & Hartlaut's Marsh Widoubird \\
\hline & & & & & & & & & & & & & & & & & & 1265 & Lnng-tailed Widowbird \\
\hline 2 & 5 & 2 & 4 & 10 & 7 & 10 & 3 & 0 & 5 & 0 & 2 & 4 & 3 & 5 & 5 & 1 & 10 & \multicolumn{2}{|c|}{ Total species recorded } \\
\hline
\end{tabular}




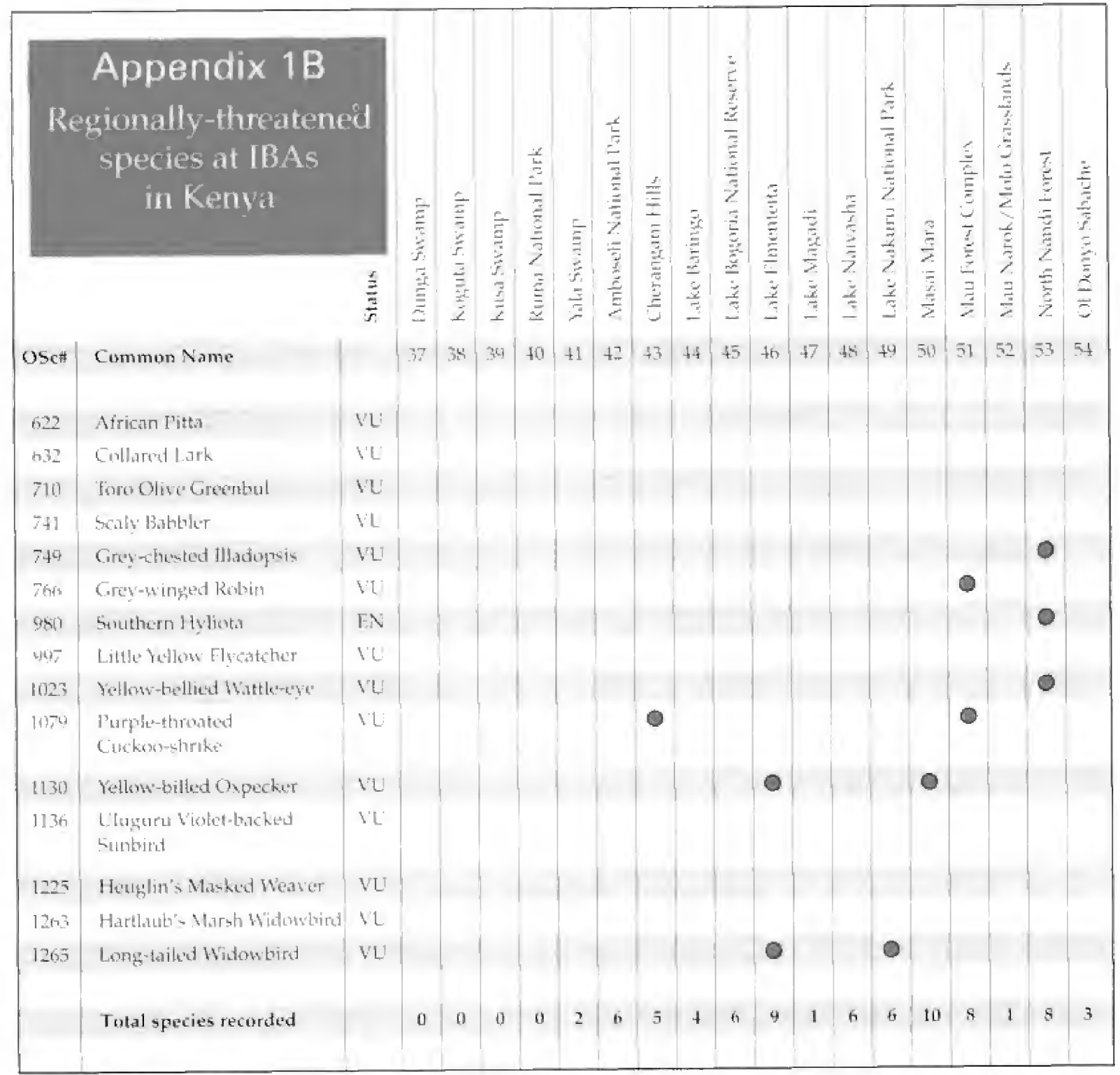




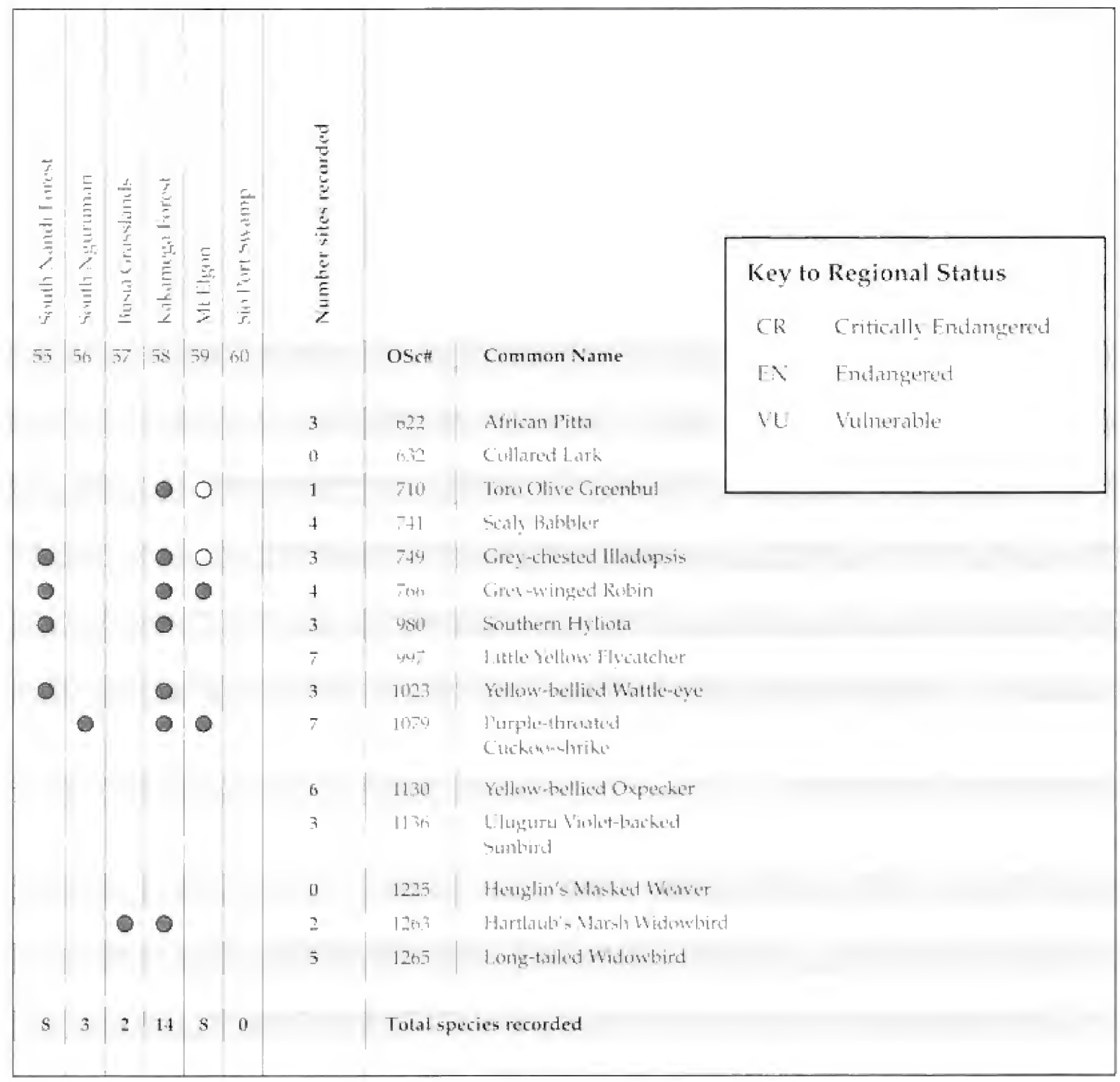



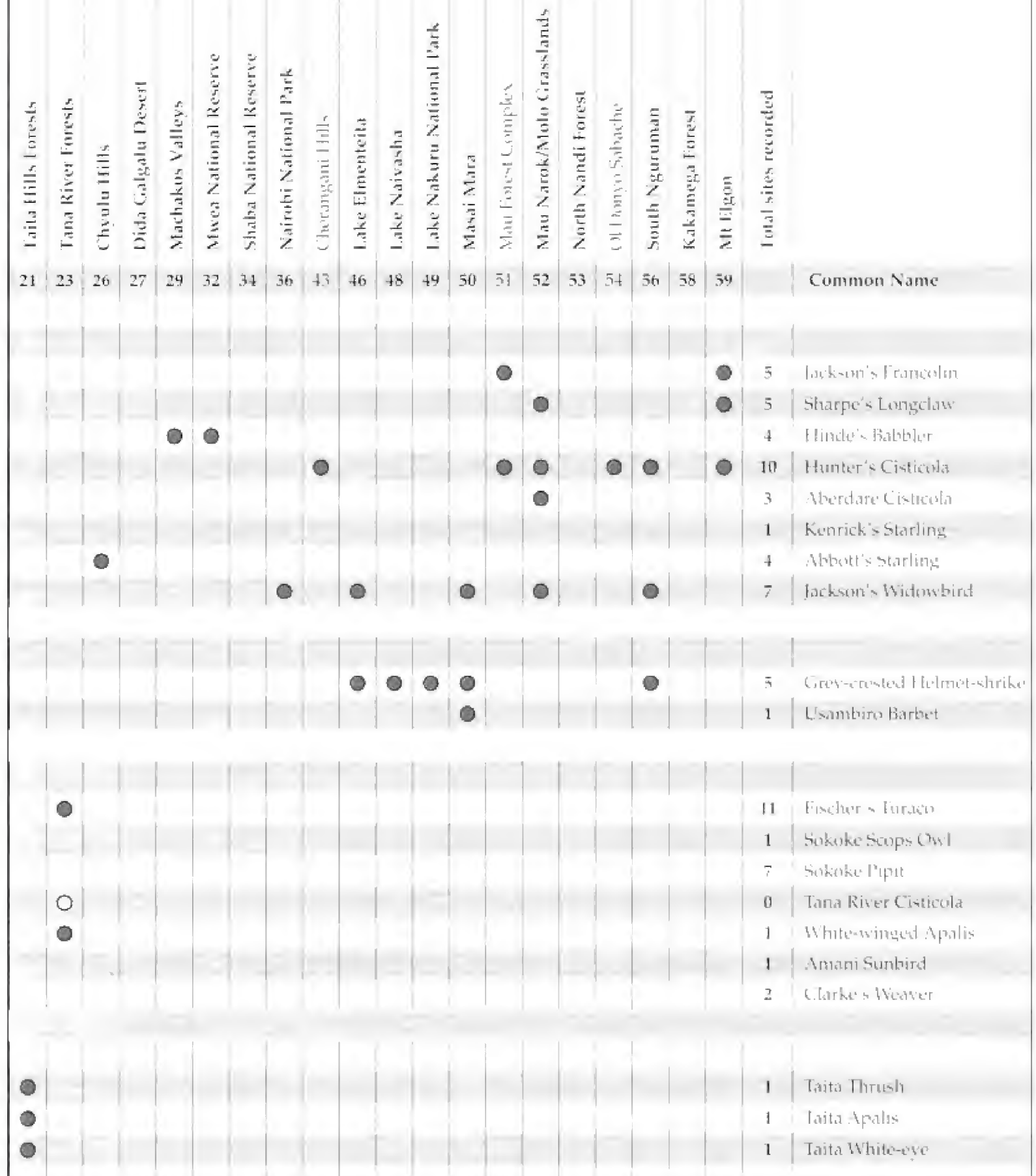


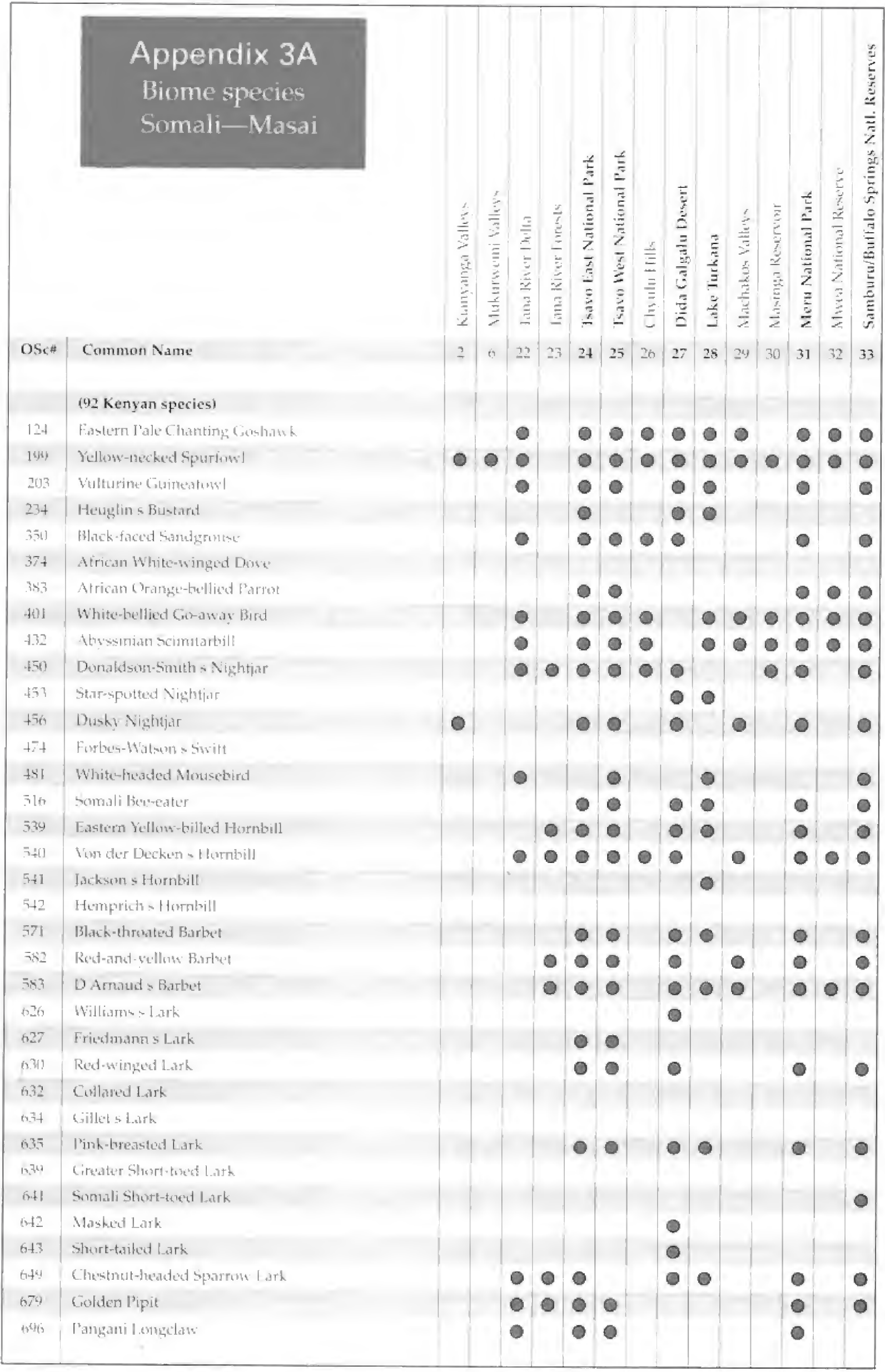




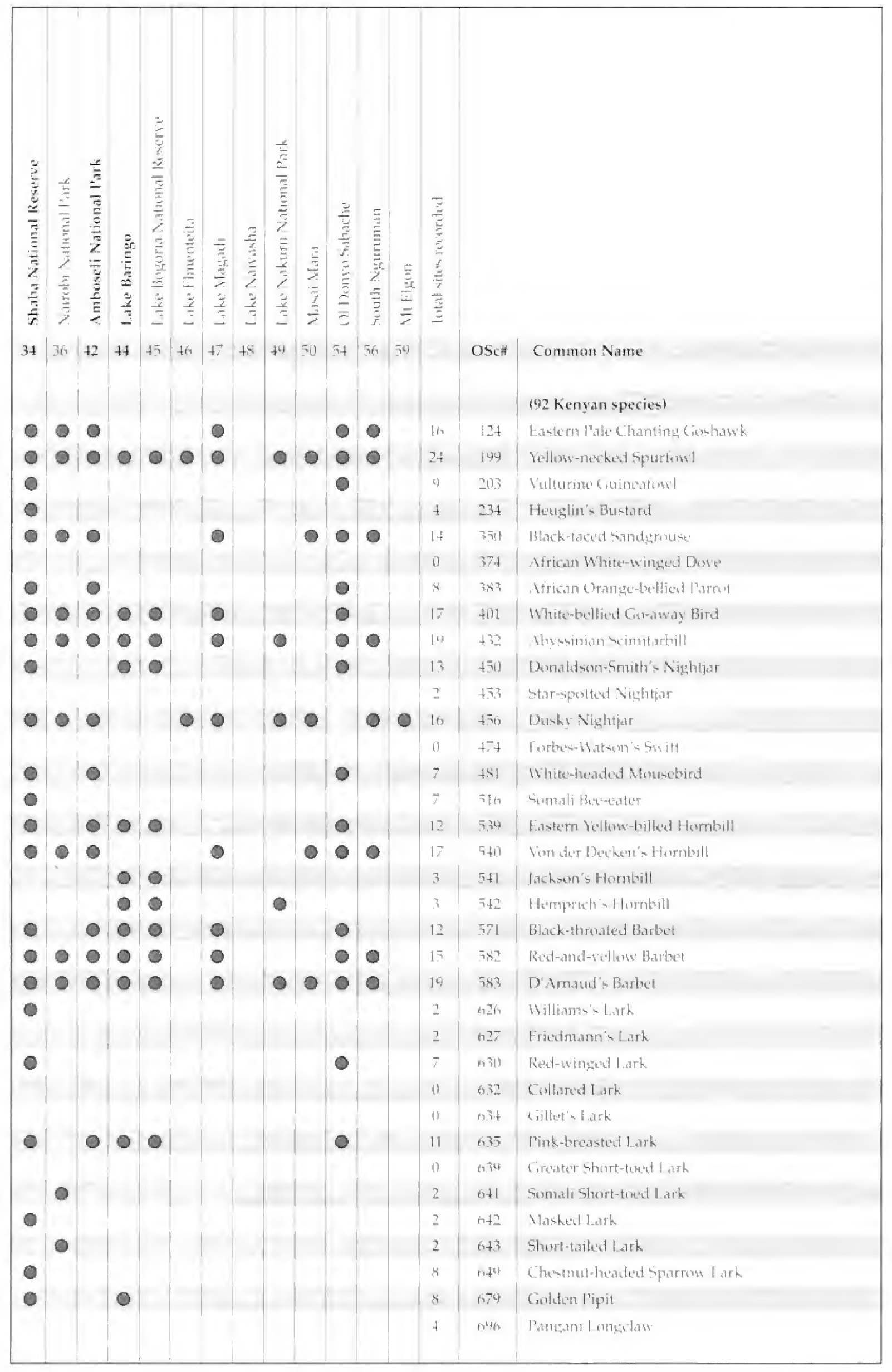

Appendix 3A 281 


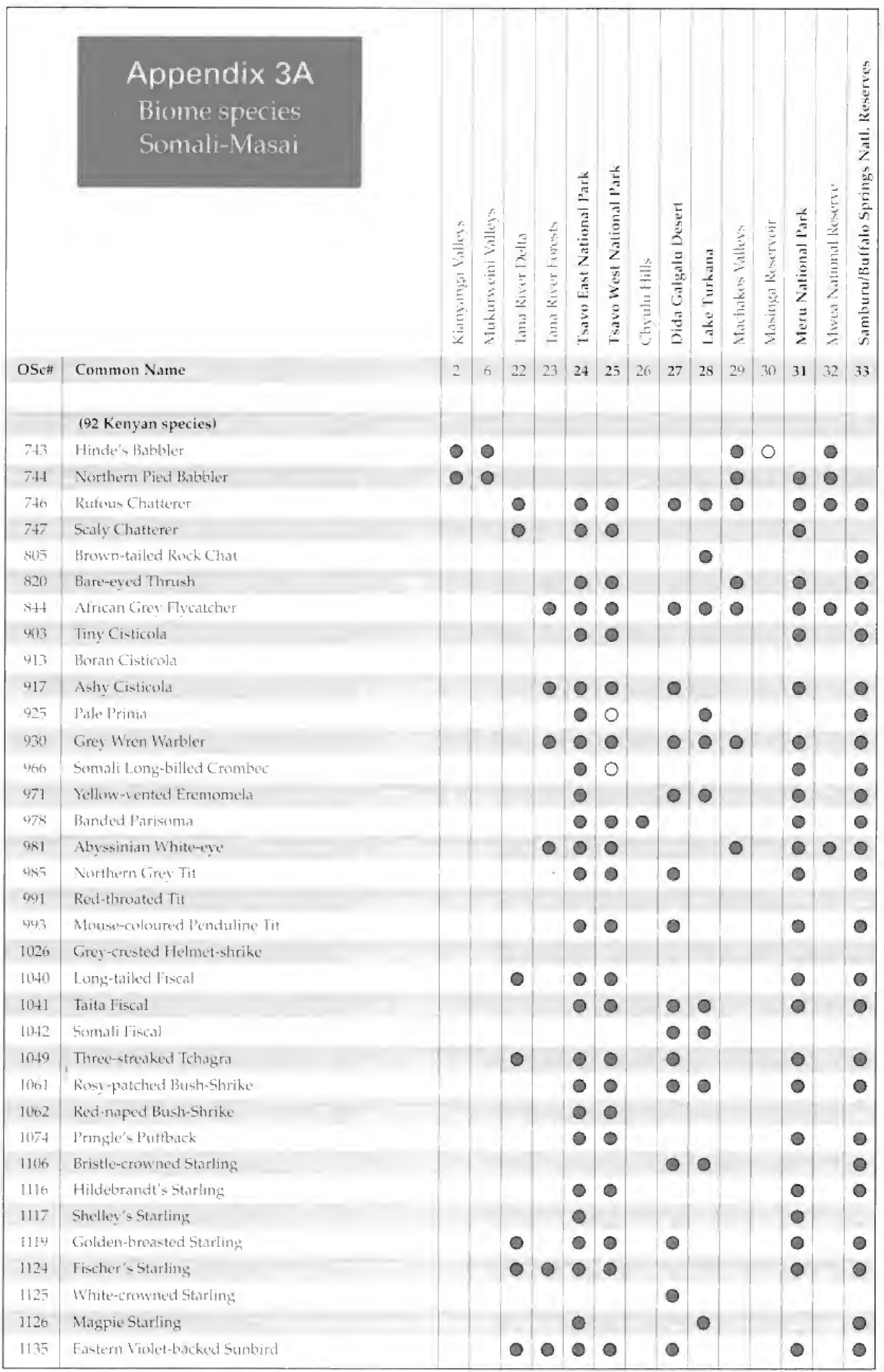




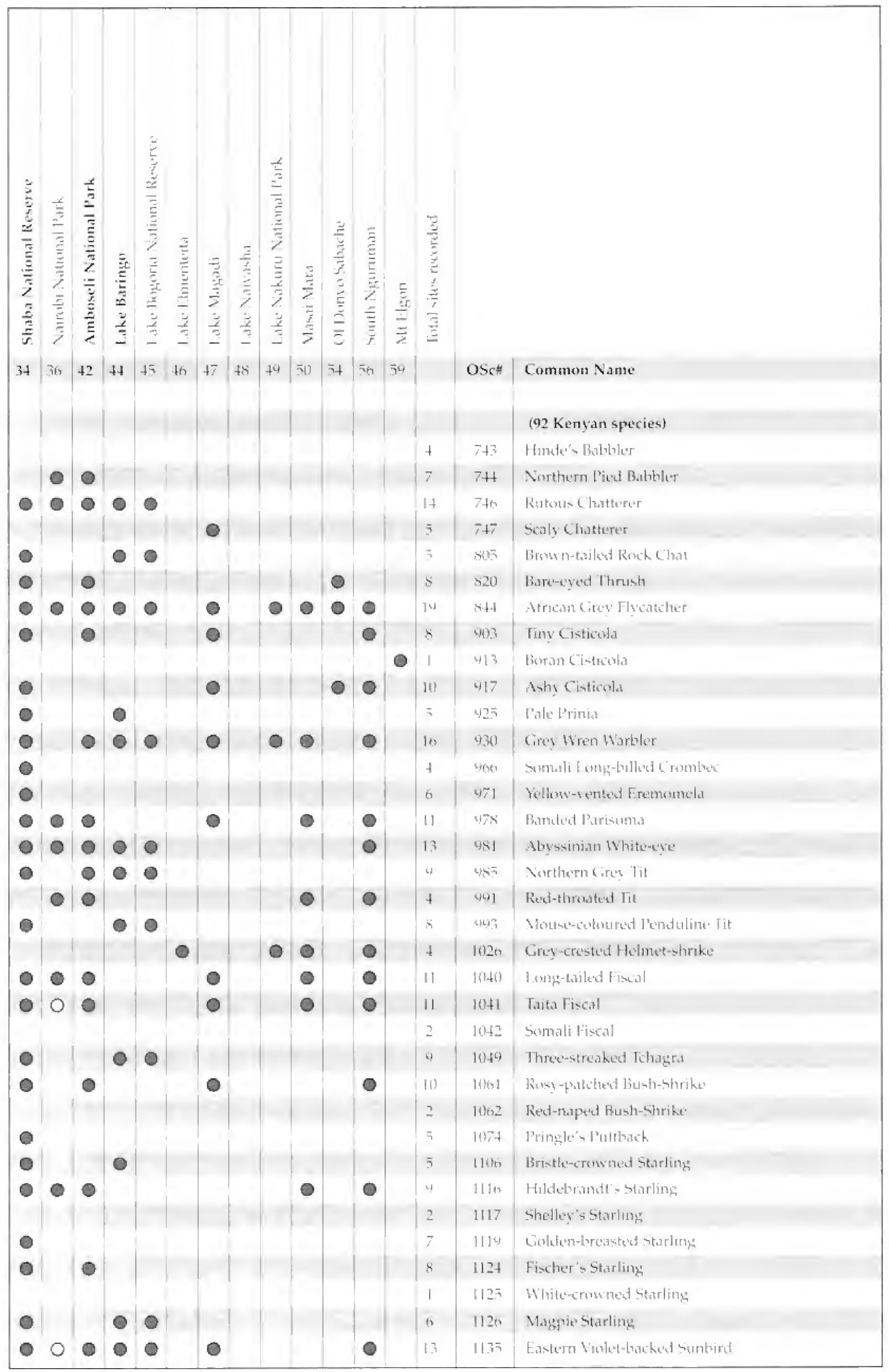

Appendix 3A 283 


\begin{tabular}{|c|c|c|c|c|c|c|c|c|c|c|c|c|c|c|c|}
\hline & $\begin{array}{l}\text { Appendix 3A } \\
\text { Biome species } \\
\text { Somali-Masai }\end{array}$ & 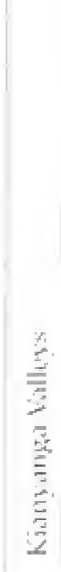 & 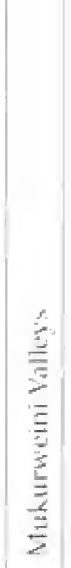 & 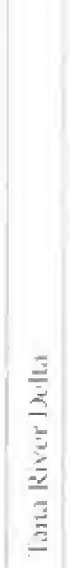 & 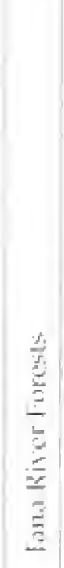 & 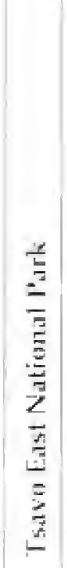 & 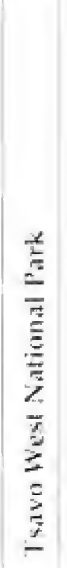 & 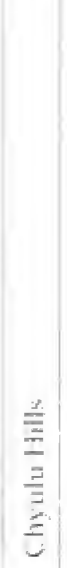 & 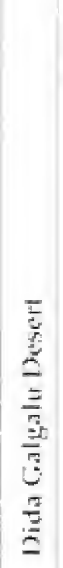 & 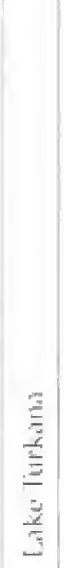 & 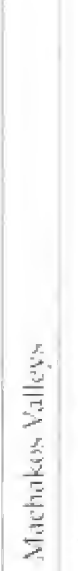 & 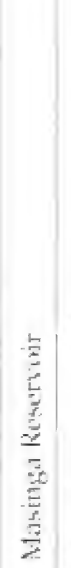 & 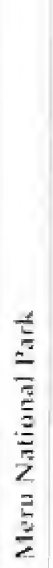 & 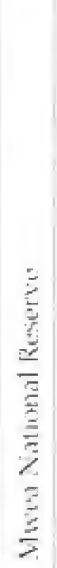 & 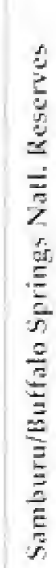 \\
\hline \multirow[t]{2}{*}{ OS $\#$} & Common Name & 2 & i & 22 & 23 & 24 & 25 & 26 & 27 & 28 & $29 \mid$ & 30 & 31 & 32 & 3,3 \\
\hline & (92 Kenyan species) & & & & & - & - & & & & & & & & \\
\hline 11 디 & Hunter's Sunbird & & & & & 0 & 0 & & 0 & 0 & & & 0 & & 0 \\
\hline 1171 & Black-bellied Sunbirt & & & & 0 & 0 & 0 & & & & & & 0 & & 0 \\
\hline 1174 & Shining Suntird & & & & & & & & & 0 & & & 0 & & 0 \\
\hline $11 \mathrm{~B}$ & Sondali Spantrom & & & & & & & & & 0 & & & & & \\
\hline 1193 & White-heated Buitabe Fhadser & & & ○ & 0 & 0 & 0 & & 0 & 0 & & & 0 & & 0 \\
\hline 1196 & Donalkson-Smith's Sparrew lyeater & & & & & 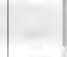 & & & 0 & 0 & & & & & 0 \\
\hline 1201 & 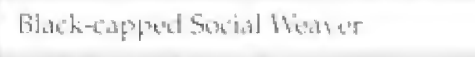 & & & & & 0 & - & & & & & & 0 & & ○ \\
\hline 1216 & Golden Palm Weraver & & & 0 & 0 & & & & & & & & 0 & & 0 \\
\hline 1217 & 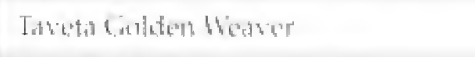 & & & & & & 0 & & & & & & & & \\
\hline 1221 & Rüppenl's Weaver & & & & & & & & & & & & & & \\
\hline 1283 & Juba heaver & & & & & & & & & & & & & & \\
\hline 1230 & Speke's Weaver & 0 & & & & & & & & & 0 & & & & \\
\hline 1252 & 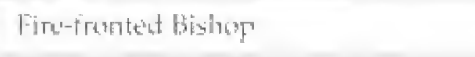 & & & & & 0 & 0 & & & & & & ○ & & 0 \\
\hline 1310 & Bhue-capped Corduntbleu & & & & & 0 & 0 & & - & & & & 0 & & 0 \\
\hline 1311 & 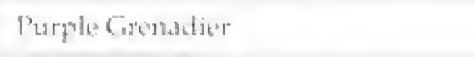 & & & 0 & & 0 & 0 & & 0 & ○ & 0 & & 0 & 0 & 0 \\
\hline 1317 & Grey-lweated silver-bill & & & & & 0 & 0 & & & & & & 0 & & 0 \\
\hline 1377 & Sted-blat Whydah & & & & & 0 & & & ○ & & & & 0 & 0 & 0 \\
\hline 1,329 & Straw-tailed Whycdah & & & & & 0 & 0 & & 0 & & 0 & & 0 & & 0 \\
\hline $137 \mathrm{n}$ & Whete-betled Camary. & & & & & 0 & 0 & & & & & & & & 0 \\
\hline 1338 & Southern Grostuak Canary & & & & & . & 0 & & & & & & & & \\
\hline 1324 & 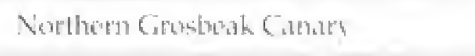 & & & & & & & & 0 & 0 & & & & & \\
\hline 1335 & Sontali Goldertbreastesd Funting & & & & & 0 & 0 & & 0 & 0 & & & 0 & & 0 \\
\hline & lintal species recotded & 5 & 3 & 22 & 16 & 60 & 55 & 8 & 44 & 34 & 18 & +3 & 57 & 14 & 61 \\
\hline
\end{tabular}




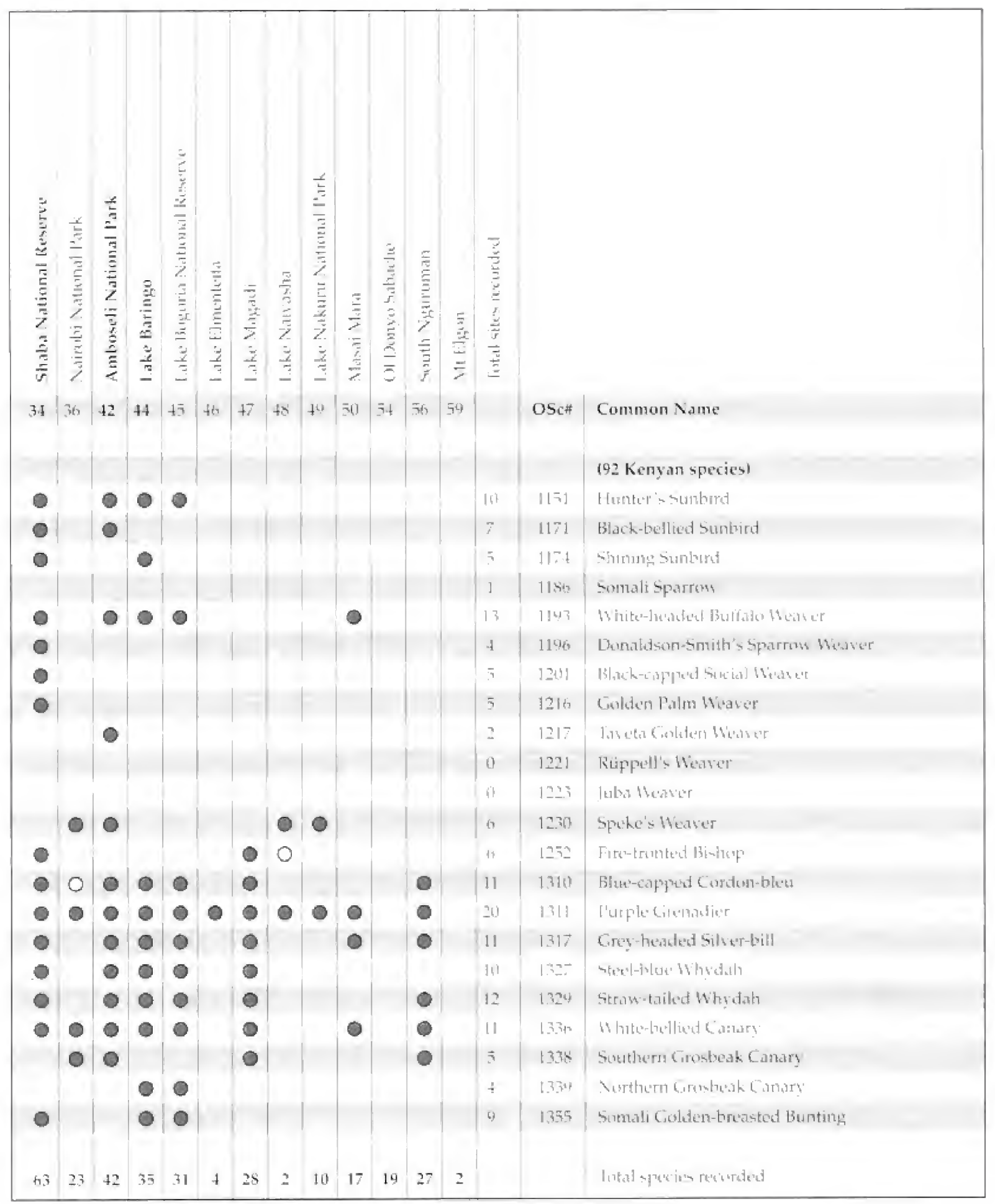

Appendix 3A 285 


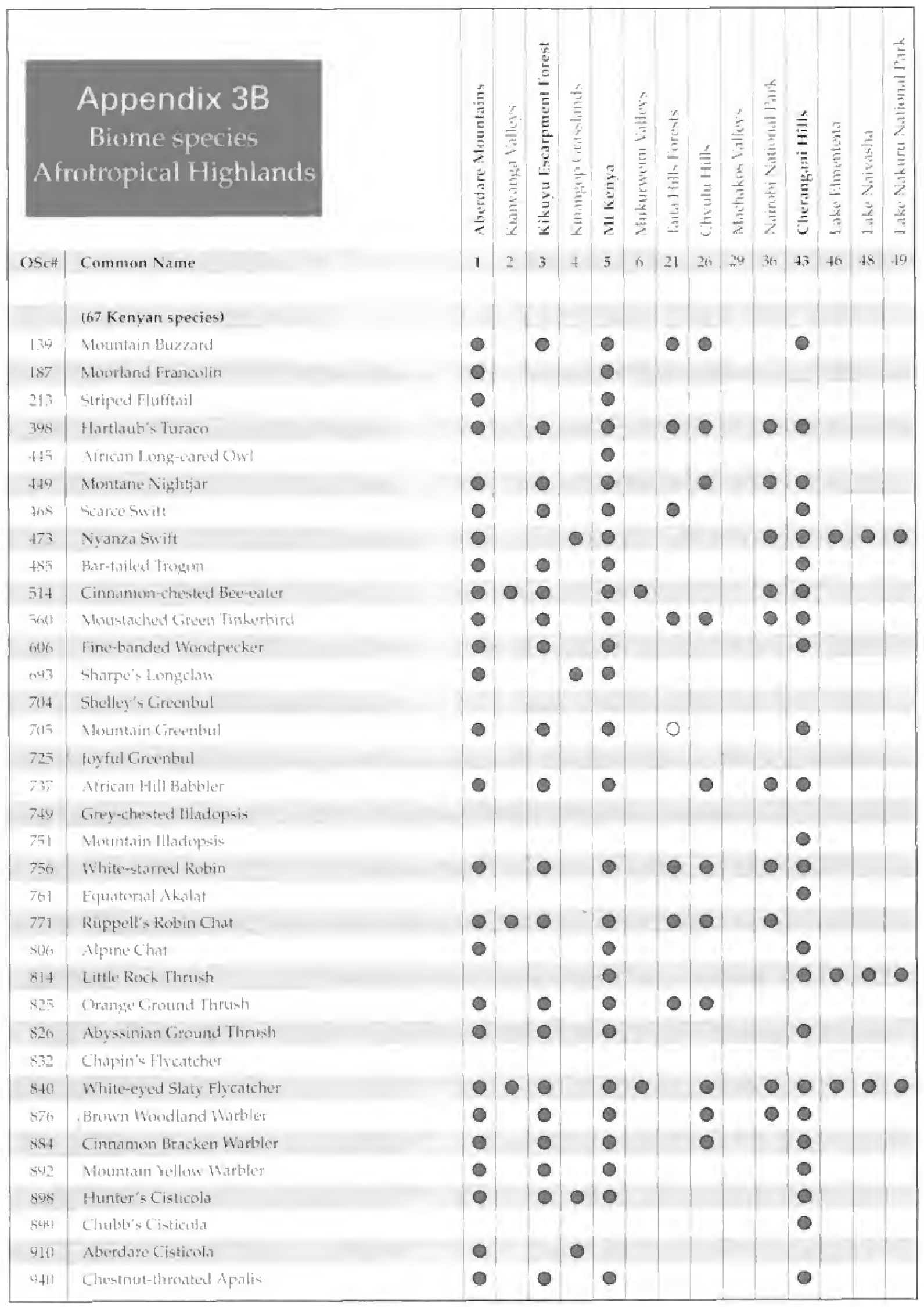




\begin{tabular}{|c|c|c|c|c|c|c|c|c|c|c|c|}
\hline 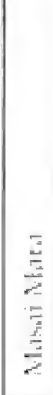 & 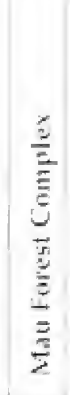 & 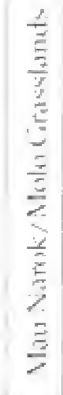 & 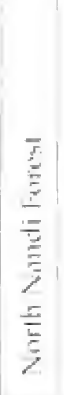 & 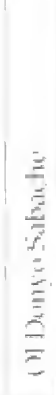 & 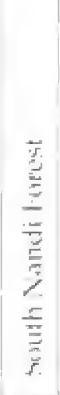 & 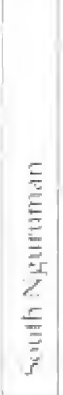 & 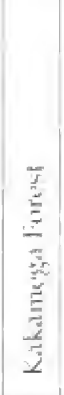 & 音 & 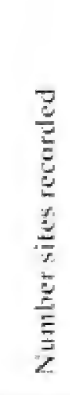 & & \\
\hline \multirow[t]{7}{*}{50} & 51 & 52 & 53 & 54 & 55 & Th & $5 s$ & 59 & & OSt $\#$ & Common Name \\
\hline & & & 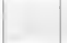 & & 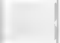 & & 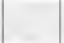 & & & & (67 Kenyan Species) \\
\hline & - & - & & & & & 0 & 0 & 10 & $13 \%$ & Nountain Fumpard \\
\hline & \multirow[t]{2}{*}{0} & O & & 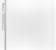 & & 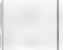 & & 0 & 3 & 187 & Mimrland Francolin \\
\hline & & & & & & 0 & & 0 & 4 & 213 & Stripes laftut \\
\hline & \multirow[t]{2}{*}{0} & & 0 & & 0 & 0 & 0 & 0 & 12 & 398 & Hartlaub's Teraces \\
\hline & & & & & & & & & 1 & $+4=$ & 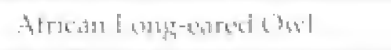 \\
\hline 0 & 0 & 0 & & & & 0 & 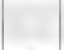 & 0 & 10 & 449 & Mont:arse Nightipar \\
\hline 0 & 0 & & & & & & 0 & 0 & 9 & 400 & Sourcestsitt \\
\hline \multirow[t]{2}{*}{ 0 } & 0 & ○ & & & & & & & 11 & 473 & Ny̆anza swif́ \\
\hline & 0 & & & & 0 & & 0 & ○ & $s$ & 485 & Bor-tailed Iromgan \\
\hline \multirow[t]{2}{*}{0} & 0 & 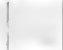 & 0 & & 0 & 0 & 0 & 0 & 14 & 514 & Cinuamun-thested Bee-eater \\
\hline & 0 & & & & 0 & 0 & & 0 & 11 & Fis:l & Mulstachud cireen linkertherd \\
\hline \multirow[t]{4}{*}{ ○ } & O & 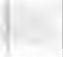 & & & & 0 & 0 & 0 & 8 & 606 & Fine-tanded Wiondpreckir \\
\hline & & 0 & & & & & & 0 & 5 & $\left(x^{2 i 3}\right.$ & Shetpes Longelas \\
\hline & - & & 0 & & 0 & & 0 & 0 & 4 & 714 & Shelley"s Greenhut \\
\hline & 0 & & & & & 0 & & 0 & 7 & 药 & 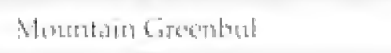 \\
\hline 0 & 0 & & 0 & & 0 & 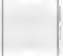 & 0 & 0 & 6 & 725 & Joyful Greentul \\
\hline \multirow[t]{11}{*}{0} & 0 & & 0 & & 0 & 0 & 0 & 0 & 12 & 737 & Sirik:an Hill listhelker \\
\hline & & & 0 & & 0 & & 0 & 0 & 3 & 799 & Grey-chested Illantopsis \\
\hline & ? & & 0 & & 0 & & 0 & 0 & 6 & +1 & 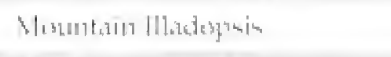 \\
\hline & 0 & & 0 & & 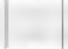 & 0 & & 0 & 11 & 736 & White-starred Robin \\
\hline & 0 & & 0 & & 0 & & 0 & 0 & 6 & $7 h 1$ & Equaturiat ahathat \\
\hline & & & & & & & & & 7 & $\pi 1$ & Rüppell's Rotrin Chat \\
\hline & & & & & & & & 0 & 4 & sulon & Aprome Clo.3t \\
\hline & & & & 0 & & & & & 6 & 814 & Little Rock Thrush \\
\hline & & & & & & & & & 5 & 82.7 & Orange cianund Thasush \\
\hline & 0 & & 0 & & & 0 & & 0 & 8 & 826 & Alyssimian Ground Thrusty \\
\hline & & & 0 & & & & 0 & & 2 & $\$ 32$ & Chapin's riscoteduer \\
\hline \multirow[t]{8}{*}{ ○ } & 0 & & 0 & & 10 & 0 & 0 & 0 & 18 & $s+10$ & White-eyed Slaty flycatcher \\
\hline & 0 & & 0 & & 0 & 0 & & 0 & 11 & 874 & Bromen thendlathe Warbler \\
\hline & 0 & & 9 & & 0 & 0 & 0 & 0 & 10 & 884 & Cinnarnon Bracken Workles \\
\hline & 0 & & 0 & & 0 & 0 & & 0 & 9 & 442 & 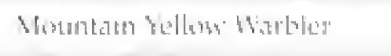 \\
\hline & 0 & ○ & & 0 & & 0 & & 0 & 10 & 898 & Hunter's Cisticolal \\
\hline & 0 & & 0 & & 0 & & 0 & 0 & is & sisy & Chubles Cisturalat \\
\hline & & 0 & & & & & & & 3 & 910 & Aberdare Cisticola \\
\hline & 0 & & & & 0 & 0 & & 0 & $s$ & 440 & Chestnut-throwed Apalis \\
\hline
\end{tabular}




\begin{tabular}{|c|c|c|c|c|c|c|c|c|c|c|c|c|c|c|c|}
\hline Af & $\begin{array}{l}\text { Appendix } 3 \mathrm{~B} \\
\text { Biome species } \\
\text { otropical Highlands }\end{array}$ & 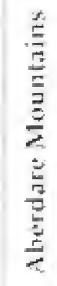 & 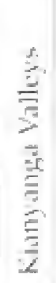 & 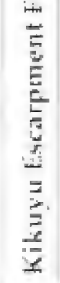 & 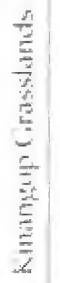 & 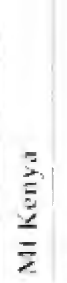 & 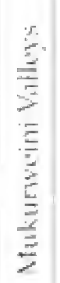 & 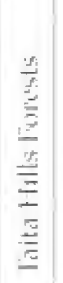 & 点 & 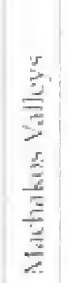 & 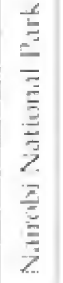 & 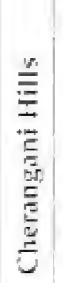 & 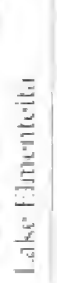 & 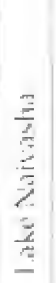 & \\
\hline OSc\# & Common Name & 1 & 2 & 3 & 4 & 5 & 6 & 21 & 26 & 29 & 36 & 43 & to & 49 & \\
\hline & (67 Kenyan spectes) & & & & & & & & & & & & & & \\
\hline $4=0$ & Bsack-collareut Apalis & 0 & 0 & 0 & & 0 & & & & & ○ & 0 & & & \\
\hline 952.1 & Taita Apalis & & & & & & & - & & & & & & & \\
\hline 467 & White-housted Cresmber & 0 & & 0 & & ○ & & & & & 0 & - & & & \\
\hline 977 & Brown Parisoma & & & & 0 & & & & & & 0 & 0 & 0 & 0 & \\
\hline 42 & Montane twhite-eye & 0 & & 0 & & 0 & & & 0 & & 0 & 0 & & & \\
\hline 982.1 & Taita. White-eye & & & & & & & - & & & & & & & \\
\hline 9872 & Kulal White'-"ye & & & & & & & & & & & & & & \\
\hline 1602 & White-tailed Crested Flycatcher & 0 & & 0 & & 0 & & 0 & 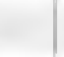 & & & 0 & & & \\
\hline 1055 & Doheretrs Bush Shrike & 0 & & & & 0 & & & & & & 0 & & & \\
\hline 1080 & Grey Cuckno Shrike & 0 & & 0 & & 0 & & 0 & & & 0 & 0 & & & \\
\hline 1088 & Mombane Orisle & 0 & & 0 & & ○ & & & & & - & O & & & \\
\hline 1100 & Stuhlmaun's Starling & & & & & & & & & & & 0 & & & \\
\hline 1101 & Kenritk - Starling & & & & & 0 & & & & & & & & & \\
\hline 1102 & Waller's Starling & 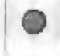 & & 0 & & 0 & & & & & & 0 & & & \\
\hline 105 & Slender-trilled Starling & 0 & & & & 0 & & & & & & - & & & \\
\hline 1122 & Abbott's Starling & 0 & & 0 & & 0 & & 0 & 0 & & & & & & \\
\hline 1123 & Shampers starling & 0 & & 0 & & 0 & & 0 & ○ & & & 0 & & & \\
\hline 1159 & Northern Double-collared Sunterd & 0 & & 0 & & 0 & & & & & 0 & 0 & & & \\
\hline 1161 & 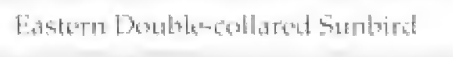 & 0 & & 0 & & 0 & & 0 & - & & & 0 & & & \\
\hline 1177 & Tacazze Stunbird & 0 & & & & 0 & & & & & & 0 & & & \\
\hline 1179 & 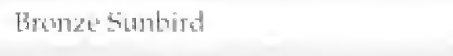 & O & 0 & 0 & & - & 0 & & 0 & & ○ & - & 0 & ○ & 0 \\
\hline 1180 & Golden-twinged Suntirit & 0 & & 0 & & 0 & & & 0 & 0 & 0 & 0 & 0 & O & 0 \\
\hline 11,82 & Scarlet-tutted Walachite Susturd & 0 & & & & 0 & & & & & & & & & \\
\hline 1205 & Baglafecht Weaver & 0 & 0 & 0 & & 0 & 0 & 0 & 0 & O & ○ & 0 & ○ & 0 & ○ \\
\hline 1211 & Black-tulled Weatur & 0 & & & & & & & & & & 0 & & & \\
\hline $12+0$ & Brown-capped Weaver & 0 & & 0 & & 0 & & & & & 0 & ० & & & \\
\hline 1266 & Jakkon's Widowbirt & 0 & & 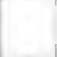 & 0 & 0 & & & & & 0 & & 0 & & - \\
\hline 1279 & Abyssinian Crimonn-wing & 0 & & 0 & & 0 & & & 0 & & 0 & 0 & & & \\
\hline 1.333 & Airican Citril & 0 & 0 & 0 & & 0 & 0 & & - & & 0 & 0 & 0 & & 0 \\
\hline 1340 & Strenty Sced-etater & 0 & 0 & 0 & & 0 & & & 0 & & 0 & 0 & 0 & 0 & 0 \\
\hline $13+4$ & Thich-billed seed-enter & 0 & & 0 & & ○ & & & & & & 0 & & & \\
\hline \multirow[t]{2}{*}{1344} & Orible finch & $\bullet$ & & & & 0 & & & & & & 0 & & & \\
\hline & Total species recorded & 52 & s & 39 & 6 & 53 & 5 & 14 & 21 & 2 & 25 & 49 & 10 & 8 & 9 \\
\hline
\end{tabular}




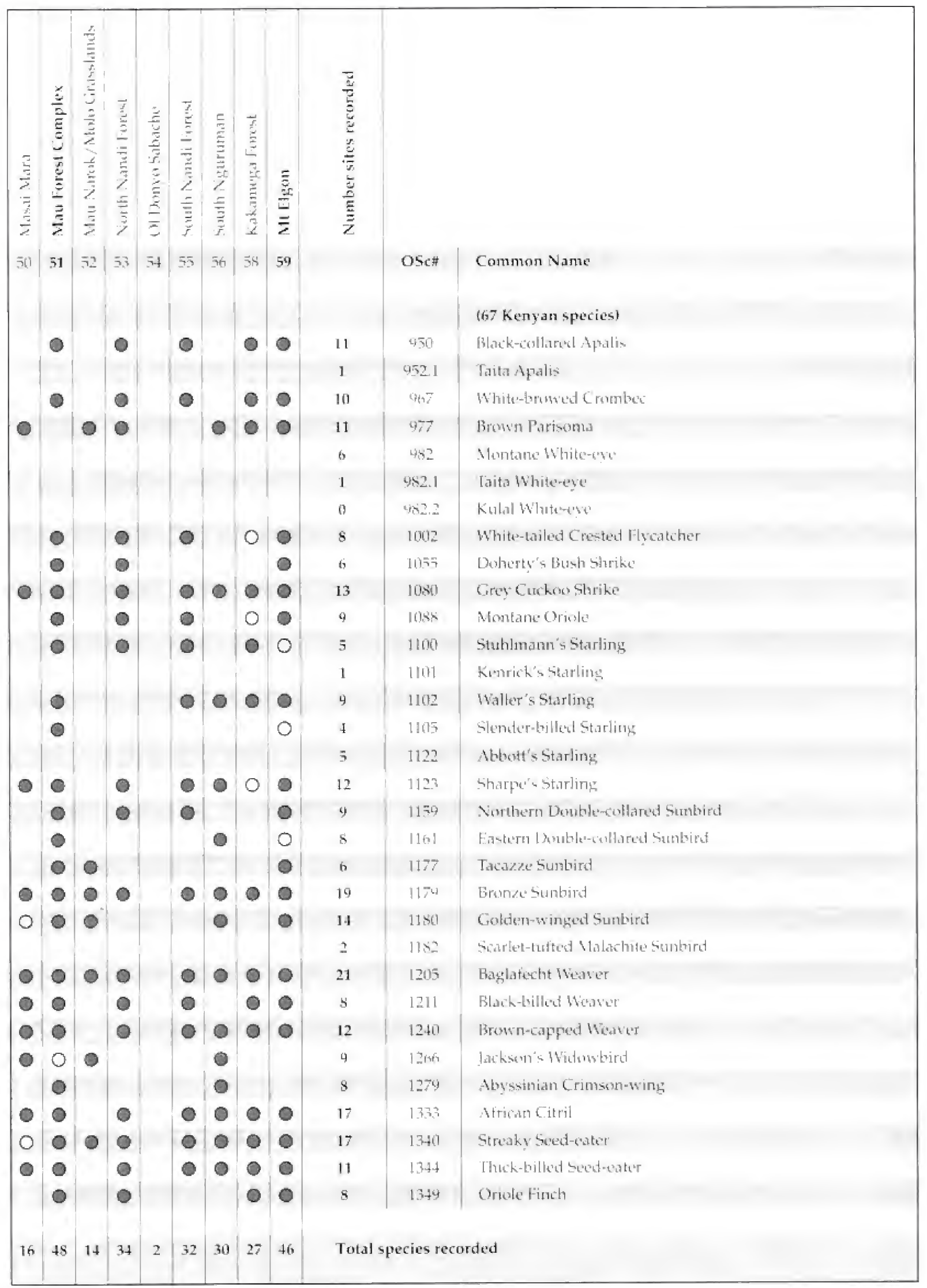









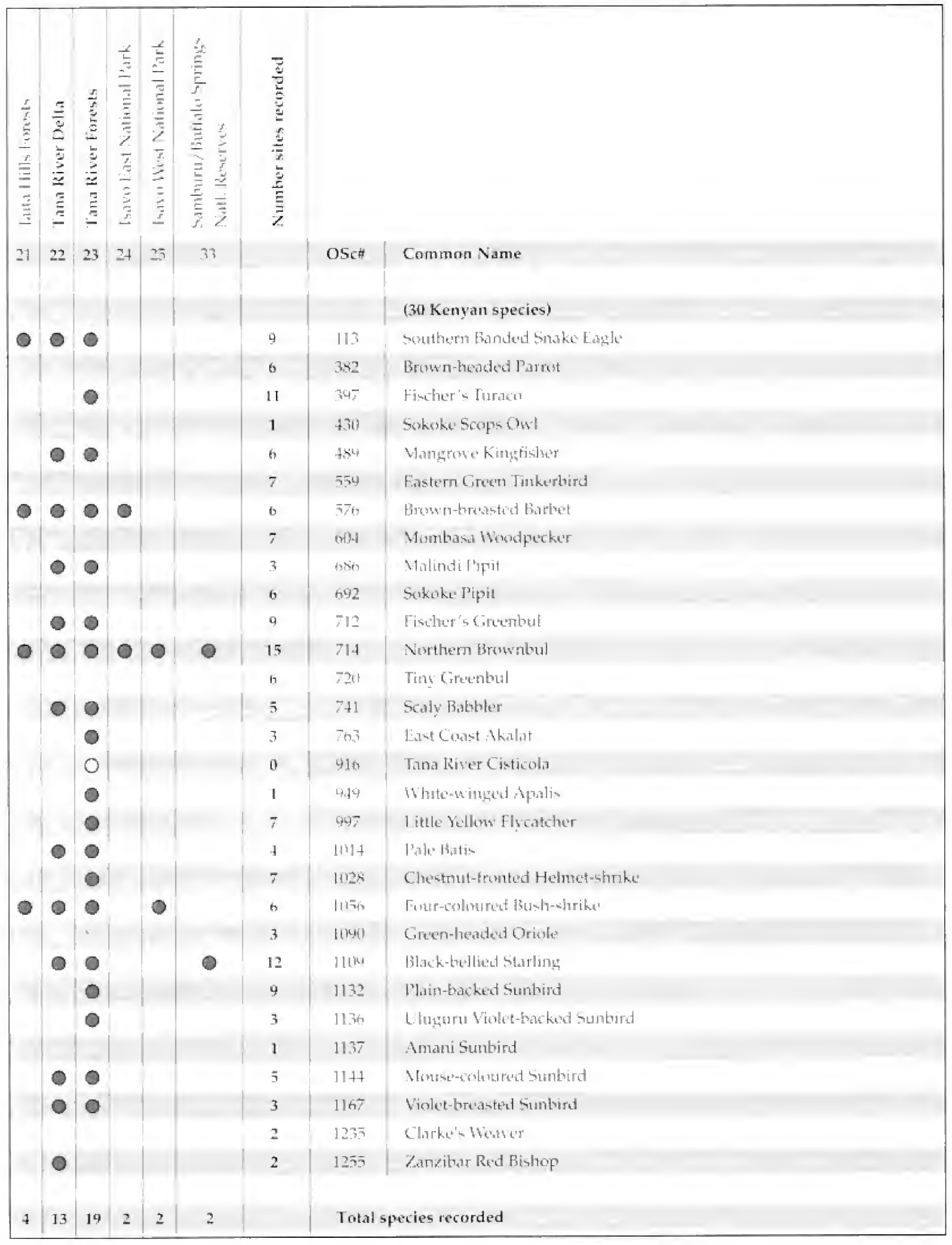




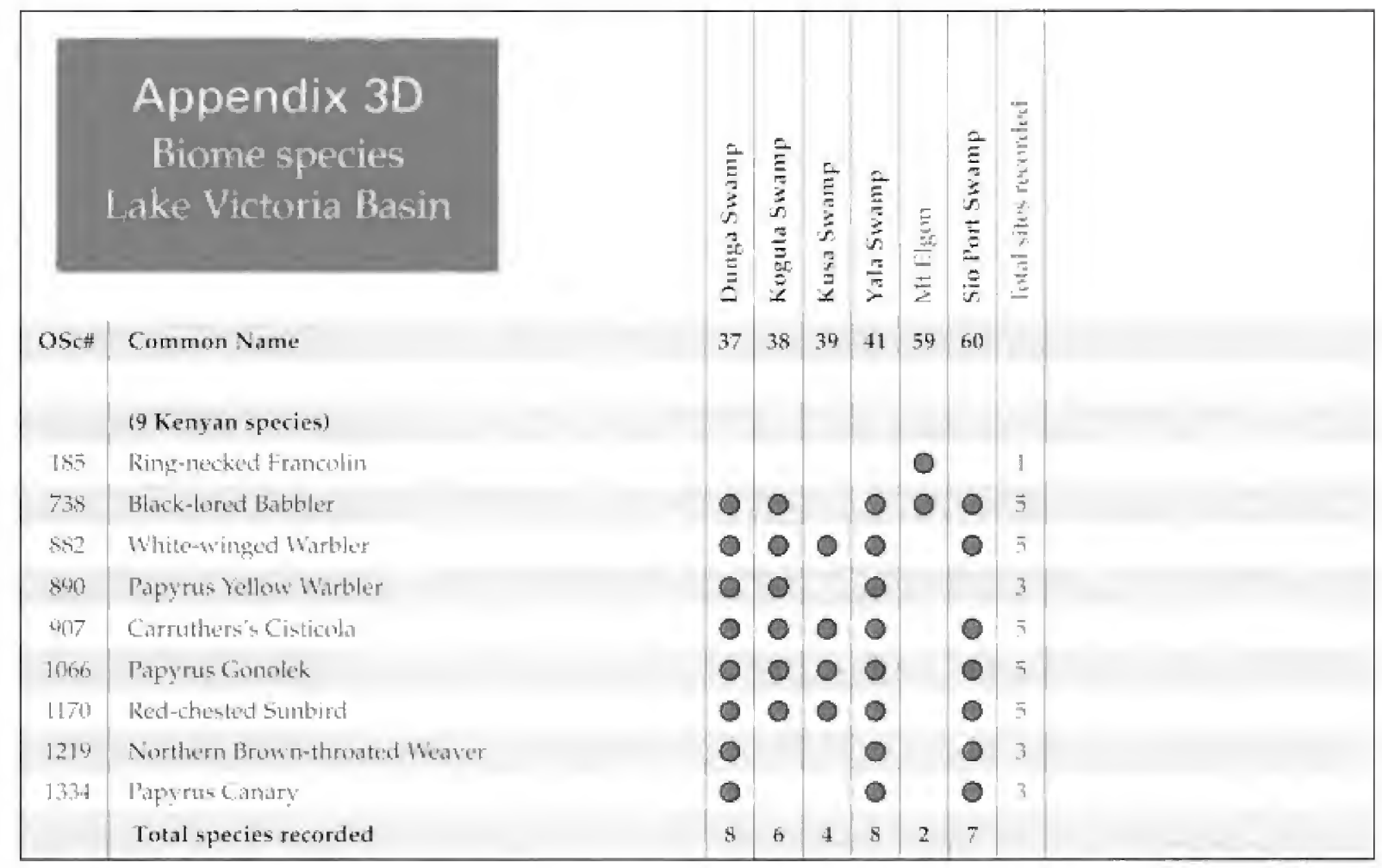

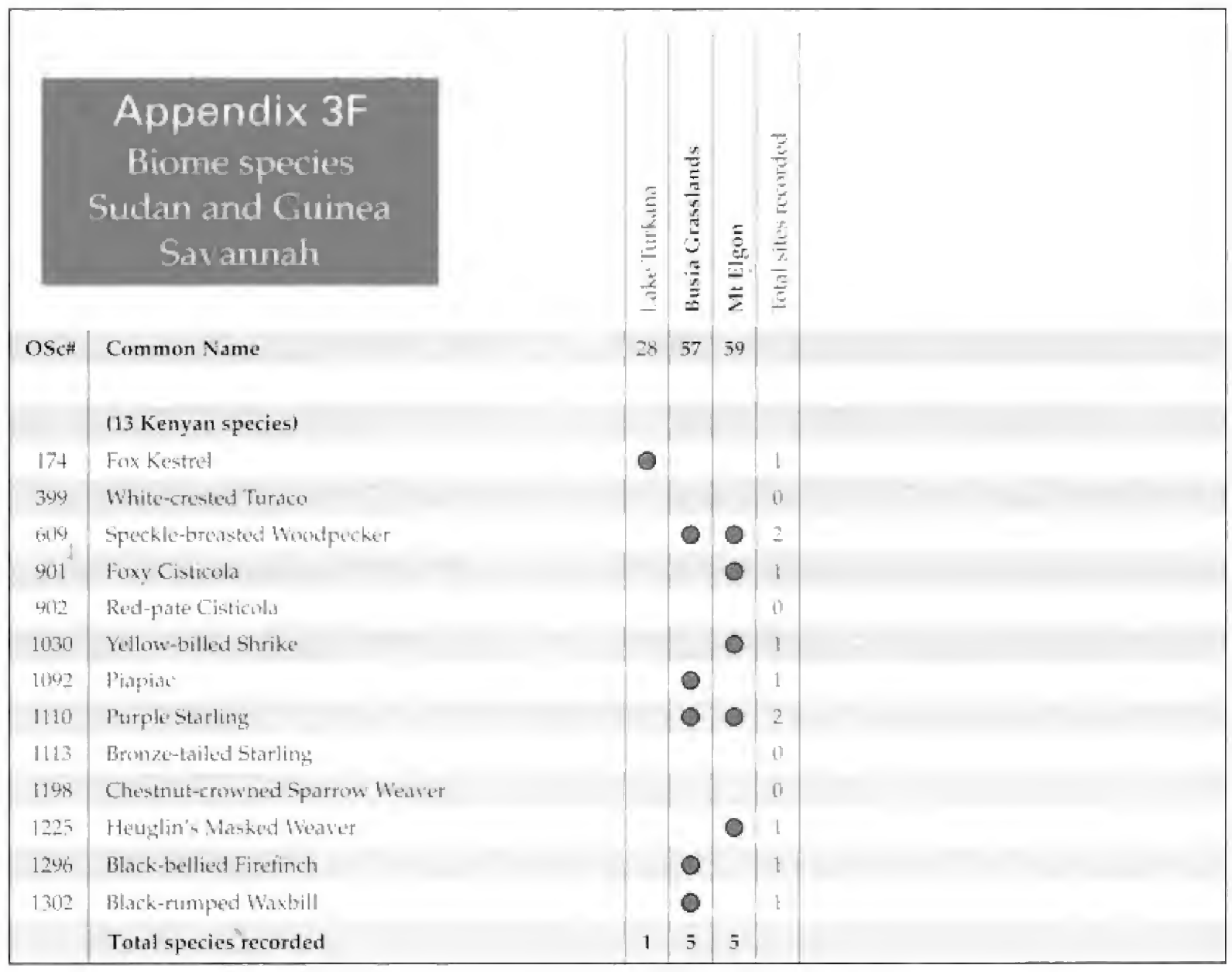




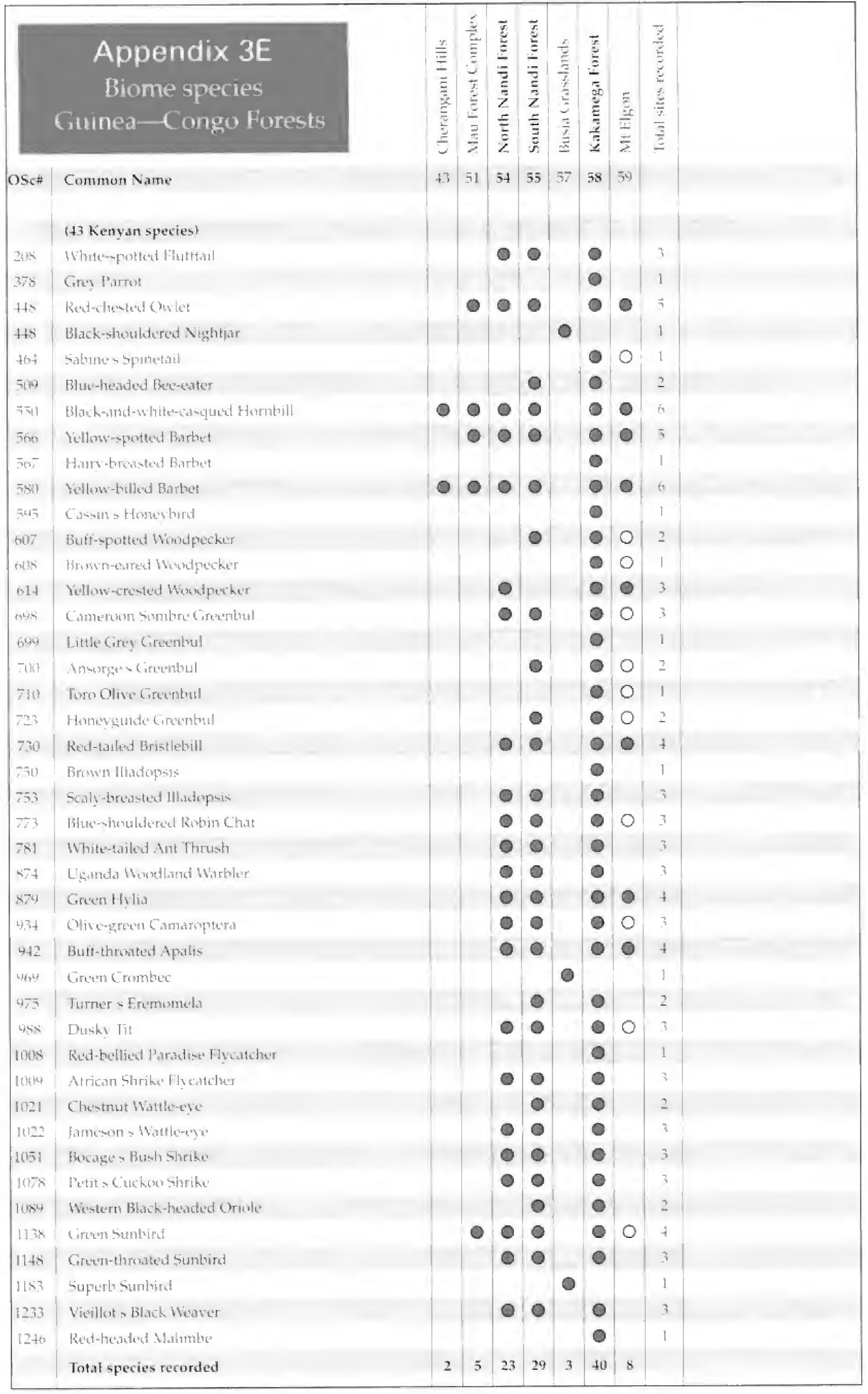




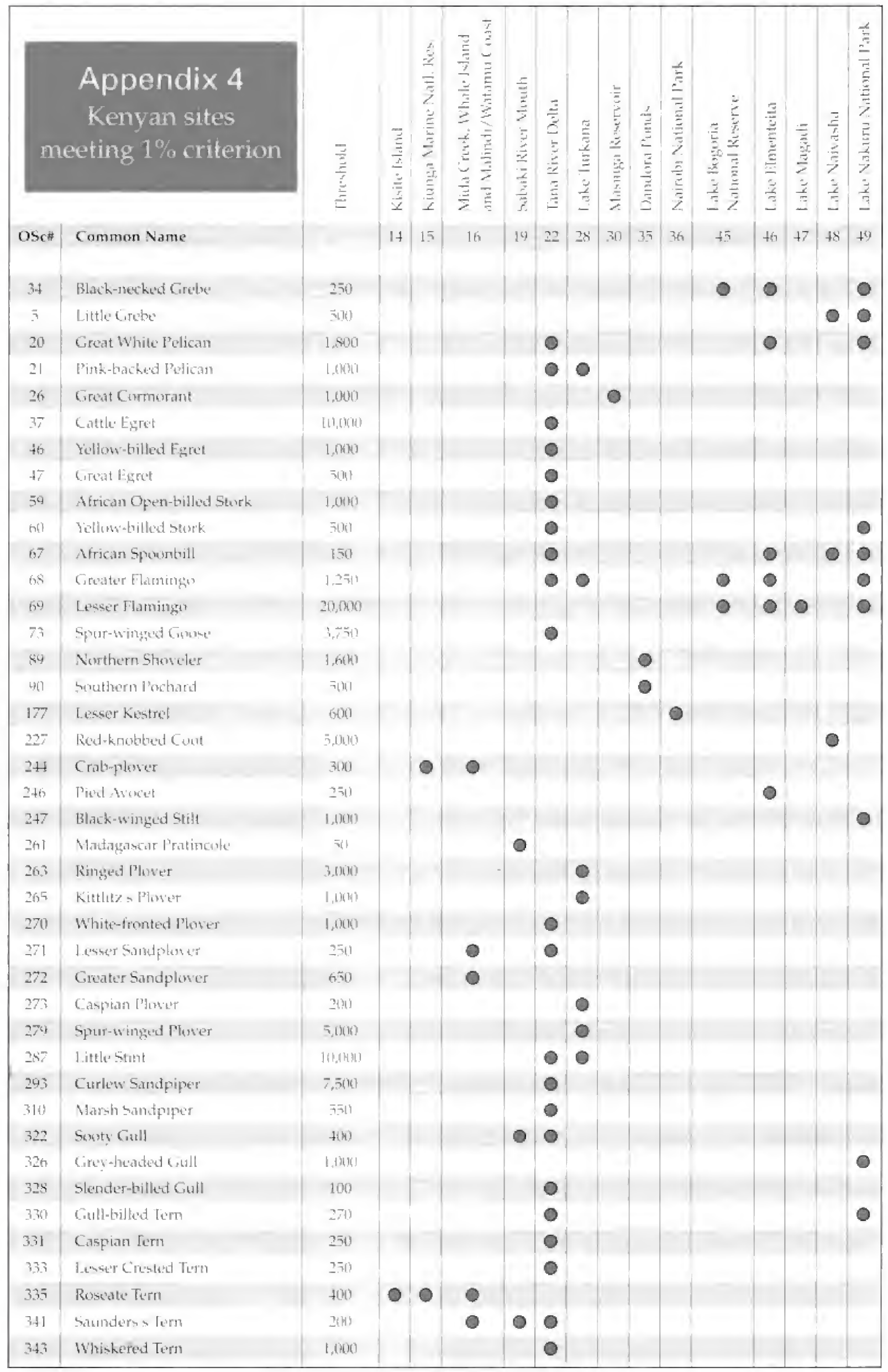




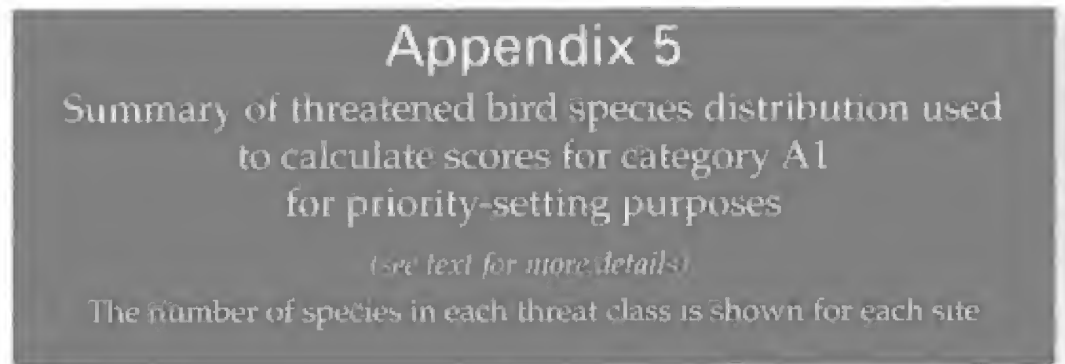

Critically Findangaterd

Endangered

Vulneralse.

or Data Detieient

Near-titruatened

Regionally threatuned

\begin{tabular}{|c|c|c|c|c|c|c|c|c|c|c|c|c|c|c|c|}
\hline & & 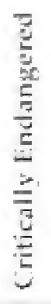 & 焉 & $\frac{\frac{\partial}{n}}{\frac{N}{5}}$ & 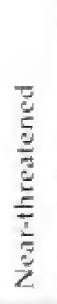 & 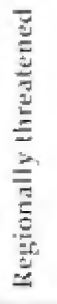 & $\frac{H}{3}$ & & & 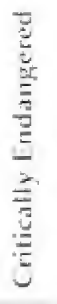 & 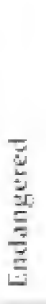 & $\frac{5}{\frac{E}{2}}$ & 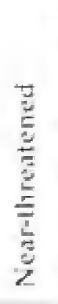 & 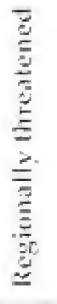 & $\frac{\xi}{\xi}$ \\
\hline 1 & Aberdare Mountains & a & 0 & 3 & 1 & 4 & 4.4 & 32 & Mrata Naturaal Remere & $n$ & 0 & 0 & 1 & 3 & 0.8 \\
\hline$\geq$ & Kiansaga valleys & 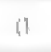 & 1 & 〔1 & $(1$ & 11 & 20 & \multirow{2}{*}{37} & \multirow{2}{*}{ 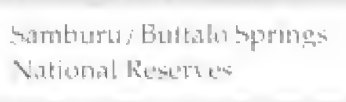 } & \multirow[t]{2}{*}{ (1) } & \multirow[t]{2}{*}{$" 1$} & \multirow[t]{2}{*}{2} & \multirow[t]{2}{*}{ (]) } & \multirow[t]{2}{*}{$\overline{7}$} & \multirow[t]{2}{*}{2.5} \\
\hline 3 & Kikuy u Exarpment forest & 0 & 0 & 1 & 0 & 4 & 1.4 & & & & & & & & \\
\hline+ & Kindstgqup Girabslands & 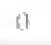 & 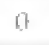 & 2 & 2 & I & 3.1 & 34 & Shatos National Remerver & 0 & !1 & ] & [] & $\overline{3}$ & 1.5 \\
\hline 5 & Mt Kenya & 0 & 0 & 3 & 1 & $s$ & 4.3 & 35 & Dondiora Ponds & 0 & 0 & 0 & (1) & 1 & 0.1 \\
\hline 6 & Mukurmeting Valleys & $n$ & 1 & $n$ & 0 & 0 & 2.0 & $3+$ & Natrobli National Park & 0 & 0 & 2 & 3 & 10 & 4.5 \\
\hline 7 & Arabuko-Solkotac Forrest & $\theta$ & 1 & 7 & 3 & 5 & 9.0 & 7 & Dunga Swamp & 0 & !) & 1 & 1 & $\|$ & 1.5 \\
\hline$s$ & Dakatcha Houdlant & 4 & 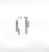 & 2 & 1 & u & 2.5 & 38 & Koguta Swanip & 0 & 0 & 1 & 1 & 1) & 1.5 \\
\hline 9 & Diani Forest & 0 & 1 & 1) & 2 & 3 & 3.3 & 34 & Kus, Swants & 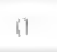 & (1) & (1) & 1 & 1) & 0.5 \\
\hline 10 & Drameno Hill Forrest & 0 & 0 & 1 & 2 & I & 2.1 & 40) & Ruma National Park & 0 & 0 & 1 & (1) & () & 1.0 \\
\hline \multirow[t]{2}{*}{11} & \multirow{2}{*}{$\begin{array}{l}\text { Gede Ruins } \\
\text { Nativnal Montument }\end{array}$} & \multirow[t]{2}{*}{0} & \multirow[t]{2}{*}{1} & \multirow[t]{2}{*}{ ) } & \multirow[t]{2}{*}{3} & \multirow[t]{2}{*}{3} & \multirow[t]{2}{*}{3.8} & 41 & 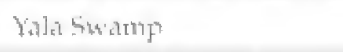 & 0 & 0 & 1 & 2 & 2 & 2.2 \\
\hline & & & & & & & & 47 & Ambustid Natjonal Park & 0 & 0 & 1 & 2 & 6 & 2.6 \\
\hline 12 & Kaya Candari & 19 & 1 & 1 & 3 & 11 & 4.5 & +7 & Cherangand flill: & () & 8 & 0 & (3) & $\overline{7}$ & 0.5 \\
\hline 13 & Kaya twa & 0 & 1 & 0 & 1 & $\theta$ & 2,5 & 14 & I.akt Buringen & 0 & 0 & 1 & 1 & 4 & 1.9 \\
\hline 14 & Kisite lstand & $n$ & (1) & "1 & $n$ & I & 0.1 & $d \bar{c}$ & L.athe: Bisguria & 0 & 4 & 1 & 3 & is & 3.1 \\
\hline \multirow[t]{2}{*}{15} & \multirow{2}{*}{$\begin{array}{l}\text { Kiungala Marine } \\
\text { National Regerve }\end{array}$} & \multirow[t]{2}{*}{ 0) } & \multirow[t]{2}{*}{0} & \multirow[t]{2}{*}{0} & \multirow[t]{2}{*}{0} & \multirow[t]{2}{*}{2} & \multirow[t]{2}{*}{0.2} & & 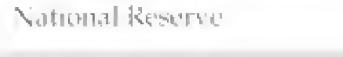 & & & & & & \\
\hline & & & & & & & & 40 & Lathe Fimenteita & 0 & 0 & 2 & 2 & S & 3.8 \\
\hline 16 & Mudacreven, Whale Island & 1) & $\|$ & t) & 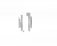 & 2 & 0.2 & 47 & Lake Mandi & i) & (1) & 0 & 1 & 1 & 0.6 \\
\hline & d Walind Wistanu Cowst & & & & & & & 48 & 1. $\mathrm{kL}$ Nairatu & 0 & 0 & 1 & I & h & 2.1 \\
\hline 17 & Marenji Forest & 0 & 0 & 1 & $\geq$ & 1 & 2.1 & 49 & Lake \akura & a & (1) & 2 & * & is & 4.1 \\
\hline 1,19 & Grtmat Hill Finest & () & 1 & 1) & 2 & 1 & 3.1 & & Vatgchatel l'ark & & & & & & \\
\hline 19 & Sabaki Kiver Mouth & 0 & 0 & [3] & 1 & 2 & 0.7 & 51) & Masiai Mara & 1) & 0 & 3 & + & 10 & 6.0 \\
\hline 241 & shimbalitills & 11 & 1 & 2 & 3 & Э & 60,0 & 51 & Man Foress Cimples & 11 & (9) & 17 & at & $i$ & 0.5 \\
\hline 21 & Taita Hilis foncests & 3 & $1)$ & 2 & 1 & 2 & 11.7 & 52 & Man Narok/Mtolo & 1) & 19 & 3 & 3 & 1 & 4.6 \\
\hline 20 & Tanat Riker Deelta & 0 & (1) & 0 & 3 & 4 & 1.9 & & Grastlands & & & & & & \\
\hline 23 & Tant? River Fore'sts & 0 & 0 & 2 & 4 & (1) & $\overline{5} .0$ & 53 & Xorth Nandi Furezet & 1) & 19 & 1 & il & $b$ & 1.8 \\
\hline 24 & 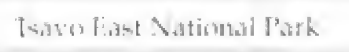 & 0 & 0 & 1 & 2 & 7 & 2.7 & 54 & Ol Donyou Subuctue & 11 & 19 & 1 & 0 & 3 & 1,3 \\
\hline 257 & Trasua West National Park & 0 & 0 & 2 & 3 & [1] & 4.5 & 55 & Geutla Narnditorest & 0 & [1) & 1 & 10 & $x$ & I.S \\
\hline 2 h & Chyolu Hids Furesti & 0 & 0 & I & [] & 3 & 1.3 & 5 & South Vguraman & Q & [] & 1 & 2 & 3 & 2.3 \\
\hline 27 & Dida Conlgalu Desert & 1) & 0 & 0 & 1 & 0 & 0.5 & $\vec{y}$ & Lüusin Grraksilands & 1) & !) & 1 & I & 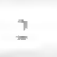 & 1.7 \\
\hline 24 & Lake lurkatou & [] & 0 & 1) & I & $\overline{7}$ & 1.0 & 58 & Kakamegna Fores & (1) & 1) & 2 & 0 & 15 & 3.5 \\
\hline 267 & Ahithatur Valleys & 0 & 1 & 0 & 0 & 0 & 2.0 & $7^{6}$ & 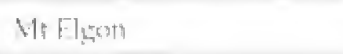 & 19 & 11 & 1 & 11 & is & I.s \\
\hline 30 & Masinga Reseratar & 0 & 0 & (1) & 0 & 2 & 0.2 & bi) & Sio l'ort Swamp & (1) & 0 & 1) & I & 0 & 0.5 \\
\hline 31 & Meru National Park & 0 & 0 & 0 & 0 & 4 & 0.4 & & & & & & & & \\
\hline
\end{tabular}




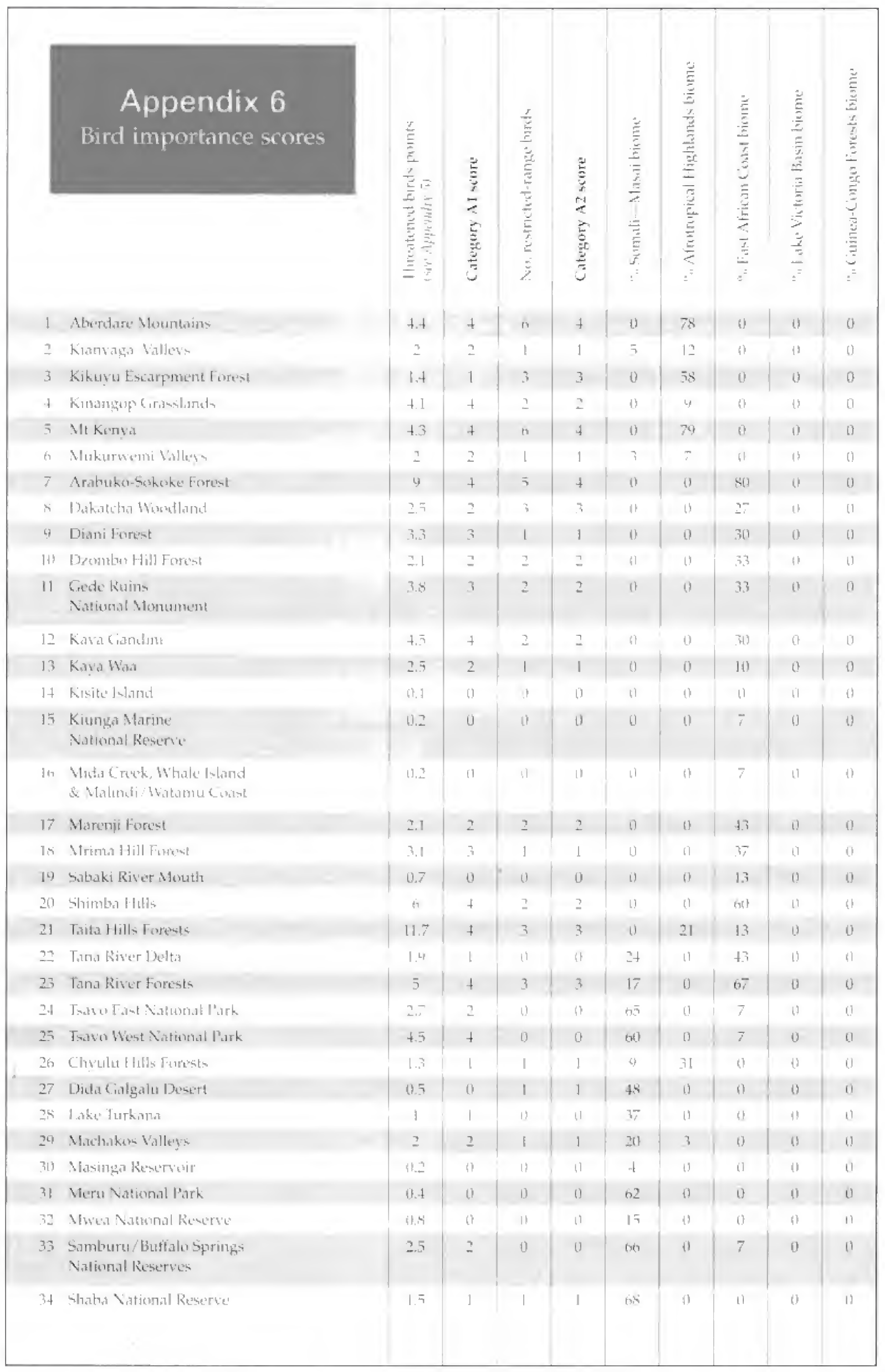




\begin{tabular}{|c|c|c|c|c|c|c|c|c|c|c|c|}
\hline 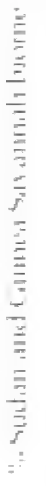 & 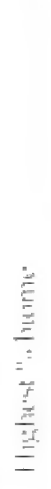 & 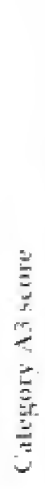 & 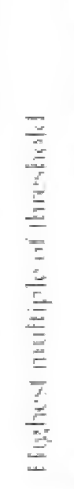 & 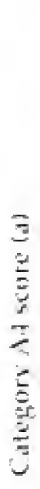 & 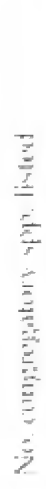 & 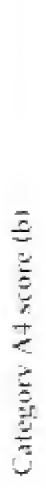 & 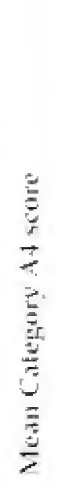 & $\frac{\vec{E}}{\vec{E}}$ & 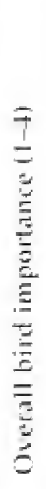 & & \\
\hline 0 & $7 \mathrm{~K}$ & + & 0.0 & 0 & 0 & 0 & 0,0 & $1 \geq 0$ & 4 & 1 & Arerdate Muuntamb \\
\hline 1) & $1 ?$ & 11 & kn & b) & (1) & 11 & 10,11 & 3,61 & 2 & 2 & 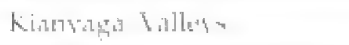 \\
\hline (1) & 58 & 3 & 0.13 & a & 1) & 0 & (0.1) & 7.81 & 4 & 3 & Kikuyu Exarememat Furest \\
\hline 11 & 4 & 1 & knt & it & 14 & "1 & 0.11 & $\$ 1.61$ & 3 & + & 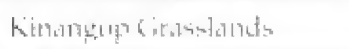 \\
\hline () & 74 & 4 & an & 0 & 1) & $\emptyset$ & {$[0,11$} & 12.0 & 4 & 5 & Nikersya \\
\hline 11 & $?$ & 11 & an & in & $\$ 4$ & १1 & 0.11 & .3 .61 & 2 & in & 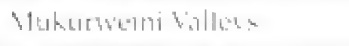 \\
\hline 1) & 80 & 4 & 00 & 0 & (1) & 0 & 0.11 & 12.11 & 4 & 7 & Arabukin-Solktikt? Furest \\
\hline 11 & $2=$ & 1 & Whis & n & 4 & $\vdots 1$ & $(1), 11$ & $\{7.11$ & $\overline{7}$ & 8 & 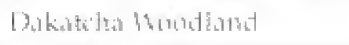 \\
\hline 0 & 30 & 1 & 0.0 & 0 & 0) & $\Leftrightarrow$ & 10.0 & 5.0 & 3 & 4 & Diani Forest \\
\hline 11 & 33 & 1 & 421 & $\|$ & 11 & n & 1111 & 50 & i & 161 & 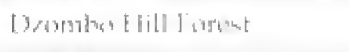 \\
\hline 0 & 33 & 1 & 0.11 & 0 & 1) & 0 & 0.11 & 6.0 & 3 & 11 & 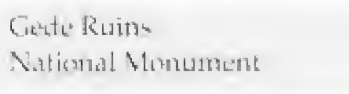 \\
\hline 11 & 311 & & 18.13 & 11 & 11 & औ & 11.11 & 7.14 & + & 12 & Rayin 5 a d17.tมn: \\
\hline 0 & 10 & (1) & 0.0 & 0 & ఏ1 & a & 0.0 & 3.0 & 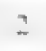 & 13 & Kay̆ Wax \\
\hline 11 & (1) & 11 & $2=$ & t & । & 1 & 11 & 1.19 & 1 & $1+$ & 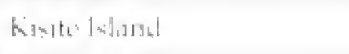 \\
\hline 0 & $\bar{l}$ & 0 & 125 & 3 & 2 & 2 & 25 & $2 . \overline{3}$ & 1 & 15 & 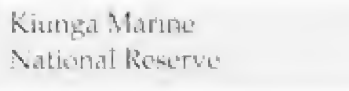 \\
\hline 11 & $\bar{z}$ & $\emptyset$ & 34.5 & 4 & $\overline{-}$ & + & 40 & (1.) & \pm & & 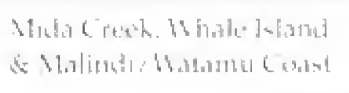 \\
\hline 11 & 4.3 & 2 & (a) & 0 & 0 & 0 & 0,0 & 6.0 & 3 & 17 & Marengi Fortst \\
\hline 11 & หr. & I & $(0,13$ & 14 & 4 & 13 & i., 11 & $m, 1)$ & 3 & $1 \mathrm{k}$ & Mrimat Hill Hurenel \\
\hline 0 & 1.3 & 11 & 5010 & 4 & 4 & + & 4.51 & 4.0 & 2 & 14 & Sotholki kivere Munuth \\
\hline 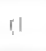 & 1311 & 3 & 11,19 & 11 & $\mathrm{t}$ & it & 51.4 & 4.11 & + & 29 & Shimball Hill= \\
\hline 0 & 21 & 1 & 0.0 & 0 & 6 & 1) & bat & 8.0 & + & 31 & Taita Hills Forests \\
\hline$!$ & $1^{7}$ & 2 & $24 \overline{5}$ & 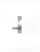 & ביב & 4 & 4.11 & 7.10 & ! & 22 & 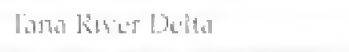 \\
\hline (1) & 19 & 3 & 0.0 & 0 & 6 & 0 & 0.0 & 100 & + & 23 & Tasa River Forests \\
\hline 11 & $19 \overline{7}$ & 3 & 41,11 & 11 & 1 & it & ant & 5,11 & I & $2+$ & 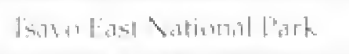 \\
\hline 0 & 60 & 3 & 0.11 & 0 & 6 & 0 & a. at & 7.0 & + & 25 & Torgo West Vational Park \\
\hline it & 31 & । & 11.17 & 11 & $\xi$ & 1) & nat & 3,11 & । & 217 & 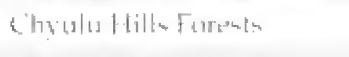 \\
\hline 0 & 48 & $=$ & 0.0 & (1) & u & (3) & 0.0 & 3,0 & 1 & 27 & Dus, Golggalu Détedt \\
\hline$\therefore$ & 3 & $=$ & 11.3 & 3 & - & 4 & 3.7 & $\$ 7.5$ & 3 & 24 & 1_ske: Iturkagth \\
\hline 0 & 20 & 1 & 0.19 & 0 & (1) & (1) & $\{1,1\}$ & 4.0 & 2 & 26 & Wathaken Vatleys \\
\hline औ & 4 & 11 & 2.2 & 1 & 2 & $=$ & $1 \div$ & 1.5 & I & \$1 & 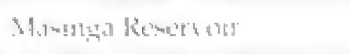 \\
\hline 0 & 62 & 3 & 0.0 & 0 & 0 & 0 & 0.4 & $3.1)$ & 2 & 31 & Meru Natumal l'ark \\
\hline\{ & $1 \approx$ & i & îli & i) & औ & 门 & thet & 11,0 & । & 32 & Whed Datural kewerte \\
\hline 1) & $H(i)$ & 3 & 0.0 & 0 & 11 & 0 & (1). 19 & 5.0 & 3 & 33 & $\begin{array}{l}\text { Samburu/Bublalesprings } \\
\text { National Reserven }\end{array}$ \\
\hline 1) & 6 & 4 & $(1.4)$ & 11 & \# & 11 & $13,1)$ & 6,11 & 3 & 34 & 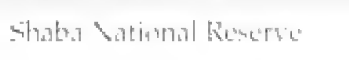 \\
\hline
\end{tabular}




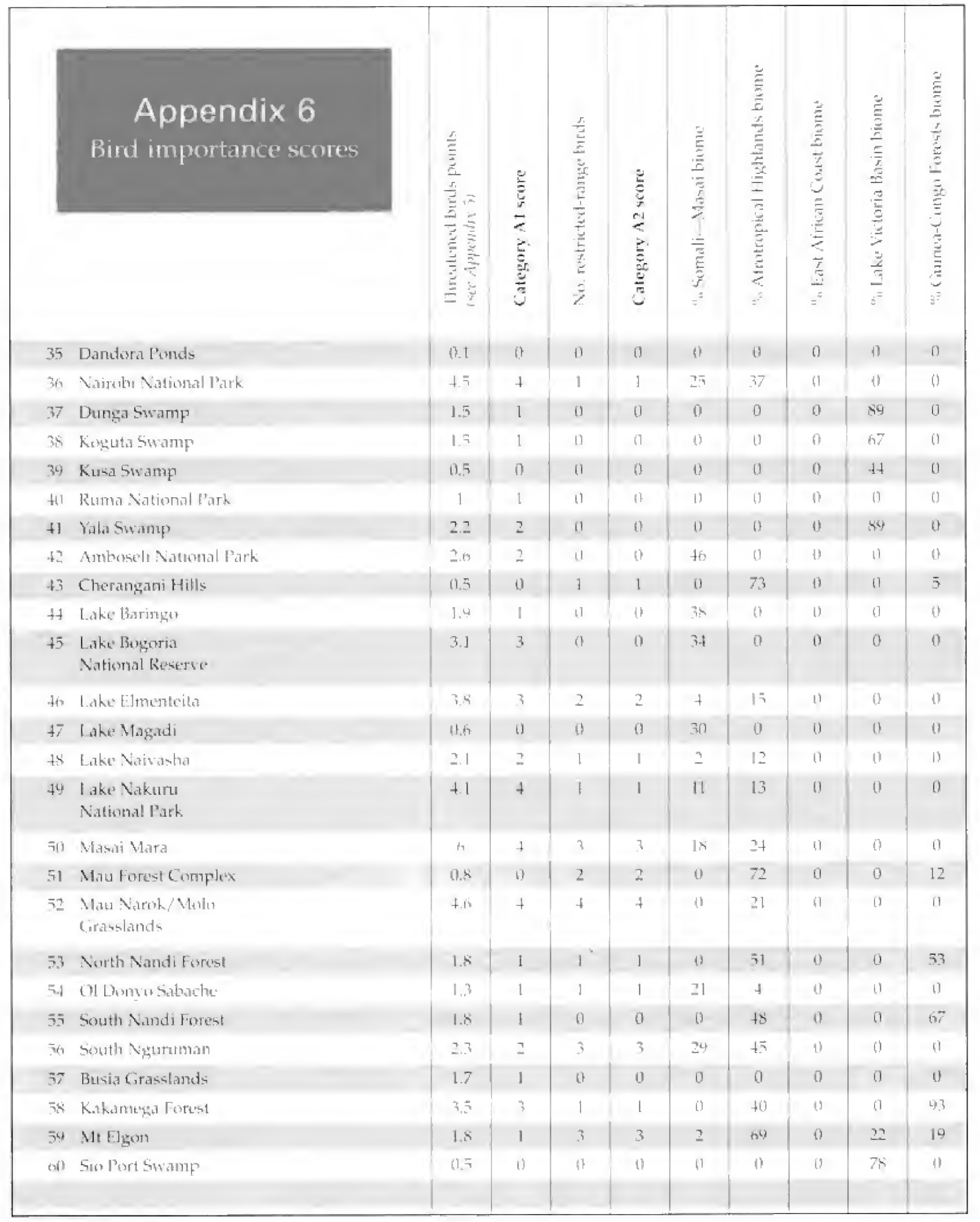




\begin{tabular}{|c|c|c|c|c|c|c|c|c|c|c|c|}
\hline 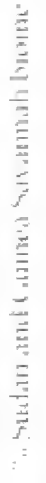 & 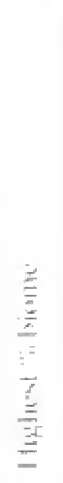 & 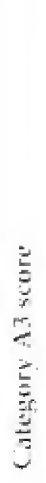 & 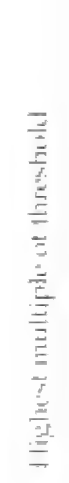 & 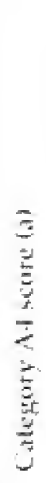 & 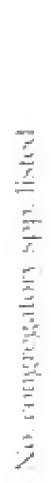 & 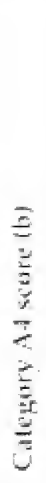 & 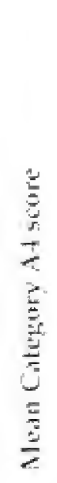 & $\frac{5}{\frac{5}{5}}$ & 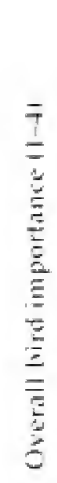 & & \\
\hline () & 0 & 0 & a. 4 & 2 & 2 & 2 & 2,0 & 2.11 & 1 & 35 & Dinutera l'omets \\
\hline 1) & $r$ & $I$ & 8.3 & 3 & I & 1 & 2,11 & y.ti & + & 3 & 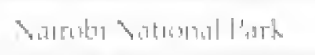 \\
\hline (1) & 84 & 4 & 0.0 & 0 & 0 & 0 & 0.0 & 50 & 3 & 37 & Dunga bHamp \\
\hline 11 & $\mathrm{~m}^{-}$ & $i$ & 11.11 & $\sharp$ & 11 & 4 & 11,11 & +19 & 2 & is & Kongutia Gusionp \\
\hline (1) & $H$ & $\beth$ & 0.0 & in & 0 & 11 & 0.01 & 2.0 & 1 & 30 & KL, \\
\hline 11 & at & 11 & 19.19 & (1) & 11 & 11 & 11,11 & 1.19 & 1 & 40 & 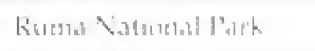 \\
\hline (1) & sit & 4 & 0.0 & 0 & () & 0 & [1. 0 & 10.19 & 3 & +1 & Yatด รพเม \\
\hline (1) & the & $\beth$ & 19.11 & $\because 1$ & $\because$ & 11 & 41,41 & +11 & $\underline{2}$ & 47 & 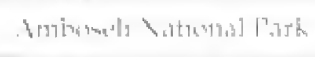 \\
\hline (1) & 73 & 4 & $19.1]$ & 11 & (t) & 0 & 0.01 & 50 & $i$ & 43 & Cherautgani Hilla \\
\hline i) & is & $\simeq$ & $\lfloor, 11$ & 1) & 4 & is & 4h.t. & 3,11 & $\therefore$ & $+t$ & l.ake fral ringes? \\
\hline 0 & 34 & 1 & 750 & + & 3 & 3 & 35 & 7.5 & 4 & 45 & 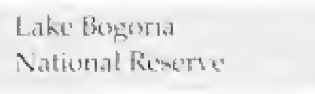 \\
\hline ఏ & $1 \overline{4}$ & $\because$ & 27 & + & h & 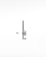 & +.11 & 4.0 & 4 & tin & I.the I"Imuente'nta \\
\hline 0 & 30 & 1 & 2.5 & 1 & 1 & 1 & 1.0 & 20 & 1 & $\vec{t} \vec{\imath}$ & Lahe Naganti \\
\hline 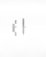 & 19 & 1 & 3,4 & 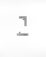 & 3 & 11 & 1.13 & 4.11 & 2 & the & L.ahe Nurtiontho \\
\hline is & 13 & 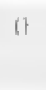 & 72,4 & 4 & 8 & 4 & 4.0 & 4,0 & 4 & 44 & $\begin{array}{l}\text { L.thet Nahurta } \\
\text { Nationtal J'urk }\end{array}$ \\
\hline$\|$ & $2+$ & 1 & (1).1) & 0 & $n$ & 11 & (1.1) & $8.6 !$ & 4 & 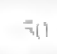 & 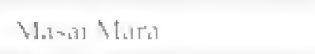 \\
\hline 0 & 72 & 1 & 00 & 0 & 0 & 1) & (a.) & 6.6) & 3 & 51 & Mat Furest Complex \\
\hline 1) & 21 & । & 41.01 & 11 & 11 & 11 & 1311 & 467 & 4 & 52 & 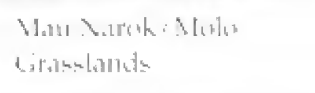 \\
\hline 0) & $53^{\circ}$ & 4 & (1:0) & 0 & 0 & 0 & (1). 1 ) & 0.0 & 3 & 53 & Sorth Nandi Furest \\
\hline 11 & 71 & 1 & a. & 11 & 11 & 11 & 11,11 & 3,4 & 3 & 7 & DDombuthache \\
\hline 0 & bi & $t$ & 0.0 & 0 & 0 & 0 & 13.0 & 5.11 & 3 & $5 \overline{5}$ & Sorutly Nandif Forest \\
\hline $1 i$ & $4 \overline{5}$ & $=$ & mis & it & $1)$ & 11 & $(1,11)$ & $-i n$ & + & $\overline{\text {. }}$ ถ & 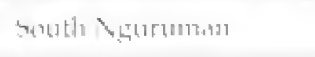 \\
\hline 38 & $3 x$ & 2 & 10.0 & 1) & 0 & (1) & 0.0 & 3.0 & 2 & 57 & Butata Grasilands \\
\hline ii & $2 i 3$ & t & $\mid 3,1\}$ & it & 11 & it & 0.07 & 80 & 4 & & 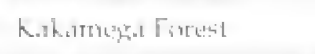 \\
\hline 38 & $(3,4$ & 4 & vol & 0 & () & 0 & 0.01 & 8,0 & 4 & 54 & Mt F] gan \\
\hline 11 & $\therefore$ & + & 111 & $\|$ & 11 & H! & 61,61 & +11 & $\ddot{7}$ & और & 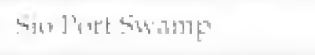 \\
\hline
\end{tabular}

Appendix 6 


\title{
7 IN DICES
}

\section{Index 1}

\section{Bird species in the site accounts and appendices, indexed by scientific name}

\author{
Nomendature follows os-c (1996). \\ Numbers refer to IBA or (if prefixed by 'A') appendix numbers, not page numbers
}

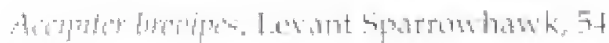

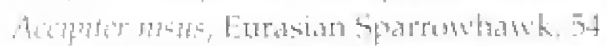

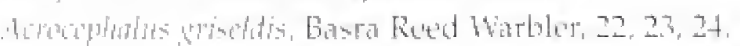

$25,39,46,42$

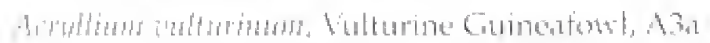

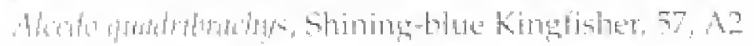

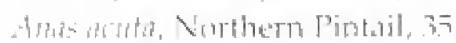

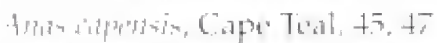

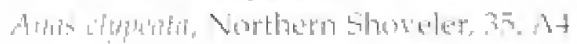

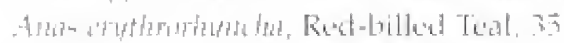

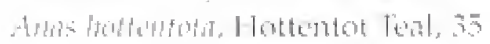

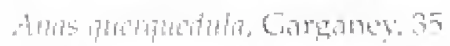

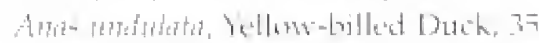

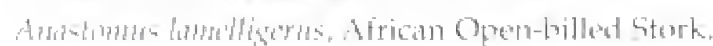

23. At

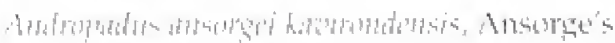

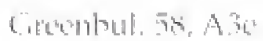

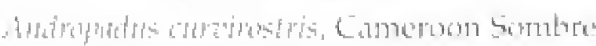

Circentrul. A to

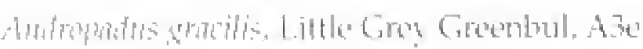

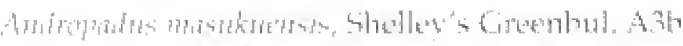

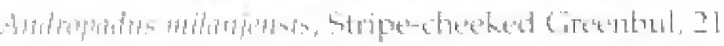

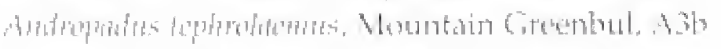

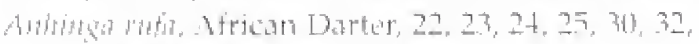

$33,34,36,42,44,45,46,30,42$

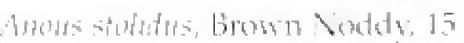

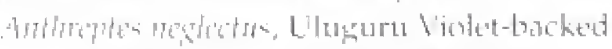

sumbind, 9.211, 73, Al, A3E

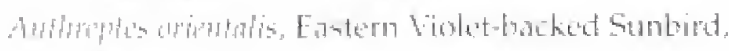

$\begin{cases}3 \\ x\end{cases}$

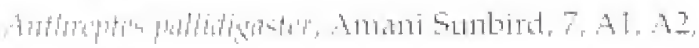
130

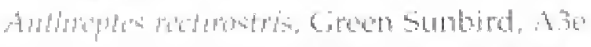

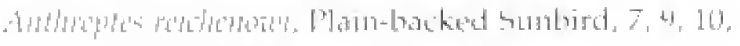

$11,12,17,18,20,23, A 1, A, 2$

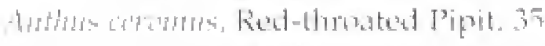

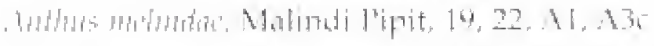

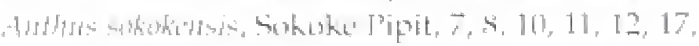

20, $1,1,2,13 x^{2}$

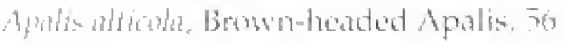

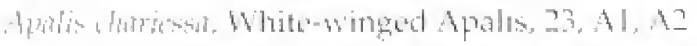

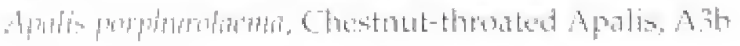

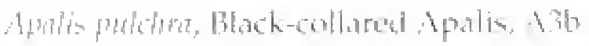

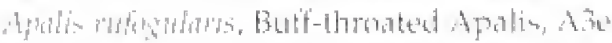

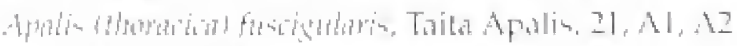

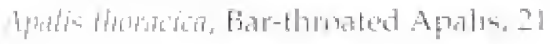

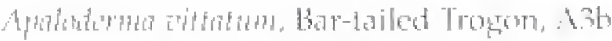

Apremper Eurasian Swit, 4

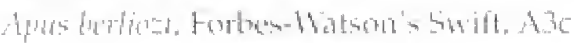

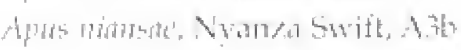

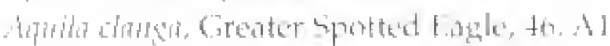

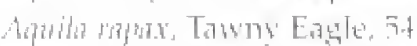

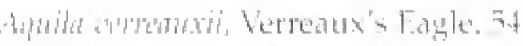

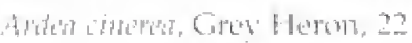

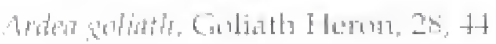

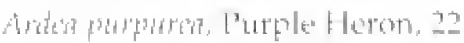

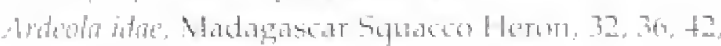
$+4,44,80,4$

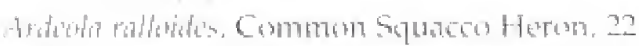

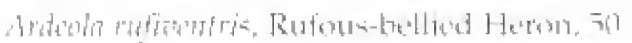

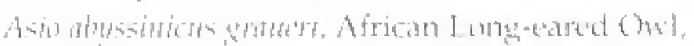

5. Aib

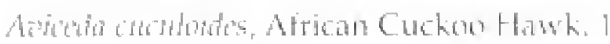

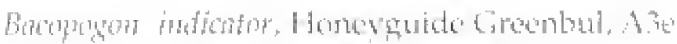

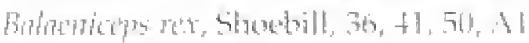

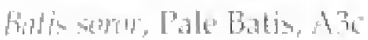

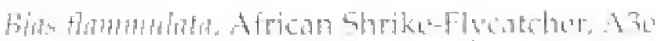

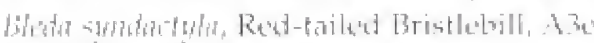

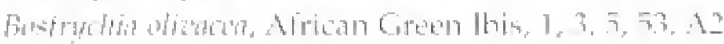

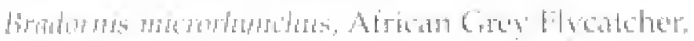

$A B$

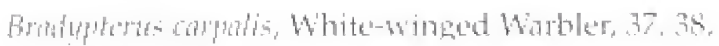

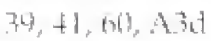

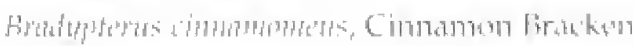

Worlaler' 26

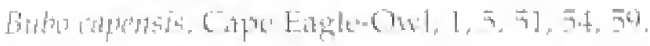

$$
\text { A1. A.t. }
$$

Buthulers inis, Cattle [y lest, 22

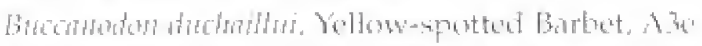

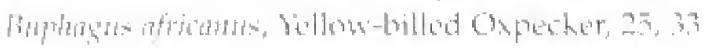

$74,76,464,501$

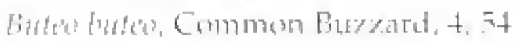

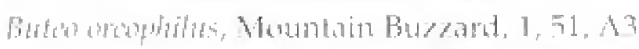

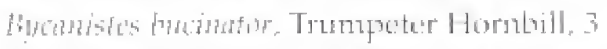

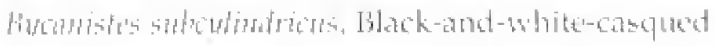

Horntill, A 3 :

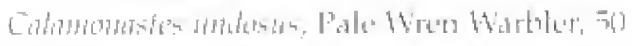

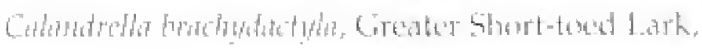

$17 \mathrm{a}$

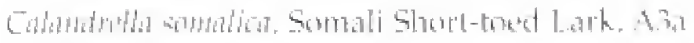

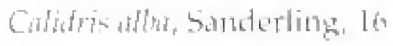

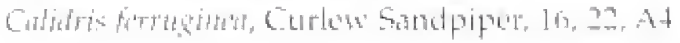




\section{Important Bird Areas in Kenya}

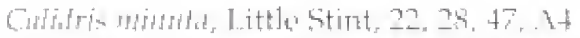

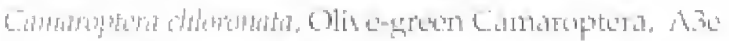

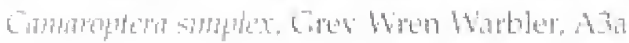

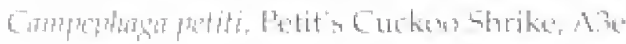

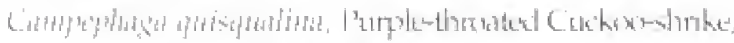

$5,43,51,70,58,79,81$

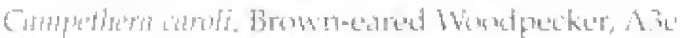

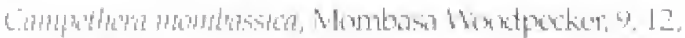
i. 7 .

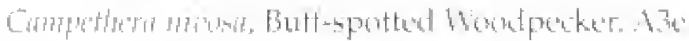

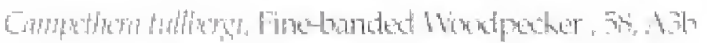

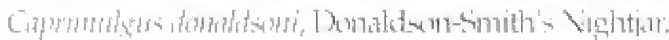
$\therefore, 3$.

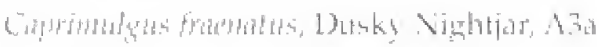

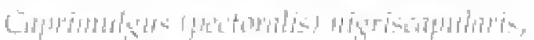

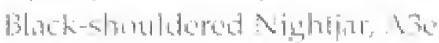

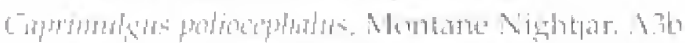

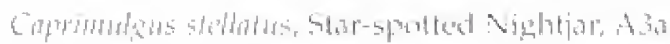

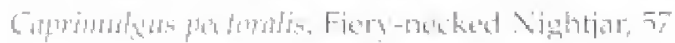

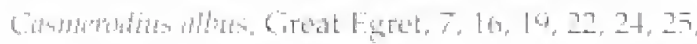

$28,30,37,34,7 \overline{7}, 37,41,42,44,40,47,45,40$ 7), $\mathrm{xH}$

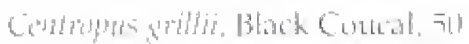

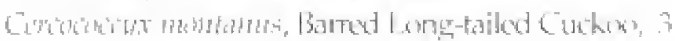

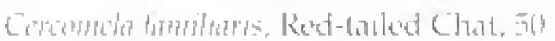

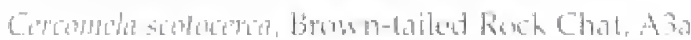

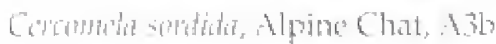

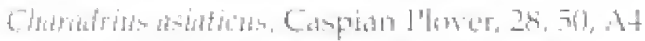

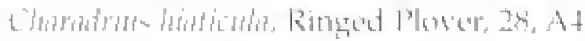

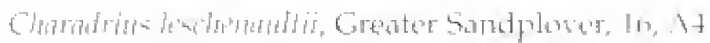

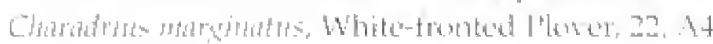

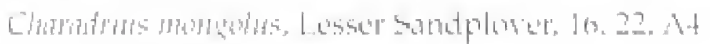

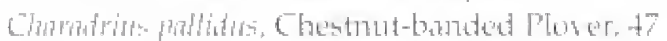

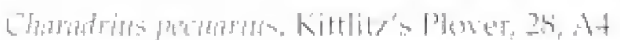

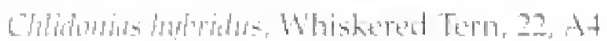

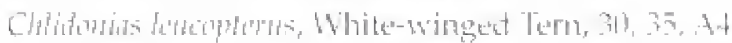

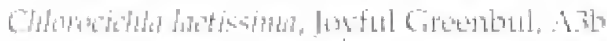

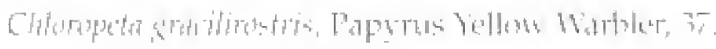

$39,34,41,60,4,1,3 \mathrm{~d}$

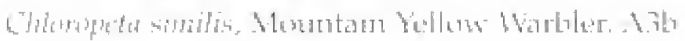

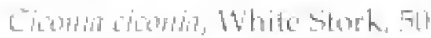

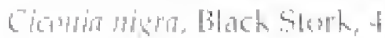

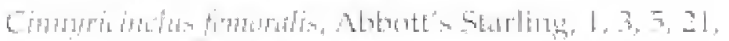

$26,41,42,43$

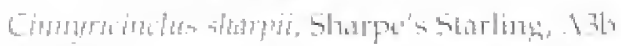

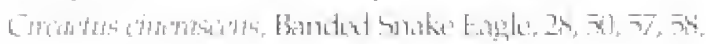

A 1

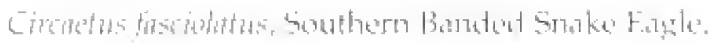

$7,8,4,11,21,21,22,23,41,43$

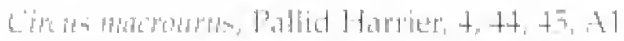

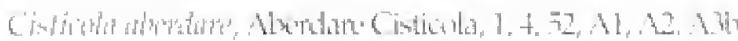

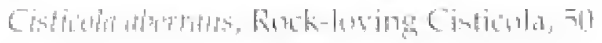

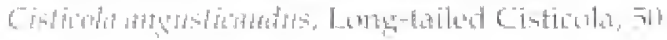

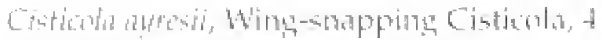

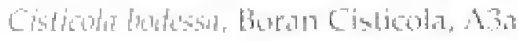

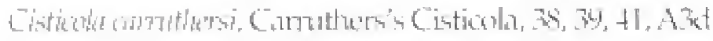

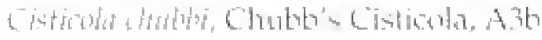

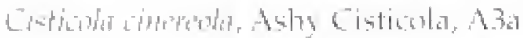

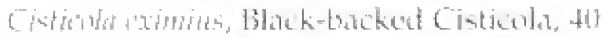

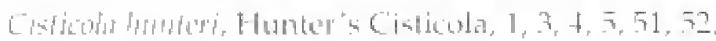

$54,50,54,12$

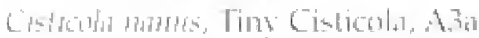

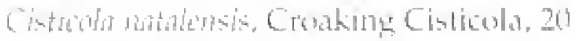

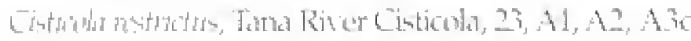

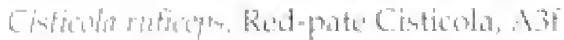

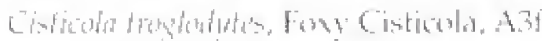

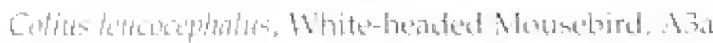

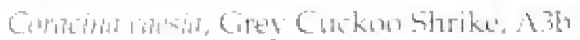

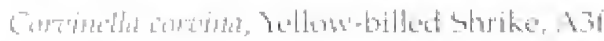

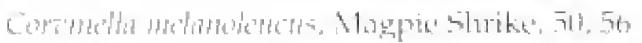

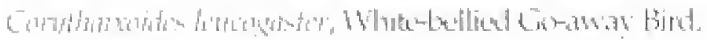

$\sin$

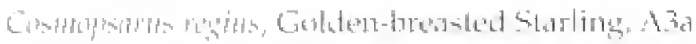

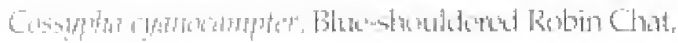
A $x^{3}$

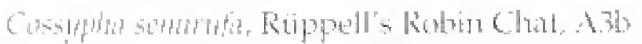

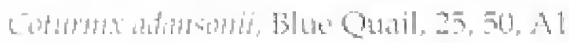

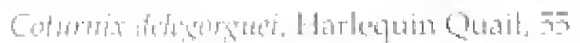

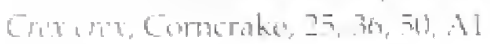

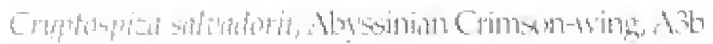

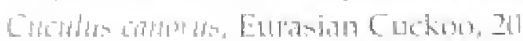

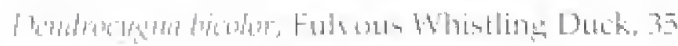

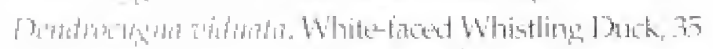

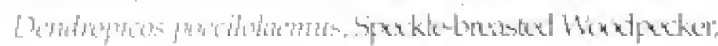
in

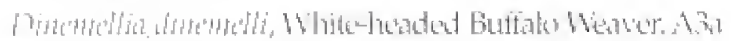

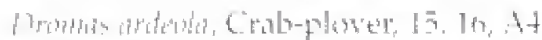

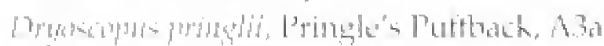

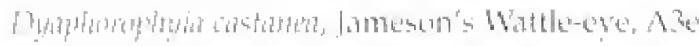

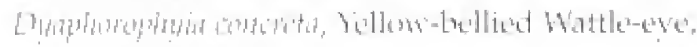
$57,55,58,41$

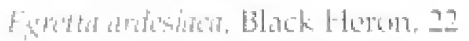

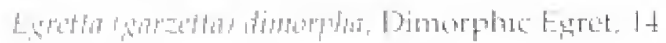

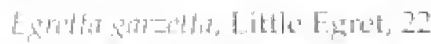

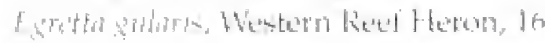

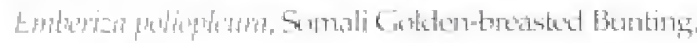
$\lambda \mathrm{x}_{\mathrm{s}}$

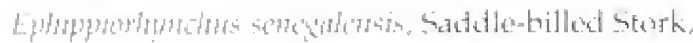
$23,23,24,26,31,35,44,48,50,41$

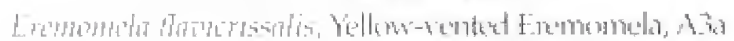

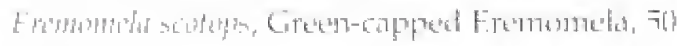

5. How

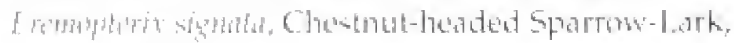
$27, A, 3,4$

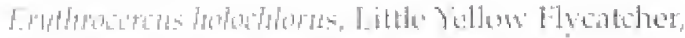
$7,4,11,17,18,20,23, A 1, A 30$ 


\section{Index of bird species: scientific names}

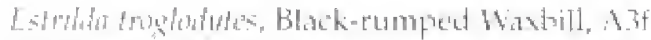

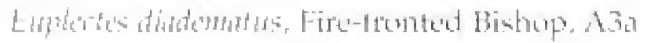

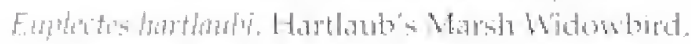

$57,58, \mathrm{Al}$

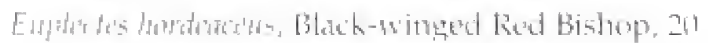

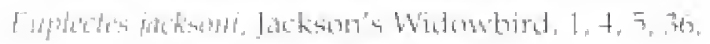

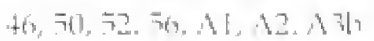

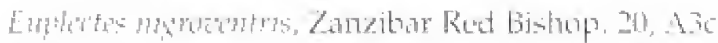

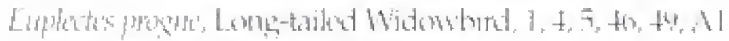

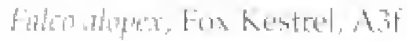

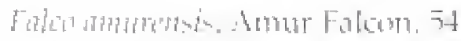

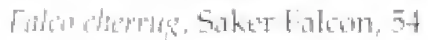

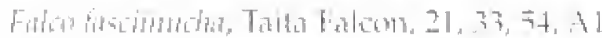

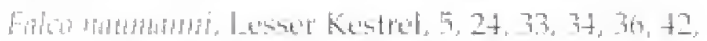

$44,45,46,44,50,52, \mathrm{~A}, 1,4$

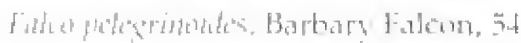

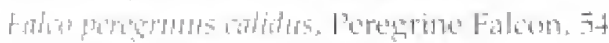

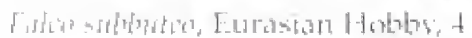

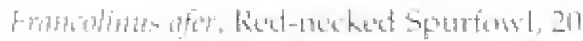

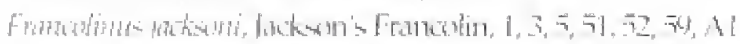

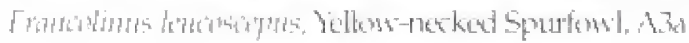

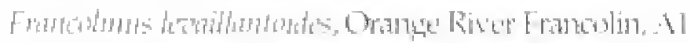

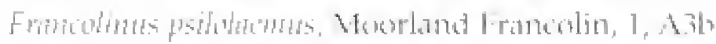

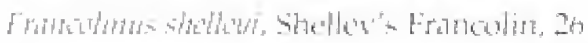

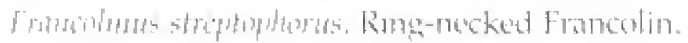

$54, A 1, A 3$.

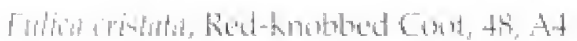

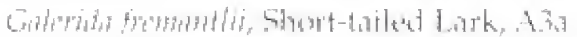

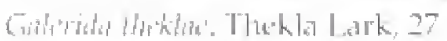

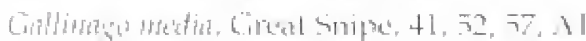

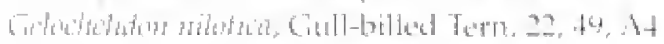

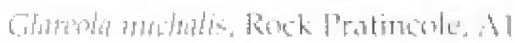

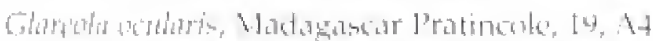

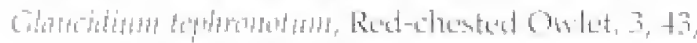

引1, $57,55,58,74$, \& 1

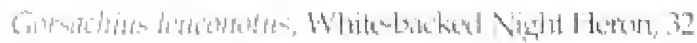

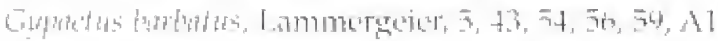

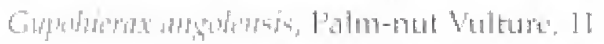

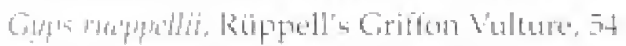

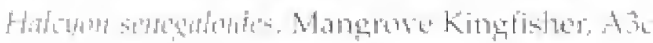

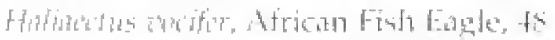

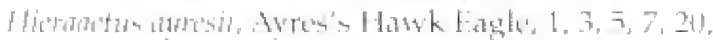

$21,23,26,73,46,20,57,54,74,42$

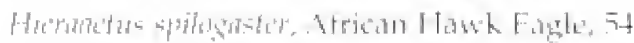

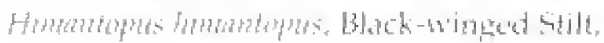

$4+4,4+4 t$

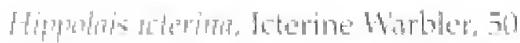

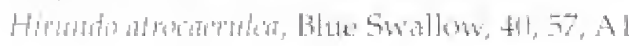

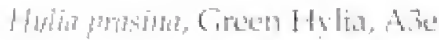

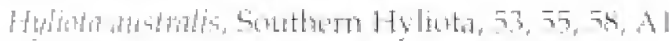

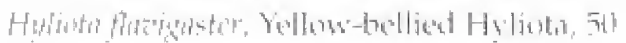

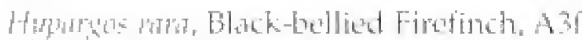

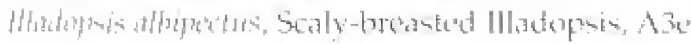

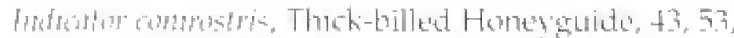
$55,78,54,1$

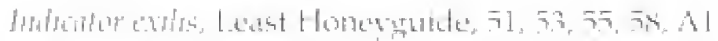

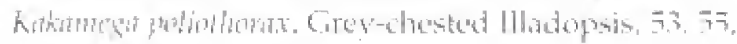

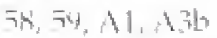

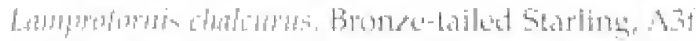

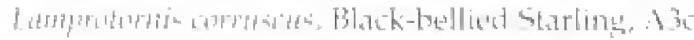

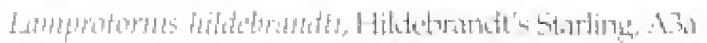

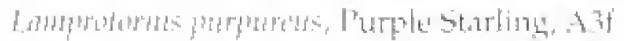

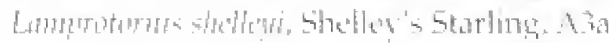

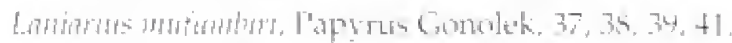
(1) $11, \wedge 3\}$

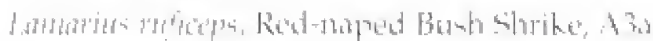

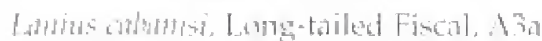

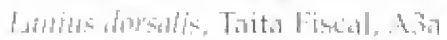

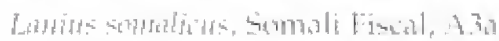

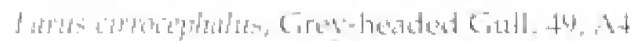

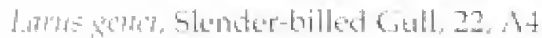

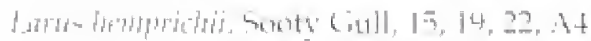

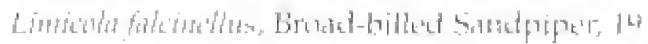

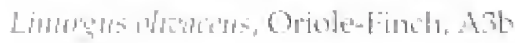

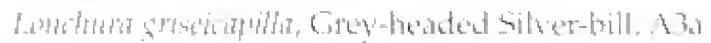

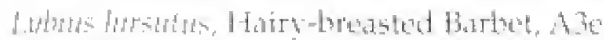

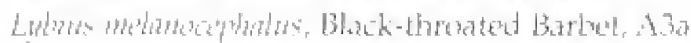

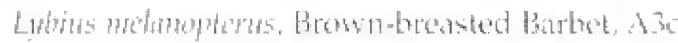

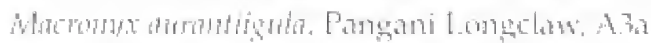

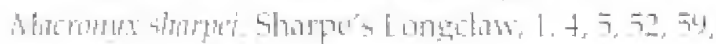

A., $12,4.34$

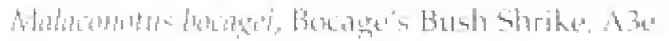

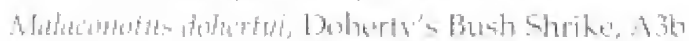

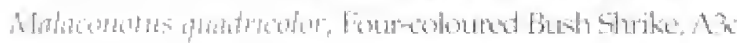

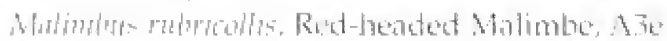

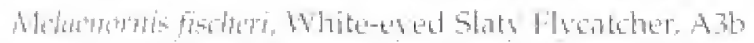

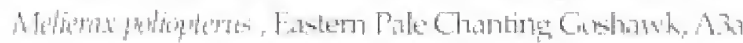

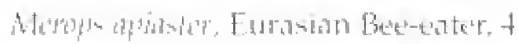

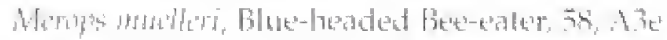

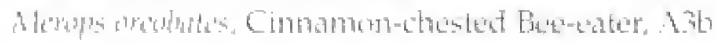

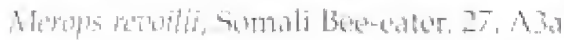

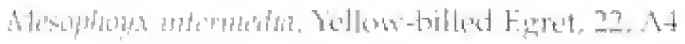

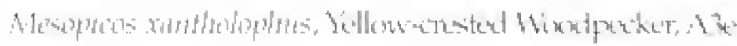

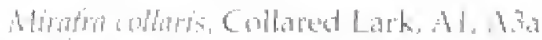

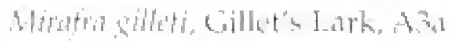

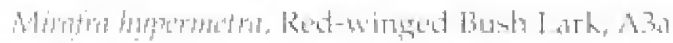

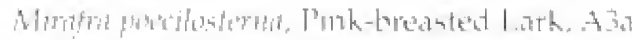

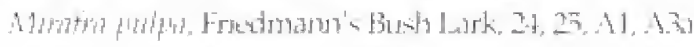

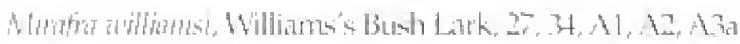

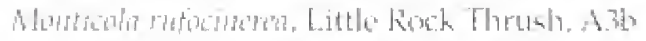

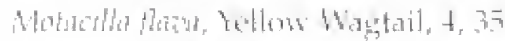

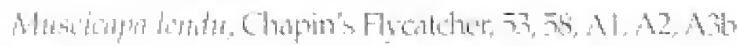

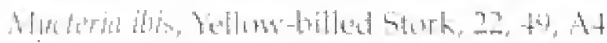

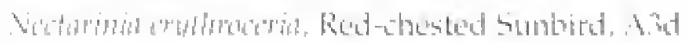

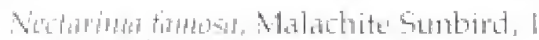

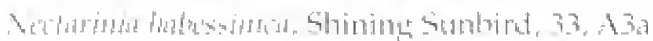




\section{Important Bird Areas in Kenya}

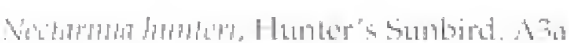

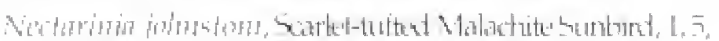
존

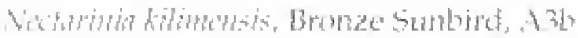

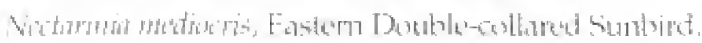
1. $\mathrm{B}$ 3

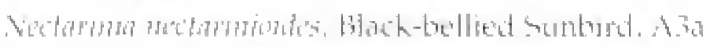

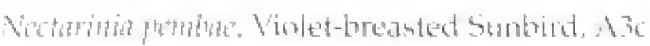

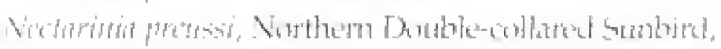
A. .7 ไา

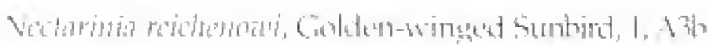

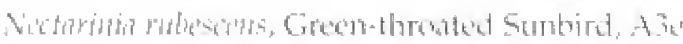

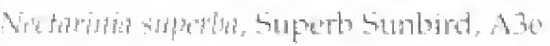

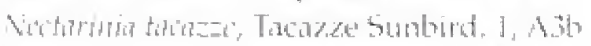

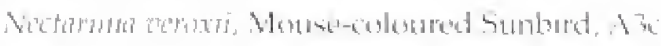

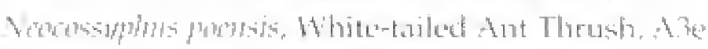

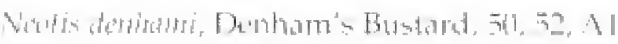

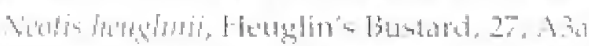

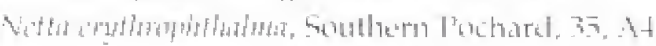

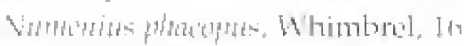

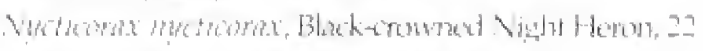

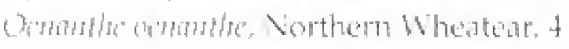

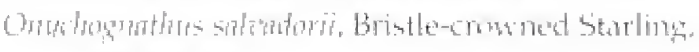

$$
73,41,13 \mathrm{a}
$$

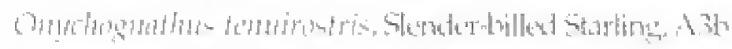

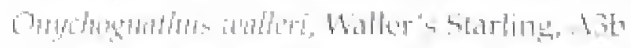

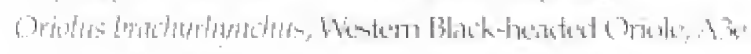

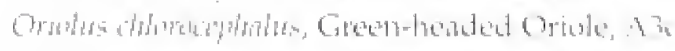

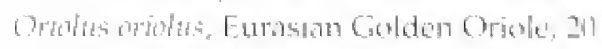

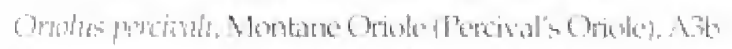

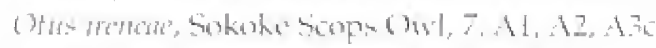

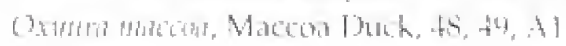

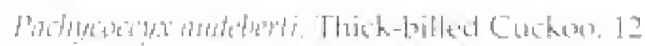

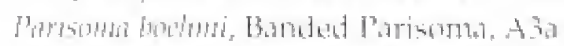

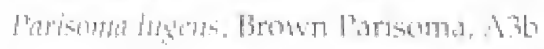

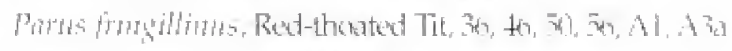

Pontherens, Dusky Tit, As?

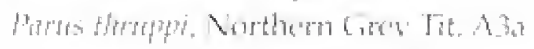

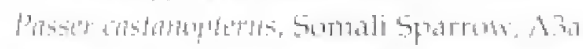

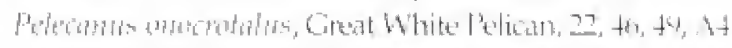

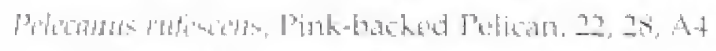

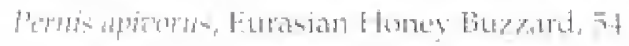

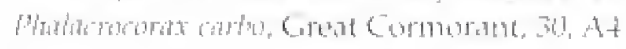

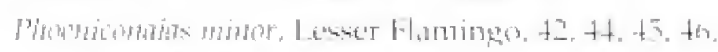

$\left[7,+5,44, A_{1}, A .4\right.$

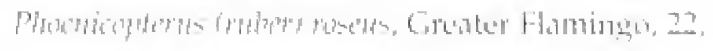

$2 x, 45,46,-44,24$

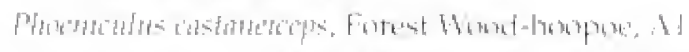

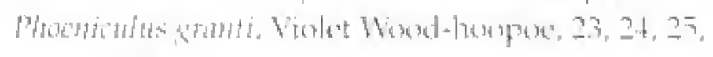

$31, \mathrm{Al}$

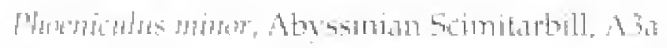

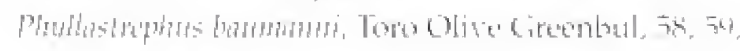
A1, $37^{\circ}$

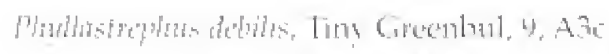

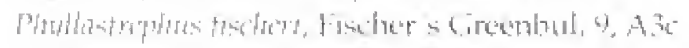

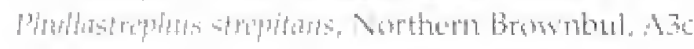

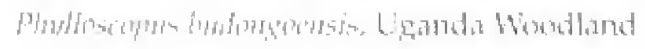

Wartioler A. A

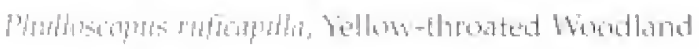

Wartelter: 21

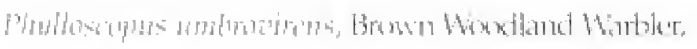
事祘

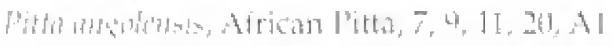

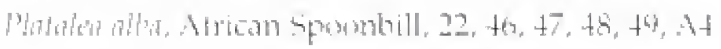

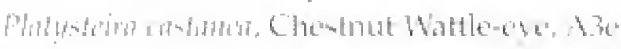

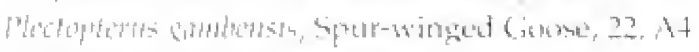

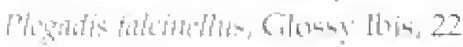

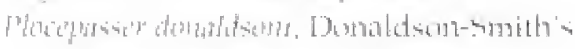

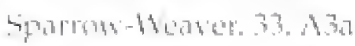

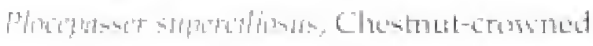

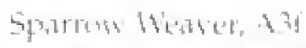

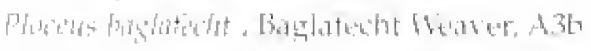

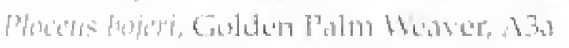

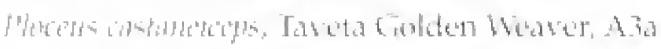

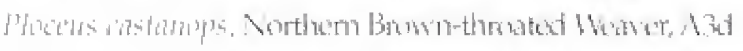

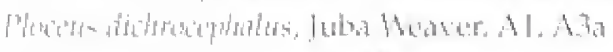

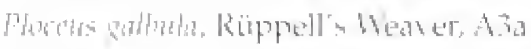

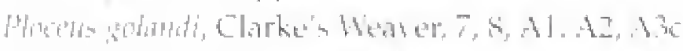

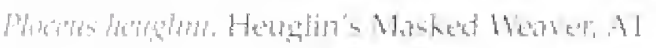

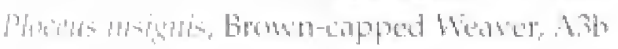

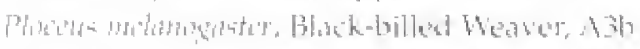

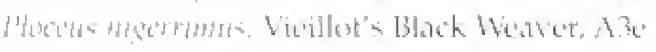

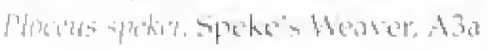

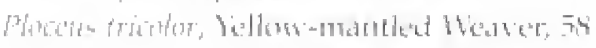

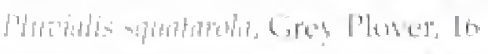

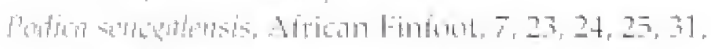

$36, \overline{71}, \mathrm{Al}$

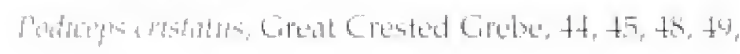

ㄱ. Al

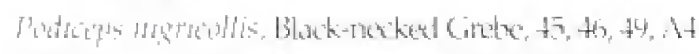

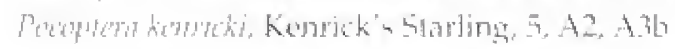

Pอำ

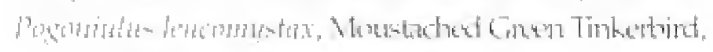

A. 旅

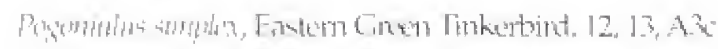

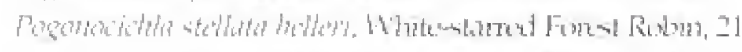

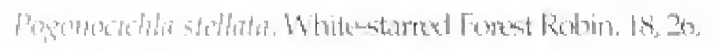

1.2⿰亻⿱丶⿻工二口力

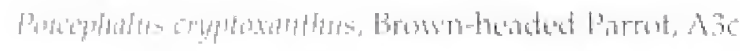

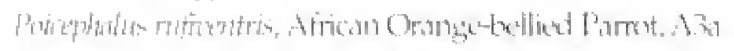

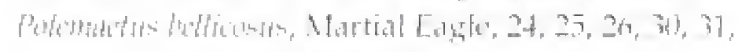

$32,33,34,30,42,4+4,45,46,+4,517,54, A 1$

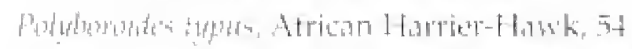

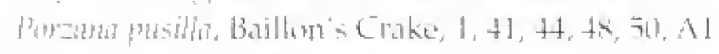

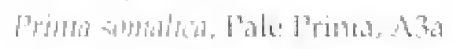

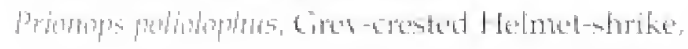

\section{Index 1}




\section{Index of bird species: scientific names}

$46,45,+4,50,56,43, A_{2}, 4,31$

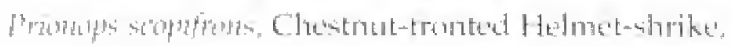
12. $A 3$

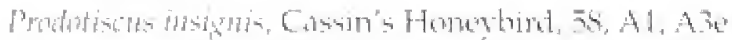

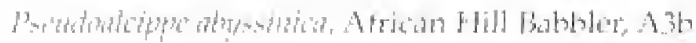

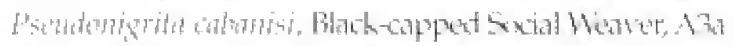

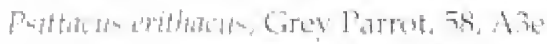

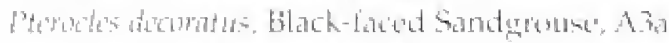

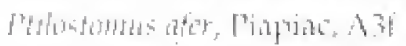

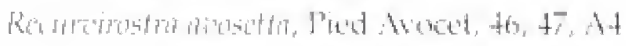

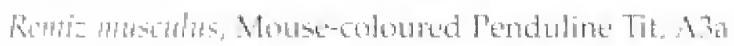

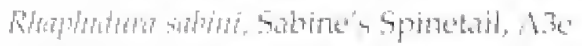

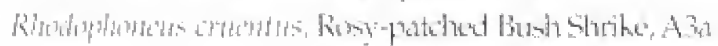

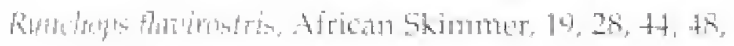
난, $A 1$

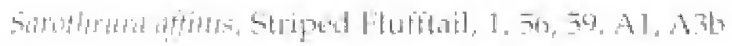

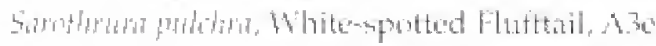

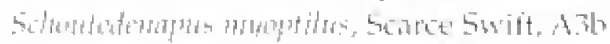

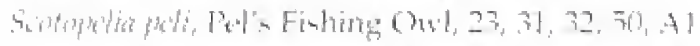

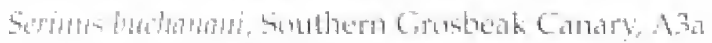

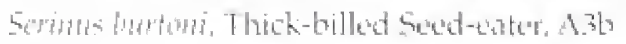

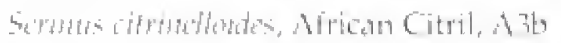

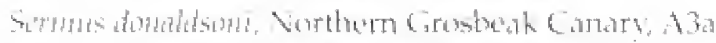

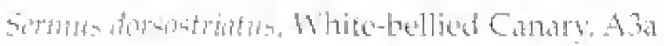

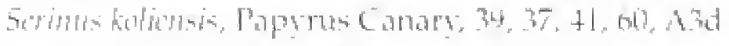

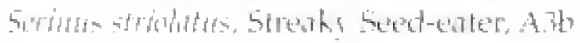

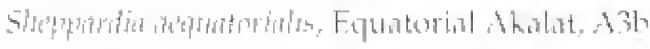

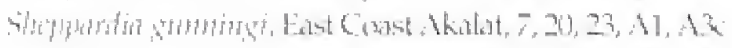

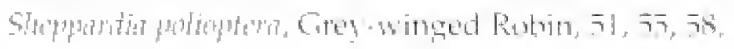
$59, \mathrm{~A} 1$

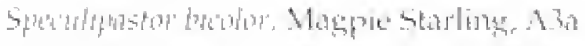

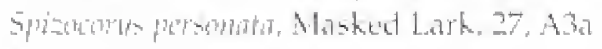

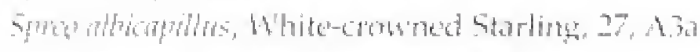

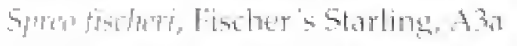

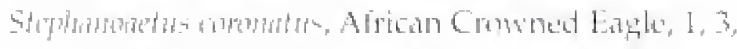
$5,-7,9,10,2(1,21,23,20,34,47,46,50,51,73$,

$54,57,36,78,-39, \mathrm{Al}$

5teme of

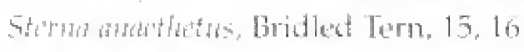

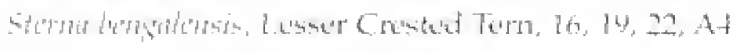

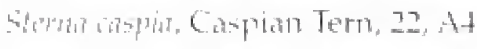

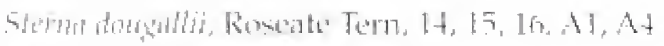

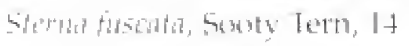

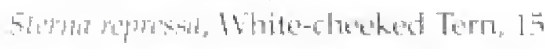

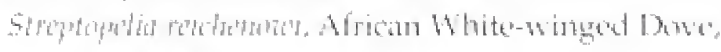
A $1 . \mathrm{ABH}_{3}$

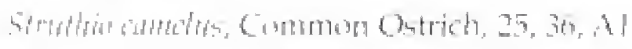

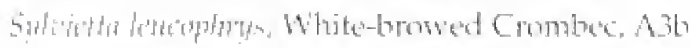

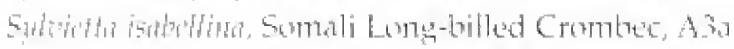

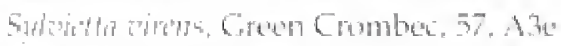

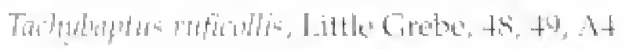

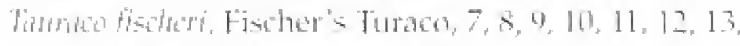
$17,18,20,27,41,2,430$

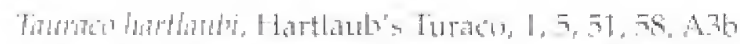

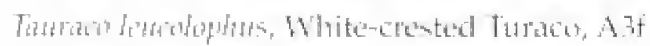

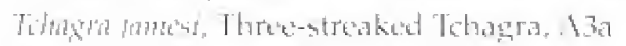

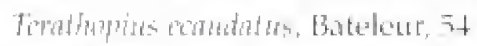

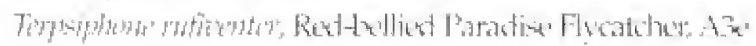

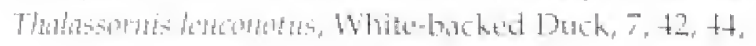
$45,48, \mathrm{~A} 1$

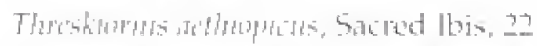

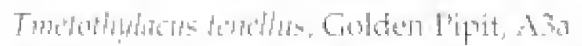

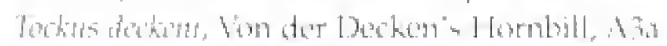

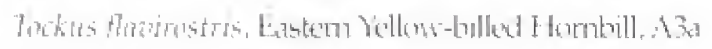

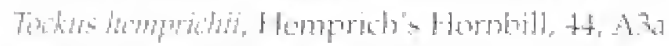

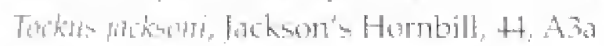

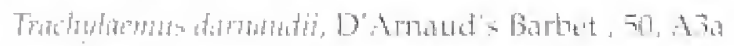

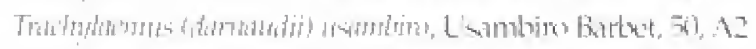

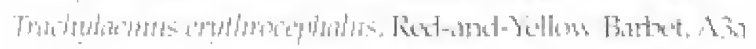

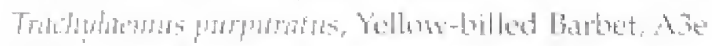

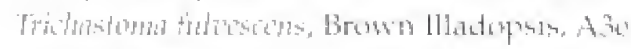

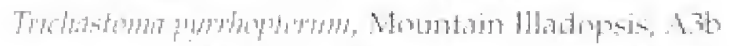

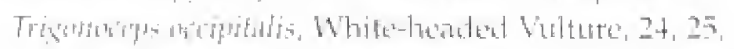
$3,3,3,42,4,4,7,-43,5(1, A 1$

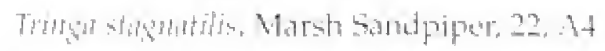

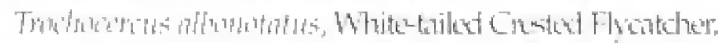
Alb

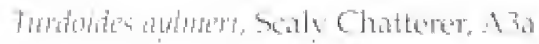

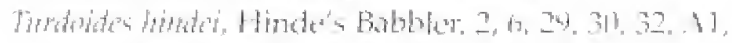
12. 12,3

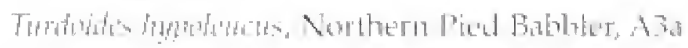

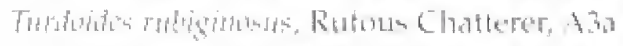

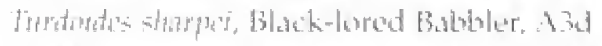

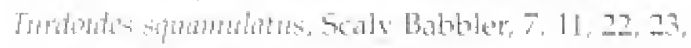
A. $A$ t. 76

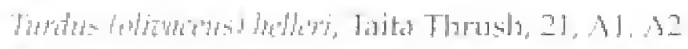

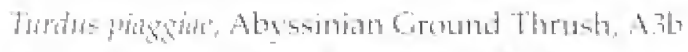

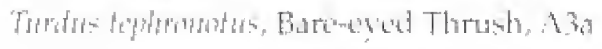

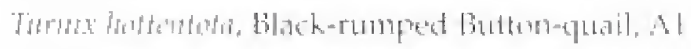

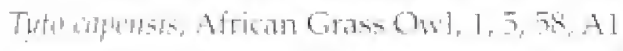

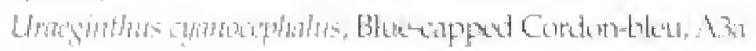

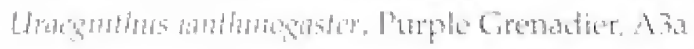

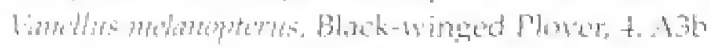

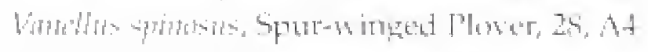

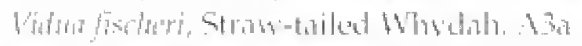

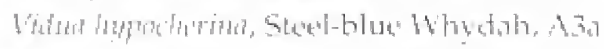
Linels

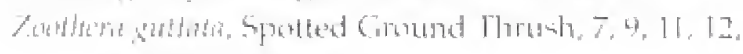

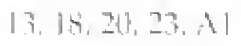

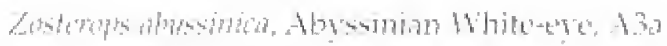

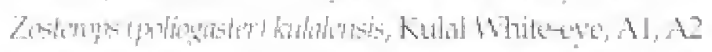

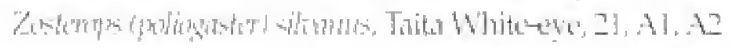

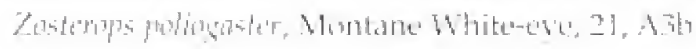




\title{
Index 2
}

\section{Bird species in the site accounts and appendices, indexed by English name}

\author{
Nommencialute follows OS-C (1496). \\ Numbers refer to 1BA or (ii prefixed by " $A$ ') appendix numbers, not page numbers
}

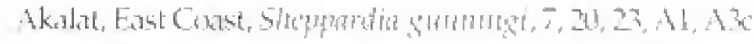

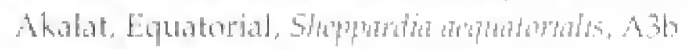

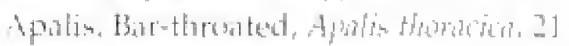

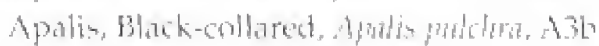

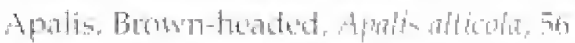

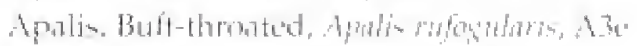

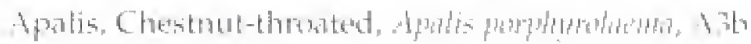

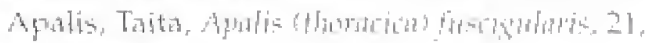

A1, $A 2$

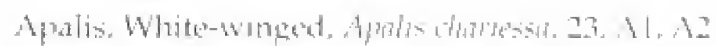

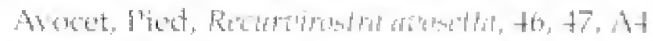

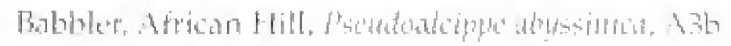

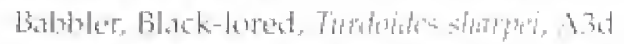

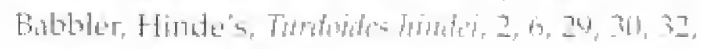

At, A2, A3

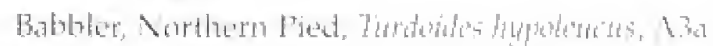

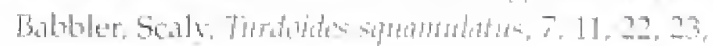

$$
\text { A. A. } 3 \mathrm{C}
$$

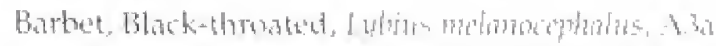

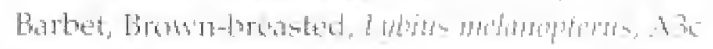

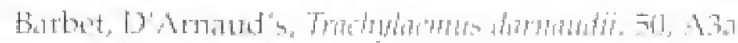

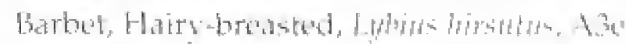

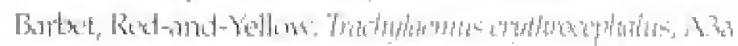

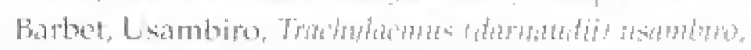
Bi), $A 2$

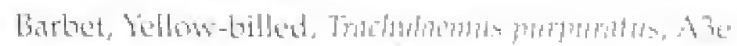

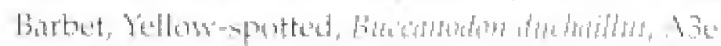

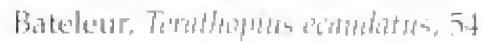

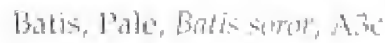

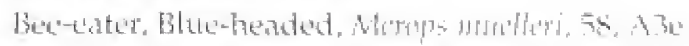

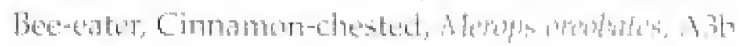

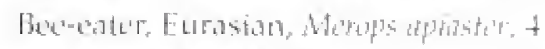

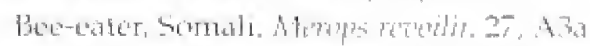

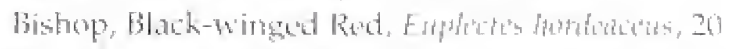

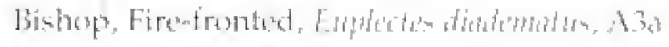

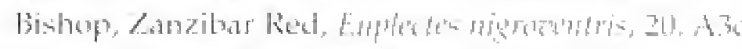

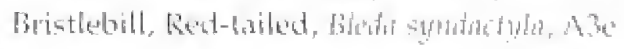

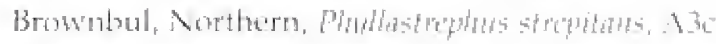

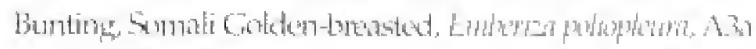

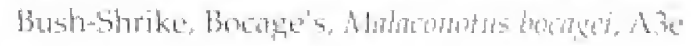

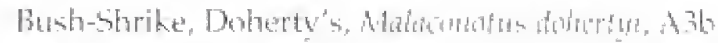

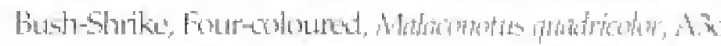

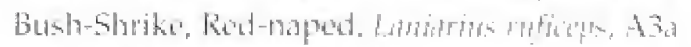

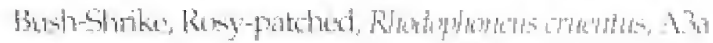

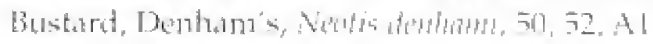

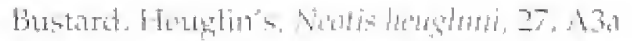

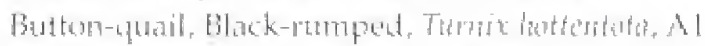

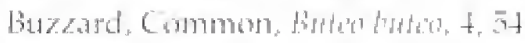

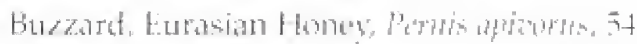

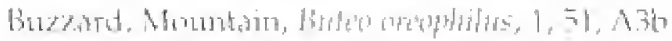

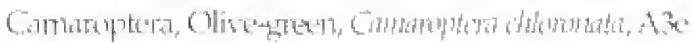

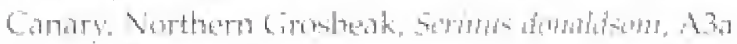

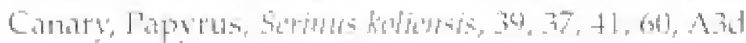

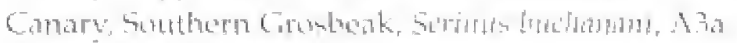

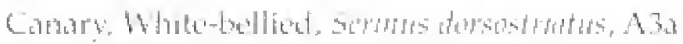

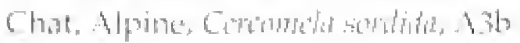

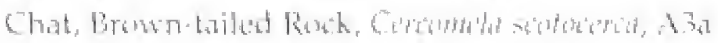

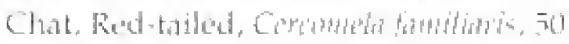

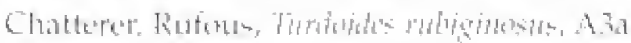

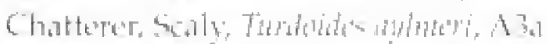

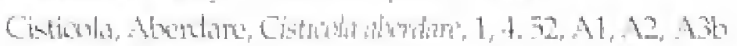

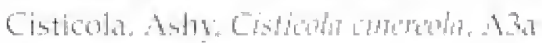

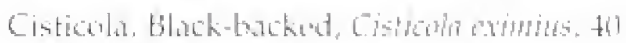

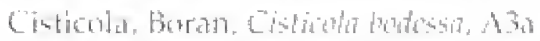

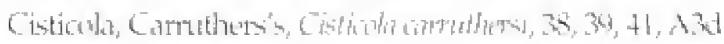

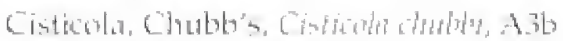

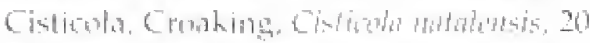

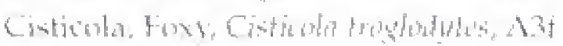

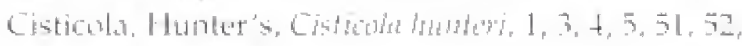
$5+50,54,12$

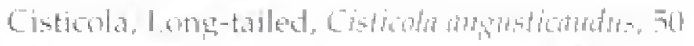

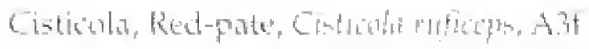

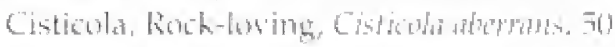

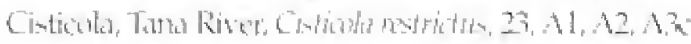

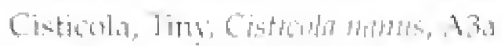

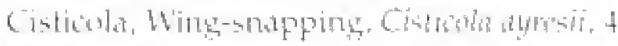

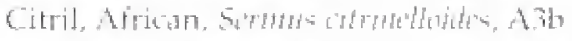

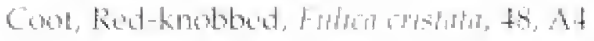

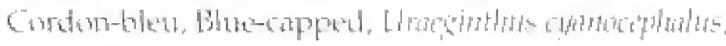
$\sqrt{3} 3$

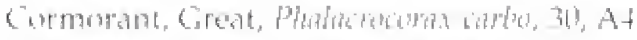

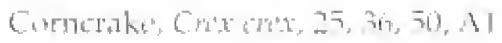

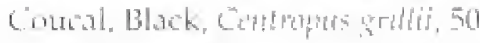

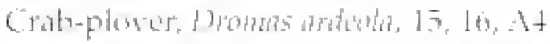

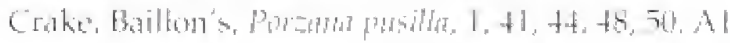

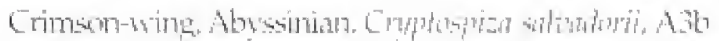

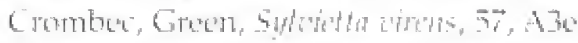

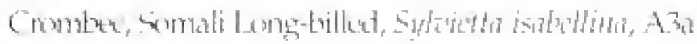

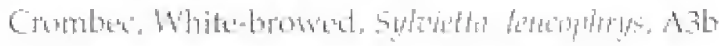

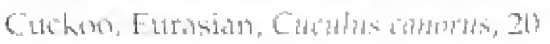




\section{Index of bird species: English names}

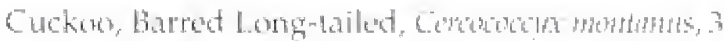

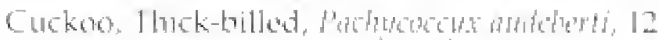

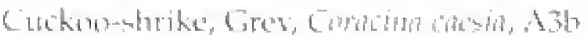

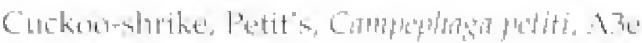
Darter, Alrican, Anbight ruth, 22, 23, 24, 25, 30, 32, $33,34,7(9,42,4+45,48,30, \mathrm{~A}$

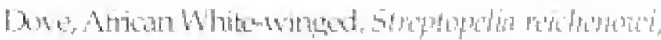
A1.A.

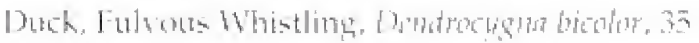

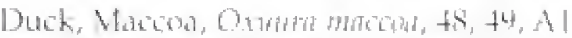

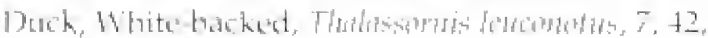
$4.45,48, A$

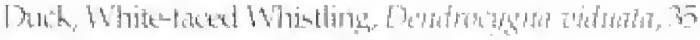

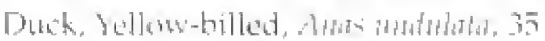

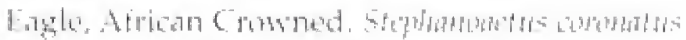
$1,3,5,7,4,110,20,21,23,217,36,43,40,70,51$, $53,54,55,50,5,5,52, A 1$

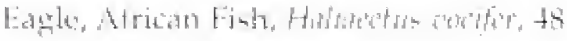

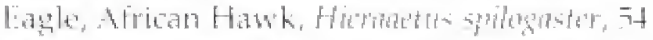

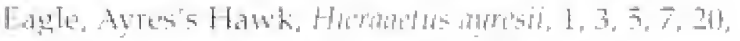
$21,23,26,36,46,50,51,54,58,4]$

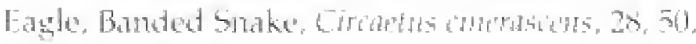
$57,58, \mathrm{~A}$

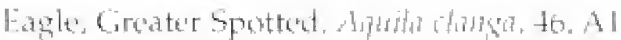

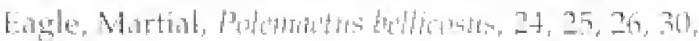
$31,32,73,34,317,42,44,45,46,49,50,54,11$

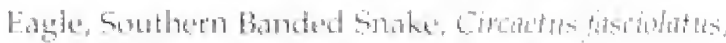
$7,8,9,11,21,21,22,23,11,130$

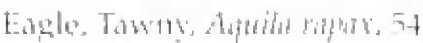

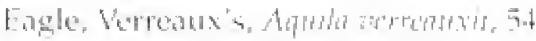

Egred, Cattle: Bubluters itis, 22

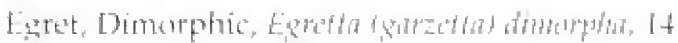

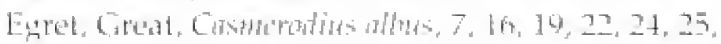
$28,361,33,3+, 35,36,+1,42,44,41,47,45,44$ $7(0, A+$

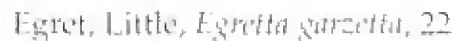

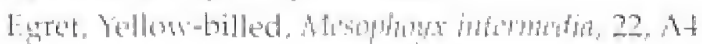

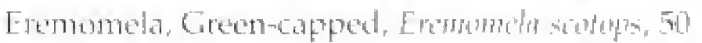

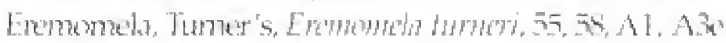

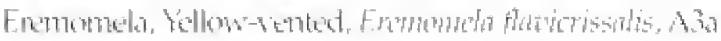

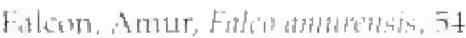

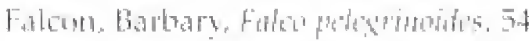

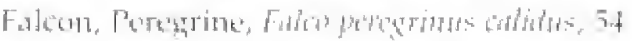

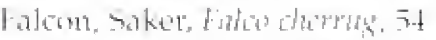

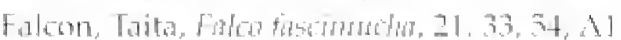

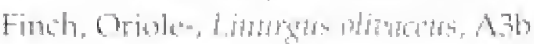

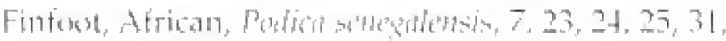
3. $50, \mathrm{Al}$

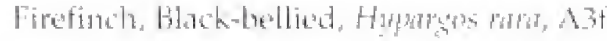

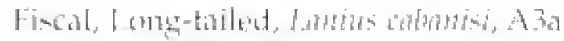

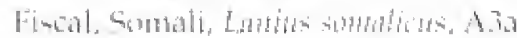

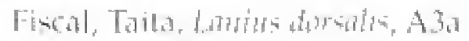

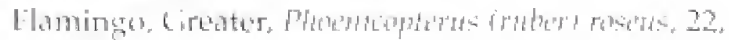

$28,47,46,19,24$

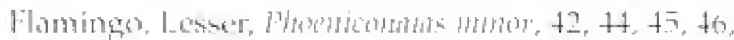
$4 \overline{7}, 48,44,81,24$

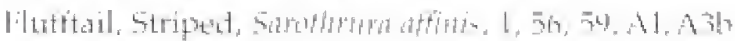

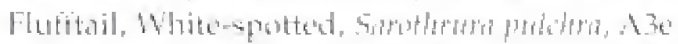

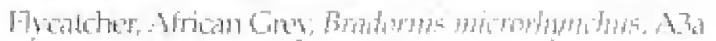

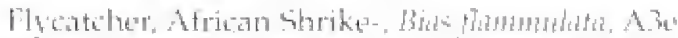

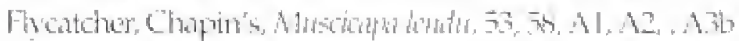

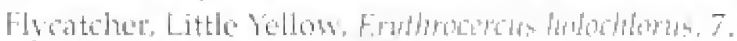
$4,11,17,18,20,27,41, A, 36$

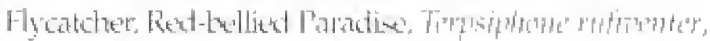
$A$ A.

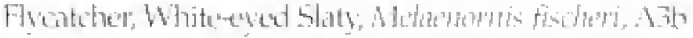

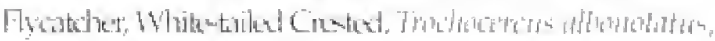
A 3 औै?

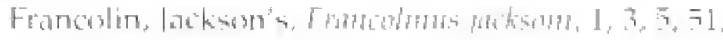
$52,54,12$

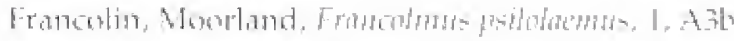

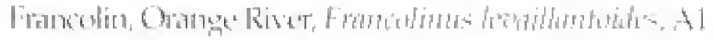

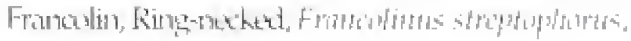
99, A I, Ant

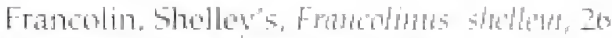

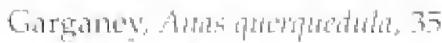

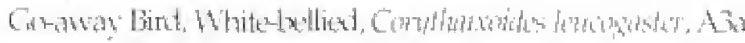

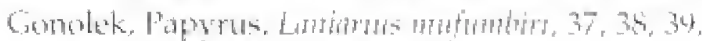
41. 60 , A1, A ind

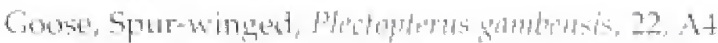

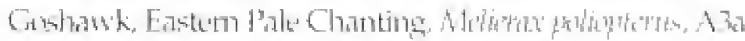

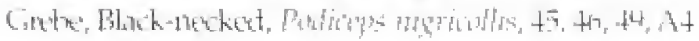

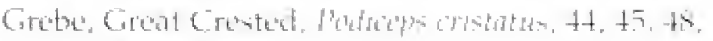
$44,52,+1$

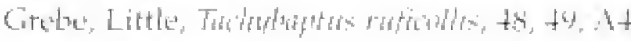

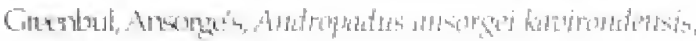
5. 12

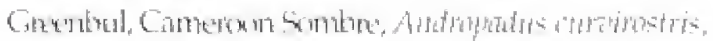
$A$,

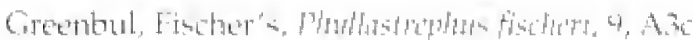

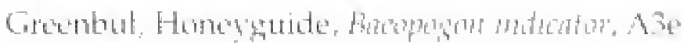

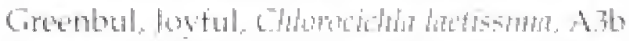

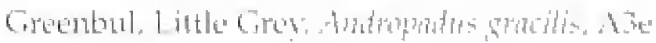

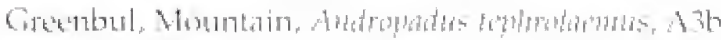

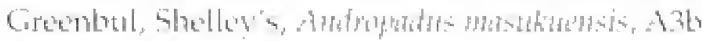

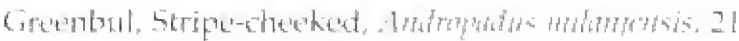

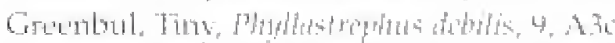

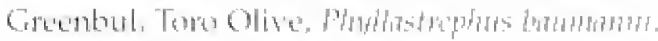
59, $54, A 1, A 3 E^{2}$

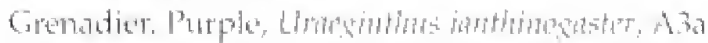

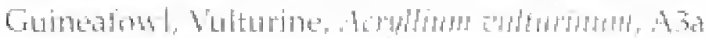

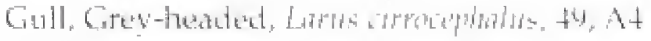

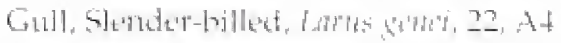

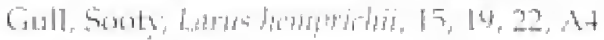

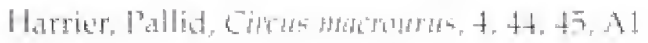

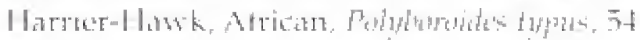




\section{Important Bird Areas in Kenya}

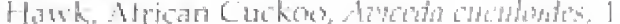

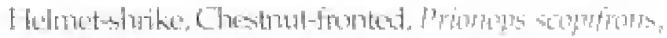
$12, A$

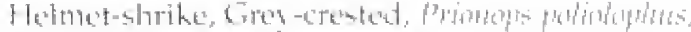
$417,45,44,50,51, A], 12,13$ a

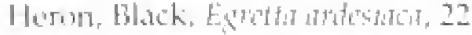

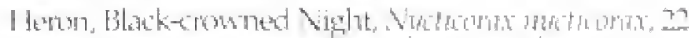

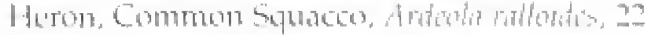

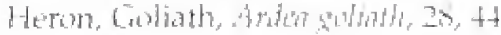

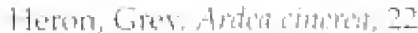

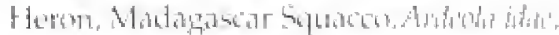

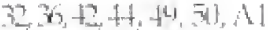

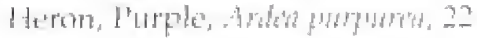

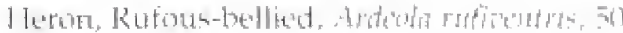

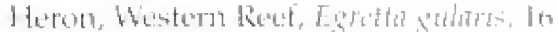

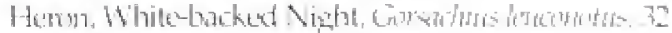

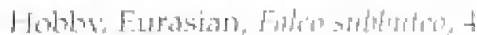

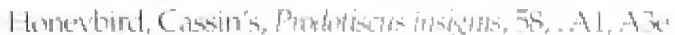

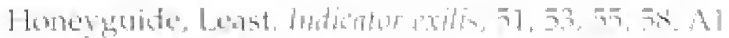

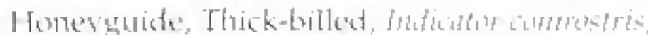
$4.7,73,3 \overline{7}, 58,54,11$

I Lombill, Black-and-ribite-arapued.

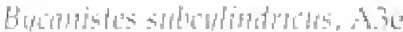

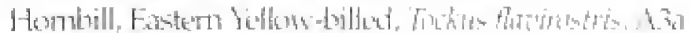

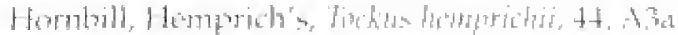

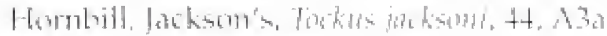

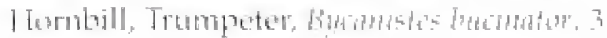

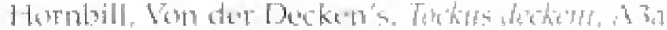

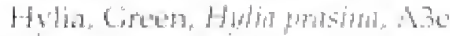

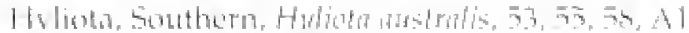

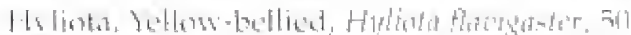

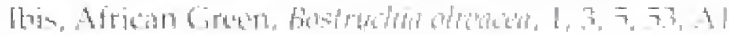

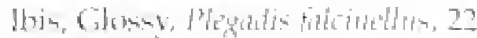

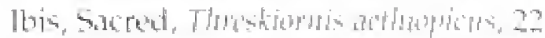

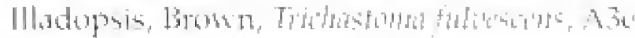

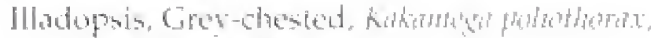
$53,55,58,54,1, \wedge 30$

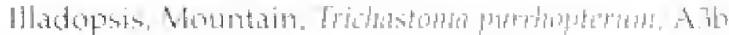

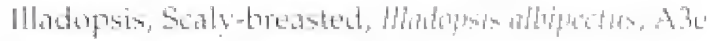

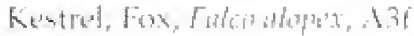

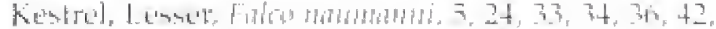
$44,45,40,46,50,52,4, A 4$

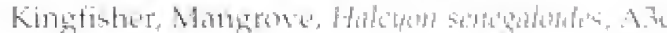

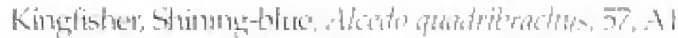

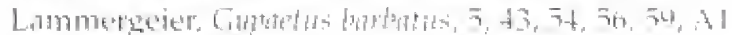

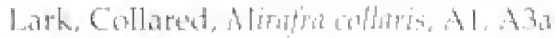

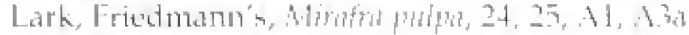

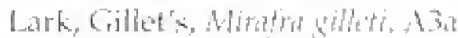

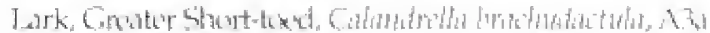

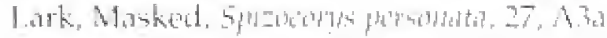

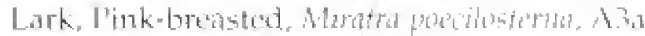

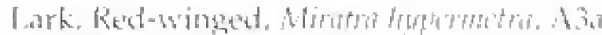

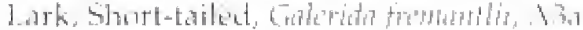

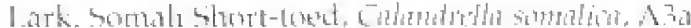

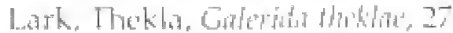

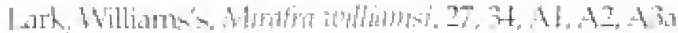

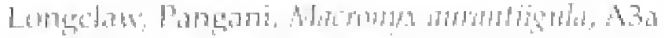

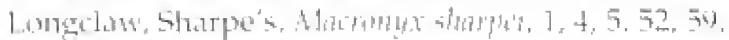
A1, $12, A, 377$

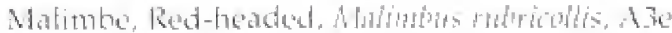

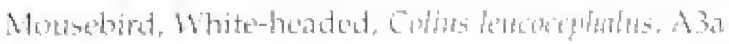

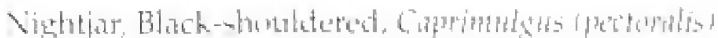

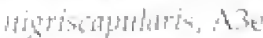

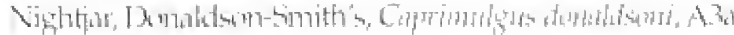

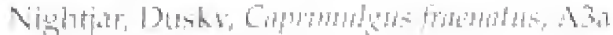

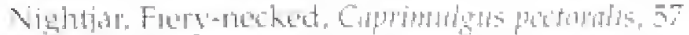

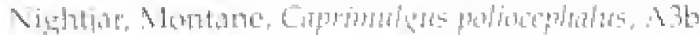

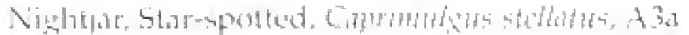

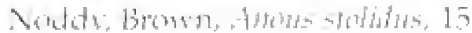

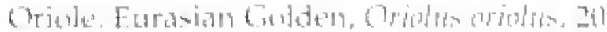

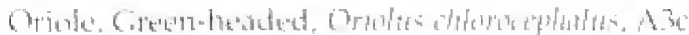

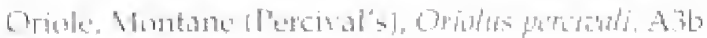

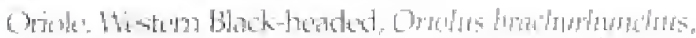
13.

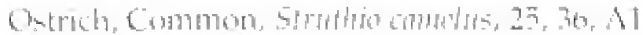

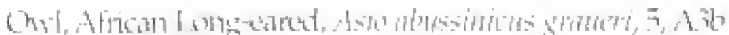

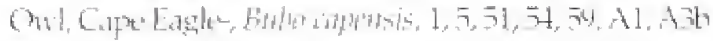

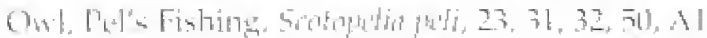

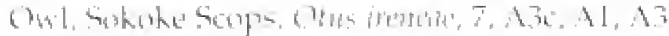

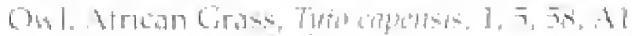

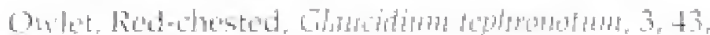
$51,5,55,58,54,1$

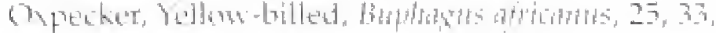
$7-4,7(2,4), 5(1)$

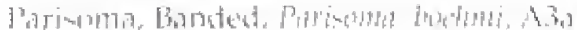

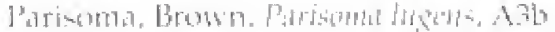

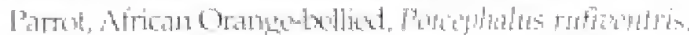

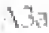

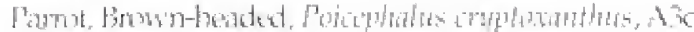

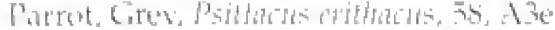

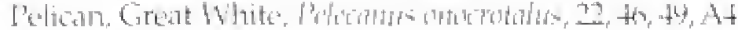

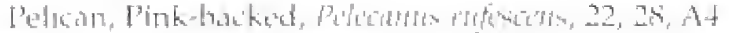

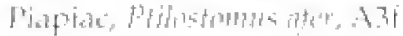

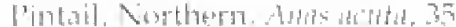

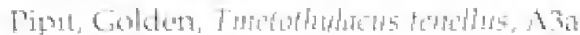

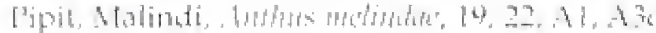

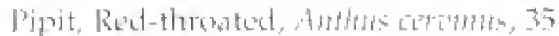

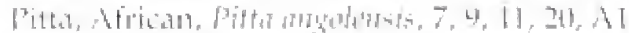

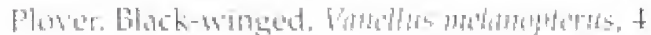

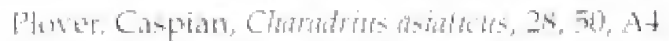

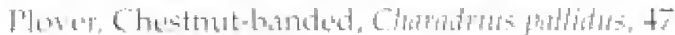

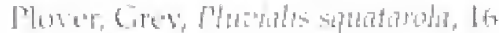

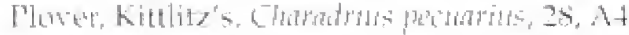

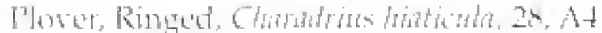

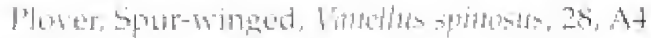

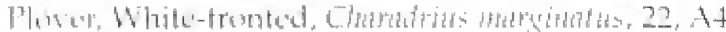




\section{Index of bird species: English names}

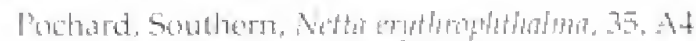

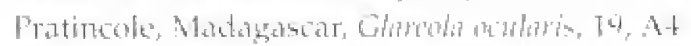

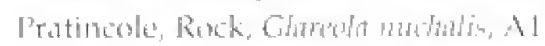

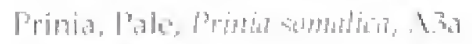

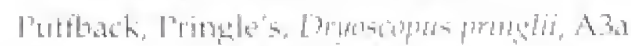

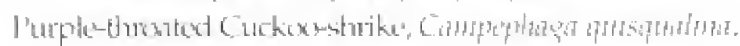
$5,43,51,56,56,59,41$

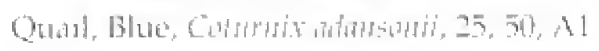

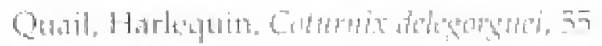

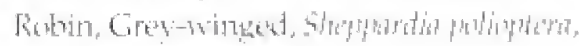
$51,55,58,54,41$

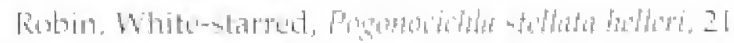

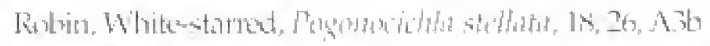

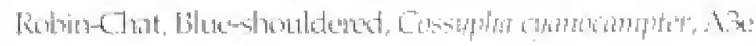

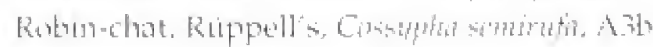

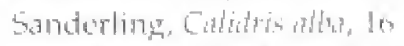

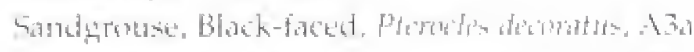

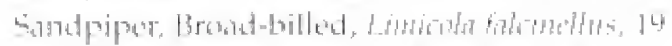

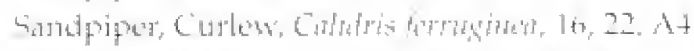

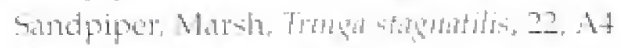

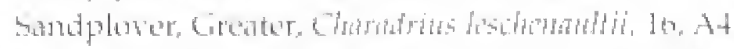

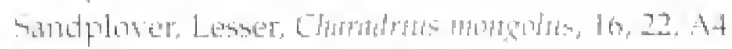

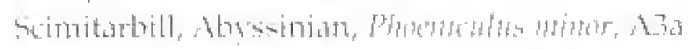

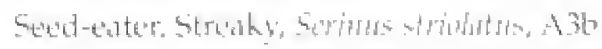

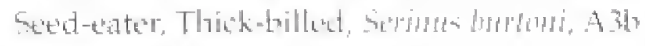

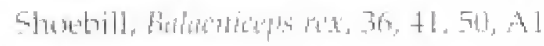

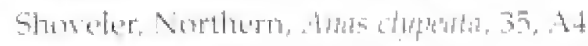

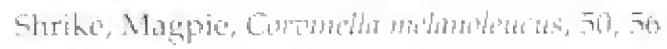

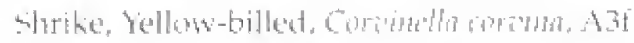

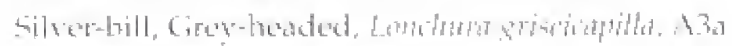

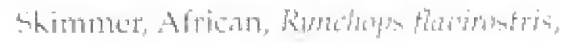
$14,28,44,48,49, A 1$

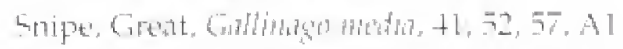

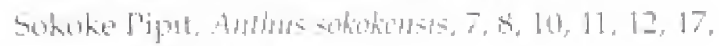
20, $A 1, A 2, A B$

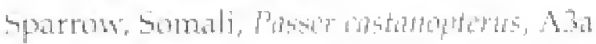

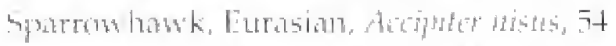

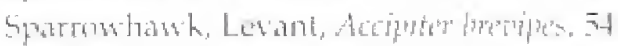

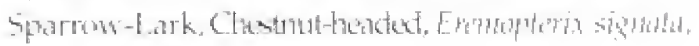

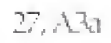

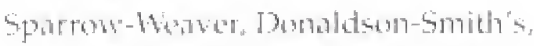

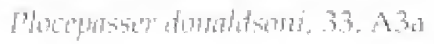

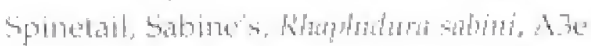

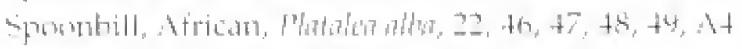

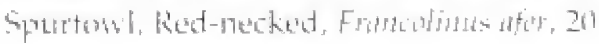

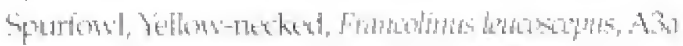

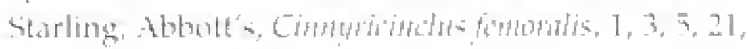
2th, $A 1, A 2, A 30$

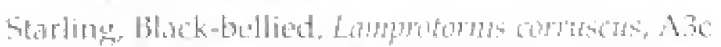

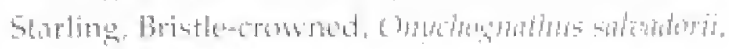
$33,4+4,4 a$

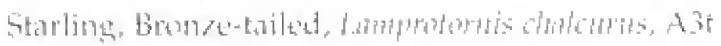

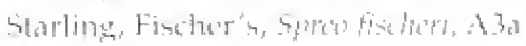

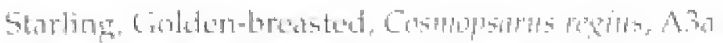

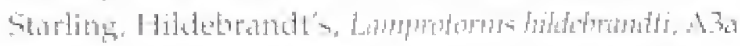

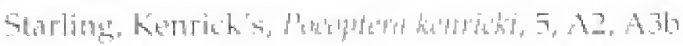

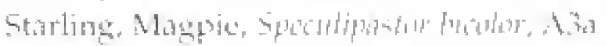

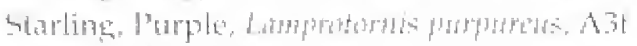

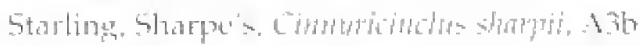

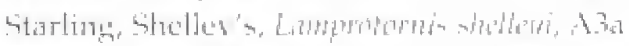

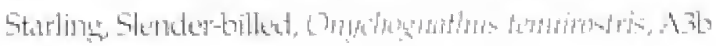

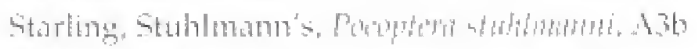

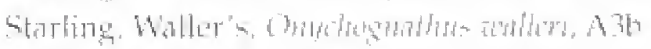

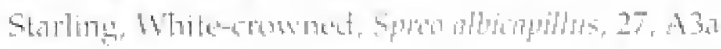

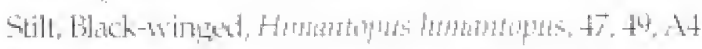

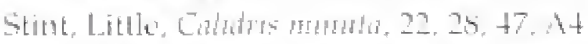

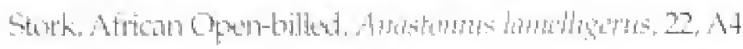

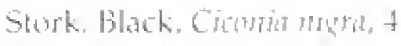

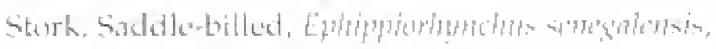
272, 23, 24, 28, 31, 367, 4t, 48, 7(1, A I

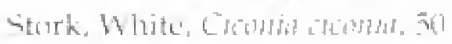

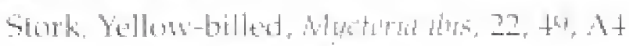

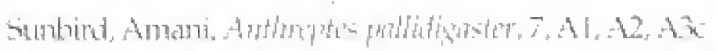

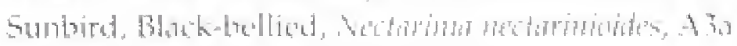

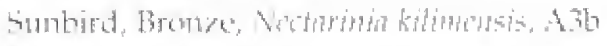

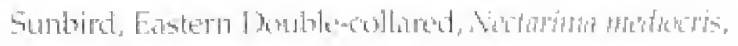
$1, x_{1}$ in

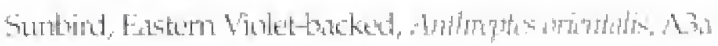

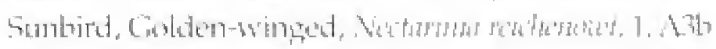

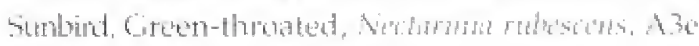

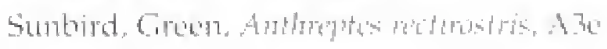

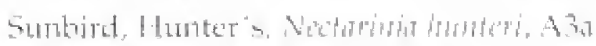

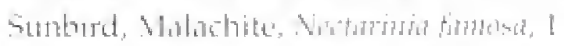

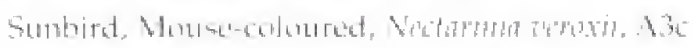

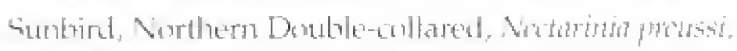
s.to

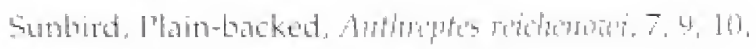
$11,12,17,18,201,23,41, A 3$

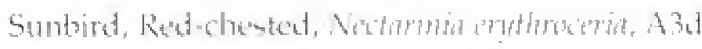

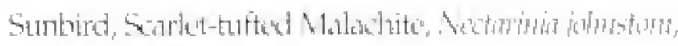
$1.5,5.75$

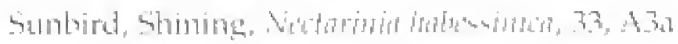

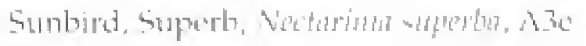

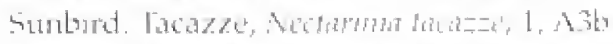

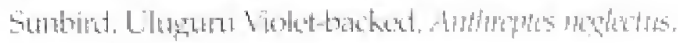
ㄴ. $20,27,41, A 30$

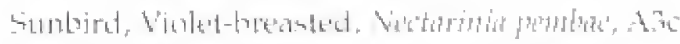

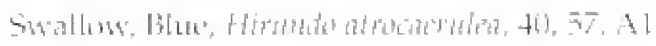

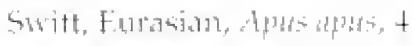

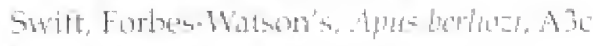

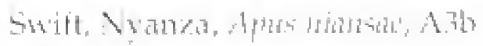

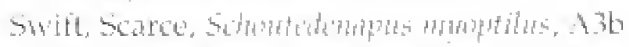

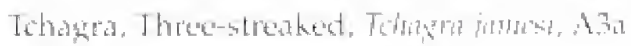

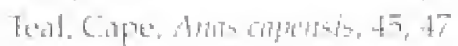

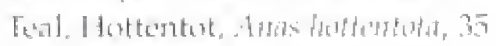

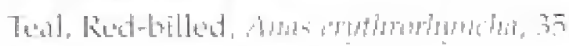




\section{Important Bird Areas in Kenya}

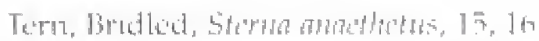

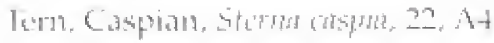

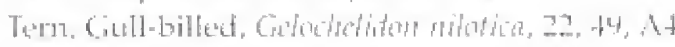

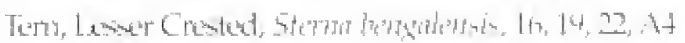

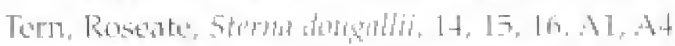

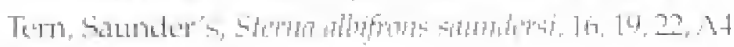

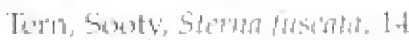

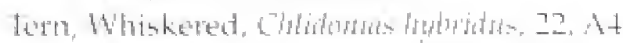

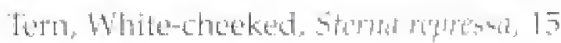

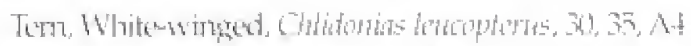

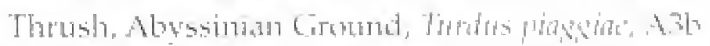

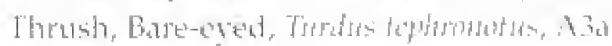

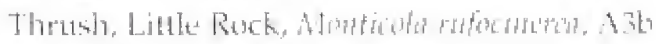

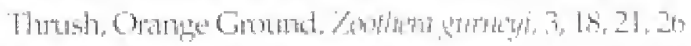

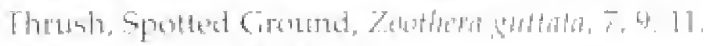

$12,13,18,20,23, \mathrm{~A} \mid$

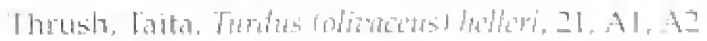

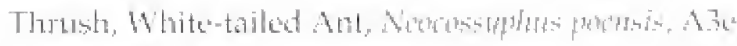

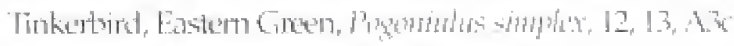

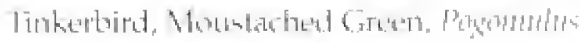

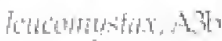

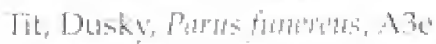

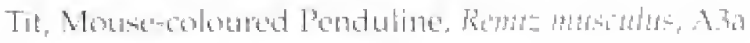

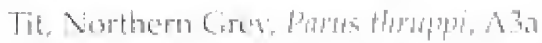

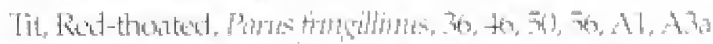

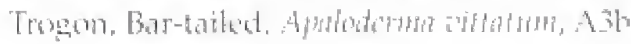

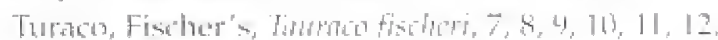

$13,17,18,20,23, A 1, A 2, A 3$

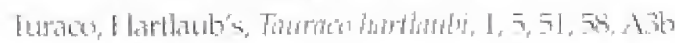

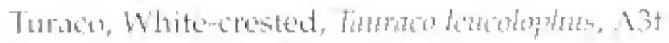

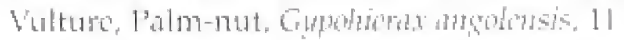

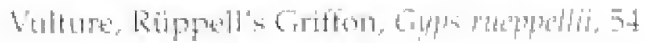

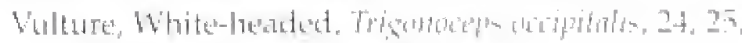
$33,34,42,+4,45,46,50, \lambda 1$

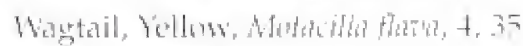

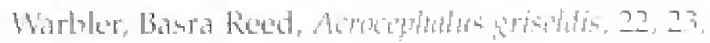
$24,25,36,48, A 1$

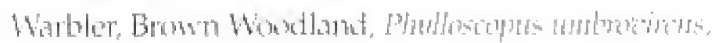
A.3b

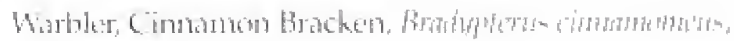
26

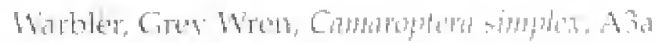

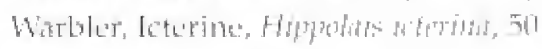

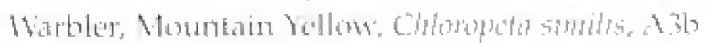

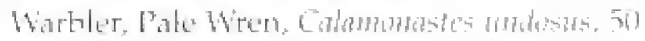

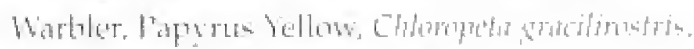
$37,35,34,41,60, \mathrm{~A}, \mathrm{~A}, \mathrm{~A}, \mathrm{C}$

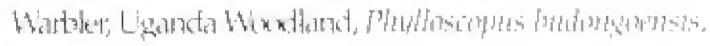
$2 x^{2}$

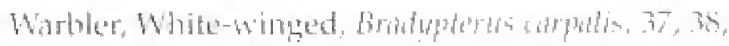
$30,41,60, A_{3}, 4$

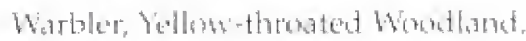

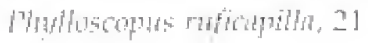

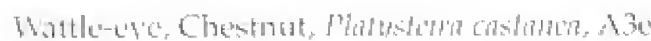

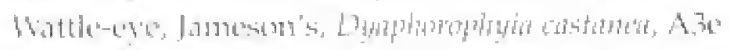

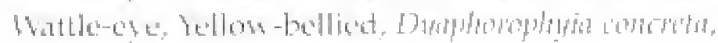
$5,5,5,58, \mathrm{~A}$

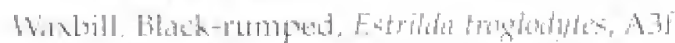

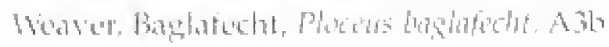

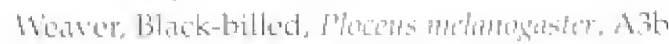

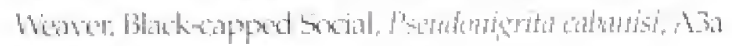

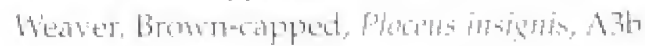

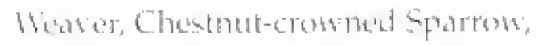

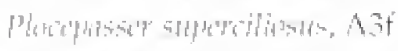

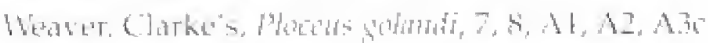

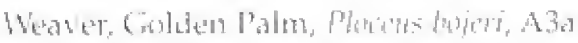

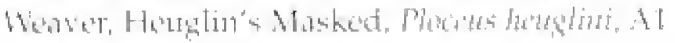

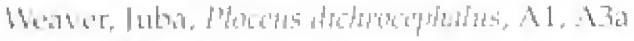

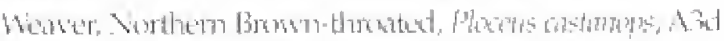

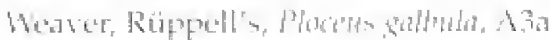

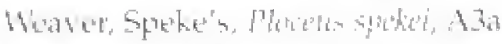

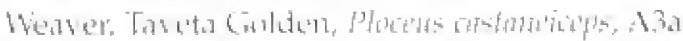

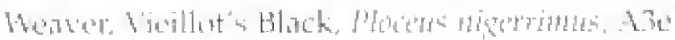

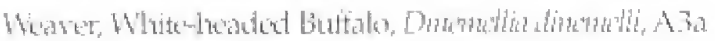

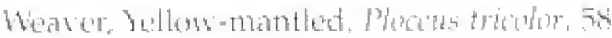

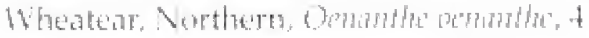

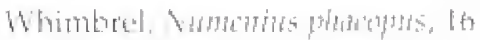

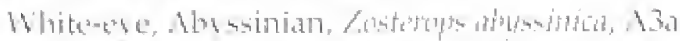

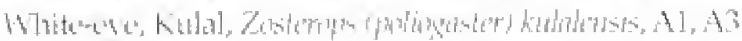

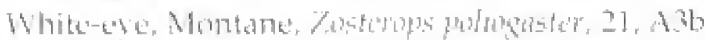

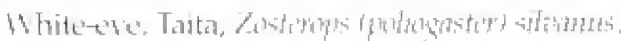
39. $1, \times 3$

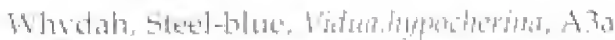

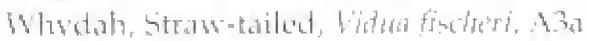

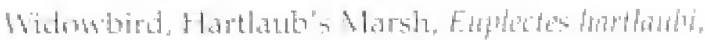
57,5 , Al

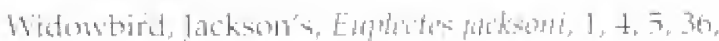
$4(x, 80,52,50,41,42,436$

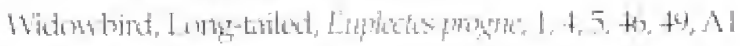

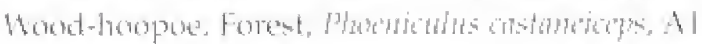

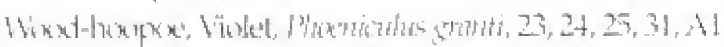

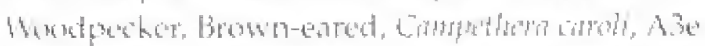

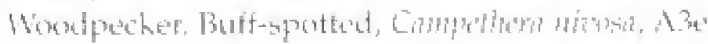

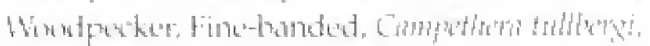
38, $A 3$

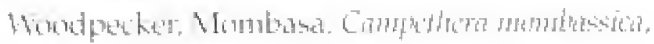
4. 12,43

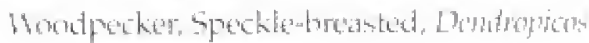

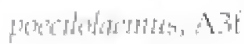

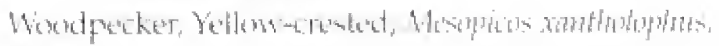
$3 x^{2}$ 


\section{Index 3}

\section{Index 3}

\section{Index of other animals and plants}

Numbers refer to ] BA numbers, not prate numbers

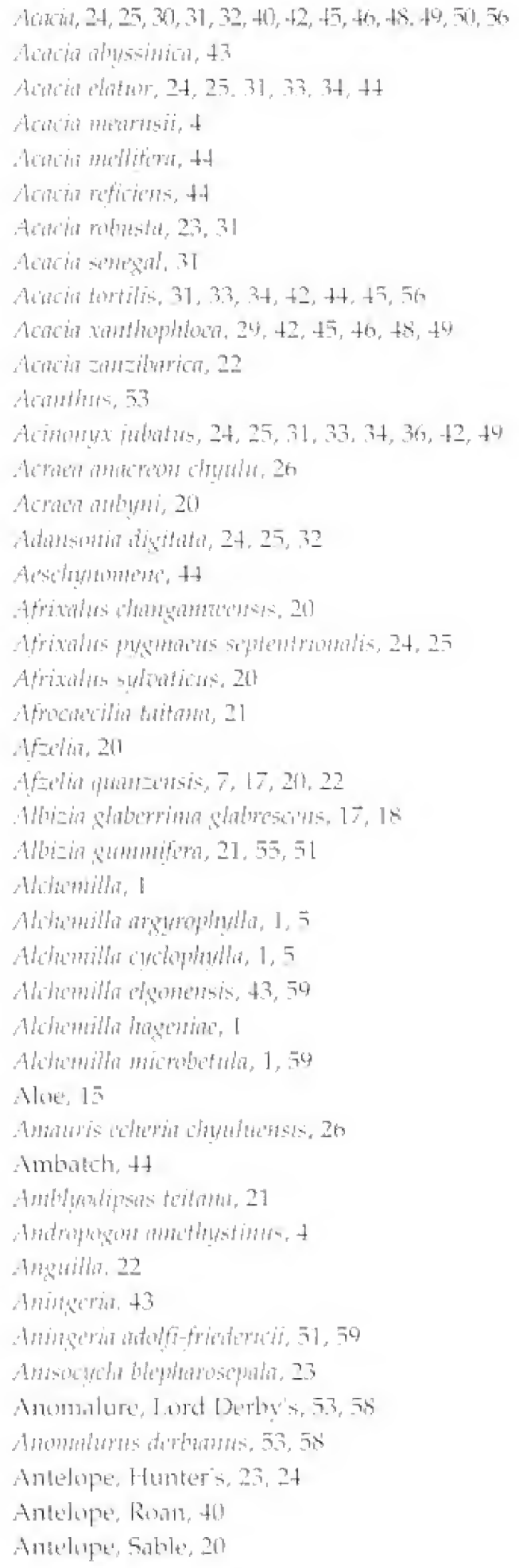




\section{Important Bird Areas in Kenya}

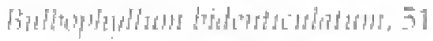

Gustr-yper, Prickly, 58

Bushlucuck, 5, 26, 44, 76

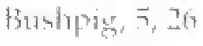

Cabbage, Nike, 22

Cacciliatr, I1

Cumberdam, 36

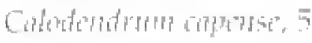

Cатирият, 5

Commatis soldita, 1,5

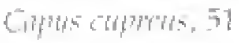

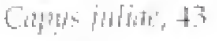

Conth Methensis, 5

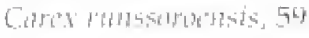

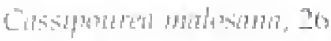

Cat, Mitican Goldetr, 1, 7, 51, 59

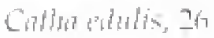

Colth is

Cittion atromen, 53,55

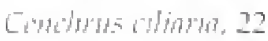

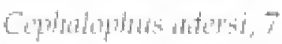

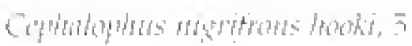

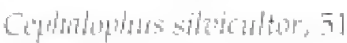

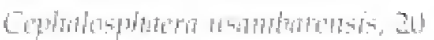

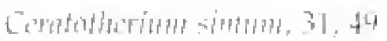

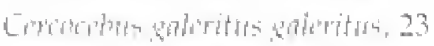

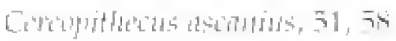

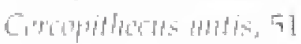

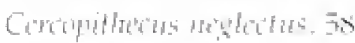

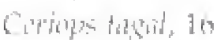

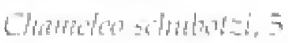

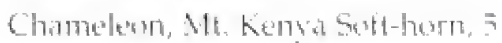

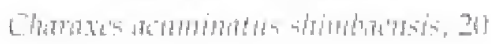

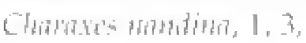

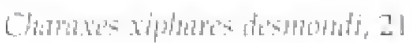

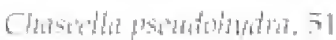

Chete.th, 2-4, 25, 31, 37, 3t 34, 42, 49

Chermom whitis, 15,22

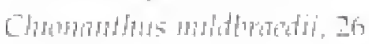

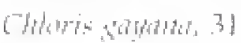

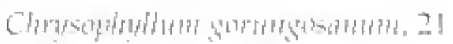

Cinct, Atrian Palm, 57

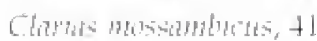

Cobra Goldt's a

Coutene, 2,6

Confertiblaria, 2]

Colditariatis, In). 22

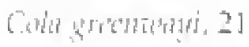

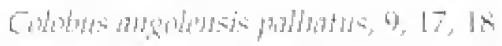

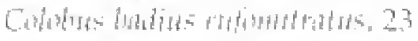

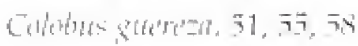

Colsobur, Angola, 4, 17, 18

Colobus, Black \& white, 51,53 , in
Coildous, Red, 23

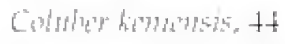

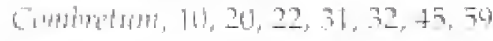

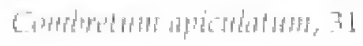

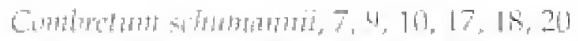

(-

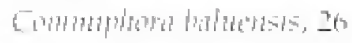

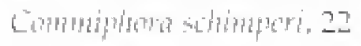

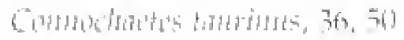

Cowhdortriciona, 17, 18

Cimpte th

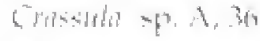

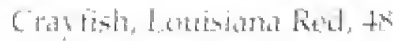

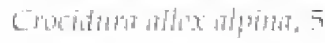

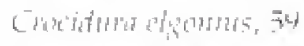

Crondite, vile, 22, 28, 34) $32,35,44$

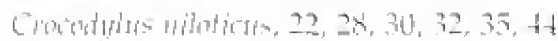

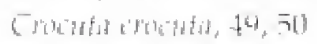

(5)

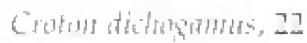

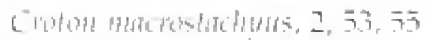

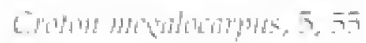

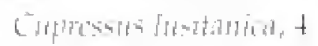

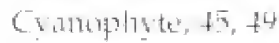

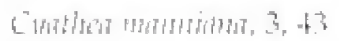

crade it

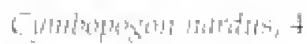

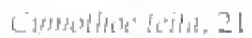

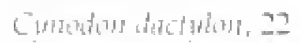

Gorlentedti, $7,8,12,13$

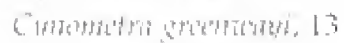

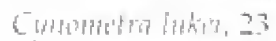

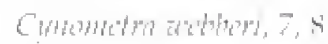

Cupters, 31, 35

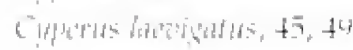

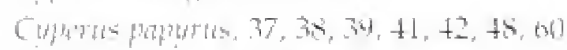

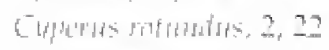

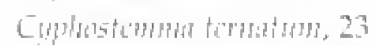

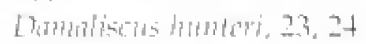

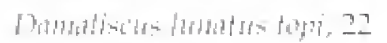

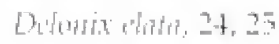

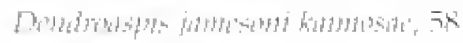

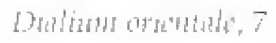

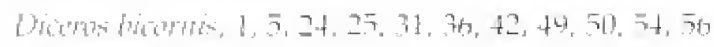

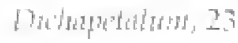

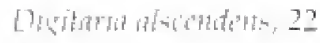

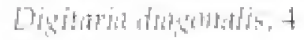

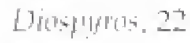

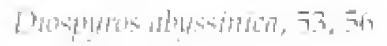

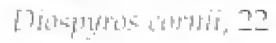

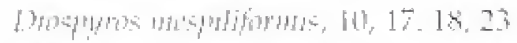

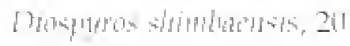

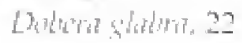

\section{Index 3}




\section{Index of other animals and plants}

Doys. Humking, 34, 30, 54, 50

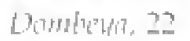

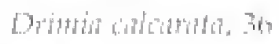

Drufents, 13,43

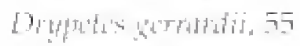

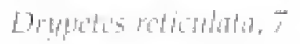

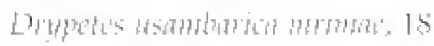

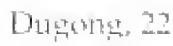

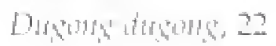

Duiker, ather", 7

Duiker, Blach-1ponfed, 5

Duiker, Yellown-barktat. 31

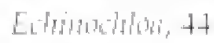

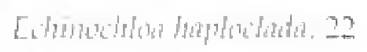

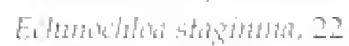

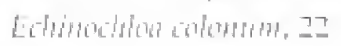

Fet. 23

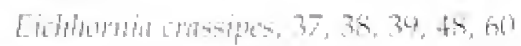

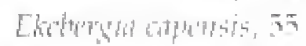

Eland, C. пา1 m)

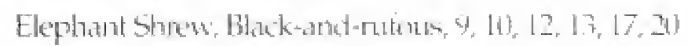

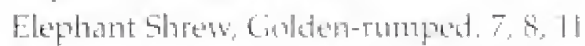

Elephant, Arican, $1,3,5,7,20,24,25,26,31,32,33$, $34,42,50,51, \overline{7}+, 54$

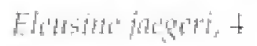

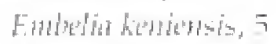

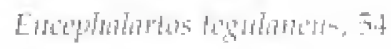

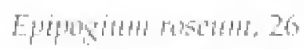

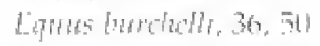

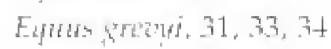

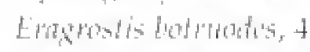

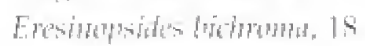

Erotholutus imbricitia, 15,22

Erion, 1, 5

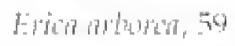

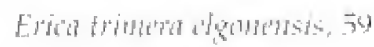

Ertoflerina, 54

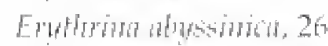

Erytam soldenti 18

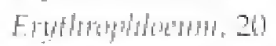

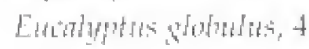

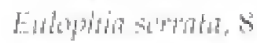

Lupledtat wer, 58

Enplartyat 45, t4

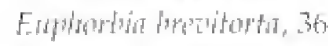

Euphomito catatelalyan, 22

Firtituma, 1,7,51, 5y

Festuth piforiti, 59

Ficus, 23, 26, +4, 56

Fetes watersts, 45

Fous Hatalcusts, 2

Finds sugumorts. 31

Ficus thombirit, a
Gataug, Zanzitant, ls

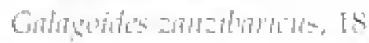

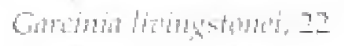

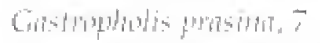

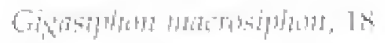

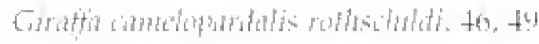

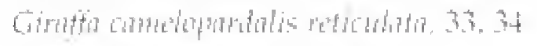

Giratere, Reticulated, 33,34

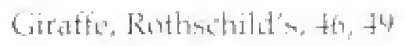

Gilidere, Tatika, 21

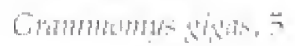

Givion, 22, 45

Cretion simuliz, 22

Giround aul, Giant, 5 it

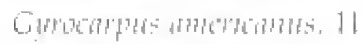

Has remat $1,5,43$

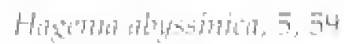

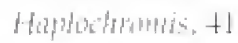

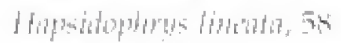

Heath, Giant, 5it

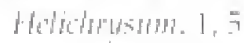

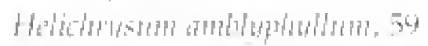

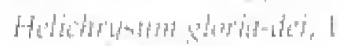

Heriterititititutis, 22

Hirola, 24, 23

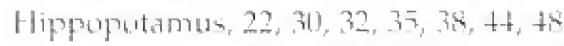

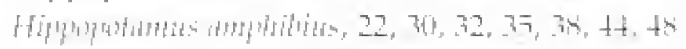

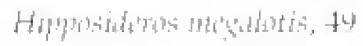

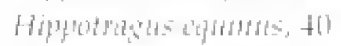

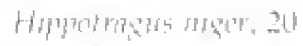

Hoge, Gijunt forrent, 1, $\overline{7}, \overline{7}, \overline{8}$

Hyacinth, Wheter, $37,38,39,48$, , 01

Hyacma, spotted, 40, जो

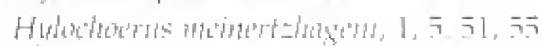

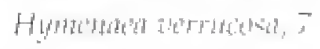

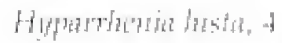

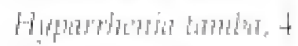

Mupretome 5

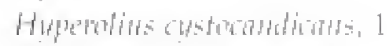

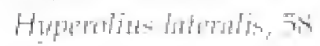

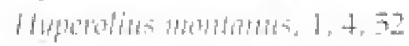

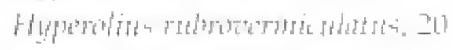

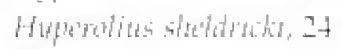

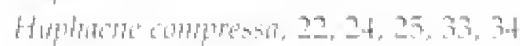

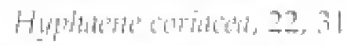

Hyral, Rath, $\bar{y}$

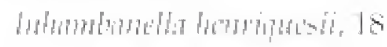

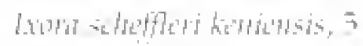

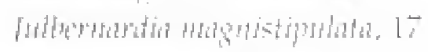

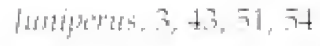

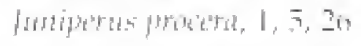

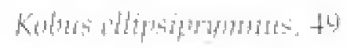

Kuad, Giteder, 45, 54 


\section{Important Bird Areas in Kenya}

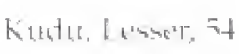

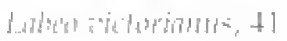

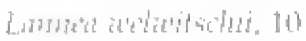

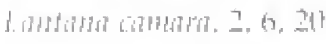

Laters motidus, th

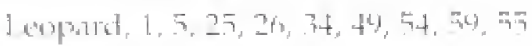

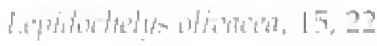

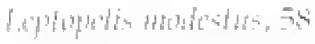

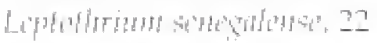

T.inn, 1, 49, 50

1.i/ard, chon koul-bellied, 7

buthes ath

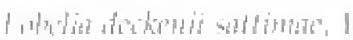

L.

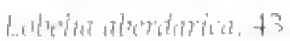

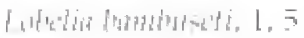

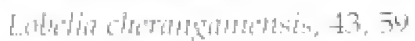

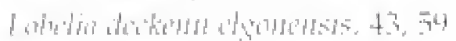

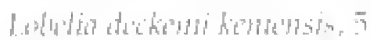

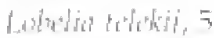

1.thentiat, Giant, I, 43, 54

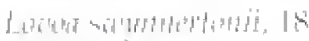

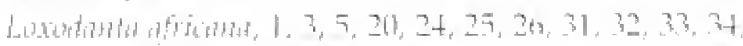
+2., 51, 51, 54, 54

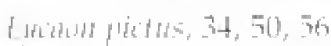

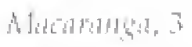

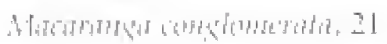

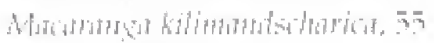

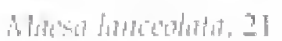

Vaira: 2, 4, 4, 22, 57

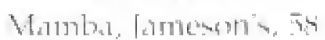

Vangabey, Tana kisuer, 23

Hanterose 22

Aamikira, 20

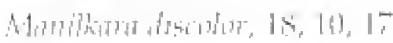

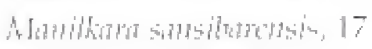

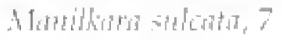

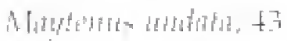

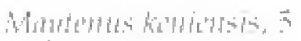

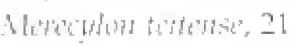

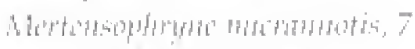

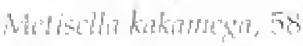

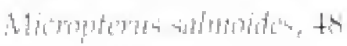

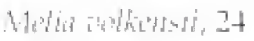

Alikis. 20

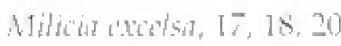

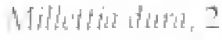

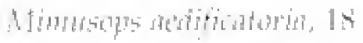

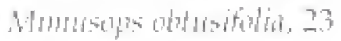

Mliraba, Zab

Solement, At Kencia, 5

Shole-tat, therdare, I

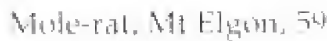

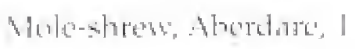

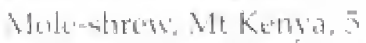

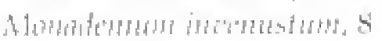

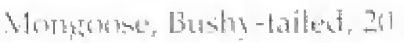

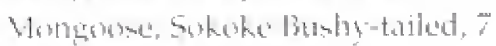

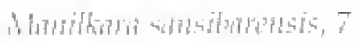

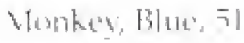

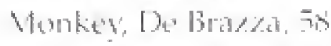

Wonkey, Ret-tailed, 71,58

whentate +1

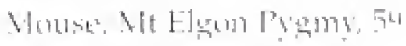

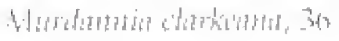

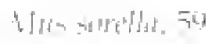

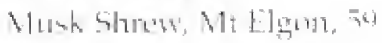

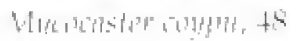

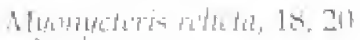

virce pedimathe dis

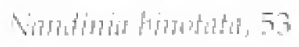

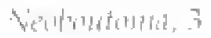

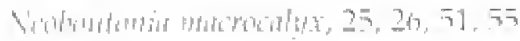

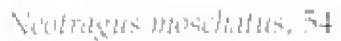

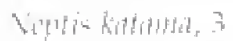

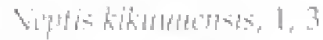

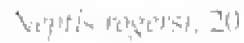

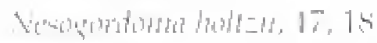

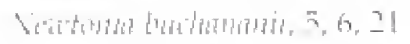

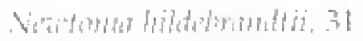

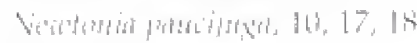

visht 43

Vum

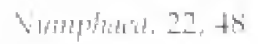

(bak, Vheru, 7

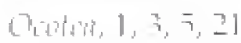

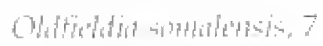

(3) $1,51,50$

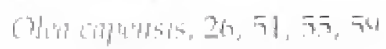

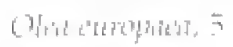

Orhat, $\overline{3}$

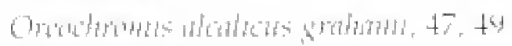

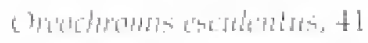

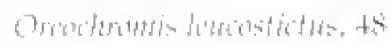

Oribi, 40

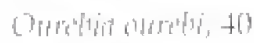

$[4] \mathrm{m}, 10 \mathrm{tum}, 33,34,22,31,24$

['] In, Wild Dole, 22

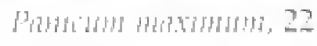

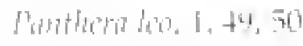

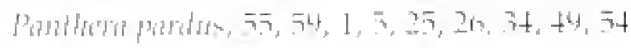

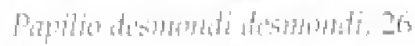

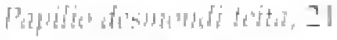

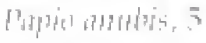

Pap, rus, 37, 38, 34, 41, 42, 48, 611

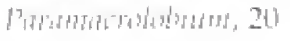

\section{Index 3}




\section{Index of other animals and plants}

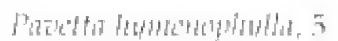

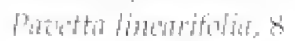

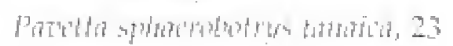

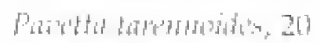

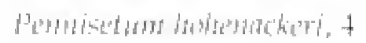

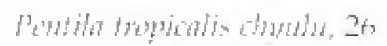

Ierch, Nide, +1

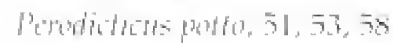

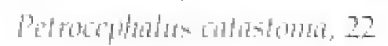

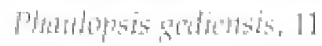

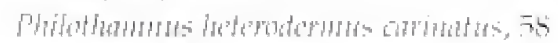

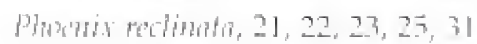

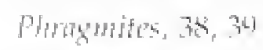

Pomanites matritams, 41

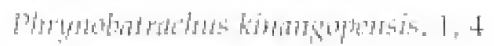

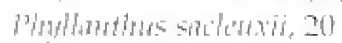

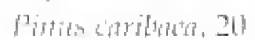

Pards ritiontat

Pistia somotints, 22

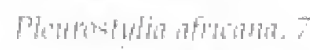

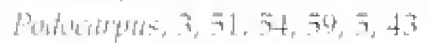

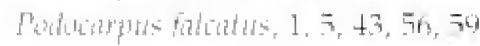

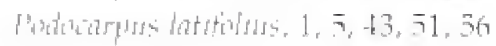

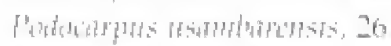

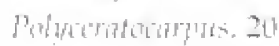

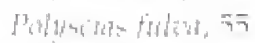

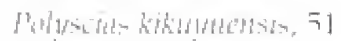

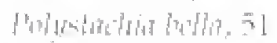

Poptear, Tama River, 31, 33

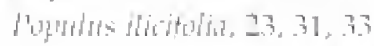

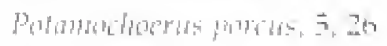

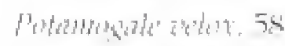

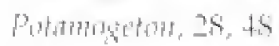

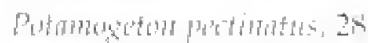

Peotater, +

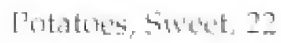

['uttu, $51,53,58$

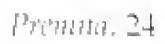

Promatrams chatis, to

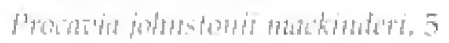

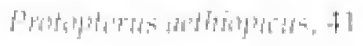

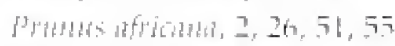

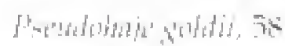

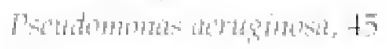

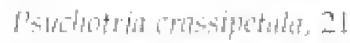

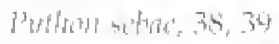

Pythom, Arricist, 34, 34

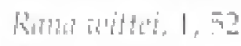

Burpaticat, 4 ?

Buplisa tiridufera, 25, 31

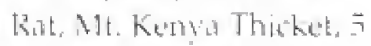

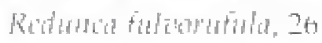

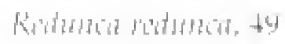

Rectlouk, balur, th

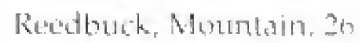

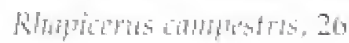

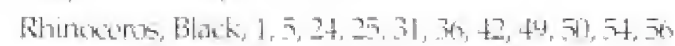

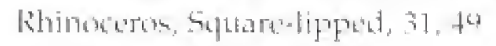

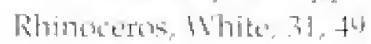

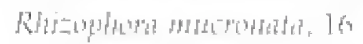

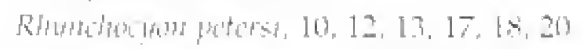

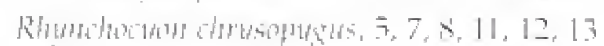

Rite. 2 ?

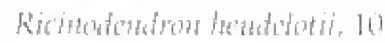

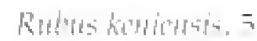

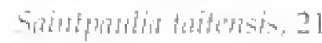

5alietrume. 15

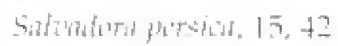

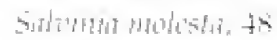

simsereterat, 15

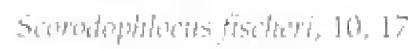

sipherte 1

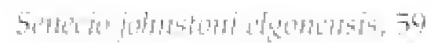

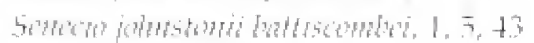

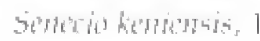

Gerech hemotemdrom, 1, 5

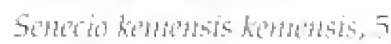

Seringmopsts. 24

Shrew, Giant Otter, 58

Shaw, Highdand Nusk,

Shu"us, Mlole, 5

Sitatumyar, 35,39

Srake, Back-lined Greert, 58

snetke, Lake Baringer th

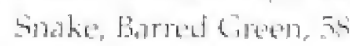

somateration ather 10

Sorghum, कx

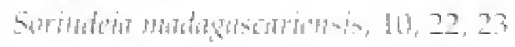

Soy Beand की

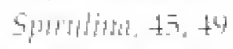

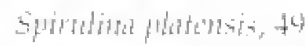

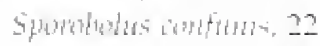

Spomblins haterdis. 27

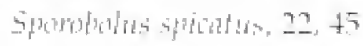

Sterinkink, 26

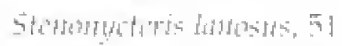

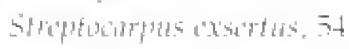

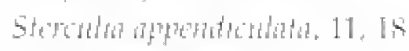

5trumberin, 3, 43 .

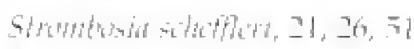

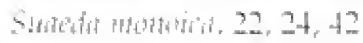

Sugutciande, 57

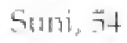

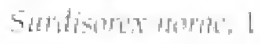

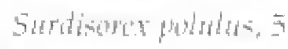

Swallowtail, Tata 21 


\section{Important Bird Areas in Kenya}

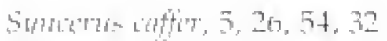

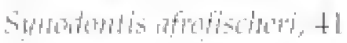

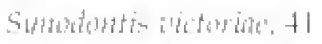

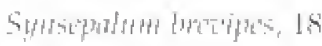

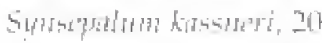

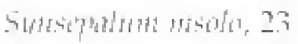

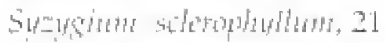

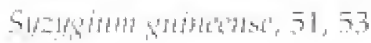

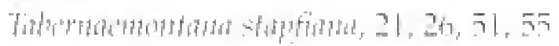

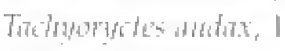

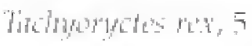

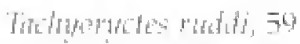

Tirmoninters indicia, 116

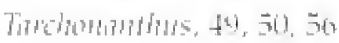

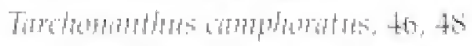

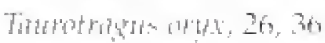

$T_{e, 1}, 58$

Titminalia, 12, 4 t

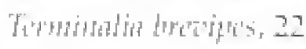

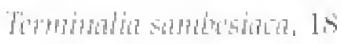

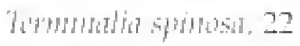

Whateria triandra, th

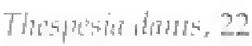

Tilupiat, 4 si

Tiranh =ilh, 48

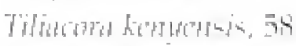

Trand, Bunty s Dusti, 7

Thipi, 2?

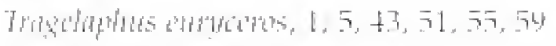

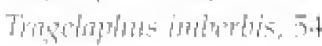

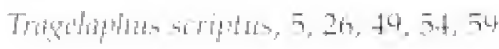

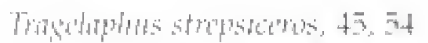

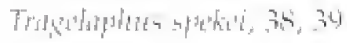

Prom, 78

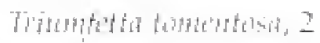

Fretset, th

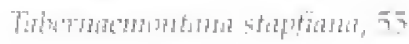

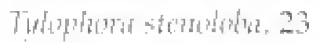

$74+4,25,35,44,45,48,44$

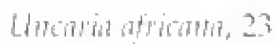

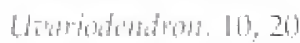

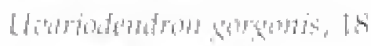

Viogles, atritall, 21

Viper, Greess Butht, 58

Viper, Kenta llormed, $t, 40,40,52$

Viper, Montane, 1, 5

Viner, Mt. Kunga Bush, 5

Viper, Rhitocerss-hormed, 58

Ijophindis, 1, 5

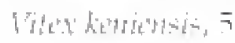

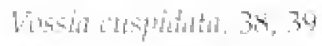

Watertruck. 1)otansed, fy

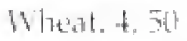

Nildebeest. 30,70

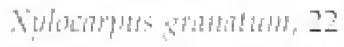

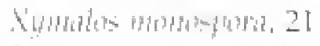

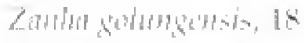

Zathlutumb, 5s

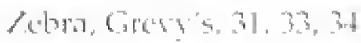

Zabra, Planess, 36, 50

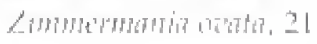

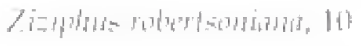

\section{$316 \quad$ Index 3}


Index $4 \mathrm{~A}$

Index $4 \mathrm{~A}$

\section{Index of Site Accounts by site code}

Site"

Conte: Site name

Abrotare Mountains

Kianyaga Valley

Kikuvu Escaproment Foresi

Kinangop Cirastalatats

Nit Kenly

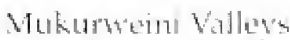

Arabukn-Sokuke For'tint

Dakatelos Worotlatad

Diani Forest

Danndar Hill Forest

Gede Ruins National Homument

Kava Gainditif

Kay has

Kisite Is]and

Kiunga Marione Vational Reserte

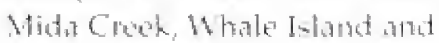

Malindi/Antamb Cons.

Marenji forest

Hrima Hidl Forest

Sabaki Rivict Mouth

Shimba tlills

Taita Hilla Ferests

Tana Biver Delta

Tana River Forresta

Tando East Vational Jayk

Tatyo West National Park

Chyulu Hills Forests

Dida Galgalu Deatert

lakke Tutrketua

Mothous vallexs

Masinga Rescrouit

Neru Natjonal Park

Nwera Sational Reserte

Sambertu and Buffalo Springe

National Rementes

Shaba National Reserver

Dandera Pounds

Naimbi Vational Park

Dutga sisatip

Kogeate Griamp

Kusd Suamp?

Ruma National Park
4 Yala Swamp tse

Page nex.

63

67

69

72

75

80

82

86

34

!)

43

$y=$

97

(4).

102

1045

42

Amboseli National Tark

184

43

Cherangani Hillo

187

Lake bariogne?

19)

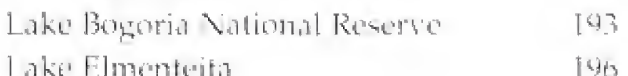

I.ake Magati 200$\}$

l.ahe Naitanta 263

lakt Nakura Natiomal frark zas

Mamai Mąral 212

Mate Forent Complex 217

Matu Varok/MoloGiassands 221

North Nandi torese 224

Ol Donwo Suthethe $\quad 227$

Gouth Nandi Forest 229

South Nytartment $\quad 233$

Busia Grassandats 236

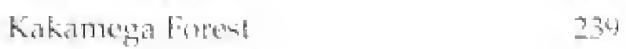

Mt Eligon 243

6i) Sio Port Suanpe $24 \%$
$1(14)$

111

114

116

121

[26

1.33

1.37

$1+0$

1.43

$1+6$

148

151

153

154

157

160

16.3

Info

] $6 x$

173

175

177

174 
Important Bird Areas in Kenya

\section{Index $4 \mathrm{~B}$}

\section{Index of Site Accounts by site name}

\begin{tabular}{|c|c|c|}
\hline Site' navare & $\begin{array}{l}\text { Site } \\
\text { conde }\end{array}$ & $\begin{array}{l}\text { Page } \\
\text { (1) }\end{array}$ \\
\hline Aberdare Motumatins & 1 & 6.3 \\
\hline Amboseli National l'ark & +2 & 184 \\
\hline Arabuko-Soknke Foremit & 7 & $\therefore 2$ \\
\hline Busia Cirasslands & 57 & 236 \\
\hline Cheransatani Hills & 43 & 187 \\
\hline Chyulu Hill: Foresits & 26 & 143 \\
\hline Dakaticha Whatland & 8 & 86 \\
\hline Dandora Ponds & $3 \bar{x}$ & 166 \\
\hline Diani Forest & 난 & si) \\
\hline Dida Gatgealu Desert & 27 & $1+4$ \\
\hline Dutnga Sntomp & 37 & 173 \\
\hline Demble Hill Farest & 111 & 41 \\
\hline Gede Ruins Notional Nombment & $1 t$ & 43 \\
\hline Kakimega Forest & 78 & 239 \\
\hline Kaval Gandini & 12 & $4 \pi$ \\
\hline Kaya Wiat & 13 & 97 \\
\hline Kianyaga Valleg's & 2 & 67 \\
\hline Kikuy Esonpment Fores & 3 & $0^{4}$ \\
\hline Kinangop Getasolands & + & 72 \\
\hline Kisite Jsland & $1+$ & 94 \\
\hline Kianga Narine National Rebersé & 15 & 102 \\
\hline Kogula Swamp & 30 & $17 \overline{7}$ \\
\hline Kusa Swamp & 39 & $17 \overline{7}$ \\
\hline Lake Barnago & 44 & 190 \\
\hline I.akk bogntio Nationtal Reserve & 45 & 193 \\
\hline Lakterlmenteital & 40 & 140 \\
\hline Laket Magati & 47 & 200 \\
\hline Iaki Naisasha & 48 & 203 \\
\hline Lakt Nakuru Natismal Park & 44 & 2178 \\
\hline Lake Turhama & $2 \mathrm{~A}$ & 148 \\
\hline Nachasurs Vallerys & 20 & 151 \\
\hline Maremil Forent & 17 & 109 \\
\hline Masai Mava & if & 212 \\
\hline Masinga Reserwor & 30 & $1,5,3$ \\
\hline Nau Forent Complex & 71 & 217 \\
\hline Mau Narok/Molo Grasslando & 52 & 221 \\
\hline Merta National Park & 31 & 1.54 \\
\hline 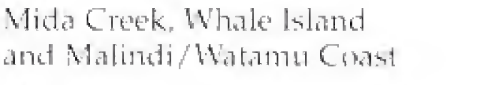 & 16 & $10 \overline{9}$ \\
\hline Mrima Hill Forest & is & 111 \\
\hline Mt Elgon & 54 & 243 \\
\hline Mt Kenya & 5 & 75 \\
\hline Mukurwerai valleys & $h_{1}$ & 80 \\
\hline
\end{tabular}

\begin{tabular}{|c|c|}
\hline Mwea Nationt Reserte" & 32 \\
\hline Nainotri Vationsl Iark & 314 \\
\hline Vurtla Ninndi Forremet & 53 \\
\hline [1] Dompos Salbache & 3 \\
\hline Ruma Sational l'ark & 40 \\
\hline Salataki liviner Mouth & 16 \\
\hline 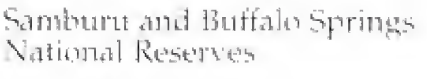 & 33 \\
\hline 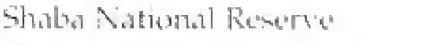 & 34 \\
\hline Shimba 1Hills & 20 \\
\hline Sio l'out Swatrey & act \\
\hline Soutba Vandi Forest & $5 \overline{3}$ \\
\hline South Vyouruman & 70 \\
\hline Thita Hills Forests & 21 \\
\hline Tathat kiver Detal & 22 \\
\hline 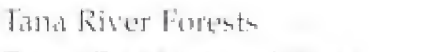 & 23 \\
\hline Tharo East National Path & 24 \\
\hline Tando Mist Natmonal l'ark & 2ラ \\
\hline hadas swants & +1 \\
\hline
\end{tabular}

\section{Index $4 \mathrm{~B}$}




\section{What is BirdLife International?}

Over 1,200 bird species actoss the world ate currently under threat of extinction in some way - over 12 of the world's birds. BirdLife International is a worldwide Partnership of conservation organisations that seeks to conserve all wild bird species and their habitats. Through this work, Bindlife strives to protect the world's biological diversity and supports the sustainable use by humans of the world's natural resources. BindLife is a national force in 64 countries around the workd and is represented in a further 27 nations.

\section{BirdLife aims to:}

- Prevent the extinction of any bird species

- Reduce the number of species that are globally threatened

- Enhance the comservation status of all birt species

- Conserve crucial sites and habitats for birds

\section{The unique Partnership of bird-oriented non-governmental organisations works towards these aims by:}

- Identifying prorities for bird and biodiversity conservation through scientific rescarch and data collection

- Promoting conservation action for birds, using the Partnership to create a strong voice for birds to govermments and other decision makers. The BirdLife Partnership can save many more birds that any one national organdation can in isolation

- Carrying out national programmes of actions for birds, including managing species. sites and habitats, education and enlisting public support.

Birdlife divides the overall global task into progranmes with a regiomal focus. Programmes agreed by the regional partners are now up and running in Europe, Africa (including associated islands), Asia, the Americas and the Middle East. The programme's aims and objectives are always achieved by working through local communities.

BirdLife is now the world's leading authority an the status of the world's birds, theit habitats and the urgent problems that face them.

The Partnership is coordinated by staff in Cambridge (UK), Wageningen (The Netherlands), Quito (Eeuador), Bogor (Indonesia), Brussels (Belgium) and Amman (Jordan).

Bird Life International is a UK registered charity no. 1042125

Birdlife International

Wellbrook Courk

Girton Road

Cambridge

CB3 ONA, UK

Tel. $+44(0) 1223277318$

$\mathrm{Fax}+4+(0) 1223277200$

E-mail: birdlifeobirdlifeorg.uk

http://wwwbirdlifenet 
This book is a contribution to identifying Kenya's biodiversity conservation priorities. It describes in detail 60 sites in Kenya that meet the criteria for Important Bird Areas (IBAs) - places of global significance for bird and biodiversity conservation.

Iniportant Bird Areas in Kenya will interest environmental planners, policy makers, wildlife managers, students and researchers. It will also be an essential source of information for birdwatchers wanting to locate Kenya's special birds.

This Kenya IB A directory is a joint project of

Nature Kenya

(the East Africa Natural History Society),

the Kenyan Partner of BirdLife International
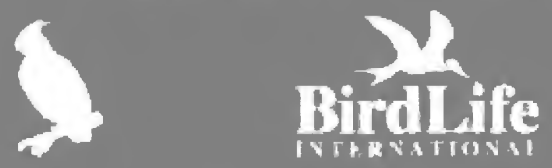

and the Ornithology Department, National Museums of Kenya
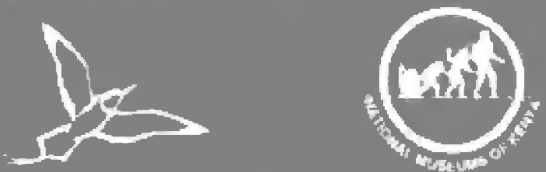

sponsored by the Royal Society for the Protection

of Birds, the BirdLife Partner in the United Kingdom

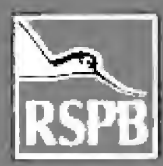

with additional support from the Glubal Environment Facility through the United Nations Development Programme project,

'African NGO-Government Partnerships for Sustainable Biodiversity Conservation'
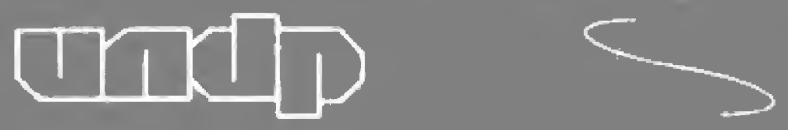

NatureKenya

Museum Hill, P O Box 44486, Nairobi, Kenya

Telephone +254 (0) $2749957 \bullet$ Fax +254 (0) $2741049 \bullet$ E-mail eanhs@africannline.co.ke 